\title{
FLORA OF PANAMA CHECKLIST AND INDEX
}




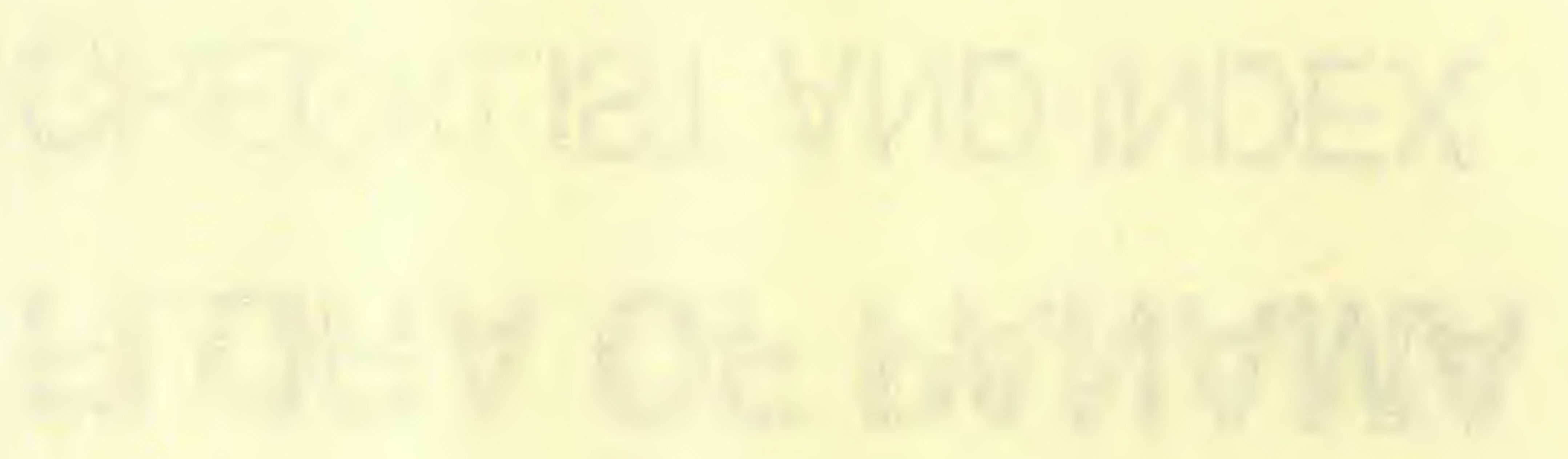




\section{FLORA OF PANAMA CHECKLIST AND INDEX}

W. G. D'ARCY

PART I: THE INTRODUCTION AND CHECKLIST

Missouri Botanical Garden

Saint Louis, Missouri

1987 
MONOGRAPHS IN SYSTEMATIC BOTANY

from the Missouri Botanical Garden

Vol. 17. 1987

\section{Flora of Panama: Checklist and Index}

William G. D'Arcy, Missouri Botanical Garden, P.O. Box 299. St. Louis, MO 63166. U.S.A.

Copyright (C) 1987 by the Missouri Botanical Garden

All rights reserved

Printed in the United States of America by Braun-Brumfield, Inc., Ann Arbor, Michigan.

\section{Library of Congress Cataloging in Publication Data}

D'Arcy, William G., 1931-

Flora of Panama

(Monographs in systematic botany from the Missouri Botanical Garden, ISSN 8661874; vol. 17-18, 1987)

Flora of Panama appeared in the Annals of the Missouri Botanical Garden from 1943-1981.

Bibliography: $p$.

Includes index.

1. Botany-Panama. 2. Phytogeography-Panama. 3. Annals of the Missouri Botanical Garden-Indexes.

4. Botany-Periodicals-Indexes. 5. Botany-Classification-Periodicals-Indexes.

I. Missouri Botanical Garden. II. Title. III. Series: Monographs in systematic botany from the Missouri Botanical Garden; v. 17, etc. QK221.D37 $1987 \quad 581.97287 \quad 87-14210$

ISBN 0-915279-08-8 (Paperback:set)

This publication constitutes Part I of the Flora of Panama, containing general information regarding the project.

Copies of the Flora of Panama: Checklist and Index can be ordered from:

Department 11, Missouri Botanical Garden, Box 299, St. Louis, MO 63166. U.S.A. (314) 577-5170.

ISBN: 0-915279-08-8 (Paperback:set) 


\section{CONTENTS}

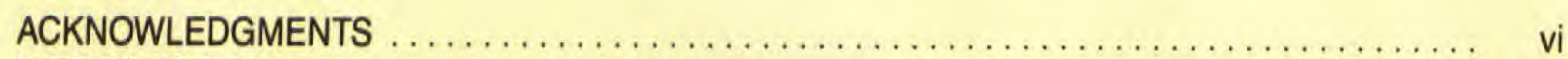

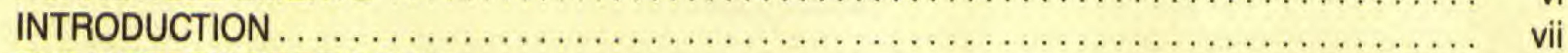

THE HISTORY OF PROJECT $\ldots \ldots \ldots \ldots \ldots \ldots \ldots \ldots \ldots \ldots \ldots \ldots \ldots \ldots \ldots \ldots \ldots \ldots \ldots \ldots, \ldots \ldots \ldots$

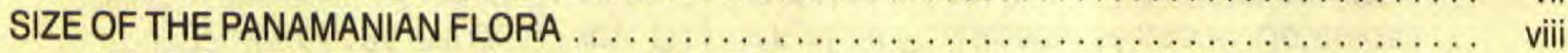

Table 1. Panama Plant Families-Numbers of Species $\ldots \ldots \ldots \ldots \ldots \ldots \ldots \ldots \ldots \ldots \ldots$ ix

Table 2. Flora Size of Selected Areas . . . . . . . . . . . . . . . . . . . . . . . .

Table 3. Numbers of New Taxa since Flora of Panama $\ldots \ldots \ldots \ldots \ldots \ldots \ldots \ldots \ldots \ldots \ldots \ldots$ xii

Table 4. Increase in Flora since Panama Treatments $\ldots \ldots \ldots \ldots \ldots \ldots \ldots \ldots \ldots \ldots \ldots \ldots$ xii

Table 5. Increase in Species for Parts of Panama . . . . . . . . . . . . . . . . . . . . xiii

SOME CHARACTERISTICS OF THE PANAMANIAN FLORA $\ldots \ldots \ldots \ldots \ldots \ldots \ldots \ldots \ldots \ldots$ xiii

Table 6. Major Life Forms $\ldots \ldots \ldots \ldots \ldots \ldots \ldots \ldots \ldots \ldots \ldots \ldots \ldots \ldots \ldots \ldots \ldots \ldots \ldots \ldots \ldots \ldots$

Table 7. Species Occurring at Different Elevations $\ldots \ldots \ldots \ldots \ldots \ldots \ldots \ldots \ldots \ldots \ldots \ldots$ xiv

Table 8. Species Occurring in Different Geographic Regions of Panama $\ldots \ldots \ldots \ldots \ldots \ldots$ xiv

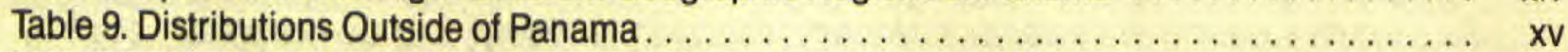

NAMING OF THE FLORA $\ldots \ldots \ldots \ldots \ldots \ldots \ldots \ldots \ldots \ldots \ldots \ldots \ldots \ldots \ldots \ldots \ldots \ldots \ldots \ldots \ldots, \ldots \ldots$

Panamanian Plant Names $\ldots \ldots \ldots \ldots \ldots \ldots \ldots \ldots \ldots \ldots \ldots \ldots \ldots \ldots \ldots \ldots \ldots \ldots$

Table 10. Most Used Names for Panamanian Species $\ldots \ldots \ldots \ldots \ldots \ldots \ldots \ldots \ldots \ldots \ldots \ldots$ xv

Authors of Panamanian Plant Names $\ldots \ldots \ldots \ldots \ldots \ldots \ldots \ldots \ldots \ldots \ldots \ldots \ldots \ldots \ldots \ldots \ldots$

Table 11. Botanists who have Named the Most Panamanian Species $\ldots \ldots \ldots \ldots \ldots \ldots \ldots$. xvi

Table 12. Botanists who have Named the Most Panamanian Genera . . . . . . . . . . . xvii

Synonymy $\ldots \ldots \ldots \ldots \ldots \ldots \ldots \ldots \ldots \ldots \ldots \ldots \ldots \ldots \ldots \ldots \ldots \ldots \ldots \ldots \ldots \ldots \ldots \ldots$

CONTRIBUTORS TO DATABASE REVISION $\ldots \ldots \ldots \ldots \ldots \ldots \ldots \ldots \ldots \ldots \ldots \ldots \ldots \ldots \ldots \ldots$

Table 13. Major Contributions by Specialists $\ldots \ldots \ldots \ldots \ldots \ldots \ldots \ldots \ldots \ldots \ldots \ldots \ldots \ldots \ldots \ldots$

Table 14. Specialists Making Contributions to the Database $\ldots \ldots \ldots \ldots \ldots \ldots \ldots \ldots \ldots$ xviii

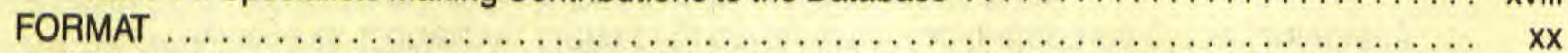

The Checklist Part I . . . . . . . . . . . . . . . . . .

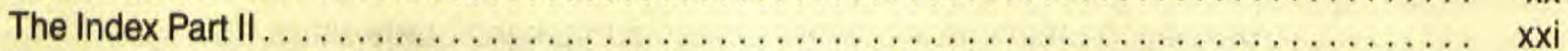

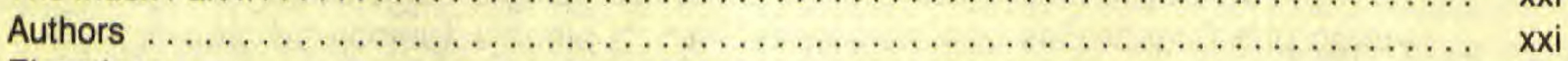

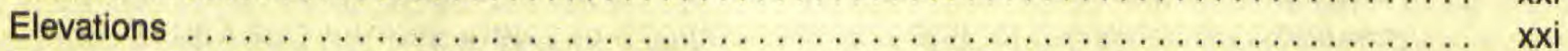

Geographical Ranges Outside of Panama $\ldots \ldots \ldots \ldots \ldots \ldots \ldots \ldots \ldots \ldots \ldots \ldots \ldots \ldots \ldots \ldots \ldots$

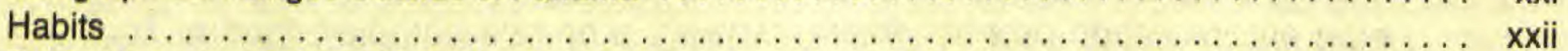

Terms Used for Habit and Life Form $\ldots \ldots \ldots \ldots \ldots \ldots \ldots \ldots \ldots \ldots \ldots \ldots \ldots \ldots \ldots \ldots \ldots \ldots \ldots$

Table 15. Major Life Forms (Habits) $\ldots \ldots \ldots \ldots \ldots \ldots \ldots \ldots \ldots \ldots \ldots \ldots \ldots \ldots \ldots \ldots \ldots \ldots$

Table 16. Modifiers of Habit Type $\ldots \ldots \ldots \ldots \ldots \ldots \ldots \ldots \ldots \ldots \ldots \ldots \ldots \ldots \ldots \ldots \ldots \ldots \ldots \ldots \ldots$

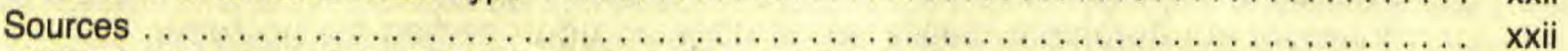

BIBLIOGRAPHY RELATING TO FLORA OF PANAMA DATABASE $\ldots \ldots \ldots \ldots \ldots \ldots \ldots \ldots \ldots$ xiii

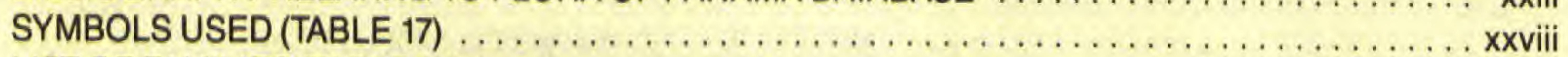

LIST OF FAMILIES $($ TABLE 18$) \ldots \ldots \ldots \ldots \ldots \ldots \ldots \ldots \ldots \ldots \ldots \ldots \ldots \ldots \ldots \ldots \ldots \ldots$

CHECKLIST $\ldots \ldots \ldots \ldots \ldots \ldots \ldots \ldots \ldots \ldots \ldots \ldots \ldots \ldots \ldots \ldots \ldots \ldots \ldots \ldots \ldots \ldots \ldots$

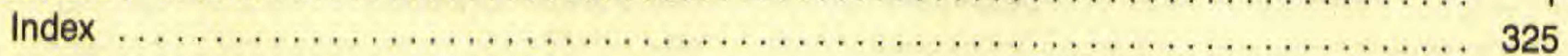




\section{ACKNOWLEDGMENTS}

Preparation of this index and checklist reflects the efforts of many people. Those who contributed to the analyses of various plant groups and made changes and additions to the taxonomy are noted in the section below: CONTRIBUTORS TO DATABASE REVISION.

Many others helped in preparation of the listing, including the following Missouri Botanical Garden staft. Sheri Murphy (Davis) prepared an initial card file of the Flora, and Ball Phillips made an initial search of the Missouri Botanical Garden herbarium. The Flora of Panama was entered into the computer by Andrea Solomon and Julie Gentry. Correction and update of the flora. computer entry, and herbarium and library verification was done by Julie Gentry and Virginia Kefec in addition, support staff at the Missouri Botanical Garden, particularty Ronald L. Liesner, helped with segregating and determining Panamanian collections in the Gardens main plant collection.

Mireya D. Correa A., University of Panama, facilitated use of the University of Panama herbarium and was supportive in other ways.

The computerization of the Checklist and index made use of the Olfice of Computing and Talecommunications at the University of Missouri-Si. Louis. which provided tochnical support and oquipment for the project. The project made unexpectedily larpe calls on stafl time and patience. which were always pleasantly met, and the size of the project at times taxed the physical resources of the uni. Arrangements and programing were begun through Harold L. Mack and Wuilam Woung. and the lask was later continued and brought to completion by Larry Piciett and Pam Hagec Expertise for instaling the Database on the Mistoui Botanical Gardents recently acoulired Mentor 5000 for final edisorial verifleation was provided by Robert E. Magll, who also assiated in many cher ways.

The proied was Aunded by National Science Foundation grants to sugpert work on the Fora of Panama Project. the most recent of which bore Crast Number BSA 6908425 (WQ. DAvey. Principal Imvestigaton). Other National Science Foundseion Grants that haiped supooet the project include G-774

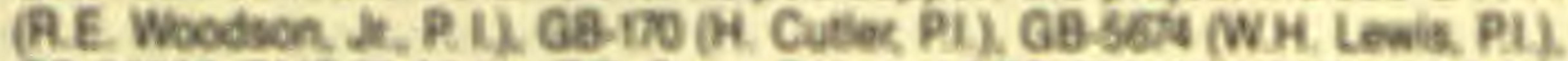
CB36200. Bus 7202441 (TR Croat, PL) and DEB 704300 DEB 7222192 BSA-ajost2s (WO. DArex. PI). Marshall A. Crosby, Missouri Betanical Garden, helped greatly in arranging supplementay financing for tho project. 


\title{
FLORA OF PANAMA CHECKLIST AND INDEX
}

\author{
W.G. D'Arcy
}

\section{INTRODUCTION}

The Flora of Panama (Woodson et al. 1943-81) is a multivolume publication that aimed to describe all the higher plants occurring in Panama. Almost as soon as various parts of the Flora were issued, new plants were found in Panama and critical studies resulted in name changes, making the publication increasingly less comprehensive as time continued. Access to the new information is difficult, for publication of new finds and changes has taken place in a wide variety of scientific books and journals, and many of the new items have not yet reached the literature but are still documented only by herbarium specimens. The Flora of Panama has hitherto lacked an index, although most families were accompanied by individual indices when they were published.

As a step toward making information about the Panamanian flora more accessible to the public, the contents of the Flora of Panama were entered into a computer-based Flora of Panama Database. Library searches for articles affecting the Panamanian flora and herbarium searches for new items and newly annotated collections resulted in many increases and changes to this Database. A large group of collaborators revised and reviewed individual plant families. The present reports are the first to be published from the Database.

This publication consists of two reports: the Checklist (Part I), and the index (Part II). The Index lists all names, accepted or synonymous, used in the Flora of Panama and elsewhere for Panamanian plants. If the name is a synonym, its accepted name is given. Where appropriate, the volume and page where the name appeared in the Flora of Panama is given. In other cases reference is given to another publication where the name has been treated or to a herbarium voucher, thus indicating a basis for acceptance or synonymy of a name. The Checklist lists only accepted names, and intormation is given about range and habit. Thus to find information for a given name, it may be necessary to consult both parts, the Index to ascertain under which name it appears in the Checklist or to find the source of the information, and the Checklist to find distributional or other information about the species concerned. Issued simultaneously in a microfiche format is a Taxonomic Listing which provides the information from both Part I and Part II in a taxonomic arrangement.

\section{HISTORY OF THE PROSECT}

Plant collecting has taken place in Panama since the arrival of the Europeans. Dwyer (1981), in his history of plant collecting in Panama, notes that the earliest herbarium specimens are from the first part of the 18 th century. By the time Carl Linnaeus was giving plant description its modern form with his Species Plantarum of 1753, he had material in hand to allow him to describe many plants occurring in Panama. Since then, description of Panamanian plants has taken place in a wide array of publications from many countries. But Panamanian botany has benefited by relatively few floras or florulas that compile the many descriptions and revisions of individual plant species and groups into a form of general utility. The first published flora that included Panama was the Flora of the Isthmus of Panama by Seemann in 1854. In the present century, Paul C. Standley published florulas covering Barro Colorado Island (1927) and the Panama Canal Zone (1928). The Flora of Panama (Woodson et al. 1943-1981) covered the entire country and is the subject of the present publication. Although published after the Flora of Panama was begun, the Floras of San José Island by Johnston (1949) and of Barro Colorado Island by Croat (1978) are important scientific statements about the plants of parts of Panama.

The Flora of Panama (Woodson et al. 1943-81) was begun in 1943 by Robert E. Woodson, Jr. and Robert W. Schery after collecting in Panama had resulted in an accumulation of significant specimens and the description of new species in the 1920's and 1930's. A short history of the project is given by D'Arcy (1981a). Schery left the project in 1952, but Woodson continued it until his death in 1963. The Flora was then carried on by Hugh C. Cutler, Walter H. Lewis, D. M. Porter, and Thomas B. Croat, with W.G. D'Arcy bringing it to completion in 1981. Beginning about 1965, work on the Flora was accelerated with initiation of a strong collecting program and expansion of the group of contributors to the Flora. The Flora of Panama, when completed in 1981 , comprised 6818 printed pages and included 5314 species. By then many of the earlier parts were out-of- print, although some parts had been reprinted. The accelerated collecting of the 1970's and 80's made the earlier publication increasingly less comprehensive as new species were described and names were changed following more critical studies. Parts of the Flora of Panama were generally out-of-date as 
soon as they were issued, and of course, the whole work seriously understated the flora at the time it was completed in 1981. In spite of this, the prospect of issuing a complete new and revised Flora was strongly counter-indicated at that time. The large amount of recently collected material was yielding numerous changes to the state of floristic knowledge about Panama, and any Flora that might be issued would suffer the same fate as the first one in being seriously out-ofdate as soon as it appeared. The magnitude of this continuing alteration and addition is large, amounting to hundreds of changes each year. To date there has been no abatement in the rate of change, testimony to the richness of the Panamanian flora and the diligence of specialists making new assessments of already recorded Panamanian taxa. The time has still not come for the preparation of a new edition of the Flora of Panama.

The present publication is a compromise intended to permit access to new taxonomic information about Panamanian plants and to ease the task of those wishing to revise different plant groups. It provides references to descriptions of the plants but does not present new descriptions or keys. Most plant groups have been reviewed or revised by specialists or by the author, and to this extent the listing is a reassessment of the taxonomy of the flora. In a series of tables, a concise statement is presented about the size of the flora and its distribution in other regions. Analyses of this kind of new data that are available from the Database is to be provided later.

The computerized Database continues to be maintained and revised, and it is anticipated that these reports will be reissued in three to five years. This report is provisional both in the sense that there is much more information still to come and in the sense that errors remain to be corrected in future editions. The author welcomes corrections or advice that will strengthen future editions.

\section{SIZE OF THE PANAMANIAN FLORA}

The Panamanian flora now includes 7345 species in 195 families. Table 1 shows the numbers of species now recorded from Panama by family.

The Panamanian flora is one of the most diverse in the world. With
7345 species of flowering plants now known in a land area of 75,902 $\mathrm{km}^{2}\left(29,306 \mathrm{mi}^{2}\right)$, the floristic average is 0.0953 species per $\mathrm{km}^{2}$ or 0.252 per $\mathrm{mi}^{2}$. Relating this to the average for other regions presents the problem that as land areas diminish, the floristic average tends to increase, so that continental size areas, even rich ones, have a low average, while quite small areas, for example, a one hectare disturbed hillside, may have an extremely large value. Nevertheless, some such comparisons of species richness and area may be useful if one keeps in mind this statistical bias and other factors that may influence the average and merely looks at the richness in species. Table 2 shows the species richness of several regions.

In addition to the 7345 species of flowering plants now known from Panama there are some 800 pteridophyte species (Lellinger 1986, pers. comm.) making a total of over 8100 species of vascular plants. Since 1981 over 330 species of flowering plants have been newly described from material collected in Panama, and no diminution of this rate of discovery has been seen to date. Thus the total flora of Panama may include $8500-9000$ flowering plants and 900 pteridophytes. These numbers will decrease as habitats are eliminated from Panama and species are extirpated or extinguished. The bryophytes include some 350 species of mosses (Allen \& Crosby, pers. comm.) plus an unknown number of hepatic and hornwort species. Hepatics are abundant, especially in cooler, moist parts of the country.

\section{RECENT INCREASE IN SIZE OF THE KNOWN PANAMANIAN FLORA}

Since completion of the Flora of Panama, there has been a substantial increase in the number of taxa known from the country. Because the parts of the Flora were issued over a four decade period, changes in the numbers of recorded species conceal a varied rate of increase. Nevertheless, a few comparisions (Tables 3-5) indicate the great improvement of knowledge since the Flora was prepared. The Flora of Panama recognized 5314 species and a total of 5597 taxa of species rank or below, compared with the 7345 species recognized now. This increase of 2009 species does not include the 776 species recognized in the Flora of Panama that have been reduced to synonymy or excluded from the flora. 
TABLE 1

PANAMA PLANT FAMILIES - NUMBERS OF SPECIES

\section{FAMILY IDENTIFICATION}

\begin{tabular}{|c|c|c|c|c|c|c|}
\hline $\begin{array}{c}\text { Dalla } \\
\text { Torre \& } \\
\text { Harms } \\
\end{array}$ & Family Name & $\begin{array}{c}\text { Acronym } \\
\text { (Weber } \\
1982 \text { ) }\end{array}$ & $\begin{array}{c}\text { (Flora } \\
\text { of } \\
\text { Panama) } \\
\end{array}$ & $\begin{array}{l}\text { Native } \\
\text { Species }\end{array}$ & $\begin{array}{l}\text { Introduced } \\
\text { Species }\end{array}$ & $\begin{array}{c}\text { Total } \\
\text { Species }\end{array}$ \\
\hline 1 & CYCADACEAE & CCD & (1) & 8 & 2 & 10 \\
\hline 5 & PODOCARPACEAE & PDC & (2) & 3 & & 3 \\
\hline 6 & ARAUCARIACEAE & ARU & $(-)$ & & 1 & 1 \\
\hline 7 & GNETACEAE & GNE & (2a) & 1 & & 1 \\
\hline 8 & TYPHACEAE & TYP & (3) & 1 & & 1 \\
\hline 11 & POTAMOGETONACEAE & РОT & (3a) & 4 & & 4 \\
\hline 12 & NAJADACEAE & NAJ & (3B) & 2 & & 2 \\
\hline 15 & ALISMATACEAE & ALI & (4) & 9 & & 9 \\
\hline 16 & BUTOMACEAE & BUT & (5) & 2 & & 2 \\
\hline 17 & HYDROCHARITACEAE & HDC & (5a) & 4 & 1 & 5 \\
\hline 18 & TRIURIDACEAE & TRI & (6) & 2 & & 2 \\
\hline 19 & GRAMINEAE & GRM & (7) & 344 & 15 & 359 \\
\hline 20 & CYPERACEAE & CYP & (8) & 164 & 1 & 165 \\
\hline 21 & PALMAE & PAL & (9) & 95 & 8 & 103 \\
\hline 22 & CYCLANTHACEAE & CYC & (10) & 33 & & 33 \\
\hline 23 & ARACEAE & ARA & (11) & 234 & & 234 \\
\hline 24 & LEMNACEAE & LMN & (12) & 7 & & 7 \\
\hline 28 & MAYACACEAE & MAY & (13) & 2 & & 2 \\
\hline 29 & XYRIDACEAE & XYR & (14) & 3 & & 3 \\
\hline 30 & ERIOCAULACEAE & ERO & (15) & 9 & & 9 \\
\hline 31 & RAPATEACEAE & RPT & (16) & 1 & & 1 \\
\hline 32 & BROMELIACEAE & BML & (17) & 136 & & 136 \\
\hline 33 & COMMELINACEAE & CMM & (18) & 31 & & 31 \\
\hline 34 & PONTEDERIACEAE & PON & (19) & 8 & & 8 \\
\hline 36 & JUNCACEAE & JUN & (20) & 3 & & 3 \\
\hline 38 & LILIACEAE & LIL & (21) & 15 & 1 & 16 \\
\hline 39 & HAEMODORACEAE & HAE & (23) & 1 & & 1 \\
\hline 40 & AMARYLLIDACEAE & AML & (24) & 26 & 1 & 27 \\
\hline 41 & VELLOZIACEAE & VLL & (25) & 1 & & 1 \\
\hline 43 & DIOSCOREACEAE & DSC & (26) & 14 & 2 & 16 \\
\hline 44 & IRIDACEAE & IRI & (27) & 10 & & 10 \\
\hline 45 & MUSACEAE & MUS & (29) & 37 & 1 & 38 \\
\hline 46 & ZINGIBERACEAE & ZIN & (30) & 36 & 6 & 42 \\
\hline 47 & CANNACEAE & CNA & (31) & 3 & 1 & 4 \\
\hline 48 & MARANTACEAE & MRN & (32) & 49 & & 49 \\
\hline 49 & BURMANNIACEAE & BMN & (28) & 15 & & 15 \\
\hline 50 & ORCHIDACEAE & ORC & (33) & 891 & 2 & 893 \\
\hline 51 & CASUARINACEAE & CAS & $(-)$ & 1 & & 1 \\
\hline 53 & PIPERACEAE & PIP & (34) & 213 & & 213 \\
\hline 54 & CHLORANTHACEAE & CLR & (35) & 9 & & 9 \\
\hline 55 & LACISTEMATACEAE & LCS & (36) & 3 & & 3 \\
\hline 56 & SALICACEAE & SAL & (37) & 1 & & 1 \\
\hline 57 & MYRICACEAE & MYR & (38) & 2 & & 2 \\
\hline 60 & JUGLANDACEAE & JUG & (39) & 3 & & 3 \\
\hline
\end{tabular}

FAMILY IDENTIFICATION

\begin{tabular}{|c|c|}
\hline $\begin{array}{l}\text { Dalla Family Name } \\
\text { Torre \& } \\
\text { Harms }\end{array}$ & $\begin{array}{l}\text { Acronym (Flora } \\
\text { (Weber of } \\
\text { 1982) Panama) }\end{array}$ \\
\hline
\end{tabular}

61 BETULACEAE $\quad$ BET $(40)$

62 FAGACEAE

63 ULMACEAE

64 MORACEAE

65 URTICACEAE

66 PROTEACEAE

67 LORANTHACEAE

71 OPILIACEAE

72 OLACACEAE

73 BALANOPHORACEAE

74 ARISTOLOCHIACEAE

75 RAFFLESIACEAE

77 POLYGONACEAE

78 CHENOPODIACEAE

79 AMARANTHACEAE

80 NYCTAGINACEAE

81 BATACEAE

83 PHYTOLACCACEAE

84 AIZOACEAE

85 PORTULACACEAE

86 BASELLACEAE

87 CARYOPHYLLACEAE

88 NYMPHAEACEAE

89 CERATOPHYLLACEAE

91 RANUNCULACEAE

93 BERBERIDACEAE

94 MENISPERMACEAE

95 MAGNOLIACEAE

95a WINTERACEAE

98 ANNONACEAE

99 MYRISTICACEAE

101 MONIMIACEAE

102 LAURACEAE

103 HERNANDIACEAE

104 PAPAVERACEAE

104a FUMARIACEAE

105 CRUCIFERAE

106 TOVARIACEAE

107 CAPPARACEAE

108 RESEDACEAE

109 MORINGACEAE

112 DROSERACEAE

113 PODOSTEMACEAE

115 CRASSULACEAE
NUMBERS OF SPECIES

Native Introduced Total

Species Species Species

$\begin{array}{lr}\text { BET } & (40) \\ \text { FAG } & (41) \\ \text { ULM } & (42) \\ \text { MOR } & (43) \\ \text { URT } & (44) \\ \text { PRT } & (45) \\ \text { LOR } & (46) \\ \text { OPI } & (47) \\ \text { OLC } & (48) \\ \text { BNP } & (49) \\ \text { ARS } & (50) \\ \text { RAF } & (50 a) \\ \text { PLG } & (51) \\ \text { CHN } & (52) \\ \text { AMA } & (53) \\ \text { NYC } & (54) \\ \text { BAT } & (55) \\ \text { PHT } & (56) \\ \text { AIZ } & (57) \\ \text { POR } & (58) \\ \text { BAS } & (58 \mathrm{a}) \\ \text { CRY } & (59) \\ \text { NYM } & (60) \\ \text { CTP } & (61) \\ \text { RAN } & (62) \\ \text { BER } & (63) \\ \text { MNS } & (64) \\ \text { MAG } & (65) \\ \text { WIN } & (66) \\ \text { ANN } & (67) \\ \text { MYS } & (68) \\ \text { MNM } & (69) \\ \text { LAU } & (70) \\ \text { HRN } & (71) \\ \text { PAP } & (72) \\ \text { FUM } & (-) \\ \text { CRU } & (74) \\ \text { TOV } & (73 a) \\ \text { CPP } & (73) \\ \text { RSD } & (75) \\ \text { MRG } & (76) \\ \text { DRS } & (76 a) \\ \text { PDS } & (78) \\ \text { CRS } & (77)\end{array}$

\begin{tabular}{|c|c|c|}
\hline 1 & 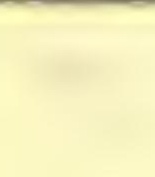 & 1 \\
\hline 11 & & 11 \\
\hline 7 & & 7 \\
\hline 96 & 3 & 99 \\
\hline 52 & 1 & 53 \\
\hline 5 & & 5 \\
\hline 51 & & 51 \\
\hline 1 & & 1 \\
\hline 10 & & 10 \\
\hline 3 & & 3 \\
\hline 14 & & 14 \\
\hline 3 & & 3 \\
\hline 33 & 1 & 34 \\
\hline 3 & 1 & 4 \\
\hline 31 & 4 & 35 \\
\hline 13 & 2 & 15 \\
\hline 1 & & 1 \\
\hline 10 & & 10 \\
\hline 3 & & 3 \\
\hline 4 & & 4 \\
\hline 1 & 1 & 2 \\
\hline 10 & 2 & 12 \\
\hline 4 & 1 & 5 \\
\hline 1 & & 1 \\
\hline 8 & & 8 \\
\hline 1 & & 1 \\
\hline 22 & & 22 \\
\hline 4 & & 4 \\
\hline 1 & & 1 \\
\hline 62 & 1 & 63 \\
\hline 16 & & 16 \\
\hline 19 & & 19 \\
\hline 78 & & 78 \\
\hline 3 & & 3 \\
\hline & $t$ fo & \\
\hline 2 & & 2 \\
\hline 4 & 6 & 10 \\
\hline see 1 & PP/ & \\
\hline 36 & 2 & 38 \\
\hline 1 & & 1 \\
\hline 1 & & 1 \\
\hline 1 & & 1 \\
\hline 12 & & 12 \\
\hline 1 & 1 & 2 \\
\hline
\end{tabular}


TABLE 1

PANAMA PLANT FAMILIES - NUMBERS OF SPECIES (continued)

FAMILY IDENTIFICATION

\begin{tabular}{|c|c|c|c|c|c|c|}
\hline \multicolumn{4}{|c|}{ FAMILY IDEINIIIICAIIUN } & \multicolumn{3}{|c|}{ NUMBERS UF SPECIES } \\
\hline $\begin{array}{l}\text { Dalla } \\
\text { Torre \& } \\
\text { Harms }\end{array}$ & Family Name & $\begin{array}{c}\text { Acronym } \\
\text { (Weber } \\
1982)\end{array}$ & $\begin{array}{c}\text { (Flora } \\
\text { of } \\
\text { Panama) }\end{array}$ & $\begin{array}{l}\text { Native } \\
\text { Species }\end{array}$ & $\begin{array}{l}\text { Introduced } \\
\text { Species }\end{array}$ & $\begin{array}{c}\text { Total } \\
\text { Species }\end{array}$ \\
\hline 117 & SAXIFRAGACEAE & SAX & (79) & 9 & & 9 \\
\hline 119 & BRUNELLIACEAE & BNL & (80a) & 2 & & 2 \\
\hline 120 & CUNONIACEAE & CUN & (80) & 4 & & 4 \\
\hline 126 & ROSACEAE & ROS & (81) & 39 & 1 & 40 \\
\hline 127 & CONNARACEAE & CNN & (82) & 11 & 1 & 12 \\
\hline 128 & LEGUMINOSAE & LEG & (83) & 430 & 6 & 436 \\
\hline 129 & GERANIACEAE & GER & (85) & 3 & & 3 \\
\hline 130 & OXALIDACEAE & $\mathrm{OXL}$ & (84) & 17 & 2 & 19 \\
\hline 131 & TROPAEOLACEAE & TRP & (86) & 3 & 1 & 3 \\
\hline 133 & HUMIRIACEAE & $\mathrm{HOU}$ & (87a) & 4 & & 4 \\
\hline 134 & ERYTHROXYLACEAE & ERX & (87) & 11 & 1 & 12 \\
\hline 135 & ZYGOPHYLLACEAE & ZYG & (88) & 4 & 1 & 5 \\
\hline 137 & RUTACEAE & RUT & (89) & 31 & 6 & 37 \\
\hline 138 & SIMAROUBACEAE & SMR & (90) & 11 & & 11 \\
\hline 139 & BURSERACEAE & BRS & (91) & 14 & 1 & 15 \\
\hline 140 & MELIACEAE & MEL & (92) & 31 & 2 & 33 \\
\hline 141 & MALPIGHIACEAE & MLP & (93) & 62 & 1 & 63 \\
\hline 142 & TRIGONIACEAE & TRG & (94) & 2 & & 2 \\
\hline 143 & VOCHYSIACEAE & VOC & (95) & 7 & & 7 \\
\hline 145 & POLYGALACEAE & PGL & (96) & 24 & & 24 \\
\hline 146 & DICHAPETALACEAE & $\mathrm{DCH}$ & (96a) & 7 & & 7 \\
\hline 147 & EUPHORBIACEAE & EUP & (97) & 134 & 1 & 135 \\
\hline 148 & CALLITRICHACEAE & CLL & (98) & & not yet found & \\
\hline 149 & BUXACEAE & BUX & (99) & 1 & & 1 \\
\hline 151 & CORIARIACEAE & CRR & (100) & 1 & & 1 \\
\hline 153 & ANACARDIACEAE & ANA & (101) & 11 & 3 & 14 \\
\hline 154 & CYRILLACEAE & CYR & (101a) & 2 & & 2 \\
\hline 157 & AQUIFOLIACEAE & $\mathrm{AQF}$ & (102) & 9 & & 9 \\
\hline 158 & CELASTRACEAE & CEL & (103) & 18 & & 18 \\
\hline 159 & HIPPOCRATEACEAE & HPC & (104) & 23 & & 23 \\
\hline 161 & STAPHYLEACEAE & STP & (105) & 1 & & 1 \\
\hline 162 & ICACINACEAE & ICC & (106) & 7 & & 7 \\
\hline 164 & HIPPOCASTANACEAE & HCS & (107) & 2 & & 2 \\
\hline 165 & SAPINDACEAE & SAP & (108) & 85 & 1 & 86 \\
\hline 166 & SABIACEAE & SAB & (109) & 12 & & 12 \\
\hline 168 & BALSAMINACEAE & BLS & (110) & 1 & 1 & 2 \\
\hline 169 & RHAMNACEAE & RHM & (111) & 15 & 1 & 16 \\
\hline 170 & VITACEAE & VIT & (112) & 12 & & 12 \\
\hline 171 & ELAEOCARPACEAE & ELC & (113) & 5 & & 5 \\
\hline 174 & TILIACEAE & TIL & (114) & 20 & & 20 \\
\hline 175 & MALVACEAE & MLV & (115) & 79 & 8 & 87 \\
\hline 177 & BOMBACACEAE & BOM & (116) & 25 & & 25 \\
\hline 178 & STERCULIACEAE & STR & (117) & 24 & 1 & 25 \\
\hline 180 & DILLENIACEAE & DLL & (118) & 18 & 2 & 20 \\
\hline
\end{tabular}

FAMILYIDENTIFICATION

\begin{tabular}{|c|c|c|c|c|c|c|}
\hline \multirow[b]{2}{*}{$\begin{array}{l}\text { Dalla } \\
\text { Torre \& } \\
\text { Harms }\end{array}$} & & & & \\
\hline & Family Name & $\begin{array}{c}\text { Acronym } \\
\text { (Weber } \\
\text { 1982) }\end{array}$ & $\begin{array}{c}\text { (Flora } \\
\text { of } \\
\text { Panama) }\end{array}$ & $\begin{array}{l}\text { Native } \\
\text { Species }\end{array}$ & $\begin{array}{l}\text { Introduced } \\
\text { Species }\end{array}$ & $\begin{array}{l}\text { Total } \\
\text { Species }\end{array}$ \\
\hline 182 & OCHNACEAE & $\mathrm{OCH}$ & (119) & 15 & & 15 \\
\hline 183 & CARYOCARACEAE & CCR & (120) & 3 & & 3 \\
\hline 184 & MARCGRAVIACEAE & MRC & (121) & 22 & & 22 \\
\hline 185 & QUIINACEAE & Qll & (121a) & 3 & & 3 \\
\hline 186 & THEACEAE & TEA & (122) & 9 & & 9 \\
\hline 187 & GUTTIFERAE & GUT & (123) & 61 & & 61 \\
\hline $187 a$ & HYPERICACEAE & HYP & (123a) & 8 & & 8 \\
\hline 183 & $\begin{array}{l}\text { CISTACEAE } \\
\text { - }\end{array}$ & CIS & (124) & \multicolumn{3}{|c|}{ not yet found } \\
\hline 194 & BIXACEAE & $\mathrm{BIX}$ & (125) & 1 & 1 & 2 \\
\hline 195 & COCHLOSPERMACEAE & $\mathrm{CCH}$ & (126) & 2 & & 2 \\
\hline 198 & VIOLACEAE & VIO & (127) & 27 & 1 & 28 \\
\hline 199 & FLACOURTIACEAE & FLC & (128) & 46 & 1 & 47 \\
\hline 201 & TURNERACEAE & TNR & (129) & 6 & & 6 \\
\hline 203 & PASSIFLORACEAE & PAS & (130) & 42 & & 42 \\
\hline 205 & CARICACEAE & CRC & (131) & 8 & & 8 \\
\hline 206 & LOASACEAE & LOA & (132) & 7 & & 7 \\
\hline 208 & BEGONIACEAE & $B E G$ & (133) & 35 & & 35 \\
\hline 210 & CACTACEAE & CAC & (134) & 23 & 1 & 24 \\
\hline 214 & THYMELAEACEAE & THY & (135) & 2 & & 2 \\
\hline 216 & $\begin{array}{l}\text { LYTHRACEAE } \\
\text { - }\end{array}$ & LYT & (136) & 17 & 5 & 22 \\
\hline 219 & LECYTHIDACEAE & LCY & (137) & 31 & & 31 \\
\hline 220 & RHIZOPHORACEAE & $\mathrm{RHZ}$ & (138) & 5 & & 5 \\
\hline 221 & COMBRETACEAE & CMB & (139) & 18 & 2 & 20 \\
\hline 222 & $\begin{array}{l}\text { MYRTACEAE } \\
\text { MYCA }\end{array}$ & MRT & (140) & 51 & 13 & 64 \\
\hline 223 & MELASTOMATACEAE & MLS & (141) & 244 & & 244 \\
\hline 224 & ONAGRACEAE & ONA & (142) & 23 & 2 & 25 \\
\hline 225 & HALORAGACEAE & HAL & (143) & 2 & & 2 \\
\hline 227 & ARALIACEAE & ARL & (144) & 29 & & 29 \\
\hline 228 & UMBELLIFERAE & UMB & (145) & 19 & 3 & 22 \\
\hline 229 & CORNACEAE & COR & (146) & 2 & & 2 \\
\hline 230 & CLETHRACEAE & CLE & (147) & 3 & & 3 \\
\hline 233 & ERICACEAE & ERI & (148) & 81 & & 81 \\
\hline $235 a$ & THEOPHRASTACEAE & TEO & (151) & 5 & & 5 \\
\hline 236 & MYRSINACEAE & MRS & (150) & 134 & & 134 \\
\hline 237 & PRIMULACEAE & PRM & (152) & 1 & & 1 \\
\hline 238 & PLUMBAGINACEAE & PLB & (153) & & 2 & 2 \\
\hline 239 & SAPOTACEAE & SPT & (154) & 45 & 1 & 46 \\
\hline 240 & EBENACEAE & EBN & (155) & 5 & & 5 \\
\hline 241 & STYRACACEAE & STY & (156) & 2 & & 2 \\
\hline 242 & SYMPLOCACEAE & SYM & (157) & 3 & & 3 \\
\hline 243 & $\begin{array}{l}\text { OLEACEAE } \\
\text { - }\end{array}$ & OLE & (158) & 2 & 7 & 9 \\
\hline 245 & LOGANIACEAE & LOG & (159) & 19 & & 19 \\
\hline 246 & GENTIANACEAE & GEN & (160) & 32 & & 32 \\
\hline $246 a$ & MENYANTHACEAE & MNY & (161) & 1 & & 1 \\
\hline
\end{tabular}


TABLE 1

PANAMA PLANT FAMILIES - NUMBERS OF SPECIES (continued)

\begin{tabular}{|c|c|c|c|c|c|c|}
\hline \multicolumn{4}{|c|}{ FAMILY IDENTIFICATION } & \multicolumn{3}{|c|}{ NUMBERS OF SPECIES } \\
\hline $\begin{array}{l}\text { Dalla } \\
\text { Torre \& } \\
\text { Harms } \\
\end{array}$ & Family Name & $\begin{array}{c}\text { Acronym } \\
\text { (Weber } \\
1982)\end{array}$ & $\begin{array}{c}\text { (Flora } \\
\text { of } \\
\text { Panama) }\end{array}$ & $\begin{array}{l}\text { Native } \\
\text { Species }\end{array}$ & $\begin{array}{l}\text { Introduced } \\
\text { Species }\end{array}$ & $\begin{array}{c}\text { Total } \\
\text { Species }\end{array}$ \\
\hline 247 & APOCYNACEAE & APO & $(162)$ & 74 & 4 & 78 \\
\hline 248 & ASCLEPIADACEAE & ASC & (163) & 47 & 4 & 51 \\
\hline 249 & CONVOLVULACEAE & CNV & (164) & 65 & 1 & 66 \\
\hline 250 & POLEMONIACEAE & PLM & (165) & 4 & & 4 \\
\hline 251 & HYDROPHYLLACEAE & HYD & (166) & 2 & & 2 \\
\hline 252 & BORAGINACEAE & $\mathrm{BOR}$ & (167) & 44 & & 44 \\
\hline 253 & VERBENACEAE & VRB & (168) & 81 & 9 & 90 \\
\hline $253 a$ & AVICENNIACEAE & $\mathrm{AVI}$ & (168a) & 3 & & 3 \\
\hline 254 & LABIATAE & $\mathrm{LAB}$ & (169) & 46 & 1 & 47 \\
\hline 256 & SOLANACEAE & SOL & (170) & 138 & 16 & 152 \\
\hline 257 & SCROPHULARIACEAE & SCR & (171) & 52 & 2 & 54 \\
\hline 258 & BIGNONIACEAE & BIG & (172) & 80 & 10 & 90 \\
\hline 259 & PEDALIACEAE & PED & (173) & 1 & & 1 \\
\hline 260 & MARTYNIACEAE & MAR & $(-)$ & \multicolumn{3}{|c|}{ see 259 PEDALIACEAE } \\
\hline 261 & OROBANCHACEAE & ORO & (174) & 2 & & 2 \\
\hline 262 & GESNERIACEAE & GSN & (175) & 159 & 2 & 161 \\
\hline 264 & LENTIBULARIACEAE & LNT & (176) & 14 & & 14 \\
\hline 266 & ACANTHACEAE & ACA & (177) & 110 & 2 & 112 \\
\hline 269 & PLANTAGINACEAE & PTG & (178) & 2 & 1 & 3 \\
\hline 270 & RUBIACEAE & RUB & (179) & 431 & 5 & 436 \\
\hline 271 & CAPRIFOLIACEAE & CPR & (180) & 5 & & 5 \\
\hline 273 & VALERIANACEAE & VAL & (181) & 6 & & 6 \\
\hline 275 & CUCURBITACEAE & CUC & (182) & 44 & 8 & 52 \\
\hline 276 & CAMPANULACEAE & CAM & (183) & 44 & 1 & 4 \\
\hline \multirow[t]{2}{*}{280} & COMPOSITAE & CMP & (184) & 272 & 7 & 279 \\
\hline & TOTALS: & & & $\overline{7123}$ & $\overline{222}$ & $\overline{7345}$ \\
\hline
\end{tabular}

TABLE 2

FLORA SIZE OF SELECTED AREAS

\begin{tabular}{|c|c|c|c|}
\hline Country (latitude') & $\begin{array}{c}\text { Area }{ }^{2} \\
\left(1,000^{\prime} \mathrm{km}^{2}\right)\end{array}$ & $\begin{array}{l}\text { No. of } \\
\text { species }\end{array}$ & Ref. \\
\hline California Floristic Province & & & \\
\hline$\left(29^{\circ}-43^{\circ} \mathrm{N}\right)$ & 324 & 4452 & 3 \\
\hline California $\left(32^{\circ}-42^{\circ} \mathrm{N}\right)$ & 411 & 5046 & 3 \\
\hline Missouri $\left(36^{\circ}-41^{\circ} \mathrm{N}\right)$ & 180 & 2438 & 4 \\
\hline $\operatorname{Texas}\left(26^{\circ}-37^{\circ} \mathrm{N}\right)$ & 692 & 4196 & 5 \\
\hline Cuba $\left(20^{\circ}-24^{\circ} \mathrm{N}\right)$ & 115 & 5800 & 6 \\
\hline Jamaica $\left(17^{\circ}-18^{\circ} \mathrm{N}\right)$ & 11 & 2888 & 7 \\
\hline Panama $\left(7^{\circ}-10^{\circ} \mathrm{N}\right)$ & 76 & 7345 & \\
\hline Barro Colorado Is., Panama $\left(8^{\circ} \mathrm{N}\right)$ & .016 & 1099 & 8 \\
\hline Cape Floristic Region $\left(31^{\circ}-35^{\circ} \mathrm{S}\right)$ & 89 & 8550 & 9 \\
\hline
\end{tabular}

[Sources: 1. Latitudes from The Odyssey World Atlas. 2. Areas from The World Almanac \& Book of Facts 1981. 3. Raven \& Axelrod 1978. 4. Steyermark 1963. 5. Correll \& Johnston 1970. 6. León 1966. 7. Adams 1972; Asprey \& Robbins 1953. 8. Croat 1978. 9. Goldblatt 1978.] 
Table 3 shows the numbers of taxa recorded in the Flora of Panama and now known from Panama through the Database. The ranks between family and genus and between genus and species are not included as they are not generally useful for making comparisons. Ranks below species also may be quite incomplete, as contributors vary in their consideration of these ranks.

TABLE 3

NUMBERS OF NEW TAXA SINCE FLORA OF PANAMA

\begin{tabular}{lrrr}
\hline Taxon & Flora & Present & $\begin{array}{r}\text { Percent } \\
\text { Change }\end{array}$ \\
& & & \\
\hline Families & 192 & 195 & $1.6 \%$ \\
Genera & 1520 & 1813 & 19.3 \\
Species & $5314^{*}$ & 7345 & 38.2 \\
$\quad$ subspecies & 35 & 89 & \\
$\quad$ varieties & 236 & 310 & \\
$\quad$ subvarieties & 0 & 0 & \\
$\quad$ forms & 12 & 9 & \\
subforms & 0 & 0 & \\
Infra specific ranks & 283 & 408 & $44.2 \%$ \\
\hline
\end{tabular}

*26 of these recognized implicitly by recognition of infraspecific taxa

Some families have shown much greater increase in known taxa since publication of the Flora than others. Generally those families completed early show the biggest increase. Table 4 shows the increase in species for the largest plant families.

\section{TABLE 4 \\ INCREASE IN FLORA SINCE FLORA OF PANAMA TREATMENTS SPECIES NUMBERS FOR FAMILIES WITH MORE THAN 100 SPECIES}

Increase In Species For Selected Groups

Family (date)

Gramineae (1943)

Cyperaceae (1943)

Palmae (1943)

Araceae (1944)

Bromeliaceae (1944)

Orchidaceae (1946-49)

All monocotyledons

Piperaceae (1950)

Leguminosae $(1950-80)$

Euphorbiaceae (1967)

Melastomataceae (1958)

Myrsinaceae (1971)

Solanaceae (1973)

Gesneriaceae (1979)

Acanthaceae (1978)

Rubiaceae (1980)

Compositae (1975)

All dicotyledons

All families
Species Species Per cent

in Flora Now Increase

Per Cent of Total Flora

In Flora Now

$276 \quad 359+30.1 \%$

$\begin{array}{lll}102 & 165 \quad 61.8\end{array}$

$\begin{array}{lll}71 & 103 & 45.1\end{array}$

$\begin{array}{lll}74 & 234 & 316.2\end{array}$

$\begin{array}{lll}85 & 136 & 60.0\end{array}$

$\begin{array}{lll}469 & 893 & 91.5\end{array}$

$\begin{array}{lll}267 & 2246 & 77.3\end{array}$

$23.8 \% \quad 30.6 \%$

$\begin{array}{lll}171 & 213 & 24.5\end{array}$

$\begin{array}{lll}401 & 436 & 8.7\end{array}$

$\begin{array}{lll}100 & 135 & 35.0\end{array}$

$\begin{array}{lll}141 & 244 & 73.0\end{array}$

$\begin{array}{lll}69 & 134 & 94.2\end{array}$

$\begin{array}{lll}139 & 152 & 9.3\end{array}$

$\begin{array}{lll}146 & 161 & 10.3\end{array}$

$\begin{array}{lll}107 & 112 & 4.7\end{array}$

$\begin{array}{lll}414 & 436 & 5.3\end{array}$

$\begin{array}{lll}252 & 279 & 10.7\end{array}$

$\begin{array}{lll}4047 & 5099 & 30.0\end{array}$

\begin{tabular}{ll}
76.1 & 69.4 \\
\hline
\end{tabular}

$53147345+38.2 \% \quad 100.0 \% \quad 100.0 \%$ 
Plant collecting has been more intense and more complete in some parts of the country than others. Table 5 shows the increases in recorded plant diversity for several regions since completion of the Flora. Three geographical subdivisions, the Canal Area, and Chiriqui and Panama Provinces have long had the most intense botanical exploration. Since completion of the Flora, there has been great effort to collect in other, more poorly explored parts of the country. The low absolute numbers in the Azuero Peninsula reflect in large part the reduction of the flora by human activities before the original flora could be sampled.

\section{TABLE 5 \\ INCREASE IN SPECIES FOR PARTS OF PANAMA}

\begin{tabular}{lrrr}
\hline Region & $\begin{array}{r}\text { Species } \\
\text { in Flora }\end{array}$ & $\begin{array}{r}\text { Species } \\
\text { Now }\end{array}$ & $\begin{array}{r}\text { Per cent } \\
\text { Increase }\end{array}$ \\
\hline & 1888 & 2545 & 34.8 \\
Canal Area & 2050 & 3057 & 49.1 \\
$\begin{array}{l}\text { Chiriquí } \\
\text { Panama }\end{array}$ & 2225 & 3246 & 45.9 \\
$\begin{array}{l}\text { Western Panama } \\
\quad \text { (Bocas del Toro, Chiriqui) }\end{array}$ & 2679 & 3921 & 46.4 \\
$\begin{array}{l}\text { Central Provinces } \\
\text { (Canal Area, Coclé, Colón, Herrera, }\end{array}$ & 3302 & 5146 & 55.8 \\
$\quad \begin{array}{l}\text { Los Santos, Panamá, Veraguas) } \\
\text { Azuero Peninsula } \\
\quad \text { (Herrera, Los Santos) }\end{array}$ & 654 & 801 & 22.5 \\
$\begin{array}{l}\text { Eastern Panama } \\
\quad \text { (Darién, San Blas) }\end{array}$ & 1754 & 2563 & 46.1 \\
$\begin{array}{l}\text { All Panama } \\
\text { Pama }\end{array}$ & 5314 & 7345 & 38.2 \\
\hline
\end{tabular}

The increases for provinces and regions are larger than that for the entire country, because they reflect new province records that are not new for the country.

\section{SOME CHARACTERISTICS OF THE PANAMANIAN FLORA}

The following series of tables summarizes some characteristics of the Panamanian flora. These data are subject to some of the limita- tions indicated in the section on FORMAT (page xx). Analyses of these characteristics and others will be presented in another publication.

\section{TABLE 6}

MAJOR LIFE FORMS

Number Habit (unmodified) Percentage* of Known Cases

\begin{tabular}{|c|c|c|}
\hline & & \\
\hline 895 & Climber & $12.3 \%$ \\
\hline 195 & Epiphyte & 2.7 \\
\hline 2 & Hemiepiphyte & 0.03 \\
\hline 3126 & Herb & 42.9 \\
\hline 11 & Parasite & 0.2 \\
\hline 2057 & Shrub & 28.2 \\
\hline 189 & Subshrub & 2.6 \\
\hline 11 & Shrublet & 0.2 \\
\hline 1952 & Tree & 26.8 \\
\hline 53 & Treelet & 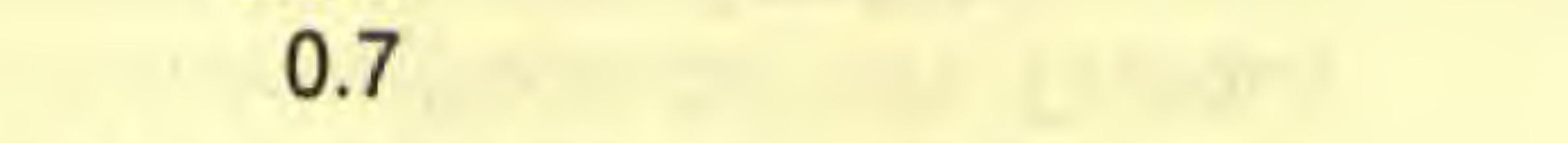 \\
\hline 56 & Without Habit ( & (not available from present data) \\
\hline Number & $\begin{array}{l}\text { Percentage * } \\
\text { of Known } \\
\text { Cases }\end{array}$ & Habit (modified) \\
\hline 3459 & $47.4 \%$ & Woody Species (shrubs, trees, etc.) \\
\hline 1709 & 23.5 & $\begin{array}{l}\text { Climbing Plants (lianas, vines, epiphytes, } \\
\text { hemiepiphytes) }\end{array}$ \\
\hline 3830 & 52.5 & $\begin{array}{l}\text { Herbaceous Plants (herbaceous minus } \\
\text { woody plants) }\end{array}$ \\
\hline 4171 & 57.2 & $\begin{array}{l}\text { Herbaceous Plants (herbs, climbers, } \\
\text { epiphytes, hemiepiphytes, parasites, } \\
\text { aquatics, marine plants) }\end{array}$ \\
\hline
\end{tabular}

Total species in flora: 7345 . Total species with known habit: 7289 . *Do not total to $100 \%$ because of multiple categories. 
TABLE 7

SPECIES OCCURRING AT DIFFERENT ELEVATIONS

Number Elevation Level

Percentage*

of Known Cases

$\begin{array}{rll}5875 & 0-1000 \mathrm{~m} \text { Elevation } & 83.5 \% \\ 2215 & 1000-2000 \mathrm{~m} \text { Elevation } & 31.5 \\ 610 & 2000-3000 \mathrm{~m} \text { Elevation } & 8.7 \\ 155 & \text { Over 3000m Elevation } & 2.2 \\ & \text { Elevation Range } & \\ 6817 & \text { Below 2000m } & 96.9 \% \\ 6989 & \text { Below 3000m } & 99.4 \\ 666 & \text { Above 2000m } & 9.5 \\ 2463 & \text { Above 1000m } & 35.0 \\ 2418 & \text { Between 1000m and 3000m } & 34.4 \\ 312 & \text { Without Elevation (not available from present data) } \\ \end{array}$

Total species in flora: 7345 . Species with known elevation: 7033.

"Do not total to $100 \%$ because of multiple categories.

\section{TABLE 8 \\ SPECIES OCCURRING IN DIFFERENT GEOGRAPHIC DIVISIONS OF PANAMA}

Number Geographical Division Percentage ${ }^{*}$ of Known Cases

$\begin{array}{rlc}1661 & \text { Bocas Del Toro } & 23.1 \% \\ 2545 & \text { Canal Area } & 35.5 \\ 3057 & \text { Chiriquí } & 42.6 \\ 2174 & \text { Coclé } & 30.3 \\ 1586 & \text { Colón } & 22.1 \\ 2188 & \text { Darién } & 30.5 \\ 444 & \text { Herrera } & 6.2 \\ 556 & \text { Los Santos } & 7.7 \\ 3246 & \text { Panamá } & 45.2 \\ 1003 & \text { San Blas } & 14.0 \\ 1437 & \text { Veraguas } & 20.0 \%\end{array}$

166 Without Geographical Division (not available from present data)

$392254.6 \%$ Western Panama (Bocas del Toro, Chiriqui)

$2563 \quad 35.7$ Eastern Panama (Darién, San Blas)

514671.7 Central Panama (Canal Area, Coclé, Colón, Herrera, Los Santos, Panamá, Veraguas)

3061 Occur In 1 Province 320 Occur In 6 Provinces 1298 Occur In 2 Provinces 263 Occur In 7 Provinces 832 Occur In 3 Provinces 174 Occur In 8 Provinces 603 Occur In 4 Provinces 138 Occur In 9 Provinces 406 Occur In 5 Provinces 72 Occur In 10 Provinces

Total species in flora: 7345 .

Total species with known geography: 7179 .

*Do not total to $100 \%$ because of multiple categories. 


\section{NAMING OF THE FLORA}

TABLE 9

DISTRIBUTIONS OUTSIDE OF PANAMA

Distribution

Number Percentage

Native species:

To the south of Panama . . . . . . . . . . . . . . . . .4034

To the south only* ................ 1006

To the north of Panama . . . . . . . . . . . . . .5569

To the north only ${ }^{*} \ldots \ldots \ldots \ldots \ldots \ldots \ldots .2433$

Central America (Guatemala, El Salvador,

Belize, Honduras, Nicaragua, Costa Rica) .5107

Central America only . . . . . . . . . . . 2322

In the Antilles (excluding Trinidad \& Tobago) . . 1298 Antilles only ${ }^{*} \ldots \ldots \ldots \ldots \ldots \ldots \ldots .66$

Endemic species ${ }^{*}$................... 1230

Species restricted as above $\left({ }^{*}\right)$. . . . . . . . . . 4735

Total native species . . . . . . . . . . . . . . . . 7123

Introduced species . . . . . . . . . . . . . . . . 222

Total flora . . . . . . . . . . . . . . . . . . . . . . . . . .7345

"Species restricted in distribution outside of Panama. of Native

Species

$56.6 \%$

14.1

\section{PANAMANIAN PLANT NAMES}

The names that have been given to Panamanian plant species were derived from many different sources, but most were derived in one of three ways; they note the localities where the plants were first found, they honor a botanist, usually the botanist who first found the species, or they are descriptive of the plants themselves. To assess the frequency of use of species names, or epithets, it is most useful to compare the parts of the epithets lacking the gender-reflecting ending (usually the roots or stems). Thus panamensis, panamaensis, panamana are all treated as equivalent, and dressleri and dressleriana are also treated as equivalent. There is a convention, not always followed, that in naming a species after a person, the endings $-i,-u m$, or ae are used for a person who collected or otherwise worked with the plant, and the endings -anus, -anum or -ana for honoring a person who had little or nothing to do with the species at hand. Table 10 shows the names that have been used most often for the species accepted in this treatment.

66.4

$100.0 \%$
TABLE 10 MOST USED NAMES FOR PANAMANIAN SPECIES

$\begin{array}{lll}\text { NAME } & \text { NO. OF } & \text { COMMEMORATES } \\ \text { PART } & \text { TIMES USED } & \end{array}$

$\begin{array}{lrl}\text { panam- } & 138 & \text { Panama } \\ \text { costaric- } & 84 & \text { Costa Rica } \\ \text { pittier- } & 58 & \text { Henri François Pittier } \\ \text { allen- } & 43 & \text { Paul Hamilton Allen } \\ \text { chiriqu- } & 40 & \text { Chiriqui Province, Panama } \\ \text { american- } & 34 & \text { America (New World) } \\ \text { mexican- } & 33 & \text { Mexico } \\ \text { macrophylla- } & 32 & \text { large leaved } \\ \text { darien- } & 28 & \text { Darien Province, Panama } \\ \text { latifol- } & 27 & \text { broad leaved } \\ \text { dressler- } & 23 & \text { Robert Louis Dressler } \\ \text { standley- } & 23 & \text { Paul Carpenter Standley }\end{array}$

All other names have been used fewer than 20 times for species recognized as occurring in Panama. 


\section{AUTHORS OF PANAMANIAN PLANT NAMES}

A large component of the Panama flora consists of widespread species, and a great majority of species occurring in Panama were named on the basis of material collected in other countries. In a history of plant collecting in Panama, Dwyer (1981) notes that early collecting in Panama was conducted by Europeans, so it is no surprise that European botanists have been prolific in naming plants that occur in Panama. The botanist who named by far the greatest number of Panamanian species was Carl Linnaeus of Sweden, who initiated the present practice of using binomial names for plants and animals. During his lifetime (1707-1778) Linnaeus named about 7700 species (Stearn 1971), and the fact that nearly eight per cent of these now occur in Panama is a striking testimony to the strong representation of neotropical plants in European studies in the mid 18th century. The next most prolific namer of Panamanian plants was Paul Standley of the Field Museum of Natural History, who wrote floras for parts of Panama and for other parts of Central America. Table 11 lists botanists who were prolific in naming Panamanian species.

\section{TABLE 11 \\ BOTANISTS WHO HAVE NAMED \\ THE MOST PANAMANIAN SPECIES}

\begin{tabular}{|c|c|c|}
\hline BOTANIST & $\begin{array}{c}\text { NO. OF } \\
\text { SPECIES } \\
\text { DESCRIBED }\end{array}$ & MAIN HOME LOCATION \\
\hline Carl Linnaeus & 629 & Stockholm, Sweden \\
\hline $\begin{array}{l}\text { Paul Carpenter Standley } \\
\text { Humboldt, Bonpland \& }\end{array}$ & 436 & Chicago, U.S.A. \\
\hline $\begin{array}{l}\text { Kunth (H.B.K.) } \\
\text { Augustin Pyramus de }\end{array}$ & 268 & Berlin/Paris \\
\hline Candolle & 251 & Geneva, Switzerland \\
\hline George Bentham & 225 & London, England \\
\hline $\begin{array}{l}\text { Heinrich Gustav } \\
\text { Reichenbach (Jr.) }\end{array}$ & & \\
\hline $\begin{array}{l}\text { Reichenbach (Jr.) } \\
\text { *John Duncan Dwyer }\end{array}$ & 204 & Hamburg, Germany \\
\hline $\begin{array}{l}\text { "John Duncan Dwyer } \\
\text { Nikolaus Joseph }\end{array}$ & 171 & St. Louis, U.S.A. \\
\hline Jacquin & 160 & Vienna, Austria \\
\hline Olof Peter Swartz & 154 & Stockholm, Sweden \\
\hline - Thomas Bernard Croat & 142 & St. Louis, U.S.A. \\
\hline John Lindley & 138 & London, England \\
\hline -Cyrus Longworth & & \\
\hline Lundell & 117 & Austin, Texas \\
\hline Casimire de Candolle & 107 & Geneva, Switzerland \\
\hline
\end{tabular}

No other botanist has named more than 100 species. This tabulation includes species first named or later transferred by the respective worker, and it includes names created in co-authorship with others.

As seen in Table 12, Linnaeus also excelled at the generic level, describing 417 genera that occur in Panama. No other botanists have described more than 25 of Panama's genera. 


\section{CONTRIBUTORS TO DATABASE REVISION}

TABLE 12

BOTANISTS WHO HAVE NAMED

THE MOST PANAMANIAN GENERA

$\begin{array}{cc}\text { BOTANIST } & \text { NO. OF } \\ \text { GENERA } \\ \text { DESCRIBED }\end{array}$

Carl Linnaeus

417

Jean Baptiste Christophe

Fusée Aublet

Hipolito Ruiz \& José

Antonio Pavón

Augustin Pyramus de

Candolle

John Lindley

George Bentham

Olof Peter Swartz
88

43

56

39

34

27
Stockholm, Sweden

Paris, France \& colonies

Madrid, Spain

Geneva, Switzerland

London, England

London, England

Stockholm, Sweden

\section{SYNONYMY}

Many names previously used for Panamanian plants are considered to be synonymous with the names accepted in this treatment. Practice in listing synonyms in the Flora of Panama differed greatly according to the particular author, some choosing to list all synonyms on a global basis, while others listed only synonyms directly germane to the Panamanian flora. Names listed in synonymies in the Flora included both validly published names and manuscript or other "ined" names. For these reasons, ratios of synonyms to accepted names in the present listing must be considered as only a gross approximation to the actual redundancy in naming. In the present Database, there are 10542 synonyms at species rank for the 7345 recognized species and 2477 synonyms for the 1813 recognized genera.
Help with parts of the Checklist was freely given by many specialists, some of whom undertook major updatings. Families that benefited from major input by specialists are listed in Table 13; other families were reviewed by the author.

\section{TABLE 13 \\ MAJOR CONTRIBUTIONS BY SPECIALISTS}

\section{Monocotyledons:}

ALISMATACEAE- Haynes; AMARYLLIDACEAE- Gereau; ARACEAECroat; BROMELIACEAE- Burt-Utley; BURMANNIACEAE-Van Benthem \& Maas; BUTOMACEAE- Haynes; CANNACEAE- Maas; COMMELINACEAE- Faden; CYCLANTHACEAE- Hammel; CYPERACEAE-Adams/Davidse; GRAMINEAE- Davidse; HYDOCHARITACEAE- Haynes; IRIDACEAE- Goldblatt/Henrich; JUNCACEAE-Balsev; LEMNACEAE- Haynes; LILIACEAE-Gereau; MUSACEAE- Andersson/Kress/Stiles; NAJADACEAE- Haynes; ORCHIDACEAE- Atwood/Barringer/Dressler; PALMAEBalick/Dransfield; PONTEDERIACEAE- Barrett; POTAMOGETONACEAE- Haynes; TRIURIDACEAE- Haynes; TYPHACEAE- Haynes; XYRIDACEAE- Kral.

\section{Dicotyledons:}

ACANTHACEAE- Durkee; ANNONACEAE- Maas/Mennenga; APOCYNACEAE- Leeuwenburg; AQUIFOLIACEAE- Hahn; ARISTOLOCHIACEAE- Barringer; ASCLEPIADACEAE-Stevens; BEGONIACEAE-Burt-Utley; BIGNONIACEAE- Gentry; BOMBACACEAE-Alverson; BORAGINACEAE-Miller; CACTACEAEBravo/Scheinvar; CAMPANULACEAE- Wilbur; CERATOPHYLLACEAE- Haynes; CHLORANTHACEAE-Todzia; COMPOSITAE- King/Strother; CONVOLVULACEAE- Austin; CORIARIACEAE-Skog; CRUCIFEREAE-Rollins; CUCURBITACEAE- Wunderlin; ERICACEAE- Luteyn; ERYTHROXYLACEAE- Plowman; EUPHORBIACEAE- Huft/Webster; GENTIANACEAE- Maas; GESNERIACEAE-Skog; GUTTIFERAEHammel; HYPERICACEAE- Robson; ICACINACEAE- Howard; JUGLANDACEAE-Stone; LABIATAE- Harley; LAURACEAE-Van der Werff; LEGUMINOSAE- Barneby; LORANTHACEAE- Kjuit; 
TABLE 13 (continued)

MAJOR CONTRIBUTIONS BY SPECIALISTS

MALVACEAE- Fryxell; MELASTOMATACEAE- Almeda; MENISPERMACEAE- Barneby; MONIMIACEAE-Antonio; MORACEAE- Berg; MYRSINACEAE-Pipoly; NYMPHAEACEAEHaynes; ONAGRACEAE-Berry/Dietrich/Ramamoorthy/Raven/Zardini; OROBANCHACEAE- Haynes; PIPERACEAE-Humphries; POLYGALACEAE- Wendt/Wurdack; RANUNCULACEAE- Duncan; RUBIACEAE- Dwyer; SABIACEAE- Gentry; SCROPHULARIACEAEBarringer; TILIACEAE- Meijer; UMBELLIFERAE-Constance.

\section{TABLE 14}

SPECIALISTS MAKING CONTRIBUTIONS TO THE DATABASE

(Does not include the many others who contributed through publications or various herbarium determinations)

Name -Institution

Taxon

Adams, C. D. -Missouri Botanical Garden Allen, B. -Missouri Botanical Garden

Allorge, L. -Museum National d'Histoire Naturelle, Paris

Almeda, F. -California Academy of Sciences

Andersson, L. -Herbarium, Botanical Museum, Göteborg, Sweden

Antonio, T.M. -Chicago Botanical Garden Atwood, J. -Marie Selby Botanic Garden Austin, D. -Florida Atlantic University Balick, M.J. -New York Botanical Garden Balsev, H. - University of Aarhus,

Denmark MUSACEAE

MONIMIACEAE

ORCHIDACEAE

CONVOLVULACEAE

PALMAE

JUNCACEAE
Taxon

Barneby, R.C. -New York Botanical Garden

Barrett, S. - University of Toronto

Barringer, K. -Brooklyn Botanic Gardens

Bedell, H.G. -Arnold Arboretum, Harvard University

Berry, P. -Universidad Simón Bolivar, Caracas

Berg, C.C. - Institute for Systematic Botany, Utrecht

Bravo, H. - Universidad Nacional Autónomó de México

Burt-Utley, C. -University of New Orleans

Constance, L. -University of California (Berkeley)

Croat, T.B. -Missouri Botanical Garden Crosby, M.R. -Missouri Botanical Garden

Davidse, G. -Missouri Botanical Garden

Dietrich, W. -Botanisches Institut der Universität, Düsseldorf

Dransfield J. -British Museum (N.H.)

Dressler, R.L. -Smithsonian Tropical Research Institute

Duncan, T. - University of California (Berkeley)

SCROPHULARIACEAE, Durkee, L.A. -Grinnell College
Dwyer, J.D. -Missouri Botanical Garden Edmonds, J. -Cambridge University

Faden, R. -Smithsonian Institution

Forero, E. -Missouri Botanical Garden

Fryxell, P.A. -Dept. Soil \& Crop Science, Texas A. \& M. University
MENISPERMACEAE, LEGUMINOSAE PONTEDERIACEAE ORCHIDACEAE, SCROPHULARIACEAE, ARISTOLOCHIACEAE MARCGRAVIACEAE

ONAGRACEAE

MORACEAE

CACTACEAE

BROMELIACEAE UMBELLIFERAE

ARACEAE

MUSCI

GRAMINEAE,

CYPERACEAE

ONAGRACEAE

PALMAE

ORCHIDACEAE

RANUNCULACEAE

ACANTHACEAE

RUBIACEAE

SOLANACEAE

COMMELINACEAE

CONNARACEAE

MALVACEAE 
TABLE 14 (continued) SPECIALISTS MAKING CONTRIBUTIONS TO THE DATABASE

Name -Institution

Taxon

Gentry, A.H. -Missouri Botanical Garden

Goldblatt, P. -Missouri Botanical Garden Graham, S.A. -Kent State University Hahn, W. -Cornell University

Hammel, B. -Missouri Botanical Garden

Haynes, R.R. -University of Alabama

Hekking, W.H.A. -Institute for Systematic Botany, Utrecht

Henrich, J. -Missouri Botanical Garden Huft, M.J. -Missouri Botanical Garden

King, R.M. -Smithsonian Institution Kral, R. -Vanderbilt University Kress, J. -Marie Selby Botanic Garden Kuijt, J. -University of Lethbridge Leeuwenberg, A.J.M. -Laboratory for

Plant Taxonomy, Wageningen Lellinger, D. -Smithsonian Institution Liesner, R.L. -Missouri Botanical Garden Luer, C. -Missouri Botanical Garden Luteyn, J.L. -New York Botanical Garden Maas, P.J. -Institute for Systematic Botany, Utrecht

Meacham, C.A. -University of Georgia Meijer, W. -University of Kentucky Mennega, E.A. -Institute for Systematic Botany, Utrecht

Miller, J. -Missouri Botanical Garden Moldenke, H.N.

BIGNONIACEA
SABIACEAE IRIDACEAE LYTHRACEAE AQUILFOLIACEAE GUTTIFERAE, CYCLANTHACEAE NYMPHAEACEAE, LEMNACEAE, VIOLACEAE

IRIDACEAE URTICACEAE, EUPHORBIACEAE COMPOSITE XYRIDACEAE MUSACEAE LORANTHACEAE APOCYNACEAE

PTERIDOPHYTA

Various ORCHIDACEAE ERICACEAE

ANNONACEAE, MUSACEAE, BURMANNIACEAE, ZINGIBERACEAE, GENTIANACEAE RANUNCULACEAE TILIACEAE ANNONACEAE

BORAGINACEAE VERBENACEAE
Taxon

Murphy, H. -Field Museum of Natural History

Pilz, G.E. -Escuela Agricola Panamericana, Zamorana, Honduras

Pipoly, J. -New York Botanical Garden

Plowman, T. -Field Museum of Natural History POTAMOGETONACEAE
Press, J.R. -British Museum (N.H.)

Ramamoorthy, T.P. -Universidad Nacional Autónomó de México

Raven, P.H. -Missouri Botanical Garden

Robson, N. -British Museum (N.H.)

Rollins, R. -Gray Herbarium, Harvard University

Schatz, G. -Missouri Botanical Garden

Scheinvar, L. -Universidad Nacional Autónomó de México

Skog, L. -Smithsonian Institution

Sleumer, H.O. -Rijksherbarium, Netherlands

Stevens, W.D. -Missouri Botanical Garden Stevenson, D. -New York Botanical Garden

Stiles, G. -Universidad de Costa Rica

Strother, J. -University of California (Berkeley)

Sullivan, E. -Louisiana State University Todzia, C. -Missouri Botanical Garden Utley, J.F. - University of New Orleans

Van Benthem \& P.J. Maas -Institute for Systematic Botany, Utrecht

Van der Werff, H. -Missouri Botanical Garden

Wagner, W. -Bishop Museum

Webster, G. - University of California (Davis)

Wendt, T.L. -Escuela Nacional de

Agricultura, Chapingo, Mexico

Wunderlin, D. -University of South Florida

Zardini, E. -Missouri Botanical Garden
Wilbur, R.L. -Duke University
ASCLEPIADACEAE

NYCTAGINACEAE

MYRSINACEAE

ERYTHROXYLACEAE

AMARYLLIDACEAE

ACANTHACEAE,

ONAGRACEAE

ONAGRACEAE

HYPERICACEAE

CRUCIFERAE

\section{ANNONACEAE}

CACTACEAE

GESNERIACEAE, CORIARIACEAE

LACISTEMATACEAE, FLACOURTIACEAE, OLACACEAE ASCLEPIADACEAE CYCADACEAE MUSACEAE COMPOSITE

RUBIACEAE CHLORANTHACEAE MARCGRAVIACEAE BURMANNIACEAE

LAURACEAE

ONAGRACEAE EUPHORBIACEAE POLYGALACEAE

CAMPANULACEAE CUCURBITACEAE ONAGRACEAE 


\section{FORMAT}

\section{THE CHECKLIST Part I.}

The Checklist is an annotated listing of the names accepted as correct for the seed plants of Panama. For each name information is added on range inside and outside of Panama, on elevation and on habit. Plants that are introduced, naturalized or cultivated are identified as such. Whether or not the name was used in the Flora of Panama is also indicated.

This report includes only names accepted as correct for the flora of Panama: synonyms or names for taxa that do not occur in Panama are omitted.

The asterisk (*) on the left of an entry means that the taxon is not represented in the Flora of Panama, at least under this name. Referral to the Index (Part II) will usually indicate if the taxon is in the Flora under a different name.

Authors are presented in the abbreviated form following the International Code of Botanical Nomenclature (ICBN 1983) Recommendations 46D.1 and 46E.1 : amplifications of authorities by use of $e x$ and in are omitted.

Under the heading "Provinces" the Canal Area (ca) and the Comarca de San Blas (sa) are recognized. They are legal divisions in the country, although they are not provinces.

Ranges outside of Panama were entered as they were encountered (not corrected), thus in many cases both individual and general range codes appear. For example, a taxon might have cag (Central America General) and also a code for each or some of the countries in this region.

Habits were entered as they were encountered but were later standardized into the categories in Tables 15 and 16.

\section{SAMPLE ENTRIES (extracted from page 1):}

1 (1) CYCADACEAE
Explanation: 1 is the Dalla Torre \& Harms family number. (1) is the Flora of Panama family number. CYCADACEAE is the name of the family.

\section{"Cycas \\ "circinalis L. intr. cult}

\section{PROV: pa 000m: 0-1 RANGE: asi tree}

Explanation: "Cycas indicates that the first genus, Cycas, was not reported as an accepted genus in the Flora of Panama, at least not under this name. "circinalis indicates that the species Cycas circinalis was not reported in the Flora of Panama under this name. The author for the species name is L. (Carl Linnaeus). The species is introduced to Panama and is cultivated there. It occurs in Panama (pa) Province, it occurs between sea level and $1000 \mathrm{~m}$ elevation (0-1), and outside of Panama it occurs in Asia (asi). It is reported to be a tree.

\section{Podocarpus \\ "guatemalensis Standl. \\ "-var. allenii (Standl.) Buch. \& Gray PROV: bo cc etc. $\quad 000 \mathrm{~m}: 0-1$ etc.}

RANGE: cr gua tree

Explanation: Podocarpus is a genus that was treated in the Flora of Panama. However, the asterisk $\left({ }^{*}\right)$ before each of the entries under Podocarpus (4 in the actual Checklist) indicates that none of the present names were accepted in that treatment. This means that the names used in the Flora of Panama have been changed and perhaps new ones added. The Flora itself would give the names used there, and reference to the Index (Part II) will show the present correct names for the changed (reduced or excluded) names. The species Podocarpus guatemalensis Standl. is represented in Panama by the variety allenii. This variety was first described by Standl. (P. C. Standley in R. E. Woodson \& R. W. Schery, if the author is cited in full) but it was changed to its present status as a variety of Podocarpus guatemalensis by Buch. \& Gray (J. T. Buchholtz and N. E. Gray). The variety occurs in Bocas Del Toro (bo) and Coclé (cc) (also Chiriquí, Herrera, Panamá, and Veraguas Provinces), it occurs between sea level and $1000 \mathrm{~m}$ (and also between 1000 and $2000 \mathrm{~m}$ ), and outside of Panama it occurs in Costa Rica (cr) and Guatemala (gua). It is reported to be a tree. 


\section{THE INDEX Part II.}

This report includes all scientific names that were used in the Flora of Panama plus scientific names that have since been used for Panamanian plants. It includes accepted names, synonyms, names incidentally mentioned, and a few names for organisms other than vascular plants.

Names underlined are not currently correct: they are synonyms of other names. The indication "(FP: $x x x)$ " with an accepted name indicates that it was known as $x x x$ in the Flora of Panama.

All taxonomic ranks have been recognized as encountered. Because different authors adopted differing standards of coverage for other than the main ranks, the inclusion of these other ranks is uneven across the family spectrum.

Authors are cited in full (including ex and in embelishments).

The Source of Entry provides the reference to placement in the Flora of Panama, to other literature that affected the use of the name, or to a herbarium voucher. Unless otherwise indicated, all vouchers are deposited at the Missouri Botanical Garden (MO). Underlined numbers in this column refer to names mentioned incidentally in the Flora of Panama.

Families are identified by the Weber (1982) acronym and by the Dalla Torre \& Harms (1900-1907) number.

\section{AUTHORS}

Authors were standardized using Stafleu \& Cowan (1976-) as a main reference. In numerous cases, an author's name is abbreviated differently in a compound than when alone. For example, Ruiz is abbreviated when it is in the compound R. \& P. (Ruiz \& Pavón), Bentham becomes Benth. when alone and B. in the compound B. \& H. (Bentham \& Hooker). Where several authors have the same surname, it was sometimes possible to indicate which is meant by the use of initials, but as authorities were mainly entered as encountered without recourse to the literature to check details, such differentiating initials were often omitted. Although this ignores the grace of attributing many names to their founders, it seldom or never causes difficulties in the roles for which they are intended: the separation of homonyms published by different authors and as an aid in bibliographic referral. There are few cases, if any, where homonyms affecting the Flora of Panama were established by two different authors with the same surname, and perhaps none where referral to a Smith or a Fries without indicating which is the one meant will cause any confusion. In most cases, the different Smiths or Frieses worked on quite different plants at different times, and no confusion occurs.

In the Checklist, authors are presented in abbreviated form following recommendations (46C \& 46D of ICBN 1966, 1972; 46D \& 46E of ICBN 1978, 1983) of the Code of botanical nomenclature, that is, amplifications of authorities by use of ex and in are omitted. In the Index, the authorities are generally presented in full, although they are presented as they were encountered, and little attempt was made to ascertain whether still more amplified authorities might be derived. Thus, Willd. may appear as merely Willd. when it might have been expanded to (author) ex Willd. in L. (Sp. PI. ed. 4), or Benth. rather than Benth. in Benth. \& Hook. f. (Genera Plantarum).

\section{ELEVATIONS}

Four elevational ranges are recognized: 0-1000 $\mathrm{m}(0-3000 \mathrm{ft}), 1000-$ $2000 \mathrm{~m}(3000-6000 \mathrm{ft}), 2000-3000 \mathrm{~m}(6000-9000 \mathrm{ft})$, and $3000+\mathrm{m}$ (over $9000 \mathrm{ft}$ ). These ranges are a compromise between the ranges that might be most useful and the ranges that could be ascertained most reliably from the sources. Where the elevational range for a taxon is not known, the symbol we (without elevation) appears.

\section{GEOGRAPHICAL RANGES OUTSIDE OF PANAMA}

Geographical ranges outside of Panama were entered as they were encountered, thus in many cases both individual and general range codes appear. For example, a taxon might have cag (Central America General) and also a code for each country in this region. Most of the ranges indicated have been documented or verified during the course of this project. Thus it is believed that the Database errs on the side of omission rather than misstatement. 
A list of range symbols appears in Table 17 on page xxviii. For future publications based on this Database, it is hoped to add other coded distributions, for example; Chocó-Colombia, Brazil, and Mexico south of the Gulf of Tehuantepec/Rio San Juan depression which includes the states of Campeche, Chiapas, Quintana Roo, Tabasco, Yucatán. These regions are not separated at present. Brazil appears within the category sao (South America Other).

\section{HABITS}

Habits were generally entered as they were encountered with little verification. They were standardized in Table 15. It was immediately apparent that at present there is insufficient accepted vocabulary available to describe even some of the commonest growth habits. To describe such forms as bananas, pineapples, barrel cacti, and philodendrons as herbs, or acaulous palms and bamboos as shrubs is a distortion of the usual or traditional meanings of the terms and disguises the actual nature of the plants. Botanists working in the tropics are faced with the dilemma of insufficient habit type terminology. Many will apply well known or "standardized" terms in non-standard ways, or they will adopt more parochial terminology for the difficult cases they commonly encounter. For example, the term palmetto may be used to cover a wide range of dwarf palm-like forms, but the term has limited currency and uncertain definition beyond the particular palms it was derived to describe. The major life forms (nouns) are modified by adjectives listed in Table 16.

In this Database, any plants that are scandent are referred to as climbers, and this includes lianas, vines, hemiepiphytes, twiners, clamberers, and other ascendent or voluble habit concepts. Hemiepiphytes are separately identified in the few cases where they were clearly denoted in the Flora or literature: their number is greatly understated at this time.

\section{STATISTICS}

As it is being developed and modified, the Database is changed on a daily basis, and statistics drawn from it also change. Thus the statistics presented in this publication may differ slightly from the reports; they represent the numbers as the reports were requested but before editorial corrections.
TERMS USED FOR HABIT AND LIFE FORM

TABLE 15

MAJOR LIFE FORMS (HABITS)

$\begin{array}{ll}\text { climber } & \text { shrub } \\ \text { epiphyte } & \text { shrublet } \\ \text { hemiep(iphyte) } & \text { subshrub } \\ \text { herb } & \text { tree } \\ \text { parasite } & \text { treelet }\end{array}$

TABLE 16 MODIFIERS OF HABIT TYPE

\begin{tabular}{llll} 
Adjective & Form used & Adjective & Form used \\
\hline annual & ann & marine & marine \\
aquatic & aquat & parasite & paras \\
bushy & bushy & perennial & per \\
clambering & clamb & root(-climber) & root \\
climbing & climb & saprophyte & sapro \\
coarse & coarse & saxicole & saxic \\
epiphytic & epiph & succulent & succ \\
fresh-water & aquat & terrestrial & terr \\
giant & giant & twining & viny \\
hemiepiphytic & hemiep & vining & viny \\
& & woody & woody \\
\hline
\end{tabular}

\section{SOURCES}

Generally, source citations are restricted to the most recent revisions or to those where names were actually changed or records reported. Sources have been maintained within the Database documenting new province records, and this is to be found in the Taxonomic Listing (microfiche). One voucher is recorded for each name. 


\section{BIBLIOGRAPHY RELATING TO FLORA OF PANAMA DATABASE}

The following listing includes cited literature for all parts of this publication; the Introductions to Part I (this Part) and to the Taxonomic Listing (published separately on microfiche), to the Checklist (in this Part), to the Index (Part II), and to the Taxonomic Listing itself (microfiche).

Abalo, J. E. \& T. M. Antonio. 1981. A new Heliconia from Panama. Baileya 21: 139-142.

Adams, C. D. 1972. Flowering Plants of Jamaica. Univ. West Indies: Mona, Jamaica.

Agostini, G. 1980. Una nueva clasificación del género Cybianthus (Myrsinaceae). Acta Biol. Venez. 10: 129-185.

Alain, H. 1962 [1963]. Flora de Cuba. vol. 5. Editorial Universitaria. Univ. Puerto Rico: Rio Piedras.

Allorge, L. 1983. Espèces et combinaisons nouvelles d'ApocynacéesAmericaines. Bull. Soc. Bot. France 130, Lettres Bot. (4-5) 339-351.

Almeda, F. 1980. Central American novelties in the genus Blakea (Melastomataceae). Rhodora 82: 609-615.

1980. Pilocosta, A new genus of tropical American Melastomataceae. Syst. Bot. 5: 294-311.

1981. The Mexican and Central American species of Adelobotrys (Melastomataceae). Ann. Missouri Bot. Gard. 68: 204-212.

1981. New and reconsidered species of Miconia

(Melastomataceae) from Costa Rica and Panama. Proc. Calif. Acad. Sci. 42: 303-314.

1983. Three new Mesoamerican species of Miconia (Melastomataceae). Brittonia 35: 42-48.

1984. New and noteworthy additions to the Melastomataceae of Panama. Proc. Calif. Acad. Sci. 43: 269-282.

Anderson, L. C., R. L. Hartman \& T. F. Stuessy. 1979. Morphology, anatomy, and taxonomic relationships of Otopappus australis (Asteraceae). Syst. Bot. 4: 44- 56.

Andersson, L. 1977. The genus /schnosiphon (Marantaceae). Opera Bot. 43:1-114.

Asprey, G. F. \& R. G. Robbins, 1953. The vegetation of Jamaica. Ecol. Monogr. 23: 359-412.

Austin, D. F., D. A. Powell \& D. H. Nicolson. 1978. Stictocardia tiliifolia (Convolvulaceae) re-evaluated. Brittonia 30: 195. \& G. W. Staples. 1983. Additions and changes in the neotropical Convolvulaceae- notes on Merremia, Operculina, and Turbina. J. Arnold Arboretum 64: 483-489.
Badillo, V. M. 1971. Monografía de la familia Caricaceae. 1-221. Assoc. Profesores: Maracay.

Barringer, K. 1983. Polygala dukei (Polygalaceae), a new species from Panama. Ann. Missouri Bot. Gard. 70: 203-204.

1983. Notes on Central American Aristolochiaceae. Brittonia 35: $171-174$.

1984. A new species of Guatteria (Annonaceae) from

Panama. Ann. Missouri Bot. Gard. 71: 1186-1187. 1985. Three new species of Elleanthus (Orchidaceae) from Central America. Brittonia 37: 286- 290.

Bockemuhl, L. 1984. Die Gattung Odontoglossum H.B.K. Studien zu einer natürlichen Gliederung (5. Fortsetzung). Die Orchidee 35: 57- 58.

Breedlove, D. E., P. E. Berry \& P. H. Raven. 1982. The Mexican and Central American species of Fuchsia (Onagraceae) except for sect. Encliandra. Ann. Missouri Bot. Gard. 69: 209-234.

Burger, W. 1973. Notes on the flora of Costa Rica, 3: new species in the Moraceae. Phytologia 26: 421-434.

1977. Flora Costaricensis. Fagaceae in Fieldiana Bot. 40: 59-82. 1983. Flora Costaricensis. Fieldiana Bot. N.S. 13: 1-255.

Burt-Utley, K. 1982. New species in Begonia section Gireoudia (Begoniaceae) from Central America. Brittonia 34: 189- 198.

Churchill, S. P. \& W. R. Buck. 1982. A taxonomic investigation of Leptotheca (Rhizogoniaceae). Brittonia 34: 1-11.

Correll, D. S. \& M. C. Johnston. 1970. Manual of the Vascular Plants of Texas. Texas Research Foundation: Renner.

Croat, T. B. 1978. Flora of Barro Colorado Island. Stanford Univ. Press: Stanford.

1979. Matayba apetala (Sapindaceae) new for the Flora of Panama. Phytologia 44: 381-383. 1981. A revision of Syngonium (Araceae). Ann. Missouri Bot. Gard. 68: 565- 651. 1981. Studies in Araceae III: new species of Anthurium from Central America. Selbyana 5: 315-31. 1983. A revision of the genus Anthurium (Araceae) of Mexico and Central America. Part I: Mexico and Middle America. Ann. Missouri Bot. Gard. 70: 211-420. 1985. The large monocots of Panama. Pp. 5-12 in W. G. D'Arcy \& M. D. Correa A., The Botany and Natural History of Panama: La Botánica e Historia Natural de Panamá. Monogr. Syst. Bot. Missouri Bot. Gard. Vol. 10. 1986. A revision of the genus Anthurium (Araceae) of Mexico and Central America. Part III: Panama. Monogr. Syst. Bot. Missouri Bot. Gard. Vol. 14. 
\& R. L. Baker. 1979. The genus Anthurium. Brenesia 16 Suppl. 1: 1 - 174.

Cuatrecasas, J. 1982. Miscellaneous notes on Neotropical Flora XV. Phytologia 52: 166-177.

Dalla Torre, C. G. \& H. Harms. 1900-1907. Genera Siphonogamarum. G. Engelmann: Leipzig.

D'Arcy, W. G. 1975. New names and species of neotropical plants: Compositae. Phytologia 30: 5- 6.

1981a. The Flora of Panama: historical outline and selected bibliography. Ann. Missouri Bot. Gard. 67: v-viii.

1981b. A new species of Hernandia (Hernandiaceae) from Panama. Ann. Missouri Bot. Gard. 68: 224-225.

1985. Publication of Verbesina fuscasiccans. Phytologia 56: 500.

\& R. L. Liesner. 1981. Hedyosmum (Chloranthaceae) in Panama. Syst. Bot. 6: 74- 86.

\& B. Hammel. 1985. The plants of 'Ocoquili' Island, San Blas Coast, Panama. Ann. Missouri Bot. Gard. 72: 264-267.

J. L. Gentry \& J. E. Averett. 1981. Recognition of Brachistus (Solanaceae). Ann. Missouri Bot. Gard. 68: 227-228.

\& M. D. Correa A. 1985. The Botany and Natural History: La Botánica e Historia Natural de Panamá. Monogr. Syst. Bot. Missouri Botanical Garden. Vol. 10.

Davidse, G., T. R. Soderstrom \& R. P. Ellis. 1986. Pohlidium petiolatum (Poaceae: Centotheceae), a new genus and species from Panama. Syst. Bot. 11: 131-144.

Dietrich, H. \& M. A. Diaz D. 1984. Eine neue blattlose Orchidee für Panama: Campylocentrum dressleri H. Dietrich et M. Diaz. Die Orchidee 35: 28-30.

Dodson, C. H. in C. H. Dodson. 1980. Icones Plantarum Tropicarum pl. 155.

Dressler, R. L. 1972. Terrestrial plants of Panama. Bull. Biol. Soc. Washington 2: 179-186.

1979. A distinctive new Hexisea from Panama: Hexisea arctata Dressler. Orquidea (Mex.) 7: 222-225.

1979. Una Sievekingia llamativa de Panamá. Orquideol. 13: 221-227.

1980. A new name for the dwarf purple Anthurium. Aroideana 3: 55.

1980. Checklist of the orchids of Panama. Pp. I-XXVI in

Orchids of Panama. Monogr. Syst. Bot. Missouri Bot. Gard.

Vol. 4: 1-26.
1981. Maxillaria insolita, especie nueva Panameña.

Orquideol. 14: 203-208.

1981. El género Neowilliamsia Garay. Orquidea (Mex.) 8:

27-36.

1981. Dos Kefersteinia nuevas de Panamá. Orquideol. 16: 4762.

1983. Palmorchis in Panama mit einer neuen Art, Palmorchis nitida, an einem unerwarteten Standort. Die Orchidee 34: 25-31.

1983. Eine charakteristische neue Cochleanthes aus Panama: Cochleanthes anatona. Die Orchidee 34: 157-161. 1983. Otra nueva Neowilliamsia de Panamá. Orquidea (Mex.) 9: $32-30$.

Dwyer, J. D. 1985. The history of plant collecting in Panama. Pp. 179-183 in W. G. D'Arcy \& M. D. Correa A., The Botany and Natural History of Panama: La Botánica e Historia Natural de Panamá. Monogr. Syst. Bot. Missouri Bot. Gard. Vol. 10.

Edmonds, J. M. 1986. Biosystematics of Solanum sarrachoides Sendtner and S. physalifolium Rusby (S. nitidibaccatum Bitter). Bot. J. Linn. Soc. (London) 92: 1-38.

Fallen, M. E. 1983. A systematic revision of Anechites (Apocynaceae). Brittonia 35: 222-231.

Fontella Pereira, J. 1977. Revisão taxonômica do gênero Tassadia Decaisne (Asclepiadaceae). Arquivos Jard. Bot. Rio de Janeiro 21: 235-359.

Fosberg, F. R. 1965. Revision of Albizia sect. Pachysperma (Leguminosae-Mimosoideae). Reinwardtia 7: 71-90.

Fryxell, P. A. 1981. Revision and expansion of the neotropical genus Wercklea (Malvaceae). J. Arnold Arboretum 62: 457-486.

Gates, B. 1982. Banisteriopsis, Diploterys (Malpighiaceae). FI. Neotrop. Monogr. 30.

Gentry, A. H. 1980. Bignoniaceae: Part 1. FI. Neotrop. 25: 1-117. 1982. New or noteworthy species of Middle American Bignoniaceae. Wrightia 7: 83-89. 1985. Studies in Bignoniaceae 48: new South American species of Bignoniaceae. Phytologia 57: 240-248.

Glassman, S. F. 1972. A revision of B. E. Dahlgren's Index of American Palms. Phanerog. Monogr. 6. Verlag Von Cramer.

Goldblatt, P. 1978. An analysis of the flora of southern Africa: its characteristics, relationships, and origins. Ann. Missouri Bot. Gard. 65: 369-436.

Gómez P., L. 1982. Plantae Mesoamericanae Novae. VI. Phytologia 52: $153-156$. 
Gould, F. W. 1979. The genus Bouteloua (Poaceae). Ann. Missouri Bot. Gard. 66: 348- 413.

Grayum, M. \& B. Hammel. 1982. Three new species of Cyclanthaceae from the Caribbean lowlands of Costa Rica. Syst. Bot. 7: 221-229.

Hamilton, C. W. 1985. Notes on and descriptions of seven new species of Mesoamerican Clethraceae. Ann. Missouri Bot. Gard. 72: 539-543.

Hammel, B. \& W. G. D'Arcy. 1981. New taxa from the uplands of western Panama. Ann. Missouri Bot. Gard. 68: 213-217.

Hamer, F. \& C. H. Dodson in C. H. Dodson. 1982. Icones Plantarum Tropicarum PI. 800

Harling, G. 1958. Monograph of the Cyclanthaceae. Act. Hort. Berg. 18: 1-428.

Hatfield, G. M. et al. 1981. An investigation of Panamanian ipecac: botanical source and alkaloid analysis. J. Nat. Prod. 44: 452-456.

Hekking, W. H. A. 1979. Studies on neotropical Violaceae tribe Rinoreae I. Phytologia 43: 461-489.

Holmes, W. C. \& S. McDaniel. 1979. Notes on Mikania (Compositae) V. Phytologia 41: 187.

ICBN. 1966. International Code of Botanical Nomenclature. Regnum Veg. 46. Int. Bureau for Plant Taxonomy and Nomenclature: Utrecht.

ICBN. 1972. International Code of Botanical Nomenclature. Regnum Veg. 82. A. Ooosthoek's Uitgev.: Utrecht.

ICBN. 1978. International Code of Botanical Nomenclature. Regnum Veg. 97. Bohn, Scheltema \& Holkema: Utrecht.

ICBN. 1983. International Code of Botanical Nomenclature. Regnum Veg. 111. Bohn, Scheltema \& Holkema: Utrecht.

Iltis, H. 1981. Studies in the Capparidaceae XV: Capparis panamensis, n. sp. Ann. Missouri Bot. Gard. 68: 681- 685.

Irwin, H. S. \& R. C. Barneby. 1978. Monographic studies in Cassia (Leguminosae-Caesalpinioideae). III. Sections Absus and Grimaldia. Mem. New York Bot. Gard. 30: 1-300. \& ___ 1982. The American Cassiinae: a synoptical revision of Leguminosae tribe Cassieae subtribe Cassiinae in the New World. Mem. New York Bot. Gard. 35(1-2): 1-918.

Janzen, D. H. 1974. Swollen-thorn acacias of Central America. Smithsonian Contr. Bot. 13: 1-101.

Jenny, R. 1983. Die Gongorinae, 6. Gongora Teil VI. Gongora gibba, Gongora aromatica, Gongora grossa, Gongora fulva. Die Orchidee 34: 147-157.
1984. Die Gongorinae, 6. Gongora Teil IX. Gongora claviodora und Gongora odoratissima. Die Orchidee 35: 172-176.

Johnson, I. M. 1949. The botany of San José Island. Sargentia 8: 1-306.

Johnston, M. C. \& L. A. Johnston. 1978. Rhamnus. FI. Neotrop. Monogr. 20: 1-96.

Kaastra, R. C. 1982. Pilocarpinae (Rutaceae). FI. Neotrop. 33: 1- 185.

Keil, D. \& T. Stuessy 1981. Systematics of /socarpha (Compositae: Eupatorieae). Syst. Bot. 6: 258-287.

1981. Pectis linifolia (Composite: Tageteae) added to the flora of Panama. Ann. Missouri Bot. Gard. 68: 225.

Kennedy, H. 1978. Notes on Central American Marantaceae III. New species of Calathea from Costa Rica and Panama. Brenesia 8: 351.

1978. Calathea elegans and C. spiralis - new Panamanian Marantaceae. Bot. Not. 131: 349.

1978. Systematics and pollination of the "closed-flowered" species of Calathea (Marantaceae). Univ. Calif. Pub. Bot. 71: 41.

King, R. M. \& H. Robinson. 1983. Studies in the Eupatorieae (Asteraceae) CCXVI: various new species from the Andes and Panama. Phytologia 54: 36-51.

1985. Studies in the Eupatorieae (Asteraceae). CCXXI. Additional species from tropical America. Phytologia 58: 258-272.

Knapp, S. D. 1986. A revision of Solanum section Geminata (G. Don) Walpers. Ph.D. thesis, Cornell Univ.

Kress, J. 1981. New Central American taxa of Heliconia (Heliconiaceae). J. Arnold Arboretum 62: 244-260. 1984. Systematics of Central American Heliconia (Heliconiaceae) with pendent inflorescences. J. Arnold Arboretum 64: 429- 532.

Krukoff, B. A. 1979. Supplementary notes on the American species of Strychnos XVI. Phytologia 41: 201-238.

1982. Supplementary notes on American Menispermaceae XVII. Phytologia 50: 80- 111.

Leeuwenberg, A. J. M. 1974 [1975]. The Loganiaceae of Africa XII. A revision of Mitreola. Meded. Landbrouwhogeschool 74-23:1-28.

1976. The Apocynaceae of Africa I: Tabernaemontana L. Adansonia sér. 2, 16: 383-392.

León, J. 1966. Central American and West Indian species of Inga (Leguminosae). Ann. Missouri Bot. Gard. 53: 115-264.

Linnaeus, C. 1753. Species Plantarum. Stockholm.

Lleras, E. 1978. Trigoniaceae. FI. Neotrop. Monogr. 19:1- 73. 
Luer, C. A. 1979. Icones Pleurothallidinarum: miscellaneous new species in the Pleurothallidinae. Selbyana 5: 145-196. 1981. Miscellaneous new species and combinations in the Pleurothallidinae (Orchidaceae). Phytologia 49: 197-240. 1981. Miscellaneous new species and combinations in the Pleurothallidinae (Orchidaceae). Phytologia 55: 1175-203. 1981. Miscellaneous new species and combinations in the Pleurothallidinae (Orchidaceae). Phytologia 57: 59-64. 1982. Miscellaneous new species and combinations in the Pleurothallidinae (Orchidaceae). Selbyana 7: 100-128. 1983. Miscellaneous new species in the Pleurothallidinae (Orchidaceae). Phytologia 54: 379-380.

Lundell, C. 1979. Neotropical Myrsinaceae. Wrightia 5:277-300. 1980. Neotropical Myrsinaceae. Wrightia 6: 101-119. 1981. Studies of American plants XX. Phytologia 48: 131-136. 1981. Neotropical Myrsinaceae IV. Phytologia 48: 137-142. 1981. Neotropical Myrsinaceae VI. Phytologia 49: 341-354. 1982. Neotropical Myrsinaceae VII. Wrightia 7: 38-50. 1983. Neotropical Myrsinaceae X. Phytologia 54: 285. 1984. Neotropical Myrsinaceae XI. Phytologia 55: 235-242. 1984. Neotropical Myrsinaceae XII. Phytologia 56: 19-27. 1985. Neotropical species of the genus Perrottetia (Celastraceae). Phytologia 57: 231-238. 1985. Neotropical Myrsinaceae XVI. Phytologia 57: 449-452. 1985. Neotropical Myrsinaceae XVII. Phytologia 58: 273-278.

Luteyn, J. L. 1983. Ericaceae-Part 1. Cavendishia. FI. Neotrop. Monogr. 35: 1-290.

Markgraf, F. et al. 1981. Two new species of Bonafousia (Apocynaceae) from Panama and Colombia-Ecuador. Ann. Missouri Bot. Gard. 68: 677-680.

Maas, P. J. M. 1981. On the true identity of Lagenanthus parviflorus Ewan (Gentianaceae). Ann. Missouri Bot. Gard. 68: 685- 686.

Marques, Maria do Carmo Mendes. 1979. Revisão das espécies do gênero Polygala L. (Polygalaceae) do Estado do Rio de Janeiro. Rodriguésia 31: 69-341.

Mathias, M. E. \& L. Constance. 1962. The Andean genus Niphogeton (Umbelliferae) revisited. Brittonia 14: 154. 1973. New and reconsidered Mexican Umbelliferae. Contr. Univ. Mich. Herb. 11: 1-24.

\& W. L. Theobald. 1981. A revision of the genus Hyperbaena (Menispermaceae). Brittonia 33: 81-104.

McDade, L. A. 1982. Three new species of Aphelandra (Acanthaceae) from Central America. Ann. Missouri Bot. Gard. 69: 402-411.
Mears, J. A. 1977. The nomenclature and type collections of the widespread taxa of Alternanthera (Amaranthaceae). Proc. Acad. Nat. Sci. Philadelphia 129: 1-21. 1980. The Linnaean species of Gomphrena L. (Amaranthaceae). Taxon 29: 85-95.

Moldenke, H. N. 1979. Additional notes on the genus Congea II. Phytologia 45: 270-280. 1983. Notes on Eriocaulaceae. Phytologia 54: 69-81. 1983. Sixth summary supplement. Phytologia 54: 228-245.

Murphy, H. 1986. A revision of the genus Fischeria (Asclepiadaceae). Syst. Bot. 11: 229-241.

Odessy 1966. The Odessy World Atlas. Golden Press: New York.

Ornduff, R. 1979. Neotropical Nymphoides (Menyanthaceae). Brittonia 21: 346-352.

Pennington, T. D. 1981. Meliaceae. FI. Neotrop. Monogr. 28: 1-470.

Pipoly, J. J. \& C. L. Lundell. 1982. Contributions toward a monograph of Cybianthus (Myrsinaceae) II. Wrightia 7: 52-54.

Pilz, G. E. 1981. Sapotaceae of Panama. Ann. Missouri Bot. Gard. 68: $172-203$.

Polhill, R. M. \& W. T. Stearn. 1976. Linnaeus's notes on Plumier drawings with special reference to Mimosa latisiliqua. Taxon 25: 323-325.

\& P. H. Raven. 1981. Advances in Legume Systematics. 2 vols. Royal Botanic Gardens: Kew.

Porter, D. M. 1970. A new Tetragastris (Burseraceae) from Panama. Madroño 20: 346-347.

Prance, G. T. 1983. Additions to neotropical Dichapetalaceae. Brittonia 35: 49-54.

Prance, G. T. \& S. A. Mori. 1979. Lecythidaceae-Part 1 The Actinomorphic-flowered New World Lecythidaceae (Asteranthos, Gustavia, Grias, Allantoma, \& Cariniana). FI. Neotrop. Monogr. 21: 1-270.

Raven, P. H. \& D. I. Axelrod. 1978. Origin and relationships of the California Flora. Univ. Calif. Pub. Bot. 72: 1-134.

Rhodes, D. G. A revision of the genus Cissampelos. Phytologia 30: 415-484.

Robertson, K. 1982. Odonella, a new genus of Convolvulaceae from tropical America. Brittonia 34: 417-423.

Robbrecht, R. 1982. The identity of the Panamanian genus Dressleriopsis (Rubiaceae). Ann. Missouri Bot. Gard. 69: 429.

Rodríguez-Carrasquilla, H. A. 1980. Studies in neotropical Sapotaceae III. Phytologia 45: 287-288.

Rohwer, J. 1982. A taxonomic revision of the genera Seguieria Loefl. and Gallesia Casar. Mitt. Staatssaml. Bot. München 18: 231-288. 
Rudd, V. E. 1981. Two new species of Paramachaerium (Leguminosae) and a brief résumé of the genus. Brittonia 33 435-440.

. 1982. Dalbergia darienensis (Leguminosae), a new species from Panama. Phytologia 50: 183-184.

Seemann, B. 1854. The Botany of the H.M.S. Herald (Flora of the Isthmus of Panama). London.

Senghas, K. \& L. Bockemuhl. 1984. Brassavola glauca Lindl. 1839. Die Orchidee 35: 107.

Seymour, F. C. 1981. Bipinnate Leguminosae of Nicaragua. Phytologia 48: 1-72.

Seigerist, E. 1984 [1985]. Herbarium specimens of the Orchidaceae of Central Central America. Selbyana 7: 281-311.

Sheffer, R.C., W. L. Theobald \& H. Kamemoto. 1980. Taxonomy of Anthurium scandens (Araceae). Aroideana 3: 86-93.

Smith, L. B. \& R. W. Read. 1979. Notes on Bromeliaceae XL. Phytologia 41: 329-345.

Snelders, H. C. in P. J. M. Maas \& H. C. Snelders. 1981. Burmanniaceae in Notes on New World saprophytes II. Acta Bot. Neerl. 30: 140-143.

Soderstrom, T. R. 1982. Cryptochloa dressleri (Poaceae), a new bambusoid grass from Panama. Brittonia 34: 25-29. \& F. O. Zuloaga. 1985. New species of grasses in Arberella, Cryptochloa, and Raddia (Poaceae: Bambusoideae: Olyreae). Brittonia 37: 22-35.

Sohmer, S. H. 1977. A revision of Chamissoa. Bull. Torrey Bot. Club 104: 111-126.

Stafleu, F. A. \& R. S. Cowan. 1976-- Taxonomic Literature. Regnum Veg. $94,98,105,110,112,115$. Bohn, Scheltema \& Holkema: Utrecht.

Standley, P. C. 1927. The flora of Barro Colorado Island. Smithsonian Misc. Collect. 78(8): 1-132.

1928. Flora of the Panama Canal Zone. Contr. U. S. Natl. Herb. 27: 1-416.

Stearn, W. T. 1971. Linnaean classification, nomenclature, and method. Appendix, Pp. 242-249 in W. Blunt. The Compleat Naturalist: A Life of Linnaeus. Viking: New York.

Steyermark, J. A. 1963. Flora of Missouri. lowa Univ. Press: Ames. 1984. Piperaceae. Vol 2, part 2. Flora de Venezuela. Inst. Nac. Parques.

Taylor, C. M. \& D. Lorence. 1985. Lectotypification of Palicourea galeottiana M. Martens (Rubiaceae) and a new name for this common species. Taxon 34: 669.

The World Almanac \& Book of Facts 1981. Newspaper Enterprise Association, Inc: New York.
Torres, A. M. 1968. Revision of Jaegeria (Compositae- Heliantheae). Brittonia 20: 52- 73.

Tucker, G. C. 1983. The taxonomy of Cyperus (Cyperaceae) in Costa Rica and Panama. Syst. Bot. Monogr. 2: 1-85. 1986. The species of Cyperus described by Liebmann in "Mexicos halvgraes." Syst. Bot. 11: 14-19.

Utley, J. F. 1983. A revision of the Middle American thecophylloid Vrieseas (Bromeliaceae). Tulane Stud. Zool. Bot. 24: 1-81.

Van Beek, T. A., R. Verpoorte, A. Baerheim Svendsen, A. J. M. Leeuwenberg, \& N. G. Bisset. 1984. Tabernaemontana L. (Apocynaceae): a review of its taxonomy, phytochemistry, ethnobotany and pharmacology. J. Ethnopharmacol. 10: 1-156.

Van der Werff, H. 1984. Notes on neotropical Lauraceae. Ann. Missouri Bot. Gard. 71: 1180-1183.

Weber, W. A. 1982. Mnemonic three-letter acronyms for the families of vascular plants: a device for more effective herbarium curation. Taxon 31: 74-88.

Whalen, M. D. et al. 1981. Taxonomy of Solanum section Lasiocarpa. Gentes Herb. 12: 1-129.

Wilbur, R. L. 1981. Additional Panamanian species of Burmeistera (Campanulaceae: Lobelioideae). Ann. Missouri Bot. Gard. 68: 167-171.

\& J. L. Luteyn. 1981. Additions to the Ericaceae of Panama. Missouri Bot. Gard. 68: 154-166.

Wilder, G.J. 1978. Two new species and a new subgenus of Cyclanthaceae. J. Arnold Arboretum 59: 74-102.

Williams, L.O. \& P.H. Allen. 1980. Orchids of Panama. Monogr. Syst. Bot. Missouri Botanical Garden. Vol. 4. [facsimile reprint of Orchidaceae fascicles from Flora of Panama with checklist by R.E. Dressler.]

Willis, J. C. 1966. A Dictionary of Flowering Plants and Ferns. ed. 7, rev. H. K. Airy Shaw. Cambridge Univ. Press: Cambridge.

Windler, D. R. 1966. A revision of the genus Neptunia (Leguminosae). Austral. J. Bot. 14: 379-420.

Wit, H. C. D. de. 1974. Typification of Leucaena leucocephala (Lam.) de Wit, Lysiloma latisiliquum (L.) Bth., and Acacia glauca (L.) Moench. Taxon 24: 349-352.

Woodson, Robert E., Jr. 1943-1981. Flora of Panama. Ann. Missouri Bot. Gard., various issues, vols. 30-67.

Wunderlin, R. P. 1973. The tribe Cerceae and a revision of Bauhinia subgenus Bauhinia in Middle America and Antilles. Ph.D. thesis. Pp. 360. St. Louis Univ.

Wurdack, J. J. 1981. Certamen Melastomataceis XXXII. Phytologia 48: $238-252$. 
TABLE 17

SYMBOLS FOR OCCURRENCES OUTSIDE OF PANAMA
1. BY SYMBOL.

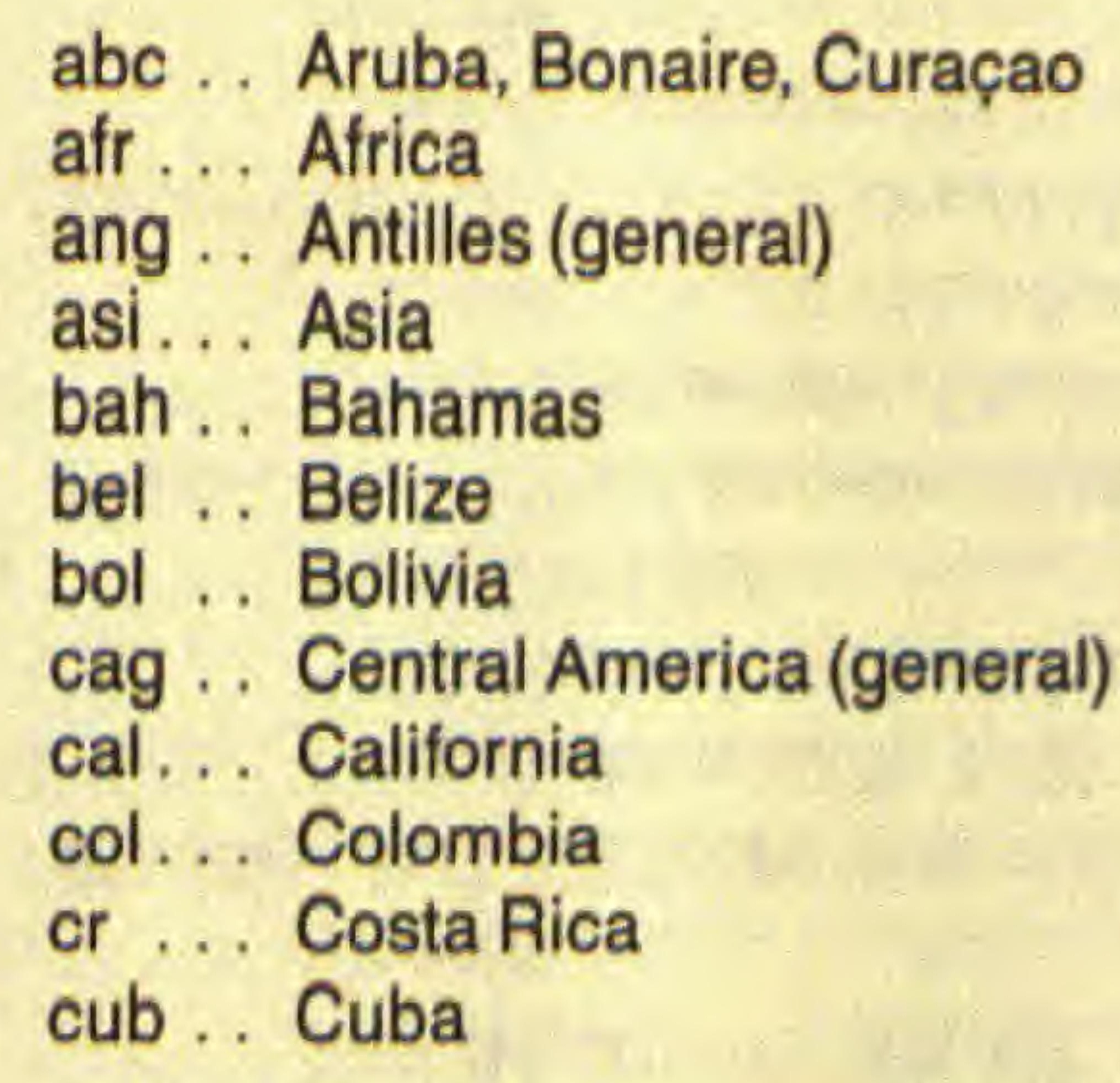

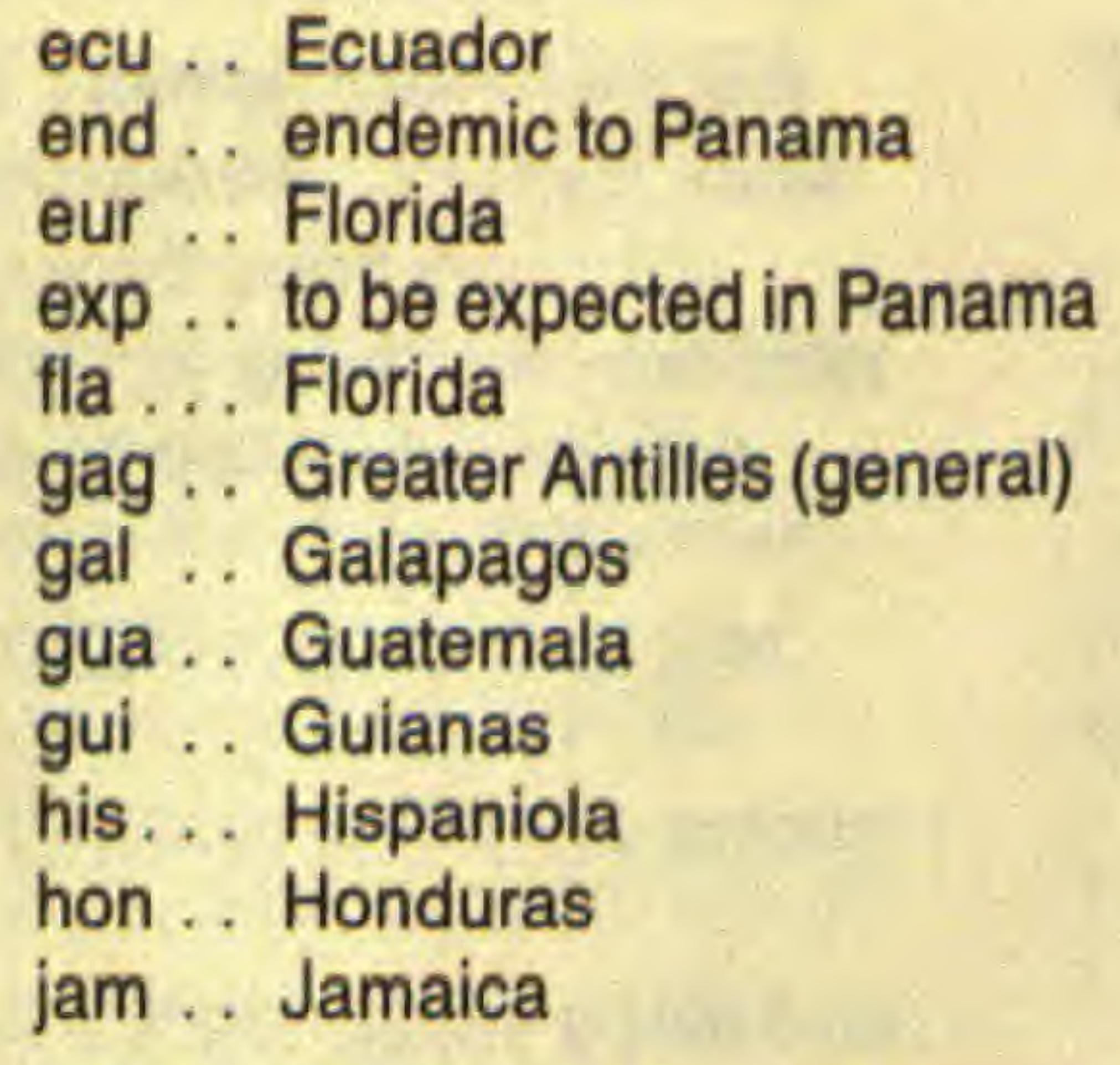

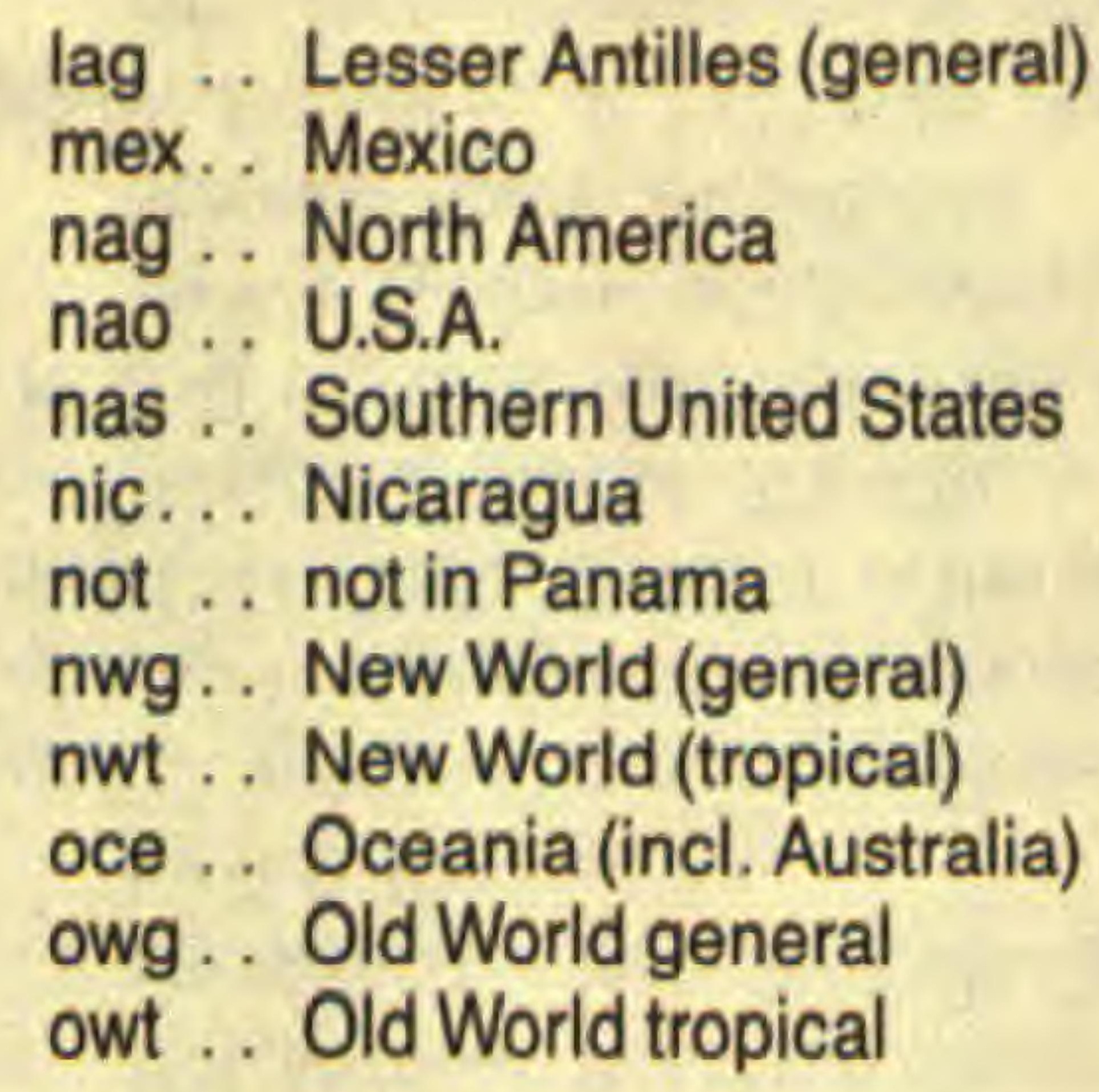

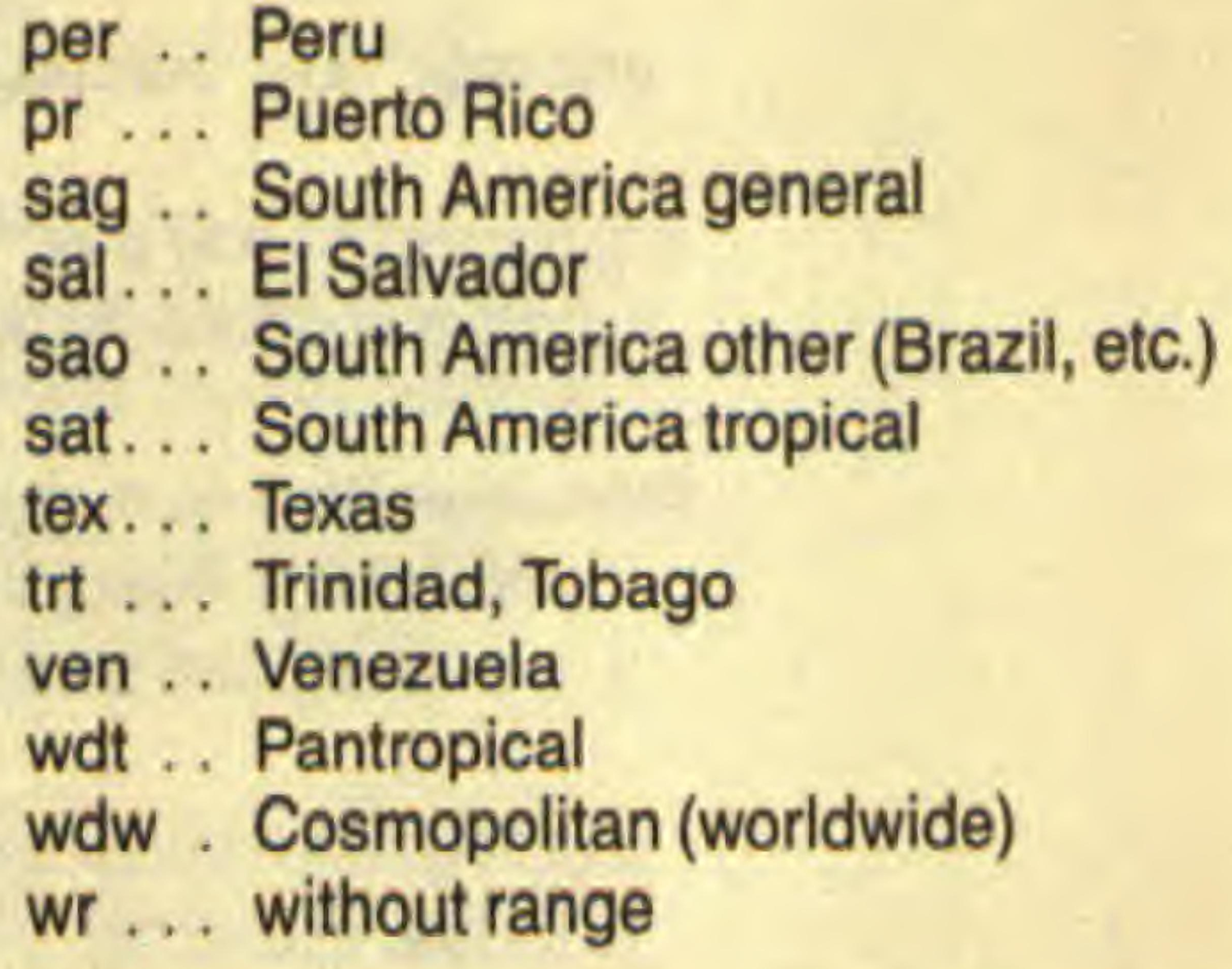

\section{SYMBOLS FOR OCCURRENCES OUTSIDE OF PANAMA}

\section{PANAMA}

endemic ............ en

to be expected in Panama

not in Panama

end

$\exp$ not

\section{CENTRAL AMERICA}

Costa Rica

Nicaragua ........... nic

El Salvador ........... sal sal

Guatemala .......... gua

Belize .............. bel

Central America general .

ELEVATION SYMBOLS

0 . below $1000 \mathrm{~m}$

1. . 1,000-2,000 m

2. . $2,000-3,000 \mathrm{~m}$

3. $3,000 \mathrm{~m}$ and over

we. . elevation not available

\section{NORTH AMERICA \\ SOUTH AMERICA}

Florida ............ fla

Texas ............... tex

California ........... ca

Mexico .............. mex

Southern United States . . nas

United States.......... nao

(North America other)

North America ......... nag

(North America general,

includes Canada)

\section{GENERAL}

Pantropical ........... wdt (worldwide, tropical)

Cosmopolitan (worldwide) wdw Without range ......... Wr
Colombia ..........

Venezuela ...........

Guianas ........... gui

Ecuador ........... ecu

Galapagos ......... gal

Peru .............. per

Bolivia ........... bol

South America tropical sat

South America general sag

South America other

(includes Brazil)

\section{BY REGION.}

ANTILLES

Bahamas .......... bah

Cuba ............. cub

Hispaniola .......... his

Puerto Rico.......... pr

Trinidad, Tobago .......

Aruba, Bonaire, Curaçao

Greater Antilles general.

Lesser Antilles general .

Antilles general.......
OLD WORLD

Africa..........

Europe .........

Asia ...........

Oceania

Old World general

Old World tropical

\section{NEW WORLD}

New World tropical New World general 
TABLE 18

LIST OF FAMILIES

Family Name

Acronym Dalla (Flora Family Name (Weber Torre of

1982) \& Harms Panama)

ACANTHACEAE

AIZOACEAE

ALISMATACEAE

AMARANTHACEAE

AMARYLLIDACEAE

ANACARDIACEAE

ANNONACEAE

APOCYNACEAE

AQUIFOLIACEAE

ARACEAE

ARALIACEAE

ARISTOLOCHIACEAE

ARAUCARIACEAE

ASCLEPIADACEAE

AVICENNIACEAE

BASELLACEAE

BATACEAE

BEGONIACEAE

BERBERIDACEAE

BETULACEAE

BIGNONIACEAE

BIXACEAE

BALSAMINACEAE

BROMELIACEAE

BURMANNIACEAE

BRUNELLIACEAE

BALANOPHORACEAE

BOMBACACEAE

BORAGINACEAE

BURSERACEAE

BUTOMACEAE

BUXACEAE

CACTACEAE

$\begin{array}{lr}\text { ACA } & 266 \\ \text { AIZ } & 84 \\ \text { ALI } & 15 \\ \text { AMA } & 79 \\ \text { AML } & 40 \\ \text { ANA } & 153 \\ \text { ANN } & 98 \\ \text { APO } & 247 \\ \text { AQF } & 157 \\ \text { ARA } & 23 \\ \text { ARL } & 227 \\ \text { ARS } & 74 \\ \text { ARU } & 6 \\ \text { ASC } & 248 \\ \text { AVI } & 253 a \\ \text { BAS } & 86 \\ \text { BAT } & 81 \\ \text { BEG } & 208 \\ \text { BER } & 93 \\ \text { BET } & 61 \\ \text { BIG } & 258 \\ \text { BIX } & 194 \\ \text { BLS } & 168 \\ \text { BML } & 32 \\ \text { BMN } & 49 \\ \text { BNL } & 119 \\ \text { BNP } & 73 \\ \text { BOM } & 177 \\ \text { BOR } & 252 \\ \text { BRS } & 139 \\ \text { BUT } & 16 \\ \text { BUX } & 149 \\ \text { CAC } & 210\end{array}$

(177)

(57)

(4)

(53)

(24)

(101)

(67)

(162)

(102)

(11)

(144)

(50)

$(-)$

(163)

(168a)

(58a)

(55)

(133)

(63)

(40)

(172)

(125)

(110)

(17)

(28)

(80a)

(49)

(116)

(167)

(91)

(5)

(99)

(134)

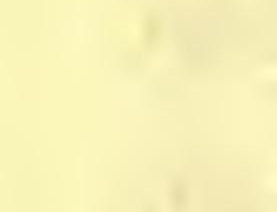

1982) \& Harms Panama)

982) \& Harms Panama)
CASUARINACEAE

CYCADACEAE

COCHLOSPERMACEAE

CARYOCARACEAE

CELASTRACEAE

CHENOPODIACEAE

CISTACEAE

CLETHRACEAE

CALLITRICHACEAE

CHLORANTHACEAE

COMBRETACEAE

COMMELINACEAE

COMPOSITAE

CANNACEAE

CONNARACEAE

CONVOLVULACEAE

CORNACEAE

CAPPARACEAE

CAPRIFOLIACEAE

CARICACEAE

CORIARIACEAE

CRASSULACEAE

CRUCIFERAE

CARYOPHYLLACEAE

CERATOPHYLLACEAE

CUCURBITACEAE

CUNONIACEAE

CYCLANTHACEAE

CYPERACEAE,

CYRILLACEAE

DICHAPETALACEAE

DILLENIACEAE
Acronym Dalla (Flora Family Name

(Weber Torre of

CAM 276

CAS 51

CCD 1

$\mathrm{CCH} \quad 195$

CCR 183

CEL $\quad 158$

CHN 78

CIS 193

CLE 230

CLL 148

CLR 54

CMB 221

CMM 33

CMP 280

CNA 47

CNN 127

CNV

COR 229

CPP 107

CPR 271

CRC 205

CRR 151

CRS 115

CRU 105

CRY 87

CTP 89

CUC 275

CUN 120

CYC 22

CYP 20

CYR 154

DCH 146

DLL $\quad 180$

\section{(183)}$$
\text { (-) DIOSCOREACEAE }
$$

(1) EBENACEAE

(126) ELAEOCARPACEAE

(120) ERICACEAE

(103) ERIOCAULACEAE

(52) ERYTHROXYLACEAE

(124) EUPHORBIACEAE

(147) FAGACEAE

(98) FLACOURTIACEAE

(35) GENTIANACEAE

(139) GERANIACEAE

(18) GNETACEAE

(184) GRAMINEAE

(31) GESNERIACEAE

(82) GUTTIFERAE

(164) HAEMODORACEAE

(146) HALORAGACEAE

(73) HIPPOCASTANACEAE

(180) HYDROCHARITACEAE

(131) HUMIRIACEAE

(100) HIPPOCRATEACEAE

(77) HERNANDIACEAE

(74) HYDROPHYLLACEAE

(59) HYPERICACEAE

(61) ICACINACEAE

(182) IRIDACEAE

(80) JUGLANDACEAE

(10) JUNCACEAE

(8) LABIATAE

(101a) LAURACEAE

(96a)

(118)
Acronym Dalla (Flora

(Weber Torre of

1982) \& Harms Panama)
DRS 112

DSC 43

EBN 240

ELC 171

ERI 233

ERO 30

ERX 134

EUP 147

FAG 62

FLC 199

GEN 246

GER 129

GNE 7

GRM 19

GSN 262

GUT 187

HAE 39

HAL 225

HCS 164

HDC 17

HOU 133

HPC $\quad 159$

HRN 103

HYD 251

HYP $\quad 187$

ICC 162

IRI 44

JUG $\quad 60$

JUN 36

LAB $\quad 254$

LAU 102

LCS 55

LCY 219 (76a)

$(148,149)$ 
TABLE 18

LIST OF FAMILIES (continued)

\begin{tabular}{|c|c|c|c|c|c|c|c|c|c|c|c|}
\hline Family Name & $\begin{array}{c}\text { Acronym } \\
\text { (Weber } \\
1982)\end{array}$ & $\begin{array}{l}\text { Dalla } \\
\text { Torre } \\
\text { \& Harms }\end{array}$ & $\begin{array}{l}\text { (Flora } \\
\text { of } \\
\text { Panama) }\end{array}$ & Family Name & $\begin{array}{c}\text { Acronym } \\
\text { (Weber } \\
1982)\end{array}$ & $\begin{array}{l}\text { Dalla } \\
\text { Torre } \\
\text { \& Harms }\end{array}$ & $\begin{array}{c}\text { (Flora } \\
\text { of } \\
\text { Panama) }\end{array}$ & Family Name & $\begin{array}{l}\text { Acronym } \\
\text { (Weber } \\
\text { 1982) \& }\end{array}$ & $\begin{array}{l}\text { Dalla } \\
\text { Torre } \\
\text { \& Harms }\end{array}$ & $\begin{array}{l}\text { (Flora } \\
\text { of } \\
\text { Panama) }\end{array}$ \\
\hline LEGUMINOSAE & LEG & 128 & (83) & OLEACEAE & OLE & 243 & (158) & RUTACEAE & RUT & 137 & (89) \\
\hline LILIACEAE & LIL & 38 & $(21,22)$ & ONAGRACEAE & ONA & 224 & (142) & SABIACEAE & SAB & 166 & (109) \\
\hline LEMNACEAE & LMN & 24 & (12) & OPILIACEAE & $\mathrm{OPI}$ & 71 & (47) & SALICACEAE & SAL & 56 & (37) \\
\hline LENTIBULARIACEAE & LNT & 264 & (176) & ORCHIDACEAE & ORC & 50 & (33) & SAPINDACEAE & SAP & 165 & (108) \\
\hline LOASACEAE & LOA & 206 & (132) & OROBANCHACEAE & ORO & 261 & (174) & SAXIFRAGACEAE & SAX & 117 & (79) \\
\hline LOGANIACEAE & LOG & 245 & (159) & OXALIDACEAE & OXL & 130 & (84) & SCROPHULARIACEAE & SCR & 257 & (171) \\
\hline LORANTHACEAE & LOR & 67 & (46) & PALMAE & PAL & 21 & (9) & IMAROUBACEAE & SMR & 138 & (90) \\
\hline LYTHRACEAE & LYT & 216 & (136) & ERACEAE & PAP & 104 & (72) & OLANACEAE & SOL & 256 & (170) \\
\hline MAGNOLIACEAE & MAG & 95 & $(6$ & LORACEAE & PAS & 203 & (130) & TACEAE & SPT & 239 & (154) \\
\hline MARTYNIACEAE & MAR & 260 & $(-)$ & PODOCARPACEAE & PDC & 5 & 2) & YLEACEAE & STP & 161 & (105) \\
\hline MAYACACEAE & MAY & 28 & (13) & IACEAE & PDS & 113 & (78) & TERCULIACEAE & STR & 178 & (117) \\
\hline EAE & MEL & 140 & (92) & IACEAE & PED & 259 & (173) & TYRACACEAE & STY & 241 & (156) \\
\hline ALPIGHIACEAE & MLP & 141 & (93) & POLYGALACEAE & PGL & 145 & (96) & SYMPLOCACEAE & SYM & 242 & (157) \\
\hline MELASTOMATACEAE & MLS & 223 & (141) & PHYTOLACCACEAE & PHT & 83 & (56) & THEACEAE & TEA & 186 & (122) \\
\hline MALVACEAE & MLV & 175 & (115) & PIPERACEAE & PIP & 53 & (34) & THEOPHRASTACEAE & TEO & $135 a$ & (151) \\
\hline MONIMIACEAE & MNM & 101 & (69) & PLUMBAGINACEAE & PLB & 238 & (153) & THYMELAEACEAE & THY & 214 & (135) \\
\hline MENISPERMACEAE & MNS & 94 & (64 & POLYGONACEAE & PLG & 77 & $(5$ & TILIACEAE & TIL & 174 & (114) \\
\hline MENYANTHACEAE & MNY & $546 a$ & (161) & POLEMONIACEAE & PLM & 250 & (165) & ERACEAE & TNR & 201 & (129) \\
\hline MORACEAE & MOR & 64 & (43) & ACEAE & PON & 34 & (19) & IACEAE & TOV & 106 & (73a) \\
\hline MARCGRAVIACEAE & MRC & 184 & (121) & ACEAE & POR & 85 & (58) & TRIGONIACEAE & TRG & 142 & (94) \\
\hline MORINGACEAE & MRG & 109 & (76) & POTAMOGETONACEAE & POT & 11 & (3a) & TRIURIDACEAE & TRI & 18 & (6) \\
\hline MARANTACEAE & MRN & 48 & (32) & PRIMULACEAE & PRM & 237 & (152) & TROPAEOLACEAE & TRP & 131 & (86) \\
\hline MYRSINACEAE & MF & 236 & (150) & ACEAE & PRT & 66 & (45) & TYPHACEAE & TYP & 8 & (3) \\
\hline MYRTACEAE & MRT & 222 & (140) & QUIINACEAE & QII & 185 & $(121$ & ULMACEAE & ULM & 63 & (42) \\
\hline MUSACEAE & MUS & 45 & $(28$ & ESIACEAE & RAF & 75 & (50a) & LLIFERAE & UMB & 228 & (145) \\
\hline MYRICACEAE & MYR & 57 & (38) & ULACEAE & RAN & 91 & $(62)$ & UNKNOWN & UNK & 500 & $(-)$ \\
\hline MYRISTICACEAE & MYS & 99 & (68) & CEAE & RHM & 169 & (111) & FAMILY UNKNOWN & UNP & 300 & $(-)$ \\
\hline NAJADACEAE & NA & 12 & (3B) & RHIZOPHORACEAE & $\mathrm{RHZ}$ & 220 & (138) & (PTERIDOPHYTE) & & & \\
\hline NYCTAGINACEAE & NY & 80 & (54) & ROSACEAE & ROS & 126 & (81) & NOT VASCULAR PLANT & UNV & 501 & $(-)$ \\
\hline NYMPHAEACEAE & NYM & 88 & (60) & RAPATEACEAE & RPT & 31 & (1t & URTICACEAE & URT & 65 & (44) \\
\hline OCHNACEAE & $\mathrm{OCH}$ & 182 & (119) & ACEAE & RSD & 108 & (75) & VALERIANACEAE & VAL & 273 & (181) \\
\hline OLACACEAE & OLC & 72 & (48) & RUBIACEAE & RUB & 270 & (179) & VIOLACEAE & VIO & 198 & (127) \\
\hline
\end{tabular}




\section{1 (1) CYCADACEAE}

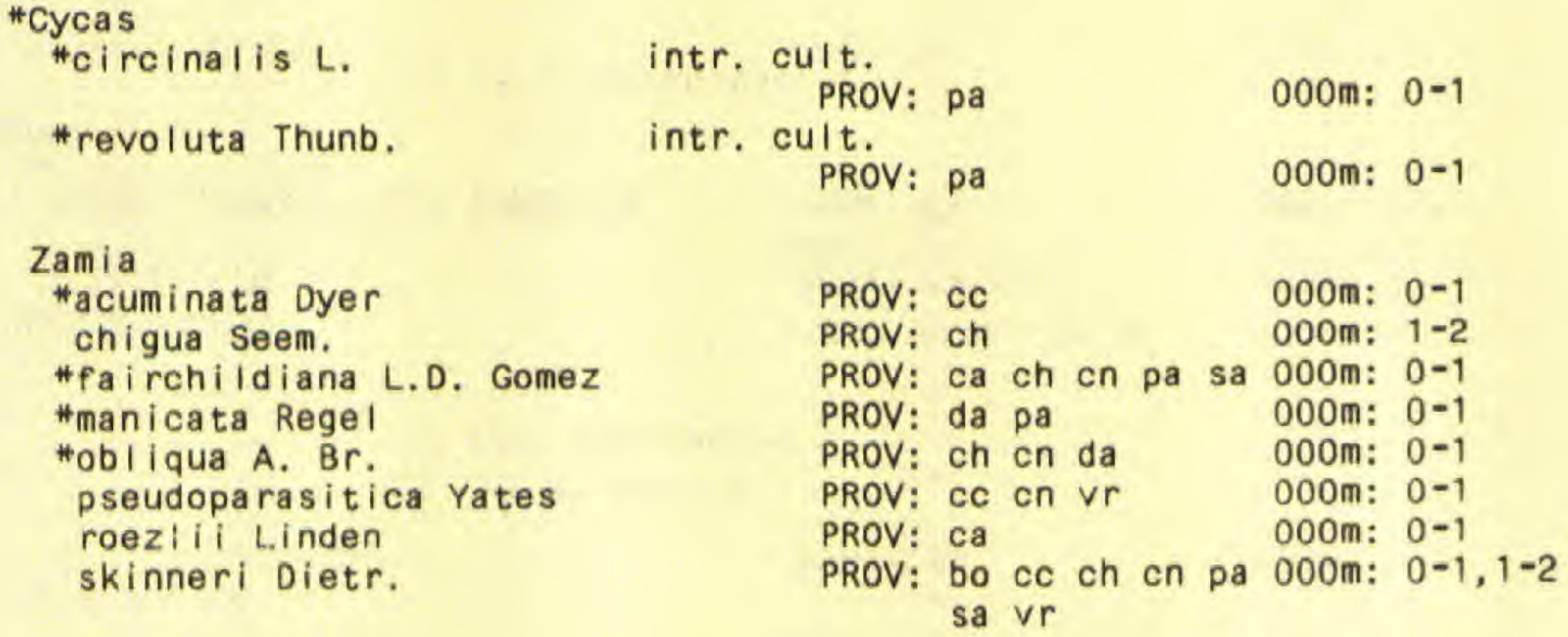

5 (2) PODOCARPACEAE

Podocarpus

* gua tema lens is StandI.

*--var. allenii (Stand.l.) Buch, \& Gray

PROV: bo cc cr. he pa 000m: $0-1,1-2$ $\checkmark r$

PROV: da pa

000m: $0-1,1-2$

*magnifolius Buch. \& N.E. Gray PROV: $c h$

$\begin{array}{ll}\text { RANGE: as } \mathrm{i} & \text { tree } \\ \text { RANGE: as } \mathrm{i} & \text { tree } \\ & \\ \text { RANGE: } c r \text { nic vr } & \text { herb } \\ \text { RANGE: col } & \text { subshrub } \\ \text { RANGE: cr ven } & \text { subshrub } \\ \text { RANGE: col } & \text { herb } \\ \text { RANGE: col } & \text { subshrub } \\ \text { RANGE: end } & \text { epiphyte } \\ \text { RANGE: col } & \text { subshrub } \\ \text { RANGE: cr nic } & \text { subshrub }\end{array}$

6 ARAUCARIACEAE

\section{*Araucaria}

*excelsa R. Br. intr. cult.

PROV: ca $\quad 000 \mathrm{~m}: 0-1$

RANGE: as

treelet

7 (2A) GNETACEAE

Gnetum

leyboldii Tul.

--var. Woodsonianum Markgr. 


\section{8 (3) TYPHACEAE}

Typha

*domingens is pers.
PROV: bo ca lo

000m: $0-1$

1 (3A) POTAMOGETONACEAE

Ha lodule wrightii Aschers.

PROV: cn pa

$000 \mathrm{~m}: 0-1$

Potamogeton

il 1 inoens is Morong

PROV: $c c$

PROV: ca he pa

PROV: sa filiforme kutz.

\section{Ruppia}

maritima $L$.

12 (3B) NAJADACEAE

\section{Najas}

a rguta H.B.K.

guadalupens is (Spr.) Magnus

$14(--)$ JUNCAGINACEAE

15 (4) ALISMATACEAE

$\begin{array}{ll}\text { PROV: ca da } & 000 \mathrm{~m}: 0-1 \\ \text { PROV: bo } & 000 \mathrm{~m}: 0-1\end{array}$

RANGE: $h$ is sat

RANGE: nWg

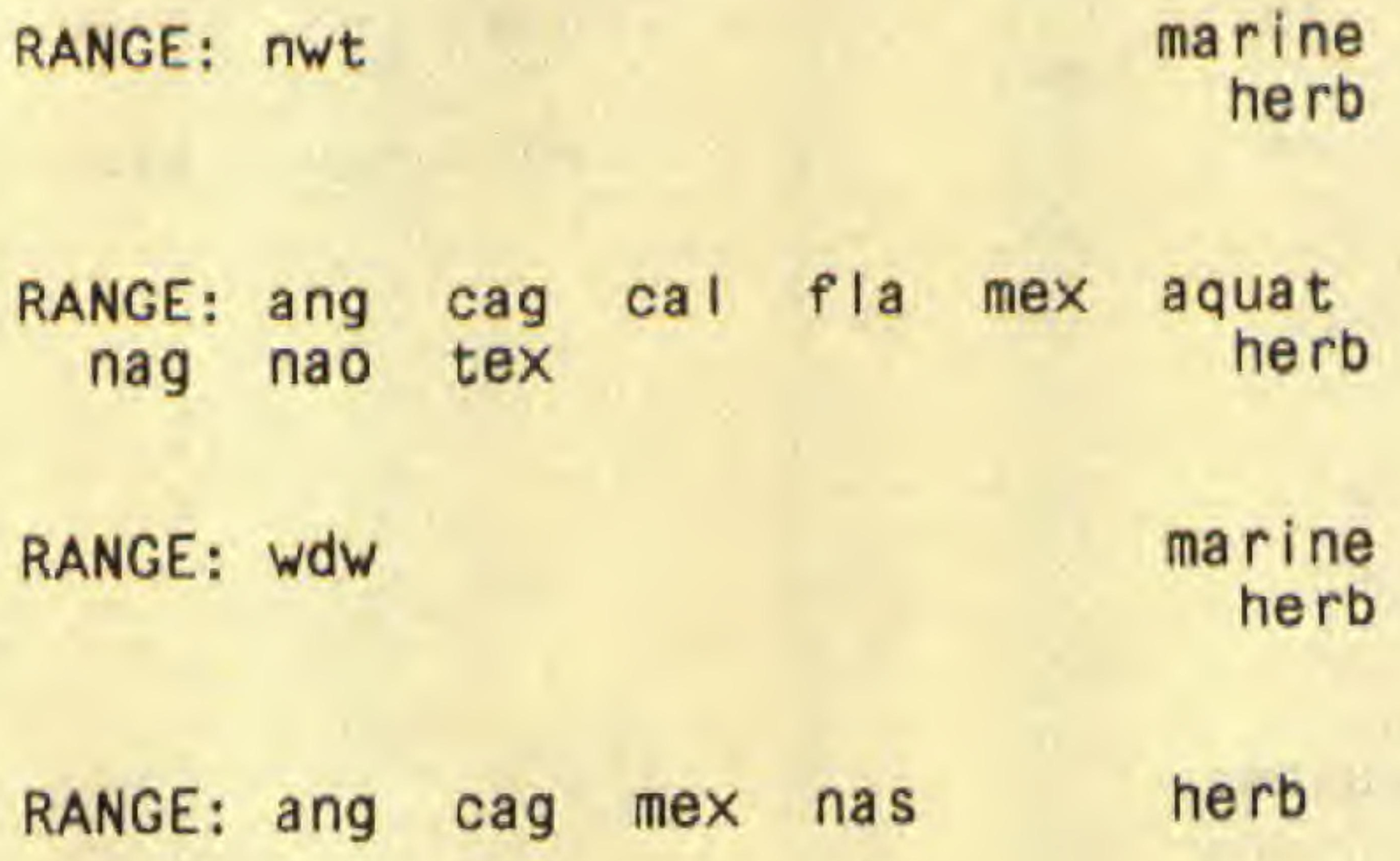

herb

(n)

ANGE: afr cub ecu nas per herb
sao wdt 


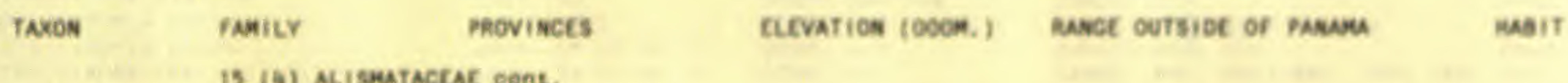

is (a) Alismitactar eent.

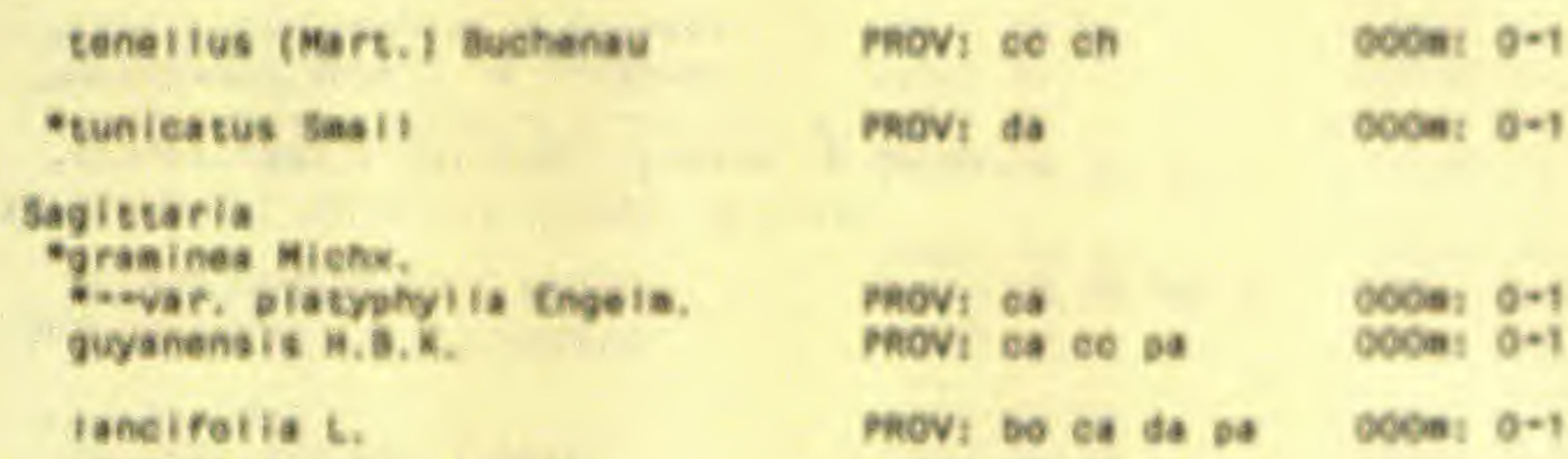

18 (5) Butcucer

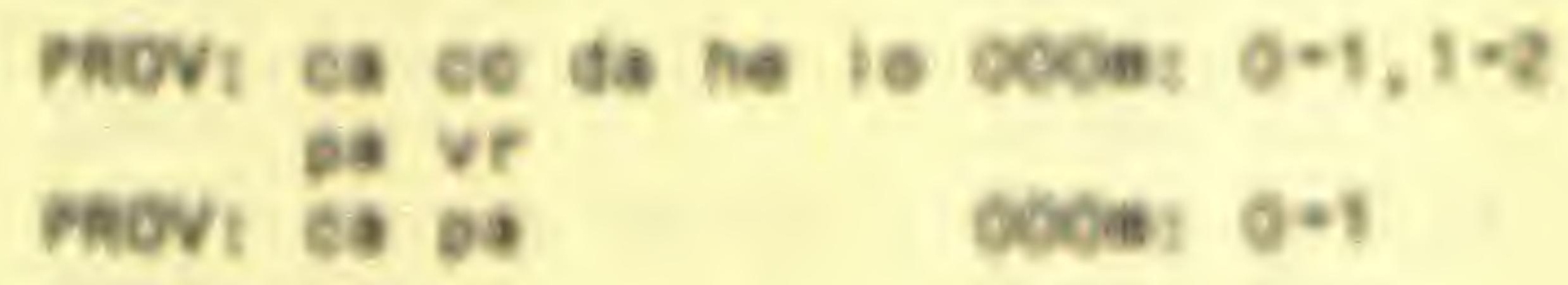

$060 \mathrm{~s}: 6-1$

\section{7 (54) imoncound itacear}

\section{Halophitia \\ garitionis Diekie}

decipiens ostent.

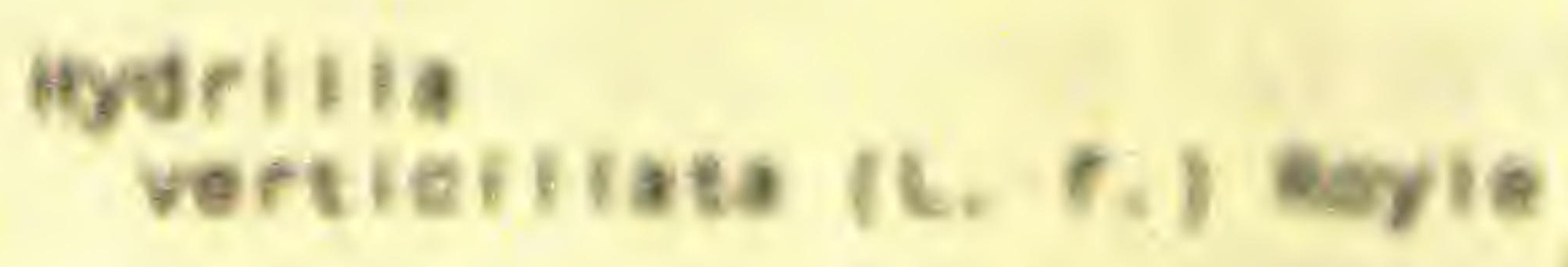

\section{Hovi vr}

neovi sa

intr. hovi ea

Limee iun

*sotoniterua iQ. meyer) Griseb. Movi ca

\section{The tosala}

testud inum Koenig phov: is is

\begin{abstract}
ocona: 0*1
ocom: 0-1
\end{abstract}

ookn $0+3$

ocon: g-1

coon: $0 * 1$

\begin{tabular}{|c|c|c|c|}
\hline $\begin{array}{c}\text { Rabet: } \\
\text { sao }\end{array}$ & $\begin{array}{l}\operatorname{cog} 7 \mathrm{col} \\
\operatorname{ven}\end{array}$ & nag & 509734 \\
\hline exect: & lat & & \\
\hline
\end{tabular}

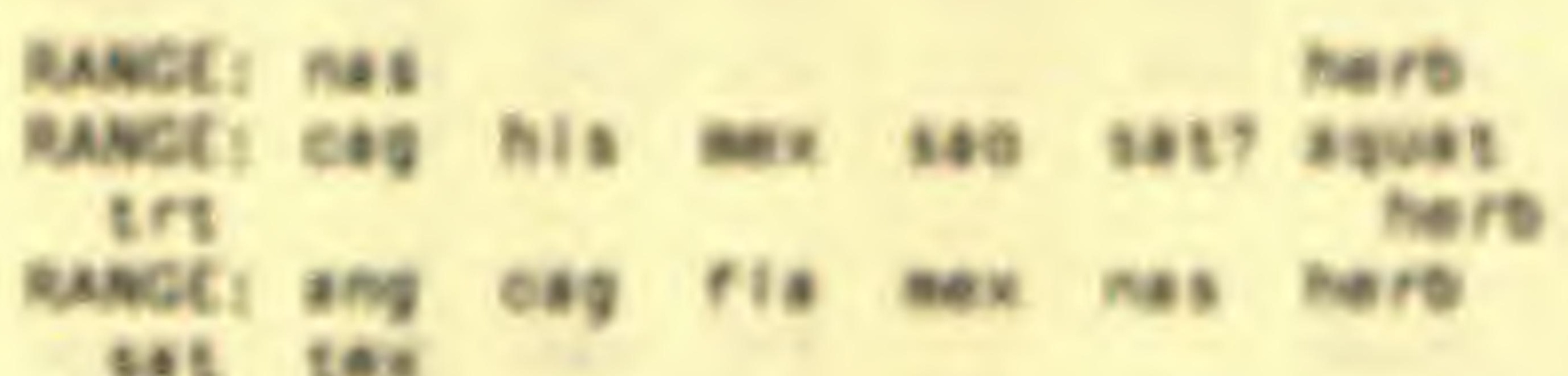

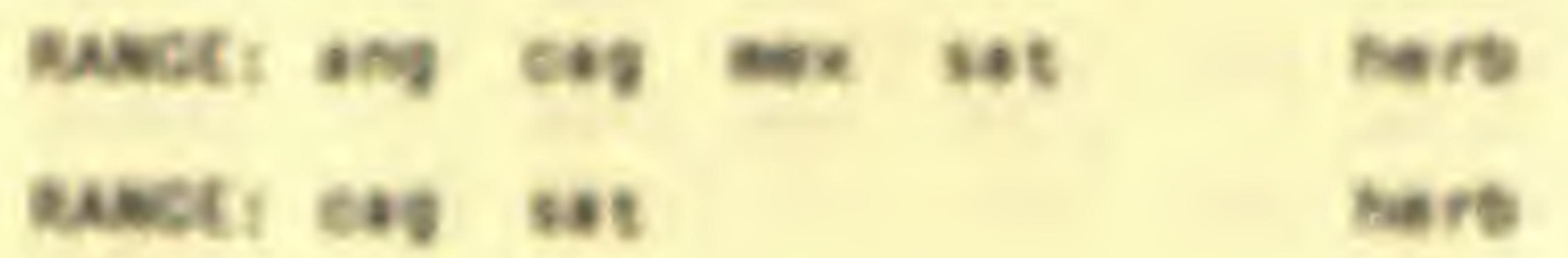

kaber eas sot

nert

\begin{tabular}{|c|c|c|c|c|c|c|}
\hline $\operatorname{secsti}$ & 106 & mo & & & & $\begin{array}{l}\text { ba ritite } \\
\text { nars }\end{array}$ \\
\hline enet: & ang & sai & ace & 306 & & $\begin{array}{l}\text { na rithe } \\
\text { hers }\end{array}$ \\
\hline based: & atr & 281 & sur & nos & act & $\begin{array}{l}\text { sovere } \\
\text { Mers }\end{array}$ \\
\hline eaver: & and & Fos & ais & set & & $\begin{array}{l}\text { buset } \\
\text { harerts }\end{array}$ \\
\hline $\begin{array}{c}\text { eascr: } \\
\text { nas }\end{array}$ & 20 & bah & $6 a 9$ & ris & men & $\begin{array}{l}\text { acrithe } \\
\text { hers }\end{array}$ \\
\hline
\end{tabular}




\section{8 (6) TRIURIDACEAE}

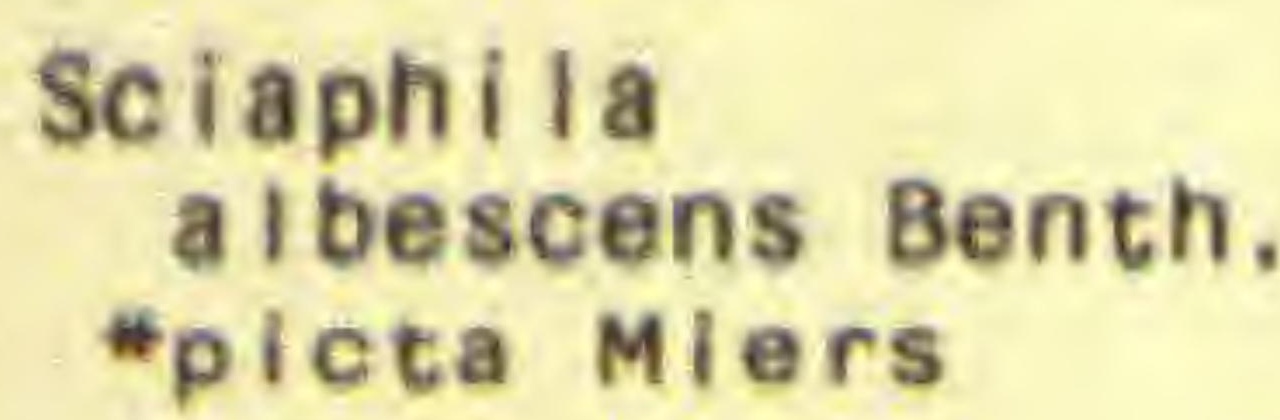

\section{PROV: Sa}

PROV: en 000m: $0-1$

000m: $0-1,1-2$
RANGE: gui sag sao ven l herb
RANGE: col

\section{9 (7) GRAMINEAE}

\section{*Acroceras}

zizanioides (H,B.K.) Dandy

\section{Aegopogon \\ cenchroides Willd. \\ *tenellus (DC.) Trin.}

\section{Agrast is}

hoffmannil Mez

*tolucens is Kunth

virescens H. B. K.

\section{And ropogon}

angustatus (PresI) Steud.

bicornis L.

glomeratus (WaIt,) B.S.P

leucostachyus Kunth

selloanus (Hack.) Hack.

virginicus $L$.

\section{Anthephora}

hermaphrodita (L.) Kuntze
PROV: bo ca ch cn pa 000m: $0-1$

$\begin{array}{ll}\text { PROV: ch } & \begin{array}{l}000 \mathrm{~m}: 1-2 \\ \text { O00m: } 1-2\end{array} \\ \text { PROV: ch } & \\ & \\ \text { PROV: ch } & 000 \mathrm{~m}: \text { we } \\ \text { PROV: ch } & 000 \mathrm{~m}: 2-3,3+ \\ \text { PROV: ch } & 000 \mathrm{~m}: 3+\end{array}$

* Arberella

* costaricensis (Hitchc.) Soders. I Calderon

dressleri Soders. \& Calderon

* lancifolia Soders. \& Zuloaga

\begin{abstract}
Aristida
capillacea Lan.

jorullensis Kunth

orizabens is Fourn.

planifolla Swallen

recurvata H.B.K.

ternipes Cav.
\end{abstract}

tincta Trin, \& Rupr.
PROV: cn

PROV: CC Pa

PROV: ca cc ch pa PROV: ca cc ch pa

PROV: $\mathrm{CC}$

PROV: ch

PROV: ca pa

PROV: ca pa

PROV: ca pa 000m: $0-1$

000m: $0-1$

000m: $0-1$

000m: $0-1$

000m: $0-1$

000m: $0-1$

000in: $0-1,1-2$

000m: $0-1$

000m: $0-1$

000m: $0-1$
RANGE: ang cag? mex sag? sao herb

RANGE: bol cag? mex sag? herb

RANGE: mex

RANGE: $\mathrm{cr}$ herb

RANGE: cag sat herb

RANGE: bol cag? mex sag? herb

RANGE: cag cub sag herb

RANGE: ang bol sao herb

RANGE: ang cag fla herb

RANGE: ang cag mex sag herb

RANGE: ang bel sag herb

RANGE: ang cag nag herb

RANGE: nWt herb

RANGE: $\mathrm{cr}$ herb

RANGE: end herb

RANGE: end herb

RANGE: bol cag? mex sag? sao herb

$\begin{array}{ll}\text { RANGE: cag? mex } & \text { herb } \\ \text { RANGE: cag? mex } & \text { herb }\end{array}$

RANGE: col herb

RANGE: bel col sao herb

RANGE: bah cag? col cub nao herb

RANGE: or sag? sao herb 


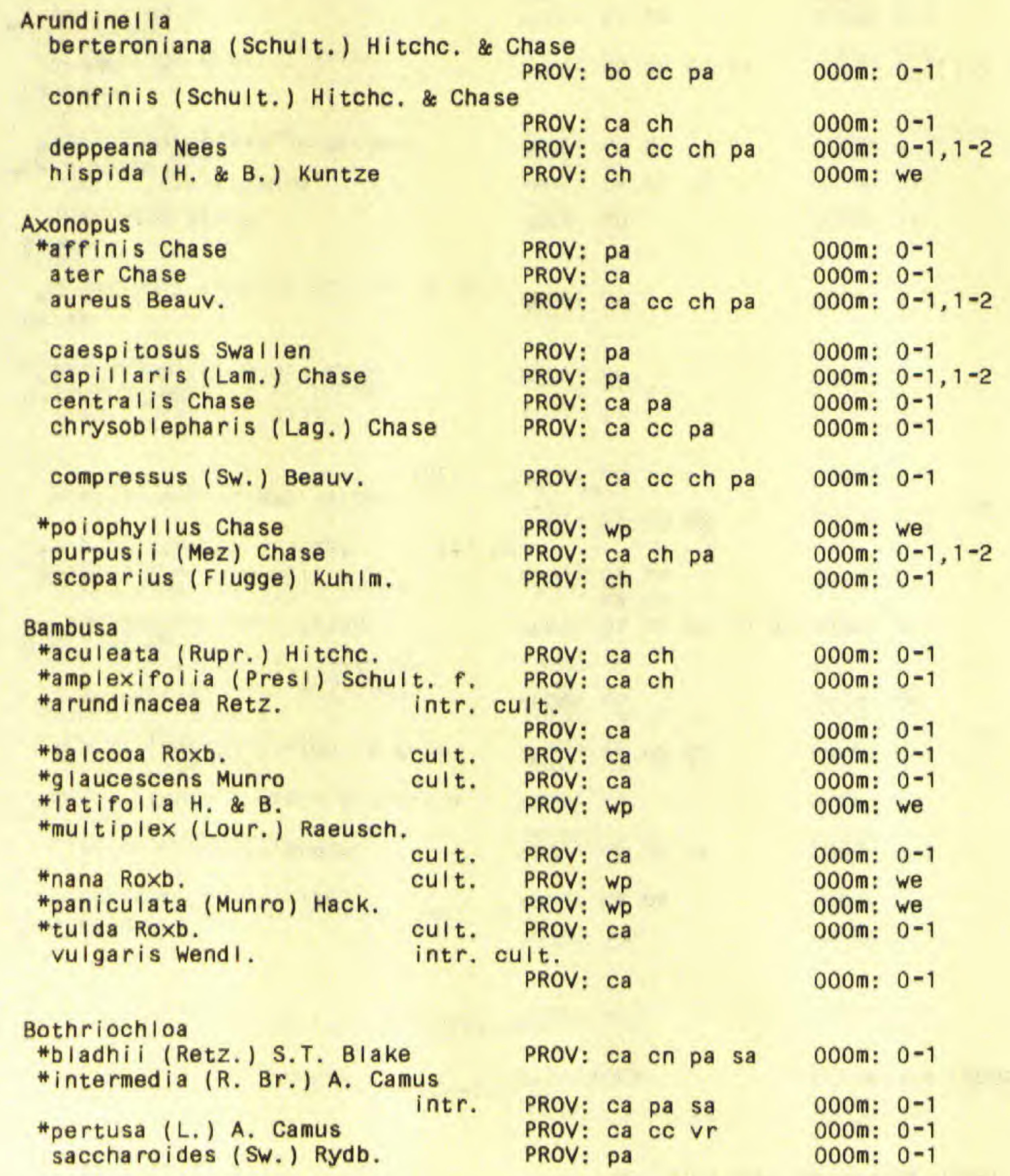

RANGE: ang cag? mex sag? sao herb
RANGE: ang cag? mex sag? sao herb
RANGE: ang cag? mex sag? sao herb
RANGE: sag? sao




\section{Boute loua}

americana (L.) Scribn.

disticha (Kunth) Benth.

*media (Fourn.) Gould \& Kapadia

repens (Kunth) Scribn. \& Merr.

"s implex Lag.

\section{Brachiaria}

* fasciculata (SW.) Parodi

*molitis (SW.) Parodi

*mutica (Forssk.) Stapf cult. nat.

$$
\text { intr. PROV: pa }
$$

mexicanum (R, S.) Link

\section{*Briza}

*minor L.

Bromus

exaltatus Bernh.

* Ca Ide rone I la

-syivatica Soders. \& Decker

Cenchrus

brownit R. \& S.

echinatus L.

-incertus M.A. Curtis

\section{* Chaetium}

*bromoides HemsI.

Chloris

inflata Link

radiata (L.) SW.

virgata Sw.
PROV: ca pa

PROV: ca cc pa

PROV: WP

PROV: ca cc pa

PROV: WP

PROV: bo ca ch cn da 000m: $0-1$ pa sa

PROV: ca pa

000m: $0-1$

000m: $0-1$

000m: $0-1$

PROV: ch

000m: $3+$

000m: $0-1$

000m: 0-1

000m: we

000m: $0-1$

ooom: we

PROV: ch

PROV: ch

PROV: en sa

PROV: ca ch en pa

PROV: ca pa

PROV: pa

PROV: ch

PROV: ca cn pa

PROV: bo ca ch pa

PROV: ca

000m: $0-1$

000m: $0-1$

000m: $0-1$

000m: $0-1$

000m: $0-1$

000m: $0-1$

RANGE: ang cag? cr gua hon herb
mex nic sao sat?
RANGE: col cr cub ecu gua herb
hon mex nic per ven
RANGE: cr gua hon mex nic herb
sat
RANGE: cag col gag mex nag herb
Ven
RANGE: cag mex nao sag sao herb
RANGE: ang cag? ecu fla sag? herb
Sao tex
RANGE: cag? mex sag? sao
RANGE: nwg herb
RANGE: bol cag? mex nag sag? herb
SaO
sao

RANGE: bol cag? mex sag? herb

RANGE: WdW herb

RANGE: gua mex herb

RANGE: end herb

000m: $0-1,1-2$

$000 m: 1-2,2-3$
RANGE: ang bol cag? fla mex herb oce sag? sao

RANGE: ang cag? nag sag? sao herb RANGE: cag cag? nag sag? sao herb

RANGE: $\operatorname{mex}$

herb

RANGE: ang cag? mex sag? sao herb RANGE: ang bol cag? mex sag? herb

Sao
RANGE: ang mex nag owg sag herb 
TAXON

FAMILY

PROVINCES

19 (7) GRAMINEAE cont. 000m: $0-1$

000m: $1-2,2-3$

$000 \mathrm{~m}: 1-2$

000m: $0-1$

000m: $1-2$

000m: $3+$

$000 \mathrm{~m}: 0-1,1-2$ 000m: $0-1$

000m: $0-1,1-2$

000m: $3+$

$000 \mathrm{~m}: 0-1,1-2$

$000 \mathrm{~m}: 0-1$

000m: $0-1$

000m: $0-1,1-2$

000m: $0-1,1-2$

000m: $0-1$

000m: $0-1$

000m: $0-1$

000m: $0=1$

000m: $0-1$

000m: we
RANGE: afr as i

herb

RANGE: or mex

RANGE: $c r$ mex

RANGE: or gua

RANGE: $\mathrm{cr}$

gua

shrub

herb

shrub

herb

RANGE: cag? mex per sag?

herb

RANGE: $\mathrm{cr}$ sag

RANGE: cag col

herb

herb

RANGE: owg

herb

RANGE: $\mathrm{cr}$ per sag?

herb

RANGE: $\mathrm{cr}$

RANGE: end

RANGE: end

RANGE: cag

RANGE: COI

RANGE: cag

RANGE: ang asi cag sag

herb

RANGE: $w d W$

herb

RANGE: WdW

herb

RANGE: as $\mathrm{i}$

RANGE: af $r$ ang as cult. PROV: ca 
19 (7) GRAMINEAE cont.

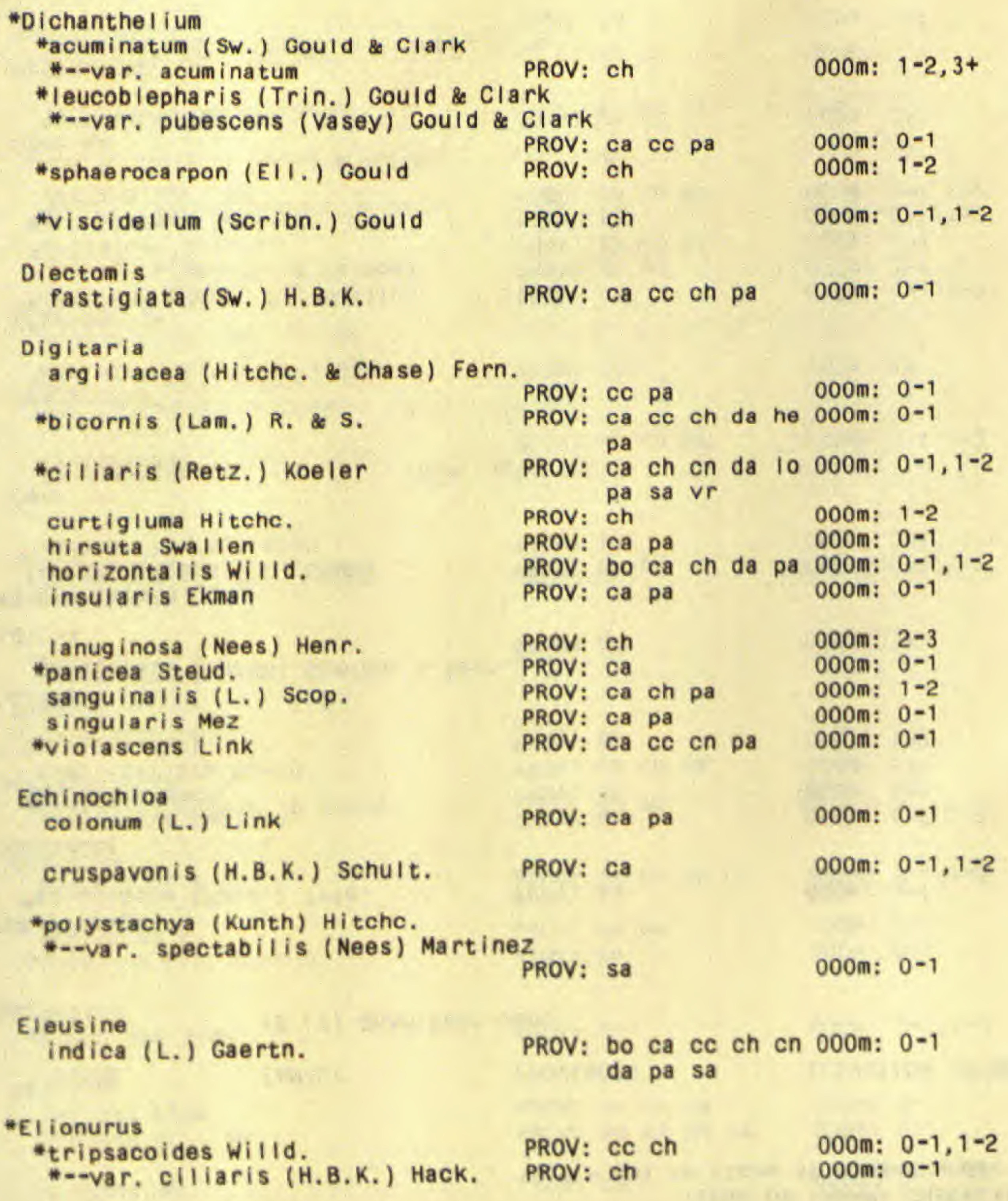

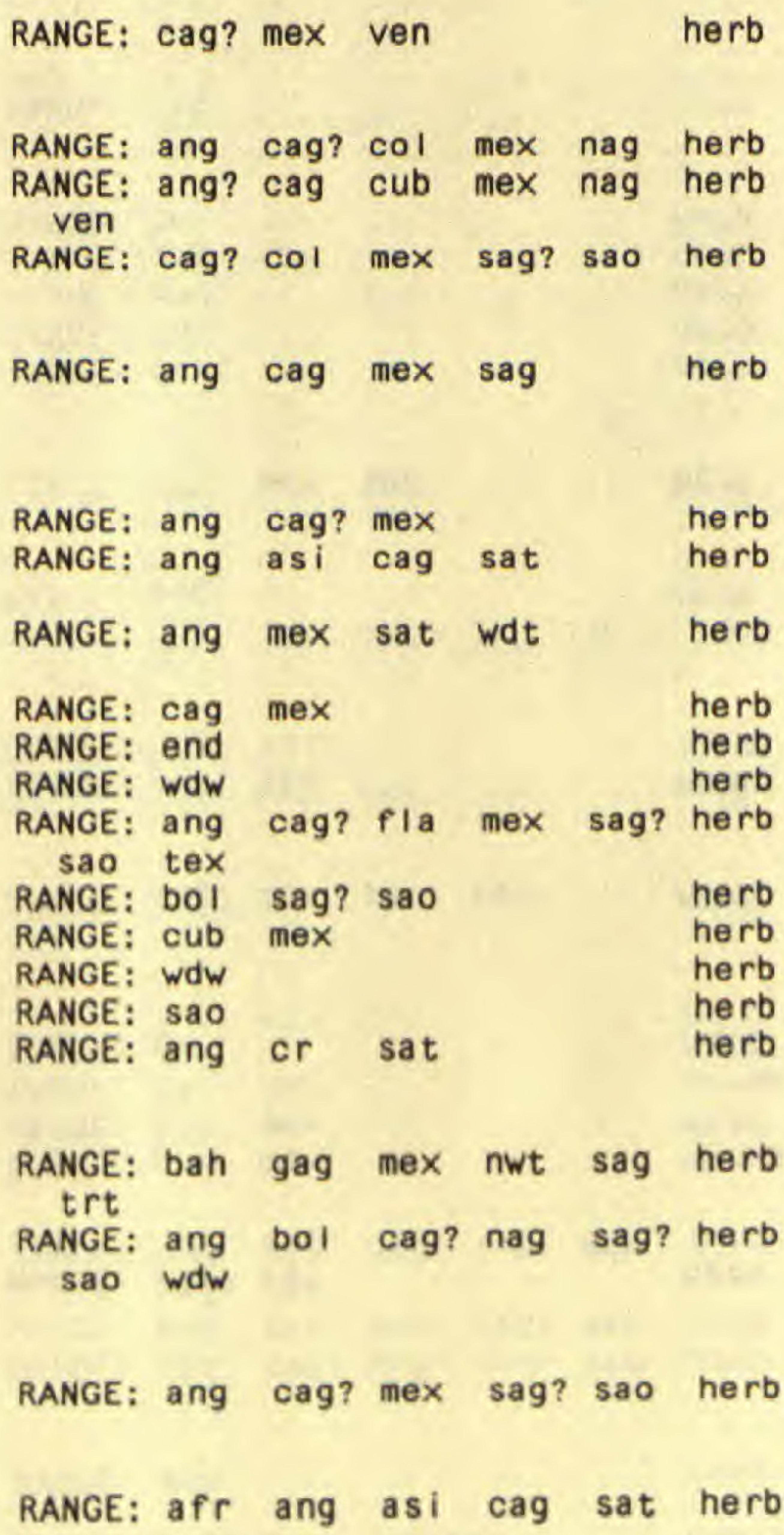




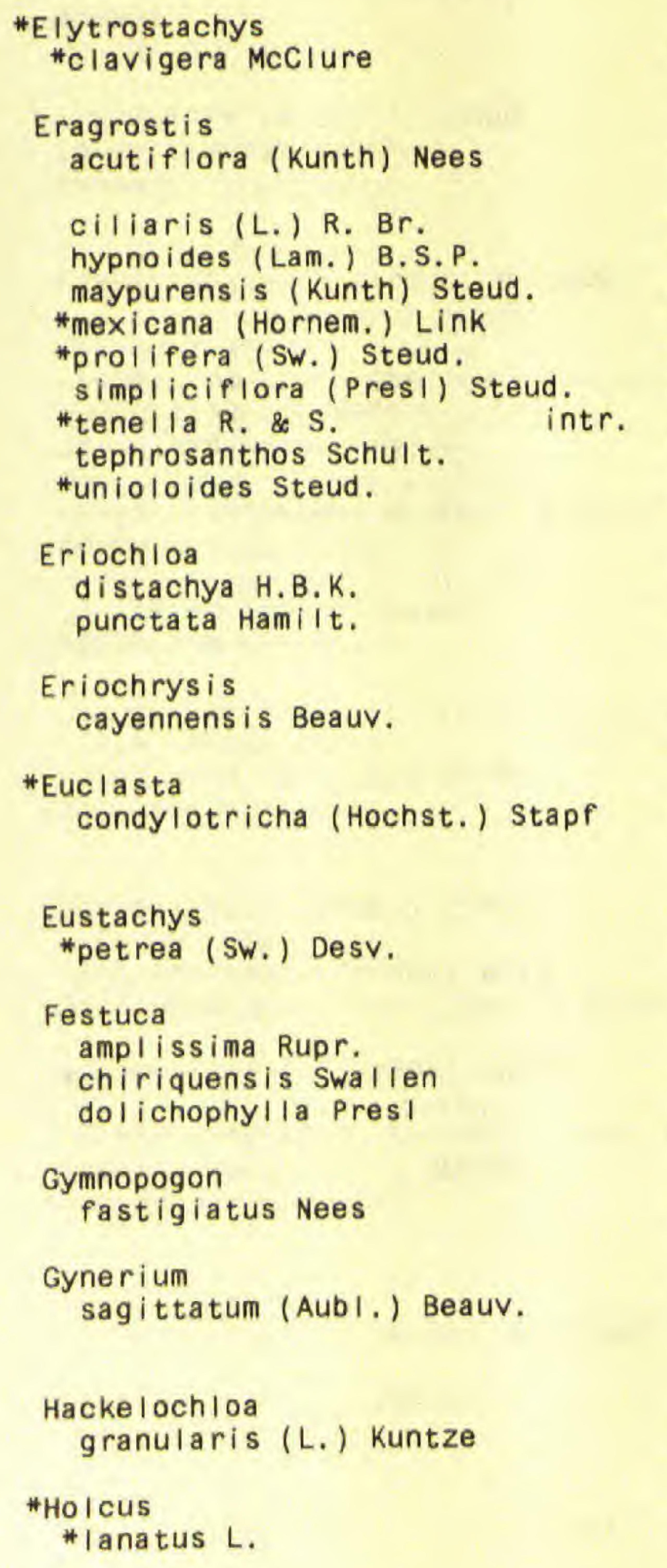

PROV: $v r$

000m: $0-1$

PROV: ca cc ch pa $000 \mathrm{~m}$ : $0-1$

PROV: bo ca ch cn pa 000m: 0-1 PROV: ca cc cn 000m: $0-1$ PROV: ca cc ch pa 000m: $0-1$ PROV: ch 000m: 2-3 PROV: he pa $000 \mathrm{~m}: 0-1$ PROV: cc pa $\quad 000 \mathrm{~m}: 0-1$ PROV: ca ch cn 000m: $0-1$ PROV: bo ca ch cn pa 000m: $0-1$ PROV: ca ch 000m: $0-1$

PROV: cc pa

PROV: bo da

000m: $0-1$ 000m: $0-1$

PROV: ch

PROV: ca

000m: $0-1$

PROV: ca cn sa

000m: $0-1$

PROV: $c h$

PROV: $\mathrm{ch}$

PROV: ch

PROV: $\mathrm{CC}$

PROV: ca da

PROV: ca cc ch pa

000m: $0-1$

PROV: bo $\mathrm{ch}$

$000 \mathrm{~m}: 1-2$

000m: $3+$

000m: $0-1$ 000m: $0-1,1-2$

RANGE: $\mathrm{cr}$ Sao

herb

RANGE: bel bol cag? sag? sao herb trt

RANGE: $w d w$

Eag sao herb

RANGE: bol mex sag? sao herb

RANGE: cag per ven herb

RANGE: ang cag? col mex herb

RANGE: cag mex herb

RANGE: Wdw herb

RANGE: ang cag? nag sag? sao herb

RANGE: bol ven herb

RANGE: cag? gua sag? sao herb RANGE: ang cag? nag sag? sao herb

RANGE: ang cag? mex sag? sao herb

RANGE: af $r$ ang cag? col mex herb ven

RANGE: ang mex nag trt herb

RANGE: cag mex herb

RANGE: $\mathrm{cr}$ herb

RANGE: sag? sao herb

RANGE: $\mathrm{cr}$ Sao

herb

RANGE: ang cag mex sag sao aquat

RANGE: $W d W$

herb

RANGE: $\mathrm{cr}$ sag sat

herb 


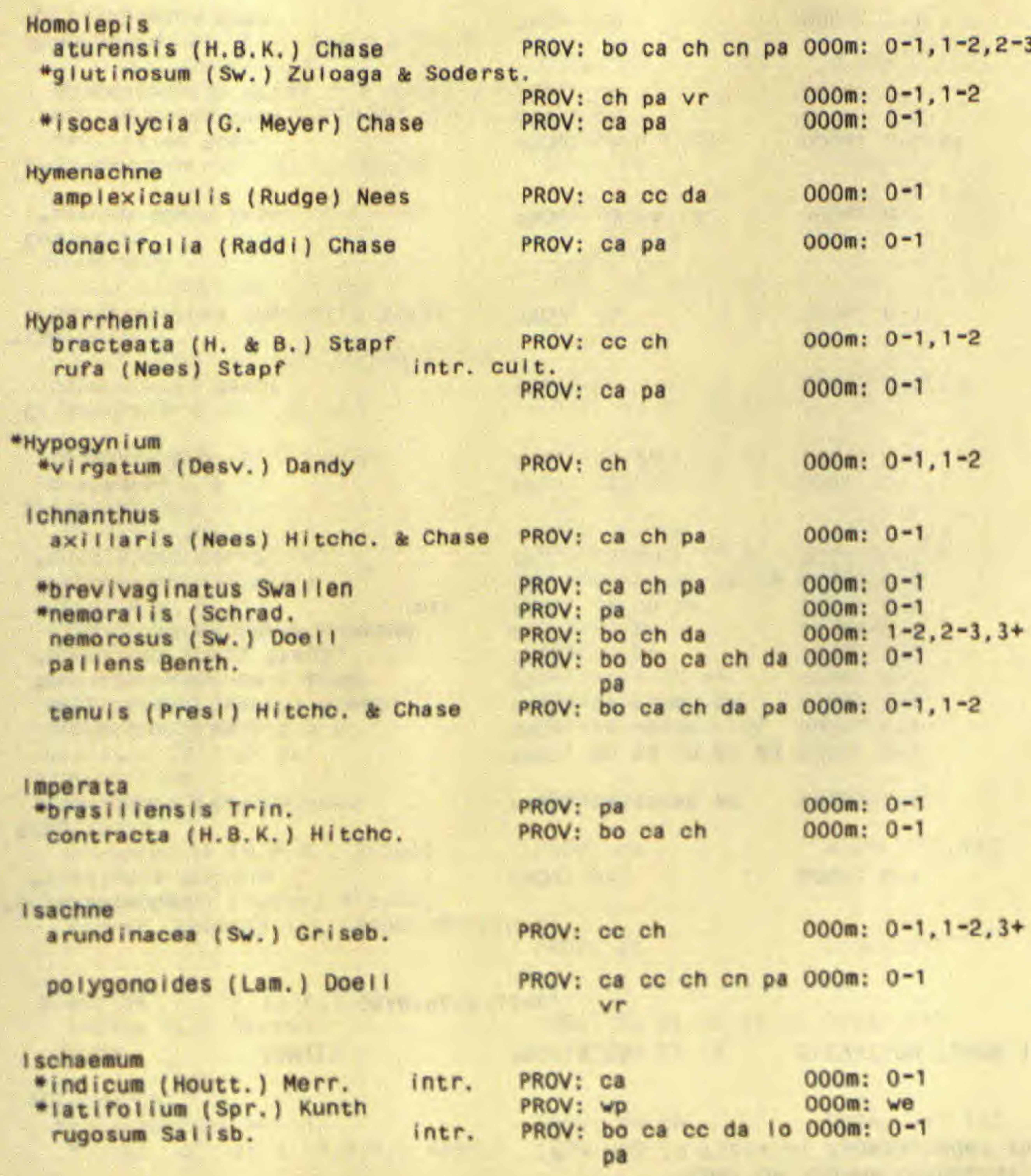

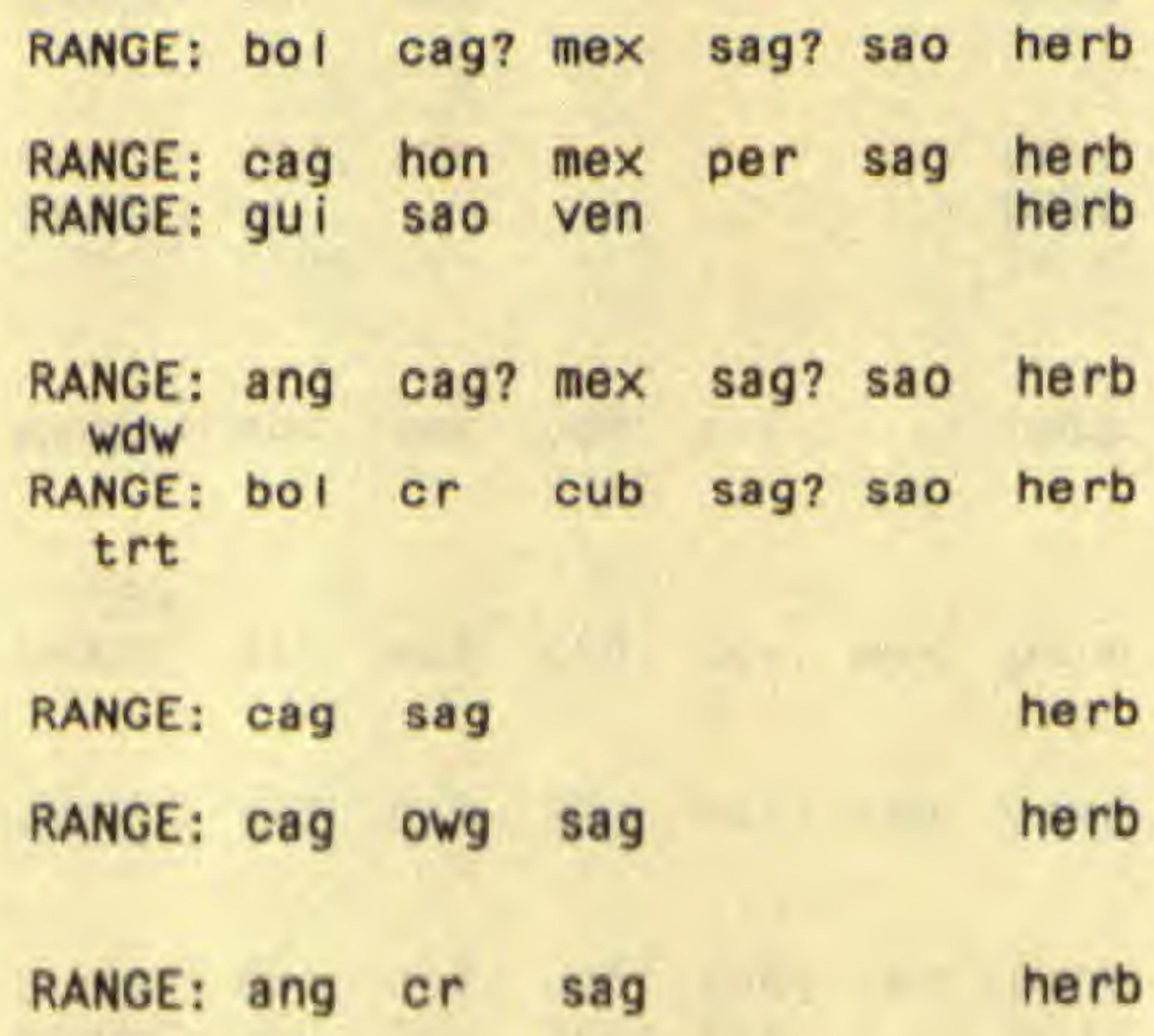

RANGE: asi gui herb

RANGE: cag sat herb

RANGE: ang ecu mex owg sao herb 


\section{Jouvea}

straminea Fourn.

Lasiaeis

divarieata (L.) witcho.

-evar, divarieata

\section{*i inearis swation}

*niegra Davidse

oaxacensis (staud.) Witche.

* *war. maxonil (swairen) gav orocerrima (Hack, ) witohe.

-rhizophora (Fourn.) Mitche. * rugeili (Criseb.) witche. - wevar, pohilil sualien ruscifoila (H, B, K.) Hitehe.

scabrier Witche.

sorgholdea (Desv.) Hitche.

\section{Chase}

standiayi witehe,

\section{Leersia}

hexand $r a$ iv.

- ilgularis Trin.

*-var. grandifiera (Doell) hyrah phovi on

\section{Ceptochies}

filfroris (thas.) Beaur.

*longa Griseb.

* seabra Nees.

virgata (L.) Deaur.

Leptocoryphican Ianatual $(\mathrm{H}, \mathrm{B}, \mathrm{K}$,$\} toes$

Lithachne

paveifiora thoir.

Lol in

*aul tiflerua can.
Phov: pa

000a: 0-1

RROV: ea on pa oconi 0*1

PRovi sh

PhOV: ce on pa goon: $0+1,1+2$

Phov: bo ca ch da ba 000n: 0-1

Phovi ch 000n: 1 -2

Phovi bo ea ee on en 000a: $0-1,1-2,2-1$ dea na

PAOV: ich

000a: $1+2$

hov: be ee pa ocons 0-1 Phov: bo ea be eh he 000n: 0-1 lo pa Vr

erov! be ce on da la 000es $0-1$ pa sa vr

prov! bo ea oe eh en 0000: 0-1 da 10 ou sa vr

MoV! ca en da he pa Doon: 0-1,1-2 ve

paovi da de pa obon! 0-1

ooon: we

\section{Prov: ca os beon: $0-1$}

Prov? sa ocon: 0-1

PhoV: bo ch is be goons ow1

Phov: be ea ch en da booin: 0-1 Io pa

Phov: ca

000 n: $0-1,1-2$

Phov: ca ch of

000in: $0-1,5 * 2$

OOOin: $3 *$ ance: cagt mex

herb

\begin{tabular}{|c|c|c|c|c|c|}
\hline RANGE; & ang & bel & $\operatorname{cag}$ & ria & $\operatorname{sex} x$ \\
\hline 380 & wet & & & & \\
\hline NANCE: & eag & & & & \\
\hline 2ANGE: & eag & $\cos x$ & sat & & \\
\hline eNabe: & ang & $\operatorname{cog}$ & col & ocu & nex \\
\hline per & $\operatorname{sat} 7$ & ven & & & \\
\hline Mot & or & nie & & & \\
\hline
\end{tabular}

uwci: or nie hers

Ravel? cag nex por sat hart

kescti cos col sex harb

eavers or gua nie herb eavcli cap col ecu ges hex harb oer ven

eavort cag col ecu gua hes. nerb ber

auser ang bol cog atex say herb

wabr: eag cel er ecu nex herb ven

2ukct: ong cagt nag sagt sao herb

zact: cagt nax sagt sao herb

suct: cag? nag sagt sao herb

Wuck: irt nert

euct: cag sas sat tre herb

aube: ing cagt fia sagt sao herb tex vor

Nuck: ang bag? nex sugt sab harb

Rusct: ang cag? nax sag? suo herb

raver: is:

nerb 


\section{Luziola}

subintegra Swallen

\section{*Maclurolyra}

*tecta Calderon \& Soders.

\section{*Melinis}

*minutiflora Beauv.

\section{Mesosetum}

pittieri Hitche.

\section{Muhlenbergia}

* implicata (Kunth) Kunth

* lehmanniana Henr.

setarioides Fourn.

tenella (Kunth) Trin.

tenuissima (PresI) Kunth

\section{*Neurolepis}

*pittieri Mcclure

\section{Olyra}

* cauda ta Trin.

*ecaudata DoeII

* fasiculata Trin.

* lateralis (PresI) Chase

latifolia L.

*standleyi Hitchc.

Op I ismenus

burmannii (Retz.) Beauv.

hirtellus (L.) Beauv.

*setarius (Lam.) R. \& S.

\section{Orthoclada}

laxa (Rich.) Beauv.

\section{oryza \\ * I ta Swallen \\ latifolia Desv. \\ perennis Moench}

sativa L.
PROV: ca

000m: $0-1$

000m: $0-1$

RANGE: ang per

herb

PROV: cc cn pa sa

RANGE: $C O$

herb

PROV: ca ch pa vr

000m: $0-1,1-2,2-3,3+$ RANGE: ang hon sat

herb

PROV: cc pa

000m: $0-1$

RANGE: cag

herb

PROV: $\mathrm{ch}$

PROV: $\mathrm{cc}$ ch

PROV: $\mathrm{ch}$

PROV: CC ch

000m: $1-2,2-3$

$000 \mathrm{~m}: 1-2$

000m: we

000m: $0-1,1-2$

000m: $0-1$

PROV: $\mathrm{ch}$

000m: 2-3

000m: $0-1,1-2$

$000 \mathrm{~m}: 0-1$

000m: $0-1$

000m: $0-1,1-2$

PROV: cn

PROV: he pa

PROV: bo ca ch cn da 000m: $0-1$ pa sa $\vee r$

000m: $0-1,1-2$

PROV: pa

PROV: bo ca ch da lo $000 \mathrm{~m}$ : $0-1$ pa $v r$

PROV: bo ca cc ch da 000m: 0-1,2-3

PROV: $\mathrm{pa}$

000m: $1-2$

PROV: bo ca ch cn pa $000 \mathrm{~m}$ : $0-1$

PROV: ca

PROV: bo ca cn da pa 000m: $0-1$

PROV: $\mathrm{CC} \quad 000 \mathrm{~m}: 0-1$
RANGE: cag ven

RANGE: cag nag herb

RANGE: cag? mex herb

RANGE: cag? mex herb

RANGE: mex herb

RANGE: ven herb

RANGE: $c r$ herb

RANGE: per sao ven herb

RANGE: $w r$ herb

RANGE: bol or $\mathrm{cr}$ sao sat herb

RANGE: ang bol cag? fla mex tree

sag? Sao

RANGE: ven

herb

RANGE: ang cag? mex sag? sao herb

WdW

RANGE: ang cag? mex sag? sao herb

RANGE: col cub his jam sag herb sao ven

RANGE: cag? mex per sag? sao herb

RANGE: hon mex sat herb

RANGE: ang bel cag? sag? sao herb

RANGE: cub his sag? sao herb 


\section{Panicum}

a tum Hitehe. Chase

arundinariae Fourn.

cayennense Lan.

cordovense fourn.

*dichotenifiarum Michx,

* elephantipes Nees

frondescens 0 . Meyor

ghlesbregheif Fourn.

grande Hitche, \& Chase

haenkeanum Pres !

nirsutua SN.

hirticaule Presi

laxum SW.

naxinum Jaca.

Mertansii Aoth

inilieriorum Hitche. Chase

pantrichua Hack.

pilosua SN.

polygonatua sehult.

bulchelfum Maddi

pyrulariue Hitehe. Chase rudgei R, $\mathrm{s}$.

\section{schiffneri Hack.}

sellovil Mees

stagnatile Mitche. Chase

stenodoides F.T. Mubb.

stoioniferum poir.

* sublaeve sualion

Erichanthum Nees

trichoides 5W.

\section{Pariana}

"aurita swailen

* campestris Aubl.

simuians fut in

strigosa sualien
PROV: pa

PROV: oh

PROV: ch pa

PROV: on

PhOV: ca pa

PROV: vp

prov: on ch pa ch sa 000a: 0-1

Phov: ca en da pa 000es: 0 -1

PROV: ca ch da

000 : $0-1$

PhOV: bo ca pa sa 000es: 0-1

Phov: ca da 000as: 0-

PROV: be ca ce ch on 000a: $0-1,1-2$ da pa

intr, cult,

phov: ea ce da he la 000nt 0-1 pa $v r$

Phov: ca pa

PhoV: ca pa

BhOV: is

000n: $0-1$

D00a: we

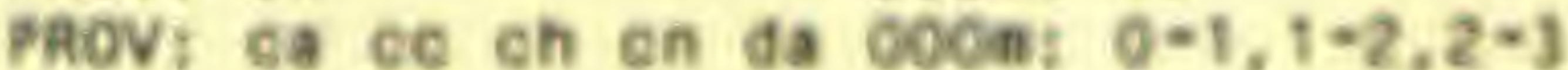
pon sa

PROV r bo ca ce en da $000 \mathrm{n}$ : $0-1,1-2$ pa sa

PAOV: bo ca eh en lo 000a: $0^{-1}$

$$
\text { pa }
$$

PROV: ch

PROV: ca pa

coon: 0-1

PAOV: $\mathrm{ch}$

PROV: $\mathrm{ch}$

PROV: bo ca

Phov: ca pa

prov: bo ca pa

Prov: pa

000n: $0-1$

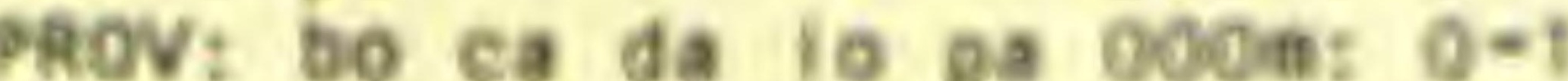

PHOV: bo ca ce ch on 000nt $0-1,1-2$ da pa sa vr

000 : $1 * 2,2-1$

0007: $0=1$

000in: $0-1$

000in: $0^{-1}$

000 an: $0-1$

PrOV: ca en pa sa 000 : $0-1,1-2$

Phov: sa

Provt ca on

Prov: ca da sa

ooon: $0-1$

000n: $0-1$

000n: 0 -
RUwCE: ang? bel cag? trt herb

RWCE: cag sex wex sag? sao herb

MANCE: boi cag? sex sag? sao hert

Ravct: cag? sex sag? sao herb

Rubet ang col gua sat her hert

WhCE: cagt mex sag? sao herb

duvce: ang bol cagt sex sag? herti

$$
\text { soo }
$$

aver: cagt nie tre ven herb

eaveE: cagl col gui mex sao herb

ven

Mrof: ang hon sax

axce: bot cagt nag sag? hert

\begin{tabular}{|c|c|c|c|c|c|}
\hline $\begin{array}{l}\text { Not: } \\
\text { nexes }\end{array}$ & $\begin{array}{l}\text { ofr } \\
\text { sagt }\end{array}$ & ing & bol & cagt & fla \\
\hline RUNCE: & $\cos 9$ & cub & $\sec x$ & sag? & 390 \\
\hline RANCE: & bei & sag? & sao & & \\
\hline
\end{tabular}

avoc: cagl nex sag? sad hert

Mavcr: sag? sao sao herb

RAwCE: ong cag? sex sag? sao hert

RUWCE: cag7 mex sag? sab hert

Rusce; cag lag aex sag? sao herb

vasce: col sao ven herb

Rukbt: ang? cagt col gua jan hert seg? 300

auct: ong cagt aex sag? sao hert

uect: ong cagt nax sag? sao hert

earor: cag? aex mert

Ravet: bei or sao tre hert

dunct: cagi ecu gus sagt sao herb

RAKCE: and herb

wack: ing cag? mex sagt sao hero

2awCE: ang cag? nex per sag? hert 140

sawer: per

ance: 500

aAwct: col or herb

QANGC: and herb 
19 (7) GRAMINEAE cont.

* Paspalidium

*geminatum (Forssk.) Stapf

Pa spa I um

acutum Chase

*blodgeti i Chapm.

boscianum Flugge

*brunneum Mez

candidum (H. \& B.) Kunth

centrale Chase

clavul iferum C. Wright

conjugatum Bergius

--var. pubescens Doell

contractum Pilger

convexum $H, B$.

coryphaeum Trin.

decumbens SW.

densum Poir.

fasciculatum Flugge

fimbriatum H.B.K.

gardnerianum Nees

heterotrichon Trin.

hitchcockii Chase

humboldt i anum Flugge

jimenezi i Chase

lineare Trin.

melanospermum Desv.

microstachyum Pres

*mill legrana Schrad.

minus Fourn.

multicaule Poir.

notatum Flugge

nutans Lam.

orbiculatum Poir.

paniculatum L.

parviflorum Flugge

pectinatum Nees

pilosum Lam.

plicatulum Michx.

*pulchel l um Kunth
PROV: ca

000m: $0-1$

000m: $0-1$

PROV: ca

PROV: sa

PROV: ch pa

PROV: ca

PROV: $\mathrm{ch}$

PROV: ca co

PROV: ca pa

000m: $0-1$

000m: $0-1$

000m: $0-1$

000m: $1-2,2-3,3+$

000m: $0-1$

000m: $0-1$

PROV: bo ca ch cn da 000m: $0-1,1-2$ he pa sa

PROV: bo

PROV: $C C$

PROV: $\mathrm{CC}$ ch

000m: $0-1$

000m: $0-1$

000m: $0-1,1-2$

PROV: pa

000m: $0-1$

PROV: bo ca cc ch cn 000m: $0-1$ he pa

PROV: ca cc pa $\quad 000 \mathrm{~m}: 0-1$

PROV: bo ca $\quad 000 \mathrm{~m}: 0-1$

PROV: ca

PROV: cc pa

PROV: ch

PROV: pa

PROV: ch

PROV: pa

PROV: ch

000m: $0-1$

o00m: $0-1$

000m: we

000m: $0-1$

000m: $1-2,2-3$

000m: $0-1$

000m: $1-2$

PROV: ca

000m: $0-1$

PROV: bo ca ch pa 000m: $0-1$

PROV: ca pa

000m: $0-1$

000m: $0-1$

PROV: ca ch pa

000m: $0-1$

PROV. ca cc ch pa

000m: 0-1

PROV: ca ch

000m: $0-1,1-2$

PROV: bo ca cn pa 000m: 0-1

PROV: bo ca ch cn da 000m: $0-1,1-2$

$$
\text { pa sa }
$$

PROV: pa

PROV: ch pa

000m: $0-1$

PROV: ca ch pa

000m: $0-1$

PROV: ca cc ch cn da 000m: $0-1,1-2$

he pa sa

PROV: $\mathrm{CC}$

000m: $0-1$
RANGE: $w d w$

herb

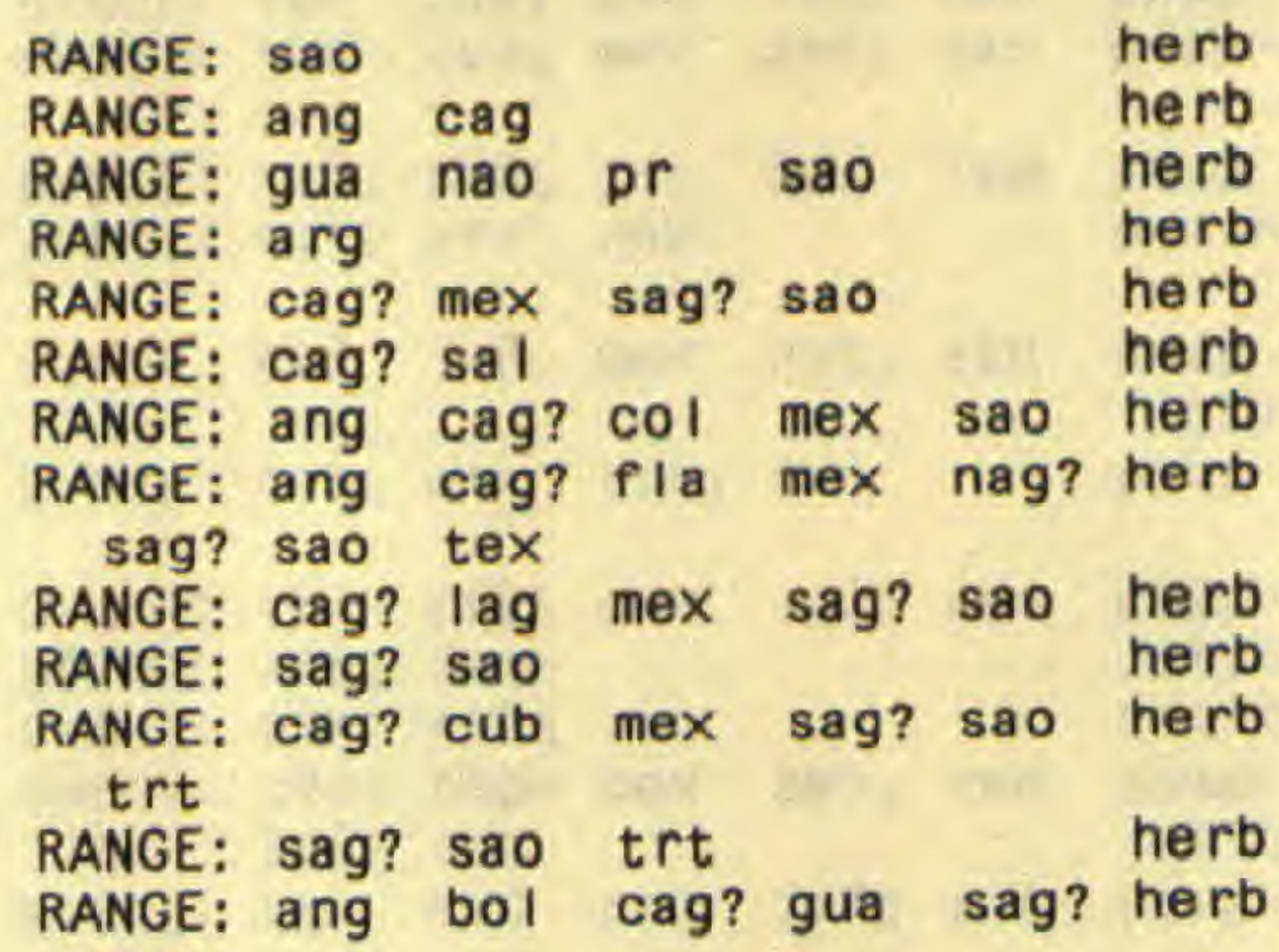$$
\text { Sao }
$$

RANGE: ang bol sag? sao herb

RANGE: cag? ecu mex sag? sao herb

RANGE: ang col nao sag? sao herb

RANGE: sag? sao

RANGE: his per sag? sao herb

RANGE: $\mathrm{col}$ herb

RANGE: cag? mex sag? sao herb

RANGE: $\mathrm{cr}$ herb

RANGE: or cub sag? sao herb

RANGE: bol lag? sag? sao herb

RANGE: cag? ecu gua sag? sao herb

RANGE: ang cag sao ven herb

RANGE: ang bol cag? mex sag? herb

SaO

RANGE: bol cag? mex sag? sao herb trt

RANGE: ang cag? mex sag? sao herb

RANGE: afr cag? hon lag sag? herb$$
\text { SaO }
$$

RANGE: ang cag? mex sag? sao herb

RANGE: afr ang cag? mex oce herb

sag? Sao

RANGE: pr sag? sao herb

RANGE: cag? hon sag? sao herb

RANGE: bol cr sag? sao herb

RANGE: ang cag? nag sag? sao herb

RANGE: cag sat 
19 (7) GRAMINEAE cont.

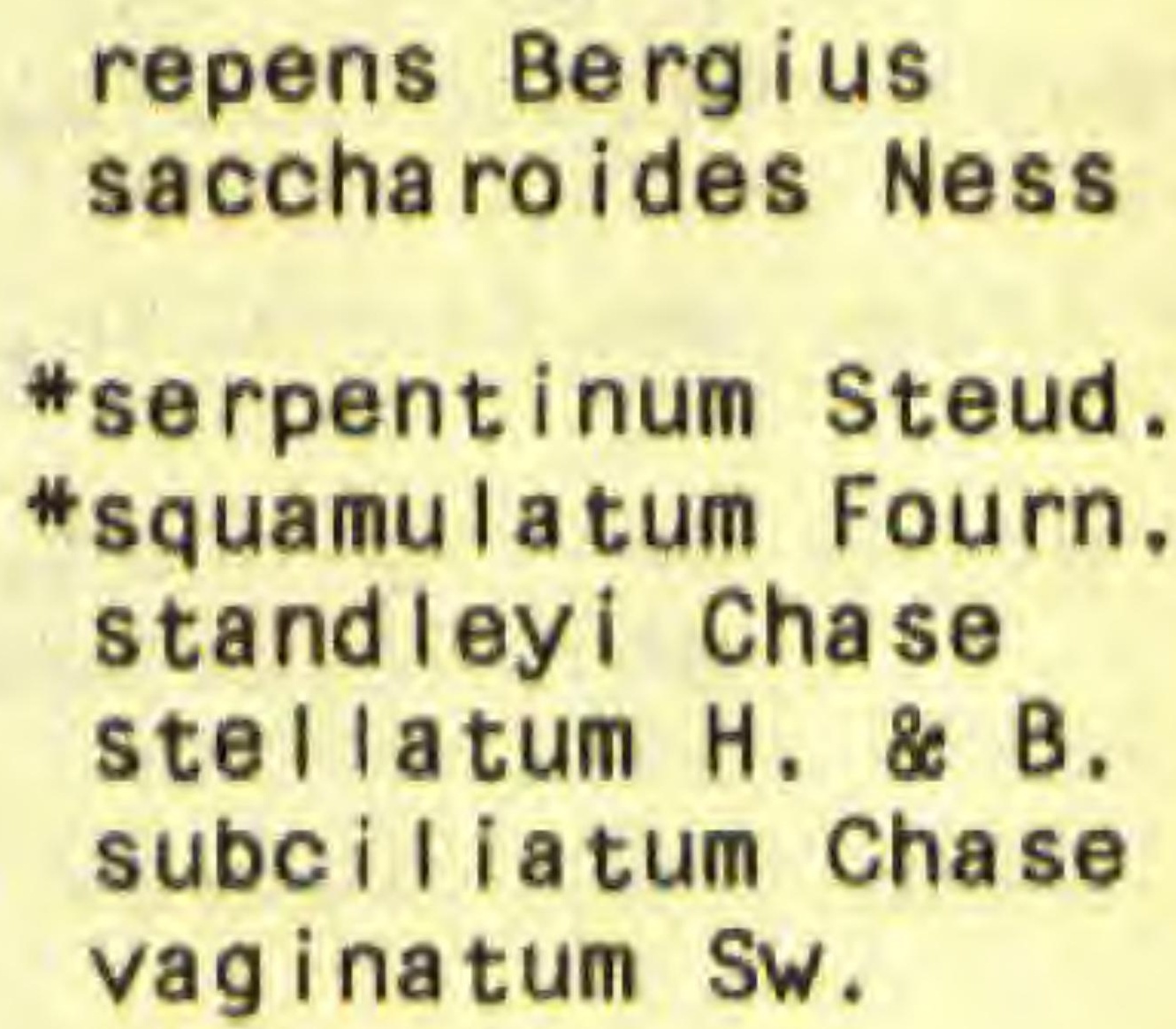

virgatum L.

\section{Pennisetum \\ complanatum (Nees) Hems I. \\ *purpureum Schum.}

setosum (Sw.) Rich.

\section{Pereilema}

crinitum Pres I

Pharus

cornutus Hack. glaber H.B.K.

latifolius L.

longifolius Swallen parvifolius Nash

virescens Doell

Phragmites

*australis Steud.

Poa

annua $L$.
PROV: ca pa

000m: $0-1$ PROV: ca cc ch cn pa $000 \mathrm{~m}: 0-1,1-2$ PROV: $\mathrm{cc}$

PROV: pa

PROV: pa

PROV: CC Vr

000m: $0-1$

000m: $0-1,1-2$

000m: $0-1$

000m: $0-1$

$000 \mathrm{~m}: 0-1$

PROV: ca cn pa $\quad 000 \mathrm{~m}: 0-1$

PROV: bo ca ch cn da 000m: 0-1 pa sa

PROV: $\mathrm{ch}$

PROV: bo ca ch cn da 000m: $0-1,1-2$ pa sa

000m: $0-1$

PROV: ca pa

000m: $0-1$

PROV: $c C$

000m: $0-1$

PROV: bo

$000 \mathrm{~m}: 0-1$

PROV: ch

$000 \mathrm{~m}: 0-1$

PROV: bo

PROV:

PROV: ca da

000m: $0-1$

$000 \mathrm{~m}: 0-1$

PROV: pa

000m: $0-1$

PROV: ca ch cn da pa 000m: $0-1$

000m: $3+$

000m: $0-1,1-2$

$000 m: 1-2,2-3$
RANGE: cag nag nao sag sao herb RANGE: bol $\mathrm{cr}$ lag sag? herb

RANGE: cag sao herb

RANGE: ang cag? fla mex herb

RANGE: ecu hon herb

RANGE: cag? his mex sag? sao herb

RANGE: sao
RANGE: cag? nag? nao owg sag? herb

SaO cag? nag? nao

RANGE: ang cag? mex sag? sao herb teX

RANGE: cag? mex herb

RANGE: ang cag sat herb

RANGE: ang bol cag? fla mex herb sag? sao

RANGE: cag? ecu mex sag? herb

RANGE: $\mathrm{cr}$ herb

RANGE: ang cag? col mex sag? herb Sao

RANGE: ang bel cag? per sag? herb SaO

RANGE: $\mathrm{CO}$ I herb

RANGE: ang cag? or mex sag? herb Sao

RANGE: cag? gua his per sag? herb Sao

RANGE: $w d w$

herb

RANGE: cag eur nag sag herb

RANGE: end

herb

RANGE: cag? mex sag? sao

herb 
19 (7) GRAMINEAE cont.

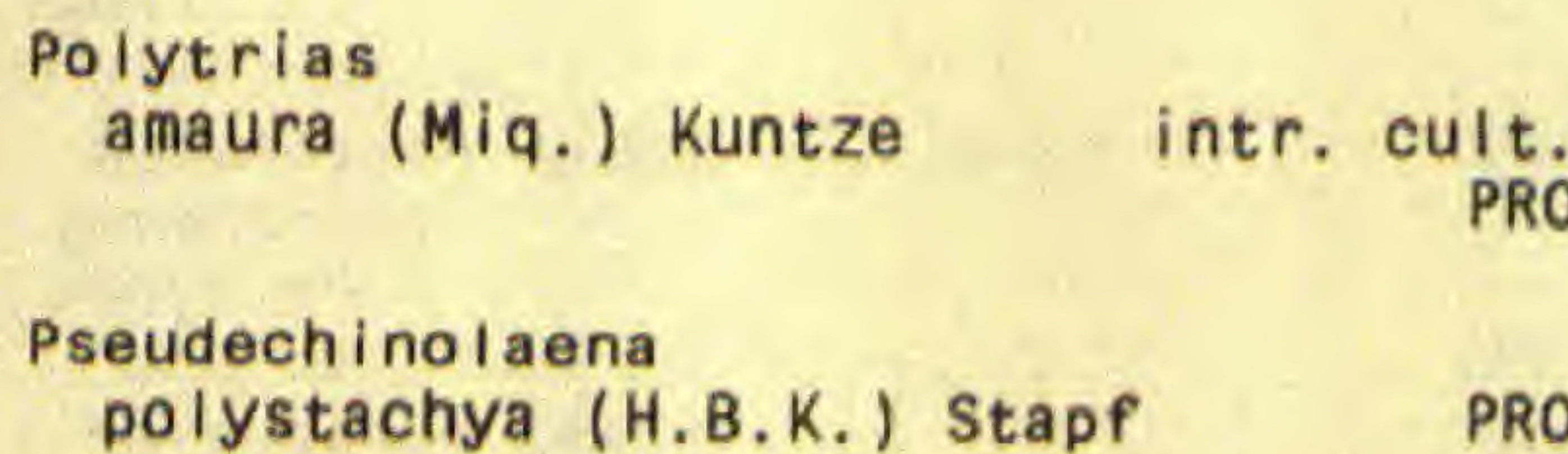

*repens (WiIId,) C.E. Hubb. Rupr. PROV: bo ca ch 000m: 0-1

"Rhynche lyt rum

\section{*Rottboelia \\ *exaltata L. f.}

PROV: ca ch da pa sa $000 \mathrm{~m}: 0-1$

Saccharum

officinarum $L$.

*spontaneum L.

cult.

PROV: ca

PROV: ca cn

000m: $0-1$

000m: $0-1$

Sacciolepis myuros (Lam.) Chase

striata (L.) Nash

*Schizachyrium

*brevifol ium Kunth

*hirtiflorum Nees

*microstachyum (Desv.) Roseng. semiberbe Nees

\section{*tenerum Nees}

Setaria

barbata (Lam.) Kunth intr. geniculata (Lam.) Beauv.

paniculifera (Steud.) Fourn. scandens Schult.

PROV: ca cc pa 000m: $0-1$

PROV: ca 000m: $0-1$

PROV: ca cc da he pa 000m: $0-1$ PROV: ch pa $000 \mathrm{~m}: 0-1,1-2$ PROV: ca cc ch pa 000m: $0-1$ PROV: ca pa $000 \mathrm{~m}: 0-1$ PROV: cc ch 000m: $0-1,1-2$

PROV: ca

PROV: bo ca cc ch da 000m: $0-1,1-2$ PROV: bo ca cc da pa 000m: $0-1,1-2$ PROV: pa pa sa

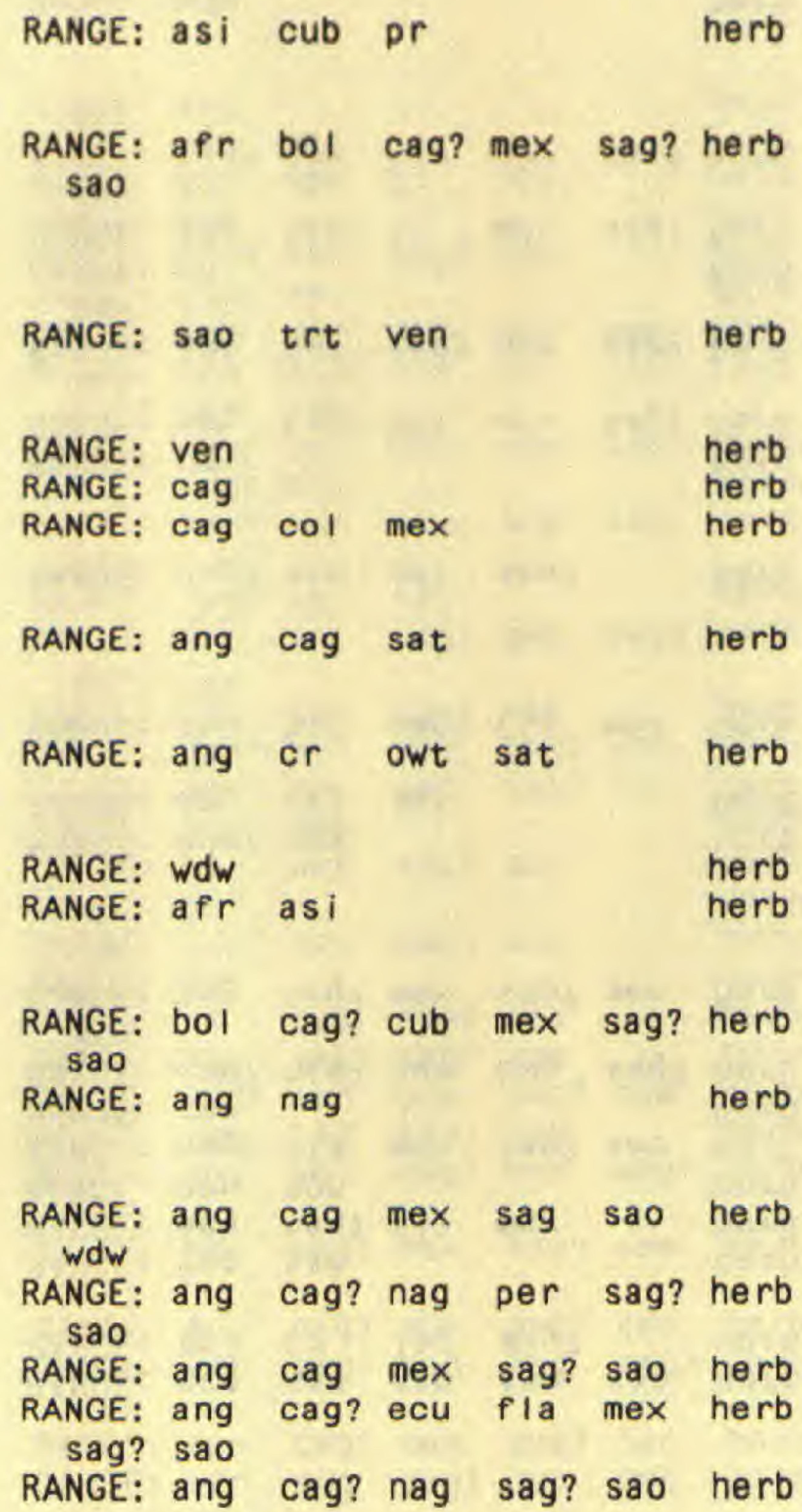


tenacissina schult.

tenax (Rich,) Desv.

vulpiseta (Lam.) R, s.

\section{Sorghast rum \\ Sorghum \\ - bicolor (L.) Moench \\ halepense (L.) Pers. \\ - Spartina \\ * spartinae (Trin.) Merr. \\ Sporobolus \\ cillatus Presi \\ cubens is Mitche. \\ indicus (L.) R. Br. \\ jacquenontil Kunth}

incompletum (Presi) Nash

*setosua (Griseb.) Mitehe.

"purpurascens (SW.) Hasiit.

* pyrainidatus (Las.) Hitcho.

- tenuissimus (Schrank) kuntze virginicus (L.) Kunth

\section{Stenotaphrua \\ secundatum (WaIt.) Kuntze}

\section{Streptochaeta \\ sodi roana Hack. \\ spicata Nees}

\section{St reptogyne}

*americana C.E. Hubb.

- Swa ti lenochioa

*vulcanalis Soders. Walderon

Thrasya

ciliatifolia Svalien

gracilis Swallen

hitchcockit Chase

petrosa (trin.) Chase

viliosa Hitche.
PROV: ch 000a: $0-1$

PROV: pa 000n: $0-1$

PROV: bo ca da pa sa 000 n: $0-1$

PROV: ca ce ch pa 000s: $0-1$ PROV: oh 000n: ve

PROV: ca da he pa vr 000ns 0-1

PROV: ca

000n: $0-1$

PROV: sa ve

000ล: $0-1$

PROV: ca ce ch da

000n: $0-1$

PROV: ca

000n: $0-1$

Phov: ca ce eh on da 000n: 0 he pa ve

Prov: ca ce on he sa 000e: $0-1$ phovi vr

PAOV: ch

PhOV: he

PhoV: ca

000n: $1-2$

PROV: ca on sa 000as: $0-1$

PROV: bo ca ch pa sa 000n: 0-1

000n: $0-1$

PRoV: ca ch da pa 000n: $0-1$

PROV: ca da pa

000n: $0-1$

PROV: sh

000n: 2-1

PROV: ca

PROV: ch

PROV: pa

PAOV: ca ch pa

PAOV: on

RANCE: ang cag? gua sag? sao herb
RANCE: cag sat
RuNcE: ang cag? aex per sag? herb
sao




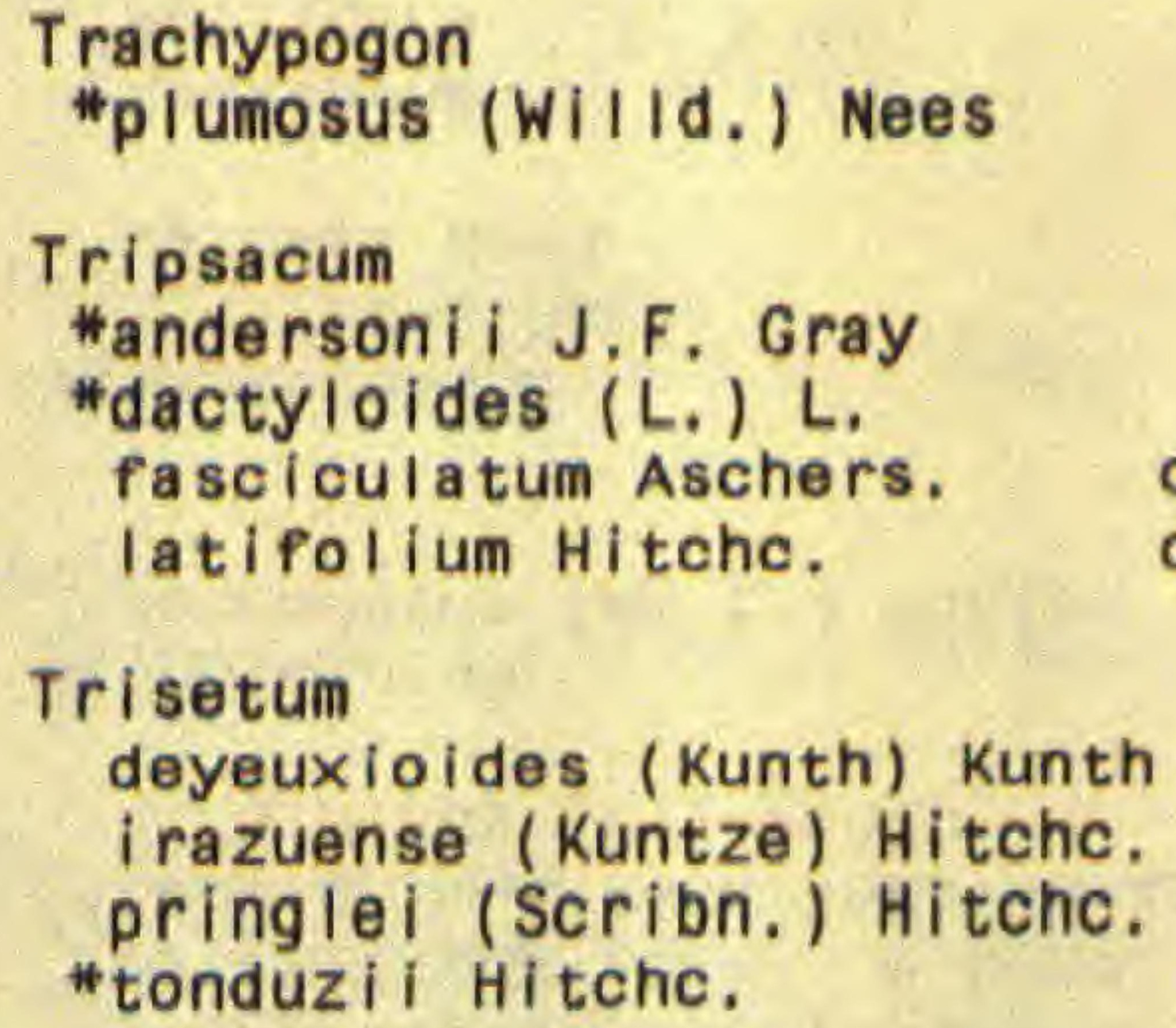

\section{Zeugites}

mexicana Steud. panamensis Swallen

pittieri Hack.

\section{0 (8) CYPERACEAE}

\section{Abildgaardia}

*ovata (Burm. f.) Kral

Ascolepis

brasiliensis C.B. Clarke

*Becquerel ia

*cymosa Brongn.

Bulbostyl is

*acutangula Roxb.

*capillaris (L.) C.B. Clarke

junciformis S. Moore

*juncoides (VahI) Kueken.

paradoxa (Spr.) Lindm.

pubescens (PresI) Sven.

tenuifolia (Rudge) Macbr.

$\begin{array}{ll}\text { PROV: ca he pa } & 000 \mathrm{~m}: 0-1 \\ \text { PROV: ch } & 000 \mathrm{~m}: 1-2 \\ & \\ \text { PROV: ca cn pa } & 000 \mathrm{~m}: 0-1 \\ & \\ \text { PROV: } v r & 000 \mathrm{~m}: \text { we } \\ \text { PROV: wp } & 000 \mathrm{~m}: 0-1,1-2 \\ \text { PROV: ca cc ch pa } & 000 \mathrm{~m}: 0-1,1-2 \\ \text { PROV: ch } & 000 \mathrm{~m}: 1-2 \\ \text { PROV: ca cc pa vr } & 000 \mathrm{~m}: 0-1 \\ \text { PROV: wp } & 000 \mathrm{~m}: \text { we } \\ \text { PROV: ca cc ch pa } & 000 \mathrm{~m}: 0-1,2-3\end{array}$

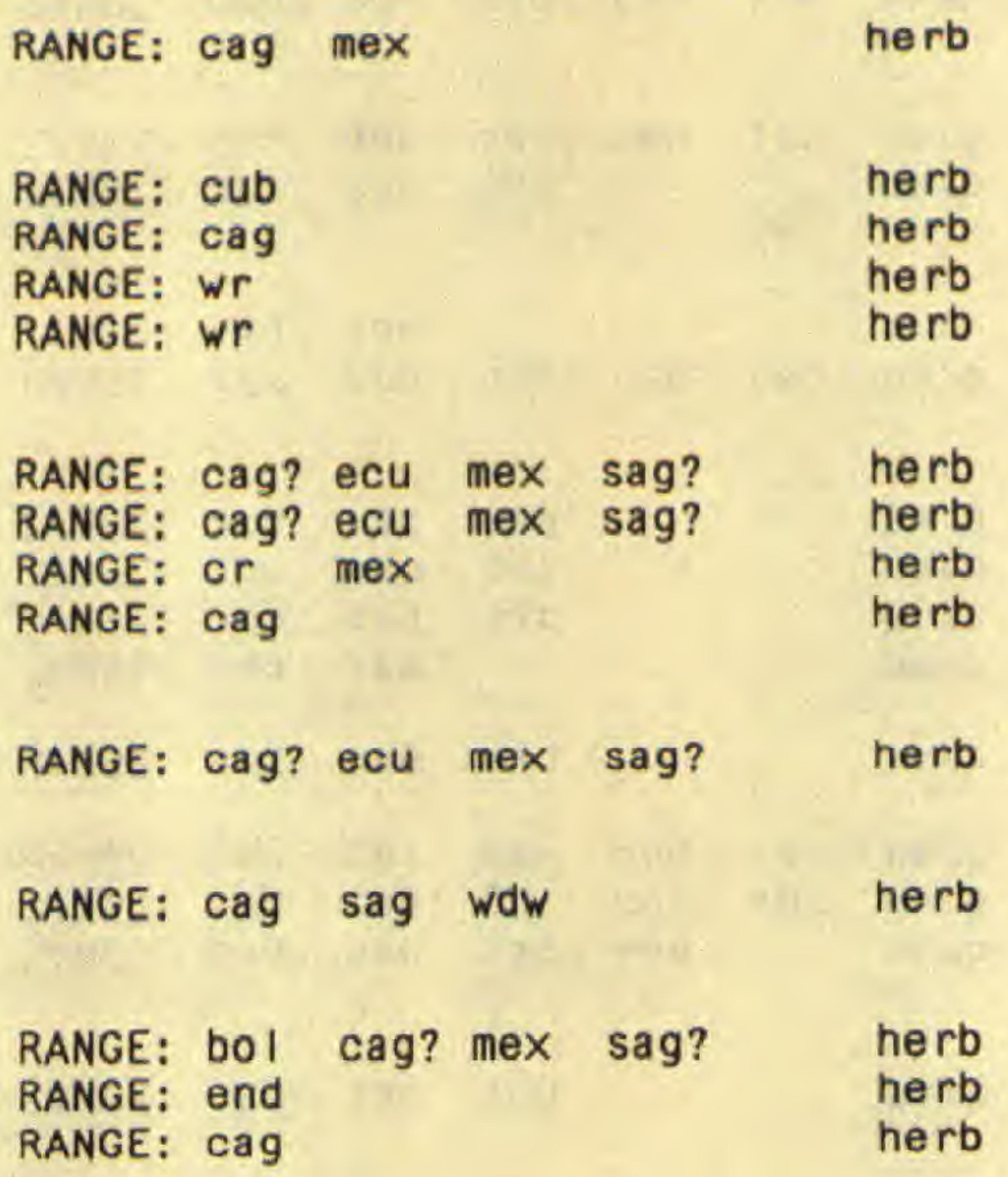

RANGE: WdW

herb

RANGE: af $r$ sao ven

herb

RANGE: $\mathrm{cr}$ sat herb

RANGE: $W r$

RANGE: bol cag? cub hon mex herb

sag? sao

RANGE: cag ecu sao ven herb

RANGE: $c r$ cub sat herb

RANGE: $\mathrm{COI}$ mex herb

RANGE: ang cag mex sag? herb 


\section{Ca lypt roca rya}

glomerulata (Brongn.) Urb.

\section{"poeppigiana Kunth}

\section{Carex}

* bonplandii kunth donnell-smithii Bailey

humboldtiana Steud.

*jamesonii Boott

lemanniana Boott

polystachya Wahl

* polysticha Boeck.

* standleyana Steyerm.

Cladium

jama icense Crantz

\section{Cyperus}

a I bomarginatus (Nees) Steud.

*amabilis Vahl

*articulatus L.

* bipartitus Torr.

cayennensis (Lam.) Britt.

* chorisanthos C.B. Clarke

compressus L.

*dentoniae Tucker

difformis $L$.

* digitatus Roxb.

esculentus L.

int $r$. cult.

PROV: bo ca ce en en 000m: $0-1$

$$
\text { da pa }
$$

PROV: cc da

000m: $0-1$

PROV: bo

PROV: ch

PROV: $\mathrm{ch}$

PROV: ch

PROV: $\mathrm{ch}$

PROV: $\mathrm{ca} \mathrm{cc}$ ch he $000 \mathrm{~m}: 0-1,1-2$

PROV: ch

PROV: $\mathrm{ch}$

000m: $1-2$

PROV: ca cn

000m: $0-1$

PROV: ca da lo pa 000m: 0-1

PROV: ca

000m: $0-1$

PROV: cn pa

000m: $0-1$

PROV: ch

000m: $1-2$

PROV: bo ca cc ch he 000m: $0-1,1-2,3+$ lo pa $v r$

PROV: da

PROV: bo ca cn da lo $000 \mathrm{~m}: 0-1$

pa $\mathrm{Vr}$

PROV: $c h$

PROV: ca

PROV: ca ch lo pa

000m: $0-1$

000m: $0-1$

000m: $0-1$

PROV: ca pa

flavescens $L$.

--var. flavescens

--var. piceus (Liebm.) Fern.

giganteus VahI

globulosus AubI.

haspan L.

hayes i i Standi.

hermaphroditus (Jacq, ) Stand I.

* imbricatus Retz.

* iria L.

* ischnos Schitr.
PROV: cc ch pa vr 000m: 0-1

PROV: WP

PROV: bo ca ch pa 000m: $0-1,1-2$

PROV: ca lo pa sa

PROV: ca cc ch cn pa 000m: $0-1,1-2$

$$
\text { vr }
$$

PROV: $\mathrm{CC}$

PROV: ca ch pa

PROV: ca pa

PROV: ca da

PROV: $c h$ 000m: $0-1$

000m: $0-1,1-2,2-3,3+$ RANGE:

$000 \mathrm{~m}: 0-1$

000m: $0-1$

000m: $1-2$
RANGE: cag sag? sat

herb

RANGE: per sat

herb

RANGE: cag sat herb

RANGE: or gua hon herb

RANGE: cag? mex sag? sao herb

RANGE: cag ven herb

RANGE: $\mathrm{col} \mathrm{cr}$ ecu herb

RANGE: ang cag? mex per sag? herb

RANGE: $w r$ herb

RANGE: gua hon herb

RANGE: cag? fla sag? herb

RANGE: bol cr mex nao nas herb

Sao wdt

RANGE: afr ang cag or gua herb

hon sal sao

RANGE: ang cag sat wdt herb

RANGE: cag cr gua mex nag herb

nic

RANGE: cag mex sag sao tex herb

RANGE: $\mathrm{cr}$ nic herb

RANGE: bol cag nag nao owg herb

$\begin{aligned} & \text { sag sao } \\ & \text { RANGE: } \mathrm{cr} \text { gua mex nic sal herb }\end{aligned}$

RANGE: mex nic owg herb

RANGE: $w d t$

RANGE: WdW herb

RANGE: WdW herb

RANGE: $\mathrm{cr}$ gua mex nic herb

RANGE: ang cag mex sag? sao herb

RANGE: ang cag $\mathrm{cr}$ nao herb

RANGE: WdW herb

RANGE: $\mathrm{cr}$ gua hon herb

RANGE: cag mex sat

RANGE: $\mathrm{cr}$ wdw herb

RANGE: bel nas owg herb 


\section{* anceolatus Poir.}

* axus Lam.

ligularis L.

Iuzulae (L.) Retz.

*manimae H.B.K.

*multifolius Poepp. \& Kunth

*mut isi $i$ (H.B.K.) Griseb.

niger R. \& $P$.

*nubigenus Britt. \& Standl.

*ochraceus VahI

odoratus L.

\#pallens (Liebm.) Stand I. \& Steyerm.

panamens is Standl.

polystachyos Rottb.

prolixus H.B.K.

rotundus $L$.

simplex H,B, K.

sphacelatus Rottb.

surinamensis Rottb.

tenerrimus Pres I

tenuis SW.

*tetraformis EII.

tolucensis H.B.K.

unioloides R. Br.

*virens Michx.

*Diplacrum

*capitatum (WilId.) Boeck.

*Diplasia

*karatifolia Rich.

leocharis

*elegans (H.B.K.) R. \& S.
PROV: $\operatorname{cc} \mathrm{ch} \mathrm{vr} \quad$ 000m: $0-1,1-2$

PROV: sa

PROV: bo ca ce ch cn 000m: $0-1,1-2$ da lo pa sa

PROV: bo ca cc ch cn $000 \mathrm{~m}$ : $0-1,1-2$ da he lo pa sa vr

PROV: $\mathrm{ch}$ 000m: $1-2$

PROV: da 000m: $0-1$

PROV: ca ch $v r \quad 000 \mathrm{~m}: 0-1,1-2$

PROV: ch

PROV: $c h$

000m: $1-2$

PROV: ch da $000 \mathrm{~m}: 0-1$

PROV: bo ca cc ch cn 000m: $0-1$ da he pa sa $v r$

PROV: da

000m: we

PROV: ca cc he pa vr 000m: $0-1$

PROV: bo ca ce cn da 000m: 0-1 pa sa

000m: $1-2$

PROV: ch

PROV: bo ca ch cn da 000m: $0-1$ lo pa $v r$

PROV: ca da pa $v r$ 000m: $0-1$ PROV: ca cn lo pa vr 000m: $0-1$ PROV: bo ca CC cn pa 000m: $0-1,1-2$

PROV: pa $\quad 000 \mathrm{~m}: 0-1$ PROV: bo ca cc ch cn 000m: $0-1,1-2$ da he pa sa vr

PROV: ch

000m: $1-2$

PROV: bo ca $\mathrm{cc}$ ch cn 000m: $0-1$

da pa sa $v r$

PROV: $\mathrm{cc}$ ch

000m: $0-1,1-2$

PROV: ch

$000 m: 1-2$

PROV: $\mathrm{CC}$

000m: $0-1$

PROV: da

000m: $0-1$

$000 \mathrm{~m}: 0-1,1-2,2-3$

\begin{tabular}{|c|c|c|c|c|c|}
\hline RANGE: & ang & bel & $\mathrm{cr}$ & gua & \\
\hline $\begin{array}{l}\text { mex } \\
\text { RANGE: }\end{array}$ & $\begin{array}{l}\text { nas } \\
\text { ecu }\end{array}$ & $\begin{array}{l}\text { sat } \\
\text { per }\end{array}$ & sau & & \\
\hline $\begin{array}{l}\text { RANGE: } \\
\text { RANGE: }\end{array}$ & afr & cag & $\operatorname{mex}$ & nao & nas \\
\hline $\begin{array}{l}\text { sag } \\
\text { RANGE: }\end{array}$ & ang & cag & sag & sao & \\
\hline RANGE: & cag & nas & sag & ven & \\
\hline RANGE: & $\mathrm{cr}$ & sat & & & \\
\hline $\begin{array}{c}\text { RANGE: } \\
\text { hon }\end{array}$ & ang & bel & Dol & $c r$ & guad \\
\hline $\begin{array}{l}\text { hon } \\
\text { RANGE: }\end{array}$ & mex & nic & sag & & \\
\hline RANGE: & bol & cag & cal & sag & saco \\
\hline RANGE: & $\mathrm{col}$ & $\mathrm{cr}$ & ecu & & \\
\hline RANGE: & $\begin{array}{l}\text { cag } \\
\text { wdw }\end{array}$ & mex & nas & sagy & \\
\hline
\end{tabular}

RANGE: WdW

herb

RANGE: cag cal ecu mex per herb

ven

per sal

RANGE: cag nas sag sao wdt herb

RANGE: bel col cr gua hon herb

mex sag sao herb

RANGE: $W d W$ her

RANGE: cag mex sat trt herb

RANGE: afr nwg fla sag sao herb

RANGE: ang cag fex herb

RANGE: cag col mex mex sag herb

SAO

RANGE: $w r$ herb

RANGE: afr bol cag cub mex herb

OWg sag sao
RANGE: cag cal mex owg ven herb

$w d t$

RANGE: ang cag nas sag herb

RANGE: bol sao trt ven herb

RANGE: $\mathrm{cr}$ sat herb

RANGE: nWt herb 
20 (8) CYPERACEAE cont.

\section{filiculmis Kunth}

fistulosa (Poir.) Link geniculata (L.) R. \& S.

interstincta (VahI) R. \& S. minima Kunth

*montana (H.B.K.) R. \& S. mutata (L.) R, \& S nigrescens (Nees) Steud. nodulosa (Roth) Schult.

*pachystyla (C. Wright) C.B. Clarke licarhachis (Griseb.) Svens, * radicans (Poir.) Kunth retroflexa (Poir.) Urb.

*sel lowiana Kunth

Fimbristylis annua $(A \mid I) R,, \& S$.

*autumnalis (L.) R. \& S. * camptotricha C. Wright complanata (Retz.) Link * cymosa R. Br.

*dichotoma (L.) VahI ferruginea (L.) VahI miliacea (L.) Vahl

"pallidula L.

*quinquangularis Kunth spadicea (L.) VahI

\section{Fui rena}

* Camptotricha C. Wright

incompleta Nees

robusta kunth

umbellata Rotto.
PROV: ca cc ch pa vr 000m: $0-1,1-2$

PROV: cc ch pa $\quad 000 \mathrm{~m}: 0-1$

PROV: bo ca cc ch cn 000m: $0-1,1-2$ he pa sa vr

PROV: ca cc ch cn pa 000m: $0-1,1-2$

PROV: ca cc pa $\quad 000 \mathrm{~m}$ : $0-1$

$\begin{array}{ll}\text { PROV: WP } & 000 \mathrm{~m}: 0-1,1-2 \\ \text { PROV: bo ca } & 000 \mathrm{~m}: 0-1\end{array}$

PROV: bo ca

PROV: CC pa

000m: $0-1$

PROV: ca cc ch da pa 000m: $0-1,1-2$

PROV: WP

PROV: ca Pa

PROV: ch

PROV: bo ca cc ch cn 000m: $0-1,1-2$

pa

pa $\mathrm{vr}$

000m: we

000m: $0-1$

$000 \mathrm{~m}: 1-2$

PROV: ch vr

000m: $1-2$

PROV: bo ca ce ch cn 000m: 0-1

da pa sa

PROV: ca 000m: $0-1$

PROV: cn sa 000m: $0-1$

PROV: cc ch pa $v r$ 000m: $0-1,1-2$

PROV: bo ca cn he lo $000 \mathrm{~m}$ : $0-1$

$$
\text { pa sa } v r
$$

PROV: ca cc ch cn da 000m: 0-1 he pa sa $v r v r$

PROV: ca cn sa

000m: $0-1$

PROV: bo ca cc ch cn 000m: 0-1 Prov: da pa

PROV: ca $\quad 000 \mathrm{~m}: 0-1$

PROV: da $\quad 000 \mathrm{~m}: 0-1$

PROV: ca cn da pa sa $000 \mathrm{~m}: 0-1$

PROV: cn sa $\quad 000 \mathrm{~m}: 0-1$

PROV: ch $000 \mathrm{~m}: 1-2$

PROV: ca 000m: $0-1$

PROV: bo ca cc cn pa 000m: 0-1

\begin{tabular}{|c|c|c|c|c|c|c|}
\hline $\begin{array}{c}\text { RANGE: } \\
\text { sao }\end{array}$ & cag & cub & his & mex & sag & herb \\
\hline RANGE: & af $r$ & as $i$ & nwg & & & herb \\
\hline RANGE: & ang & cag? & $\operatorname{mex}$ & sag? & sao & herb \\
\hline $\begin{array}{c}\text { RANGE: } \\
\text { tex }\end{array}$ & cag & fla & $\operatorname{mex}$ & nao & sag? & herb \\
\hline RANGE: & cag & cub & jam & $\operatorname{mex}$ & nao & herb \\
\hline sag? & sao & tex & & & & \\
\hline RANGE: & ang & be 1 & nao & sat? & & herb \\
\hline RANGE: & $a f r$ & nwg & & & & herb \\
\hline RANGE: & af $r$ & bel & cag? & hon & nag? & herb \\
\hline nao & sag? & sao & & & & \\
\hline $\begin{array}{c}\text { RANGE: } \\
\text { tex }\end{array}$ & cag & fla & nwg & sag? & sao & herb \\
\hline RANGE: & cag & cub & his & $p r$ & sat & herb \\
\hline RANGE: & cag? & cub & $\operatorname{mex}$ & sag? & sao & herb \\
\hline RANGE: & oce & & & & & herb \\
\hline RANGE: & cag? & $\mathrm{col}$ & cub & $\operatorname{mex}$ & nao & herb \\
\hline $\begin{array}{l}\text { Sao } \\
\text { RANGE: }\end{array}$ & $\begin{array}{l}\text { Wdt } \\
\text { cag }\end{array}$ & sag & & & & herb \\
\hline RANGE: & wdw & & & & & herb \\
\hline RANGE: & ang & cag & $\operatorname{mex}$ & nao & sat & herb \\
\hline RANGE: & bel & gua & hon & $\operatorname{mex}$ & & herb \\
\hline $\begin{array}{l}\text { RANGE: } \\
\text { RANGE: }\end{array}$ & $\begin{array}{l}w d w \\
w d t\end{array}$ & & & & & $\begin{array}{l}\text { herb } \\
\text { herb }\end{array}$ \\
\hline RANGE: & $w d t$ & & & & & herb \\
\hline $\begin{array}{c}\text { RANGE: } \\
\text { wdt }\end{array}$ & ang? & cag? & cub & owg & sag? & herb \\
\hline RANGE: & wdw & & & & & herb \\
\hline RANGE: & $\operatorname{mex}$ & nic & & & & herb \\
\hline RANGE: & af $r$ & as $\mathrm{i}$ & sat & & & herb \\
\hline RANGE: & nwg & & & & & herb \\
\hline RANGE: & cub & mex & sat & & & herb \\
\hline RANGE: & be! & gua & hon & $\operatorname{mex}$ & sao & herb \\
\hline $\begin{array}{l}\text { RANGE: } \\
\text { RANGE: }\end{array}$ & $\underset{\text { wdw }}{g u i}$ & sao & & & & erb \\
\hline & & & & & & \\
\hline
\end{tabular}




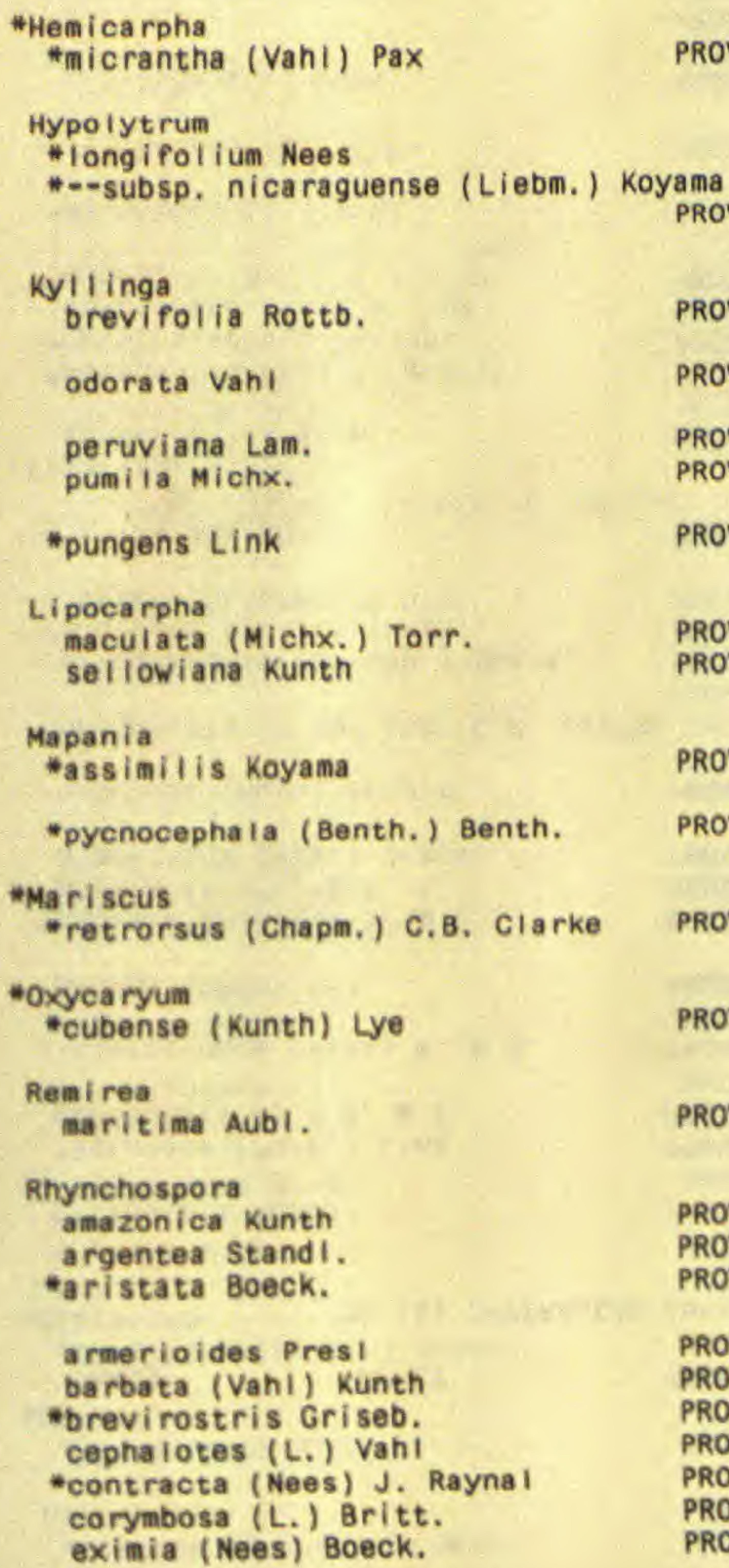

PROV: bo ca cc ch on 000m: $0-1,1-2$ da pa sa $v r$

PROV: ca ch pa $v r$ 000m: $0=1,1-2$

PROV: bo ca cn sa 000m: $0-1$

PROV: ca ca ch cn da 000m: $0-1,1-2$ he lo pa sa

PROV: cn pa vr

000m: $0-1$

PROV: ca cC pa vr 000m: 0-1 PROV: $\mathrm{cC}$ ch

$000 \mathrm{~m}: 0-1,1-2$

PROV: bo ce cn da pa 000m: $0-1,1-2$ sa vr

000m: $0-1$

PROV: cc pa

000m: $0-1$

PROV: CC

000m: $0-1$

PROV: ca pa

000m: $0-1$

PROV: ca

$\begin{array}{ll}\text { PROV: pa } & 000 \mathrm{~m}: 0-1 \\ \text { PROV: cc pa sa } & 000 \mathrm{~m} ; 0-1\end{array}$

PROV: $\mathrm{ch}$ 000m: $1-2,2-3$

PROV: pa $\quad 000 \mathrm{~m}: 0=1$

PROV: CC ch pa 000m: $0-1,1-2$

PROV: WP 000m: we

PROV: ca en pa sa vr 000m: $0-1$

PROV: ca lo pa 000m: $0-1$

PROV: bo ch cn 000m: $0-1,1-2,2-3$

PROV: cc pa
RANGE: afr ang cag nao sao herb

RANGE: cag sao sat herb

RANGE; ang cag nas owg sat herb

WdW af as i cag? fla nao herb

RANGE: afr a

sag? sao WdW

RANGE: afr nWt herb

RANGE: afr nwg herb

RANGE: hon herb

RANGE: cag? nag? sag? herb

RANGE: hon mex sag sao herb

RANGE: col or gui ven herb

RANGE: col or ven herb

RANGE: $w r$ herb

RANGE: afr cag gag nwg? sag herb

RANGE: WdW herb

RANGE: sag? herb

RANGE: end herb

RANGE: or gua hon mex nic herb

Ven

RANGE: or sag? herb

RANGE: ang cag sat herb

RANGE: afr cr hon mex ven herb

RANGE: ang cag mex sat herb

RANGE: afr ang cag nWg herb

RANGE: wdw herb

RANGE: cub hon mex herb 
20 (8) CYPERACEAE cont.

globosa (H.B.K.) R. S.

hirsuta Vahi

*holoschoenoides (Rich.) Herter

*kunthil Nees

Iocuples C.B. Clarke

macrochaeta Steud.

-mexicana (Liebm.) Steud.

*nervosa (Vahi) Boeck.

* orizabensis Kueken.

polyphylla (VahI) VahI

- pubera (Vahl) Boeck.

* radicans (S. C. C.) Pfeiff.

robusta (Kunth) Boeck.

* rugosa (VahI) Gale

*schiedeana Kunth

tenerrima Spr.

*tenuis Link

*trichodes C.B. Clarke

twatsonii (Britt.) Davidse

Scirpus

-inundatus (R, Br, ) Poir.

Scteria

*anceps Liebm.

bracteata Cav.

* distans Poir.

eggersiana Boeck.

interrupta Rich.

- lagoensis Boeck.

latifolia SW.

I ithosperma (L.) SW.

macrophyila Presi

melaleuca Schlecht, \& Cham.

\section{microcarpa Nees}

micrococca (Liebm,) Steud.

mitis Bergius

*polyphyil ia Steud.

purdiei C.B. Clarke

* reticularis Michx.

secans (L.) Urb.
PROV: cc ch pa

PROV: pa

PROV: pa

PROV: ch

PROV: ch

PROV: ch

PROV: pa

ch cn da 000m: $0-1$ lo pa vr

PROV: ch

00m: $0-1,1=2$

000m: $0-1,1-2$

000m: $0-1$

000m: $1-2$

000m: $1-2$

000m: $2-3,3+$

000m: $1-2$

PROV: bo ca pa sa 000m: $0=1$

PROV: $\mathrm{ca} \mathrm{CC}$ ch cn da 000m: $0=1,1-2$ he 10 pa sa vr

PROV: $\mathrm{CC} \mathrm{ch}$

PROV: ch pa

000m: $0-1,1-2$

PROV: ch

000m: $0-1,1-2$

000m: $1-2,2-3$

PROV: pa

PROV: ch

PROV: pa

000m: $0-1$

000m: $1-2$

PROV: ca da pa vr 000m: $0-1$

PROV: ch

000m: $1-2$

PROV: pa

PROV: $\mathrm{ca} \mathrm{cc}$ ch cn da 000m: $0-1$ he lo pa vr

PROV: ce ch

PROV:

000m: $0-1,1-2$

PROV: ca pa

000m: $0-1$

000m: $0-1$

PROV: pa

000m: $0-1$

PROV: ca cc ch da pa 000m: $0-1,1-2$

PROV. $\mathrm{vr}$

PROV: ca pa vr 000m: 0-1

PROV: ca ch pa vr 000m: 0-

PROV: bo ca cc ch da 000m: $0-1$ he lo pa sa vr

PROV: cn

000m: $0-1$

PROV: bo ca cn pa pa 000m: $0-1$

PROV: bo ca cc cn pa 000m: $0-1$ sa

PROV: pa

000m: $0-1,1-2$

000m: $0-1$

PROV: ca ch cn da pa 000m: $0-1$ vr

\begin{tabular}{|c|c|c|c|c|c|c|}
\hline RANGE: & cag & cub & $\begin{array}{l}\text { cag? } \\
\text { sat }\end{array}$ & a & $9:$ & herb \\
\hline RANGE: & af $r$ & ang & cag & $\operatorname{mex}$ & sag & herb \\
\hline sao & & & & & & \\
\hline ANGE: & $w r$ & & & & & herb \\
\hline RANGE: & col & cr & ecu & & & herb \\
\hline RANGE: & er & sag & & & & 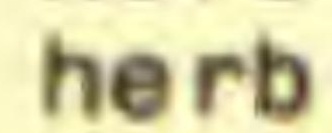 \\
\hline RANGE: & his & $\operatorname{mex}$ & & & & herb \\
\hline RANGE: & ang & cag? & $\operatorname{mex}$ & sag? & & herb \\
\hline ANGE: & or & gua & hon & $\operatorname{mex}$ & & 0 \\
\hline RANGE: & ang & $\mathrm{cag}$ & $\mathrm{col}$ & $\operatorname{mex}$ & ven & $b$ \\
\hline RANGE: & sag? & & & & & b \\
\hline RANGE: & ang & cag & sat & & & herb \\
\hline RANGE: & cag & his & jam & $\operatorname{mex}$ & sag? & b \\
\hline RANGE: & $w d t$ & & & & & b \\
\hline $\begin{array}{l}\text { RANGE: } \\
\text { meX }\end{array}$ & col & $\mathrm{cr}$ & ecu & gua & hon & \\
\hline RANGE: & ang & bel & gua & hon & sat & \\
\hline RANGE: & $w r$ & & & & & \\
\hline RANGE: & $p r$ & sag & & & & \\
\hline ANGE: & cag & $\mathrm{col}$ & ecu & & & \\
\hline RANGE: & as i & or & gua & oce & sag & herb \\
\hline RANGE: & gua & hon & mex & & & herb \\
\hline $\begin{array}{l}\text { RANGE: } \\
\text { SaO }\end{array}$ & ang & bol & cag? & $\operatorname{mex}$ & sag? & herb \\
\hline RANGE: & afr & nwg & & & & he \\
\hline RANGE: & ang & cag & gui & nag? & & \\
\hline RANGE: & ang & bel & $\mathrm{cr}$ & hon & sat & \\
\hline RANGE: & col & $\mathrm{cr}$ & sao & ven & & he \\
\hline RANGE: & cag & lag & $\operatorname{mex}$ & sag & & herb \\
\hline RANGE: & $w d w$ & & & & & h \\
\hline RANGE: & bol & cag? & $\operatorname{mex}$ & sag? & sao & \\
\hline RANGE: & nwt & & & & & \\
\hline RANGE: & cag & cub & mex & sag & sao & \\
\hline RANGE: & cag & cub & $\operatorname{mex}$ & sag & sao & herb \\
\hline RANGE: & ang & cag & cub & sag & sao & 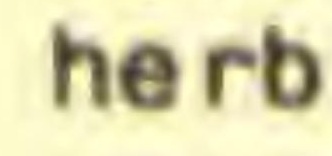 \\
\hline RANGE & $\sqrt{1}$ & & & & & \\
\hline ANGE: & $\mathrm{col}$ & ven & & & & \\
\hline RANGE: & gua & hon & & & & \\
\hline RANGE: & ang & bol & cag & $\operatorname{mex}$ & sao & \\
\hline
\end{tabular}

RANGE: ang bol

sat 
setacea Poir.

setuloso-ciliata Boeck. *tenel la Kunth

*verticillata Muhl.

PROV: pa

PROV: ca da pa

PROV: $c c$

PROV: pa

$000 \mathrm{~m}: 0-1$

000m: $0-1$

$000 \mathrm{~m}: 0-1$

000m: $0-1$

PROV: $\mathrm{cn}$

000m: $0-1$

PROV: ch da $v r$

000m: $1-2,3+$

RANGE: ang cag fla nag? nas herb
Sag? tex
RANGE: cag cub mex sag sao herb
RANGE: ang cag sat
RANGE: ang cag mex nag sag herb

RANGE: cag gag mex sat herb

RANGE: ang cag? mex sag? sao herb
21 (9) PALMAE

Acrocomia panamensis L.H. Bailey

Aiphanes

fuscopubens L.H. Bailey

Asterogyne

martiana Burret

\section{Ast roca ryum}

a la tum Loomis

* confertum Bur.

standleyanum L.H. Bailey

\section{*Atta lea}

*allenii H. Moore

Bactris

al Ieniana L.H. Bailey

aureodrupa L.H. Ba iley

* ba i leyana H. Moore

barronis L.H. Bailey

coloniata L.H. Bailey

coloradonis L.H. Bailey

divisicupula L.H. Bailey

fuscospina L.H. Bailey

* gas ipaes H.B.K.

intr. cult.

PROV: ca ch he pa vr 000m: $0-1$

PROV: pa

000m: $0-1$

PROV: bo cc en da pa 000m: 0-1 sa

PROV: $c a$ cc cn $\mathrm{vr}$ 000m: $0-1$ PROV: WP

000m: we

PROV: ca sa

000m: $0-1$

PROV: ca cn sa

000m: $0-1$

PROV: bo ca cc ch

000m: $0-1$

PROV: CC

PROV: ch

PROV: ca pa $\vee r$

PROV: ca cn pa sa

PROV: ca

on $\quad 000 \mathrm{~m}: 0-1$

PROV: pa

*guineens is (L.) H. Moore major Jacq.

PROV: ca pa

PROV: WP

000m: we

PROV: ca da lo pa 000m: $0-1$

PROV: ca cc lo pa 000m: $0-1$

PROV: cc cn pa sa 000m: $0-1$
RANGE: end

tree

RANGE: end

treelet

RANGE: cag col ven

shrub

RANGE: $\mathrm{cr}$

RANGE: $\mathrm{cr}$

RANGE:

RANGE: $c O$

nic sat

tree

tree

tree

tree

RANGE: nic

RANGE: end

RANGE: $\mathrm{cr}$

RANGE: end

RANGE: sat

RANGE: end

RANGE: $c r$

RANGE: $w r$

shrub

shrub

shrub

shrub

shrub

shrub

shrub

shrub

RANGE: cag

RANGE: cag

RANGE: cag

RANGE: end

tree

wh

shrub

shrub

shrub 


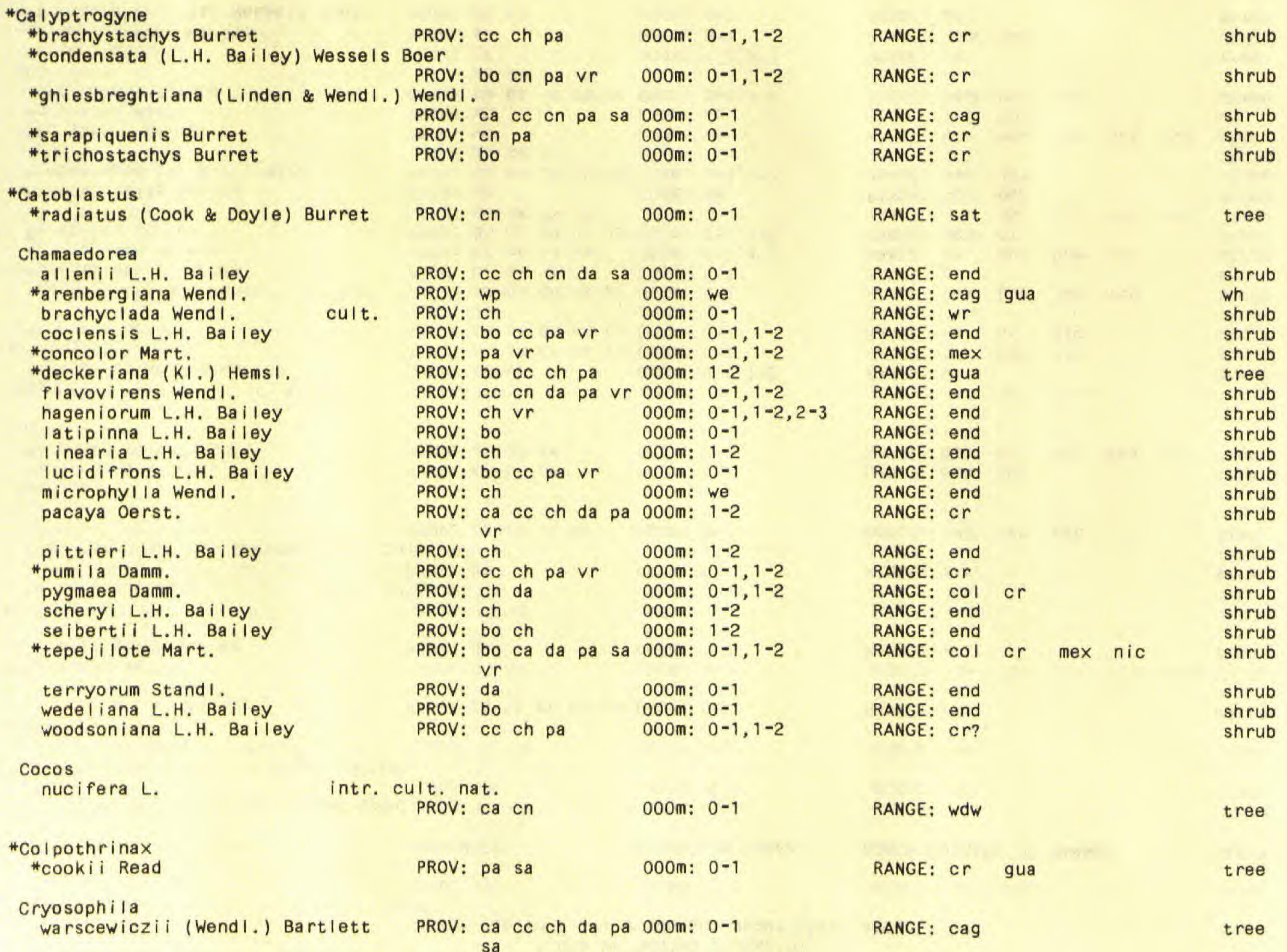




\section{Desmoncus}

isthmius L.H. Bailey

"Dictyoca ryum

"platysepalum Burret

PROV: ca ch cn da pa 000m: 0-1

PROV: da

000m: $1-2$

intr. cult.

PROV: cn

000m: $0-1$

tguineensis Jacq.

Holeifera (H.B.K.) Cortes intr. cult.

PROV: ca ch cn da 000m: $0-1$

\section{Euterpe}

PROV: cc sa vr 000m: $0-1$ macrospadix 0erst.

*precatoria Mart.

\section{Geonoma \\ *calyptrogynoidea Burret \\ congesta Spruce \\ *cuneata Spruce \\ *deversa (Poit.) Kunth \\ *epetiolata H. Moore \\ ferruginea Spruce \\ *gracilis Spruce}

theinrichsiae Burret

*interrupta (R. \& P.) Mart.

* Iehmannii Burret

*oxycarpa Mart.

procumbens Spruce

*seleri Burnet

simplicifrons Willd.

*triandra (Burret) Wessels Boer

$\begin{array}{ll}\text { PROV: ca cn pa } & 000 \mathrm{~m}: 0-1 \\ \text { PROV: } & 0-1\end{array}$

PROV: bo $v r \quad 000 \mathrm{~m}: 0-1,1-2$

PROV: $c c$ cn da pa vr 000m: $0-1$

PROV: bo ca cc cn pa 000m: $0-1$

sa

PROV: bo ce cn da pa 000m: $0-1$

PROV: $c$ c ch cn pa 000m: $0-1,1-2$

PROV: bo $c c$ en da pa $000 \mathrm{~m}: 0-1,1-2$ sa $\vee r$

PROV: WP 000m: we

PROV: bo ca ch cn da 000m: $0-1,1-2$

pa sa $\vee r$

PROV: WP 000m: we

PROV: $w P$ 000m: we

PROV: bo ca cc cn da 000m: $0-1,1-2$

pa $v r$

PROV: $c h$

PROV: da

000m: $1-2,2-3$

000m: $0-1$

PROV: da sa

000m: $0-1$

\section{Hyospathe}

* concinna H. Moore

*elegans Mart.

lehmannii Burret

PROV: $\mathrm{CC}$

PROV: CC

PROV: $C C$

PROV: da pa sa

000m: $0-1$

000m: $0-1$

000m: $0-1$

* simplex Burret

PROV: da

000m: 1-2

PROV: pa

000m: $0-1$
RANGE: end

climber

RANGE: $w r$

tree

RANGE: OWt

tree

RANGE: $c r$ owt sat

tree

RANGE: cag gua tree

RANGE: col gui per sao trt tree

ven

RANGE: $w r$

RANGE: $\mathrm{cr}$

RANGE: $C O I$

hon nic

shrub

shrub

RANGE: or shrub

RANGE: or gua hon nic shrub

RANGE: $\mathrm{col} \mathrm{cr}$ shrub

RANGE: col ecu shrub

RANGE: cag sat shrub

RANGE: col ecu per sat ven shrub

RANGE: cag col $\quad$ wh

RANGE: $w r$ tree

$\begin{array}{ll}\text { RANGE: sao ven } & \text { shrub } \\ \text { RANGE: col } & \end{array}$

RANGE: end

shrub

RANGE: sat

RANGE: col shrub

RANGE: COI shrub

RANGE: col tree

RANGE: $\mathrm{cr}$ tree corneto (Karst.) WendI.

*gigantea Burret 


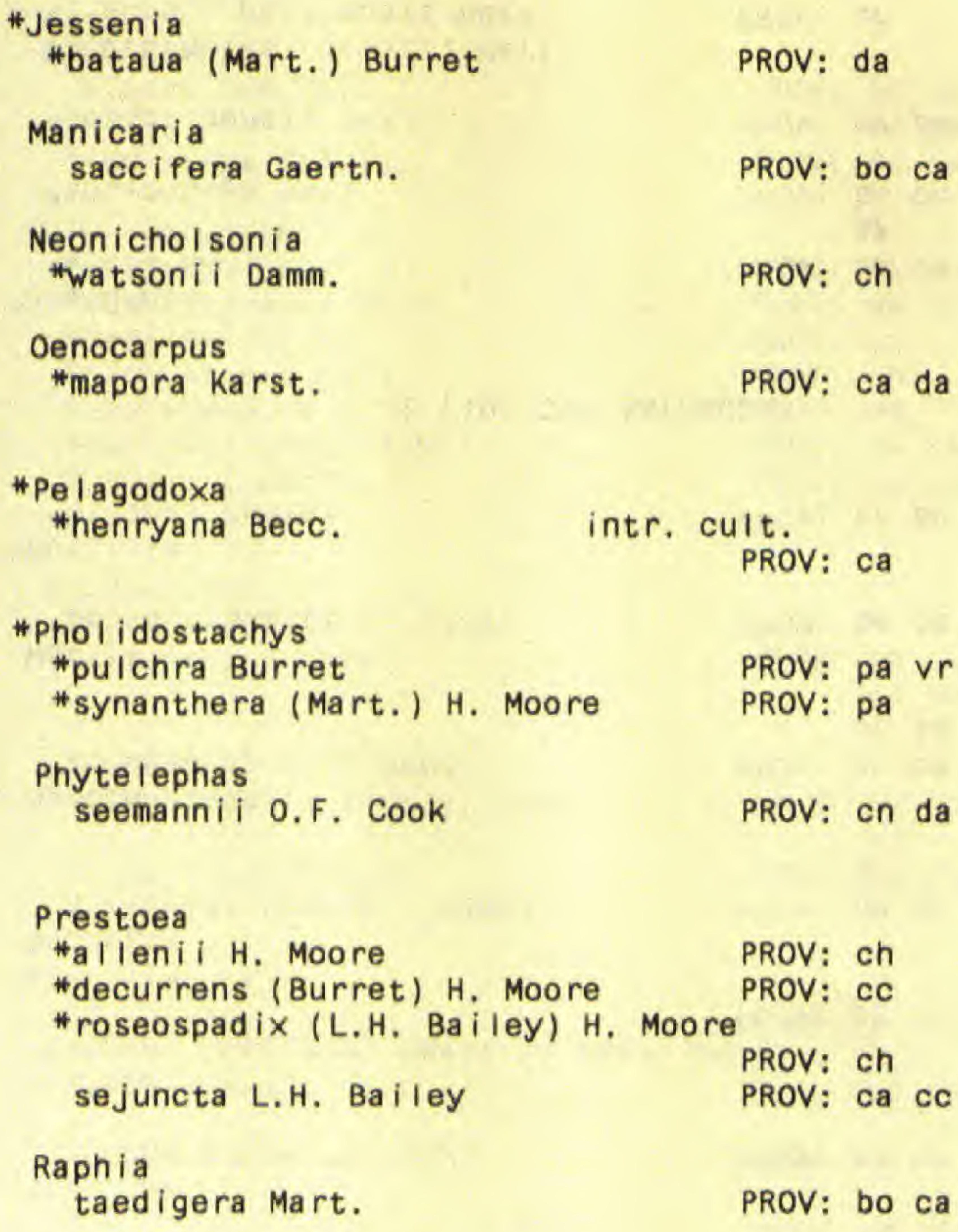

PROV: da

PROV: ch

intr. cult.

PROV: ca

PROV: pa vr

PROV: pa

PROV: cn da

*allenii H. Moore PROV: ch
*decurrens (Burret) H. Moore PROV: cc
* roseospadix (L,H. Bailey) H, Moore

*allenii H. Moore
*decurrens (Burret) H. Moore PROV: ch
* roseospadix (L.H. Bailey) H, Moore

* roseospadix (L.H. Bailey) H. Moore

sejuncta L.H. Bailey

PROV: ca

PROV: bo ca

PROV: bo ca cn sa

PROV: ca da pa sa 000m: $0-1$

000m: $0-1$

000m: $0-1$

000m: $0-1$

000m: $0-1$

000m: $0-1$

000m: $0-1$

000m: $0-1$

000m: we 000m: $0-1,1-2$

000m: $1-2,2-3$

000m: $0-1$

000m: $0-1$

000m: 0-1

000m: $0-1$ 000m: $0-1$

000m: $0-1$

000m: $0-1$
RANGE: sat

tree

RANGE: ang? cag?

tree

RANGE: $\mathrm{cr}$

shrub

RANGE: bol col cr ecu per tree sao ven

RANGE: OWt

tree

RANGE: $\mathrm{cr}$

RANGE: $\mathrm{CO}$ I ecu per

treelet treelet

RANGE: end

treelet shrub

RANGE: end

RANGE: $w r$

wh

tree

RANGE: end

tree

RANGE: sat

tree

RANGE: afr col cr nic nwg? tree sao

shrub

RANGE: cag

RANGE: $\mathrm{cr}$

shrub

RANGE: Iag? trt

tree

RANGE: cub 
(* - not in Flora of Panama under this name)

TAXON

FAMILY

PROVINCES

ELEVATION (OOOM.)

RANGE OUTSIDE OF PANAMA

HAB IT

21 (9) PALMAE cont.

Saba I

$$
\text { allenii L.H. Bailey }
$$

Schee lea

zonens is L.H. Bailey

e renoa

* repens (Bartram) Small intr. cult.

durissima (Oerst.) Wend I.

Synechanthus

warscewiczianus Wend I.

Welfia

georgii Burret

"Wettinia

*hirsuta Burret
PROV: ca

000m: $0-1$

PROV: bo ca en da pa 000m: 0-1 sa

PROV: da pa

000m: $0-1$

PROV: ca cn da pa 000m: $0-1$

PROV: bo ca $c c$ en da 000m: $0-1,1-2$ pa sa $v r$

PROV: bo ca ce pa 000m: 0-1

PROV: en pa sa

000m: $0-1$

\section{2 (10) CYCLANTHACEAE}

\section{*Aspl und ía}

*a lata Harl.

*antioquiae Harl.

* costaricensis Harl.

* microphylla (Oerst.) Harl. *--subsp. panamensis Harl. * pittieri (Woods.) Harl.

* sleeperae Grayum \& Hammel

*vagans Harl.

\section{Carludovica}

drudei Mast.

palmata R. \& P.

rotundifolia Hook. $f$.

*--subsp. pachyspadix Harl.
PROV: ca ch cn da pa 000m: $0-1,2-3$

PROV: bo cc 000m: $0-1,1-2$

PROV: cc pa sa

000m: $0-1$

PROV: ch

PROV: Sa

PROV: $C C$

000m: $1-2,2-3$

000m: $0-1$

000m: $0-1$

PROV: $\mathrm{CC}$

000m: $0-1$

PROV: ca ch da sa 000m: $0-1$

PROV: bo ca cc cn da 000m: $0-1,1-2$ pa

PROV: ch

$000 m: 1-2,2-3$
RANGE: end

RANGE: $\mathrm{cr}$

RANGE: $\mathrm{fI}$ a

RANGE: $\mathrm{cr}$ nic sat

tree

RANGE: $\mathrm{COI}$ cr ecl

shrub

RANGE: $\mathrm{COI} \mathrm{cr}$

tree

RANGE: $c O$ I

tree

tree

tree

shrub

ee shrub

cl imber

epiph

herb

epiph

herb

RANGE: or climber

RANGE: $c r$ shrub

RANGE: $c r$ herb

RANGE: $\mathrm{COl} \mathrm{cr}$ ecu gua per epiph

ven

$\begin{array}{lll}\text { RANGE: } \mathrm{cr} \text { mex } & \text { shrub } \\ \text { RANGE: bol cag gag mex per shrub }\end{array}$

RANGE: $c r$ 
TAXON

22 (10) CYCLANTHACEAE cont.

--subsp, rotundifolia

Cyclanthus

bipartitus poit.

*indivisus Schult.

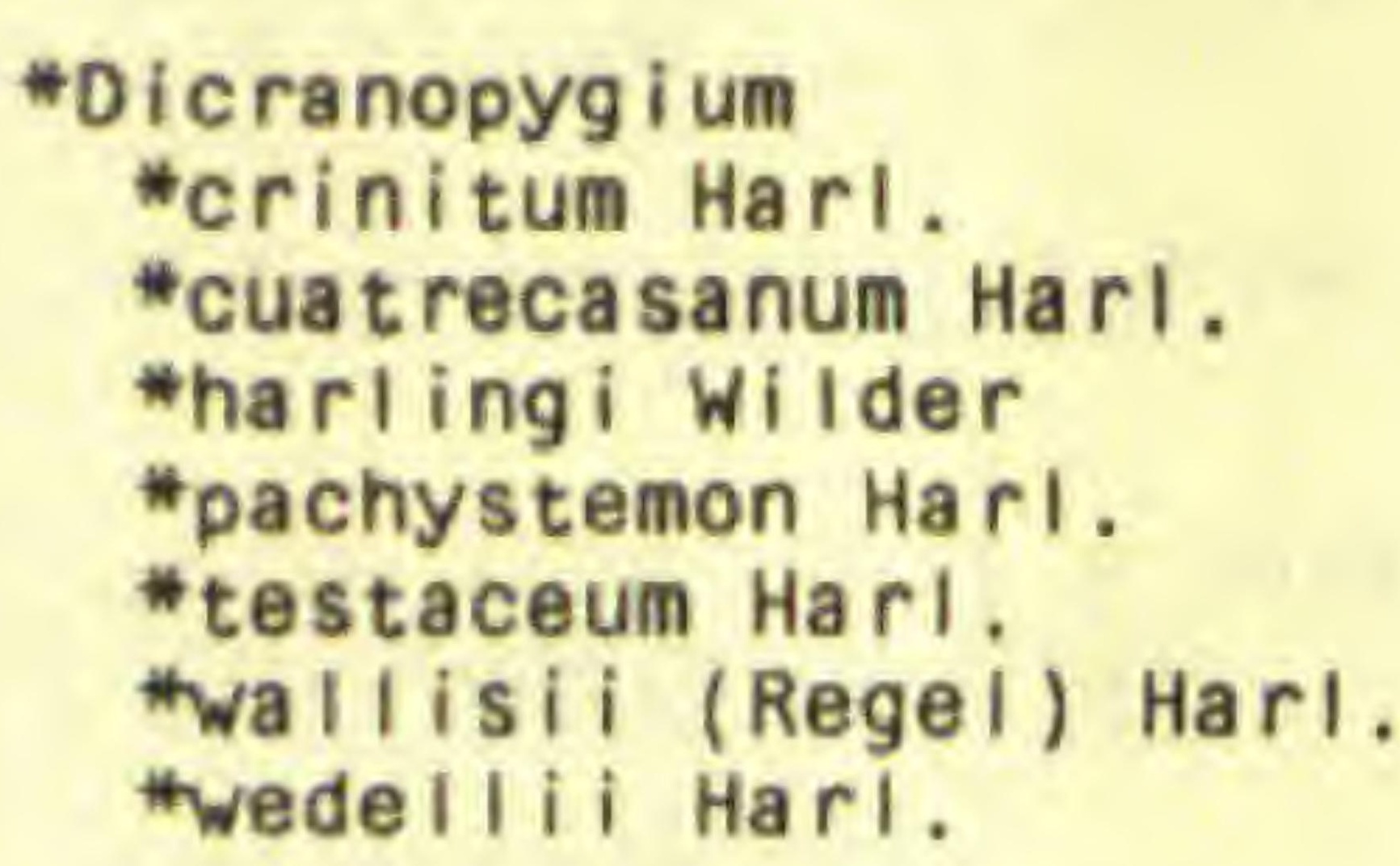

twoodsoni i Harl.
PROV: bo

$000 \mathrm{~m}: 0-1$

PROV: bo ca cc ch cn 000m: $0-1,1-2$ da pa sa vr

PROV: pa sa

000m: $0-1$

PROV: ca cn pa sa 000m: $0-1,2-3$

PROV: CC Vr $000 \mathrm{~m}: 0-1$

PROV: pa 000m: $0-1$

PROV: cn

PROV: da

000m: $0-1$

PROV: da pa

$000 \mathrm{~m}: 0-1$

PROV: bo cc da pa vr 000m: $0-1,1-2$

PROV: bo cc cn da pa 000m: 0-1 sa sa $\mathrm{vr}$

PROV: ca cc cn da pa 000m: 0-1 $\mathrm{vr}$

PROV: cn pa

000m: $0-1$

PROV: cc pa

000m: $0-1,1-2$

000m: $1-2,2-3$

PROV: CC ch da

000m: $2-3$

PROV: ch

PROV: cc ch pa

000m: $0-1,1-2$

PROV: ch

PROV: da

PROV: ch

PROV: $\mathrm{ch}$

PROV: pa

PROV: $\mathrm{CC}$

PROV: cc cn da

000m: $1-2,2-3$

$000 \mathrm{~m}: 0-1$

000m: $0-1$ onom: $0-1$

RANGE: or hon

shrub

RANGE: cag gua lag per sag shrub

Sao trt

RANGE: $\mathrm{CO}$

shrub

RANGE: end

RANGE: $\mathrm{COI}$

RANGE: end

RANGE: per

RANGE: $c O$

RANGE: $\mathrm{COI}$

RANGE: cag? $\mathrm{cr}$ gag hon herb

herb

herb

herb

herb

herb

herb

shrub

RANGE: cag? $\mathrm{cr}$ hon per sao climber trt

RANGE: $\mathrm{COI} \mathrm{cr}$ ecu

RANGE: col per sao

climber

shrub

epiph

herb

RANGE: end

epiph

herb

RANGE: end

RANGE: end

RANGE: $\mathrm{cr}$

RANGE: ecu

RANGE: $C O I$

RANGE: end

RANGE: ecU

RANGE: $\mathrm{COI}$

RANGE: end terr

herb

herb

epiph

herb

herb

shrub

herb

herb

epiph

herb

epiph

herb

RANGE: col gui per sag sao climber 


\section{*Alocasia}

*macrorrhiza Schott

\section{cult}

Anthurium

acutangulum Engl.

\#acutifolium Engl.

\section{Halticola Croat}

*amnicola Dressler

*angust il obum Croat

*angustispadix Croat Baker

*antonioanum Croat

Hbakeri Hook. $f$.

*barryi croat

"bicol lectivum Croat

*brevispadix Croat

*browni i Mast.

*ca loveboranum Croat

*caperatum Croat \& Baker

*carnosum Croat \& Baker

* cartiense Croat

* cerrocampanense Croat

* cerropirrense Croat

chiriquense standi.

*chorrense Croat

*chromostachyum Croat

*cineraceum Croat

*cinereopetiolatum Croat

*circinatum Croat

*clavigerum Poepp.

*clidemioides StandI. 000m: $0-1$

RANGE: as i

herb

PROV: bo bo ca cn pa 000m: $0-1,1-2$

PROV. sa

000m: $0-1$

PROV: bo $\mathrm{ch}$ da

000m: $1-2$

PROV: $\mathrm{CC}$

PROV: pa

000m: $0-1$

000m: $0-1$

000m: $0-1,1-2$

PROV: ch da

000m: 1-2

PROV: bo ca cc en da 000m: $0-1$ pa sa $v r$

PROV: da 000m: $0-1$

PROV: ca ce ch cn da 000m: $0-1$

pa $v r$

PROV: $\mathrm{CC}$

RANGE: $c r$ hon nic

RANGE: $\mathrm{cr}$

RANGE: $\mathrm{COI}$

RANGE: end

RANGE: end

RANGE: $c r$

RANGE: end

RANGE: be I

nic

RANGE: end

RANGE: end

RANGE: $\mathrm{CO}$

$\begin{array}{ll}000 \mathrm{~m}: & 0-1 \\ 000 \mathrm{~m}: & 0-1,1-2\end{array}$

PROV: ca

PROV: bo cc ch $v r \quad 000 m: 0-1,1-2$
PROV: $c c$ ch da pa $v r$ 000m: $0-1,1-2$

PROV: 00 ch da pr $000 m: 0-1,1-2$

RANGE: $c r$

PROV: bo $\mathrm{ch}$

o00m: $0-1,1-2,2-3$

RANGE: $c r$

PROV: cn pa sa

000m: $0-1$

PROV: cc cn pa

000m: $0-1$

PROV: da

PROV: ch

PROV: pa

PROV: da

000m: $0-1,1-2$

000m: $1-2$

000m: $0-1$

000m: $0-1$

000m: $0-1$

PROV: da

000m: $0-1$

PROV: pa

000m: $1-2$

PROV: bo $\mathrm{ch}$

PROV: bo ca cc ch cn 000m: $0-1$ da pa sa

000m: $0-1$
RANGE: end RANGE: end

RANGE: end

RANGE: end

RANGE: end

RANGE: end

RANGE: $\mathrm{CO}$ I

RANGE: end

RANGE: end

RANGE: bol col or ecu gui epiphyte

nic per sao sat ven

RANGE: $\mathrm{cr}$

terr

herb

terr

epiph

herb

epiph

herb

terr

ep iph
herb epiph

herb

terr

epiph

herb

herb

herb

epiph

herb

col cr gua mex epiphyte

epiphyte

ep iphyte

epiphyte

epiphyte

ep iphyte

terr

ep iph

her

epiph

climber

epiphyte

ep iphyte

epiphyte

epiphyte

epiphyte

PROV: bo 
23 (11) ancrot soet.

tiveranas Great

*uovinasir Croat

tetahenge Crost

celonisuan R. Rrause

*taligradensa croas

toneiahatein Schott

*isuhealar K. Krause

todhscb inum Sehot:

*ceprase croas

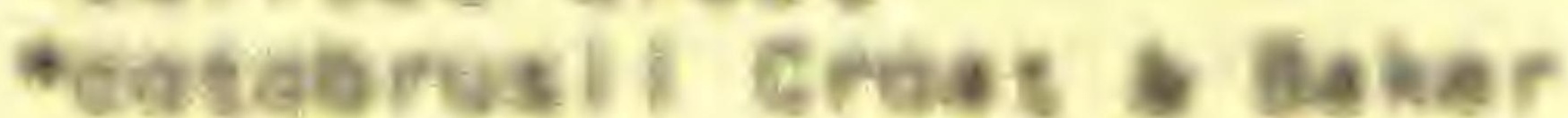

*opastianinum Croas

- Grassimalix Croat

- wovar, erassiradix

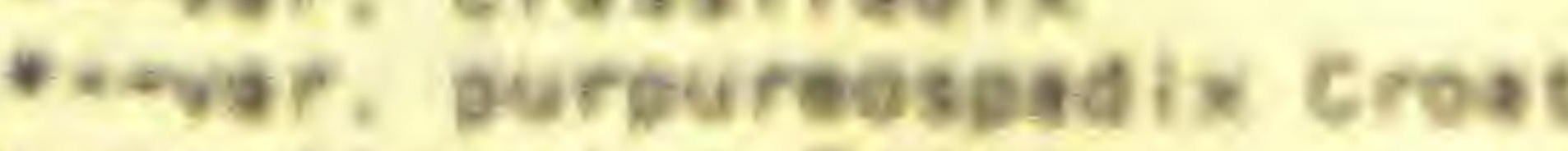

cerassibapalium Croat

*arystali inum Linden andre

*auas fitanuan Crogt

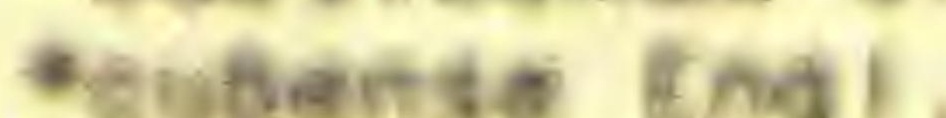

- suevi fiseathuan Croat

*tuneatisaimum $\{($ heis $)$ Croat

*eurvilaninein Croat

*arvisoadix Crast

*evalidatush Mast.

+davidaentas standi.

-ifichrophyi i uis Great

on rassieri croat.

thuka i Creat

- Avyari Sroat

- arysarestachyua croat

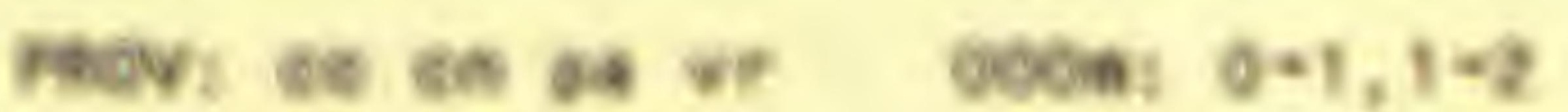

ocom! 0-1

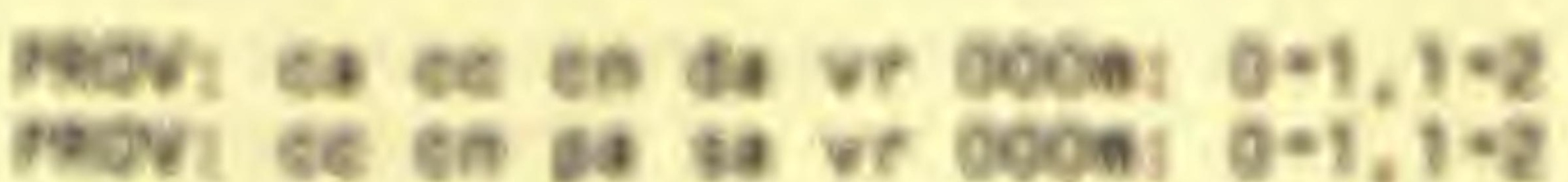

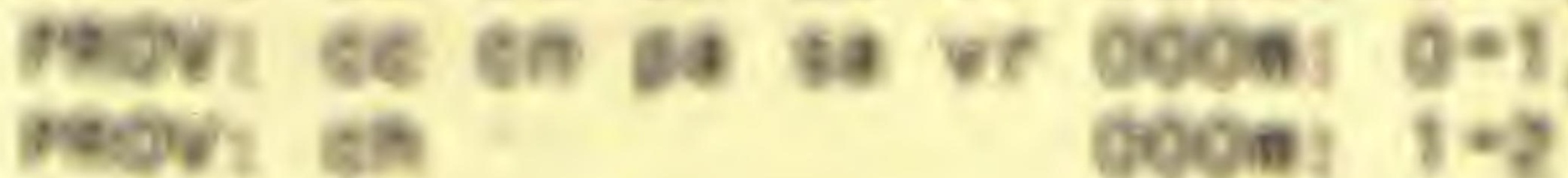

Hark es ah ga

006 : $0 * 1,2 * 3$

movi be ea en

Hev? vi

thovy: as

agon! $0^{-1}$

000 nt $0-1$

oogen! $1=2$

movr be

600n! $0-1$

abor ce en pa

000a: $0-1$

Doon: $0-1$

000 en: $1=2$

000 n! $1=2$

goon: $t=2$

nobvy ta

phov: is

onom: $0-1$

nowi sh

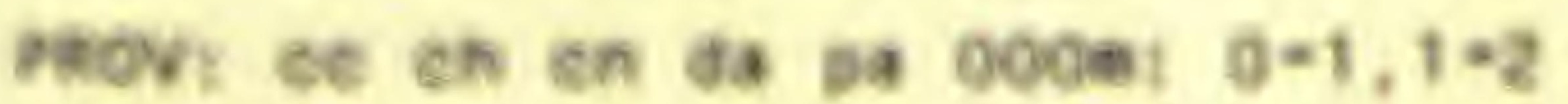
ver

peory se ch

000n: $0 * 1$

howi se oa

000 n: $0-1$

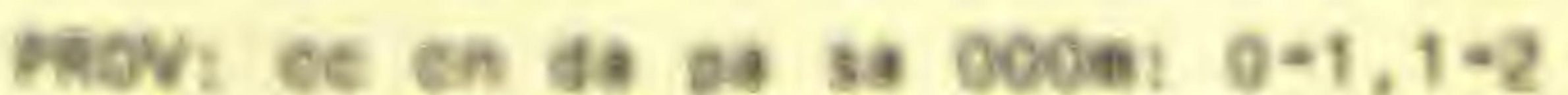

$$
\text { ir }
$$

novy bo es eh en at gocen: $0 * 1,1 * 2$ Da sa vr

phick: se ch

oobes: $1+2$

now: vr

000 a $1+2$

packi ea en as sa

000 : $0=1$

Dhov: $4 a$

000 a: $0-1,1=2$

rov: ec en ds a sa 000n? $0<1,1-2$

nov: is

ooon: i-2 aver: ond

nacr, end

vodit ens

vesti end

exwori and

enect er

anect col

ewor! cag

eavor: end

eascr: er

2awof: end

akor: and

awor: end

ance: en:

eakcf: col

aAwoE: ond

aruct: cer

nic ven

pavcr: end

ancr, of

awce: ind

Ravor! end

anor: col

er

ocy

anber of

ANGr: end

ANot; col

ANCE: and

AANGE! COI

ANACE: Col cotion

terr

hers

torr

nert

es iebrits

epienyrte

terr

nefs

epibh

nert

piehyrte

epishyrte

opiphyse

tert

opiph

nerb

epiph

norb

ebiohyste

epiphyte

epiphyte

eo ishyte

epiphyte

err

herb

opiphyte

poiphyre

epiph

terr

epiphyto

terr

epiph

herb

terr

nerb

epishyte

terr

nerb

epiohyte

epiphyte

epiph

tere 
23 (11) ARACEAE cont.

\section{*fatoense K. Krause}

*fendieri Schott

*flexile Schott

* fol somianum Croat

* foreroanum Croat

*formosum Schott

* fragrant iss imum Croat

*friedrichsthalii Schott

\section{*fus iforme Croat}

garagaranum StandI.

\section{* gentryi Croat}

*globosum Croat

*gracile (Rudge) Lindl.

*gracililaminum Croat

* gracil ispadix Croat

*hacumense Engl.

*hammelii croat

"hebetatum Croat

*hoffmannii Schott

\section{*hornitense Croat}

*hutchisoni i Croat

* impolitum Croat

* interruptum Sod.

\section{*jefense Croat}

*ka I l unkiae Croat

*kamemotoanum Croat
PROV: bo ce ch on vr 000m: $0-1$ PROV: $V r$ $000 \mathrm{~m}: 0-1$

PROV: bo ca ce ch da 000m: 0-1

PROV: pa

000m: $0-1$

PROV: da

000m: $1-2$

PROV: bo $c c$ ch cn $v r$ 000m: $0-1,1-2$

PROV: cc cn da pa vr 000m: $0-1,1-2$

PROV: bo ca ce ch cn 000m: 0-1 da pa sa $\vee r$

PROV: $\mathrm{ch}$

000m: $1-2$

PROV: cc cn da pa sa 000m: $0-1,1-2$

PROV: $V r$

000m: $0-1$

PROV: bo $\mathrm{ch}$

000m: $1-2,2-3$

PROV: bo ca da pa 000m: $0-1$

PROV: ch

PROV: bo cc ch

$000 \mathrm{~m}: 0-1,1-2$

PROV: ca ce ch cn da 000m: $0-1,1-2$

pa sa vr

PROV: bo *

PROV: CC Vr

PROV: $\mathrm{cc}$ ch cn $\mathrm{vr}$

000m: $1-2$

000m: $0-1,1-2$

o00m: $0-1,1-2$

PROV: ch vr

000m: $1-2$

PROV: $c c$ ch cn da pa 000m: $0-1,1-2$ sa $\mathrm{vr}$

PROV: cn da pa sa PROV: $\mathrm{cc}$ ch da $v \mathrm{r}$

000m: $0-1$

$000 \mathrm{~m}: 0-1,1-2$

PROV: pa

000m: $0-1,1-2$

PROV: $\vee r$

PROV: pa 000m: $1-2$

000m: $0-1$
RANGE: $c r$

RANGE: col ven

ep iphyte

terr

epiph

RANGE: bel cag or gua hon epiphyte

mex nic

RANGE: end

RANGE: end

RANGE: COI

cr ecu

terr

ep iph

epiphyte

epiph

terr

herb

RANGE: $\mathrm{CO}$

RANGE: cag col cr gua

piph

herb

epiphyte

RANGE; end

RANGE: $\mathrm{COI}$

RANGE: end

RANGE: end

ep iphyte

epiph

herb

epiphyte

epiph

terr

RANGE: ang bel cag col or epiphyte

gua gui hon per sat trt

RANGE: end

RANGE: end

RANGE: COI

cr

RANGE: end

RANGE: end

RANGE: $\mathrm{cr}$

RANGE: end

RANGE: COI

RANGE: end

RANGE: bel

$$
\text { nic }
$$

RANGE: end

col cr ecu gua

RANGE: end

RANGE: end epiphyte

epiph

herb

ep iphyte

epiphyte

epiphyte

epiph

terr

herb

epiph

herb

terr

epiph

herb

herb

epiph

herb

epiph

herb

ep iphyte

epiph

terr 
*kunthil Poepp.

* lactifructum Croat

* I ancifol ium Schott

*--var. a Ibifructum Croat

*--var. I ancifolium

* lentii Croat \& Baker

* leptocaule croat

* I lanense Croat

* Iong istipitatum Croat

* Iuteyni i Croat

*madisonianum Croat

*mal ianum Croat

*me lastomat is Croat

michelii Guillaumin

microspadix Schott

myosuroides (H.B.K.) Endl, *nervatum Croat

*niqueanum croat

*obtusilobum Schott

ochranthum K. Koch

*oxystachyum Croat

*pageanum Croat

pallens Schott

"pa I udosum Engl.

*panamense Croat

*panduriforme Schott

*papillilaminum Croat

*pauc iflorum Croat
PROV: bo ca cc cn da 000m: $0-1$ pa sa

PROV: da 000m: $0-1$

PROV: ch 000m: $1-2$

PROV: bo $\mathrm{cc} \mathrm{ch}$ cn da $000 \mathrm{~m}$ : $0-1,1-2$ pa sa $\vee r$

PROV: cc ch cn da pa 000m: $0-1,1-2$ sa $v r$

PROV: bo cc ch vr 000m: $0-1,1-2$

PROV: cn pa sa $000 \mathrm{~m}: 0-1$

PROV: bo $\mathrm{ch}$

000m: $1-2$

PROV: CC da $v r$

000m: $0-1$

PROV: ch Vr

000m: $0-1,1-2,2-3$

PROV: da

$000 \mathrm{~m}: 1-2$

$000 \mathrm{~m}: 0-1,1-2$

PROV: cc sa

PROV: bo $\mathrm{cc}$ ch $\mathrm{cn}$ da 000m: $0-1,1-2$ pa sa $\vee r$

PROV: ch $v r$

000m: $0-1,1-2,2-3$

PROV: ca cc pa

PROV: $c c$ ch $V r$

PROV: da

000m: $0-1$

000m: $0-1,1-2$

000m: $1-2$

PROV: bo ch da

000m: $0-1,1-2$

PROV: bo ca ce ch cn 000m: $0-1,1-2$ da pa sa vr

PROV: $\mathrm{cC}$

PROV: bo cc pa vr

PROV: bo $\mathrm{ch}$

000m: $0-1$

000m: $0-1,1-2$

000m: $1-2$

PROV: bo ca cc cn da 000m: $0-1$ pa sa

PROV: bo cc vr

000m: $0-1$

PROV: bo cc ch da pa $000 \mathrm{~m}$ : $0-1,1-2$

PROV: cn

000m: $0-1$

000m: $1-2$

\begin{tabular}{|c|c|c|c|c|c|c|}
\hline $\begin{array}{l}\text { RANGE: } \\
\text { RANGE: }\end{array}$ & $\begin{array}{l}\text { col } \\
\text { end }\end{array}$ & $\mathrm{cr}$ & ecu & nic & per & $\begin{array}{l}\text { epiph } \\
\text { herb } \\
\text { epiphyte }\end{array}$ \\
\hline RANGE: & end & & & & & herb \\
\hline RANGE: & $\mathrm{col}$ & $\mathrm{cr}$ & nic & & & $\begin{array}{l}\text { epiph } \\
\text { terr } \\
\text { herb }\end{array}$ \\
\hline RANGE: & $\mathrm{col}$ & $\mathrm{cr}$ & & & & $\begin{array}{l}\text { epiph } \\
\text { terr } \\
\text { herb }\end{array}$ \\
\hline $\begin{array}{l}\text { RANGE: } \\
\text { RANGE: }\end{array}$ & $\begin{array}{l}\text { end } \\
\text { end }\end{array}$ & & & & & $\begin{array}{l}\text { herb } \\
\text { ep iphyte }\end{array}$ \\
\hline RANGE: & end & & & & & ep iphyte \\
\hline RANGE: & end & & & & & $\begin{array}{c}\text { epiph } \\
\text { terr } \\
\text { herb }\end{array}$ \\
\hline RANGE: & end & & & & & ep iphyte \\
\hline RANGE: & $\mathrm{col}$ & & & & & epiphyte \\
\hline RANGE: & end & & & & & $\begin{array}{l}\text { epiph } \\
\text { terr } \\
\text { herb }\end{array}$ \\
\hline RANGE: & $\mathrm{col}$ & $\mathrm{cr}$ & & & & $\begin{array}{l}\text { epiph } \\
\text { terr } \\
\text { herb }\end{array}$ \\
\hline $\begin{array}{c}\text { RANGE: } \\
\text { hon }\end{array}$ & $\begin{array}{l}\text { bel } \\
\text { mex }\end{array}$ & $\begin{array}{l}\text { col } \\
\text { nic }\end{array}$ & $\mathrm{cr}$ & ecu & gua & $\begin{array}{l}\text { epiph } \\
\text { terr } \\
\text { herb }\end{array}$ \\
\hline $\begin{array}{l}\text { RANGE: } \\
\text { RANGE: }\end{array}$ & col & & & & & epiphyte \\
\hline $\begin{array}{l}\text { RANGE: } \\
\text { RANGE: }\end{array}$ & $\begin{array}{l}\text { end } \\
\text { end }\end{array}$ & & & & & $\begin{array}{c}\text { ep iphyte } \\
\text { epiph } \\
\text { herb }\end{array}$ \\
\hline RANGE: & $\mathrm{col}$ & $\mathrm{cr}$ & & & & $\begin{array}{c}\text { epiph } \\
\text { terr } \\
\text { herb }\end{array}$ \\
\hline RANGE: & $\mathrm{cr}$ & & & & & herb \\
\hline RANGE: & end & & & & & epiphyte \\
\hline RANGE: & end & & & & & epiphyte \\
\hline RANGE: & cr & & & & & $\begin{array}{l}\text { epiph } \\
\text { herb }\end{array}$ \\
\hline RANGE: & $\mathrm{col}$ & $\mathrm{cr}$ & & & & $\begin{array}{l}\text { epiph } \\
\text { herb }\end{array}$ \\
\hline RANGE: & end & & & & & $\begin{array}{l}\text { epiph } \\
\text { herb }\end{array}$ \\
\hline RANGE: & col & $\mathrm{cr}$ & & & & $\begin{array}{l}\text { epiph } \\
\text { herb }\end{array}$ \\
\hline $\begin{array}{l}\text { RANGE: } \\
\text { RANGE: }\end{array}$ & $\begin{array}{l}\text { end } \\
\text { end }\end{array}$ & & & & & $\begin{array}{l}\text { herb } \\
\text { epiphyte }\end{array}$ \\
\hline
\end{tabular}


23 (11) ARACEAE cont.

"pendens Croat

*pentaphy I lum (Aub I.) G. Don

PROV: cn da pa sa $000 \mathrm{~m}$ : $0-1$

*--var, bombacifolium (Schott)

"pirrense Croat

pittieri Engl.

*--var. moril Engl.

--var. pittieri

*platyrhizum croat

*pluricostatum 'Croat \& Baker

"protensum Schott

*--subsp, a rcuatum croat

\section{"pseudospectabile Croat}

"purpureospathum Croat

\section{ramonense $K$. Krause}

*ranchoanum Engl.

* ravenil croat \& Baker

* redolens Croat

* roseospadix Croat

* rotund ist igmatum Croat

* rubrifructum Croat

*rupicola Croat

* sagawae croat

*salviniae HemsI.

*sanctifidense Croat

* sapense Croat

scandens (AubI.) Engl.

*--subsp. pusillum Sheffer

--subsp. scandens

* seibertii Croat \& Baker

\section{Madison}

PROV: ca ce ch cn da 000m: 0-1 pa sa

000m: $1-2$

PROV: bo cc ch vr 000m: $0-1,1-2,2-3$

PROV: cc ch cn pa vr 000m: $0-1,1-2,2-3$

PROV: on sa 000m: $0-1$

PROV: ch on pa $\quad 000 \mathrm{~m}: 0-1$

PROV: bo ch $\vee r \quad 000 \mathrm{~m}$ : $0-1,1-2$

PROV: ch

PROV: bo $\mathrm{ch}$

000m: $1-2$

$000 \mathrm{~m}: 0-1,1-2$

PROV: bo ca cc cn da 000m: $0-1,1-2$

$$
\text { pa sa } v r
$$

000m: $0-1,1-2,2-3$

PROV: ca cc ch cn da 000m: $0-1$

$$
\text { pa sa vr }
$$

PROV: cc da pa 000m: $0-1,1-2,2-3$

PROV: ca cc ch pa vr 000m: $0-1$

PROV: da pa $v r \quad 000 \mathrm{~m}: 0-1$

PROV: da

$000 \mathrm{~m}: 1-2$

PROV: $\mathrm{CC} \vee$

000m: $0-1$

PROV: $\mathrm{pa}$

000m: $0-1$

PROV: ca ch da pa 000m: $0-1,1-2$

PROV: $c c$ ch cn da pa $000 \mathrm{~m}: 0-1$

sa vr

000m: $0-1$

PROV: cc ch da pa vr 000m: $0-1,1-2,2-3$

PROV: bo ca cc ch cn 000m: $0-1,1-2,2-3$ da pa sa vr

PROV: ch
RANGE: $\mathrm{COI}$

ep iphyte

RANGE: bel $\mathrm{cr}$ gua hon mex epiph

nic

RANGE: end

herb

epiphyte

RANGE: end

RANGE: $c r$

RANGE: end

RANGE: $c r$

per

terr

epiph

herb

ep iphyte

epiphyte

herb

RANGE: $\mathrm{cr}$

RANGE: end

RANGE: end

epiph

herb

ep iphyte

epiph

terr

herb

RANGE: $c r$ nic

epiph

herb

RANGE: $c r$

epiph

terb

RANGE: COI C

herb

RANGE: end

RANGE: end

RANGE: end

RANGE: end

RANGE: end

RANGE: end

piphyte

epiph

herb

ep iphyte

epiph

climber

herb

epiph

RANGE: cag col cr gua hon epiphyte

mex nic

RANGE: end

herb

RANGE: end

epiphyte

RANGE: col hon ven

epiph

RANGE: ang bel cag cr gag epiph

gua gui hon mex nic sal herb

sao trt ven

RANGE: $\mathrm{cr}$ 
23 (11) ARACEAE cont.

*spathiphyI I um N.E. Br.

* subrotundum Croat

* suprag l andulum Croat

* syt smae Croat

*taca rcunense Croat

*teribense Croat

*terryae StandI. \& L. O. Wms.

*testaceum Croat \& Baker

*t il a ranense Stand I.

*tonduzii Engl.

trinerve Miq.

"trisectum Sod.

*tutense Croat

*tysoni i croat

validifolium K. Krause

\#va I lense Croat

*watermaliense L.H. Bailey

twedel ianum Croat

\#--subsp. viridispadix Croat

*--subsp. wedel i anum

twend lingeri G. Barroso

williamsii K. Krause

Ca ladium

* I inden i i (Andre) M. Madison on

*Colocasia

*esculenta (L.) Schott

Dieffenbachia

aurantiaca Engl.

longispatha Engl. \& K. Krause

oerstedii Schott
PROV: bo ch da pa sa $000 \mathrm{~m}: 0-1$

PROV: ch

$\begin{array}{ll}\text { PROV: bo cc } & 000 \mathrm{~m}: 0-1 \\ \text { PROV: da } & 000 \mathrm{~m}: 1-2 \\ \text { PROV: pa } & 000 \mathrm{~m}: 0-1 \\ \text { PROV: cc } & 000 \mathrm{~m}: 0-1 \\ \text { PROV: da pa } & 000 \mathrm{~m}: 0-1,1-2 \\ \text { PROV: bo } & 000 \mathrm{~m}: 0-1 \\ \text { PROV: da } & 000 \mathrm{~m}: 0-1,1-2 \\ \text { PROV: bo ch } & 000 \mathrm{~m}: 0-1,1-2,2-3\end{array}$

PROV: cc ch da pa vr 000m: $0-1,1-2$ PROV: $c c$ ch da $v r$ 000m: $0-1,1-2$

PROV: bo ca cc ch cn 000m: $0-1,1-2$ PROV: cc ch $v r$ sa $v r$ 000m: $0-1$

PROV: Cc ch $v r$ 000m: $0-1,1-2$

PROV: pa 000m: $0-1$

PROV: ch

joom: $1-2$

PROV: cc cn pa $v r \quad 000 \mathrm{~m}$ : $0-1,1-2$

PROV: bo ch vr

000m: $0-1,1-2,2-3$

PROV: $c c$ en vr

000m: $0-1,1-2$

000m: $0-1$

PROV: bo

$000 \mathrm{~m}: 0-1$

PROV: bo $C C$ da pa $\vee r$ 000m: $0-1,1-2$

PROV: ca

000m: $0-1$

000m: $0-1$

PROV: bo ca cc ch cn 000m: $0-1$

PROV: bo ca ch cn da 000m: $0-1$

lo pa
RANGE: $\mathrm{cr}$

RANGE: end

RANGE: end

RANGE: end

RANGE: end

RANGE: end

RANGE: end

RANGE: $\mathrm{cr}$

RANGE: $\mathrm{cr}$

RANGE: $\mathrm{cr}$

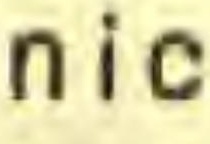

iphyte

herb

ep iphyte

epiph

herb

epiph

herb

ep iphyte

ep iphyte

epiph

terr

epiphyte

epiph

terr

RANGE: bel cr gua gui hon epiph

nic sao cr herb

RANGE: col $\mathrm{cr}$ ecu herb

RANGE: end epiphyte

RANGE: end herb

RANGE: end

epiph

herb

RANGE: $\mathrm{CO}$ I

epiph

terr

RANGE: $\mathrm{col} \mathrm{cr}$

herb

RANGE: end

RANGE: end

RANGE: $\mathrm{cr}$

RANGE: $\mathrm{COI} \mathrm{cr}$ ecu

ep iphyte

ep iphyte

ep iphyte

epiph

herb

RANGE: gui nwg?

herb

RANGE: $w r$

terr

RANGE: wdt

terr

herb

RANGE: $\mathrm{cr}$ herb

RANGE: col? $\mathrm{cr}$ hon herb

RANGE: gua herb 
TAXON

FAMILY

PROVINCES

23 (11) ARACEAE cont.

pittieri Engl. \& K. Krause

PROV: ca ce sa

000m: $0-1,1-2$

Dracontium

costaricense Engl.

*dressleri Croat

*Heteropsis

*oblongifolia Kunth

PROV: ca sa

PROV: ca cn da

000m: $0-1$

000m: $0-1$

PROV: ch on da pa sa $000 \mathrm{~m}$ : $0-1$

PROV: bo pa sa

000m: $0-1$

"wallisii Regel

wend landii schott

\section{Monstera}

*adansonii Schott

*--var. I aniata (Schott) M. Madison

PROV: bo ca cc ch cn 000m: $0-1,1-2$

$$
\text { da lo pa } \mathrm{vr}
$$

deliciosa Liebm.

PROV: ca ch vr

000m: $0-1,1-2$

*dilacerata (K. Koch \& Sello) K. Koch

PROV: ca cc ch da pa 000m: $0-1,1-2$ sa

*dubia (H.B.K.) Engl. \& K. Krause

* lechleriana Schott

PROV: ca ch pa $\quad 000 m$ : $0-1$

PROV: pa

000m: $0-1$

*membranacea M. Madison

*minima M. Madison

\#obI iqua Miq.

*oreophila M. Madison

pittieri Engl.

Hpunctulata (Schott) Engl.

PROV: ch

000m: $0-1$

PROV: sa

000m: $0-1$

PROV: bo cc da pa sa $000 \mathrm{~m}: 0-1$

PROV: $\mathrm{VC}$

000m: $0-1,1-2,2-3$

PROV: ca cc cn

000m: $0-1$

PROV: $\mathrm{ch}$ da

$000 m: 1-2$

*spruceana (Schott) Engl.

PROV: pa sa

000m: $0-1$

"tenuis K. Koch

PROV: ch

000m: $1-2$

*tuberculata Lund.

\#--var. brevinodum (StandI. \& W. O. Wms.) M. Madison

PROV: bo

000m: $0-1$

PROV: bo ca cn da sa $000 \mathrm{~m}$ : $0-1$

RANGE OUTSIDE OF PANAMA

HABIT

RANGE: end

herb

RANGE: $\mathrm{cr}$

RANGE: $\mathrm{cr}$

herb

herb

RANGE: $c r$

cl imber

RANGE: $W r$

RANGE: $\mathrm{COI} \mathrm{cr}$

Wh

climber

RANGE: abc ang cag col cr epiph ecu gui hon lag mex nic climber

per sag sao trt ven

RANGE: cag mex

epiph

climber

RANGE: bel cag col cr gua epiph

nic per sag sao ven herb

RANGE: bol cr sag sao trt epiph

RANGE: cag sat cli

RANGE: $\mathrm{cr}$

epiph

climber

RANGE: end

cl imber

RANGE: col gui per sao trt epiph

ven herb

RANGE: $c r$

epiph

RANGE: $\mathrm{cr}$

RANGE: cag mex

RANGE: $\mathrm{cr}$ sag

RANGE: $c r$ nic

herb

epiph

climber

epiph

herb

herb

epiph

herb

RANGE: bel or gua mex

climber

RANGE: bel gua gui lag

aquat 
TAXON

23 (11) ARACEAE cont.

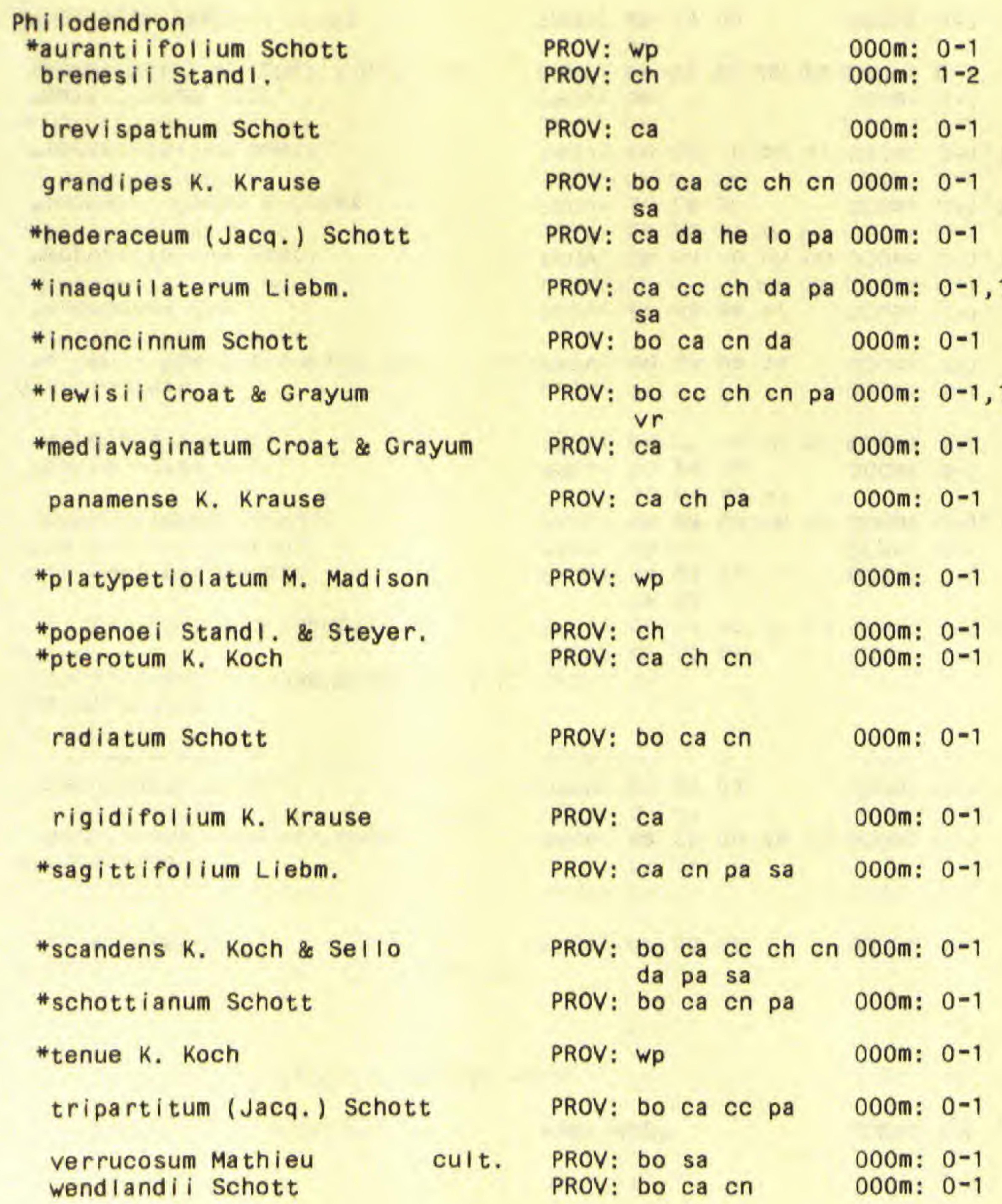

\begin{tabular}{|c|c|c|c|c|c|c|}
\hline RANGE: & $\mathrm{cr}$ & & & & & climber \\
\hline RANGE: & $\mathrm{cr}$ & & & 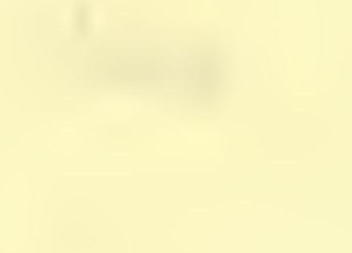 & & $\begin{array}{l}\text { epiph } \\
\text { climber }\end{array}$ \\
\hline RANGE: & $\mathrm{cr}$ & nic & ven & & & $\begin{array}{l}\text { epiph } \\
\text { climber }\end{array}$ \\
\hline RANGE: & $\mathrm{cr}$ & ecu & nic & & & $\begin{array}{l}\text { epiph } \\
\text { herb }\end{array}$ \\
\hline $\begin{array}{l}\text { RANGE: } \\
\text { ven }\end{array}$ & cag & $\mathrm{col}$ & cub & gui & mex & $\begin{array}{l}\text { hemiep } \\
\text { cl imber }\end{array}$ \\
\hline $\begin{array}{l}\text { RANGE: } \\
\text { ven }\end{array}$ & $\mathrm{col}$ & $\mathrm{cr}$ & ecu & $\operatorname{mex}$ & per & $\begin{array}{l}\text { hemiep } \\
\text { climber }\end{array}$ \\
\hline RANGE: & $\mathrm{col}$ & $\mathrm{cr}$ & nic & & & $\begin{array}{l}\text { hemiep } \\
\text { cl imber }\end{array}$ \\
\hline RANGE: & $\mathrm{cr}$ & & & & & $\begin{array}{l}\text { hemiep } \\
\text { cl imber }\end{array}$ \\
\hline RANGE: & $\mathrm{cr}$ & & & & & $\begin{array}{l}\text { hemiep } \\
\text { cl imber }\end{array}$ \\
\hline RANGE: & cr & ecu & hon & nic? & & $\begin{array}{l}\text { epiph } \\
\text { hemiep } \\
\text { cl imber }\end{array}$ \\
\hline RANGE: & $\mathrm{cr}$ & ecu & & & & $\begin{array}{l}\text { epiph } \\
\text { herb }\end{array}$ \\
\hline RANGE: & $\mathrm{cr}$ & hon & nic & ( & & climber \\
\hline RANGE: & $\mathrm{cr}$ & & & & & $\begin{array}{l}\text { epiph } \\
\text { hemiep } \\
\text { climber }\end{array}$ \\
\hline RANGE: & cag & $\operatorname{mex}$ & & & & $\begin{array}{l}\text { epiph } \\
\text { hemiep } \\
\text { climber }\end{array}$ \\
\hline RANGE: & $\mathrm{cr}$ & nic & & & & $\begin{array}{l}\text { hemiep } \\
\text { climber }\end{array}$ \\
\hline RANGE: & cag & $\mathrm{col}$ & $\mathrm{cr}$ & $\operatorname{mex}$ & & $\begin{array}{l}\text { epiph } \\
\text { hemiep } \\
\text { climber }\end{array}$ \\
\hline $\begin{array}{l}\text { RANGE: } \\
\text { mex }\end{array}$ & $\begin{array}{l}\text { ang } \\
\text { sag }\end{array}$ & bol & cag & $\mathrm{cr}$ & gua & climber \\
\hline $\begin{array}{l}\text { RANGE: } \\
\text { ven }\end{array}$ & bel & $\mathrm{cr}$ & gui & per & sao & $\begin{array}{l}\text { epiph } \\
\text { climber }\end{array}$ \\
\hline RANGE: & $\mathrm{col}$ & ecu & nic & ven & & $\begin{array}{l}\text { epiph } \\
\text { cl imber }\end{array}$ \\
\hline RANG & bel & cag & $\mathrm{col}$ & gua & jam & epiph \\
\hline 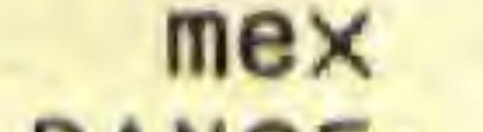 & sao & ven & & & & $\mathrm{climber}$ \\
\hline RANG & $\mathrm{col}$ & $\mathrm{cr}$ & & & & climber \\
\hline RANC & $\mathrm{cr}$ & & & & & epiph \\
\hline
\end{tabular}


Pistia

stratiotes $L$.

Rhodospatha

*moritziana (Schott) Croat

twend landil schott

Spathiphy I I um

ploribundum (Linden \& Andre) N.E. Br.

friedrichsthalii Schott

fulvovirens schott

*ka Ibreyeri Bunting phrynifolium Schott

"quindiuense Engl. twend landii Schott

Stenospermation

*allenil Gomez \&e Croat

*andreanum Engl.

*angustifolium HemsI.

*Iuteynil Gomez \& Croat

*ma rant ifolium Hems।.

*multiflorum Engl.

*multiovulatum (Engl.) N.E. Br.

* robustum Engl.

sessile Engl.

spruceanum Schott

\section{Syngon ium}

*angustatum Schott

cult.

*erythrophyl I um Bunting

PRO
PROV: ca da pa

000m: $0-1$

PROV: ca ch cn sa $000 \mathrm{~m}$ : $0-1$

PROV: ca ce da

PROV: bo ca ce ch cn 000m: $0-1,1-2$

da sa

PROV: bo cc sa $\quad 000 \mathrm{~m}$ : $0-1$

PROV: da 000m: $0-1$

PROV: bo ca co ch cn 000m: $0-1,1-2,2-3$

lo pa sa vr

PROV: cc pa sa $\quad 000 \mathrm{~m}: 0-1$

PROV: ch

$000 \mathrm{~m}: 1-2$

PROV: ce en pa sa 000m: $0-1$

PROV: cc da pa vr 000m: 0-1

PROV: bo ca ce ch cn 000m: $0-1,1-2$ da pa $v r$

000m: $0-1,1-2$

PROV: cc ch pa

PROV: bo ec en pa vr 000m: $0-1,1-2$

PROV: sa

000m: $0-1$

PROV: bo ca cc cn pa 000m: $0-1$

Vr

$000 \mathrm{~m}: 0-1,1-2$

PROV: bo ca cc ch pa 000m: $0-1,1-2$

PROV: bo ce ch ch da 000m: $0-1,1-2$ pa sa $v r$

RANGE: $w d w$

RANGE: $\mathrm{col}$ or ven

RANGE: cag? col? cr mex? aquat

herb

terr

herb

epiph

climber

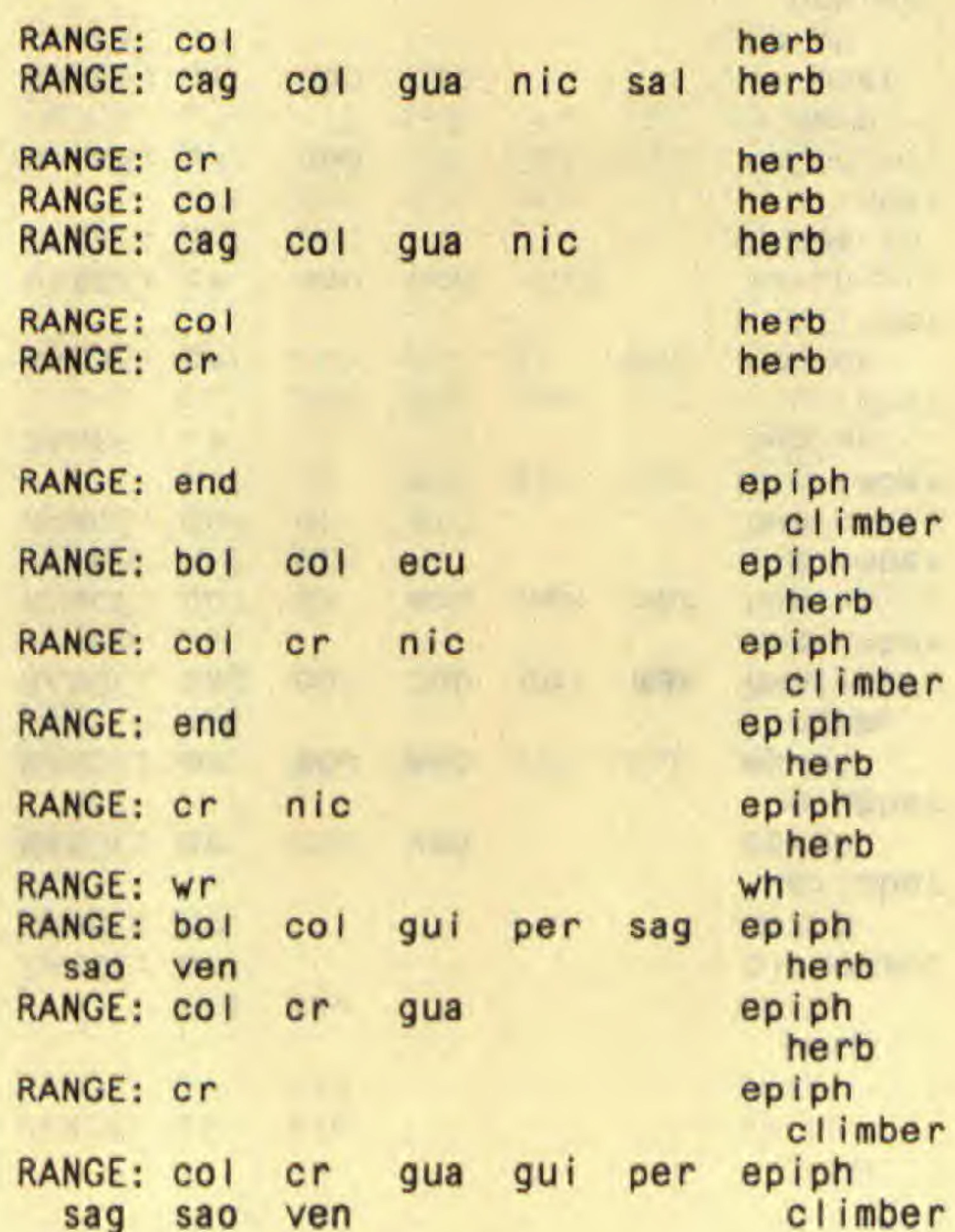

RANGE: bel cr gua hon mex hemiep

nic climber

RANGE: end hemiep 


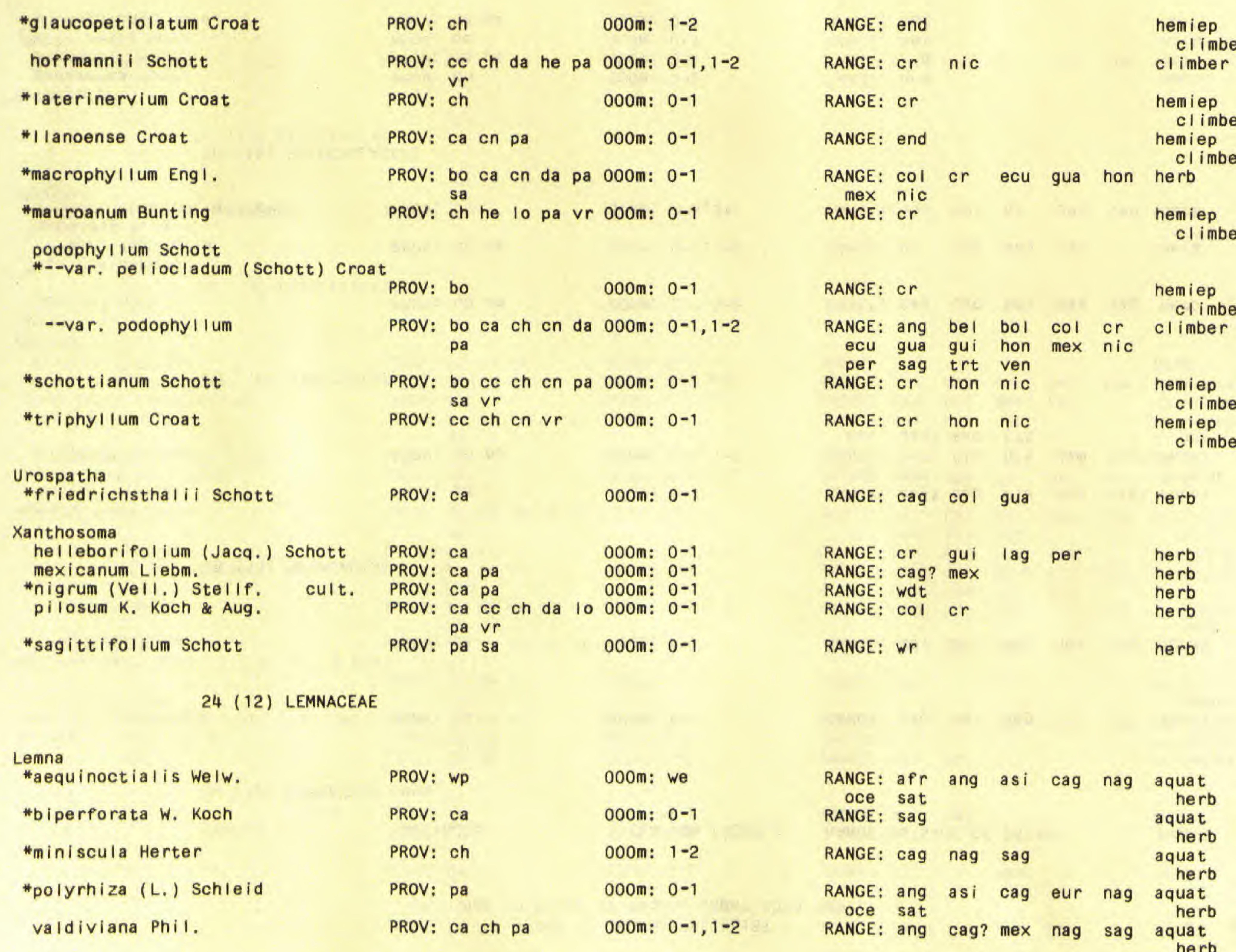


24 (12) LEMNACEAE cont.

\section{Wolffia \\ *brasiliensis Wedd.}

PROV: ca

PROV: ca

lingulata $\mathrm{Hg} \mathrm{Im}$.

28 (13) MAYACACEAE

\section{Mayaca}

aubleti Michx.

fluviatilis Aubl.

PROV: pa

000m: $0-1$

PROV: ch pa

000m: $0-1,1-2$

29 (14) XYRIDACEAE.

000m: $0-1$

000m: $0-1$

$$
\text { Xyris }
$$

jupica i Rich.

* Iaxifolia Mart.

*--var. laxifolía

* subulata R. \& $P$.

*--var. acutifolía Hiemerl.
RANGE: cag mex sag

RANGE: cal mex

\section{0 (15) ERIOCAULACEAE}

\section{Eriocaulon \\ panamense Mold. \\ seemannii Mold. \\ williamsil Mold.}

woodsonianum Mold.

\section{Paepalanthus \\ * costaricensis Mold.}

lamarcki i Kunth

"pilosus (H.B.K.) Kunth

Syngonanthus

pittierl Mold.
PROV: ch

PROV: ca pa

PROV: $C C$

PROV: he pa

PROV: bo

PROV: $C C$

PROV: bo

PROV: $\mathrm{ch}$
RANGE: cag? cub fla mex nag? aquat
nao tex
RANGE: cub fla his jam nag? aquat
nao sag? sao trt 000m: $0-1,1-2$

000m: $0-1,1-2$

000m: $2-3,3+$

$\begin{aligned} & \text { RANGE: cag gag mex nas sag herb } \\ & \text { Sao }\end{aligned}$
RANGE: or gua sag ven herb
RANGE: bol col $\mathrm{cr}$ per ven herb

RANGE: end

RANGE: end

RANGE: bel

RANGE: end

herb

000m: $0-1$

000m: $0-1$

000m: $0-1$

000m: $2-3$

000m: $0-1$

000m: $2-3$

000m: $0-1,1-2$
RANGE: or RANGE: beI

trt

RANGE: $c r$

cag? cub his sao herb

herb
RANGE: end

herb 
TAXON FAMILY

PROVINCES

30 (15) ERIOCAULACEAE cont.

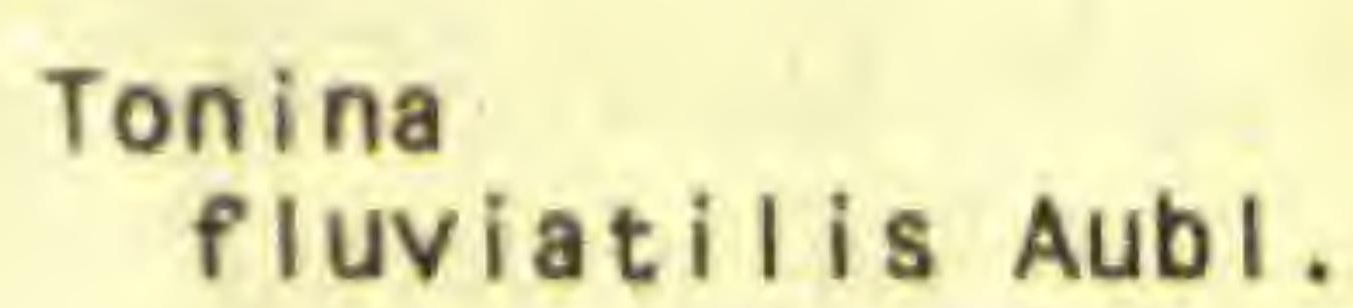

PROV: ch

000m: $1-2$

\section{1 (16) RAPATEACEAE}

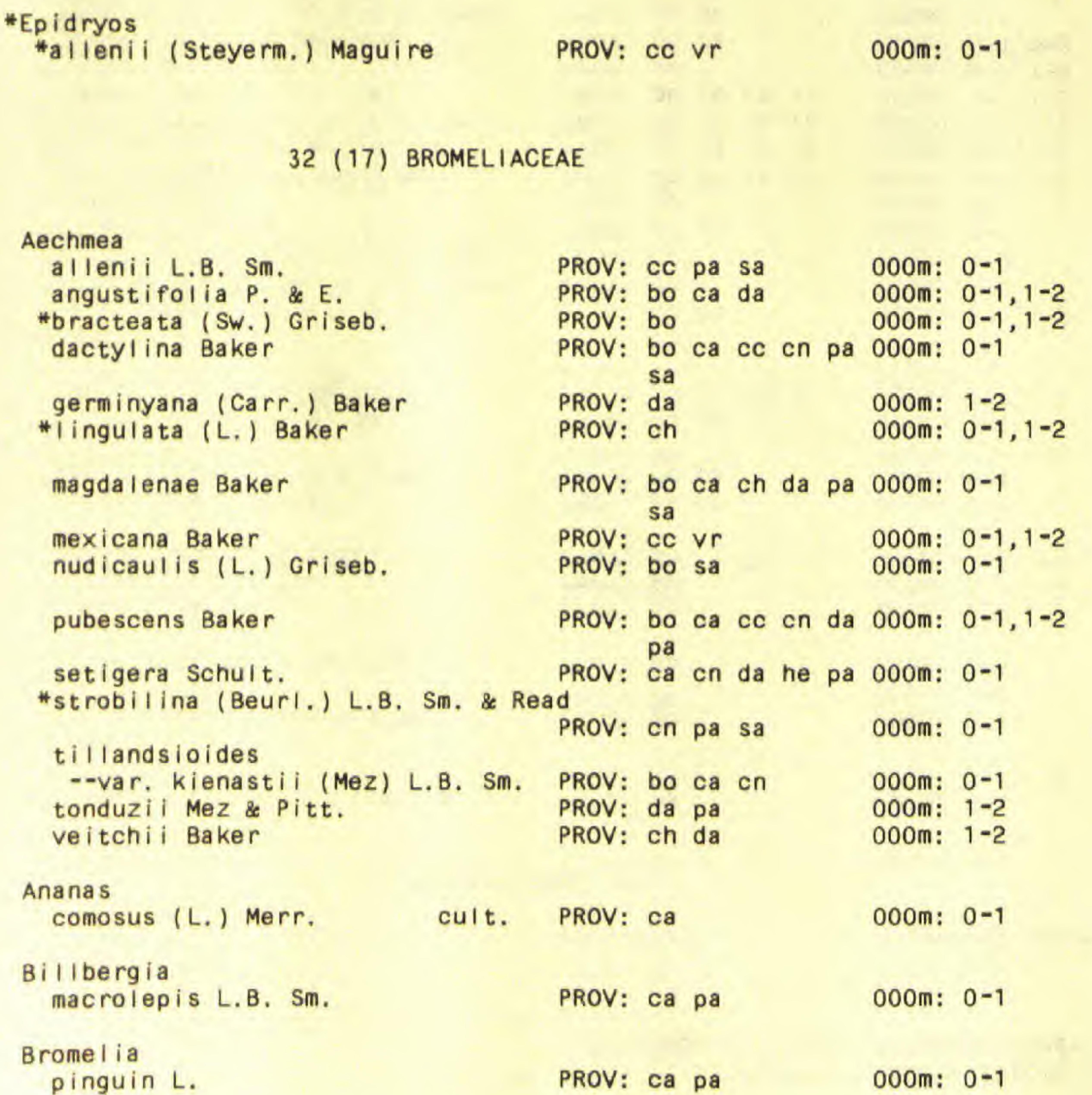

$$
\begin{aligned}
& \text { RANGE: bel cag? col cub sao aquat } \\
& \text { trt }
\end{aligned}
$$

\section{epiph}

herb

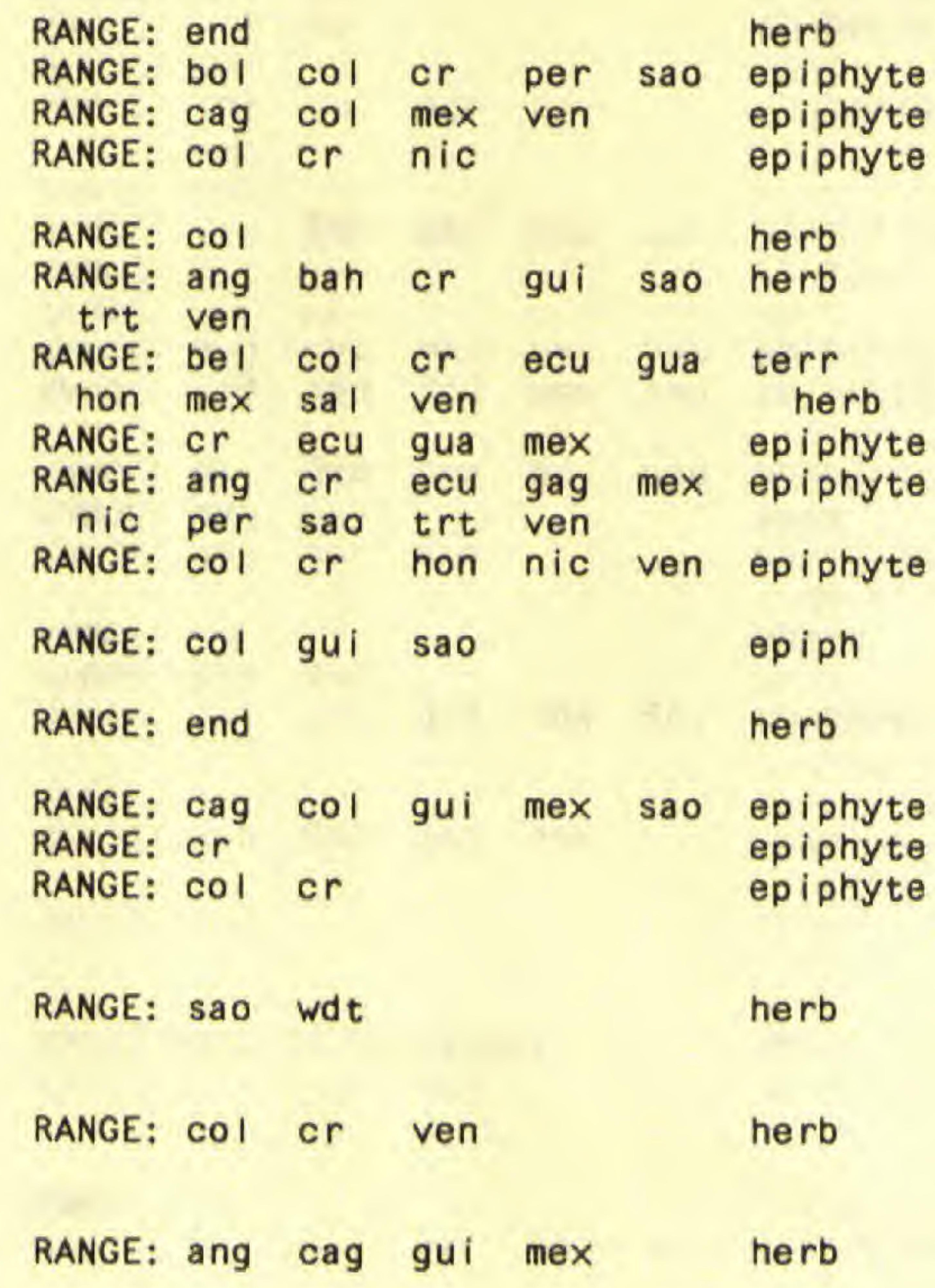


32 (17) BROMELIACEAE cont.

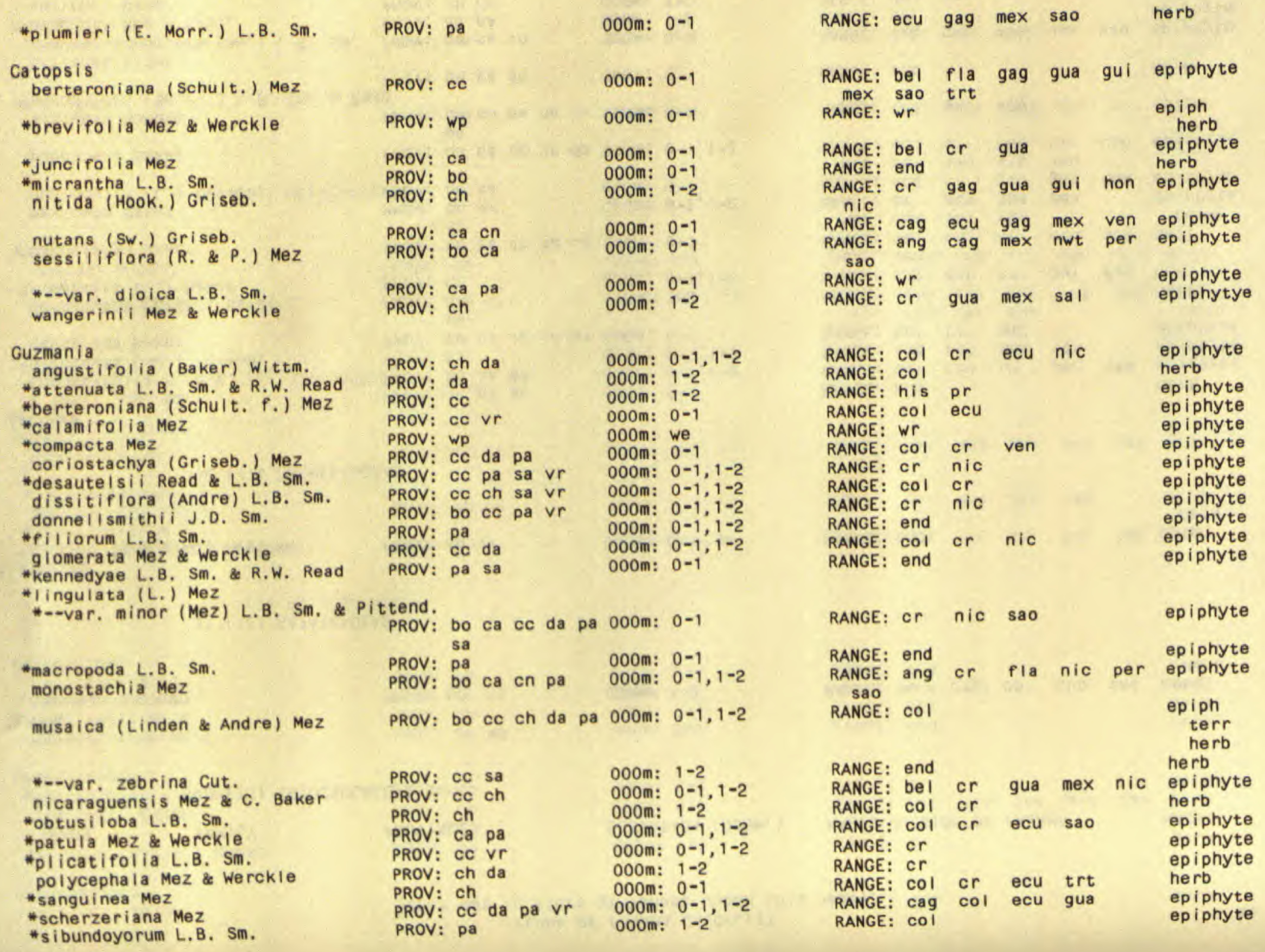


32 (17) BROMELIACEAE cont.

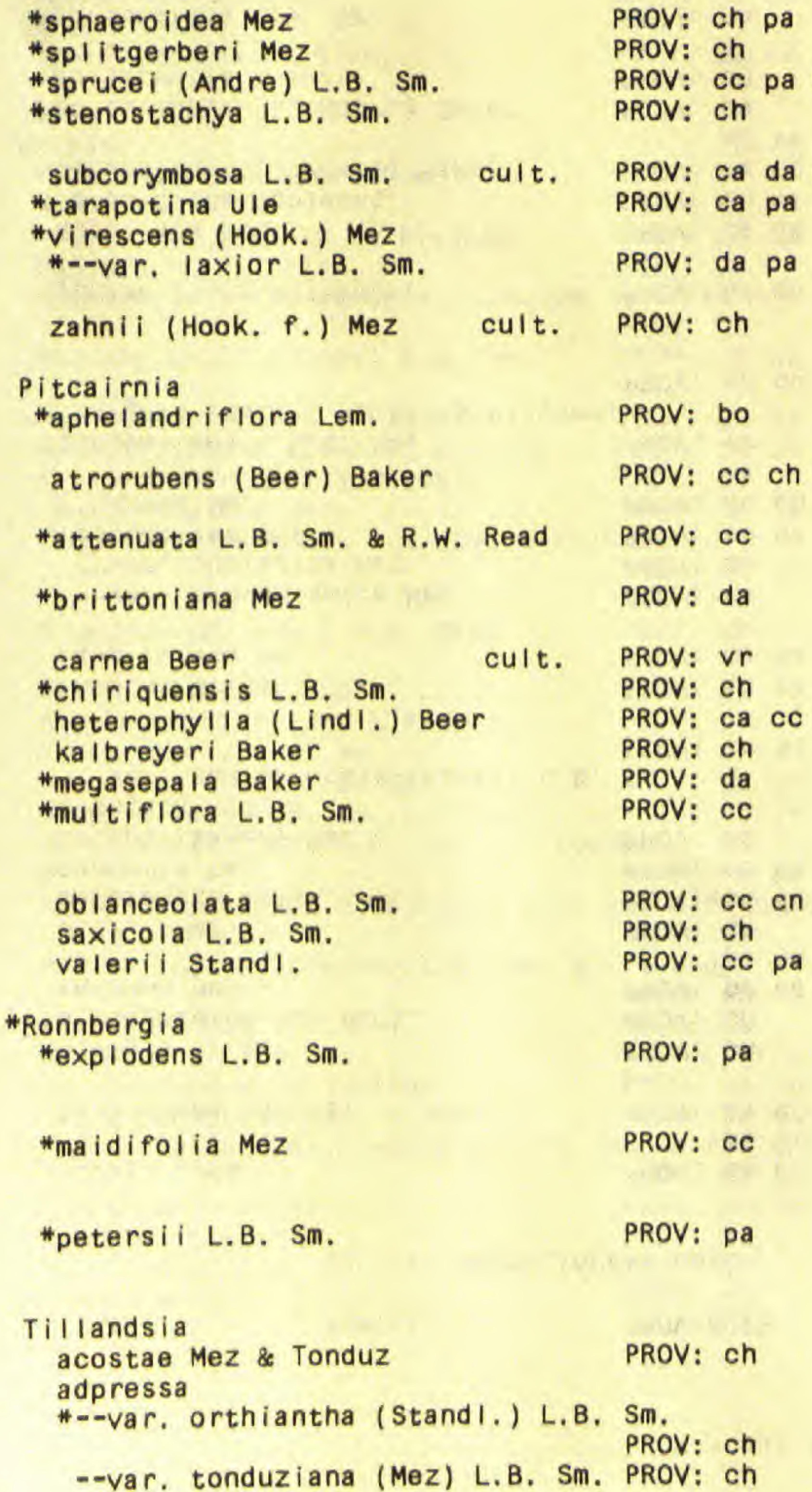

000m: $0-1,1-2$

000m: $1-2$

000m: $0-1,1-2$

000m: $1-2$

000m: $0-1$

000m: $0-1$

000m: $0-1,1-2$

000m: $1-2,3+$

000m: $0-1$

000m: $0-1,1-2$

000m: $0-1$

000m: $1-2$

000m: we

000m: $0-1$

000m: $0-1,1-2$

000m: $1-2$

000m: $0-1,1-2$

000m: $0-1$

000m: $0-1,1-2$

000m: $0-1,2-3$

$000 \mathrm{~m}: 0-1,1-2$

000m: $0-1$

000m: $0-1,1-2$

000m: $0-1$

000m: $0-1$

000m: $1-2,2-3$

000m: $1-2,2-3$

\begin{tabular}{|c|c|c|c|c|c|c|}
\hline $\begin{array}{l}\text { RANGE: } \\
\text { RANGE: } \\
\text { RANGE: } \\
\text { RANGE: }\end{array}$ & $\begin{array}{l}\mathrm{col} \\
\mathrm{cr} \\
\mathrm{col} \\
\mathrm{cr}\end{array}$ & trt & & the & & $\begin{array}{l}\text { ep iphyte } \\
\text { herb } \\
\text { epiphyte } \\
\text { epiph } \\
\text { herb }\end{array}$ \\
\hline $\begin{array}{l}\text { RANGE: } \\
\text { RANGE: }\end{array}$ & col & $\begin{array}{l}\text { cr } \\
\text { per }\end{array}$ & & & & $\begin{array}{l}\text { herb } \\
\text { epiphyte }\end{array}$ \\
\hline NGE: & ecu & per & 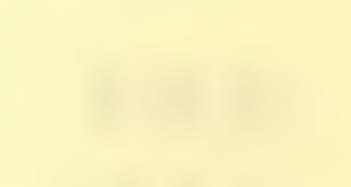 & sit & & 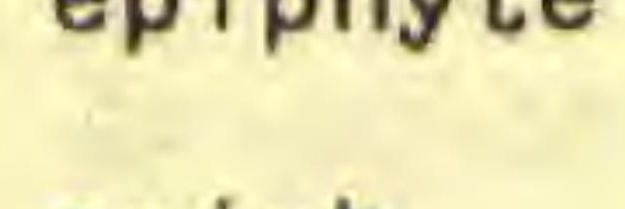 \\
\hline RANGE: & end & & & $x^{x+1}$ & & $\begin{array}{l}\text { epiph } \\
\text { herb }\end{array}$ \\
\hline RANGE: & $\mathrm{cr}$ & nic & & & & epiphyte \\
\hline RANG & ecu & per & & & & $\begin{array}{l}\text { terr } \\
\text { herb }\end{array}$ \\
\hline RANGE: & $\mathrm{col}$ & $\mathrm{cr}$ & gua & hon & $\operatorname{mex}$ & $\begin{array}{l}\text { terr } \\
\text { herb }\end{array}$ \\
\hline RANGE: & par & sent & (1) & it & & $\begin{array}{l}\text { terr } \\
\text { herb }\end{array}$ \\
\hline RANGE: & $\mathrm{cr}$ & & 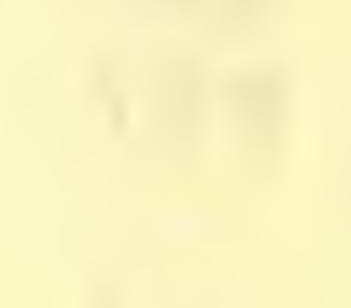 & & & $\begin{array}{l}\text { epiph } \\
\text { herb }\end{array}$ \\
\hline $\begin{array}{l}\text { RANGE: } \\
\text { RANGE: }\end{array}$ & $\begin{array}{l}\text { end } \\
\text { end }\end{array}$ & & & & & $\begin{array}{l}\text { herb } \\
\text { herb }\end{array}$ \\
\hline RANGE : & $\mathrm{cag}$ & ecu & $\operatorname{mex}$ & per & ven & herb \\
\hline RANGE: & $\mathrm{col}$ & $\mathrm{cr}$ & 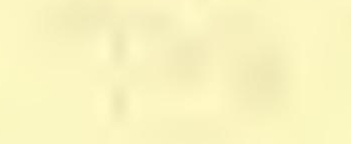 & $x+1$ & & herb \\
\hline $\begin{array}{l}\text { RANGE: } \\
\text { RANGE: }\end{array}$ & $\begin{array}{l}\mathrm{COI} \\
\mathrm{col}\end{array}$ & $\mathrm{cr}$ & & & & $\begin{array}{l}\text { herb } \\
\text { epiph }\end{array}$ \\
\hline & 1) & $x^{2}$ & & & & $\begin{array}{l}\text { terr } \\
\text { herb }\end{array}$ \\
\hline RANGE: & $\mathrm{col}$ & cr & & & 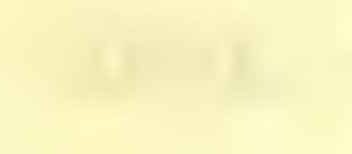 & herb \\
\hline RANGE: & gua & $\operatorname{mex}$ & & & & herb \\
\hline RANGE: & $\mathrm{cr}$ & & & & & herb \\
\hline & 2. & nititat & & & ' & 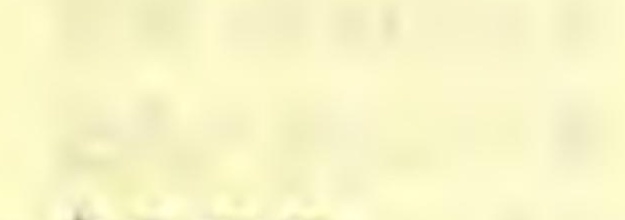 \\
\hline RANGE: & per & $=1$ & 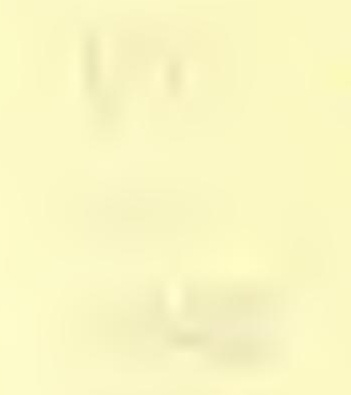 & (c) & & $\begin{array}{l}\text { terr } \\
\text { epiph } \\
\text { herb }\end{array}$ \\
\hline RANGE: & $\mathrm{Col}$ & & & & & terr \\
\hline & & $x^{2}$ & & & & $\begin{array}{l}\text { epiph } \\
\text { herb }\end{array}$ \\
\hline RANGE: & end & & & & & $\begin{array}{l}\text { epiph } \\
\text { herb }\end{array}$ \\
\hline RANGE & $c r$ & gua & $\operatorname{mex}$ & & & epiphyte \\
\hline ANC & $r$ & & & & & \\
\hline & & & & & & pipnyte \\
\hline
\end{tabular}


32 (17) BROMELIACEAE cont.

anceps Lodd.

balbisiana schult.

\#biflora R. \& P.

"brachycaulos Schlecht.

bulbosa Hook.

\section{butzii Mez}

complanata Benth.

* contorta Mez

crispa (Baker) Mez

*elonga ta H.B.K.

*--var. subimbricata (Baker) L.B. Sm

\section{PROV: cn da pa}

PROV: bo ch $\mathrm{Vr}$

exce Isa Griseb.

fasciculata SW.

--var. convexispica Mez

--var, uncispica Mez

festucoides Mez

flexuosa SW.

\section{PROV: ca da pa}

\section{PROV: ·ca}

PROV: bo

PROV: bo da

PROV: ca ch en da lo $000 \mathrm{~m}: 0-1$

PROV: $\mathrm{VA}$

*gua tema lens is L.B. Sm.

*insignis (Mez) L.B. Sm. \& Pittend. 000m: $1-2$

PROV: bo $\mathrm{ccch}$

PROV: bo ch cn da pa 000m: $1-2$

PROV: ca da

PROV: ch Vr 000m: $0-1,1-2,2-3$

PROV: ca cc en da pa 000m: $0-1$ sa $v r$

PROV: pa

PROV: ch

000m: $0-1$

000m: $1-2$

multicaulis Steud.

*oerstediana L.B. Sm.

*pinnata Mez \& Sod.

punctulata Schlecht. \& Cham.

rubra R. \& P.

--var. costaricensis (Mez) Mez

singularis Mez \& Werckle

* spiculosa Griseb.

*--var. spiculosa

subulifera Mez

*tricolor Schlecht. \& Cham.

- -var, melanocrater (L.B. Sm.) L.B. Sm.

PROV: ch 000m: 1 -2

PROV: pa

PROV: ch vr

000m: $0-1$

000m: $1-2,2-3$

PROV: $c h$

000m: $1-2$

PROV: bo cc pa $v r$ 000m: $0-1$

PROV: ca ch cn da pa 00cm: 0-1 PROV: $c a$

$000 \mathrm{~m}: 0-1$

\begin{tabular}{|c|c|c|c|c|c|c|}
\hline RANGE: & bel & col & $\mathrm{cr}$ & gua & gui & epiphyte \\
\hline hon & nic & trt & ven & & & \\
\hline RANGE: & bah & cag & cub & fla & his & epiphyte \\
\hline $\begin{array}{l}\text { jam } \\
\text { RANGE: }\end{array}$ & $\begin{array}{l}\operatorname{mex} \\
\text { bol }\end{array}$ & $\mathrm{col}$ & $\mathrm{cr}$ & ecu & ven & epiphyte \\
\hline RANGE: & cag & $\operatorname{mex}$ & & & & ep iphyte \\
\hline RANGE: & ang & cag & col & gui & $\operatorname{mex}$ & epiphyte \\
\hline sao & trt & ven & & & & \\
\hline RANGE: & cag & $\operatorname{mex}$ & & & & epiphyte \\
\hline RANGE: & ang & bol & $\mathrm{cr}$ & gui & per & epiphyte \\
\hline RANGE: & cr & & & & & epiphyte \\
\hline RANGE: & $\mathrm{col}$ & ecu & per & & & herb \\
\hline RANGE: & $\mathrm{col}$ & cub & jam & $\operatorname{mex}$ & nic & epiphyte \\
\hline per & sao & trt & & & & \\
\hline RANGE: & cag & cub & jam & & & ep iphyte \\
\hline $\begin{array}{l}\text { RANGE: } \\
\text { mex }\end{array}$ & ang & cag & col & fla & gui & herb \\
\hline RANGE: & bel & gua & jam & & & herb \\
\hline RANGE: & cub & gua & his & lag? & & epiphyte \\
\hline RANGE: & cag & fla & gag & mex & & epiphyte \\
\hline RANGE: & ang & $\mathrm{col}$ & fla & gui & ven & epiphyte \\
\hline RANGE: & cag & $\operatorname{mex}$ & & & & epiphyte \\
\hline RANGE: & $\mathrm{cr}$ & & & & & epiph \\
\hline & & & & & & $\begin{array}{l}\text { terr } \\
\text { herb }\end{array}$ \\
\hline RANGE: & bol & cag & col & cub & fla & epiphyte \\
\hline his & jam & $\operatorname{mex}$ & per & trt & & \\
\hline RANGE: & $\mathrm{col}$ & gui & sao & ven & & epiphyte \\
\hline RANGE: & cag & $\operatorname{mex}$ & & & & epiphyte \\
\hline RANGE: & cag & $\mathrm{col}$ & ecu & gui & $t r t$ & herb \\
\hline RANGE: & $w r$ & & & & & epiphyte \\
\hline $\begin{array}{c}\text { RANGE: } \\
\text { nic }\end{array}$ & be I & $\mathrm{cr}$ & gua & hon & $\operatorname{mex}$ & epiphyte \\
\hline $\begin{array}{c}\text { nic } \\
\text { RANGE: }\end{array}$ & sa I & 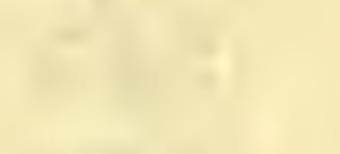 & & & & \\
\hline $\begin{array}{l}\text { RANGE: } \\
\text { RANGE: }\end{array}$ & $\mathrm{cr}$ & re & pris & & & ep iphyte \\
\hline $\begin{array}{l}\text { RANGE: } \\
\text { RANGE: }\end{array}$ & ecu & & & & & epiphyte \\
\hline RANGE: & cag & gui & mex & & & epiphyte \\
\hline RANGE: & $\mathrm{cr}$ & (a) & & & & epiphyte \\
\hline RANGE: & $\mathrm{cr}$ & & & & & epiphyte \\
\hline RANGE: & bol & $\mathrm{col}$ & cr & his & sao & ep iphyte \\
\hline RANGE: & trt & & 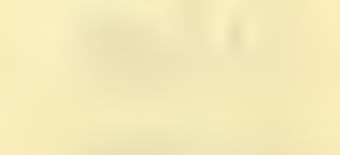 & & & nero \\
\hline & & & gua & & & epiphyte \\
\hline
\end{tabular}


TAXON

FAMILY

PROVINCES

ELEVATION (OOOM.)

RANGE OUTSIDE OF PANAMA

HAB IT

32 (17) BROMELIACEAE cont.

usneoides (L.) L.

valenzuelana A, Rich.
PROV: bo ca ch da pa 000m: $0-1,1-2,2-3$

PROV: bo ca

000m: $0-1$

000m: $0-1$

PROV: $v r$

PROV: $c h$

PROV: $C C$ ch $V r$

PROV: ch

* Ita Mez

*bicolor L.B. Smith

* capitata (Mez \& Werckle) L.B. Sm. \& Pittend.

*chontelens is (Baker) L.B. Sm.

PROV: $p$ a

PROV: ch da $\mathrm{vr}$

*diffusa L.B. Sm. \& Pittend.

gladioliflora (Wendl.) Antoine

* graminifolia Mez \& Werckle

PROV: ch da pa

PROV: ca ch

PROV: ch

PROV: bo ca da pa

hel iconioides Walp.
*hyg rometrica (Mez) L.B. Sm. \& Pittend.

*hyg rometrica (Mez) L.B. Sm. \& Pittend.

PROV: ch $v r$

*incurva (Griseb.) R.W. Read

PROV: $c h$

\#kill ipiana L.B. Sm.

* leptopoda L.B. Sm. \& Pittend.

* I eucophyl Ia L.B. Sm.

*monstrum (Mez) L.B. Sm.

PROV: pa

PROV: ch

PROV: $c h$

PROV: pa sa

*nephrolepis L.B. Sm. \& Pittend.

*ororiensis (Mez) L.B. Sm. \& Pittend.

ROV: $c h \mathrm{vr}$

PROV: bo $\mathrm{cc} \mathrm{ch}$

*pedicellata (Mez \& Werckle) L.B. Sm. \& Pittend.

PROV: ch

\#picta (Mez \& Werckle) L.B. Sm. \& Pittend.

*pittieri Mez

PROV: ch

PROV: pa

ringens (Griseb.) Harms

sanguinolenta Cogn. Marchal

* stenophylla (Mez \& Werckle) L.B. Sm.

subsecunda Wittm.

*umbrosa L.B. Sm.

*williamsii L.B. Sm.

woodsoniana L.B. Sm.
PROV: ca cn da PROV: bo ca ch pa Sm. \& Pittend. PROV: $\mathrm{cc}$ ch pa $\mathrm{vr}$ PROV: ch da PROV: pa

PROV: cn

PROV: ch 000m: $0-1,1-2,2=3$

000m: $0-1$

000m: $1-2,2-3$

000m: $0-1$

000m: $0-1,1-2$

000m: $0-1,1-2$

000m: $0-1,1-2$

000m: $1-2,2-3$

000m: $0-1$

000m: $0-1,1-2$

000m: $1-2$

000m: $0-1$

000m: $1-2$

000m: $1-2$

000m: $0-1$

000m: $0-1,1-2$

000m: $0-1,1-2,3+$

000m: $1-2$

000m: $2-3$

000m: $0-1$

000m: $0-1,1-2$

000m: $0-1$

000m: $0-1,1-2$

000m: $1-2$

000m: $0-1$

000m: $1-2,2-3$

000m: $1-2$

RANGE: ang cag nao sag sao epiph
tex
RANGE: bol cag col fla gag herb
mex ven

RANGE: $c r$

RANGE: jam

RANGE: $\mathrm{cr}$

RANGE: $\mathrm{cr}$

herb

herb

RANGE: $\mathrm{cr}$

RANGE: $w r$

RANGE: $c r$

RANGE: be

RANGE: $\mathrm{cr}$

RANGE: bol

RANGE: $c O$

Ven

RANGE: bol

jam ven

RANGE: col

RANGE: $\mathrm{cr}$

RANGE: $c r$

RANGE: $\mathrm{COI}$

RANGE: $\mathrm{cr}$

RANGE: $c r$

RANGE: $\mathrm{cr}$

hon nic

epiphyte

herb

ep iphyte

epiph

herb

ep iphyte

col cr gua epiphyte

gua herb

cag? gua sag? sao herb

cr ecu gua mex epiph

col cr cub gag epiphyte

epiphyte

epiphyte

epiphyte

epiphyte

ep iphyte

ep iphyte

ep iphyte

RANGE: $\mathrm{cr}$

RANGE: $w r$

RANGE: ang

RANGE: $\mathrm{CO}$

$\mathrm{col} \mathrm{cr}$

cr cub jam

ep iphyte

epiph

herb

epiphyte

RANGE: $\mathrm{cr}$

RANGE: $c r$

RANGE: $\mathrm{cr}$

RANGE: $\mathrm{cr}$

RANGE: end epiphyte

ep iphyte

herb

ep iphyte

herb

herb 


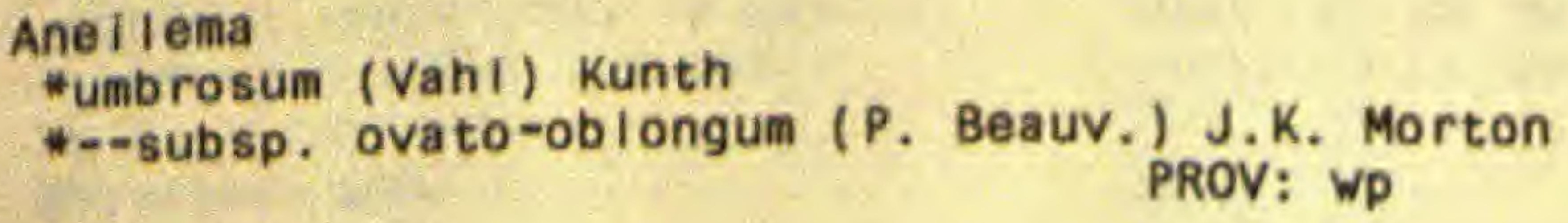

PROV: bo ca cc ch da 000m: $0-1,1-2$ pa

PROV: bo sa

000m: $0-1$

PROV: bo ca cc ch da 000m: $0-1,1-2,2-3$

PROV: ca

000m: $0-1$

PROV: ca cc ch da he 000m: $0-1,1-2$

pa sa

PROV: WD

000m: we

PROV: pa

000m: 0-1

PROV: bo ca ce ch cn 000m: $0-1,1-2$ da lo pa sa

PROV: bo

000m: $0-1$

000m: $0-1$

000m: $0-1$

000m: $0-1$
RANGE: Wr

herb

RANGE: mex nWt

herb

RANGE: COI RANGE: $W r$

herb herb

RANGE: bol gag mex nwt sao herb

RANGE: bol cr? ecu nic epiph herb

RANGE: $W d W$

RANGE: $W r$

RANGE: PIa tex wdw

herb

herb nerb

RANGE: $w r$ herb RANGE: $w r$

nerb

RANGE: $w r$

herb

RANGE: gua nwt sao

herb

RANGE: cag nic per sag sao herb

RANGE: mex nwt sao

herb

RANGE: mex nwt sao

herb

RANGE: $W r$ 


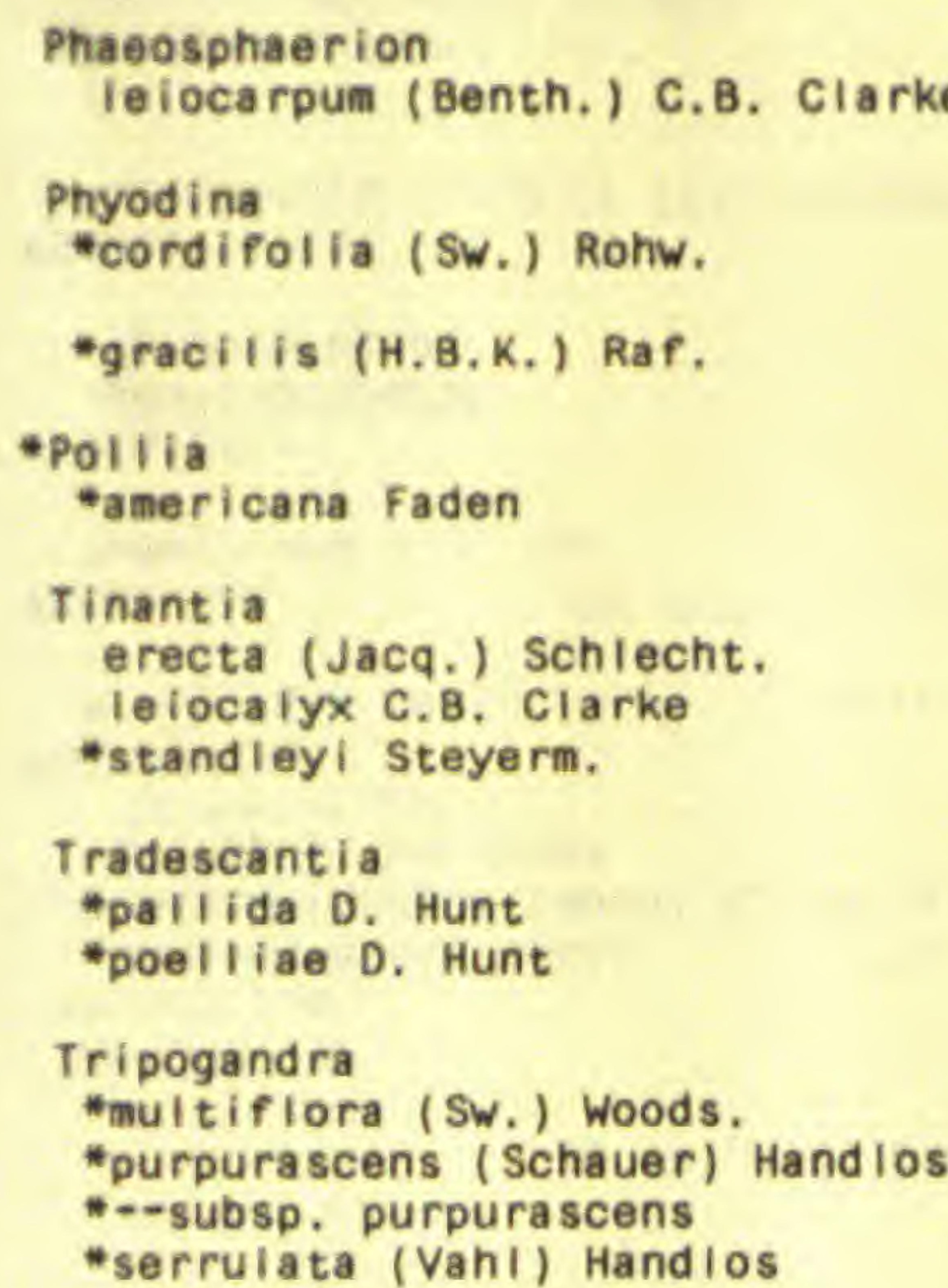

000m: $0-1$ 000m: $0-1$

34 (19) PONTEDERIACEAE

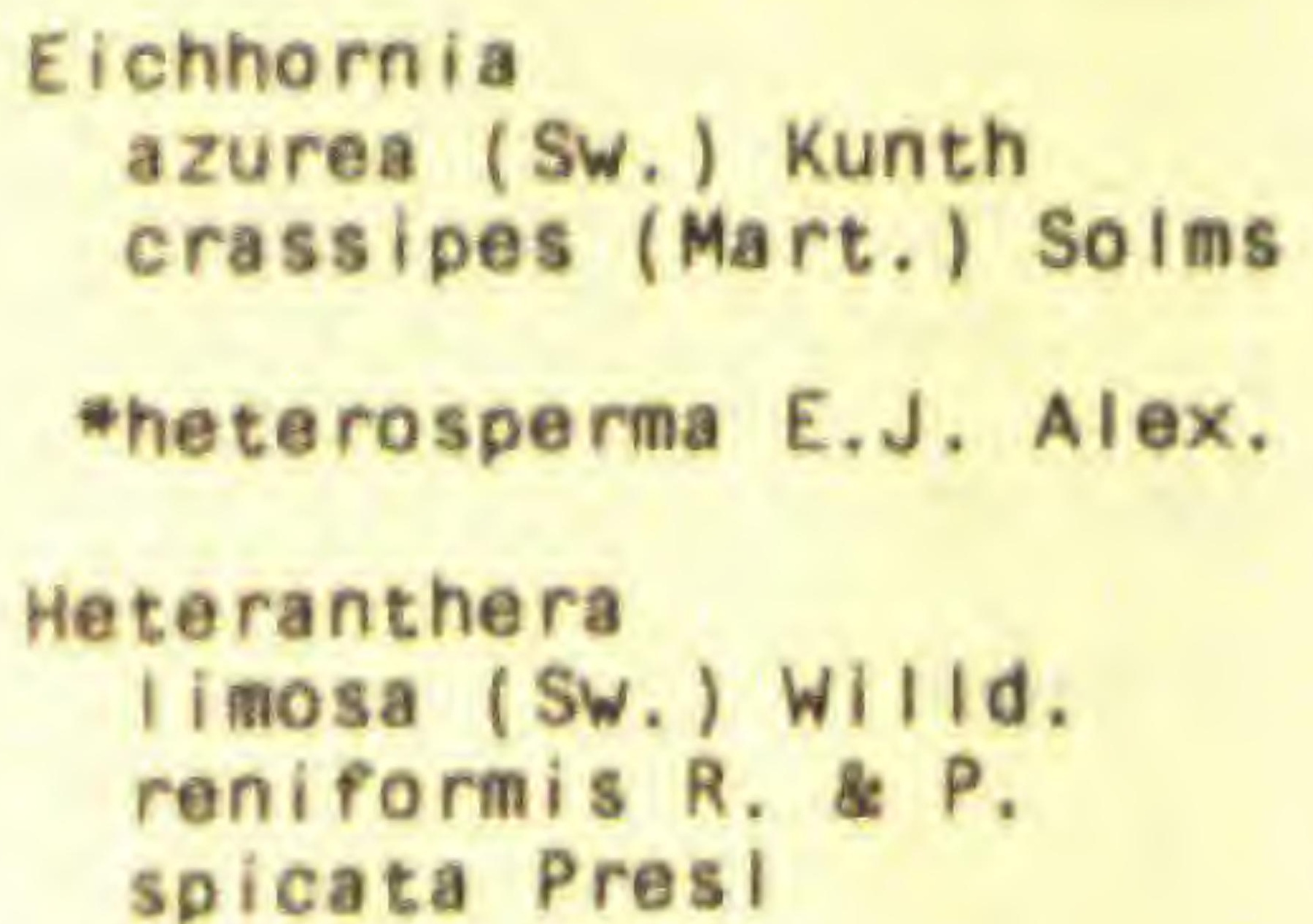

Pontederia parviflora E.J. Alex.

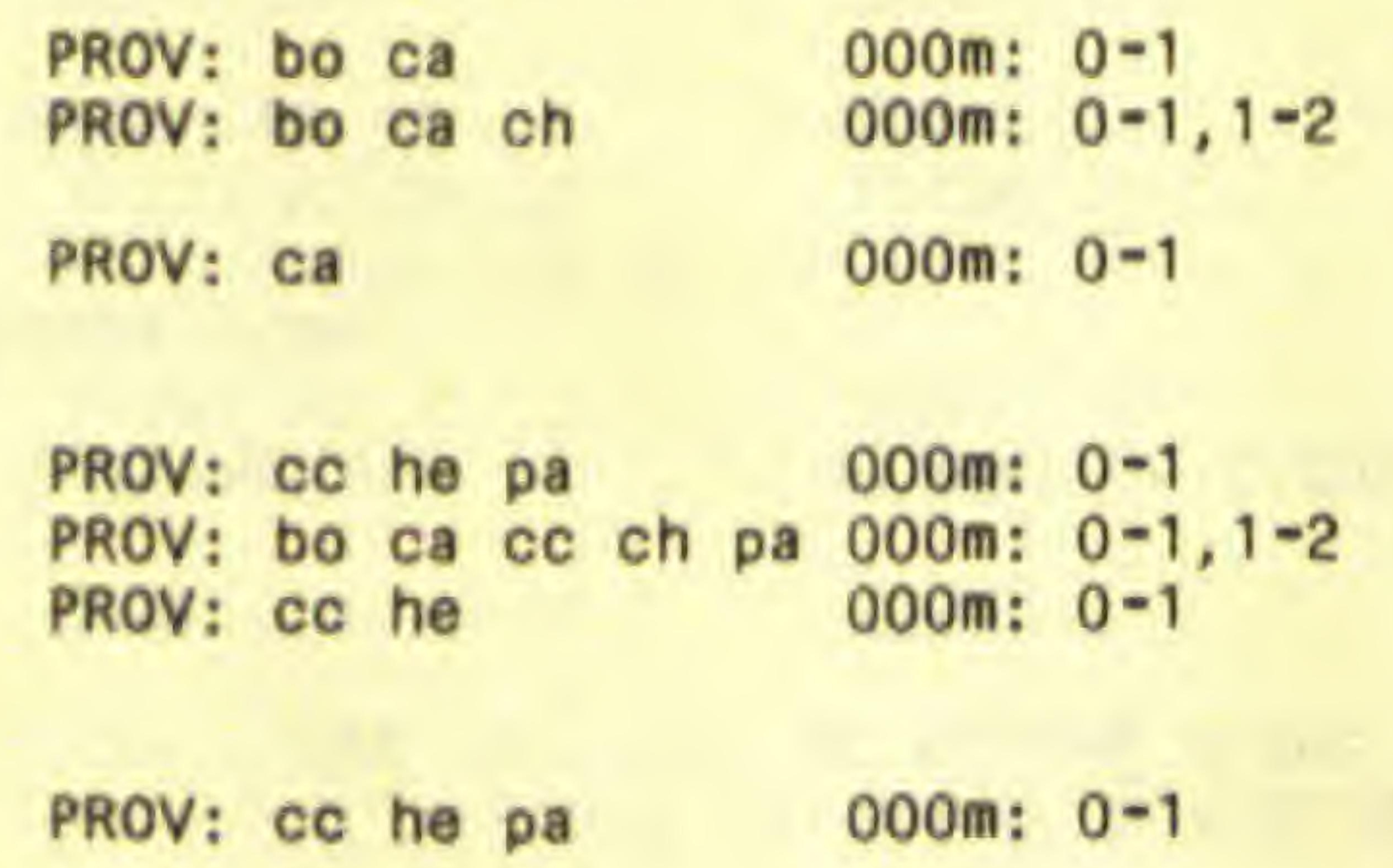

RANGE: cag mex ven
RANGE: bel cag col ecu fla herb
gag hon mex per ven
RANGE: per ven
RANGE: end

RANGE: ang cag mex sag sao herb RANGE: ang cag? nag sag? sao aquat WdW
RANGE: col sao trt ven herb

RANGE: cag nag sag sao herb RANGE: cag nag sag sao herb RANGE: cub his mex herb

RANGE: nWt ven 
34 (19) PONTEDERIACEAE cont.

rotundifolia $L$. $f$.

PROV: ca da 000m: $0-1$

RANGE: cag? mex herb

36 (20) JUNCACEAE

*Juncus

* I ebmanni i Macbr.

*--var. I iebmanni i

*mic rocephalus H.B.K.

PROV: bo

PROV: ch

000m: $3+$

000m: $1-2$

PROV: ch

000m: $3+$
$38(21,22)$ LILIACEAE

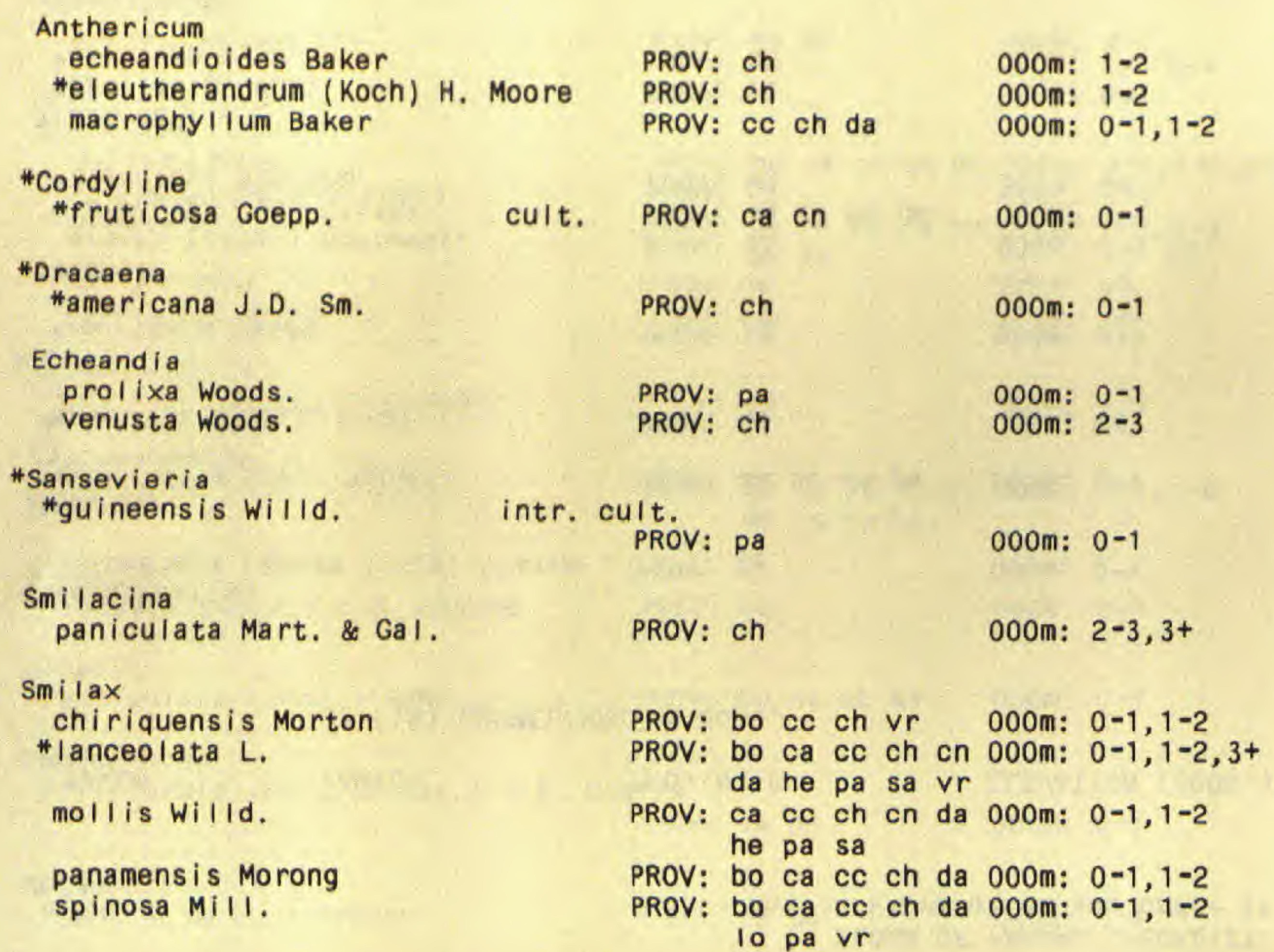

RANGE: mex

RANGE: cao sao

herb

herb

RANGE: $\mathrm{cr}$ gua mex

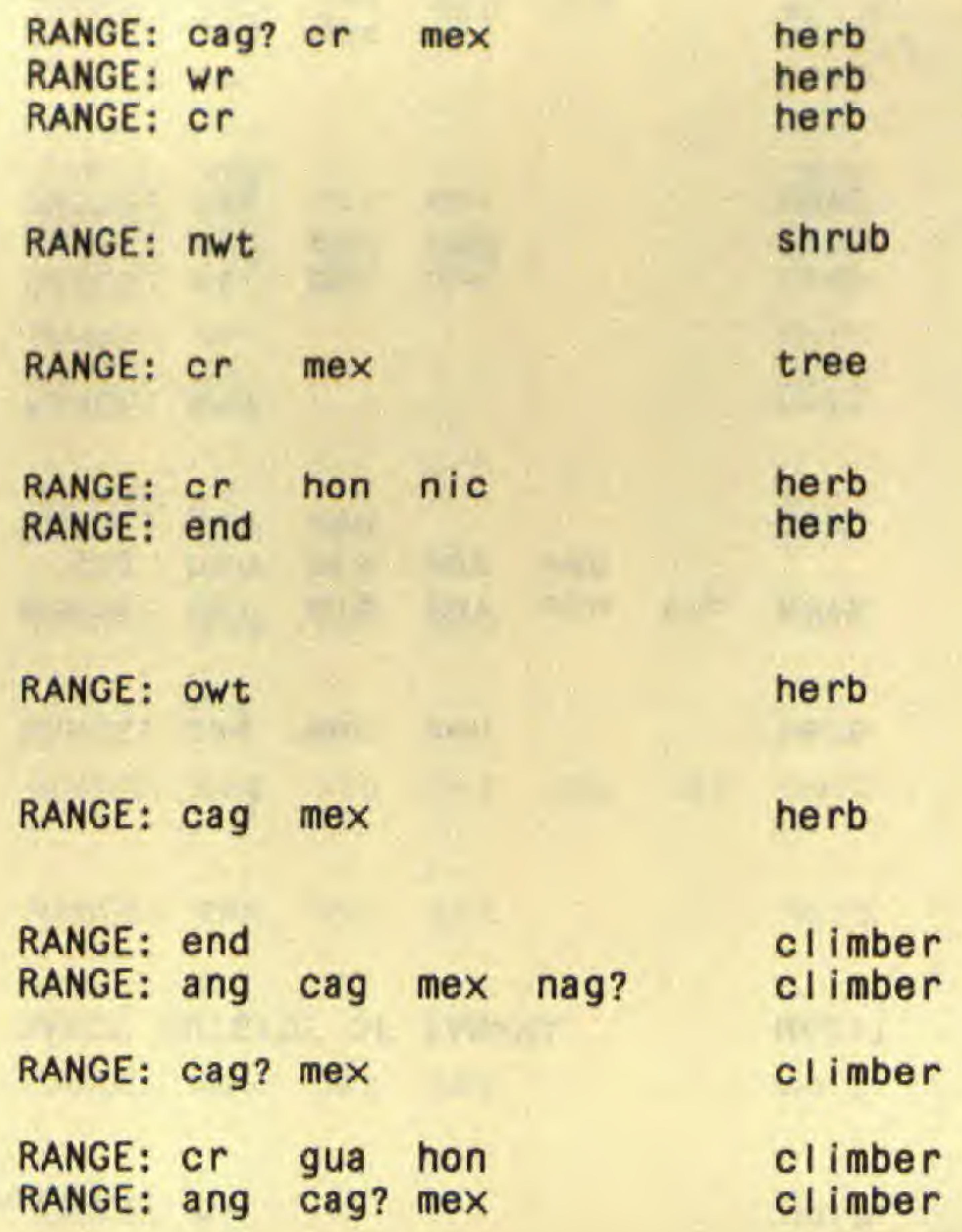


TAXON

\section{FAMILY}

PROVINCES

$38(21,22)$ LILIACEAE cont.

spissa Killip \& Morton

subpubescens A. DC.
PROV: ca cc da pa vr 000m: $0-1,1-2$ PROV: bo ch pa 000m: $0-1,1-2$
RANGE: or RANGE: cag mex
HAB IT

\section{9 (23) HAEMODORACEAE}

Xiphidium
caeruleum Aubl.

PROV: bo ca cc ch cn 000m: $0-1,1-2$ da lo pa sa vr
RANGE: ang bol cag? mex sag? herb climber

climber

\section{0 (24) AMARYLLIDACEAE}

\begin{tabular}{|c|c|c|c|c|c|c|c|c|c|c|c|c|c|c|}
\hline \multicolumn{15}{|l|}{ Agave } \\
\hline panamana Trel. & & PROV: & ca & 10 & pa & 000m: & $0-1$ & RANGE: & end & & & & & herb \\
\hline \multicolumn{15}{|l|}{ *Ama ryI I is } \\
\hline \multicolumn{15}{|l|}{ Boma rea } \\
\hline *acuminata Baker & & PROV: & bo & $\mathrm{ch}$ & & 000m: & $2-3$ & RANGE: & $\mathrm{cr}$ & & & & & herb \\
\hline *acutifolia & & PROV: & bo & $\mathrm{ch}$ & & 000m: & $2-3$ & RANGE: & $\mathrm{cr}$ & gua & hon & $\operatorname{mex}$ & sal & climber \\
\hline alleni Killip & & PROV: & cc & pa & $v r$ & 000m: & $0-1$ & RANGE: & sat & & & & & $\begin{array}{l}\text { climber } \\
\text { herb }\end{array}$ \\
\hline $\begin{array}{l}\text { *andreana Baker } \\
\text { *caldas i i (H.B.K.) Aschers. }\end{array}$ & \& Graeb & $\begin{array}{l}\text { PROV: } \\
\text { bn. }\end{array}$ & bo & & & 000m: & $2-3$ & RANGE : & $\mathrm{col}$ & & & & & herb \\
\hline & & PROV: & $\mathrm{ch}$ & & & 000m: & $2-3$ & RANGE: & $\mathrm{col}$ & $\mathrm{cr}$ & ecu & a & & herb \\
\hline chiriquina killip & & PROV: & $\mathrm{ch}$ & & & 000m: & $1-2,2-3$ & RANGE: & $\mathrm{cr}$ & & & & & climber \\
\hline chontalensis Seem. & & PROV: & bo & & & 000m: & $0-1$ & RANGE: & cag? & nic & & & & climber \\
\hline *dent iculata (R. \& P.) Herb & & PROV: & $\mathrm{ch}$ & & & 000m: & $2-3$ & RANGE: & $\mathrm{pr}$ & & & & & herb \\
\hline $\begin{array}{l}\text { edulis (Tuss.) Herb. } \\
\text { hirsuta (H.B.K.) Herb. }\end{array}$ & & PROV: & $\mathrm{cc}$ & $\mathrm{ch}$ & & 000m: & $0-1,1-2$ & RANGE: & ang & cag & $\operatorname{mex}$ & sao & & climber \\
\hline - -var. concolor (Cufod.) & $k i l l i p$ & PROV: & bo & $\mathrm{ch}$ & & 000m: & $1-2,2-3,3+$ & RANGE: & $\mathrm{cr}$ & & & & & climber \\
\hline - -var. hirsuta & & PROV: & ch & & & 000m: & $2-3$ & RANGE: & col & $\mathrm{cr}$ & ecu & & & climber \\
\hline "obovata Herb. & & PROV: & bo & & & 000m: & $0-1$ & RANGE: & bol & ecu & per & & & herb \\
\hline \multicolumn{15}{|l|}{ Crinum } \\
\hline darienelisis Woods. & & PROV: & $\mathrm{cc}$ & da & pa & 000m: & $0-1$ & RANGE: & end & & & & & herb \\
\hline $\begin{array}{l}\text { erubescens Ait. } \\
\text { longiflorum Herb. }\end{array}$ & $\begin{array}{l}\text { cult. } \\
\text { cult. na }\end{array}$ & $\begin{array}{l}\text { PROV: } \\
\text { at. }\end{array}$ & bo & ca & cc da sa & 000m: & $0-1$ & RANGE: & nwg? & & & & & herb \\
\hline & & PROV: & bo & & & 000m: & $0-1$ & RANGE: & jam & & & & & herb \\
\hline \multicolumn{15}{|l|}{ Curculigo } \\
\hline scorzoneraefolia (Lam.) Ba & Baker & PROV: & pa & & & 000m: & $0-1$ & RANGE: & ang & $\mathrm{cag}$ & $\operatorname{mex}$ & sag & & herb \\
\hline $\begin{array}{l}\text { Eucharis } \\
\text { bouchei Woods. \& Allen }\end{array}$ & & PROV: & $\mathrm{cc}$ & pa & & 000m: & $0-1$ & RANGE: & end & & & & & herb \\
\hline
\end{tabular}


TAXON

40 (24) AMARYLLIDACEAE cont.

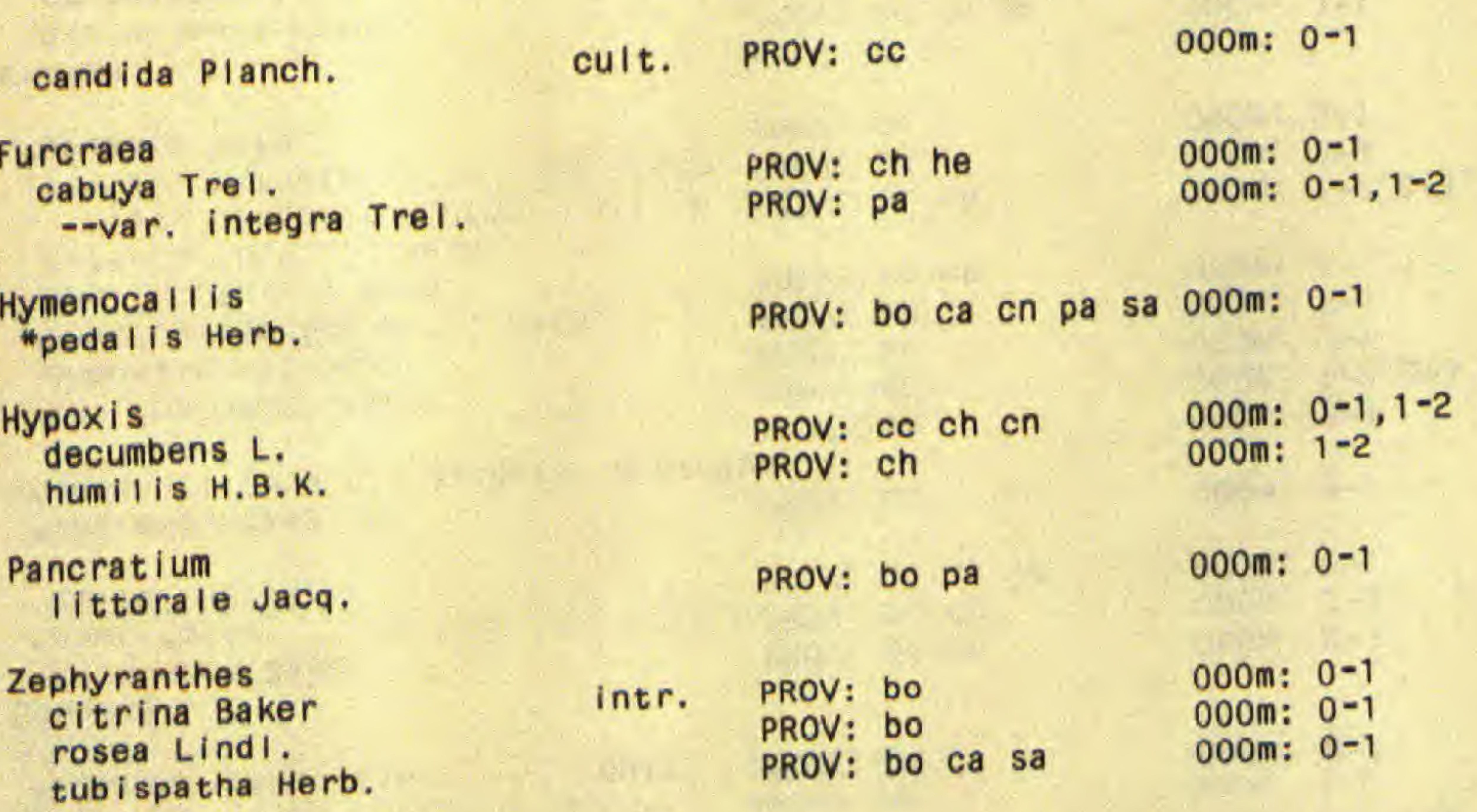

41 (25) VELLOZIACEAE

Vellozia

panamensis Stand $I$.

PROV: $\mathrm{ch}$

000m: $0-1$

42 TACCACEAE

43 (26) DIOSCOREACEAE

\author{
Dioscorea \\ *alata L. \\ *carionis Prain \& Burkill \\ convolvulacea Schlecht. \& Cham. \\ --var. glabra Knuth \\ cymosula Hems I. \\ * deami i Matuda \\ *haenkeana PresI \\ lepida Morton \\ macrostachya Benth.
}

*mexicana scheidw.

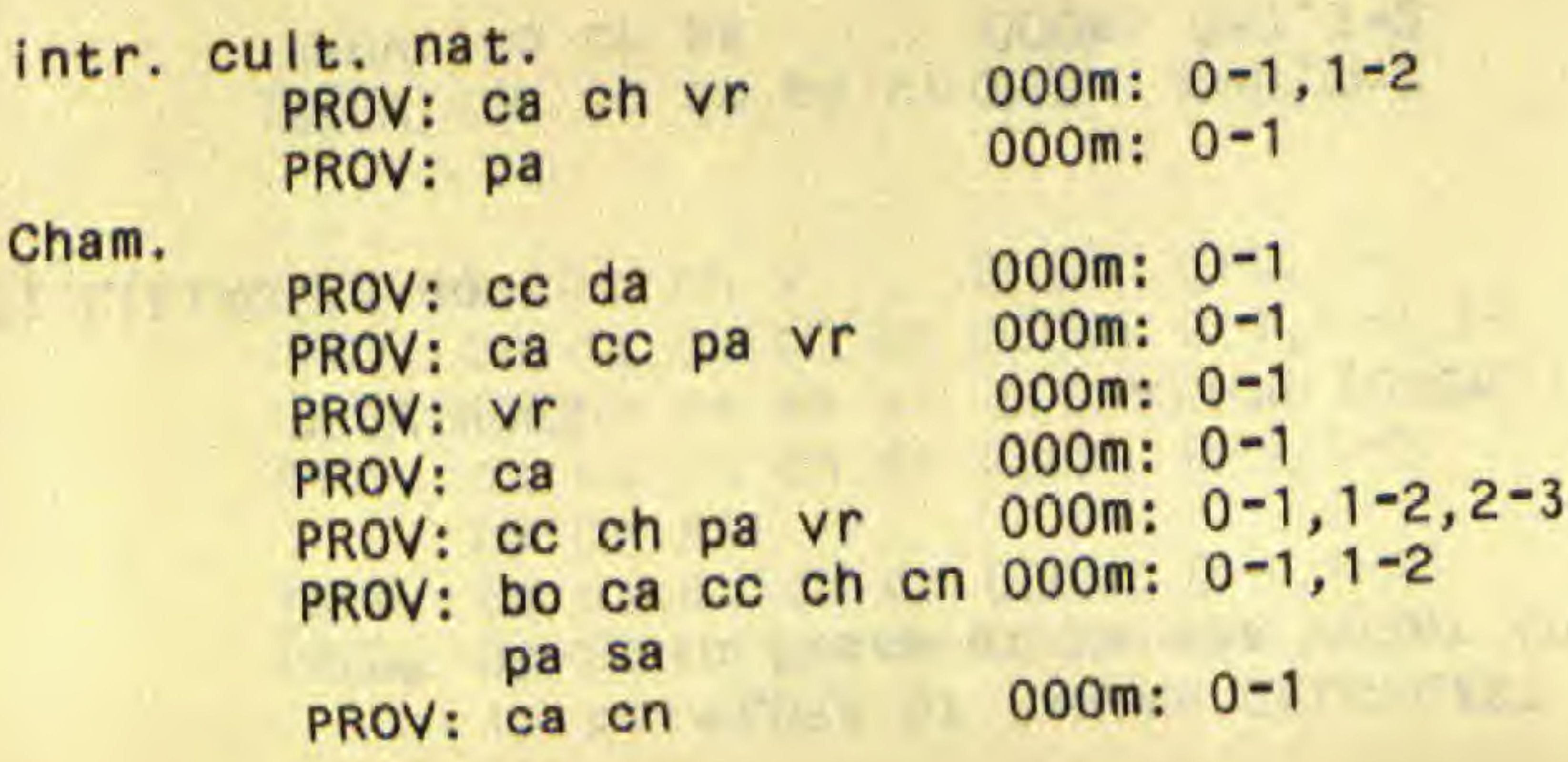

PROV: cc ch pa vr $000 \mathrm{~m}: 0-1,1-2,2$
PROV: bo ca cc ch cn $000 \mathrm{~m}: 0-1,1-2$

000m: $0-1,1-2$

000m: $0-1$

000m: $0-1$

000m: $0-1$

000m: $0-1$

$000 \mathrm{~m}: 0-1$

000m: $0-1$

\section{RANGE: $\mathrm{cOI}$}

nerb

RANGE: $c r$ RANGE: $w r$

\section{herb}

herb

RANGE: bel sao herb

RANGE: ang cag? mex sag? sao herb RANGE: cag? mex sag? sao herb

RANGE: ang cag? nag? sag? herb

RANGE: cub gui? nwg? trt herb RANGE: ang cub herb RANGE: cag jam per sao? trt herb

RANGE: end

shrub

RANGE: as i

climber

RANGE: mex

cl imber

RANGE: cag mex trt climber

RANGE: end

RANGE: bel

cl imber

RANGE: per climber

RANGE:

climber

RANGE: cag mex

climber

RANGE: $\mathrm{cr}$ hon mex

climber 


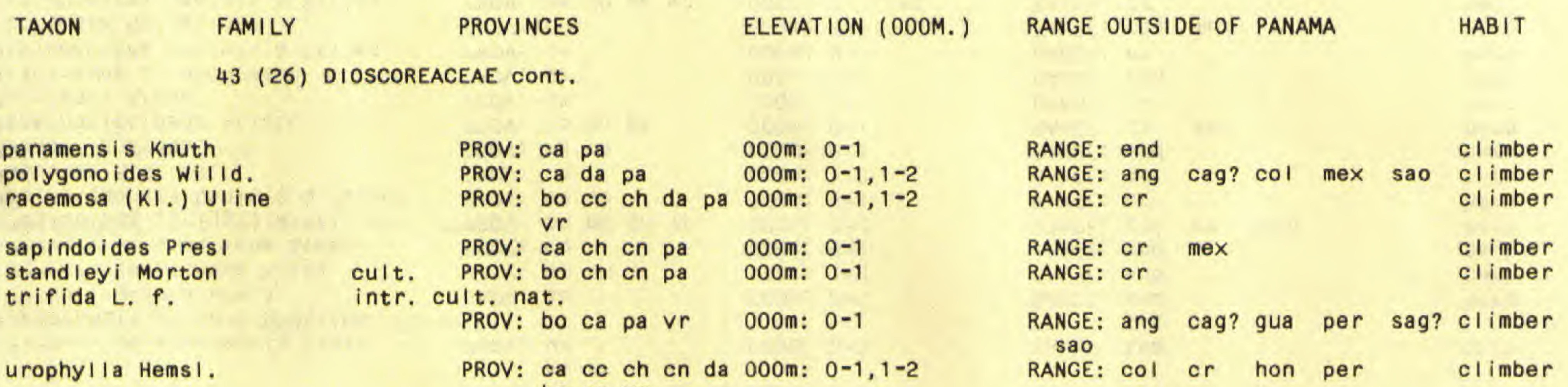

44 (27) IRIDACEAE

Cipura

paludosa AubI.

PROV: ca cc ch lo pa $000 \mathrm{~m}: 0-1$

000m: $2-3$

PROV: ch

ROV: bo $000 \mathrm{~m}$ : $0-1$

PROV: bo ca cc cn he lo pa vr

Orthrosanthus
*monadelphus Ravenna

PROV: $\mathrm{ch}$

$000 m: 2-3,3+$

Sisyrinchium

chiricanum Woods.

convolutum Nocca

mandoni Baker

micranthum Cav.

tinctorium H.B.K. cult

Prof

RANGE: ang? cag? cub mex sag? herb Sao

RANGE: ecu per sao

herb

RANGE: gui sao herb RANGE: cag mex sat herb

RANGE: bol cag? mex per sag? herb

RANGE: end herb

RANGE: cag? mex per sag? herb

RANGE: bol col herb

RANGE: bol cag? mex sag? herb

RANGE: bol cag? mex per sag? herb

45 (29) MUSACEAE

Heliconia

*atropurpurea Daniels \& Stiles

* colgantea Daniels \& Stiles

curtispatha O.G. Peters. $\begin{array}{ll}\text { PROV: ch } v r & 000 \mathrm{~m}: 0-1 \\ \text { PROV: ch da pa } & 000 \mathrm{~m}: 0-1 \\ \text { PROV: ca cc ch cn da } & 000 \mathrm{~m}: 0-1\end{array}$ pa sa vr
RANGE: $c r$

RANGE: $c r$

RANGE: $\mathrm{cr}$ nic

herb

herb

herb 
hirsuta L. $f$.

*ignescens Daniels \& Stiles

imbricata (Kuntze) Baker

* irrasa R.R. Sm.

lankesteri Stand I. --var, rubra Daniels \& Stiles lat ispatha Benth.

* Iong iflora R.R. Sm.

* Iophocarpa Daniels \& Stiles * maculata Kress

* magnifica Kress

marginata (Griggs) Pitt. mariae Hook. f.

*mathiasiae Daniels \& Stiles *metallica Hook.

*monteverdensis Daniels \& Stiles *necrobracteata Kress

nutans Woods.

*osaensis Cufod.

platystachys Baker

*pogonantha Cufod.
*--var. holerythra Daniels \& Stiles

PROV: ca da pa sa $000 \mathrm{~m}: 0-1$
PROV: bo

PROV: bo ca cc ch cn 000m: $0-1$ da

PROV: ca cc ch cn da 000m: 0-1,1-2 pa $v r$

PROV: $\mathrm{ch} \quad 000 \mathrm{~m}: 1-2$

PROV: ch 000m: we

PROV: bo ca cc ch cn 000m: $0-1$ da pa sa

PROV: cc cn da pa sa $000 \mathrm{~m}: 0-1$

PROV: $c c$ Vr O00m: $0-1$

PROV: cn 000m: $0-1$

PROV: pa 000m: $0-1$

PROV: da $000 \mathrm{~m}: 0-1$

PROV: bo ca cn da pa $000 \mathrm{~m}$ : $0-1$

$s a$

000m: $0-1$

PROV: bo $000 \mathrm{~m}$ : we

PROV: ca cc cn da pa 000m: 0-1 pa

PROV: ch vr 000m: $1-2$

PROV: $C C \quad 000 \mathrm{~m}: 0-1$

PROV: ch Vr $\quad$ 000m: $0-1,1-2,2-3$

PROV: ch

PROV: ca ce ch cn da 000m: $0-1$

$$
\text { lo pa sa }
$$

PROV: bo ca cc cn da 000m: $0-1$

PROV: $v r$ pr $v r$ 000m: $0-1$

\#--var. veraguasensis Kress

* ramonensis Daniels \& Stiles

*--var. glabra Kress

*--var. Ianuginosa Kress

*--var. xanthotricha Kress

reticulata (Griggs) Winkl.

* sarapiquensis Daniels \& Stiles

* secunda R.R. Sm.

*sessilis Kress

* spathocircinada Arist.

-stilesil Kress

* taca rcuna L. Andersson

* ta lamancana Daniels \& Stiles

tortuosa Griggs

*trichocarpa Daniels \& Stiles

*vaginalis Benth.

wagneriana 0,G. Peters.
PROV: $\mathrm{CC}$

PROV: bo ch

PROV: $c c$

PROV: bo $\mathrm{CC}$

PROV: $\mathrm{ch}$

PROV: ca cn

PROV: bo CC pa

PROV: ch

PROV: da

PROV: $v r$

PROV: $\mathrm{ch}$

000m: $0-1$

$000 \mathrm{~m}: 0-1$

000m: $0-1$

000m: $1-2$

000m: $0-1$

000m: $0-1$

000m: $0-1$

000m: we

000m: $0-1$

PROV: bo ca ce ch cn 000m: $0-1$

da pa sa

PROV: bo ca cc ch cn 000m: $0-1$ da sa

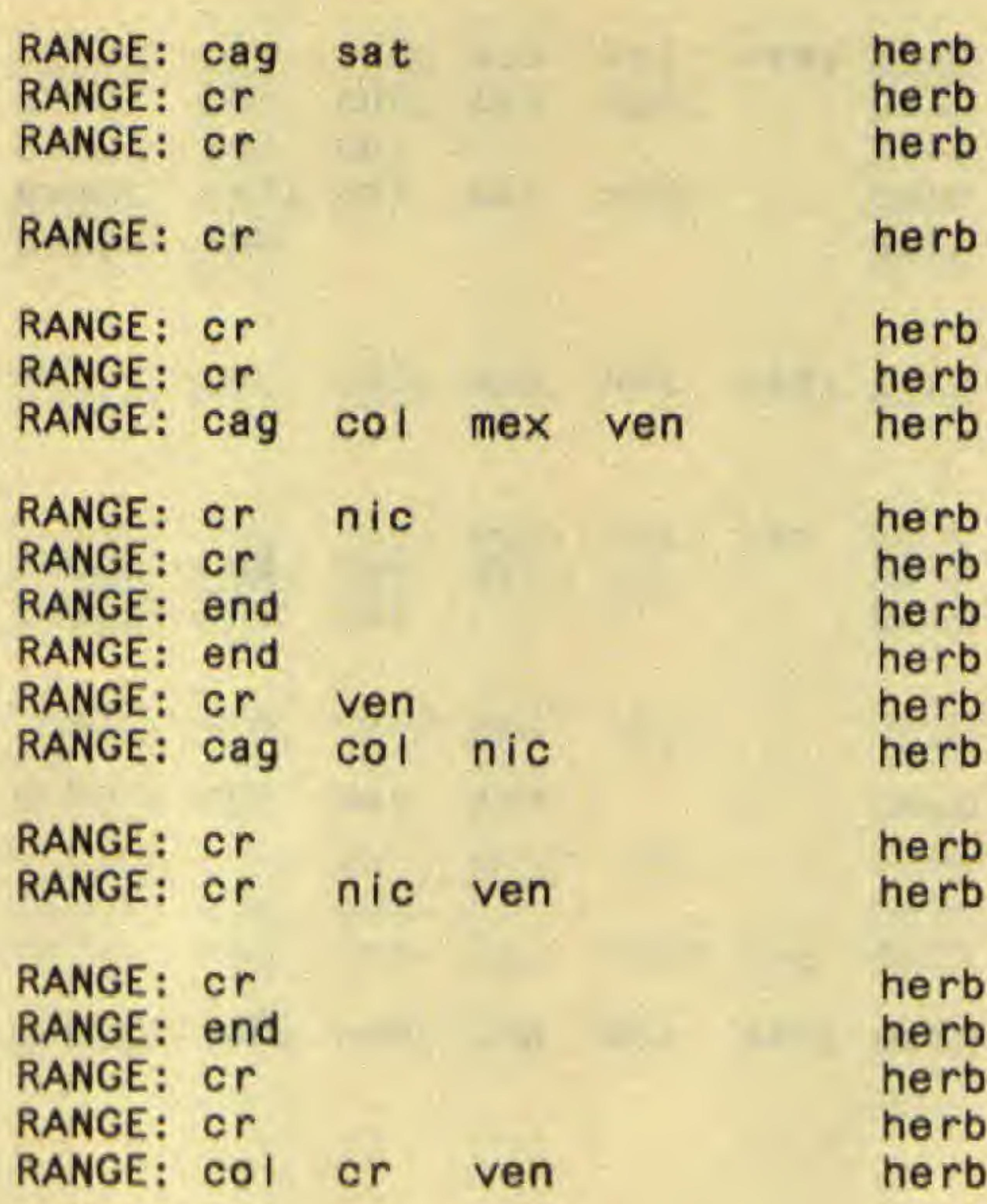

RANGE: $\mathrm{cr}$

herb

RANGE: end

herb

RANGE: end

RANGE: end

RANGE: end

RANGE: $\mathrm{CO}$ I

RANGE: $\mathrm{cr}$

RANGE: $\mathrm{cr}$

RANGE: end

RANGE: $\mathrm{cr}$

RANGE: $\mathrm{cr}$

RANGE: end

RANGE: $c r$

RANGE: cag mex

RANGE: $\mathrm{Cr}$

RANGE: cag

col ecu mex

herb

herb

herb

herb

herb

herb

herb

herb

herb

herb

herb

herb

herb

herb

RANGE: cag col cr

herb 
45 (29) MUSACEAE cont.

*xanthovillosa Kress

PROV: pa

000m: $0-1$

intr, cult

*sapientum L.

\section{6 (30) ZINGIBERACEAE}

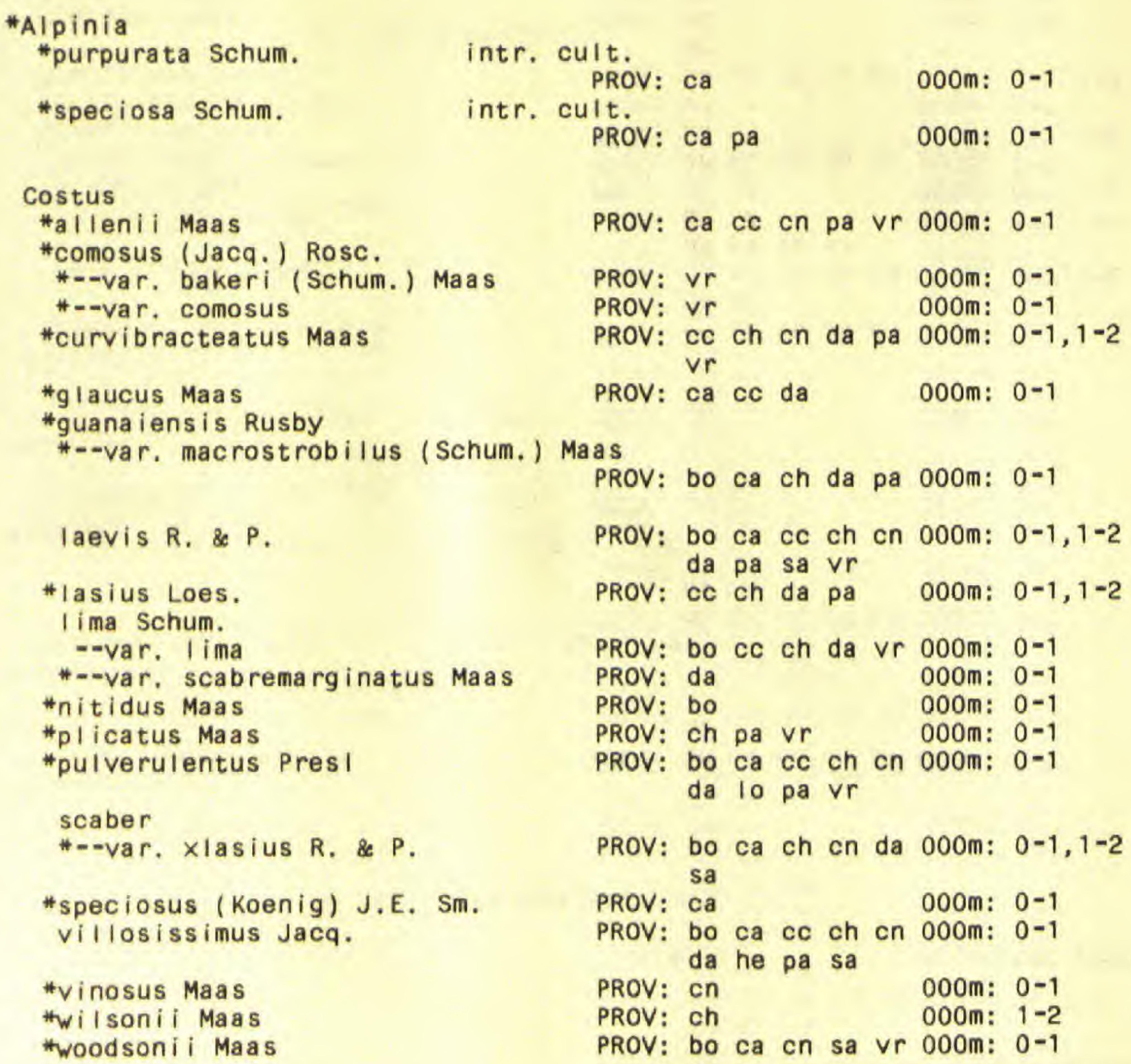

RANGE: oWt

herb

RANGE: owt

RANGE: col ecu ven

herb

RANGE: col gua mex sal herb

RANGE: $\mathrm{col} \mathrm{cr}$ ven herb

RANGE: $\mathrm{cr}$ herb

RANGE: $\mathrm{col} \mathrm{cr}$ herb

RANGE: bel col ecu gua gui herb

$\begin{array}{lll}\text { per pr sao trt ven } & \\ \text { RNGE: bel col cr ecu gua herb }\end{array}$

nic per sat cr

RANGE: col cr per sao herb

RANGE: $\mathrm{col} \mathrm{cr}$ ecu nic herb

RANGE: col ecu ven herb

RANGE: $\mathrm{cr}$ herb

RANGE: $c r$ herb

RANGE: cag col ecu gua mex herb sat ven

RANGE: end herb

RANGE: bel cr herb RANGE: col cr ecu gua gui herb jam lag per sag? ven

RANGE: end

RANGE: $c r$

RANGE: or nic 


\section{Dimerocostus}

strobilaceus Kuntze

\section{*Hedychium}

"coronarium koenig

int $r$, cult. nat.

Prov: bo ca ce ch on 000a: $0-1$ da 10

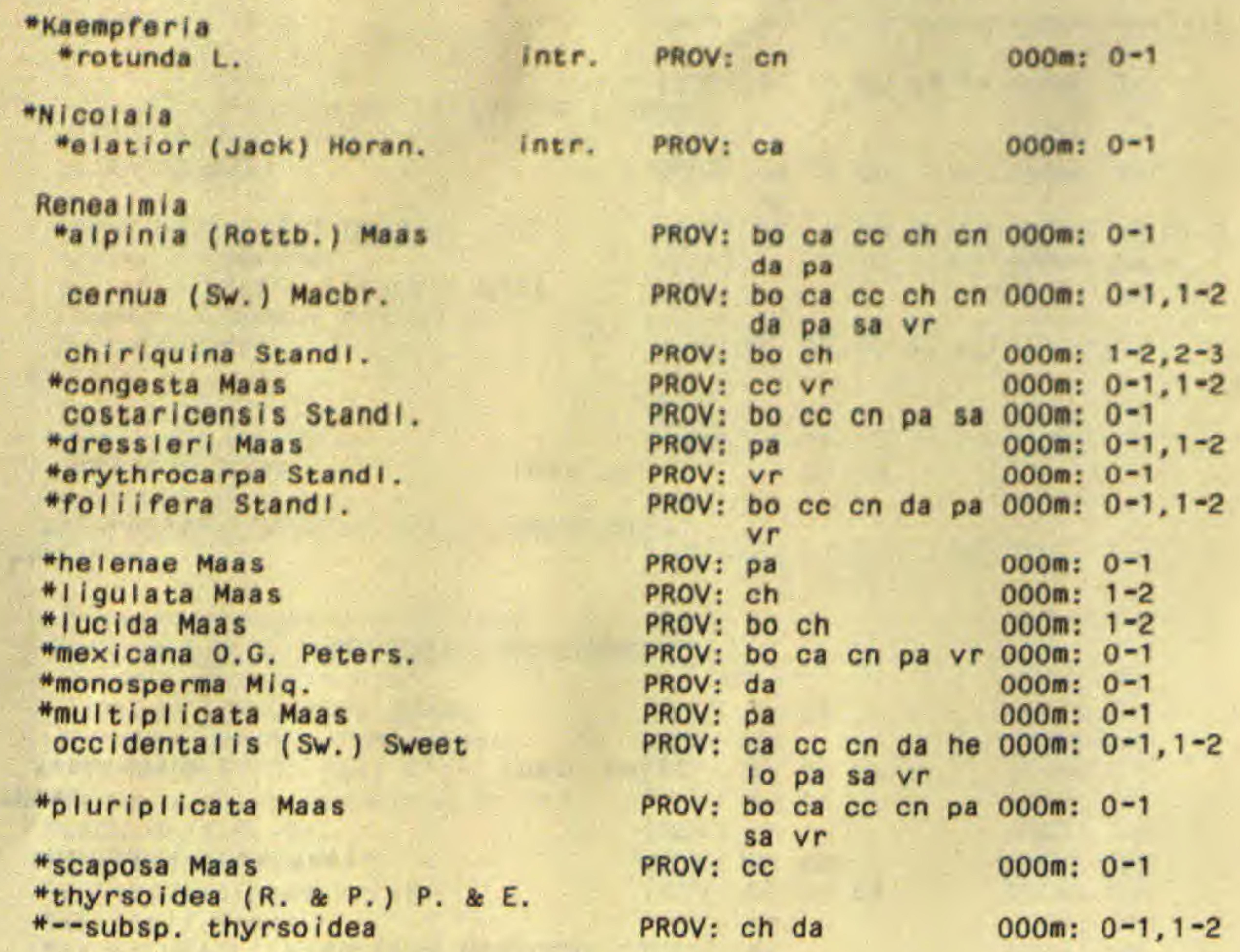

\section{*Zing iber}

Hofficinale Rosc. intr. cult.

PROV: ca $000 \mathrm{~m}: 0-1$

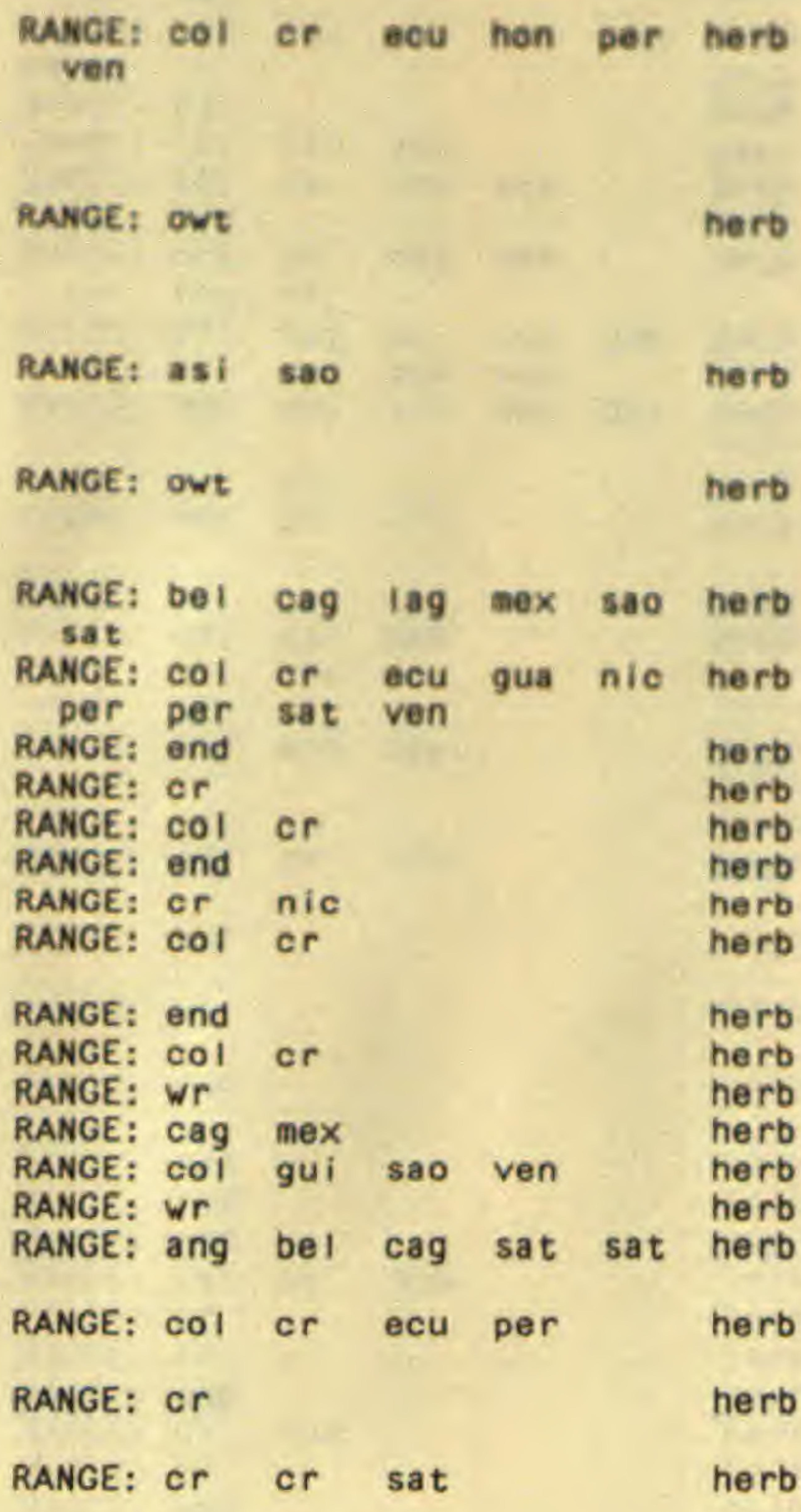

RANGE: $w d t$ 


\section{7 (31) CANNACEAE}

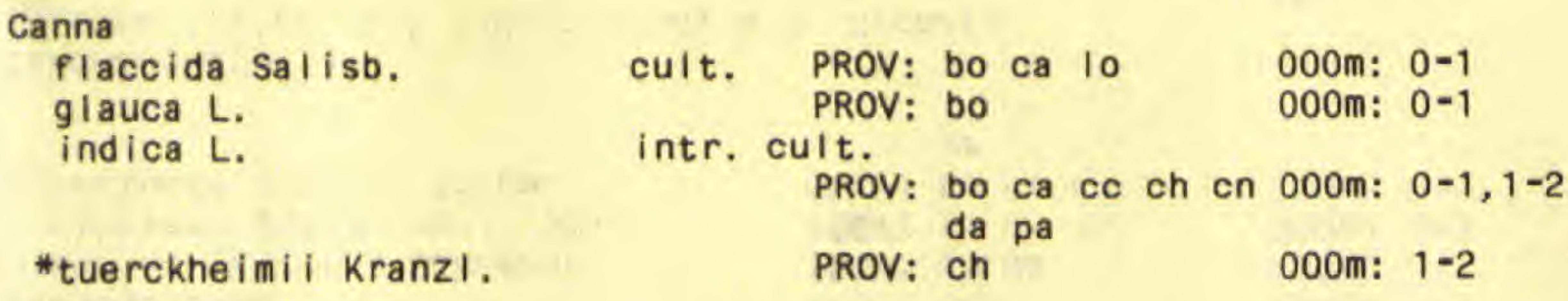

*tuerckheimii Kranzl.

ROV: $\mathrm{ch}$

\section{8 (32) MARANTACEAE}

Ca l a thea

al Ienii Woods.

*brenes i i Standl.

* caesariata H. Kennedy

*cleistantha Stand I.

* coriacea H. Kennedy

* crotal ifera Wats.

* cuneata H. Kennedy

* donnell-smithii schum.

*dressleri H. Kennedy

*elegans $H$. Kennedy

foliosa Woods.

*guzmanioides L.B. Sm. \& Idrobo

* gymnocarpa H. Kennedy

indecora Woods.

PROV: Pa

PROV: pa

PROV: ch

PROV: $V r$

PROV: $V r$

$\begin{array}{llll}\text { RANGE: ang fla nao nao } & \text { herb } \\ \text { RANGE: ang gui sag? sao } & \text { herb } \\ \text { RANGE: ang cag? mex sag? sao herb } & \text { herb } \\ \text { RANGE: cag mex } & & \text { her }\end{array}$

* inocepha la (Kunt

insignis 0.G. Peters.

*killipii L.B. Sm, \& Idrobo

lasiostachya J.D. Sm.

* Iatifolia (Link) KI.

* leucostachys Hook. f.

Iutea (Aub I.) G. Meyer

*macrocepha la Schum.

*marantifolia Standi.

micans (Mathieu) Koern.

*nitidifolia H. Kennedy panamens is StandI.
PROV: cc cn pa sa $v r$ 000m: $0-1$ PROV: ch Vr

000m: $0-1$

PROV: cc pa

000m: $0-1$

$000 \mathrm{~m}: 0-1$

000m: $0-1$

000m: $0-1$

000m: $0-1$

PROV: $000 \mathrm{~m}: 0=1$

PROV: pa sa $\quad 000 \mathrm{~m}: 0-1$

PROV: bo $000 \mathrm{~m}: 0-1$

PROV: bo cn $000 \mathrm{~m}: 0-1$

PROV: bo ch vr $000 \mathrm{~m}: 0-1$

. Nichols.

PROV: bo ca cc ch cn 000m: 0-1

PROV: bo ca ce ch en 000m: 0-1,1-2

da pa sa $v r$

PROV: pa pa sa $\mathrm{Vr}$ 000m: $0-1$

PROV: bo ca cc cn da $000 \mathrm{~m}$ : $0-1$

PROV: ca ch cn da he 000m: $0-1$

$$
\text { pa sa }
$$

PROV: cn 000m: $0-1$

PROV: bo ca ce ch cn 000m: $0-1$ da lo pa

PROV: $\mathrm{ch}$ lo $\mathrm{Vr} \quad$ 000m: 0-1

PROV: bo ca cc ch cn 000m: $0-1$ da pa sa $v r$

PROV: bo ca cc cn da 000m: 0-1 pa sa $\vee r$

PROV: $v r$

000m: $0-1$

PROV: ca da lo pa 000m: $0-1$

\begin{tabular}{|c|c|c|c|c|c|c|}
\hline $\begin{array}{l}\text { RANGE: } \\
\text { RANGE: }\end{array}$ & $\begin{array}{l}\text { end } \\
\mathrm{cr}\end{array}$ & & & u & & $\begin{array}{l}\text { herb } \\
\text { herb }\end{array}$ \\
\hline RANGE: & $\mathrm{cr}$ & & & & & herb \\
\hline RANGE: & $\mathrm{cr}$ & & & & & herb \\
\hline RANGE: & end & & & & & erb \\
\hline RANGE: & $\mathrm{cr}$ & & & & & ierb \\
\hline RANGE: & $\mathrm{cr}$ & & & & & erb \\
\hline RANGE: & $\mathrm{cr}$ & & & & & \\
\hline RANGE: & end & & & & & \\
\hline RANGE: & $\mathrm{COI}$ & $\mathrm{cr}$ & & & & \\
\hline RANGE: & cr? & & & & & \\
\hline RANGE: & $\mathrm{cr}$ & & & & & \\
\hline RANGE: & $\mathrm{cr}$ & & & & & \\
\hline RANGE: & $\mathrm{cr}$ & & & & & \\
\hline $\begin{array}{r}\text { RANGE: } \\
\text { sag? }\end{array}$ & cag? & $\mathrm{cr}$ & gua & gui & per & herb \\
\hline RANGE: & cag & $\mathrm{col}$ & $\operatorname{mex}$ & per & & arb \\
\hline RANGE : & col & & & & & \\
\hline RANGE: & cr & & & & & \\
\hline RANGE: & $\mathrm{col}$ & trt & ven & & & \\
\hline RANGE: & $c r$ & nic & & & & \\
\hline $\begin{array}{c}\text { RANGE: } \\
\text { sao }\end{array}$ & ang & bel & cag? & per & sag? & \\
\hline RANGE: & cag & & & & & D \\
\hline RANGE & bel & per & sat & & & \\
\hline $\begin{array}{c}\text { RANGE: } \\
\text { Sao }\end{array}$ & cag? & $\mathrm{cr}$ & gua & per & sag? & \\
\hline $\begin{array}{l}\text { RANGE: } \\
\text { RANGE: }\end{array}$ & $\begin{array}{l}\mathrm{cr} \\
\mathrm{cr}\end{array}$ & & & & & \\
\hline
\end{tabular}


48 (32) MARANTACEAE cont

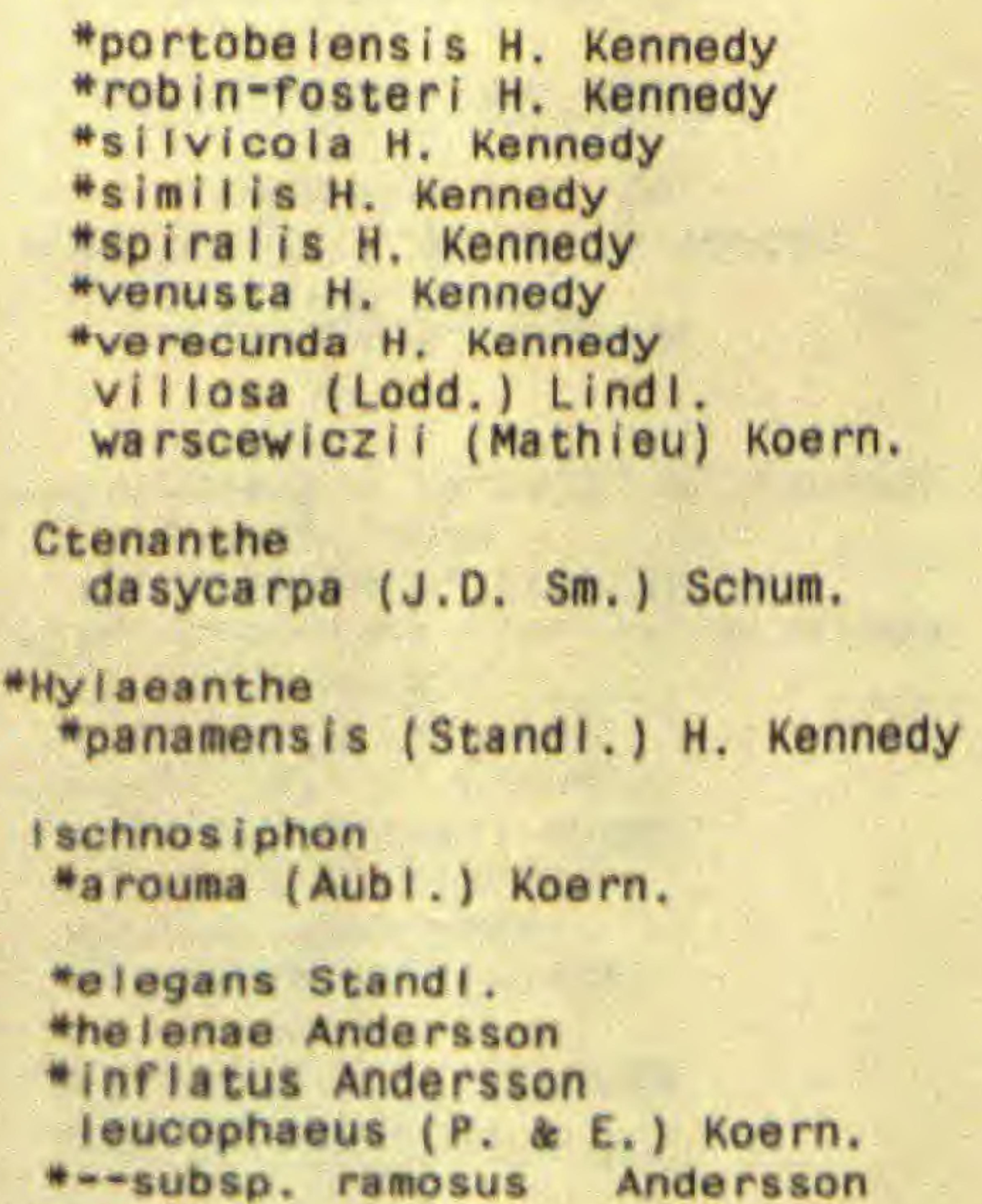

\section{Maranta}

a rund inacea $\mathrm{L}$.

\section{Pleiostachya}

pitcieri standi.

pruinosa (Regei) schum. morlae (Eggers) Schumann

$\begin{array}{ll}\text { PROV: ca cn pa } & 000 \mathrm{~m}: 0-1 \\ \text { PROV: cn } & 000 \mathrm{~m}: 0-1 \\ \text { PROV: ch cn pa vr } & 000 \mathrm{~m}: 0-1 \\ \text { PROV: bo } & 000 \mathrm{~m}: 0-1 \\ \text { PROV: cc ch da Vr } & 000 \mathrm{~m}: 0-1,1-2 \\ \text { PROV: cn } & 000 \mathrm{~m}: 0-1 \\ \text { PROV: pa } & 000 \mathrm{~m}: 0-1 \\ \text { PROV: ca pa } & 000 \mathrm{~m}: 0-1 \\ \text { PROV: bo } & 000 \mathrm{~m}: 0-1 \\ & \\ \text { PROV: bo cc pa vr } & 000 \mathrm{~m}: 0-1\end{array}$

PROV: ca da pa

000m: $0-1$

PROV: ca cc cn

000m: $0-1$

PROV: bo

PROV: ce en pa $\quad 000 \mathrm{~m}: 0=1$

PROV: bo on pa 000m: $0=1$

PROV: bo ca da pa sa 000m: 0-1 vr

$\begin{array}{ll}\text { PROV: bo da } & 000 \mathrm{~m}: 0-1 \\ \text { PROV: sa } & 000 \mathrm{~m}: 0-1 \\ \text { PROV: bo ca ch da pa 000m: } 0-1\end{array}$

yr
PROV: ca ce ch pa sa 000m: 0-1

* Jacquinif (R. \$.) H, Kennedy \& D, Nichois.

*tonekat (AubI.) Eichl.

PROV: ca on da pa sa 000m: 0-1 PROV: da

000m: $1-2$

Thatia

genieulata L.
PROV: ca ch da he pa 000m: 0-1

\section{RANGE: end \\ RANGE: end \\ RANGE: $w r$ \\ RANGE: $w r$ \\ RANGE: end \\ RANGE: or \\ RANGE: end \\ RANGE: $\mathrm{cr}$}

RANGE: $\mathrm{cr}$

sag? sao

hon

RANGE: $\mathrm{cr}$

RANGE: $\mathrm{COI} \mathrm{cr}$

herb

RANGE: col ecu gui lag sat herb trt ven

RANGE: or nic

RANGE: sat

RANGE: $\mathrm{COI} \mathrm{cr}$

RANGE: col cr nic ven

RANGE: cag nag? sag

RANGE: sag?

RANGE: COI?

RANGE: beI

nerb

herb

herb

RANGE: col or ven RANGE: ven

nerb

herb

herb

herb

herb

herb

herb

herb

herb

herb

herb

herb

herb

rb

RANGE: ang cag fla mex sag? herb sao 
49 (28) BURMANNIACEAE

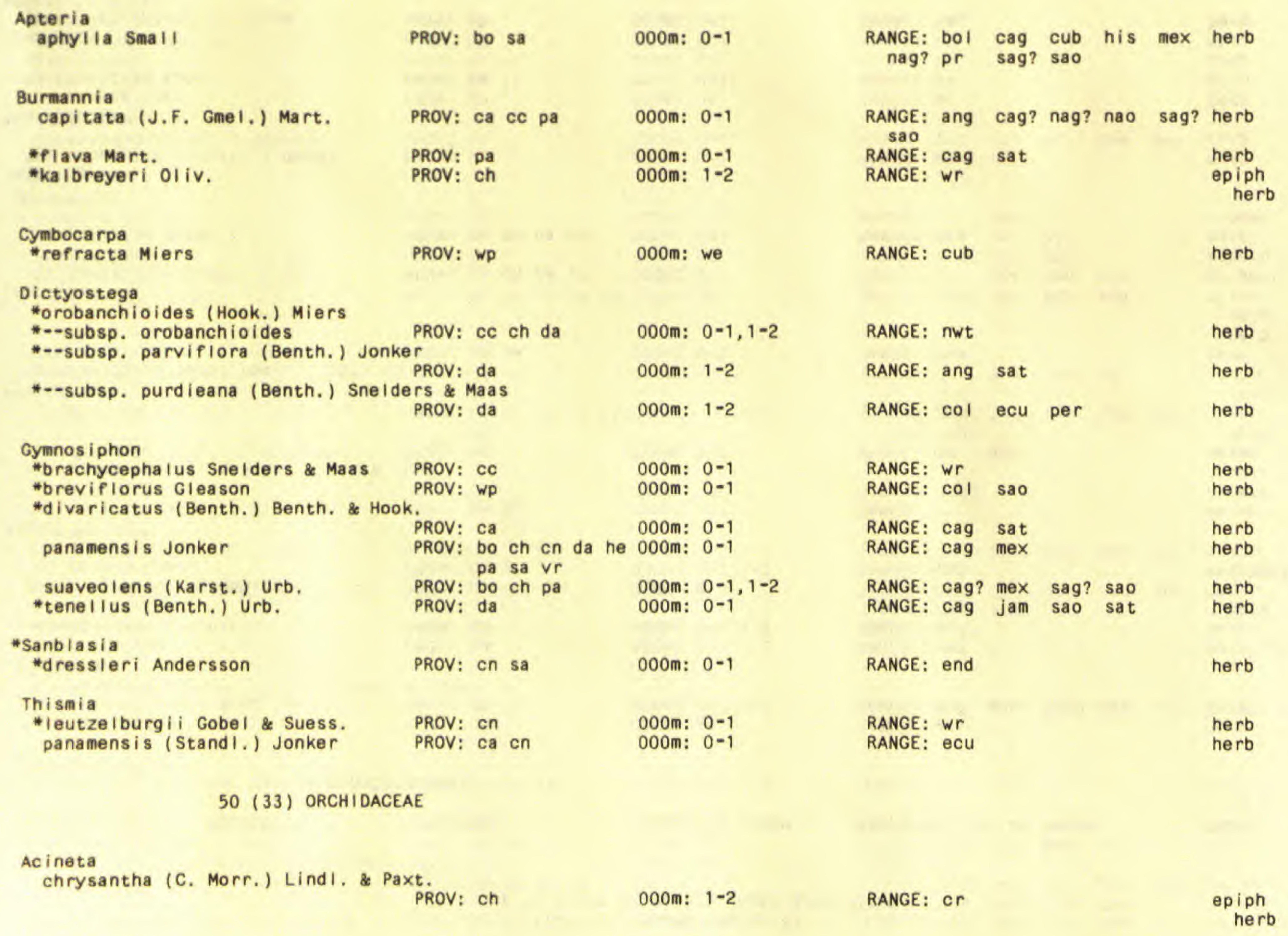


50 (33) ORCHIDACEAE cont.

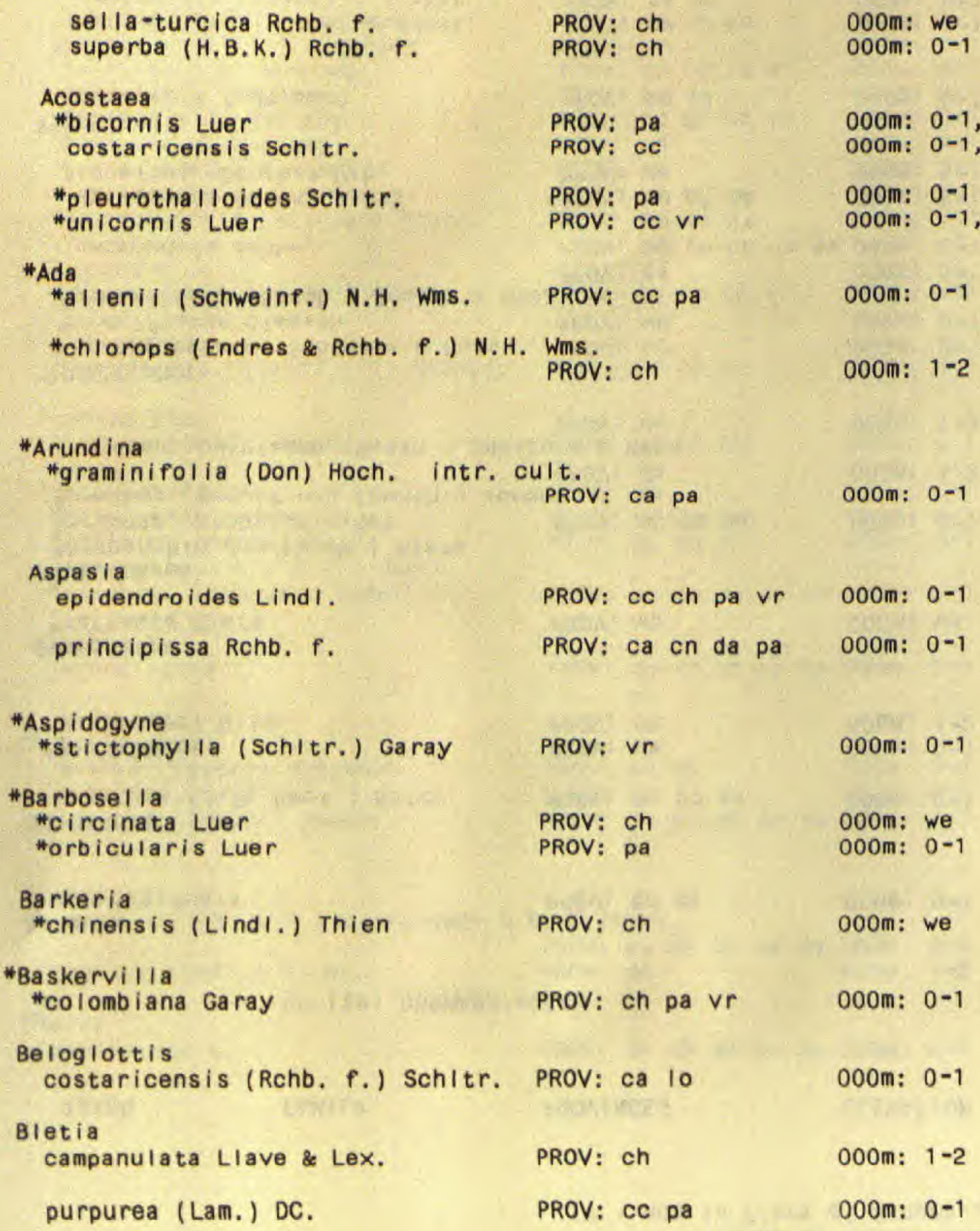

$\begin{array}{lll}\text { RANGE: wr } \\ \text { RANGE: col ecu herb ven } & \begin{array}{c}\text { herb } \\ \text { epiph }\end{array} \\ \text { RANGE: end } \\ \text { RANGE: cr } \\ \text { RANGE: cr } \\ \text { RANGE: end }\end{array}$




\begin{tabular}{|c|c|c|c|c|c|c|c|}
\hline 1070 in & & & & & & & \\
\hline "rolsomil Dressier & PROV: & ce & & & & 000m: & $0-1$ \\
\hline *kuhniarum Dressier & PROV: & ce & & & & 000m: & $0-1$ \\
\hline "pusiliua A. S. & PROV: & ch & $\mathrm{vr}$ & & & 000m: & $0-1$ \\
\hline *valeriol A. $\mathrm{s}$. & PROV: & on & vr & & 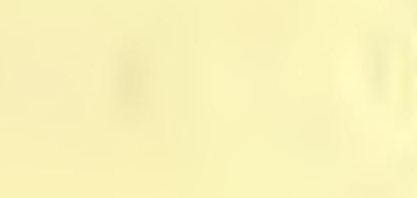 & 000m: & $0-1$ \\
\hline Brachystele & & - & $x^{2}$ & & & & 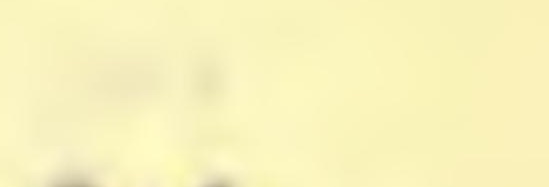 \\
\hline guyanensis (Lindi,) Schitr. & PROV: & ca & & & & 000a: & $0-1$ \\
\hline Brassavola & & 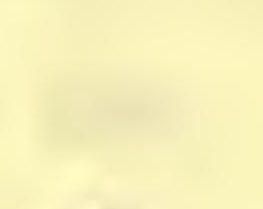 & & & & & 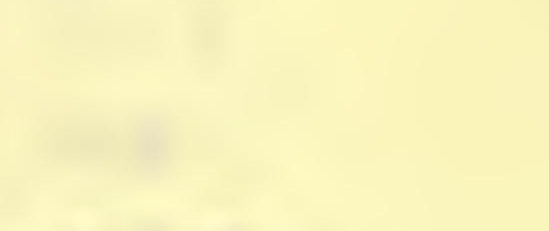 \\
\hline acaul is Lindi. & PROV: & ch & & & & 000n: & $1-2$ \\
\hline "glauca Lindi. & PROV: I & $W p$ & & & & 000m: & wo \\
\hline nodosa (L.) LindI. & PROV: & bo & ca & $\operatorname{cec}$ & pa $v r$ & 000n: & $0-1$ \\
\hline Brassia & & & & & & & \\
\hline *areuigera Reho. $r$. & PROV: & $\infty$ & pa & & & 000n: & $0-1$ \\
\hline caudata (L.) LindI. & PROV: & $\begin{array}{l}\text { ca } \\
\text { vr }\end{array}$ & on & da & 10 pa & $000 \mathrm{se}:$ & $0-1,1-2$ \\
\hline giraoudiana Rchb, f, W Warse. & PROV: & en & & & & 000a: & $1+2$ \\
\hline *lanceana Lindl. & PROV: & $W p$ & & & & $000 \mathrm{~s}:$ & ve \\
\hline Bu I bophyl ium & & & & & & & \\
\hline aristatum (Rohb. F.) Hens). & PROV: & co & ch & pa & & 000a: & $0-1,1-2$ \\
\hline pachyrrhachis (Achb. r.) Griseb. & PROV: & ca & pa & vr & & 000a: & $0-1$ \\
\hline Wagneri schitr. & PROV: & $W p$ & & & & $000 \mathrm{n}:$ & ve \\
\hline Ca I anthe & & & & & & & \\
\hline "calanthoides (Rich. WaI.) Maner & $\begin{array}{l}\text { La Ga } \\
\text { phov: }\end{array}$ & bo & ch & & & 000m: & $1 \cdot 2,2 \cdot 3$ \\
\hline Campylacent rum & & & & & & & \\
\hline brenesil schitr. & PROV: & on & pa & vr & & 000n: & $0-1,1=2$ \\
\hline *dressleri Dietrich $\&$ Diaz & PROV: & da & & & & 000n: & $0-1$ \\
\hline faseiola (Lind I, ) Cogn. & Prov: & da & pa & & & $000 \mathrm{~m}:$ & $0-1$ \\
\hline - Iong ical lca ratua Ames schwe inf. & PROV: & $v p$ & & & & 000n: & ve \\
\hline micranthua (Lind 1.$)$ Ro ife & PROV: & bo & ca & da & pa & 000n: & $0-1$ \\
\hline "pachyrrhizus (Rehb, f.) Roife & Prov: & ca & pa & & & $000 \mathrm{n}:$ & $0-1$ \\
\hline
\end{tabular}

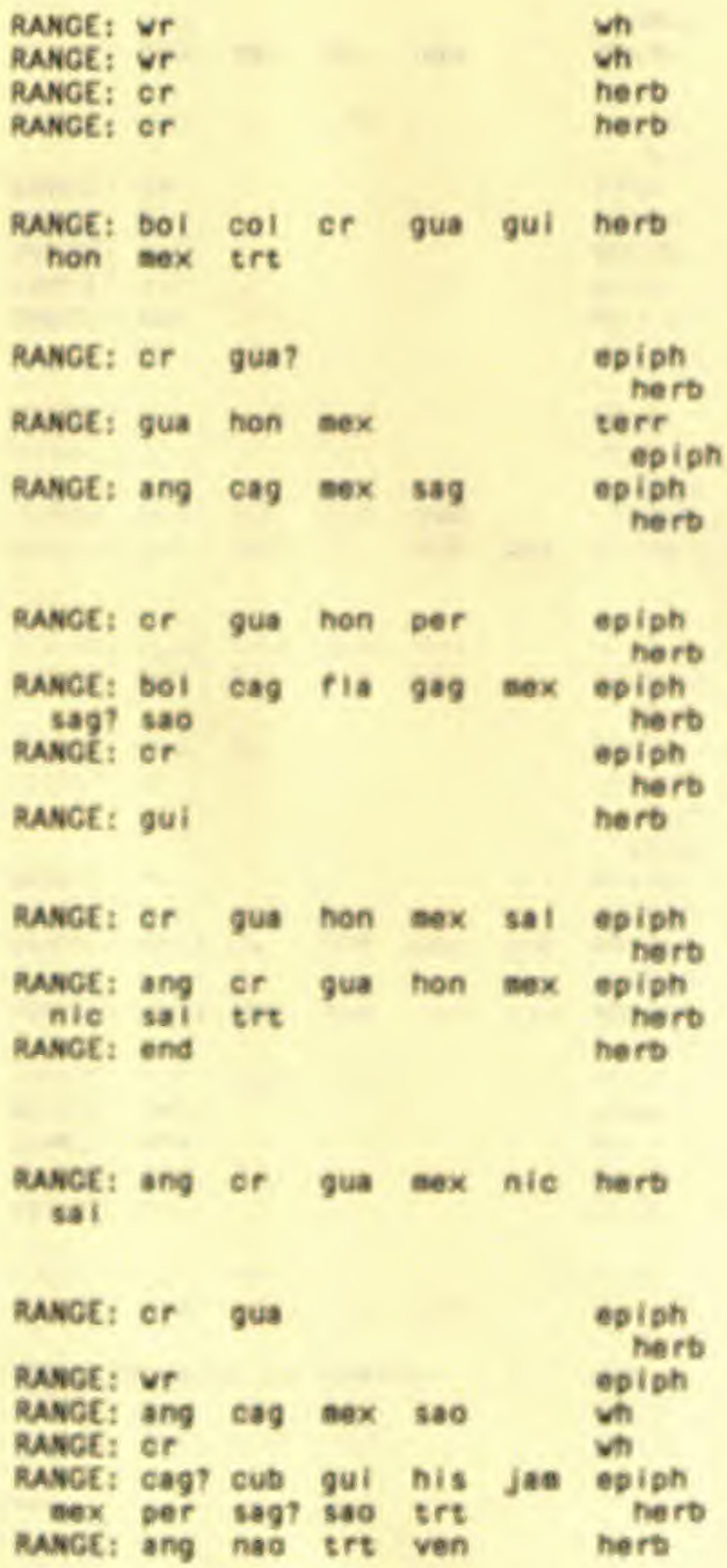


50 (33) ORCHIDACEAE cont.

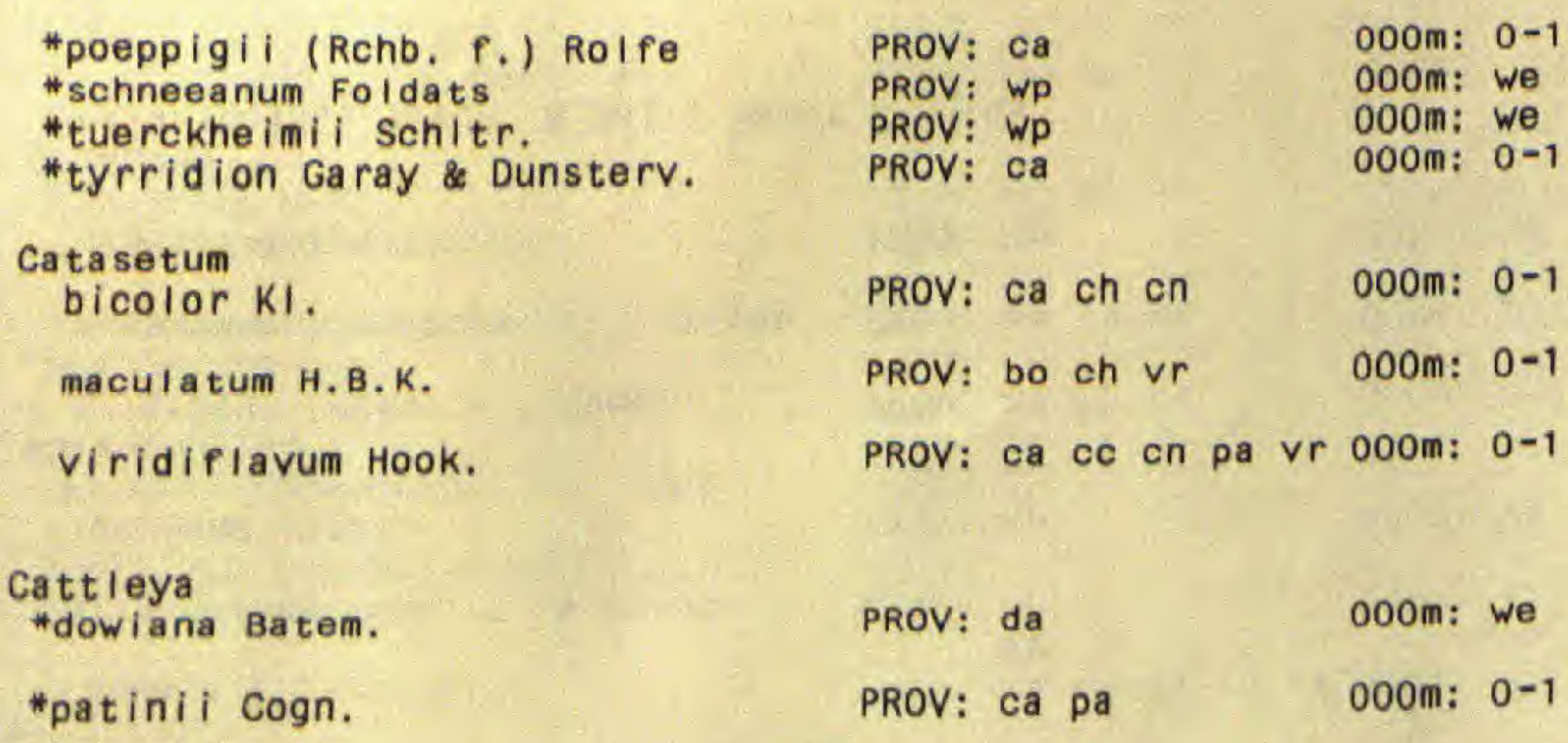

*Caula rthron

"bilamel la tum (Rchb. f.) R.E. Schult.

PROV: bo ca ch en da 000m: $0-1$ pa

\section{*Chaubardiell la}

* chasmatochila (Fowlie) Garay

PROV: pa

000m: $0-1$

\section{Chond rorhyncha}

*albicans Rolfe

* bicolor Rolfe

* crassa Dressier

* eburnea Dressier

* reichenbachiana Schitr.

\section{PROV: bO \\ PROV: $c 0$ ch $V r$ \\ PROV: $\mathrm{CC}$ ch \\ PROV: $\mathrm{CC}$ \\ PROV: cc da}

(n)

000m: we

000m: $0-1$

$000 \mathrm{~m}: 0-1$

000m: $0-1$

000m: $0-1$

Chys is

aurea Lind.

--var, maculata Hook.

PROV: ca

$000 \mathrm{~m}: 0-1$

000m: $0-1$

*dasyandra (Rchb, f.) Dressler \& N.H. Wms,
PROV: ch pa
"pusilla (C. Schweinf.) Dressler \& N.H. Wms.

PROV: $\mathrm{cc}$ ch da

000m: $0-1$

\section{Cleistes}

rosea Lind I.

\begin{tabular}{|c|c|c|c|c|c|c|}
\hline $\begin{array}{l}\text { RANGE: } \\
\text { RANGE: } \\
\text { RANGE: } \\
\text { RANGE: }\end{array}$ & $\begin{array}{l}\text { wr } \\
\text { ven } \\
\text { gua } \\
\text { ven }\end{array}$ & $=1-4$ & 5 & $20=$ & & $\begin{array}{l}\text { herb } \\
\text { wh } \\
\text { wh } \\
\text { herb }\end{array}$ \\
\hline RANGE: & col? & sao & ven & & & $\begin{array}{l}\text { epiph } \\
\text { herb }\end{array}$ \\
\hline RANGE: & col? & $\mathrm{cr}$ & gua & hon & nic & $\begin{array}{l}\text { epiph } \\
\text { herb }\end{array}$ \\
\hline RANGE: & $w r$ & & & & & $\begin{array}{l}\text { epiph } \\
\text { herb }\end{array}$ \\
\hline RANGE: & col & cr & & & & $\begin{array}{l}\text { epiph } \\
\text { herb }\end{array}$ \\
\hline RANGE: & col & $\mathrm{cr}$ & trt & ven & n. & herb \\
\hline $\begin{array}{l}\text { RANGE: } \\
\text { mex }\end{array}$ & $\begin{array}{l}\text { bel } \\
\text { nic }\end{array}$ & $\begin{array}{l}\text { cag } \\
\text { sal }\end{array}$ & $\begin{array}{l}\text { cr } \\
\text { trt }\end{array}$ & $\begin{array}{l}\text { gua } \\
\text { ven }\end{array}$ & hon & $\begin{array}{l}\text { epiph } \\
\text { herb }\end{array}$ \\
\hline RANGE: & $w r$ & it & in & & & herb \\
\hline RANGE: & $\mathrm{cr}$ & & 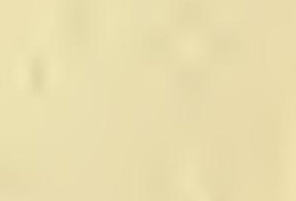 & & & wh \\
\hline RANGE: & $\mathrm{cr}$ & & & & & herb \\
\hline RANGE: & $w r$ & 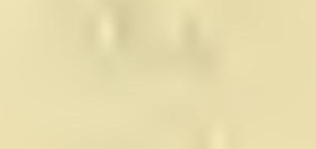 & 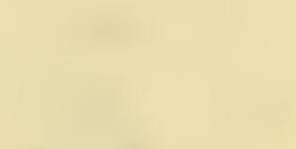 & & & epiph \\
\hline RANGE: & $w r$ & 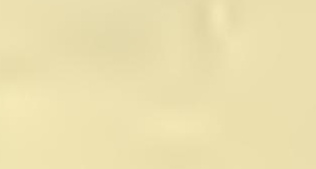 & $1 x^{2}$ & 10 & te & epiph \\
\hline RANGE: & $\mathrm{cr}$ & & & & & herb \\
\hline & & & 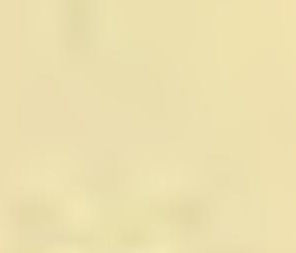 & & & 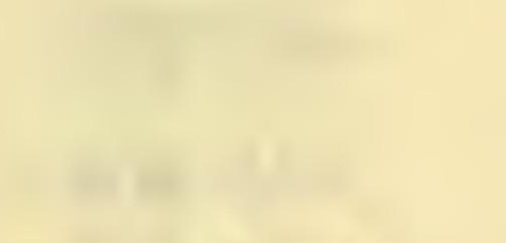 \\
\hline RANGE: & cag & $\mathrm{col}$ & $\mathrm{cr}$ & ven & & $\begin{array}{l}\text { epiph } \\
\text { herb }\end{array}$ \\
\hline RANGE: & $w r$ & & & & & herb \\
\hline RANGE: & $\mathrm{cr}$ & & 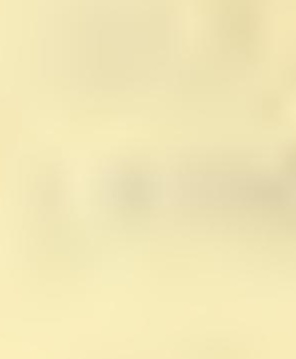 & & & $\begin{array}{l}\text { epiph } \\
\text { herb }\end{array}$ \\
\hline RANGE: & ang & col & $c r ?$ & gui & ven & herb \\
\hline
\end{tabular}


50 (33) ORCHIDACEAE cont.

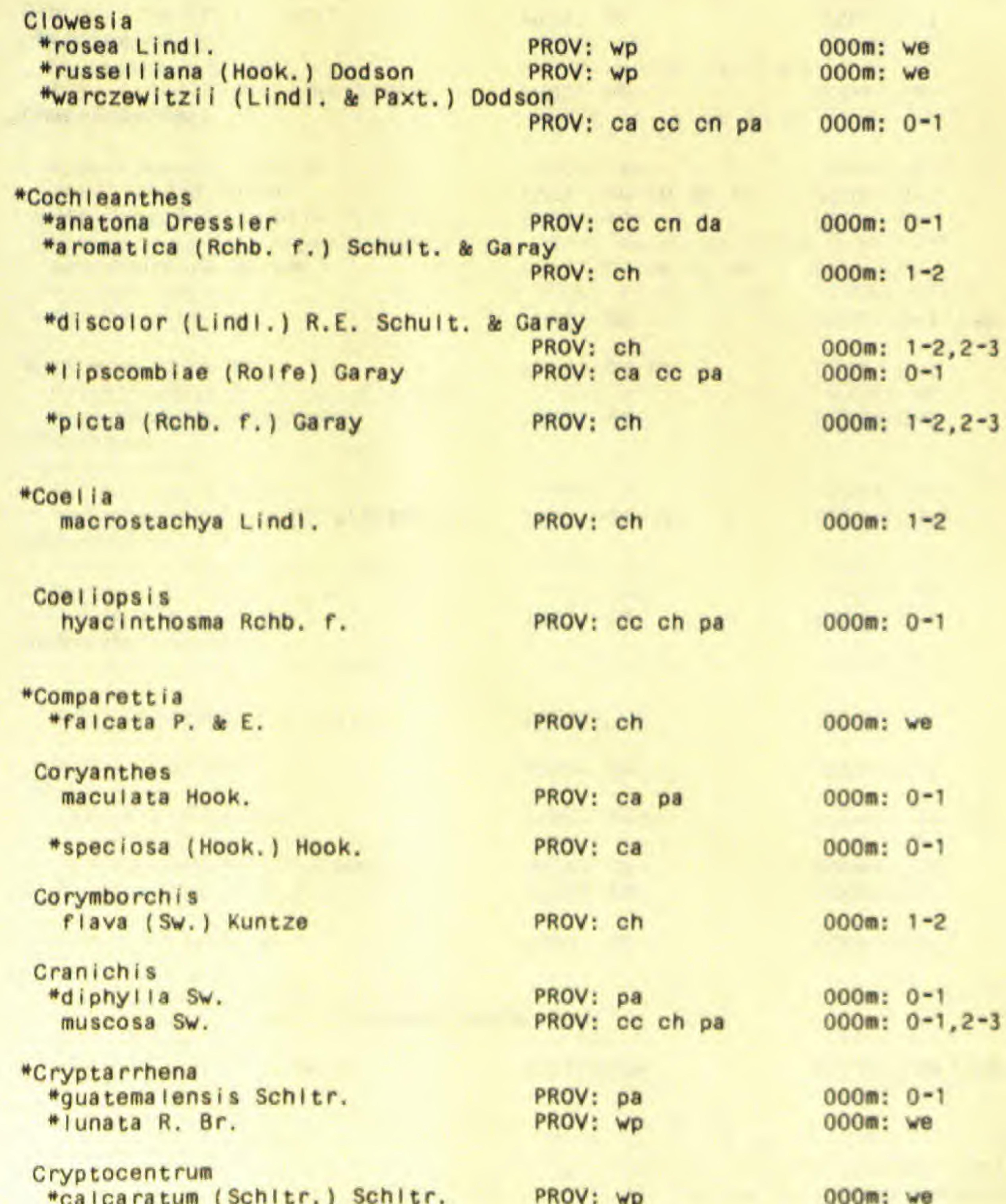

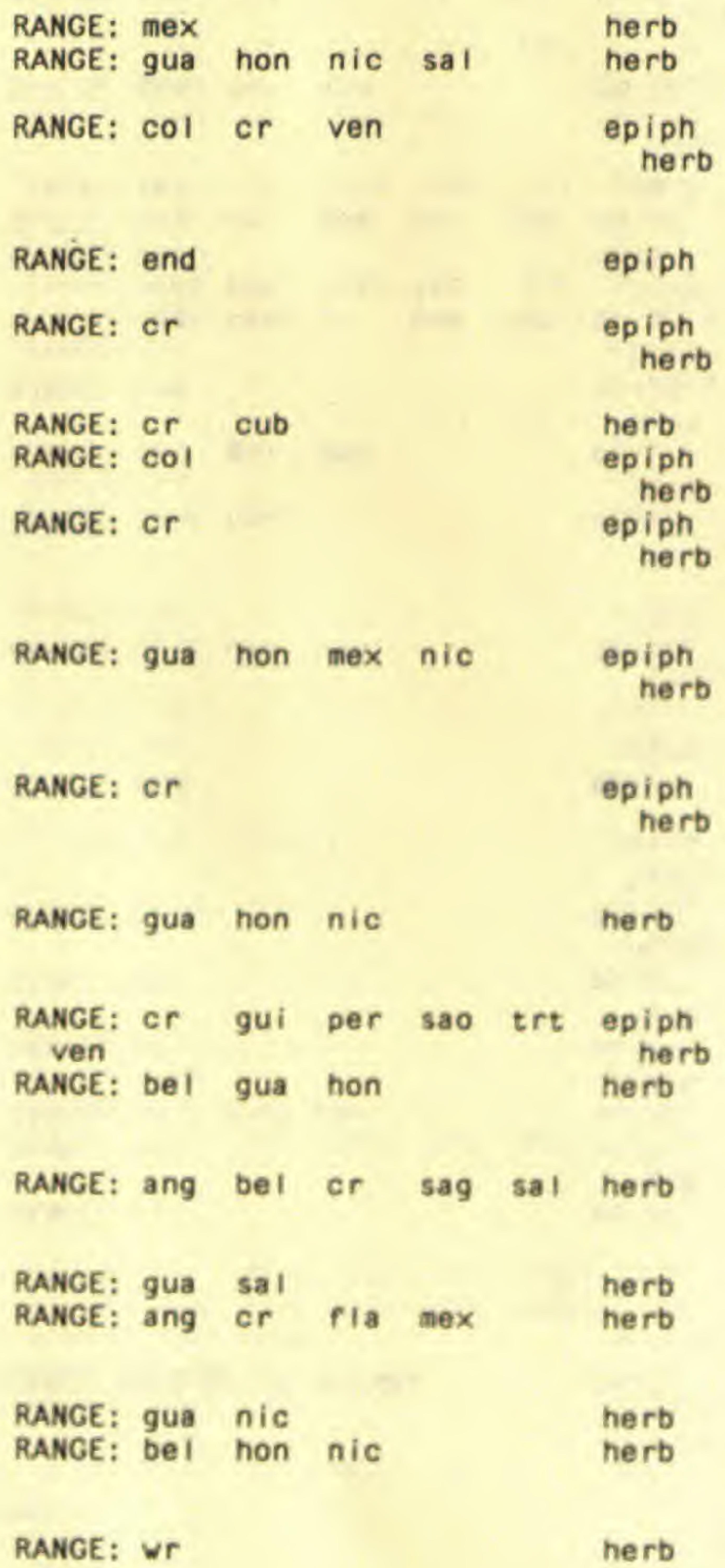


TAXON

\section{FAMILY}

PROVINCES

ELEVATION (OOOM.)

RANGE OUTSIDE OF PANAMA

HAB IT

50 (33) ORCHIDACEAE cont.

gracilipes Schltr.

\#gracillimum A, \& S.

inaequi sepa I um C. Schwe inf.

latifolium Schltr.

*minus Schlecht.

standleyi Ames

\section{Cryptophoranthus}

lepidotus L.O. Wms.

Cyclopogon

prasophyl Ium (Rehb, f.) SchItr. PROV: ca cc

Cycnoches

a ureum Lind I. \& Paxt.

chlorochilon Kl.

dianae Rchb. $f$.

egertonianum Batem.

stenodactylon Schitr.

ventricosum Batem.

\section{*Cymbiglossum}

* stell latum (LindI.) Halbinger

Cyrtopodium

punctatum (L.) LindI.

\section{Dichaea}

*brachypoda Rchb. f.

* costaricensis Schltr.

* dammeriana Kranzl.

*hystricina Rchb. f.

* lobata (Ames \& Corr.) L.O. Wms. morrisil Fawc. \& Rendl.
PROV: $c C$

PROV: WP

PROV: $\mathrm{ch}$

PROV: $\mathrm{CC}$

PROV: vr

PROV: $\mathrm{cC}$

PROV: $c c$

ooom: $0-1$

000m: $0-1$

000m: we

000m: $0-1,1-2$

000m: $0-1$

000m: $0-1$

PROV: $\mathrm{cc}$

000m: $0-1$

PROV: ch pa

000m: $0-1$

PROV: $c h$

PROV: ca cc ch pa

$000 \mathrm{~m}: 0-1,1-2$

000m: $0-1$

$\begin{array}{ll}\text { PROV: Wp } & 000 \mathrm{~m}: \text { we } \\ \text { PROV: } c c \text { ch da } V r & 000 \mathrm{~m}: 0-1\end{array}$

PROV: $c c$ ch da $v r$

000m: we

000m: $0-1$

000m: $0-1$

000m: we

000m: we

000m: $1-2$

000m: we

PROV: ch 000m: $0-1$

000m: $1-2$ 000m: $1-2$

RANGE: $c r$

RANGE: $\mathrm{cr}$

RANGE: col? ecu? per

RANGE: $\mathrm{cr}$

RANGE: per

RANGE: $c r$

RANGE: end

epiph

herb

herb

epiph

epiph

herb

epiph

herb

epiph

herb

epiph

herb

RANGE: $c r$ gua hon

epiph herb

RANGE: $\mathrm{cr}$ hon

epiph herb epiph

RANGE: col gui ven

herb

RANGE: cr?

epiph

RANGE: cag col cr gua hon epiph

mex nic per sal sao herb

RANGE: end

RANGE: cag

cr gua hon mex

nic sai

epiph

hert

RANGE: gua mex ven epiph

RANGE: ang cag col cr fla herb

gua gui mex per sao ven

RANGE: $\mathrm{cr}$ nic

epiph

RANGE: $\mathrm{cr}$

RANGE: $\mathrm{nIC}$

RANGE: $\mathrm{cr}$ gua hon

herb

herb

herb

RANGE: $c r$

RANGE: $\mathrm{cr}$ 
TAXON

FAMILY

ELEVATION (OOOM.)

RANGE OUTSIDE OF PANAMA

HAB IT

50 (33) ORCHIDACEAE cont.

muricata (Sw.) Lind I.

*oxypetala Schltr.

panamensis Lind I.

\#trulla Rehb. f.

"tuerckheimil Schltr.

Dimerandra

*emarginata ( $G$. Meyer) Hoehne

*isthmi Schltr.

*Dracula

*erythrochaete (Rchb. f.) Luer

*pusilla (RoIfe) Luer

*Dress Ierel I a

*eIvallensis Luer

*hispida (L.0. Wms.) Luer

*pertusa (Dressier) Luer

"powelli i (Ames) Luer

\section{*Dressleria}

*dilecta (Rchb. P.) Dodson

* suavis (A. \& S.) Dodson

\section{* Dryade I la \\ * gnoma (Luer) Luer \\ * I nearifolia (Ames) Luer \\ *pusiola (Rchb. P.) Luer \\ * simula (Rchb. P.) Luer}

El leanthus

aurantiacus (Lind I.) Rchb. $P$.

capitatus (P. \& E.) Rchb. f.

* caricoides Nash

*curtii Schltr.

*glaucophyllus Schitr.

* graminifolius (Barb. Rodr.) Lojt hymenophorus Rchb. $f$.

\# jimenezii (Schltr.) C. Schweinf. * I ancifolium PresI
PROV: $\mathrm{cc} \mathrm{ch} \mathrm{cn}$

$000 \mathrm{~m}: 0-1,1-2$

PROV: ch

000m: $2-3$

PROV: bo ca cc cn pa $000 \mathrm{~m}: 0-1$

vr

PROV: bo ca cn da pa 000m: $0-1$

PROV: pa

000m: $0-1$

PROV: bo ca da he pa 000m: $0-1$

PROV: bo ca da pa $000 \mathrm{~m}$ : $0-1$

PROV: ch

PROV: ch

000m: we

000m: we

PROV: $C C$

PROV: CC pa

000m: $0-1$

PROV: pa

PROV: WP

000m: $0-1$

000m: 0-1

$000 \mathrm{~m}$ : we

PROV: Vr

000m: $0-1$

PROV: pa

000m: $0-1$

PROV: bo

PROV: pa

PROV: pa

PROV: ca

000m: we

000m: $0-1$

000m: $0-1$

000m: $0-1$

PROV: cc ch pa

PROV: $c c$ ch Vr

PROV: ch

PROV: WP

PROV: bO

PROV: $c c$ cn pa vr

PROV: $\mathrm{cC} \mathrm{ch}$

PROV: da 000m: $0-1,1-2,2-3$

000m: $0-1,1-2$

000m: $2-3$

000m: we

$000 \mathrm{~m}: 2-3$

$000 \mathrm{~m}: 0-1,1-2$
$000 \mathrm{~m}: 0-1,1-2$

000m: $0-1,1-2$

000m: $0-1$

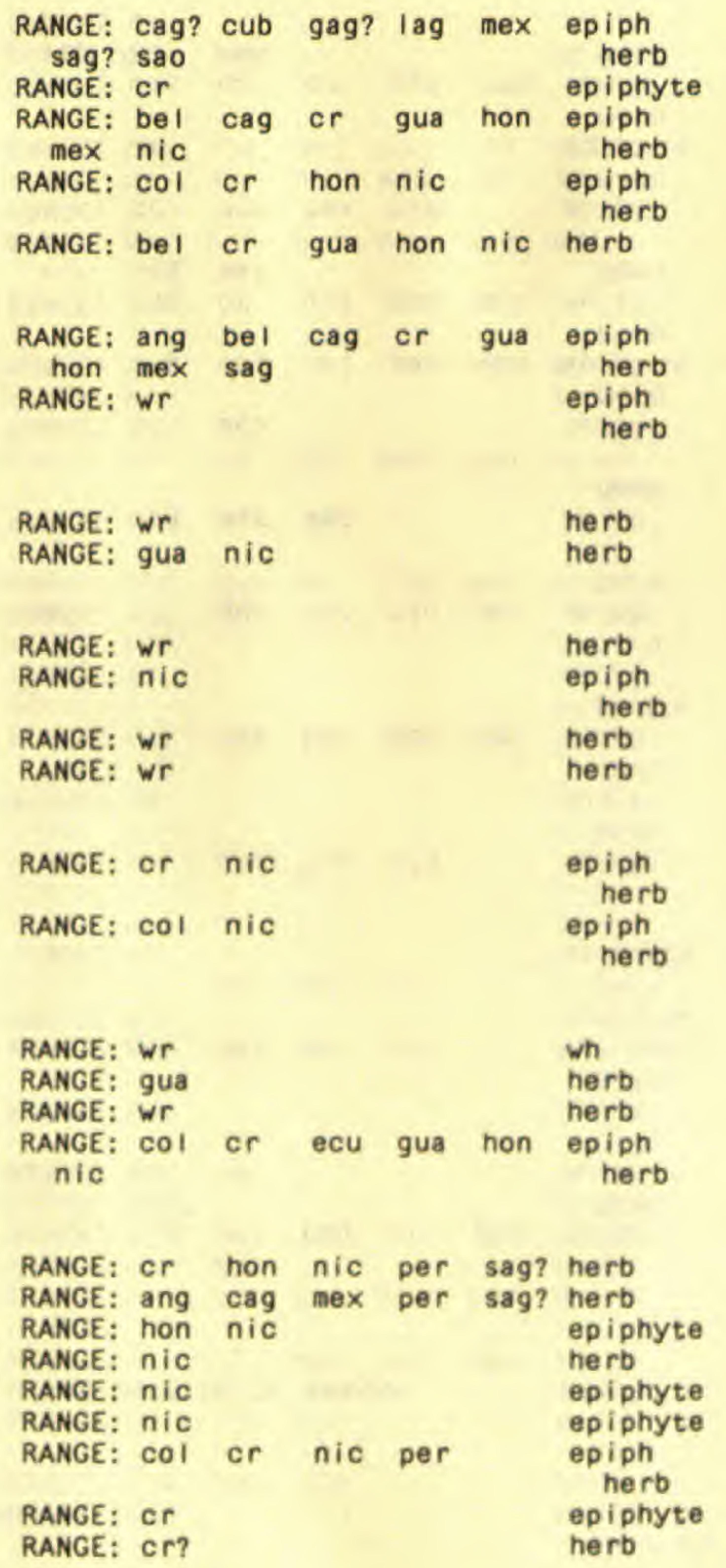


|inifolius Pres|

PROV: ca cc on da pa 000m: 0-1

* long ibracteatus (Griseb.) Fawe. muscicola Schltr.

*poiformis Schltr.

*stolonifer Barringer

*tillandsioides Barringer

Encycila

*abbreviata (Schltr.) Dressler

*amanda (Ames) Dressler

*brassavolae (Rchb. f.) Dressler campylostalix (Rchb, f.) Schltr.

*ceratistes (LindI.) Schltr.

*chacaoensis (Rehb. f.) Dressler \& Pollard

PROV: ca ch lo vr 000m: $0-1,1-2$

*chimborazoensis (Schltr.) Dressler

PROV: ca ce cn pa sa 000m: 0-1 PROV: da

000m: we

*cochleata (L.) Lemee

*cordigera (H.B.K.) Dressler

* fortunae Dressler

gravida (LindI.) Schltr.

* ionocentra (Rchb.) Dressler

* I ivida (LindI.) Dressler

*mooreana (Rolfe) Schitr.

PROV: cc ch pa

000m: 0-1

PROV: ch

PROV: ch pa

000m: we

000m: $0-1$

PROV: ca da pa

000m: $0-1$

PROV: ch

$000 m: 1-2$

PROV: $\mathrm{ch}$

$000 m: 1-2,2-3$

*prismatocarpa (Rchb. f.) Dressler

$$
\text { cult. PROV: ch }
$$

*pseudopygmaea (Finet) Dressler \& Pollard

*pygmaea (Hook.) Dressler

$\begin{array}{ll}\text { PROV: } c h & 000 \mathrm{~m}: 1-2 \\ \text { PROV: cc ch pa } & 000 \mathrm{~m}: 0-1 \\ \text { PROV: cc da pa sa } & 000 \mathrm{~m}: 0-1,1-2\end{array}$

\begin{tabular}{|c|c|c|c|c|c|c|}
\hline $\begin{array}{c}\text { RANGE: } \\
\text { per }\end{array}$ & $\begin{array}{l}\text { ang } \\
\text { sag? }\end{array}$ & bel & cag & $\mathrm{cr}$ & gua & $\begin{array}{l}\text { epiph } \\
\text { herb }\end{array}$ \\
\hline RANGE: & col & cr & & & & $\begin{array}{l}\text { epiph } \\
\text { herb }\end{array}$ \\
\hline RANGE: & $\mathrm{cr}$ & & & & & $\begin{array}{l}\text { epiph } \\
\text { herb }\end{array}$ \\
\hline $\begin{array}{l}\text { RANGE: } \\
\text { RANGE: }\end{array}$ & $\begin{array}{l}\text { gua } \\
\text { cr }\end{array}$ & nic & & & & $\begin{array}{c}\text { ep iphyte } \\
\text { ep iphyte } \\
\text { herb }\end{array}$ \\
\hline RANGE: & cr & & & & & epiphyte \\
\hline RANGE: & cr & gua & hon & nic & & $\begin{array}{l}\text { epiph } \\
\text { herb }\end{array}$ \\
\hline RANGE: & $w r$ & & & & & $\begin{array}{l}\text { epiph } \\
\text { herb }\end{array}$ \\
\hline RANGE: & $\mathrm{cr}$ & gua & hon 1 & mex & sa I & $\begin{array}{l}\text { epiph } \\
\text { herb }\end{array}$ \\
\hline RANGE: & $\mathrm{cr}$ & & & & & $\begin{array}{l}\text { epiph } \\
\text { herb }\end{array}$ \\
\hline RANGE: & $\mathrm{cr}$ & gua & hon & nic & sal & $\begin{array}{l}\text { epiph } \\
\text { herb }\end{array}$ \\
\hline RANGE: & cag & $\operatorname{mex}$ & ven & & & $\begin{array}{l}\text { epiph } \\
\text { herb }\end{array}$ \\
\hline RANGE: & col & ecu & & & & $\begin{array}{l}\text { epiph } \\
\text { herb }\end{array}$ \\
\hline RANGE: & ang & cag & $\mathrm{col}$ & mex & ven & $\begin{array}{l}\text { epiph } \\
\text { herb }\end{array}$ \\
\hline RANGE: & ang & cr & gua & hon & $\operatorname{mex}$ & epiph \\
\hline $\begin{array}{l}\text { nic } \\
\text { RANGE: }\end{array}$ & $\begin{array}{l}\text { sag } \\
w r\end{array}$ & sal & & & & $\begin{array}{l}\text { herb } \\
\text { wh }\end{array}$ \\
\hline RANGE: & $\mathrm{cr}$ & hon & $\operatorname{mex}$ & nic & & $\begin{array}{l}\text { epiph } \\
\text { herb }\end{array}$ \\
\hline RANGE: & $w r$ & 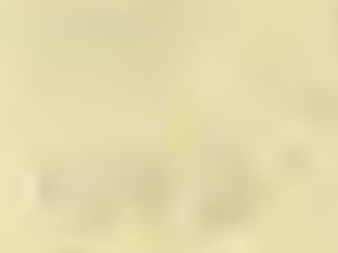 & $y$ & 1,8 & $x^{1}$ & $\begin{array}{l}\text { epiph } \\
\text { herb }\end{array}$ \\
\hline $\begin{array}{c}\text { RANGE: } \\
\text { max }\end{array}$ & cag & $\begin{array}{l}\text { col } \\
\text { ven }\end{array}$ & cr & gua & hon & $\begin{array}{l}\text { epiph } \\
\text { herb }\end{array}$ \\
\hline RANGE: & $\mathrm{cr}$ & ven & (1) & & ( & $\begin{array}{c}\text { epiph } \\
\text { herb }\end{array}$ \\
\hline RANGE: & $\mathrm{cr}$ & & in & & & $\begin{array}{l}\text { epiph } \\
\text { herb }\end{array}$ \\
\hline RANGE: & gua & sa I & & & & herb \\
\hline RANGE: & ang & bol & cag & fla & $\operatorname{mex}$ & epiph \\
\hline $\begin{array}{l}\text { sag? } \\
\text { RANGE. }\end{array}$ & $\begin{array}{l}\text { sao } \\
w r\end{array}$ & & & & & herb \\
\hline
\end{tabular}


50 (33) ORCHIDACEAE cont.

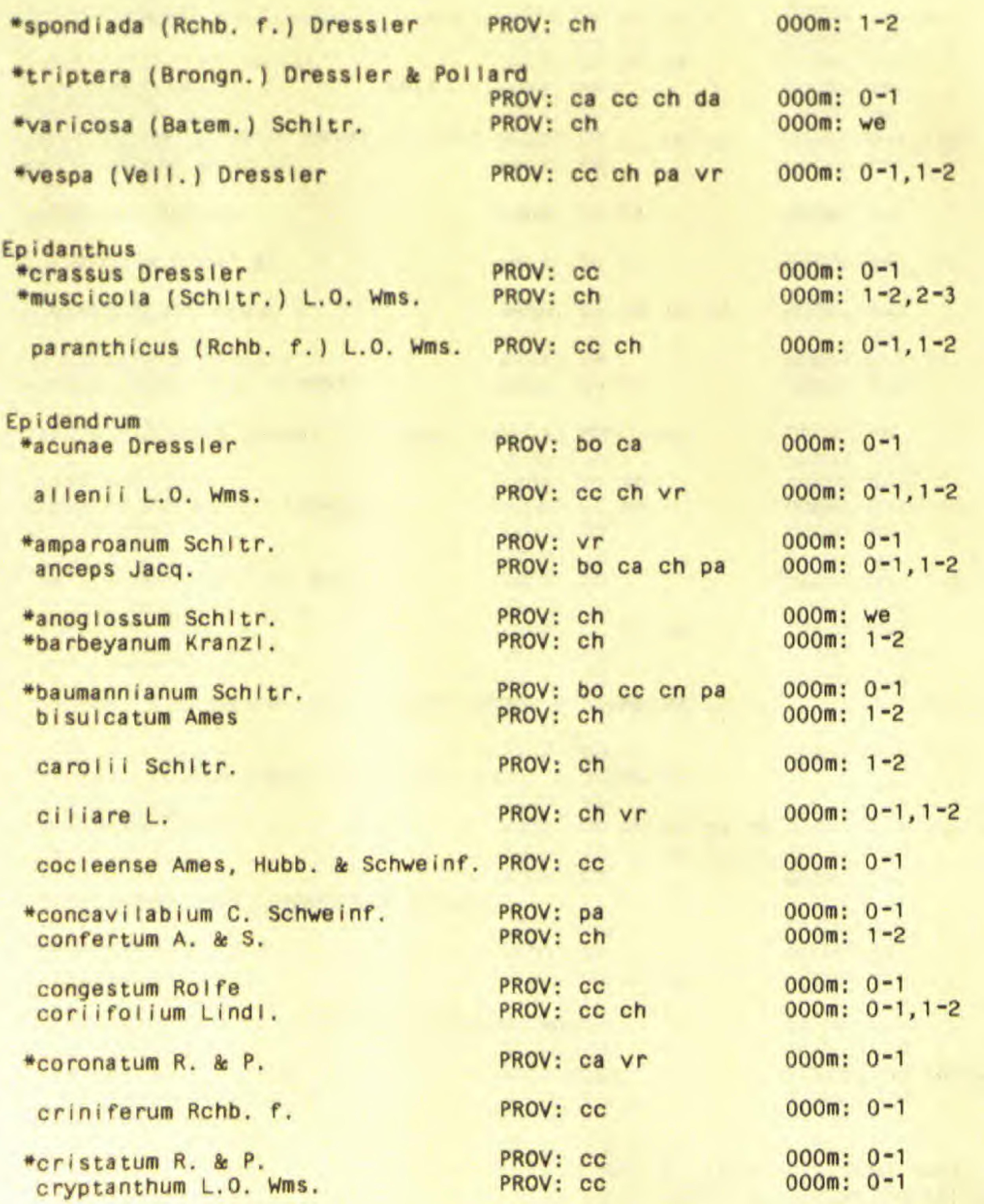

\begin{tabular}{|c|c|c|c|c|c|c|}
\hline RANGE: & $\mathrm{cr}$ & jam? & & & & $\begin{array}{l}\text { epiph } \\
\text { herb }\end{array}$ \\
\hline RANGE: & ang & fla & $\operatorname{mex}$ & sao & trt & epiphyte \\
\hline RANGE: & er & gua & hon & mex & sa I & $\begin{array}{l}\text { epiph } \\
\text { herb }\end{array}$ \\
\hline $\begin{array}{l}\text { RANGE: } \\
\text { Sao }\end{array}$ & ang & $\mathrm{cr}$ & hon & nic & sag & $\begin{array}{l}\text { epiph } \\
\text { herb }\end{array}$ \\
\hline RANGE: & $w r$ & & $x^{2}$ & & & ep iphyte \\
\hline RANGE: & $\mathrm{cr}$ & & & & s. & $\begin{array}{l}\text { epiph } \\
\text { herb }\end{array}$ \\
\hline RANGE: & $\mathrm{cr}$ & gua & hon & mex & nic & $\begin{array}{l}\text { epiph } \\
\text { herb }\end{array}$ \\
\hline RANGE: & ang & cag & $\mathrm{cr}$ & fla & gua & epiph \\
\hline $\begin{array}{l}\text { mex } \\
\text { RANGE: }\end{array}$ & $\begin{array}{l}\text { sag? } \\
\text { end }\end{array}$ & sao & & & & $\begin{array}{l}\text { herb } \\
\text { epiph } \\
\text { herb }\end{array}$ \\
\hline RANGE: & $\mathrm{cr}$ & & & & & epiphyte \\
\hline RANGE: & ang & cag & fla & $\operatorname{mex}$ & per & epiph \\
\hline sag? & sao & & & & & herb \\
\hline RANGE: & $\mathrm{cr}$ & & & & & herb \\
\hline RANGE: & cr & & & & & $\begin{array}{l}\text { epiph } \\
\text { herb }\end{array}$ \\
\hline RANGE: & $\mathrm{col}$ & & & & & herb \\
\hline RANGE: & end & & & & & $\begin{array}{l}\text { epiph } \\
\text { herb }\end{array}$ \\
\hline RANGE: & $\mathrm{cr}$ & hon & mex & sa 1 & & $\begin{array}{l}\text { epiph } \\
\text { herb }\end{array}$ \\
\hline RANGE: & ang & cag & mex & sag & & $\begin{array}{l}\text { epiph } \\
\text { herb }\end{array}$ \\
\hline RANGE: & end & & & & & $\begin{array}{l}\text { epiph } \\
\text { herb }\end{array}$ \\
\hline RANGE: & $\mathrm{cr}$ & & & & & herb \\
\hline RANGE: & $\mathrm{cr}$ & & & & & $\begin{array}{l}\text { epiph } \\
\text { herb }\end{array}$ \\
\hline RANGE: & $\mathrm{cr}$ & hon & & & & herb \\
\hline $\begin{array}{l}\text { RANGE: } \\
\text { ven }\end{array}$ & $\mathrm{cr}$ & ecu & gua & $\operatorname{mex}$ & per & $\begin{array}{l}\text { epiph } \\
\text { herb }\end{array}$ \\
\hline RANGE: & $\mathrm{Col}$ & cr & ecu & gua & mex & epiph \\
\hline per & sao & trt & ven & & & herb \\
\hline RANGE: & $\mathrm{cr}$ & nic & per & & & $\begin{array}{l}\text { epiph } \\
\text { herb }\end{array}$ \\
\hline $\begin{array}{l}\text { RANGE: } \\
\text { RANGE: }\end{array}$ & $\begin{array}{l}\text { gua } \\
\text { end }\end{array}$ & hon & nic & & & $\begin{array}{l}\text { herb } \\
\text { epiph }\end{array}$ \\
\hline
\end{tabular}


dentiferum A. \& S.

PROV: da

000m: $2-3$

dentil obum Ames, Hubb. \& Schwe inf.

PROV: ca

000m: $0-1$

PROV: ca cc ch da pa 000m: $0-1,1-2,2-3$ $r$

difforme Jacq.

--var, firmum (Rchb, f.) Ames, Hubb, \& Schweinf. PROV: ch

$000 \mathrm{~m}: 1-2$

--var. simulacrum (Ames) Ames, Hubb, \& Schweinf. PROV: ch

$000 \mathrm{~m}: 1-2$

PROV: bo ca cn

000m: $0-1$

eburneum Rchb. $f$.

PROV: $C C$

$000 \mathrm{~m}: 0-1,1=2$

texile Ames

\# flexuosiss imum C. Schwe inf. fragrans SW.

fuscopurpureum Schltr.

\#hel lerianum A.D. Hawkes hunterianum Schltr.

ima tophy I I um LindI.

incomptum Rchb. f.

i some rum Schltr.

* Iancilabium Schltr.

* latifolium (Lindl.) Garay \& Sweet PROV: cc

* I a ucheanum Rolfe

lividum Lind I.

lockhartioides Schltr.

cult. PROV: Wp

PROV: ca cc pa

PROV: ch

PROV: da

PROV: cc Vr

PROV: bo ca ce ch cn 000m: $0-1,1-2$

microphy I l um LindI.

nocturnum Jacq.

obesum Ames

PROV: ch

000m: $1-2$

*obliquifolium Ames, Hubb. \& Schweinf.

oerstedii Rchb. f.
PROV: ch 000m: we

PROV: $\mathrm{ch}$ 000m: 1 -2

\begin{tabular}{|c|c|c|c|c|c|c|}
\hline & & & & & & $\begin{array}{l}\text { epiph } \\
\text { herb }\end{array}$ \\
\hline RANGE: & $w r$ & & & & & $\begin{array}{l}\text { epiph } \\
\text { herb }\end{array}$ \\
\hline RANGE: & ang & cag & fla & $\operatorname{mex}$ & nwt & epiph \\
\hline per & sag? & sao & & & & herb \\
\hline RANGE: & cr & gua & & +4 & & $\begin{array}{l}\text { epiph } \\
\text { herb }\end{array}$ \\
\hline RANGE: & $w r$ & & & & & $\begin{array}{l}\text { epiph } \\
\text { herb }\end{array}$ \\
\hline RANGE: & hon & nic & & 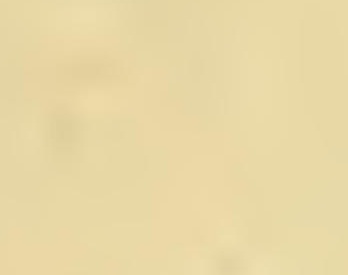 & & $\begin{array}{l}\text { epiph } \\
\text { herb }\end{array}$ \\
\hline RANGE: & end & & & & & epiph \\
\hline RANGE: & $\mathrm{cr}$ & & & & & $\begin{array}{l}\text { nerb } \\
\text { herb }\end{array}$ \\
\hline RANGE: & end & & & & & herb \\
\hline RANGE: & ang & $\mathrm{cr}$ & gua & $\operatorname{mex}$ & per & epiph \\
\hline $\begin{array}{l}\text { sag? } \\
\text { RANGE: }\end{array}$ & $\begin{array}{l}\text { sao } \\
\text { end }\end{array}$ & 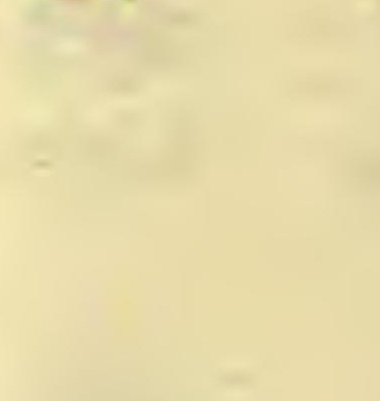 & & & & $\begin{array}{l}\text { herb } \\
\text { epiph } \\
\text { herb }\end{array}$ \\
\hline RANGE: & nic & 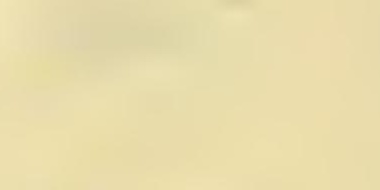 & & & & herb \\
\hline RAN & $\mathrm{cr}$ & t & thent & 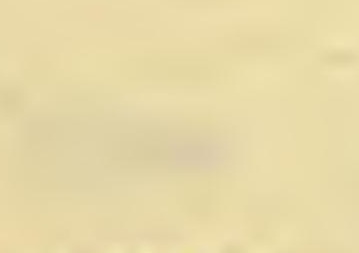 & $\ln ^{7} 7$ & $\begin{array}{l}\text { epiph } \\
\text { herb }\end{array}$ \\
\hline $\begin{array}{c}\text { RANGE: } \\
\text { SaO }\end{array}$ & $\begin{array}{l}\text { cag } \\
\text { trt }\end{array}$ & $\mathrm{col}$ & gui & $\operatorname{mex}$ & per & herb \\
\hline RANGE: & $\mathrm{cr}$ & gua & mex & & & $\begin{array}{l}\text { epiph } \\
\text { herb }\end{array}$ \\
\hline $\begin{array}{l}\text { RANGE: } \\
\text { mex }\end{array}$ & $\begin{array}{l}\text { bel } \\
\text { nic }\end{array}$ & cag & $\mathrm{cr}$ & gua & hon & $\begin{array}{l}\text { epiph } \\
\text { herb }\end{array}$ \\
\hline RANGE: & cr & - & & & - & herb \\
\hline RANGE: & gua & hon & & & & herb \\
\hline RANGE: & gua & hon & nic & sa I & 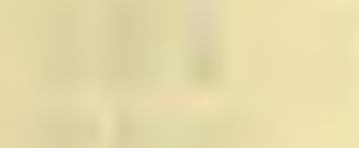 & herb \\
\hline RANGE: & $\mathrm{COI}$ & $\mathrm{cr}$ & ven & & - & herb \\
\hline RANGE: & $\mathrm{cr}$ & 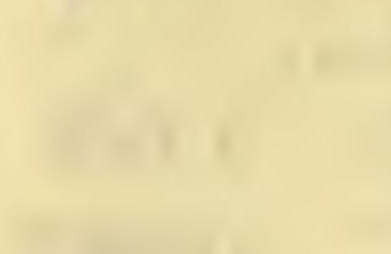 & & 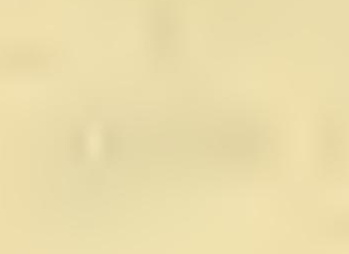 & & $\begin{array}{l}\text { epiph } \\
\text { herb }\end{array}$ \\
\hline RANGE: & $\mathrm{cr}$ & 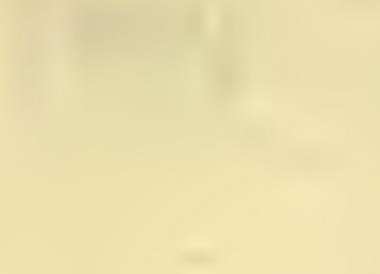 & & & & $\begin{array}{l}\text { epiph } \\
\text { herb }\end{array}$ \\
\hline $\begin{array}{l}\text { RANGE: } \\
\text { RANGE: }\end{array}$ & $\begin{array}{l}\text { wr } \\
\text { nic }\end{array}$ & & & & & $\begin{array}{l}\text { herb } \\
\text { herb }\end{array}$ \\
\hline $\begin{array}{r}\text { RANGE: } \\
\text { sag? }\end{array}$ & af $r$ & ang & cag & fla & $\operatorname{mex}$ & $\begin{array}{l}\text { epiph } \\
\text { herb }\end{array}$ \\
\hline RANGE & $\mathrm{cr}$ & ecu? & nic & & & $\begin{array}{l}\text { epiph } \\
\text { herb }\end{array}$ \\
\hline $\begin{array}{l}\text { RANGE: } \\
\text { RANGE: }\end{array}$ & $\begin{array}{l}\mathrm{cr} \\
\mathrm{cr}\end{array}$ & hon & nic & & & $\begin{array}{l}\text { herb } \\
\text { epiph } \\
\text { herb }\end{array}$ \\
\hline
\end{tabular}


50 (33) ORCHIDACEAE cont.

oncidioides Lindl.

--var. ramonense (Rchb, f.) Ames, Hubb. \& Schweinf.

Hubb. \& Schwe inf. 000m: $0-1,1-2$
PROV: cc ch

000m: $0-1$

panamense Schltr.

PROV: pa

paniculatum R. \& P.

PROV: ca cc ch pa 000m: $0-1,1-2$

parkinsonianum Hook.

PROV: ch

000m: 1-2

pendens L.0. Wms.

PROV: $\mathrm{cc}$

000m: $0-1$

\#peperomia Rchb. $f$.

PROV: ca ch

000m: $0-1,1-2$

\#phyl locharis Rchb. f.

physodes Rchb. f.

platystigma Rchb. $f$.

PROV: $\mathrm{cc}$ ch

PROV: bo

PROV: $\mathrm{ch}$

000m: $0-1$

000m: $0-1$

polyanthum Lindl.

PROV: ch

$000 \mathrm{~m}: 2-3,3+$

--var, myodes (Rchb. f.) Ames, Hubb. \& Schwe inf.

PROV: $\mathrm{ch}$

000m: $1-2$

powellii schltr.

PROV: $\mathrm{cc} \mathrm{ch}$

000m: $0-1,1-2$

probiflorum Schltr.

PROV: ch

000m: we

pseudepidendrum Rchb. $f$.

PROV: ch

000m: $1-2$

pudicum Ames

PROV: ch

$000 \mathrm{~m}: 1-2$

radicans Lind I.

PROV: bo ca cc ch pa 000m: $0-1,1-2,2-3$

ramosum Jacq.

PROV: $\mathrm{cc} \mathrm{ch}$

000m: $0-1,1-2$

repens Cogn.

PROV: $\mathrm{ch}$

000m: $2-3$

rigidum Jacq.

PROV: bo ca cn da pa $000 \mathrm{~m}$ : $0-1$

--var. angusti segmentum L. O. Wms.

rousseauae schltr.

PROV: bo

000m: $0-1$

PROV: ca ch pa

000m: $0-1$

* rugosum Ames

sanchoii Ames

PROV: ch

PROV: ch

000m: we

schlechterianum Ames

PROV: ca vr

000m: $1-2$

sculptum Rchb. $f$.

PROV: ca cn pa

000m: $0-1$

*selaginella Schltr.

PROV: cc ch vr

000m: $0-1$

000m: $0-1$

\begin{tabular}{|c|c|c|c|c|c|c|}
\hline ANGE: & $\mathrm{cr}$ & hon & 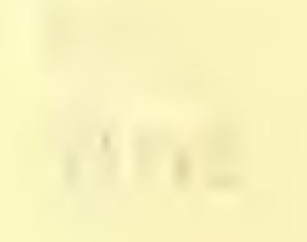 & th & (1784t) & $\begin{array}{l}\text { epiph } \\
\text { herb }\end{array}$ \\
\hline ANGE: & $w r$ & & & & & $\begin{array}{l}\text { epiph } \\
\text { herb }\end{array}$ \\
\hline RANGE: & cag & mex & sag & sao & & herb \\
\hline $\begin{array}{c}\text { RANGE: } \\
\text { sa I }\end{array}$ & $\mathrm{cr}$ & gua & hon & $\operatorname{mex}$ & nic & $\begin{array}{l}\text { epiph } \\
\text { herb }\end{array}$ \\
\hline ANGE: & $\mathrm{cr}$ & $25+1$ & 9 & & & $\begin{array}{l}\text { epiph } \\
\text { herb }\end{array}$ \\
\hline RANGE: & $\mathrm{cr}$ & gua & hon & $\operatorname{mex}$ & ven & $\begin{array}{l}\text { epiph } \\
\text { herb }\end{array}$ \\
\hline ANGE: & $w r$ & & & & & herb \\
\hline RANGE: & $\mathrm{cr}$ & gua & & & & herb \\
\hline RANGE: & $\mathrm{cr}$ & & 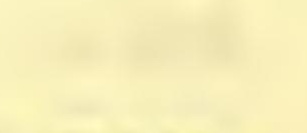 & & & herb \\
\hline RANGE: & cag & $\mathrm{cr}$ & gua & hon & nic & epiph \\
\hline sao & ven & & & & & herb \\
\hline RANGE: & $\mathrm{cr}$ & hon & & & & epiph \\
\hline RANGE: & $w r$ & & & & & $\begin{array}{l}\text { epiph } \\
\text { herb }\end{array}$ \\
\hline RANGE: & end & & & & & $\begin{array}{l}\text { epiph } \\
\text { herb }\end{array}$ \\
\hline RANGE: & $\mathrm{cr}$ & & & & & $\begin{array}{l}\text { epiph } \\
\text { herb }\end{array}$ \\
\hline AN & nic & & & & & $\begin{array}{l}\text { epiph } \\
\text { herb }\end{array}$ \\
\hline
\end{tabular}

RANGE: cag col gui mex per herb

RANGE: ang cag sat epiph

RANGE: ang cag cr gua gua epiph

hon mex nic sal ven herb

RANGE: ang bel cag fla gua epiph

hon mex nic nwt sag sat herb

RANGE: end

RANGE: end

epiph

herb

RANGE: $\mathrm{cr}$

RANGE: $\mathrm{cr}$

epiph

RANGE: cag gui jam mex per herb SaO

RANGE: cr? gui hon?

epiph

herb

RANGE: $n i c$ 
TAXON FAMILY

PROVINCES

ELEVATION (OOOM.)

RANGE OUTSIDE OF PANAMA

HABIT

50 (33) ORCHIDACEAE cont.

* sma ragd inum Lind $t$.

stamfordianum Batem.

stangeanum Rchb. $f$.

strobiliferum Rchb. $f$.

subnutans $A, \& S$.

trachythece Schltr.

triangulabium A. \& S.

\#vincent inum Lind $I$.

volutum Lindl. \& Paxt.

warszewiczii Rchb. P.

Eriopsis

\#bil oba Lindl.

\section{Erythrodes}

killipil Ames

*querceticola Ames

*vesicifera (Rchb. f.) Ames

Eulophia

alta (L.) Fawc. \& Rendl.

\section{*Eurystyles}

*cotyledon Wawra

Gal eandra

baueri Lindl.

*beyrichii Rchb. f.

*styl Iomisantha (VeII.) Hoehne

\section{Gongora}

a rmeniaca (Lindl.) Rchb. $f$.

*atropurpurea Hook.

* cha ront is Rchb. f.

*claviodora Dressler

fulva Lindl.

*gibba Dressier
PROV: cn

PROV: $\mathrm{ch}$

PROV: ca

PROV: ca pa

PROV: $\mathrm{ch}$

PROV: ch

PROV: co pa

PROV: da

PROV: ch

PROV: pa

PROV: ce ch pa

PROV: $c c$ ch

PROV: $c n$

PROV: $\mathrm{CC}$

PROV: ca cc cn pa

PROV: ch

PROV: bo

PROV: $\mathrm{CC}$

PROV: pa

PROV: ch

PROV: da

PROV: da

PROV: wp

PROV: ca cc cn pa

PROV: $\mathrm{Cc}$ on sa
$000 \mathrm{~m}: 0-1,1-2$

000m: $0-1$

000m: $0-1$

000m: $0-1$

000m: $0-1$

000m: $1-2$

000m: $1-2$

000m: $0-1$

000m: we

000m: $1-2$

000m: $0-1$

000m: $0-1,1-2$

000m: $0-1$

$000 \mathrm{~m}: 0-1$

000m: $0-1$

000m: we

000m: we

000m: $0-1$

000m: $0-1$

000m: $1-2$

000m: we

000m: we

000m: we

000m: $0-1$

000m: $0-1$

\begin{tabular}{|c|c|c|c|c|c|c|}
\hline $\begin{array}{l}\text { RANGE: } \\
\text { RANGE: }\end{array}$ & $\begin{array}{l}W r \\
\text { cag }\end{array}$ & $\mathrm{col}$ & mex & ven & & $\begin{array}{l}\text { herb } \\
\text { epiph } \\
\text { herb }\end{array}$ \\
\hline RANGE: & $\mathrm{cr}$ & hon & nic & & & $\begin{array}{l}\text { epiph } \\
\text { herb }\end{array}$ \\
\hline RANGE: & ang & cag & fla & gui & mex & epiph \\
\hline per & sao & ven & & & & herb \\
\hline RANGE: & $\mathrm{cr}$ & & & & & $\begin{array}{l}\text { epiph } \\
\text { herb }\end{array}$ \\
\hline RANGE: & cr & gua & hon & nic & sa I & $\begin{array}{l}\text { eploh } \\
\text { herb }\end{array}$ \\
\hline RANGE: & $\mathrm{cr}$ & nic & & $t^{2}$ & & $\begin{array}{l}\text { epiph } \\
\text { herb }\end{array}$ \\
\hline RANGE: & $w r$ & & & & & herb \\
\hline RANGE: & $w r$ & & & & & \\
\hline RANGE: & cr? & & & & & $\begin{array}{l}\text { epiph } \\
\text { herb }\end{array}$ \\
\hline RANGE: & $w r$ & & & & & $\begin{array}{l}\text { epiph } \\
\text { herb }\end{array}$ \\
\hline RANGE: & $\mathrm{cr}$ & & & that & & herb \\
\hline RANGE: & $w r$ & & & & & $\begin{array}{l}\text { terr } \\
\text { herb }\end{array}$ \\
\hline RANGE: & gua & nic & & & & herb \\
\hline RANGE: & ang & be 1 & col & fla & gua & herb \\
\hline mex & per & sao & ven & & & \\
\hline RANGE: & $w r$ & & & & & herb \\
\hline $\begin{array}{l}\text { RANGE: } \\
\text { meX }\end{array}$ & bel & cag & gua & gui & hon & $\begin{array}{l}\text { epiph } \\
\text { herb }\end{array}$ \\
\hline RANGE: & $w r$ & & & & & herb \\
\hline RANGE: & gui & sao & ven & & & herb \\
\hline RANGE: & $\mathrm{cr}$ & nic & $x$ & & & $\begin{array}{l}\text { epiph } \\
\text { herb }\end{array}$ \\
\hline RANG & $w r$ & & & & & Wh \\
\hline RANG & $w r$ & & & & & Wh \\
\hline RANGE: & $\mathrm{cr}$ & nic & & & & epiph \\
\hline $\begin{array}{l}\text { RANGE: } \\
\text { RANGE: }\end{array}$ & $\begin{array}{l}\text { col } \\
\text { end }\end{array}$ & per & & & & $\begin{array}{l}\text { herb } \\
\text { epioh }\end{array}$ \\
\hline
\end{tabular}


*horichiana Fowlie quinquenervis R. \& P.

\section{Govenia \\ cililabia A. \& S.}

Il iacea Lindl.

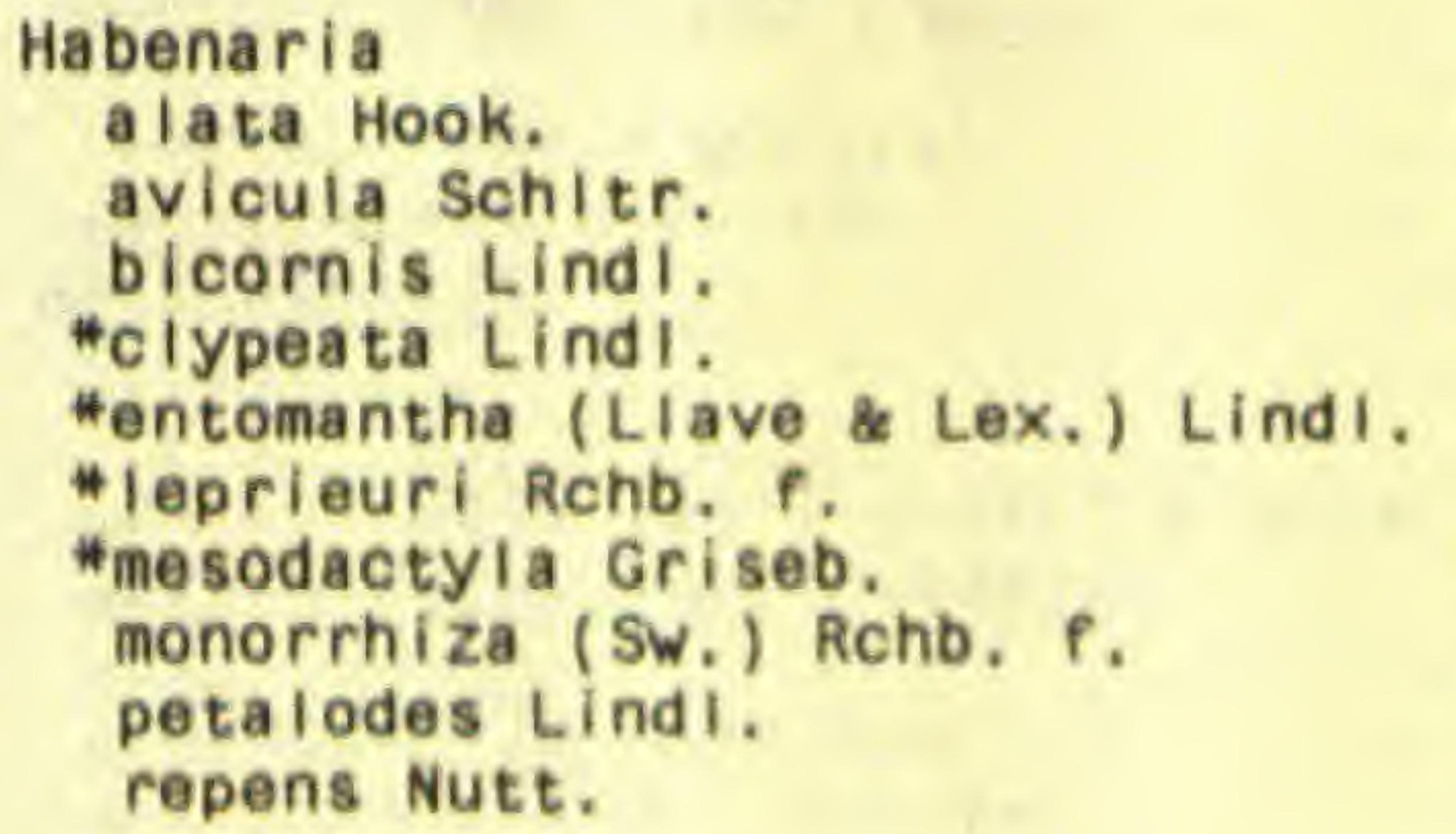

PROV: $v r \quad 000 \mathrm{~m}: 0-1$ PROV: bo ca cc cn pa 000m: $0-1$

PROV: ch

PROV: ch

000m: $1-2$

$000 \mathrm{~m}: 1-2,2-3$

PROV: ca ch

PROV: ca pa

PROV: ca pa

PROV: $\mathrm{ch}$

PROV: $\mathrm{ch}$

PROV: pa

PROV: $\mathrm{CC}$ ch

PROV: ca cc pa

PROV: ca

PROV: ch pa

PROV: ch

PROV: ch Vr PROV: ch pa Vr

PROV: ca

PROV: ch

PROV: oh

PROV: bo $\mathrm{CC}$

PROV: pa

PROV: Cc da pa

PROV: cc da pa

PROV: CC 000m: $0-1$

000m: $0-1$

000m: $0-1$

000m: 2-3

000m: $0-1$

000m: $0-1$

000m: $0-1$

000m: $0-1,1-2$

000m: $0-1$

000m: $0-1$

000m: $0-1$

000m: we

000m: 0-1

000a: $0-1,1-2$

000a: $0-1$

000m: we

000m: ve

000m: $0-1$

000m: $0-1$

000m: $0-1$

000m: $0-1$

000m: $0-1$
RANGE: $\mathrm{cr}$ sag? sao cag? ecu mex per epiph

RANGE: $\mathrm{cr}$ sal herb RANGE: ang bol cag or gua herb hon mex sal sao sao ven

RANGE: WdW herb

RANGE: end herb

RANGE: ang herb

RANGE: gua hon nic herb

RANGE: bel gua hon nic sal herb

RANCE: col gui sao ven herb

RANGE: bel herb

RANGE: ang cag gua sag herb

RANGE: col or sao herb

RANGE: ang bel or gua hon herb

nag nic sag

RANGE: cag mex sag sao herb

RANGE: gua hon nic nic sal herb

RANGE: $W r$ herb

RANGE: bel col or gui hon epiph

mex nic herb

RANGE: col or gua hon mex epiph

nic per ven herb

RANGE: $\mathrm{cr}$ herb

RANGE: gua hon nic sal herb

RANGE: $W r$

wh

RANGE: $\because r$

RANGE: nic

nerb

herb

RANGE: col cr sao epiph

RANGE: beI nerb 


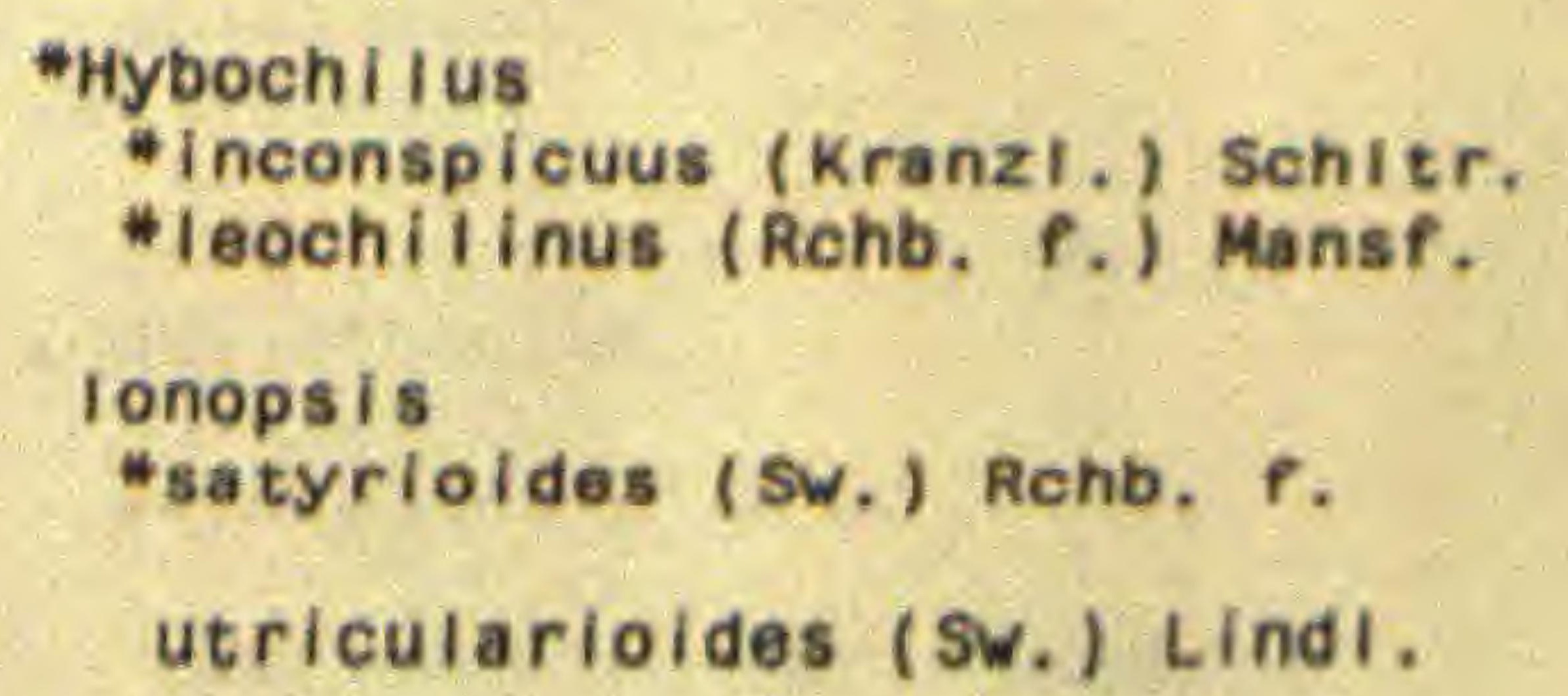

\section{PROV; ch}

PROV: ch

PROV: ca ch cn

PROV: ca da pa

PROV: ch

PROV: $v r$

PROV: ca ch

PROV: cc ch on pa

PROV: cn

PROV: ch

PROV: ch Vr

PROV: CC

PROV: cn

PROV: ch

PROV: ch

PROV: WP

PROV: da

PROV: $\mathrm{cC}$ ch

PROV: $C C$

PROV: $c n$

PROV: cc pa

PROV: ch 000m: $0-1$

000m: $0-1$

000m: $0-1$

000m: we

000m: ve

$000 \mathrm{~m}: 1-2$

000m: $0-1$ $000 \mathrm{~m}: 0-1,1-2$

000m: $0-1$

000m: $0-1$

000m: we

000m: $0-1,1-2$

000m: $0-1$

000m: $0-1$

000m: we

000m: we

000m: we

$000 \mathrm{~m}$ : we

000m: $0-1$

000m: $0-1$

000m: $0-1$

000m: we

\begin{tabular}{|c|c|c|c|c|c|c|}
\hline $\begin{array}{l}\text { RANGE; } \\
\text { RANGE: }\end{array}$ & $\begin{array}{l}w r \\
w r\end{array}$ & & & te & & $\begin{array}{l}\text { herb } \\
\text { herb }\end{array}$ \\
\hline $\begin{array}{c}\text { RANGE: } \\
\text { sat: }\end{array}$ & $\begin{array}{l}\text { ang } \\
\text { ven }\end{array}$ & bel & or & gua & hon & herb \\
\hline $\begin{array}{c}\text { RANGE: } \\
\text { sao }\end{array}$ & ang & cag & fla & $\operatorname{mex}$ & sag? & $\begin{array}{l}\text { epiph } \\
\text { herb }\end{array}$ \\
\hline r & -1 & & $z$ & 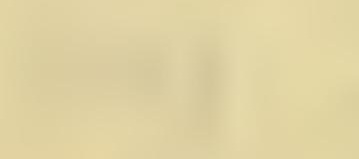 & in & $t=$ \\
\hline $\begin{array}{c}\text { RANGE: } \\
\text { nic }\end{array}$ & cr & gua & hon & jam & $\operatorname{mex}$ & $\begin{array}{l}\text { epiph } \\
\text { herb }\end{array}$ \\
\hline & & &. & to & 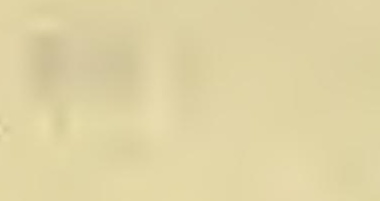 & $x^{1}$ \\
\hline RANGE: & $w r$ & & & & & herb \\
\hline RANGE: & bel & hon & $\operatorname{mex}$ & & & $\begin{array}{l}\text { epiph } \\
\text { herb }\end{array}$ \\
\hline RANGE: & gua & hon & nic & & & herb \\
\hline RANGE: & $w r$ & te & atear & $x=$ & & $\begin{array}{c}\text { epiph } \\
\text { herb } \\
\text { herb }\end{array}$ \\
\hline $\begin{array}{l}\text { RANGE: } \\
\text { RANGE: } \\
\text { ven }\end{array}$ & ang & gua & hon & $\operatorname{mex}$ & nic & $\begin{array}{l}\text { epiph } \\
\text { herb }\end{array}$ \\
\hline & $\sqrt{15}$ & & & & & \\
\hline RANGE: & $w r$ & & & & & epiphyt \\
\hline RANGE: & cr & nic & & & & $\begin{array}{l}\text { epiph } \\
\text { herb }\end{array}$ \\
\hline RANGE: & $w r$ & & & 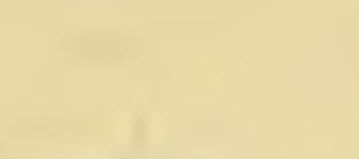 & & herb \\
\hline RANGE: & cr & & 1 & $-4 x^{4}$ & 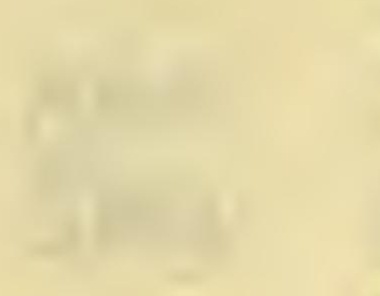 & $\begin{array}{l}\text { epiph } \\
\text { herb }\end{array}$ \\
\hline $\begin{array}{l}\text { RANGE: } \\
\text { RANGE: }\end{array}$ & $\begin{array}{l}w r \\
w r\end{array}$ & 20 & & & & Wh \\
\hline RANGE: & $w r$ & & & & & herb \\
\hline & -1 & & a & $x^{2}$ & $6 x^{1}$ & \\
\hline RANGE: & gui & jam & trt & & & $\begin{array}{l}\text { epiph } \\
\text { herb }\end{array}$ \\
\hline RANGE: & $\mathrm{cr}$ & & & & & $\begin{array}{l}\text { epiph } \\
\text { herb }\end{array}$ \\
\hline RANGE: & $\mathrm{col}$ & gui & sao & ven & & herb \\
\hline
\end{tabular}

RANGE: $w r$ 


\section{Laelía}

rubescens LindI.

Leochilus

labiatus (SW.) Kuntze

scriptus (Scheidw.) Rchb, f.

\section{Lepanthes \\ *brunnescens Luer \\ * ca loptera Luer \\ chiriquensis Schltr.}

\section{* coelog lossa Luer \\ *demissa Luer \\ eciliata Schltr.}

elata Rchb. f.

\section{*emp is Luer}

eximia Ames

*floripecten (Rchb, f.) Ames

*horrida Rchb, $f$.

* infund ibulum Luer

insectiflora C. Schweinf.

* I ind leyana Oerst. \& Rchb. f.

*maduroi Luer

*mariposa Luer

maxonii Schltr.

\section{*pachyglossa Luer \\ *prora Luer}

* psyche Luer

rotundifolia L.O. Wms.

* schizocardia Luer

* setos Luer

*trichidion Luer

turialvae Rchb. $f$

Leucohyle

warczewiczii KI.
PROV: ch

000m: we

PROV: $c c$

000m: $0-1$

PROV: ca pa vr

000m: $0-1$

PROV: pa

PROV: pa

PROV: $\mathrm{ch}$

PROV: ch

PROV: ch

PROV: ch

PROV: ch

PROV: ch

PROV: ch

PROV: WP

PROV: bo

PROV: ch

PROV: ch

PROV: WP

PROV: ch

PROV: ch

PROV: ch

PROV: pa

PROV: pa

PROV: ch

PROV: CC

PROV: pa

PROV: $c h$

PROV: ch

PROV: $\mathrm{cc} \mathrm{ch}$

PROV: WP

000m: $0-1$

000m: $0-1$

000m: $1-2$

000m: we

000m: we

000m: $1-2$

000m: we

000m: $1-2$

000m: we

000m: 0-1

000m: we

000m: 1-2

000m: we

000m: we

000m: we

000m: $2-3$

000m: $0-1$

000m: we

000m: we

000m: we

000m: we

$\begin{aligned} & \text { RANGE: cag or gua hon mex epiph } \\ & \text { nic sal }\end{aligned}$
RANGE: ang hon nic sal trt epiph
RANGE: or gua hon nic sal epiph

000m: 1-2

000m: $0-1$

000m: $0-1$

000m: $0-1$

000m: $0-1,1-2$ wh

RANGE: $w r$

RANGE: $w r$

RANGE: $\mathrm{cr}$

RANGE: $w r$

RANGE: $w r$

RANGE: end

RANGE: $c r$

RANGE: $w r$

RANGE: $c r$

RANGE: $w r$

RANGE: $w r$

RANGE: $w r$

RANGE: end

RANGE: $c r$

RANGE: $w r$

RANGE: $w r$

RANGE: end

RANGE: $w r$

RANGE: $w r$

RANGE: $w r$

RANGE: $\mathrm{cr}$ nic

RANGE: $w r$

RANGE: $w r$

RANGE: $w r$

RANGE: cag mex wh

epiph

wh

wh

epiph

herb

epiph

wh

epiph

herb

herb

epiphyte

wh

epiph

herb

herb

wh

herb

epiph

$w^{h e}$

herb

wh

epiph

herb

wh

wh

epiph

herb epiphyte 


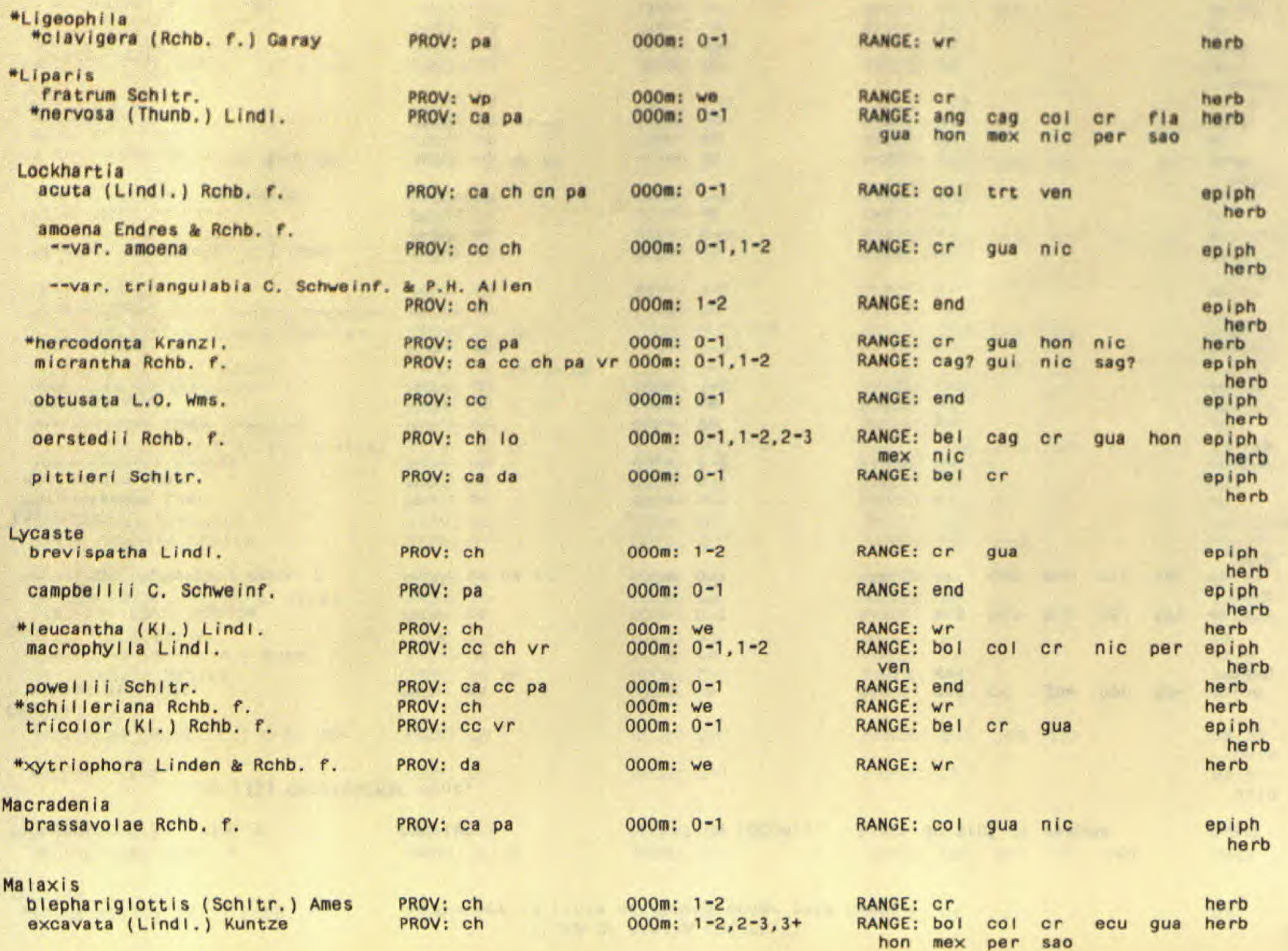


50 (33) ORCHIDACEAE cont.

fastigiata (Rchb. f.) Kuntze PROV: ch pa

*histionantha (Link, KI. \& Otto) Garay \& Dunsterv. PROV: bo ca ch

majanthemifolia Cham. \& Schlecht. PROV: ch pittieri (Schltr.) Ames

simillima (Rchb. f.) Kuntze PROV: ch

soulei L.0. Wms.

PROV: ch

tipuloides (Lindl.) Kuntze

PROV: ch pa

wendlandi i (Rchb. f.) L.O. Wms.

woodsonii L.O. Wms,

PROV: ch

PROV: ch

Masdeval I ia

attenuata Rchb. $f$.

chontalensis Rchb. $f$

collina L.O. Wms.

ecaudata Schltr.

\#erinacea Rchb, $p$.

* lata Rchb. f.

livingstoneana Rchb. $f$.

*molossoides Kranzl.

*nidifica Rchb. f.

*pelecaniceps Luer

*picturata Rchb. f.

*pleurothalloides Luer

*scabrilinguis Luer

*schizopetala Kranzl.

* tonduzii Woolw.

* zahlbruckneri kranzl.

PROV: $\mathrm{ch}$

PROV: $c c$ ch $v r$

PROV: $\mathrm{CC}$

PROV: ch

PROV: ch cn pa vr 000m: $0-1$

PROV: ch

PROV: ca cn pa

000m: we

000m: $0-1$

PROV: ch

PROV: ch

PROV: pa

PROV: ch

PROV: pa

PROV: ch

PROV: bo

PROV: bo

PROV: ch

PROV: pa

000m: $0-1,1-2$

000m: $0-1,1-2$

000m: $1-2,2-3,3+$

000m: $2-3$

000m: $1-2$

000m: $1-2,2-3,3+$

ooom: $0-1,1-2,2-3$

000m: $1-2$

000m: 1-2

000m: $1-2$

000m: $0-1,1-2$

000m: $0-1$

000m: $1-2$

000m: we

000m: we

000m: $0-1$

000m: we

000m: $0-1$

000m: we

000m: we

000m: we

000m: we

000m: $0-1$

Maxillaria

* adend robi um (Rchb. f.) Dressler

a lba (Hook.) Lind I.

PROV: pa

000m: $0-1$

allenii L.O. Wms.

PROV: cc pa

000m: $0-1,1-2$

angustisegmenta $A . \& S$.

PROV: ch

000m: $0-1,1-2$

000m: $1-2$

000m: $0-1$
PROV: cn

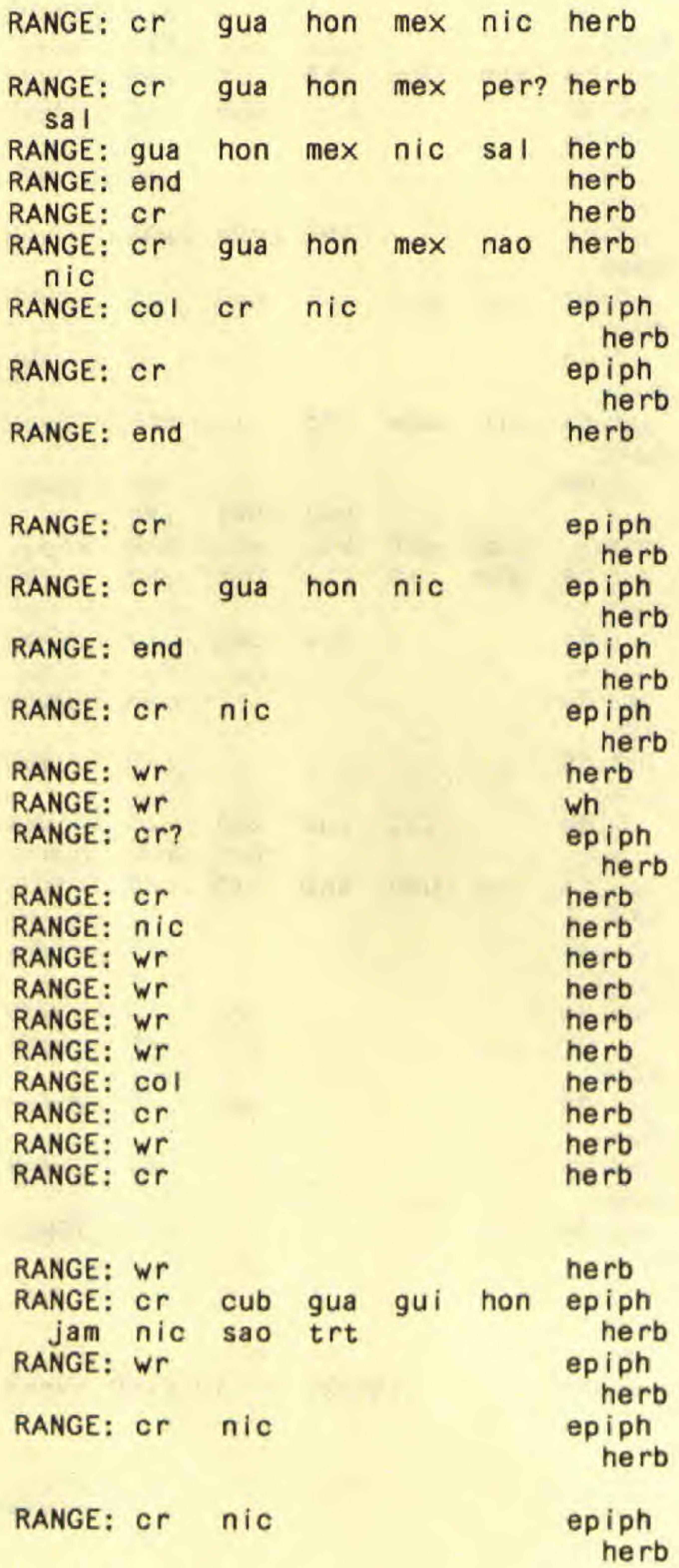


TAXON

50 (33) ORCHIDACEAE cont.

\section{a rachnitiflora $A$, \& $S$.}

biolleyi (Schltr.) L.O. Was.

brachybuibon schltr.

*bracteata (Schltr.) Ames corr. bradeorum (Schltr.) L.0. Wins.

brevipes schltr.

"brunnea Linden \& Rchb. $\mathrm{f}$.

camaridii Rchb. $P$.

chartacifolía A, \&.

conduplicata (A. \& S.) L.0. Wms.

confusa A. \& $\mathrm{S}$.

crassifolia (Lind), Rchb, f.

ctenostachya Rchb. $\mathrm{P}$.

cucullata LindI.

dendrobioides (Schltr.) L.O. Wms. PROV: bo ch

diuturna A. \& S.

exaltata (Kranzl.) C. Schweinf.

*falcata Ames \&e Corr.

\#flava Ames, Hubb. \& Schwe inf.

*foliosa A. \& S.

friedrichsthalii Rchb. $f$.

fulgens (Rchb. f.) L.0. Wms.

\section{*hedwigae Hamer \& Dodson}

*horichii Senghas

inaudita Rchb. $f$.

\section{*insolita Dressler}

* Inearifolia A, \& S.

longipetiolata A. \& S.

PROV: ch
PROV: $C C$

PROV: pa

PROV: bo

PROV: $\mathrm{CC}$ ch

PROV: $\mathrm{CC}$

PROV: ce ch da pa

PROV: ca ce ch pa

PROV: CC

PROV: ch

PROV: $\mathrm{CC}$ Vr

PROV: bo ca ch da pa 000m: $0-1,1-2$

PROV: ch

PROV: ch

000m: $1-2$

000m: $1-2$

000m: $0-1,1-2$

000m: $0-1$

000m: $0-1,1-2$

000m: we

000m: we

000m: $0-1$

PROV: ch

PROV: pa

PROV: ca cc ch da pa 000m: $0-1,1-2$

PROV: bo cc ch da pa $000 \mathrm{~m}$ : $0-1,1-2,3+$ sa $v r$

PROV: bo

PROV: ch

PROV: $v r$

PROV: pa

PROV: ch

PROV: pa 000m: we

000m: we

000m: $0-1$

000m: $0-1$

000m: we

000m: $0-1$
RANGE: $\mathrm{cr}$

RANGE: or

RANGE: or hon

RANGE: $w r$

RANGE: COI or

RANCE: or

RANGE: bel $\mathrm{cr}$ gua

ANGE: bel cr gua hon mex epiph
nic per sag?

RANGE: or gua gui trt

herb

herb

RANGE: $w r$

RANGE: or hon nic

RANGE: bel cag col cr cub
fla gua his hon jam mex

nic sal sao ven

RANGE: $\mathrm{cr}$

RANGE: cag or gua mex nic

RANGE: $\mathrm{cr}$

RANGE: $\mathrm{cr}$ nic

RANGE: col? ecu? per

RANGE: $c r$

RANGE: $W r$

RANGE: $\mathrm{cr}$

nic sag?

RANGE: nic

RANGE: $w r$

RANGE: $c r$

RANGE: $\mathrm{cr}$

RANGE: $w r$

RANGE: $c r$

RANGE: $w r$ epiph

epiph

herb

epiph

wh

epiph

nerb

iph

piph

piph

epiph

hero

epiph

herb

epiph

epiph

herb

epiph

herb

epiph

herb

epiph

herb

epiph

herb

herb

herb

epiphyte

epiph

herb

herb

wh

herb

epiph

herb

epiph

herb

herb

epiph 
TAXON

FAMILY

50 (33) ORCHIDACEAE COnt.

* Iueri Dodson

I uteo-a I ba LindI.

maleolens schitr.

minor (Schltr.) L.O. Wms.

"nasuta Rchb. f.

neglecta (Schltr,) L.O. Wms.

oreocharis schltr.

parvilabia A. \&e S.

pittieri (Ames) L.O. Wms.

planicola C. Schweinf.

powellii Schltr.

* reichenheimiana Rchb. $f$.

repens L. 0 . Wms.

rufescens Lindl.

*sanguinea Rolfe

* speciosa Rchb. f.

*splendens $P$. \& E E.

*tonduzii (Schltr.) Ames \& Corr.

umbratilis L. O. Wms.

uncata Lind I.

vagans A. \& S.

valenzuelana (A. Rich.) Nash

variabilis Lindl.

wercklei (schltr.) L.0. Wms.

Hwrightii (Schltr.) Ames \& Corr, PROV: cc

*Mendonce I la

*grandiflora (A. Rich.) A.D. Hawkes
PROV: $c$

PROV: cc pa

PROV: ca cc

PROV: $\mathrm{CC}$

PROV: ch da

PROV: ca ce ch cn lo 000m: $0-1,1-2,2-3$

PROV: ch

000m: $1-2$

PROV: ch

$000 \mathrm{~m}: 1-2$

PROV: ch

000m: $1-2$

PROV: ca cn pa

000m: $0-1$

PROV: ca cc pa

000m: $0-1,1-2$

PROV: $c h$

PROV: $\mathrm{CC}$

PROV: $c c$

000m: $0-1,1-2$

000m: $0-1$

PROV: bo

PROV: CC Vr

PROV: pa

PROV: $\mathrm{CC}$ ch

PROV: ch

000m: We

$000 \mathrm{m:} 0-1$

000m: $0-1,2-3$

0000m: $1-2$,

PROV: bo ca $\mathrm{cc}$ cn da 000m: 0-1, 1-2 pa $v r$

PROV: cc ch pa

000m: $0-1,1-2$

PROV: ch

000m: $1-2$

PROV: ca ce ch cn pa 000m: $0-1,1-2$

PROV: $\mathrm{CC}$

000m: $0-1$

000m: $0-1$

$000 \mathrm{~m}: \quad 0-1,2-3$

PROV: ch da
RANGE OUTSIDE OF PANAMA

HAB IT

\begin{tabular}{|c|c|c|c|c|c|c|}
\hline ANGE: & $w r$ & & & & & wh \\
\hline AANGE: & $\mathrm{col}$ & $\mathrm{cr}$ & ecu & & & $\begin{array}{l}\text { epiph } \\
\text { herb }\end{array}$ \\
\hline ANGE: & $c r$ & hon & nic & & & $\begin{array}{l}\text { epiph } \\
\text { herb }\end{array}$ \\
\hline ANGE: & $\mathrm{cr}$ & ws & & & & $\begin{array}{l}\text { epiph } \\
\text { herb }\end{array}$ \\
\hline RANGE: & hon & nic & & & & $\begin{array}{l}\text { epiph } \\
\text { herb }\end{array}$ \\
\hline $\begin{array}{c}\text { RANGE: } \\
\text { nic }\end{array}$ & bel & cag & $\mathrm{cr}$ & gua & hon & $\begin{array}{l}\text { epiph } \\
\text { herb }\end{array}$ \\
\hline RANGE: & $\mathrm{cr}$ & nic & & & & $\begin{array}{l}\text { epiph } \\
\text { herb }\end{array}$ \\
\hline RANGE: & $\mathrm{cr}$ & & 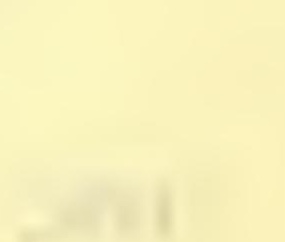 & & & $\begin{array}{l}\text { epiph } \\
\text { herb }\end{array}$ \\
\hline RANGE: & $\mathrm{cr}$ & & & & & $\begin{array}{l}\text { epiph } \\
\text { herb }\end{array}$ \\
\hline RANGE: & $w r$ & & & & & $\begin{array}{l}\text { epiph } \\
\text { herb }\end{array}$ \\
\hline RANGE: & end & & & & & $\begin{array}{l}\text { epiph } \\
\text { herb }\end{array}$ \\
\hline $\begin{array}{l}\text { RANGE: } \\
\text { RANGE: }\end{array}$ & $\begin{array}{l}\text { cr } \\
\text { end }\end{array}$ & ecu & nic & & & $\begin{array}{l}\text { herb } \\
\text { epiph }\end{array}$ \\
\hline RANGE: & cag & cr & cub & gua & aui & epiph \\
\hline $\mathrm{hi}$ & hon & jam & nic & per & 0 & herb \\
\hline $\begin{array}{c}\text { trt } \\
\text { RANGE: }\end{array}$ & cr & & & & & herb \\
\hline RANGE: & $w r$ & & & & & herb \\
\hline RANGE: & $w r$ & & & & & herb \\
\hline RANGE: & $\mathrm{cr}$ & & & & & herb \\
\hline RANGE: & $\mathrm{cr}$ & & & & & $\begin{array}{l}\text { epiph } \\
\text { herb }\end{array}$ \\
\hline RANC & bel & cag & $\mathrm{cr}$ & gua & gui & epiph \\
\hline hon & nic & per & sao & & & herb \\
\hline RANGE: & $\mathrm{cr}$ & hon & & & & $\begin{array}{l}\text { epiph } \\
\text { herb }\end{array}$ \\
\hline RANC & ang & cag & col & $\mathrm{cr}$ & cub & epiph \\
\hline 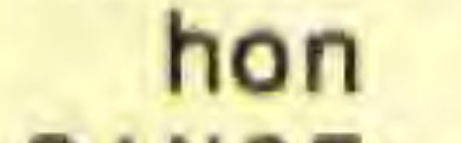 & nic & sao & sat & ven & & herb \\
\hline RANGE: & bel & cag & $\mathrm{cr}$ & gua & gui & epiph \\
\hline ho & mex & nic & sal & ven & & herb \\
\hline RANGE: & col & $\mathrm{cr}$ & & & & $\begin{array}{l}\text { epiph } \\
\text { herb }\end{array}$ \\
\hline ANC & $\mathrm{Cr}$ & nic & & & & herb \\
\hline
\end{tabular}




\section{Mesospinidium *endresii (Kranzl.) Garay}

"panamense Garay

\section{"Mil toniopsis}

*roezlif (Rchb. f,) Godefr, -Leb, PROV: ce ch da pa vr 000m: 0-1,1-2

warscewiczil (Rchb, f, ) Garay \& Dunsterv.

$$
\text { PROV: oh }
$$

$000 \mathrm{~m}: 1-2$

\section{Monophyliorchis \\ *maculata Garay \\ Mormodes \\ at ropurpureum Lind I. \\ *cartonil Hook. \\ colossus Rohb. f. \\ hookeri Lem. \\ igneum Lind I. \& Paxt. \\ * Iancilabris Pabst \\ powellit Schltr.}

\section{*Myoxanthus \\ *affinis (Lindl.) Luer \\ *hirsuticaulis (A \&.) Luer \\ *octomeriae (SchItr.) Luer}

"parahybunensis (Barb. Rodr.) Luer PROV: ch

* scandens (Ames) Luer

*speciosus (Luer) Luer

*trachychlamys (Schitr.) Luer

\section{Neomoorea}

irrorata Rolfe

* Neowill iamsia

* alfaroi (A. \& S.) Dressler

* cuneata Dressler
PROV: ca cc en vr 000m: 0-1

PROV: $\mathrm{ch}$

000m: $0-1$

PROV: pa

000m: $0-1$

PROV: ca cc

000m: $0-1$

PROV: WP

000m: we

PROV: ca ce ch pa $v r$ 000m: $0-1$

PROV: $\mathrm{CC}$

000m: $0-1$

PROV: ca ce cn 000m: $0-1$

$\begin{array}{ll}\text { PROV: Wp } & 000 \mathrm{~m}: \text { we } \\ \text { PROV: Vr } & 000 \mathrm{~m}: 0-1 \\ \text { PROV: ce ch } & 000 \mathrm{~m}: 0-1 \\ \text { PROV: ch } & 000 \mathrm{~m}: \text { we } \\ \text { PROV: cn pa } & 000 \mathrm{~m}: 0-1 \\ \text { PROV: ch } & 000 \mathrm{~m}: \text { we } \\ \text { PROV: ca da pa } & 000 \mathrm{~m}: 0-1\end{array}$

PROV: ca

000m: $0-1$

PROV: $\mathrm{ch}$

PROV: $v r$ 000m: we

000m: $0-1$
RANGE: $W r$

RANGE: $c r$

RANGE: $c r$

per

epiph herb

RANGE: $c r$

epiph

herb

epiph

herb

RANGE: $w r$

herb

RANGE: $c r$

RANGE: $w r$

RANGE: $c r$

RANGE: $\mathrm{cr}$

RANGE: $\mathrm{col} \mathrm{cr}$ hon

RANGE; end

RANGE: end

RANGE: $w r$

RANGE: $W r$

RANGE: $c r$

hon?

RANGE: $w r$

RANGE; $w r$

RANGE: end

RANGE: $c r$

per? ven

RANGE: $c O I$$$
\text { epiph }
$$

herb

RANGE: $c r$ RANGE: $w r$ herb

wh 
TAXON

FAMILY

PROVINCES

50 (33) ORCHIDACEAE cont.

ELEVATION (OOOM.)

RANGE OUTSIDE OF PANAMA
HABIT

\section{* tenuisulcata Dressler}

wercklei (Schltr.) Dressler

\section{Nidema}

boothil (Lind1.) Schltr.

ottonis (Rchb, f.) Britt. \& Millsp. 000m: $0-1$

000m: we

000m: $0-1$

000m: $0-1$

Notylia

*a Ibida KI.

barkeri LindI.

cordesii L.O. Wms.

*juncta Dressler

* Iankesteri Ames

latilabia A. \& S.

*I inearis A. \& S.

pentachne Rchb. $f$.

* ramonensis Schltr.

* simplex Dressier

*octomeria

*valeriol A. \& S.

Odontoglossum

\#bictoniense (Batem.) Lindl.

* krameri Rchb. $f$.

*--var. a Ibum (Halb.) Halb.

* maculata (Lindl,) LindI.

oerstedii Rchb. f.

\section{* Oerstede I la}

*caligaria (Rchb. f.) Hags.

centradenia Rchb. $P$.

centropetala (Rchb, f.) Rchb, f

*crescentiloba (Ames) Hags.

*endresii (Rchb. f) Hags.
PROV: $C C$

PROV: $\mathrm{ch}$

PROV: bo

PROV: bo pa

PROV: ca cn da

PROV: ca ch vr

PROV: bo

PROV: pa

PROV: WP

PROV: ca

PROV: cc da pa

PROV: ca da vr

PROV: $c h$

PROV: pa

PROV: ch

PROV: ch

PROV: ch pa

PROV: bo

PROV: $\mathrm{ca}$

PROV: ch

PROV: $v r$

PROV: ch

PROV: $\mathrm{ch}$

PROV: ch

PROV: ch 000m: $0-1$

000m: $0-1,1-2$

000m: $0-1$

000m: $0-1$

000m: we

000m: $0-1$

000m: $0-1,1-2$

000m: $0-1$

000m: we

000m: $0-1$

000m: we

000m: we

000m: $0-1$

000m: we

000m: $0-1$

000m: 2-3,3+

000m: $0-1$

000m: $1-2$

000m: $1-2$

000m: $2-3$

000m: $1-2$

RANGE: end
RANGE: cr
RANGE: ang cag cag mex sag? epiph
herb
$\begin{gathered}\text { RANGE: ang col hon nic per epiph } \\ \text { ven }\end{gathered}$

ven

RANGE: COI

epiph

RANGE: cag mex

herb

mex epiph

RANGE: $\mathrm{cr}$

RANGE: $W r$

RANGE: $c r$

RANGE: end

RANGE: $\mathrm{cr}$

RANGE: hon nic

RANGE: $\mathrm{cr}$

RANGE: $w r$

epiph

herb

herb

herb

epiph

herb

herb

epiph

herb

herb

herb

RANGE: nic

herb

RANGE: gua hon sal

RANGE: $\mathrm{n}$ ic

RANGE: $w r$

RANGE: gua

RANGE: or nic

herb

herb

ep iphyte

herb

epiph

RANGE: end

epiph

herb

RANGE: $c r$

RANGE: $c r$ gua hon

piph

epiph

herb

RANGE: $w r$

terr

RANGE: $\mathrm{cr}$ 
*exasperata (Rchb. f.) Hags.

*fuscina Dressler

*intermixta (A. \& S.) Hags.

* lactea (Dressier) Hags.

*ornata Dressler

*pajitense (C. Schweinf.) Hags.

"pansamalae (Schltr.) Hags.

* pinnifera (C. Schweinf.) Hags.

"pseudo-wallisif (Schltr.) Hags.

\#pumila (Rolfe) Hags.

* schumanniana (Schltr.) Hagsater

*tetraceros (Rchb, f.) Hags.

Hallisii (Rohb, f.) Hags.

Oncidium

*abortivum Rchb. $f$.

amp I i a tum Lindi.

ansiferum Rchb. $f$.

baueri Lindl.

bracteatum Warsc, \& Rchb. f.

cabagrae Schltr.

cariniferum (Rchb. f.) Beer

carthaginense (Jacq.) SW.

chei rophorum Rchb. $f$.

crista-ga III Rchb. f.

ensatum Lind I.

*exa l a tum Hags.

"fuscatum Rchb. f.

globuliferum H.B.K.

heteranthum P. \& E.

isthmi Schltr.

klotzschianum Rchb. $f$.

* Iuteum Rolfe

\begin{tabular}{|c|c|c|c|c|c|}
\hline PROV: & $\mathrm{cc}$ & $\mathrm{ch}$ & & 000m: & $0-1,2-$ \\
\hline PROV: & $\mathrm{cc}$ & & & 000m: & $0-1$ \\
\hline PROV: & ch & $v r$ & & 000m: & $0-1$ \\
\hline PROV: & $v r$ & & & 000m: & $\begin{array}{l}0=1 \\
0-2\end{array}$ \\
\hline PROV: & $\mathrm{ch}$ & & & 000m: & $2-3$ \\
\hline PROV: & cc & & & 000m: & $0-1$ \\
\hline PROV: & CC & & & 000m: & $0=1$ \\
\hline PROV: & $\mathrm{ch}$ & & & 000m: & we \\
\hline ROV: & pa & & & 000m: & $0-1$ \\
\hline $\begin{array}{l}\text { ROV: } \\
\text { ROV: }\end{array}$ & $\begin{array}{l}\mathrm{ch} \\
\mathrm{cc}\end{array}$ & & & $\begin{array}{l}\text { 000m: } \\
\text { 000m: }\end{array}$ & $\begin{array}{l}\text { We } \\
0-1\end{array}$ \\
\hline v. & & & & & \\
\hline PROV: & ch & & & 000m: & $1-2$ \\
\hline PROV: & $\mathrm{cc}$ & pa & & $000 \mathrm{~m}:$ & $0-1$ \\
\hline PROV: & ch & & & 000m: & $1-2$ \\
\hline PROV: & ca & cc pa & $v r$ & 000m: & $0-1$ \\
\hline PROV: & ch & & & 000m: & $1-2$ \\
\hline PROV: & ch & pa & & 000m: & $0-1$ \\
\hline PROV: & wp & & - & 000m: & we \\
\hline PROV: & $\mathrm{cc}$ & ch pa & & 000m: & $0-1,1-2$ \\
\hline PROV: & $\mathrm{ch}$ & & & 000m: & $1-2,2-3$ \\
\hline PROV: & $\mathrm{ca}$ & ch da & pa & 000m: & $0-1$ \\
\hline PROV: & $\mathrm{cc}$ & ch & & 000m: & $0-1,1-2$ \\
\hline PROV: & cc & & & 000m: & $0-1$ \\
\hline PROV: & pa & 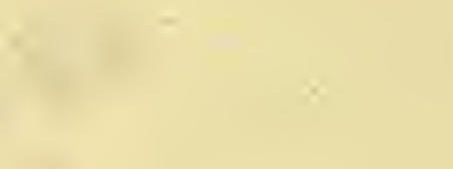 & & 000m: & $0-1$ \\
\hline PROV: & $v r$ & & & 000m: & $0-1$ \\
\hline PROV: & pa & & & 000m: & $0-1$ \\
\hline PROV: & $\mathrm{cc}$ & ch da & $p a v r$ & 000m: & $0-1,1-2$ \\
\hline PROV: & ch & & & 000m: & $1-2$ \\
\hline PROV: & ca & da pa & & 000m: & $0-1$ \\
\hline PROV: & $w p$ & & & 000m: & we \\
\hline OV: & & $v r$ & & & $0-1$ \\
\hline
\end{tabular}

\begin{tabular}{|c|c|c|c|c|c|c|}
\hline RANGE: & $\mathrm{cr}$ & & & & & $\begin{array}{l}\text { epiph } \\
\text { herb }\end{array}$ \\
\hline RANGE: & $w r$ & & & & & epiphyte \\
\hline RANGE: & $w r$ & 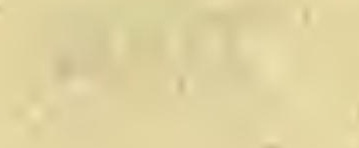 & 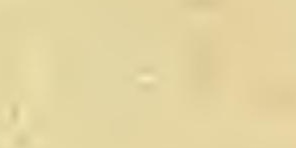 & & & herb \\
\hline RANGE: & $w r$ & & & 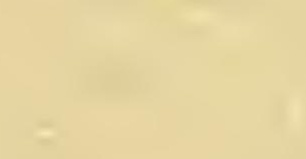 & & herb \\
\hline RANGE: & $w r$ & & & & & eplphyte \\
\hline RANGE: & $w r$ & & & & & herb \\
\hline RANGE: & $w r$ & $y+1$ & $s^{2}$ & & & herb \\
\hline RANGE: & $w r$ & $x_{1}$ & & & & herb \\
\hline RANGE: & $w r$ & & & & & epiphyte \\
\hline RANGE: & $w r$ & & & & & herb \\
\hline RANGE: & $\mathrm{cr}$ & & & & & $\begin{array}{l}\text { epiph } \\
\text { herb }\end{array}$ \\
\hline RANGE: & $\mathrm{cr}$ & & & & & $\begin{array}{l}\text { epiph } \\
\text { herb }\end{array}$ \\
\hline RANGE: & $w r$ & & & & & herb \\
\hline & wr & $=$ & & & & aninhyta \\
\hline $\begin{array}{l}\text { RANGE: } \\
\text { RANGE: }\end{array}$ & gua & hon & nic & per & trt & $\begin{array}{l}\text { epipnyte } \\
\text { epiph }\end{array}$ \\
\hline ven & & & & & & herb \\
\hline RANGE: & $\mathrm{cr}$ & gua & & & & $\begin{array}{l}\text { epiph } \\
\text { herb }\end{array}$ \\
\hline RANGE: & cag? & lag? & $\operatorname{mex}$ & per & sag? & epiph \\
\hline $\begin{array}{l}\text { Sao } \\
\text { RANGE: }\end{array}$ & $\mathrm{cr}$ & & & & & $\begin{array}{l}\text { herb } \\
\text { epiph } \\
\text { herb }\end{array}$ \\
\hline RANGE: & $\mathrm{cr}$ & & & & & $\begin{array}{l}\text { epiph } \\
\text { herb }\end{array}$ \\
\hline RANGE: & $\mathrm{cr}$ & ven & & & & $\begin{array}{l}\text { epiph } \\
\text { herb }\end{array}$ \\
\hline $\begin{array}{c}\text { RANGE: } \\
\text { ven }\end{array}$ & ang & cag & fla & mex & sao & $\begin{array}{l}\text { epiph } \\
\text { herb }\end{array}$ \\
\hline RANGE: & col & $\mathrm{cr}$ & nic & sal & 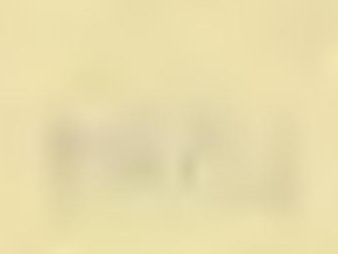 & $\begin{array}{l}\text { epiph } \\
\text { herb }\end{array}$ \\
\hline RANGE: & cag & $\mathrm{col}$ & $\operatorname{mex}$ & per & & $\begin{array}{l}\text { epiph } \\
\text { herb }\end{array}$ \\
\hline $\begin{array}{l}\text { RANGE: } \\
\text { RANGE: }\end{array}$ & $\begin{array}{l}\text { be I } \\
\text { end }\end{array}$ & cag? & & & & $\begin{array}{l}\text { herb } \\
\text { epiphyte }\end{array}$ \\
\hline RANGE: & $w r$ & & 4 & & & herb \\
\hline RANGE: & cr & per & ven & & & $\begin{array}{l}\text { epiph } \\
\text { herb }\end{array}$ \\
\hline RANGE: & bol & $\mathrm{col}$ & cr & per & & $\begin{array}{l}\text { epiph } \\
\text { herb }\end{array}$ \\
\hline RANGE: & $\mathrm{cr}$ & & & & & $\begin{array}{l}\text { epiph } \\
\text { herb }\end{array}$ \\
\hline RANGE: & gua & $\operatorname{mex}$ & & & & $\begin{array}{l}\text { epiph } \\
\text { herb }\end{array}$ \\
\hline RANGE: & $w r$ & & & & & herb \\
\hline
\end{tabular}


TAXON

50 (33) ORCHIDACEAE cont.

\section{*nudum Batem.}

obryza tum Rchb. f.

ochma tochil um Rchb. f.

*ornithorhynchum H.B.K.

panamense Schltr.

panduriforme A. \& S.

parviflorum L. 0 . Wms

powellif schltr.

* schroederianum Garay \& Stacey

stenotis Rchb. $f$.

stipitatum Lindl.

teres A. \& S.

warscewiczii Rchb. f.
PROV: da pa

PROV: $\mathrm{cc} \mathrm{ch}$

PROV: $\mathrm{cC}$ ch

PROV: $\mathrm{ch}$

PROV: ca pa

PROV: ch

PROV: cc pa

PROV: ca cc

PROV: ch

PROV: bo ch

PROV: ca ch cn pa

PROV: ch vr

PROV: ch

PROV: da

PROV: ca da pa vr

PROV: cc

PROV: $\mathrm{ch}$

PROV: ca pa

PROV: ch

osmoglossum

egertoni i (Lindl.) Schltr. *otog los sum

*chiriquense (Rchb. P.) Garay \& Dunsterv.

PROV: bo ch pa vr
$000 m: 1-2$

$000 \mathrm{~m}: 0-1,1-2$

000m: $0-1,1-2$

000m: $0-1,1-2$

$000 \mathrm{~m}: 1-2$

000m: $0-1$

000m: $1-2$

000m: $0-1$

000m: $0-1$

000m: we

000m: $0-1$

000m: $0-1$

000m: $0-1$

000m: $2-3$

000m: we

000m: $0-1$

000m: $0-1$

000m: 1-2

000m: $0-1$

$000 \mathrm{~m}: 0-1,1-2,2-3$
RANGE: $w r$

epiph

RANGE: col cr ecu gua per epiph

Sal

RANGE: col cr gua nic per epiph

RANGE: gua nic sal epiphyte

RANGE: nic epiph

RANGE: $\mathrm{cr}$ epiph

RANGE: $w r$ epiph

RANGE: nic epiph

epiph

RANGE: $w r$ herb

RANGE: $c r$ hon nic epiph

RANGE: bel hon nic epiph

RANGE, hon hic herb

epiph

RANGE: $\mathrm{cr}$ epiph

herb

RANGE: $w r \quad$ herb

RANGE: cag col cr ecu gua epiph

hon mex nic ven herb

RANGE: end epiph

RANGE: bel or gua hon mex epiph
nic

RANGE: end epiph

herb

RANGE: or gua hon mex nic epiph herb

RANGE: $\mathrm{COI}$ or per

epiph herb 
50 (33) ORCHIDACEAE cont.

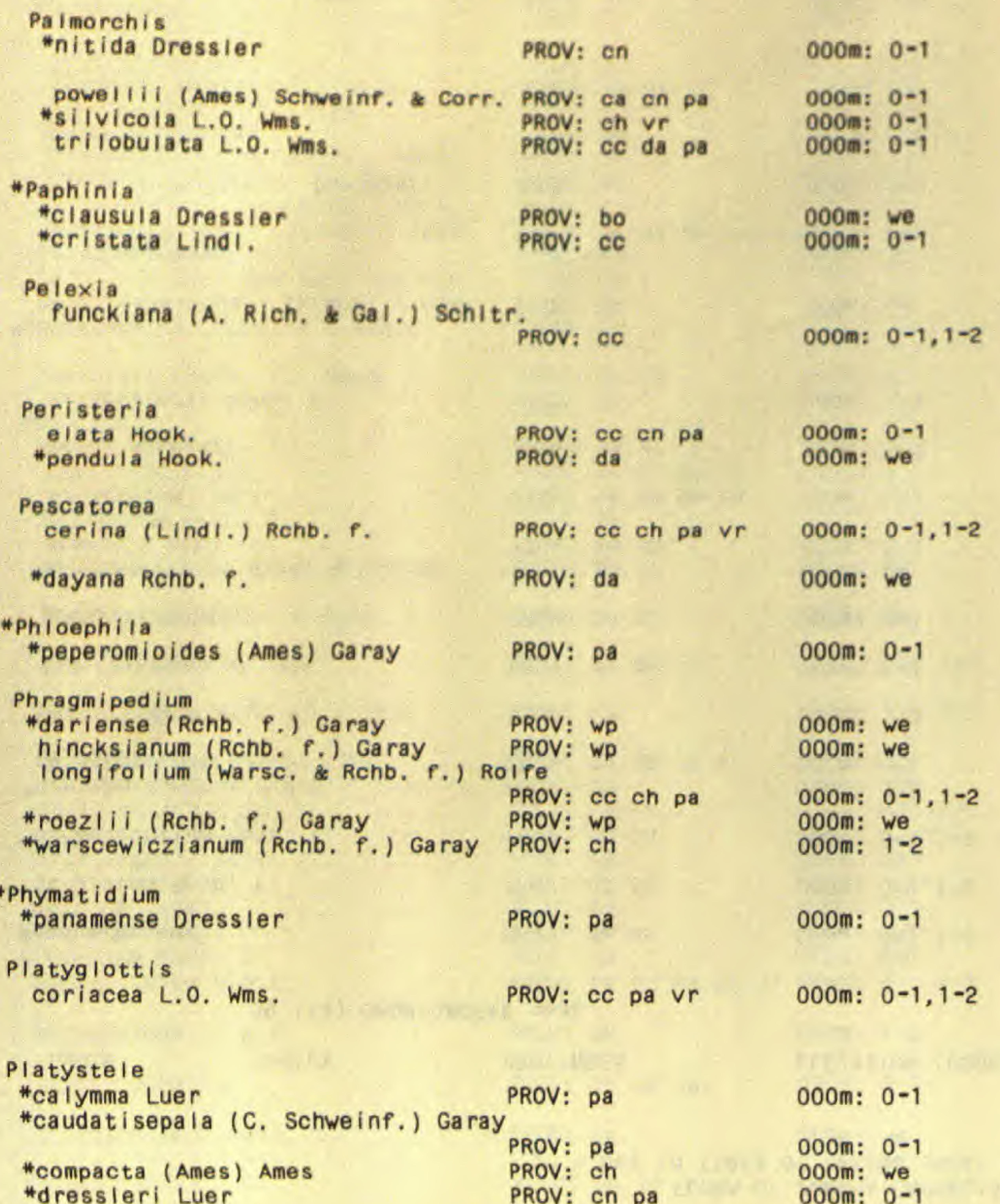

\begin{tabular}{|c|c|c|c|c|c|c|}
\hline RANGE: & end & & & & & $\begin{array}{c}\text { ep iph } \\
\text { herb }\end{array}$ \\
\hline RANGE: & end & & & & & herb \\
\hline $\begin{array}{l}\text { RANGE: } \\
\text { RANGE: }\end{array}$ & $\begin{array}{l}\text { nic } \\
\text { end }\end{array}$ & 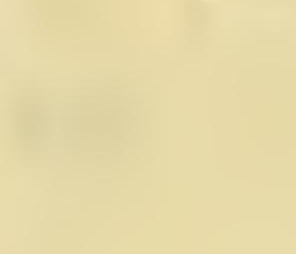 & & 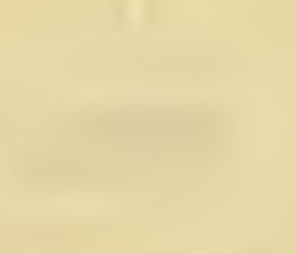 & & $\begin{array}{l}\text { herb } \\
\text { herb }\end{array}$ \\
\hline RANGE: & $w r$ & - & & & & herb \\
\hline RANGE: & $w r$ & 't & & it & 't & herb \\
\hline $\begin{array}{c}\text { RANGE: } \\
\text { nic: }\end{array}$ & $\begin{array}{l}\text { bel } \\
\text { sag? }\end{array}$ & cag & gua & hon & mex & herb \\
\hline RANGE: & col & cr & sal & ven & & herb \\
\hline RANGE: & $w r$ & & & & & herb \\
\hline RANGE: & or & & & & & epiph \\
\hline RANGE: & $w r$ & & & & & herb \\
\hline Dumer. & & & & & & \\
\hline RANGE: & $w r$ & & & & & epiphyte \\
\hline RANGE: & $w r$ & & 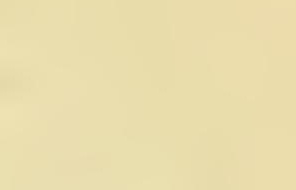 & & & herb \\
\hline RANGE: & $\mathrm{cr}$ & & & & & herb \\
\hline RANGE: & col & cr & 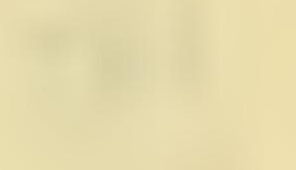 & & & herb \\
\hline RANGE: & $\mathrm{col}$ & $\mathrm{cr}$ & & & 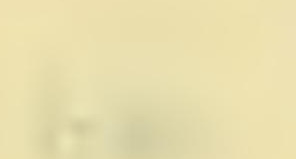 & herb \\
\hline RANGE: & gua & hon & r & & & herb \\
\hline & trat & trat & 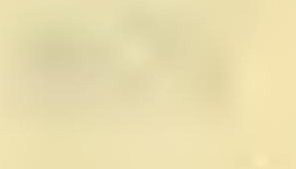 & 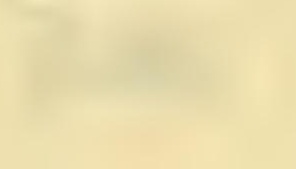 & nt & 19 \\
\hline RANGE: & $w r$ & r & & & & herb \\
\hline RANGE: & end & vate & & & & $\begin{array}{l}\text { epiph } \\
\text { herb }\end{array}$ \\
\hline RANGE: & $w r$ & & & & & herb \\
\hline $\begin{array}{l}\text { RANGE: } \\
\text { RANGE: } \\
\text { RANGE: }\end{array}$ & $\begin{array}{l}w r \\
b e l \\
w r\end{array}$ & gua & hon & nic & & $\begin{array}{l}\text { herb } \\
\text { herb } \\
\text { herb }\end{array}$ \\
\hline
\end{tabular}


50 (33) ORCHIDACEAE cont.

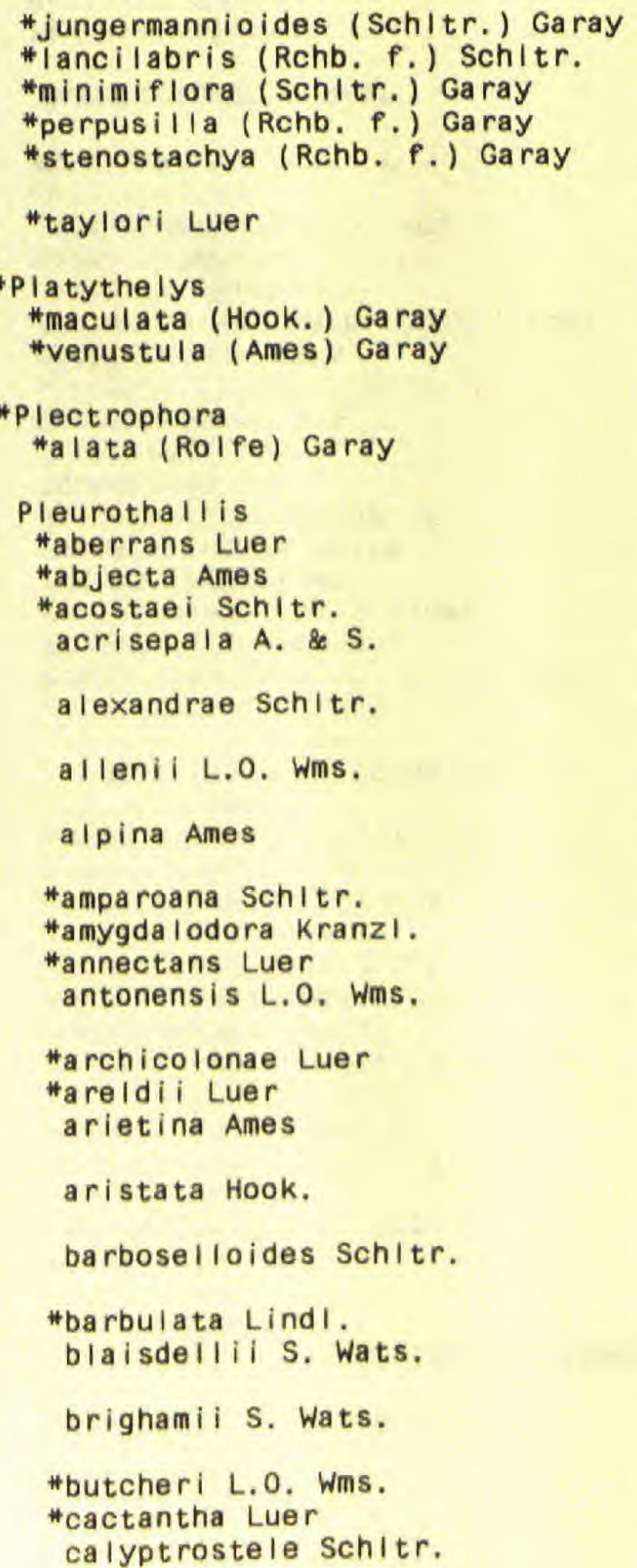

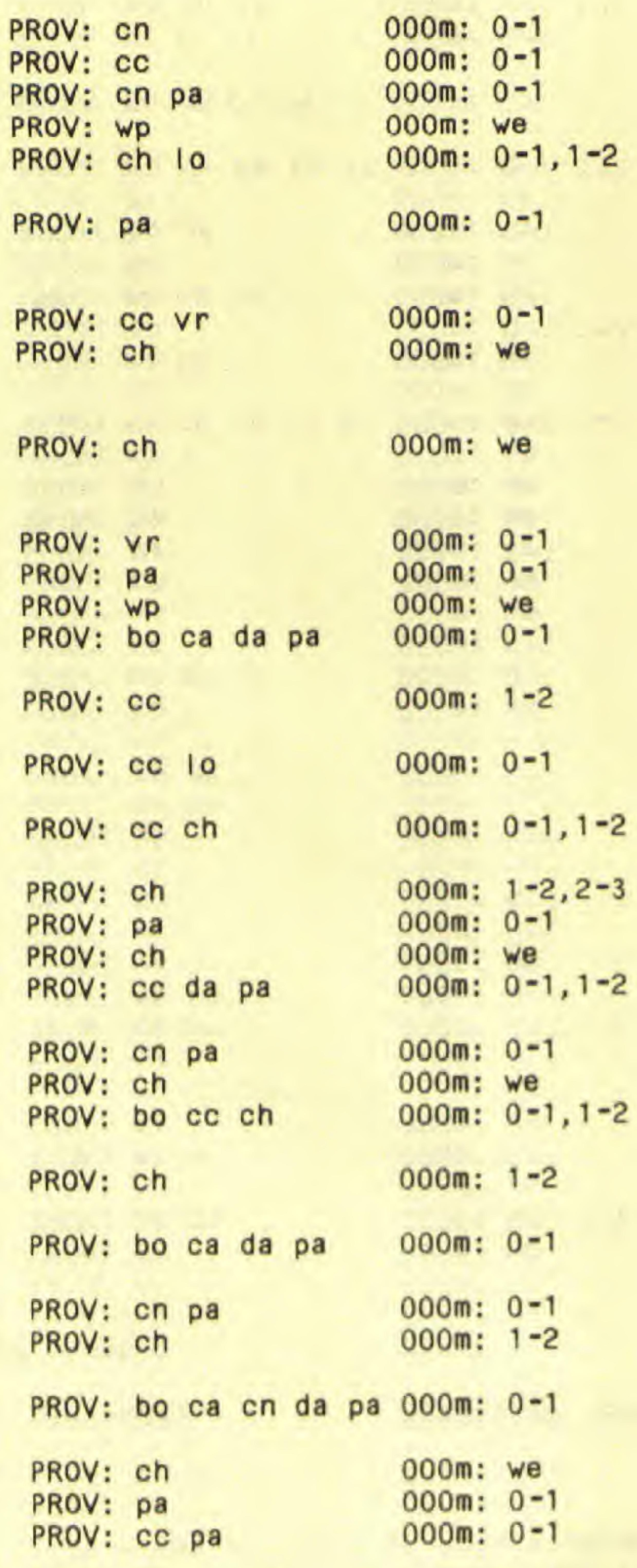

\begin{tabular}{|c|c|c|c|c|c|c|}
\hline RANGE: & gua & & & & & herb \\
\hline RANGE: & gua & hon & & & & $\begin{array}{l}\text { herb } \\
\text { herb }\end{array}$ \\
\hline $\begin{array}{l}\text { RANGE: } \\
\text { RANGF: }\end{array}$ & $\begin{array}{l}w r \\
w r\end{array}$ & & & & & $\begin{array}{l}\text { nerb } \\
\text { herb }\end{array}$ \\
\hline $\begin{array}{l}\text { RANGE: } \\
\text { RANGE: }\end{array}$ & $\begin{array}{l}\text { Wr } \\
\text { bel }\end{array}$ & cag & $\mathrm{cr}$ & gua & hon & epiph \\
\hline mex & nic & & & & & herb \\
\hline RANGE: & $w r$ & & & & & herb \\
\hline & 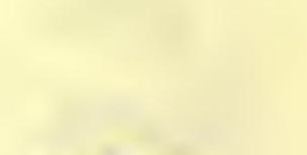 & & & & & \\
\hline $\begin{array}{l}\text { RANGE: } \\
\text { RANGE: }\end{array}$ & $w r$ & & & & & herb \\
\hline RANGE: & gua & hon & nic & & & herb \\
\hline RANGE: & gua & & & & & herb \\
\hline & $1=$ & & & & & \\
\hline RANGE: & end & & & & & herb \\
\hline RANGE: & gua & hon & nic & & & herb \\
\hline RANGE: & $\mathrm{cr}$ & 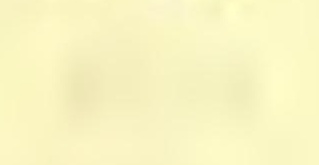 & & 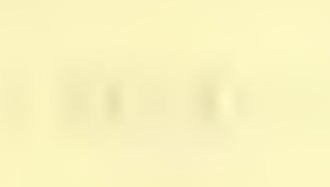 & & herb \\
\hline RANGE: & end & & & & & $\begin{array}{l}\text { epiph } \\
\text { herb }\end{array}$ \\
\hline RANGE: & $\mathrm{cr}$ & & & & & $\begin{array}{l}\text { epiph } \\
\text { herb }\end{array}$ \\
\hline RANGE: & hon & nic & & & & $\begin{array}{l}\text { epiph } \\
\text { herb }\end{array}$ \\
\hline RANGE: & $\mathrm{cr}$ & & & & & $\begin{array}{l}\text { epiph } \\
\text { herb }\end{array}$ \\
\hline RANGE: & cr & & & & & epiphyte \\
\hline RANGE: & per & & & & & herb \\
\hline RANGE: & end & & & & & herb \\
\hline RANGE: & end & & & & & $\begin{array}{l}\text { epiph } \\
\text { herb }\end{array}$ \\
\hline $\begin{array}{l}\text { RANGE: } \\
\text { RANGE: }\end{array}$ & $\begin{array}{l}\text { end } \\
\text { end }\end{array}$ & & & & & $\begin{array}{l}\text { herb } \\
\text { herb }\end{array}$ \\
\hline $\begin{array}{l}\text { RANGE: } \\
\text { RANGE: }\end{array}$ & $\begin{array}{l}\text { end } \\
\mathrm{cr}\end{array}$ & gua & & & & $\begin{array}{l}\text { epiph } \\
\text { herb }\end{array}$ \\
\hline RANGE: & ang & $\mathrm{cr}$ & & & & $\begin{array}{l}\text { epiph } \\
\text { herb }\end{array}$ \\
\hline RANGE: & end & & & & & $\begin{array}{l}\text { epiph } \\
\text { herb }\end{array}$ \\
\hline RANGE: & nic & & & & & herb \\
\hline $\begin{array}{c}\text { RANGE: } \\
\text { sa I }\end{array}$ & bel & $\mathrm{cr}$ & hon & $\operatorname{mex}$ & nic & $\begin{array}{l}\text { epiph } \\
\text { herb }\end{array}$ \\
\hline RANGE: & cag & gua & & & & $\begin{array}{l}\text { epiph } \\
\text { herb }\end{array}$ \\
\hline RANGE: & $w r$ & & & & & herb \\
\hline RANGE: & end & & & & & herb \\
\hline RANGE: & $\mathrm{cr}$ & & & & & $\begin{array}{l}\text { epiph } \\
\text { herb }\end{array}$ \\
\hline
\end{tabular}


* campicola Luer

cardiochila L.O. Wims.

"cardiothallis Rchb. P.

*carinilabia Luer

*carnosilabia Heller \& Hawkes

"carpinterae Schitr.

*cedralensis Ames

*ciliaris (LindI.) L.0. Wms.

*citrophila Luer

cobraeformis L.O. Wms.

cogniauxiana Schitr.

concaviflora C. Schweinf.

\section{* condy lata Luer}

* congruens Luer

* conval laria Schltr.

* corniculata (Sw.) LindI.

*corynophora Luer

* costaricensis Rolfe

* crocodiliceps Rchb. $f$.

"cucumeris Luer

*cuspidata Luer

*decipiens A. \& S.

*dentipeta la Ames

*deregularis (Barb. Rodr.) Luer

* dressleri Luer

*dura LindI.

el I ipsophyila L.O. Wms.

*endotrachys Rchb. $f$.

eumecocaulon SchItr.

*excavata Schitr.

* foliata Griseb.

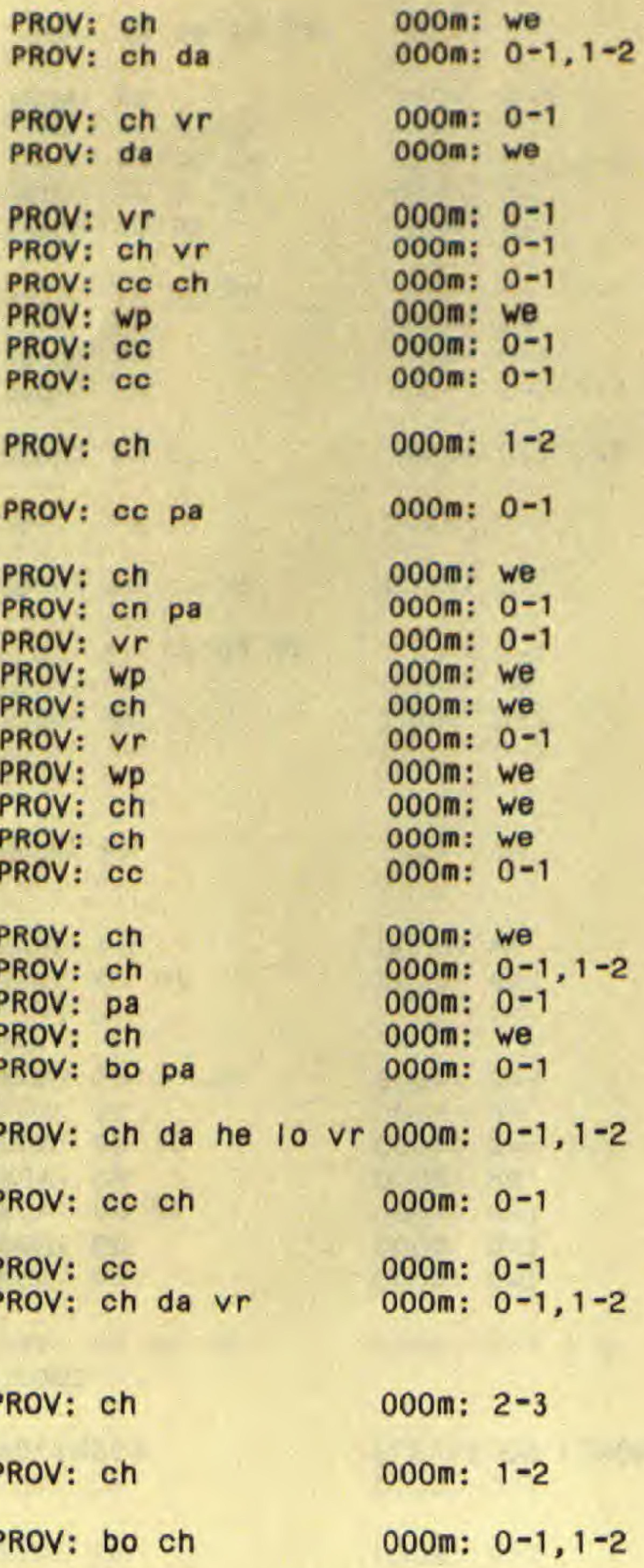

fuegii Rchb. $f$.

--var. echinata L. 0 . Wms.

fulgens Rchb. $f$.

PROV: bo $\mathrm{ch}$

000m: $0-1$

\begin{tabular}{|c|c|c|c|c|c|c|}
\hline $\begin{array}{l}\text { RANGE: } \\
\text { RANGE: }\end{array}$ & $\begin{array}{l}\text { end } \\
\text { gua }\end{array}$ & hon & nic & sal & & $\begin{array}{l}\text { herb } \\
\text { eplph } \\
\text { herb }\end{array}$ \\
\hline $\begin{array}{l}\text { RANGE: } \\
\text { RANGE: }\end{array}$ & $\begin{array}{l}\text { gua } \\
\text { end }\end{array}$ & hon & nic & sal & & $\begin{array}{l}\text { herb } \\
\text { epiph } \\
\text { herb }\end{array}$ \\
\hline RANGE: & nic & & & & & herb \\
\hline RANGE: & cr & & & & & herb \\
\hline RANGE: & cr & & & & & herb \\
\hline RANGE: & gua & hon & nic & & & herb \\
\hline RANGE: & end & & & & & herb \\
\hline RANGE: & end & & & & & $\begin{array}{l}\text { epiph } \\
\text { herb }\end{array}$ \\
\hline RANGE: & $\mathrm{cr}$ & nic & & & & $\begin{array}{l}\text { epiph } \\
\text { herb }\end{array}$ \\
\hline RANGE: & cr & & & & & $\begin{array}{l}\text { epiph } \\
\text { herb }\end{array}$ \\
\hline RANGE: & end & & & & & herb \\
\hline RANGE: & end & & & & & herb \\
\hline RANGE: & nic & & & & & herb \\
\hline RANGE: & $\mathrm{cr}$ & gua & hon & nic & & herb \\
\hline RANGE: & $w r$ & & & & & wh \\
\hline RANGE: & cr & & & & & herb \\
\hline RANGE: & $w r$ & & & & & herb \\
\hline RANGE: & end & & & & & herb \\
\hline RANGE: & end & & & & & herb \\
\hline RANGE: & end & & & & & $\begin{array}{l}\text { epiph } \\
\text { herb }\end{array}$ \\
\hline RANGE: & $\mathrm{cr}$ & & & & & herb \\
\hline RANGE: & cr & hon & nic & & & herb \\
\hline RANGE: & end & & & & & herb \\
\hline RANGE: & $w r$ & & & & & herb \\
\hline RANGE: & end & & & & & $\begin{array}{l}\text { epiph } \\
\text { herb }\end{array}$ \\
\hline $\begin{array}{c}\text { RANGE: } \\
\text { Sal }\end{array}$ & bel & cag & cr & hon & $\operatorname{mex}$ & $\begin{array}{l}\text { epiph } \\
\text { herb }\end{array}$ \\
\hline RANGE: & $\mathrm{cr}$ & 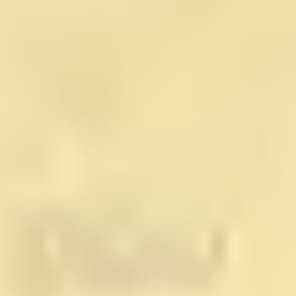 & & & & $\begin{array}{l}\text { epiph } \\
\text { herb }\end{array}$ \\
\hline RANGE: & $\mathrm{cr}$ & & & & & herb \\
\hline RANGE: & ang & $\mathrm{cr}$ & hon & mex & nic & $\begin{array}{l}\text { epiph } \\
\text { herb }\end{array}$ \\
\hline RANGE: & end & & & & & epiph \\
\hline RANGE: & $\mathrm{cr}$ & nic & & & & $\begin{array}{l}\text { epiph } \\
\text { herb }\end{array}$ \\
\hline $\begin{array}{l}\text { RANGE: } \\
\text { mex }\end{array}$ & $\begin{array}{l}\text { ang } \\
\text { nic }\end{array}$ & $\begin{array}{l}\text { bel } \\
\text { sag }\end{array}$ & cag & fla & hon & $\begin{array}{l}\text { epiph } \\
\text { herb }\end{array}$ \\
\hline RANGE: & C & & & & & epiph \\
\hline
\end{tabular}


50 (33) ORCHIDACEAE cont.

glandulosa Ames

grobyi Lind $\mathrm{l}$.

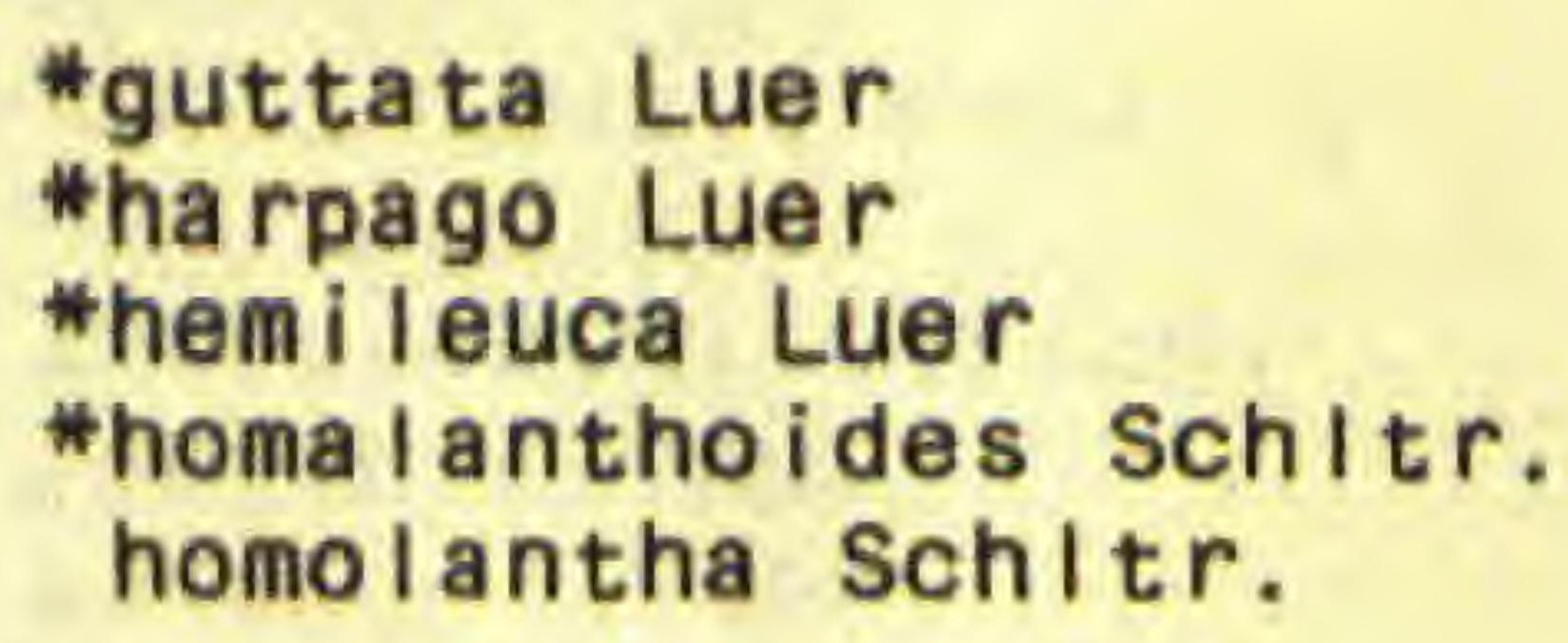

* gutta ta Luer

*harpago Luer

*hem i leuca Luer

*homa lanthoides Schltr.

homolantha Schltr.

* imago Luer

* immersa Linden \& Rchb. f.

*imraei Lind I.

* instar Luer

* intricata Lind I.

* isthmica Luer

* jaretiae Luer

*johnsonii Ames

* lappiformis Heller \& L.O. Wms.

* lateral is L.O. Wms.

lepidota L. 0 . Wms.

\section{\# lewisae Ames}

* longipedicellata A. \& S

* Ioranthophyl Ia Rchb. f.

* I uctuosa Rchb. f.

macrantha L.O. Wms.

* mammi I la ta Luer

*membraniflora C. Schweinf.

*memor Rchb. $f$.

*microphylla A. Rich, \& GaI.

*minutipetala $A$, \& $S$.

*monocardia Rchb. $f$

*moschata Rchb. $f$.

muricata Schltr.

*mystax Luer

*nitida Luer

*operculata Luer

*arbicularis (LindI.) LindI.

*oscitans Ames

ovatilabia A, \& S.

*pallida Luer
PROV: ca lo pa

000m: $0-1$

PROV: ca ch cn pa vr 000m: $0-1$

PROV: pa

PROV: da

PROV: ch

PROV: WP

PROV: cc ch sa vr

$0-1$

000m: we

000m: we

000m: we

PROV: $000 \mathrm{~m}: 0-1$

PROV: ca cc ch da pa $000 \mathrm{~m}: 0-1,1-2$

Vr

PROV: ch

PROV: pa

PROV: $\mathrm{ch}$

PROV: $c c$

PROV: cc cn pa

PROV: CC

PROV: ch

PROV: ca pa

PROV: $c h$

PROV: ch

PROV: ch

PROV: cn pa

PROV: WP

PROV: ch pa

PROV: WP

PROV: WP

PROV: WP

PROV: WP

PROV: CC Vr

PROV: $v r$

PROV: ch

PROV: CC

PROV: $C C$

PROV: cc pa

PROV: $\mathrm{ch}$

PROV: $\mathrm{CC}$ 000m: we

000m: $0-1$

000m: we

000m: $1-2$

000m: 0-1

000m: $0-1$

000m: $0-1$

000m: $1-2$

000m: 0-1

000m: we

000m: $0-1$

000m: we

000m: $1-2$

000m: $0-1$

000m: we

000m: $0-1,1-2,2-3$

000m: we

000m: we

000m: we

000m: we

000m: $0-1$

000m: 0-1

000m: we

000m: $0-1$

000m: $0-1$

000m: $0-1$

000m: 0-1

000m: $0-1$

\begin{tabular}{|c|c|c|c|c|c|c|}
\hline RANGE: & $\mathrm{cr}$ & gua & sa I & & & $\begin{array}{l}\text { herb } \\
\text { epiph } \\
\text { herb }\end{array}$ \\
\hline RANGE: & ang & cag & mex & nwt & sag & $\begin{array}{l}\text { epiph } \\
\text { herb }\end{array}$ \\
\hline RANGE: & end & & & & & herb \\
\hline RANGE: & end & & - & & & herb \\
\hline RANGE: & $w r$ & t & the & & & wh \\
\hline RANGE: & $\mathrm{cr}$ & & 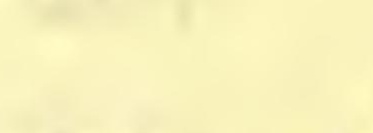 & & & herb \\
\hline $\begin{array}{c}\text { RANGE: } \\
\text { sa I }\end{array}$ & bel & cag & $\mathrm{cr}$ & hon & nic & $\begin{array}{l}\text { epiph } \\
\text { herb }\end{array}$ \\
\hline RANGE: & end & & & & & herb \\
\hline RANGE: & gua & hon & nic & sal & & herb \\
\hline RANGE: & $w r$ & & & & & herb \\
\hline RANGE: & end & & & & & herb \\
\hline RANGE: & $w r$ & & & & & herb \\
\hline RANGE: & end & & & & & herb \\
\hline RANGE: & end & & & & & $\begin{array}{l}\text { epiph } \\
\text { herb }\end{array}$ \\
\hline RANGE: & gua & hon & sal & 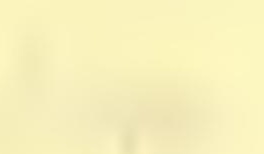 & & herb \\
\hline RANGE: & nic & & & & & herb \\
\hline RANGE: & $c r$ & & & & & epiphyte \\
\hline RANGE: & gua & & & & 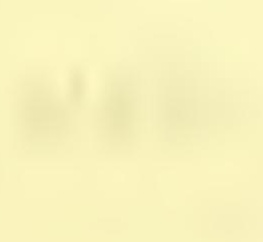 & $\begin{array}{l}\text { epiph } \\
\text { herb }\end{array}$ \\
\hline RANGE: & gua & hon & nic & & & herb \\
\hline RANGE: & $\mathrm{cr}$ & & & & & herb \\
\hline RANGE: & $w r$ & & & & & herb \\
\hline RANGE: & nic & & & & & herb \\
\hline RANGE: & end & & & & & herb \\
\hline RANGE: & end & & & & & herb \\
\hline RANGE: & $\mathrm{cr}$ & & & & & herb \\
\hline RANGE: & $\mathrm{cr}$ & nic & & & & $\begin{array}{l}\text { epiph } \\
\text { herb }\end{array}$ \\
\hline RANGE: & hon & nic & & & & herb \\
\hline RANGE: & $\mathrm{cr}$ & & & & & herb \\
\hline RANGE: & $w r$ & & & & & herb \\
\hline RANGE: & $w r$ & & & & & herb \\
\hline RANGE: & cag & $\operatorname{mex}$ & per? & & & $\begin{array}{l}\text { epiph } \\
\text { herb }\end{array}$ \\
\hline RANGE: & end & & & & & herb \\
\hline RANGE: & end & & & & & herb \\
\hline RANGE: & end & & & & & $\begin{array}{l}\text { epiph } \\
\text { herb }\end{array}$ \\
\hline RANGE: & $c r$ & nic & & & & $\begin{array}{l}\text { ep iph } \\
\text { herb }\end{array}$ \\
\hline RANGE: & hon & nic & & & & herb \\
\hline RANGE: & $\mathrm{cr}$ & hon & & & & $\begin{array}{l}\text { epiph } \\
\text { herb }\end{array}$ \\
\hline RANGE: & end & & & & & herb \\
\hline
\end{tabular}


50 (33) ORCHIDACEAE cont.

"pan Luer

* peculiaris Luer

* pergrata Ames

"phyil ocardia Rchb. f.

phyl locardioides Schitr. picta Lindl.

pittieri Schitr.

*platysepala Schltr.

"polygonoides Griseb.

*polysticha Luer

powellii Schltr.

\section{praegrandis Ames}

pruinosa Lindl.

"pubescens LindI.

racemiflora Lodd.

*ramonensis Schitr.

rhodoglossa Schltr.

rowleei Ames

* rubel lia Luer

ruscifolia (Jacq.) R. Br.

\section{*sanchoi Ames}

* scitula Luer

* sclerophyl Ia Lindi.

segoviensis Rchb. f.

*segregatifolia A. \& S.

* sempergemmata Luer

simulans L.O. Wms.

spectabilis A, \& S.

\section{*tantil la Luer}

*telamon Luer

*thymochils Luer

*titan Luer

*tonduzi i Schltr.

triangulabia $c$. Schweinf.

$\begin{array}{ll}\text { PROV: pa vr } & 000 m: 0-1 \\ \text { PROV: vr } & 000 m: 0-1 \\ \text { PROV: cc } & 000 m: 0-1 \\ \text { PROV: ch } & 000 m: 1-2,2-3 \\ \text { PROV: da } & 000 m: \text { we } \\ \text { PROV: ca ch ch pa vr } 000 m: 0-1\end{array}$

PROV: ca ch cn pa vr 000m: $0-1$

PROV: $\mathrm{CC} \mathrm{ch}$

000m: $0-1$

PROV: WP

000m: we

PROV: da 000m: we

PROV: ch pa

000m: $0-1,1-2$

PROV: da

000m: $1-2$

PROV: ch

000m: $1-2$

PROV: pa

PROV: ch pa $v r$

000m: $0-1$

$000 \mathrm{~m}: 0-1,1-2$

PROV: $\mathrm{cc} \mathrm{ch}$

000m: $0-1$

PROV: ch

000m: $1-2$

PROV: ch da

000m: $1-2$

PROV: ch

PROV: ce ch cn da pa 000m: $0-1,1-2$

PROV: $\mathrm{ch}$

PROV: ch

PROV: ch

000m: we

000m: we

000m: $2-3,3+$

PROV: cc ch da $v r \quad 000 m: 0-1,1-2$

PROV: WP

PROV: ch

PROV: CC

000m: we

000m: we

000m: $0-1$

PROV: $v r$

000m: 0-1

PROV: cc pa

PROV: $C C$

PROV: pa

PROV: ch

PROV: ch

PROV: $\mathrm{ch}$ 000m: 0-1

000m: $0-1$

000m: $0-1$

000m: we

000m: we

000m: $1-2,2-3$

\begin{tabular}{|c|c|c|c|c|c|c|}
\hline RANGE: & end & & & & & herb \\
\hline RANGE: & end & & & & & herb \\
\hline RANGE: & $\mathrm{cr}$ & & 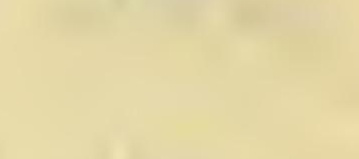 & & & herb \\
\hline RANGE: & $\mathrm{er}$ & & & & & epiphyte \\
\hline $\begin{array}{l}\text { RANGE: } \\
\text { RANGE: }\end{array}$ & $\begin{array}{l}\text { hon } \\
\text { wr }\end{array}$ & nic & & & & herb \\
\hline & & $\theta$ & & & & herb \\
\hline RANGE: & $w r$ & 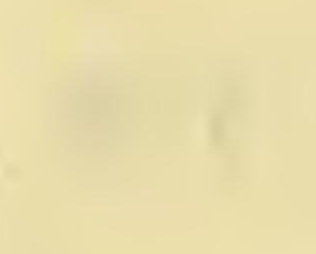 & & & & $\begin{array}{l}\text { epiph } \\
\text { herb }\end{array}$ \\
\hline RANGE: & $\mathrm{COl}$ & & . & & & herb \\
\hline RANGE: & $w r$ & & & & & herb \\
\hline RANGE: & end & & & & & herb \\
\hline RANGE: & $c r$ ? & & 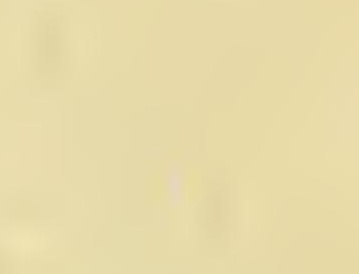 & & & $\begin{array}{l}\text { epiph } \\
\text { herb }\end{array}$ \\
\hline RANGE: & end & & & & & $\begin{array}{l}\text { epiph } \\
\text { herb }\end{array}$ \\
\hline $\begin{array}{l}\text { RANGE: } \\
\text { per? }\end{array}$ & ang & $\mathrm{cr}$ & gui & hon & nic & $\begin{array}{l}\text { epiph } \\
\text { herb }\end{array}$ \\
\hline RANGE: & hon & nic & & & & epiphyte \\
\hline $\begin{array}{l}\text { RANGE: } \\
\text { mex }\end{array}$ & ang & bel & cr & gua & hon & $\begin{array}{l}\text { epiph } \\
\text { herb }\end{array}$ \\
\hline RANGE: & $\mathrm{cr}$ & & & & & $\begin{array}{l}\text { epiph } \\
\text { herb }\end{array}$ \\
\hline RANGE: & end & & & & & $\begin{array}{l}\text { epiph } \\
\text { herb }\end{array}$ \\
\hline RANGE: & $\mathrm{cr}$ & & & & & $\begin{array}{l}\text { epiph } \\
\text { herb }\end{array}$ \\
\hline RANGE: & end & & & & & herb \\
\hline RANGE: & ang & $\mathrm{cr}$ & gua? & sag & sal? & $\begin{array}{l}\text { epiph } \\
\text { herb }\end{array}$ \\
\hline $\begin{array}{l}\text { RANGE: } \\
\text { RANGE: }\end{array}$ & $\begin{array}{l}\text { cr } \\
\text { end }\end{array}$ & & & & What & $\begin{array}{l}\text { herb } \\
\text { herb }\end{array}$ \\
\hline RANGE: & $\mathrm{cr}$ & gua & mex & & & $\begin{array}{l}\text { epiph } \\
\text { herb }\end{array}$ \\
\hline RANGE: & $\mathrm{cr}$ & gua & hon & $\operatorname{mex}$ & nic & $\begin{array}{l}\text { epiph } \\
\text { herb }\end{array}$ \\
\hline $\begin{array}{l}\text { RANGE: } \\
\text { RANGE: }\end{array}$ & $\begin{array}{l}\text { hon } \\
\text { end }\end{array}$ & nic & sal & $3 x+1$ & tes & $\begin{array}{l}\text { herb } \\
\text { herb }\end{array}$ \\
\hline RANGE: & end & whing & - & che & & $\begin{array}{l}\text { epiph } \\
\text { herb }\end{array}$ \\
\hline RANGE: & $\mathrm{cr}$ & & & & & $\begin{array}{l}\text { epiph } \\
\text { herb }\end{array}$ \\
\hline RANGE: & end & & & & & herb \\
\hline RANGE: & end & & & & & herb \\
\hline RANGE : & end & & 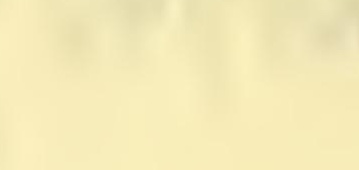 & $\cdots$ & & herb \\
\hline RANGE: & end & & & & & herb \\
\hline RANGE: & $\mathrm{cr}$ & 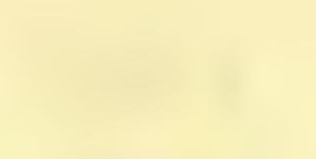 & 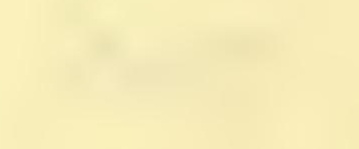 & - & 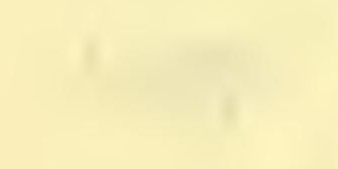 & herb \\
\hline RANGE: & $c r$ & 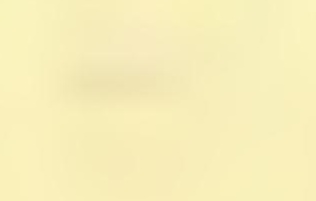 & & & & $\begin{array}{l}\text { epiph } \\
\text { herb }\end{array}$ \\
\hline
\end{tabular}


50 (33) ORCHIDACEAE cont.

tribuloides (SW.) LindI.

\#tripterantha Rchb. $f$.

*tropida Luer

tuerckheimi schltr.

uncinata Fawc.

*vaginata Schltr.

velaticaulis Rchb. f.

*veraguacensis Luer

verecunda Schltr.

vittata Lind I.

*volcanica Luer

Polycycnis

barbata (Lindl.) Rchb. $f$.

gratiosa Endres \& Rchb. f.

* Iehmanni i Rolfe

*musicfera (Lindl. \& Paxt.) Rchb. $f$

\section{*ornata Garay}

* tortuosa Dressler

Polys tachya

\# fol iosa (Hook.) Rchb. f.

masayens is Rchb. $f$.

Ponthieva

brenesii schltr.

ephippium Rchb. f.

maculata Lindl.

racemosa (Walt.) Mohr

Prescottia

oligantha (SW.) LindI.

stachyodes (SW.) LindI.
PROV: ch

PROV: ch

PROV: $\mathrm{CC}$

PROV: ch pa

PROV: $\mathrm{cc} \mathrm{ch}$

PROV: ch

PROV: $\mathrm{cc} \mathrm{ch}$

PROV: vr

PROV: ca cn

PROV: cC

PROV: ch

PROV: $C C$

PROV: cc pa

PROV: da

PROV: ch

PROV: da

PROV: CC

PROV: ca cc ch pa

PROV: ca cc ch cn

PROV: WP

PROV: $\mathrm{ch}$

PROV: ch

PROV: cc ch pa

PROV: cc ch

PROV: ca cc 000m: $1-2$

000m: 1-2

000m: $0-1,1-2$

000m: $0-1,1-2$

$000 \mathrm{~m}: 0-1,1-2$

000m: $0-1$

$000 \mathrm{~m}: 0-1,1-2,2-3$

000m: $0-1$

000m: $0-1$

000m: $0-1$

000m: we

000m: $0-1,1-2$

000m: $0-1$

000m: we

000m: we

000m: we

000m: $0-1$

$000 \mathrm{~m}: 0-1,1-2$

000m: $0-1$

000m: we

000m: $1-2$

000m: $2-3$

000m: $0-1,3+$

000m: $0-1$

000m: $0-1$

\begin{tabular}{|c|c|c|c|c|c|c|}
\hline $\begin{array}{c}\text { RANGE: } \\
\text { hon }\end{array}$ & $\begin{array}{l}\text { ang } \\
\text { mex }\end{array}$ & bel & cag & $\mathrm{cr}$ & gua & $\begin{array}{l}\text { epiph } \\
\text { herb }\end{array}$ \\
\hline RANGE: & $\mathrm{cr}$ & $\operatorname{mex}$ & & trat & & herb \\
\hline RANGE: & end & & & & & $\begin{array}{l}\text { epiph } \\
\text { herb }\end{array}$ \\
\hline RANGE: & cag & $\mathrm{cr}$ & gua & $\operatorname{mex}$ & & $\begin{array}{l}\text { epiph } \\
\text { herb }\end{array}$ \\
\hline RANGE: & ang & $\mathrm{cr}$ & nic & & & $\begin{array}{l}\text { epiph } \\
\text { herb }\end{array}$ \\
\hline RANGE: & $\mathrm{cr}$ & & & & & herb \\
\hline RANGE: & ang & $\mathrm{cr}$ & per & sag? & ven & $\begin{array}{l}\text { epiph } \\
\text { herb }\end{array}$ \\
\hline RANGE: & end & & & & & herb \\
\hline RANGE: & $\mathrm{cr}$ & & & & & $\begin{array}{l}\text { epiph } \\
\text { herb }\end{array}$ \\
\hline RANGE: & $\mathrm{cr}$ & gua & hon & $\operatorname{mex}$ & ven? & $\begin{array}{l}\text { epiph } \\
\text { herb }\end{array}$ \\
\hline RANGE: & end & & & & & herb \\
\hline RANGE: & col & cr & sao & ven & & $\begin{array}{l}\text { epiph } \\
\text { herb }\end{array}$ \\
\hline RANGE: & $w r$ & & & & & $\begin{array}{l}\text { epiph } \\
\text { herb }\end{array}$ \\
\hline RANGE: & $w r$ & & & & & herb \\
\hline RANGE: & $w r$ & & & & & herb \\
\hline RANGE: & ecu & & & & & herb \\
\hline RANGE: & $w r$ & & & & & nero \\
\hline RANGE: & ang & cag & $\operatorname{mex}$ & sag? & & $\begin{array}{l}\text { epiph } \\
\text { herb }\end{array}$ \\
\hline $\begin{array}{l}\text { RANGE: } \\
\text { mex }\end{array}$ & $\begin{array}{l}\text { bel } \\
\text { nic }\end{array}$ & cag & or & gua & hon & $\begin{array}{l}\text { epiph } \\
\text { herb }\end{array}$ \\
\hline RANGE: & $\mathrm{cr}$ & & & & & herb \\
\hline RANGE: & gua & hon & $\operatorname{mex}$ & & & herb \\
\hline $\begin{array}{c}\text { RANGE: } \\
\text { sa I }\end{array}$ & $\begin{array}{l}\text { col } \\
\text { ven }\end{array}$ & $\mathrm{cr}$ & ecu & gua & $\operatorname{mex}$ & herb \\
\hline RANGE: & ang & $\mathrm{cr}$ & fla & gua & hon & herb \\
\hline mex & nag? & nao & nao & per & sag? & \\
\hline $\begin{array}{l}\text { RANGE: } \\
\text { mex }\end{array}$ & $\begin{array}{l}\text { ang } \\
\text { ven }\end{array}$ & $\mathrm{col}$ & $\mathrm{cr}$ & fla & gua & herb \\
\hline $\begin{array}{c}\text { RANGE: } \\
\text { hon }\end{array}$ & $\begin{array}{l}\text { ang } \\
\text { mex }\end{array}$ & $\begin{array}{l}\text { bel } \\
\text { sao }\end{array}$ & cag & $\mathrm{col}$ & gua & herb \\
\hline
\end{tabular}




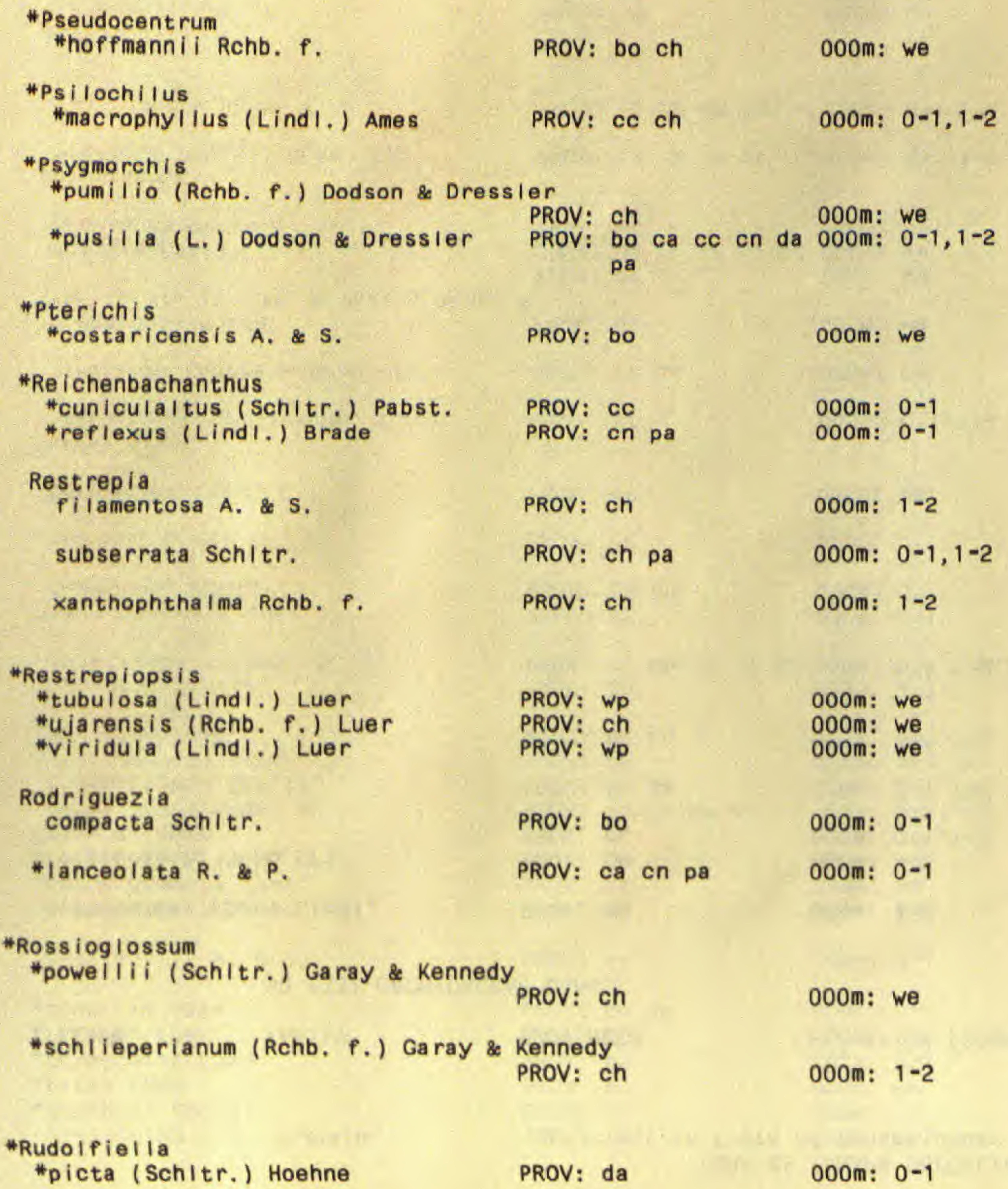

\begin{tabular}{|c|c|c|c|c|c|c|}
\hline RANGE: & $w r$ & & & & & wh \\
\hline RANGE: & gua & nic & & & & herb \\
\hline & . & y & $-4 x^{2}$ & -1 & & +1 \\
\hline RANGE: & hon & nic & & & & herb \\
\hline $\begin{array}{c}\text { RANGE: } \\
\text { trt }\end{array}$ & bol & cag & $\operatorname{mex}$ & sag? & sao & $\begin{array}{l}\text { epiph } \\
\text { herb }\end{array}$ \\
\hline RANGE: & $\mathrm{cr}$ & & & & & herb \\
\hline RANGE: & $w r$ & & & & & \\
\hline RANGE: & or & & & & & herb \\
\hline RANGE: & $w r$ & & & & & epiph \\
\hline RANGE: & $\mathrm{cr}$ & & & $-x^{2}$ & & \\
\hline RANGE: & $\mathrm{cr}$ & gua & hon & $\operatorname{mex}$ & nic & $\begin{array}{l}\text { epiph } \\
\text { herb }\end{array}$ \\
\hline RANGE: & $w r$ & & 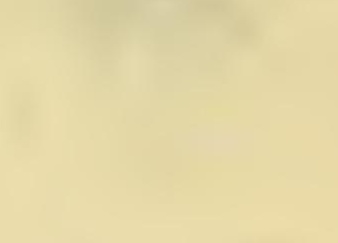 & & & herb \\
\hline RANGE: & gua & & 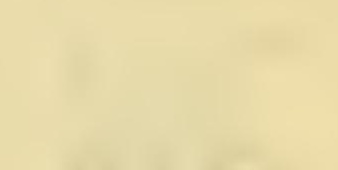 & & & herb \\
\hline RANGE: & $w r$ & & ntre & & & herb \\
\hline & ry & o & 11/F & $x+x$ & & \\
\hline RANGE: & $\mathrm{cr}$ & nic & & & & $\begin{array}{l}\text { epiph } \\
\text { herb }\end{array}$ \\
\hline RANGE: & $\mathrm{col}$ & gui & trt & ven & & $\begin{array}{l}\text { epiph } \\
\text { herb }\end{array}$ \\
\hline$x^{-}$ & and & $a s$ & tha & 16 & nit & diero \\
\hline RANGE: & $w r$ & 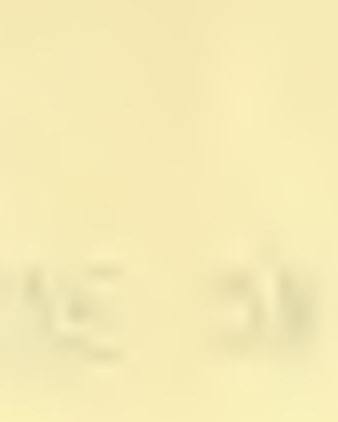 & bent & & & $\begin{array}{l}\text { epiph } \\
\text { herb }\end{array}$ \\
\hline RANGE: & $\mathrm{cr}$ & & & & & $\begin{array}{l}\text { epiph } \\
\text { herb }\end{array}$ \\
\hline RANGE: & col & & & & & $\begin{array}{l}\text { epiph } \\
\text { herb }\end{array}$ \\
\hline
\end{tabular}




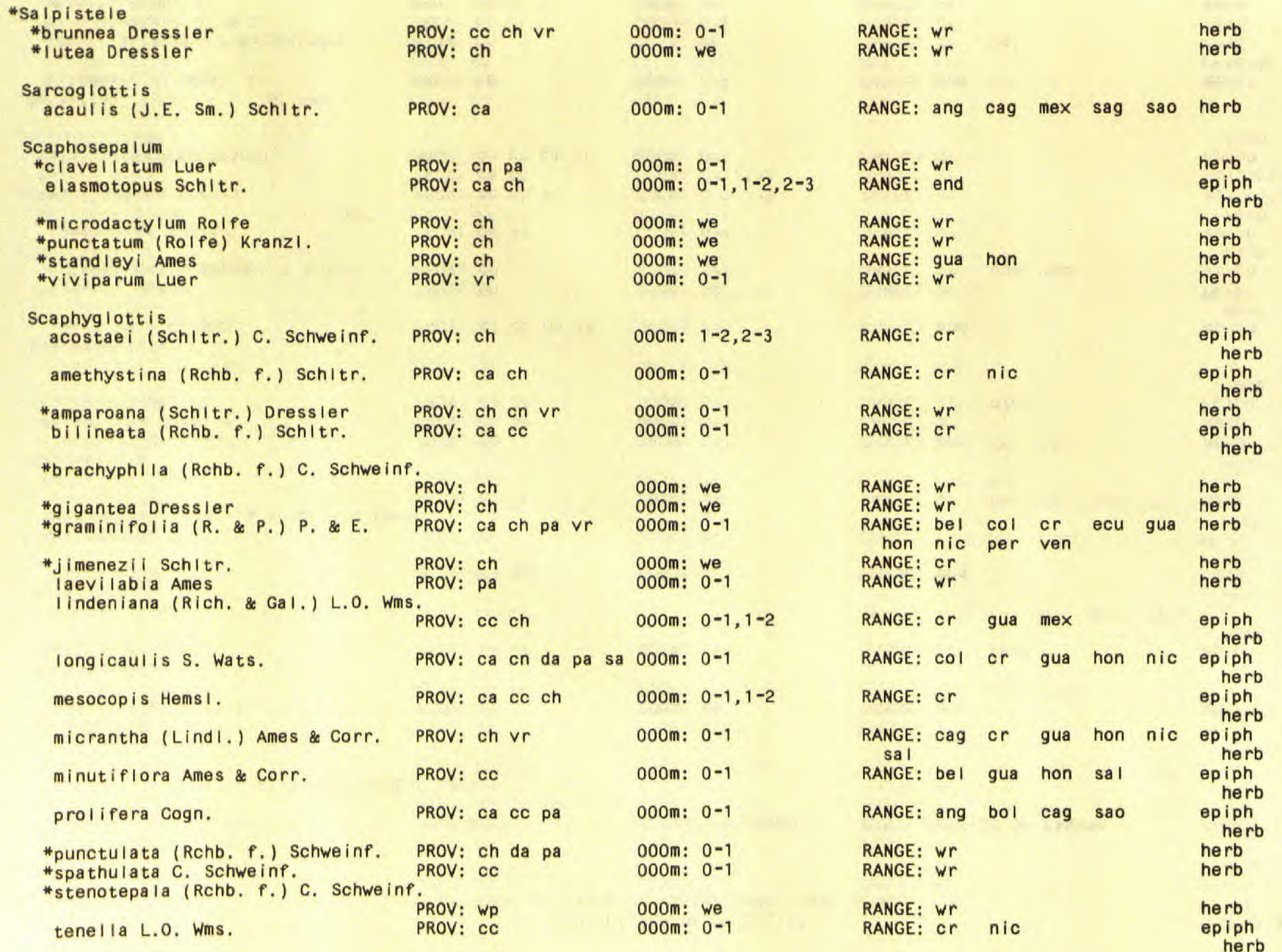




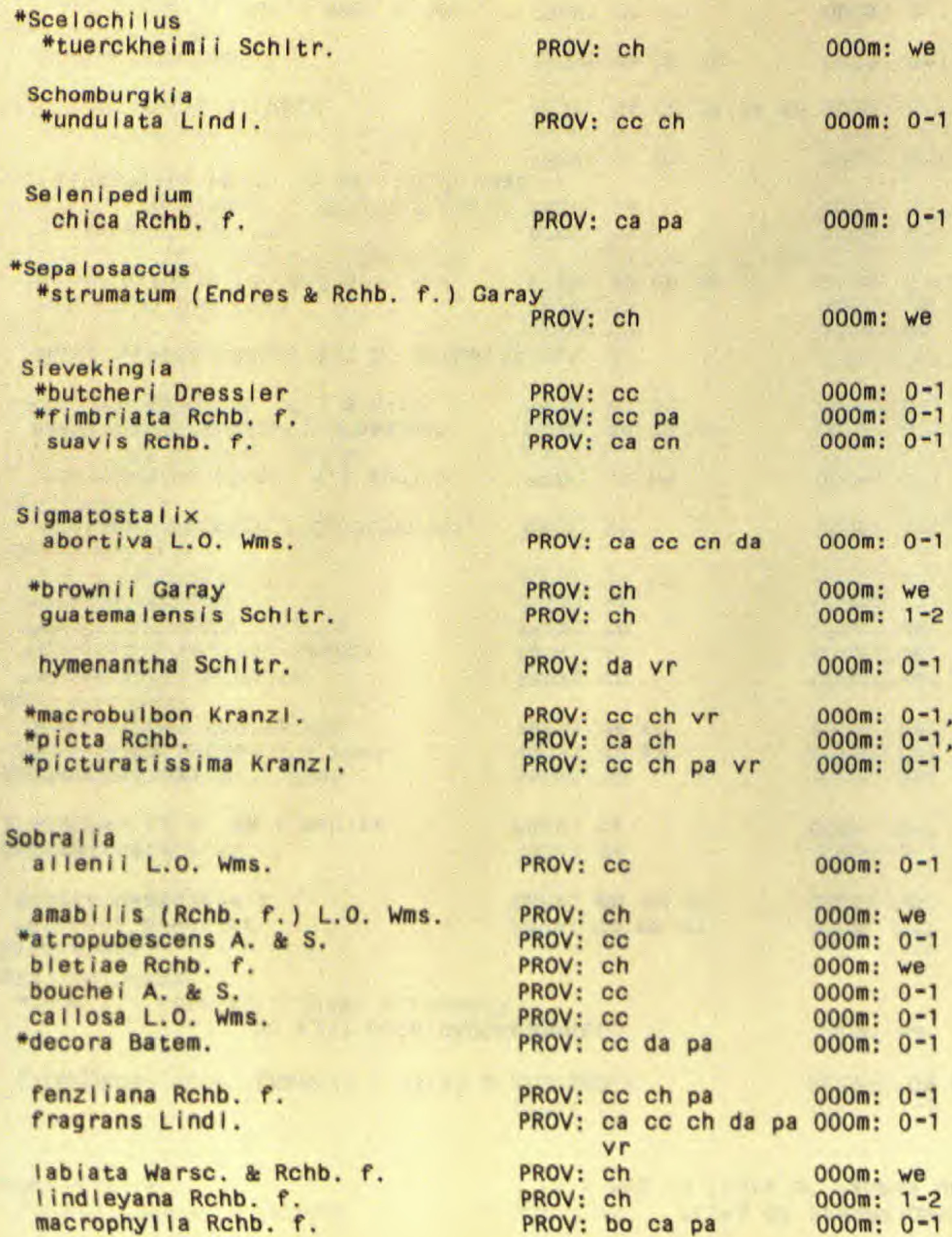

\begin{tabular}{|c|c|c|c|c|c|c|}
\hline RANGE: & gua & & & & & wh \\
\hline RANGE: & $\mathrm{cr}$ & ven? & & & & $\begin{array}{l}\text { epiph } \\
\text { herb }\end{array}$ \\
\hline RANGE: & end & & & & & herb \\
\hline & & +4 & 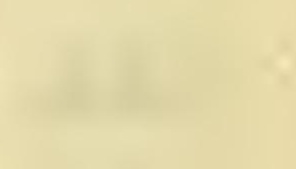 & & & \\
\hline & & 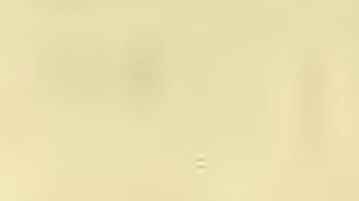 & $\cdots$ & & wat & $2=$ \\
\hline RANGE: & $w r$ & & & & & herb \\
\hline RANGE: & end & & & & & herb \\
\hline RANGE: & $p r$ & & & & & herb \\
\hline RANGE: & cr & nic & & & & $\begin{array}{l}\text { epiph } \\
\text { herb }\end{array}$ \\
\hline RANGE: & end & & & & & $\begin{array}{l}\text { epiph } \\
\text { herb }\end{array}$ \\
\hline RANGE: & $w r$ & & & & & nerb \\
\hline RANGE: & cr & gua & $\operatorname{mex}$ & & & $\begin{array}{l}\text { epiph } \\
\text { herb }\end{array}$ \\
\hline RANGE: & $\mathrm{cr}$ & & & & & $\begin{array}{l}\text { epiph } \\
\text { herb }\end{array}$ \\
\hline RANGE: & cr & 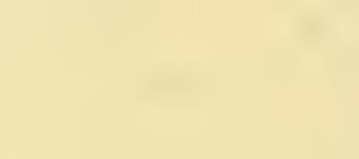 & & & & epiphyte \\
\hline RANGE: & $w r$ & & & & & epiphyte \\
\hline RANGE: & or & & & & & $\begin{array}{l}\text { epiph } \\
\text { herb }\end{array}$ \\
\hline & 4' & 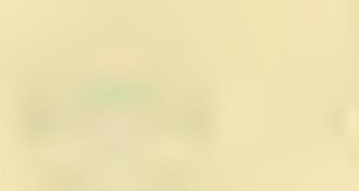 & $=$ & 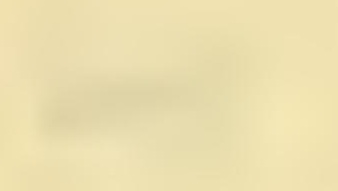 & $y^{-}$ & 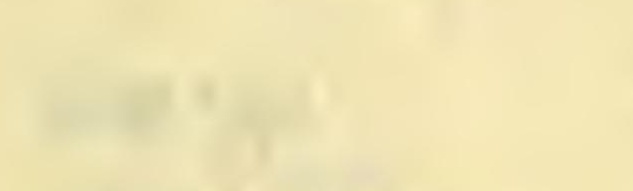 \\
\hline RANGE: & end & & & & & $\begin{array}{l}\text { epiph } \\
\text { herb }\end{array}$ \\
\hline RANGE: & $\mathrm{cr}$ & sal & & & & herb \\
\hline RANGE: & $c r$ & & & & & herb \\
\hline RANGE: & $w r$ & & & & & herb \\
\hline RANGE: & nic & & & & & herb \\
\hline RANGE: & end & & & & & herb \\
\hline $\begin{array}{c}\text { RANGE: } \\
\text { Sa I }\end{array}$ & bel & cag & gua & hon & nic & epiphyte \\
\hline RANGE: & $w r$ & & & & & herb \\
\hline RANGE: & bel & $\mathrm{cr}$ & gua & hon & & herb \\
\hline RANGE & $w r$ & & & & & herb \\
\hline $\begin{array}{l}\text { RANGE: } \\
\text { RANGF: }\end{array}$ & $\mathrm{cr}$ & nic & & & & herb \\
\hline
\end{tabular}


TAXON

FAMILY

PROVINCES

50 (33) ORCHIDACEAE cont.

powellit schltr.

suaveolens Rchb, f

*undatocarinata C. Schweinf.

valida Rolfe

warszewiczi i Rchb. f.

*wercklei (SchItr.) L.O. Wms.

*Sol enocentrum

*costaricense Schltr.

PROV: cc da pa
PROV: ca pa
PROV: ch
PROV: da
PROV: ch
PROV: vr

PROV: ch

*Spathoglottis

intr. PROV: ca cn

*Sphyrasty I is

*cryptantha (Schweinf. \& Allen) Garay

PROV: cc

Spiranthes

woodsonil L. O. Wms.

PROV: ch

Stanhopea

* cirrhata LindI.

costaricensis Rchb. $f$.

*ecornuta Lem.

graveolens Lindl,

oculata (Lodd.) LindI.

pulla Rchb. f.

wardi $\mathrm{i}$ Lind I.

\section{Stelis}

aemula Schitr.

allenii L.O. Wms

*aprica LindI.

*argentata Lindı.

atrorubens L.0. Wms.

*biserrula Lind I.

*butcheri Luer

PROV: Ch

PROV: bo

PROV: ca pa

PROV: cc

PROV: ca cc vr

PROV: $\mathrm{cc} \mathrm{ch}$

PROV: $\mathrm{cc} \mathrm{ch}$

PROV: ch pa

PROV: cc da pa

PROV: $c C$

PROV: $c c$ vr

PROV: $c h$
ELEVATION (OOOM.)

RANGE OUTSIDE OF PANAMA

HAB IT

000m: $0-1$

000m: $0-1$

000m: we

000m: $0-1$

000m: 1-2

000m: $0-1$

000m: we

000m: $0-1$

000m: $0-1,1-2$

RANGE: $w r$

herb

RANGE: $\mathrm{cr}$

RANGE: $\mathrm{nIC}$

RANGE: $\mathrm{cr}$

RANGE: end

RANGE: be I

RANGE: Wr

RANGE: $c r$

herb

herb

herb

herb

wh

herb

RANGE: $w r$

herb

000m: $1-2$

RANGE: end

000m: we

000m: we

000m: we

000m: $0-1$

000m: $0-1$

000m: $0-1$

$000 \mathrm{~m}: 0-1,1-2$

000m: $0-1,1-2$

000m: $0-1$

000m: $0-1,1-2$ 000m: $0-1,1-2$

000m: $0-1$

000m: $0-1$

$000 \mathrm{~m}: 2-3$
RANGE: hon herb

RANGE: $\mathrm{cr}$ hon nic epiph

RANGE: hon nic herb

RANGE: $\mathrm{cr}$ gua hon mex sal epiph

RANGE: cag? mex sag?

RANCE: cag? mex sag? herb

RANGE: or epiph

RANGE: cag? cr gua mex sag? epiph herb

RANGE: or gua? hon? epiph

RANGE: end herb

herb

RANGE: cag or gua hon mex epiph

nic sal
RANGE: or gua mex nic sag? epiph

RANGE: herb

RANGE: end epiph

RANGE: $w r$ herb

RANGE: end epiph 
collina Schltr.

*cooperi schitr. crescenti icola schltr.

\section{despectans Schltr.}

\#dressleri Luer

* fimbriata R.K. Baker

*gratiosa Luer

inaequalis Ames

leucopogon Rchb. f.

* loculifera Luer

longipetiolata Ames

maxonii Schltr.

microchila Schitr.

montana L.O. Wms.

panamensis schltr.

powellii schltr.

*purpurea (R. \& P.) Willd.

* sa rcophyl la Luer

* semperflorens Luer

skutchii Ames

*spathulata P. \& E. storki i Ames

*triaristata Luer

* umbell iformis Hespenh. \& Dressler PROV: vestita Ames

williamsii Ames

*Ste I I ilabium

*minutiflorum (Kranzl, ) Garay
PROV: pa

000m: 0-1

PROV: ch PROV: ca ce cn da pa 000m: $0-1$

PROV: ch

000m: 2-3

PROV: $v r$

000m: $0-1$

PROV: pa

PROV: pa

PROV: Pa

000m: $0-1$

000m: $0-1$

000m: 0-1

PROV: $\mathrm{cc} \mathrm{ch}$

000m: $0-1,1-2$

PROV: ch

000m: $1-2$

PROV: ch

PROV: ch

PROV: $\mathrm{CC}$

PROV: ch

PROV: pa

PROV: bo $\mathrm{ch}$

PROV: pa

PROV: ch

PROV: pa

PROV: ch

PROV: $c h$

PROV: cc pa

PROV: pa

PROV: $\mathrm{ch}$

PROV: da

PROV: cc ch pa 000m: $2-3$

$000 m: 1-2,2-3,3+$

000m: $0-1$

000m: $2-3$

000m: $0-1$

000m: $0-1,1-2$

000m: $0-1$

000m: $1-2$

000m: $0-1$

000m: $1-2$

000m: $1-2$

000m: $0-1$

000m: $0-1$

000m: $0-1$

000m: 1-2

000m: $1-2$
RANGE: end

RANGE: $\mathrm{cr}$ RANGE: $\mathrm{cr}$

RANGE: or

RANGE: end

RANGE: $w r$

RANGE: $w r$

RANGE: hon

RANGE: $\mathrm{cr}$

RANGE: end

RANGE: end

RANGE: $c r$ nic

RANGE: cr gua

RANGE: end

RANGE: $c r$

RANGE: $\mathrm{cr}$

RANGE: $w r$

RANGE: end

RANGE: end

RANGE: $\mathrm{cr}$

RANGE: $w r$

RANGE: $c r$

RANGE: $w r$

RANGE: $w r$

RANGE: $\mathrm{cr}$

RANGE: hon epiph

herb herb

epiph

herb

epiph

herb

epiph

herb

herb

epiph

herb

epiph

herb

epiph

epiph

herb

epiph

herb

epiph

epiph

herb

epiph

herb

herb

herb

epiph

herb

epiph

epiph

epiph

herb

epiph

herb

herb

herb

ep iph

herb

epiph

herb 


\section{Stenoptera}

costaricensis Schltr.

*Stenorrhynchos

*I anceolatum Spr.

navarrensis Ames

*speciosum Spr.

*standleyi Ames

*Systelog lossum

Hpanamense Dressler \& N.H. Wms.

\section{Telipogon \\ *ampliflorus C. Schweinf. \\ dendriticus Rchb. $f$.}

*endresianum kranzl.

"parvulus C. Schweinf.

radiatus Rchb. $P$.

\section{*Teuscheria}

*pickiana (Schltr.) Garay

*Trevoria

"zahibruckneriana (Schltr.) Garay PROV: vr

Trichocentrum

*caloceras Endres \& Rchb, $f$.

*capistratum Rchb. $f$.

Trichopilia

leucoxantha L.0. Wms,

maculata Rehb. $f$.

marginata Henfr.

--var, a Iba Rchb. f.

--var. Iepida Veitch

--var. ol ivacea Rchb. f.
PROV: $\mathrm{ch}$

$000 m: 1-2,2-3$

PROV: ca ch cn pa sa $000 \mathrm{~m}$ : $0-1$

PROV: bo cc ch vr PROV: ch da pa vr

PROV: cc ch pa

000m: $0-1,1-2,2-3$

000m: $0-1,1-2$

000m: $0-1,1-2$

PROV: ch pa vr

PROV: WP

PROV: ch

PROV: ch

PROV: ch

PROV: ch

PROV: ca ch cn

000m: 0-1

000m: 0-1

PROV: ch

PROV: ca cc en pa

000m: we

000m: $0-1$

PROV: CC

000m: $0-1$

PROV: ca cc pa

000m: 0-1

000m: $1-2$

000m: we

000m: we

000m: we
RANGE: or sag?

herb

RANGE: ang cag fla mex sag herb
Sao
RANGE: cr
RANGE: ang cr gua hon mex herb
nic sag
RANGE: cr

herb

RANGE: end

herb

RANGE: $c r$

RANGE: $c O$

RANGE: $c r$

RANGE: $c r$

RANGE: $\mathrm{cOl}$ per

herb

epiph

herb

herb

ep iphyte

epiph herb

RANGE: hon nic

herb

RANGE: nic

herb

RANGE: $W r$

RANGE: $\mathrm{col} \mathrm{cr}$ ven

herb

epiph
herb

RANGE: $w r$

epiph

RANGE: hon

herb

epiph

herb

RANGE: col cr gua hon nic epiph

RANGE: $w r$

epiph

RANGE: $w r$

herb

epiph

herb

RANGE: $w r$

epiph 
TAXON

50 (33) ORCHIDACEAE cont.

suavis Lind 1 , \& Paxt.

subulata (Sw.) Rchb. f.

turialvae Rchb. $f$.

\section{Trigonidium}

egertonianum Lind I.

lankesteri Ames
PROV: $\mathrm{ch}$

$000 \mathrm{~m}: 1-2$

PROV: ca cc ch pa

PROV: $v r$

000m: $0-1$

000m: $0-1$

PROV: bo ca ce cn da 000m: $0-1$ pa $\vee r$

PROV: $\mathrm{cc}$

$000 \mathrm{~m}: 0-1$

Triphora

*gentianoides (Sw.) Ames \& Schltr. PROV: ca

mexicana ( $S$, Wats.) Schltr. PROV: ca wagneri schitr.

PROV: ch $v r$

000m: $0-1$

000m: $0-1$

000m: $0-1$

\section{*Trisetella}

*dressleri (Luer) Luer

*huebneri (Luer) Luer

*tenuissima (C. Schweinf.) Luer

*triaristella (Rchb, f.) Luer

"tridactylites (Rchb. f.) Luer

\section{Trizeuxis}

falcata Lind I.

\#Uleiorchis

*ulaei (Cogn.) Handro

Vanilla

*pauciflora Dressler

* paraviana Rchb. $f$.

planifolia Andr.

pompona Scheide

cult.

\section{Warrea}

costaricensis Schltr.

*Warreops is

"parviflora (L.0. Wms.) Garay
PROV: cc pa

PROV: bo vr

PROV: $\mathrm{CC}$

PROV: $v r$

PROV: CC

PROV: ch $v r$

PROV: ca

PROV: pa

PROV: $\mathrm{ch}$

PROV: ca pa sa

PROV: ca

PROV: ch

PROV: ch 000m: $0-1$

000m: $0-1$

000m: $0-1$

000m: $0-1$

000m: $0-1$

000m: $0-1$

000m: $0-1$

000m: $0-1$

000m: $0-1$

000m: we

000m: $0-1$

000m: $0-1$

000m: $1-2$

\begin{tabular}{|c|c|c|c|c|c|c|}
\hline RANGE: & $\mathrm{col}$ & $\mathrm{cr}$ & & & & epiph \\
\hline RANGE: & $\mathrm{col}$ & cub & jam & per & trt & epiph \\
\hline ANGE: & $\mathrm{cr}$ & nic & & & & $\begin{array}{l}\text { epiph } \\
\text { herb }\end{array}$ \\
\hline RANGE: & bel & cag & $\mathrm{col}$ & cr & ecu & epiph \\
\hline $\begin{array}{l}\text { gua } \\
\text { ven }\end{array}$ & hon & mex & nic & per & sal & \\
\hline RANGE: & $\mathrm{cr}$ & & & & & he \\
\hline
\end{tabular}

RANGE: cub fla gua hon mex herb RANGE: gua hon mex herb RANGE: end

herb

RANGE: end

RANGE: $w r$

RANGE: end

herb

herb

RANGE: $w r$

epiph

RANGE: $w r$

herb

herb

RANGE: bol cr per sao trt epiph
ven

RANGE: $w r$ herb

RANGE: $c r$ ?

RANGE: bel gua hon herb

RANGE: ang? cag mex herb

RANGE: ang bol cag ecu gui herb

mex ven

RANGE: $c r$ gua

herb

RANGE: $w r$

herb 


\section{"WuI I sch l aege I ia \\ *aphyila (SW.) Rehb. P.}

"calcarata Benth.

PROV: $c C$

PROV: cn pa

PROV: en

PROV: $c c$ ch do

PROV: ca pa

PROV: ch

PROV: co pa *atropurpureua (Lindi.) Luer 000n: $0-1$

000a: $0-1$

000a: $0-1$

000 a $: 0-1,1-2,2=3$

000: $0-1$

000 a : $1-2$

000n: $0-1$

\section{CASUARIMACEAE}

\section{* Casuarina}

*equisetifolia L. intr. cult.

prov: pa

000n: $0-1$

53 (34) PIPERACEAE

Anderssonlopiper

panamense Tre L.

PROV: wp

000n: we

Peperosia

acuainata R. P.

al bescens irel.

* amphitricha Trel.

- angularis C. OC.

*angustata H.B. K.

barbinodis Trel.

bifrons Trei.

- biephariphyila Trel. Kuncker

PROV: on

PROV: ce

PROV:

PROV: oc ch lo pa 000 in: $0-1,1-2$

PROV: ch 000n: $1-2,2-1$

PROV: ch

PROV: ch

Prov: pa

brevipeduncula Trel.

caudulitimba C. DC.

PROV: da pa

Prov: bo ca ce da

var. cylindribacca (C. DC.) Yuncker

chiriquiensis Yuncker

Prov: on

chrysleri Yuncker

OROV: bo

PROV: ce pa

$000 \mathrm{n}: 1-2$

000 a: $1-2$

$000 n: 2-1$

$000 \mathrm{n}: 0-1$

000n: $0-1$

000n: $0-1$

000n: $1-2,2-1$

$000 \mathrm{~m}: 0-1$

000 : $0-1,1-2$
RANCE: gua hon herb

RANCE: $v r$ herb

RANCE: $v r$ herb

RANCE: or gua nic eplph

RANCE: cag col gui hon jas epiph

sex nic per sag? herb

auve: or nic epiph

herb

RANCE: $\vee r$

市

RAMCE: 000

zree

RACE: end

shrob

Wuck: ang gua per sag sat herb

baver: and herb

aAvCE: or hert

Whot: cag sat herb

RANOE: or
RANOE: or herb

M4Or! and

GHCE: vr epish

herb

herb

herb

Pusce: and

nerb

RANGE: end

auser: end

awce: and

nerts

nerb

he $r$ o 
TAXON

FAMILY

PROVINCES

ELEVATION (OOOM.)

RANGE OUTSIDE OF PANAMA

HABIT

53 (34) PIPERACEAE cont.

clilolibractea C. DC.

cocleana Trel.

cordulata C, DC.

cordulatiformis Trel.

*costaricensis C. DC.

eyclophyl Ia Miq.

davidsoni i Yuncker

digitinervia Trel.

distachya (L.) A. Dietr.

*dotana Trel.

*ebingeri Yuncker

elata Schroeder

*ema rginel la (Sw.) C. DC.

*emi li ana C. DC.

flexinervia Yuncker

*galioides H.B.K.

glabella (SW.) A. Dietr.

--var. glabelía

- -var. nervulosa (C. DC.) Yuncker

"guapilesiana Trel.

*hernandifolia (Vahl) A. Dietr. PROV: cc ch da vr 000m: 0-1,1-2

hispidula (SW, ) A, Dietr.

thoffmannii C. DC.

hyg rophiloides C. DC,

insueta Trel.

killipi Trel.

*Ianceolato-peltata C, DC.

* I ancifolia Hook.

* Iancifolioidea Burger

$\begin{array}{ll}\text { PROV: ca da pa } & 000 \mathrm{~m}: 0-1 \\ \text { PROV: cc a } & 000 \mathrm{~m}: 0-1 \\ \text { PROV: ca cn } & 000 \mathrm{~m}: 0-1 \\ \text { PROV: ca da pa } & 000 \mathrm{~m}: 0-1 \\ \text { PROV: da } & \end{array}$

PROV: ca ch da pa vr 000m: 0-1

PROV: ch 000m: 2-3

PROV: $c c$ cn cn pa vr 000m: 0-1

PROV: bo cc pa $\vee r$ 000m: $0-1,1-2$

PROV: ch $v r$

PROV: ca $v r$

PROV: ch $V r$

PROV: cc da pa $v r$

000m: $1-2,2-3$

$000 \mathrm{~m}: 0-1$

000m: $1-2$

PROV: bo da

000m: $0-1$

PROV: bo cc ch $\mathrm{vr}$ 000m: $0-1$ PROV: $\mathrm{ch}$

000m: $1-2,2-3$

PROV: ca ch cn da pa 000m: $0-1,1-2$

PROV: ca da

PROV: bo pa

000m: $0-1$

000m: $0-1$

PROV: ch

000m: $1-2$

PROV: ch

PROV: $\mathrm{ch}$

000m: $0-1,1-2$

$000 \mathrm{~m}: 1-2$

PROV: ca ce cn da pa 000m: $0-1$

sa

PROV: ca ch cn da lo 000m: $0-1$ pa

PROV: cc lo pa

000m: $0-1,1-2$

PROV: da pa $v r$

000m: $0-1,1-2$

PROV: cc cn pa

000m: $0-1$ 000m: $0-1,1-2$

\begin{tabular}{|c|c|c|c|c|c|c|}
\hline $\begin{array}{l}\text { RANGE: } \\
\text { RANGE: } \\
\text { RANGE: }\end{array}$ & $\begin{array}{l}\text { end } \\
\text { end } \\
\text { end }\end{array}$ & (n) & & & & $\begin{array}{l}\text { herb } \\
\text { herb } \\
\text { epiph } \\
\text { herb }\end{array}$ \\
\hline RANGE: & end & & & & & herb \\
\hline RANGE: & cr & gua & & 4.4 & & $\begin{array}{c}\text { epiph } \\
\text { terr } \\
\text { herb }\end{array}$ \\
\hline $\begin{array}{c}\text { RANGE: } \\
\text { per }\end{array}$ & $\begin{array}{l}\text { ang } \\
\text { sao }\end{array}$ & $\begin{array}{l}\text { bol } \\
\text { sat }\end{array}$ & cag & cr & gua & herb \\
\hline $\begin{array}{l}\text { per } \\
\text { RANGE: } \\
\text { RANGE: }\end{array}$ & $\begin{array}{l}\text { sao } \\
\text { end } \\
\text { end }\end{array}$ & sat & & & & $\begin{array}{l}\text { herb } \\
\text { epiph } \\
\text { herb }\end{array}$ \\
\hline $\begin{array}{l}\text { RANGE: } \\
\text { per }\end{array}$ & $\begin{array}{l}\text { ang } \\
\text { sao }\end{array}$ & $\begin{array}{l}\text { cag } \\
\text { ven }\end{array}$ & ecu & gui & $\operatorname{mex}$ & herb \\
\hline RANGE: & $\mathrm{col}$ & cr & ecu & ven & & herb \\
\hline RANGE: & cr & gua & & & & herb \\
\hline RANGE: & $\mathrm{cr}$ & hon & & & & herb \\
\hline RANGE: & ang & $\mathrm{cr}$ & sat? & & & epiphyte \\
\hline RANGE: & cag & & & & & $\begin{array}{l}\text { epiph } \\
\text { herb }\end{array}$ \\
\hline RANGE: & end & & & & & herb \\
\hline $\begin{array}{l}\text { RANGE: } \\
\text { Ven }\end{array}$ & ang & cag & cr & $\operatorname{mex}$ & sat & herb \\
\hline $\begin{array}{r}\text { RANGE: } \\
\text { sat? }\end{array}$ & $\begin{array}{l}\text { ang } \\
\text { ven }\end{array}$ & cag & $\mathrm{col}$ & $\mathrm{cr}$ & gua & herb \\
\hline RANGE: & ang & cag & sat & & & herb \\
\hline RANGE: & $\mathrm{cr}$ & 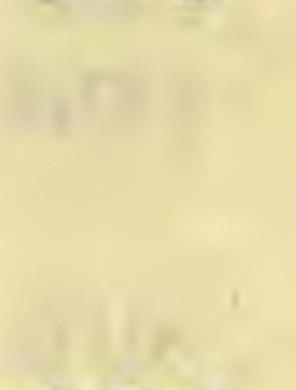 & & & & $\begin{array}{l}\text { epiph } \\
\text { terr } \\
\text { herb }\end{array}$ \\
\hline $\begin{array}{c}\text { RANGE: } \\
\text { sao }\end{array}$ & ang & cag & col & $\mathrm{cr}$ & $\operatorname{mex}$ & $\begin{array}{l}\text { epiph } \\
\text { cl imb } \\
\text { herb }\end{array}$ \\
\hline $\begin{array}{c}\text { RANGE: } \\
\text { sat }\end{array}$ & $\begin{array}{l}\text { ang } \\
\text { ven }\end{array}$ & cag & $\mathrm{cr}$ & gua & sao & herb \\
\hline $\begin{array}{l}\text { RANGE: } \\
\text { RANGE: }\end{array}$ & $\begin{array}{l}\text { cr } \\
\text { end }\end{array}$ & gua & sao & & & $\begin{array}{l}\text { herb } \\
\text { epiph } \\
\text { herb }\end{array}$ \\
\hline RANGE: & end & & & & & herb \\
\hline RANGE: & end & & & & & herb \\
\hline RANGE: & cag & sat & ven & & & $\begin{array}{l}\text { epiph } \\
\text { herb }\end{array}$ \\
\hline $\begin{array}{c}\text { RANGE: } \\
\text { oe } r\end{array}$ & $\begin{array}{l}\text { col } \\
\text { sao }\end{array}$ & $\begin{array}{l}\mathrm{cr} \\
\text { sat }\end{array}$ & $\begin{array}{l}\text { ecu } \\
\text { ven }\end{array}$ & gui & $\operatorname{mex}$ & herb \\
\hline RANGE: & $\mathrm{cr}$ & & & & & herb \\
\hline
\end{tabular}


53 (34) PIPERACEAE cont.

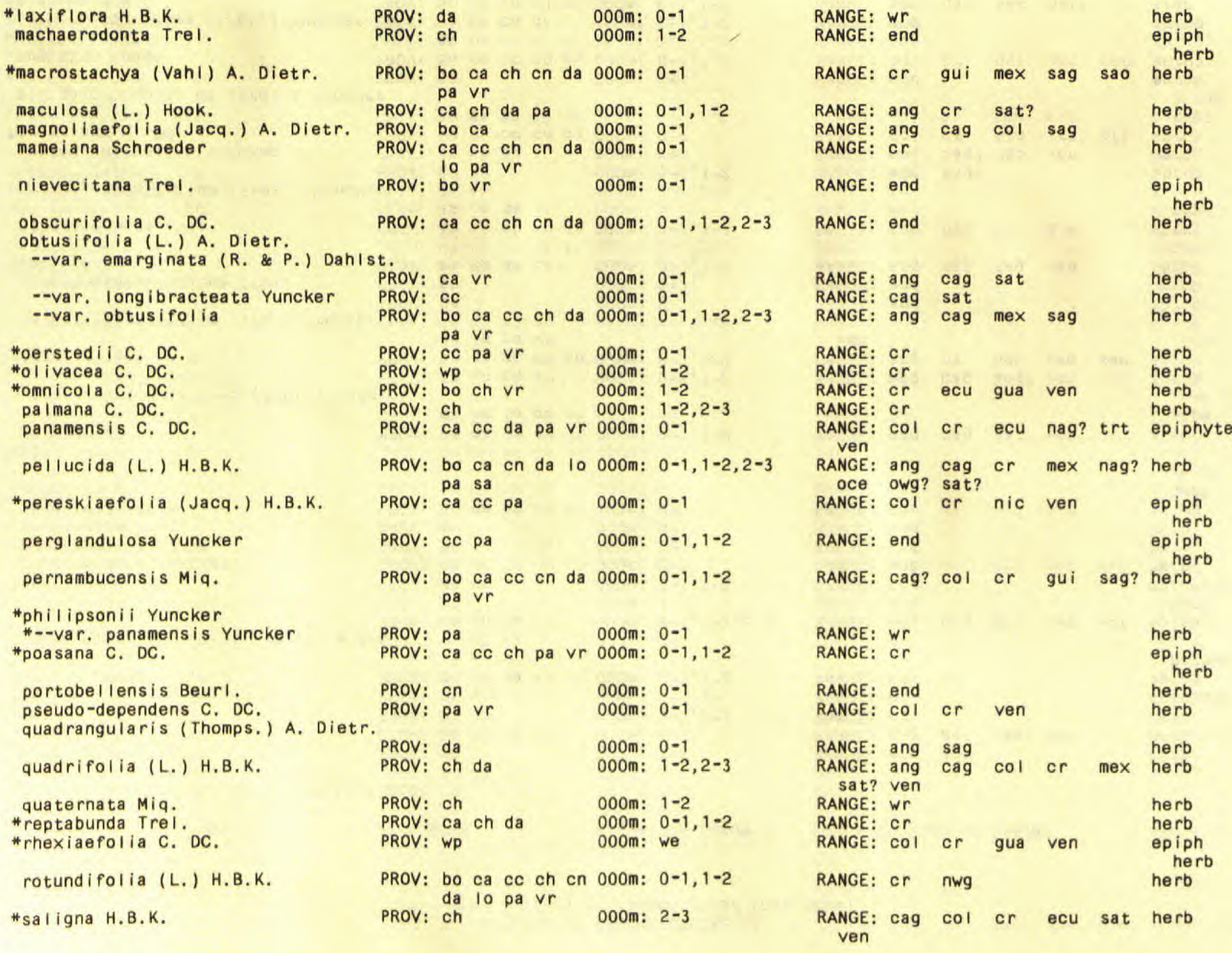


53 (34) PIPERACEAE cont.

seemanniana Miq. serpens (SW.) Loud. substriata C. DC.

*syringifolia C. DC.

*tenellaeformis Trei.

"tetraphylla (G. Forst.) Hook. \& Arn.

$\begin{array}{ll}\text { PROV: ch } & 000 \mathrm{~m}: 1-2 \\ \text { PROV: bo ca da pa } & 000 \mathrm{~m}: 0-1 \\ \text { PROV: bo ch } & 000 \mathrm{~m}: 1-2,2-3 \\ \text { PROV: ch } v r & 000 \mathrm{~m}: 0-1,3+ \\ \text { PROV: cc ch pa } & 000 \mathrm{~m}: 0-1,1-2\end{array}$

thakiana C, DC,

PROV: $c a$ ch da

umbrigaudens Yuncker

urocarpoides C. DC.

valliculae Trel.

wedelil Yuncker

Piper

aduncum L.

PROV: bo ca cc ch cn 000m: $0-1,1-2$ da he lo pa $v r$

- -var. brachyarthrum (Trel.) Yuncker

PROV: ca ce ch $v r$ 000m: $0-1,1-2$ aequale VahI

PROV: bo ca cc ch cn $000 \mathrm{~m}: 0-1,1-2$ da lo pa vr

--var. laurifolium (Trel.) Yuncker

albopunctulatissimum Trel.

*ama lago L.

a rboreum Aubl.

--var. falcifolium (Trel.) Yuncker

--var. hirtellum Yuncker

*arieianum C. DC.

aristolochiaefolium (Trel.) Yuncker

augustum Rudge --var. cocleanum (Trel.) Yuncker PROV: ca cc ch da 000m: 0-1,1-2 auritum H.B.K.

*biauritum C. DC.

"biolleyi C, DC.

*bisasperatum Trel. bogotense C. DC.

*boquetense Yuncker

*bredemeyeri Jacq.
PROV: ca ch da pa $000 \mathrm{~m}: 0-1,1-2$

PROV: ca ce ch cn da 000m: $0-1,1-2$ lo pa sa

PROV: ch pa 000m: $0-1,1-2$

PROV: ca ch pa 000m: $0-1$

PROV: bo ca cc cn da 000m: 0-1 he pa sa

PROV: ca 000m: $0-1$

PROV: bo ca co cn da 000m: $0-1$

PROV: bo ca cc ch en $000 \mathrm{~m}: 0-1,1-2$ da pa sa $v r$

PROV: bo ch da pa vr 000m: $0-1,1-2$ PROV: bo 000m: $0-1$

PROV: ch Vr

PROV: WP

000m: $0-1,1-2$ 000m: we

PROV: ch PROV: $\mathrm{ch}$ 000m: 1-2 000m: $1-2$

\begin{tabular}{|c|c|c|c|c|c|c|}
\hline $\begin{array}{l}\text { RANGE: } \\
\text { RANGE: } \\
\text { RANGE: } \\
\text { RANGE: } \\
\text { RANGE: }\end{array}$ & $\begin{array}{l}\text { cr } \\
\text { ang } \\
\text { cr } \\
\text { cr } \\
\text { cr }\end{array}$ & $\begin{array}{l}\text { gua } \\
\text { per }\end{array}$ & sag? & sao & & $\begin{array}{l}\text { herb } \\
\text { herb } \\
\text { herb } \\
\text { epiphyte } \\
\text { epiph } \\
\text { herb }\end{array}$ \\
\hline $\begin{array}{l}\text { RANGE: } \\
\text { RANGE: } \\
\text { RANGE: } \\
\text { RANGE: } \\
\text { RANGE: } \\
\text { RANGE: }\end{array}$ & $\begin{array}{l}\text { ang } \\
\text { cr } \\
\text { end } \\
\text { end } \\
\text { end } \\
\text { end }\end{array}$ & cag & $\operatorname{mex}$ & sag & $w d t$ & $\begin{array}{l}\text { epiph } \\
\text { herb } \\
\text { epiphyte } \\
\text { herb } \\
\text { herb } \\
\text { herb } \\
\text { epiph } \\
\text { herb }\end{array}$ \\
\hline RANGE: & ang & cag & sat? & ven & & $\begin{array}{l}\text { shrub } \\
\text { tree }\end{array}$ \\
\hline $\begin{array}{c}\text { RANGE: } \\
\text { RANGE: } \\
\text { Ven }\end{array}$ & $\begin{array}{l}\text { ang } \\
\text { ang }\end{array}$ & $\begin{array}{l}\text { cag } \\
\mathrm{cr}\end{array}$ & $\begin{array}{l}\text { sag? } \\
\text { hon }\end{array}$ & $\begin{array}{l}\text { ven } \\
\text { sag }\end{array}$ & sao & $\begin{array}{l}\text { shrub } \\
\text { shrub }\end{array}$ \\
\hline $\begin{array}{l}\text { RANGE: } \\
\text { RANGE: } \\
\text { RANGE: }\end{array}$ & $\begin{array}{l}\text { end } \\
\text { end } \\
\text { ang }\end{array}$ & cag & sag & ven & & $\begin{array}{c}\text { shrub } \\
\text { shrub } \\
\text { shrub } \\
\text { tree }\end{array}$ \\
\hline RANGE: & ang & cag & cr & gua & & $\begin{array}{l}\text { shrub } \\
\text { tree }\end{array}$ \\
\hline $\begin{array}{c}\text { RANGE: } \\
\text { RANGE: } \\
\text { RANGE: } \\
\text { ven }\end{array}$ & $\begin{array}{l}\text { ang } \\
\text { gui } \\
\text { col }\end{array}$ & $\begin{array}{l}\text { sag? } \\
\text { sag? } \\
\text { cr }\end{array}$ & $\begin{array}{l}\text { sao } \\
\text { nic }\end{array}$ & $\begin{array}{l}\text { ven } \\
\text { trt }\end{array}$ & trt & $\begin{array}{l}\text { shrub } \\
\text { herb } \\
\text { shrub } \\
\text { tree }\end{array}$ \\
\hline $\begin{array}{l}\text { RANGE: } \\
\text { RANGE: }\end{array}$ & $\begin{array}{l}\text { end } \\
\text { col }\end{array}$ & $\mathrm{cr}$ & gui & sat & ven & $\begin{array}{l}\text { shrub } \\
\text { shrub }\end{array}$ \\
\hline $\begin{array}{l}\text { RANGE: } \\
\text { RANGE: }\end{array}$ & $\begin{array}{l}\text { end } \\
\text { ang }\end{array}$ & cag & $\operatorname{mex}$ & sat? & & $\begin{array}{l}\text { shrub } \\
\text { tree }\end{array}$ \\
\hline $\begin{array}{l}\text { RANGE: } \\
\text { RANGE: } \\
\text { RANGE: } \\
\text { RANGE: }\end{array}$ & $\begin{array}{l}\text { cr } \\
\text { cr } \\
\text { cr } \\
\text { sag? } \\
\text { end } \\
\text { col }\end{array}$ & nic & when & ven & & $\begin{array}{l}\text { shrub } \\
\text { shrub } \\
\text { treelet } \\
\text { shrub } \\
\text { shrub } \\
\text { tree } \\
\text { shrub } \\
\text { shrub }\end{array}$ \\
\hline
\end{tabular}


campanum Yuncker
* canaense Standi.

"carpinteranum C. DC.

carrilloanum C. DC.

cativalense Trel.

changuinolanum Trel.

* chrysostachyum C. DC.

* coilostachum C. DC.

colon-insulae Trel.

colonense C. DC.

*concepcionis Trel.

cooperi Yuncker

cordulatum C. DC.

* crassinervium H.B.K.

*--var. crassinervium

crassispicatum Opiz

culebranum C. DC.

"curtispicum C. DC.

*daguanum C. DC.

darienense C. DC.

davidsonii Yuncker

dilatatum Rich.

distigmatum Yuncker

*dryadum C. DC.

* dukei C. DC.

*enganyanum Trel, \& Yuncker

*epigynium C. DC.

erubescentispicum Trel.

exiguicaule Yuncker

* fimbriulatum C. DC.

*flagel licuspe Trel. \& Yuncker

*friedrichsthalif C. DC.

ga ragaranum C. DC.

* glabrescens (Miq.) C. DC.
PROV: cc en pa 000m: $0-1$

PROV: bo cc on da pa $000 \mathrm{~m}: 0-1,1-2$

PROV. $\mathrm{Vr}$

000m: $1-2$

PROV: bo ca ch cn da $000 \mathrm{~m}$ : $0-1,1-2$

pa

PROV: cn

PROV: bo

PROV: $c h$ Vr

PROV: ch

PROV: bo

ROV: ca ch cn pa sa 000m: $0-1,1-2$

PROV: da pa Vr $000 \mathrm{~m}: 0-1$

PROV: bo

000m: $0-1$

PROV: ca cn pa vr 000m: $0-1,1-2$

PROV: ch

000m: $1-2$

PROV: WP

000m: we

PROV: ca cn pa $\quad 000 \mathrm{~m}: 0-1$

PROV: $C C$ cn da pa vr 000m: $0-1$

PROV: cc cn da vr 000m: $0-1$

PROV: bo ca ch da pa 000m: $0-1$

PROV: ch

000m: $1-2$

PROV: ca cc ch cn da 000m: $0-1$$$
\text { lo pa }
$$

000m: $1-2$

PROV: ch

000m: $0-1$

PROV: ce da vr

000m: $0-1$

PROV:

PROV: $c C$ 000m: $0-1$

PROV: bo

000m: $0-1$

$000 \mathrm{m:}: 0-1$

000m: $0-1$

PROV: pa

000m: $0-1$

PROV: bo ca cc ch cn 000m: $0-1,1-2$

$$
\text { lo ver }
$$

PROV: ca cc cn da pa 000m: 0-1 vr

PROV: bo ch da vr 000m: $0-1,1-2$

\begin{tabular}{|c|c|c|c|c|c|c|}
\hline $\begin{array}{l}\text { RANGE: } \\
\text { RANGE: }\end{array}$ & $\begin{array}{l}\text { end } \\
\text { col }\end{array}$ & & s. & & & $\begin{array}{l}\text { shrub } \\
\text { shrub }\end{array}$ \\
\hline RANGE: & $\mathrm{cr}$ & trits & 1 & & & $\begin{array}{l}\text { herb } \\
\text { shrub }\end{array}$ \\
\hline RANGE: & $\mathrm{col}$ & cr & ecu & nic & & shrub \\
\hline RANGE: & end & & & & & shrub \\
\hline RANGE: & end & & & & & shrub \\
\hline RANGE: & cr & 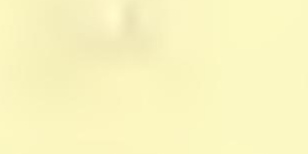 & & & & shrub \\
\hline RANGE: & $w r$ & & & & & shrub \\
\hline RANGE: & end & & & & & shrub \\
\hline RANGE: & cr & & & & & shrub \\
\hline RANGE: & $\mathrm{cr}$ & ecu & & & & shrub \\
\hline RANGE: & end & t & & & & $\begin{array}{l}\text { shrub } \\
\text { tree }\end{array}$ \\
\hline RANGE: & end & & & & & $\begin{array}{c}\text { shrub } \\
\text { tree }\end{array}$ \\
\hline RANGE: & col & or & per & sao & ven & $\begin{array}{c}\text { shrub } \\
\text { tree }\end{array}$ \\
\hline RANGE: & end & & & & & shrub? \\
\hline RANGE: & $c r$ & & & 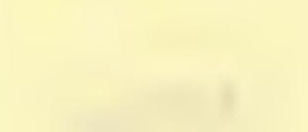 & & shrub \\
\hline RANGE: & $\mathrm{cr}$ & & & 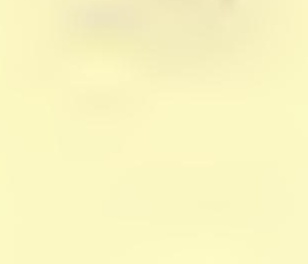 & 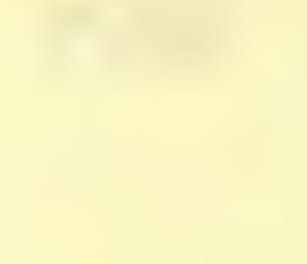 & $\begin{array}{l}\text { shrub } \\
\text { herb }\end{array}$ \\
\hline RANGE; & $w r$ & & & & & shrub \\
\hline RANGE: & $\mathrm{col}$ & $\mathrm{cr}$ & nic & ven & & shrub \\
\hline RANGE: & end & & & & & shrub \\
\hline RANGE: & ang & $\mathrm{cr}$ & gui & $\operatorname{mex}$ & sag & shrub \\
\hline sao & trt & & & & & \\
\hline RANGE: & end & & & & & $\begin{array}{r}\text { shrub? } \\
\text { tree }\end{array}$ \\
\hline RANGE: & $\mathrm{cr}$ & & & & & $\begin{array}{l}\text { shrub } \\
\text { cl imber }\end{array}$ \\
\hline RANGE: & $\mathrm{col}$ & $\mathrm{cr}$ & & & & shrub \\
\hline RANGE: & $w r$ & & & & & subshrub \\
\hline RANGE: & cr & & & & & $\begin{array}{l}\text { shrub } \\
\text { treelet }\end{array}$ \\
\hline RANGE: & end & & & & & shrub \\
\hline RANGE: & $w r$ & & & & & shrub \\
\hline RANGE: & or & & & & & shrub \\
\hline RANGE: & $w r$ & & & & & $\begin{array}{r}\text { climber } \\
\text { shrub }\end{array}$ \\
\hline RANGE: & $\mathrm{cr}$ & & & & & shrub \\
\hline RANGE: & cr & nic & & & & shrub \\
\hline ANGE & $\mathrm{s}$ & c & ecu & gua & nic & shrub \\
\hline
\end{tabular}


gonoca rpum Trel. grande Vah!

hartwegianum (Benth.) C. DC.

*haughtil Trel, \& Yuncker

*hebetifolium Burger

theterotrichum $C$. OC.

hirtellipetiolun C. OC.

- -var. harveyanum (Trel.) Yuncker

--var. hirtellipetiofun
--var. tapianum (Trei.) Yuncker

hispidum SW.

-var. ellipticifolium Yuncker

- rvar. hispidum

- -var, trachydermua (Trel.) Yuncker

humorigaudens Trel.

imperiale (Miq.) C. DC.

infraluteum Trel.

* jacquemontianum Kunth

* I acunosum. H. B. K.

* Ianceaefol ím H.B.K.

latibracteum C. DC.

leptocladum C. DC.

*ittorale C. DC.

I ucigaudens C. DC.

- -var. alieni (TreI.) Yuncker magnantherum $C$. DC. marginatum Jacq.

--var. anisatum (H.B.K.) C. DC. PROV: ch da pa 000m: 0-1

--var. catalpaefolium (H.B.K.) C. DC.

--var, clausum Yuncker

- -var. marginatum

maxoni i C, DC.

--var, varium (Trel.) Yuncker minute-scabiosum Trei.

*multiplinervium C. DC.

*munchanum C. DC.
PROV: ca 000m: $0-1$

PROV: ca ce ch ch da 000s: $0-1$ 10 pa sa $\mathrm{Vr}$

PROV: WP

ooon: ve

PROV: Ce

PROV: vr

000a: $0-1,1=2$

0000: $0-1$

000an: $1-2$

PROV: bo ca ce on pa 000m: $0-1,1-2$ PROV: bo ca ch da pa 000m: $0-1$

PROV: pa

000n: $0-1$

PROV: $\mathrm{cc}$

000m: $0-1$

PROV: bo ca ce ch en 000a: $0-1,1-2$ da lo pa sa vr

PROV: ca ce en pa 000m: $0-1$ PROV: $C C$ 000m: $0-1$

PROV: ca ce ch en da 000m: $0-1,1=2$ pa sa PROV: WP 000m: We PROV: bo ca cn $\quad 000 m: 0-1$

PROV: ch 000m: $1-2,2-3$

PROV: da 000m: $0=1,1=2$

PROV: ca cn pa 000m: $0-1$

PROV: bo cn 000m: $0-1$

PROV: bo ca cc ch vr 000m: $0-1,2-3$

PROV: da

$000 \mathrm{~m}: 0-1$

PROV: da

000m: $0-1$

PROV: ca da pa $000 \mathrm{~m}: 0-1$ PROV: ca pa 000m: $0-1$

PROV: ca cc ch cn da 000m: $0-1$ he lo pa sa vr

PROV: ch

000m: $1-2$

PROV: $\mathrm{ch}$

000m: $1-2$

PROV: $\mathrm{cC}$ cn Vr

000m: $0-1$

PROV: ca da pa vr

000m: $0-1,1-2$

PROV: da
QAYCE: and

MWOE: col

RANICE: col

RANGE: $W r$

RANGE: $w r$

RANCE: Ur

RANGE: end

RANGE: and

RANOE: and

RANGE: end RANGE: ang

RANGE: ang RANGE: end RANGE: er

RANGE: end

RANGE: or RANGE: COI

RANCE: CO RANGE: $w r$

RANGE: $O r$

RANGE: $C O$

RANGE: end

RANGE: end

RANGE: end

RANGE: ang

RANGE: ang

RANGE: $w r$

RANGE: ang

RANGE: $\mathrm{cr}$

RANGE: end RANGE: end

RANGE: col cr ven

RANGE: ven

cag sag shrub col or nic ven shrub

tree

shrub

tree

shrub

shrub

shrub

shrub

shrub

shrub

cag or sag ven shrub

shrub

shrub

shrub

tree

shrub

shrub

shrub

tree

shrub

shrub

shrub

shrub

shrub

tree

tree

shrub?

shrub

shrub

cag ecu sag? sao shrub

tree

shrub

tree

shrub

shrub

tree

imber

shrub 
*nudiforíra C. DC.

*obi iquea $R_{2}$ \& $p_{\text {s }}$

*otophopuas C. OC.

*attanisafoliua C. DC.

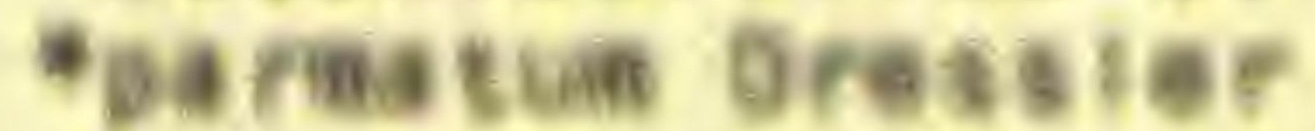

geracuenthatuin C. OC.

eserevieaula hunckar

*war. derserevicauis

-rvar. subglabritimbine noneker

perlasense venever

corbutoulatian C. DC.

beruligersan tre?.

parvelucimas troi.

befrenewah G. OC.

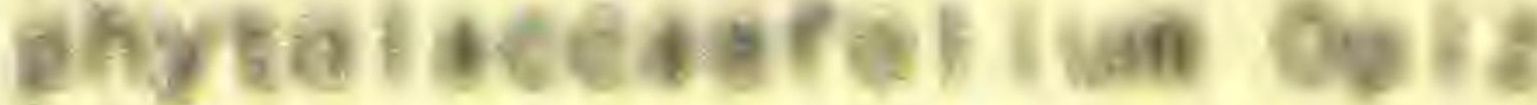

Dinoganerie tirel.

pinteri 6. 68 .

- peasanua C. OC.

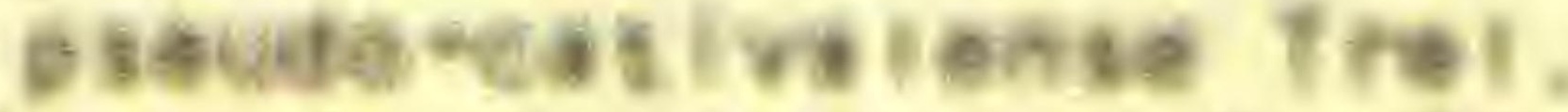

Cosesudo*fuligimesun C. BC

oseude zaragaratewin Trai.

bubissigursin $C$. OC.

regtabunduin C. De

ret ieglatua L.

*riparense 6 . D.

sanbuarean C. De.

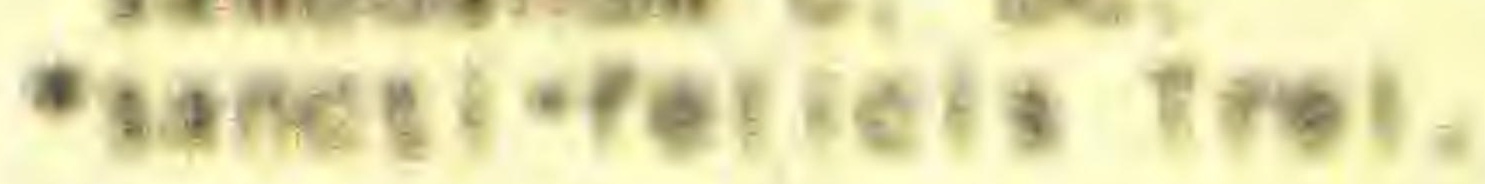

*agansali tres. Funcker

* goegriringesh C. De

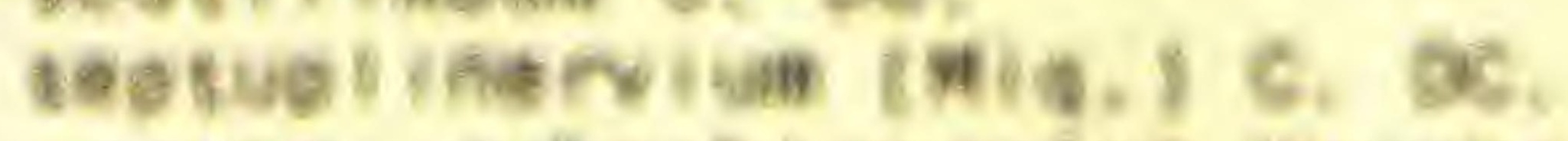

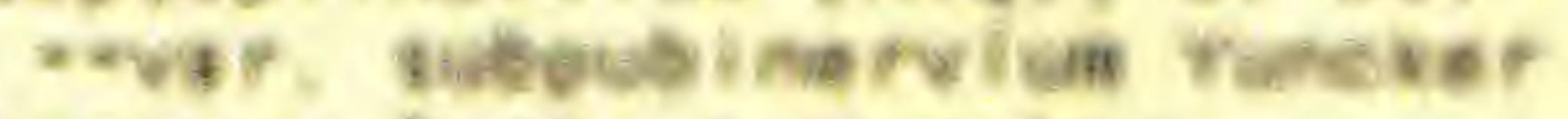

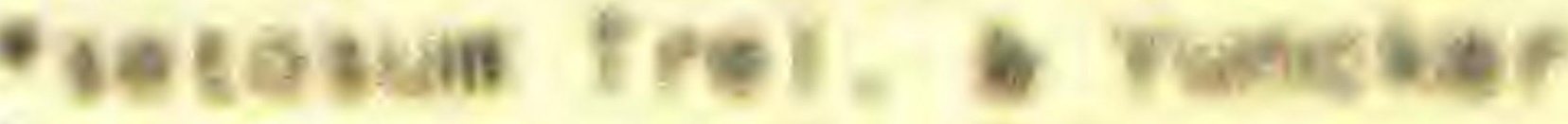

* sivavaguar C. DC.

*a i aupavidena E. ac.

*sembir nuscaser

atevenai trei.

agerail fires

aubeaudetist frot.

* subetedate iral. * Puracker

subqui mavene rive frei

aborepens trot

Whovi bo ee ch on os bodes $0+1,1-2$

$$
\text { pa sa }
$$

reovt bo ea ce eh on obon: $0+1,1+2,2+1$ da on sa vr

phovi da en da ou sa oober o-1

phovi on da ba ve godas $0-1, t-2$

Plavi on

Cocan: $0-1$

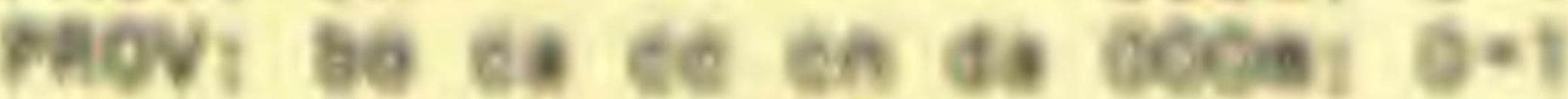
pa ve 
53 (34) PIPERACEAE cont.

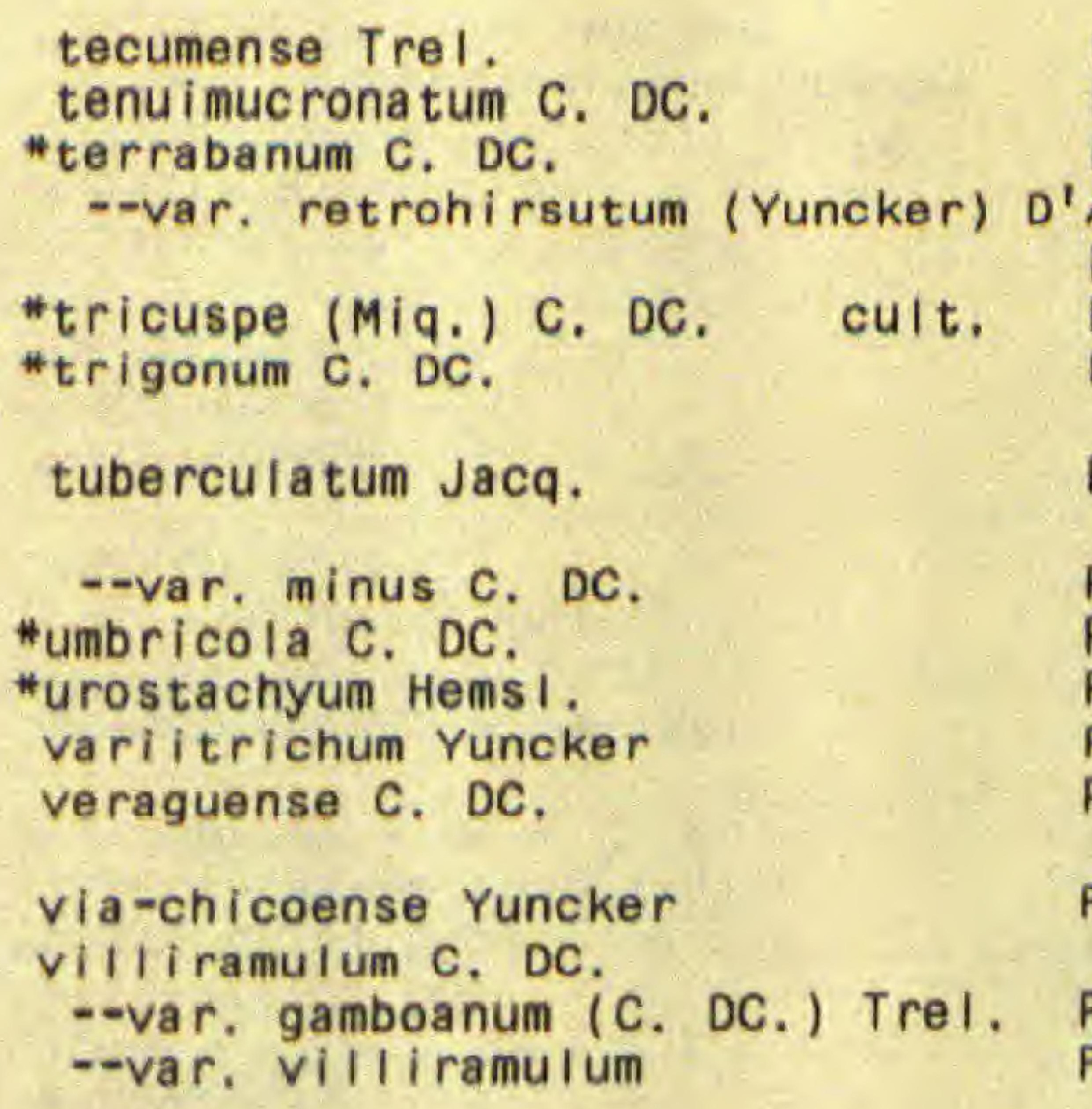

PROV: ec ch da pa 000m: $0-1,1-2$

PROV: ec ch da pa 000m: $0-1,1-2$

PROV: vr

000m: $0-1$

000m: $1-2$

000m: $0-1$

PROV:

Arcy

PROV: bo 000m: $0-1$

PROV: bo ce en da sa 000m: $0-1,1-2$

$\checkmark r$

PROV: ca cc ch cn da 000m: 0-1 lo pa sa

PROV: ca cc $000 \mathrm{~m}: 0-1$

PROV: $\mathrm{ch} \quad 000 \mathrm{~m}: 2-3$

PROV: bo cc da sa 000m: $0-1$

PROV: da $000 \mathrm{~m}: 0-1$

PROV: WP $000 \mathrm{~m}: 0-1$

PROV: ca da pa $000 \mathrm{~m}: 0-1$

PROV: ca da pa 000m: $0-1$

PROV: bo ca ch cn da 000m: $0-1$

PROV: lo pa sa

PROV: Ca

PROV: ch 000m: 1 -2

PROV: bo ch da pa $000 \mathrm{~m}$ : $0-1,1-2,2-3$

PROV: bo ca pa 000m: $0-1$

PROV: bo ca cc ch cn 000m: $0=1,1-2$ da lo pa sa

PROV: cc ch pa vr 000m: 0-1, 1-2

000m: $0-1$

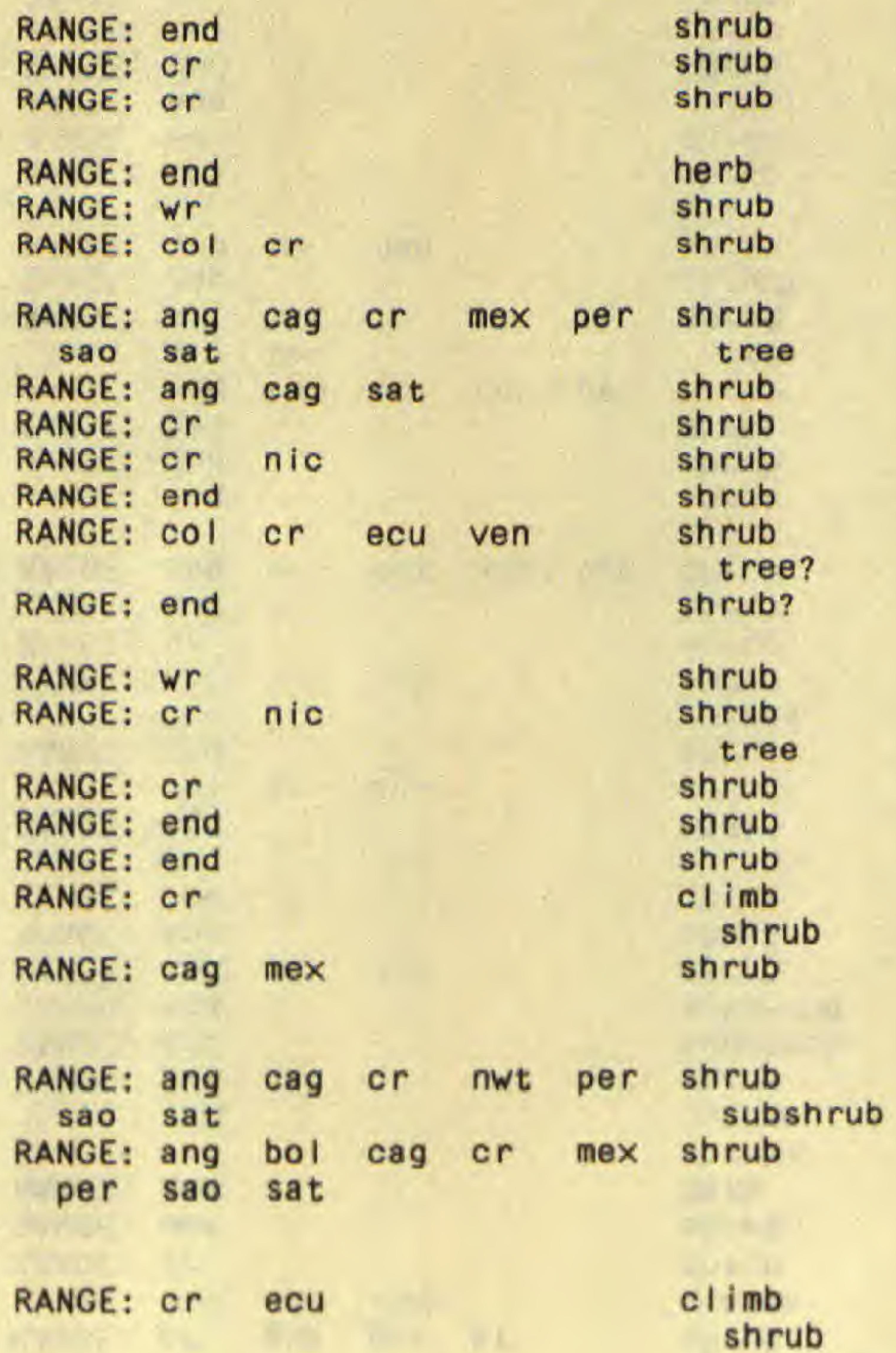

RANGE: $w r$ shrub

\section{4 (35) CHLORANTHACEAE}

\section{Hedyosmum}

*bonplandianum H.B.K.

brenesii Standl.

*burgerianum D'Arcy \& Liesner
PROV: bo ce ch pa PROV: bo ch da

PROV: $\mathrm{ch}$
$000 \mathrm{~m}: 0-1,1-2$ $000 \mathrm{~m}: 0-1,1-2$ 000m: $1-2$
RANGE: $\mathrm{col} \mathrm{cr}$ nic RANGE: $\mathrm{cr}$ hon $\mathrm{nic}$

tree RANGE: end shrub

tree 


TAXON FAMILY PROVINCES ELEVATION (OOOM.) RANGE OUTSIDE OF PANAMA HABIT

54 (35) CHLORANTHACEAE cont.

$\begin{array}{lll}\text { *correanum D'Arcy \& Liesner } & \text { PROV: ch } & \text { 000m: } 1-2 \\ \text { \#costaricense Wood } & \text { PROV: ch Vr } & 000 \mathrm{~m}: 1-2,2-3 \\ \text { \#gentryi D'Arcy \& Liesner } & \text { PROV: da } & 000 \mathrm{~m}: 1-2 \\ \text { \#goudotianum } & \text { PROV: ch } & 000 \mathrm{~m}: 1-2,2-3 \\ \text { "mexicanum Cord. } & \text { PROV: ch } & 000 \mathrm{~m}: 2-3 \\ \text { scaberrimum StandI. } & \text { PROV: cc sa } & 000 \mathrm{~m}: 0-1\end{array}$

scaberrimum Standl.

PROV: cc sa

\begin{tabular}{|c|c|c|c|c|}
\hline RANGE: & end & & . & $\begin{array}{c}\text { shrub } \\
\text { tree } \\
\text { shrub }\end{array}$ \\
\hline RANGE: & $\mathrm{col}$ & ven & & tree \\
\hline RANGE: & $\mathrm{cr}$ & nic & sat & shrub \\
\hline RANGE: & cag & $\operatorname{mex}$ & & shrub \\
\hline RANGE: & col & $\mathrm{cr}$ & nic & $\begin{array}{c}\text { shrub } \\
\text { tree }\end{array}$ \\
\hline
\end{tabular}

55 (36) LACISTEMATACEAE

Lac istema

aggregatum (Berg) Rusby

\section{Lozania}

*mustisiana R, \& S.

"pittieri (Blake) L.B. Sm.

\section{6 (37) SALICACEAE}

Sa I ix

humboldtiana Willd.

PROV: ch

$000 m: 1-2$

$000 m: 1-2,2-3$

PROV: bo ca cn pa sa 000m: $0-1,1-2$

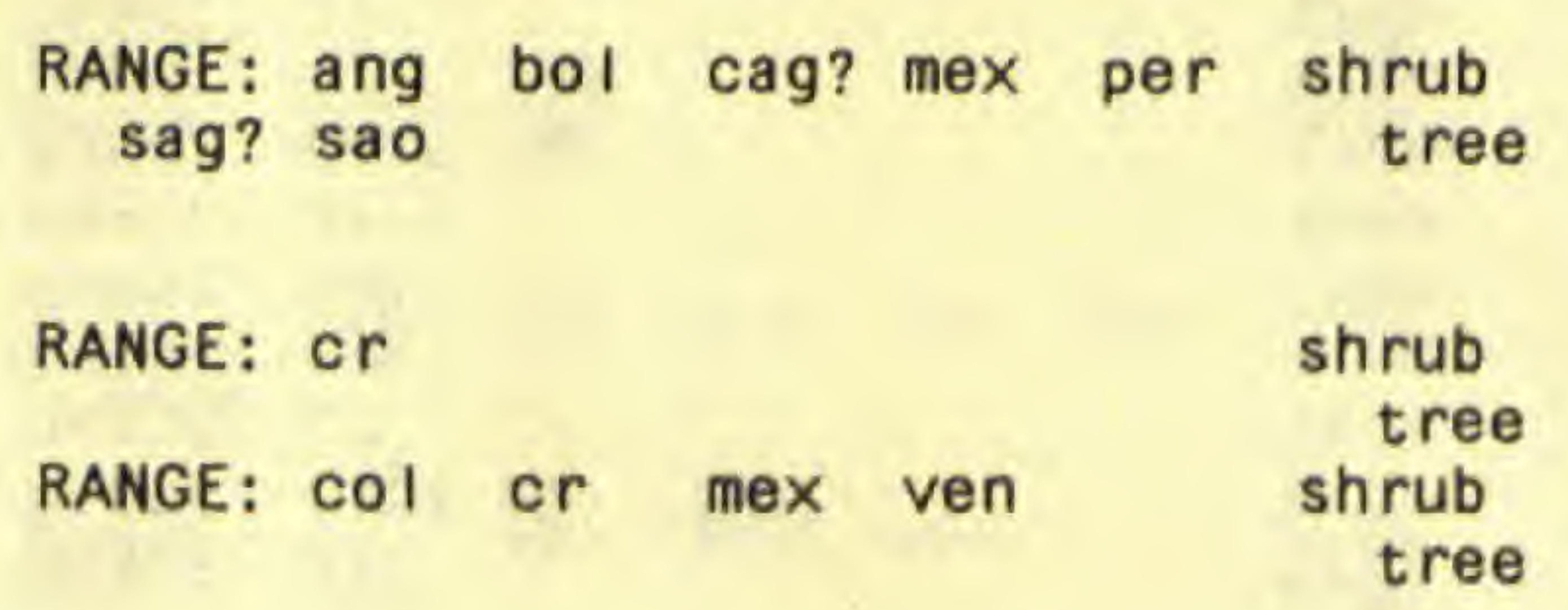

RANGE: cag mex sag

shrub

tree

57 (38) MYRICACEAE
PROV: ch

PROV: $c c$ pa 000m: $2-3$

000m: $0-1$
RANGE: fla mex nas

RANGE: cag mex tree

tree

60 (39) JUGLANDACEAE

\section{Alfaroa}

costaricensis StandI.

*williamsii A. Molina
PROV: ch vr

PROV: $\mathrm{ch}$ 000m: $1-2$

000m: $1-2$
RANGE: cr gua mex

RANGE: $\mathrm{cr}$

shrub
tree
shrub


60 (39) JUGLANDACEAE cont.

"oreomunnea

*mexicana (Standi.) Leroy
PROV: $\mathrm{CC}$ ch

61 (40) BETULACEAE
Alnus

"acuminata H. B, K.
PROV: ch

000m: $1-2,2-3$
RANGE: er

tree

\section{2 (41) FAGACEAE}

Quercus

copeyens is C.H. Mull.

corrugata Hook.

davidsoniae Stand I.

gulielmi-treleasei C.H. Mull.

humboldtil Bonpl.

*insignis Mart. \&al.

ooca rpa Liebm.

*pilarius Trel.

* rapurahuensis Trel.

seemannil Liebm.

seibertil C.H. MulI.
PROV: ch

PROV: ch

PROV: ch

PROV: $\mathrm{ch}$

PROV: da

PROV: ch

PROV: $\mathrm{ch}$

PROV: $\mathrm{ch}$

PROV: $\mathrm{ch}$

PROV: ch

PROV: ch
$000 \mathrm{~m}: 0-1,1-2$

RANGE: gua mex nic shrub

tree

\section{3 (42) ULMACEAE}

\section{Ampe locera}

*edentula KuhIm.

hottlei (Standi.) Standi.

Celt is

iguanaeus (Jacq.) Sarg.

*schippil standl.

Lozanel la

enantiophylla (J.D. Sm.) Killip \& Mort.
$000 \mathrm{~m}: 2-3$

000m: 2-3

000m: $1-2$

000m: $1-2$

000m: $1-2$

000m: $1-2$

000m: $1-2$

000m: $1-2$

000m: $1-2,2-3$

000m: 2-3

000m: $1-2$

RANGE: $\mathrm{Cr}$

RANGE: cag? $\mathrm{cr}$ mex

RANGE: $c r$

RANGE: $\mathrm{cr}$

RANGE: $\mathrm{COI}$

RANGE: $\mathrm{cr}$

RANGE: cag? mex

RANGE: $\mathrm{cr}$ mex

RANGE: $\mathrm{cr}$

RANGE: $c r$

RANGE: $\mathrm{cr}$

tree

tree

tree

tree

tree

tree

tree

tree

tree

tree

tree

\section{Trema}

micrantha (L.) BI ume
PROV: ch

PROV: cn

PROV: cn da pa sa

000m: $0-1$

PROV: ca da pa

000m: $0-1$

PROV: Ca

000m: $0-1$

$000 m: 1-2$
RANGE: bol ven tree

RANGE: cag tree

RANGE: ang cag? mex sag? sao shrub

RANGE: ecu

tree tree

RANGE: cag? mex per sag?

tree 
TAXON

UImus

mexicana (Liebm.) Planch.

PROV: ch

000m: $1-2$

\section{4 (43) MORACEAE}

RANGE: cag mex

tree

RANGE: oce tree

RANGE: as $\mathrm{i}$ tree

RANGE: $\mathrm{cr}$ tree

RANGE: col per tree

RANGE: cag? cub ecu jam mex tree sag?

\begin{tabular}{|c|c|c|c|c|c|c|}
\hline RANGE: & $\begin{array}{l}\mathrm{cr} \\
\text { end }\end{array}$ & 9 & s & s & & $\begin{array}{l}\text { tree } \\
\text { tree }\end{array}$ \\
\hline ANGE: & cr & & & & & tree \\
\hline ANG & bel & cag? & ven & & & tree \\
\hline ANS & bel & bol & per & sao & ven & 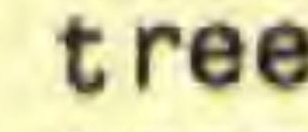 \\
\hline NGE: & col & per & sao & & & e \\
\hline NG & $\mathrm{cr}$ & gua? & sal? & & & \\
\hline MC & $\mathrm{cc}$ & & & & & \\
\hline
\end{tabular}

RANGE: WdW

herb

RANGE: cag? col ecu mex tree

RANGE: $\mathrm{cr}$

RANGE: bel cag? tree

RANGE: sat tree

RANGE: $w r$ tree

RANGE: $\mathrm{col}$ col or or nic tree$$
\text { nic }
$$

RANGE: end

RANGE: end

RANGE: cag? mex sag? tree

RANGE: cag? gag mex sag? tree 
64 (43) MORACEAE cont.

\section{* scutata Cuatr.}

Chlorophora

tinctoria (L.) Gaud.

\section{Clarisia \\ "biflora R, \& P. \\ * racemosa $R$. \& $\dot{P}$.}

\section{Coussapoa}

*asperifolia Trecul

*--subsp, magnifolia (Trecul) Akkermans Berg

brevipes pitt.

* crass ivenosa Mildbr.

*echinata Akkermans \& Berg

*glaberrima Burger

*ovalifolia Turcz.

panamensis Pitt.

"parviceps Standi.

\#villosa P. \& E.

Dorstenia

contrajerva L.

*panamens is C. Berg

Ficus

americana AubI.

*benghalensis L.

*benjamina L.

bullenei I.M. Johnst.

*cervantesiana Stand

citrifolia Mill.

*colubrinae StandI.

* coronaria King costaricana (Liebm.) Miq. davidsoniae Stand I.

dendrocida H.B.K.

*dugand $i i$ Stand $I$.

*elastica Hornem.
PROV: ca
PROV: pa sa

000m: $0-1$

000m: $0-1$

PROV: pa vr

000m: $0-1$

PROV: ca ch da he lo 000m: 0-1 pa

PROV: $c c$ ch en pa

000m: $0-1$ PROV: ch 000m: $0-1$

PROV: pa

PROV: pa

000m: $0-1$ $000 \mathrm{~m}: 0-1$

000m: $0-1$

000m: 1-2

PROV: sa

PROV: bo ca en pa

000m: $0-1$

$000 \mathrm{~m}: 0-1,1-2$

000m: $0-1$
PROV: cc ch pa vr

PROV: bo ca cc da pa 000m: $0-1,1-2$

PROV: cn

PROV: ca cn he pa vr 000m: $0-1,1-2$

cult.

PROV: $c a$

PROV: ca

PROV: ca da pa

PROV: cn

PROV: ca da pa

PROV: bo ca cc $\mathrm{cn}$ da $000 \mathrm{~m}$ : $0=1,1-2$

cult. PROV: ca sa $\mathrm{cr}$

PROV: ca ch

PROV: cc ch

PROV: pa

PROV: ca cc cn 000m: $0-1$ 000m: $0=1$

000m: $0-1$

000m: $0-1$

000m: $0-1$

000m: $0-1$

000m: $0-1,1-2$

000m: $0-1$

000m: $0-1$

000m: $0-1$

RANGE: $w r$

tree

RANGE: ang cag? mex sag? sao tree

RANGE: col or mex per RANGE: gui per

tree

shrub

\begin{tabular}{ll} 
RANGE: per sag? sao & \multicolumn{1}{c}{$\begin{array}{l}\text { tree } \\
\text { epiph } \\
\text { RANGE: end }\end{array}$} \\
RANGE: bol ecu & $\begin{array}{l}\text { tree } \\
\text { hemiep } \\
\text { tree }\end{array}$ \\
RANGE: $w r$ & wh \\
RANGE: $w r$ & tree \\
RANGE: $w r$ & tree \\
RANGE: cag? mex sag? & epiph \\
RANGE: $w r$ & tree \\
RANGE: cr hon & epiph \\
shrub & tree
\end{tabular}

RANGE: ang cag? mex sag? herb RANGE: $w r$ herb

RANGE: ecu hon lag per epiph

RANGE: owt tree

RANGE: OWt

RANGE: or

RANGE: $c r$

RANGE: ang? cag? col fla gag tree

gui hon sag? sao ven

RANGE: bel gua

hemiep

RANGE: OWt

RANGE: cag? cr gua

RANGE: cag? sal

RANGE: $\mathrm{COI}$

RANGE: col $\mathrm{cr}$ ven

000m: $0-1$

RANGE: OWt tree

tree

tree

tree

tree

hemiep

tree 
TAXON

64 (43) MORACEAE cont.

eximia Spr.

glabrata H.B.K.

hartwegii (Miq.) Miq.

insipida Willd.

* Iutea VahI

macbride $i$ Stand I.

maxima Mill.

*microcarpa L. f.

nymphaeaefolia Mill.

obtusifolia H.B.K.

*oerstediana (Miq.) Miq.

paraensis (Miq.) Miq.

perforata $\mathrm{L}$.

pertusa L. $f$.

popenoei Standl.

* retusa L.

* schippii Standl.

tonduzii stand I.

trachelosyce Dugand

trigonata $\mathrm{L}$.

tuerckheimi i StandI.

*turrialbana Burger

*yoponensis Desv.

* zarzalens is Dugand

*Hel icostyl is

*tomentosa (P. \& E, ) Rusby

* tovarensis (KI. \& Karst.) Berg

Maquira

* costaricana (Standl.) C. Berg

*Morus

*insignis Bur.

* Nauc leops is

*ulei (Warb.) Ducke

Olmedia

aspera R. \& $P$.

Perebea

*angustifolia (P. \& E.) C. Berg
PROV: $\mathrm{CC}$

PROV: ca

000m: $0-1$

PROV: bo ca cc $000 \mathrm{~m}: 0-1$

PROV: bo ca cc ch cn 000m: $0-1$ da pa

PROV: ca

PROV: bo en $000 \mathrm{~m}: 0-1$

PROV: bo ca cc pa $000 \mathrm{~m}: 0-1$

PROV: $\mathrm{ca} \mathrm{cc} \quad 000 \mathrm{~m}: 0-1$

PROV: ca cn da $v r$ 000m: $0-1$

PROV: ca sa $v r \quad 000 \mathrm{~m}: 0-1,1-2$

PROV: cc cn da pa 000m: $0-1$

PROV: ca cn da pa $000 \mathrm{~m}$ : $0-1$

PROV: ca cc ch da lo 000m: $0-1$

pa $V$

PROV: bo ca ch 000m: $0-1,1-2$

PROV: ca cc pa $\quad 000 \mathrm{~m}: 0-1$

cult.

PROV: ca da he

000m: $0-1$

PROV: bo cn pa

PROV: ca ch

000m: $0-1$

PROV: $000 \mathrm{~m}: 0-1$

PROV: ca cc ch da pa $000 \mathrm{~m}: 0-1$

PROV: bo ca cc ch he $000 \mathrm{~m}$ : $0-1$

PROV: 10

000m: $0-1$

PROV: ca ch 000m: $0-1$

PROV: da 000m: we

PROV: bo

PROV: da

000m: $0-1$

000m: 1-2

PROV: bo ca da pa sa $000 \mathrm{~m}$ : $0-1$

PROV: ch

$000 m: 1-2,2-3$

PROV: cn pa

000m: $0-1$

PROV: bo ca cc ch cn 000m: $0-1$ da $10 \mathrm{pa}$

PROV: cn da pa sa 000m: 0-1
RANGE: $w r$

RANGE: cag

RANGE: $\mathrm{col}$ gua

RANGE: cag mex sag? sao tree

RANGE: afr tree

RANGE: $c r$ per sat tree

RANGE: cag? cub jam mex sag? tree

RANGE: sat $r$ sag? sao trt tree

RANGE: $\mathrm{col} \mathrm{cr}$ sag? sao

Ven

ANGE: cag? col cr gua mex tree

per sag?

RANGE: hon tree

RANGE: cag? mex per sag? sao shrub

RANGE: bah cag? col gag gua shrub

RANGE: jam mex sao

RANGE: bel cag? col tree

RANGE: OWt tree

RANGE: sat tree

RANGE: $\mathrm{col} \mathrm{cr}$ ecu hon tree

RANGE: $c O l \mathrm{cr}$ tree

RANGE: $\mathrm{col}$ gag mex tree

RANGE: bel cag? tree

RANGE: $\mathrm{cr}$

RANGE: col mex ven tree

RANGE: $w r$ tree

RANGE: sat shrub

RANGE: sat tree

RANGE: or nic per tree

RANGE: $\mathrm{col} \mathrm{cr}$ ven tree

RANGE: sat treelet

RANGE: bol $\mathrm{cr}$ sat tree

shrub

RANGE: cag sat tree 
guianensis Aubl.

xanthochyma Karst.

\section{Poulsenia \\ a rmata (Miq.) Standi.}

\section{Pourouma}

\#guianensis Aubl.

johnstonil Woods.

* minor Benoist

oraria Standl, \& Cuatr.

radula Benoist

scobina Benoist

Pseudolmedia

* laevigata Trec.

spuria (Sw.) Griseb.

Sorocea

affinis Hems I.

* cufodontisii Burger

* faustiana Cuatr.

pubivena Hems I.

*trophoides Burger

*--subsp. trophoides

\section{Trophis}

*mexicana (Liebm,) Bur.

racemosa (L.) Urb.
PROV: bo ca pa sa 000m: $0-1$
PROV: bo ca cn da pa $000 \mathrm{~m}: 0-1,1-2$ sa

PROV: bo ca en da pa $000 \mathrm{~m}$ : $0-1$ sa

$\begin{array}{ll}\text { PROV: da } & 000 \mathrm{~m}: 0-1 \\ \text { PROV: ca } & 000 \mathrm{~m}: 0-1 \\ \text { PROV: cc } & 000 \mathrm{~m}: 0-1 \\ \text { PROV: wP } & 000 \mathrm{~m}: \text { we } \\ \text { PROV: wp } & 000 \mathrm{~m}: \text { we } \\ \text { PROV: cc da } & 000 \mathrm{~m}: 0-1 \\ & \\ \text { PROV: da } & 000 \mathrm{~m}: 1-2 \\ \text { PROV: bo } & 000 \mathrm{~m}: 0-1\end{array}$

PROV: bo ca ce ch cn 000m: 0-1 da pa sa vr

PROV: bo ch 000m: $0-1$

PROV: $000 \mathrm{~m}: 0-1$

PROV: bo $\mathrm{ch} \quad 000 \mathrm{~m}: 0-1$

PROV: ch $\vee r \quad 000 m: 1-2$

PROV: $v r \quad$ 000m: $0-1$ PROV: bo ca cc ch cn 000m: $0-1$ da pa $\vee r$

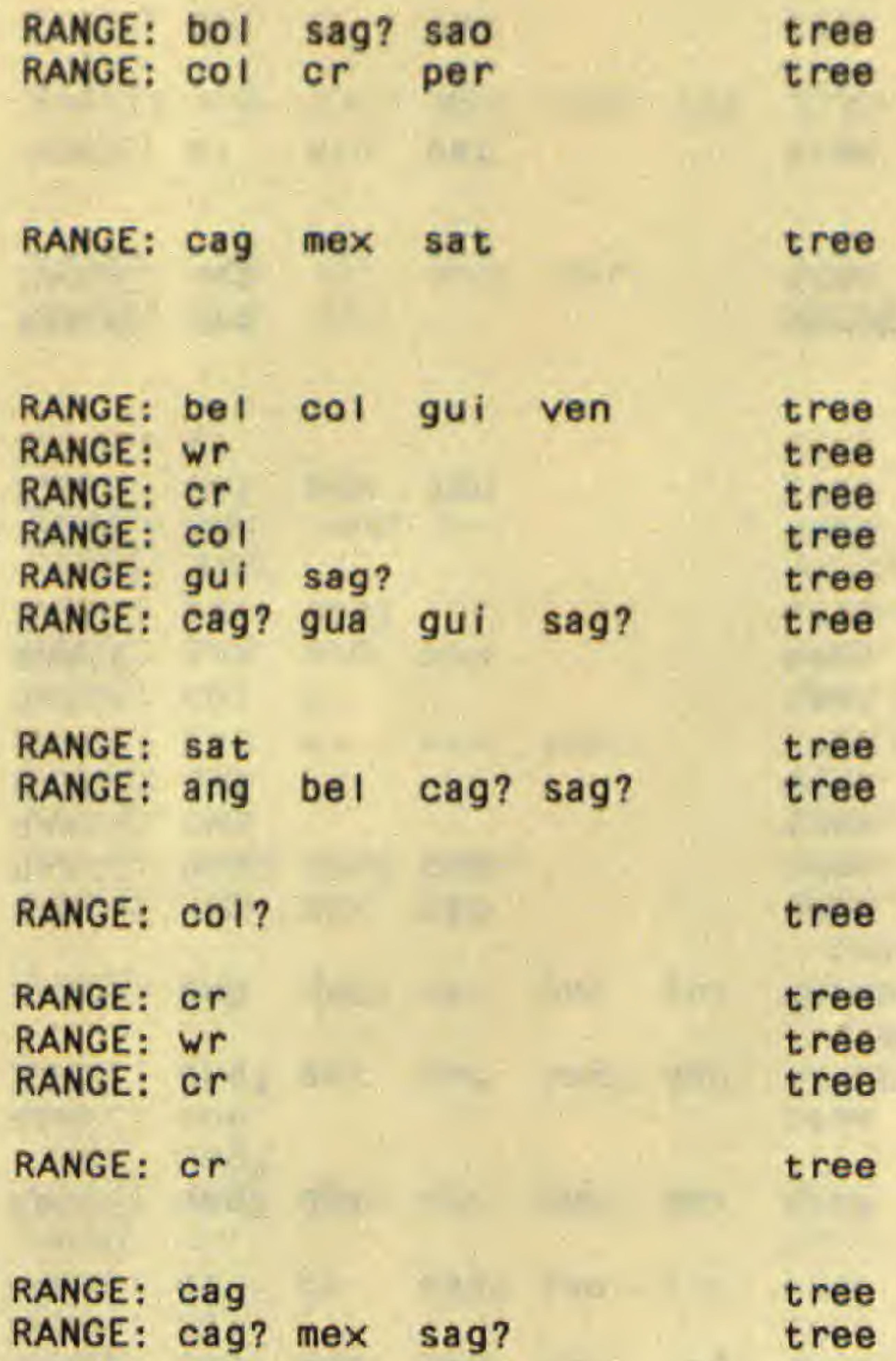

\section{5 (44) URTICACEAE}

\section{Boehmeria}

aspera Wedd.

cylindrica (L.) Sw.

\#nivea Gaud.

pavoni i Wedd.

* ramiflora Jaca.

uImifolia Wedd.
PROV: ch

PROV: bo ca ch

PROV: pa

PROV: $\mathrm{ch}$

PROV: pa vr

PROV: bo ch da $v r$

\section{0m: $1-2$}

$000 \mathrm{~m}: 0-1,1-2$

000m: $0-1$

000m: $1-2$

000m: $0-1,1-2$
RANGE: $o r$ per sag?

RANGE: ang cag mex nag? nao

SaO

RANGE: $\mathrm{col}$

RANGE: bol gua

RANGE: ang

000m: $0-1,1-2,2-3,3+$ RANGE: cag mex

shrub

herb

herb

shrub

shrub

tree

shrub

herb

shrub 
TAXON

65 (44) URTICACEAE cont.

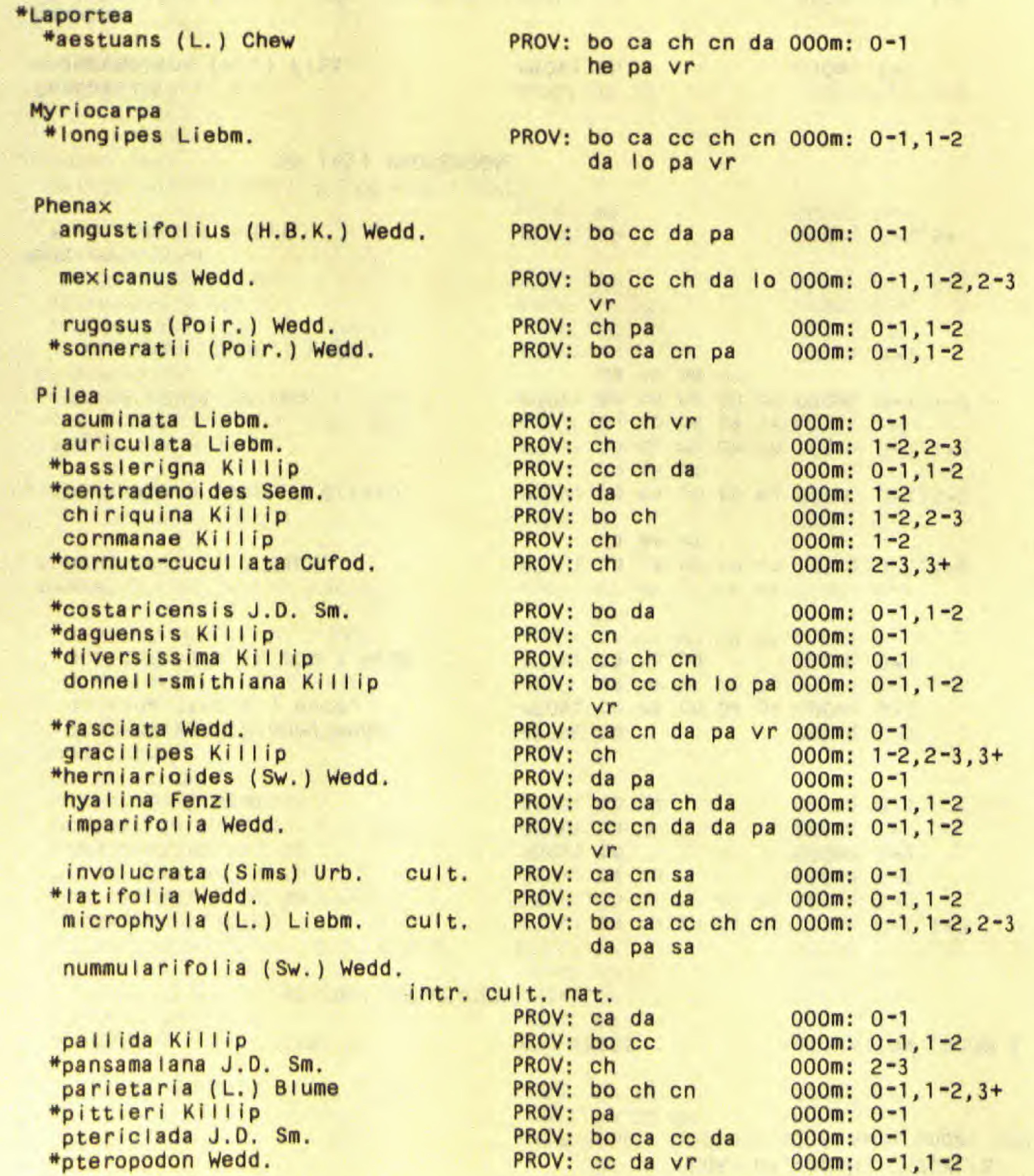

RANGE: $w d t$

herb

RANGE: cag? gua sat

treelet shrub

\begin{tabular}{|c|c|c|c|c|c|c|}
\hline $\begin{array}{l}\text { RANGE: } \\
\text { RANGE: }\end{array}$ & bol & $\begin{array}{l}\mathrm{cr} \\
\operatorname{mex}\end{array}$ & per & sag? & & $\begin{array}{l}\text { shrub } \\
\text { herb } \\
\text { shrub }\end{array}$ \\
\hline $\begin{array}{l}\text { RANGE: } \\
\text { RANGE: }\end{array}$ & $\begin{array}{l}\text { bol } \\
\text { ang }\end{array}$ & $\begin{array}{l}\text { cag } \\
\text { cag }\end{array}$ & mex & sag? & ven & $\begin{array}{l}\text { shrub } \\
\text { shrub }\end{array}$ \\
\hline RANGE: & cag & col & $\operatorname{mex}$ & & & herb \\
\hline RANGE: & $c r$ & gua & mex & 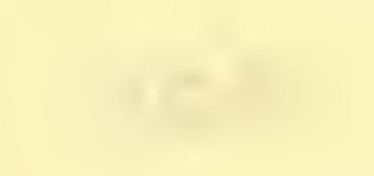 & & herb \\
\hline RANGE: & sat & & & & & herb \\
\hline RANGE: & sat & & & & & herb \\
\hline RANGE: & end & 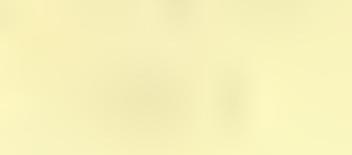 & & & & herb \\
\hline RANGE: & $\mathrm{cr}$ & & & & & herb \\
\hline RANGE: & $\mathrm{cr}$ & & & & & $\begin{array}{l}\text { epiph } \\
\text { herb }\end{array}$ \\
\hline RANGE: & $c r$ & & & & & shrub \\
\hline RANGE: & sat & t & & & & herb \\
\hline RANGE: & $\mathrm{cr}$ & +. & (xt) thit & 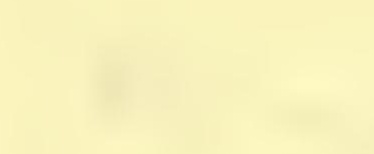 & $(x-1)$ & herb \\
\hline RANGE: & $\mathrm{cr}$ & & & & & herb \\
\hline RANGE: & sat & & & & & herb \\
\hline RANGE: & cag? & gua & & & & herb \\
\hline RANGE: & ang & $\mathrm{cr}$ & fla & & & herb \\
\hline RANGE: & cag? & lag & $\operatorname{mex}$ & sag? & sao & herb \\
\hline RANGE: & col & gui & per & sao & & herb \\
\hline RANGE: & ang & $\mathrm{col}$ & ven & & & herb \\
\hline RANGE: & sat & & & & & herb \\
\hline RANGE: & nWt & & & & & herb \\
\hline RANGE: & ang & cag? & per & ven & & herb \\
\hline RANGE: & $\mathrm{Cr}$ & & & & & herb \\
\hline RANGE: & gua & & & & & herb \\
\hline RANGE: & ang & cag? & gua & & & herb \\
\hline RANGE: & $c r$ & & & & & herb \\
\hline RANGE: & $c r$ & & & & & herb \\
\hline
\end{tabular}


65 (44) URTICACEAE cont.

\begin{abstract}
pubescens Liebm.
purulensis J.D. Sm.

"rhombifolia Kililip

rugosissima Kili ip

* seemannil Killip

trianaeana Wedd.
\end{abstract}

Pouzolzia

guatemalana (Blume) Wedd.

obliqua (Poepp,) Wedd.

occidentalis (Liebm.) Wedd.

\section{Urera}

baccifera (L.) Gaud.

caracasana (Jacq.) Gríseb.

*eggersil hieron.

elata (SW.) Griseb.

laciniata (Goud.) Wedd.

\section{*urtica}

* leptophyl Ia H.B.K.

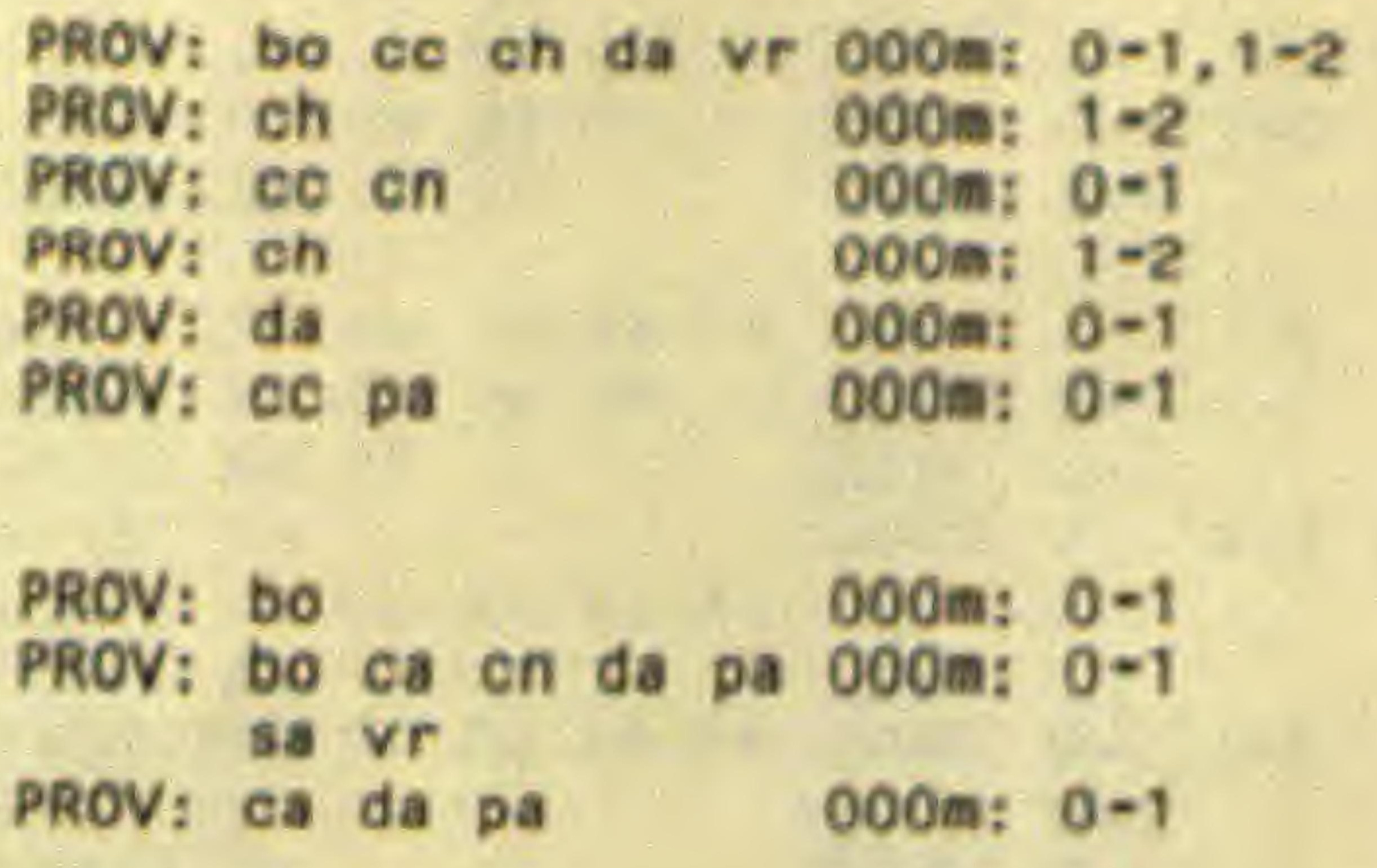

PROV: bo ca ce ch ch 000m: $0-1,1-2$ da pa vr

PROV: bo ca ch cn da 000m: $0-1,1-2$ Io pa vr

PROV: bo ca cc ch cn 000m: 0-1,1-2 da lo pa sa vr

PROV: bo ca ce ch cn 000m: $0-1,1=2$ da lo pa vr

PROV: ca

000m: $0-1$

PROV: ch

$000 m: 2-3,3+$

66 (45) PROTEACEAE

Panopsis

suaveolens (KI.) Pitt.

Roupa I a

"glaberrima Pitt.

montana Aubi.

*percoriacea A. Gentry

*suaveolens (KI,) Pitt.
PROV: ch

000m: $1-2$

PROV: $\mathrm{ch}$

pa Vr

PROV: da pa

PROV: pa 000m: $0-1$

$000 \mathrm{~m}: 0-1$

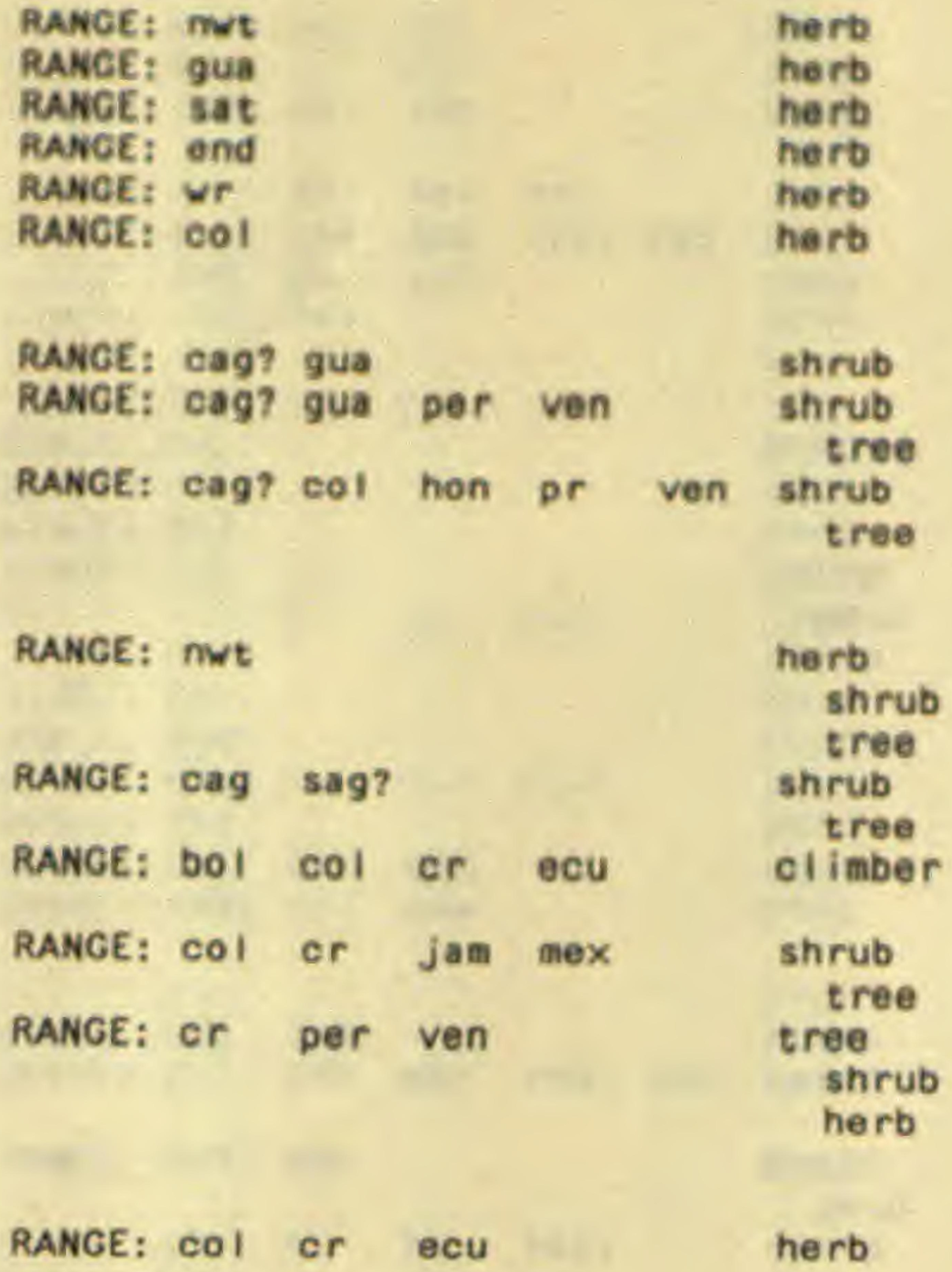

RANGE: col or ecu ven

tree

RANGE: $C r$

RANGE: nWt

tree

tree

RANGE: end

tree

RANGE: ven tree


67 (46) LORANTHACEAE

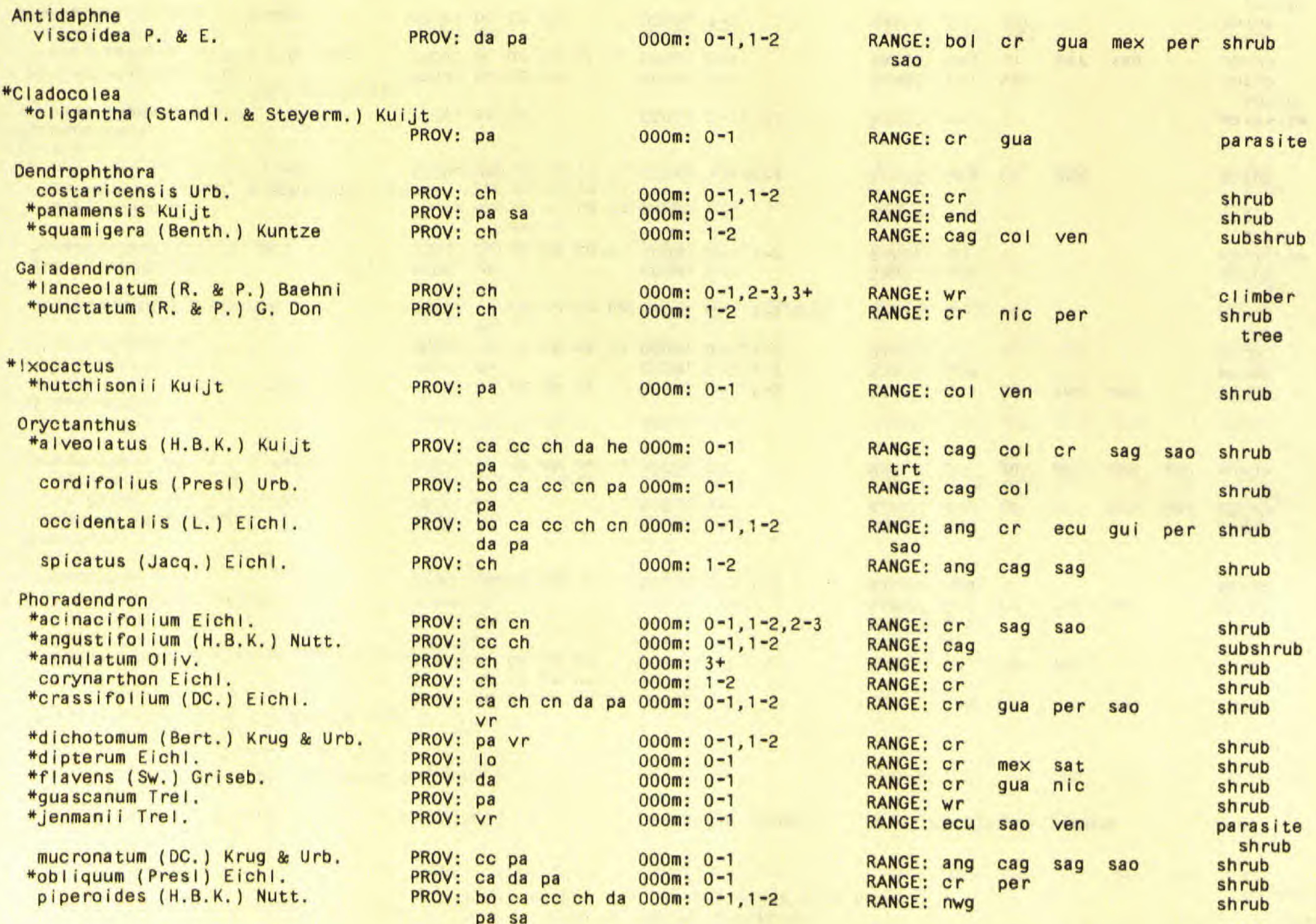


*quadrangulare (H.B.K.) Krug \& Urb.

\section{* robust issimum EichI. tonduzii Trel. \\ trinervium Griseb. \\ undulatum (Pohl) Eichl. woodsoni i Trel.}

\section{Phthi rusa}

pittieri Krause

pyrifolía (H.B.K.) Eichl.

"retroflexa (R, \& P.) Kuijt

Psittacanthus

*calyculatus (DC.) G. Don

*hamul ifer Kuijt

* krameri kuijt

"nodosus (Desr.) G. Don

"pusillus kuijt

* ramiflorus (DC.) G. Don

scheryi Woods.

schiedeanus (Cham, \& Schlecht.) Blume

PROV: bo ca ce en da 000m: 0-1

he lo pa vr

PROV: $c c$ ch to pa

PROV: ch

PROV: bo

PROV: ch

PROV: cc ch pa

000 : $0-1,1-2$

$000 \mathrm{~m}: 0-1$

000m: $1-2$

000 m: $0-1,1-2$

PROV: cn

PROV: ca

000m: 0-1

000m: $0-1$

PROV: bo ca ch he lo 000m: $0-1$ pa $v r$

PROV: ce ch he pa 000m: $0-1,1-2$ PROV: da 000m: $1-2,2-3$

PROV: cc ch cn pa sa 000m: $0-1,1-2$

PROV: $c c$ ch cn da pa 000m: $0-1,1-2,2-3$

PROV: $V r$

000m: $0-1$

PROV: bo ca cc cc ch 000m: $0-1,1-2$ $v r$

000m: $1-2$

PROV: $\mathrm{ch}$

000m: $1-2,2-3$

Struthanthus

"costaricensis StandI.

PROV: ch cn

$000 \mathrm{~m}: 0-1,1-2$

* dichotrianthus Eichl.

* leptostachyus (H.B.K.) G. Don

PROV: bo $\mathrm{cc} \mathrm{Vr}$

000m: $0-1$

PROV: bo ce cn pa sa 000m: $0-1$

marginatus (Desr.) Blume

$$
\mathrm{vr}
$$

$000 \mathrm{~m}: 1-2$

orbicularis (H,B,K.) Blume

PROV: bo ca cc ch da 000m: $0-1$ he lo pa sa vr

"panamensis (Rizz.) Barlow \& Wiens PROV: ch 000m: $1-2$

"quercicola (Cham. \& Schlecht.) Blume

PROV: bo ch da $\mathrm{vr}$

rotundatus Rizz.

PROV: $\mathrm{ch}$

PROV: bo 000m: $0-1,1-2,2-3$

$000 \mathrm{~m}: 0-1$ 000m: $1-2,2-3$

\begin{tabular}{|c|c|c|c|c|c|c|}
\hline ZANGE: & ang & col & er & ven & & shrub \\
\hline RANGE: & or & $\operatorname{mex}$ & ven & & & shrub \\
\hline RANCE: & er & & & & & shrub \\
\hline RANGE: & ang & & & & & shrub \\
\hline RANGE: & bol & or & nic & sao & & shrub \\
\hline RANGE: & end & & & & & shrub \\
\hline RANGE: & end & - & & & $2=$ & shrub \\
\hline $\begin{array}{c}\text { RANGE: } \\
\text { ven }\end{array}$ & ang & col & or & ecu & gui & shrub \\
\hline $\begin{array}{c}\text { RANGE: } \\
\text { ven }\end{array}$ & or & gui & per & sag & sao & shrub \\
\hline RANGE: & $\mathrm{col}$ & cr & $\operatorname{mex}$ & ven & & shrub \\
\hline RANGE: & end & & & & & shrub \\
\hline RANGE: & $w r$ & & & & & $\begin{array}{l}\text { epiph } \\
\text { tree }\end{array}$ \\
\hline RANGE: & cr & ecu & te & & & shrub \\
\hline RANGE: & end & & ( & & & shrub \\
\hline RANGE: & cr & ( & wante & & & $\begin{array}{c}\text { parasite } \\
\text { shrub }\end{array}$ \\
\hline RANGE: & er & & & & & shrub \\
\hline RANGE: & cag & cr & mex & & & shrub \\
\hline & & at & & & & - \\
\hline RANGE: & $\mathrm{cr}$ & & & & & $\begin{array}{c}\text { parasite } \\
\text { shrub }\end{array}$ \\
\hline RANGE: & gui & ven & & & & shrub \\
\hline RANGE: & cag & $\mathrm{cr}$ & per & sag & the & shrub \\
\hline RANGE: & $\mathrm{cr}$ & sao & 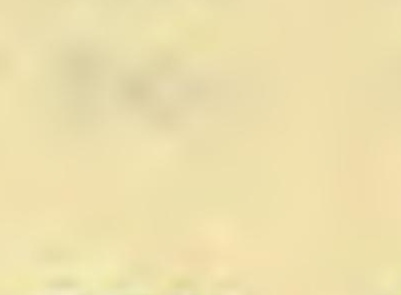 & $x=$ & & $\begin{array}{l}\text { epiph } \\
\text { shrub }\end{array}$ \\
\hline RANGE: & cag & $\mathrm{col}$ & ecu I & per & sao & climber \\
\hline RANGE: & end & & & & & shrub \\
\hline RANGE: & $\mathrm{cr}$ & mex & & & & shrub \\
\hline $\begin{array}{l}\text { RANGE: } \\
\text { RANGE: }\end{array}$ & $\begin{array}{l}\text { sat } \\
\text { cr }\end{array}$ & $7 y$ & 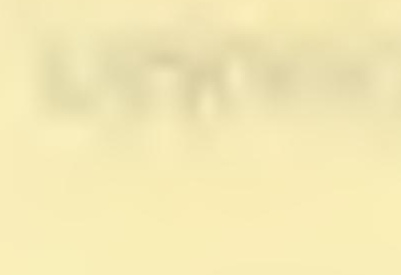 & & & $\begin{array}{l}\text { cl imber } \\
\text { shrub }\end{array}$ \\
\hline
\end{tabular}


TAXON

FAMILY

PROVINCES

ELEVATION (OOOM, )

RANGE OUTSIDE OF PANAMA

HAB IT

71 (47) OPILIACEAE

Agonand ra

brasiliensis B. \& H.

PROV: cc

000m: $0-1$

RANGE: cag sag

tree

72 (48) OLACACEAE

\section{Heisteria}

*acuminata (H. \& B.) Engl.

concinna StandI.

costaricensis J.D. Sm.

* cyanocarpa Poepp.

macrophyl la Oerst.

*scandens Ducke

\section{Minquartia}

guianensis Aubl.

Schoepfia

schreberi J.F. Gmel.

*vacciniiflora HemsI.

\section{Ximenia}

americana $\mathrm{L}$.
PROV: bo ca cc ch cn 000m: $0-1,1-2$ da he pa sa vr

PROV: ca ch cn da he 000m: $0-1$

lo pa vr

PROV: bo ca en sa $000 \mathrm{~m}: 0-1$

PROV: bo

000m: $0-1$

PROV: bo ca cc ch cn 000m: $0-1,1-2,2-3$

pa $v r$

PROV: bo ca ch en 000m: $0-1,1-2$

PROV: bo ca ch

000m: $0-1$

PROV: cc ch pa $v r$ 000m: $0-1,1-2$

PROV: ch

000m: $1-2$

PROV: bo ca ch da he 000m: $0-1$ pa

73 (49) BALANOPHORACEAE

\section{Corynaea}

crassa Hook, f.

--var. sprucei (Eichl.) B. Hansen

PROV: ch

000m: $3+$

Helosis

* cayennens is (SW.) Spr.

*---var. cayennens is

PROV: ca ce da $\mathrm{vr}$

000m: $0-1,1-2$

\#--var. mexicana (Liebm.) B. Hansen

PROV: ch pa

000m: $2-3$

\begin{tabular}{|c|c|c|c|c|c|c|}
\hline $\begin{array}{l}\text { RANGE: } \\
\text { RANGE: }\end{array}$ & $\begin{array}{l}\mathrm{col} \\
\mathrm{cr}\end{array}$ & $\mathrm{cr}$ & $\operatorname{mex}$ & & & $\begin{array}{l}\text { shrub } \\
\text { tree } \\
\text { tree }\end{array}$ \\
\hline RANGE: & $\mathrm{cr}$ & & & & & shrub \\
\hline RANGE: & $\mathrm{col}$ & $\mathrm{cr}$ & sty & & & $\begin{array}{c}\text { shrub } \\
\text { tree } \\
\text { shrub }\end{array}$ \\
\hline RANGE: & cag & 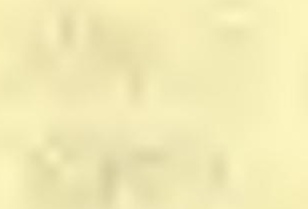 & 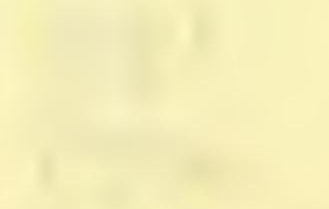 & & Lat & \\
\hline RANGE: & cr & per & sao & & & $\begin{array}{c}\text { shrub } \\
\text { tree }\end{array}$ \\
\hline & $1=-1$ & 2049 & 1. & & & \\
\hline RANGE: & $\mathrm{cr}$ & ecu & nic & sao & & tree \\
\hline RANGE: & ang & cag & $\mathrm{cr}$ & fla & sag & $\begin{array}{c}\text { shrub } \\
\text { tree }\end{array}$ \\
\hline RANGE: & $w r$ & (2) & 1 1 & & & tree \\
\hline & 1.15 & & & & & \\
\hline RANGE & ow & & & & & tree \\
\hline
\end{tabular}

RANGE: bol col cr ecu per parasite
ven
RANGE: bol cag cr mex sag root sao
parasite

RANGE: cag col cub ecu mex parasite 
73 (49) BALANOPHORACEAE cont.

Langsdorffia

hypogaea Mart.

74 (50) ARISTOLOCKIACEAE

\begin{tabular}{|c|c|c|c|c|c|c|c|c|c|}
\hline anguiclida Jacq. & & PROV: & co & 10 & & & & 000n: & $0-1$ \\
\hline "constricta Criseb. & & PROV: & bo & en & pa & vr & & 000n: & $0-1,1-2$ \\
\hline "cordifiora H.B.K. & & PROV: & bo & ca & $\mathrm{ce}$ & da & pa & 000a: & $0-1$ \\
\hline grandifiora SW. & & PROV: & en & da & pa & & & 000an: & $0=1$ \\
\hline Inflata H.B.K. & & PROV: & ca & $\mathrm{CC}$ & he & pa & & 000m: & $0-1$ \\
\hline maxima Jacq. & & PROV: & ca & pa & & & & 000m: & $0-1$ \\
\hline odoratissima L. & & PROV: & bo & ce & da & & & 000): & $0-1$ \\
\hline panamens is Standi. & & PROV: & ca & ch & en & da & & 000m: & $0-1$ \\
\hline *pelferi Barringer & & PROV: & on & & & & & 000m: & $0-1$ \\
\hline "pilosa H.B.K. & & PROV: & bo & $\mathrm{ca}$ & en & da & Da & 000急: & $0-1$ \\
\hline "ringens Vahl & cult. & PROV: & pa & ( & & & & 000m: & $0-1$ \\
\hline "tonduzif Schmidt & & PROV: & $\begin{array}{l}\text { ca } \\
\text { pa }\end{array}$ & ce & $\mathrm{ch}$ & da & 10 & 000m: & $0-1$ \\
\hline trflobata L. & & PROV: & Do & ca & & & & 000า: & $0-1$ \\
\hline veraguens is i & & PROV: & da & vr & & ( & & 000m: & $0-1$ \\
\hline
\end{tabular}

75 (50A) RAFFLESIACEAE

\section{Apodanthes \\ caseariae Poit. \\ * flacourtiae Karst.}

Pilostyles

blanchetii (Gardn,) R. Br.

77 (51) POLYGONACEAE

\begin{tabular}{|c|c|c|c|c|c|c|}
\hline $\begin{array}{l}\text { ANGE: } \\
\text { niC }\end{array}$ & per & $\begin{array}{l}\text { col } \\
\operatorname{sac}\end{array}$ & $\begin{array}{l}\text { or } \\
\text { ven }\end{array}$ & gua & hon & clisber \\
\hline RANCE: & er & nie & & & & elinber \\
\hline RANIGE: & $\mathrm{col}$ & gui & ven & & & el inbor \\
\hline RANGE: & ang & cag & or & sex & & climber \\
\hline RANGE: & eag & & & & & cli laber \\
\hline RANGE: & cag & or & sag & & & al imber \\
\hline RANOE: & cag & or & sag & & & ber \\
\hline RANGE: & col & $\operatorname{mex}$ & & & & subshrut \\
\hline RANCE: & end & & & & & ber \\
\hline RANGE: & cag & $\mathrm{cr}$ & sat & & & ber \\
\hline RANGE: & or & cub & gua & jaa & pr & ber \\
\hline RANGE: & cag & or & sat & & & climber \\
\hline & & & & & & \\
\hline & $\mathrm{cr}$ & a & & & & climbi \\
\hline
\end{tabular}

RANGE: bel cr sat

parasite parasite

000m: $0-1$

RANGE: sat

parasite

\section{Antigonon}

grandiflorum (Bertol.) Robinson PROV: WP

guatemalense Meisn.

leptopus H. A. intr. cult.

cult. PROV: Io 000m: $0-1$

000m: $0-1$

RANGE: ang or gua mex nic climber
Sal




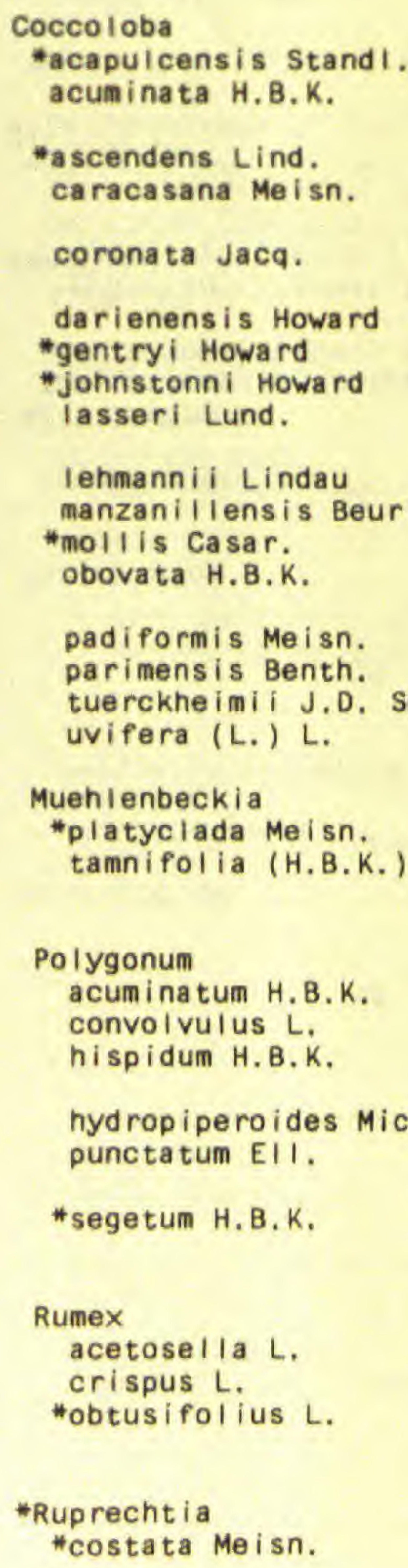

PROV: ca cn 000m: $0-1$ PROV: ca cc cn da pa 000m: $0-1,1-2$

PROV: cc da sa 000m: $0-1$ PROV: ca ch lo pa 000m: $0-1$

PROV: ca cc ch cn da 000m: $0-1$ he pa sa vr

PROV: $\mathrm{CC}$ da

$000 \mathrm{~m}: 0-1$

PROV: cn Pa

PROV: ca cc cn he lo 000m: $0-1$ pa $\vee r$

PROV: bo $\mathrm{cc}$ da $\quad 000 \mathrm{~m}: 0=1$

PROV: ca cn da pa 000m: $0-1$

PROV: $000 \mathrm{~m}: 0-1$

PROV: ca cc ch da lo $000 \mathrm{~m}: 0-1$

PROV: ca cc ch da lo 000m: $0-1$ PROV:

PROV: Ca Da $000 \mathrm{~m}: 0-1$

PROV: bo cc en pa $000 \mathrm{~m}: 0-1$

PROV: bo cn sa $000 \mathrm{~m}: 0-1$

PROV: ch

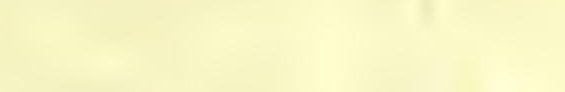

PROV: ch

$000 \mathrm{~m}: 1-2$
PROV: ca en 000m: $0-1$
PROV: ch 000m: $1-2$
PROV: ca ch cn pa 000m: $0=1$
PROV: bo ca lo $000 \mathrm{~m}$ : $0-1$
PROV: ca cc ch cn da 000m: $0-1,1-2$
pa
PROV: cn 10
000m: $0-1$

PROV: ch

PROV: ch

PROV: ch

000m: $0-1,1-2$

000m: $1-2$

000m: $2-3,3+$

\begin{tabular}{|c|c|c|c|c|c|c|}
\hline RANGE: & cag & mex & & & & tree \\
\hline RANGE: & col & $\mathrm{cr}$ & ecu & gui & per & shrub \\
\hline $\begin{array}{r}\text { SaO } \\
\text { RANGE: }\end{array}$ & $\begin{array}{l}\text { Ven } \\
\text { I aq }\end{array}$ & & & & & climber \\
\hline RANGE: & col & $\mathrm{cr}$ & gua & mex & nic & tree \\
\hline sa I & ven & & & & & \\
\hline $\begin{array}{c}\text { RANGE: } \\
\text { ven }\end{array}$ & $\mathrm{col}$ & gua & lag? & per & trt & shrub \\
\hline RANGE: & $w r$ & & & & & tree \\
\hline RANGE: & $w r$ & 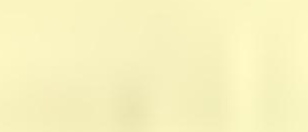 & & 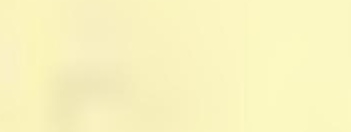 & 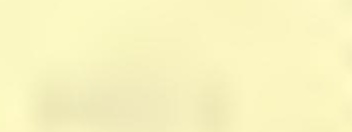 & tree \\
\hline RANGE: & $w r$ & & & & & treelet \\
\hline RANGE: & end & & & & & tree \\
\hline RANGE: & $\mathrm{col}$ & $\mathrm{cr}$ & ven & & & tree \\
\hline RANGE: & end & & & & & tree \\
\hline RANGE: & $\mathrm{col}$ & sao & , & $x^{2}$ & it & tree \\
\hline RANGE: & $\mathrm{col}$ & $\mathrm{cr}$ & & & & shrub \\
\hline RANGE: & col & cr & ven & & & tree \\
\hline RANGE: & col & per & sao & & & tree \\
\hline RANGE: & $\mathrm{cr}$ & gua & hon & nic & & tree \\
\hline RANGE: & ang & cag & $\operatorname{mex}$ & sag? & & tree \\
\hline RANGE: & $w r$ & & & 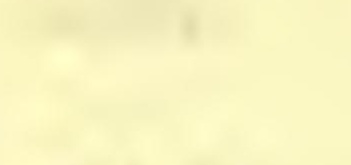 & & shrub \\
\hline $\begin{array}{c}\text { RANGE: } \\
\text { Sao }\end{array}$ & cag & $\mathrm{col}$ & $\mathrm{cr}$ & mex & sag? & climber \\
\hline RANGE: & cag & gua & mex & sag & sao & herb \\
\hline RANGE: & cag & eur & nag & sag & & herb \\
\hline RANGE: & ang & cag & sag & & & $\begin{array}{l}\text { aquat } \\
\text { herb }\end{array}$ \\
\hline RANGE : & cag & nag & nao & sag & & herb \\
\hline RANGE: & cag & nag & nao & sag & sao & herb \\
\hline RANGE: & cag & gua & mex & & & $\begin{array}{l}\text { aquat } \\
\text { herb }\end{array}$ \\
\hline RANGE: & as i & $c r$ & eur & nwg? & & herb \\
\hline RANGE: & as i & $\mathrm{cr}$ & eur & gua & nwg? & herb \\
\hline RANGE: & bol & $\mathrm{col}$ & $\mathrm{cr}$ & gua & $\operatorname{mex}$ & herb \\
\hline sao & ven & & & & & \\
\hline RANGE: & & gua & & & & tree \\
\hline
\end{tabular}


TAXON

77 (51) POLYGONACEAE cont.

\section{Triplaris} americana $L$. cumingiana Fischer \& Meyer
PROV: da

cult. PROV: ca da pa sa
$000 \mathrm{~m}: 0-1$

000m: $0-1$

78 (52) CHENOPODIACEAE

\author{
Chenopodium \\ *a I bum L. \\ ambrosioides L. \\ murale L.
}

pratericola Rydb.

$\begin{array}{lll}\text { intr. } & \text { PROV: } c h & 000 \mathrm{~m}: 1-2 \\ & \text { PROV: } \mathrm{ch} \text { da he pa } & 000 \mathrm{~m}: 0-1,1-2 \\ & \text { PROV: pa } & 000 \mathrm{~m}: 0-1 \\ \text { PROV: pa } & 000 \mathrm{~m}: 0-1\end{array}$

79 (53) AMARANTHACEAE

\section{Achyranthes}

aspera L.

*tenel la Colla

\section{Alternanthera}

* costaricensis Kuntze

halimifolla (Lam.) Standl.

Iaguroides (StandI.) StandI.

* lanceolata (Benth.) Schinz

mexicana (Schlecht.) Hieron.

paronychioides St. Hil.

polygonoides (L.) R. Br.

*pubiflora (Benth.) Kuntze

repens (L.) Gmel.

sessilis (L.) R. Br.

\section{Ama ranthus}

californicus (Moq.) S. Wats. cruentus L.

dubius Mart.

cult.

hybridus L.
PROV: bo ca cc ch he 000m: 0-1 $10 \mathrm{pa}$

PROV: bo ca ch

000m: $0-1$

PROV: bo cn vr $\quad 000 \mathrm{~m}: 0-1$ PROV: WP $000 \mathrm{~m}: 0-1$

PROV: $v r \quad$ 000m: $0-1,1-2$

PROV: WP 000m: $1-2$

PROV: WP 000m: we

PROV: ca ce ch en da $000 \mathrm{~m}: 0-1$ $10 \mathrm{pa}$

PROV: $10 \quad 000 \mathrm{~m}: 0-1$

PROV: ca cn da he lo 000m: $0-1$ pa

PROV: WP 000m: we

PROV: bo ca cc ch cn 000m: $0-1,1-2$ da 10

PROV: pa

PROV: wp $000 \mathrm{~m}$ : we

PROV: bo ca cn da he 000m: $0-1$ pa

PROV: ch da pa $v r$ 000m: $0-1,1-2$

\section{RANGE: col per sag? sao tree}

RANGE: col or ecu sag? tree

$\begin{array}{ll}\text { RANGE: eur nwg } & \text { herb } \\ \text { RANGE: cag cr mex } & \text { herb } \\ \text { RANGE: afr asi eur nao nwg? herb } \\ \text { RANGE: mex nag? sao } & \text { herb }\end{array}$

RANGE: cr gua hon mex sal herb
WdW
RANGE: cr
RANGE: cr


TAXON

79 (53) AMARANTHACEAE cont.

spinosus L.

viridis L.

\section{Blutaparon}

"vermiculare (L.) Mears

\section{Celosia}

a rgentea $L$.

*grandifolia Moq.
PROV: bo ca cc ch cn 000m: $0-1,1-2$ da he lo pa sa

PROV: bo ch pa

000m: $0-1$

PROV: bo ca cn sa

000m: $0-1$

intr. cult.

PROV: bo ca ch pa 000m: $0-1,1-2$

PROV: bo ch

RANGE: cag cr mex wdw

RANGE: nag? owg RANGE: afr bel or fla mex shrub
sag? sao tex

RANGE: af $r$ cag mex

RANGE: per sao

herb

herb

acuminata Mart.

*--var. maximilianii (Moq.) Sohmer

PROV: bo da

000m: $0-1$

*--var. Swansoni i Sohmer

altissima (Jacq.) H.B.K.

\section{PROV: bo $000 \mathrm{~m}: 0-1$}

PROV: bo ca cc ch cn $000 \mathrm{~m}$ : $0-1$

da lo pa sa vr

\section{Cyathula}

achyranthoides (H.B.K.) Moq.

ROV: bo ca cc ch cn $000 \mathrm{~m}$ : $0-1$ da he lo pa sa $v r$

prostrata (L.) Blume intr. nat.

PROV: bo ca $\mathrm{cc}$ ch cn 000m: $0-1$ da pa $v r$

\section{Gomphrena \\ globosa L.}

*serrata L.

\section{I resine}

angustifolia Euphr.

completa UIine \& Bray

diffusa $H$, \& B.

hassleriana chod.

Pfafia

grandiflora (Hook.) R.E. Fries PROV: da

paniculata (Mart.) Kuntze intr. PROV: co

intr. cult.

PROV: WP
PROV: bo ca 000m: $0-1$

PROV: bo ca ch da lo $000 \mathrm{~m}: 0-1$

pa sa $v r$

PROV: ca cc cn da he 000m: 0-1

$000 \mathrm{~m}: 0-1$

PROV: bo ca ce ch da $000 \mathrm{~m}: 0-1,1-2,2-3,3+$ RANGE: cag? gua

lo pa vr

000m: we

$000 \mathrm{~m}: 0-1,1-2$

000m: $0-1$

\begin{tabular}{|c|c|c|c|c|c|c|}
\hline RANGE: & $\mathrm{col}$ & cr & hon & mex & nic & herb \\
\hline per & sag & sao & & & & $\begin{array}{l}\text { shrub } \\
\text { cl imber } \\
\text { herb }\end{array}$ \\
\hline
\end{tabular}

RANGE: cag mex sag sao shrub

climber
RANGE: ang cag gua hon mex herb

RANGE: af $r$ as $i$ herb

RANGE: as i gua wdw? herb

RANGE: ang bol cag? gua mex herb sao sao

RANGE: cag ecu mex sao herb

RANGE: bol col per sao shrub

RANGE: cag? or gui mex per herb sag?

RANGE: per sao sao 
TAXON

FAMILY

PROVINCES

ELEVATION (OOOM.)

RANGE OUTSIDE OF PANAMA

HABIT

79 (53) AMARANTHACEAE cont.

\section{Pleuropeta I um}

pleiogynum (Kuntze) Standi.

sprucei (Hook. f.) Standl.

\section{PROV: ca ch da pa 000m: 0-1}

PROV: bo ch da vr
$000 \mathrm{~m}: 0-1,1-2$

\section{0 (54) NYCTAGINACEAE}

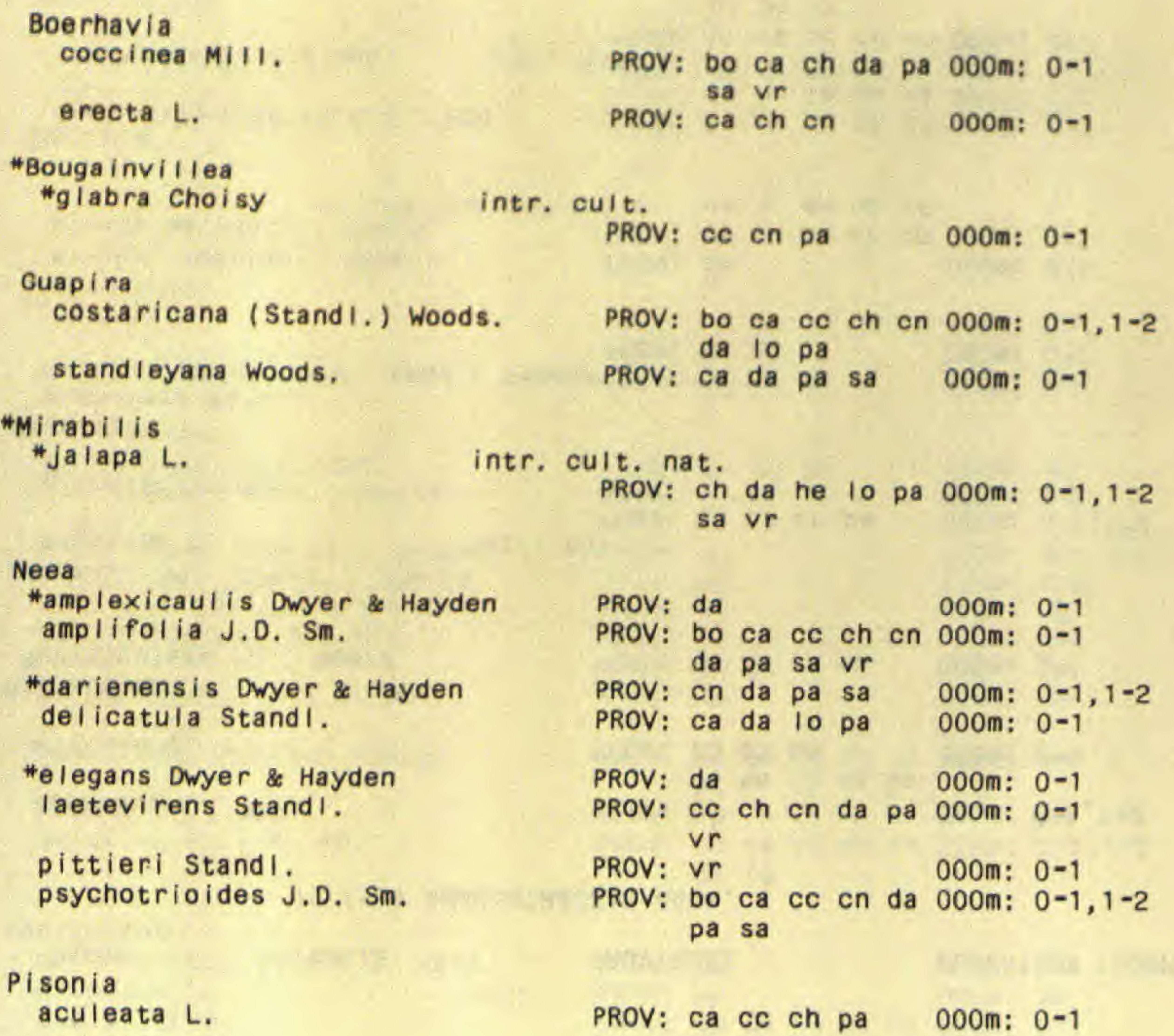

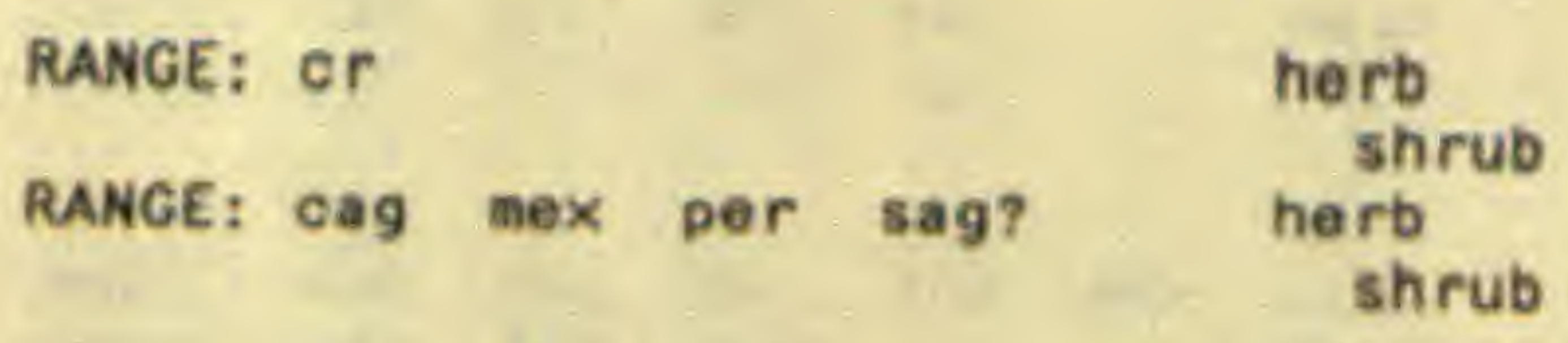

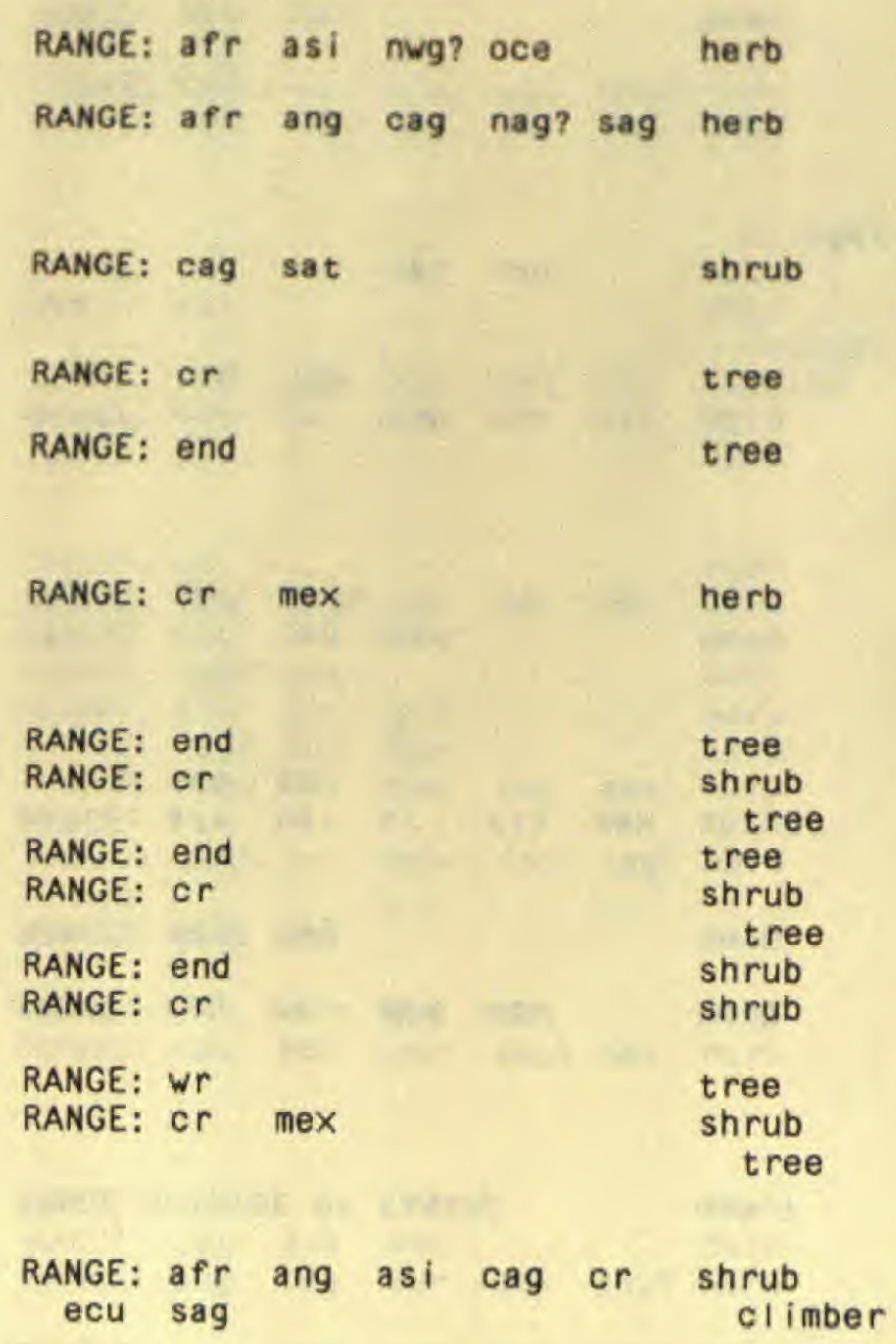


TAXON

81 (55) BATACEAE

Batis

maritima L.
PROV: pa
RANGE: ang cag? nao pr sag? shrub

83 (56) PHYTOLACCACEAE

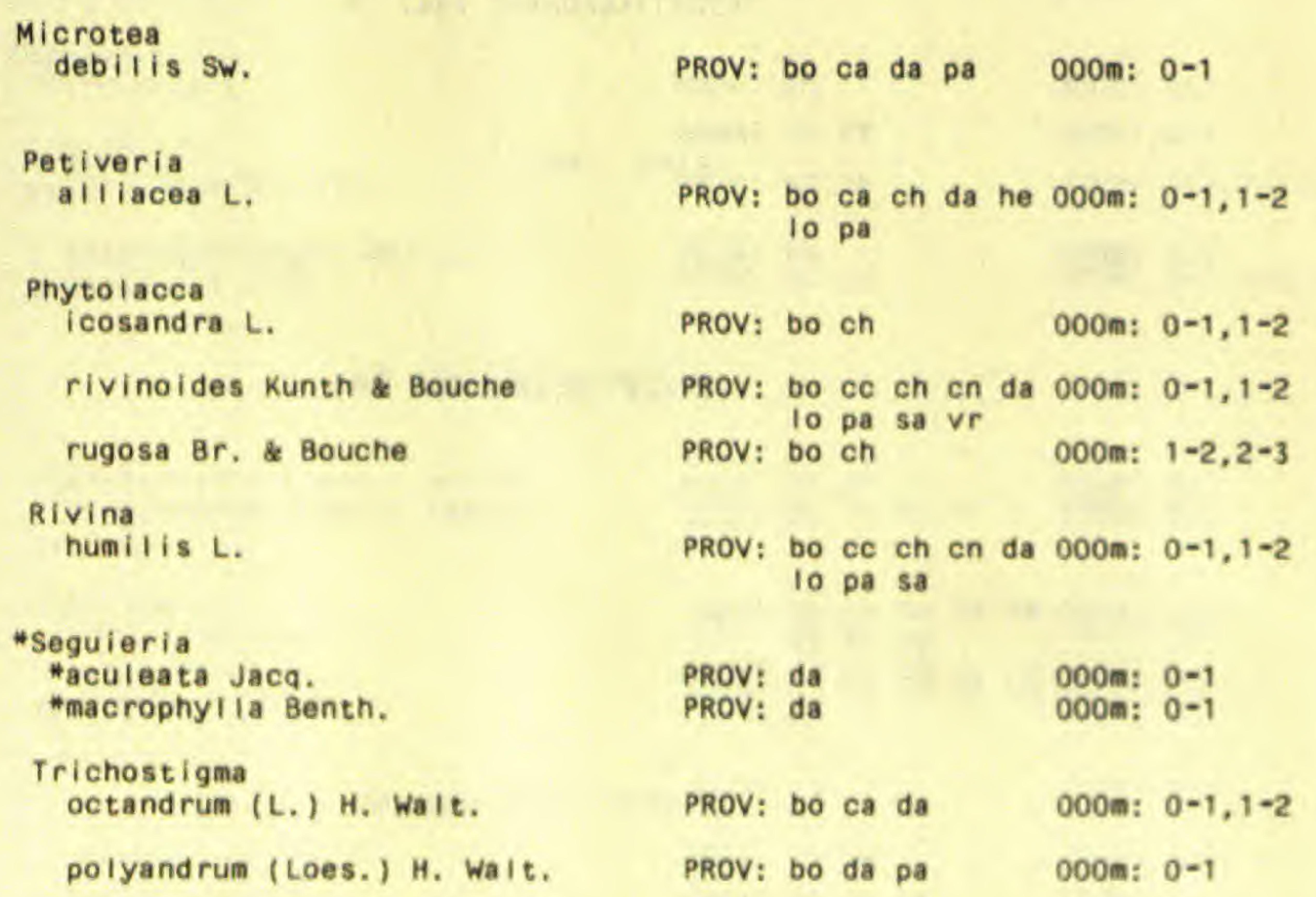

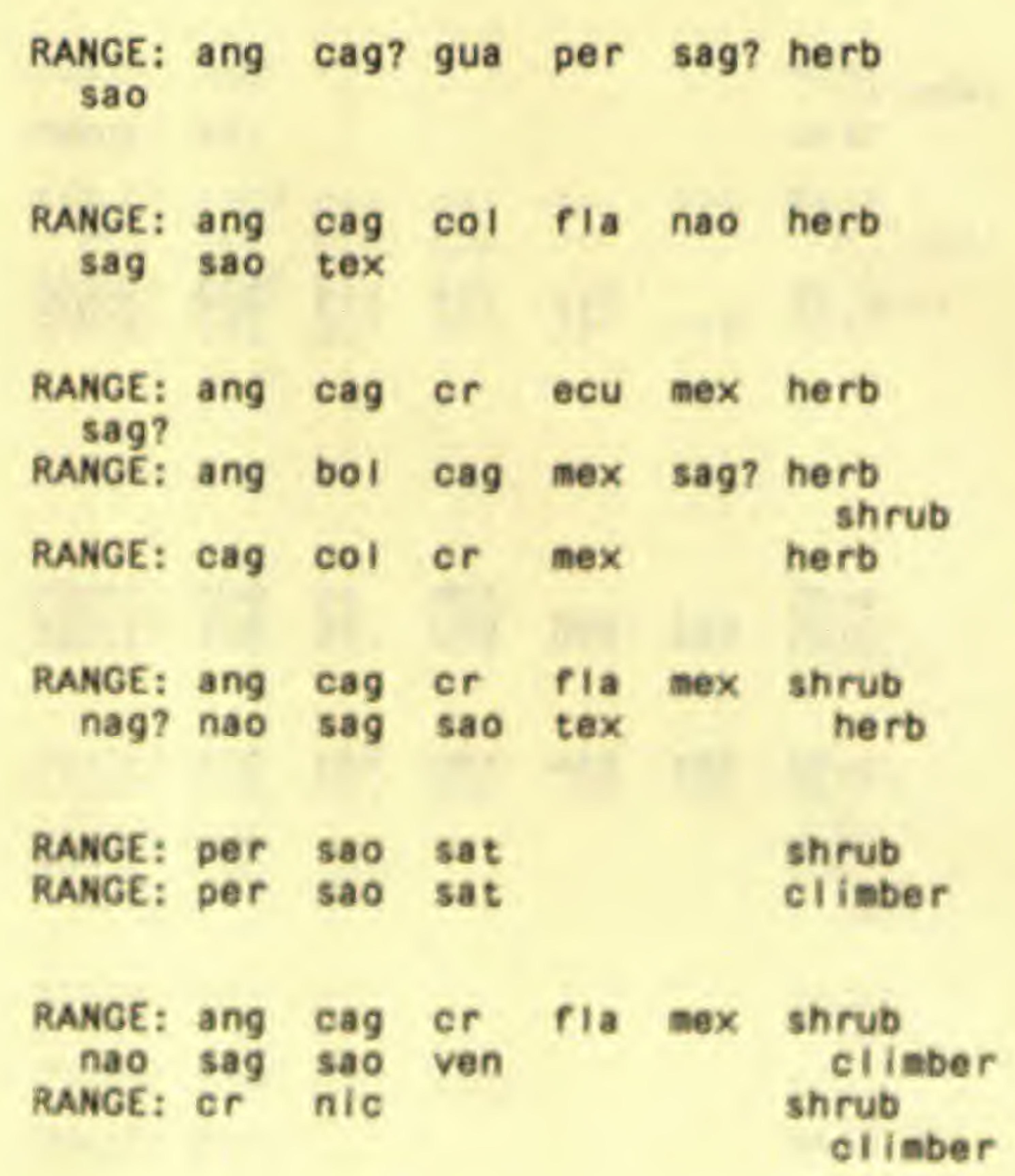

84 (57) AIZOACEAE

Mol I ugo verticellata $L$.

Sesuvium

portulacastrum (L.) L.
PROV: bo ca pa $000 \mathrm{~m}: 0-1$

PROV: bo ca cn he pa 000a: 0-1
RANGE; WdW

nerb

RANCE: wdW 
TAXON

84 (57) AIZOACEAE cont.

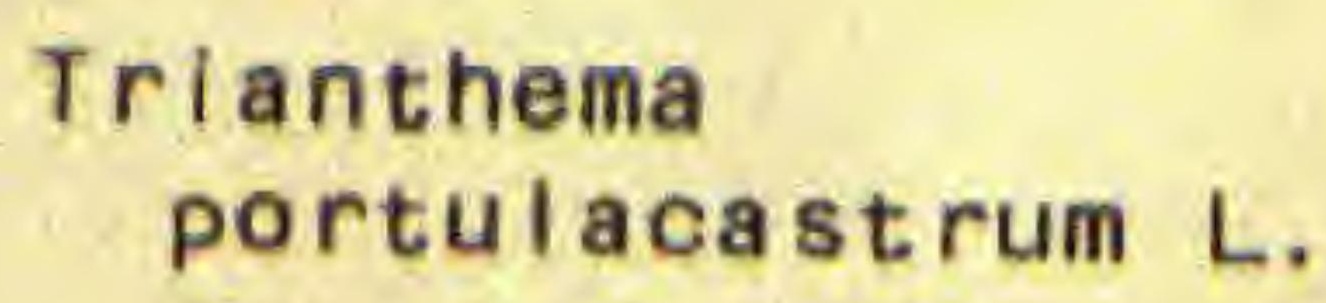

\section{Portulaca \\ oleracea L. \\ *pilosa L.}

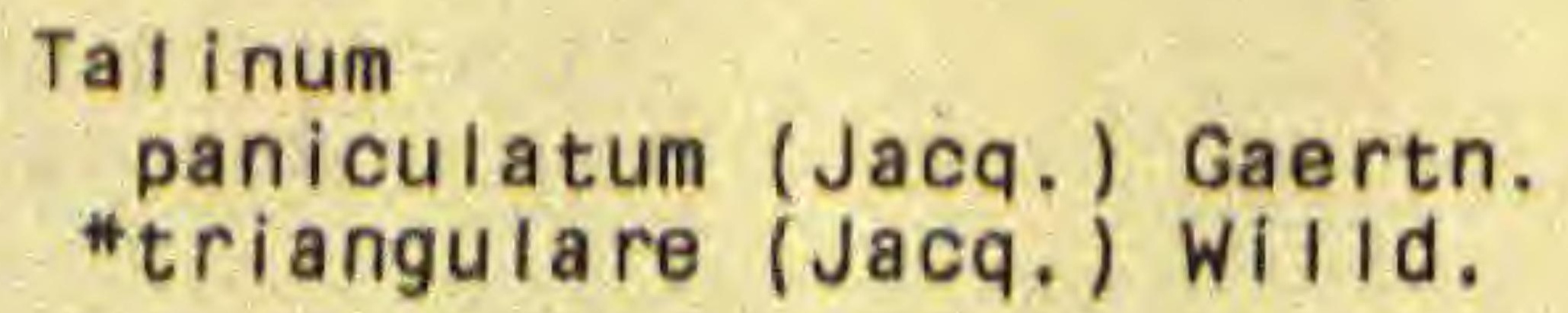

\section{Anredera}

vesicaria (Lam.) Gaertn. f. PROV: pa 000m: 0-1 Basella a Iba L. intr. cult. PROV: ca da

PROV: bo ca ce ch cn 000m: $0-1$ PROV: cc ch cn da pa 000m: $0=1$

\section{PROV: pa}

PROV: ch pa

000m: $0-1$ 000m: $0=1$

000m: $0-1$
RANGE: WdW

herb
RANGE: cag

herb

RANGE: ang

ca

RANGE: ang RANGE: ang asi cag nas cr mex

sag herb

\section{7 (59) CARYOPHYLLACEAE}

\section{Arenaria}

lanuginosa (Mich.) Rohrb.

--var. guatemalensis (Standl. \& Steyerm.) Duke

--var. saxosa (A. Gray) Maguire PROV: ch --subsp. Ianuginosa PROV: ch

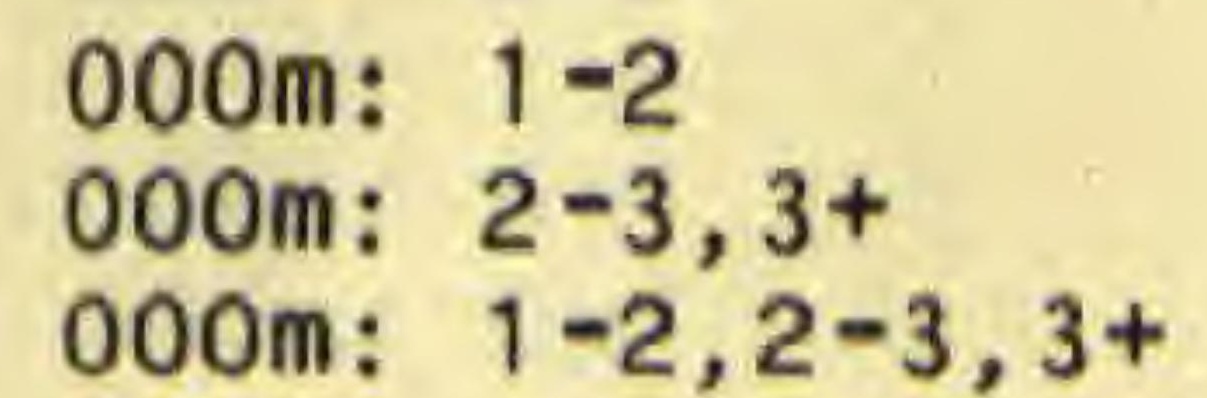

PROV: ch

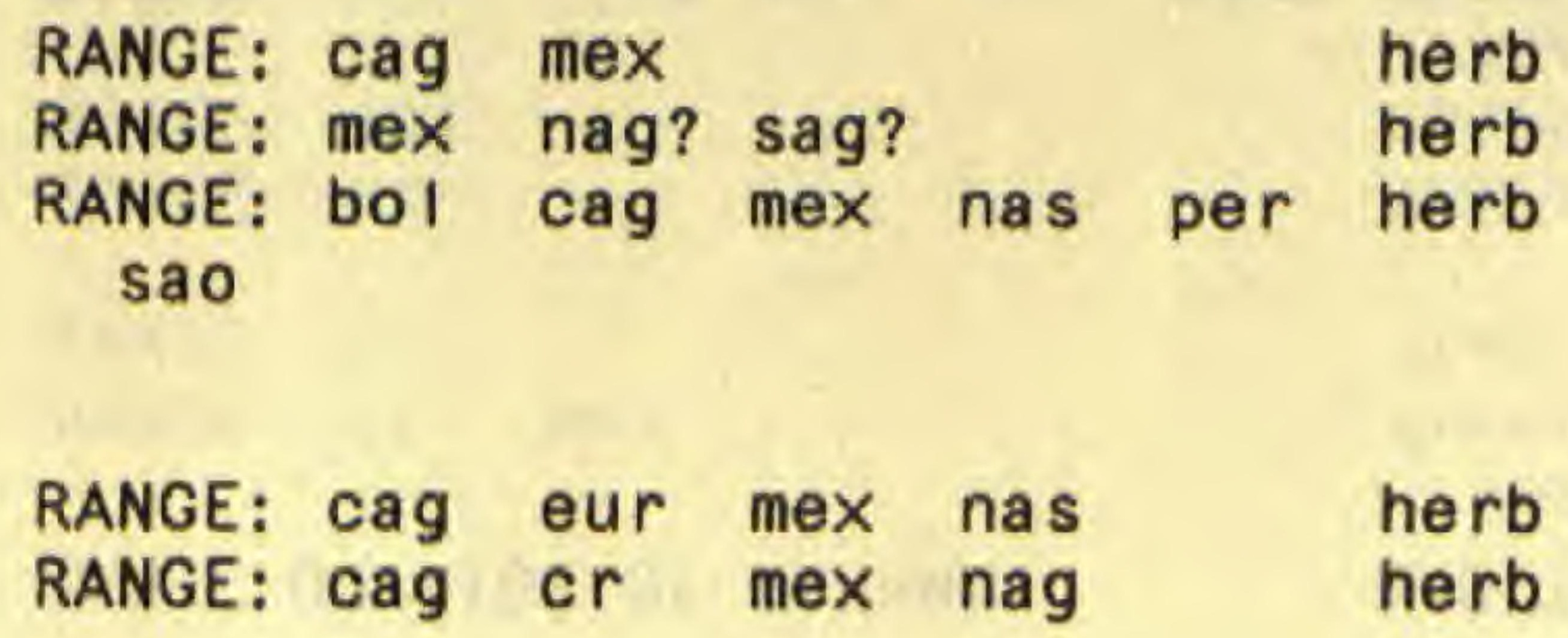


TAXON

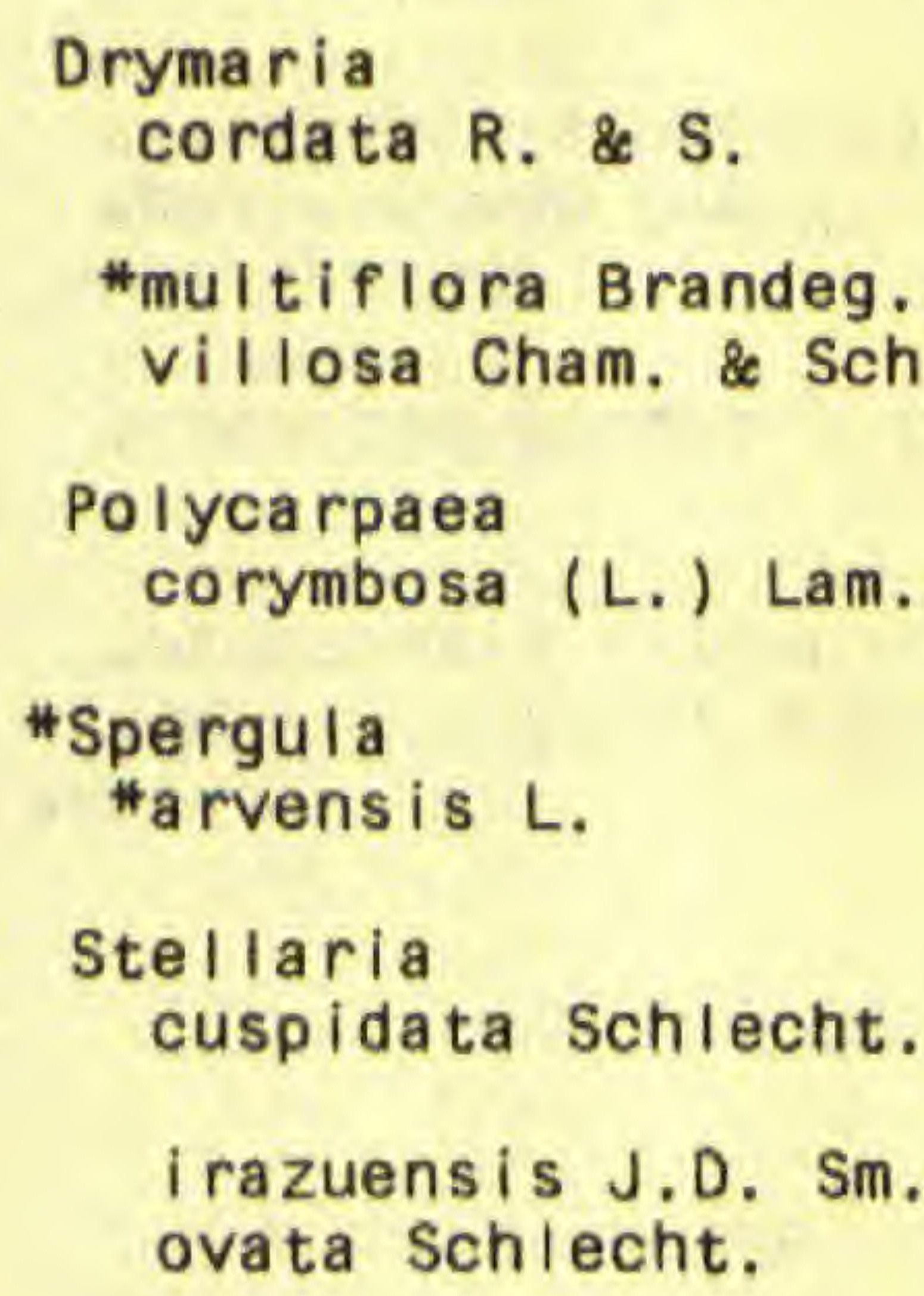

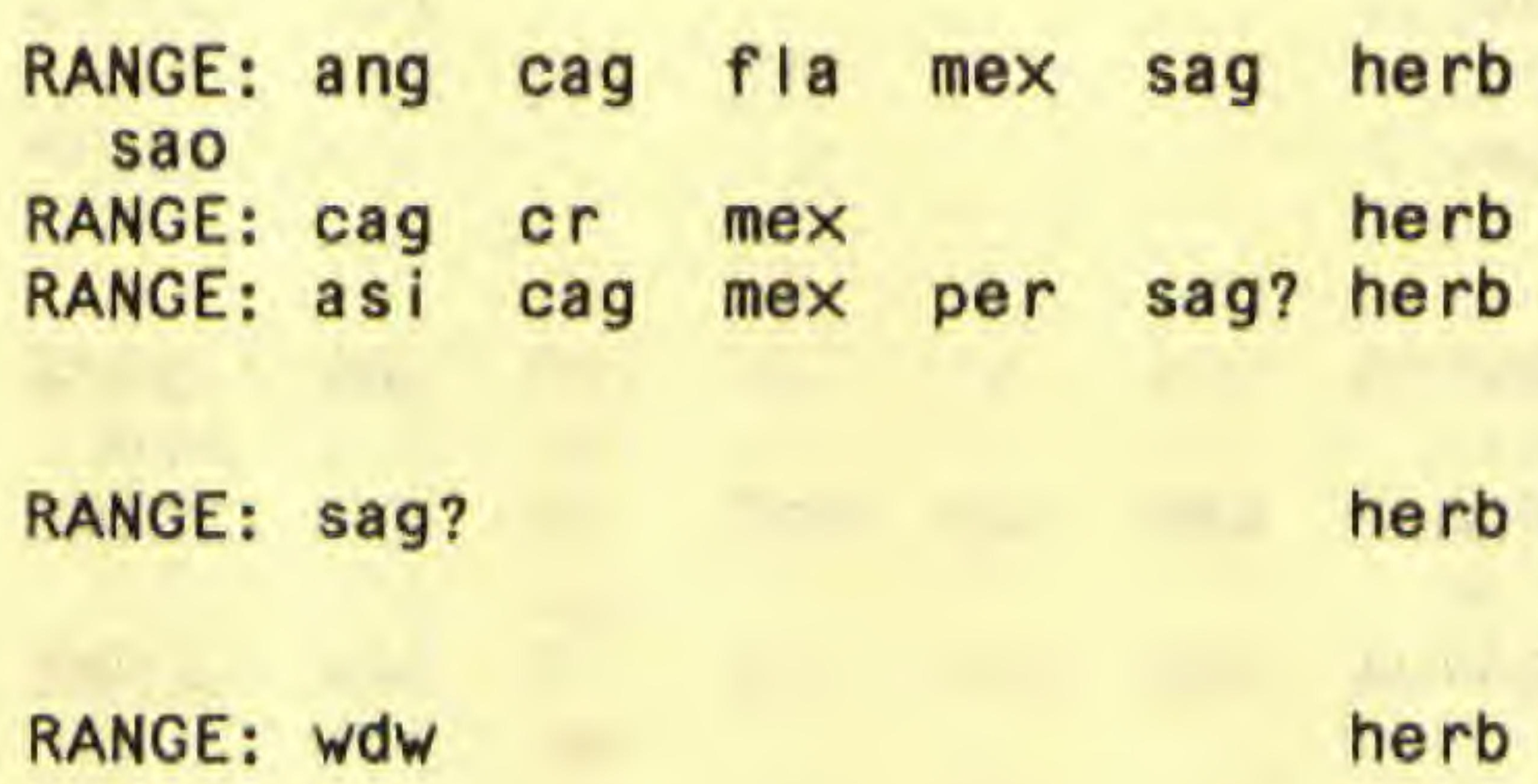

88 (60) NYMPHAEACEAE

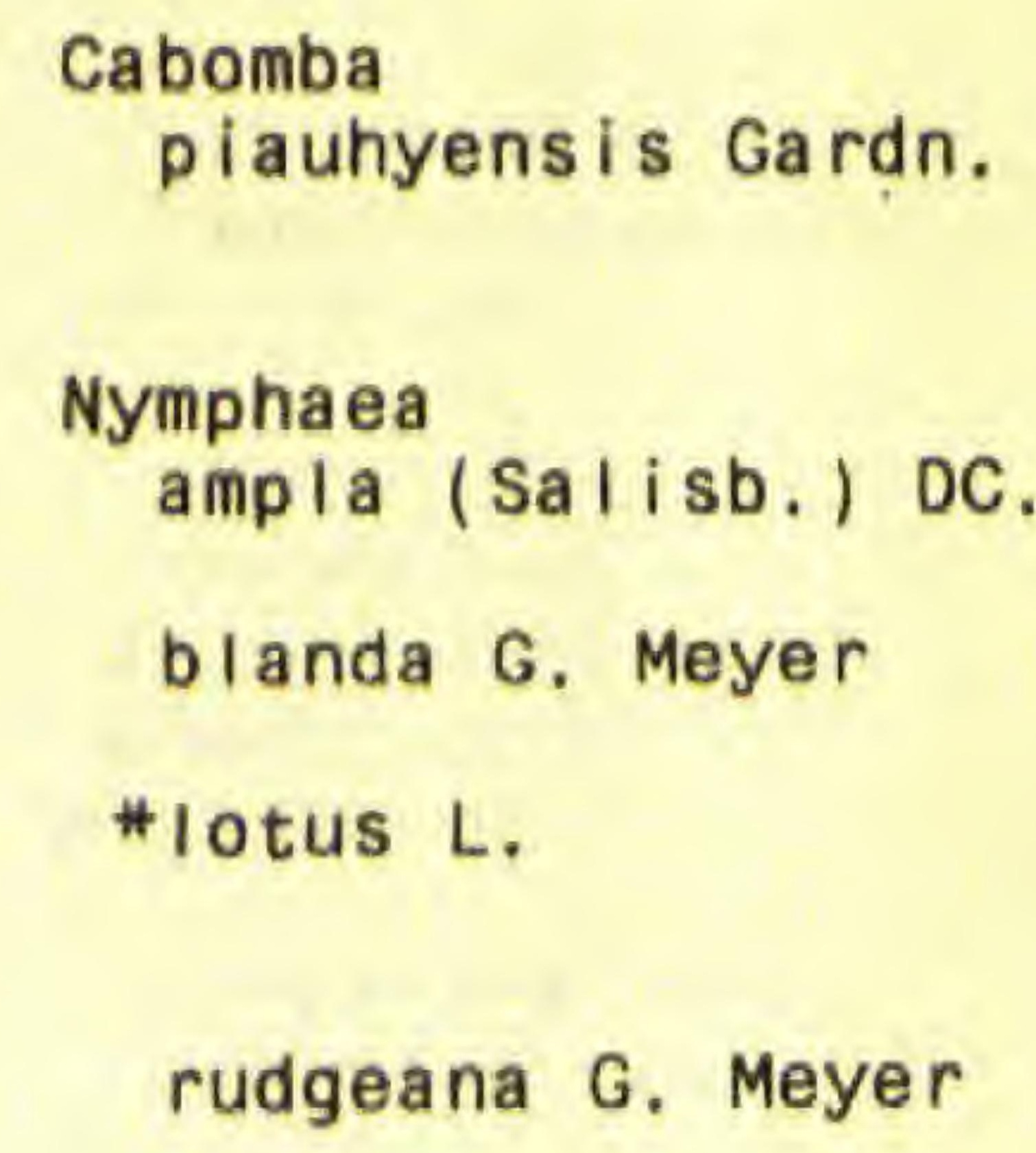

\section{PROV: ca cn da pa 000m: $0-1$}

PROV: ca pa $\quad 000 \mathrm{~m}: 0-1$

PROV: ca ce da pa sa 000m: 0-1 intr. nat.

PROV: pa $000 \mathrm{~m}: 0-1$

PROV: ca pa $000 \mathrm{~m}: 0-1$

89 (61) CERATOPHYLLACEAE

\section{Ceratophy I I um}


91 (62) RANUNCULACEAE

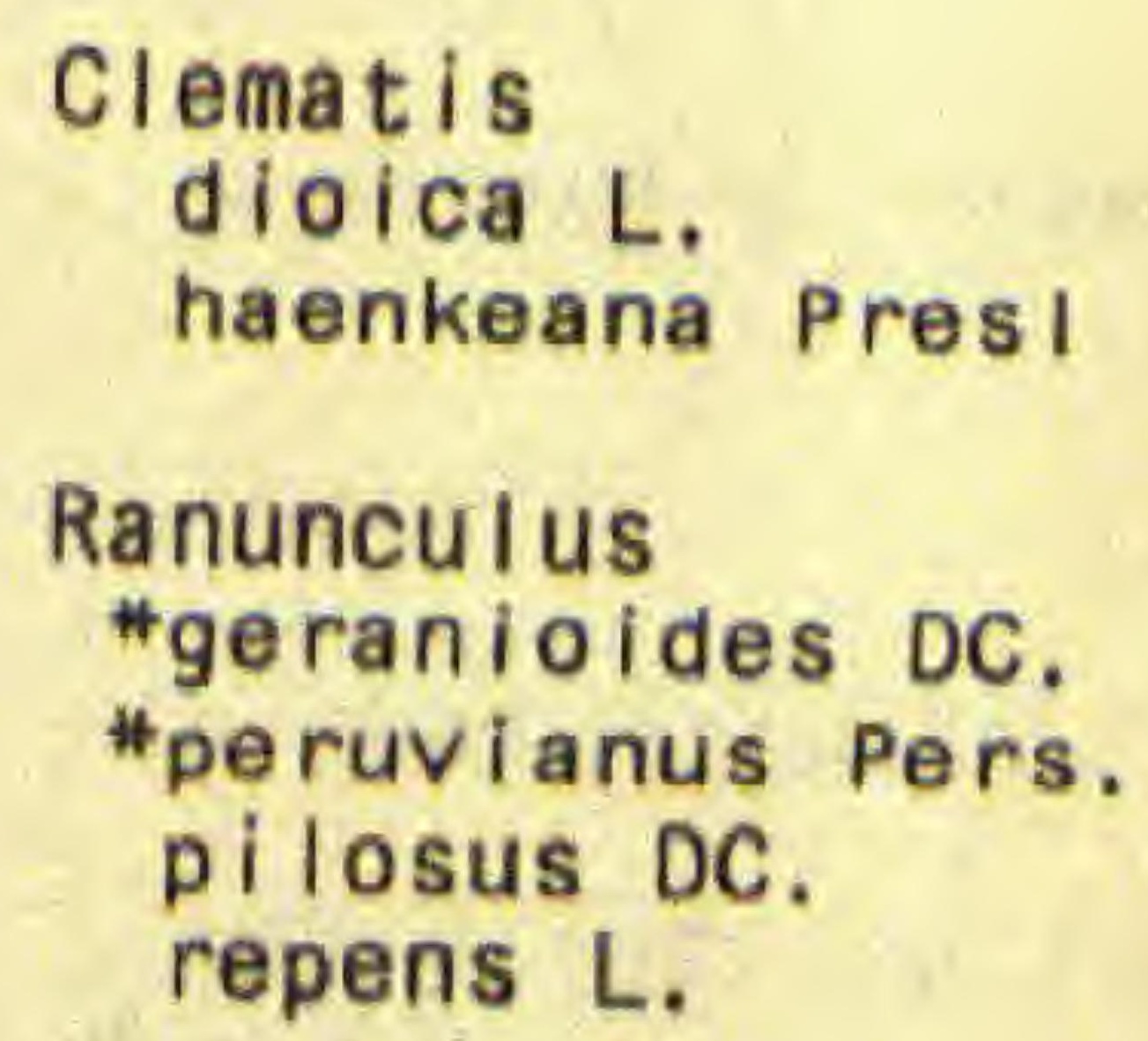

PROV: cc ch pa PROV: ce ch Vr

000m: $0-1,1-2$ 000m: $0-1,1-2$

PROV: ch

PROV: ch

PROV: ch

PROV: ch

$000 \mathrm{~m}: 1-2,2-3$

000m: $3+$

$000 \mathrm{~m}: 1-2,2-3$

Tha I ict rum

panamense Stand I.

viridulum Boivin

PROV: ch

PROV: $\mathrm{ch}$

000m: 1-2

000m: $1-2$

000m: $1-2$

\section{3 (63) BERBERIDACEAE}

\section{Berberis}

nigricans Kuntze 000m: $2-3,3+$

PROV: ch
94 (64) MENISPERMACEAE

\begin{tabular}{|c|c|c|c|c|c|}
\hline RANGE: & ang & cag & $\operatorname{mex}$ & sag & cl imber \\
\hline & cag & $\operatorname{mex}$ & sag & sao & climber \\
\hline RANGE: & $\mathrm{col}$ & ecu & & & herb \\
\hline RANGE: & sat & & & & herb \\
\hline RANGE: & cag & mex & & & herb \\
\hline RANGE: & eur & nag & sag & 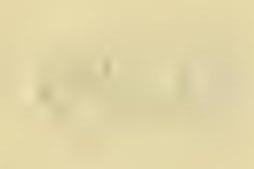 & herb \\
\hline ANGE: & & & & & $\begin{array}{l}\text { herb } \\
\text { herb }\end{array}$ \\
\hline
\end{tabular}

RANGE: $\mathrm{cr}$

shrub

RANGE: $c r$

shrub

RANGE: bel nic

RANGE: bo or

woody

climber

000m: $0-1$

* racemosa (

PROV: ca da

col

climber

000m: $0-1$

000m: $0-1$

000m: we

000m: $1-2$

000m: $0-1$

*chloranthum Diels

*--subsp. isthmicola Kruk. \& Barneby

* reticulatum (Mart.) Eichl.

PROV: da pa

*--subsp, allenil Kruk. \& Barneby PROV: cc cn pa

\& Barneby

PROV: da

*--subsp. glabrescens Kruk. \& Barneby

PROV: da

\#--subsp. idroboi Kruk, \& Barneby PROV: cc ch cn
RANGE: co

RANGE: $\mathrm{CO}$ I

RANGE: per sao

RANGE: ven

RANGE: $\mathrm{col} \mathrm{cr} \quad \mathrm{sag}$

climber
climber
viny
tree
viny
tree
viny
tree


TAXON

\section{Chond rodend ron}

tomentosum $R$, \& $P$.

"toxicoferum (Wedd.) Kruk.

. Mold. PROV: da

PROV: pa

000m: $0-1$

000m: $0-1$

*andromorpha DC.

fasciculata Benth.

*grandifolia Tr. \& PI.

pareira L.

tropaeolifolia DC.

"Curarea
"toxicofera (Wedd.) Barneby \& Kruk.

PROV: ca cn da pa

000m: $0-1,1-2$

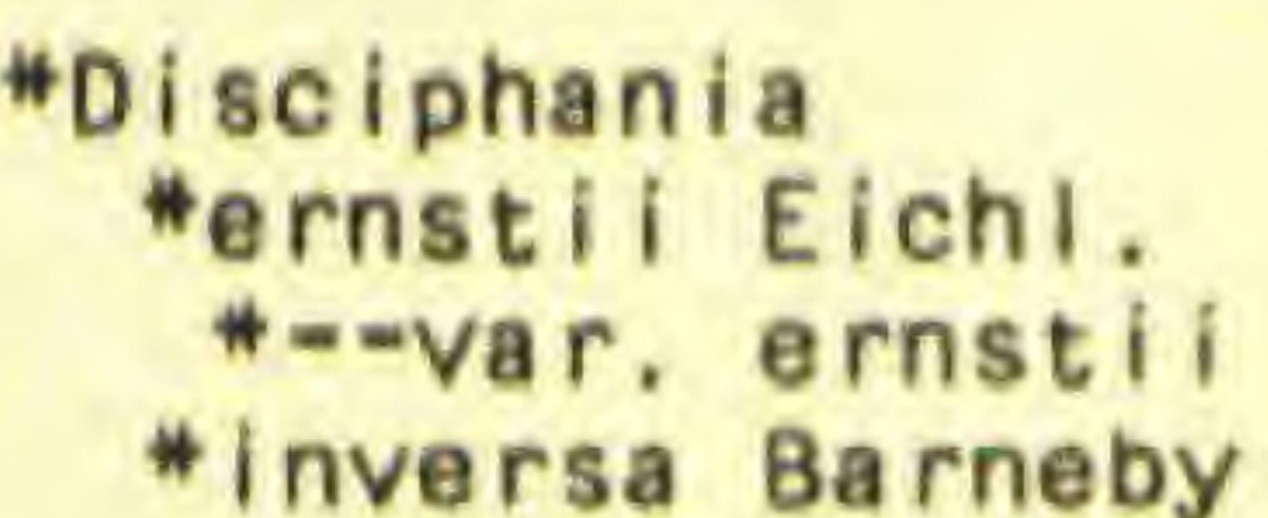

PROV: da pa

PROV: pa

PROV: $\mathrm{CC}$

PROV: 10
PROV: bo ca cc ch da 000m: 0-1, 1-2

PROV: bo ca cc ch cn 000m: $0-1,1-2$ da lo pa vr

PROV: bo ca cc ch cn 000m: $0-1,1-2$ da pa sa vr

\section{Hyperbaena}

allenii Standi.

Odontoca rya

"tamnoides (DC.) Miers

*--var. canescens (Miers) Barneby PROV: ca pa

* tripetala Diels

PROV: da pa

PROV: ca cn da

PROV: ca da

PROV: da 000m: $0-1$ 000m: $0-1$

000m: $0-1$ 000m: $0-1$

000m: $0-1$ 000m: $0-1$

000m: $0-1$

000m: $0-1$

000m: we

\begin{tabular}{|c|c|c|c|c|c|c|}
\hline ANGE: & $w r$ & & & & & climber \\
\hline ANC & bol & cao & $\mathrm{col}$ & $\mathrm{cr}$ & ecu & climb \\
\hline gi & gui & ven & & & & \\
\hline RANGE: & col & $\mathrm{cr}$ & ecu & gua & mex & viny \\
\hline $\operatorname{mex}$ & nic & sao & & & & sh \\
\hline $\begin{array}{c}\text { ANGE: } \\
\text { per }\end{array}$ & $\begin{array}{l}\mathrm{col} \\
\mathrm{pr}\end{array}$ & $\begin{array}{l}\mathrm{cr} \\
\text { ven }\end{array}$ & ecu & hon & mex & clin \\
\hline ANGE: & afr & ang & as i & cag & fla & vin \\
\hline me: & oce & sag & & & & shrub \\
\hline ANGE: & bel & boi & cag & col & $\mathrm{cr}$ & climber \\
\hline ec & gua & hon & mex & nic & per & \\
\hline sag? & sal & sao & sat & ven & & \\
\hline
\end{tabular}

RANGE: bol col ecu per sao shrub

RANGE: col ecu per sat ven herb RANGE: $w r$ climber

RANGE: end

RANGE: or

sa I

tree

tree

RANGE: lag nag?

RANGE: sat

cr

shrub

RANGE: $\mathrm{COI} \mathrm{Cr}$

climber

viny shrub

RANGE: $C O I$

viny shrub

RANGE: col ecu per sat climber 
TAXON

FAMILY

PROVINCES

ELEVATION (OOOM.)

RANGE OUTSIDE OF PANAMA

HAB I T

95 (65) MAGNOLIACEAE

Magnolia

sororum seib.

PROV: $\mathrm{cc}$ ch

$000 \mathrm{~m}: 0-1,1-2,2-3$

RANGE: end

tree

*Michel ia

* champaca L.

cult. PROV: pa

000m: 0-1

RANGE: $w r$

herb

Ta I a uma

gloriensis Pitt.

PROV: $\mathrm{CC}$

000m: 0-1

000m: $0-1,1-2$

RANGE: $c r$

RANGE: end

tree

PROV: da

95A (66) WINTERACEAE

Drimys

granadens is L. $f$.

PROV: ch da pa $v r \quad 000 m: 0-1,1-2$

RANGE: cag? mex per ven

shrub

tree

\section{8 (67) ANNONACEAE}

\section{Anaxagorea}

alleni i R.E. Fries

* crassipetala Hemsi.

panamensis Stand I.

*phaeocarpa Mart.

Annona

acuminata Saff.

* cherimola Mill.

glabra L.

hayesi i Standl.

*montana Macfad.

*muricata L.

*palmeri Saff.

*pittieri J.D. Sm.

purpurea Duna I

* reticulata $L$.

* scleroderma Saff.

spraguei Saff.

* squamosa L.
PROV: ca cn pa sa $000 \mathrm{~m}: 0-1$

PROV: 0 : $000 \mathrm{~m}: 0-1$

PROV: ca pa $000 \mathrm{~m}: 0=1$

PROV: bo da

000m: $0-1$

PROV: ca ch da pa $000 \mathrm{~m}: 0-1$

PROV: ch

000m: $2-3$

PROV: bo ca cn da pa 000m: $0-1$

sa

000m: $0-1$

PROV: ce cn lo

PROV: ca sa Vr

PROV: Wp

000m: $0-1$

000m: $0-1,1-2$

000m: we

PROV: WP

000m: we

PROV: ca ch da lo vr 000m: $0-1$

PROV: cn pa

000m: $0-1$

PROV: cn

$000 \mathrm{~m}: 0-1$

PROV: ca cn da pa $000 \mathrm{~m}: 0-1$

cult. PROV: pa

000m: $0-1$

\begin{tabular}{|c|c|c|c|c|c|c|}
\hline RANGE: & cag & sat & & & & tree \\
\hline RANGE: & cag & sat & $=$ & xar & $111^{-}$ & tree \\
\hline RANGE: & $\mathrm{cr}$ & & & & & shrub \\
\hline RANGE: & $\mathrm{cr}$ & sat & $k^{-6}$ & 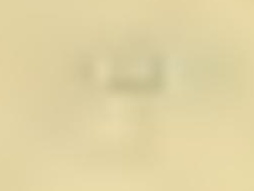 & 20 & tree \\
\hline & $-2-1$ & vate & 200 & & $3 y$ & \\
\hline RANGE: & end & 68 & & & & tree \\
\hline RANGE: & ang & cag & $\mathrm{col}$ & $\operatorname{mex}$ & (2) 7 & tree \\
\hline RANGE: & af $r$ & ang & cag & $\operatorname{mex}$ & per & tree \\
\hline sag? & sao & & & & & \\
\hline RANGE: & end & & & & & shrub \\
\hline & & $=1$ & 11 & & & tree \\
\hline RANGE: & ang & sat & & & & tree \\
\hline RANGE: & cag & sat & & & & tree \\
\hline RANGE: & $\operatorname{mex}$ & & & & & tree \\
\hline RANGE: & $w r$ & & & & & wh \\
\hline $\begin{array}{c}\text { RANGE: } \\
\text { ven }\end{array}$ & bel & cr & ecu & $\operatorname{mex}$ & trt & tree \\
\hline RANGE: & cag & sat & 8 & & & tree \\
\hline RANGE: & cag & & & & & shrub \\
\hline RANGE: & end & & & & & tree \\
\hline RANGE: & cag & sat & & & & tree \\
\hline
\end{tabular}




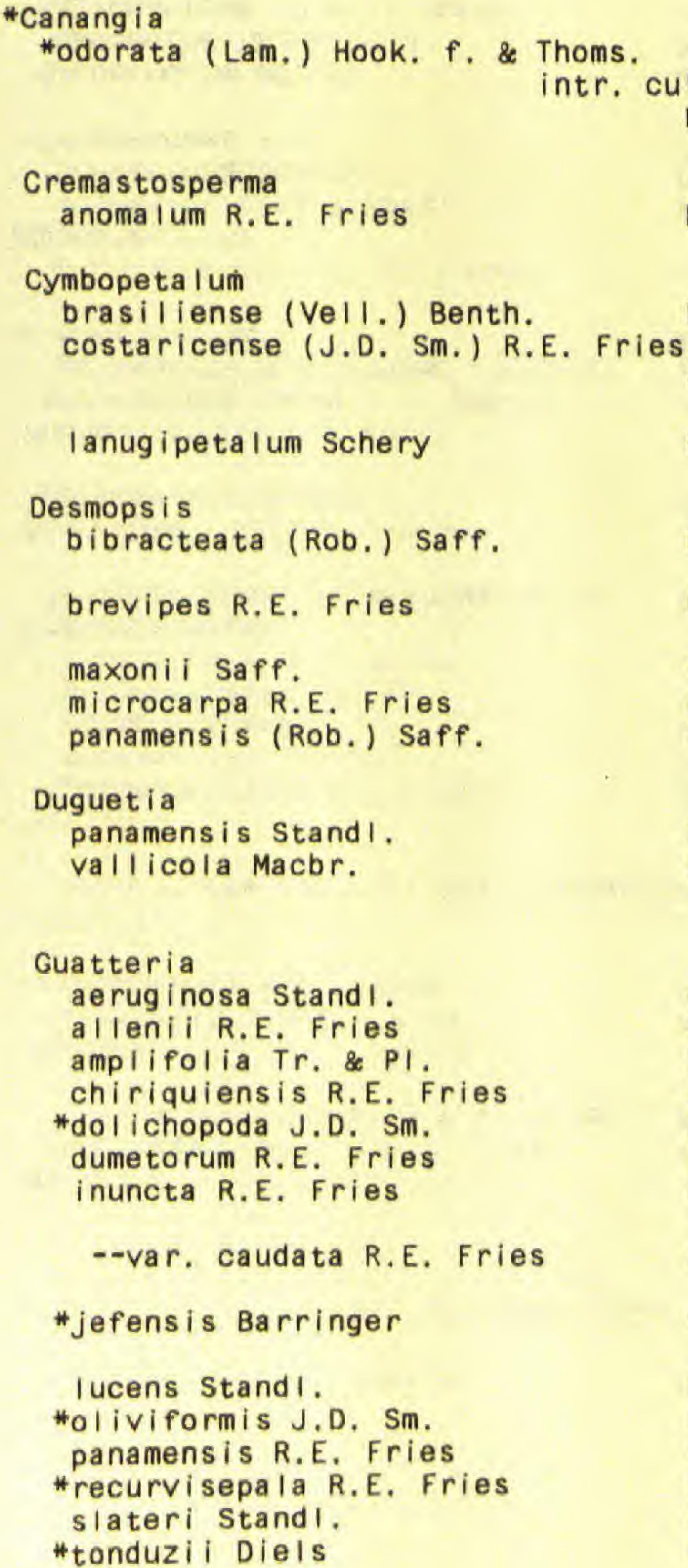

000m: $0-1$

\section{$000-1$}

$000 \mathrm{~m}: 0-1$

PROV: ch 10

PROV: bo

PROV: ch

PROV: bo pa

PROV: ca

PROV: bo pa

PROV: da

\section{PROV: bo ch da}

PROV: $\mathrm{CC}$ da

000m: $0-1,1-2$

PROV: $\mathrm{ch}$

PROV: bo da pa sa 000m: $0-1,1-2$

PROV: ca ch cn da

PROV: bo $\mathrm{cc} \mathrm{cn}$

000m: $0-1$

000m: $0-1$

PROV: bo pa

000m: $0-1$

PROV: pa

PROV: bo sa

PROV: da pa

PROV: bo 10

PROV: cn he pa vr

PROV: bo $\mathrm{ch}$

PROV: cC da pa 000m: $0-1$

000m: $0-1$

000m: $0-1$

000m: $0-1$

000m: $0-1$

000m: $1-2,2-3$

000m: $0-1,1-2$
RANGE: afr ang asi cag sat tree

RANGE: $\mathrm{COI}$

tree

RANGE: col gui sat trt ven tree

RANGE: $c r$

RANGE: cag sat

tree

shrub

tree

RANGE: $\mathrm{cr}$ nic

shrub

tree

shrub

tree

RANGE: cag

RANGE: cag

RANGE: $\mathrm{cr}$

RANGE : $\mathrm{col} \mathrm{cr}$

tree

tree

tree

RANGE: $\mathrm{COI} \mathrm{cr}$

RANGE: $\mathrm{COI}$ ven

tree

shrub

tree

RANGE: cag

RANGE: cag

RANGE: ang

RANGE: cag

RANGE: cag

RANGE: cag

RANGE: $\mathrm{cr}$

RANGE: cag

RANGE: end

RANGE: ang cag sat

RANGE: cag sat

RANGE: end

RANGE: $\mathrm{cr}$

RANGE: $\mathrm{cag}$

RANGE: $c r$ tree

tree

tree

tree

tree

tree

shrub

$$
\text { tree }
$$

shrub

tree

shrub

tree

tree

tree

tree

tree

tree

tree 


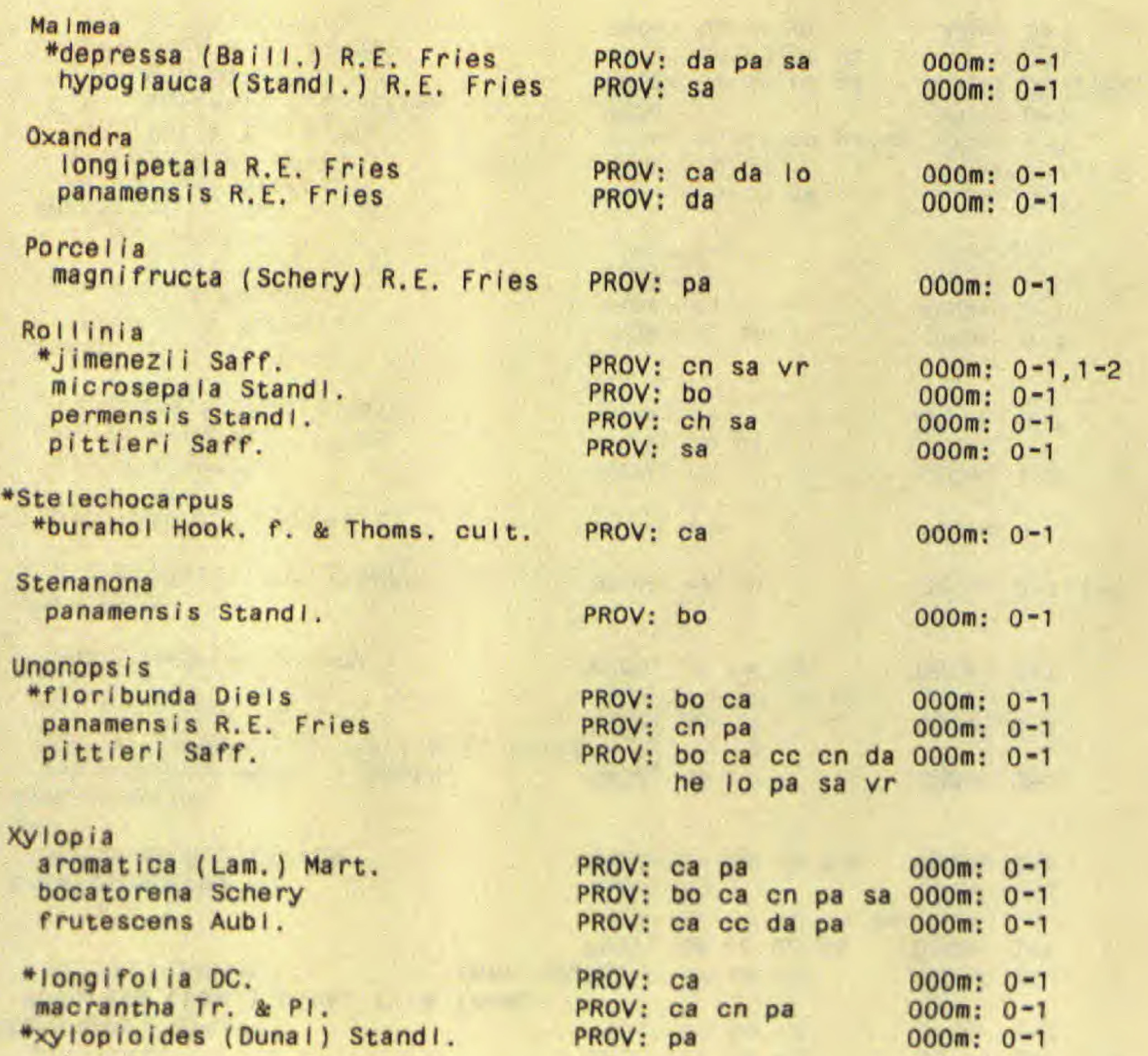

99 (68) MYRISTICACEAE

$\begin{array}{ll}\text { PROV: pa } & 000 \mathrm{~m}: 0-1 \\ \text { PROV: bo cn sa } & 000 \mathrm{~m}: 0-1\end{array}$

\begin{tabular}{|c|c|c|c|c|c|}
\hline $\begin{array}{l}\text { RANGE: } \\
\text { RANGE: }\end{array}$ & $\begin{array}{l}\text { cag } \\
\text { col }\end{array}$ & mex & & & $\begin{array}{l}\text { tree } \\
\text { tree }\end{array}$ \\
\hline RANGE: & ang & cag & & & tree \\
\hline RANGE: & ang & $\mathrm{cag}$ & & & tree \\
\hline RANGE: & ven & & & & tree \\
\hline RANGE: & $w r$ & & & & tree \\
\hline RANGE: & $\mathrm{cr}$ & & & & tree \\
\hline RANGE: & ang & cag & sat & & tree \\
\hline RANGE: & ang & cag & sat & & tree \\
\hline RANGE: & $w r$ & & & & tree \\
\hline RANGE: & end & & & & tree \\
\hline RANGE: & sat & 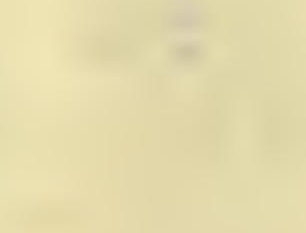 & & & tree \\
\hline RANGE: & end & & & & tree \\
\hline RANGE: & cr & & & & tree \\
\hline RANGE: & ang & cag & sat & & tree \\
\hline RANGE: & cag & & & 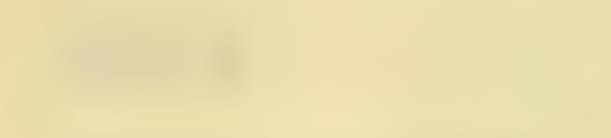 & tree \\
\hline RANGE: & bei & gua & sat & rats & $\begin{array}{l}\text { tree } \\
\text { shrub }\end{array}$ \\
\hline RANGE: & gui & & & & tree \\
\hline RANGE: & col & ven & & & tree \\
\hline RANGE: & $w r$ & & & & tree \\
\hline
\end{tabular}

RANGE: per sao

RANGE: cag mex per sag? sao shrub
* capitellata (A. DC.) Warb. sprucei (A. DC.) Warb. 
Dialyanthera

otoba (H. \& B.) Warb. "parvifolia Markgr.

\section{Myristica}

fragrans Houtt.

*osteoph loeum

*platyspermum (A, DC, ) Warb.

\section{*Otoba}

*acuminata (StandI.) A. Gentry

* Iatialata (Pitt.) A. Gentry

*novagranatensis Mold.

\section{Virola}

elongata (Benth.) Warb.

guatemalens is (HemsI.) Warb.

koschnyi Warb.

*macroca rpa A.C. Smith

*megacarpa A. Gentry

sebifera AubI.

*surinamens is (Rol.) Ward

101 (69) MONIMIACEAE

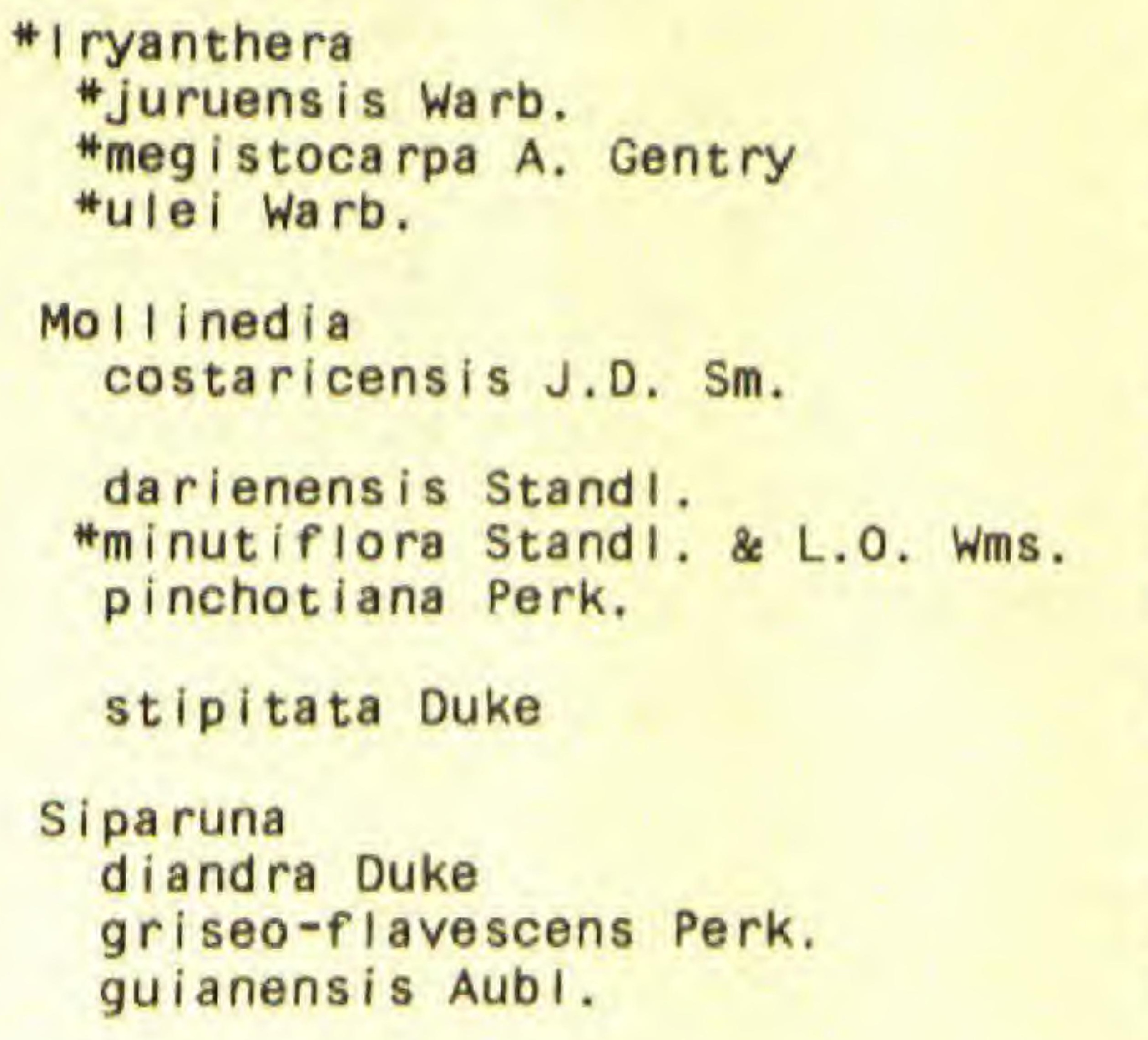

PROV: bo $\mathrm{ch}$

PROV: cn

000m: $0-1$

000m: $0=1$

PROV: bo

000m: $0-1$

PROV: $c c$

000m: $0-1$

PROV: bo cc en pa 000m: $0-1$

PROV: ca cn da pa sa $000 \mathrm{~m}: 0-1$

PROV: bo cn da $000 \mathrm{~m}: 0-1$

PROV: ca

000m: $0-1$

PROV: ch

PROV: ch da

PROV: sa

PROV: cn

PROV: bo ca ch cn da 000m: $0-1$

PROV: ca cn pa pa

cho 0000

PROV: cn

PROV: pa

PROV: da

PROV: ch

PROV: da

PROV: da

PROV: ch da

PROV: ch

PROV: da

PROV: ch pa

PROV: bo ca 000m: $0-1$

000m: $0-1$

000m: $0-1$

000m: $1-2$

000m: $0-1$

000m: $1-2$

000m: $1-2,2-3$

000m: $1-2,2-3$

000m: $0-1$

000m: $0-1$ pa $\vee r$
RANGE: $\mathrm{COI} \mathrm{cr}$

RANGE: end

tree

RANGE: asi cag?

tree

RANGE: $w r$

tree

RANGE: end

RANGE: $\mathrm{CO} 1$

RANGE: $\mathrm{COI}$

tree

tree

tree

RANGE: per sao

RANGE: cag? gua

RANGE: cag? gua

RANGE: $W r$

RANGE: end

RANGE: boI

Sao

RANGE: or gui lag sao

shrub

tree

tree

tree

wh

tree

tree sag? tree
RANGE: COI

RANGE: end

RANGE: $c 01$

RANGE: $c r$

RANGE: end

RANGE: $\mathrm{cr}$

RANGE: cag

RANGE: cag

RANGE: $\mathrm{CO}$

RANGE: $\mathrm{cr}$

RANGE: $\mathrm{CO}$ tree

tree

tree

shrub

tree

shrub

treelet

shrub

tree

shrub

shrub

shrub 
TAXON

\section{FAMILY PROVINCES}

ELEVATION (OOOM.)

RANGE OUTSIDE OF PANAMA

HAB IT

101 (69) MONIMIACEAE cont.

"macrophyl la (H,B, K.) DC,

nicaraguens is Hems i.

"paralleloneura Perk.

"patelliformis Perk.

pauciflora (Beurl.) A. DC.

riparia (TuI.) A. DC.

tetraceroides Perk.

tonduziana Perk.
PROV: da 000m: $1-2$

PROV: bo ca ce en da 000m: $0-1$

PROV: Da

PROV: pa 000m: $0-1$

PROV: bo ca ce ch cn 000m: 0-1

PRov: da pa

PROV: ch

000m: $1-2$

000m: $0-1$

PROV: bo da vr
PROV: $\mathrm{ch} \quad 000 \mathrm{~m}: 1-2$

$\begin{array}{ll}\begin{array}{l}\text { RANGE: col } \\ \text { RANGE: cag mex }\end{array} & \begin{array}{c}\text { shrub } \\ \text { shrub } \\ \text { tree } \\ \text { RArub } \\ \text { RANGE: } w r\end{array} \\ \text { RANGE: } w r \\ \text { RANGE: col cr peelet } \\ \text { shrub } \\ \text { tree } \\ \text { RANGE: cag col mex }\end{array}$

102 (70) LAURACEAE

$\begin{array}{ll}\text { PROV: ch } & 000 \mathrm{~m}: 2-3 \\ \text { PROV: ch } & 000 \mathrm{~m}: 0-1,1-2 \\ \text { PROV: pa } & 000 \mathrm{~m}: 0-1 \\ \text { PROV: da } & 000 \mathrm{~m}: 0-1\end{array}$

RANGE: $\mathrm{cr}$

RANGE: end

RANGE: gui

RANGE: bo

per sao

col per sao

RANGE: hon

RANGE: ang

RANGE: $\mathrm{COI}$

cr

or per ven

RANGE: wdt

RANGE: $\mathrm{COI}$

RANGE: sag

RANGE: ang sat

tree

tree

tree

RANGE: sat

RANGE: $\mathrm{CO}$

RANGE: $c r$

RANGE: cag

RANGE: ang per ven

tree

tree

tree

col mex tree

cag col ecu gag tree

treelet 
102 (70) LAURACEAE cont.

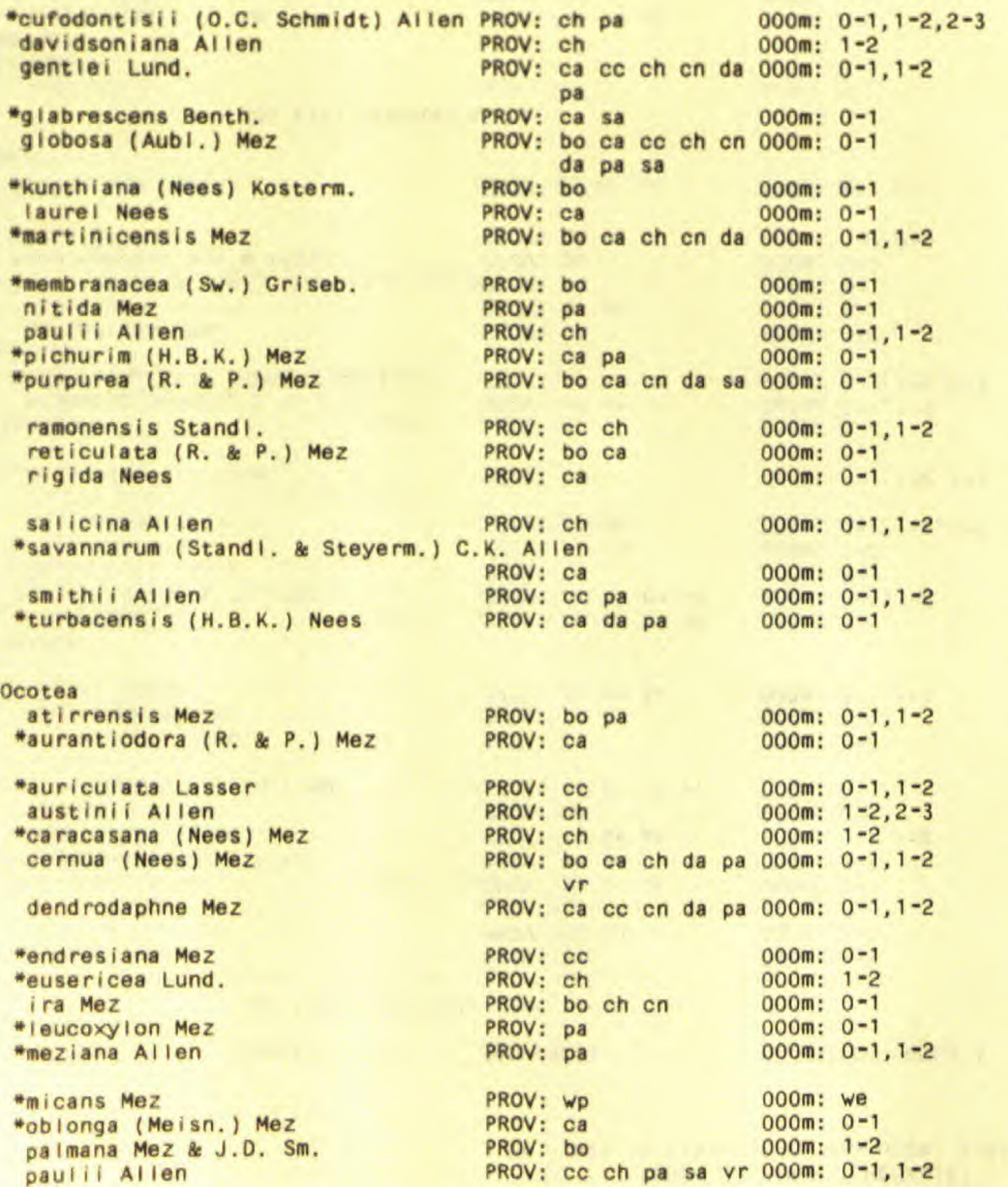

\begin{tabular}{|c|c|c|c|c|c|c|}
\hline $\begin{array}{l}\text { RANGE: } \\
\text { RANGE: } \\
\text { RANGE: }\end{array}$ & $\begin{array}{l}\text { cr } \\
\text { end } \\
\text { bel }\end{array}$ & gua & hon & mex & & $\begin{array}{l}\text { tree } \\
\text { tree } \\
\text { tree }\end{array}$ \\
\hline RANGE: & sat & & & & & tree \\
\hline $\begin{array}{c}\text { RANGE: } \\
\text { per }\end{array}$ & ang & cag & $\mathrm{cr}$ & gui & $\operatorname{mex}$ & tree \\
\hline RANGE: & col & cr & ecu & per & ven & tree \\
\hline RANGE: & cr & per & sag? & & & tree \\
\hline RANGE: & bel & $\mathrm{col}$ & or & ecu & lag & tree \\
\hline $\operatorname{mex}$ & nic & sal & trt & ven & & \\
\hline RANGE: & $c r$ & per & & & & tree \\
\hline RANGE: & mex? & & & & & tree \\
\hline RANGE: & $\mathrm{cr}$ & & & & & tree \\
\hline RANGE: & sat & & & & & tree \\
\hline RANGE: & cag & $\mathrm{col}$ & nic & sao & & $\begin{array}{l}\text { tree } \\
\text { shrub }\end{array}$ \\
\hline RANGE: & $\mathrm{cr}$ & & & & & tree \\
\hline RANGE: & cag & $\operatorname{mex}$ & per & sag & & tree \\
\hline RANGE: & sag? & sao & & & & $\begin{array}{l}\text { shrub } \\
\text { tree }\end{array}$ \\
\hline RANGE: & er & & & & & tree \\
\hline RANGE: & bel & gua & hon & & & tree \\
\hline RANGE: & $\mathrm{cr}$ & & 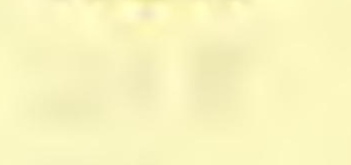 & & & tree \\
\hline RANGE: & sao & ven & & & & $\begin{array}{l}\text { tree } \\
\quad \text { shrub? }\end{array}$ \\
\hline RANGE: & $\mathrm{cr}$ & & & & & shrub \\
\hline RANGE: & gui & sao & trt & ven & & $\begin{array}{l}\text { tree } \\
\text { shrub }\end{array}$ \\
\hline RANGE: & $w r$ & 12 & & & & tree \\
\hline RANGE: & $\mathrm{cr}$ & & & & & tree \\
\hline RANGE: & ven & & & & & tree \\
\hline $\begin{array}{c}\text { RANGE: } \\
\text { Der }\end{array}$ & $\begin{array}{l}\text { ang } \\
\text { sao }\end{array}$ & cag & $\mathrm{cr}$ & gui & mex & $\begin{array}{l}\text { tree } \\
\text { shrub }\end{array}$ \\
\hline RANGE: & $\mathrm{cr}$ & gua & hon & mex & & $\begin{array}{l}\text { shrub } \\
\text { tree }\end{array}$ \\
\hline RANGE: & $\mathrm{cr}$ & & & & & tree \\
\hline RANGE: & end & & & & & tree \\
\hline RANGE: & cr & & & & & tree \\
\hline RANGE: & ang & ven & & & & tree \\
\hline RANGE: & $\mathrm{cr}$ & & $85 x^{4}$ & & & $\begin{array}{l}\text { tree } \\
\text { shrub }\end{array}$ \\
\hline RANGE: & $\mathrm{col}$ & & & & & tree \\
\hline RANGE: & gui & & & & & tree \\
\hline RANGE: & $\mathrm{cr}$ & & & & & tree \\
\hline RANGE: & cr & & & & & tree \\
\hline
\end{tabular}


TAXON

FAMILY PROVINCES

102 (70) LAURACEAE cont.

* puberula Nees

rubrinervis Mez

*skutchil Allen

* stenoneura Mez \& Pitt.

subsericea Standl.

"tonduzil standi.

veraguensis (Meisn.) Mez

"viridiflora Lund.

wedeliana Allen

whitei Woods.

\section{Persea}

americana Mill.

* caerulea (R. \& P.) Mez

*obtusifolia Kopp

rigens Allen

schiedeana Nees

veraguasens is seem.

\section{Phoebe}

brenesii standl.

* cinnamomifolia (Kunth) Nees

\section{costaricana Mez}

johnstonii Allen

* mollicella Blake

*neurophyila Mez \& Pitt.

pittieri Mez

*valeriana StandI.
PROV: ca

PROV: he 10 pa

cult. PROV: ca cn pa

PROV: ch da

PROV: cc da pa

PROV: CC

PROV: ce ch pa Vr

PROV: ch

PROV: bo $\mathrm{cn}$

PROV: $c c$ ch sa

cult.

PROV: ca ch he pa

PROV: $\mathrm{cc}$ oh he vr

PROV: ch

PROV: bo $\mathrm{ch}$

PROV: ch pa

PROV: ch

PROV: ch $V r \quad 000 \mathrm{~m}: 0-1,1-2$

PROV: ca cc ch da he 000m: $0-1,1-2,2-3$

pa

PROV: ca pa

PROV: $\mathrm{ca}$

PROV: pa

PROV: ch da

000m: $1-2$

000m: $0-1$

000m: $0-1$

000m: $0-1$

000m: $1-2,2-3$

PROV: vr

000m: we

000m: $0-1$

$00 \mathrm{~m}: 0-1$

000m: $2-3$

$00 \mathrm{~m}: 0-1,1-2$

000m: $0-1$

$000 \mathrm{~m}: 0-1,1-2$

$000 \mathrm{~m}: 2-3$
$0-1,1-2$

RANGE: bol bol gui mex sao tree

RANGE: bol per tree

RANGE: col or mex nWt tree

RANGE: or ecu tree

RANGE: or tree

RANGE: $\mathrm{Cr}$

RANGE: cag mex nic

RANGE: end

RANGE: end

RANGE: $\mathrm{cr}$

tree

tree

shrub

tree

tree

tree

RANGE: ang cag cr mex tree

RANGE: cag or sat tree

RANGE: end treelet

RANGE: end

tree

tree

shrub

tree

tree

RANGE: $\mathrm{cr}$

RANGE: cag col gua mex per tree

RANGE: $c r$

RANGE: end

RANGE: $c r$

RANGE: $c r$

RANGE: $\mathrm{cr}$

RANGE: $\mathrm{cr}$ tree

tree

tree

tree

shrub

tree

\section{3 (71) HERNANDIACEAE}

\section{Hernandia}

didymantha J.D, Sm.

*hammelii D'Arcy

stenura StandI.
PROV: bo $\mathrm{cc} \mathrm{ch}$

PROV: $C C$

PROV: bo 000m: $0-1$

000m: $0-1$

000m: $0-1$
RANGE: $c r$

RANGE: end

RANGE: $c r$ tree

tree 
104 (72) PAPAVERACEAE

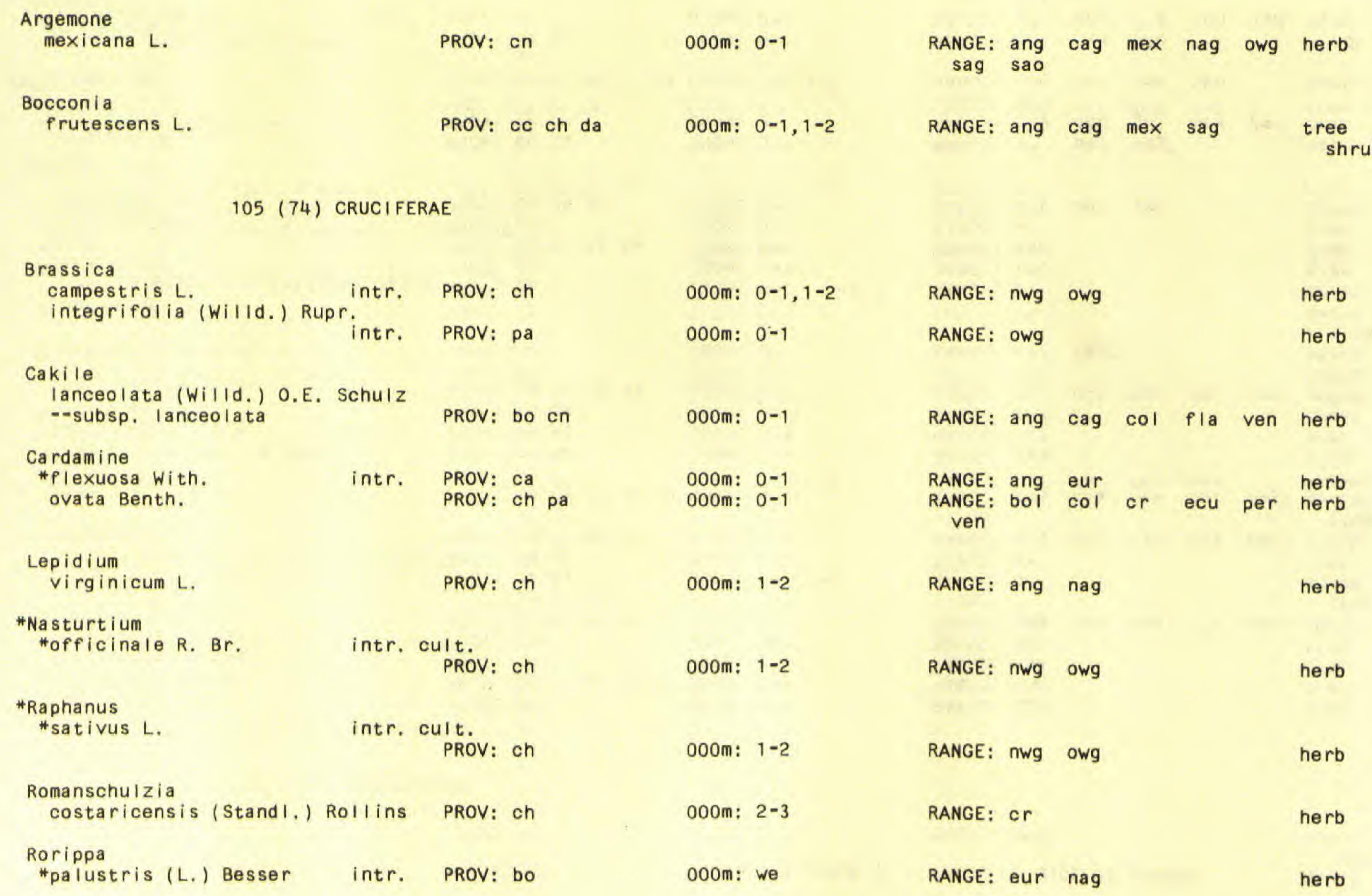




\section{7 (73) CAPPARACEAE}

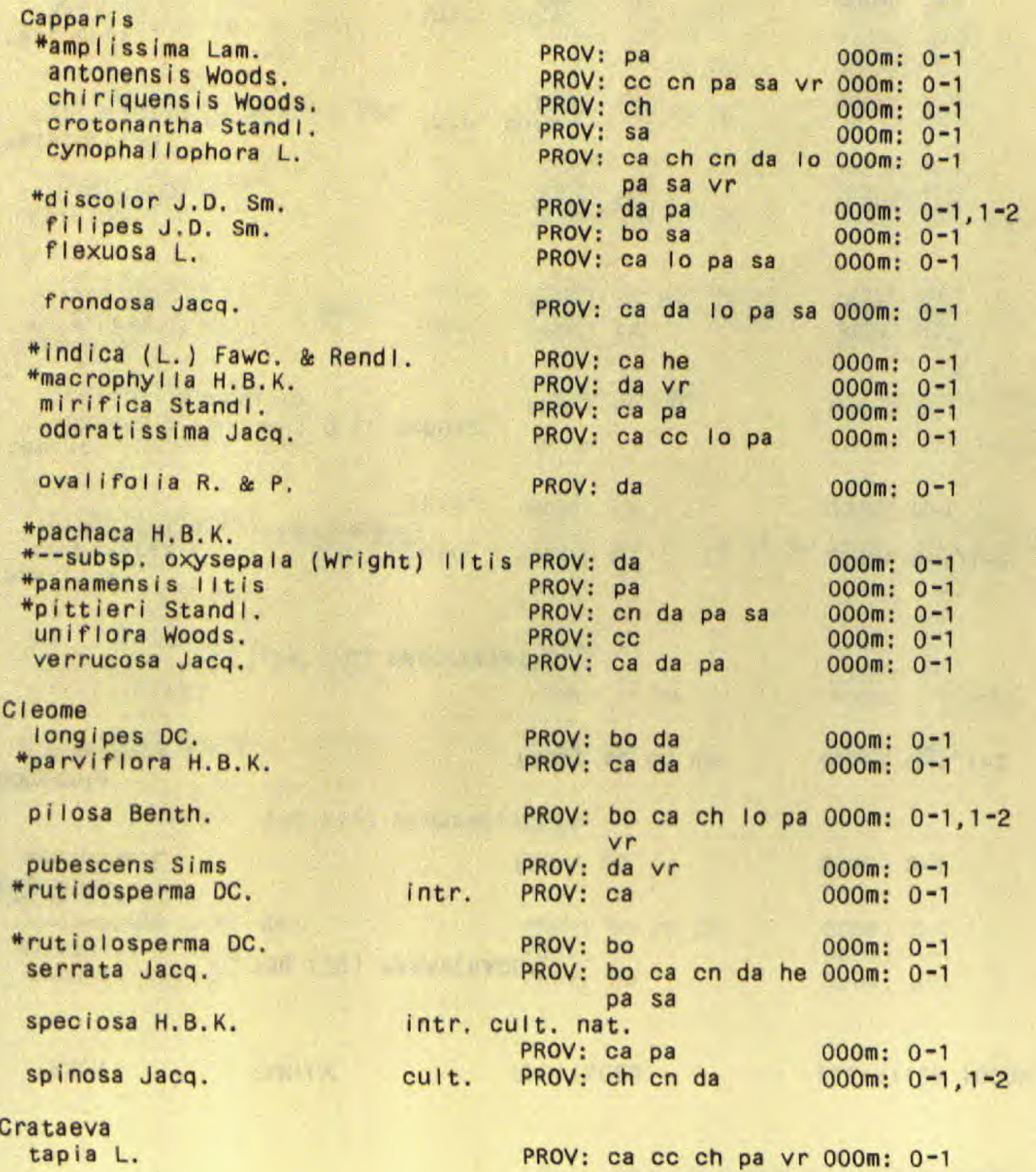

\begin{tabular}{|c|c|c|c|c|c|c|}
\hline RANGE: & gag & & & & & tree \\
\hline RANGE: & end & & & & & tree \\
\hline RANGE: & end & & & & & tree \\
\hline RANGE: & end & & & & & tree \\
\hline $\begin{array}{c}\text { RANGE: } \\
\text { ven }\end{array}$ & ang & bah & cag & fla & $\operatorname{mex}$ & $\begin{array}{l}\text { shrub } \\
\text { tree }\end{array}$ \\
\hline RANGE: & $\mathrm{cr}$ & 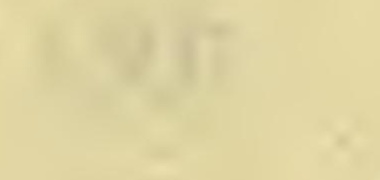 & & & & shrub \\
\hline ANGE: & $\mathrm{cr}$ & & & & & tree \\
\hline $\begin{array}{c}\text { RANGE: } \\
\text { Ven }\end{array}$ & ang & cag & fla & mex & per & $\begin{array}{l}\text { shrub } \\
\text { tree }\end{array}$ \\
\hline $\begin{array}{c}\text { RANGE: } \\
\text { sao }\end{array}$ & ang & cag & mex & per & sag? & $\begin{array}{l}\text { shrub } \\
\text { tree }\end{array}$ \\
\hline RANGE: & gag & & & & & tree \\
\hline RANGE: & sat & & & & & tree \\
\hline RANGE: & end & & & & & tree \\
\hline RANGE: & ang & cag & mex & per & ven & shruo \\
\hline RANGE: & per & sag? & & & & $\begin{array}{l}\text { shrub } \\
\text { tree }\end{array}$ \\
\hline RANGE: & $w r$ & 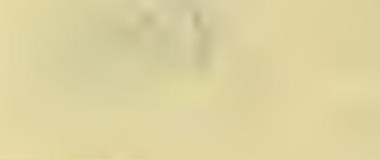 & & & & shrub \\
\hline RANGE: & end & & & & & tree \\
\hline RANGE: & cag & & 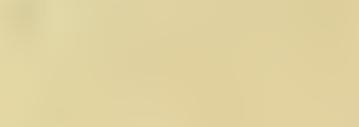 & & & tree \\
\hline RANGE: & $w r$ & & & & & tree \\
\hline RANGE: & cag & mex & ven & & & shrub \\
\hline RANGE: & $\mathrm{cr}$ & per & sag? & & & herb \\
\hline RANGE: & cag & ecu & gui & $\operatorname{mex}$ & per & herb \\
\hline sao & sat & & & & & \\
\hline KANGE: & cag & col & mex & ven & & nerb \\
\hline RANGE: & $\mathrm{cr}$ & his & sao & 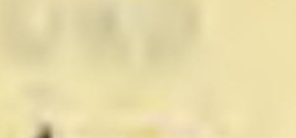 & a. & herb \\
\hline RANGE: & af $r$ & ang & fla & hon & jam & herb \\
\hline sao & trt & & & & & \\
\hline RANGE: & sao & & & & & herb \\
\hline RANGE: & cag & $\mathrm{col}$ & cub & jam & $\operatorname{mex}$ & herb \\
\hline $\begin{array}{l}\text { RANGE: } \\
\text { RANGE: }\end{array}$ & cag? & $\operatorname{mex}$ & sag? & & & herb \\
\hline RANGE: & cag? & sag? & & & & herb \\
\hline RANGE: & jam & & & & & $\begin{array}{c}\text { shrub } \\
\text { tree }\end{array}$ \\
\hline
\end{tabular}




\section{*Forchhammeria}

*trifoliata Radik.

*--subsp. grandifolia Hansen PROV: bo 000m: 0-1

\section{*Morisonia}

*americana L.

*oblongifolia Britton

PROV: ca da pa

PROV: sa

000m: $0-1$

000m: $0-1$

$000 \mathrm{~m}: 0-1,1-2$

PROV: bo $\mathrm{cc}$ ch $\mathrm{vr}$

chiriquensis (Stand I.) Woods.

* decipiens (Tr. \& PI.) Woods. PROV: Pa

*decipiens (Tr. \& PI.) Woods. PROV: pa
*--subsp. chiriquensis (Standl.) Cochrane

*--subsp, pulcherrima (Standl.) Cochrane

*formosa Cochrane

PROV: ch

PROV: ch

Steriphoma

macranthum StandI.

PROV: da

*paradoxum EndI.

PROV: da

Tovaria

pendula $R$. \& $P$.

PROV: ch da 000m: $0-1$

000m: $2-3$

000m: $0-1,1-2,2-3$

$000 \mathrm{~m}: 3+$

$000 \mathrm{~m}: 0-1$

000m: $0-1$

$000 m: 0-1,1-2$

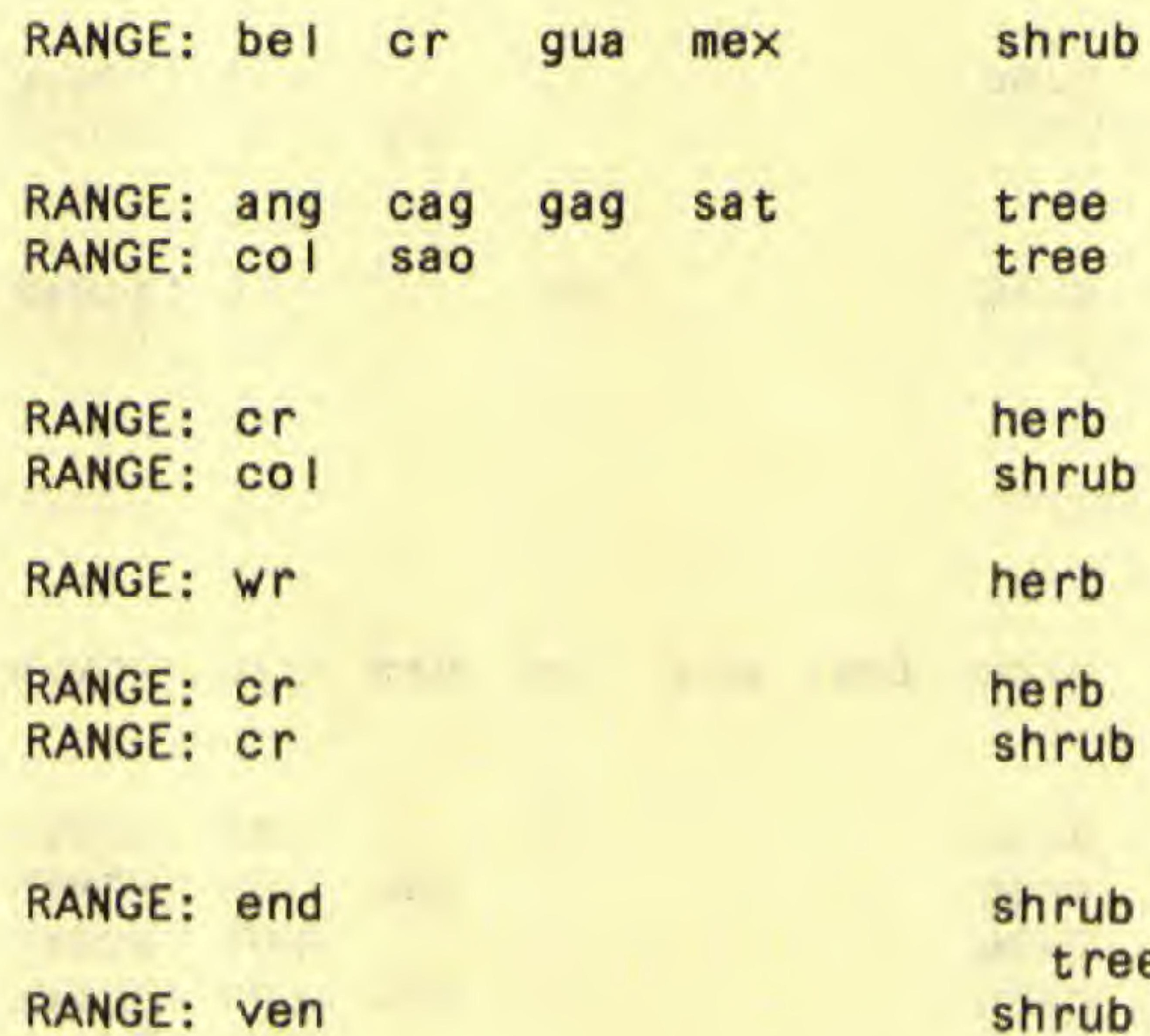

RANGE: cag mex per ven shrub

herb

shrub

108 (75) RESEDACEAE

Reseda

uteola L. intr. cult.

PROV: ch

000m: $1-2$

RANGE: owt

herb

109 (76) MORINGACEAE

Moringa

aleifera Lam. intr. cult.

PROV: wp

000m: $0-1$

RANGE: owg 
TAXON

117 (79) SAXIFRAGACEAE cont.

preslii Briq.

Philadelphus

myrtoides Bertol.

Phyl Ionoma

ruscifolia $R$. \& S.

*tenuidens Pitt.

*Ribes

* eptostachyum Benth.
PROV: $c C$

PROV: ch

PROV: ch da

PROV: ch

PROV: bo $\mathrm{ch}$ 000m: $0-1,1-2$

000m: $1-2$

000m: $1-2,2-3$

000m: $1-2$

000m: $3+$
RANGE: $\mathrm{cr}$ ecu per

RANGE: $\mathrm{cr}$ gua

RANGE: bol or sag?

RANGE: $c r$

RANGE: $c r$

119 (80A) BRUNELLIACEAE

\section{Brunell ia}

*ostaricensis StandI.

darienensis Cuatr. \& Porter

PROV: da

PROV: da

120 (80) CUNONIACEAE

\section{We inmannia}

glabra L. $f$.

PROV: ch

\#--var. caripensis (H.B.K.) Engl. PROV: ch

* Iaurina H.B.K.

*--var. pseudolaurina (Woods.) Bernardi

pinnata L.

PROV: da pa

PROV: ch da

Hercklei standl.
PROV: bo $\mathrm{ch}$ 000m: $1-2$

000m: $0-1,1-2$

RANGE: $c r$

RANGE: end

tree

tree
$000 m: 1-2,2-3$

$000 m: 1-2$

000m: $0-1,1-2$

000m: $1-2,2-3$

000m: $1-2$

RANGE: col mex per sat ven $\begin{gathered}\text { shrub } \\ \text { tree } \\ \text { shrub } \\ \text { tree }\end{gathered}$
RANGE: col ven
RANGE: end

shrub

woody

cl imber

shrub

shrub

tree

shrub

shrub 


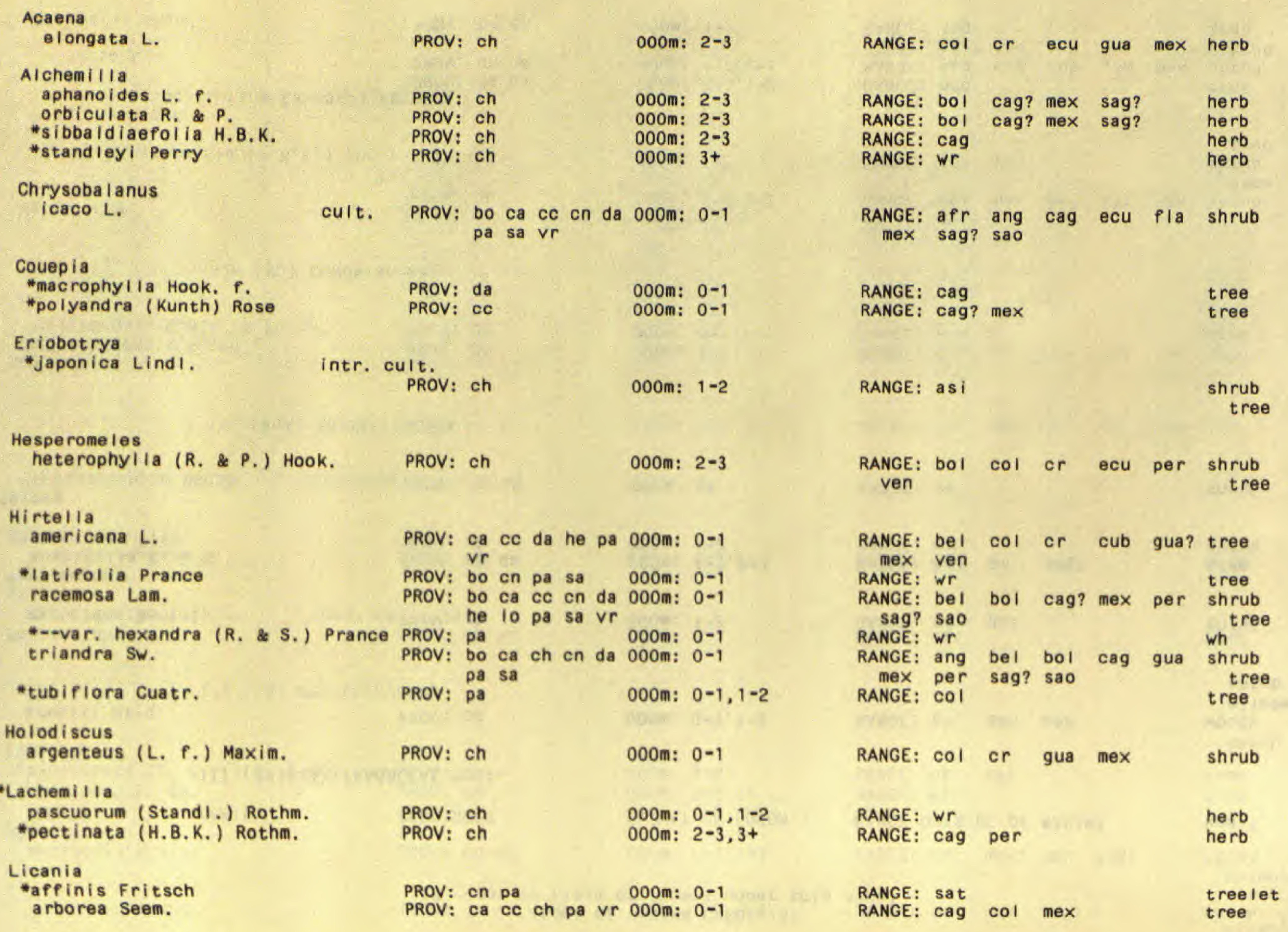


126 (81) ROSACEAE cont.

* chiriquiensis Prance
"cruegeriana Urb.
\# fasciculata Prance
hypoleuca Benth.
"jefensis Prance
"kallunki Prance
* Iongistyla (Hook. f.) Fritsch
"macroca rpa Cuatr.
"mori i Prance
platypus (Hemsl.) Fritsch cult.

$\begin{array}{ll}\text { PROV: ch } & 000 \mathrm{~m}: 1-2 \\ \text { PROV: cn pa } & 000 \mathrm{~m}: 0-1 \\ \text { PROV: cn pa } & 000 \mathrm{~m}: 0-1 \\ \text { PROV: bo ca da pa } & 000 \mathrm{~m}: 0-1 \\ \text { PROV: ca cn pa } & 000 \mathrm{~m}: 0-1 \\ \text { PROV: cn } & 000 \mathrm{~m}: 0-1 \\ \text { PROV: wp } & 000 \mathrm{~m}: \text { we } \\ \text { PROV: wp } & 000 \mathrm{~m}: \text { we } \\ \text { PROV: ca pa } & 000 \mathrm{~m}: 0-1 \\ \text { PROV: bo ca ch da lo } 000 \mathrm{~m}: 0-1\end{array}$

pa

\section{"Maranthes}

"panamens is (StandI.) Prance \& White

PROV: ca pa sa

000m: $0-1$

Prunus

annularis Koehne

*brachybot rya Zucc.

PROV: ch pa

PROV: $\mathrm{ch}$

000m: $0-1,1-2$

000m: $2-3$

*Rosa

*centifolia L.

intr. cult.

PROV: ca

000m: $0-1$

Rubus

*eriocarpus Liebm.

glaucus Benth.

PROV: ch

PROV: ch

PROV: ch

PROV: ch

PROV: ch

PROV: $c c$ ch vr panamanus Bailey

praecipuus Bailey

tantus Bailey

trichomallus schlecht. 000m: $3+$

$000 \mathrm{~m}: 1-2,2-3,3+$

000m: $2-3$

000m: $1-2$

000m: 1 -2

$000 \mathrm{~m}: 0-1,1-2$
RANGE: end

RANGE: trt

RANGE: end

RANGE: be I

RANGE: end

RANGE: end

RANGE: per

RANGE: col

RANGE: end

cag col mex

ecu per

col mex tree

tree

tree

tree

shrub

tree

wh

wh

tree

tree
RANGE: $\mathrm{cr}$ nic

tree

RANGE: cag? cr mex? sag? RANGE: $m \mathrm{X} \times$

tree tree

RANGE: OWg

herb

RANGE: cag

RANGE: $c O 1$ ecu

shrub

viny

RANGE: end

RANGE: end

RANGE: end

RANGE: cag mex shrub

viny

shrub

shrub

viny

shrub

viny

shrub

127 (82) CONNARACEAE

Cnestidium

rufescens Planch.

PROV: bo ca ce ch cn 000m: $0-1$

da he pa $v r$

RANGE: cag col cub ecu mex climber

*Cnest is

*ferruginea DC. intr. cult.

PROV: ca $\quad 000 \mathrm{~m}: 0-1$

RANGE: af $r$

shrub 
127 (82) CONNARACEAE cont.

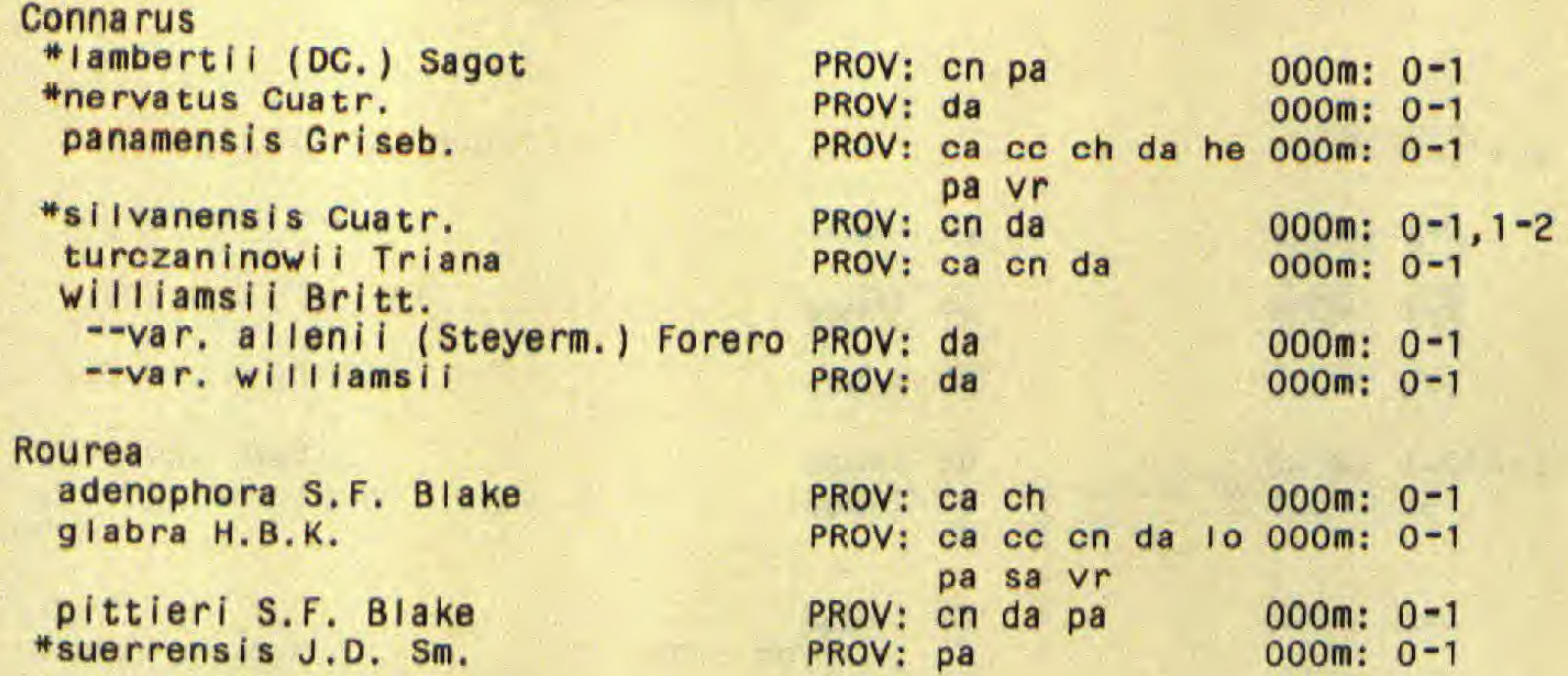

128 (83) LEGUMINOSAE

\section{Abrus}

precatorius L.

PROV: bo sa

000m: $0-1$

\section{Acacia}

*acanthophylla (Britt. \& Rose) StandI.

angustissima (Mill.) Kuntze

PROV: ca

PROV: ch

000m: $0-1$

000m: $1-2$

*auriculaeformis Benth. cult. *collinsii Saff.

PROV: ca

000m: $0-1$

PROV: ca cc ch he lo $000 \mathrm{~m}: 0-1$ pa vr

000m: $0-1$

cooki i Saff.

farnesiana (L.) Willd.

*glauca (L.) Moench

glomerosa Benth.

hayes i Benth.

macracantha WilId.

melanoceras Beurl.

cult.

PROV: ca cc pa

PROV: wP

PROV: ca

PROV: ca da pa

riparia H.B.K.

* ruddiae Janzen 000m: $0-1$

000m: $0-1$

000m: $0-1$

000m: $0-1$
PROV: ca cn pa

PROV: ca ch pa

PROV: cn 000m: $0-1$

000m: we

000m: $0-1$

000m: $0-1$

\begin{tabular}{|c|c|c|c|c|c|c|}
\hline $\begin{array}{l}\text { RANGE: } \\
\text { RANGE: }\end{array}$ & $\begin{array}{l}\text { cag } \\
\text { col? }\end{array}$ & gui & sat & trt & & $\begin{array}{l}\text { shrub } \\
\text { tree }\end{array}$ \\
\hline RANGE: & col & or & gua & nic & ven & climber \\
\hline $\begin{array}{l}\text { RANGE: } \\
\text { RANGE: }\end{array}$ & $\begin{array}{l}\text { col } \\
\text { end }\end{array}$ & & & & & $\begin{array}{l}\text { tree } \\
\text { climber }\end{array}$ \\
\hline $\begin{array}{l}\text { RANGE: } \\
\text { RANGE: }\end{array}$ & $\begin{array}{l}\text { end } \\
\text { col }\end{array}$ & & & & & $\begin{array}{l}\text { tree } \\
\text { climber }\end{array}$ \\
\hline RANGE: & hon & & & & & climber \\
\hline RANGE: & cag & cub & jam & $\operatorname{mex}$ & sao & climber \\
\hline $\begin{array}{l}\text { sat } \\
\text { RANGE: }\end{array}$ & $\begin{array}{l}\text { trt } \\
\text { col }\end{array}$ & & & & & cl imber \\
\hline RANGE: & $\mathrm{cr}$ & $\operatorname{mex}$ & nic & & & tree \\
\hline
\end{tabular}

cl imber cl imber

\begin{tabular}{|c|c|c|c|c|c|c|}
\hline RANGE: & $\mathrm{cr}$ & qua & hon & $\operatorname{mex}$ & & climber \\
\hline RANGE: & cag & nic & sag & & & $\begin{array}{l}\text { shrub } \\
\text { tree }\end{array}$ \\
\hline RANGE: & oce & & & & & tree \\
\hline ANGE: & cag & $\mathrm{col}$ & mex & & & $\begin{array}{c}\text { shrub } \\
\text { tree }\end{array}$ \\
\hline RANGE: & bel & gua & hon & nic & & $\begin{array}{c}\text { shrub } \\
\text { tree }\end{array}$ \\
\hline ANGE: & ang & cag & $\operatorname{mex}$ & nag? & nic & shrub \\
\hline sag & sao & & & & & tree \\
\hline RANGE: & ang & cag & jam & nic & & shrub \\
\hline RANGE: & $\mathrm{cag}$ & $\operatorname{mex}$ & nic & sag & sao & tree \\
\hline RANGE: & $\mathrm{cr}$ & hon & nic & & & $\begin{array}{l}\text { cl imber } \\
\text { shrub }\end{array}$ \\
\hline RANGE: & ang & cag? & $\mathrm{col}$ & nic & sag & tree \\
\hline RANGE: & $\mathrm{cr}$ & nic & & & & $\begin{array}{c}\text { shrub } \\
\text { tree }\end{array}$ \\
\hline $\begin{array}{c}\text { RANGE: } \\
\text { sao }\end{array}$ & G & $\mathrm{cr}$ & ecu & nic & sag & $\begin{array}{c}\text { shrub } \\
\text { tree }\end{array}$ \\
\hline RANGE: & $\mathrm{cr}$ & gua & & & & shrub \\
\hline
\end{tabular}


tenuifolia (L.) Willd.

PROV: da sa

000m: $0-1$

\section{Acosmium}

panamense (Benth.) Yakovl.

Adenopodia

* polystachya (L.) Dixon

Aeschynomene

americana $L$.

*--var. glandulosa (Poir.) Rudd

brasiliana (Poir.) DC.

ciliata Vog.

elegans Schlecht. \& Cham.

histrix Poir.

--var. incana (Vog.) Benth.

pratens is Small

--var. caribaea Rudd

rudis Benth.

sensitiva SW.

villosa Poir.

\section{Albizia}

adinocephala Record carbonaria Britt. \& Wils. caribaea (Urb.) Britt. \& Rose gauchapele Record

PROV: ca da pa

000m: $0-1$

PROV: bo ca ch he pa 000m: $0-1$

PROV: bo ca cc ch cn 000m: $0-1$ da he lo pa vr

PROV: bo ca ch he pa 000m: $0-1,1-2$

vr

PRoV: ca pa 000m: $0-1$

PROV: $\mathrm{ch}$

PROV: ca cc lo pa $000 \mathrm{~m}$ : $0-1$

PROV: ca cc lo pa $000 \mathrm{~m}: 0-1$

PROV: $\mathrm{cc}$ he pa $\quad 000 \mathrm{~m}: 0-1$

PROV: cc he pa 000m: $0-1$

PROV: pa 000m: $0-1$

PROV: bo ca ce cn da $000 \mathrm{~m}: 0-1$ he pa

PROV: ca pa $v r$

000m: $0-1$

lebbeck (L.) Benth. cult. PROV: ca

*procera (Roxb.) Benth. intr. cult.

PROV: ca

000m: $0-1$

000m: $0-1$

$000 \mathrm{~m}: 0-1$

000m: $0-1$

000m: $0-1$

000m: $0-1$

\section{Alysicarpus}

vaginal is (L.) DC.

PROV: bo ca cc ch cn 000m: $0-1$ he pa sa vr

*Amicia

* gl andulosa H.B.K.

PROV: da

000m: $1-2$

And i ra

inermis (W. Wright) H.B.K.

PROV: bo ca cc ch da 000m: $0-1$

he pa $\vee r$

PROV: he

000m: $0-1$

RANGE: ang cag mex nic sag climber
RANGE: cag col mex sal ven tree


Bauhinia

*aculeata L.

*beguinotil Cufod.

cumanens is H.B.K. emarginata $\mathrm{Mill}$.

\section{eucosma Blake}

* glabra Jaca.

*guianensis Aubl.

hymeneaefolia Triana

ligulata Pitt.

* microstachya (Raddi) Macbr.

monandra Kurz

obovata Blake

pauletia Pers.

*petiolata Hook.

*picta (H.B.K.) DC.

purpurea L.

reflexa schery

standleyi Rose

storki i (Rose) Schery

ungulata $\mathrm{L}$.

*variegata L.

\section{Brownea}

macrophyl Ia Linden

rosa-de-monte Berg.

Browneops is

exce Isa pitt.

Caesalpinia

coriaria (Jacq.) Willd.

crista L.

PROV: pa 000m: $0-1$

PROV: pa sa $000 \mathrm{~m}: 0-1$

PROV: WP 000m: $0-1$

PROV: cC pa $\quad 000 \mathrm{~m}: 0-1$

PROV: ca pa 000m: $0-1$

$000 \mathrm{~m}: 0-1$

PROV: bo ca ce en da 000m: $0-1$

pa sa vr

PROV: WP

000m: $0-1$

PROV: sa

000m: $0-1$

PROV: da

000m: $0-1$

intr. cult.

PROV: cc pa $000 \mathrm{~m}: 0-1$

PROV: da

PROV: ca he lo pa $000 \mathrm{~m}: 0-1$

PROV: cn

PROV: sa

000m: 0-1

$000 \mathrm{~m}: 0-1$

000m: $0-1$

$\begin{array}{ll}\text { PROV: ca da sa } & 000 \mathrm{~m}: 0-1 \\ \text { PROV: ca cc pa } & 000 \mathrm{~m}: 0-1\end{array}$

$\begin{array}{ll}\text { PROV: ca cc pa } & 000 \mathrm{~m}: 0-1 \\ \text { PROV: bo } & 000 \mathrm{~m}: 0-1\end{array}$

PROV: ca ch da he vr 000m: $0-1$

PROV: pa

000m: 0-1

PROV: ca cn da pa

000m: $0-1$

PROV: ca da sa

000m: $0-1$

PROV: da

000m: $0-1$

PROV: ca cc

000m: $0-1$

PROV: ca cc ch cn pa 000m: $0-1$

eriostachys Benth.

pulcherrima (L.) Sw.

cult.

PROV: $\mathrm{CC}$ Pa

000m: $0-1$

PROV: bo ca lo pa 000m: $0-1$

\section{Cajanus}

bicolor DC.

cult.
PROV: bo ca cc ch cn $000 \mathrm{~m}$ : $0-1$ da pa

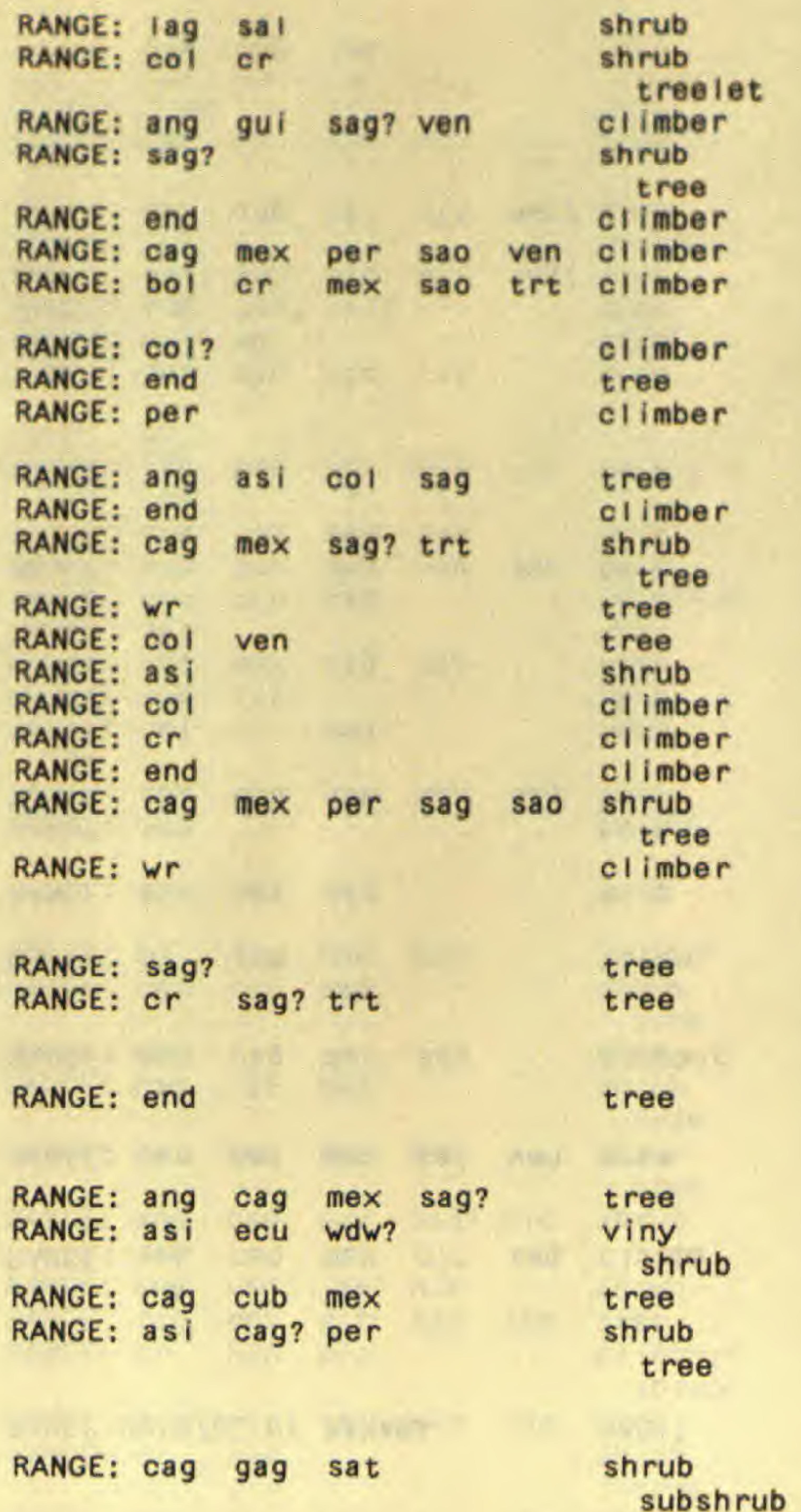




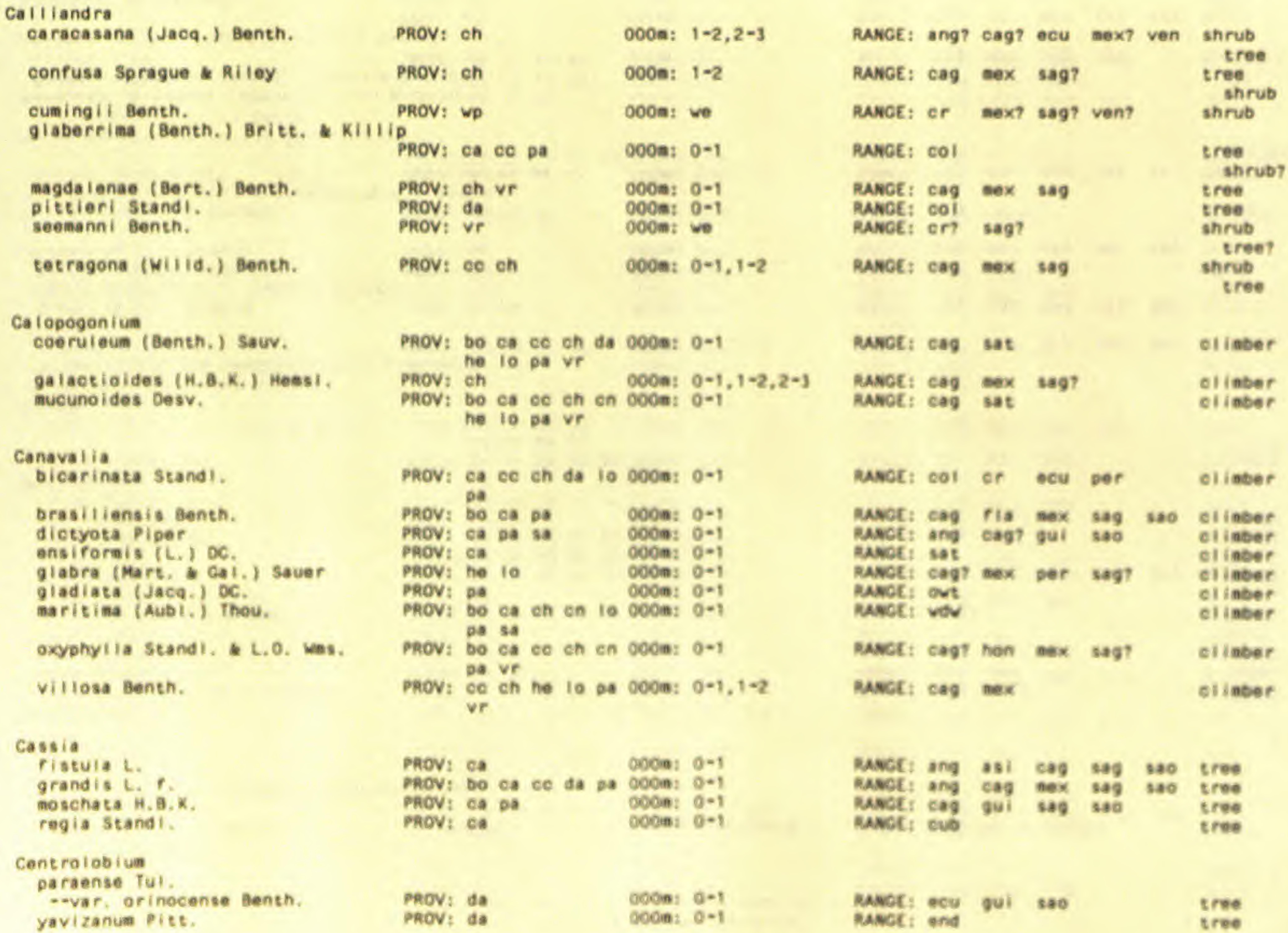


TAXON

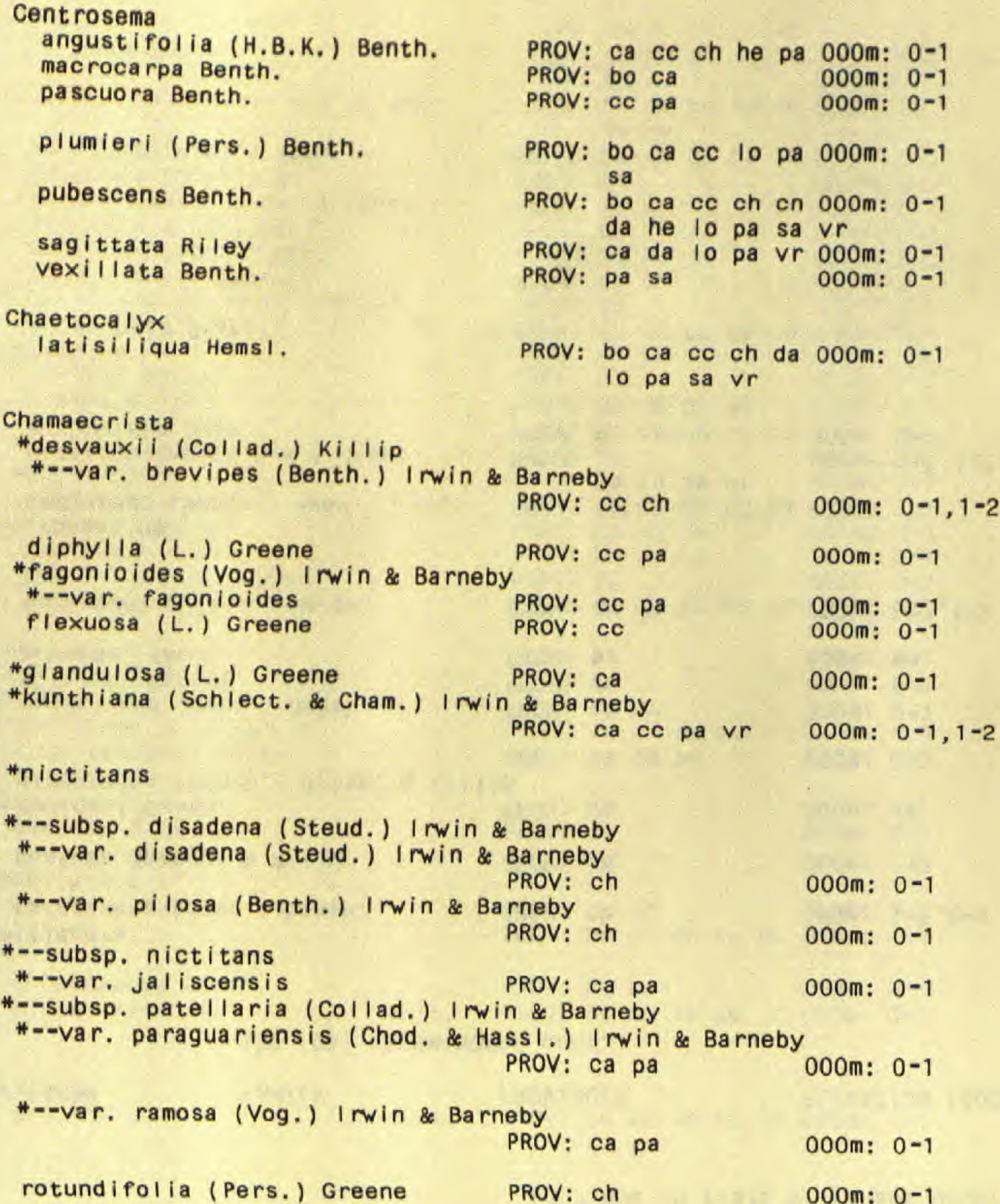

*--subsp. disadena (Steud.) I rwin \& Barneby

*--var. disadena (Steud.) I rwin \& Barneby

PROV: $\mathrm{ch} \quad 000 \mathrm{~m}: 0-1$

*--subsp. nictitans

*--var. jaliscensis

PROV: ch

$00 \mathrm{~m}: 0-1$

000m: $0-1$

PROV: ca pa

000m: $0-1$

* subsp. patellaria (Collad.) I win \& Barneby ss I.) I rwin \& Barneby PROV: ca pa 000m: $0-1$

\#--var. ramosa (Vog.) I rwin \& Barneby PROV: ca pa

000m: $0-1$

rotundifolia (Pers.) Greene

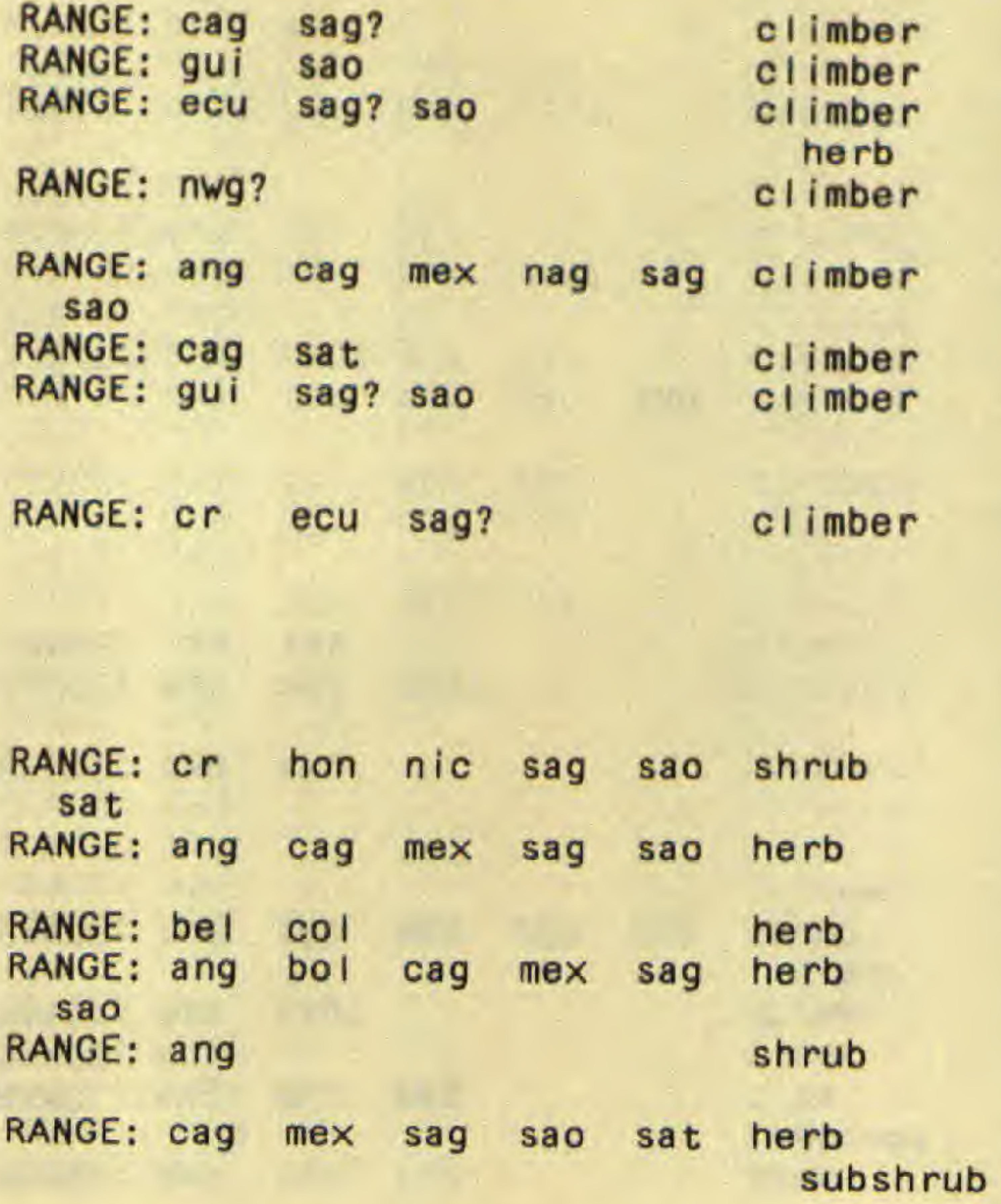

RANGE: cag mex sag sao herb

RANGE: cag or mex sao sat herb

RANGE: cag cub mex per sat herb

RANGE: bol cag col gua sao herb sat

RANGE: ang bel or mex per herb Sag sao sat
ANGE: ang cag cub mex nas herb sag sao sat 
128 (83) LEGUMINOSAE cont.

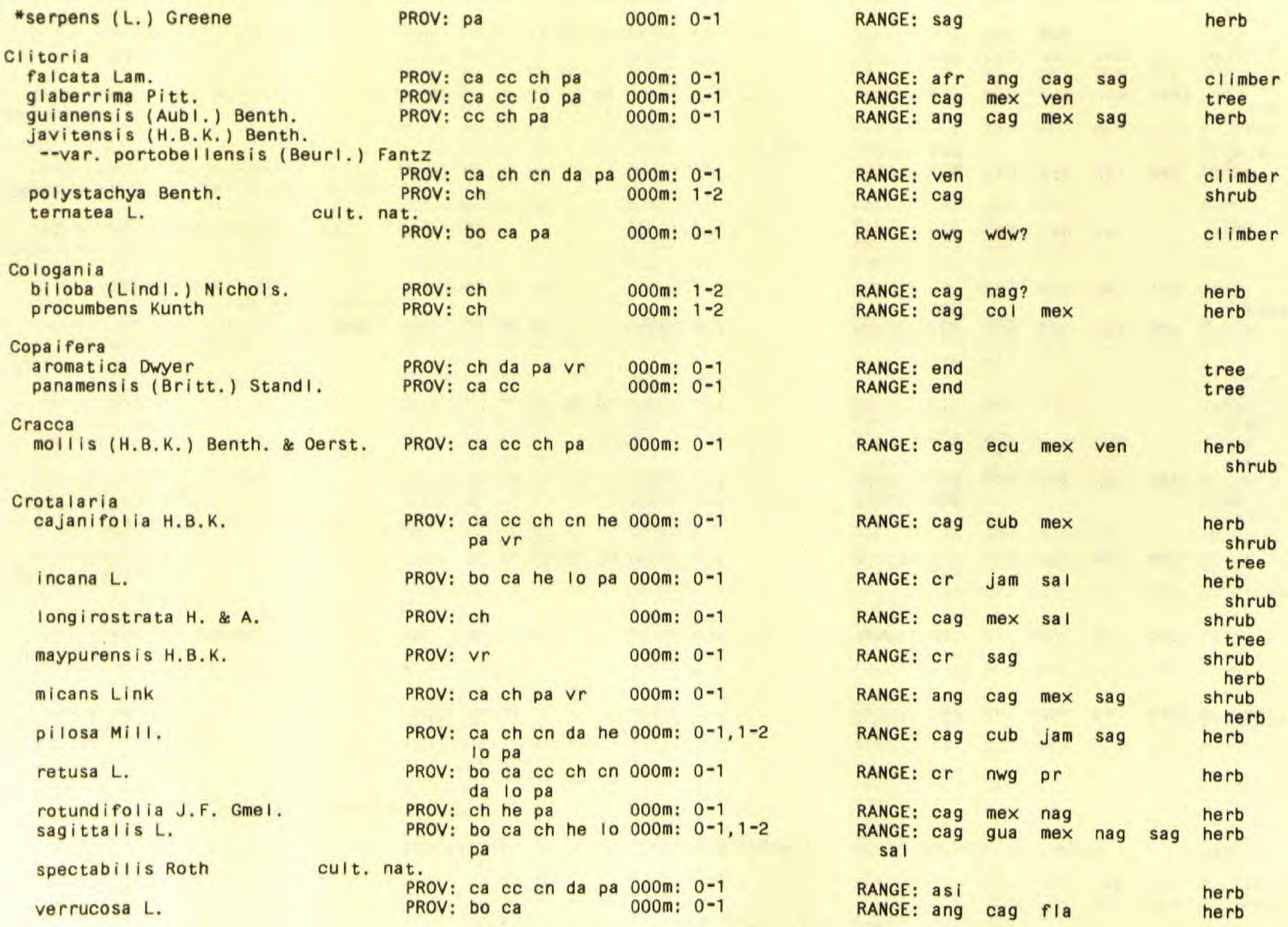


TAXON

FAMILY

PROVINCES

ELEVATION (000M.)

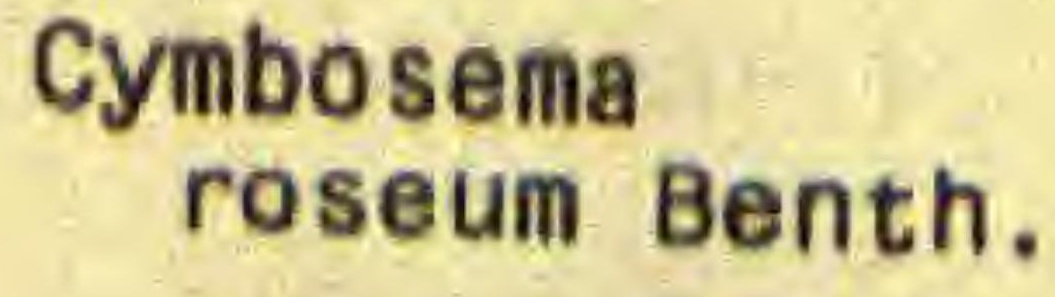

$000 m: 0-1$

PROV: ca cn pa

000m: $0-1$

$$
\text { bauhiniaefolia Benth. }
$$

\section{Dalbergia}

brownei (Jacq.) Urb.

cuscatlanica (Standl.) Standl.

*da rienens is Rudd

ecastaphylla (L.) Taub.

monetaria $L$. $f$.

retusa HemsI.

Dalea

carthagenens is (Jacq.) Macbr.

- -Var. barbata (Oerst.) Barneby PROV: cc he pa 000m: 0-1

cliffortiana Willd.

PROV: cc he lo 000m: $0-1$

Delonix

regia (Boj.) Raf.

intr.

PROV: ca

PROV: ca cc pa

000m: $0-1$

smanthus

virgatus (L.) Willd.

PROV: bo ca cn da lo 000m: $0-1$ PROV: pa

PROV: da

PROV: bo sa

000m: $0-1$

000m: $0-1$

PROV: ca cn

000m: $0-1$

PROV: ca cc cn da pa 000m: 0-1

\section{Desmodium}

adscendens (SW.) DC.

affine schlecht.

angustifolium (H.B.K.) DC.

axillare (SW.) DC.

PROV: ca ch he pa vr 000m: $0-1,1-2$

-var, acutifolium (Kuntze) Urb. PROV: ca cc ch cn da 000m: 0-1

- -var. axillare

he lo pa

PROV: bo ca cc ch da $000 \mathrm{~m}$ : $0-1,1-2$ he pa

--var. stoloniferum (Poir.) Schubert

PROV: ca ch en da lo 000m: $0-1$

barbatum (L.) Benth.

pa sa

PROV: bo ca cc ch cn 000m: $0-1,1-2$

da pa $v r$

cajanifolium (H.B.K.) DC.
PROV: bo ca cc ch cn 000m: $0-1$ pa $\vee r$

\begin{tabular}{|c|c|c|c|c|c|c|}
\hline $\begin{array}{c}\text { RANGE: } \\
\text { sao }\end{array}$ & cag & $\mathrm{col}$ & $\operatorname{mex}$ & per & sag? & climber \\
\hline $\begin{array}{c}\text { RANGE: } \\
\text { sat }\end{array}$ & $\mathrm{col}$ & $\mathrm{cr}$ & gui & per & sao & tree \\
\hline RANGE: & ang & cag & fla & $\operatorname{mex}$ & sag & $\begin{array}{c}\text { shrub } \\
\text { tree }\end{array}$ \\
\hline $\begin{array}{l}\text { RANGE: } \\
\text { RANGE: }\end{array}$ & $\begin{array}{l}\text { cr } \\
\text { end }\end{array}$ & gua & & & & \\
\hline $\begin{array}{l}\text { RANGE: } \\
\text { Sao }\end{array}$ & ang & cag & fla & $\operatorname{mex}$ & sag? & $\begin{array}{c}\text { shrub } \\
\text { tree }\end{array}$ \\
\hline $\begin{array}{l}\text { RANGE: } \\
\text { RANGE: }\end{array}$ & $\begin{array}{l}\text { ang } \\
\mathrm{cr}\end{array}$ & $\begin{array}{l}\text { cag } \\
\text { mex }\end{array}$ & $\begin{array}{l}\operatorname{mex} \\
\text { nic }\end{array}$ & sag & & $\begin{array}{c}\text { shrub } \\
\text { tree } \\
\text { tree }\end{array}$ \\
\hline $\begin{array}{c}\text { RANGE: } \\
\text { per }\end{array}$ & $\begin{array}{l}\text { ang } \\
\text { ven }\end{array}$ & cag & $\mathrm{col}$ & fla & $\operatorname{mex}$ & $\begin{array}{l}\text { shrub } \\
\text { subshrub }\end{array}$ \\
\hline RANGE: & asi & cag & ecu & $\operatorname{mex}$ & ven & herb \\
\hline RANGE: & af $r$ & ang & cag & sag & & tree \\
\hline $\begin{array}{c}\text { RANGE: } \\
\text { SaO }\end{array}$ & $\begin{array}{l}\text { ang } \\
\text { tex }\end{array}$ & cag & fla & ga I & sag & $\begin{array}{l}\text { shrub } \\
\text { herb }\end{array}$ \\
\hline & & 3 & 251 & $\ln ^{2}=x^{2}=$ & & 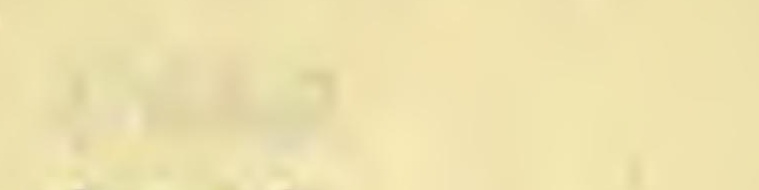 \\
\hline RANGE: & $a f r$ & ang & asi & cag? & sag? & herb \\
\hline $\begin{array}{l}\text { RANGE: } \\
\text { RANGE: }\end{array}$ & $\begin{array}{l}\text { ang } \\
\text { cag }\end{array}$ & $\begin{array}{l}\text { cag } \\
\text { mex }\end{array}$ & $\begin{array}{l}\text { mex } \\
\text { sag }\end{array}$ & sag & $=$ & $\begin{array}{l}\text { herb } \\
\text { herb }\end{array}$ \\
\hline RANGE: & ang & cag & $\mathrm{col}$ & gui & sao & herb \\
\hline RANGE: & ang & cag & $\operatorname{mex}$ & nwt & sag & herb \\
\hline RANGF. & & & 18 & & & -1 \\
\hline RANGE: & cag & gag & sag & & & herb \\
\hline RANGE: & $a f r$ & as $\mathrm{i}$ & nwg? & & & herb \\
\hline RANGE: & ang & ex & sag & & & $\begin{array}{l}\text { herb } \\
\text { shrub }\end{array}$ \\
\hline
\end{tabular}


Eanpyloeiados Honsi.

phov: ee on

000 a $0 * 1,1 * 2$

distartua (Aubi,) Nacbr.

giabrun (Mili.) DC.

incanus $\mathrm{DC}$.

infraesua $D C$.

insertua (Mi)!, urb.

wovar, apiculatum Schubert

aacrodesaus (biake) standi.

\section{aaxonil (Btand!, ) Standis

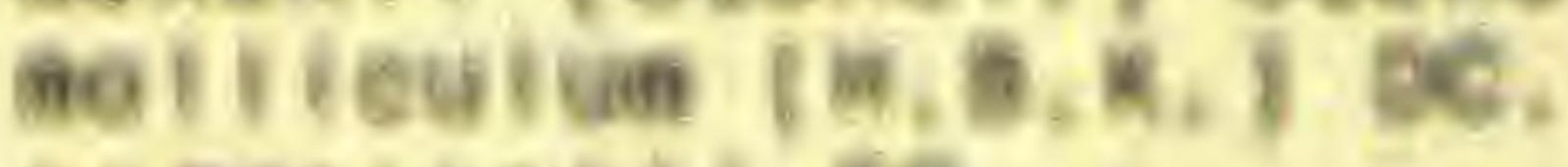

berrettetil be.

epocumbens (miis, witehe.

covar. lenglges (sehinds.) sehubers

savar, aprecumbens

hovi ea ee eh do he 000si 0-1

$$
\text { pa ve }
$$

Phovi ca ch on ba boons 0-1

phovi be ea $6 e$ en en goon: $0-1$

$$
\text { da ho la pa sa vi }
$$

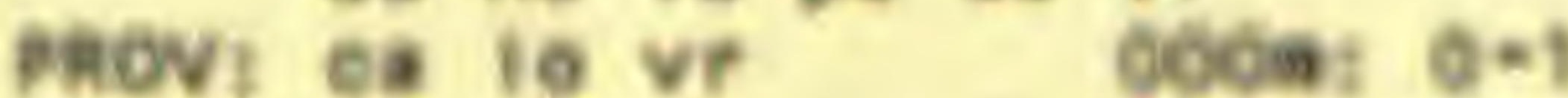

rhovi bo eh da gooin! $0+1,1+2,2+1$

Phovi vp

000 n: ve

bhov! be ce da obeni 0*1

phovi in Goon: $1+2,2 * 1$

cerori en

coon: $1+2,2 \cdot 1$

hovi ce

oconen: $0 \times 1$

MoOVi ca es on ea goton: a-1

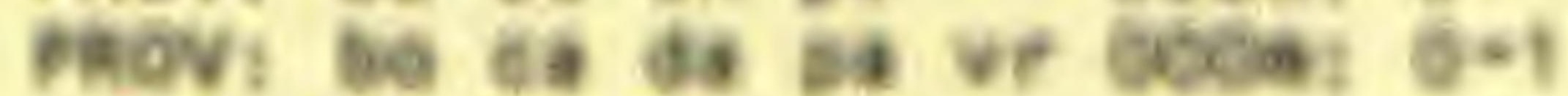

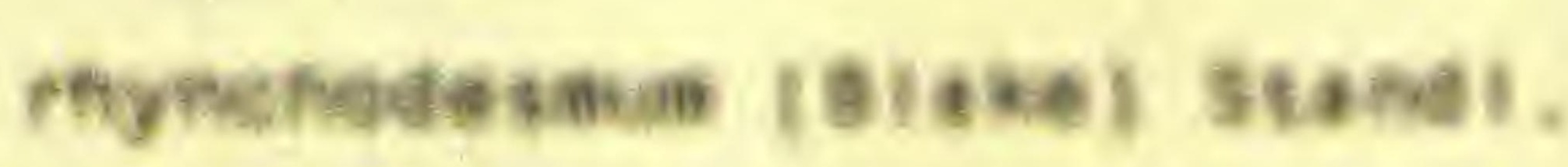

\section{macestoin sesubers.}

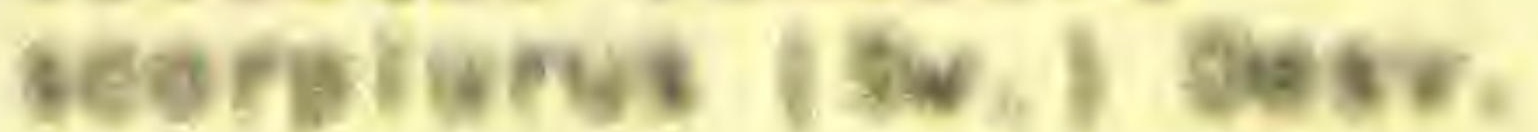

seribaghyilian Sebiacht

tertuessie $(\mathrm{N}, \mathrm{T}) \mathrm{DC}$.

teifigrse it. 1 b.

tyenustian stacut.

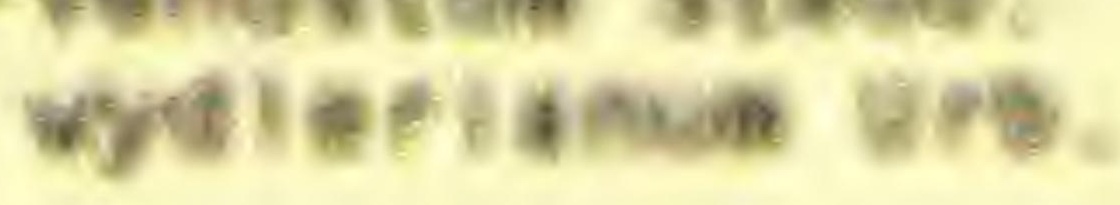

\section{dial inim}

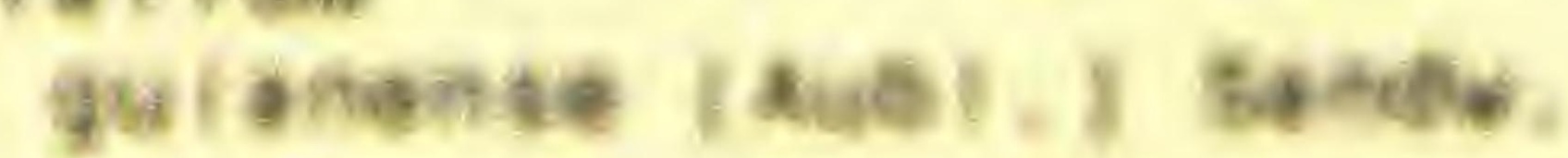

giosiod

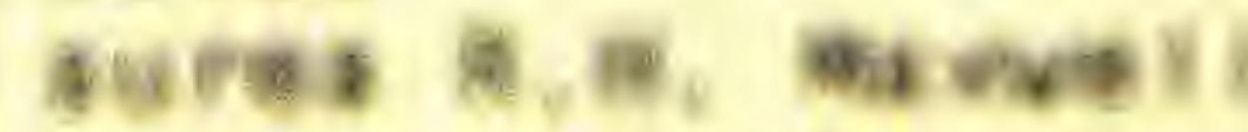

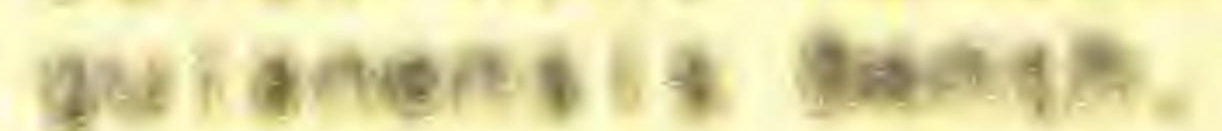

negacesed bisto

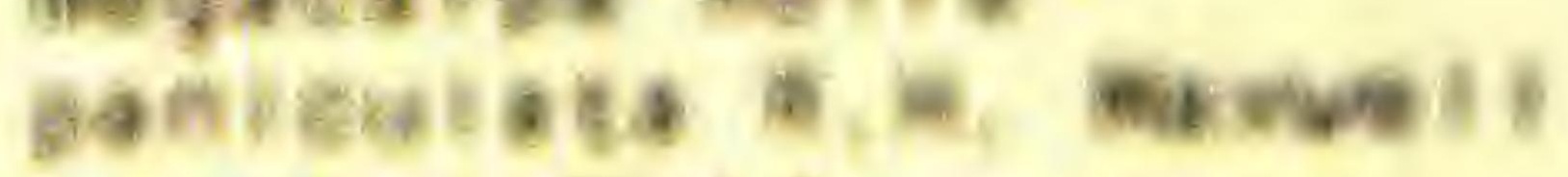

agtionts mats

replexs whis a ?

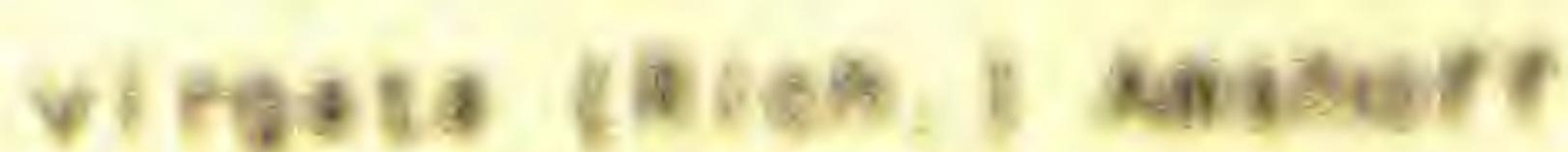

viligenth grated how: bo en cooen out

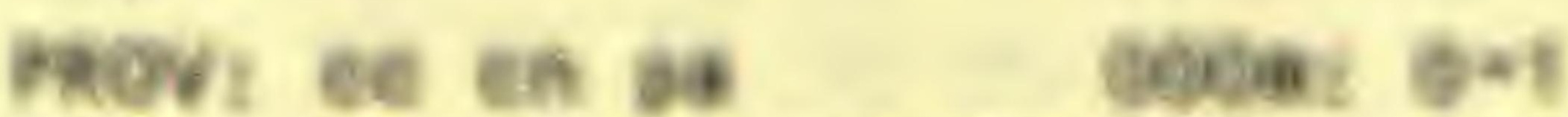

hovi bo es te ch sen coon: gut

goces ovi, $1+2$

obor en

how be ea ee en to gotal ant 18 ga

Phovi be ea ge eh se betie: Ger

Dat

Phove on

novi es ta

asos: $z+3$ Goine $9 \times 3$

Geovi de is

obelie $0+1$

raovs it

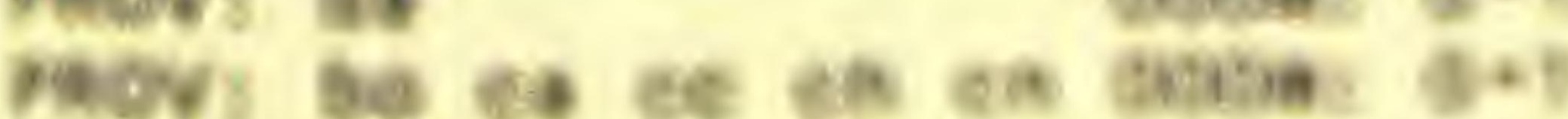
ta 0 as

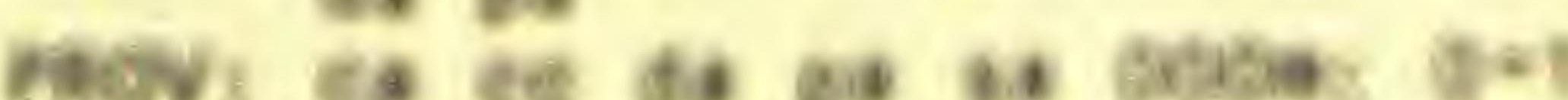

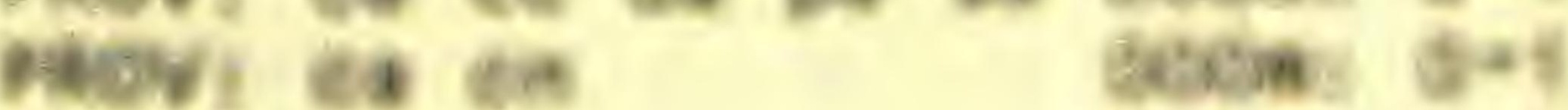

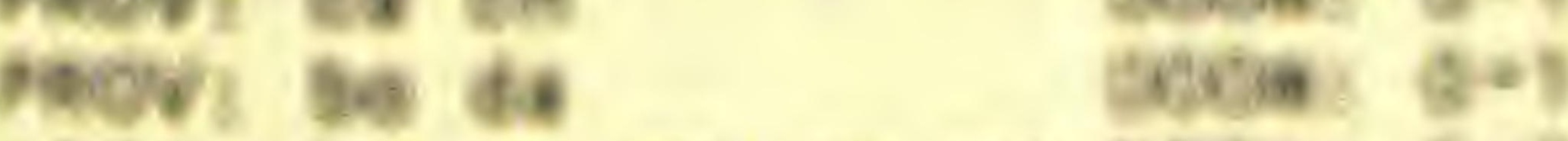

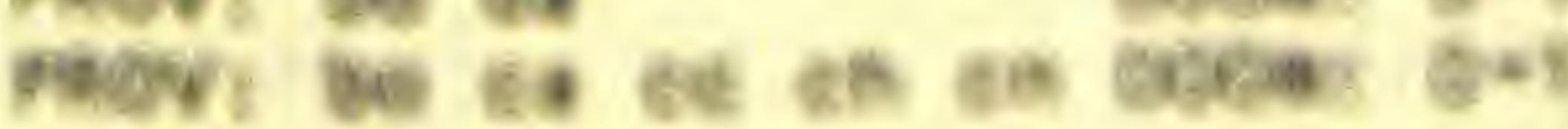

$$
\text { ta ba } \text { ba }
$$

acese $2 \times 3$

\begin{tabular}{|c|c|c|c|c|c|c|}
\hline 2uect: & $\cos$ & 601 & Der & ven & & herb \\
\hline naced: & 609 & $\operatorname{sos} x$ & $\operatorname{sag}$ & & & $\begin{array}{l}\text { hers } \\
\text { shrub }\end{array}$ \\
\hline Nect: & ong & or & 901 & $\operatorname{sex}$ & sag & nens \\
\hline DAOCH: & ing & $\cos$ & & & & herb \\
\hline EActi & eag & coi & 004 & $\max$. & ven & cilinber \\
\hline $2400 t_{1}$ & $n v t$ & & & & & here \\
\hline Nast! & $\operatorname{sen} x$ & 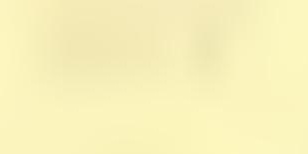 & & 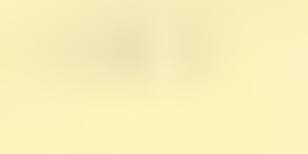 & 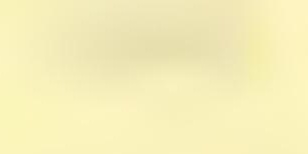 & hers \\
\hline mecti: & bei & gua & han & & & nere \\
\hline excti & $\cos$ & ans & & & & ans \\
\hline wase? & $\cos$ & aes & 309 & 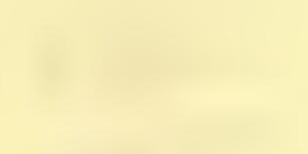 & 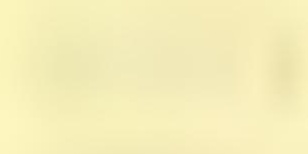 & hers \\
\hline NASE: & $30 i$ & ter & & (2) & 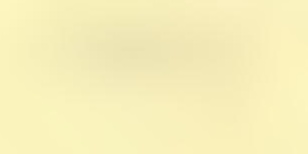 & ners \\
\hline savet! & $\cos$ & $\operatorname{mex}$ & $\operatorname{seg}$ & & & kers \\
\hline $2068 t_{1}$ & eth & 90 & 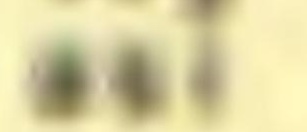 & eas & atse & nerb \\
\hline $\mathbf{* 1}$ & 40 & $m i$ & & & & \\
\hline eases: & er & & & & & $\begin{array}{l}\text { orimest } \\
\text { tusponst }\end{array}$ \\
\hline esectit & ond & & & & & cilineser \\
\hline daesf & or & $m$ & bete & & & keers \\
\hline suscefi & 604 & eel & ase & van & & ners \\
\hline ANWSE: & $\operatorname{mes}$ & & & & & Alers \\
\hline batses & $\cos$ & 263 & wt & wet & & hers \\
\hline tover: & neve & & & & & Natra \\
\hline $\begin{array}{c}\text { Aawer: } \\
\text { 3as }\end{array}$ & the & 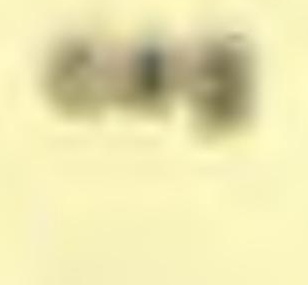 & er & $m$ & $\mathbf{m y}$ & moberve \\
\hline ta: & 99 & 3.6a & ger & 157 & 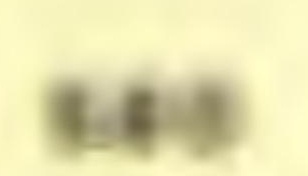 & ras \\
\hline
\end{tabular}

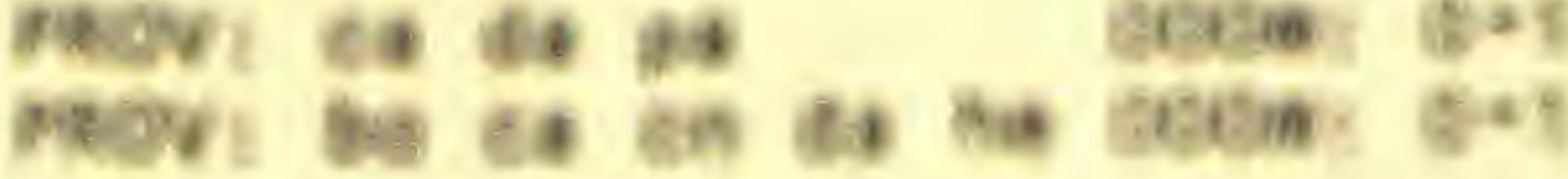

$$
\text { is } \mathrm{w} \text { vr }
$$

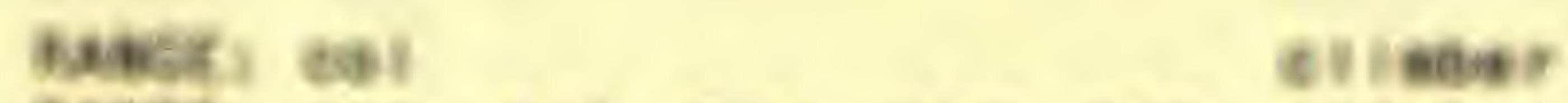

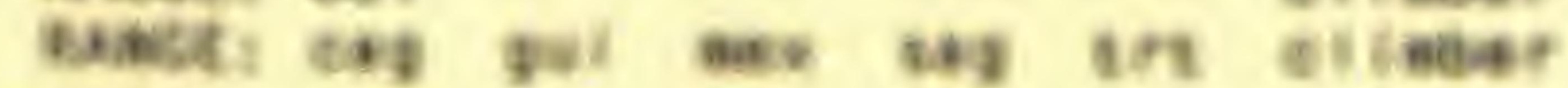

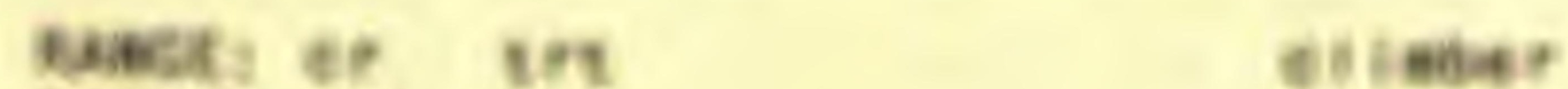

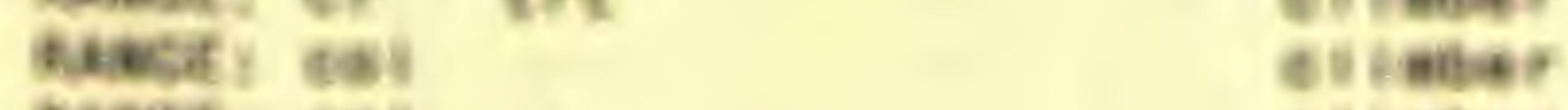

raser: teil allabe?

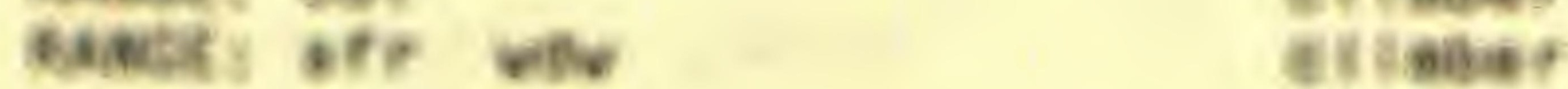

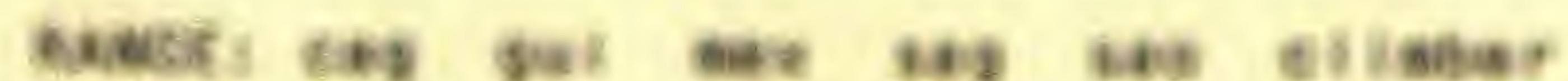

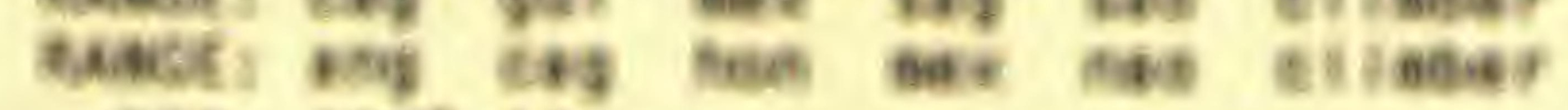
ote kes? ise 
TAXON

Diphysa

robinioides Benth.

PROV: ca ce ch da he 000m: $0-1,1-2$ lo pa sa vr

*Dipteryx

panamensis (Pitt.) Record Mell PROV: bo ca cn da pa 000m: 0-1

Dussia

cuscatIanica (StandI.) StandI. Steyerm.

PROV: ch da

$000 \mathrm{~m}: 0-1,1-2$

000m: $0-1$

\section{Entada}

* monostachya DC.

Enterolobium

cycloca rpum (Jaca.) Griseb.

schomburgkil Benth.

\section{Eriosema}

crinitum (H.B.K.) G. Don

diffusum (H.B.K.) G. Don

simplicifolium (H.B.K.) G. Don

violaceum (AUb).) G. Don

\section{Erythrina}

berteroana Urb.

chiriquensis Kruk.

costaricensis M. Mich.

cristamgalil L.

edulis Mich.

fusca Lour.

\section{cult.}

cult.

\section{gibbosa curod.}

Ianceolata StandI.

poeppigiana (WaIp.) 0,F, Cook rubrinervia H.B.K.
PROV: bo ca da pa 000m: 0-1

PROV: bo ca ce ch pa 000m: $0-1$

PROV: ch pa 000m: $0-1$

PROV: ca ce ch pa vr 000m: $0-1,1-2,2-3$

PROV: ch pa vr $\quad 000 \mathrm{~m}: 0-1,1-2$

PROV: ch

PROV: ca ce pa $\quad 000 \mathrm{~m}: 0-1$

PROV: bo ca ce ch en 000m: $0-1,1-2$ da he lo pa sa vr

PROV: ch da $000 \mathrm{~m}: 1=2,2-3$

PROV: bo ca ec eh en 000m: $0-1,1-2$

PROV: ca pa sa 000m: $0=1$

PROV: ch 000m: $0=1,1=2$

PROV: bo ca ce ch en 000m: $0-1$,

$$
\text { da pa }
$$

PROV: bo ce ch ve 000m: $0-1,1-2$

PROV: ch

000m: $0-1$

PROV: ca da

PROV: da pa vr 000m: $0-1$

000m: $0-1,1-2,2-3$
RANGE: cag mex nic

tree

RANGE: $\mathrm{COI} \mathrm{cr}$

tree

RANGE: cag gua mex sal tree

RANGE: $\mathrm{cr}$ tree

RANGE; afr ang cag sag climber

RANGE; cag mex

RANGE: cag? gua mex sag? sao tree

RANGE: bel or ven herb

RANGE: cag col mex subshrub

RANGE: cag gag gui sag? ven subshrub

RANGE: cag gag gui mex sag? herb

sao

subshrub

RANGE: ang cag col mex ven tree

RANGE: er

RANGE: col or

RANGE; sao

RANGE: bol sag?

RANGE: afr nwg? oce

RANGE: cag? or hon

RANGE: cag? or hon

RANGE: bol per sag?

RANGE: bel bol col per

\section{tree}

tree

tree

tree

tree

tree

shrub

shrub

tree

tree

shrub 

TAXON

\section{Flemingia}

strobilifera (L.) Ait.

\section{Galactia}

latisiliqua Desv.

striata (Jacq.) Ürb.

\section{Gificidia}

sepium (Jacq.) Walp.

cult

Hymenaea

courbaril L.

\section{Indigofera}

Jama icensis spr.

lespedezioides H, B, K.

panamens is Rydb.

suffruticosa MiII.

Inga

- alienil J. Leon

cocleensis pitt.

* coruscans willd.

davidsoniae Standi.

densifiora Benth.

* ragifolia Benth.

goldananif pitt.

hayesil Benth.

heterophylia Wilid.

*hintonir sandr.

onarginata wilid.

* inutula (Schery) Eilas

mucuna walp. Duchass.

multijuga genth.

pauciflora Walp. Duchass. - pezizifer Benth.
PROV: bo ca cn da pa 000m: $0-1$

PROV: $c h$ Vr

000m: $0-1$

PROV: ca ch da he lo 000m: $0-1$ pa sa vr

PROV: bo ca ch en da 000es: 0-1 he Io pa sa vr

PROV: ca ce on da he 000a: $0-1$ pa $v r$

PROV: bo ca ce ch ch 000a: $0-1,1-2$ da he 10 pa yr

PROV: ca cC ch he pa 000n: $0-1$

PROV: oa ce he OOOn: $0-1$ PRov: bo ea ce ch he 000m: $0-1$ lo pa vr

\begin{tabular}{|c|c|c|c|c|c|c|c|}
\hline PROV: & $c e$ & & & & & 000an: & $0-1$ \\
\hline PROV: & ca & ce & pa & & & 000n: & $0-1$ \\
\hline PROV: & da & & & & & 000m: & $0-1$ \\
\hline PROV: & ch & & & & & 000m: & $2-1$ \\
\hline PROV: & ce & sa & & & & 000an: & $0-1$ \\
\hline PAOV: & $\begin{array}{l}c a \\
\text { pa }\end{array}$ & $\begin{array}{l}\text { ce } \\
\forall r\end{array}$ & ch & da & he & 000an: & $0-1$ \\
\hline PROV: & ca & 10 & pa & $v r$ & & 000n: & $0-1$ \\
\hline PROV: & ca & da & pa & & & 000n: & $0-1$ \\
\hline PROV: & ce & da & & & & $000 \mathrm{en}:$ & $0-1,1-2$ \\
\hline PROV: & ca & & & & & $000 \mathrm{n}:$ & $0-1$ \\
\hline PROV: & bo & $\mathrm{ca}$ & ce & ch & da & $000 \mathrm{n}$ : & $0-1,1-2$ \\
\hline PROV: & $\begin{array}{l}\text { bo } \\
\text { da }\end{array}$ & ca & $c c$ & ch & da & 000n: & $0-1,1 \cdot 2,2 \cdot 3$ \\
\hline PROV: & bo & $\mathrm{ca}$ & da & pa & sa & 000n: & $0=1$ \\
\hline PROV: & $\begin{array}{l}c a \\
\mathrm{~V} r\end{array}$ & ch & da & pa & so & $000 \mathrm{a}:$ & $0-1$ \\
\hline PROV: & ca & da & pa & $v r$ & & 000n: & $0-1$ \\
\hline PROV: & ca & pa & & & & $000 \mathrm{n}$ : & $0-1$ \\
\hline
\end{tabular}

RANGE: as

shrub

subshrub

RANGE: col gui per? sag? herb

RANGE: afr cag nag sag sao climber Wdw?

RANCE: ang cag col sex tree

RAWGE: ang cag bex per sag tree sao

RANce: ang cag col gua jan herb

mex ven shrub

Rukce: bol cag gag mex sag? herb

sao shrub

RANCE: end shrub

RANGE: afr ang asi bol eag herb

fla nao oce sag sao tex shrub

Ravce: and

Pawce: col gua tree

uncr: end or tree

Nwort col ven

auce: ang bol cag nex sag tree

ert

Qunge: or

RuWCE: end

Qunce: sag tr:

tree

QuWcE: $\operatorname{mex}$

RUWGE: thex
RUWCE: bol or per sag? sao tree

aANCE; cag col or ecul gue tree

hon lag nex sag? sai sao

trt ven

AxGE: and

cagt hon tree

arot: and

Navet: 390 tree 
TAXON

FAMILY

PROVINCES

ELEVATION (OOOM.)

RANGE OUTSIDE OF PANAMA

HABIT

128 (83) LEGUMINOSAE cont.

portobellensis Beurl. punctata Willd.

*quaternata Poepp.

ruiziana G. Don

saffordiana Pitt.

* sapindoides Wilid.

spectabilis (Vahl) Willd.

standleyana Pitt.

"thibaudiana DC.

tumbellifera (VahI) Steud.

vera

*--subsp. spuria (Willd.) J. Leon

PROV: ca

000m: $0-1$

PROV: bo ca ce ch on $000 \mathrm{~m}$ : $0-1$ da pa sa vr

PROV: bo ca cc ch sa 000m: $0-1$

PROV: bo ca cn da pa $000 \mathrm{~m}: 0-1$

PROV: da 000m: $0-1$

PROV: bo ca cc ch da $000 \mathrm{~m}: 0-1$ pa sa

PROV: bo ca ce ch cn 000m: $0-1$ da he lo pa sa

PROV: ca cn da 000m: $0-1$

PROV: ca cn da pa 000m: $0-1$

PROV: bo ca ce ch da 000m: $0-1$ he lo pa vr

Lablab

purpureus (L.) Sweet cult. PROV: ca 000m: 0-1

Lennea

viridiflora Seem.

PROV: ca cc da $\mathrm{vr} \quad 000 \mathrm{~m}: 0-1$

Leucaena

* leucocephala (Lam.) de Wit

cult. PROV: ca

PROV: ca cc da pa 000m: $0-1$

PROV: da

\#trichodes Benth.

Lonchoca rpus

atropurpureus Benth.

calcaratus Hermann

chiricanus Pitt.

densiflorus Benth.

fendleri Benth.

guatemalensis Benth.

- var. proteranthus (Pitt.) Herman

I uteomaculatus Pitt.
monofoliaris Schery

ol iganthus Hermann

peninsularis (J,D, Sm.) Pitt.
PROV: ca cn

PROV: $\mathrm{cc}$

PROV: ch

PROV: bo ca

PROV: bo PROV: da

PROV: $\mathrm{CC}$

PROV: bo ca cn

PROV: bo

PROV: bo

PROV: bo
$000 \mathrm{~m}: 0-1$

000m: $0-1$

000m: $0-1$

000m: $0-1$

000m: $0-1$

000m: $0-1$

000m: $0-1$

$000 \mathrm{~m}: 0-1$

000m: $0-1$

000m: $0-1$

000m: $0-1$

000m: $0-1$

000m: $0-1$

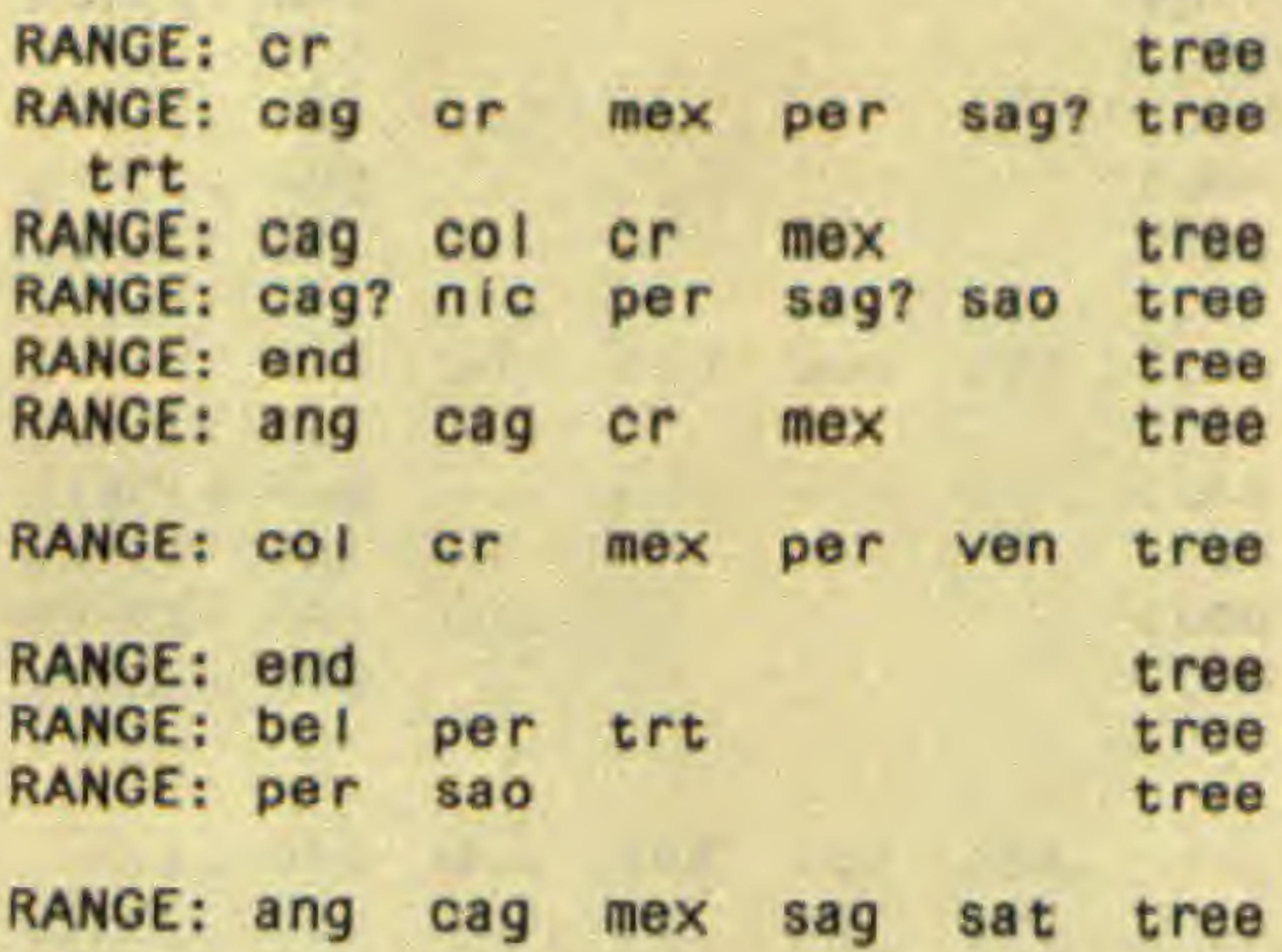

RANGE: owt

herb

RANGE: end

tree

RANGE; cag nwg? nwt per

shrub

RANGE: nic

RANGE: $\mathrm{cr}$ ecu per ven

tree

tree

shrub

RANGE: or ecu gua hon mex

ven

RANGE: end

RANGE: end

RANGE: gUi

RANGE: ven

RANGE: cag mex

shrub

tree

tree

tree

cl imb

shrub

tree

tree

tree

RANGE: end

RANGE: bel gua hon

RANGE: $c r$

RANGE: $c r$

tree

tree

tree

shrub

RANGE: cag mex

tree

shrub 
pentaphyllus (Poir.) DC.

sericeus (Poir.) DC. - -var. glabrescens Benth. velutinus seem.

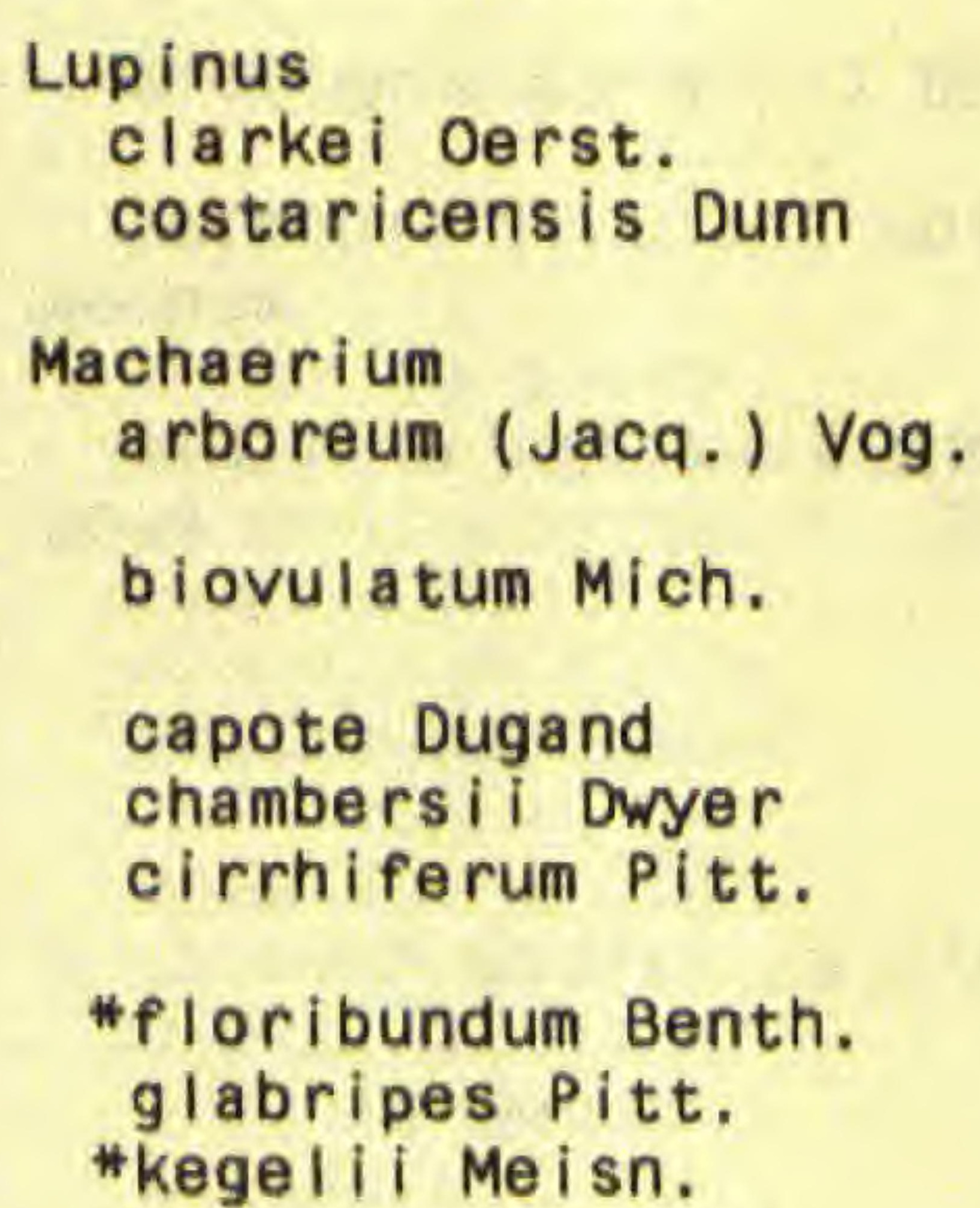

\section{Machaerium}

a rboreum (Jacq.) Vog.

biovula tum Mich.

\section{capote Dugand}

chambers i i Dwyer

cirrhiferum pitt.

*floribundum Benth.

glabripes Pitt.

*kegelii Meisn.

longifolium Benth.

I unatum (L. f.) Ducke

*microphyIIum (E, Meyer) StandI.

*milleflorum Pitt.

* riparium Brandeg.

*robini ifolium (DC.) Vog. seemanni i Seem.
PROV: bo ca cc cn pa 000m: $0-1$ sa $v r$

PROV: he $v r \quad 000 \mathrm{~m}: 0-1$

PROV: ca cn da pa 000m: 0-1

PROV: ca cc ch pa vr 000m: $0-1$

PROV: ch

PROV: ch

000m: $1-2,2-3,3+$

$000 \mathrm{~m}: 2-3,3+$

PROV: ca da

000m: $0-1$

PROV: ca cc pa

000m: $0-1,2-3$

PROV: da

PROV: da

PROV: $\mathrm{CC}$

000m: $0-1$ 000m: $0-1$ $000 \mathrm{~m}: 0-1$

PROV: ca

000m: $0-1$

PROV: $\mathrm{CC}$

000m: $0-1$

PROV: ca cc pa vr $\quad 000 \mathrm{~m}: 0-1$

PROV: da

000m: $0-1$

PROV: ca ch pa

000m: $0-1$

PROV: ca ch cn da he $000 \mathrm{~m}$ : $0-1$ pa sa $v r$

PROV: ca cc cn da pa 000m: 0-1 PROV: ca $000 \mathrm{~m}: 0-1$

PROV: da 000m: $0-1$

PROV: ca cc ch ch cn 000m: $0-1$ da pa

\footnotetext{
Macrolobium

modicopeta I um Schery

pittieri (Rose) Schery

Macropti I ium

a tropurpureum (DC.) Urb.

gracile (Benth.) Urb.
}

*colombianum (Britt. \& killip) killip

PROV: pa

PROV: bo

PROV: sa

000m: $0-1$

000m: $0-1$

000m: $0-1$

PROV: ca cc lo pa

000m: $0-1$

PROV: cc pa

\begin{tabular}{|c|c|c|c|c|c|c|}
\hline RANGE: & ang & cag & sag & & & tree \\
\hline RANGE: & af $r$ & ang & cag & $\operatorname{mex}$ & sag? & tree \\
\hline RANGE: & af $r$ & ang & cag & sag? & & tree \\
\hline RANGE: & cag & $\mathrm{col}$ & $=$ & & & tree \\
\hline RANGE: & $\mathrm{cr}$ & hon & & & & herb \\
\hline RANGE: & $\mathrm{cr}$ & & wix & & & herb \\
\hline RANGE: & $\mathrm{col}$ & $\mathrm{cr}$ & hon & $\operatorname{mex}$ & ven & shrub \\
\hline RANGE: & cag & $\operatorname{mex}$ & & & & $\begin{array}{c}\text { tree } \\
\text { shrub } \\
\text { troe }\end{array}$ \\
\hline RANGE: & $\mathrm{col}$ & 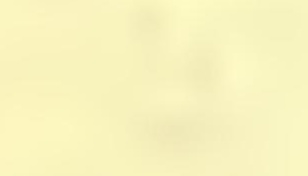 & 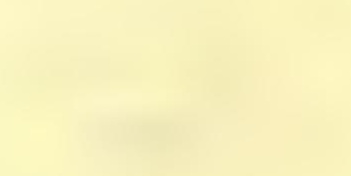 & & & tree \\
\hline RANGE: & end & & & & & tree? \\
\hline RANGE: & end & & & 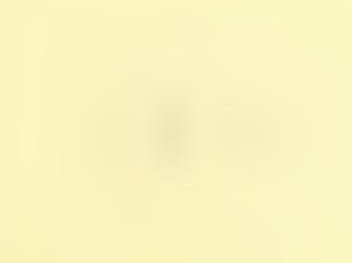 & & $\begin{array}{l}\text { tree } \\
\text { shrub }\end{array}$ \\
\hline RANGE: & $\operatorname{mex}$ & per & ven & & & cl imber \\
\hline RANGE: & end & & & & & tree \\
\hline RANGE: & cr & & & & 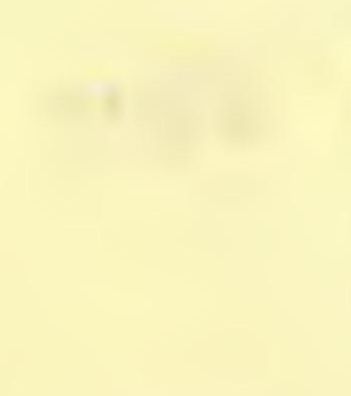 & $\begin{array}{l}\text { tree } \\
\text { shrub } \\
\text { climber }\end{array}$ \\
\hline RANGE: & $\mathrm{cr}$ & nic & sag? & & & $\begin{array}{l}\text { shrub } \\
\text { cl imber }\end{array}$ \\
\hline RANGE: & af $r$ & ang & cag & sag? & & $\begin{array}{l}\text { shrub } \\
\text { climber }\end{array}$ \\
\hline RANGE: & & $-x$ & 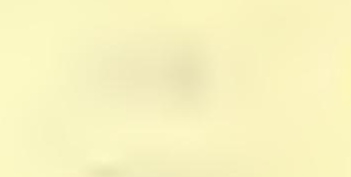 & sert & & tree \\
\hline & say & tis & & & & $\begin{array}{l}\text { shrub } \\
\text { climber }\end{array}$ \\
\hline & & & & t & & $\begin{array}{c}\text { tree } \\
\text { climber }\end{array}$ \\
\hline RANGE & bel & mex & & & & $\begin{array}{l}\text { climber } \\
\text { shrub } \\
\text { climber }\end{array}$ \\
\hline $\begin{array}{l}\text { RANC } \\
\text { RANO }\end{array}$ & 1 & ven & & & & tree \\
\hline & 01 & gua & hon & & & climber \\
\hline
\end{tabular}

RANGE: $c O 1$ ven

RANGE: sat

RANGE: end

tree

tree

RANGE: WdW

RANGE: ang

cag climber

herb 
TAXON

lathyroides (L.) Urb.

PROV: ca cc cn he lo 000m: $0-1$

longepedunculatum (Benth.) Urb. PROV: ca cc lo pa vr 000m: 0-1

\section{Mimosa}

albida Willd.

- -var. albida

*--var. glabrior Rob.

casta $L$.

"guilandinae (DC.) Barneby

invisa Mart.

"myriadena Benth.

panamens is (Benth, ) Stand I.

pigra L.

\section{polydactyla WilId}

pudica L.

pusilla Benth.

* quadrivalvis L.

*--var. hamata (H,B,K.) Beard somnians Willd.

velloziana Mart.

Mora

oleifera (Triana) Ducke

Mucuna

holtonil (Kuntze) Mold.

mutisiana (H.B.K.) DC.

rostrata Benth.

sloanei Fawc. Rendl.

urens (L.) DC.

Muel Iera

monil iformis L. f.

\section{*My rospermum}

"frutescens Jacq.

Myroxyion

ba I samum (L.) Harms

*--var, pereirae (Royle) Harms

000m: $0-1$
PROV: bo ca

$000 \mathrm{~m}: 0-1$

PROV: da pa

000m: $0-1$

PROV: bo ca cc cn da $000 \mathrm{~m}$ : $0-1,1-2$

PROV: bo ca ce da lo 000m: $0-1$ pa sa

PROV: ca cn da 000m: $0-1$

PROV: ca cc pa 000m: $0-1$

PROV: ca cc ch da 000m: $0-1,1-2$

PROV: bo ca da

000m: $0-1$

000m: $0-1$

PROV: CC

$000 \mathrm{~m}: 0-1$

PROV: da pa sa
RANGE: $\operatorname{mex}$

herb

RANGE: NWt

cl imber

$\begin{array}{lll}\text { RANGE: cag mex sag? } & \text { climber } \\ \text { RANGE: gua mex nic sal } & \text { shrub } \\ \text { RANGE: lag sao } & \text { herb } \\ \text { RANGE: col mex sao } & \text { climber } \\ \text { RANGE: ang cag mex sag sao herb }\end{array}$

RANGE: ang cag mex sag sao herb

RANGE: sat climber

RANGE: $\mathrm{col} \mathrm{cr}$ herb

RANGE: afr ang cag? sag? sao shrub

Wd $t$

RANGE: sag? sao

RANGE: ang cag owt per sag herb

RANGE: cag mex herb

RANGE: or per climber

RANGE: per

RANGE: cag mex per sag sao herb

RANGE: cag mex sag sao viny

subshrub

shrub

RANGE: $\mathrm{cO}$ I

tree

RANGE: cag col mex

climber

RANGE: $c O \mathrm{I}$ or ven

climber

RANGE: bol ecu per sao

climber

RANGE: afr ang cag

RANGE: ang $\mathrm{cr}$ cag

cl imber

RANGE: sag? trt

tree

RANGE: col cr ven

tree
RANGE: cag? mex per sag?

RANGE: mex per sao ree

tree 
TAXON

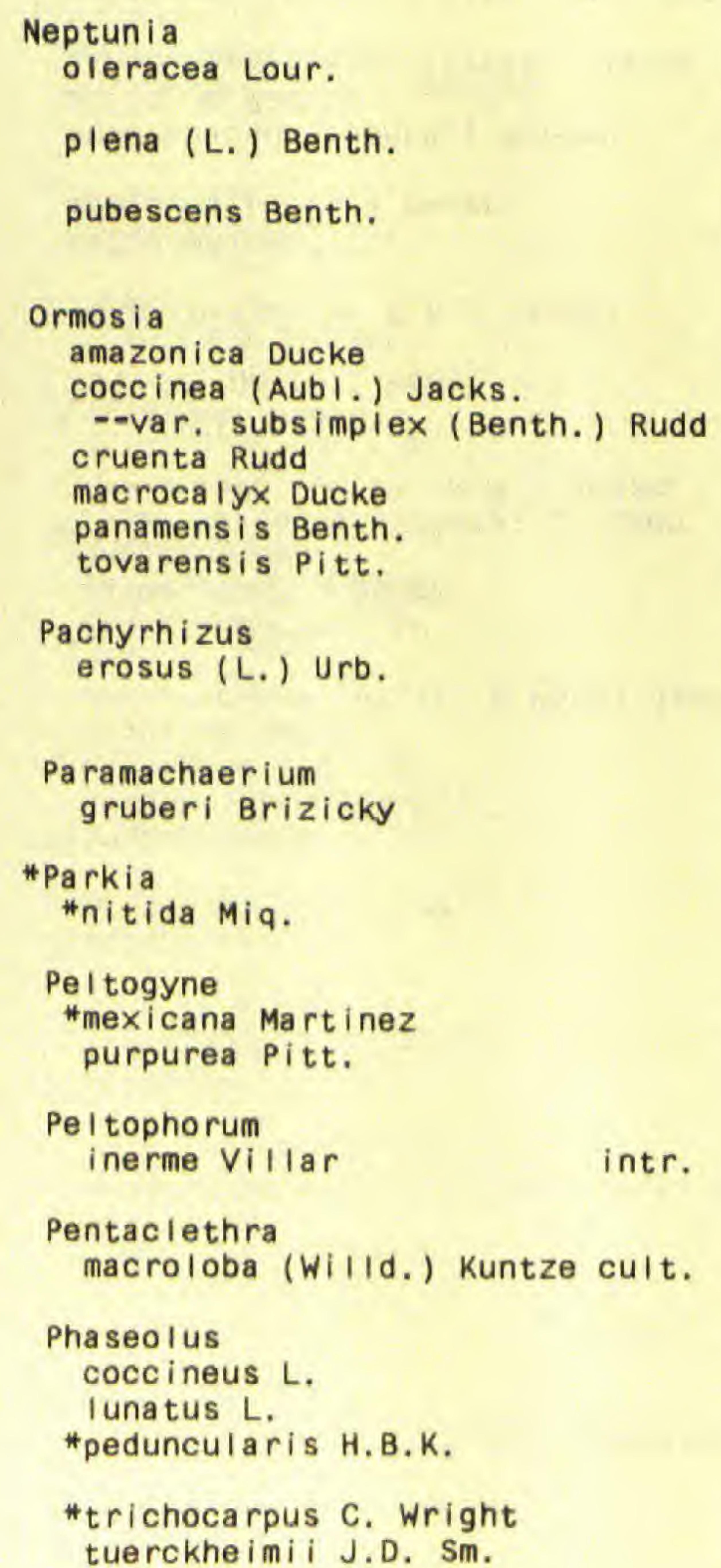

Pentaclethra macroloba (Willd.) Kuntze cult.

Phaseolus

coccineus $L$.

lunatus $L$.

*peduncularis H.B.K.

*trichocarpus C. Wright

tuerckheimi i J.D. Sm.

PROV: $\mathrm{CC}$

PROV: $\mathrm{CC}$

PROV: $\mathrm{CC}$

PROV: ca

PROV: ca sa

PROV: ca $v r$

PROV: cc ch

$000 \mathrm{~m}: 0-1,1-2$

ch pa vr 000m: $0-1$

PROV: bo ca ch

000m: $0-1$

PROV: da

000m: $1-2$

PROV: bo ca ch cn lo $000 \mathrm{~m}$ : $0-1$ pa sa

PROV: ch

000m: $0-1$

PROV: da

000m: $0-1$

PROV: da

PROV: ca da

000m: $0-1$

000m: $0-1$ 000m: $0-1$

PROV: ca

000m: $0-1$

PROV: ch

PROV: ca ch he pa vr 000m: $0-1$

PROV: bo ca cc da pa $000 \mathrm{~m}: 0-1$

PROV: ca ch da pa $000 \mathrm{~m}: 0-1$

PROV: ch 000m: $1-2,2-3$

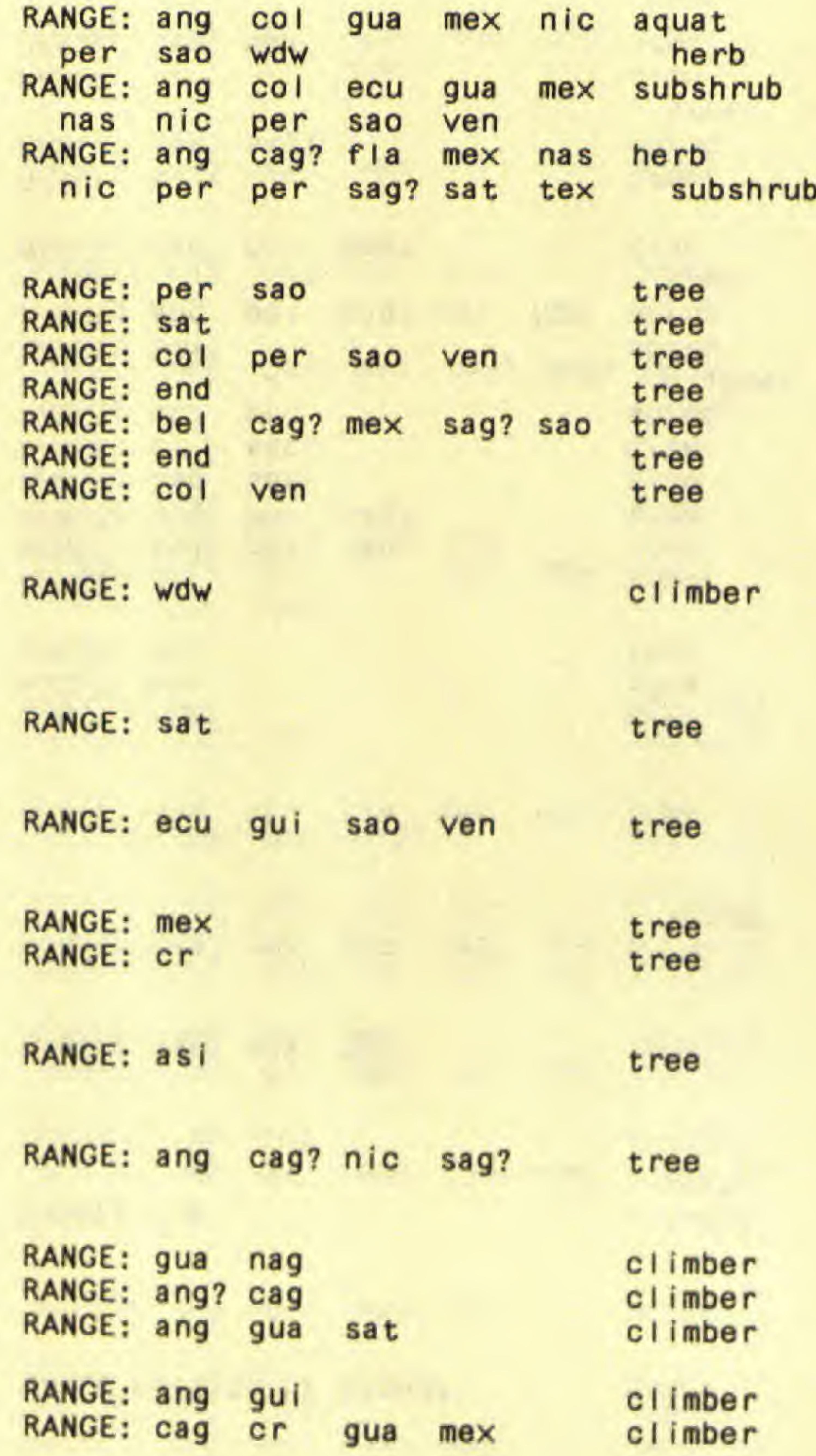


TAXON

vulgaris L.

$$
\text { cult. PROV: WP }
$$

000m: we

Phy I locarpus

septentrionalis J.D. Sm. intr. cult.

\section{PROV: ca}

PROV: $\mathrm{CC}$

000m: $0-1$

Piptadenia

Plava (Spr.) Benth.

$000 \mathrm{~m}: 0-1$

Piscidia

piscipula (L.) Sarg.

PROV: ca

00m: $0-1$

*Pi thecel lobium

$\begin{array}{lll}\text { ba rbourianum Stand I. } & \text { PROV: ca } & 000 \mathrm{~m}: 0-1 \\ \text { catenatum J.D. Sm. } & \text { PROV: bo } & 000 \mathrm{~m}: 0-1\end{array}$

cognatum Benth.

costaricense (Britt. \& Rose) Standl.

"dinizil Ducke

dulce (Roxb.) Benth.

fragrans Benth.

* giganifol iol um (Schery) J. Leon

hymeneaefolium (H. \& B.) Benth.

* johanseni StandI.

latifolium (L.) Benth.

PROV: ca

000m: $0-1$

000m: $0-1$

PROV: ch

PROV: ca

PROV: ca

PROV: $V r$

PROV: bo

PROV: ca da lo pa

$000 m: 1-2,2-3$

$000 \mathrm{~m}: 0-1$

000m: $0-1$

000m: $0-1$

000m: $0-1$

000m: $0-1$

PROV: ch

PROV: bo ca

000m: $0-1$

000m: $0-1$

longifolium (H. \& B.) Standl.

macradenium Pitt.

mangense (Jacq.) Macbr.

PROV: bo ca ce cn da $000 \mathrm{~m}$ : $0-1$ pa

PROV: ca

000m: $0-1$

PROV: vr

000m: $0-1$

membranaceum (Benth.) Schery

oblongum Benth.

PROV: ch

PROV: ca pa

000m: $0-1,1-2$

000m: $0-1$

PROV: bo

rufescens (Benth.) Pitt., non Mohl. (1966)

000m: $0-1$

PROV: ca cc cn da he 000m: 0-1

lo pa sa

--var, val lense Schery

saman (Jacq.) Benth.

tubuliferum Pitt.

PROV: $\mathrm{CC}$

PROV: bo ca he $\mathrm{vr}$

PROV: $\mathrm{cc}$ ch

000m: $0-1$

000m: $0=1$

000m: $0-1,1-2$

*unifoliatum Benth.

PROV: cn

PROV: bo 000m: $0=1$

000m: $0-1$

\section{RANGE: nwg}

climber

herb

RANGE: cag? gua hon?

tree

RANGE: cag? mex nic sag?

tree

shrub

RANGE: ang cag fla mex ven tree

RANGE: end

RANGE: $\mathrm{cr}$

RANGE: $\operatorname{mex}$

tree

tree

tree

RANGE: $c r$

RANGE: col per sao

RANGE: cag mex sag?

RANGE: cag sat

RANGE: or sag?

RANGE: Col ven

RANGE: beI?

RANGE: ang bol cag? col hon Sao

RANGE: cag? hon sag?

tree

tree

tree

tree

tree

shrub

climber

shrub

shrub

tree

RANGE: $c r$

RANGE: jam sag?

tree

RANGE: $\mathrm{cr}$

RANGE: cag mex

tree

shrub

tree

RANGE: end

tree

tree

tree

RANGE: $\mathrm{col} \mathrm{cr}$

tree

RANGE: end

RANGE: ang

RANGE: $\mathrm{COI}$

cag mex owg tree

RANGE: col ecu per ven

tree

RANGE: $\mathrm{cr}$ 


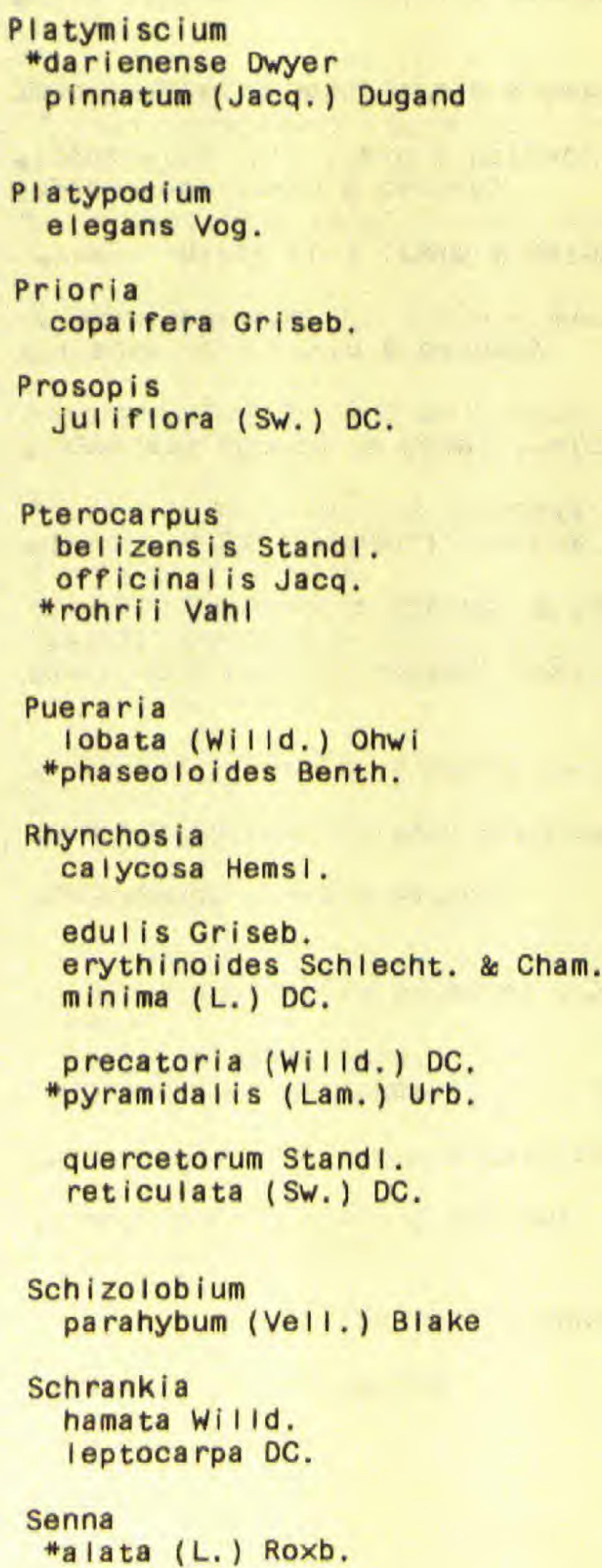

PROV: da 000m: $0-1$ PROV: ca cc ch da pa $000 \mathrm{~m}: 0-1$

PROV: ca ch da pa $000 \mathrm{~m}: 0-1$ PROV: bo ca da sa $000 \mathrm{~m}$ : $0-1$ PROV: da pa $\quad 000 \mathrm{~m}: 0-1$

PROV: bo ca $\quad 000 \mathrm{~m}: 0-1$ PROV: bo ca cn da pa 000m: $0-1$ PROV: bo ca da pa 000m: $0-1$

PROV: 000m: $0-1$ PROV: ch da 000m: $0-1$

PROV: ca ce ch da lo 000m: $0-1$ pa $v r$

PROV: pa $000 m: 0-1$ PROV: bo ca cn pa $000 \mathrm{~m}$ : $0-1$ PROV: bo ca ch lo pa $000 \mathrm{~m}$ : $0-1$ PROV: 000m: $0-1$ PROV: bo ca ce en da 000m: $0-1$ PROV: $\mathrm{ch} \quad 000 \mathrm{~m}: 1$-2 PROV: ca cc ch he pa 000m: $0-1$ $\mathrm{vr}$

PROV: ca da pa

000m: $0-1$

PROV: ca da pa

000m: $0-1$ PROV: ca cc pa 000m: $0-1$

PROV: ca cc pa vr 000m: $0-1$

\begin{tabular}{|c|c|c|c|c|c|c|}
\hline $\begin{array}{c}\text { RANGE: } \\
\text { per }\end{array}$ & col & $\begin{array}{l}c r \\
t r t\end{array}$ & $\begin{array}{l}\text { gua } \\
\text { ven }\end{array}$ & hon & nic & $\begin{array}{l}\text { tree } \\
\text { tree }\end{array}$ \\
\hline RANGE: & sag & sao & ven & 2 & 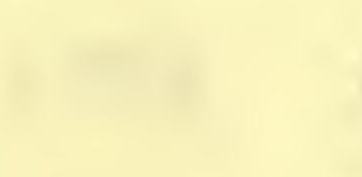 & tree \\
\hline RANGE: & ang & cag? & $\mathrm{col}$ & nic & 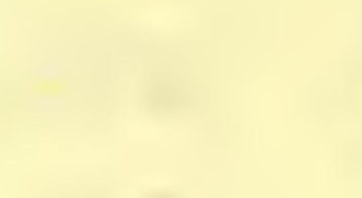 & tree \\
\hline $\begin{array}{c}\text { RANGE: } \\
\text { ven }\end{array}$ & ang & cag? & $\mathrm{col}$ & nag? & sag? & $\begin{array}{c}\text { shrub } \\
\text { tree }\end{array}$ \\
\hline RANGE: & bel & & & & & tree \\
\hline RANGE: & ang & cag & ecu & sag & & tree \\
\hline RANGE: & bel & bol & $\mathrm{cr}$ & gua & mex & tree \\
\hline nic & per & sao & & & & \\
\hline $\begin{array}{l}\text { RANGE: } \\
\text { RANGE: }\end{array}$ & $\begin{array}{l}\text { as i } \\
\text { ecu }\end{array}$ & per & - & 10 & & $\begin{array}{l}\text { cl imber } \\
\text { cl imber }\end{array}$ \\
\hline RANGE: & cag & ecu & sag? & & & climber \\
\hline RANGE: & cag & nao & sag & sao & & climber \\
\hline RANGE: & cag & $\mathrm{cr}$ & ecu & $\operatorname{mex}$ & sag & climber \\
\hline RANGE: & cag & jam & $\operatorname{mex}$ & sag & sao & climber \\
\hline RANGE: & cag & col & $\operatorname{mex}$ & 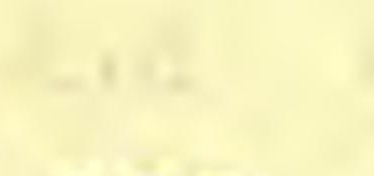 & & climber \\
\hline $\begin{array}{c}\text { RANGE: } \\
\text { sat }\end{array}$ & ang & gui & $\operatorname{mex}$ & per & sao & climber \\
\hline RANGE: & cag & $\mathrm{col}$ & & 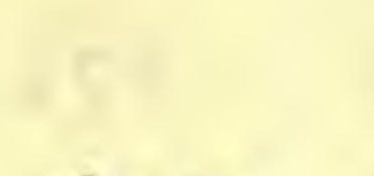 & $=$ & climber \\
\hline $\begin{array}{l}\text { RANGE: } \\
\text { sag }\end{array}$ & cag & gag & hon & jam & $\operatorname{mex}$ & climber \\
\hline RANGE: & cag & $\operatorname{mex}$ & sag & sao & & tree \\
\hline RANGE: & $\mathrm{col}$ & nic & & & & herb \\
\hline RANGE: & ang & cag & owg & sag & & herb \\
\hline
\end{tabular}




\section{TAXON}

FAMILY

PROVINCES

ELEVATION (OOOM.)

RANGE OUTSIDE OF PANAMA

HABIT

128 (83) LEGUMINOSAE cont.

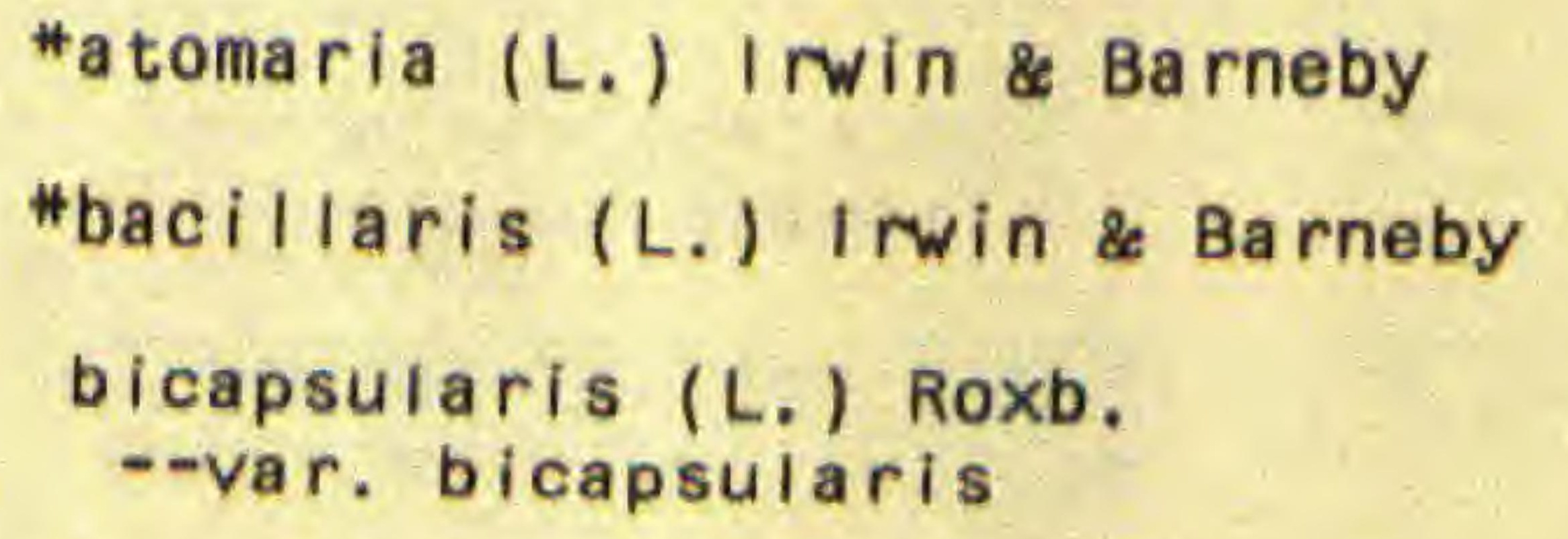

000m: $0-1$

"burkartiana (Villa Carenzo) I win \& Barneby PROV: ch

*cajamarcae I Wwin \& Barneby

PROV: ch

000m: $1-2$

$000 \mathrm{~m}: 1-2$

000m: $0-1$

* cobanensis (Britt. \& Rose) I rwin \& Barneby PROV: bo ca ch da pa 000m: $0-1,1-2$

\#dariensis (Britt. \& Rose) Irwin se Barnebr *--var. dariensis PROV: ca da pa \& Rose) I Wwin \& Barneby 000m: $0-1$

*didymobotrya (Fresen.) I rwin \& Barneby cult. PROV: ca

000m: $0-1$ 000m: $0-1$

*hayesiana (Britt. \& Rose) I rwin \& Barneby PROV: ca cc ch pa 000m: 0-1, 1-2

*hirsuta (L.) Irwin \& Barneby *--var. hirsuta PROV: bo ce da $000 \mathrm{~m}: 0-1$

*--var, hirta (L.) Irwin \& Barneby

*hypog lauca I rwin \& Barneby PROV: bo * igustrina $(L$,$) I rwin \& Barneby PROV: bo$

*macrophyl la (Kunth) Irwin \& Barneby PROV: da

*multijuga (Rich.) I rwin \& Barneby PROV: ca

000m: $0-1$

*nicaraguensis (Benth.) I rwin \& Barneby

PROV: ca pa Vr 000m: $0-1$

*obtusifolia (L.) Irwin \& Barneby PROV: bo ca cc cn da 000m: 0-1 occidentalis Link he pa $v r$ PROV: bo ca ch pa $000 \mathrm{~m}: 0-1$

*pallida (VahI) I win \& Barneby PROV: cc he pa $v r$ *papillosa (Britt. \& Rose) Irwin \& Barneby *--var. papillosa 000m: $0-1$

$000 m: 0-1,1-2$

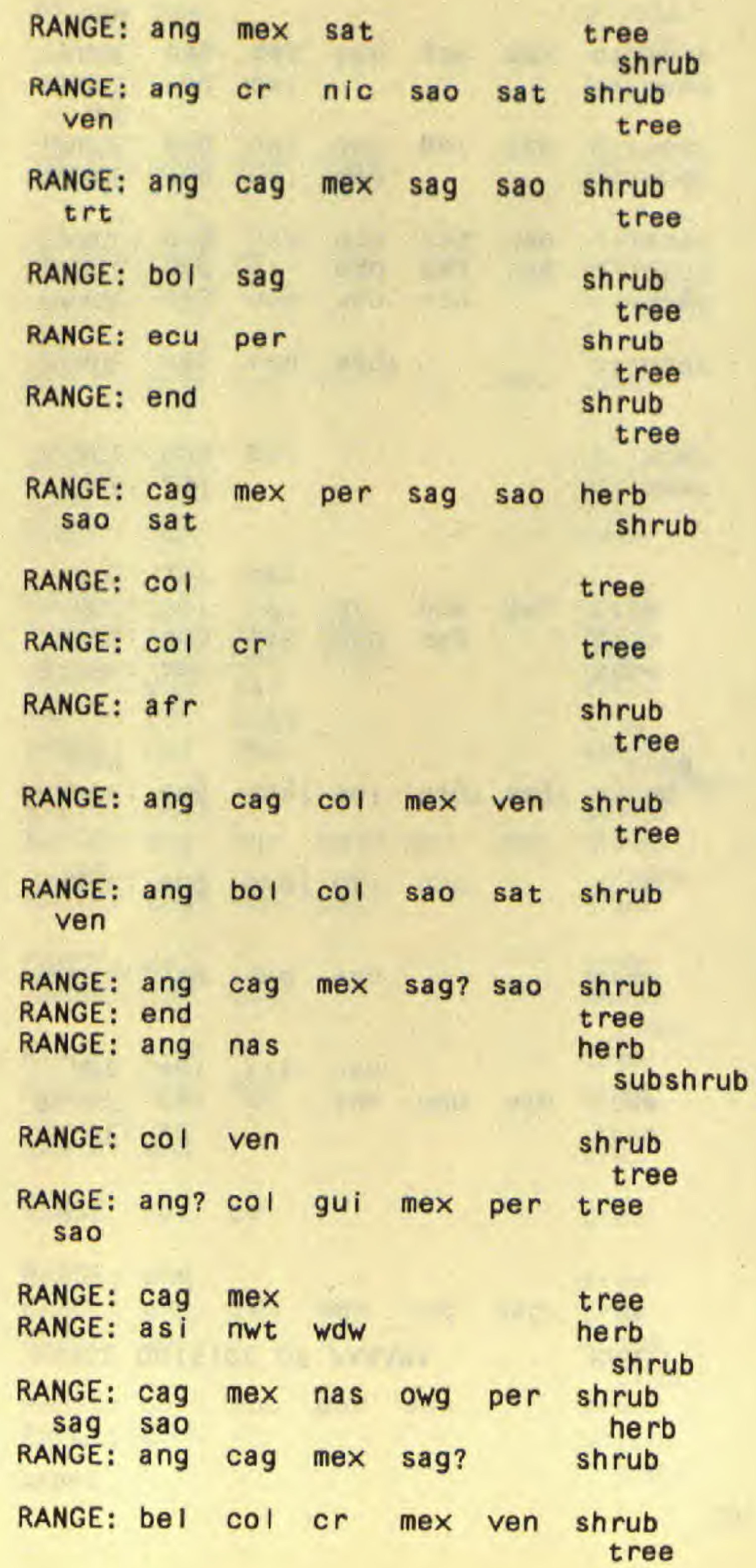


128 (83) LEGUMINOSAE cont.

*pendula (Willd.) Irwin \& Barneby

*--var. advena (Vog.) I rwin \& Barneby

PROV: ca cc pa $000 \mathrm{~m}: 0-1$

"pilifera (Vol.) I win \& Barneby

*--var. subglabra (S. Moore) I rwin \& Barneby

PROV: ca pa 000m: $0-1$

*quinquangulata (Rich.) Irwin \& Barneby PROV: ch da pa vr 000m: 0-1

*reticulata (Willd.) I win \& Barneby PROV: bo ca cc da he $000 \mathrm{~m}$ : $0-1$ $10 \mathrm{pa}$

* septemtrionalis (Viviani) Inwin \& Barneby PROV: pa

000m: $0-1$

*siamea (Lam.) I rwin \& Barneby

\section{cult. PROV: ca da pa} PROV: ca

* sophera (L.) Roxb.

* spectabilis (DC.) I Wwin \& Barneby PROV: Ch

twilliamsi (Britt, \& Rose) Irwin \& Barneby PROV: cc ch pa $000 \mathrm{~m}: 0-1,1-2$

\section{Sesbania}

emerus (Aubl.) Urb.

PROV: ca ch lo pa $v r$ 000m: $0-1$

exasperata H.B.K.

PROV: pa

000m: $0-1$

grandiflora (L.) Pers.

cult

PROV: ca cc pa

000m: $0-1$

Sophora

tomentosa L.

- -subsp. occidentalis (L.) Brummitt

PROV: bo cn sa $000 \mathrm{~m}: 0-1$

PROV: bo en sa

000m: $0-1$

Stizolobium pruriens (L.) Medik.

PROV: ca ch da lo pa 000m: $0-1$ $\mathrm{vr}$

\section{Stylosanthes}

guyanensis (Aubl.) Sw.

humil is H.B.K.

viscosa (L.) Sw.
PROV: ca cc ch he pa 000m: $0-1,1-2$

$$
\text { vr }
$$

PROV: ca cc ch cn pa 000m: 0-1

PROV: $\mathrm{vc}$ he lo pa $\vee r$ 000m: $0-1,1-2$

$$
\text { RANGE: ang cag? mex sag? shrub }
$$

\begin{tabular}{|c|c|c|c|c|c|c|}
\hline RANGE: & ang & cag? & mex & sag? & & $\begin{array}{l}\text { shrub } \\
\text { tree }\end{array}$ \\
\hline $\begin{array}{c}\text { RANGE: } \\
\text { sat }\end{array}$ & cub & mex & per & sag & sao & $\begin{array}{l}\text { shrub } \\
\text { herb }\end{array}$ \\
\hline $\begin{array}{c}\text { RANGE: } \\
\text { SaO }\end{array}$ & $\begin{array}{l}\text { bel } \\
\text { sat }\end{array}$ & $\mathrm{cr}$ & gua & mex & per & shrub \\
\hline $\begin{array}{c}\text { RANGE: } \\
\text { trt }\end{array}$ & bol & cag & $\operatorname{mex}$ & sag & sao & tree \\
\hline $\begin{array}{c}\text { RANGE: } \\
\text { per }\end{array}$ & $\begin{array}{l}\text { afr } \\
\text { sag }\end{array}$ & ang & cag & mex & owt & $\begin{array}{l}\text { shrub } \\
\text { tree }\end{array}$ \\
\hline RANGE: & as $i$ & $w d t$ & & & & tree \\
\hline RANGE: & ang & $\mathrm{col}$ & $\operatorname{mex}$ & nwt & sao & shrub \\
\hline RANGE: & ang & cag & $\operatorname{mex}$ & sag & & tree \\
\hline RANGE: & cag & mex & sag & sao & trt & shrub \\
\hline RANGE & c & & & & & $\begin{array}{c}\text { shrub } \\
\text { tree }\end{array}$ \\
\hline
\end{tabular}

RANGE: ang cag

RANGE: ang sag ven

RANGE: OWg

herb shrub herb shrub tree

RANGE: cag shrub

RANGE: afr ang bel col mex shrub tex

RANGE: WdW

climber

RANGE: cag sat

herb

RANGE: ang asi cag col mex herb oce sao ven

RANGE: cag mex sag? sao herb 
TAXON

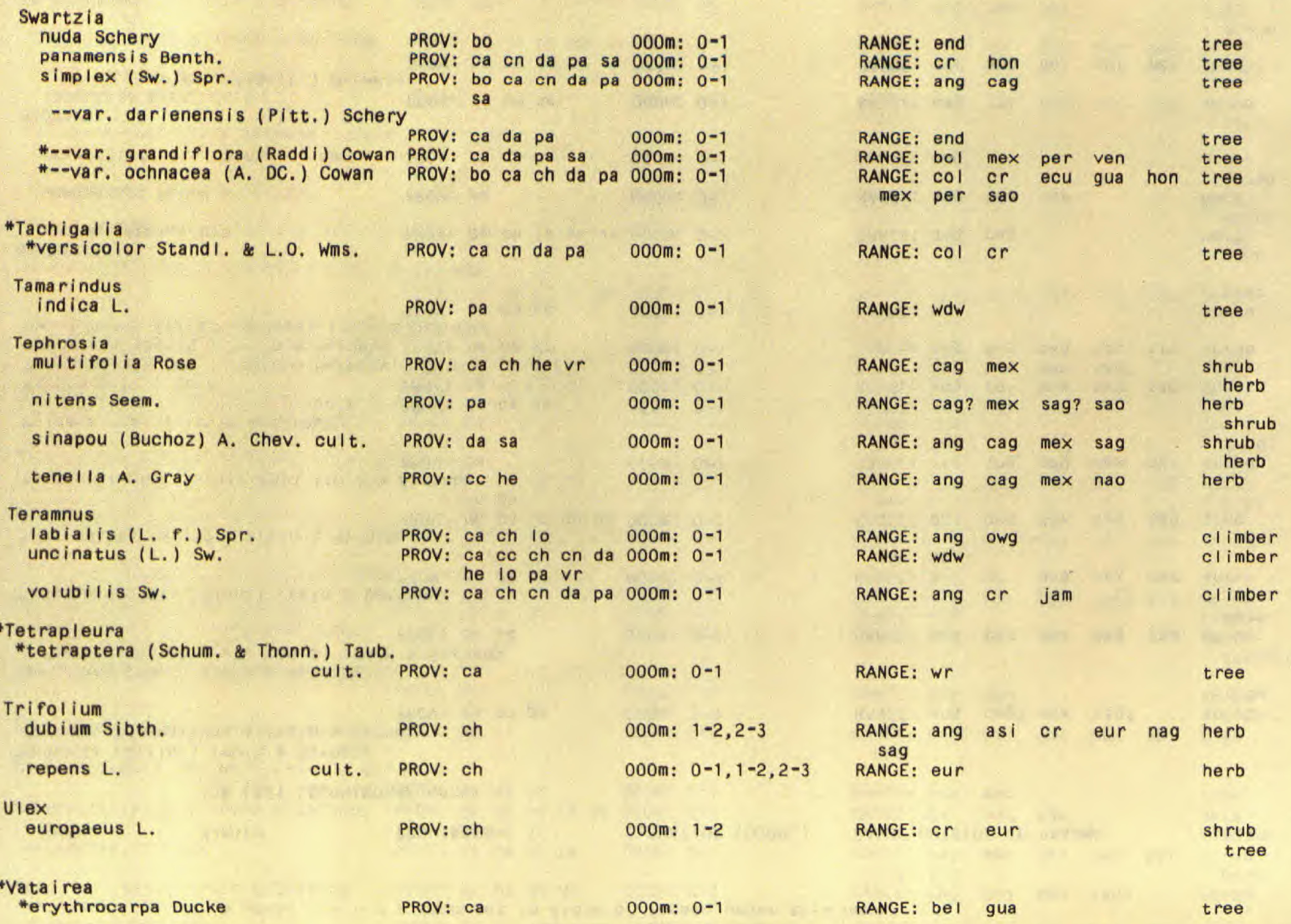


Vigna

adenantha (G. Meyer) Marechal et al.

PROV: bo ca cc ch da 000m: $0=1$ he 10 pa

PROV: bo ca $000 \mathrm{~m}$ : $0-1$ PROV: $\mathrm{ca} \quad$ 000m: $0=1$

caracalla (L.) Verdc. PROV: ce ch da to pa 000m: 0-1 juruana (Harms) Verdc. PROV: vr

I inearis (H.B.K.) Marechal et al. PROV: CC pa 000m: $0=1$ longifolia (Benth, ) Verdc. Prov: ca ch da pa 000m: $0-1$ Iuteola (Jacq.) Benth. PROV: bo ca ch cn pa 000m: $0-1$ peduncularis (H.B.K.) FawC. RendI. sa

PROV: bo ca ce cn da $000 \mathrm{~m}$ : $0-1$ retusa (Meyer) WaIp. PROV: pa sa vr 000m: $0-1$ unguiculata (L.) Walp. cult. 000m: $0-1$ vexillata (L.) A. Rich.

PROV: cn pa PROV: bo ca ch cn da 000m: 0-1 pa

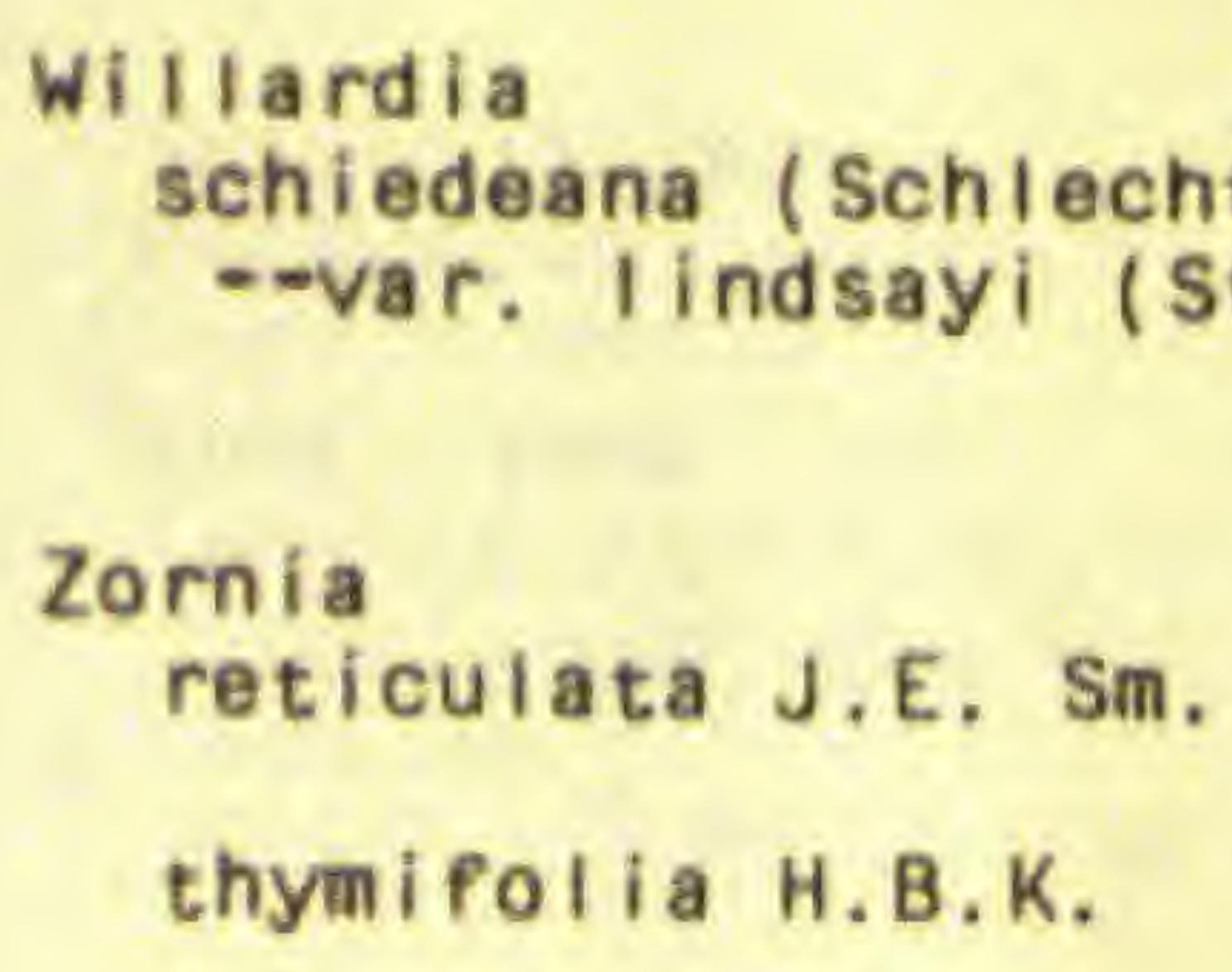

129 (85) GERANIACEAE

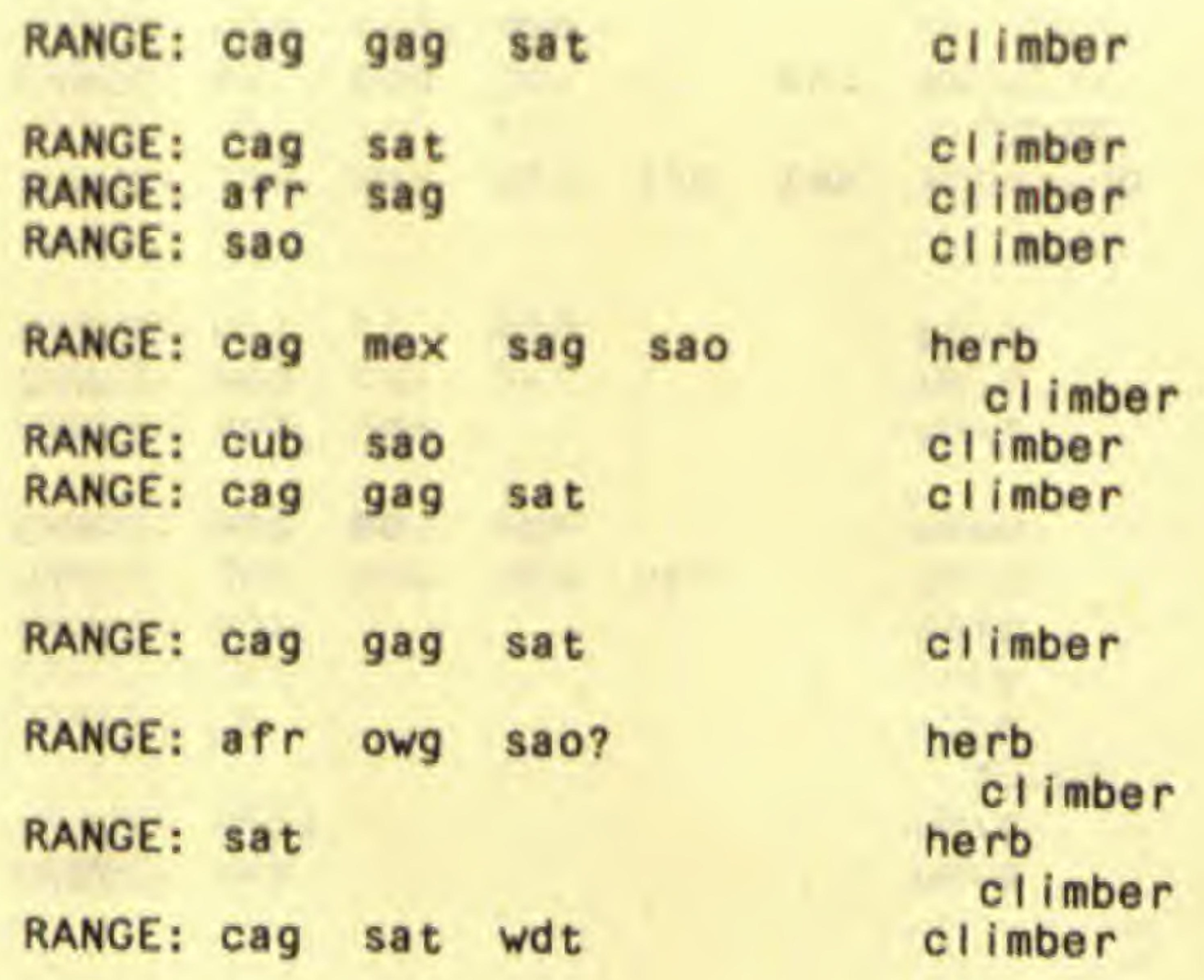

$\begin{array}{llll}\text { RANGE: cag gua mex sal } & \text { tree } \\ \text { RANGE: cr mex } & & \text { tree } \\ \text { RANGE: cag nag sag sao } & \text { herb } \\ \text { RANGE: cag mex } & & \text { herb }\end{array}$

herb 


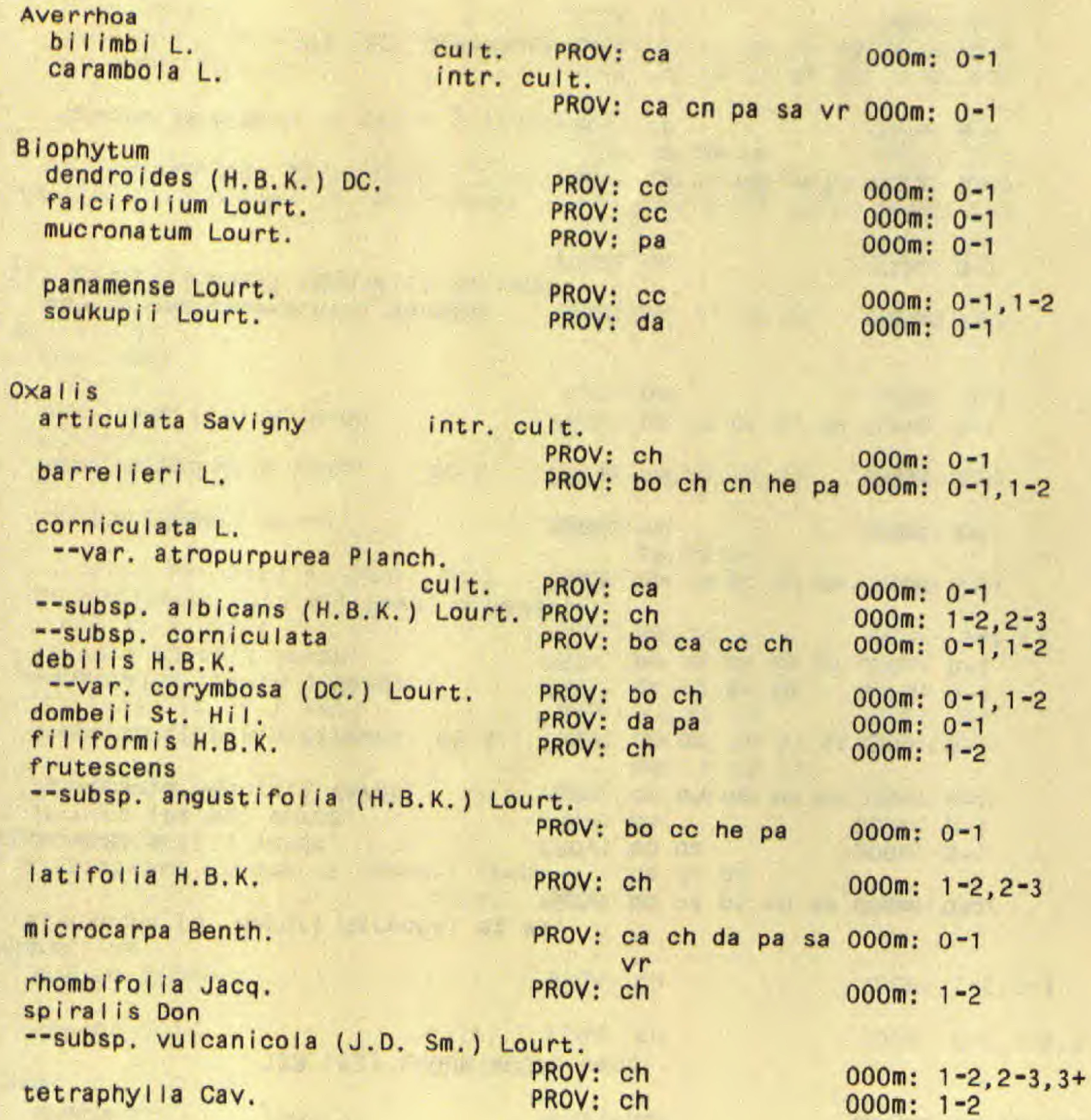

microcarpa Benth.

rhombifolia Jacq.

spiralis Don

PROV: ca ch da pa sa $000 \mathrm{~m}: 0-1$

PROV: $\mathrm{vr}$

000m: $1-2$

--subsp. vulcanicola (J.D. Sm.) Lourt.

tetraphyl Ia Cav.

PROV: ch PROV: ch

000m: $1-2,2-3,3+$ 000m: $1-2$

RANGE: as $\mathrm{i}$

tree

RANGE: as $\mathrm{i}$

tree

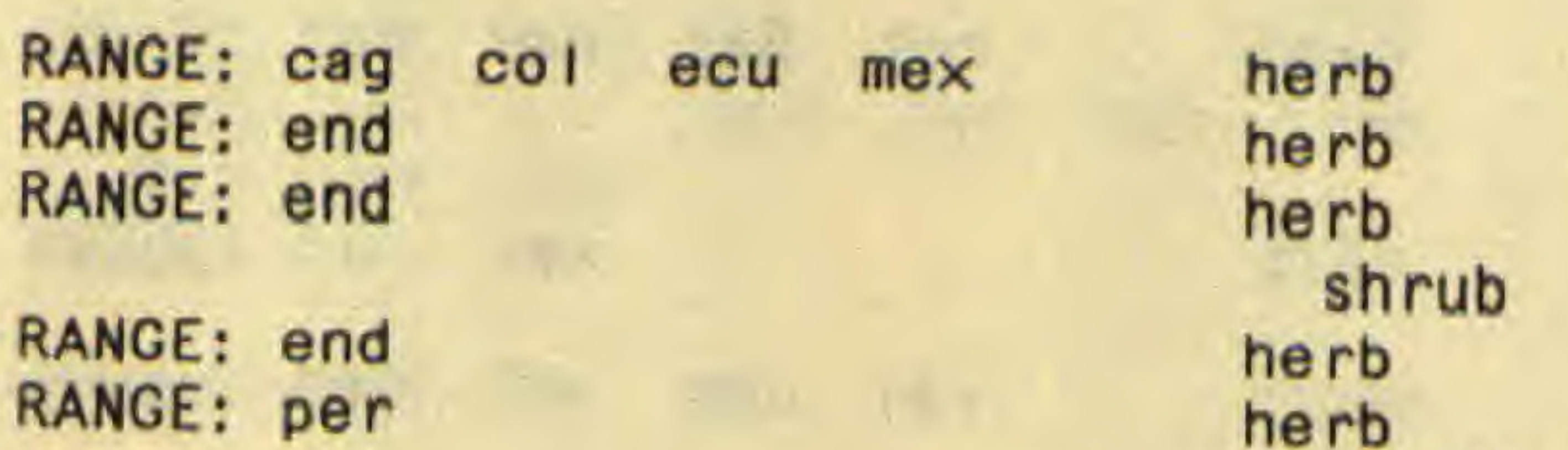

RANGE: per

subshrub

RANGE: sao
RANGE: nWg?

herb

herb

shrub

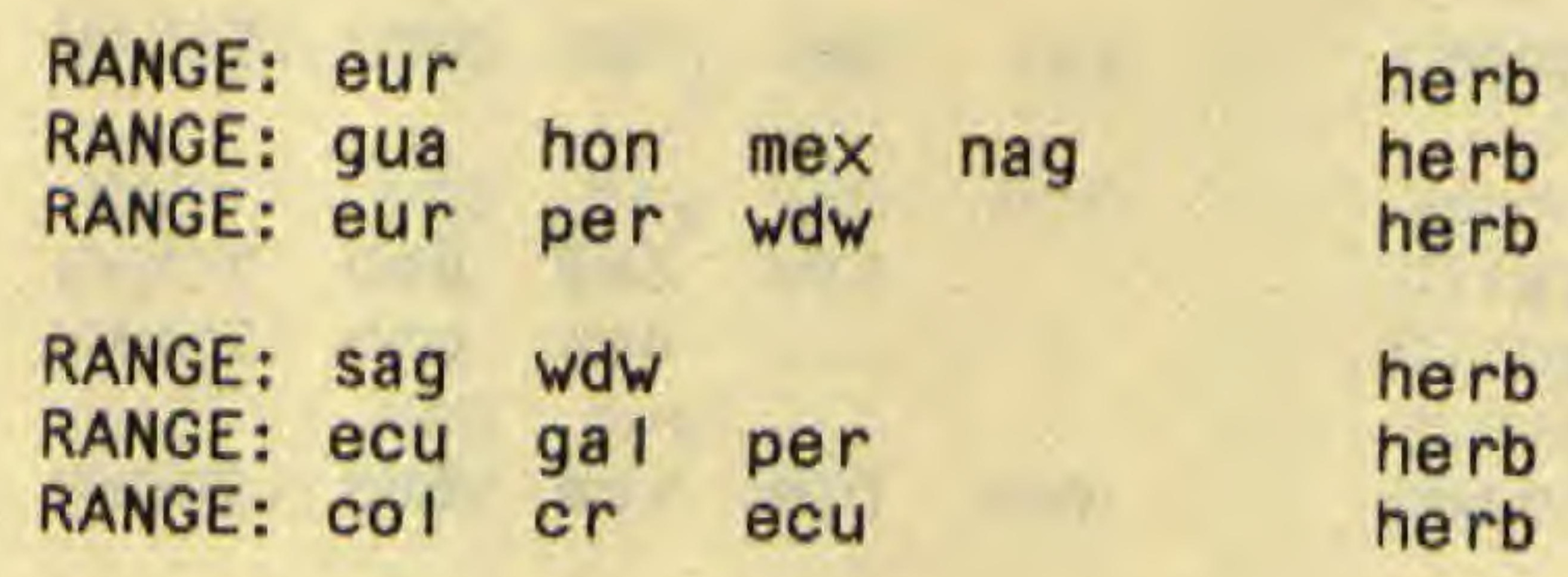

RANGE: cag mex sag sao tex subshrub

RANGE: afr ang bol shrub

cr eur herb

RANGE: cag ecu mex herb

RANGE: cag col mex ven shrub

subshrub

RANGE: cag $\mathrm{cr}$

RANGE: $\operatorname{mex} w d w$ herb 
131 (86) TROPAEOLACEAE

\section{Tropaeo I um}

emarginatum Turcz.

majus $L$.

pendulum $\mathrm{KI}$.

$\begin{array}{ll} & \text { PROV: bo ch } \\ \text { cult. } & \text { PROV: WP } \\ \text { PROV: ch }\end{array}$

PROV: ch

\author{
000m: $1-2,2-3$ \\ $000 \mathrm{~m}$ : we \\ 000m: $1-2$
}

RANGE: cag col mex
RANGE: eur per
RANGE: col cr ecu

cl imber

climber

climber

\section{3 (87A) HUMIRIACEAE}

\section{Humi riast rum}

diguense (Cuatr.) Cuatr.

PROV: ca da pa

000m: $0-1,1-2$

RANGE: $\mathrm{col} \mathrm{cr}$

tree

Sacoglottis

ovicarpa Cuatr.

trichogyna Cuatr.

PROV: cn pa sa

000m: $0-1$

000m: $0-1$

RANGE: $C O I$

RANGE: $c r$

tree

tree

000m: $0-1,1-2$

RANGE: COI

occidentalis cuatr.
occinea

PROV: ca vr

tree

\section{4 (87) ERYTHROXYLACEAE}

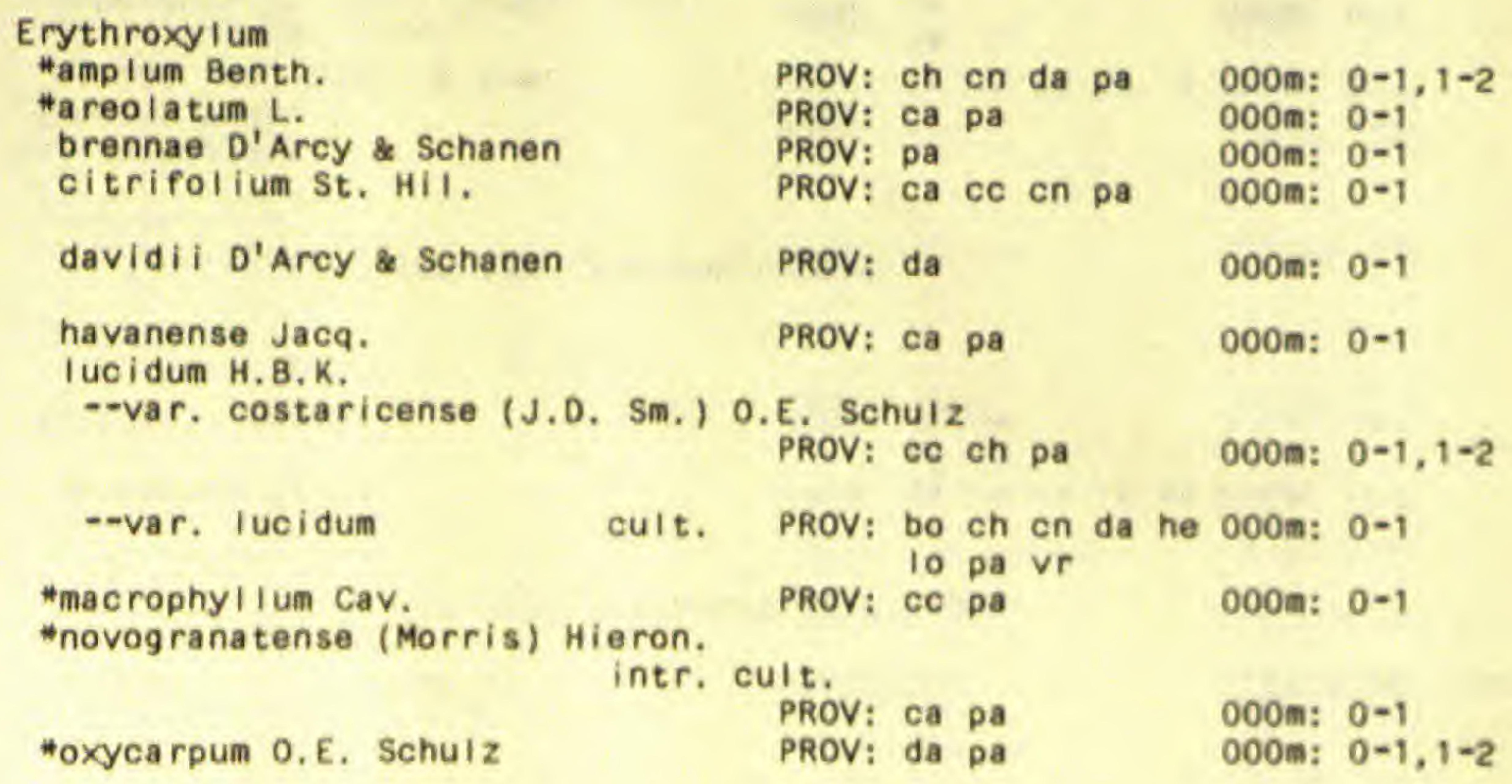

RANGE: sat

RANGE: gag mex nic

tree

RANCE: 9nd

RANGE: $\mathrm{cr}$

RANGE: end

RANGE: cag gag sat

shrub

shrub tree

tree

shrub

shrub

RANGE: $\mathrm{cr}$

RANGE: or Sao

RANGE: bel per sao ven

shrub

tree

shrub

tree

treelet

RANGE: $C O$ I

RANGE: col lag ven 
TAXON FAMILY PROVINCES ELEVATION (OOOM.) RANGE OUTSIDE OF PANAMA HABIT 134 (87) ERYTHROXYLACEAE cont.

panamense Turcz.

*skutchii Standl.

PROV: ca ch da lo pa 000m: 0-1
PROV: ca cn

000m: $0-1$

\section{5 (88) ZYGOPHYLLACEAE}

Ka I I st roemia
\# incana Rydb.

maxima (L.) Hook. \& Arn.

pubescens (G. Don) Dandy intr. PROV: pa

\section{PROV: ca}

PROV: ca

000m: $0-1$

000m: $0-1$

137 (89) RUTACEAE

\section{*Amyris}

*elemifera L.

PROV: pa

000m: $0-1$

Angostura

nica raguiens is (StandI. \&. O. Wms.) Elias

PROV: ce cn da pa 000m: 0-1

\section{Citrus}

aurantifolia (Christm.) Swingle

intr. cult.

$$
\text { PROV: ca da pa 000m: } 0-1
$$

aurantium $L$.

grandis (L.) Osbeck

IImon (L.) Burm, $f$.

reticulata Blanco

sinensis (L.) Osbeck intr, cult.

intr. cult.

PROV: bo ca ch da pa 000m: 0-1

int $r$, cult.

intr. cult.

PROV: ca ch da $000 \mathrm{~m}: 0-1$

intr, cult.

PROV: ca ch cn da
PROV: ca cc 000m: $0-1$

000m: $0-1$

$000 m: 0-1,1-2$

\author{
RANGE: col sat \\ RANGE; $c r$
}

shrub

tree

shrub

tree

RANGE: ang
RANGE: ang cag fla mex nao herb
nao sag? tex
RANGE: afr asi cag ecu fla herb
jam lag mex per sag?

RANGE: cag

shrub

RANGE: nic

shrub

tree

RANGE: as i

shrub

tree

RANGE: as i

tree

RANGE: ang asi

tree

RANGE: as i

tree

RANGE: as i

tree

RANGE: as i 
137 (89) RUTACEAE cont.

\section{Erythrochiton}

incomparabilis Riley

I indeni (Baill.) HemsI.
PROV: $\mathrm{CC} \mathrm{vr}$

PROV: bo

\section{Esenbeckia}

a lata (Karst. \& Triana) Tr. \& PI. PROV: Io pa

litoralis J.D. Sm.

panamens is Elias

\section{Ga I ipea}

panamensis Elias

trifoliata Aubl.

Hortia

colombiana Gleason

Murraya

paniculata $(L$,$) Jacks.$

cult. PROV: bo ca pa

unifoliolata Elias

* Triphasia

*trifolia (Burm. f.) P. Wils.

cult. PROV: pa

\section{Zanthoxylum}

belizense Lund.

chiriquinum Standl.

elephantiasis Macfad.

eliasii D.M. Porter

fagara (L.) Sarg.

ja ime i D.M. Porter

I imoncello Planch. \& Oerst.
PROV: da pa

PROV: $v r$

PROV: cn pa

PROV: PA

PROV: cn da

PROV: cn da pa

PROV: da

$000 \mathrm{~m}: 0-1$

000m: $0-1$

000m: $0-1$

000m: $0-1,1-2$

000m: 1-2

000m: 0-1

000m: 0-1

PROV: ca cn sa

000m: 0-1

PROV: ch

PROV: da

PROV: da

PROV: pa

PROV: da PROV: ch
$000 m: 1-2,2-3$

000m: $0-1$

000m: $0-1,1-2$

000m: $0-1$

000m: $0-1,1-2$

000m: $1-2$
RANGE: end

shrub

tree

RANGE: cag mex

shrub

tree

RANGE: sat

RANGE: cag

RANGE: cag

tree

shrub

shrub

tree

RANGE: end

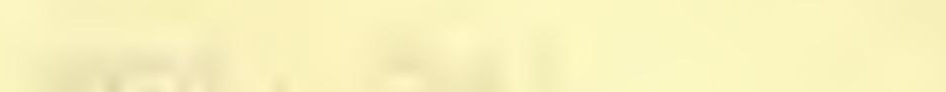

RANGE: col gui tree

RANGE: $c O$ I

tree

RANGE: as $\mathrm{i}$

shrub

tree

RANGE: cag

tree

RANGE: $w r$

shrub

RANGE: $C O I$

tree

RANGE: ang

as $\mathrm{i}$

viny

shrub

RANGE: bel col? or gua hon tree

mex nic

RANGE: $c r$

shrub

RANGE: or gag jam

RANGE: end

RANGE: cag

tree

sao tex

RANGE: end

RANGE: $c r$

shrub

tree tree shrub 
TAXON

FAMILY PROVINCES

ELEVATION (OOOM.)

RANGE OUTSIDE OF PANAMA

HAB IT

137 (89) RUTACEAE cont.

melanostictum Schlecht. \& Cham. microca rpum Griseb.

PROV: pa $v r$

$000 \mathrm{~m}: 0-1,1-2$

panamense P. Wils.

pittieri P. Wils.

procerum J.D. Sm.

pucro D.M. Porter

scheryi Lund.

setulosum P. Wils.

tripetalum (StandI.) D.M. Porter PROV: ch

PROV: bo ca ch cn da 000m: $0-1$

PROV: da

PROV: $000 \mathrm{~m}: 0-1$

PROV: $\mathrm{Vr}$

PROV: da

PROV: ch

0

$000 \mathrm{~m}: 1-2,2-3$

PROV: ca cc da he lo 000m: 0-1

$000 \mathrm{~m}: 1-2$

138 (90) SIMAROUBACEAE

Picramnia
allenil D.M. Porter

carpinterae Polak. cooperi D.M. Porter

coral lodendron Tul. dwyeri D.M. Porter

latifolia Tul.

\section{Quass ia}

amara $L$.

Simaba

cedron PIanch.

Sima rouba

amara Aubl.

*glauca DC.

\section{* Suriana}

*maritima L.
PROV: bo $\mathrm{cc} \mathrm{ch}$ 000m: $0-1,1-2$

PROV: ch

PROV: bo

000m: $1-2$

000m: $0-1$

PROV: cn da pa 000m: $0-1,1-2$ PROV: ca da pa sa $v r$ 000m: $0-1$

PROV: bo ca ch da lo 000m: $0-1$ pa sa $v r$

PROV: bo ca ce ch cn 000m: 0-1 da pa $v r$

cult. PROV: da he pa sa 000m: $0-1$

PROV: ca pa vr

$000 \mathrm{~m}: 0-1$

PROV: ca pa vr

000m: $0-1$

PROV: sa

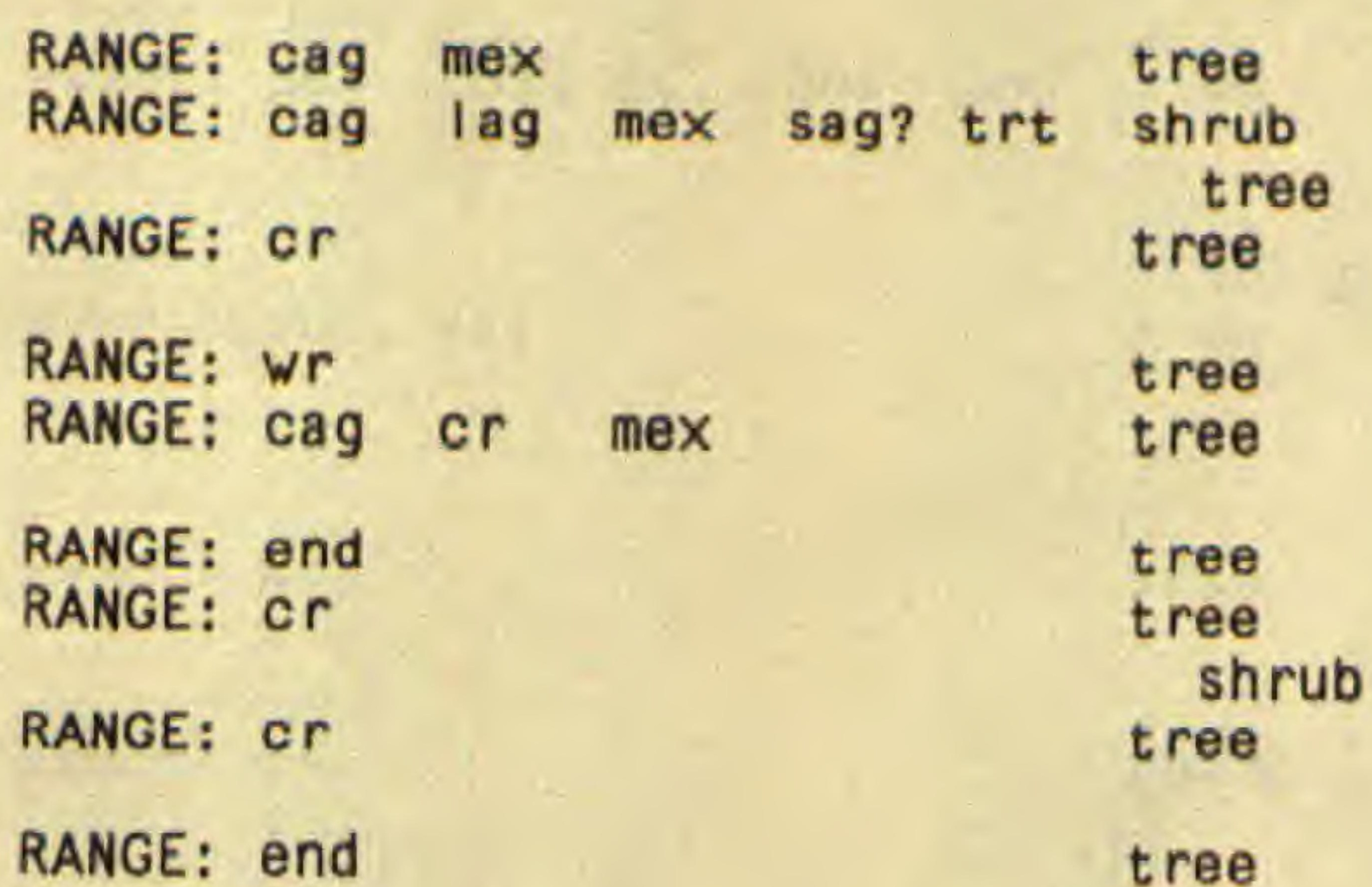

RANGE: end

\begin{tabular}{|c|c|c|c|c|c|c|}
\hline RANGE: & $\mathrm{cr}$ & & & & & $\begin{array}{c}\text { shrub } \\
\text { tree }\end{array}$ \\
\hline RANGE : & $\mathrm{cr}$ & & & & & tree \\
\hline RANGE: & cr & & & & & $\begin{array}{l}\text { shrub } \\
\text { tree }\end{array}$ \\
\hline $\begin{array}{l}\text { RANGE: } \\
\text { RANGE: }\end{array}$ & $\begin{array}{l}\text { bo I? } \\
\text { end }\end{array}$ & $\mathrm{col}$ & ecu? & per & & $\begin{array}{l}\text { tree } \\
\text { shrub } \\
\text { tree }\end{array}$ \\
\hline RANGE: & $\mathrm{col}$ & $\mathrm{cr}$ & & & & $\begin{array}{l}\text { shrub } \\
\text { tree }\end{array}$ \\
\hline RANGE: & ang & cag? & mex & sag? & sao & $\begin{array}{l}\text { shrub } \\
\text { tree }\end{array}$ \\
\hline RANGE: & cag & sao & & & & $\begin{array}{r}\text { shrub } \\
\text { tree }\end{array}$ \\
\hline $\begin{array}{r}\text { RANGE: } \\
\text { sag? }\end{array}$ & $\begin{array}{l}\text { bel } \\
\text { sao }\end{array}$ & bol & cag? & $\mathrm{cr}$ & lag & tree \\
\hline RANGE: & cag & & & & & tree \\
\hline RANGE: & ang & oce & 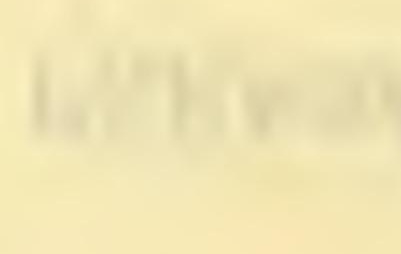 & & & shrub \\
\hline
\end{tabular}


139 (91) BURSERACEAE

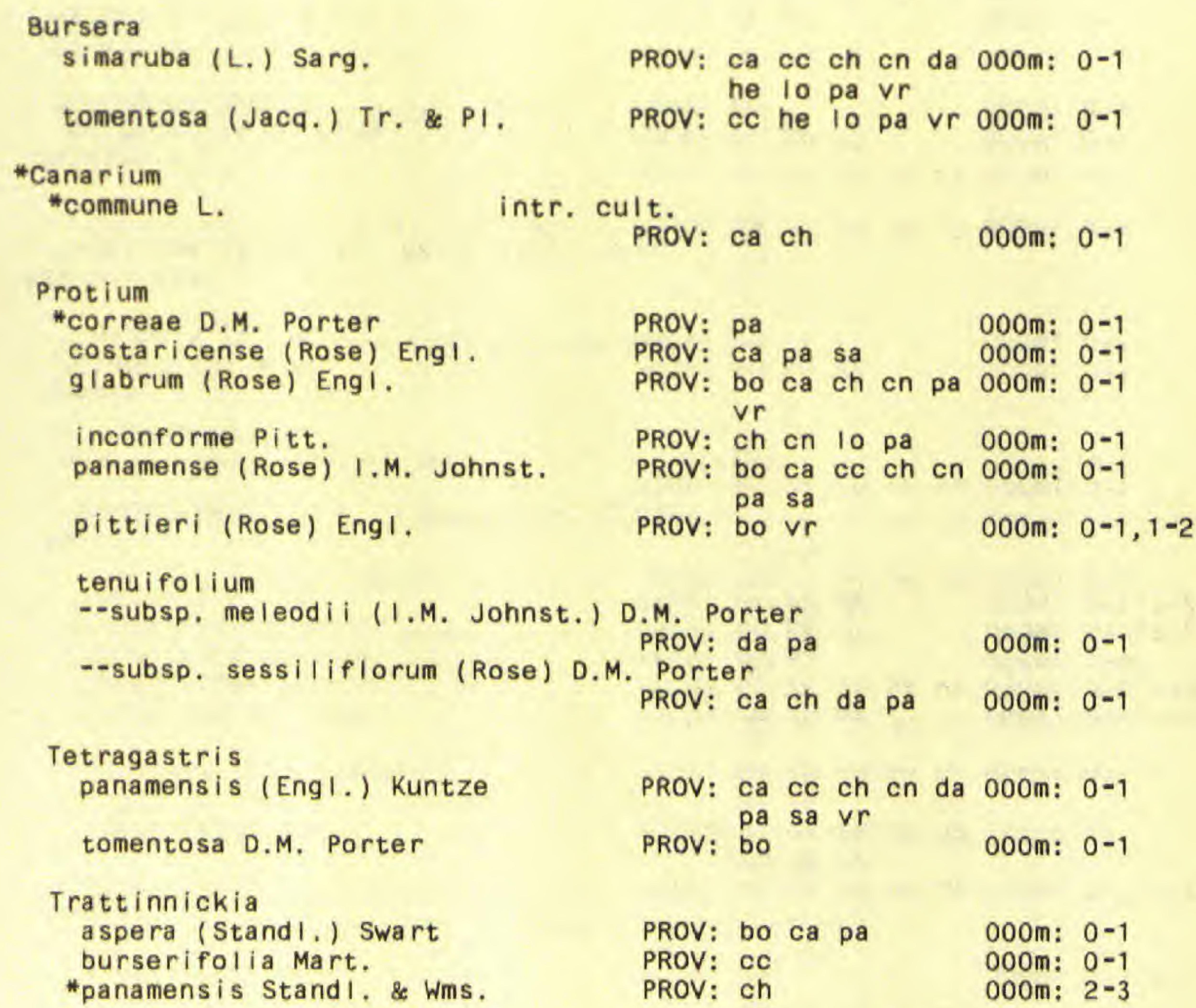

\section{0 (92) MELIACEAE}

Carapa

guianensis Aubl.

nica raguens is C. DC.

PROV: bo ca cn sa vr 000m: 0-1

PROV: bo ca da

000m: $0-1$

Cedrela

angustifolia DC.
PROV: ca cc ch da he 000m: $0-1,1-2$

RANGE: ang cag col fla mex tree
ven
RANGE: abc col cr ven

RANGE: oce

RANGE: end

tree

tree

RANGE: end tree

RANGE: end tree

shrub

tree

RANGE: end

tree

RANGE: $\mathrm{cr}$

tree

RANGE: bel cr gui hon nic tree

per sao ven tree
RANGE: end

RANGE: $C O 1$

RANGE: col gui tree

RANGE: col gui lag? sao ven tree

RANGE: ang bel cag? col cr tree
Sag? sao
RANGE: cag? ecu nic sag?

RANGE: cag? mex per sag? sao tree


TAXON FAMILY PROVINCES HABIT

140 (92) MELIACEAE cont.

*fissilis Vell. odorata $L$.

tonduzii C. DC.

Gua rea

glabra Vahl

"grandifolía DC.

guidonia (L.) Sleumer

kunthiana A. Juss.

* macropetala Penning.

*pterorhach is Harms

* rhopa locarpa Rad Ik. tonduzii C. DC.

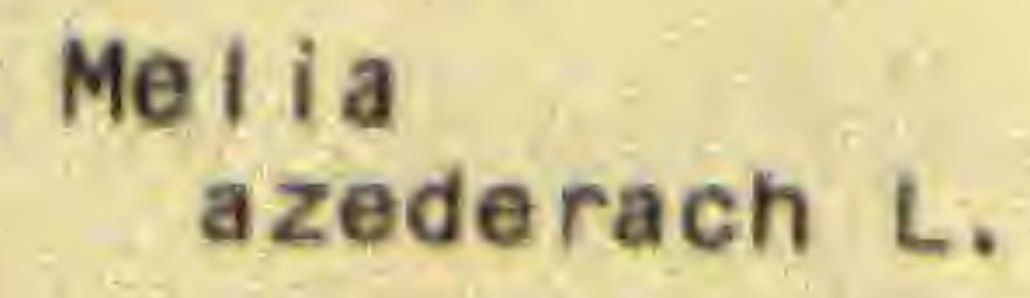

* glabra Tr. \& Pl.

*insignis iC. DC.

* Sandoricum

"koetjape (Burm. f.) Merr, intr. cult.

Swietenia

macrophyl Ia G. King

*mahagoni Jacq.

Trichilia

*acuminata (R, \& S, ) C. DC.

glabra L.

hirta L.

*martiana C. DC.

"pallida Sw.

"pleeana (A. Juss.) C. DC.

*poeppigii C. DC.

*quadrijuga H.B.K. 000m: $0-1$ 000m: $0-1$

PROV: ca $000 \mathrm{~m}: 0-1$

cult,

PROV: ca pa

PROV: ca ch 000m: $0-1$

PROV: bo ca ch cn da 000m: $0-1$ he

$000 \mathrm{~m}: 0-1,1-2$

PROV: ca cc ch cn da 000m: $0-1,1-2$ lo pa $\mathrm{vr}$

ch da 000m: 0-1 pa sa

PROV: ca ch cn da pa 000m: $0-1$

PROV: ca ch da 000m: $0-1,1=2$

PROV: bo cn da pa $v r$ 000m: $0-1,1-2$

PROV: bo pa 000m: $0-1$

PROV: cC pa $v r \quad 000 \mathrm{~m}: 0-1,1=2$

PROV: ca ch da 000m: $0-1,1-2$

cult. PROV: pa $000 \mathrm{~m}: 0-1$

PROV: da sa

000m: $0=1$

PROV: ch da

000m: $1-2$

PROV: ca ch da lo pa 000m: $0-1$ $\mathrm{vr}$

PROV: cc ch cn da lo 000m: 0-1

PROV: bo ca ch cn da 000m: $0-1,1-2$ pa vr

PROV: ca cn da lo pa 000m: 0-1

000m: $0-1$

\begin{tabular}{|c|c|c|c|c|c|c|}
\hline RANGE: & $\mathrm{cr}$ & per & sag & sao & & tree \\
\hline $\begin{array}{c}\text { RANGE: } \\
\text { Sao }\end{array}$ & ang & cag & mex & per & sag & $t r$ \\
\hline RANGE; & cag & mex & & & & $r e$ \\
\hline $\begin{array}{l}\text { RANGE: } \\
\text { meX }\end{array}$ & $\begin{array}{l}\text { ang } \\
\text { ven }\end{array}$ & bol? & cag & $\mathrm{col}$ & ecu & \\
\hline RANGE: & bol & cag? & $\operatorname{mex}$ & sag? & & \\
\hline RANGE: & nwg? & & & & & \\
\hline $\begin{array}{l}\text { RANGE: } \\
\text { RANGE: }\end{array}$ & $\begin{array}{l}\text { bol } \\
\text { end }\end{array}$ & cag? & mex & sag? & & \\
\hline $\begin{array}{l}\text { RANGE: } \\
\text { RANGE: }\end{array}$ & $\begin{array}{l}\text { per } \\
\text { cr }\end{array}$ & sao & sat & & & \\
\hline
\end{tabular}

RANGE: $O r$

tree

RANGE: ang cr owt sat tree

RANGE: cag per sat treelet

RANGE: bol cag cr per tree

RANGE: $w r \quad$ tree

RANGE: cag mex per sag? sao tree

RANGE: nwt tree

RANGE: sat

RANGE: ang cag mex

RANGE: cag tree

RANGE: cag mex sag sao tree

RANGE: bol gui sao sat trt tree

Ven

RANGE: sat tree 
140 (92) MELIACEAE cont.

*--subsp. cinerascens (C. DC.) Penning. *--subsp. quadrijuga
*septentrionalis C. DC.

PROV: bo cc cn da pa 000m: $0-1,1-2$ PROV: ch da 000m: $0-1$ PROV: ch pa sa 000m: $0-1$

*skutchil Morton \& Alten tomentosa H.B.K.

PROV: cn

000m: $0-1$ trifolia L. PROV: bo ch pa $000 \mathrm{~m}: 0-1$ tuberculata (Tr, \& PI.) C, DC. PROV: $C C$ da he pa pa 000m: $0-1$ PROV: bo ca ch cn pa 000m: $0-1$

\section{1 (93) MALPIGHIACEAE}

\section{Banisteriopsis}

*acapulcensis (Rose) Small

PROV: ca

000m: $0-1$

*elegans (Tr. \& PI,) Sandw. muricata (Cav.) Cuatr.

*nutans (Ndzu.) B. Gates

"platyptera (Griseb.) Cuatr.

"wilburii B. Gates

twurdacki i B. Gates

000m: $0-1,1-2$

PROV: bo $\mathrm{cc}$

PROV: ca cc ch he lo 000m: 0-1 pa sa $v r$

PROV: cn da pa 000m: 0-1 PROV: da $000 \mathrm{~m}: 1-2$

PROV: CO 000m: $1-2$ PROV: ca cn lo pa vr 000m: $0-1$

\section{Bunchosia}

cornifolia H,B,K.

PROV: bo ca ch cn da $000 \mathrm{~m}$ : $0-1$ lo pa vr

diphylla (Jacq.) Cuatr. \& Croat

--subsp, brevisurcularis (Dobson) Cuatr. Croat

PROV: cn da $000 \mathrm{~m}: 0-1$

dwyeri Cuatr. \& Croat

PROV: bo ca ch da pa 000m: $0-1,1-2$ vr

*hartwegiana Benth.

*--var. brevisurcularis Cuatr. \& Croat

Ianieri Wats.

PROV: da

PROV: ch

000m: $0-1$

PROV: bo cn sa

$0-1$

Iindeniana Juss.

macrophyl la Rose

odorata (Jacq.) H.B.K.

*pittieri Cuatr. \& Croat
PROV: cn 000m: $0-1$

PROV: bo ca cc ch da 000m: 0-1 he lo pa vr

000m: $0-1$

\begin{tabular}{|c|c|c|c|c|c|c|}
\hline RANGE: & cag? & $\operatorname{mex}$ & sag? & & & tree \\
\hline RANGE: & cag & per & sao & sat & & tree \\
\hline $\begin{array}{c}\text { RANGE: } \\
\text { ven }\end{array}$ & $\mathrm{cr}$ & lag & per & sao & sat & tree \\
\hline RANGE: & $w r$ & & & & & shrub \\
\hline RANGE: & cag? & $\operatorname{mex}$ & per & sag? & & tree \\
\hline RANGE: & cag? & col & $\operatorname{mex}$ & ven & & $\begin{array}{c}\text { shrub } \\
\text { tree }\end{array}$ \\
\hline
\end{tabular}

RANGE: bol cag? gua sat tree

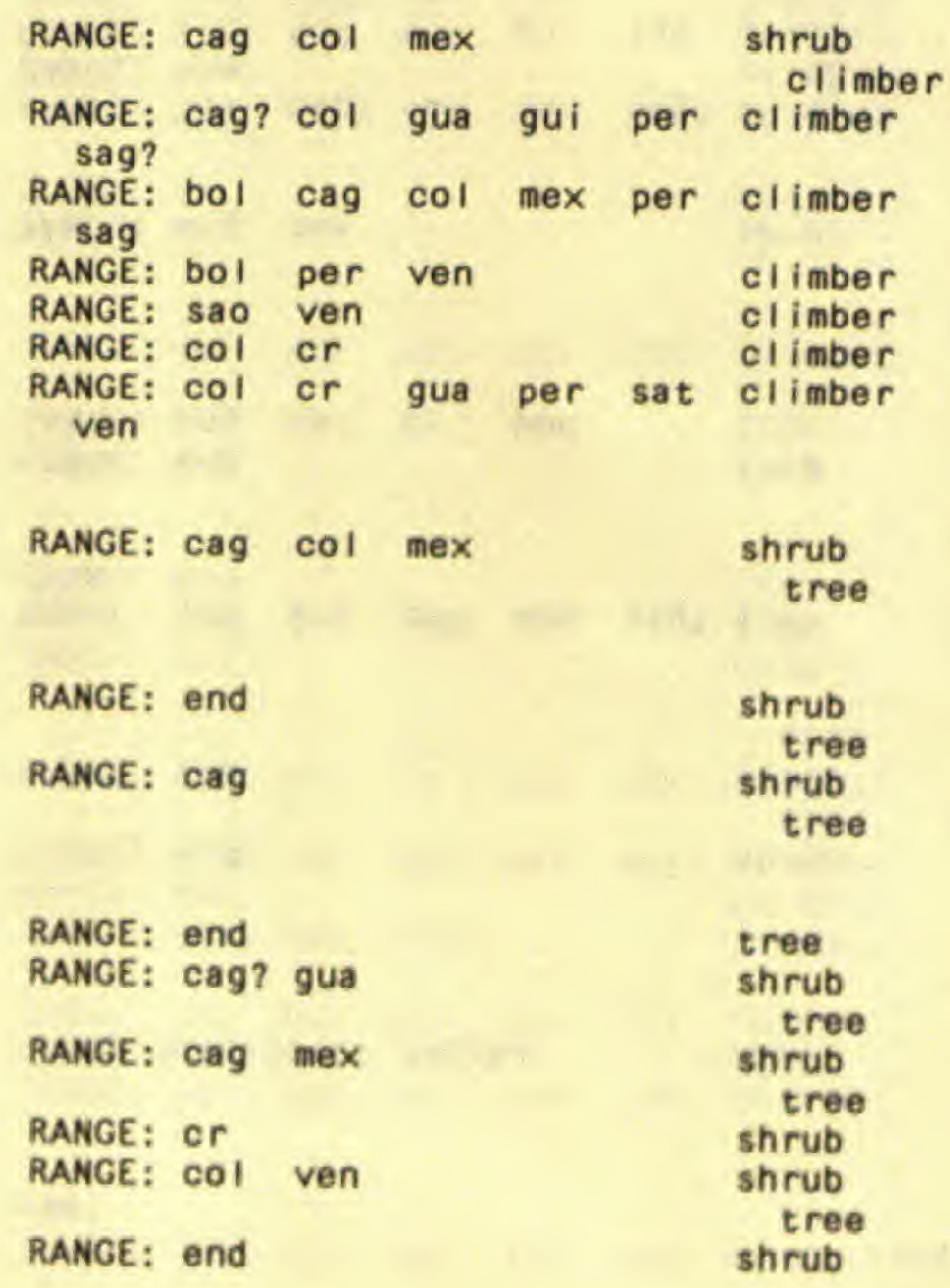


TAXON

141 (93) MALPIGHIACEAE cont.

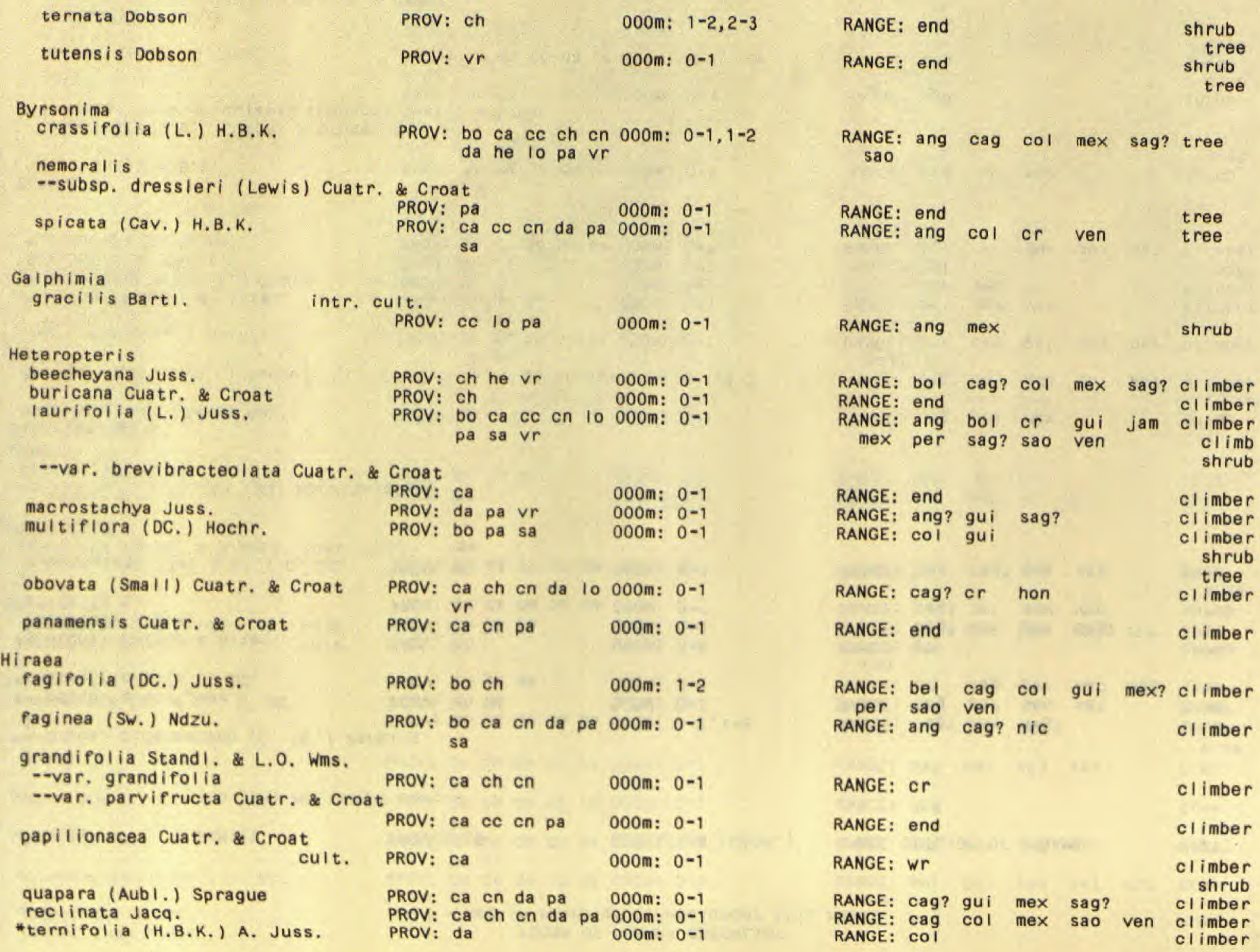




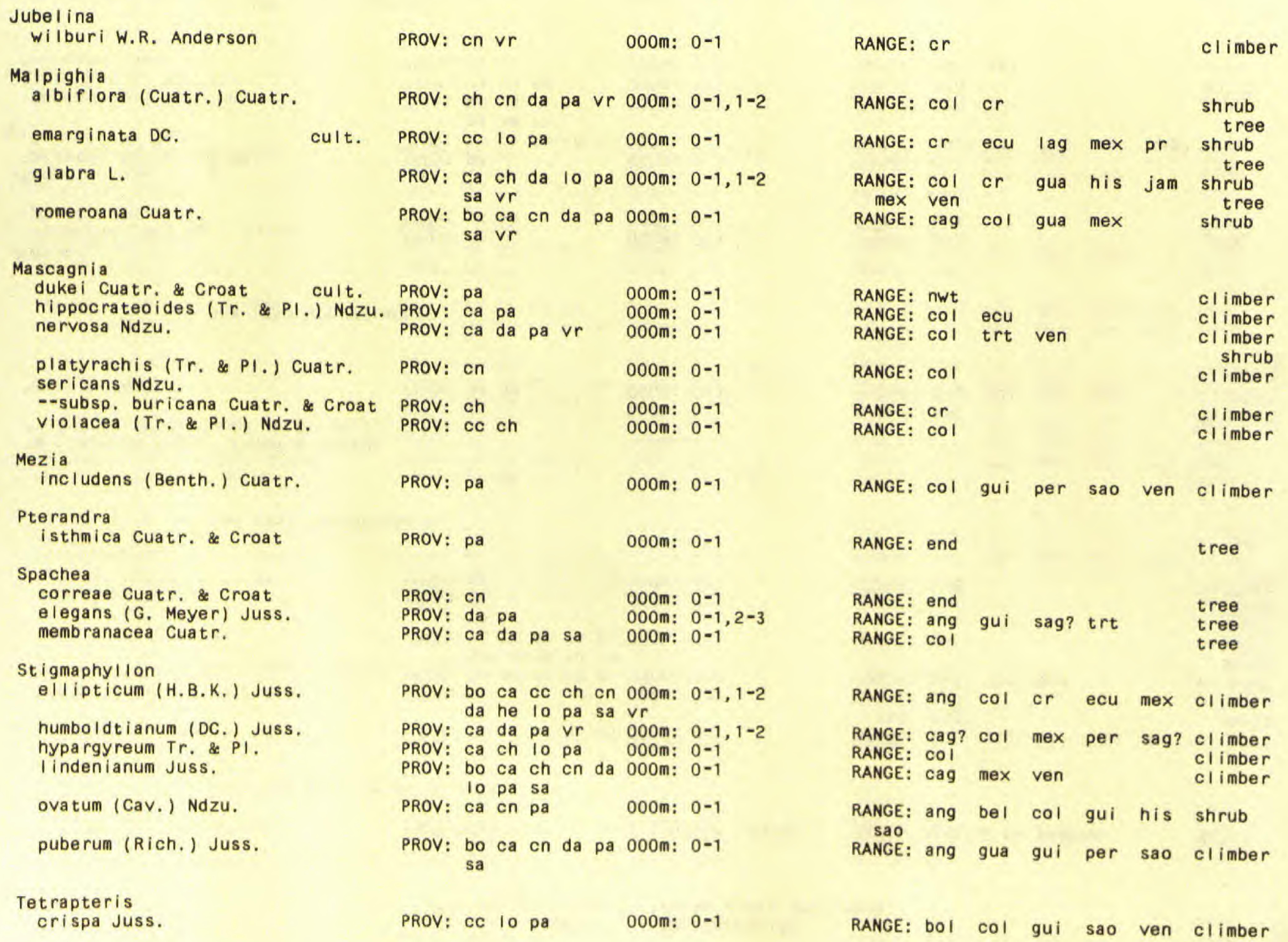


TAXON FAMILY PROVINCES ELEVATION (O0OM.) RANGE OUTSIDE OF PANAMA HABIT

141 (93) MALPIGHIACEAE cont.

discolor (G. Meyer) DC.

hirsutula cuatr. \& Croat macrocarpa Johnst.

seemannil Tr. \& PI.

subaptera Cuatr.

tysonii cuatr. \& Croat
PROV: bo ca ce ch cn 000m: $0-1$

PROV: da

000m: $0-1$

da he pa sa vr

PROV: ca ch da lo pa 000m: 0-1

PROV: $\mathrm{da}$

000m: $0=1$

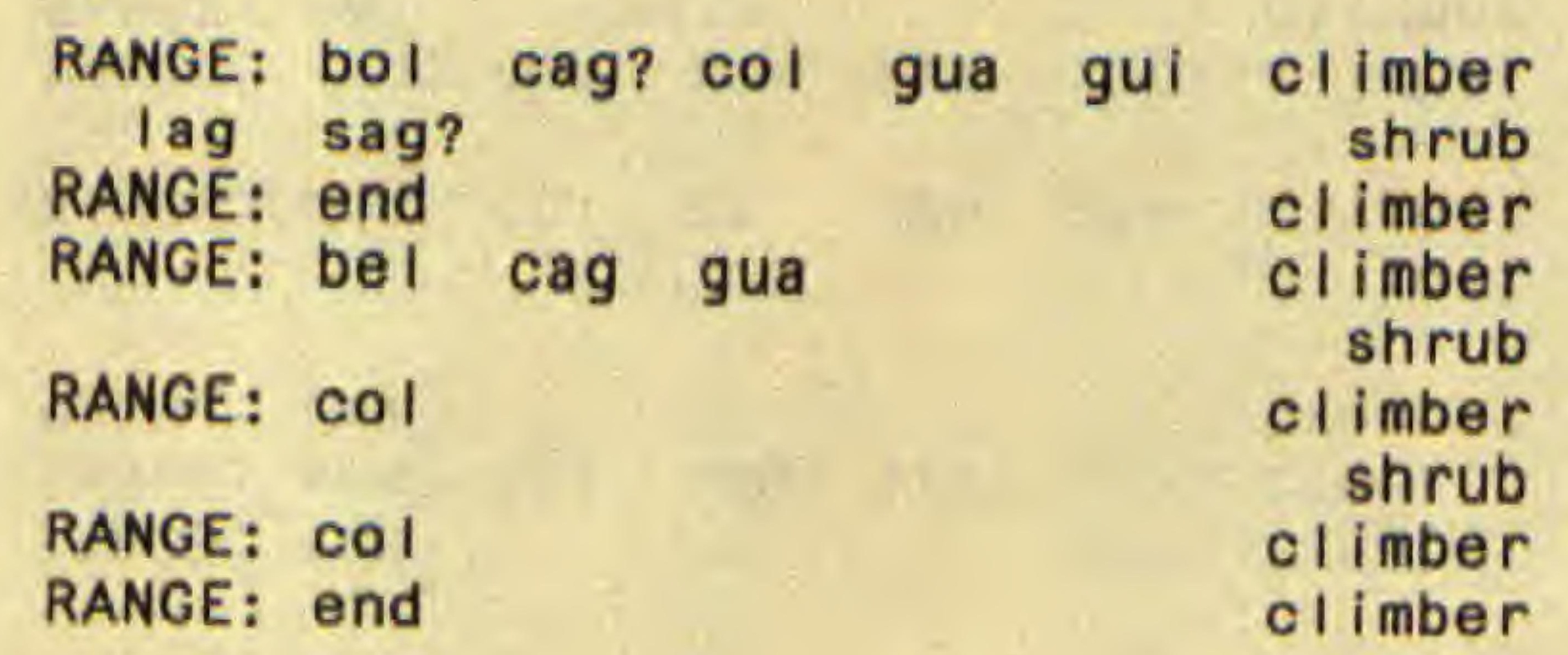

142 (94) TRIGONIACEAE

Trigonia

*eriosperma (Lam.) Fromm \& Santus

*--subsp. membranacea (A,C. Sm, ) Lleras

*rugosa Benth.

PROV: da 000m: $0-1$

PROV: ca da

000m: $0-1$

RANGE: Sao

RANGE: cag col $\mathrm{cr}$ mex

cl imber

climber

shrub

143 (95) VOCHYSIACEAE

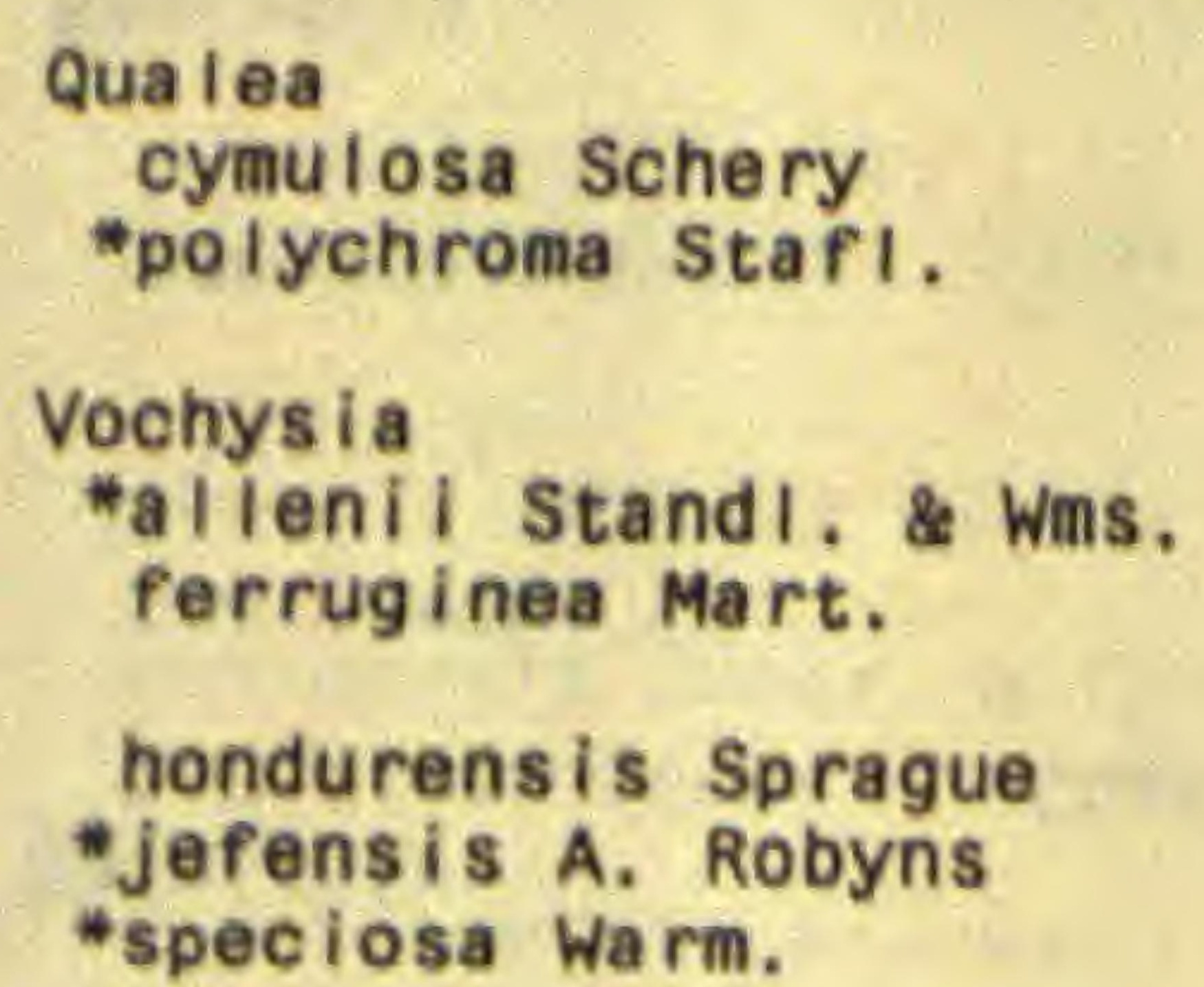

RANGE: $\mathrm{COI}$ RANGE: gui

tree

RANGE: $c 0$ I

RANGE: cag? cr

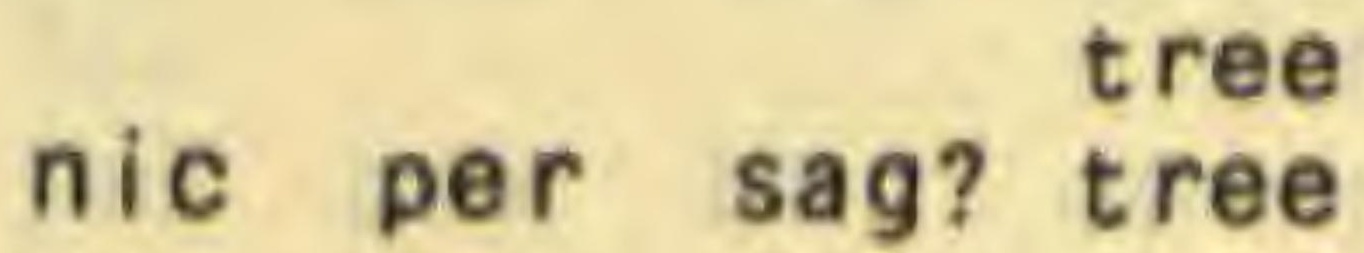

RANGE: cag mex

RANGE: end

RANGE: $\mathrm{CO} I$ tree

shrub

tree

145 (96) POLYGALACEAE

\section{*Bredemeyera}

* I ucida (Benth.) Benn.

\section{Monnina}

* deppei G. Don

* saprogena J.D. Sm.

* speciosa Tr. PI.

sylvatica Schlecht. \& Cham.
PROV: da

000m: $0-1$

PROV: $\mathrm{CC}$ IO Vr PROV: ch Vr

PROV: da

PROV: $C 0$ ch da 10
$000 \mathrm{~m}: 0-1,1-2$

000m: $1-2,2-3$

000m: $1-2$
RANGE: bel gua hon ven

climber

shrub

shrub

cl imber

shrub 
TAXON

FAMILY

PROVINCES

ELEVATION (OOOM.)

RANGE OUTSIDE OF PANAMA

$H A B \mid T$

145 (96) POLYGALACEAE cont.

Xalapens is H.B.K.

Moutabea

longifolia P. \& E.

Polygala

aparinoides Hook. \& Arn.

asperuloides H.B.K.

*dukei Barringer

fendleri Chod.

- -var. heterothrix Blake

hygrophila H.B.K.

jefens is W.H. Lewis

leptocaulis Torr. \& Gray

long icaul is H.B.K.

panamens is Chod.

paniculata $L$.

timoutou Aubl.

variabilis H.B.K.

*violacea Aubi. emend. Marq.

wurdackiana W.H. Lewis

\section{Securidaca}

* cristata A.C. Sm.

diversifolia (L.) Blake

tenuifolia Chod.
PROV: $\mathrm{ch}$

$000 \mathrm{~m}: 1-2,2-3,3+$

RANGE: cag mex

000m: $0-1$

000m: $0-1,1-2$

000m: $0-1$

000m: $0-1$

PROV: ch cn

PROV: da

000m: $0-1,1-2$

000m: $0-1,1-2$

$000 \mathrm{~m}: 0-1$

PROV: ca cc ch pa

PROV: pa $v r$

PROV: ca ce ch pa 000m: $0-1,1-2$
PROV: ca cc ch pa vr 000m: $0-1$

PROV: $\mathrm{cc}$ ch $\quad 000 \mathrm{~m}: 0-1,1-2$

PROV: bo ca cc ch cn 000m: $0-1,1-2,2-3$ da he pa sa vr

PROV: ca pa 000m: $0-1$

PROV: cc 000m: $0-1$

PROV: ca cc ch pa 000m: $0-1,1-2$

PROV: cc cn $v r$ 000m: $0-1,1-2$

PROV: pa 000m: $0-1$

PROV: bo ca cc ch cn 000m: $0-1$ da he lo pa sa

PROV: ca cn da pa $v r$ 000m: $0-1$
RANGE: cag

RANGE: bel

RANGE: beI

RANGE: end

RANGE: end

RANGE: be I

RANGE: end

RANGE: ang

RANGE: ang$$
\text { SaO }
$$

RANGE: end

RANGE: cag

RANGE: be

RANGE: cag

RANGE: ang

RANGE: ang

RANGE: sat

RANGE: cag

RANGE: trt herb

shrub

tree

shrub

gua mex herb

ol gua ven herb

tree

cr his sag? herb

herb

shrub

cag nao sag? sao herb

cag mex per sag herb

subshrub

sag? sao tex herb

cr sag? trt herb

mex sag? trt herb

cag mex sag sao herb

herb

limber

lag mex per sag? shrub

cl imber shrub

climber

146 (96A) DICHAPETALACEAE

Dichapeta I um

axillare Woods,

*donnell-smithi $i$ Engl.

PROV: cc ch sa sa $000 \mathrm{~m}: 0-1$

RANGE: $\mathrm{COI} \mathrm{cr}$

tree

*--var. chiapasense (Standl.) Prance

*--var. donnell-smithii PROV: cn
PROV: bo

*gentryi Prance

PROV: bo $\quad 000 \mathrm{~m}: 1-2$

nevermannianum stand \& Valerio PROV: bo

000m: $1-2$

$000 \mathrm{~m}: 0-1$

RANGE: cag

RANGE: cag

RANGE: end

RANGE: $\mathrm{cr}$

tree

tree

shrub

shrub

*Tapura

* colombiana Cuatr.

PROV: sa

000m: $0-1$

* cubensis (P. \& E.) Griseb.

PROV: pa

$000 \mathrm{~m}: 0-1$

RANGE: COI

tree

RANGE: CUb

tree 
TAXON

FAMILY

PROVINCES

ELEVATION (OOOM.)

RANGE OUTSIDE OF PANAMA

HAB IT

146 (96A) DICHAPETALACEAE cont.

"panamensis Prance

PROV: pa

000m: $0-1$

RANGE: end

climber

\section{7 (97) EUPHORBIACEAE}

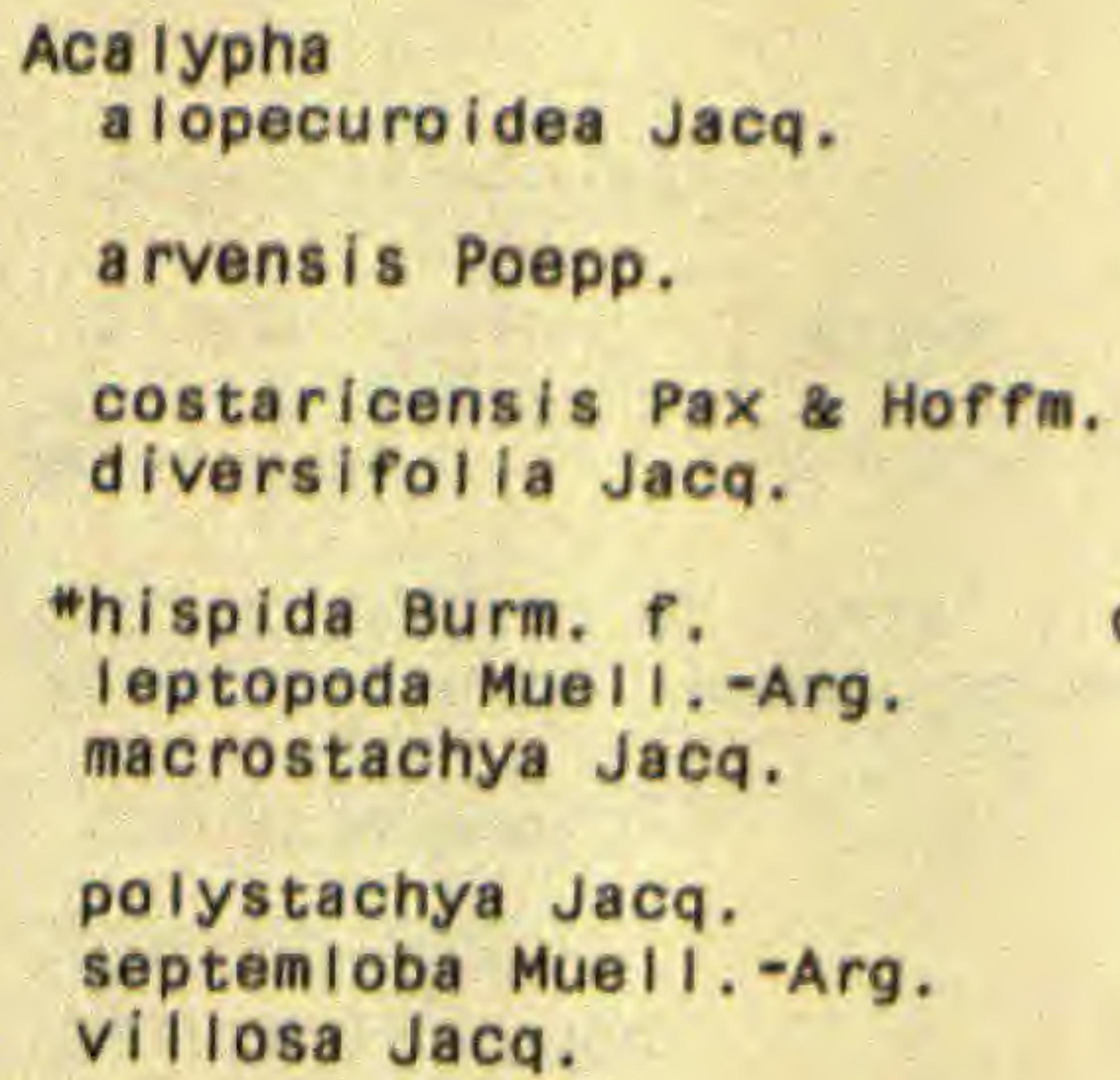

\section{Alchornea}

costaricensis Pax \& Hoffm.

glandulosa Poepp.

*--var. pittieri (Pax) Pax

*grandiflora (KI.) Muel I. -Arg. grandis Benth.

latifolia SW.

\#mega lophyl la MueII,-Arg.

*triplinervia (Spr.) Mueill.-Arg.
PROV: ca ce cn da pa 000m: 0-1

PROV: bo ca ce ch en $000 \mathrm{~m}$ : $0-1$

PROV: bo ca pa 000m: $0-1$

PROV: bo ca cc ch cn 000m: 0-1 da he lo pa vr

PROV: ca pa 000m: 0-1

PROV: ch lo 000m: $0-1,1-2$

PROV: bo ca cc cn da $000 \mathrm{~m}$ : $0-1$ he lo pa sa vr

PROV: bo $\mathrm{ch}$

000m: 0-1

PROV: ch

$000 \mathrm{~m}: 1-2$

PROV: bo ca ch da pa 000m: $0-1$ vr

000m: 0-1

PROV: cn da pa sa 000m: $0-1$

PROV: bo ca cc ch cn 000m: $0-1$ da pa sa

PROV: vr

$000 m: 0-1,1-2$

PROV: bo ca cc ch cn 000m: $0-1$ da lo pa

PROV: bo da $v r$

000m: $0-1,1-2$

PROV: ch da

000m: $0-1$

PROV: da $v r$

000m: $0-1$

PROV: bo ca ch cn pa 000m: $0-1$

PROV: da

PROV: cc da 000m: $0-1,1-2$ $000 m: 0-1,1-2$

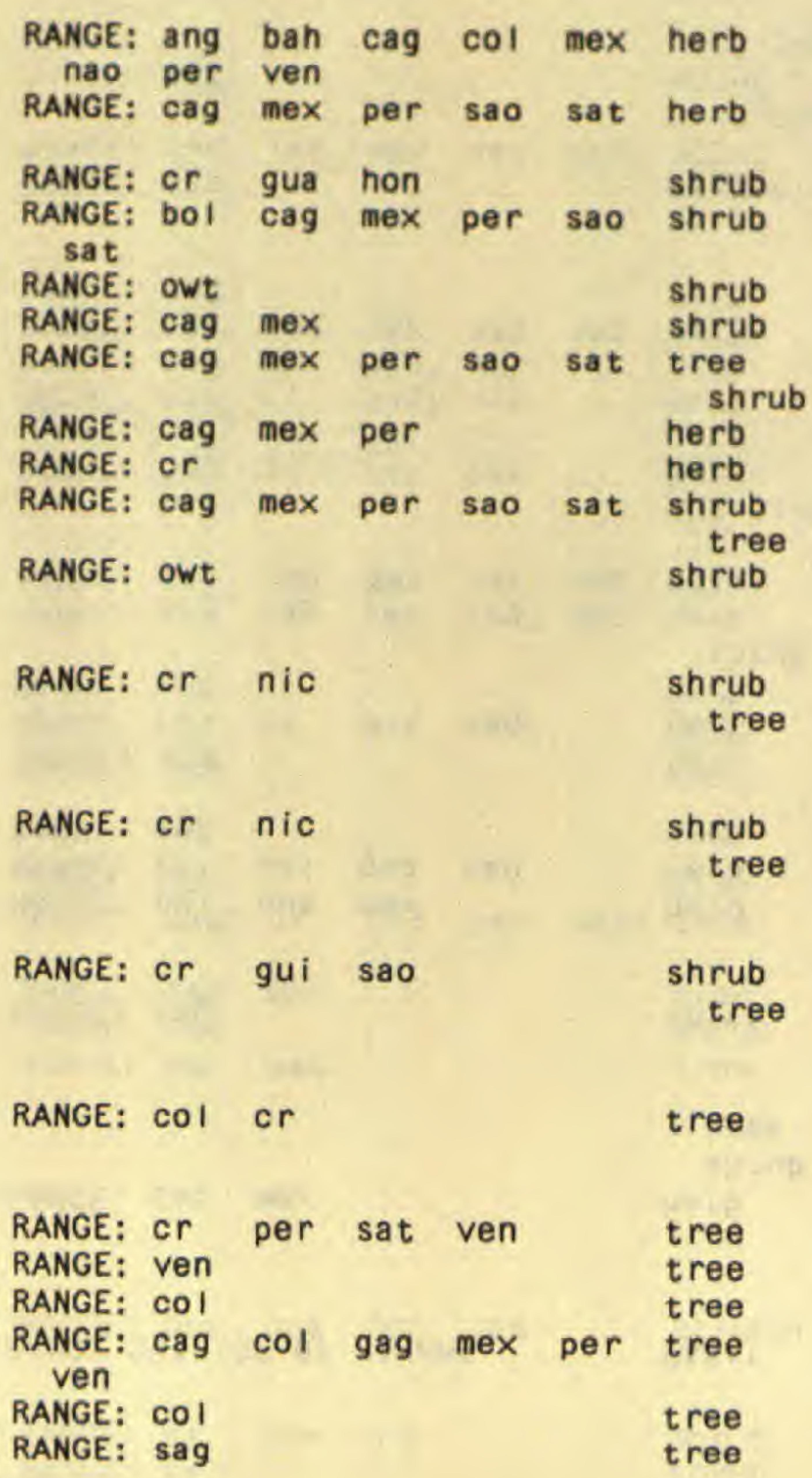


TAXON

147 (97) EUPHORBIACEAE cont.

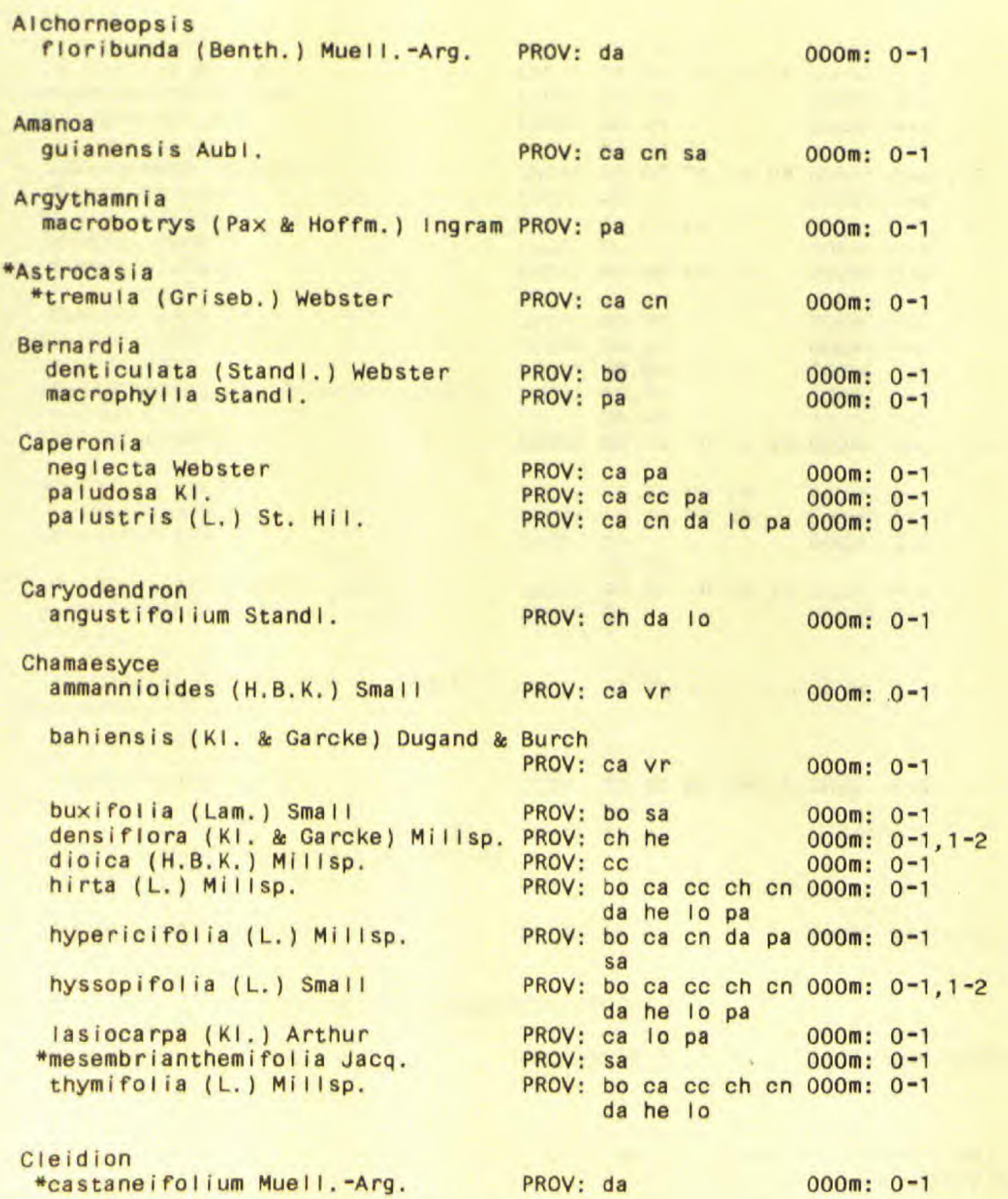

\begin{tabular}{|c|c|c|c|c|c|c|}
\hline $\begin{array}{c}\text { RANGE: } \\
\text { pr }\end{array}$ & $\begin{array}{l}\text { col } \\
\text { sao }\end{array}$ & $\mathrm{cr}$ & ecu & gui & his & tree \\
\hline RANGE: & sao & sat & ven & & & tree \\
\hline RANGE: & end & & & & & shrub \\
\hline RANGE: & cag & jam & mex & & & treelet \\
\hline $\begin{array}{l}\text { RANGE: } \\
\text { RANGE: }\end{array}$ & $\begin{array}{l}\mathrm{cr} \\
\mathrm{cr}\end{array}$ & $=$ & & & & $\begin{array}{l}\text { tree } \\
\text { shrub }\end{array}$ \\
\hline $\begin{array}{c}\text { RANGE: } \\
\text { RANGE: } \\
\text { RANGE: } \\
\text { SaO }\end{array}$ & $\begin{array}{l}\text { end } \\
\text { cag } \\
\text { cag }\end{array}$ & $\begin{array}{l}\text { gui } \\
\text { fla }\end{array}$ & $\begin{array}{l}\operatorname{mex} \\
\text { nao }\end{array}$ & $\begin{array}{l}\text { sag } \\
\text { per }\end{array}$ & sag & $\begin{array}{l}\text { herb } \\
\text { herb } \\
\text { herb }\end{array}$ \\
\hline RANGE: & end & & & & & tree \\
\hline $\begin{array}{c}\text { RANGE: } \\
\text { nic }\end{array}$ & $\begin{array}{l}\text { bel } \\
\text { sat }\end{array}$ & $\mathrm{cr}$ & cub & mex & nas & herb \\
\hline $\begin{array}{c}\text { RANGE: } \\
\text { sat }\end{array}$ & $\mathrm{cr}$ & jam & mex & nic & sao & herb \\
\hline RANGE: & ang & bah & cag & fla & ven & subshrub \\
\hline $\begin{array}{l}\text { RANGE: } \\
\text { RANGE: } \\
\text { RANGE : }\end{array}$ & $\begin{array}{l}\text { cag } \\
\text { cag } \\
\text { wdw }\end{array}$ & $\begin{array}{l}\operatorname{mex} \\
\text { mex }\end{array}$ & $\begin{array}{l}\text { sat } \\
\text { sat }\end{array}$ & & & $\begin{array}{l}\text { herb } \\
\text { herb } \\
\text { herb }\end{array}$ \\
\hline RANGE: & nwg & & & & & herb \\
\hline RANGE: & nwg & owg & & & & herb \\
\hline $\begin{array}{l}\text { RANGE: } \\
\text { RANGE: } \\
\text { RANGE: }\end{array}$ & $\begin{array}{l}\text { cag } \\
\text { nwt } \\
\text { wdw }\end{array}$ & fla & gag & $\operatorname{mex}$ & sat & $\begin{array}{l}\text { herb } \\
\text { herb } \\
\text { herb }\end{array}$ \\
\hline RANGE: & $c r ?$ & ecu & & & & tree \\
\hline
\end{tabular}


TAXON

woodsonianum croiz.

PROV: ca pa

000m: $0-1$

Cnidoscolus

aconitifolius (Mill.) I.M. Johnst.

$$
\text { cult. PROV: ca vr }
$$

000m: $0-1$

urens (L,) Arthur

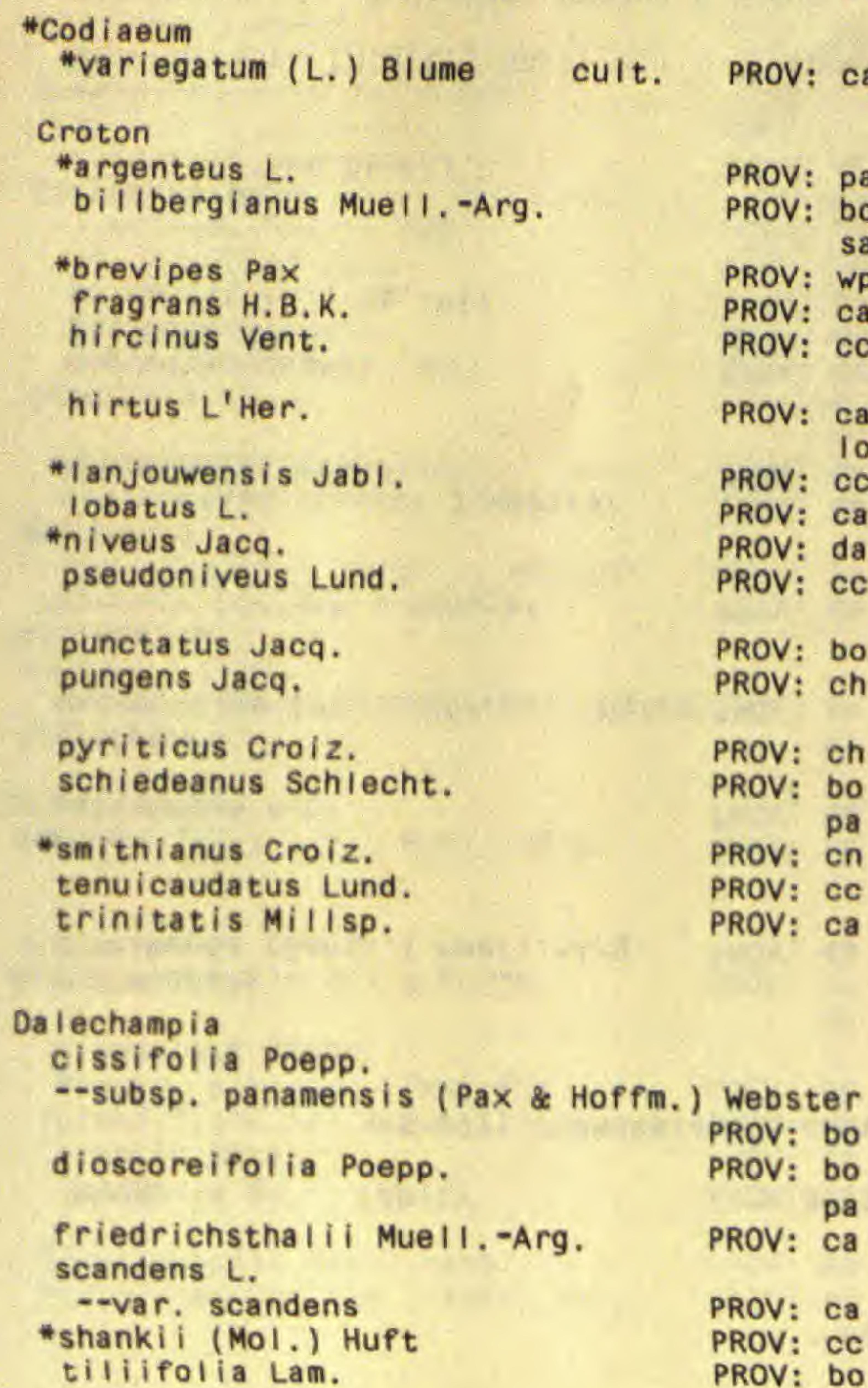

\begin{tabular}{|c|c|c|c|c|c|c|}
\hline RANGE: & per & & & & & tree \\
\hline & th & $\cos 1 \mathrm{x}$ & $8 \%$ & & & \\
\hline RANGE: & cag & $\operatorname{mex}$ & & & & $\begin{array}{l}\text { tree } \\
\text { shrub }\end{array}$ \\
\hline RANGE: & cag & $\operatorname{mex}$ & sao & sat & & $\begin{array}{l}\text { herb } \\
\text { subshru }\end{array}$ \\
\hline RANGE: & $\mathrm{cr}$ & oce & & $=$ & & tree \\
\hline $\begin{array}{l}\text { RANGE: } \\
\text { RANGE: }\end{array}$ & $\begin{array}{l}\mathrm{cag} \\
\mathrm{cr}\end{array}$ & mex & sao & tex & & $\begin{array}{l}\text { herb } \\
\text { shrub } \\
\text { tree }\end{array}$ \\
\hline $\begin{array}{l}\text { RANGE: } \\
\text { RANGE: }\end{array}$ & $\begin{array}{l}\mathrm{cr} \\
\mathrm{col}\end{array}$ & & & & & shrub \\
\hline & $\begin{array}{l}\text { col } \\
\text { lag }\end{array}$ & $\begin{array}{l}\text { ven } \\
\text { ven }\end{array}$ & $x^{2}$ & $5 y$ & 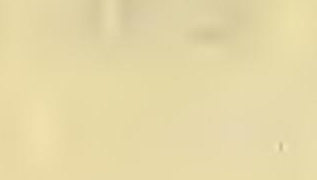 & shrub \\
\hline RANGE: & ang & cag & sat & $=215$ & & $\begin{array}{c}\text { shrub } \\
\text { tree } \\
\text { herb }\end{array}$ \\
\hline RANGE: & gui & ven & & & & tree \\
\hline RANGE: & bah & cag & fla & nwt & per & herb \\
\hline RANGE: & col & cr & gag & ven & & tree \\
\hline RANGE: & $\operatorname{mex}$ & & & & & $\begin{array}{c}\text { shrub } \\
\text { tree }\end{array}$ \\
\hline RANGE: & bah & cag & cub & nao & nas & shrub \\
\hline RANGE: & cr & per & sao & ven & & $\begin{array}{l}\text { shrub } \\
\text { tree }\end{array}$ \\
\hline RANGE: & $\mathrm{cr}$ & & & & & tree \\
\hline RANGE: & cag & $\mathrm{col}$ & $\mathrm{cr}$ & gui & $\operatorname{mex}$ & tree \\
\hline per & sao & ven & & & & \\
\hline RANGE: & col & $\mathrm{cr}$ & nic & & & tree \\
\hline RANGE: & er & & & & & tree \\
\hline $\begin{array}{c}\text { RANGE: } \\
\text { OWt }\end{array}$ & $\begin{array}{l}\text { ang } \\
\text { per }\end{array}$ & bah & ag & fla & nwt & herb \\
\hline
\end{tabular}

RANGE: cag gua per climber RANGE: col cr per ven climber

RANGE: $\mathrm{cr}$ nic climber

RANGE: nWt climber

RANGE: $c r$ climber

RANGE: cag hon mex per sao climber 
TAXON

147 (97) EUPHORBIACEAE cont.

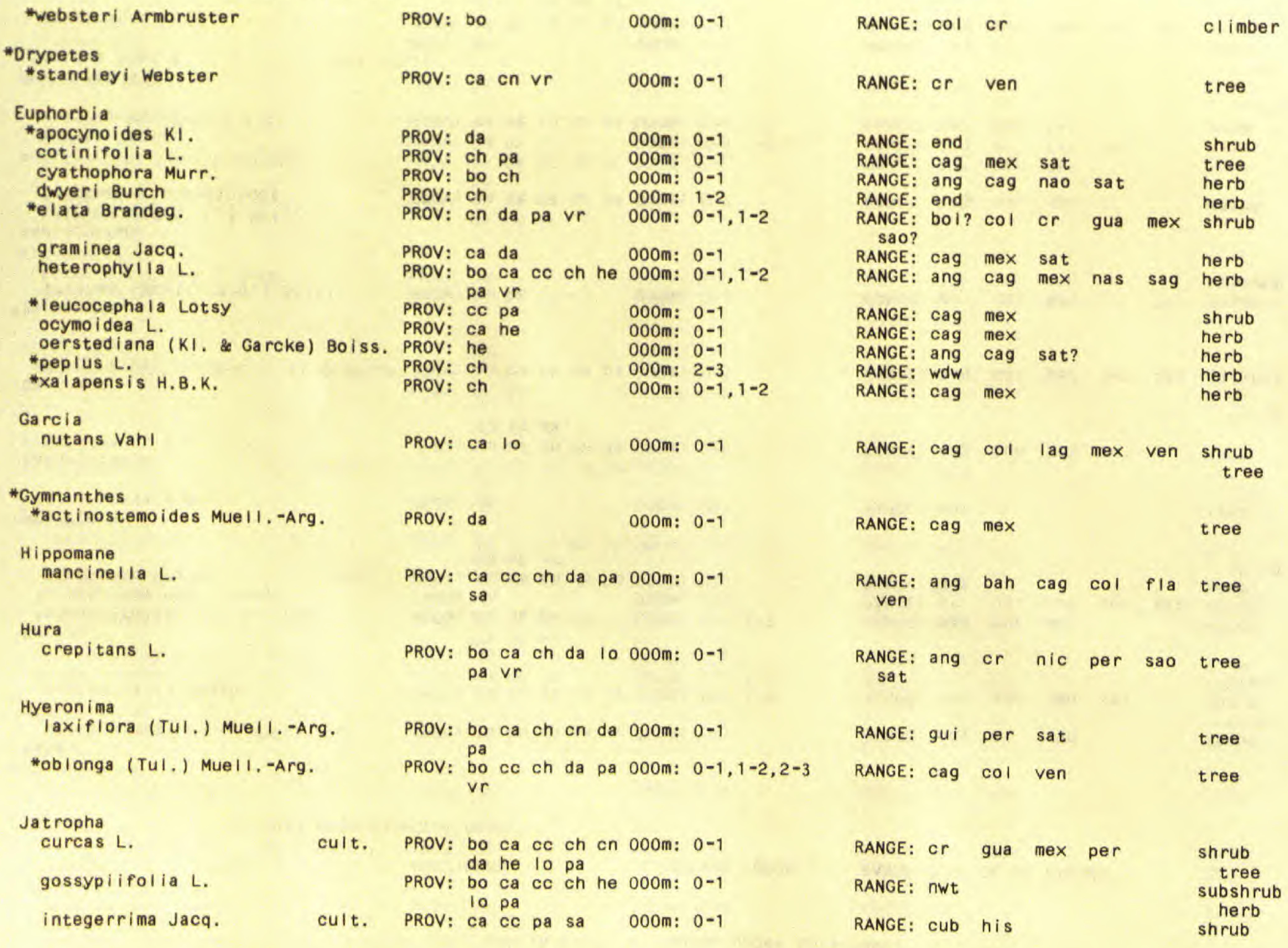


TAXON

Mabea

montana Muell, -Arg.

occidentalis Benth.

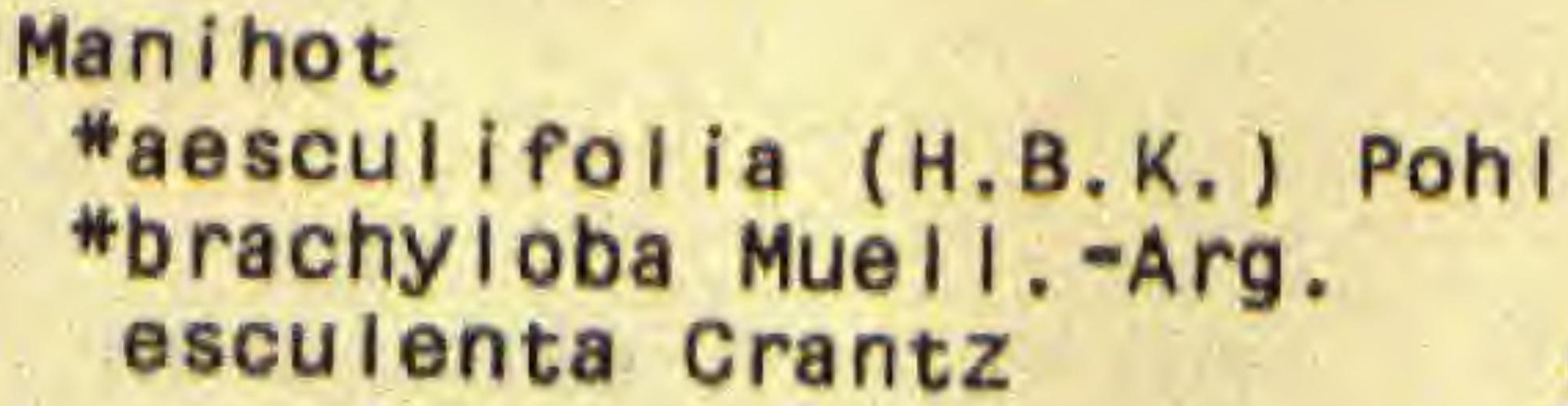

PROV: ca cc da pa vr 000m: $0-1$

PROV: ca cn da pa sa 000m: $0-1,1-2$

PROV: ca da pa $\quad 000 \mathrm{~m}: 0-1,1-2$

ch da he pa sa $\vee r$

000m: $0-1$

PROV: bo ca ch cn da $000 \mathrm{~m}: 0-1$ lo pa sa

PROV: bo ca ch da pa 000m: $0-1$ $v r$ PROV: ca ce en da pa $000 \mathrm{~m}: 0-1$
PROV: ca cc he pa vr 000m: $0=1$

PROV: $C C \quad 000 \mathrm{~m}: 0-1$

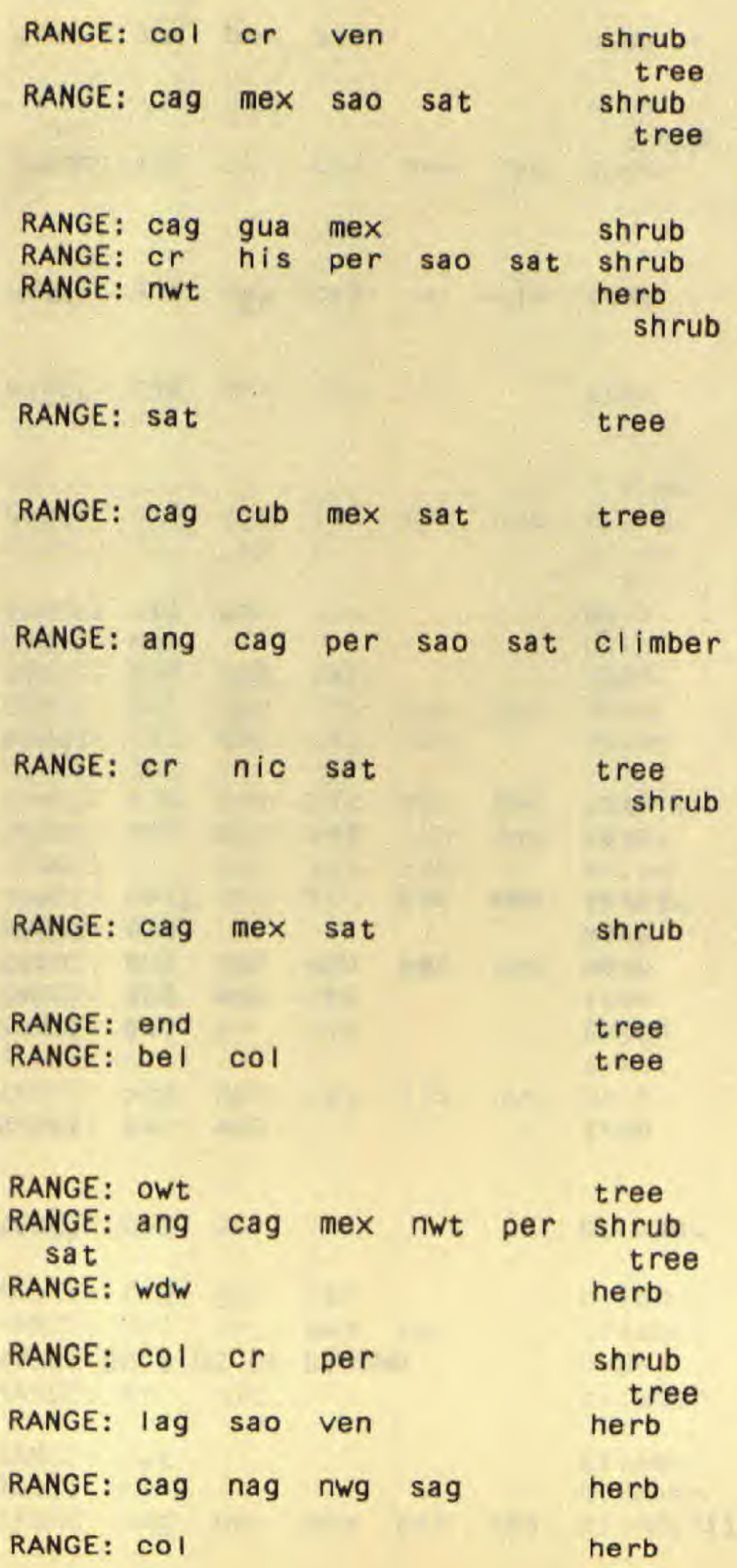


TAXON

147 (97) EUPHORBIACEAE cont.

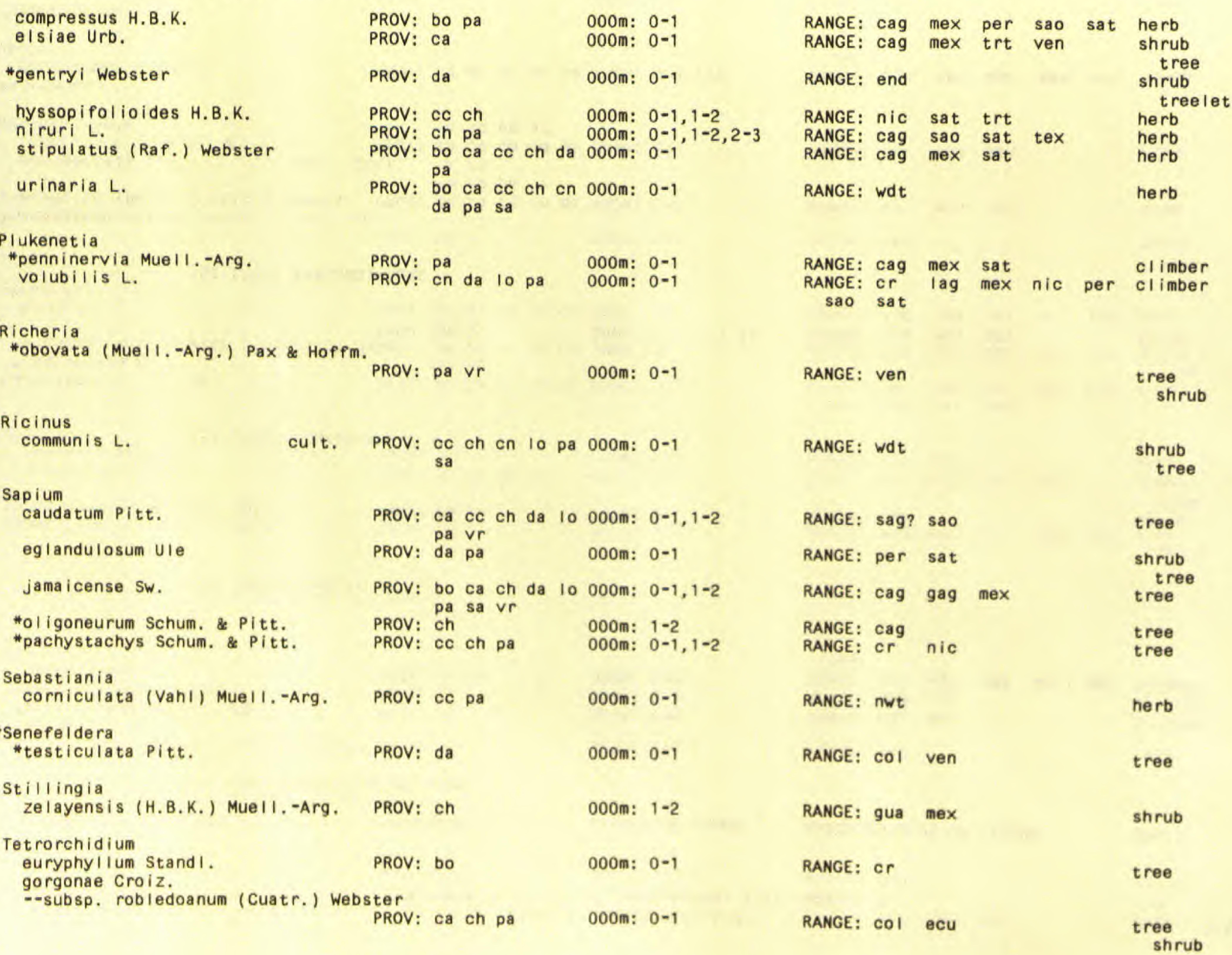


TAXON

147 (97) EUPHORBIACEAE cont.

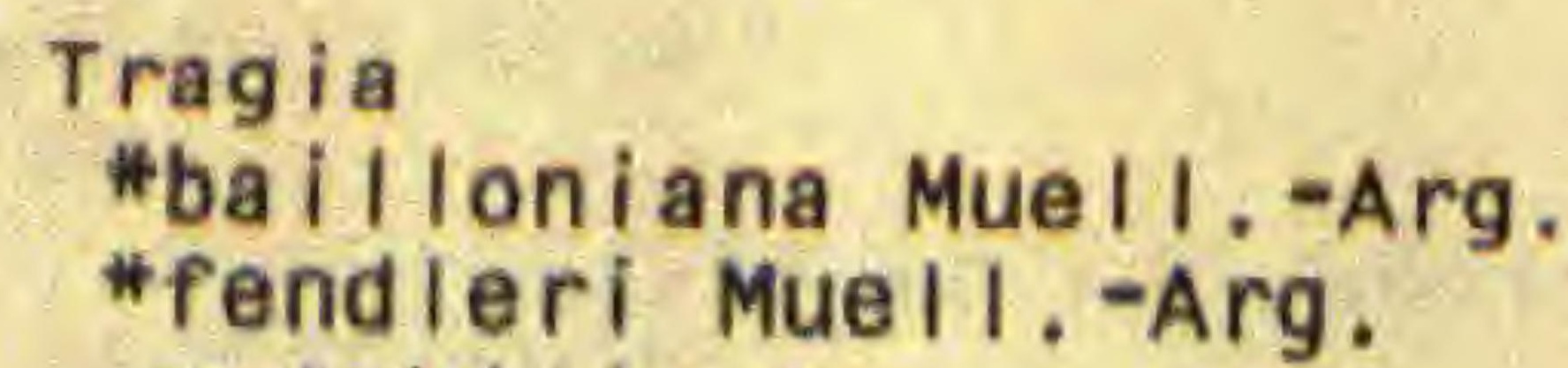

000m: $1-2$

000m: $0-1$

000m: $0-1$

149 (99) BUXACEAE

\section{Buxus}

citrifolia (WiIId.) Spr. PROV: ca pa

151 (100) CORIARIACEAE

\section{Coriaria}

* ruscifolia L.

*--subsp. microphylla (Poir.) L. Skog PROV: ch

000m: $1-2,2-3,3+$ shrub climber

RANGE: cag mex

RANGE: Ven

RANGE: cag cub mex per sao climber

RANGE: ven

\section{3 (101) ANACARDIACEAE}

\section{Anacardium}

excelsum (Bert. Balb.) Skeels PROV: bo ca cc ch da 000m: 0-1

occidentale L.

intr, cult.

PROV: ca ce ch cn da 000m: $0-1$

he lo pa vr

\section{Astronium}

graveolens Jacq.

\section{Campnosperma}

panamensis Stand I.

Mang ifera

ind ica L.
PROV: ca cc ch da pa $000 m: 0-1,1-2$

PROV: bo

000m: $0-1$
RANGE: cr ecu ven

tree

RANGE: or ecu sao
RANGE: bol cag col mex sao sao

RANGE: $\mathrm{cr}$

tree intr. cult.

PROV: ca ch lo pa sa 000m: $0-1$
RANGE: asi $\mathrm{cr}$ 
TAXON

Mauria

heterophyIIa H.B.K.

PROV: ch da

000m: $0-1,1-2$

\section{Mosqui toxylum}

jamaicense Krug \& Urb.

PROV: ca ce ch cn da 000m: 0-1

$$
\text { pa sa }
$$

Schinus

terebinthifolius Raddi intr. cult.

PROV: ca $\quad 000 \mathrm{~m}: 0-1$

Spondias

mombin L.

purpurea L.

radikoferi J.D, Sm.

cult. PROV: $\begin{aligned} & \text { da pa sa ca ch da } 000 \mathrm{~m}: 0-1,1-2 \\ & \text { lo pa }\end{aligned}$

PROV: bo ca ch cn pa 000m: 0-1

Tapirira

brenesii Standi. guianensis Aubl.

PROV: $c h$ PROV: ca cn pa sa 000m: $0-1$

Toxicodendron striatum (R. \& P) Kuntze

PROV: ch

000m: $1-2$

154 (101A) CYRILLACEAE

Cyrilia

racemiflora L.

PROV: pa $v r$

000m: $0-1$

*Purdiaea

\#belizensis (Sm. \& Standl.) Thomas PROV: vr

000m: $0-1$

157 (102) AQUIFOLIACEAE
PROV: ch

000m: 1-2

PROV: bo cc ch on he 000m: $0-1,1-2$ pa

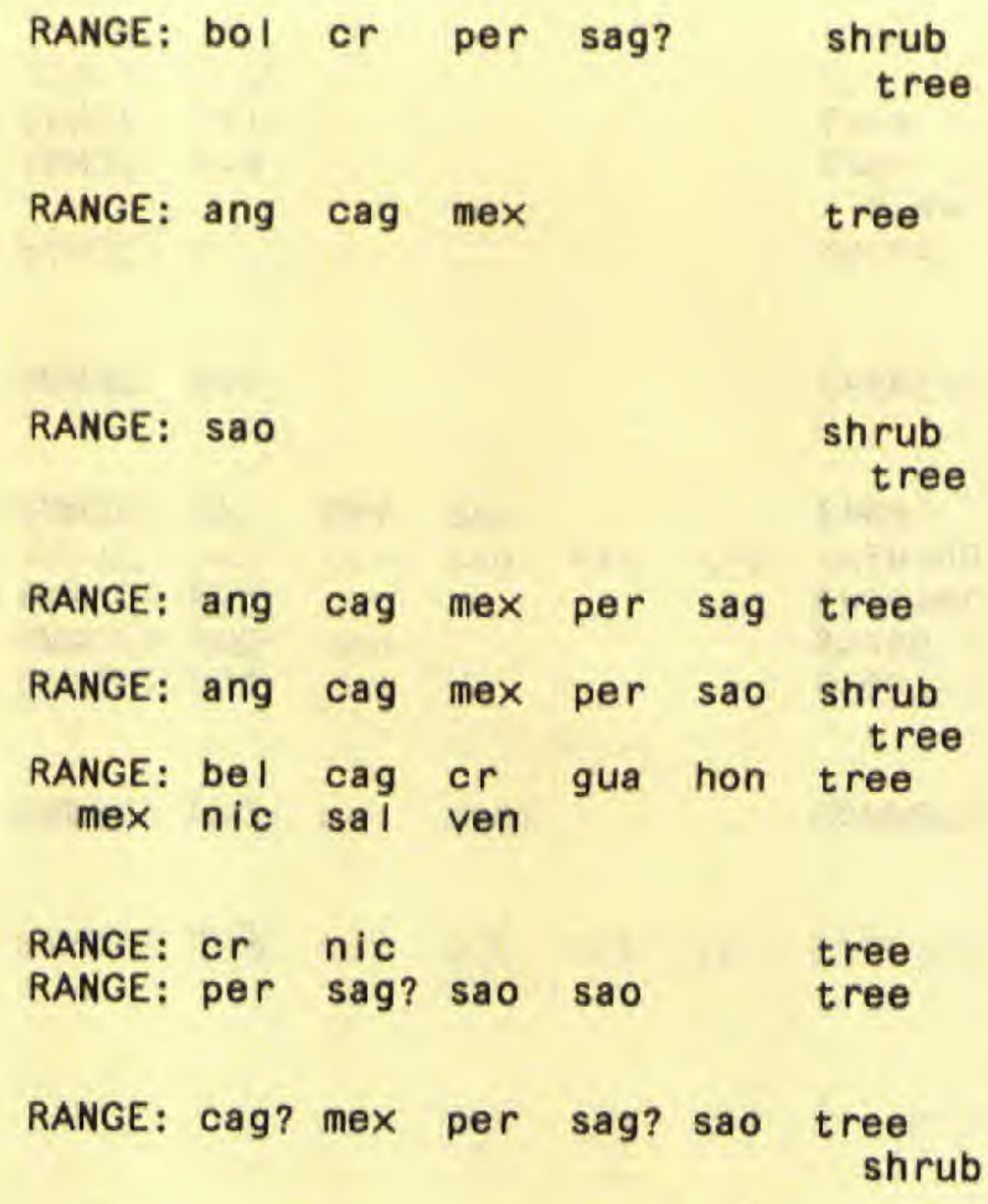

RANGE: ang bel col gui mex tree nag? sao ven

RANGE: cag

shrub

RANGE: end

RANGE: $c r$

RANGE: ang cag gui

shrub

tree

tree 
TAXON

157 (102) AQUIFOLIACEAE cont.

*kunthiana Triana I amprophyl la StandI.

*pa I I ida Stand I.

valerii Standi.

--var. ramonensis (Standl.) Edwin

- var. valeri.

*vulcanicola Standl.

*yurumanquinis Cuatr.

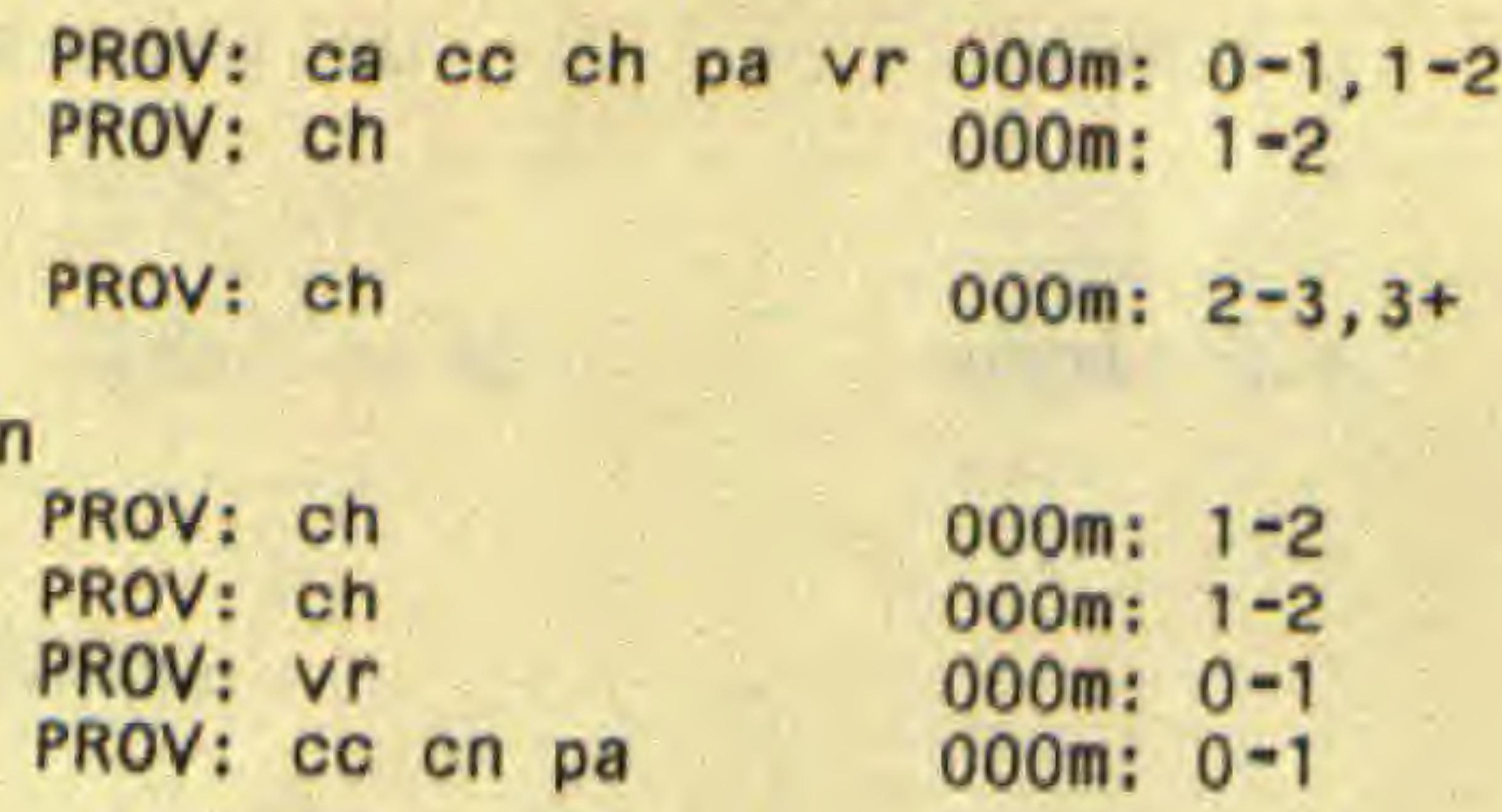

PROV: ch

PROV: ch

PROV: ch

PROV: $V r$

PROV: cc cn pa

000m: $1-2$

000m: $2-3,3+$

000m: $1-2$

$000 \mathrm{~m}: 1-2$

000m: $0-1$

000m: $0-1$

\section{8 (103) CELASTRACEAE}

\section{*Cass ine}

*xy locarpa Vent.

Celastrus

panamens is Lund.

\section{Crossopeta I um}

eucymosa (Loes. \& Pitt.) Lund. *panamense Lund.

*parviflorum (HemsI.) Lund.

"tonduzii (Loes.) Lund.

\section{*Goup ia}

*glabra Aubl.

Maytenus

*b lepha rodes (Pitt.) A. Gentry

* jefeana Lund. schippii Lund.

\section{* Perrottetia}

*exce I sa Lund.

*mul tiflora Lund.

*quinduens is H.B.K.

* sessiliflora Lund.

\section{*Quetza I ia}

Hoccidental is (Loes.) Lund.

Wimmeria

sternii Lund.
PROV: pa sa

000m: $0-1$

PROV: ch

000m: $0-1$

PROV: bo ca cn

PROV: cn pa

PROV: bo ca

PROV: ch

PROV: sa

PROV: ch

PROV: pa

PROV: ca cn da

PROV: cn

PROV: ch

PROV: da

PROV: vr

PROV: pa

PROV: $\mathrm{ch}$

000m: $0-1$

000m: $0-1$

000m: $0-1$

000m: $1-2$

000m: $0-1$

000m: 0-1

000m: $0-1$

000m: $0-1$

000m: $1-2$

000m: 1-2

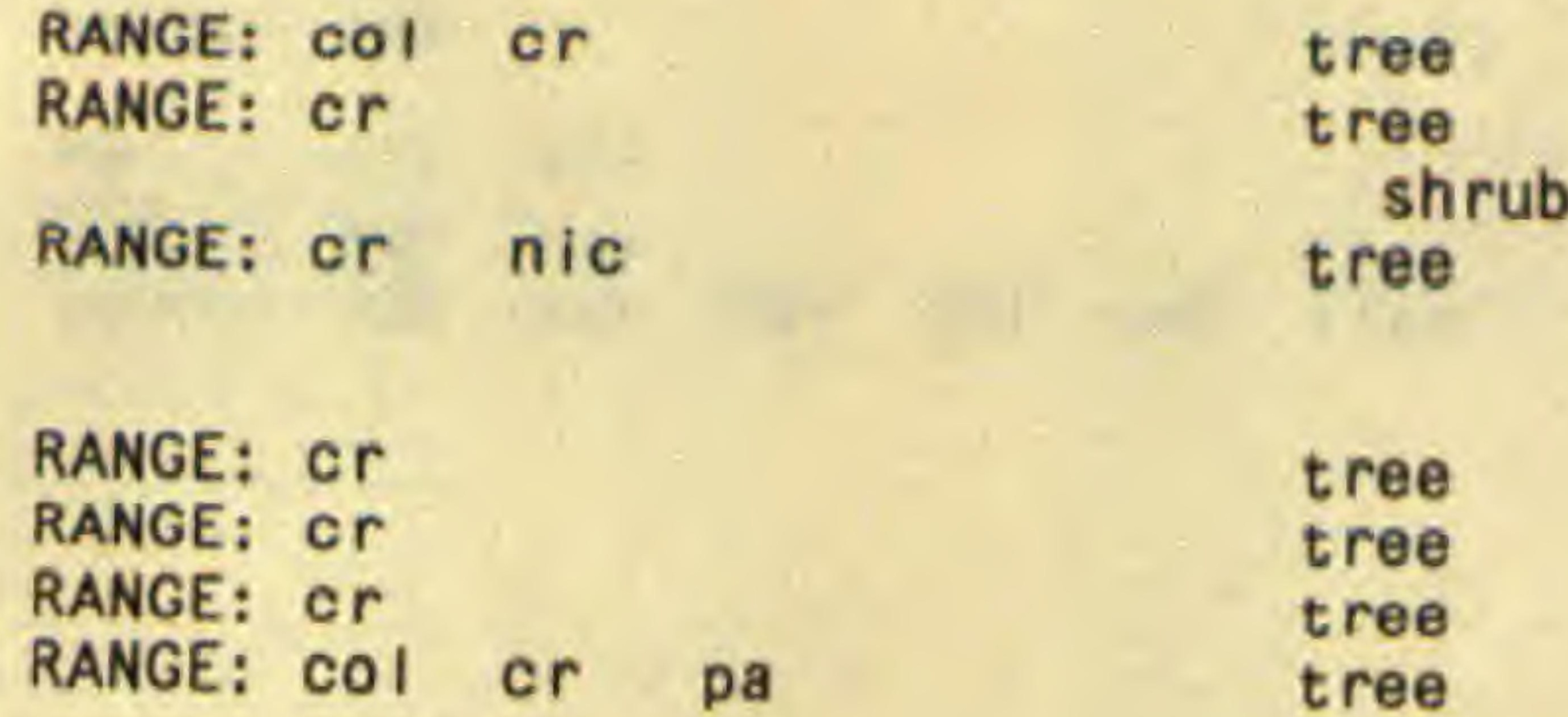

tree
$000 \mathrm{~m}: 0-1,2-3,3+$

000m: $0-1,1-2$

000m: $0-1,1-2$

$000 \mathrm{~m}: \quad 0-1,1-2$ tree

shrub

RANGE: gag

RANGE: end

RANGE: cag

RANGE: end

RANGE: end

RANGE: or mex

shrub

treelet

tree

RANGE: sat

tree

RANGE: $\mathrm{cr}$

RANGE: end

RANGE: bel

shrub

tree

tree

tree

RANGE: $e x$

RANGE: $\mathrm{cr}$

RANGE: $\mathrm{col}$

per ven

RANGE: $c r$

tree

tree

shrub

tree

RANGE: $c r$

treelet
RANGE: end 


\section{Zinowiewia}

costaricensis Lund.

*micrantha Lund.

PROV: $\mathrm{ch}$

PROV: $\mathrm{ch}$

000m: $1-2$

000m: $2-3$

159 (104) HIPPOCRATEACEAE

\section{Anthodon}

panamense A.C. Sm.

Cheiloclinium

*anoma I um Miers

cognatum (Miers) A.C. Sm.

*klugii A.C. Sm.

"podos temmum Sandw.

Cuervea

kappleriana (Miq.) A.C. Sin.

\section{El achyptera}

floribunda (Benth.) A.C. Sm.

Hemiangium

exceisum (H,B,K.) A.C. Sm.

Hippocratea

$$
\text { volubilis L. }
$$

Hylenaea

praecelsa (Miers) A.C. Sm.

Peritassa

pruinosa (Seem.) A.C. Sm.

Prionostemma

aspera (Lam.) Miers

Pristimera

*austin-smithii (Lund.) A.C. Sm. nervosa (Miers) A.C. Sm.
PROV: bo ca cn da 000m: $0-1$

PROV: pa

000m: $0-1$

000m: $0-1$

PROV: pa

000m: $0-1$

PROV: pa

C.t.

PROV: bo da sa vr 000m: $0-1$

PROV: bo ca ch on pa 000m: $0-1,1-2$ $v r$

PROV: $\mathrm{Vr}$

000m: $0-1$

PROV: bo ca cn da pa $000 \mathrm{~m}$ : $0-1$ sa

PROV: ca da pa sa 000m: $0-1$

PROV: ca da pa 000m: $0-1$

PROV: ca da

000m: $0-1$

000m: $0-1$ 000m: $0-1$

PROV: da

PROV: ca
RANGE: $c r$

RANGE: end

tree

tree

RANGE: end

cl imber

RANGE: gui per sao tree?

RANGE: bol per sag sao trt shrub

RANGE: per ven tree

RANGE: $W r$ climber

RANGE: ang or sag? climber

RANGE: cag ecu gua sag sao climber

RANCE; cag col mex sao ven climber

shrub

RANGE: cag fla mex sag sao climber

RANGE: $c O$

climber

RANGE: COI per

climber

RANGE: bol sag? trt climber

RANGE: Cr

climber

RANGE: bol gui per sao ven climber 
TAXON

FAMILY

PROVINCES

ELEVATION (OOOM.)

RANGE OUTSIDE OF PANAMA

HAB IT

159 (104) HIPPOCRATEACEAE cont.

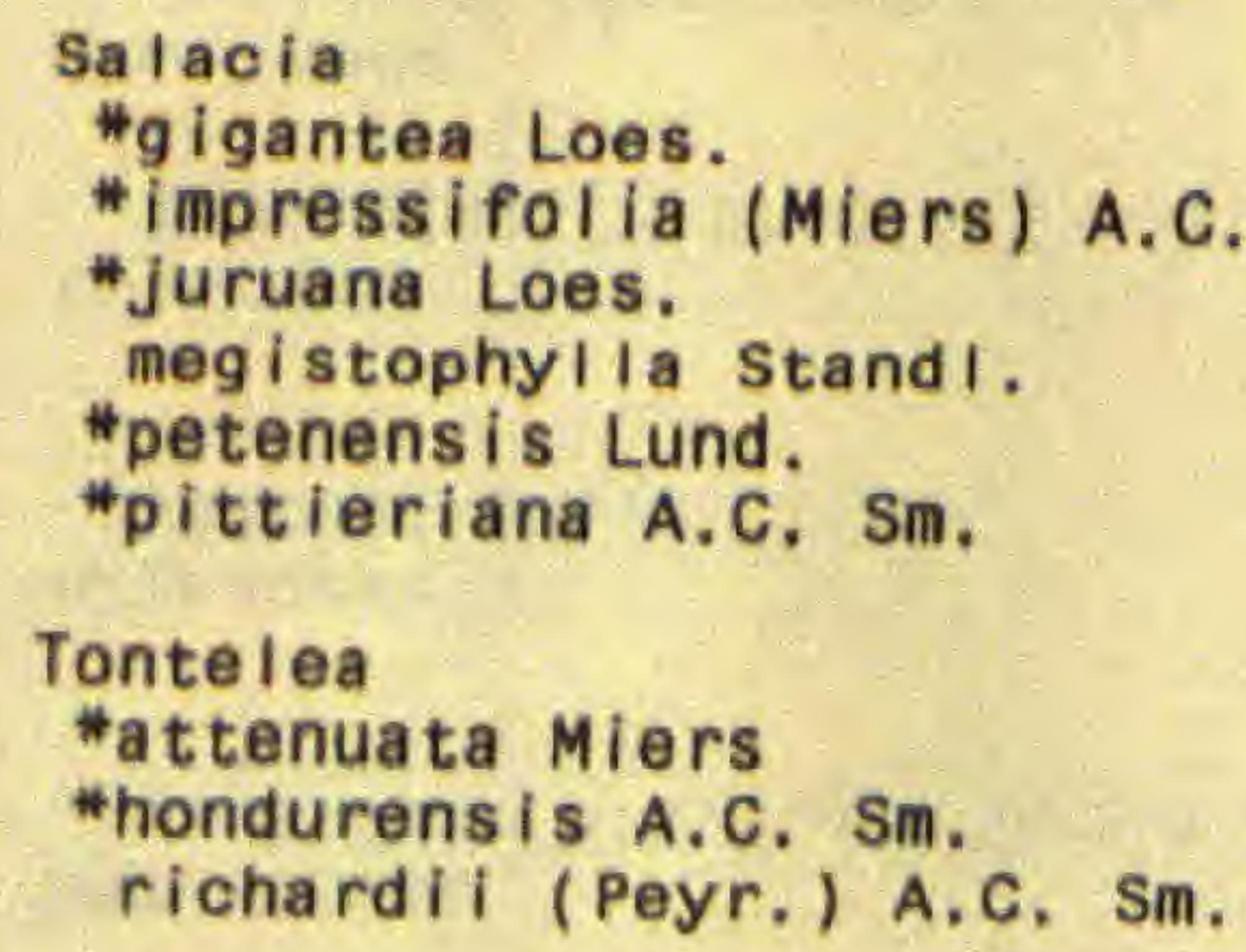

\section{$\begin{array}{ll}000 \mathrm{~m}: & 0-1 \\ 000 \mathrm{~m}: & 0-1,2-3 \\ 000 \mathrm{~m}: & 0-1\end{array}$ \\ 000m: $0-1$ \\ $000 \mathrm{~m}: 0=1,1-2$ \\ $00 \mathrm{~m}: 0-1,1-2$}

161 (105) STAPHYLEACEAE

\section{Turpinia}

occidentalis (SW.) G. Don

--subsp. breviflora Croat

--subsp. occidental is

PROV: ca ce da pa vr 000m: $0=1,1-2$ PROV: ch Vr

\begin{tabular}{|c|c|c|c|c|c|}
\hline RANGE: & per & & & & tree \\
\hline RANGE: & hon & $\operatorname{mex}$ & & & tree \\
\hline RANGE: & per & ven & & & tree \\
\hline RANGE: & ven & & & & climber \\
\hline RANGE: & cr & gua & & & tree \\
\hline RANGE: & $w r$ & & & & tree \\
\hline RANGE: & $\mathrm{col}$ & gui & sao & ven & tree \\
\hline $\begin{array}{l}\text { RANGE: } \\
\text { RANGE: }\end{array}$ & gua & hon & & & I imber \\
\hline TAIVUE: & gui & & & & climbe \\
\hline
\end{tabular}

\section{2 (106) ICACINACEAE}

\section{Calatola}

costaricensis Standl.

PROV: bo ce ch da pa 000m: $0-1,1-2$ sa $v r$

\section{Citronel la}

costaricensis (J.D. Sm.) Howard PROV: ch

000m: $1-2$

\section{Dendrobangla}

boliviana Rusby

\section{Discophora}

guianensis Miers

\section{Leretia}

cordata Vell.

\section{Mappia}

racemosa Jacq.
PROV: pa sa

000m: 0-1

PROV: bo ca da pa sa 000m: $0-1,1=2$

PROV: bo cn da sa

000m: 0-1

PROV: bo 000m: $0-1$
RANGE: ang cag col mex tree RANGE: ang cag his jam mex tree
RANGE: er

tree

RANGE: $c r$

tree

RANGE: boI gui

tree

RANGE: gui sao

tree shrub

climber tree

RANGE: cub jam pr shrub 
162 (106) ICACINACEAE cont.

Poraqueiba

guianensis AubI.

PROV: ch

000m: $1-2$

\section{4 (107) HIPPOCASTANACEAE}

\section{Billia}

columbianum Planch, \& Lind.

PROV: ch da $v r$ PROV: $\mathrm{cc}$ 000m: $1-2$ 000m: $0-1,1-2$

\section{5 (108) SAPINDACEAE}

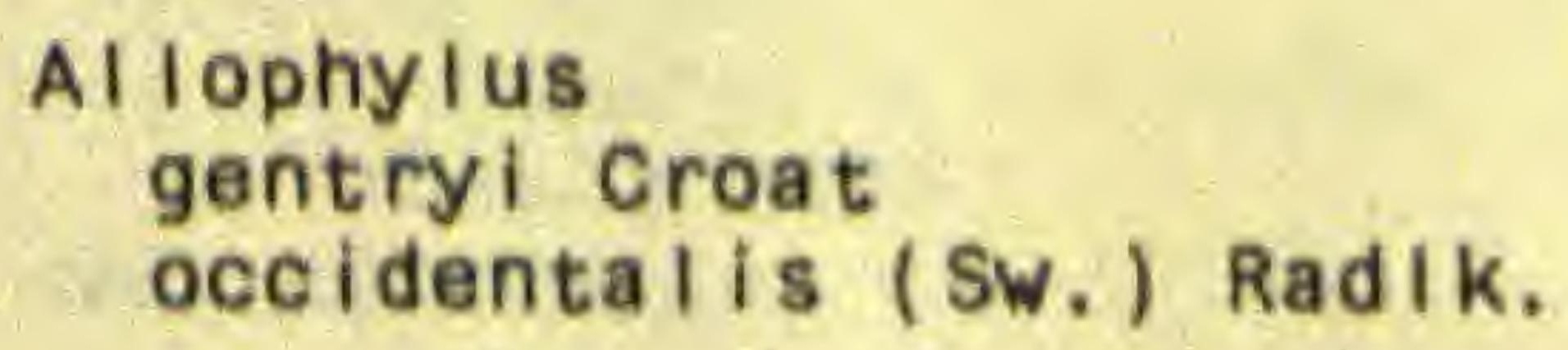

psilospermus Radik.

\section{BI ighia \\ sapida Koenig}

\section{Cardiospermum}

grandiflorum SW.

microca rpum H.B.K.

\section{Cupania}

cinerea $P$. \& $E$.

dukei croat

guatemalensis (Turcz.) Radik.

hirsuta Radik.

latifolia H.B.K.

livida (Radik.) Croat

rufescens $\mathrm{Tr}$. $\mathrm{PI}$.

scrobiculata Rich.

sylvatica Seem.

- -var. Posterí Croat

- -var. sylvatica
PROV: da pa pa $v r$

PROV: ca cc ch da lo $000 \mathrm{~m}: 0-1$ pa $v r$

PROV: bo ca

$000 \mathrm{~m}: 0-1$

PROV: bo ca ce cn pa 000m: 0-1 $\mathrm{Vr}$

PROV: ca he pa $\quad 000 \mathrm{~m}$ : $0-1$

PROV: bo ca cn da pa 000m: $0-1$

PROV: da sa 000m: $0-1$ PROV: $\mathrm{cC}$ ch da he vr 000m: $0-1$

PROV: bo

PROV: ca cn pa $\quad 000 \mathrm{~m}: 0-1$

PROV: cn 000m: $0-1$

PROV: bo ca ch da 10 000m: $0-1$ pa $v r$

PROV: ca ch on vr $000 \mathrm{~m}: 0-1$

PROV: ca pa vr

PROV: ca da pa 000m: $0-1$ 000m: $0-1$
RANGE: gui sao ven

tree

RANGE: $\mathrm{col} \mathrm{cr}$ ecu ven tree RANGE: cag tree

\begin{tabular}{|c|c|c|c|c|c|c|}
\hline $\begin{array}{c}\text { RANGE: } \\
\text { RANGE : } \\
\text { gui }\end{array}$ & $\begin{array}{l}\text { end } \\
\text { ang } \\
\text { his }\end{array}$ & bel & $\mathrm{cr}$ & cub & gua & $\begin{array}{l}\text { tree } \\
\text { shrub } \\
\text { tree }\end{array}$ \\
\hline RANGE: & ang & cag & mex & 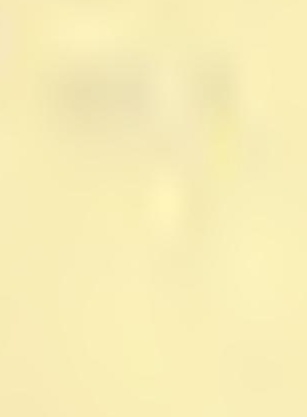 & 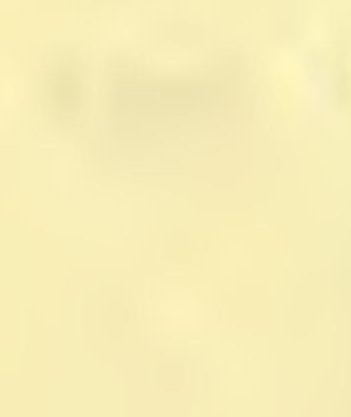 & tree \\
\hline RANGE: & a f $r$ & t) & & & & tree \\
\hline $\begin{array}{l}\text { RANGE: } \\
\text { SaO } \\
\text { RANGE: }\end{array}$ & $\begin{array}{l}\text { afr } \\
\text { ven } \\
\text { afr }\end{array}$ & $\begin{array}{l}\text { cag? } \\
\text { gui }\end{array}$ & $\begin{array}{l}\text { jam } \\
\text { ven }\end{array}$ & per & sag? & climber \\
\hline $\begin{array}{l}\text { RANGE: } \\
\text { ven }\end{array}$ & ang & bol & col & $\mathrm{cr}$ & per & tree \\
\hline $\begin{array}{l}\text { RANGE: } \\
\text { RANGE: }\end{array}$ & $\begin{array}{l}\text { end } \\
\text { cag }\end{array}$ & gua & mex & & & $\begin{array}{l}\text { tree } \\
\text { shrub } \\
\text { tree }\end{array}$ \\
\hline RANGE: & cr & gui & ven & & & tree \\
\hline $\begin{array}{l}\text { RANGE: } \\
\text { RANGE: }\end{array}$ & $\begin{array}{l}\mathrm{col} \\
\mathrm{cr}\end{array}$ & ecu & per & 520 & ven & $\begin{array}{l}\text { tree } \\
\text { tree }\end{array}$ \\
\hline $\begin{array}{c}\text { RANGE: } \\
\text { nic }\end{array}$ & $\begin{array}{l}\text { bel } \\
\text { sao }\end{array}$ & $\begin{array}{l}\text { cag } \\
\text { ven }\end{array}$ & col & gui & $\operatorname{mex}$ & tree \\
\hline RANGE: & bel & col & gui & sao & ven & $\begin{array}{c}\text { shrub } \\
\text { tree }\end{array}$ \\
\hline $\begin{array}{l}\text { RANGE: } \\
\text { RANGE: }\end{array}$ & $\begin{array}{l}\text { end } \\
\text { col }\end{array}$ & & & & & $\begin{array}{l}\text { tree } \\
\text { tree }\end{array}$ \\
\hline
\end{tabular}


TAXON

\section{ipterodend ron}

costaricense Radik.

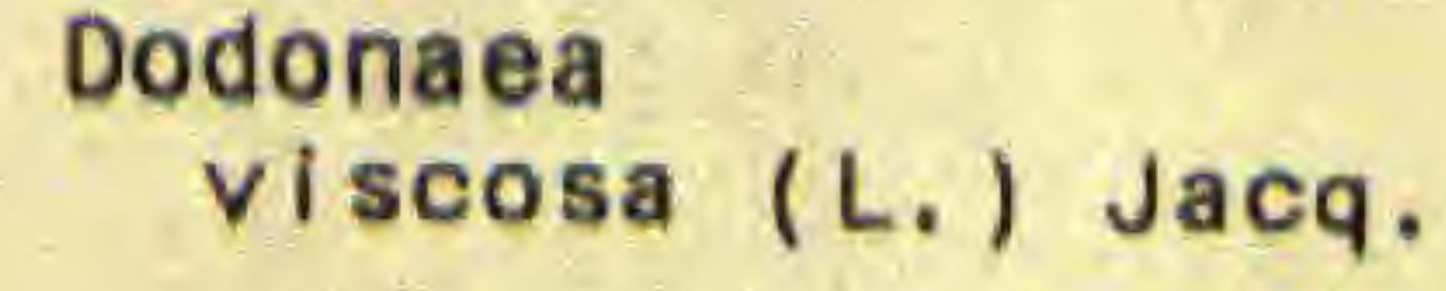

PROV: bo ch pa vr

$000 m: 0-1,1-2$

Filicium

decipiens (Wight \& Arn.) Thwaites cult.

PROV: pa

000m: $0-1$

\section{Ma tayba}

*apetala (Macfad.) RadIk. glaberrima Radik.

\section{ingaefolla standl.}

kennedyi croat

scrobiculata (H, B, K.) RadIk.

I icoccus

bijugatus Jacq. intr. cult.

\section{Paullinia \\ alata (R. \& P.) G, Don \\ allenii Standi. \\ baileyi Standl. \\ bracteosa RadIk. \\ brenesii croat \\ bristani i croat \\ buricana croat \\ correae croat \\ costaricensis Radik. \\ costata Schlecht, \& Cham.}

cururu L.

\section{dasystachya RadIk.}

dodge $i$ Standl.

dukei croat

eliasi i Croat

faginea ( $T r$. \& PI.) Radlk.

fasiculata Radik.

fibrigera Radik.

funicularis Radik.
PROV: ca pa 000m: $0-1$

PROV: ca

PROV: ca cc da lo pa 000m: $0-1,1-2$

PROV: bo

PROV: bo vr 000m: $0=1$

PROV: pa 000m: $0-1$

PROV: ca ch da lo pa $000 \mathrm{~m}: 0-1$

PROV: bo ch da pa 000m: 0-1 PROV: $\mathrm{CC}$

000m: $0-1$

PROV: bo ca cn pa 000m: 0-1

PROV: bo ca ch da lo $000 \mathrm{~m}: 0-1$

pa sa

PROV: $\mathrm{ch} \quad 000 \mathrm{~m}: 0-1,1-2$

PROV: da 000m: $0-1$

PROV: $\mathrm{ch} \quad 000 \mathrm{~m}: 0-1$

PROV: cn pa 000m: $0-1$

PROV: ca cc ch cn pa 000m: $0-1$

PROV: cc pa 000m: $0-1$

PROV: ca cc ch da he 000m: $0-1$

000m: $0-1$

PROV: da pa sa

PROV: da pa

000m: $0-1$

PROV: $c c$ cn da pa

PROV: ch pa Vr

PROV: bo cn pa

PROV: ca ch da pa

$000 \mathrm{~m}: 0-1$

000m: $0-1$

000m: $0-1,1-2$

000m: $0-1$

$000 \mathrm{~m}: 0-1$

000m: $0-1$
PROV: he
RANGE: $\mathrm{cr}$

\section{tree}

RANGE: $w d t$

shrub tree

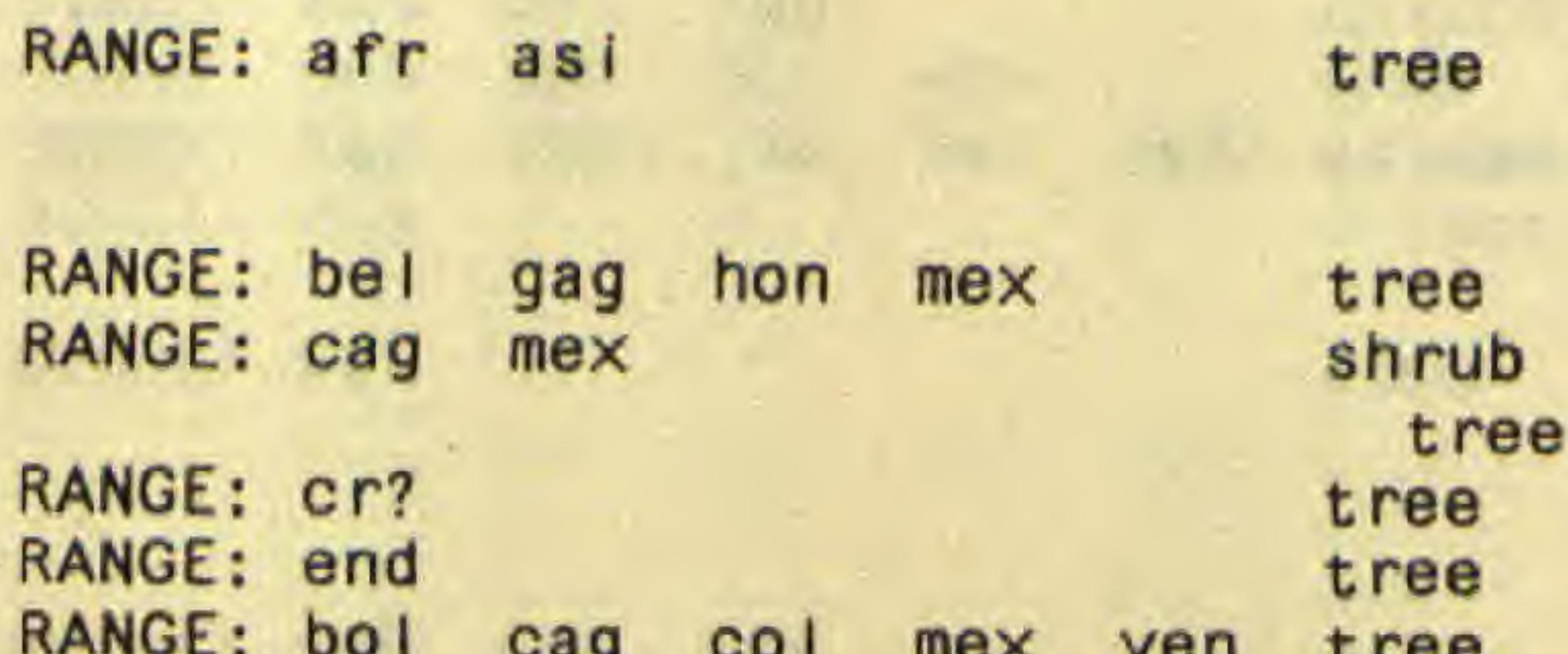

RANGE: bol cag col mex ven tree

RANGE: ang col cr gui his tree

lag nic trt

RANGE: bol col or per sag? climber

RANGE: end climber

RANGE: end tree

RANGE: bol cr per sao ven climber

RANGE: $\mathrm{cr}$ hon climber

RANGE: end climber

RANGE: end climber

RANGE: end climber

RANGE: cag or gua

RANGE: cag gua gui mex climber

RANGE: ang cag col mex sao climber

ven

RANGE: bol ecu per sao climber

RANGE: $c r \quad$ climber

RANGE: end climber

RANGE: end climber

RANGE: $\mathrm{col} \mathrm{cr}$ per climber

RANGE: $c r$ gui trt climber

RANGE: cag climber

RANGE: end 
fuscescens H.B.K.

- -var. fuscescens

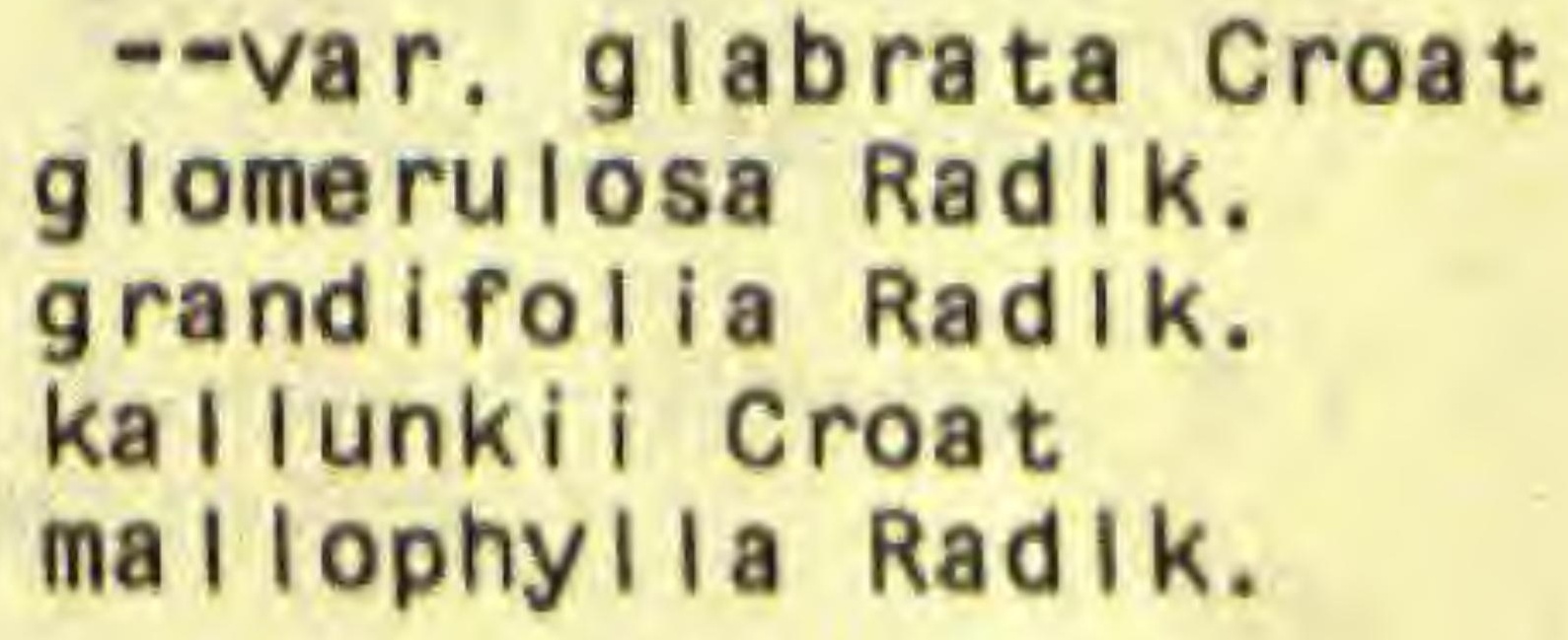

Pseudima

frutescens (AubI,) RadIk. "panamensis Croat

\section{Sapindus}

saponaria L.

Serjania

acuta $\mathrm{Tr} . \& \mathrm{PI}$.

alienii Croat

atrolineata Sauv, \& Wright

ci rcumvallata Radik.

cornigera Turcz.

darcyi Croat

decapleuria croat

grandis Seem.

insignis Radik.

mexicana (L,) Willd.
PROV: ca cc da he lo $000 \mathrm{~m}$ : $0-1$ pa $v r$

PROV: ca ch vr

PROV: bo ca da pa

000m: $0-1,1-2$

$000 \mathrm{~m}: 0-1$

PROV: cc da pa $v r$ 000m: $0-1,1-2$

PROV: da pa sa

000m: $0-1$

PROV: bo ca cc ch cn 000m: $0-1,1-2$ pa $\vee r$

PROV: $\vee r$

PROV: pa sa $\quad 000 \mathrm{~m}: 0-1$

PROV: CC 000m: $0-1$

PROV: bo ca da pa sa $000 \mathrm{~m}: 0-1$

PROV: bo ca cc ch cn $000 \mathrm{~m}$ : $0-1$ da pa

PROV: ca cn pa sa vr 000m: $0-1$ PROV: $c a c c$ ch cn da $000 \mathrm{~m}: 0-1$ pa sa $v r$

PROV: ch da pa sa 000m: $0-1$ PROV: da 000m: 0-1 PROV: cn pa $\quad 000 \mathrm{~m}: 0-1$ PROV: $c c$ da pa $000 \mathrm{~m}: 0-1$ PROV: ch $000 \mathrm{~m}: 0-1$ PROV: ca ch cn da pa $000 \mathrm{~m}: 0-1$ sa

000m: $0-1$

PROV: da sa

000m: $0-1$

PROV: da

000m: $0-1$ pa sa

PROV: $c h$ V

$000 m: 1-2$

PROV: ca cc pa 000m: $0-1$

PROV: $\mathrm{ca} \mathrm{CC}$ ch cn da 000m: $0=1$ he lo pa sa vr

PROV: bo $\mathrm{CB}$

PROV: ch 000m: $1-2$

PROV: ca 000m: $0-1$

PROV: ch lo vr 000m: 0-1

PROV: ca da pa 000m: 0-1

PROV: bo ca ce ch cn 000m: $0=1$ da he lo pa vr

\begin{tabular}{|c|c|c|c|c|c|c|}
\hline RANGE: & ang & cag & $\operatorname{mex}$ & sag? & sao & climber \\
\hline RANGE: & cag & $\operatorname{mex}$ & & & & climber \\
\hline RANGE: & ang & mex & ven & & & climber \\
\hline RANGE: & $\mathrm{cr}$ & gui & per & sao & & climber \\
\hline RANGE: & end & & & & & cl imber \\
\hline & & & & & & climber \\
\hline RANGE: & end & & & & & cl imber \\
\hline RANGE: & end & & & & & climber \\
\hline RANGE: & end & & & & & climber \\
\hline RANGE: & af $r$ & bol & cag? & gui & sag? & climber \\
\hline RANGE: & $\mathrm{col}$ & $c r$ & ecu & per & trt & climber \\
\hline RANGE: & $\mathrm{col}$ & $\mathrm{cr}$ & per & sao & & climber \\
\hline RANGE: & $\mathrm{col}$ & $\mathrm{cr}$ & per & & & climber \\
\hline RANGE: & end & - & & & & cl imber \\
\hline RANGE: & end & & & & & cl imber \\
\hline RANGE: & $\mathrm{col}$ & & & & & climber \\
\hline RANGE: & sao & & & & & climber \\
\hline RANGE: & per & sao & ven & & & climber \\
\hline RANGE: & $\mathrm{col}$ & gua & mex & & & climber \\
\hline RANGE: & gui & sao & & & & shrub \\
\hline RANGE: & $w r$ & & & & & shrub \\
\hline RANGE: & ang & cag? & fla & his & mex & tree \\
\hline sag? & sao & & & & & \\
\hline RANGE: & cag & mex & ven & & & climber \\
\hline RANGE: & end & & & & & climber \\
\hline $\begin{array}{l}\text { RANGE: } \\
\text { ven }\end{array}$ & ang & cag & $\mathrm{col}$ & cub & mex & climber \\
\hline RANGE: & col & $\mathrm{cr}$ & & & & climber \\
\hline RANGE: & cag? & hon & & & & climber \\
\hline RANGE: & end & & & 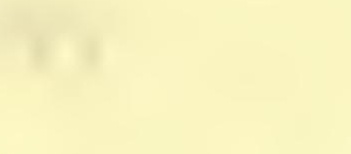 & & climber \\
\hline RANGE: & col & $\mathrm{cr}$ & & & & climber \\
\hline $\begin{array}{l}\text { RANGE: } \\
\text { RANGE: }\end{array}$ & $\begin{array}{l}\text { cag? } \\
\text { col? }\end{array}$ & hon & per & sag? & & climber \\
\hline RANGE: & cag & col & jam & mex & ven & cl imber \\
\hline
\end{tabular}


TAXON

FAMILY PROVINCES

ELEVATION (OOOM.)

RANGE OUTSIDE OF PANAMA

HABIT

165 (108) SAPINDACEAE cont.

paniculata H.B.K.

paucidentata $D C$.

pluvialiflorens Croat

rhombea Radik.

trachygona Radik.

\section{Talisia \\ allenif Croat}

dwyeri Croat

hexaphyI Ia VahI

nervosa RadIk.

princeps oliv.

\section{Thinouia}

myriantha Tr. \& PI.

Urvillea

ulmacea H.B.K.
PROV: ch

PROV: ca

000m: $0-1$ 000 m: $0=1$

PROV: bo ca

000m: $0-1$

PROV: ca cc on da lo 000m: $0-1$ pa 58

PROV: ca cn pa 000m: $0-1$

PROV: da

000m: $0-1$

PROV: pa

000m: $0-1$

PROV: ch da

000m: $0-1$

PROV: bo ca cn da pa 000m: $0-1$ vr

PROV: bo ca da pa 000m: $0-1$

PROV: ca da

000m: $0-1$

PROV: ca ch pa

$000 \mathrm{~m}: 0-1$

166 (109) SABIACEAE

\section{Lorenzanea}

* i razuens is StandI.

PROV: ch

000m: $3+$

Mel iosma

allenii StandI, \& L.0. Wms.

brenesil StandI.

cordata A. Gentry

frondosa Cuatr, \& Idrobo

glabrata (Liebm.) Urb.

grandiflora A. Gentry

idiopoda Blake

I inearifolia A. Gentry occidentalis Cuatr.

schlimi i (Turcz.) Triana

vernicosa (Liebm.) Griseb.

PROV: bo ca cn da pa 000m: $0-1$ sa $v r$

PROV: $\mathrm{cc} \mathrm{ch}$

PROV: $v r$

PROV: $v r$

PROV: bo $\mathrm{CC}$

PROV: $\mathrm{CC}$

PROV: ch

000m: $0-1$

000m: $0-1$

000m: $1-2$

000m: $0-1$

000m: $0-1$

000m: $1-2,2-3$

PROV: cn pa PROV: $\mathrm{cc}$ ch da

PROV: cc cn pa

000m: $0-1$

000m: $0-1,1-2$

000m: $0-1,1-2$

PROV: pa $v r$ 000m: $0-1$

\begin{tabular}{|c|c|c|c|c|c|c|}
\hline RANGE: & cag & col & $\operatorname{mex}$ & ven & & elimber \\
\hline $\begin{array}{c}\text { RANGE: } \\
\text { trt }\end{array}$ & cag & gui & $\operatorname{mex}$ & per & sao & olimber \\
\hline RANGE: & end & & & & & clinber \\
\hline $\begin{array}{l}\text { RANGE: } \\
\text { ven }\end{array}$ & cag & col & er & ecu & mex & climber \\
\hline RANGE: & bol & per & & & & climber \\
\hline RANGE: & $\mathrm{cr}$ & & 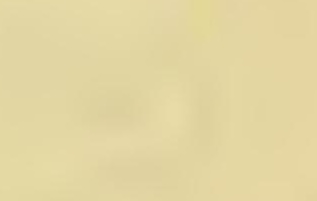 & & & shrub \\
\hline RANGE: & end & & & & & shrub \\
\hline RANGE: & bol & sao & & & & $\begin{array}{l}\text { tree } \\
\text { shrub }\end{array}$ \\
\hline RANGE: & $\mathrm{col}$ & $\mathrm{cr}$ & trint & & & $\begin{array}{l}\text { shrub } \\
\text { tree }\end{array}$ \\
\hline RANGE: & ven & & 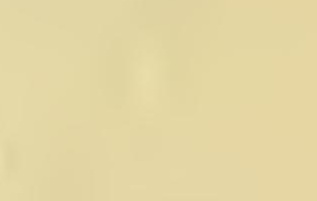 & & & tree \\
\hline RANGE: & bel & col & gui & per & ven & el imber \\
\hline RANGE: & cag? & sag? & sao & $x$ & ven & clin \\
\hline
\end{tabular}

RANGE: $\mathrm{cr}$

tree

RANGE: cag col gua mex

shrub

RANGE: $c r$

RANGE: end

RANGE: $c O I$

RANGE: $\mathrm{COI}$

RANGE: $\mathrm{cr}$

cr ecu?

cr gua mex

tree

tree

tree

tree

tree

RANGE: end

RANGE: $\mathrm{CO}$ I ecu

shrub

RANGE: $\mathrm{COl} \mathrm{cr}$

tree

tree

tree 


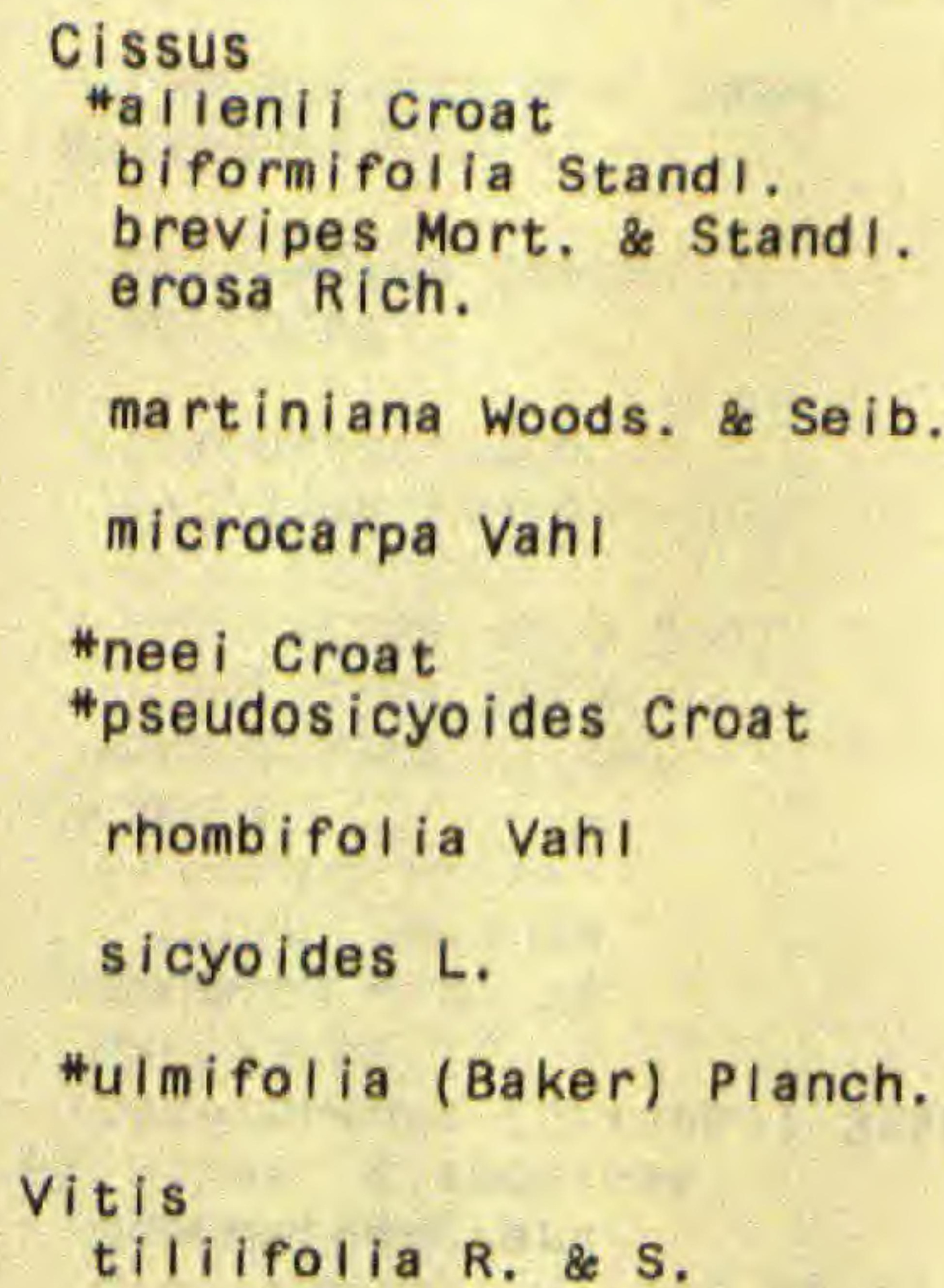

171 (113) ELAEOCARPACEAE

\section{Munting ia}

ca labura L.

\section{Sloanea}

medusula Schum. \& Pitt.

megaphylla pitt.

terniflora (DC.) StandI.

zuliaensis Pitt.
PROV: ca cc ch cn da 000m: $0-1$ he $10 \mathrm{pa}$

PROV: $\mathrm{cc} \mathrm{ch} \quad$ 000m: $0-1,1-2$

PROV: $c$ Cn $000 \mathrm{~m}: 0-1$

PROV: ca cc ch he lo 000m: 0-1 pa $\vee r$

PROV: ca cn da

\begin{tabular}{|c|c|c|c|c|c|c|}
\hline $\begin{array}{l}\text { RANGE: } \\
\text { RANGE: } \\
\text { RANGE: } \\
\text { RANGE: }\end{array}$ & $\begin{array}{l}\text { cr } \\
\text { bel } \\
\text { cr } \\
\text { ang }\end{array}$ & cag? & $\mathrm{col}$ & sag & & $\begin{array}{l}\text { cl imber } \\
\text { cl imber } \\
\text { cl imber } \\
\text { cl imber }\end{array}$ \\
\hline RANGE: & cag? & gua & $\operatorname{mex}$ & sag? & & $\begin{array}{r}\text { cl imber } \\
\text { shrub }\end{array}$ \\
\hline RANGE: & ang & cag & $\operatorname{mex}$ & sag & sao & climber \\
\hline $\begin{array}{l}\text { RANGE: } \\
\text { RANGE: }\end{array}$ & $\begin{array}{l}\text { end } \\
\text { col }\end{array}$ & cr & & - & & $\begin{array}{l}\text { climber } \\
\text { herb }\end{array}$ \\
\hline RANGE & ang & cag & $\operatorname{mex}$ & sag & & climber \\
\hline RANGE & ang & cag & nas & sag? & & climber \\
\hline RANGE & ecu & per & ${ }^{2}$ & & & climber \\
\hline ANG & & cag & $\operatorname{mex}$ & sag? & & climber \\
\hline
\end{tabular}

RANGE: nWt

tree

$\begin{array}{llr}\text { RANGE: cag col gua } & \text { tree } \\ \text { RANGE: col? cr? nic? } & \text { tree } \\ \text { RANGE: bol cag mex per sag? tree } \\ \text { Sao } \\ \text { RANGE: cr ven } & & \text { tree }\end{array}$

174 (114) TILIACEAE

\section{Ape iba}

aspera AubI.

tibourbou Aubl.
PROV: bo ca cc cn da 000m: $0-1$ pa sa

PROV: ca cc ch en da 000m: $0-1$ he he pa sa $v r$
RANGE: bol col cr sag? tree

RANGE: cag sag 
PROV: ch

000m: $0-1$

PROV: ca he pa

000m: $0-1$

PROV: $\mathrm{ca}$ cc lo pa

000m: $0-1$

PROV: bo ca cc ch cn 000m: $0-1$ da pa

PROV: bo

000m: $0-1$

PROV: ch

000m: $0-1$

PROV: ca cc ch cn da 000M: $0-1,1-2,3-2$

PROV: cc lo pa

000m: $0-1$

PROV: bo ca cn da he $000 \mathrm{~m}: 0-1$

pa sa $\vee r$

PROV: ca cc cn da he $000 \mathrm{~m}$ : $0-1$ lo pa sa vr

teyerm.

PROV: bo

PROV: cn

000m: 0-1

PROV: bo

000m: $0-1$

000m: $0-1$

PROV: ca cc ch da pa 000m: $0-1,1-2$ sa $\mathrm{vr}$

PROV: $\mathrm{CC}$

000m: $0-1$

PROV: ca ch cn lo pa 000m: $0-1,1-2$ PROV: $\mathrm{CC}$ 000m: $0-1$

PROV: bo ca cc ch cn 000m: 0-1 da he lo pa sa vr

PROV: ch
RANGE: af $r$

treelet

RANGE: nWt

RANGE: ang cag fla nao sag herb

teX

RANGE: cag? fla nwt sag? shrub

RANGE: $\mathrm{cr}$

shrub

tree

RANGE: $\mathrm{col} \mathrm{cr}$

tree

RANGE: cag mex sag sao

tree

RANGE: cag col mex ven

RANGE: $\mathrm{cag}$ col $\mathrm{cr}$

tree

tree

RANGE: cag col cub sao

tree

RANGE: $\mathrm{cr}$

RANGE: $c r$

tree

RANGE: end

shrub

treelet

shrub

RANGE: cag col ecu mex

tree

RANGE: $\mathrm{cr}$

shrub

RANGE: nWt

RANGE: NWt

tree

shrub

shrub

RANGE: nWt

shrub

RANGE: cag mex

shrub 
TAXON

175 (115) MALVACEAE

\section{Abel Imoschus}

esculentus (L.) Moench intr. cult.

PROV: ca pa 000m: 0-1

*manihot (L.) Medik.

intr. cult. nat.

PROV: Vr 000m: $0-1$

moschatus Medik.

intr, cult.
PROV: bo ca ch en da 000m: 0-1 he pa

RANGE: asi wdt
RANGE: asi wdt $\begin{gathered}\begin{array}{c}\text { woody } \\ \text { herb } \\ \text { herb }\end{array} \\ \text { RANGE: asi wdt }\end{gathered}$

Abutilion

cymosum $\mathrm{Tr}$. \& $\mathrm{PI}$.

"divaricatum Turcz.

giganteum (Jacq.) Sweet
PROV: ch
PROV: ch
000m: $0-1$
PROV: pa
000m: $0-1,1-2$

*hi rtum (Lam.) Sweet

PROV: $\mathrm{ch} \mathrm{Ba}$

000m: $1-2$

purpusii Stand I.

theophrast i Medik.

PROV: ch

000m: $0-1,1-2$

intr. PROV: pa

000m: $0-1$

*Al thea

* rosea L.

Anoda

acerifolia (Roem.) DC.

PROV: WP

000m: we

PROV: ca

000m: $0-1$

*Briquetia

"spicata (H.B.K.) Fryx.

PROV: ca pa

$000 \mathrm{~m}: 0-1$

Gossypium

barbadense $L$.

cult. PROV: ca cc he pa sa $000 m: 0-1$

\#hirsutum L.

cult. PROV: $\mathrm{cc} \mathrm{ch} \mathrm{cn}$ da 000m: 0-1

\footnotetext{
Hampea

*a Ibipetala Cuatr.

appendiculata (J.D. Sm.) StandI. --var. appendiculata

"--var. longicalyx Fryx.

* dukei A. Robyns

*micrantha A. Robyns
}

PROV: $\mathrm{CC}$

000m: $0-1,1-2$

PROV: bo ca ce ch da 000m: $0-1,1-2,2-3$

PROV: ca cc da pa 000m: $0-1$

PROV: sa

$000 \mathrm{~m}: 0-1$

PROV: cn pa pa sa $000 \mathrm{~m}: 0-1$ 
175 (115) MALVACEAE cont.

*platanifolia StandI. * punctulata Cuatr.

PROV: ch

000m: $0-1$

*Herissantia

*crispa (L.) Brizicky

PROV: ca he pa

000m: $0-1$

Hibiscus

bifurcatus Cav,

PROV: ca cn pa

000m: $0-1$

costatus A. Rich.

furcellatus Desr.

"pernambucensis Arruda

phoeniceus Jacq.

radiatus Cav.

rosa-sinensis $L$.

sabdariffa L.

schizopetalus (Mast.) Hook. $f$

intr, cult.

intr. cult.

PROV: $\mathrm{cc}$

000m: $0-1$

PROV: ca

000m: $0-1$

PROV: bo ca ch cn da 000m: $0-1$ pa sa vr

000m: $0-1$

000m: 0-1$$
\text { t. nat. }
$$$$
\text { PROV: ca }
$$

intr. cult.

PROV: bo ca pa vr $000 \mathrm{~m}: 0-1$ PROV: bo ca da pa $000 \mathrm{~m}$ : $0^{-1}$

sororius L.

PROV: ca vr

000m: $0-1$

spathulatus Garcke

ates

PROV: he pa

000m: $0-1$

kosteletzkya

*depressa (L.) Blanchard, Fryx. \& Bate

Lopimia

dasypetala (Turcz.) StandI.

PROV: ca pa

000m: $0-1$

malacophylla (Link \& otto) Mart. PROV: ca pa

000m: $0-1$

Ma I achra

alceifolia Jacq.

PROV: bo ca da he pa 000m: $0-1$

fasciata Jacq.

PROV: ca pa

000m: $0-1$

radiata (L.) L.
PROV: pa
RANGE: $\mathrm{cr}$ nIC

RANGE; $C O$

treelet

shrub

treelet

RANGE: bol cag mex nas per herb

sao sat shrub

RANGE: ang cag gui sao her

RANGE: cag cub mex shrub

woody

RANGE: ang cag fla mex sag? woody

sao herb

RANGE: nWt per shrub

RANGE: ang cag col mex ven woody

herb

RANGE: asi wdt herb

RANGE: WdW shrub

RANGE: $a f r$ wdt herb

RANGE: afr per wdt shrub

RANGE: bol cag cub mex sag woody

Sao
RANGE: gui sao herb

RANGE: ang cag ecu mex ven herb

shrub

RANGE: col or nic sao ven tree

RANGE: bol mex nic shrub

sao sao 0 tree

RANGE: ang cag mex per sao woody

woody
herb

RANGE: afr ang cag mex sag woody 
175 (115) MALVACEAE cont.

Ma I va

neglecta WalIr.

\section{Ma Ivastrum}

americanum (L.) Torr.

coromandel ianum (L.) Garcke

\section{Malvaviscus}

a rboreus Cav.

--var. lobatus A. Robyns

--var. mexicanus Schlecht.

cult. PROV: bo ca ce ch da 000m: $0-1,1-2$ $10 \mathrm{pa} v \mathrm{r}$

PROV: ce ch da $v r$ 000m: $0-1$

*long ifolius Garcke

Neobrittonia

acerifolia (G. Don) Hochr.

\section{Pavonia}

a I ba Seem.

PROV: ch

000m: $1-2$

PROV: ca pa

000m: $0-1$

fruticosa (Mill.) Fawc. \& Rendl. PROV: ca ch da pa sa 000m: 0-1,1-2

long ipes Stand 1 .

- -var. hirsuta A. Robyns

- -var. longipes

PROV: da

PROV: bo

000m: $0-1$

PROV: $c h$

PROV: bo $\mathrm{CC}$

000m: $0-1$

maxonii (Standl.) Standl. oxyphyl laria J.D. Sm.

paniculata Cav.

pendul iflora (Stand I.) StandI. rhizophorae Killip

*schiedeana Steud.

sidifolia H.B.K.

spicata Cav,

spinifex (L.) Cav.
PROV: bo ca cc ch da 000m: 0-1 pa

PROV: ch

m: $1-2,2-3$

PROV: da sa

000m: $0-1$

PROV: bo ca cc ch da 000m: $0-1$ pa

PROV: 10

000m: $0-1$

PROV: bo ca cn

PROV: WP 000m: $0-1$
RANGE: $W d W$

herb

RANGE: $w d t$

shrub

woody

herb

RANGE: WdW

$$
\text { herb }
$$

RANGE: end

shrub

tree

RANGE: cag mex per sao shrub

RANGE: col ecu per sao ven shrub

RANGE: cag mex

herb

shrub

RANGE: COI ven

woody

RANGE: ang per sao

herb

shrub

RANGE: $\mathrm{cr}$

herb

RANGE: $c r$

woody

RANGE: $c r$

RANGE; col or per sao

herb

herb

herb

RANGE: ang cag mex sag sao wh

woody

RANGE: $c r$

herb

RANGE: $\mathrm{CO}$

shrub

RANGE: ang cag col mex sao

shrub

herb

RANGE: mex per sao ven

shrub

RANGE: ang cag fla sao

woody

he rb

RANGE: ang per sag? sao 


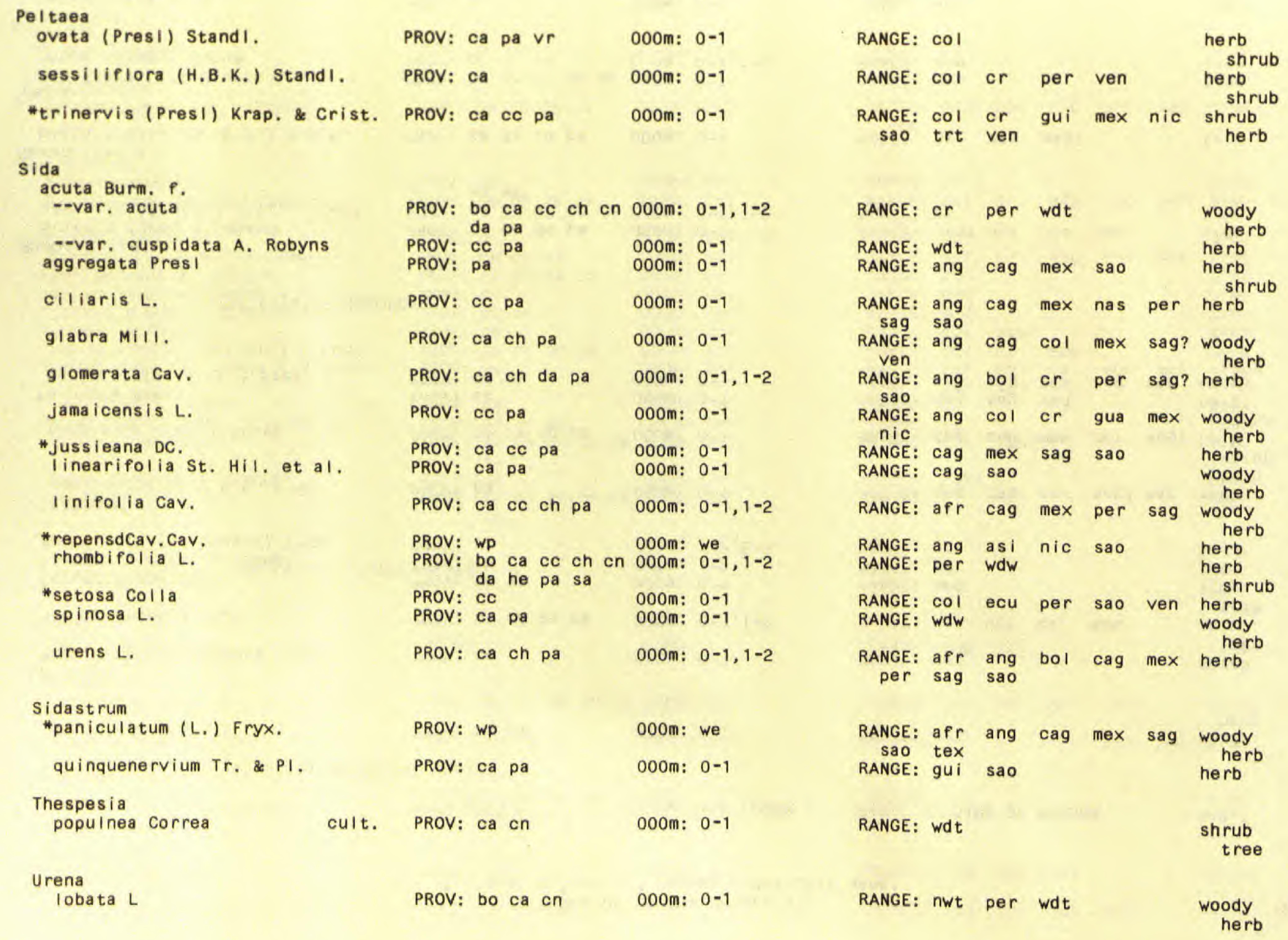


TAXON

\section{FAMILY}

PROVINCES

ELEVATION (OOOM.)

RANGE OUTSIDE OF PANAMA

HABIT

175 (115) MALVACEAE cont.

sinuata L.

\section{Wercklea}

"cocleana (A. Robyns) Fryx.

\#ferox (Hook.) Fryx.

"grandifiora Fryx.

*insignis Pitt. \& Standl.

"woodsoni i (A. Robyns) Fryx.

\section{Wissadula}

amplissima (L.) R.E. Fries

excelsior (Cav.) PresI

*hirsuta PresI

periplocifolia (L.) Presl
PROV: ca pa

000m: $0-1$

PROV: $C C$

PROV: ca cn da pa

PROV: CC Vr

PROV: ch

PROV: ch

PROV: pa

PROV: bo ca cc pa

PROV: $\mathrm{CC}$

PROV: ca pa 000m: $0-1$

000m: $0-1,1-2$

000m: 0-1

000m: $1-2$

000m: $1-2,2-3$

000m: $0-1$

000m: $0-1$

000m: $0-1$

000m: 0-1

\section{7 (116) BOMBACACEAE}

Bombacops is

quinata (Jacq.) Dugand sessilis (Benth.) Pitt.

\section{Cavanillesia}

platanifolia (H. B.) H.B.K. PROV: ca da lo pa 000m:0-1

Ceiba

pentandra (L.) Gaertn.

rosea (Seem.) Schum.

\section{Gyranthera}

darienensis Pitt.

Ochroma

pyramidale (Lam.) Urb. cult, PROV: bo ca ch on da 000m: 0-1

$$
\text { lo pa vr }
$$

\section{Pachi ra}

aquatica AubI.
PROV: ca ce da pa 000m: $0-1$

PROV: ca ce ch cn pa 000m: $0-1,1-2$ sa $v r$

PROV: bo ca ch da pa 000m: $0-1$ PROV: cC da Vr 000m: $0-1,1-2$

PROV: sa

000m: $0-1$
RANGE: nWt

woody herb

RANGE: end

tree

RANGE: bol col cr ecu shrub

RANGE: end tree

tree

RANGE: or

tree

$\begin{aligned} & \text { RANGE: ang cag mex sag? sao } \begin{array}{c}\text { woody } \\ \text { herb } \\ \text { tex }\end{array} \\ & \text { shrub }\end{aligned}$
$\begin{gathered}\text { RANGE: cag cub mex per sag? herb } \\ \text { shrub } \\ \text { sao }\end{gathered}$
$\begin{gathered}\text { RANGE: cag sag ven } \\ \text { RANGE: ang cag mex sag sao woody }\end{gathered}$

RANGE: cag? col nic ven

RANGE: $c r$

tree

RANGE: nic per sag?

tree

RANGE: WdW

RANGE: end

tree

RANGE: end

tree

RANGE: $\mathrm{cr}$ wdt

tree 


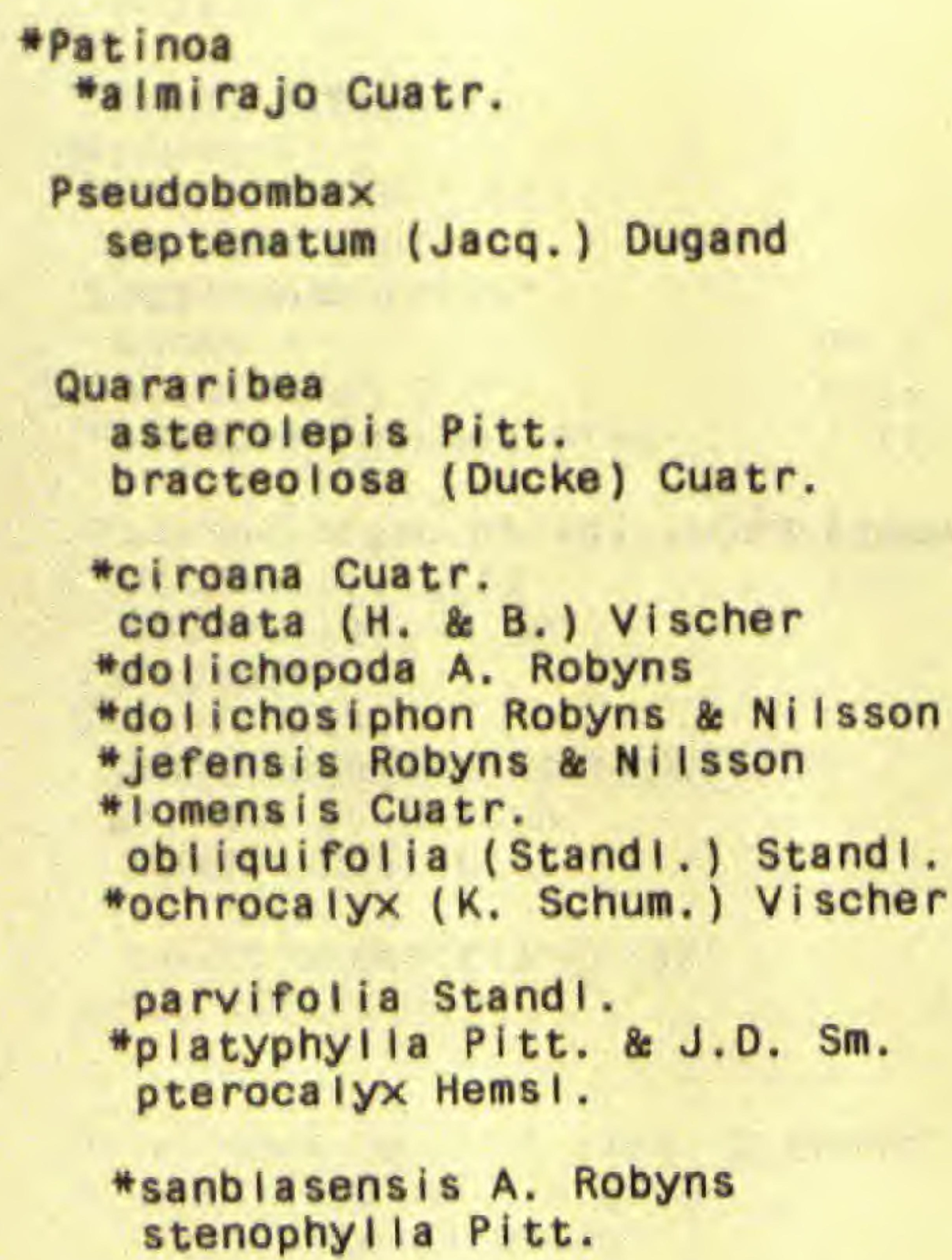

PROV: cc pa

000m: $0-1$

PROV: ca cn da pa

000m: $0-1$

PROV: da

PROV: CC

PROV: $c c$ cn da

PROV: cc cn da pa

PROV: da

PROV: $c C$ Pa

PROV: $C C$

PROV: bo $000 \mathrm{~m}: 0=1$

$\begin{array}{ll}\text { PROV: bo } & 000 \mathrm{~m}: 0-1 \\ \text { PROV: bo cn sa } & 000 \mathrm{~m}: 0-1\end{array}$

PROV: bo ca cn da pa $000 \mathrm{~m}$ : $0-1$

sa

000m: $0-1$

PROV: bo ca ce cn da $000 \mathrm{~m}$; $0-1$ pa
PROV: WP

RANGE: $c O l$

tree

RANGE: cag? col nic per sao tree ven

RANGE: $\mathrm{cr}$

SaO

RANGE: $w r$

RANGE: $\mathrm{col}$ or ecu per sao tree

tree

RANGE: end tree

$\begin{array}{ll}\text { RANGE: end } & \text { tree } \\ \text { RANGE: col ecu? } & \text { tree }\end{array}$

RANGE: $\mathrm{COl} \mathrm{cr}$ ecu tree

RANGE; bol col cr ecu gui tree

per sao ven cr tre

RANGE: $\mathrm{cr}$

RANGE: $c r$

RANGE: col ven?

tree

RANGE: col? tree

RANGE: $\mathrm{cr}$ sao tree

178 (117) STERCULIACEAE

\section{*Ayena}

*micrantha StandI.

PROV: $\mathrm{CC}$

000m: $0-1$

Byttneria

aculeata Jacq.

*cata lpaefolia Jacq.

PROV: bo ca ce da pa 000m: $0-1$

$v r$

$000 \mathrm{~m}: 0-1$

Guazuma

ulmifolia Lam.

cult. PROV: bo ca cc ch cn 000m: 0-1 da he lo pa sa

Helicteres

baruens is Jacq.

guazumaefolia H.B.K.
PROV: $c c$

000m: $0-1$

PROV: ca en pa vr

000m: $0-1$

\footnotetext{
RANGE: cr
}

$\begin{array}{llll}\text { RANGE: cag mex } & & \text { treelet } \\ \text { RANGE: bol cag mex sag? } & \text { shrub } \\ \text { RANGE: bol cr ecu per sao } & \text { tree } \\ \text { RANGE: nwt } & & & \text { tree } \\ & & & \\ \text { RANGE: cag sag? trt } & \text { shrub } \\ \text { tree } \\ \text { RANGE: ang cag mex sag? }\end{array}$


TAXON

\section{Herrania}

purpurea (Pitt.) R.E. Schult. PROV: bo ca ch cn da 000m: $0-1$ sa

\section{Melochia}

corchorifolia L.

intr.

PROV: pa

000m: $0-1$

I upulina Sw.

manducata Wright \& Sauvalle

melissifolia Benth.

nodiflora SW.

villosa (Mili.) Fawc. \&endl.

\section{Sterculia}

apetala (Jacq.) Karst.

costaricana Pitt.

*glauca A. Gentry

* guapayensis Cuatr.

\section{Theobroma}

angustifolium DC.

bernoullii pitt.

--subsp. a sclepiadiflorum (Schery)

--subsp, bernoulli

bicolor H. \& B.

cacao L.

*chocoense Cuatr.

hylaeum Cuatr.

$\begin{array}{lll} & \text { PROV: bo } & 000 \mathrm{~m}: 0-1 \\ & \text { PROV: cn sa } & 000 \mathrm{~m}: 0-1 \\ \text { cult. } & \text { PROV: bo ca da } & 000 \mathrm{~m}: 0-1 \\ \text { cult. } & \text { PROV: bo ca da he pa } 000 \mathrm{~m}: 0-1 \\ & \text { PROV: pa } & 000 \mathrm{~m}: 0-1 \\ & \text { PROV: cn } & 000 \mathrm{~m}: 0-1\end{array}$

Wa I theria

glomerata Pres।

ind ica $L$.
PROV: bo ca ce ch cn 000m: $0-1$ da pa

PROV: pa

000m: $0-1$

PROV: ca cc cn pa

PROV: ca pa

000m: $0-1$

$000 \mathrm{~m}: 0-1$

000m: $0-1$
PROV: ca cc ch da pa 000m: $0-1$

000m: $0-1$

PROV: cn pa 000m: $0-1$

PROV: cn 000m: $0-1$

PROV: ch

000m: $0-1$

PROV: ca cc ch da he 000m: $0-1$ lo pa $v r$

PROV: ca cc he pa vr 000m: $0-1$ sa
RANGE: $\mathrm{cr}$

shrub

tree

RANGE: nag? owg

herb

RANGE: ang er mex per sabshrub

erb

RANGE: col? cub

subshrub

shrub

herb

subshrub

RANGE: afr or sag?

RANGE: ang cag herb

RANGE: afr cag? sag? herb

subshrub

RANGE: ang cag mex per sag? tree

RANGE: $c r$

RANGE: $w r$

RANGE: $w r$

tree

tree

treelet

RANGE: cag

tree

RANGE: $W r$

RANGE: $w r$

RANGE: cag

RANGE: $w d t$

RANGE: $\mathrm{COI}$

RANGE: $\mathrm{COI}$

RANGE: $\mathrm{cr}$ mex

shrub

RANGE: $w d w$ 
TAXON

Davilla

kunthii St, Hil.

"nitida (Vahl) Kub.

*rugosa Poir.

\#Dillenia

*excelsa (Jaca.) Gilg intr. cult.

"suffruticosa (Griff.) Martell

$$
\text { intr. cult. }
$$

Dol ioca rpus

dentatus (AubI.) StandI.

major Gmel.

multiflorus Standi.

olivaceus StandI.

\section{"Pinzoana}

* coriacea Mart. \&e Zuco.

Saurauia

laevigata $\mathrm{Tr}$. $\mathrm{PI}$.

"pseudoscabrida Buscal.

rubiformis Vatke

seibertii Standi.

veraguasensis seem.

\section{Tetracera}

hydrophila Tr.\& PI.

portobellens is Beurl,

volubilis L.

willdenowiana steud.
PROV: ca $000 \mathrm{~m}: 0=1$

PROV: bo ca ce ch cn 000m: 0-1 da he pa $v r$

PROV: ca cc ch cn pa $000 \mathrm{~m}$ : $0-1$

000m: $0-1$

PROV: pa

000m: $0-1$

PROV: ca cc da pa 000m: $0-1$

PROV: bo ca cn da pa 000m: 0-1$$
\text { prov: sa }
$$

PROV: ca cn

PROV: ca pa

000m: $0-1$

000m: $0-1$

PROV: cn

000m: $0-1$

PROV: ca $\mathrm{CC}$ pa

PROV: ch

PROV: $\mathrm{ch}$

000m: $0-1$

000m: $1-2$

000m: $2-3$

PROV: bo $\mathrm{ch}$

000m: $1-2,2-3$

PROV: $\mathrm{ch}$

000 m: $1-2,2-3$

PROV: bo ca pa 000m: $0-1$

PROV: bo ca cn da 000m: 0-1

PROV: ca ec da pa 000m: $0-1$ PROV: bo ca pa

RANGE: ang cag mex sag? sao climber
tree
RANGE: ang cag mex per sag? climber
trao
RANGE: sao ven

RANGE: as $\mathrm{i}$

tree

RANGE: as $\mathrm{i}$

tree

RANGE: ang bel bol cag sag? climber
Sao
RANGE: per sag? sao $\begin{array}{r}\text { climber } \\ \text { RANGE: bel cag? cub gui sag? climber } \\ \text { RANGE: end } \\ \text { shrub } \\ \text { climber }\end{array}$

RANGE: bel or per sao ven climber

RANGE: cag col mex tree

RANGE: $w r$ gua tree

tree

RANGE: end tree

RANCE: or hon shrub

tree

RANGE: cag col ecu cilimber

RANCE: bol cag mex sag? sao climber

RANGE: ang cag mex sag? sao climber RANCE: cag sat climber 
TAXON

\section{Cespedezia}

macrophyl la Seem.

\section{Ouratea}

cocleens is Dwyer

curvata Dwyer

flexipedicellata Dwyer

insulae Riley

Iucens (H.B.K.) Engl.

mexicana (H. \& B.) Engl.

patelliformis Dwyer

tue rckhe imi i J.D. Sm.

*valeril Char.

\section{Sauvagesia}

elata Benth.

erecta $L$.

* paucielata Sastre

pulchella Seem.

tenel la Lam.
PROV: bo ca ce cn da 000m: 0-1 pa sa vr

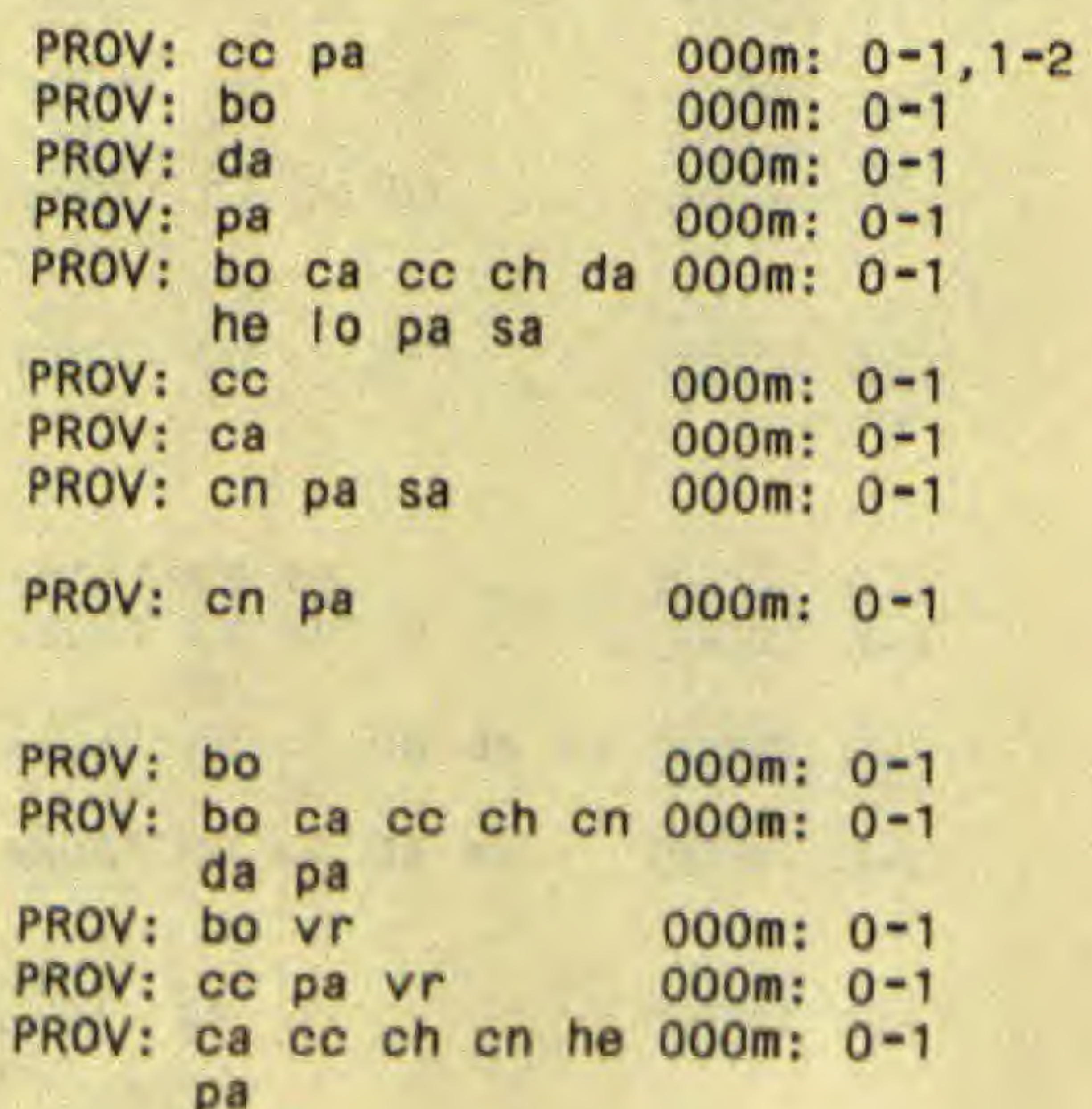

RANGE: cag sat

climber

RANGE: end

RANGE: $\mathrm{cr}$ Sao

RANGE: end

RANGE: beI

RANGE: cag mex

RANGE: $\operatorname{mex}$

RANGE; end

RANGE: gua

RANGE: $\mathrm{cr}$

RANGE: gui

RANGE: wdw

RANGE: end

RANGE: $\mathrm{cr}$ sag?

RANGE: cag cub sag $x+x$

tree

shrub

shrub?

tree

shrub

tree

shrub

tree

shrub

tree

tree

herb

herb

herb

herb

herb

183 (120) CARYOCARACEAE

\section{Ca ryocar}

amygda I iferum Mut is

costaricense J.O. Sm.

nuciferum L.
PROV: da

PROV: da 000m: 0-1

000m: $0=1$

000m: $1-2$

184 (121) MARCGRAVIACEAE

\section{Marcgravia}

affinis Hems!.

\section{atropuncta de Roon}

brownei (Tr. \& PI.) Krug \& Urb.

macrophylla (Wittm.) GiIg
PROV: bo cc da vr

PROV: $\mathrm{cc} \mathrm{ch}$

PROV: cc ch pa vr

PROV: da 000m: $0-1,1-2$

000m: $0-1$

000m: $0-1,1-2,2-3$

000m: $0-1$

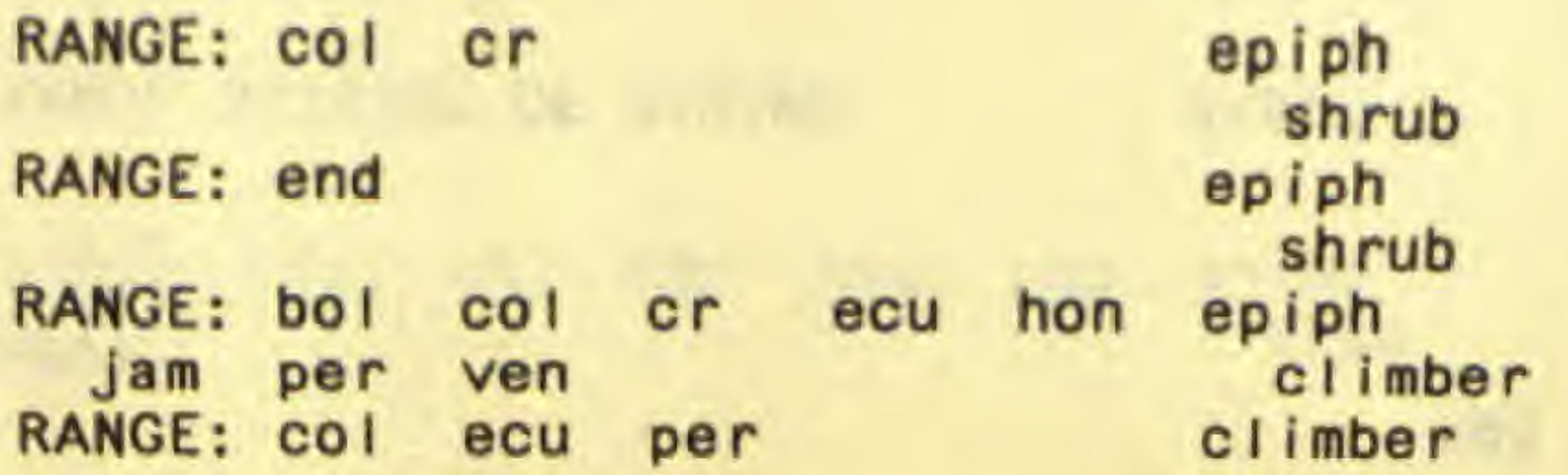

\section{RANGE: $\mathrm{COI}$ tree}

RANGE: ang gui tree 
membranacea StandI. *myriostigma Tr. \& Pi.

nepenthoides Seen.

"nervosa Tr. R PI.

epittieri cilg

* receifiora Tr. \& PI.

*-wvar. rectiflora

schippil standi.

serrae de Roon

\section{No rantea \\ costaricensis 0119}

subsessilis (Benth, J.O, Sa.

Ruyschis

phylladenis sands.

\section{Souroubea}

- bievior reenth. 1 de kodon

gilgil Al. michter

foezy \{AI. Nicheer\} to Noon

- subsp, tinima de Roon

aymeetala aris

vallieala de Roos

venesa schery

*verruges schery
PROV: $C C$ ch cn pa 000m: $0=1,1-2$

PROV: $c C$

000a: $0-1,1-2$

PROV: bo ca cn pa sa 000m: $0=1$

PROV: CC 000a: $0=1,1=2$

PROV: pa

000a: $0-1$

PROV: on

PROV: bo en da pe

PROV: on pa vr

000 : $0-1$

000 : $0-1,1-2,2-3$

rhov: pa

PAOV: bo

Whovi ee vr

Phov: be es en pa

phovi ce as

hov: ee on ve

phovi vr
000 e: $2-3,3+$

000a: $1-2,2-3$

coon: 0-1

$000 n$ : $0-1,1-2$

000n: 0-1

Docie: $0-1$

RANGE: or

RANGE: COI

RANGE: beI

RANGE: COI

RANCE: cag

RANCE: cag

RANCE: beí cag?

RANGE: or

cag

(n)

gaver: or

ance: col or

ruset or

ancert cel

excr: bet cagt

cookn: $0+1,1-2$

boon: 0-1

000in: 0-1

obon: $0+1$

060en: $0-1$

zavef: $\mathrm{cr}$

Nests and

suecr: vr climber

epiph

shrub

climber

shrub

tree

epiph

ol inber

ol inber

ol inber

opiph

shrub

clinber

apiph shrub clinter

epiph

shrub

ot inber

shrus

elimber

cilinber

wigh

shrub

elinber

oigh

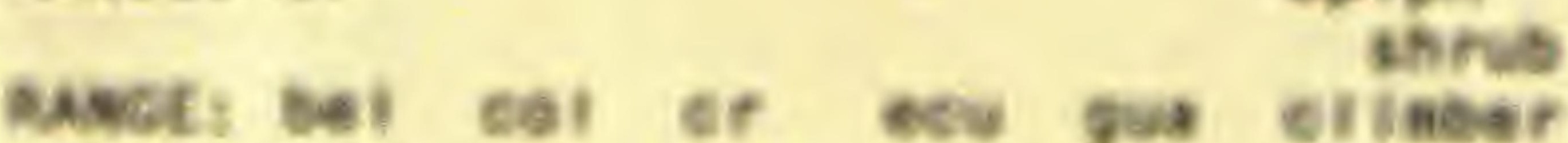

Crimear

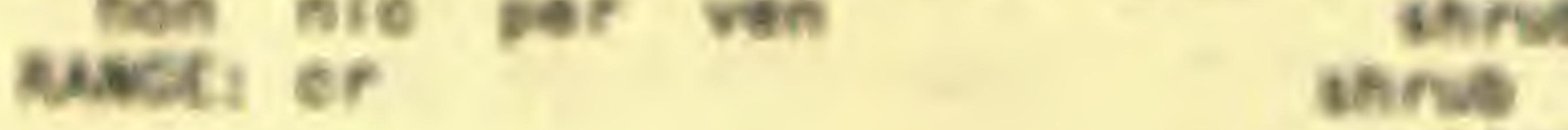

eilineser

asigh

throb

eilineser 
TAXON

Lacunaria

panamens is (StandI,) StandI.

PROV: bo pa

000m: $0-1$

PROV: $c$ n

PROV: bo pa

000m: $0-1$

000m: $0-1$

* schippii Standi.

186 (122) THEACEAE

\section{Cleyera}

theaeoides (Sw.) Choisy

Freziera

candicans Tul.

* forerorum A. Gentry

*hie ronymi Kobuski

Laplacea

fruticosa (Schrad.) Kobuski

grandis Brandeg.

Pelliciera

rhizophorae $\mathrm{Tr}$, \& PI.

Symp lococa rpon

chiriquense Kobuski

Ternst roemia

tepezapote Schlecht, \& Cham.
PROV: ch

PROV: ch

PROV: da

PROV: da

PROV: ch da

PROV: da pa

000m: $0-1,1-2$

PROV: ca da pa

000m: $0-1$

PROV: ch da

$000 \mathrm{~m}: 1-2$

PROV: bo ca ce ch en 000m: $0-1$ da he pa $v r$

$00 \mathrm{~m}: 1-2,2-3$

000m: $1-2$
RANGE: end

tree

RANGE: end

RANGE: $\mathrm{cr}$

tree

tree

RANGE: cag jam mex

tree shrub

$000 \mathrm{~m}: 1-2$

RANGE: col or ven
RANGE: col

RANGE: $W r$

RANGE: $\mathrm{cr}$ hon sag

RANGE: or hon mex

RANGE: cag sat

RANGE: end

tree

RANGE: cag mex

tree

shrub

RANGE: afr asi oce tree

RANGE: col gui per sao tree

RANGE: end

RANGE: as $i$ oce tree

shrub soulattri Burm. f.

cult.
PROV: ca cn da he pa 000m: $0-1$ sa $v r$

000m: $0-1$

000m: $0-1$ 
187 (123) GUTTIFERAE cont.

*Chrysochlamys
eclipes L. . Wms.
weberbaueri Engl.
Clusia
coclensis StandI.
congestiflora Cuatr.
croatii D'Arcy
cupulata (Maguire) Maguire
divaricata Maguire
dukei Maguire
fructiangusta Cuatr.
*gracilis StandI.
I iesneri Maguire
I ineata (Benth.) PI. \& Tr.
osseocarpa Maguire

PROV: ca cn pa sa $\begin{aligned} & 000 m: 0-1 \\ & 000 m: 1-2\end{aligned}$
PROV: da

PROV: ch da pa sa 000m: $0-1,1-2$

PROV: bo ca ce ch da 000m: $0-1,1-2$ pa

PROV: ch cn pa 000m: $0-1,1-2$

PROV: cc pa sa vr 000m: $0-1$

PROV: ch pa vr 000m: $0-1,1-2$

PROV: cc pa $\quad 000 \mathrm{~m}: 0-1$

PROV: bo $v r \quad 000 \mathrm{~m}: 0-1$

PROV: cc cn pa $v r$ 000m: $0-1$

PROV: bo ce da $v r$ 000m: $0-1,1-2$

PROV: bo $\mathrm{cn}$

000m: $0-1$ PROV: bo ca cc ch cn 000m: $0-1,1-2$
da he pa sa $v r$

PROV: bo da pa sa $000 \mathrm{~m}: 0-1$

PROV: ca da pa sa 000m: $0-1,1-2$

PROV: bo cc ch da $\vee r$ 000m: $0-1,1-2,2-3$

PROV: bo ca cc

000m: $0-1$
RANGE: end

RANGE: $\mathrm{COI}$

RANGE: $c r$

RANGE: $c O I$

RANGE: end

RANGE: end

RANGE: $\mathrm{cr}$

RANGE: end

RANGE: $\mathrm{COI}$ ecu

RANGE: $c r$

RANGE: $\mathrm{cr}$

RANGE: $\mathrm{CO}$ I

RANGE: end

RANGE: ang

cag? or sag?

RANGE: $c r$

RANGE: end

RANGE: $c r$

RANGE: cag

shrub
tree
epiph
shrub
tree
epiph
shrub
tree
epiph
tree
shrub
epiph
shrub
tree
epiph
shrub
tree
epiph
tree
epiph
shrub
tree
epiph
shrub
epiph
shrub
tree
epiph
shrub
tree
epiph
shrub
tree
epiph
tree
shrub
epiph
climber
tree
epiph
shrub
tree
epiph
tree
epiph
shrub


TAXON

\section{rosea Jacq.}

rotundata StandI.

salvinif J,D, Sa.

stenophyIla Stand I.

\section{*trifiora cuatr.}

uvitana Pitt.

*valerif standl.

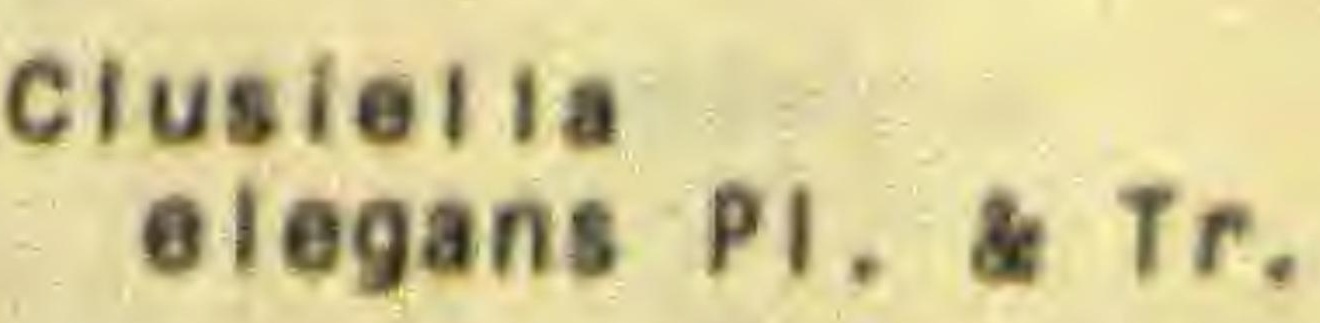

Dystovomita

pittieri (Engl.) D'Arcy

Garcinia

mangostana $\mathrm{L}$.

plctorius (Roxb.) D'Arcy cult.

Havetiopsis

flexilis PI. \& Tr.

Mammea

americana $\mathrm{L}$.

immansueta D'Arcy

Marila

domingens is Urb.

lactogena Cuatr.

Iaxifiora Rusby

cult.

(

pluricostata StandI. L. O. Wms.

\section{"Quapoya}

* sipapoana Maguire

Rheedia

acuminata $(R, \& P) P, I . \& T r$.

edulis (Seem.) PI. \& Tr.
PROV: ca ce on on he 000m: $0-1,1-2,2-3$ pa vr

PROV: ce ch en da pa 000a: $0-1,1-2,2-3$ sa vr

PROV: bo ce ch da pa 000m: $0-1,1-2$ vr.

PROV: ce cn

000m: $0-1,1-2$

PROV: $\mathrm{CC}$

PROV: en

PROV: $\mathrm{co}$ en en sa

000า: $0-1$

000m: $0-1$

000m: $0-1$

PROV: ce da pa sa vr 000m: 0-1

PROV: da sa

000m: $0-1$

PROV: ca

PROV: ca pa

\section{0m: $0-1$}

000m: $0-1$

PROV: ca ch cn

$000 m: 0-1$

PROV: ca pa

PROV: pa

000m: $0-1$

000m: $0-1$

PROV: pa sa

PROV: CC pa sa

000m: $0=1$

000m: $0-1,1-2$

PROV: ca cc cn da pa 000m: 0-1

000m: $0-1$

PROV: pa sa

000m: $0-1$

PROV: ca cc ch cn da 000m: $0-1,1-2$

PROV: bo ca ce ch da 000m: $0-1$

he lo pa vr

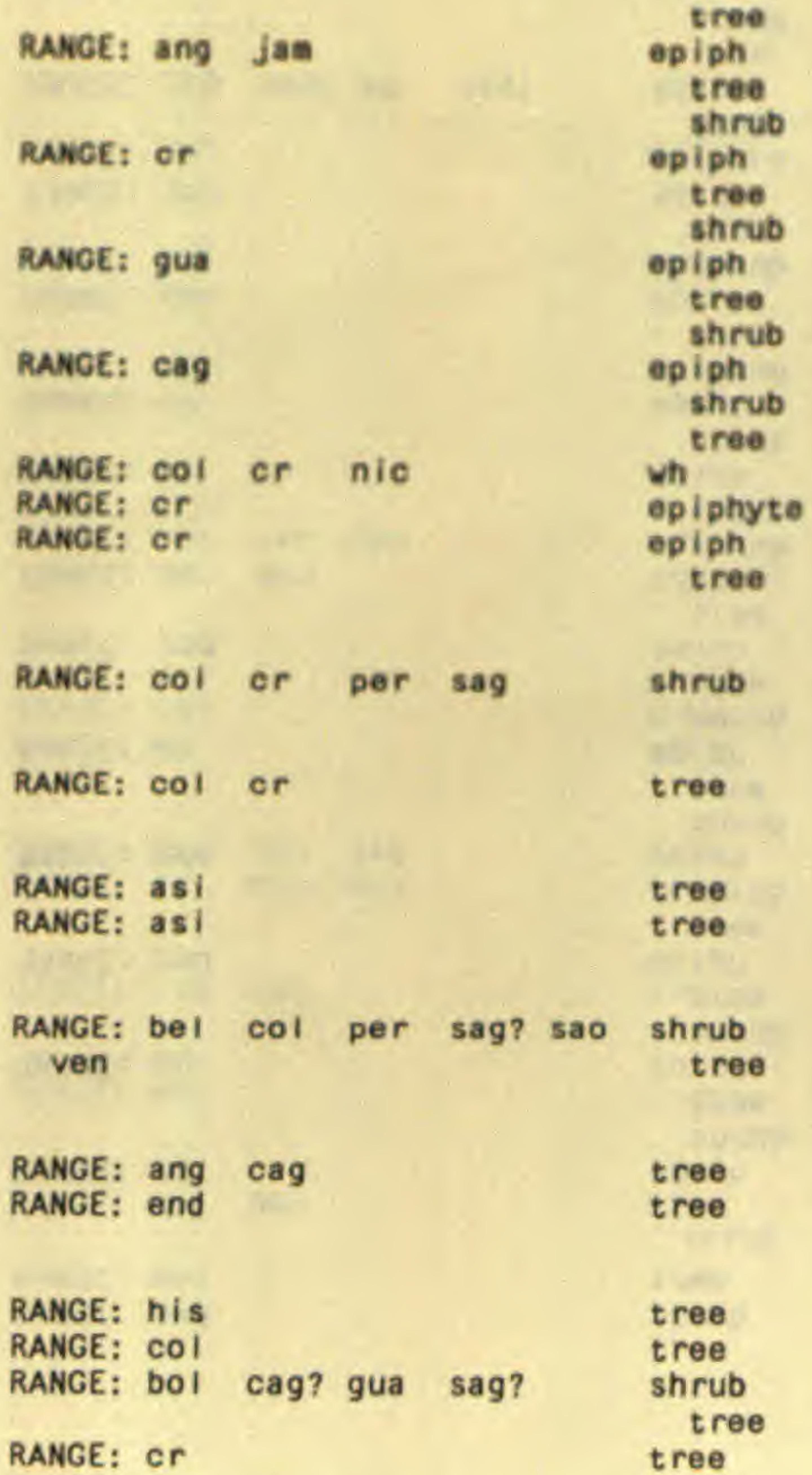

RANGE: per

hemiepiphyte

RANGE: col or gui per sag? tree

RANGE: cag mex per tree 
187 (123) GUTTIFERAE cont.

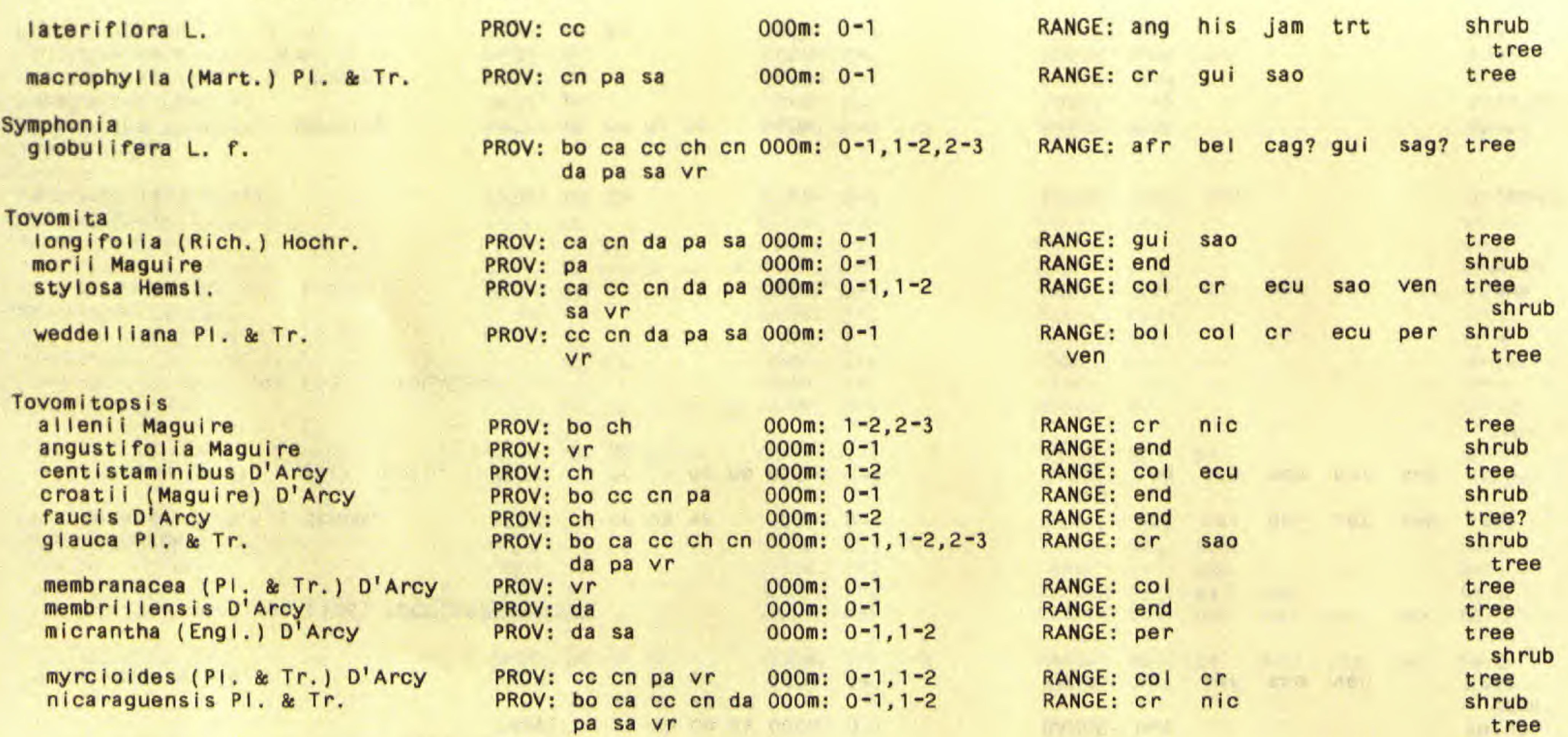

\section{7 (123a) HYPERICACEAE}

Hypericum

gnidioides Seem.

*moranense Kunth

Vismia

Hbaccifera (L.) Tr. \& PI.

billbergiana Beurl.

jefensis N. Robson

latisepala N. Robson

macrophyI Ia H.B.K.

\section{PROV: $\mathrm{cc} \mathrm{ch}$}

PROV: ch

000m: $0-1,1-2,2-3,3+$

000m: $1-2,2-3,3+$

PROV: ca cc ch cn da 000m: $0-1$ pa $\vee r$

PROV: bo ca cc ch cn 000m: $0-1,1-2$ da pa sa $v r$

PROV: pa

000m: $0-1,1-2$

PROV: ca ce cn pa sa $000 \mathrm{~m}$ : $0-1$ $v r$

PROV: bo ca cc ch cn 000m: $0-1$ da pa sa $v r$

\begin{tabular}{|c|c|c|c|c|c|c|}
\hline $\begin{array}{l}\text { RANGE: } \\
\text { RANGE : }\end{array}$ & $\begin{array}{l}\text { cag } \\
\text { cag }\end{array}$ & $\begin{array}{l}\text { cr } \\
\text { mex }\end{array}$ & hon & mex & nic & $\begin{array}{l}\text { shrub } \\
\text { herb }\end{array}$ \\
\hline $\begin{array}{c}\text { RANGE: } \\
\text { sao }\end{array}$ & $\begin{array}{l}\text { cag } \\
\text { ven }\end{array}$ & $\mathrm{col}$ & $\mathrm{cr}$ & gui & $\operatorname{mex}$ & shrub \\
\hline RANGE: & $\mathrm{col}$ & $\mathrm{cr}$ & & & & shrub \\
\hline RANGE: & end & & & & & $\begin{array}{c}\text { shrub } \\
\text { tree }\end{array}$ \\
\hline RANGE: & $\mathrm{col}$ & & & & & $\begin{array}{c}\text { shrub } \\
\text { tree }\end{array}$ \\
\hline $\begin{array}{c}\text { RANGE: } \\
\text { sao }\end{array}$ & $\begin{array}{l}\text { cag? } \\
\text { ven }\end{array}$ & $\mathrm{col}$ & $\mathrm{cr}$ & gui & per & $\begin{array}{l}\text { tree } \\
\text { shru }\end{array}$ \\
\hline
\end{tabular}


TAXON

FAMILY

PROVINCES

ELEVATION (OOOM.)

RANGE OUTSIDE OF PANAMA

HABIT

cont.

panamens is Duchass. \& Walp.

PROV: bo ca ce ch cn 000m: $0-1,1-2$ da he lo pa $v r$
RANGE: or nic

shrub

tree

\section{4 (125) BIXACEAE}

Bixa

orel Iana $\mathrm{L}$.

*urucurana Willd. intr. cult.

PROV: bo ca ch cn da 000m: 0-1 he lo pa sa vr

PROV: ca cn da pa

195 (126) COCHLOSPERMACEAE

\section{Coch lospermum}

*orinocense (H.B.K.) Steud.

PROV: ca cn da sa 000m: $0-1$

vitifolium (Willd,) Spr. cult.

PROV: ca cc cn da he 000m: 0-1 lo pa

\section{8 (127) VIOLACEAE}

Amphirrhox

longifolia (St. Hil.) Spr.

Corynostylis

a rborea (L.) Blake

Gloeospermum

*b lakeanum (Stand I.) Hekking

*eneidense Hekking

ferrugineostictum A. Robyns

portobelense A. Robyns

* sphaeroca rpum Tr. \& PI.

Hybanthus

attenuatus (Schult.) Schulze

*galeottii (Turcz.) Morton

prunifolius (Schult.) Schulze

PROV: bo

000m: $0-1$

PROV:

o

$\begin{array}{ll}\text { PROV: ca da } & 000 \mathrm{~m}: 0-1 \\ & \\ \text { PROV: cc cn da pa } & 000 \mathrm{~m}: 0-1,1-2 \\ \text { PROV: pa } & 000 \mathrm{~m}: 0-1 \\ \text { PROV: bo } & 000 \mathrm{~m}: 0-1 \\ \text { PROV: cn } & 000 \mathrm{~m}: 0-1 \\ \text { PROV: cn da } & 000 \mathrm{~m}: 0-1\end{array}$

pa sa
RANGE: nWt

shrub

RANGE: bol per sao ven

tree

tree

RANGE: bol col gui per sao tree
Ven
RANGE: ang cag mex per sag tree

RANGE: col gui per sao ven shrub tree

RANGE: cag? sag?

cl imber

RANGE: end

RANGE: cag

RANGE: end

RANGE: end

RANGE: per

shrub

treelet

tree

tree

tree

RANGE: cag mex nao per sag? herb

RANGE: meX

RANGE: col or ven 
TAXON

\author{
thiemei (J.D. Sm.) Morton
}

*Leonia

"g I ycycarpa R. \& P.

\section{Rinorea}

"blakeana Standl.

brachythrix Blake

* crenata Blake

*dasyadena A. Robyns

"hirsuta Hekking

"hummelif Sprague

* paniculata (Mart.) Kuntze

"passoura (DC.) Kuntze

roureoides Woods.

squama ta Blake

sylvatica (Seem.) Kuntze

\section{Viola}

gua tema lens is Becker

*nannel Polak.

odorata L.

scandens schuit.

intr.

stipularis SW.
PROV: $c c$

000m: $0-1$

$000 \mathrm{~m}: 0-1$

PROV: da

000m: $0-1$

PROV: on

PROV: da

PROV: cn da

000m: $0-1$

PROV: co cn pa sa vr $000 \mathrm{~m}: 0-1$

PROV: 5 :

o0m: $0-1$

PROV:

000m: $0-1$

PROV: cn pa

PROV: on

000m: $0-1$

PROV: bo ca cc en da 000m: $0=1$

pa sa

PROV: bo ca da pa 000m: $0-1$

$\begin{array}{ll}\text { PROV: ch } & 000 m: 2-3,3+ \\ \text { PROV: ch } & 000 m: 2-3 \\ \text { PROV: ch } & 000 m: 1-2 \\ \text { PROV: ch } & 000 m: 0-1\end{array}$

PROV: ce oh da 000m: $0-1,1-2$
RANGE: cag mex

herb

RANGE: sat

tree

RANGE: end

RANGE: end

RANGE: $\mathrm{cr}$

RANGE: end

RANGE: COI

RANGE: cag

RANGE: Sao

RANGE: per ven

RANGE: end

RANGE: $\mathrm{cr}$

RANGE: $\mathrm{COI}$ or

RANOE: ca9 mex

RANGE: or mex

RANGE: af $r$

RANGE: boI

ven

RANGE: COI

ven

shrub

shrub

tree

tree

shrub

tree

tree

tree

shrub

tree

shrub

shrub

erb

herb
nerb

nerb

cag col

or ecu

nerb

tree

tree

\section{9 (128) FLACOURTIACEAE}

\section{Banara}

guianensis AubI.

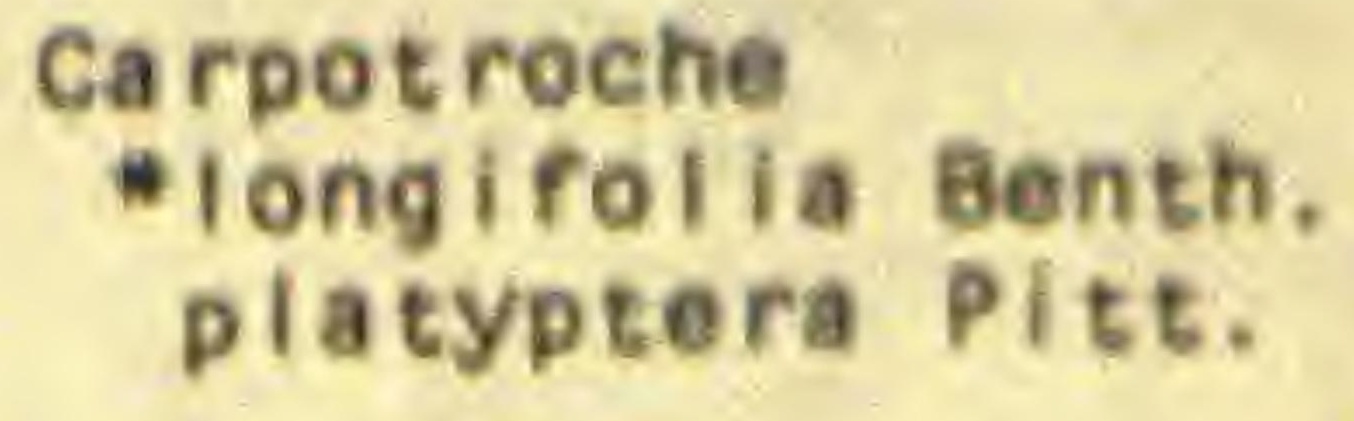

Ca rpot roche

* longifolia Benth.

platyptera Pitt.

\section{Casearia}

*aculeata Jaca.

arborea (Rich.) Urb.

a rguta $\mathrm{H}, \boldsymbol{\theta}, \mathrm{K}$.
PROV: bo ca cC ch en 000s: 0-1 lo pa vr

PROV: en pa 000n: 0-1 PROV: bo CC en da pa 000s: $0-1$ sa vr

PROV: ca ch da he lo 000et $0-1$ pa vr

PROV: ca ce en en pa 000n: $0-1$

PROV: bo ca ce ch da 000a: 0-1 he pa vr

\begin{tabular}{|c|c|c|c|c|c|c|}
\hline $\begin{array}{c}\text { RANCE: } \\
\text { sat }\end{array}$ & bol. & col & or & nic & per & $\begin{array}{l}\text { shrub } \\
\text { tree }\end{array}$ \\
\hline RANOE: & 500 & sat & & & & tree \\
\hline RANCE: & cag & ecu & gua & & & shrub \\
\hline $\begin{array}{c}\text { RANGE: } \\
\text { sat }\end{array}$ & bol & cag & sex & per & 590 & $\begin{array}{l}\text { tres } \\
\text { shrub }\end{array}$ \\
\hline RAHCE: & ang & bet & cag & gag & gui & shrub \\
\hline per & sao & sat & & & & \\
\hline RANCE: & cag & col & $\operatorname{mex}$ & per & ven & $\begin{array}{l}\text { shrub } \\
\text { tree }\end{array}$ \\
\hline
\end{tabular}


TAXON

PROVINCES

ELEVATION (OOOM.)

RANGE OUTSIDE OF PANAMA

HAB IT

199 (128) FLACOURTIACEAE cont.

\begin{abstract}
*atlantica sleumer
* cajambrensis Cuatr.

commersoniana Camb.
\end{abstract}

*coronata StandI. \& L.O. Wms. corymbosa H.B.K.

*decandra Jacq.

grandiflora Camb.

guianensis (AubI.) Urb.

nitida (L.) Jacq.

"spinescens (SW.) Griseb.

sylvestris SW.

*tacanensis Lund.

*Flacourtia

*jangomas (Lour.) Raeusch. intr. cult.

PROV: ca $\quad 000 \mathrm{~m}: 0-1$

000m: $0-1$

PROV: $\mathrm{Vr}$

PROV: $v r \quad$ 000m: $0-1$

PROV: bo ca ch cn da $000 \mathrm{~m}$ : $0-1$

10 pa sa

PROV: bo cn da pa 000m: $0-1,1-2$

PROV: ca cn da lo pa 000m: $0-1$

PROV:

000m: we

PROV: da

000m: $0-1$

PROV: bo ca cn da he $000 \mathrm{~m}: 0-1$

pa $v r$

PROV: bo ca ch cn da 000m: 0-1 pa sa vr

PROV: pa 000m: $0-1$

PROV: bo ca cc ch cn 000m: $0-1$

da pa sa $v r$

000m: $1-2$

Hasseltia

floribunda H.B.K.

Homa I i um

racemosum Jacq.

Laetia

micrantha A, Robyns

procera (Poepp.) Eichl.

thamnia $L$.

Lindackeria

|aurina Pres|

PROV: bo ca ch cn da 000m: $0-1,1-2$ pa $\vee r$

PROV: ca da

000m: $0-1$

PROV: da

PROV: ca

000m: $0-1$

PROV: ca cn lo pa sa 000m: $0-1,1-2$ vr

PROV: ca ch cn da pa 000m: $0-1$

PROV: ch pa vr PROV: cn da sa

000m: $0-1$

*mexicana Brandeg.

parviflora Benth.

piperoides StandI.
PROV: bo $\mathrm{cc} v \mathrm{r}$ 000m: $0-1$

000m: $0-1$
RANGE: end

RANGE: $c O$

RANGE: cag mex sao sat

tree

tree

RANGE: $\mathrm{cr}$

RANGE: cag col mex ven

tree

tree

shrub

RANGE: ang hon sao sat

treelet

shrub

RANGE: col gui sao ven tree

ven ang ang or sao sat shrub

RANGE: nWt

tree

shrub

RANGE: gag trt ven shrub

RANGE: nwt per shrub

RANGE: cag sat

tree

tree

RANGE: OWt

tree

RANGE: bol $\mathrm{cr}$ hon nic sat shrub

tree

RANGE: ang cag mex sao sat tree

RANGE: end

RANGE: ang bel or gua gui

cr gua gui tree

RANGE: ang cag col mex tree

RANGE: bel col or gua mex tree ven

RANGE: cag mex

RANGE: bol col or tree

sao sat

RANGE: hon

tree

shrub 


\section{*acrohasseltia}

*macroterantha (Stand I. \& L.0. Wms.) L.0. Wms.

PROV: pa vr

000m: $0-1$

\section{Mayna}

*grandifolia (Karst.) Warb.

*odorata AubI.

\section{Neosprucea}

*grandiflora (Benth.) Sleumer

\section{*Pleuranthodendron}

* I indenii (Turcz.) sleumer

Prockia

crucis $L$.

\section{Ryania}

speciosa Vahl

--var. panamens is Monachino

\section{Tetrathylacium}

johansenii StandI.

macrophyl I um Poepp.

\section{Xy losma}

an isophyI la StandI.

*chloranthum J.D. Sm.

flexuosa (H,B,K.) HemsI.

hispidula Standl.

intermedium (Seem.) Tr. \& PI.

*ol igandrum J.D. Sm.

panamensis Turcz.

* rubicundum (Karst.) Gilg

*velutinum (Tul.) Tr. \& PI.

PROV: ca da pa sa
PROV: da pa

000m: $0-1,1-2$

PROV: ca cc ch da pa 000m: $0-1,1-2$ sa

PROV: $\mathrm{CC}$

000m: $0-1$

PROV: en da $v r$

000m: $0-1$

PROV: ca pa vr

000m: $0-1$

PROV: bo ca cc cn pa 000m: 0-1

000m: $0-1$

PROV: $\mathrm{cc}$ ch

000m: $0-1$

PROV: he pa

000m: $0-1$

PROV: ca ch cn

PROV: ch pa

000m: $0-1,1-2$

$000 \mathrm{~m}: 0-1,1-2,2-3$

PROV: bo

000m: $0-1$

PROV: ch

PROV: ca cc da sa

000m: $1-2$

000m: $0-1$

PROV: bo ca cn sa $v r$ 000m: $0-1$

PROV: da $v r$

PROV: WP 000m: $0-1,1-2$

000m: we

\begin{tabular}{|c|c|c|c|c|c|c|}
\hline RANGE: & cr & hon & nic & -1 & 250 & tree \\
\hline RANGE: & $\mathrm{col}$ & per & ven & $-k$ & atia & $\begin{array}{c}\text { shrub } \\
\text { tree }\end{array}$ \\
\hline RANGE: & $\mathrm{cr}$ & hon & sat & 7at & $y$ & $\begin{array}{l}\text { shrub } \\
\text { tree }\end{array}$ \\
\hline RANGE: & $\mathrm{col}$ & ecu & per & & & $\begin{array}{l}\text { tree } \\
\text { shrub }\end{array}$ \\
\hline RANGE: & cag & ecu & per & sat & & tree \\
\hline RANGE: & ang & cag & $\operatorname{mex}$ & sag & & $\begin{array}{l}\text { shrub } \\
\text { tree }\end{array}$ \\
\hline RANGE: & end & & & & & $\begin{array}{c}\text { shrub } \\
\text { tree }\end{array}$ \\
\hline $1+4$ & 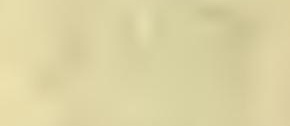 & & & & & $\mathrm{Cr}=$ \\
\hline RANGE: & $\mathrm{col}$ & $\mathrm{cr}$ & & & & tree \\
\hline RANGE: & $\mathrm{col}$ & $\mathrm{cr}$ & per & sat & 11. 4 & tree \\
\hline RANGE: & bel & $\mathrm{cr}$ & $\operatorname{mex}$ & $x=1$ & & $\begin{array}{l}\text { shrub } \\
\text { tree }\end{array}$ \\
\hline RANGE: & cag & $\mathrm{cr}$ & $\operatorname{mex}$ & & & tree \\
\hline RANGE: & cag & mex & nas & sat & & $\begin{array}{c}\text { shrub } \\
\text { tree }\end{array}$ \\
\hline RANGE: & $\mathrm{cr}$ & & & & & $\begin{array}{c}\text { shrub } \\
\text { tree }\end{array}$ \\
\hline RANGE: & cag & col & $\operatorname{mex}$ & sat & ven & shrub \\
\hline RANGE: & cag & $\operatorname{mex}$ & & & & tree \\
\hline RANGE: & cag & $\operatorname{mex}$ & & & & $\begin{array}{l}\text { shrub } \\
\text { tree }\end{array}$ \\
\hline RANGE: & $\mathrm{col}$ & ven & & & & tree \\
\hline RANGE: & cag & $\operatorname{mex}$ & sat & & & $\begin{array}{c}\text { shrub } \\
\text { tree }\end{array}$ \\
\hline
\end{tabular}

RANGE: ang cag or mex trt tree 
Erblichia

odorata Seem.

Piriqueta

cistoides steud.

\section{Turnera}

angustifolia Mill.

*diffusa Schult. panamens is Urb. ulmifolia L.
PROV: ce ch $v r$

000m: 0-1

PROV: cc pa

000m: $0-1$

PROV: bo ca sa

000m: $0-1$

PROV: $\mathrm{CC}$

PROV: ce ch lo pa vr 000m: $0=1$

\section{3 (130) PASSIFLORACEAE}

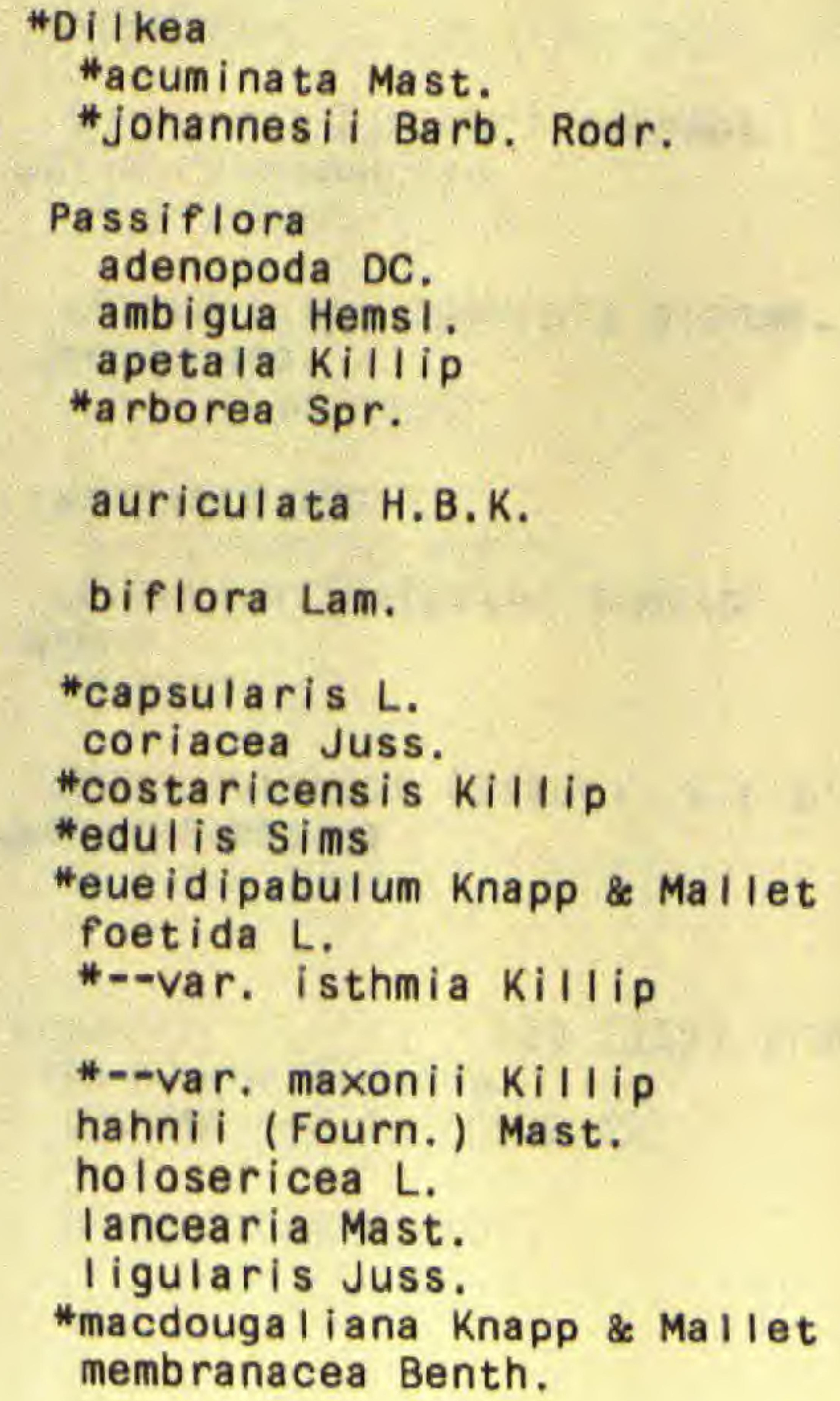

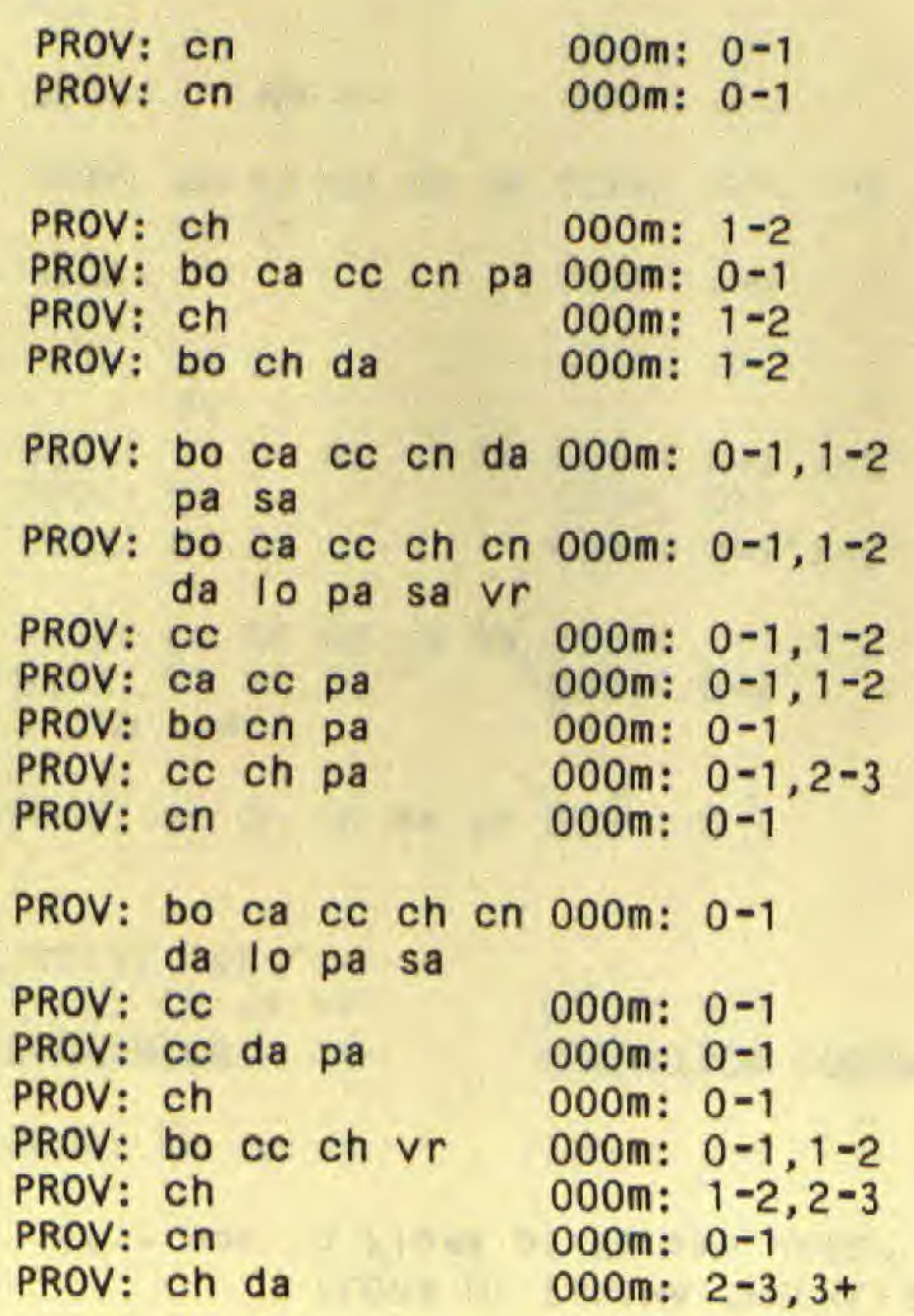

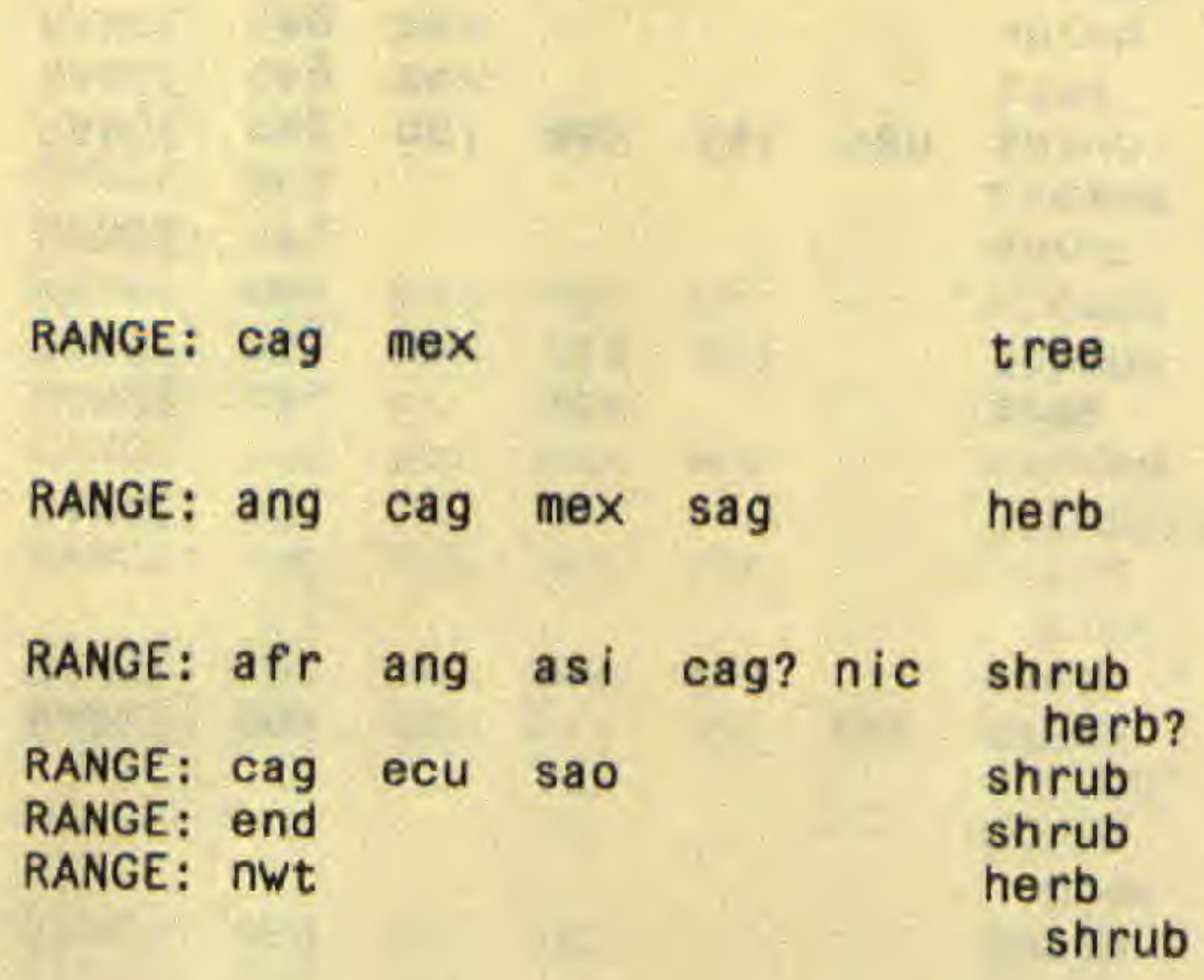

RANGE: col sao
RANGE: sao


TAXON

menispermifolia H.B.K.

misera H,B.K.

nitida H,B.K.

oerstedil Mast.

*--var, choconia panamensis Killip

pediculata Mast.

*pittieri Mast.

*platyloba Kili ip

"porphyretica Mast.

pulchella H, B, K.

punctata $L$.

quadrangularis L.

seemannil Griseb.

* serrato-digitata L.

sexflora Juss.

suberosa L.

subpel tata ort.

tiliaefolia

tryphostemma to ides Ha rms

vitifolia H,B.K.

Williamsil killip

\#Tetrastylis

* lobata killip
PROV: bo ca ch cn pa 000m: $0-1,1-2$ $v r$

PROV: ca pa 000m: $0-1$

PROV: ca cn da pa 000m: $0-1$

PROV: bo $\mathrm{cc} \mathrm{ch}$ cn da $000 \mathrm{~m}$ : $0-1,1-2$ he pa

PROV: ca 000m: $0-1$

PROV: da 000m: $0-1$

$000 \mathrm{~m}: 0-1$

000m: $0-1,1-2$

$\begin{array}{ll}\text { PROV: ch } V r & 000 \mathrm{~m}: 0-1 \\ \text { PROV: } & 0-1\end{array}$

PROV: Io pa 000m: $0-1$

PROV: ca da lo pa

PROV: ca pa pam: $0-1$

cult. PROV: bo ca cc ch cn 000m: 0-1 pa $\vee r$

ROV: bo ca ch cn da $000 \mathrm{~m}$ : $0=1,1=2,2=3$

pa sa

PROV: da $000 \mathrm{~m}: 0-1$

PROV: bo ch vr 000m: $0-1,1-2$

PROV: pa sa

000m: $0-1$

PROV: ca cc cn pa 000m: $0-1,1-2,2-3$

PROV: $\mathrm{CC}$ Vr

$000 \mathrm{~m}: 0-1$

PROV: bo ch en pa 000m: $0-1$

PROV: bo ca cc ch cn 000m: $0-1$ da pa sa vr

PROV: ca cc da lo pa 000m: 0-1

PROV: bo

$000 \mathrm{~m}: 0-1$
RANGE: cag? nic per sag? sao climber

RANGE: per

climber

RANGE: gui per sag? sao climber

RANGE: cag col mex ven climber

RANGE: cag

RANGE: $c 01$

RANGE: $c r$

RANGE: $c 01$

RANGE: cag

RANGE: mex

RANGE: cag

cag col mex nao ven

RANGE: per

RANGE: nWt

sag sao

RANGE: cag col mex climber

climber

climber

climber

climber

climber

climber

climber

climber

climber

RANGE: bol col ecu per sao climber

RANGE: cag col fla gag nex climber

RANGE: ang bah cag fla mex climber

owg sag? sao sao tex

RANGE: cag col mex ven

RANGE: $e \mathrm{CU}$

climber

climber

RANGE: col
RANGE: cag? cub jam nic per climber
climber

ven

RANGE: end

cl imber

RANGE: or

cl imber

205 (131) CARICACEAE

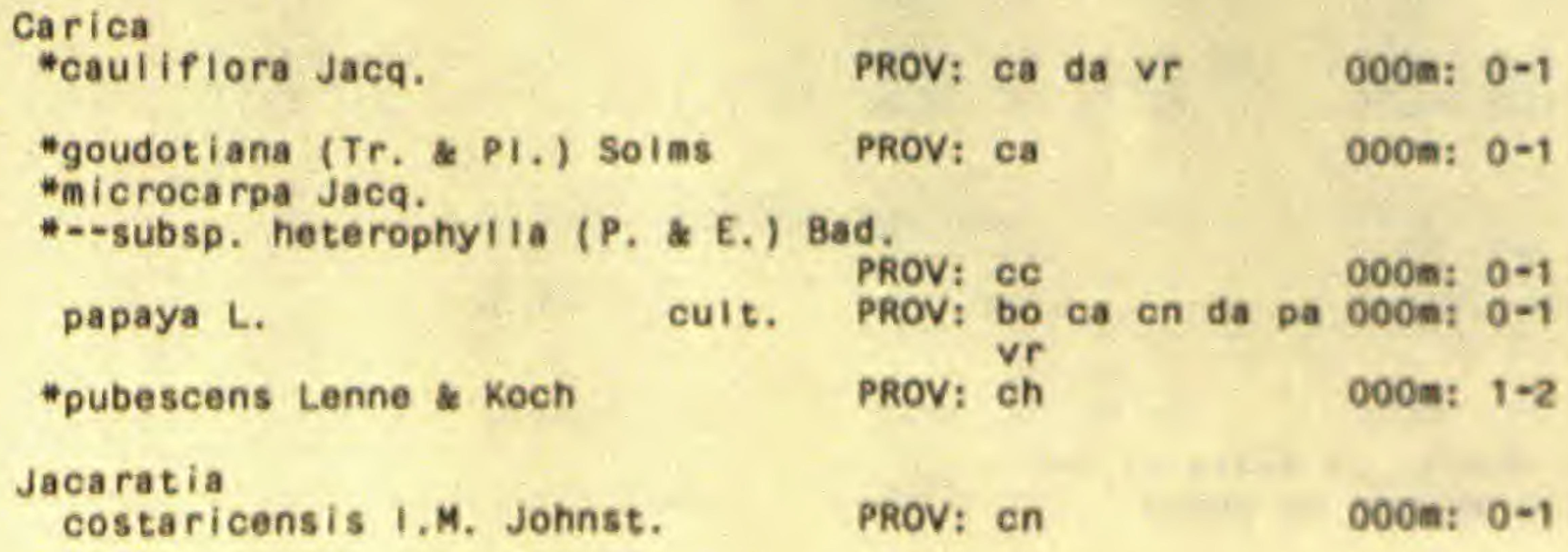

\begin{tabular}{|c|c|c|c|c|c|c|}
\hline $\begin{array}{c}\text { RANGE: } \\
\text { TrE } \\
\text { RANGE: }\end{array}$ & $\begin{array}{l}\mathrm{cag} \\
\mathrm{col}\end{array}$ & cr & $\operatorname{mex}$ & sag & sat & $\begin{array}{l}\text { tree } \\
\text { shrub } \\
\text { shrub }\end{array}$ \\
\hline $\begin{array}{l}\text { RANCE: } \\
\text { RANCE: }\end{array}$ & $\begin{array}{l}\text { col } \\
\text { nvt }\end{array}$ & ecu & per & sao & & $\begin{array}{l}\text { tree } \\
\text { tree }\end{array}$ \\
\hline RANGE: & bol & $\mathrm{col}$ & ecu & per & ven & tree \\
\hline
\end{tabular}


TAXON

dolichaula J.D. Sm.

*spinosa (Aubi.) A. DC.

PROV: bo da $v r$

PROV: ca cn da pa 000m: $0-1$ 000m: $0-1$

206 (132) LOASACEAE

Gronovia scandens $L$.

Klaprothia mentzelioides H.B.K.

\section{Loasa}

grandis Stand I. speciosa J.D. Sm.

\section{Mentzelia} aspera $L$.

\section{*Sclerothrix}

*fasciculata C. Pres! rudis Benth.

$\begin{array}{ll}\text { PROV: lo pa } & 000 \mathrm{~m}: 0-1 \\ \text { PROV: bo ch } & 000 \mathrm{~m}: 1-2 \\ \text { PROV: ca cc da } & \begin{array}{l}000 \mathrm{~m}: 0-1 \\ \text { PROV: ch }\end{array} \\ \text { PROV: ch } & 000 \mathrm{~m}: 1-2 \\ \text { PROV: ca cc pa } & 000 \mathrm{~m}: 0-1\end{array}$

208 (133) BEGONIACEAE

\section{Begonia}

brevicyma C. DC.

*buseyi Burt-Utley

carletonii Standl.

conchifolia A. Dietr.

convallariodora C. DC.

* corredorana C. DC.

"croatil Burt-Utley

davidsoniae Sm, \& Schubert

*dressleri Burt-Utley

estrellensis C. DC.

* fischeri Schrank

garaga rana C. DC.

glabra Aubl.

guaduens is H.B.K.

"heracleifolla Schlecht. \& Cham.

heydei C. DC.

*hirsuta Aubl.

involucrata Liebm.

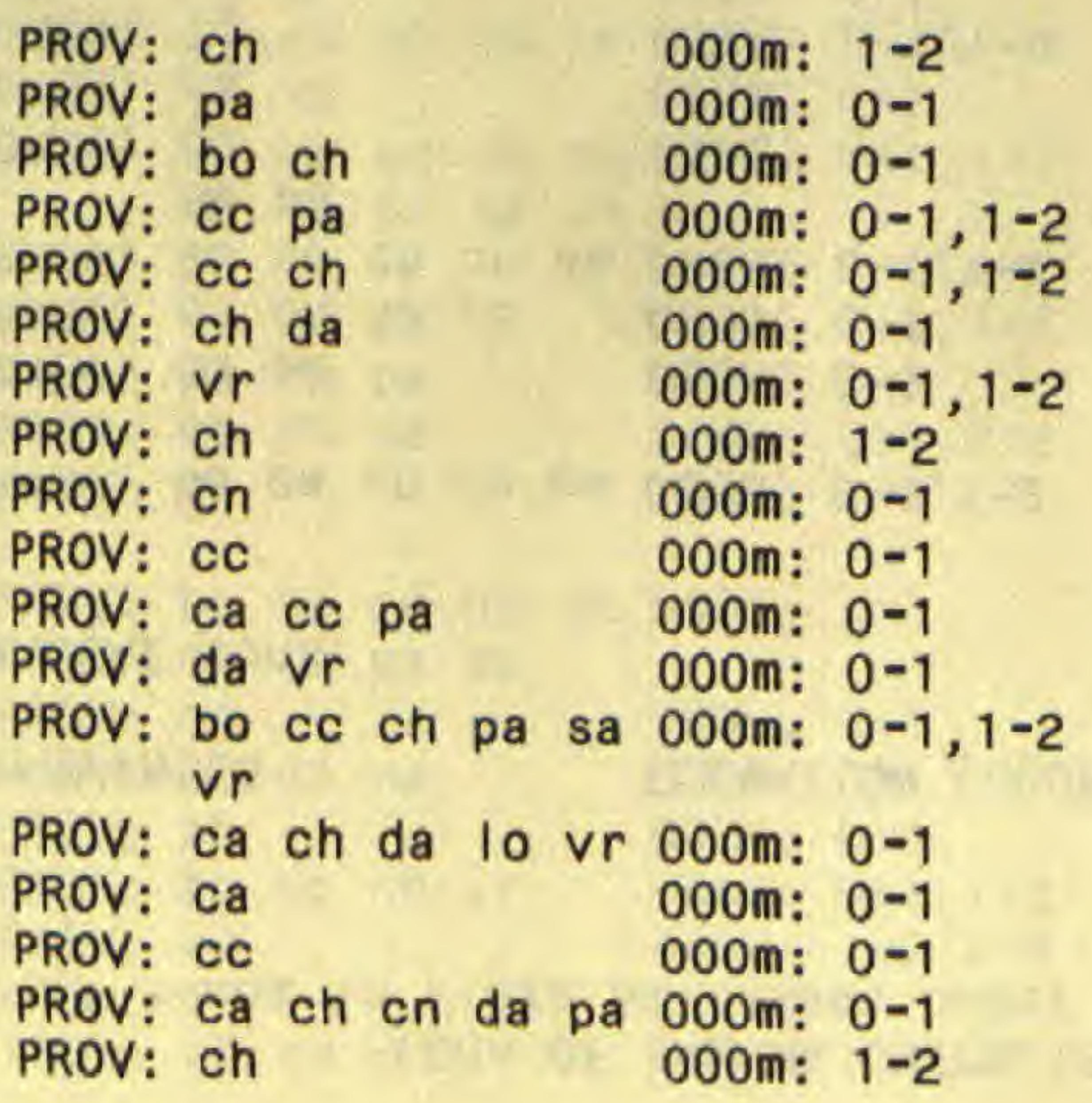
RANGE: bel cr gua mex
RANGE: or gui nic per sag tree
sao herb

herb

herb

herb

herb

herb

herb

\begin{tabular}{|c|c|c|c|c|c|c|}
\hline ANGE: & end & & & & & he \\
\hline $\begin{array}{l}\text { ANGE: } \\
\text { ANGE: }\end{array}$ & end & & & & & b \\
\hline $\begin{array}{l}\text { ANGE: } \\
\text { ANGE: }\end{array}$ & $\begin{array}{l}\mathrm{cr} \\
\mathrm{cr}\end{array}$ & & & & & herb \\
\hline ANGE: & $\begin{array}{l}\mathrm{cr} \\
\mathrm{caq}\end{array}$ & & & & & herb \\
\hline GE: & $\begin{array}{l}\text { cag } \\
\mathrm{cr}\end{array}$ & mex & & & & herb \\
\hline : & or & & & . & & herb \\
\hline : & end & 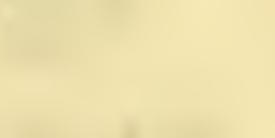 & & & & herb \\
\hline RANGE: & end & $=$ & 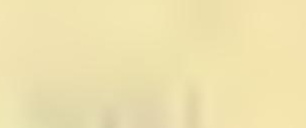 & & & herb \\
\hline RANGE: & end & & 2 & the & -205 & herb \\
\hline RANGE: & $\mathrm{Cr}$ & & & & & 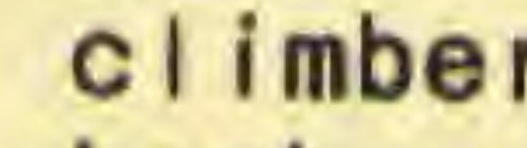 \\
\hline RANC & per & ven & & & & \\
\hline RANGE: & ang & & & & & \\
\hline per & sag? & 001 & & gui & mex & \\
\hline RANGE: & $\mathrm{col}$ & ven & $=$ & 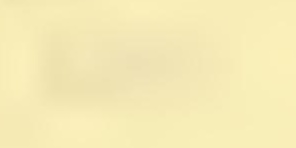 & $2+4=$ & \\
\hline RANC & cag & $\operatorname{mex}$ & & & & \\
\hline RANGE: & $\mathrm{cr}$ & gua & & & & \\
\hline RA! & $\mathrm{col}$ & $\mathrm{cr}$ & ui & nic & ven & \\
\hline ANC & $\mathrm{cr}$ & & & & & \\
\hline
\end{tabular}


208 (133) BEGONIACEAE cont.

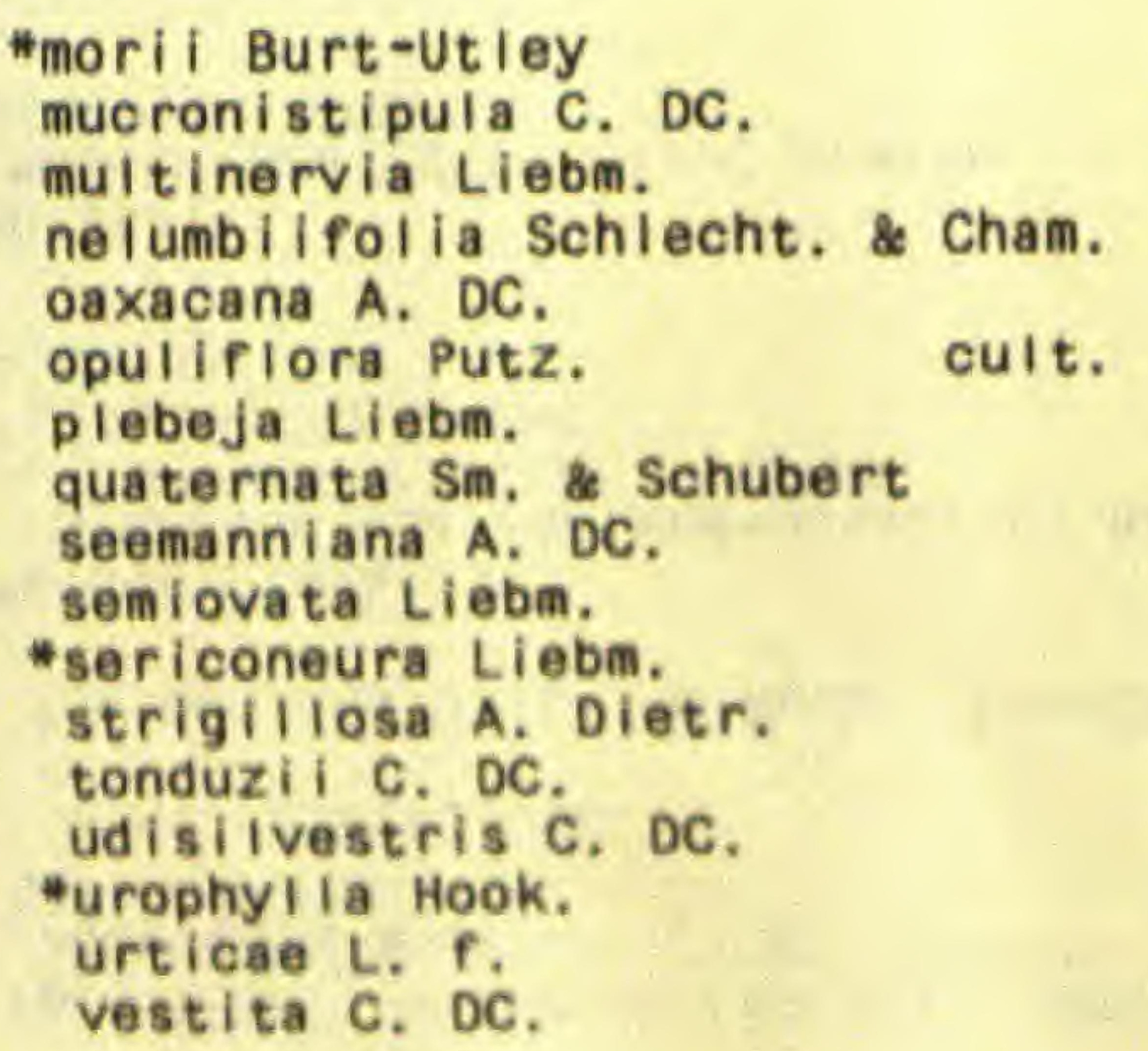

PROV: pa

PROV: ch

PROV: cC ch Vr

PROV: ca da pa

PROV: ch

cult. PROV: cC

PROV: ca cc da pa vr 000m: $0-1$

PROV: ch

PROV:

PROV: bo ca

PROV: ch Vr

PROV: ch

PROV: $c c$ da

PROV: bo ch

PROV: bo ch

PROV: cc ch 000m: $0-1$

000m: $1-2$

000m: $0-1$

000m: $0-1$

000m: $1-2,2-3$

$000 \mathrm{~m}: 0-1$

000m: $1-2,2-3$

000m: $0-1$

000m: $0=1$

000m: $2-3$

000m: $0-1,1-2$

$000 \mathrm{~m}: 1-2,2-3,3+$

000m: $0-1$

000m: $1-2,2-3$

000m: $0-1,1-2$

\begin{tabular}{|c|c|c|c|c|c|c|}
\hline $\begin{array}{l}\text { RANGE: } \\
\text { RANGE: }\end{array}$ & end & & & & & $\begin{array}{l}\text { herb } \\
\text { herb }\end{array}$ \\
\hline RANGE: & $\mathrm{cr}$ & nic & & & & herb \\
\hline RANGE: & cag & col & $\operatorname{mex}$ & & & herb \\
\hline RANGE: & cr & gua & mex & nic & & herb \\
\hline RANGE: & end & & & & & herb \\
\hline RANGE: & cag & mex & & & & herb \\
\hline RANGE: & cr & & & & & herb \\
\hline RANGE: & $\mathrm{cr}$ & & & & & shrub \\
\hline RANGE: & $\mathrm{col}$ & cr & gui & nic & per & herb \\
\hline RANGE: & cag & col & $\operatorname{mex}$ & & & herb \\
\hline RANGE: & cr & gua & $\max$ & sal & & herb \\
\hline RANGE: & er & & & & & herb \\
\hline RANGE: & or & & & & & herb \\
\hline RANGE: & col & $\mathrm{cr}$ & gua & ven & & herb \\
\hline RANGE: & col & er & per & sag? & & \\
\hline RANGE: & er & & & & & \\
\hline
\end{tabular}

$210(134)$ CACTACEAE

Acanthoce reus

pentagonus (L.) Britt. Rose PROV: bo ca pa 000a: 0-1

Cerous

costaricensis (Weber) A. Berger PROV: ca

* crenatum (LindI,) G. Don Prov: pa
*grandilobua (Weber) Britt. a Rose Prov: ch pa vi

Epiphyilue

lepidocarpun (Weber) Britt. Rose

PROV: ch

macropterua (Lea.) Britt. Hose PhOV: bo ch

phyl Ianthus (L.) Mav,

*-evar. hookeril (Mav.) Kian.

PROV: ch

- -var. phyilanthus

- var. pittieri (Wober) kien.

PROV: bo ca ec ch en 000nt $0=1$ da pa

PROV: bo ca ch

- - -var. rubrocoronatua kian.

Prov: ca
Doon: we

000n: $0-1$

$000 \mathrm{~m}: 0-1$

000n: ve

000n: $0-1$

000n: 1 -2

000n: $0-1$

000n: 0-1

\section{RANGE: ang cag col or fia shrub hon sex nic sat tex ven}

RAMCE: or

epiph

ol inber

RAYGe: gus hon mex

epiph

herb

RANGE: or

cilinber

RANCE: or

RANCE: $C$ or

epiph

shrub

epiph

shrub

RAMGE: col or ecu

epiphyte

epiph

QAWGE: or

hert

RavoE: bel bol or mex nic epiph

sag? sao sac

shrub

RANCE: or

agion

RABCE: col secu shrub

op iphyte 
TAXON

FAMILY PROVINCES

*--var. costaricensis (Weber) Kimn.

PROV: bo $\quad 000 \mathrm{~m}: 0-1$

\section{Hylocereus} monacanthus (Lem.) Britt. \& Rose PROV: ca ch da pa vr 000m: 0-1
polyrhizus (Weber) Britt, \& Rose PROV: ch pa Vr $000 m: 0-1$

*stenopterus (Weber) Britt. \& Rose PROV: ch

000m: $0-1$

Nopa lea

cochenillifera (L.) SaIm-Dyck

intr. cult.

dejecta (Salm-Dyck) SaIm-Dyck PROV: ca 000m: 0-1

cult. PrOV: ca 000m: $0-1$

Opuntia

elatior Mill.

PROV: pa

000m: $0-1$

Pereskia

aculeata Mill,

bleo (H,B. K.) DC.

*colombiana Britt. \& Rose

PROV: pa

000m: $0-1$

PROV: da pa sa

000m: $0-1$

PROV: Wp

000m: we

*Pseudorhipsalis

himantoclada (Roland-Gosselin) Britt, \& Rose

PROV: ca cc

000m: $0-1$

Rhipsalis

*baccifera (J. Miller) Stearn PROV: bo ca cc ch da 000m: 0-1

\section{* Selen icereus}

*pteranthus (Link \& Otto) Britt. \& Rose

PROV: ch

000m: we

PROV: ca ch cn $000 \mathrm{~m}: 0-1$
Webe roce reus

panamensis Britt. \& Rose
RANGE: $\mathrm{cr}$

epiph

herb

RANGE: $c O I$

RANGE: col nic

RANGE: end

epiphyte

epiph

climber

epiph

climber

RANGE: nWt

shrub

RANGE: nWt

shrub

RANGE: COI cr

shrub

RANGE: ang col fla?

RANGE: nic sag?

RANGE: $C O I$

shrub

climber

shrub

tree

shrub

RANGE: $w r$

epiph

root

climber

RANGE: afr cag mex sag? epiph succ

RANGE: mex

epiphyte

RANGE: $\mathrm{cr}$ nic epiph root cl imber 
TAXON

FAMILY PROVINCES

ELEVATION (OOOM.)

210 (134) CACTACEAE cont.

\section{"Werckleocereus}

*tonduzii Weber

\section{Wittia \\ *amazonica Schum.}

panamensis Britt. \& Rose
PROV: da

PROV: cn da pa sa
RANGE: $\mathrm{cr}$

RANGE: per

RANGE: $\mathrm{col}$ or ven epiph

climber

ep iphyte

climb

214 (135) THYMELAEACEAE

\section{Daphnopsis}

*americana (Mill.) J,R. Johnst.

\#--subsp. caribaea (Griseb,) Nevl. PROV: cc he 000m: 0-1

Schoenobiblus

panamens is Stand I. L. 0 . Wms. PROV: bo da 000m: $0-1,1-2$

tree

\section{6 (136) LYTHRACEAE}

\section{Adenaria}

floribunda H,B,K.

\section{"Alza tea}

"verticillata R. \& P.

$\begin{array}{ll} & \text { PROV: Io } \\ \text { intr. PROV: Pa }\end{array}$

*auriculata Willd.

coccinea Rot tb.

latifolia L.

Cuphea

calophylla Cham. Schlecht.

carthagenens is (Jacq.) Macbr.

*elliptica Koehne

*--var. ol igostemon Koehne

epilobifolia koehne
PROV: ch ve

PROV: ca cn da pa 000m: 0-1

000m: $1-2$

000m: $1-2$

000a: $0-1$

000a: $0-1$

PROV: pa

000m: $0-1$

PROV: bo ca cc ch ch 000m: 0-1 da pa vr

PROV: bo ca cc ch ch 000m: $0-1,1+2$ he 10 pa

PROV: ca

PROV: pa

PROV: bo ca ce ch on 000m: $0-1,1-2$ pa $v r$
RANCE: cag mex sag sao shrub

RANGE: bol per tree

RANCE: col or epiph

$$
\text { shrub }
$$

RWGE: wdt

RANCE: ang as

oce sag tex

RANGE: ang cag nag? sag herb

RANCE: cag sao herb

RANCE: ang cag nag nao sag herb

RMACE: col mex herb

RANCE: or mex nio herb

RANGE: or ven nio shrub 
TAXON

FAMILY PROVINCES

ELEVATION (OOOM.)

RANGE OUTSIDE OF PANAMA

HABIT

216 (136) LYTHRACEAE cont.

"hyssopifolia H.B.K.

infund ibulum Koehne * serpyI I ifolia H.B.K. setosa Koehne

utriculosa Koehne

\section{Lafoensla}

punicifolia DC.

\section{*Lagerst roemia \\ * ind ica $L$. \\ Hspeciosa (L.) Pers. \\ *tomentosa PresI \\ *turbinata Koehne}

\section{* Lawsonia}

*inermis L.

Rotala

mexicana Cham. \& Schlecht.

ramosior (L.) Koehne
PROV: bo ce ch da pa 000m: $0-1,1-2$ $\mathrm{vr}$

PROV: cc ch pa 000m: $0-1,1-2$

PROV: ch pa

$000 \mathrm{~m}: 0-1$

PROV: bo ca ce cn da $000 \mathrm{~m}$ : $0=1$

PROV: ca cc ch cn da 000m: 0-1 he pa $v r$

PROV: ca da he pa 000m: $0-1$

intr. cult.

PROV: pa $000 \mathrm{~m}: 0-1$

PROV: ca ch pa

PRO

PROV: ca

intr. cult.

PROV: ca

PROV: cc pa sa

PROV: ca ch pa

PROV: bo ca pa 000m: $0-1$

000m: $0-1$

000m: $0-1$

000m: $0-1$

000m: $0-1$ $000 \mathrm{~m}: 0-1$

RANGE: cag
RANGE: cr
RANGE: sat
RANGE: bol cag col per
RANGE: cag mex

\section{9 (137) LECYTHIDACEAE}

\section{Couratari}

panamensis StandI.

* scottmorii Prance

\section{Couroupita}

cutteri Mort. \& Skutch

darienensis Pitt.

idol ica Dwyer

magnifica Dwyer

nicaraguensis DC.

odoratissima Seem.

parviflora StandI.

\section{Eschweilera}

*antioquensis Dugand \& Daniel

calyculata Pitt.

garagarae Pitt.

$\begin{array}{ll}\text { PROV: bo ca pa } & 000 \mathrm{~m}: 0-1 \\ \text { PROV: sa } & 000 \mathrm{~m}: \text { we } \\ & \\ \text { PROV: bo } & 000 \mathrm{~m}: 0-1 \\ \text { PROV: da } & 000 \mathrm{~m}: 0-1 \\ \text { PROV: vr } & 000 \mathrm{~m}: 0-1 \\ \text { PROV: da } & 000 \mathrm{~m}: 0-1 \\ \text { PROV: wp } & 000 \mathrm{~m}: 0-1 \\ \text { PROV: wp } & 000 \mathrm{~m}: 0-1 \\ \text { PROV: bo } & 000 \mathrm{~m}: 0-1 \\ & \\ \text { PROV: pa } & \\ \text { PROV: bo ca } & 000 \mathrm{~m}: 0-1 \\ \text { PROV: da } & 000 \mathrm{~m}: 0-1 \\ & \end{array}$

RANGE: $\mathrm{cr}$

RANGE: end

tree tree

RANGE: end

RANGE: end

RANGE: end

RANGE: end

RANGE: $c r$

RANGE: end

RANGE: end

\section{nic}

(1)

RANGE: COI

RANGE: $\mathrm{cr}$

RANGE: end tree

tree

tree

tree

tree

tree

tree

tree

tree 


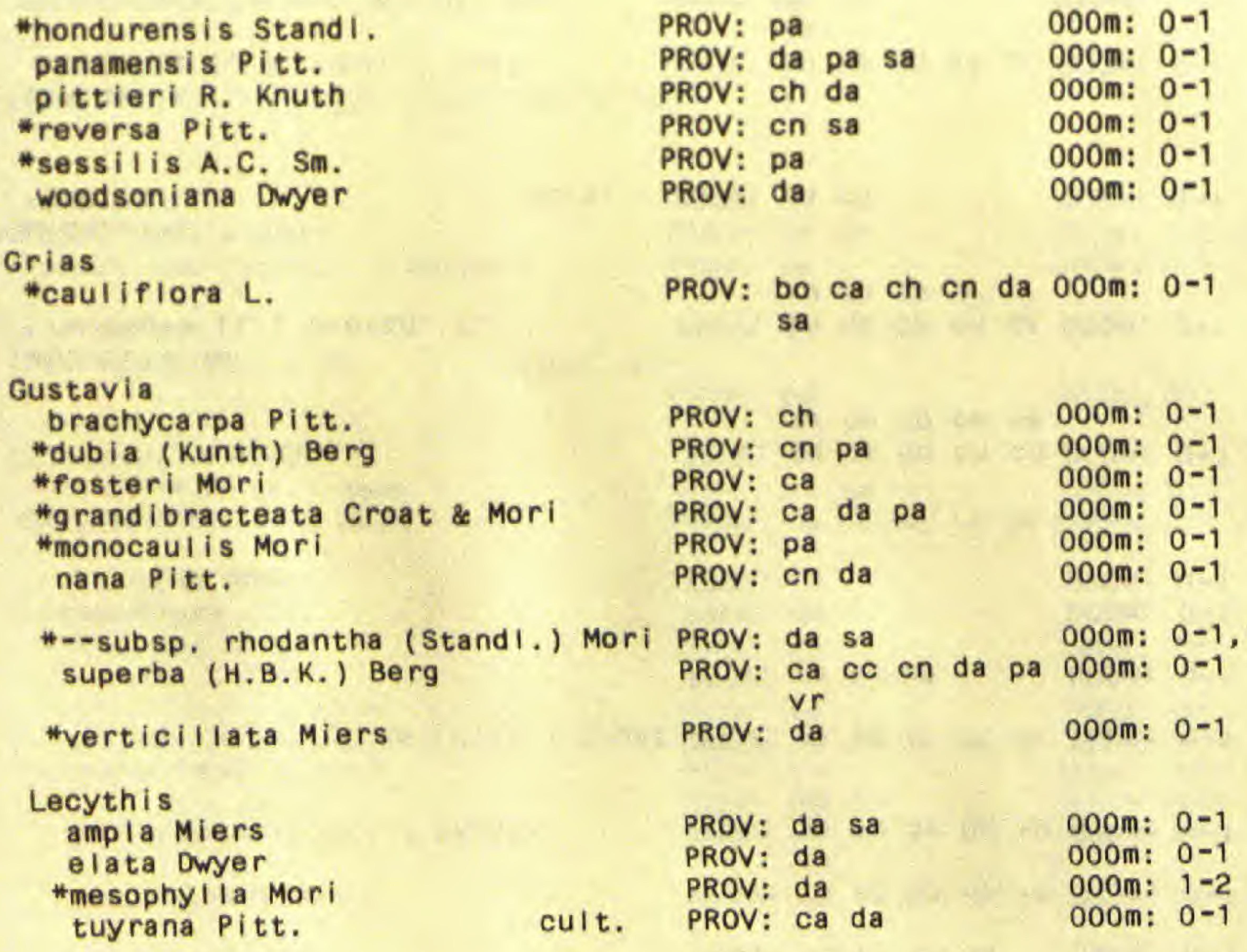

220 (138) RHIZOPHORACEAE

Cassipourea elliptica (Sw.) Poir.

\section{Rhizophora \\ brevistyla Salv. \\ \#harrisonii Leechm. \\ mangle L.}

samoens is (Hochr.) Salvoza
PROV: bo ca cc ch cn 000m: $0-1$ da pa sa

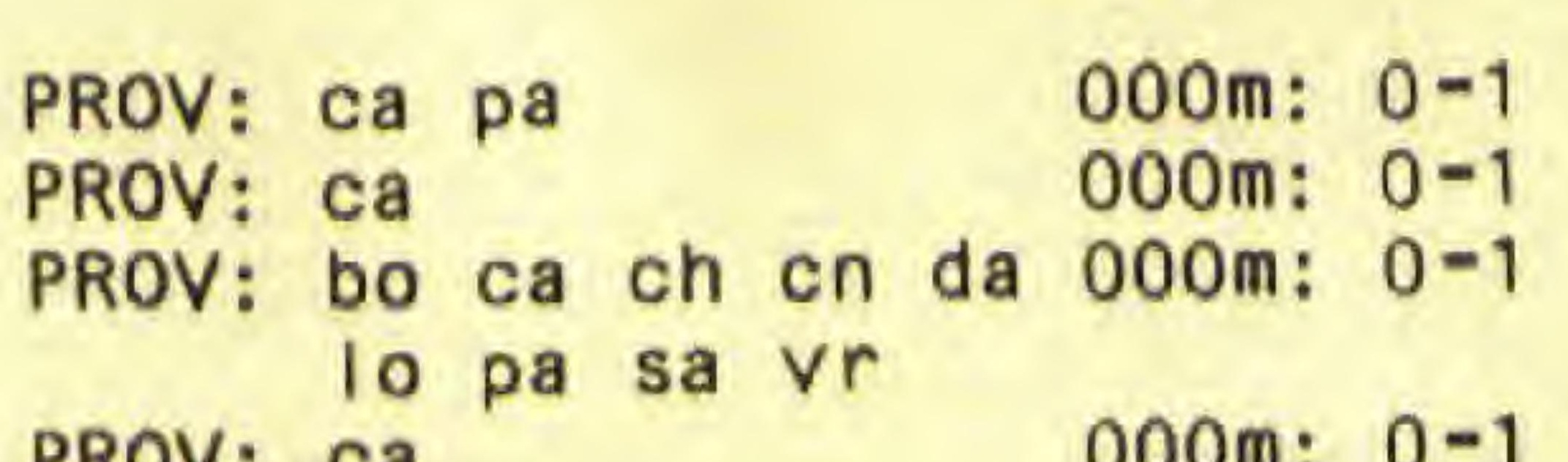

PROV: ca

000m: $0-1$

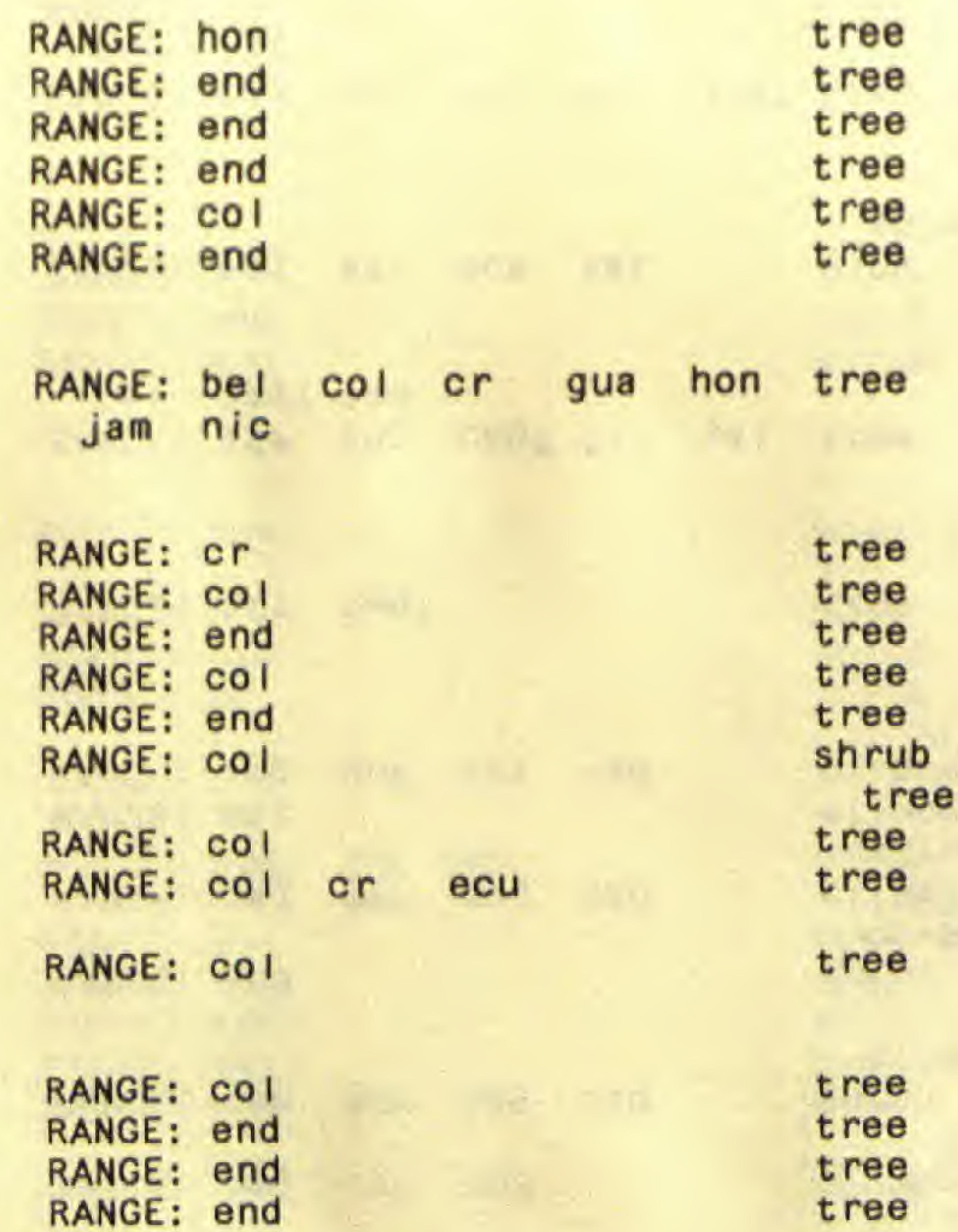

tree

$\begin{array}{ll}\text { RANGE: ang cag } & \begin{array}{c}\text { shrub } \\ \text { tree }\end{array} \\ & \\ \text { RANGE: ecu sag? } & \text { tree } \\ \text { RANGE: col ecu gui ven } & \text { tree } \\ \text { RANGE: afr ang cag? fla sag? tree } \\ \text { Sao } \\ \text { RANGE: cag ecu mex nao sag? shrub }\end{array}$


TAXON

FAMILY

PROVINCES

ELEVATION (OOOM.)

RANGE OUTSIDE OF PANAMA

HABIT

221 (139) COMBRETACEAE

Buchenavia

capitata (Vahl) Eichl.

PROV: pa vr

000m: $0-1$

Bucida

buceras $\mathrm{L}$.

Combretum

cacoucia Sandw.

decandrum Jacq.

fruticosum (LoefI.) Stuntz

I axum Jacq.

*--var. epiphyticum (Pitt.) Croat PROV: ca vr 000m: 0-1

--var. Iaxum

sambuense Pitt.

spinosum Bonpl.

Conoca rpus

erectus $L$.

--var. erectus

Laguncularia

racemosa (L.) Gaertn. $f$.

\section{*Quisqua I is}

*indica $L$.

\section{cult}

PROV: ca da pa

000m: $0-1$

PROV: da

$000 \mathrm{~m}: 0-1$

PROV: ca

000m: $0-1$

PROV: bo ca cc ch cn 000m: $0-1$ da he lo pa sa

PROV: bo ca ch cn da 000m: $0-1$ lo pa sa vr

Terminalia amazonia (J.F. Gmel.) Exel)

"bucioides StandI. \& L.O. Wms.

* ca tappa L.

*dichotoma G. Meyer

"edulis Blanco

$$
\text { intr. cult. }
$$

PROV: ca

*muelleri Benth.

intr. cult.

PROV: ca

*myriocarpa Huerck. \& MueII. - Arg. PROV: ca *oblonga (R. \& P.) Steud. 000m: $0-1$ lo pa

\begin{tabular}{|c|c|c|c|c|c|c|}
\hline RANGE: & ang & bol & sag? & sao & & tree \\
\hline RANGE: & ang & cag? & Pla & gui & sag? & tree \\
\hline $\begin{array}{l}\text { RANGE: } \\
\text { RANGE: }\end{array}$ & $\begin{array}{l}\text { bel } \\
\text { cag }\end{array}$ & $\begin{array}{l}\text { cag? } \\
\mathrm{col}\end{array}$ & $\begin{array}{l}\text { sag? } \\
\text { mex }\end{array}$ & sao & & $\begin{array}{l}\text { shrub } \\
\text { climber } \\
\text { climber }\end{array}$ \\
\hline RANGE: & cag & $\operatorname{mex}$ & sag & sao & & $\begin{array}{l}\text { shrub } \\
\text { climber }\end{array}$ \\
\hline RANGE: & end & & & & & $\begin{array}{l}\text { shrub } \\
\text { climber }\end{array}$ \\
\hline RANGE: & cag & $\operatorname{mex}$ & sag & sao & & $\begin{array}{l}\text { cl imber } \\
\text { shrub? }\end{array}$ \\
\hline RANGE: & sat & & & & & cl imber \\
\hline RANGE: & ang & cub & trt & ven & & climber \\
\hline RANGE: & afr & nwg? & & & & tree \\
\hline $\begin{array}{c}\text { RANGE: } \\
\text { per }\end{array}$ & $\begin{array}{l}\text { afr } \\
\text { sag? }\end{array}$ & $\begin{array}{l}\text { ang } \\
\text { sao }\end{array}$ & cag? & fla & gal & tree \\
\hline RANGE: & an & as & oce & sat & & $\begin{array}{l}\text { viny } \\
\text { shrub }\end{array}$ \\
\hline
\end{tabular}

RANGE: cag gui mex per sag? tree SaO trt RANGE: $\mathrm{cr}$

RANGE: cag

tree

tree

RANGE: $W r$

tree

RANGE: oce

tree

RANGE: oce

RANGE: COI Sao ven

tree

RANGE: $\mathrm{cr}$ 
TAXON

222 (140) MYRTACEAE

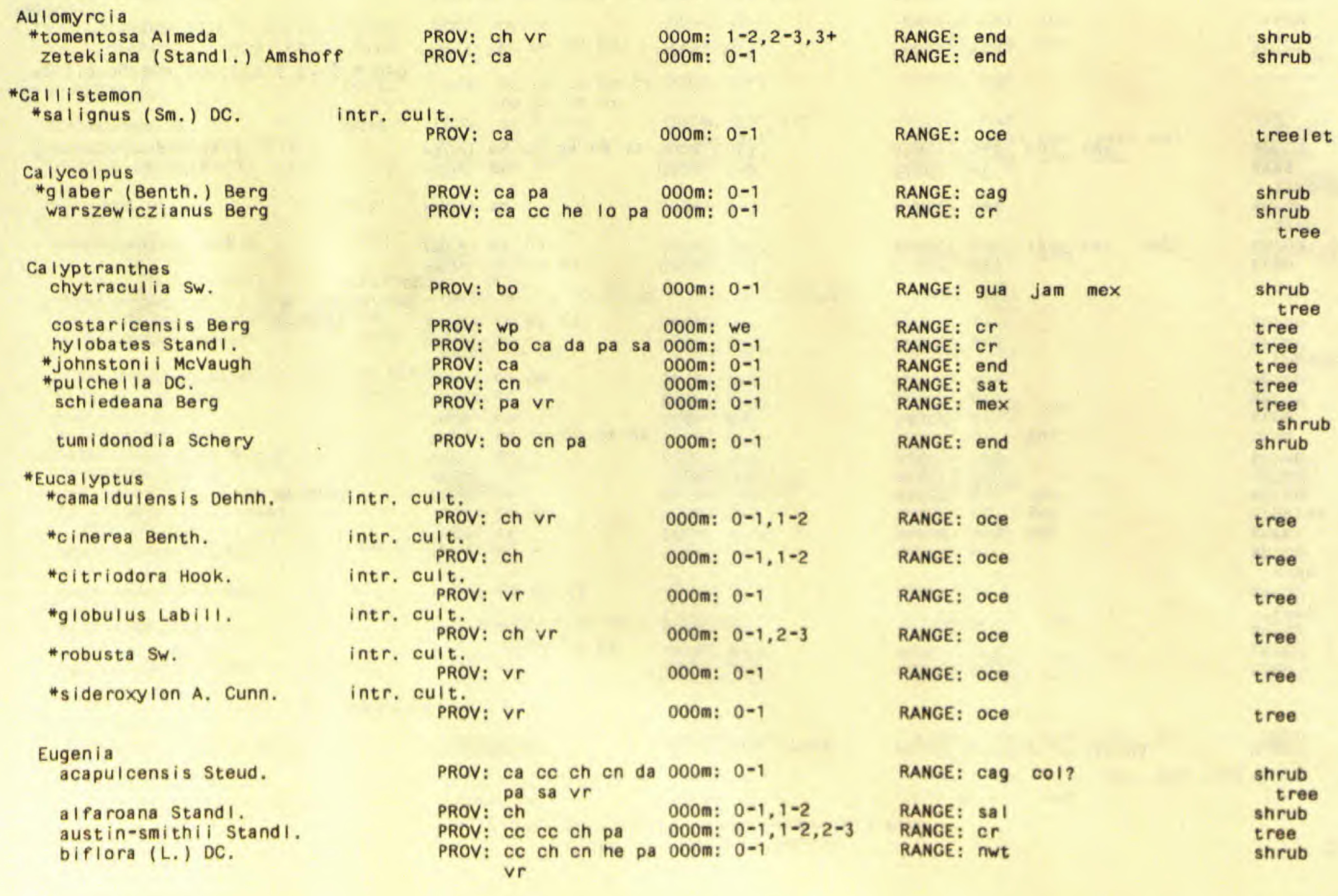


222 (140) MYRTACEAE cont.

\begin{tabular}{|c|c|c|c|c|c|c|}
\hline $\begin{array}{l}\text { cartagenens is Berg } \\
\text { chepensis Stand I. } \\
\text { chrysophyl I um Poir. }\end{array}$ & $\begin{array}{l}\text { PROV: } \\
\text { PROV: } \\
\text { PROV: }\end{array}$ & $\begin{array}{l}\text { ch } \\
\text { ca } \\
\text { bo }\end{array}$ & cn I & $10 \mathrm{p}$ & pa & $\begin{array}{l}\text { 000m: } \\
\text { 000m: } \\
000 \mathrm{~m}:\end{array}$ \\
\hline coloradensis Standl. & PROV: & ea & en & da & & 000m: \\
\hline costaricens is Berg & PROV: & da & $v r$ & & & 000m: \\
\hline (Sw.) wilid. & PROV: & ch & & & & 000m: \\
\hline "ga la lonens is (Griseb.) Urb. & PROV: & ca & pa & & & 000m: \\
\hline "monticola (Griseb.) McVaugh & PROV: & pa & & & & 000m: \\
\hline $\begin{array}{l}\text { nesiotica Standl, } \\
\text { octopleura Krua U Urb. }\end{array}$ & : & ca & & & & Om: \\
\hline $\begin{array}{l}\text { octopleura Krug \& Urb. } \\
\text { oerstedeana Berg }\end{array}$ & PROV: & ca & & & & 000m: \\
\hline $\begin{array}{l}\text { oerstedeana Berg } \\
\text { oreinoma Berg }\end{array}$ & : & ca & ch & cn & da pa & m: \\
\hline $\begin{array}{l}\text { oreinoma Berg } \\
\text { pittleri Standi. }\end{array}$ & $P F$ & ch & & & & m: \\
\hline $\begin{array}{l}\text { pittleri Standl. } \\
\text { "principlum McVaugh }\end{array}$ & PRO & pa & & & & Om: \\
\hline & & $\mathrm{ca}$ & da & & & \\
\hline $\begin{array}{l}\text { salamancana Standl. } \\
\text { *--var. hiraelfolia McVaugh }\end{array}$ & : & ca & & & & \\
\hline sysygioides (Miq.) M.R. Henderson & PROV: & 10 & pa & pa & & 000m: \\
\hline & & ca & ch & pa & & \\
\hline tapacumens is Ber & PROV: & ca & pa & & & OOm: \\
\hline uniflora L. & PROV: & ca & & & & 000m: \\
\hline $\begin{array}{l}\text { ler } \\
\text { nez }\end{array}$ & VV: & ch & & & & \\
\hline
\end{tabular}

\section{* Marl ierea}

*guildingiana (Griseb.) Krug \& Urb.

PROV: pa

000m: $0-1$

Myrcia

costaricensis Berg

coumeta (AubI.) DC.

*fallax (Rich.) DC.

*fosteri Croat

gatunens is StandI.

\#mollis (H.B.K.) DC.

*splendens (SW.) DC.

Myrciaria

floribunda (Willd.) Berg
PROV: bo cc ch pa vr 000m: $0-1,1-2$

PROV: bo

000m: $0-1$

PROV: ca ch cn $v r$ 000m: $0-1,2-3$

PROV: ca da pa 000m: $0-1$

PROV: bo ca ch $\mathrm{cn}$ da $000 \mathrm{~m}$ : $0-1,1-2$

PRov: pa

000m: $1-2$

PROV: cc ch cn da he 000m: $0-1,1-2$ lo pa

PROV: ca cc ch da lo $000 \mathrm{~m}: 0-1$

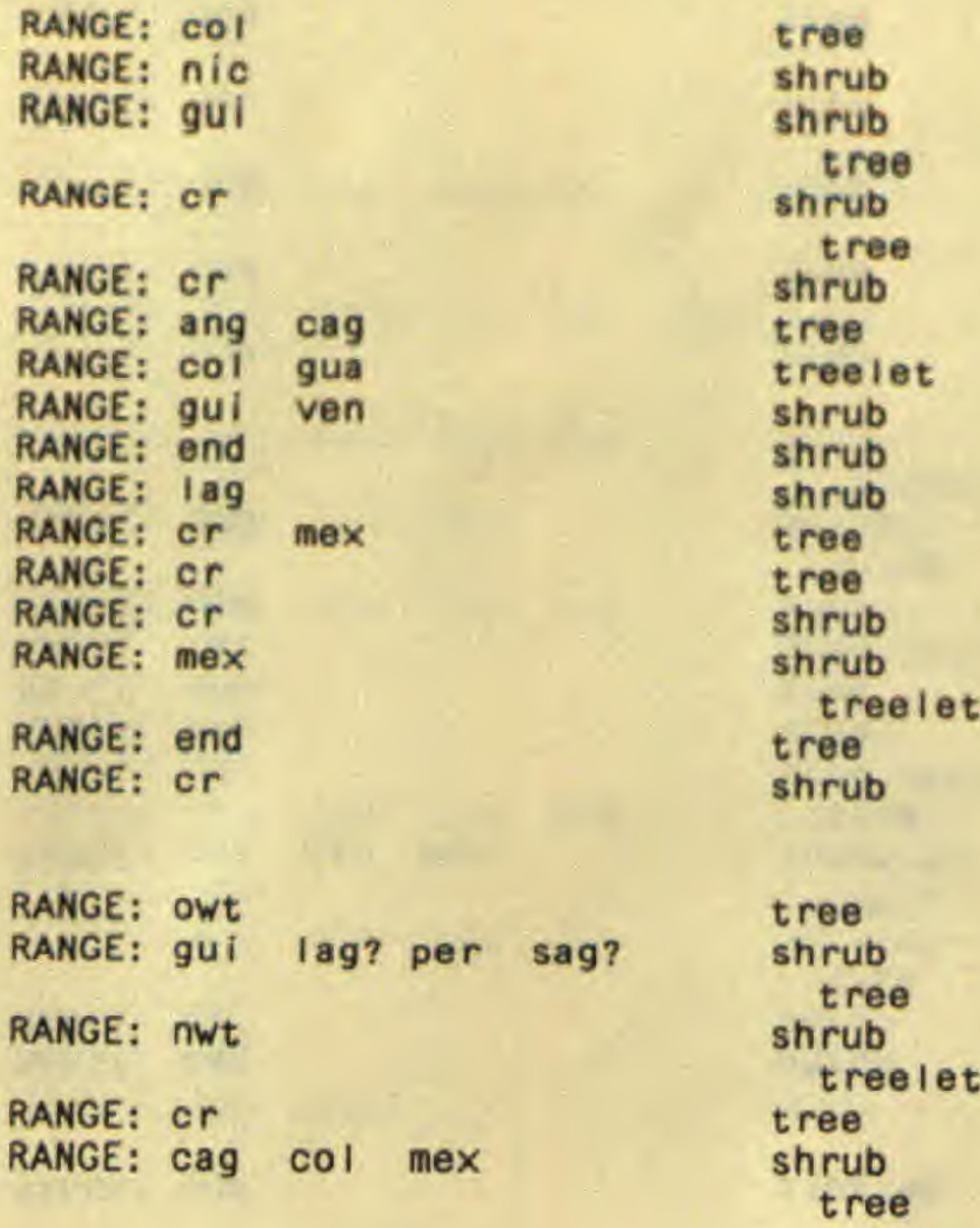

RANGE: ven

shrub

RANGE: $c r$

RANGE: cr gui

RANGE: ang sat

RANGE: end

RANGE: end

at

RANGE: $c O \mathrm{l}$ cr per RANGE: ang mex

per

RANGE: ang

ca shrub

tree

shrub

tree

treelet

tree

shrub

treelet

shrub

treelet 
TAXON

vismeaefolia (Benth.) Berg

intr. cult.

$$
\text { PROV: ca }
$$

000m: $0-1$

000m: $0-1$

Psidium

*anglohondurens is (Lund.) McVaugh friedrichsthal ianum (Berg) Nied.

PROV: bo ca ch pa 000m: $0-1$

guajava L.

guineense SW.

salutare (H.B.K.) Berg

cult. PROV: bo ca cc ch da 000m: 0-1

lo pa sa vr

sartorianum (Berg) Nied.

cult. PROV: ca cc ch

000m: $0-1,1-2$

PROV: da pa

\section{Syzyg ium \\ "cumini (L.) Skeels}

intr. cult.

000m: $0-1$

jambos (L.) Alston

PROV: bo ca ch cn pa 000m: $0-1,1-2,2-3$

malaccense (L.) Merr. \& Perry

$$
\text { intr. cult. }
$$

samaragnense (BIume) Merr. \& Perry

$$
\text { PROV: da vr } \quad 000 m: 0-1
$$

intr. cult.

PROV: ca pa

000m: $0-1$

Ugni

*montana (Benth.) Berg

warscewiczif Berg

PROV: ch

PROV: $\mathrm{ch}$

000m: $3+$

000m: $2-3,3+$ intr. cult. nat.

RANGE: gui

shrub

tree

RANGE: bel gua

RANGE: cag mex

RANGE: NWt

shrub

tree

shrub

tree

RANGE: $n W t$

RANGE: cag? hon sag? sal

shrub

subshrub

shrub

tree

\section{3 (141) MELASTOMATACEAE}

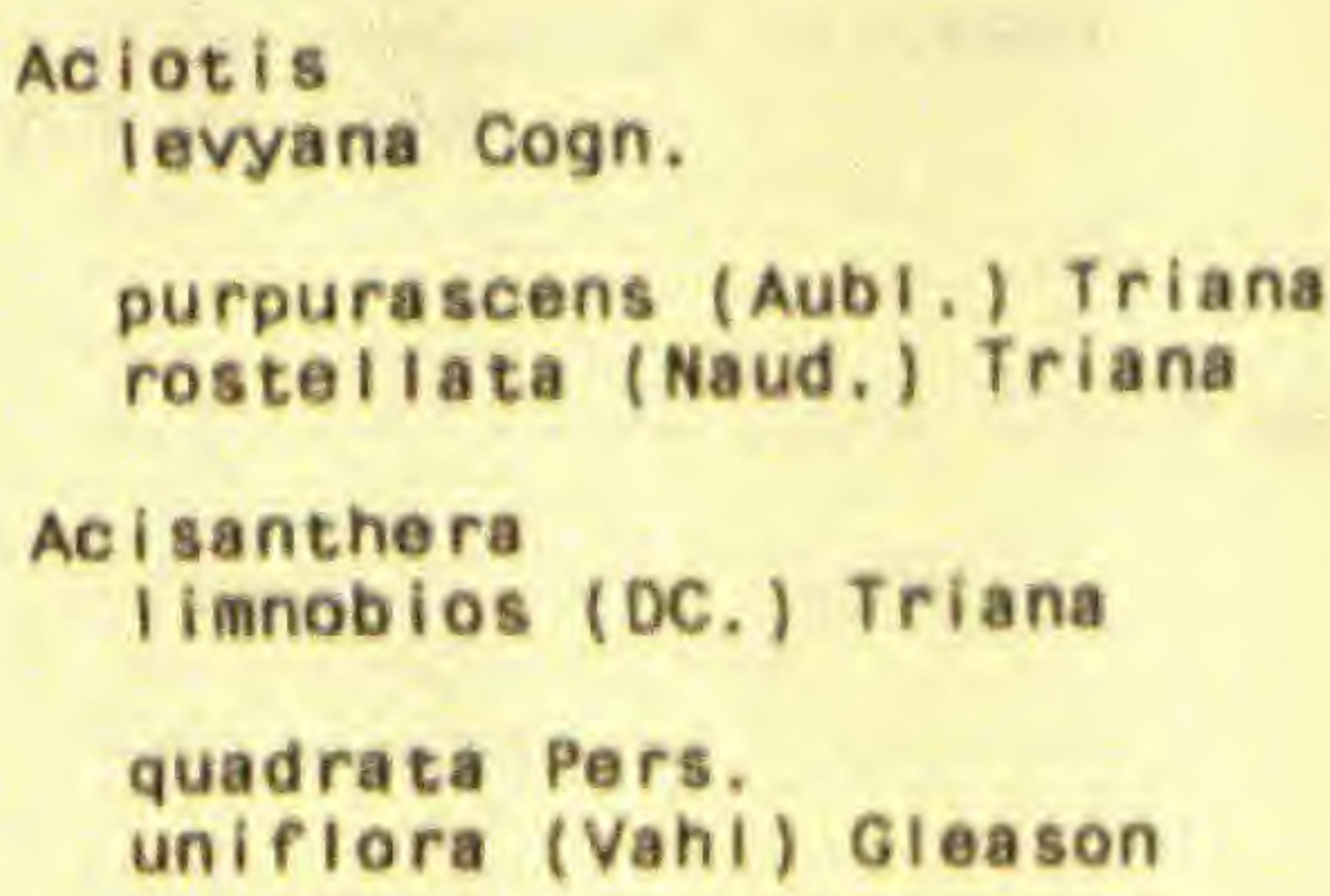

PROV: bo ca ce on pa 000m: 0-1 sa $\mathrm{Vr}$

000m: $0-1$ PROV: bo cc cn da sa 000m: $0-1$

PROV: CC

000m: $0-1$

PROV: cc ch pa vr

PROV: vr 000m: $0-1$

\section{treelet}

tree

tree

tree

shrub

shrub

$\begin{array}{ll}\text { RANGE: asi cag } & \text { treelet } \\ \text { RANGE: as } i & \text { tree } \\ \text { RANGE: as } i & \text { tree } \\ \text { RANGE: as i wdt } & \text { tree } \\ \begin{array}{ll}\text { RANGE: mex } & \text { shrub } \\ \text { RANGE: end } & \text { shrub }\end{array}\end{array}$

RANGE: col gua
RANGE: sao sat trt
RANGE: cag hex
RANGE: col cub gua gui hon herb
Sao ven herb
RANGE: cag col gag mex ven herb
RANGE: cr sag


TAXON

Ade lobotrys adscendens (SW.) Triana

\section{*jefens is Almeda}

*panamens is Almeda

\section{Arthrostema \\ a latum Triana}

*ciliatum R. \& $P$.

\section{Axinaea}

costaricensis Cogn.

Bellucia

*axinanthera Triana

* grossularioides (L.) Triana

Blakea

brunnea Woods, \& Schery

calycosa Gleason

*crassifolia Almeda

crinita Gleason

*elliptica (Gleason) Almeda

foliacea Gleason

gracilis Hemsl.

\section{*micrantha Almeda}

parvifolia Gleason

pauciflora Gleason

*tuberculata J.D. Sm.

\section{*wilburiana Almeda}

woodsonii Woods, \& Seib.
PROV: bo ca cc ch cn 000m: $0-1$ da pa sa vr

PROV: pa

$000 \mathrm{~m}: 0-1$ 000m: $0-1$

PROV: ca pa 000m: $0-1$ PROV: bo ca cc ch cn 000m: $0-1,1-2$

PROV: bo $\mathrm{ch}$

000m: $1-2,2-3$

PROV: ca ch da

PROV: bo ca da sa

000m: $0-1$

000m: $0-1$

PROV: ch

PROV: $\mathrm{CC}$

PROV: $\mathrm{CC}$

PROV: CC

PROV: bo ch

PROV: bo cc sa

PROV: bo $\mathrm{ch}$

PROV: $v r$

PROV: CC

PROV: ch pa sa

$000 \mathrm{~m}: 1-2,2-3$

000m: $0-1$

000m: $0-1$

000m: 1 -2

$000 \mathrm{~m}: 1-2,2-3$

000m: $0-1$

$000 m: 1-2,2-3$

000m: $1-2$

000m: $1-2$

000m: $1-2$

PROV: cc ch cn pa vr 000m: $0-1,1-2$

PROV: cc sa

000m: $0-1$

PROV: ch pa
$000 m: 1-2,2-3,3+$

\begin{tabular}{|c|c|c|c|c|c|c|}
\hline $\begin{array}{c}\text { RANGE: } \\
\text { hon } \\
\text { RANGE: } \\
\text { RANGE: }\end{array}$ & $\begin{array}{l}\text { bel } \\
\text { jam } \\
\text { end } \\
\text { end }\end{array}$ & $\begin{array}{l}\text { cag } \\
\text { mex }\end{array}$ & $\begin{array}{l}\mathrm{cr} \\
\mathrm{nic}\end{array}$ & $\begin{array}{l}\text { gua } \\
\text { per }\end{array}$ & $\begin{array}{l}\text { gui } \\
\text { sat }\end{array}$ & $\begin{array}{l}\text { climber } \\
\text { cl imber } \\
\text { tree }\end{array}$ \\
\hline $\begin{array}{c}\text { RANGE: } \\
\text { RANGE: } \\
\text { mex }\end{array}$ & $\begin{array}{l}\text { cr } \\
\text { bol } \\
\text { per }\end{array}$ & $\begin{array}{l}\text { mex } \\
\text { cag } \\
\text { trt }\end{array}$ & $\begin{array}{l}\text { sal } \\
\text { col } \\
\text { ven }\end{array}$ & $\begin{array}{l}\text { ven } \\
\text { ecu }\end{array}$ & gui & $\begin{array}{l}\text { herb } \\
\text { herb }\end{array}$ \\
\hline RANGE: & $\mathrm{cr}$ & & & & & tree \\
\hline $\begin{array}{l}\text { RANGE: } \\
\text { RANGE: }\end{array}$ & $\begin{array}{l}\text { cag } \\
\text { cag }\end{array}$ & $\begin{array}{l}\operatorname{mex} \\
\text { mex }\end{array}$ & $\begin{array}{l}\text { per } \\
\text { per }\end{array}$ & $\begin{array}{l}\text { sat } \\
\text { sao }\end{array}$ & & $\begin{array}{l}\text { tree } \\
\text { treelet }\end{array}$ \\
\hline $\begin{array}{l}\text { RANGE: } \\
\text { RANGE: }\end{array}$ & $\begin{array}{l}\text { end } \\
\text { cr }\end{array}$ & I & & & & $\begin{array}{l}\text { tree } \\
\text { epiph }\end{array}$ \\
\hline RANGE: & $\mathrm{cr}$ & & & & & $\begin{array}{l}\text { shrub } \\
\text { epiph } \\
\text { shrub }\end{array}$ \\
\hline $\begin{array}{l}\text { RANGE: } \\
\text { RANGE: }\end{array}$ & $\begin{array}{l}\text { end } \\
\text { end }\end{array}$ & -7 & -17 & 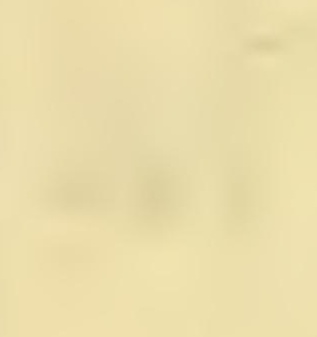 & & $\begin{array}{l}\text { shrub } \\
\text { epiph }\end{array}$ \\
\hline RANGE: & $\mathrm{cr}$ & & & & & $\begin{array}{l}\text { Shrub } \\
\text { epiph } \\
\text { shrub }\end{array}$ \\
\hline RANGE: & $\mathrm{cr}$ & & & & & $\begin{array}{l}\text { epiph } \\
\text { shrub }\end{array}$ \\
\hline RANGE: & end & 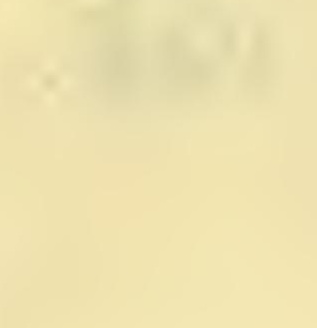 & & & & $\begin{array}{l}\text { epiph } \\
\text { shrub }\end{array}$ \\
\hline RANGE: & end & & & & & tree \\
\hline RANGE: & $\mathrm{cr}$ & & & & & $\begin{array}{l}\text { tree } \\
\text { shrub }\end{array}$ \\
\hline $\begin{array}{l}\text { RANGE: } \\
\text { RANGE: }\end{array}$ & end & ( & & & & $\begin{array}{l}\text { epiph } \\
\text { shrub } \\
\text { epiph } \\
\text { shrub }\end{array}$ \\
\hline & & & & & & $\begin{array}{l}\text { shrub } \\
\text { tree }\end{array}$ \\
\hline
\end{tabular}

Centradenia

inaequilateralis (Cham. \& Schlecht.) G. Don

*paradoxa (Kranzl.) Almeda

PROV: bo $\mathrm{cc} \mathrm{ch}$

000m: $0-1,1-2$

000m: $0-1$ 
223 (141) MELASTOMATACEAE cont.

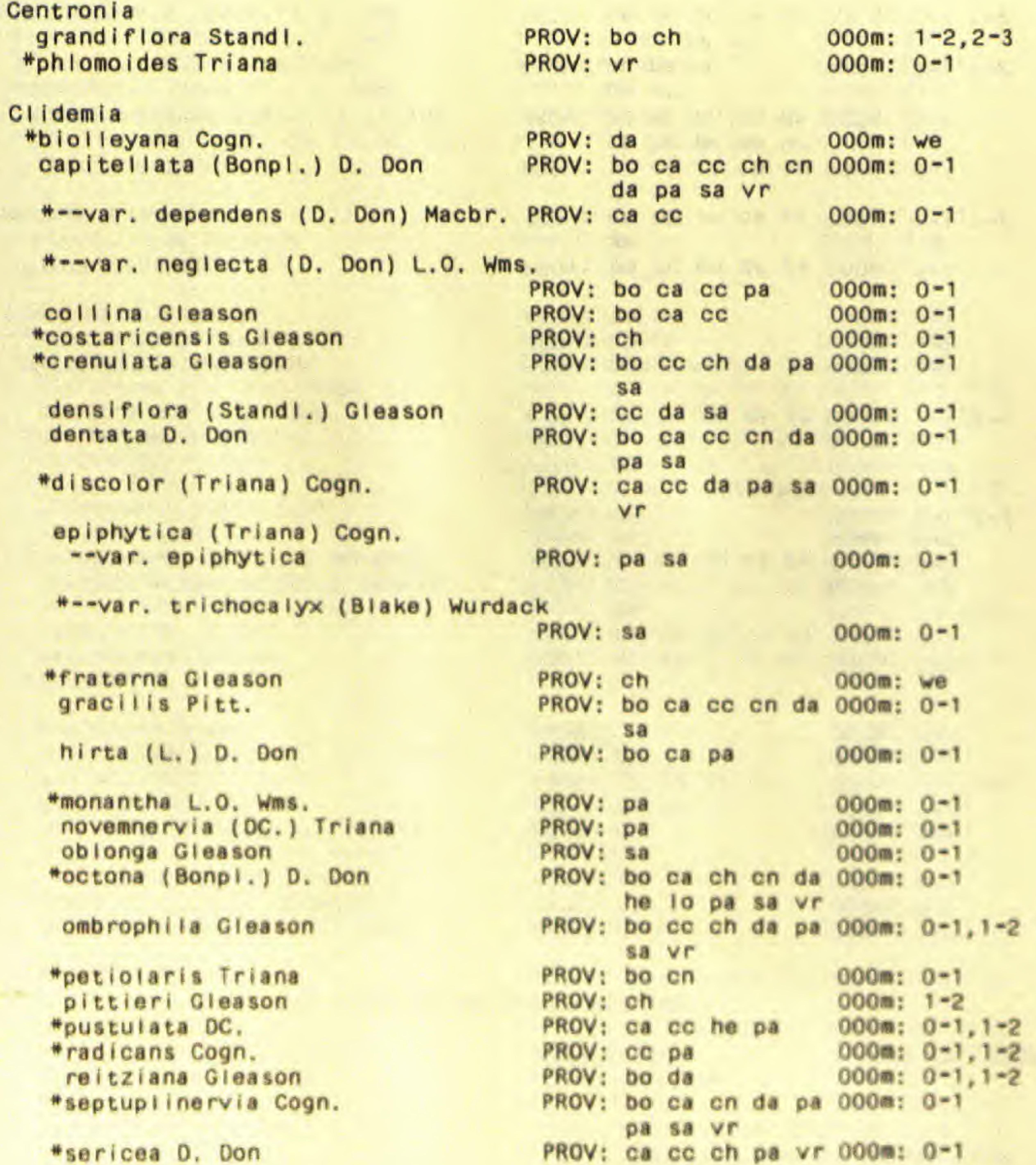

RANGE: end RANGE: $c r$

tree tree

RANGE: $c r$ RANGE: ang cag col mex per shrub RANGE: bol cag cub mex per shrub sag sao

RANGE: bel cag cub sag sao shrub RANGE: end shrub RANGE: or $\quad$ shrub RANGE: col or shrub

RANGE: cag col ecu shrub RANGE: bol cag mex per sao shrub

RANGE: col or ecu per shrub

RANGE: bol cag col per epiph

RANGE: cag col ecu per epiph

RANGE: Shrub

RANGE: $\mathrm{COI}$ shrub

RANGE: ang bol cag mex per shrub sag. sao hon mex nic ven shrub

RANGE: cag sag shrub

RANGE: end shrub

RANGE: cag cub jam mex per shrub

sag sao

RANGE: or nic shrub

RANGE: cag mex shrub

RANCE: end mex shrub

RANGE: col cr nic trt shrub

RANGE: or eou nic per shrub

RANGE: or nic shrub

RANCE: bel cag col ecu jan shrub

per sag sao col nex sao shrub 
TAXON

FAMILY

PROVINCES

ELEVATION (OOOM.)

223 (141) MELASTOMATACEAE cont.

\section{* sessilliflora (Naud.) Cogn. setosa (Triana) Gleason strigiliosa (SW.) DC.}

\section{taurina Gleason \\ * tetrapetal a Almeda \\ *trichosantha Almeda \\ *urceola ta DC. \\ *ut leyana Almeda \\ Conostegia \\ *attenuata Triana \\ bracteata Triana \\ hirsuta Gleason \\ * icosandra (SW.) Urb. \\ macrantha Triana \\ micrantha Stand $I$. \\ montana (SW.) D. Don \\ *montealegreana Cogn. \\ oerstediana Triana \\ polyandra Benth.}

chiriquensis Woods. \& Schery * cinnamonea (Beurl.) Wurdack

* rufescens Naud.

\section{setosa Triana}

speciosa Naud.

subcrustulata (BeurI.) Triana

*tenuifolia J.D. Sm.

Xa lapens is (Bonpl.) D. Don

\section{* Graffenrieda}

*bel la Almeda

"galeottil (Naud.) L.0. Wms.

*gracilis (Triana) L.0. Wms.

*micrantha (Gleason) L.O. Wms.
PROV: da 000m: $1-2$

PROV: CC 000m: $0-1$

PROV: pa $\quad 000 \mathrm{~m}: 0-1$

PROV: bo

PROV: bo $\mathrm{ch}$

Prov: cc pa sa $v r$ 000m: $0-1,1-2$

PROV: pa

$000 \mathrm{~m}: 0-1$

PROV: ch

$000 \mathrm{~m}: 0-1$

PROV: $C C \mathrm{Vr}$

000m: $0-1$

PROV: bo ca ch cn da 000m: $0-1$

PROV: ch

PROV: $c$ a ce cn da $P a$ o $000 \mathrm{~m}: 0-1$

PROV: bo

Om: $0-1$

PROV: $c h$

000m: $0-1$

PROV: ch

000m: $1-2$

PROV: bo CC O00m: $0=1$

PROV: bo ch cn da pa $000 \mathrm{~m}$ : $1=2,2-3$

PROV: $\mathrm{cc}$

000m: $0-1$

000m: $1-2$

PROV: bo on 000m: $0-1$

PROV: bo ce en da pa 000m: $0-1$

$$
\text { sa }
$$

PROV: cc ch cn da pa 000m: $0-1,1-2$ sa vr

PROV: bo ca ec cn en 000m: $0-1$ da he pa sa vr

PROV: bo ce ch en da 000m: 0-1 pa vr

000m: $0-1,1-2$

PROV: $\mathrm{CC}$ da

PROV: bo ca ce ch cn 000m: $0-1,1-2$ da pa sa vr
PROV: ch vr

PROV: pa vr

PROV: Vr

PROV: bo
$000 \mathrm{~m}: \quad 0-1,1=2,2-3$

000m: $0-1,1-2$

000m: $0-1$

000m: $0-1,1-2$

\begin{tabular}{|c|c|c|c|c|c|c|}
\hline RANGE: & col & cr & ecu & per & & shrub \\
\hline RANGE: & cag & cr & $\operatorname{mex}$ & & & \\
\hline $\begin{array}{c}\text { RANGE: } \\
\text { sag }\end{array}$ & ang & bel & cag & gua & per & shrub \\
\hline RANGE: & end & 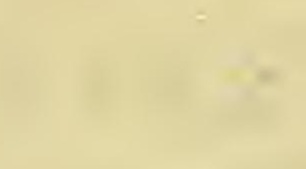 & & & & shrub \\
\hline RANGE: & end & & & & & shrub \\
\hline RANGE: & end & & & & & shrub \\
\hline RANGE: & bel & hon & sao & ven & 1 & shrub \\
\hline RANGE: & cr & & & & & shrub \\
\hline RANGE: & $\mathrm{col}$ & $c r$ & ecu & & & shrub \\
\hline RANGE: & cr & nic & & & & shrub \\
\hline RANGE: & end & & & & & tree \\
\hline RANGE: & $\mathrm{col}$ & nic & & $\mathrm{H}$ & & shrub \\
\hline RANGE: & end & & & & & shrub \\
\hline $\begin{array}{c}\text { RANGE: } \\
\text { nic }\end{array}$ & $\begin{array}{l}\text { bel } \\
\text { ven }\end{array}$ & $\mathrm{col}$ & $\mathrm{cr}$ & hon & $\operatorname{mex}$ & tree \\
\hline RANGE: & $\mathrm{cr}$ & nic & & & & tree \\
\hline RANGE: & $c r$ & nic & & & & tree \\
\hline RANGE: & ang & cag & $\mathrm{col}$ & cr & ven & tree \\
\hline RANGE: & $\mathrm{cr}$ & & & & & shrub \\
\hline $\begin{array}{l}\text { RANGE: } \\
\text { RANGE: }\end{array}$ & $\begin{array}{l}\mathrm{cr} \\
\mathrm{col}\end{array}$ & nic & & & & $\begin{array}{l}\text { tree } \\
\text { shrub }\end{array}$ \\
\hline RANGE: & ang & col & $\mathrm{cr}$ & nic & & $\begin{array}{c}\text { tree } \\
\text { shrub } \\
\text { tree }\end{array}$ \\
\hline RANGE: & cag & $\mathrm{col}$ & $\operatorname{mex}$ & ven & ill & shrub \\
\hline RANGE: & cag & col & $\operatorname{mex}$ & ven & wit & shrub \\
\hline RANGE: & $\mathrm{col}$ & $\mathrm{cr}$ & nic & & & shrub \\
\hline RANGE: & cr & nic & & & & shrub \\
\hline RANGE: & cag & $\mathrm{col}$ & cub & mex & & $\begin{array}{c}\text { tree } \\
\text { shrub } \\
\text { tree }\end{array}$ \\
\hline RANGE: & end & & & & & tree \\
\hline & 1 & ecu & gua & $\operatorname{mex}$ & per & $\begin{array}{l}\text { shrub } \\
\text { tree }\end{array}$ \\
\hline $\begin{array}{l}\text { RANGE: } \\
\text { RANGE: }\end{array}$ & $\begin{array}{l}\text { bol } \\
\text { end }\end{array}$ & col & ecu & per & ven & $\begin{array}{l}\text { shrub } \\
\text { shrub } \\
\text { tree }\end{array}$ \\
\hline
\end{tabular}




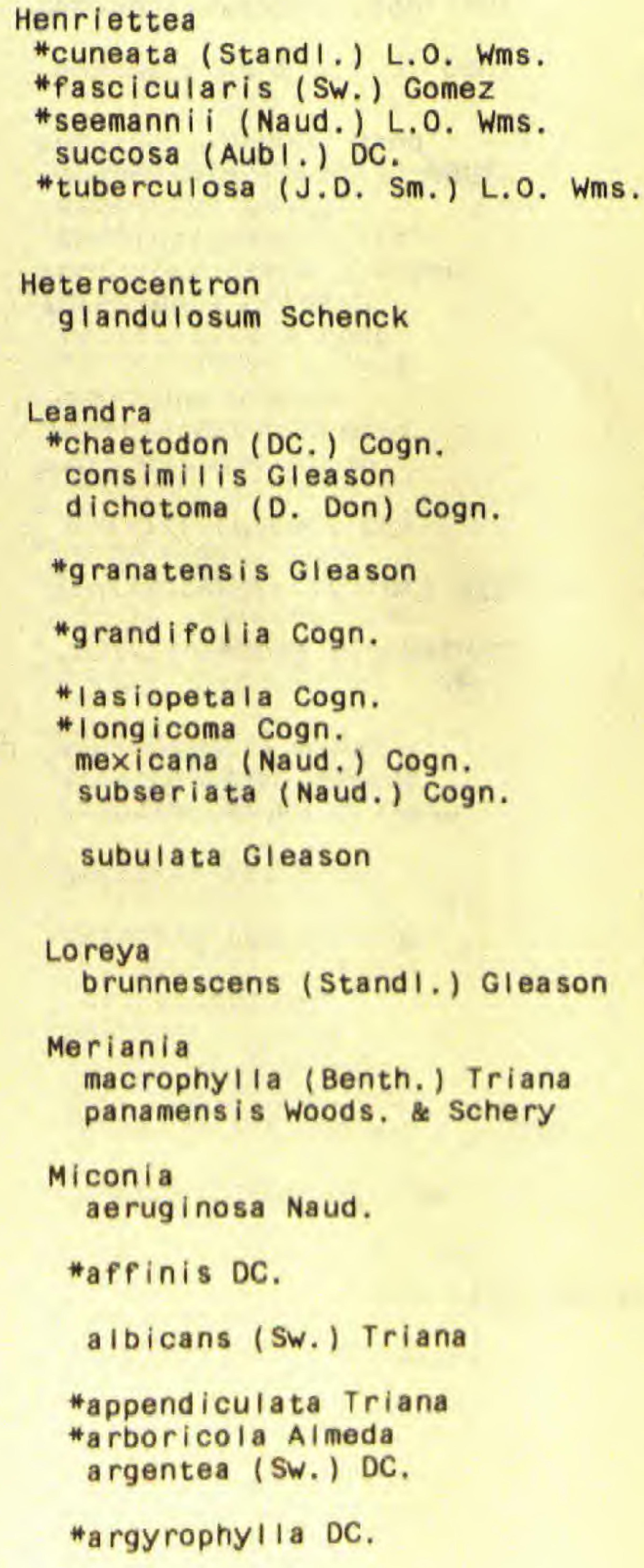

PROV: $c c$ da

PROV: bo ca $c c$ cn da $000 \mathrm{~m}$ : $0-1,1-2$ pa sa $\vee r$

PROV: bo ca cc ch cn 000m: $0-1$

PROV: ca ce ch da pa 000m: $0-1,1-2$ $v r$

PROV: $v r$

PROV: da

PROV: bo

000m: $0-1$

$000 \mathrm{~m}: 0-1$

PROV: bo cc ch da pa 000m: $1-2,2-3$

PROV: bo

000m: $0-1$

PROV: ch

000m: $1-2$

PROV: ch

000m: $2-3,3+$

PROV: ch cn

000m: $0-1,1-2$

PROV: bo ca cc on da 000m: $0-1,1-2$ pa sa

PROV: cc ch da pa vr 000m: $0-1,1-2$

PROV: cn pa sa $\quad 000 m: 0-1$

PROV: $c c$ ch 000m: $0-1,1-2$

PROV: ca ce ch cn da 000m: $0-1,1-2$ lo pa sa vr

PROV: da

000m: $1-2$ co $00 \mathrm{~m}: 0-1$

\begin{tabular}{|c|c|c|c|c|c|c|}
\hline RANGE: & bel & $\mathrm{cr}$ & gua & & & shrub \\
\hline RANGE: & ang & cag & & & & tree \\
\hline RANGE: & col & $\mathrm{cr}$ & nic & & & tree \\
\hline RANGE: & cag & gui & $\operatorname{mex}$ & sao & trt & tree \\
\hline RANGE: & $\mathrm{cr}$ & nic & & & & $\begin{array}{c}\text { shrub } \\
\text { tree }\end{array}$ \\
\hline $\tan ^{2}$ & t & & & & & $45=$ \\
\hline RANGE: & $\mathrm{cr}$ & gua & sa I & & & $\begin{array}{c}\text { subshrub } \\
\text { herb }\end{array}$ \\
\hline RANGE: & $\mathrm{col}$ & ecu & per & sao & ven & shrub \\
\hline RANGE: & $\mathrm{cr}$ & ven & & & & tree \\
\hline RANGE: & cag & mex & sag & $x^{2}=2$ & i. & shrub \\
\hline RANGE: & $\mathrm{col}$ & $\mathrm{cr}$ & nic & ven & 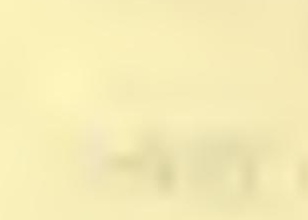 & shrub \\
\hline RANGE: & $\mathrm{cr}$ & nic & & & & shrub \\
\hline RANGE: & $\mathrm{cr}$ & & & & & shrub \\
\hline RANGE: & cag & $\mathrm{col}$ & ecu & per & sao & shrub \\
\hline RANGE: & cag & col & $\operatorname{mex}$ & & & shrub \\
\hline RANGE: & cag & col & ecu & mex & ven & $\begin{array}{c}\text { shrub } \\
\text { tree }\end{array}$ \\
\hline RANGE: & $\mathrm{cr}$ & $x_{1}$ & $x \rightarrow$ & & & $\begin{array}{l}\text { epiph } \\
\text { shrub }\end{array}$ \\
\hline & $=4$ & here & 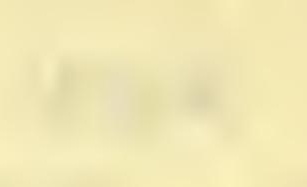 & & & $y^{2}$ \\
\hline RANGE: & or & nic & thent & -2 & $1=-7$ & tree \\
\hline $\begin{array}{l}\text { RANGE: } \\
\text { RANGE: }\end{array}$ & $\begin{array}{l}\text { cr } \\
\text { end }\end{array}$ & gua & mex & ven & & $\begin{array}{l}\text { tree } \\
\text { tree }\end{array}$ \\
\hline RANGE: & cag & col & mex & ven & & $\begin{array}{c}\text { shrub } \\
\text { tree }\end{array}$ \\
\hline $\begin{array}{c}\text { RANGE: } \\
\text { sao }\end{array}$ & $\begin{array}{l}\text { cag } \\
\text { trt }\end{array}$ & $\mathrm{col}$ & $\operatorname{mex}$ & per & sag & tree \\
\hline RANGE: & ang & bol & cag & mex & sao & $\begin{array}{c}\text { shrub } \\
\text { tree }\end{array}$ \\
\hline $\begin{array}{l}\text { RANGE: } \\
\text { RANGE: }\end{array}$ & $\begin{array}{l}\mathrm{cr} \\
\mathrm{cr}\end{array}$ & & nic & per & ven & $\begin{array}{l}\text { tree } \\
\text { climber }\end{array}$ \\
\hline RANGE: & cag & mex & & & & $\begin{array}{c}\text { shrub } \\
\text { tree }\end{array}$ \\
\hline RANGE: & per & & & & & shrub \\
\hline
\end{tabular}


223 (141) MELASTOMATACEAE cont.

*astroplocama J.D. Sm.

barbinervis (Benth.) Triana

* benthamiana Triana

*biperulifera Cogn.

*brenesi i Standi.

calvescens DC.

caudata (Bonpl.) DC.

* centrodesma Naud.

* centronioides Gleason

* centrosperma Almeda

* chionophil a Naud.

\section{*chiriquiensis Almeda}

*chrysophyila (Rich.) Urb.

ciliata (Rich.) DC.

*cionotricha Uribe

*coloradens is Almeda

* concinna Almeda

costaricensis Cogn.

* cremadena Gleason

* curvipetiolata Gleason

cuspidatissima pitt.

* desmantha Benth.

*dodecandra (Desr.) Cogn.

*dol ichorrhyncha Naud.

*donaeana Naud.

*donnell-smithii Cogn.

* dorsil oba Gleason

*elata (SW.) DC

*flavida Cogn.

*gentryi Wurdack

goniostigma Triana

gracilis Triana

*grandidentata Almeda

* gratissima Benth.

*holosericea (L.) DC.
PROV: vr

000m: $0-1$

PROV: bo $\mathrm{cc}$ da

PROV: $V r$

PROV: bo

PROV: ch

000m: $0-1$

000m: 1-2

000m: 2-3

000m: $0-1$

PROV: da

000m: $0-1$

PROV: $\mathrm{cc} \mathrm{ch}$

000m: $1-2$

PROV: ca cc cn da pa 000m: 0-1 sa vr

PROV: CC sa

000m: $0-1$

PROV: bo

000m: $0-1$

000m: $3+$

PROV: bo ch

$000 m: 1-2,2-3$

PROV: da

000m: $0-1,1-2$

PROV: ca da pa $v r$

000m: $0-1$

PROV: cn da

PROV: bo ch

000m: $0-1$

000m: $1-2$

000m: $1-2,2-3$

PROV: bo ch da

000m: $1-2,2-3$

PROV: ch

PROV: pa

PROV: $c h$

PROV: ch da

PROV: cc da pa $v r$

PROV: $\mathrm{ch}$ da

$000 \mathrm{~m}: 2-3$

000m: $0-1$

000m: $1-2,2-3$

000m: $0-1$

$000 \mathrm{~m}: 0-1,1-2$

000m: $0-1$

PROV: ca cc ch cn 000m: $0-1,2-3$

PROV: da

PROV: cc da pa $v r$ 000m: $0-1,1-2$

PROV: ca pa

PROV: $c h$

PROV: da

PROV: da pa

PROV: bo cc cn da pa 000m: $0-1$ sa

PROV: $V r$

$000 \mathrm{~m}: 0-1$

\begin{tabular}{|c|c|c|c|c|c|c|}
\hline RANGE: & 0. & $c$ & per & & & $\begin{array}{c}\text { shrub } \\
\text { tree }\end{array}$ \\
\hline RANGE: & cag & nic & per & sao & & shrub \\
\hline RANGE: & col & er & ecu & per & & shrub \\
\hline RANGE: & $\mathrm{er}$ & & & & & shrub \\
\hline RANGE: & $\mathrm{cr}$ & nic & & & & shrub \\
\hline $\begin{array}{l}\text { RANGE: } \\
\text { Sao }\end{array}$ & bel & bol & $\mathrm{cag}$ & $\operatorname{mex}$ & sag & tree \\
\hline RANGE: & $\mathrm{Col}$ & gua & $\operatorname{mex}$ & & & shrub \\
\hline RANGE: & cag & per & sag & 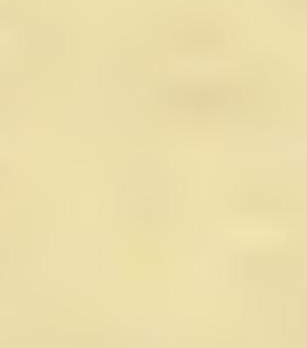 & & $\begin{array}{l}\text { tree } \\
\text { shrub } \\
\text { tree }\end{array}$ \\
\hline RANGE: & col & ecu & & & & tree \\
\hline RANGE: & end & & & 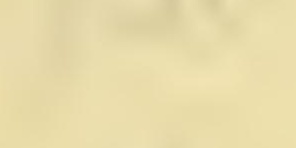 & ret & treelet \\
\hline RANGE: & $\mathrm{cr}$ & ecu & ven & $=1$ & & $\begin{array}{l}\text { subshrub } \\
\text { herb } \\
\text { shrub }\end{array}$ \\
\hline RANGE: & $\mathrm{cr}$ & & & & & $\begin{array}{c}\text { shrub } \\
\text { tree }\end{array}$ \\
\hline $\begin{array}{l}\text { RANGE: } \\
\text { ven }\end{array}$ & bel & gui & mex & nic & sao & treelet \\
\hline $\begin{array}{c}\text { RANGE: } \\
\text { SaO }\end{array}$ & ang & cag & mex & per & sag & shrub \\
\hline RANGE: & $\mathrm{col}$ & ven & & & & shrub \\
\hline $\begin{array}{l}\text { RANGE: } \\
\text { RANGE: }\end{array}$ & $\begin{array}{l}\text { end } \\
\text { end }\end{array}$ & & & 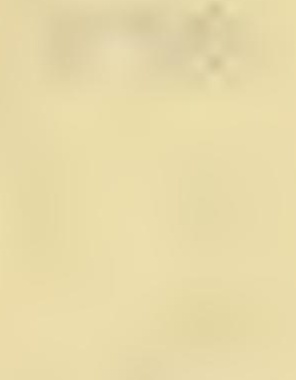 & & $\begin{array}{l}\text { herb } \\
\text { epiph } \\
\text { shrub }\end{array}$ \\
\hline RANGE: & $\mathrm{cr}$ & $\operatorname{mex}$ & & & & shrub \\
\hline RANGE: & $\mathrm{cr}$ & & 21 & & & tree \\
\hline RANGE: & cc & & & & & tree \\
\hline RANGE: & end & & & & & tree \\
\hline RANGE: & $\mathrm{cr}$ & gua & hon & $\operatorname{mex}$ & & tree \\
\hline RANGE: & ang & cag & $\operatorname{mex}$ & per & sag & tree \\
\hline RANGE: & $\mathrm{col}$ & $\mathrm{cr}$ & per & & & $\begin{array}{c}\text { shrub } \\
\text { tree }\end{array}$ \\
\hline RANGE: & per & & & & & $\begin{array}{l}\text { shrub } \\
\text { tree }\end{array}$ \\
\hline RANGE: & $\operatorname{mex}$ & & & 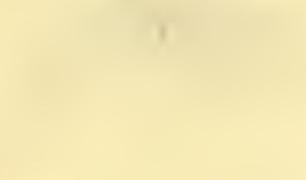 & & treelet \\
\hline RANGE: & col & $\mathrm{cr}$ & ecu & & & shrub \\
\hline RANGE: & ang & $\operatorname{mex}$ & ven & & & tree \\
\hline RANGE: & $\mathrm{cr}$ & & & & & shrub? \\
\hline RANGE: & $\mathrm{COI}$ & & & 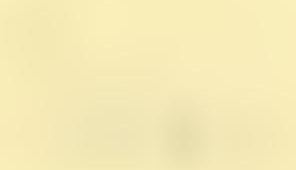 & & tree \\
\hline RANGE: & $\mathrm{col}$ & ecu & -10 & 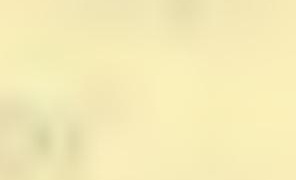 & & shrub \\
\hline RANGE: & col & $\mathrm{cr}$ & nic & & & shrub \\
\hline $\begin{array}{l}\text { RANGE: } \\
\text { RANGE: }\end{array}$ & $\begin{array}{l}\text { col } \\
\text { sao }\end{array}$ & & & & & $\begin{array}{l}\text { shrub } \\
\text { shrub }\end{array}$ \\
\hline $\begin{array}{c}\text { RANGE: } \\
\text { sao }\end{array}$ & $\begin{array}{l}\text { bel } \\
\text { trt }\end{array}$ & bol & cag & $\operatorname{mex}$ & sag & $\begin{array}{l}\text { tree } \\
\text { shrub }\end{array}$ \\
\hline
\end{tabular}


hondurensis J,D, Sm. baguens is (Bonpl.) Triana

impetiolaris (SW.) D. Don

--var. impetiolaris

-mar, pandurifolia Naud.

insularis gieason

\section{*intricata Triana}

lacera (Bonpl.) Naud.

laterifiora Cogn.

* I enchophyil ia Naud.

* longlfolia (Aub).) DC.

- ioreyoides Triana

melanotricha (Triana) gleason - -var. selanotricha - -var, panamensis oleason *ninutirlora (Bonol.) OC.

*auleipilinervia Cogn.

*ibultispicata Naud.

nervosa (Sin.) Triana

*nutans J.0. Sin.

oinechrophyilia J.D. Sin.

panamens is dieasion.

* phaeophyila iriana

* pileata DC.

* pineterum naud.

Diteieri Cogn.

* poeppigir teiana

prasina $(54$,$) DC.$

*punctata (Desvi) 0, Oon

* reducens telana

* robinsoniana cogn.

rubiginass (AOAnd.] OC.

rufosentiniaga pits.

* santarigensis Alonedo
PROV: ca da pa 000m: $0=1$

PROV: $c c$ ch pa vr 000m: $0-1,1-2$

PROV: bo ca cC ch da 000m: 0-1 pa vr

PROV: bo ca da pa 000m: 0-1

PROV: ce pa

000n: $0-1$

PROV: cC Vr

000m: $0-1$

PROV: bo ca ce ch en 000a: 0-1 da he pa sa vr

PROV: bo ca ce ch da 000n: 0-1

PROV: ca ch da pa 000n: $0-1$

PROV: da

000as: $0-1,1-2$

PROV: ch 000n: $1=2,2-3$

PROV: ch 000n: $1+2,2-1$

PHOV: ca ec ch on he 000m: $0-1$

pa vr

PROV: ch da Vr O00an: $1-2$

Peovi sa 000mi $0-1$

PAOV: bo ea ch en da $000 \mathrm{e}$ : $0-1$ pa vr

PROV: ch

200an: $1-2$

provi ce on da pa sa oocen: o-1

phov: bo g00m: o-1

PACV: da 000n: 0-1

PeVt en pa

PROV: da

Hov: ich

000n: $0+1,1-2$

provt ca ce da sa gooes: $0-1,1-2$

Chovi bo ea ce ch on boon: 0-1 de he pa vr

Provt pa

900m: $0-1$

Shov: se da be

Soen: $0+1,1 * 2$

PAOV: de

000in: $0+1$

Phovi se pa

000m: $0-1$

Provi ca da pa

0004: $0-1$

ooven: $0+1$

\begin{tabular}{|c|c|c|c|c|c|c|}
\hline ANGE: & bel & cag & & & & tre \\
\hline RANGE: & bol & cag & cub & mex & per & shrub \\
\hline sag & sao & & & & & tree \\
\hline ANGE: & ang & cag & col & $\operatorname{mex}$ & per & shrub \\
\hline ANGE: & cag & col & & & & shrub \\
\hline incel & cag & ecu & & & & tree \\
\hline & & & 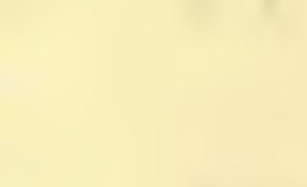 & & & $\begin{array}{l}\text { snrub } \\
\text { tree }\end{array}$ \\
\hline MCE: & col & & & 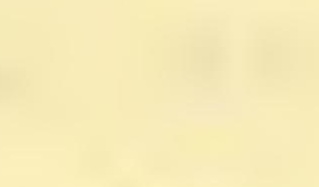 & & eplph \\
\hline $\begin{array}{l}\text { ANGE: } \\
\text { trt }\end{array}$ & ang & $\operatorname{cog}$ & $\operatorname{mex}$ & per & $\operatorname{sag}$ & $\begin{array}{l}\text { shrub } \\
\text { shrub }\end{array}$ \\
\hline $\begin{array}{c}\text { RANGE: } \\
\text { trt }\end{array}$ & bel & $\operatorname{cog}$ & col & gui & sao & shrub \\
\hline QANOE: & col & or & ven & & & shrub \\
\hline WGE: & 901 & & & & & shrub \\
\hline WEGE & cel & or & ecu & per & & shrub \\
\hline No & col & er & & & & shrub \\
\hline Mec & end & & & & & shrub \\
\hline W6 & $\operatorname{cag}$ & cub & $\sec x$ & $\operatorname{sag}$ & & $\begin{array}{l}\text { shrub } \\
\text { tree }\end{array}$ \\
\hline Wot: & col & er & & & & shrub \\
\hline Wac & or & ven & & & & shrub \\
\hline $\mathrm{Mact}$ & $\operatorname{cag}$ & $\operatorname{sen} x$ & per & $\operatorname{sag}$ & sht & shrub \\
\hline 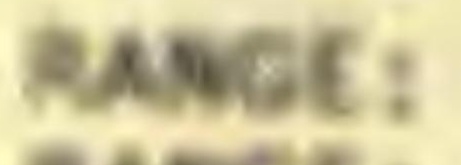 & col & er & ecu & nit & & shrus \\
\hline Ne. & Be! & 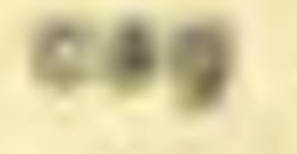 & cot & $\operatorname{sen} x$ & & shreb \\
\hline RNACE: & end & & & & & shrub \\
\hline Noct & sol & gui & Det & $\$ 90$ & ven & $\begin{array}{l}\text { shrus } \\
\text { tree }\end{array}$ \\
\hline 06 & 8 & $\operatorname{sing}$ & & & & ahrob \\
\hline $\mathcal{W}$ & nowe & & & & & shrubt \\
\hline Woc & Cr & & & & & shrve \\
\hline $\begin{array}{l}\text { Wots: } \\
\text { oer }\end{array}$ & 800 & $\operatorname{col}$ & gus & gui & non & trese \\
\hline RAOBK: & shy & be I & bol & $\operatorname{sag}$ & $n \in=x$ & shrue \\
\hline ber & 6.93 & 390 & & & & trots \\
\hline $\begin{array}{c}\text { RawaE: } \\
\text { 9ab }\end{array}$ & 639 & 909 & nex & jer & 993 & trese \\
\hline Awok: & bel & $\operatorname{col}$ & nie & & & shrob \\
\hline 2ANG: & ect & 921 & & & & $\begin{array}{l}\text { traeiet } \\
\text { shrus }\end{array}$ \\
\hline s.6. & & Do! & or & or & 843 & $\begin{array}{l}\text { shrvb } \\
\text { tree }\end{array}$ \\
\hline & & & & & & thrus \\
\hline wat & and & & & & & trea \\
\hline
\end{tabular}


schímii Triana

*serrulata (DC.) Naud.

shattuckii Standl.

\section{*simplex Triana}

* sma ragdina Naud.

stenostachya DC.

*ternatifolia Triana theaezans (Bonpl.) Cogn.

"tomentosa DC.

"tonduzil cogn.

*trinervia Loud.

triplinervis R. \& P.

*valeriana (StandI.) Wurdack

Monochaetum

*compactum Almeda

* cordatum Almeda

*exa I ta tum Almeda

*floribundum (Schlecht.) Naud.

*neglectum Almeda

*trichophyl I um Almeda

*vulcanicum Cogn.

\section{Monolena}

"primulaeflora Hook. f.

Mouriri

*coibens is Morley

completens (Pitt.) Burret

* cyphoca rpa Standi.

*exil is Gleason

*myrtilloides (SW.) Poir.

*--subsp. parvifolia (Benth.) Morley

PROV: bo ca ce ch da 000m: 0-1 lo pa

Nepsera

aquatica (AubI.) Naud.
PROV; bo ec ch da 000m: $0-1,1-2$

PROV: bo ca cn da pa $000 \mathrm{~m}$ : $0-1$ sa

PROV: ca cc da pa 000m: 0-1

PROV: bo ca cc en pa 000m: $0-1$ sa

PROV: bo cc cn pa 000m: 0-1

PROV: ca $000 \mathrm{~m}: 0-1$

PROV: da 000m: 0-1

PROV: bo $\mathrm{cc}$ ch pa sa 000m: $0-1,1-2$

PROV: WP

000m: $0-1$

PROV: ch

000m: $1-2$

PROV: pa

000m: $0-1$

PROV: bo cn

000m: $0-1$

PROV: da

000m: we

PROV: ch

PROV: ch

PROV: ch

PROV: ch

PROV: ch

PROV: $\mathrm{ch}$

PROV: $c h$

$000 \mathrm{~m}: 1-2,2-3,3+$

000m: $1-2,2-3$

000m: $1-2,2-3$

000m: $0-1,1-2,2-3$

000m: $1-2,2-3$

000m: $1-2,2-3$

000m: 1-2

PROV: $c c$ da

000m: $0-1,1-2$

PROV: $c n v r$

PROV: da sa

PROV: pa

$00 \mathrm{~m}: 0-1$

000m: $0-1$

000m: $0-1$

000m: $0-1$

PROV: cn
PROV: bo cc $v r$ 000m: 0-1

\begin{tabular}{|c|c|c|c|c|c|c|}
\hline $\begin{array}{l}\text { RANGE: } \\
\text { RANGE: }\end{array}$ & $\begin{array}{l}\text { bel } \\
\text { ang }\end{array}$ & $\begin{array}{l}\text { cag } \\
\text { cag }\end{array}$ & $\begin{array}{l}\text { col } \\
\text { mex }\end{array}$ & per & & $\begin{array}{l}\text { tree } \\
\text { tree }\end{array}$ \\
\hline RANGE: & $\mathrm{col}$ & nic & & & & shrub \\
\hline RANGE: & $\mathrm{col}$ & or & & & & $\begin{array}{l}\text { tree } \\
\text { shrub }\end{array}$ \\
\hline RANG & & & & & & treele \\
\hline ZANGE & bol & cag & $\operatorname{mex}$ & sag & sao & shrub \\
\hline $\begin{array}{r}\text { trt } \\
\text { RANGE }\end{array}$ & ecu & per & sao & & & $\begin{array}{l}\text { tree } \\
\text { tree }\end{array}$ \\
\hline RANGE & ang & bol & cag & jam & per & shrub \\
\hline sag & sao & & & & & tree \\
\hline RANGE: & bel & bol & cag & cub & per & tree \\
\hline sag & trt & 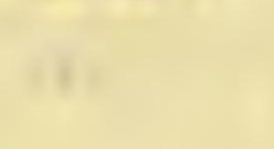 & & & & shrub \\
\hline RANGE; & cr & & & & & shrub \\
\hline RANGE: & $\begin{array}{l}\text { bol } \\
\text { per }\end{array}$ & $\begin{array}{l}\text { cag } \\
\text { ven }\end{array}$ & col & ecu & jam & tree \\
\hline $\begin{array}{l}\operatorname{mex} \\
\text { RANGE: }\end{array}$ & $\begin{array}{l}\text { pe } \\
\text { bo }\end{array}$ & $\begin{array}{l}\text { ven } \\
\text { cag }\end{array}$ & jam & mex & sao & $\begin{array}{c}\text { shrub } \\
\text { tree } \\
\text { shrub }\end{array}$ \\
\hline
\end{tabular}

RANGE: end

RANGE: $\mathrm{cr}$

RANGE: end

RANGE: cag

nic sai

RANGE: $\mathrm{cr}$

RANGE: end

RANGE: or

shrub

shrub

shrub

or gua hon mex shrub

shrub

shrub

shrub

RANGE: col ecu per sao herb

RANGE: end

RANGE: COI

RANGE: gua nic

RANGE: bel gua

tree

tree

treelet

RANGE: bol cag col ecu mex shrub per sag? sao ven

RANGE: bel cag col gui his shrub jam lag pr sao subshrub 
223 (141) MELASTOMATACEAE cont.

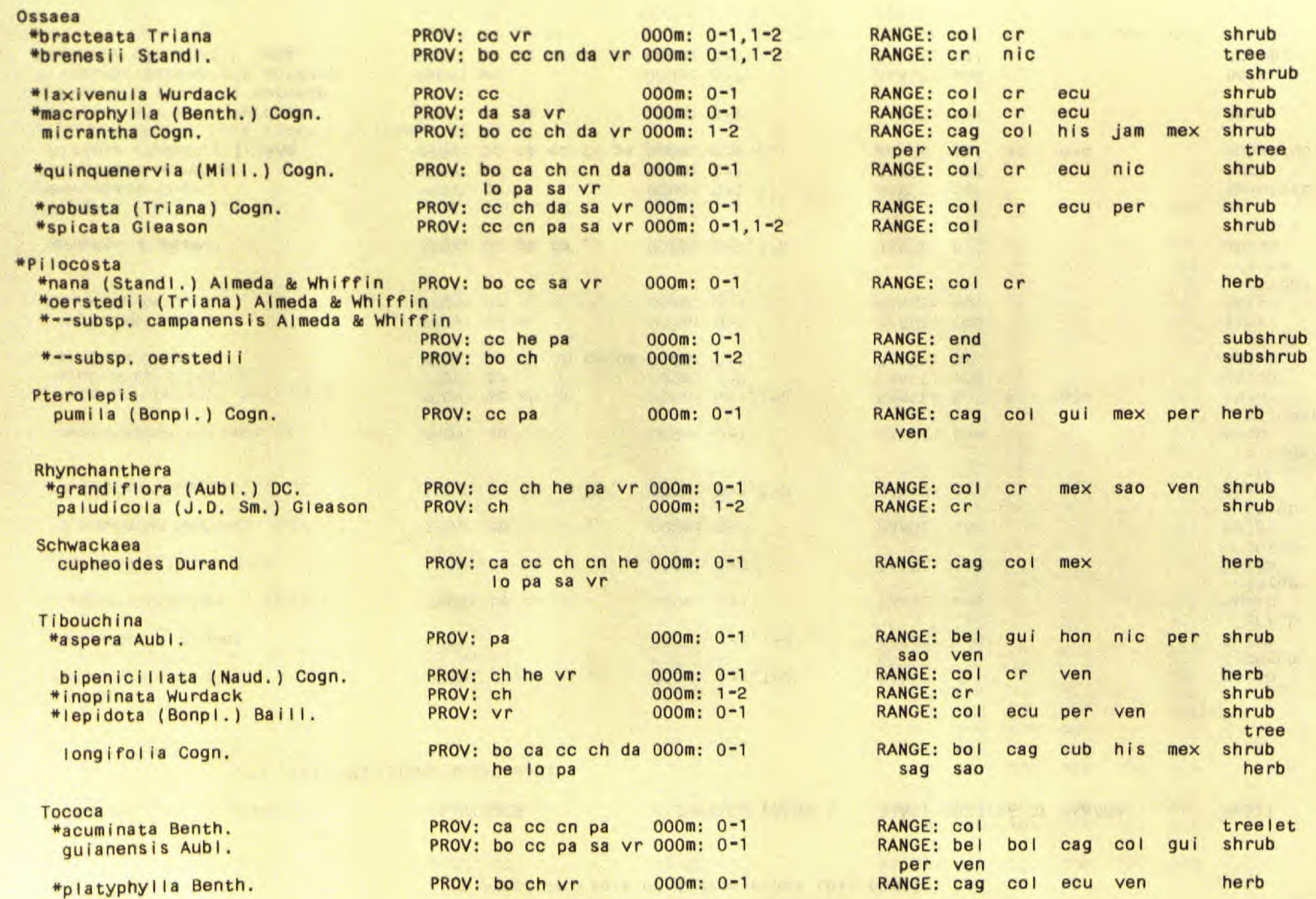


TAXON

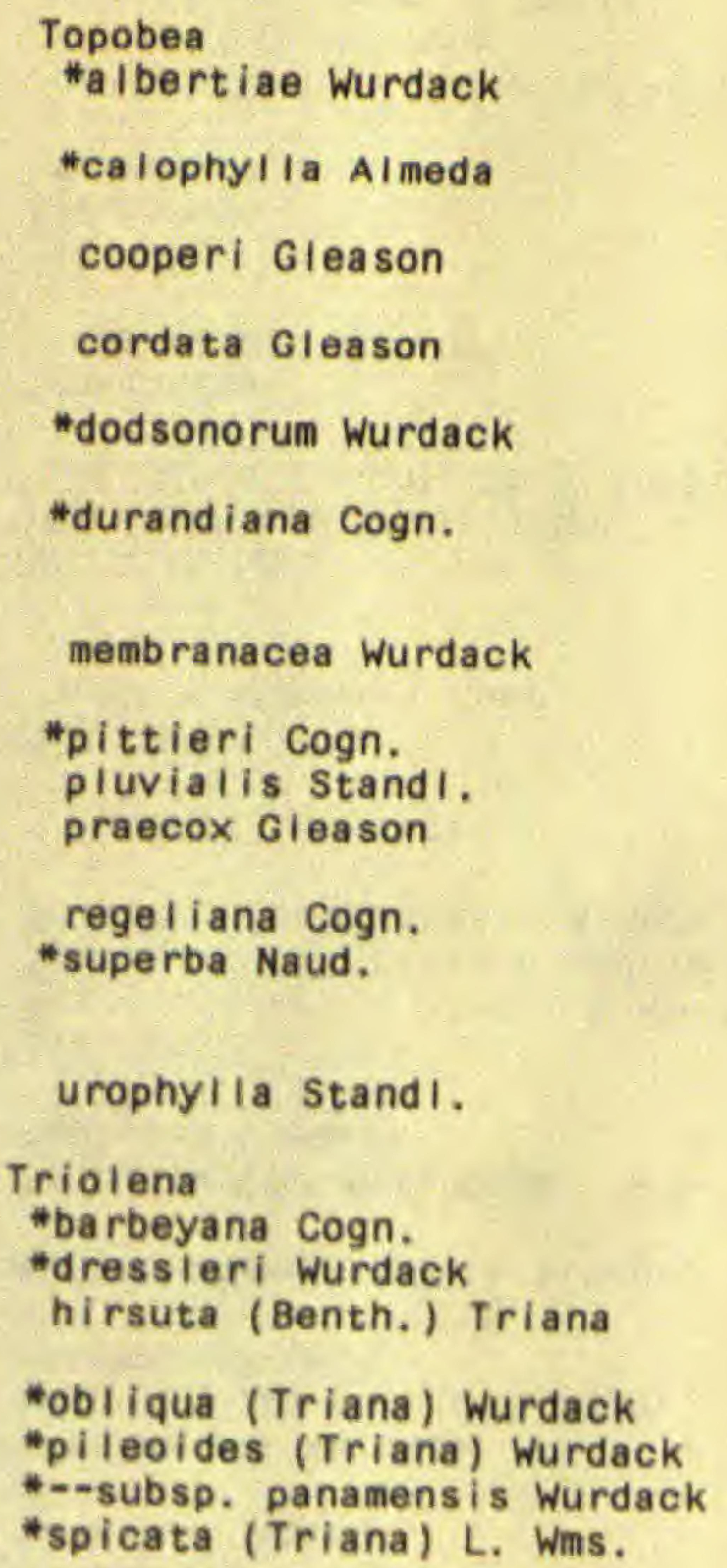

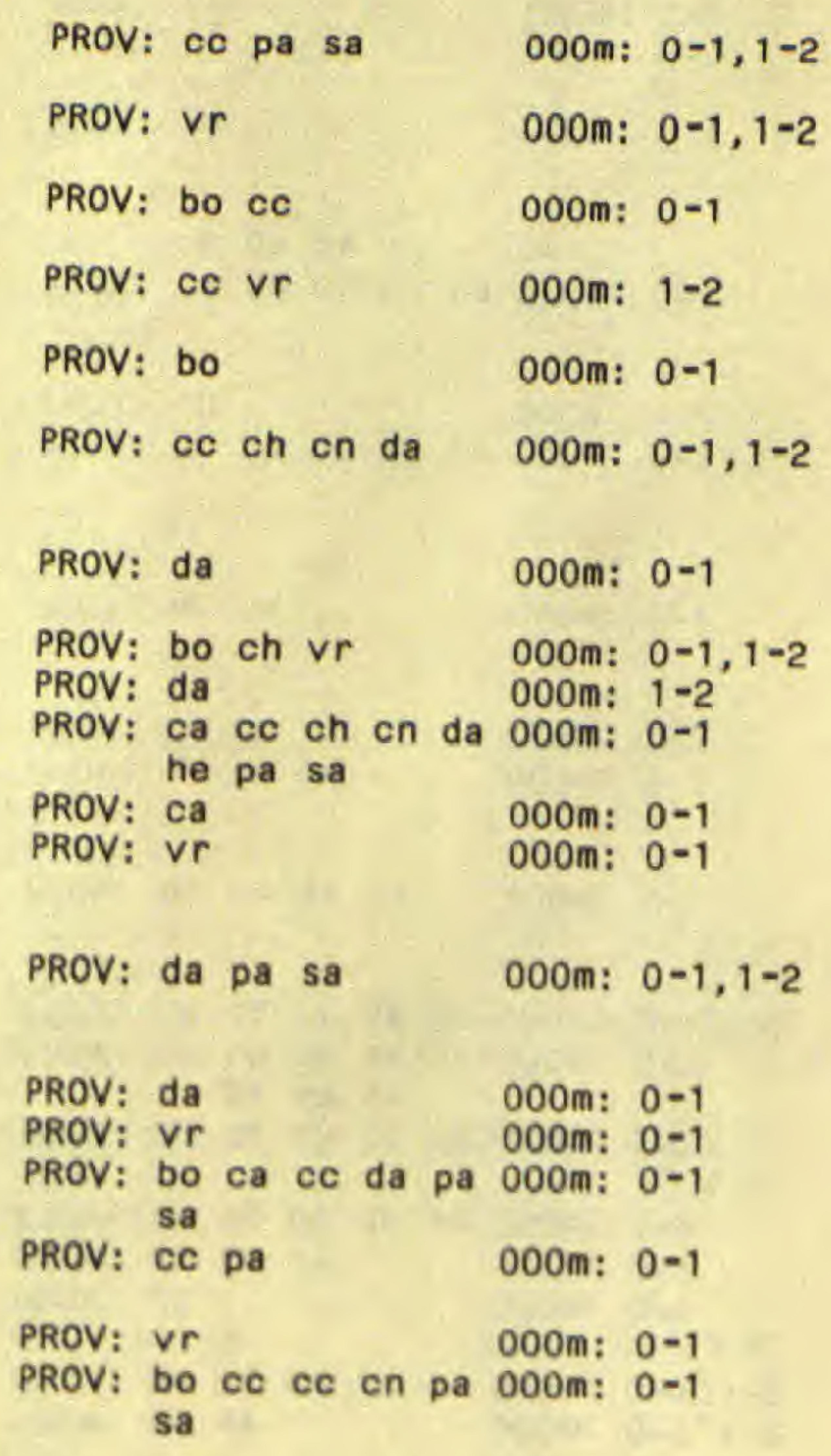

RANGE: COI

RANGE: end

RANGE: end

RANGE: end

RANGE: ecu

RANGE: $\mathrm{cr}$

RANGE: co

RANGE: end

RANGE: $\mathrm{cr}$

RANGE: end

RANGE: $C O I$

RANGE: nic

RANGE: per

RANGE: end

RANGE: COI or nic

RANGE: col

RANGE: end

RANGE: cOI
RANGE: $\mathrm{COI}$

epiph
shrub
epiph
shrub
epiph
shrub
epiph
shrub
epiph
shrub
epiph
shrub
climber
tree
climber
shrub
shrub
epiph
shrub
tree
epiph
shrub?
tree
shrub

224 (142) ONAGRACEAE

\section{Fuchsia}

*hybrida Sieb, \& Voss intr. cult.

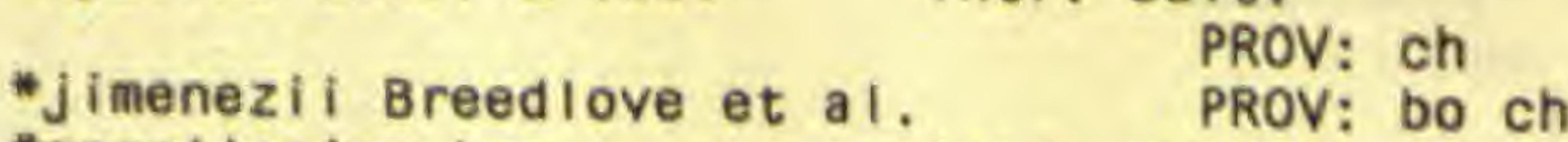

* mage I lanica Lam. intr. cult.

$\begin{array}{ll}\text { ROV: ch } & 000 \mathrm{~m}: 2-3 \\ \text { ROV: bo ch } & 000 \mathrm{~m}: 1-2\end{array}$

PROV: ch 000m: $1-2$
RANGE: $c r$ gua mex shrub

RANGE: $\mathrm{cr}$ shrub
*microphyI la H.B.K.
RANGE: $\operatorname{mex}$ 


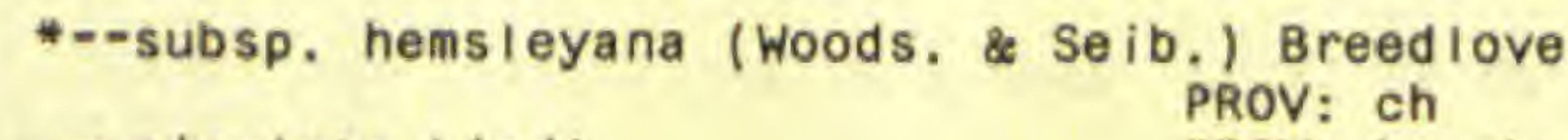

000m: $0-1$ 000m: $0-1$
RANGE: $\mathrm{cr}$

herb

\begin{tabular}{|c|c|c|c|c|c|c|}
\hline $\begin{array}{l}\text { ANGE: } \\
\text { sag? }\end{array}$ & $\begin{array}{l}\text { ang } \\
\text { sao }\end{array}$ & bol & cag? & gua & per & $\begin{array}{l}\text { herb } \\
\text { shrub }\end{array}$ \\
\hline RANGE: & ang & bol & cag & $\mathrm{col}$ & $\mathrm{cr}$ & herb \\
\hline ecu & gua & gui & hon & $\operatorname{mex}$ & nao & \\
\hline nic & per & $\mathrm{sag}$ & sa I & sao & ven & \\
\hline RANGE: & ang & i & cag & $\mathrm{col}$ & $c r$ & erb \\
\hline ecu & fla & gua & gui & hon & $\operatorname{mex}$ & \\
\hline nic & owg & per & sa I & sao & sat & \\
\hline trt & ven & & & & & \\
\hline RANGE: & cag & $\operatorname{mex}$ & per & sag & sao & erb \\
\hline RANGE: & ang & cag? & $\mathrm{col}$ & $\mathrm{cr}$ & $\operatorname{mex}$ & $a r b$ \\
\hline owg & per & sag? & sao & & & \\
\hline AANGE: & cag & $\mathrm{col}$ & $\mathrm{cr}$ & cub & gua & erb \\
\hline gui & hon & jam & $\operatorname{mex}$ & nic & sal & \\
\hline sao & sat & ven & & & & \\
\hline RANGE: & $\mathrm{col}$ & $\mathrm{cr}$ & ecu & gui & nic & herb \\
\hline per & sao & sat & trt & ven & & \\
\hline RANGE: & ang & cag & $\operatorname{mex}$ & nag? & per & \\
\hline sag? & sao & & & & & nruc \\
\hline RANGE: & bel & cag & $\mathrm{col}$ & gui & hon & UD \\
\hline nic & per & sao & sat? & ven & & ree \\
\hline RANGE: & ang & cag & $\operatorname{mex}$ & nag? & owg & 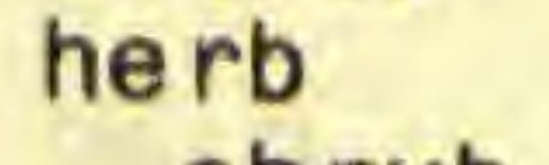 \\
\hline per & sag? & sao & ven & & & irub \\
\hline RANGE: & ang & $\mathrm{co}$ & gua & $\operatorname{mex}$ & nag? & \\
\hline RANGE: & bel & bol & cag & $\mathrm{col}$ & $\mathrm{cr}$ & $r b$ \\
\hline cub & ecu & fla & gua & his & jam & shrub \\
\hline mex & mex & nic & per & $p r$ & sag & \\
\hline sa I & sao & $\mathrm{tr}$ & ven & & & \\
\hline RANGE: & bel & bo & cag & $\mathrm{col}$ & $\mathrm{cr}$ & 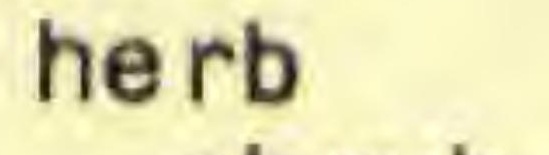 \\
\hline $\begin{array}{l}\text { gua } \\
\text { ven }\end{array}$ & gui & hon & $\operatorname{mex}$ & nic & sat & 15 \\
\hline RANGE: & bol & co & cub & gua & gui & herb \\
\hline $\begin{array}{l}\text { hon } \\
\text { ven }\end{array}$ & jam & $x$ & ic & sa I & sao & \\
\hline RANGE: & & & cub & gui & sao & \\
\hline
\end{tabular}


TAXON

FAMILY PROVINCES

ELEVATION (OOOM.)

RANGE OUTSIDE OF PANAMA

HAB IT

224 (142) ONAGRACEAE cont.

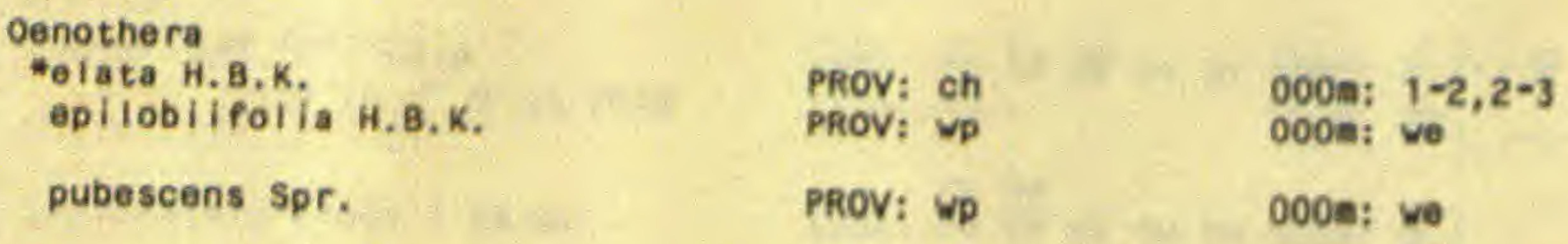

225 (143) HALORAGACEAE

\section{Gunnera}

insignis (Oerst.) A. DC.

PROV: bo ch

PROV: ch
000: $1-2,2-3$

000m: $3+$

\section{7 (144) ARALIACEAE}

Dendropanax

alberti-smithii Nevi.

arboreus (L.) Dec. Planch.

darienens is Seem.

gonatopodus (J.D. Sm.) A.C. Sm.

* leptopodus (J.0. Sm.) A.C. Sm.

praestans Standi.

querceti J.D. Sm.

sessiliflorus (Standl. \& A.C. Sm.) A.C. Sm.

stenodontus (StandI.) A.C. Sm.

\section{PROV: $c c$ ch sa vr}

PROV: ca cn sa

PROV: $\mathrm{CC}$

PROV: bo ce en pa

000m: $0-1$

PRoV: bo ca cc ch da 000m: $0-1,1-2$

PROV: da

PROV: bo ch da

PROV: bo ce ch

PROV: ch

$000 \mathrm{~m} ; 1-2$

000m: $0-1,1-2$

000m: 1-2, 2-3

000m: $0-1,1-2$

*tessmannii Harms

Didymopanax

morototoni (Aubl.) Dec. \& Planch. PROV: $\underset{v r}{\mathrm{~V}}$ cn da pa sa 000m: 0-1

pittieri Marcha I

PROV: ch

$000 m: 1-2,2-3$

Oreopanax

tanchicayanum Cuatr.

capitatus (Jacq.) Decne. \& Planch.

PROV: da

000m: $0-1,1-2$

PROV: bo ca cc ch da 000m: $0-1,1-2,2-3$

costaricens is Marcha I

I iebmanni Marcha I

*oerstediana Marcha
PROV: ch

PROV: bo $\mathrm{ch}$

PROV: $\mathrm{ch}$
$0-1,1-2$

000m: $0-1$

000m: $0-1,1-2$

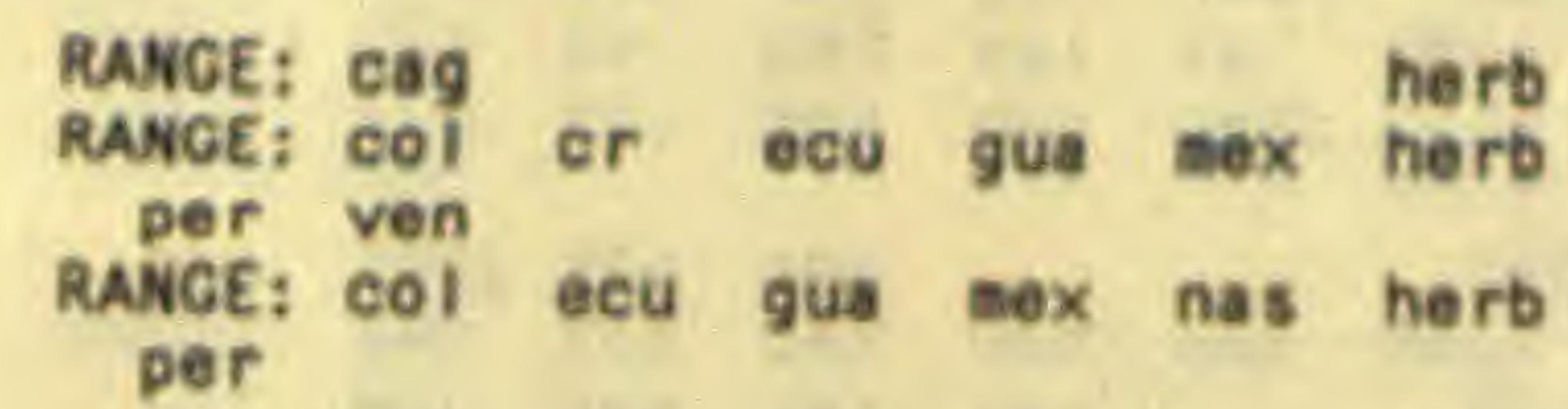

per

RANGE: Cr RANGE: er

herb herb

RANGE: COI

RANGE: CUb

or

tree

RANGE: end

RANGE: $\mathrm{Cr}$

RANGE: hon

RANGE: or

RANGE: $\mathrm{cr}$

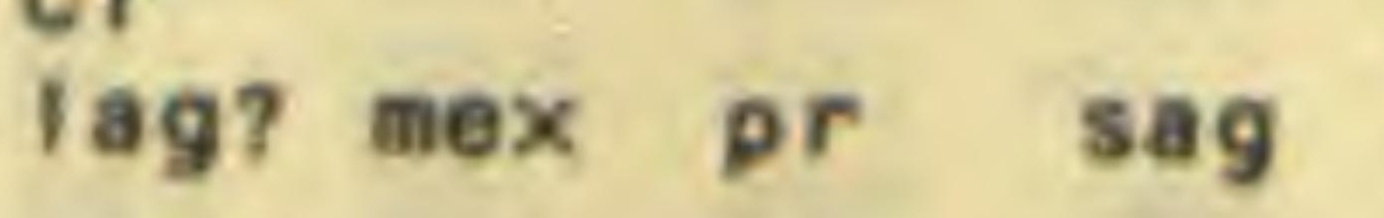

thrub

tree

tree

tree

tree

tree

shrub

tree

RANGE: end

RANGE: mex

tree

shrub

shrub

tree

RANGE: ang

cag

sa

tree

RANGE: $\mathrm{cr}$

tree

RANGE: sat

climber

RANGE: ang cag cub his mex tree

sag

RANGE: $c r$

RANGE: cag mex

RANGE: $\mathrm{cr}$ tree

tree

tree 
TAXON

FAMILY PROVINCES

vestitus A.C. Sm.

PROV: ch

xalapensis (H.B.K.) Decne. \& Planch.

PROV: ch

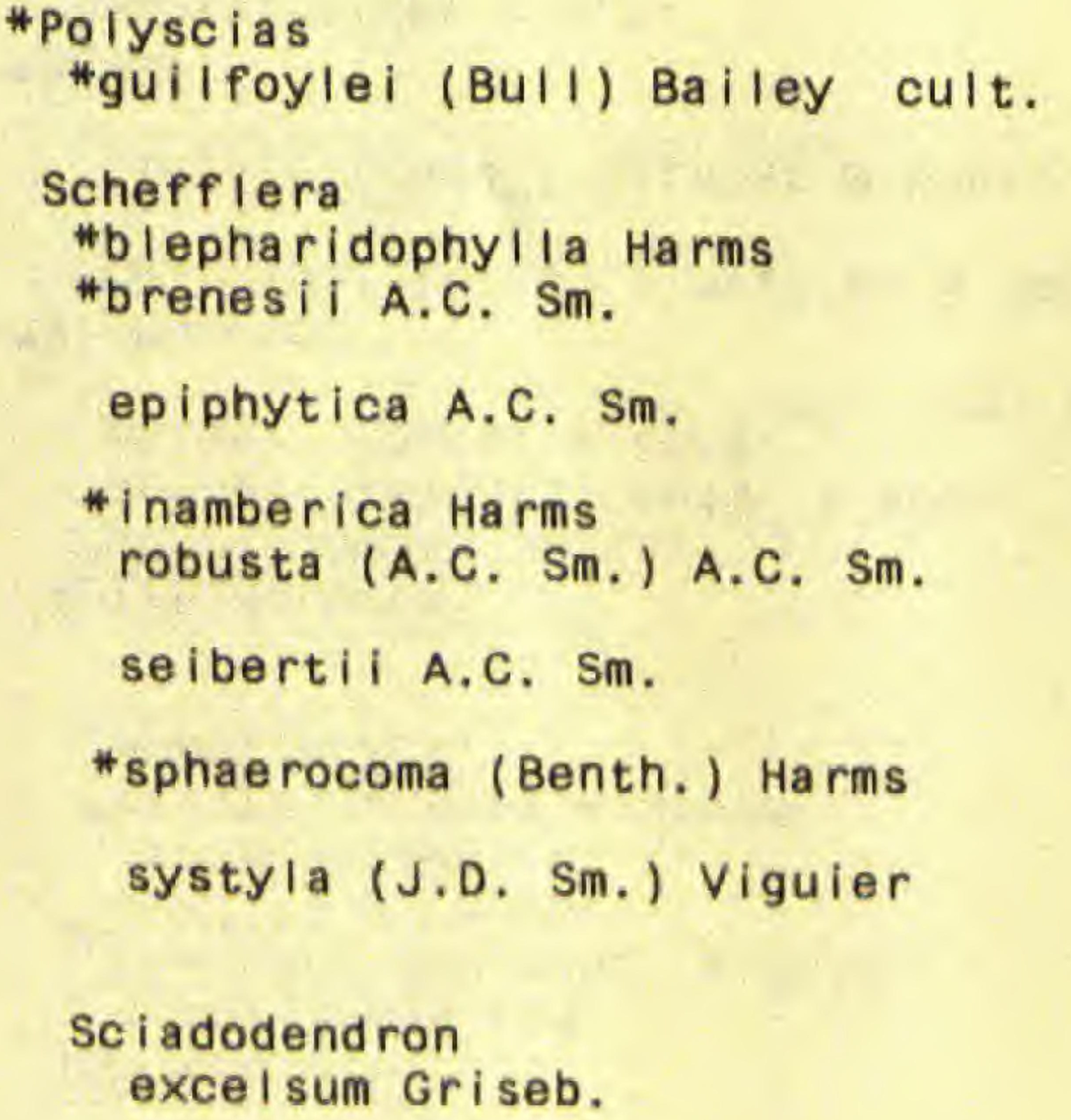

228 (145) UMBELLIFERAE

\section{Ane thum}

graveolens $L$. intr, cult.

$$
\text { PROV: bo }
$$

$000 \mathrm{~m}: 1-2$

RANGE: wdw

herb

*aegopodioides (H.B.K.) Coult. \& Rose

PROV: ch

$000 m: 1-2,2-3,3+$

RANGE: or gua mex

herb

Cyc lospermum

leptophyl Ium (Pers.) Sprague PROV: ch

$000 m: 1-2,2-3$

RANGE: $w d t$

herb

Daucus

*carota L. intr. cult.

climber
tree
shrub
climber
shrub
tree
shrub

tree
shrub
tree
epiph
shrub
tree
shrub
tree
shrub
tree
epiph
shrub
shrub
tree
shrub
tree

rub

rub

000m: $0-1$,

tree

shrub

shrub

tree

iph

hrub

(1)

000m: $0-1,1-2$ RANGE: $\mathrm{cr}$ shrub


TAXON

FAMILY PROVINCES

\section{\#Donnel I smithia}

*juncea (Spr.) Mathias \& Const. PROV: wp 000m: 1-2,2-3

Eryng ium

carlinae Delar. $f$.

foetidum L.

intr. cult.

PROV: $c h$

000m: $1-2$

PROV: bo ca cc ch cn 000m: $0-1,1-2$ da lo pa sa $v r$

"humile Cav.

Hydrocotyle

bonariensis Lam.

mexicana Schlecht. \& Cham.

pusilla A. Rich.

ranunculoides L. $f$.

*ribifolia Rose \& Standl.

umbel lata L.

$000 m: 2-3,3+$

PROV: ca ch cn sa vr 000m: $0-1$

PROV: bo $\mathrm{ch}$ da $000 \mathrm{~m}$ : $1-2,2-3$

PROV: bo

PROV: bo

000m: $2-3$,
PROV: bo ca ch 000m: $0-1,1-2,2-3$

000m: $0-1,1-2,2$

PROV: bo ca cc ch cn 000m: $0-1,1-2,2-3$

PROV: bo ch
PROV: bo

PROV: ch

*donneli-smithii Coult. \& Rose

maxonit Coult, \& Rose

*Niphogeton

*chirripoi (Suess.) Mathias \& Const.

* i ingula (Wedd, PROV: bo ch

* toa

*oenanthoides H.B.K.

PROV: ch

PROV: bo ch da $\mathrm{vr}$

PROV: ca ch
$000 \mathrm{~m}: 3+$

000m: $2-3,3+$

000m: $2-3,3+$

000m: $3+$

000m: $3+$

$000 m: 0-1,1-2,3+$

000m: $0-1,1-2$ 000m: $3+$
RANGE: cag mex sat

herb

RANGE: cag or mex herb

RANGE: cag mex sag wdt herb

RANGE: col cr ecu per ven herb

RANGE: nwt sao herb

RANGE: cag mex herb

RANGE: $\mathrm{cr}$ gag nic sat herb

RANGE: cag gag nag sag herb

RANGE: or herb

RANGE: cag gag nag sag herb

RANGE: $\mathrm{cr}$ herb

RANGE: $\mathrm{cr}$ gua mex tree

RANGE: end tree

RANGE: $\mathrm{col} \mathrm{cr}$ ven herb

RANGE: $\mathrm{col} \mathrm{cr}$ herb

RANGE: col cr ecu gua mex herb ven

RANGE: cag mex per sag sao herb

RANGE: nWt

herb

229 (146) CORNACEAE

Cornus

disciflora DC.

PROV: ch

000m: $2-3,3+$

RANGE: cag mex

shrub 
TAXON

FAMILY PROVINCES

ELEVATION (OOOM.)

RANGE OUTSIDE OF PANAMA

HAB IT

229 (146) CORNACEAE cont.

\section{*Garrya}

* Iaurifolia Hartw.

PROV: ch

000m: $3+$

RANGE: cag

tree

230 (147) CLETHRACEAE

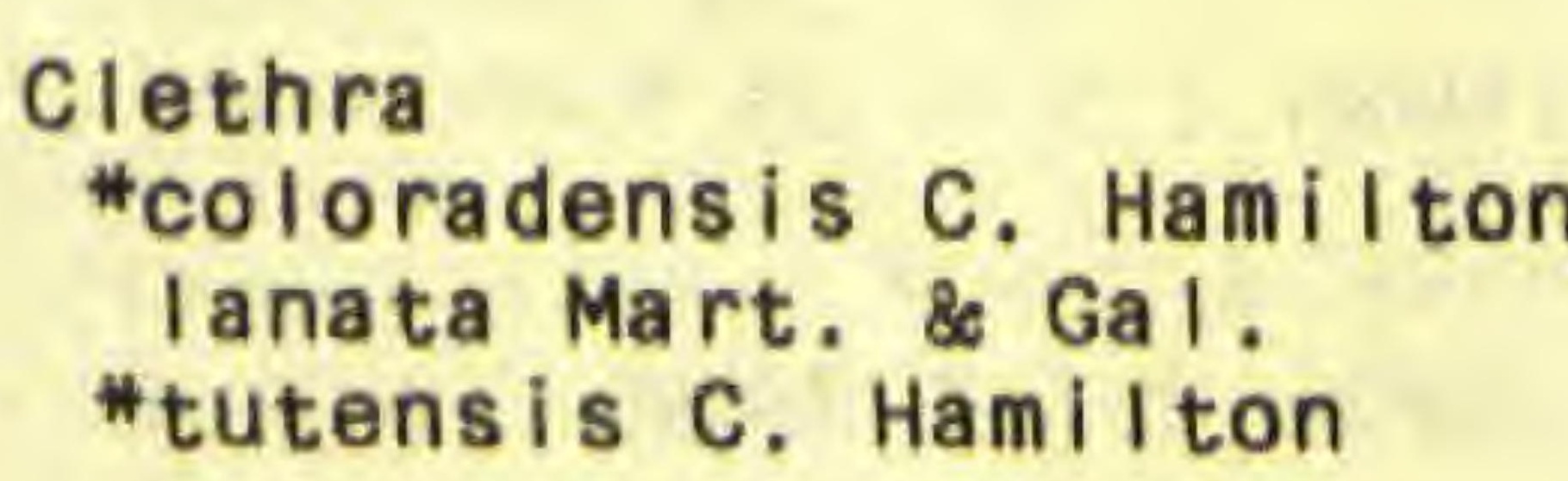

000m: $0-1,1-2$

000m: $1-2$

PROV: $V r$

(n)

\section{$233(148,149)$ ERICACEAE}

\section{Anthopteropsis}

insignis A.C. Sm.

PROV: cc pa

000m: $0-1$

Anthopterus

wardii Ball

\section{Befaria}

*aestuans L.

Cavend ishia

*aberrans Luteyn

allenii A.C. Sm.

*arizonensis Luteyn

atroviolacea Luteyn

*--var. folsomii Luteyn

axillaris A.C. Sm.

callista J.D. Sm.

calycina A.C. Sm.

capitulata J.D. Sm.

chiriquiensis A.C. Sm.

PROV: da 000m: $0-1,1-2$

PROV: ch vr

000m: $0-1$

PROV: $c c$

000m: $0-1,1-2$

PROV: cc en pa sa

000m: $0-1$

PROV: $v r$

000m: 1-2

PROV: ch

$000 \mathrm{~m}: 1-2,2-3$

PROV: cc cn

000m: $0-1$

PROV: $c c$ ch da $\mathrm{vr}$

000m: $0-1,1-2$

PROV: ce cn da pa sa $000 \mathrm{~m}: 0-1,1-2$

PROV: $\mathrm{Vr}$

000m: 1-2

PROV: bo cc ch da pa 000m: $0-1,1-2,2-3$

PROV: bo

000m: $1-2$
RANGE: end

RANGE: cag mex

RANGE: end

tree

tree

tree

RANGE: end

shrub

RANGE: $\mathrm{col}$ ecu

shrub

tree

RANGE: ven

tree

RANGE: end

epiph

shrub

epiph

shrub

epiph

shrub

ep iph

shrub

epiph

shrub

terr.

epiph

RANGE: $\mathrm{col} \mathrm{cr}$ ecu

shrub

RANGE: col cr gua nic sao Ven

RANGE: $w r$

terr

RANGE: $\mathrm{COI} \mathrm{cr}$

ep iph

shrub
shrub

RANGE: end

shrub 
TAXON

$233(148,149)$ ERICACEAE cont.

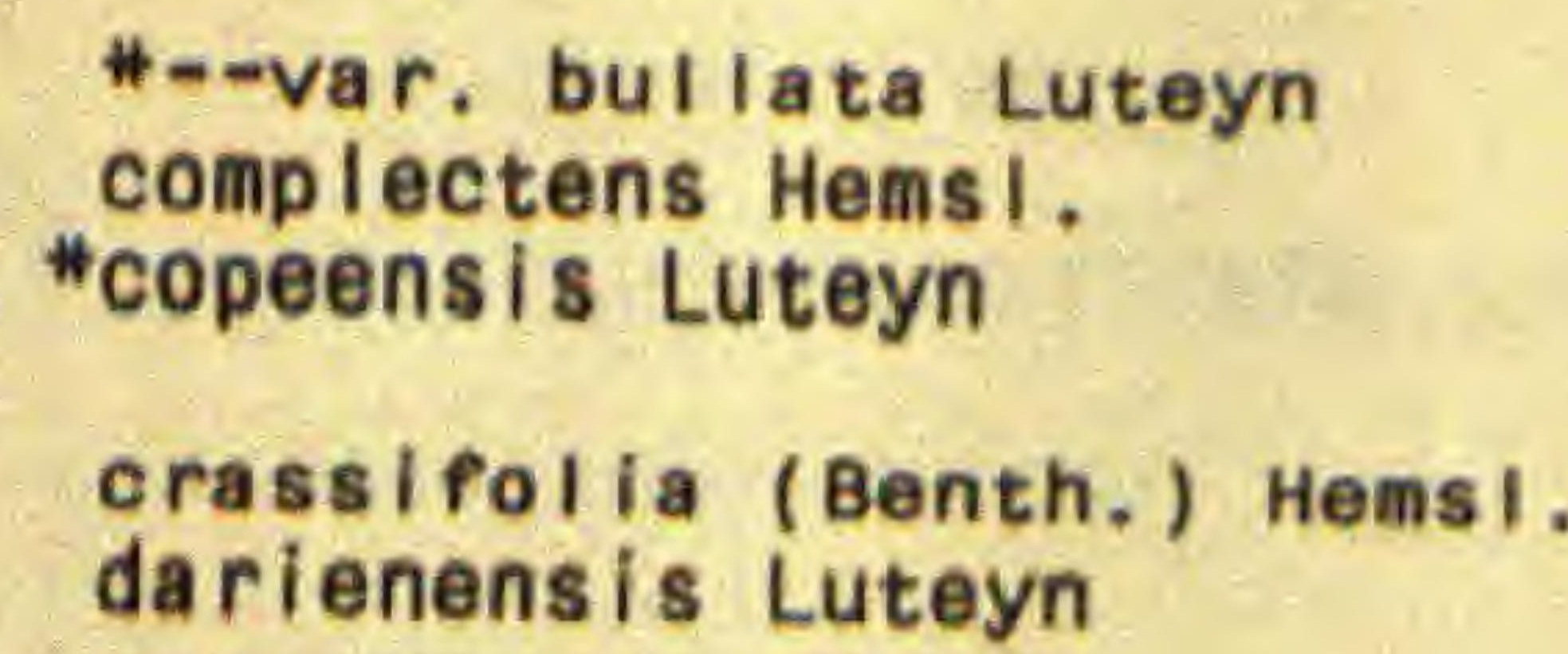

PROV: cc ch

PROV: ch da

PROV: CC

PROV: bo $\mathrm{cc}$ ch $\mathrm{vr}$

PROV: da

PROV: ch

PROV: da

PROV: da

PROV: da

PROV: bo $\mathrm{ch}$

PROV: $c c$

PROV: ch

PROV: da

PROV: $c h \mathrm{Vr}$ PROV: bo $v r$

PROV: $\mathrm{ch}$

PROV: $\mathrm{ch}$

PROV: ch

PROV: cc pa

PROV: $v r$

PROV: cc pa

PROV: ch Vr 000m: $0-1,1-2$

000m: $0-1,1-2$

000m: $0-1$

RANGE: end

RANGE: col or nic
RANGE: end

000m: $0-1,1-2,2-3,3+$ RANGE: cag or gua mex

000m: $0-1,1-2$

RANGE: col

000m: $1-2$

$000 m: 0-1,1-2$

000m: $1-2$

000m: 1-2

000m: $1-2,2-3$

000m: 0-1

000m: $1-2$

$000 \mathrm{~m}: 0-1,1-2$

000m: $1-2$

000m: $0-1$

000m: $1-2$

000m: $1-2$

$000 \mathrm{~m}: 1-2$

000m: $0-1$

000m: $0-1,1-2$

000m: $0-1$

000m: $0-1,1-2,2-3$
RANGE: end

RANGE: col ecu

RANGE: end

RANGE: col gua mex

RANGE: end

RANGE: end

RANGE: end

RANGE: end

RANGE: end

RANGE: end

RANGE: bo

RANGE: $\mathrm{cr}$

RANGE: $\mathrm{COI} \mathrm{cr}$

RANGE: end epiph

RANGE: end

RANGE: end

RANGE: end

$$
\begin{aligned}
& \text { shrub } \\
& \text { shrub } \\
& \text { hemiep } \\
& \text { shrub } \\
& \text { shrub } \\
& \text { terr } \\
& \text { epiph } \\
& \text { shrub } \\
& \text { epiph } \\
& \text { shrub } \\
& \text { epiph } \\
& \text { shrub } \\
& \text { epiph } \\
& \text { shrub } \\
& \text { terr } \\
& \text { epiph } \\
& \text { shrub } \\
& \text { epiph } \\
& \text { shrub } \\
& \text { epiph } \\
& \text { shrub } \\
& \text { epiph } \\
& \text { shrub } \\
& \text { epiph } \\
& \text { shrub } \\
& \text { shrub } \\
& \text { epiph } \\
& \text { terr } \\
& \text { shrub } \\
& \text { shrub } \\
& \text { terr } \\
& \text { epiph } \\
& \text { shrub } \\
& \text { terr } \\
& \text { epiph } \\
& \text { shrub } \\
& \text { terr } \\
& \text { shrub } \\
& \text { epiph } \\
& \text { shrub } \\
& \text { terr } \\
& \text { epiph } \\
& \text { shrub } \\
& \text { terr } \\
& \text { epiph } \\
& \text { shrub }
\end{aligned}
$$


$233(148,149)$ ERICACEAE cont.

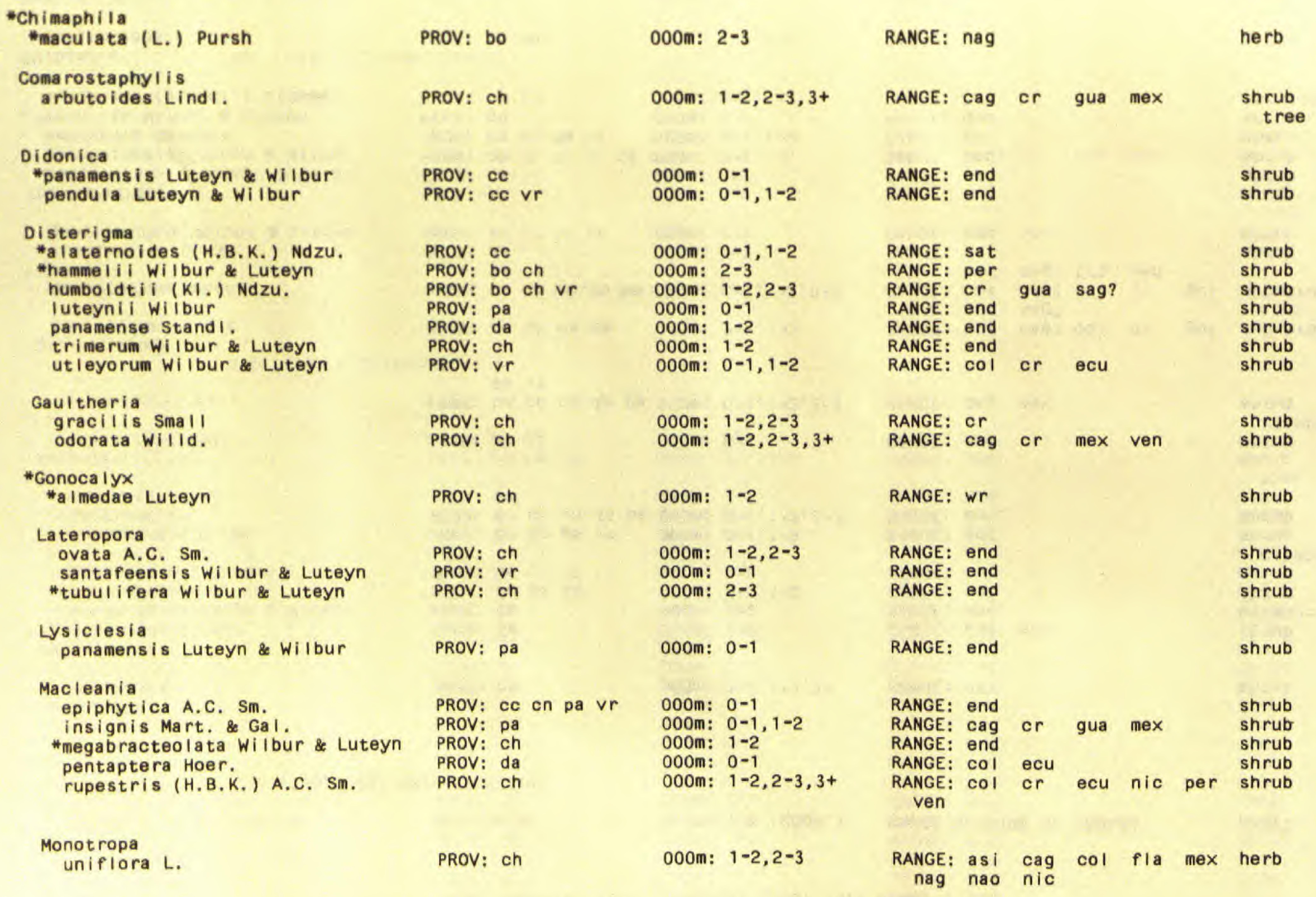


TAXON

$233(148,149)$ ERICACEAE cont.

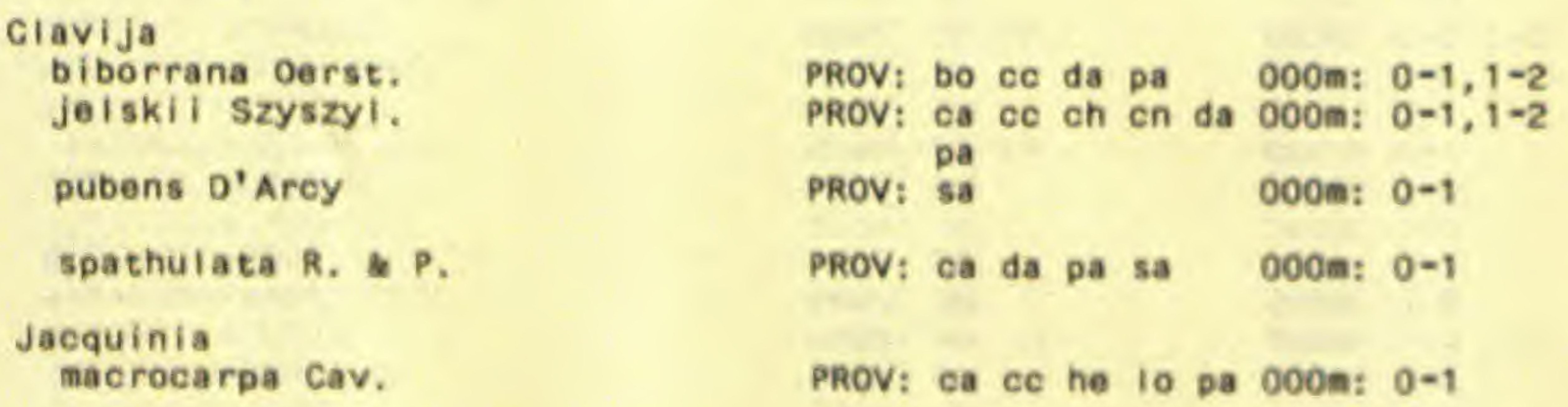

PROV: bo CC da pa 000m: $0=1,1-2$ PROV: $c a$ ce ch cn da 000m: $0-1,1-2$ PROV: sa

000m: $0-1$

PROV: ca da pa sa 000an: $0-1$

PROV: ca ce he lo pa 000m: 0-1

RANGE: or RANGE: cag? or per sag?

RANGE: end

RANGE: per ven

RANGE: or mex shrub shrub

shrub

tree

tree

shrub

tree

236 (150) MYRSINACEAE

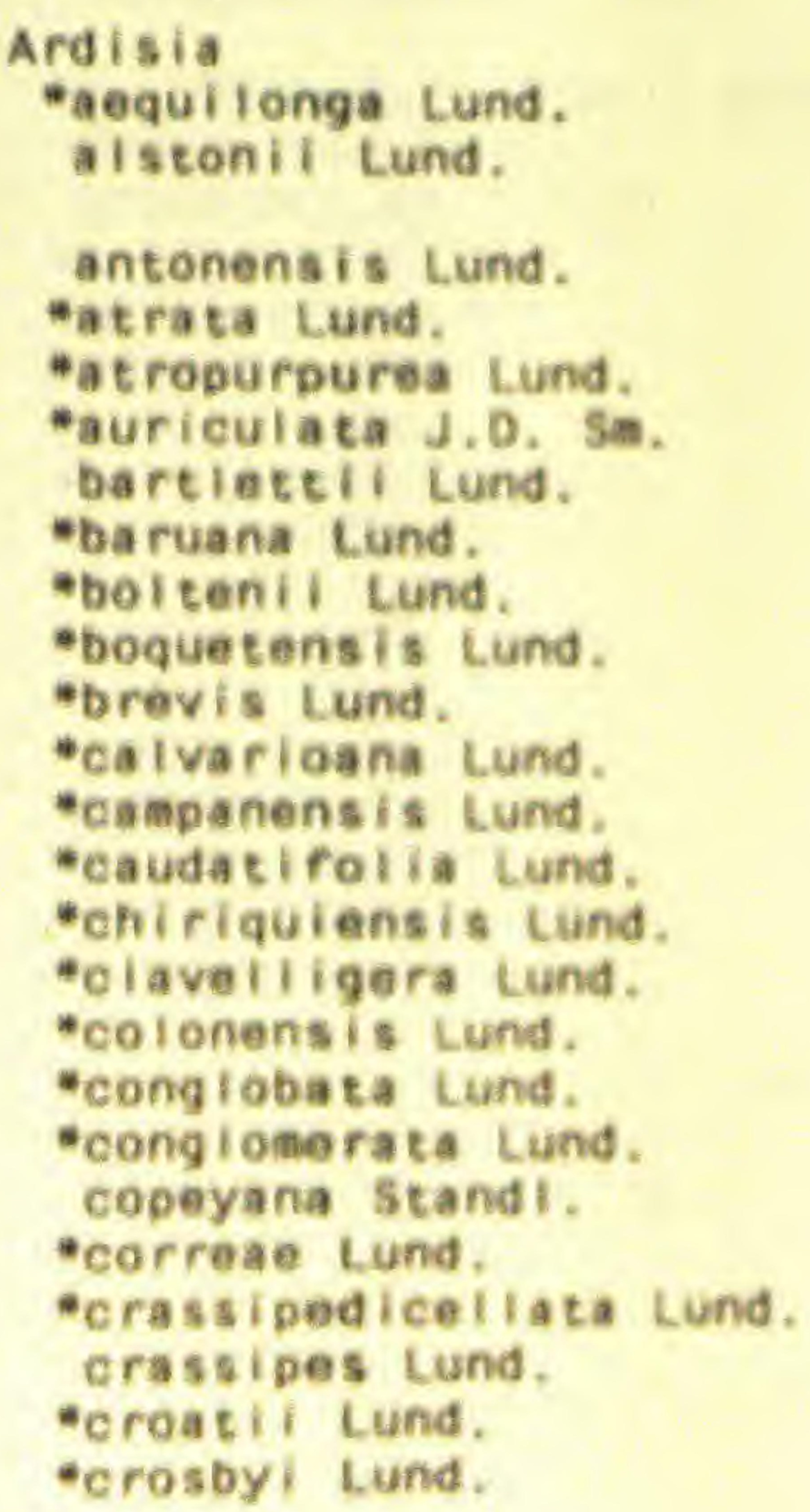

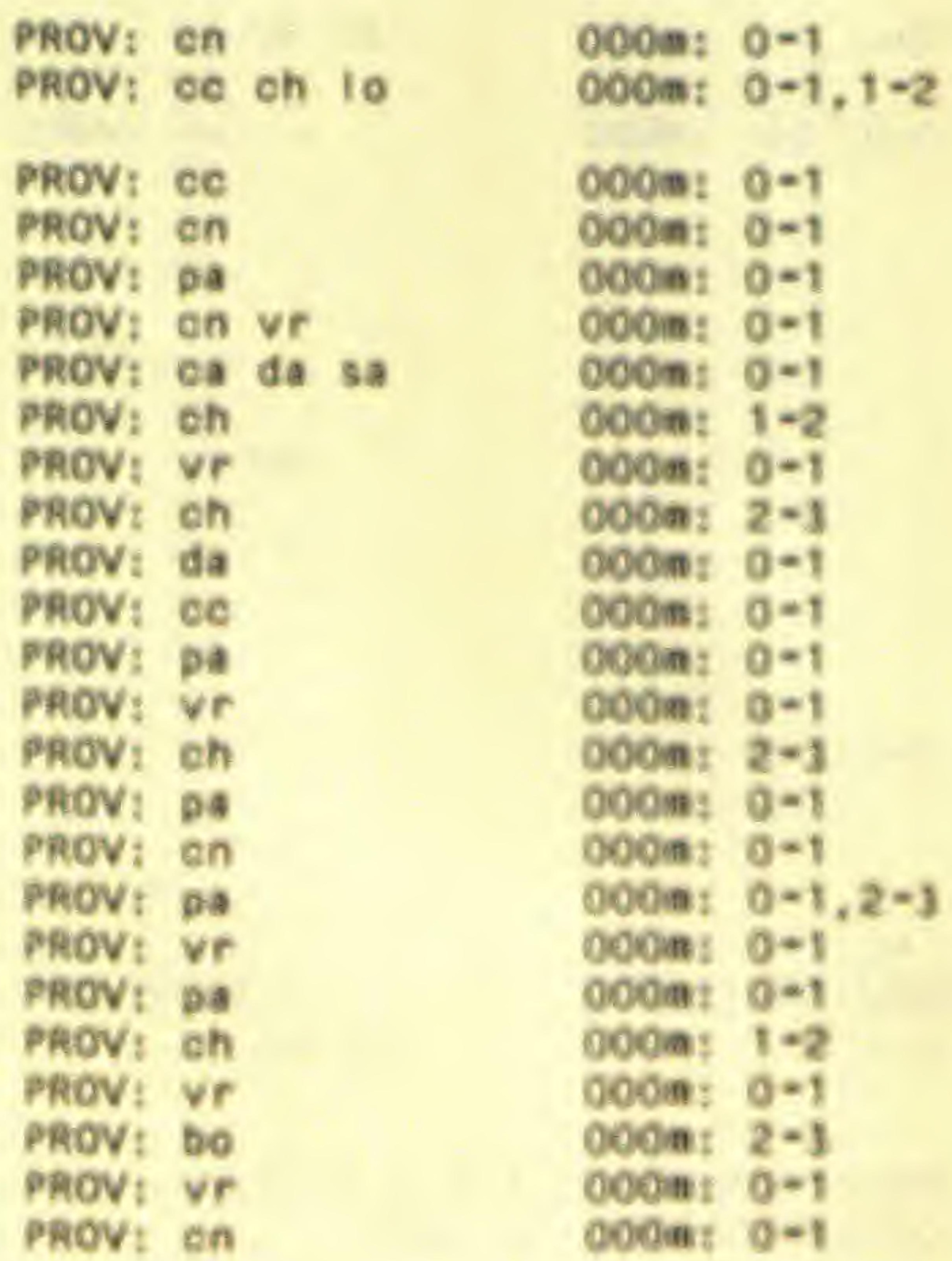

RANOE: end RawOE: end

\section{Pavar: end}

PANGE: ind

avor: and aANOE: or

unce: and

QANoE: end

Ruce: nio

Raver: end

eavor: ond

MWOE: and

RAMOE: and

uwot: and

ANCE: and

RANGE: and

MNOE: and

AwCE: end

MWOE: end

RANCE! cr

Mwar: end

ruscr: ond

Rancer: end

Awor: and

MAnot: and tree

shrub

tree

tree

tree

shrub

tree

shrut

shrus

shrub

inrut

sree

shrub

shrub

shrub

tree

tree

tree

tree

tree

trea

tree

three

tree

tree

tree

shrub 
TAXON

236 (150) MYRSINACEAE cont.

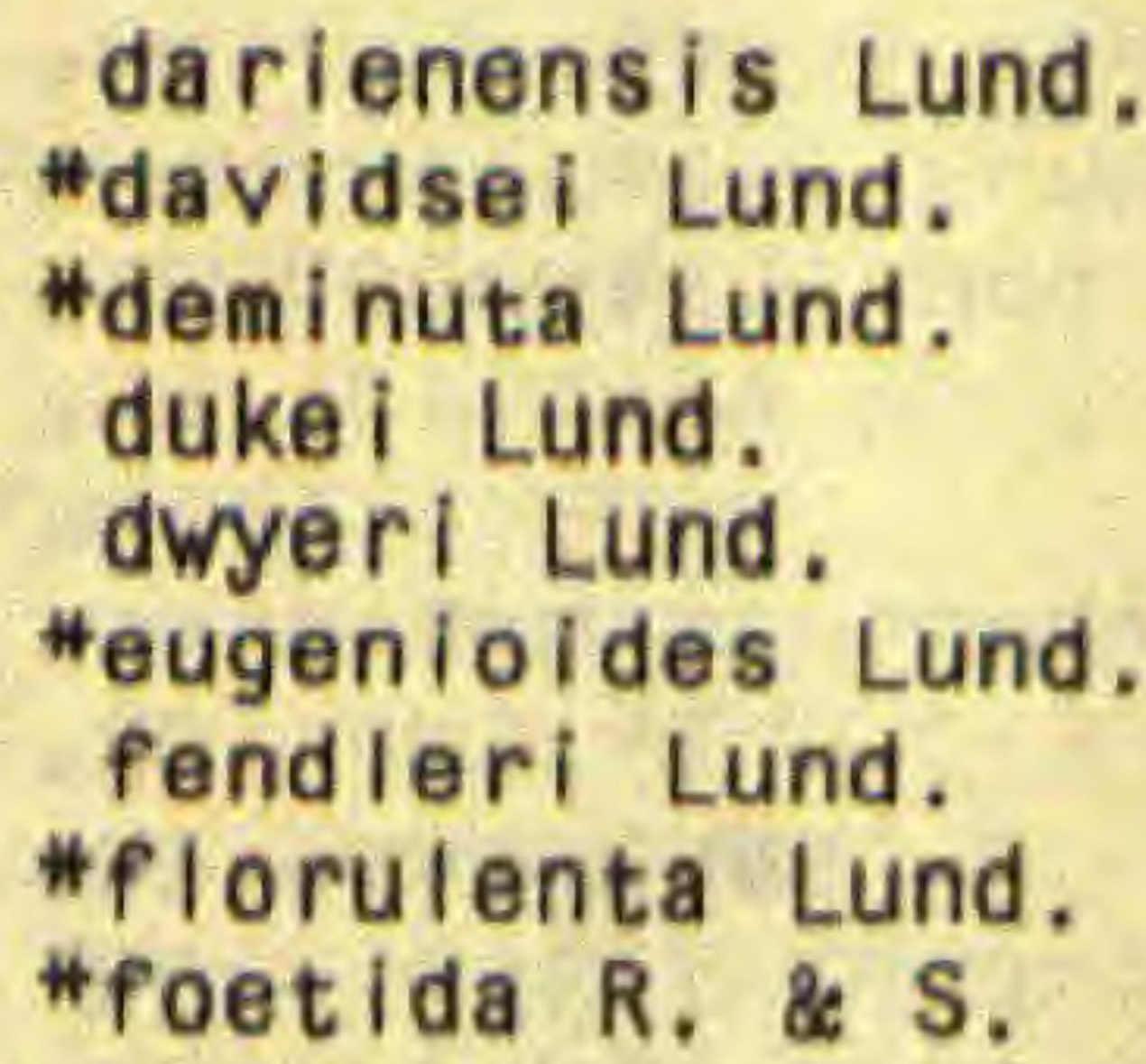

*oerstediana Lund. opaca Lund. opegrapha Oerst.

pa Imana J.D. Sm. panamens is Lund.
PROV: da

PROV: ch

PROV: pa

PROV: da

PROV: pa

PROV: ch

PROV: $\mathrm{cc}$ cn

PROV: ch

PROV: da

PROV: $\mathrm{CC}$

PROV: $V r$

PROV: ca

PROV: ch

PROV: ch

PROV: ch da pa

000m: $0-1,1-2$

000m: $1-2$

000m: $0-1$

000m: $0-1$

000m: $0-1$

000m: 2-3

000m: $0-1$

000m: 2-3

000m: $0-1$

000m: $0-1$

000m: $0-1$

000m: $0-1$

000m: $1-2,2-3$

000m: $0-1$

000m: $0-1,1-2,2-3$

PROV: $c c$

PROV: da

$000 \mathrm{~m}: 0-1$

000m: $0-1$

000m: $0-1$

PROV: cn

PROV: bo ca pa sa

000m: $0-1$

PROV: ch

PROV: cn

PROV: ch

PROV: pa

000m: $1-2$

000m: $0-1$

000m: 2-3

000m: $0-1$

PROV: cn

PROV: cn sa

PROV: pa

PROV: pa

PROV: $\mathrm{cc}$ ch

000m: $0-1$

000m: $0-1$

000m: $0-1$

000m: $0-1$

000m: $0-1,1-2,2-3$

PROV: $v r$

PROV: $\mathrm{ch}$

PROV: $\mathrm{CC}$

PROV: ch

PROV: pa

PROV: CC

PROV: ca pa

PROV: bo

PROV: $v r$

PROV: ch da

PROV: bo cc ch pa vr $000 \mathrm{~m}: 0-1$

PROV: ch pa

PROV: $\mathrm{ch}$ 000m: $0-1$

000m: $0-1,1-2$

$000 \mathrm{~m}: 0-1$

000m: $1-2$

000m: $0-1$

000m: $0-1$

000m: $0-1$

000m: $0-1$

000m: 0-1

000m: $1-2$

000m: $0-1,1-2$

000m: we
RANGE: end

RANGE: end

RANGE: end

RANGE: end

RANGE: end

RANGE: end

RANGE: end

RANGE: end

RANGE: COI

RANGE: end

RANGE: end

RANGE: end

RANGE: end

RANGE: end

RANGE: $\mathrm{cr}$

RANGE: end

RANGE: $\mathrm{COI}$

RANGE: end

RANGE: ang

RANGE: end

RANGE: end

RANGE: end

RANGE: end

RANGE: ven

RANGE: end

RANGE: end

RANGE: end

RANGE: COI

RANGE: end

RANGE: end

RANGE: end

RANGE: end

RANGE: end

RANGE: end

RANGE: be

RANGE: end

RANGE: end

RANGE: $\mathrm{cr}$

nic

RANGE: $c r$

RANGE: end

tree
tree
shrub
tree
shrub
tree
tree
tree
shrub
tree
shrub
tree
shrub
tree
tree
tree
shrub
tree
shrub
tree
shrub
shrub
tree
tree
shrub
tree
shrub
tree
shrub
shrub
tree
shrub
shrub
tree
shrub
tree
shrub
tree
tree
shrub
shrub
shrub
tree
shrub
tree
shrub
tree
tree
tree
trien


236 (150) MYRSINACEAE cont.

pel lucida Oerst.

*pergracilis Lund.

*pilonens is Lund.

*pirreana Lund.

pittieri Mez

pleurobotrya J.D. Sm.

*pluriflora Lund.

pulverulenta Mez

reflexiflora Lund,

* retusa Lund.

revoluta Kunth

* rimiformis Lund.

romeroi cuatr.

* rufa Lund.

* santafeana Lund.

* sapoana Lund.

scheryi Lund.

solanacea Roxb.

stenophylla J.D. Sm.

subsessilifolia Lund.

*tacarcunana Lund.

*tenuicaulis Lund.

tenuis Lund.

*tuirana Lund.

tysonil Lund.

*ursina Lund.

*veraguasens is Lund.

*vesca Lund.

wedelii Lund.

whitei Lund.

woodsonil Lund,

* Conomorpha

*gentryi Lund.

* panamens is Lund.

PROV: ca da pa

PROV: pa

PROV: $\mathrm{cC}$

PROV: da

PROV: cc

PROV: ch

PROV: $\mathrm{ch}$

PROV: $v r$

PROV: ch

PROV: $v r$

PROV: ch

PROV: pa

PROV: da

PROV: ch pa

PROV: vr

PROV: da

PROV: ch

PROV: ca

PROV: bo

PROV: ch

PROV: da

PROV: ch

PROV: da

PROV: da

PROV: pa

PROV: pa

PROV: $v r$

PROV: $c c$

PROV: bo

PROV: ch

PROV: ch

PROV: pa

PROV: ch

PROV: ch

*ba ruana (Lund.) Pipoly

* costaricanus HemsI.

*--subsp. costaricanus

* gentryi Lund.

*montanus (Lund.) Agostin

PROV: bo ch sa

PROV: da

PROV: ca pa

*occigranatensis (Cuatr.) Agostini PROV: da 000m: $0-1$

000m: $0-1$

$000 \mathrm{~m}: 0-1$

$000 \mathrm{~m}: 1-2$

000m: $0-1$

000m: $2-3$

000m: $1-2$

000m: we

000m: 1-2

000m: $1-2$

000m: 2-3

000m: $0-1$

000m: $0-1$

$000 \mathrm{~m}: 0-1,2-3$

000m: $0-1$

000m: $1-2$

000m: $1-2,2-3$

000m: $0-1$

000m: $0-1$

000m: $1-2,2-3$

000m: 1-2

000m: $1-2$

000m: $0-1,1-2$

000m: $0-1$

000m: $0-1$

000m: $0-1$

000m: $0-1$

000m: $0-1$

000m: $0-1$

000m: $2-3$

000m: $1-2$

000m: $0-1$

000m: $2-3$

$000 m: 2-3,3+$

000m: $1-2,2-3$

$000 m: 1=2$

000m: $0-1$

000m: $1-2$

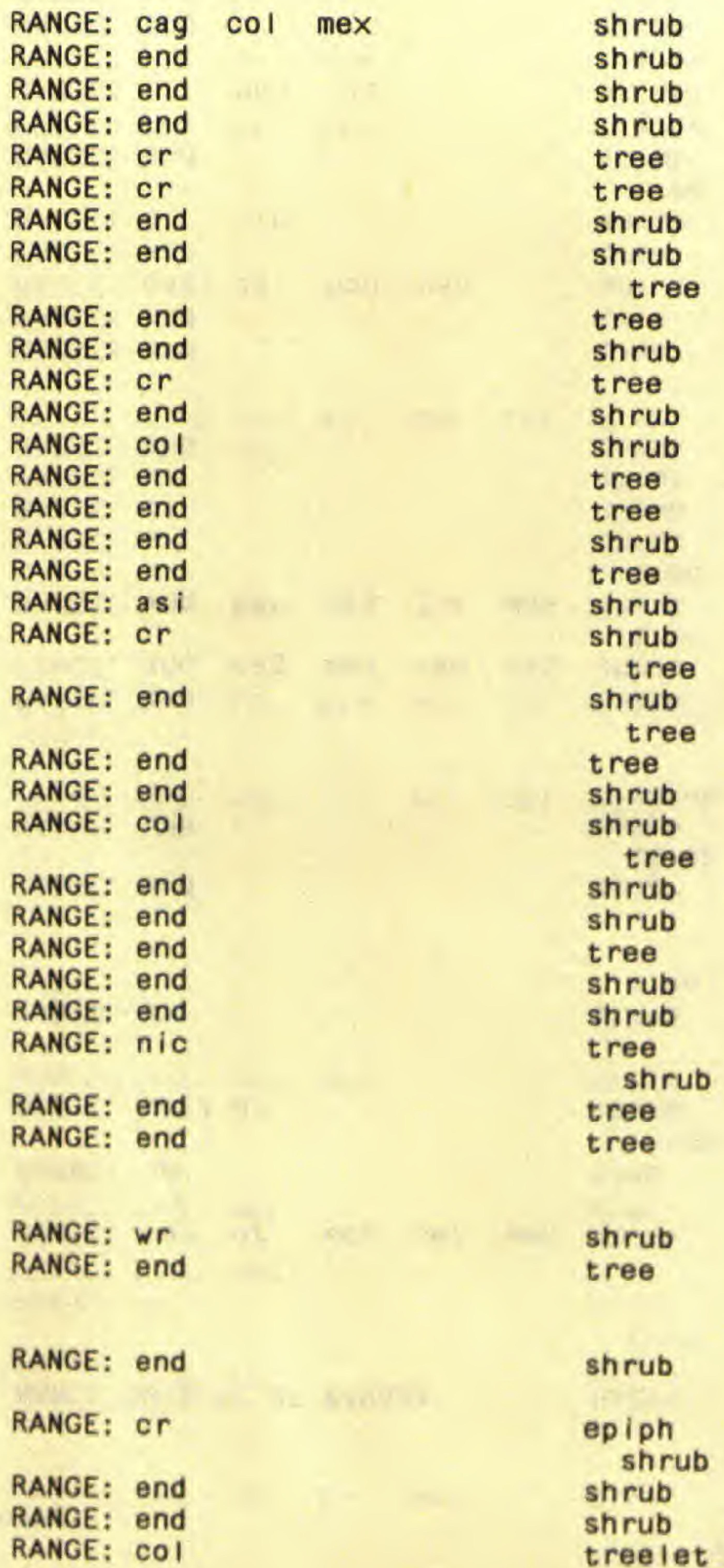


TAXON

FAMILY

PROVINCES

ELEVATION (000M.)

RANGE OUTSIDE OF PANAMA

HABIT

236 (150) MYRSINACEAE cont.

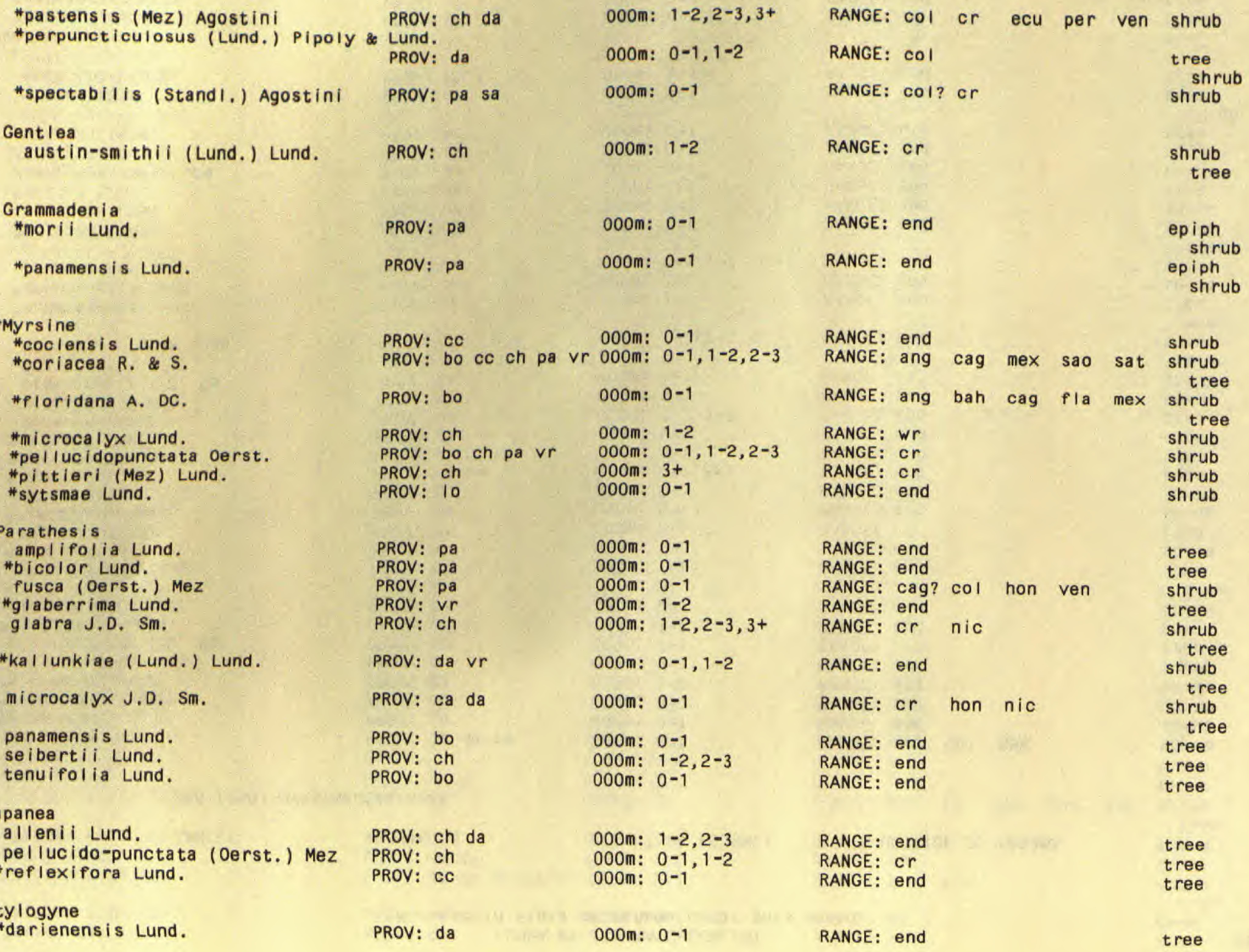


TAXON

FAMILY

PROVINCES

ELEVATION (OOOM.)

RANGE OUTSIDE OF PANAMA

HAB IT

236 (150) MYRSINACEAE cont.

hayesif Mez

standleyi Lund.

turbacensis (Kunth) Mez
PROV: da pa 000m: $0-1$

PROV: bo ca da pa $000 \mathrm{~m}: 0-1$

PROV: ca cc ch he lo 000m: $0-1$
RANGE: end

RANGE: cag col ven shrub

shrub

tree

shrub

\section{7 (152) PRIMULACEAE}

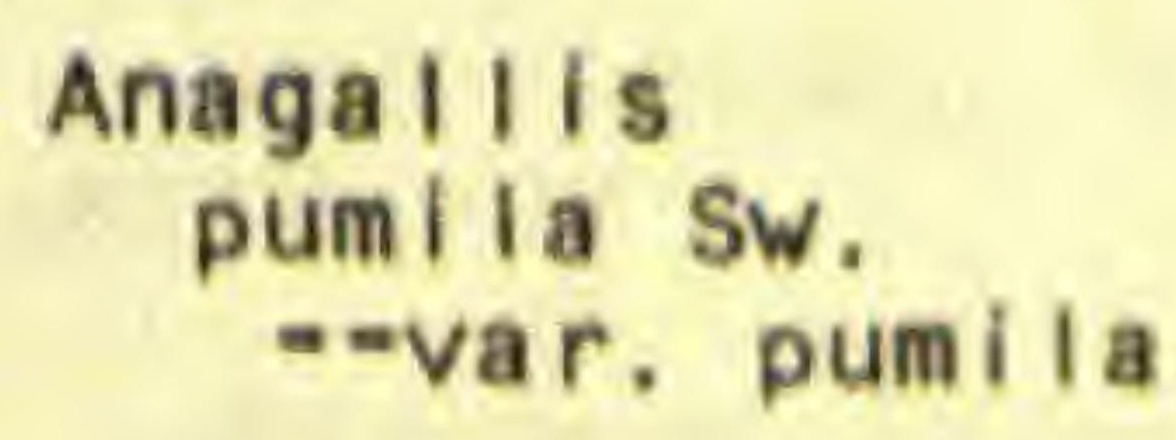

$000 \mathrm{~m}: 0-1,1-2$

238 (153) PLUMBAGINACEAE

\section{Plumbago}

auriculata Lam.

intr. cult.

PROV: vr

$000 m: 1-2$

scandens L.

intr. cult. nat.

PROV: ca cc he lo pa 000m: $0-1$

\section{$239(154)$ SAPOTACEAE}

Bume I ia

persimilis Heasi.

- -subsp. persimitis

PROV: $\mathrm{cc} 10 \mathrm{pa}$

000m: $0-1$

Chrysophy I I um

cainito L.

"exce I sum Huber

*hirsutum Crong

* mexicanum Standi.

*Cynodendron

* panamense (Pitt.) Aubrev. cult. PROV: bo ca co ch da 000m: 0-1

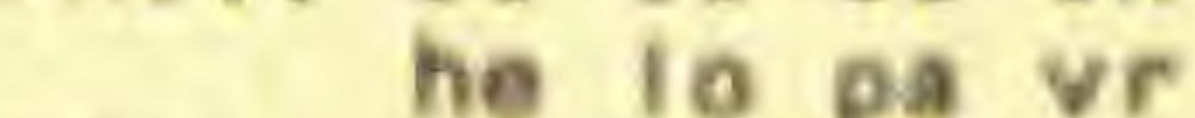

PROV: pa

PROV: ch pe

000n: $0-1$

PROV: CE da

000n: $0-1$

PROV: bo ca cn da pa 000a: $0-1$

RANGE: afr ang asi cag fla herb
mex oce sag


TAXON

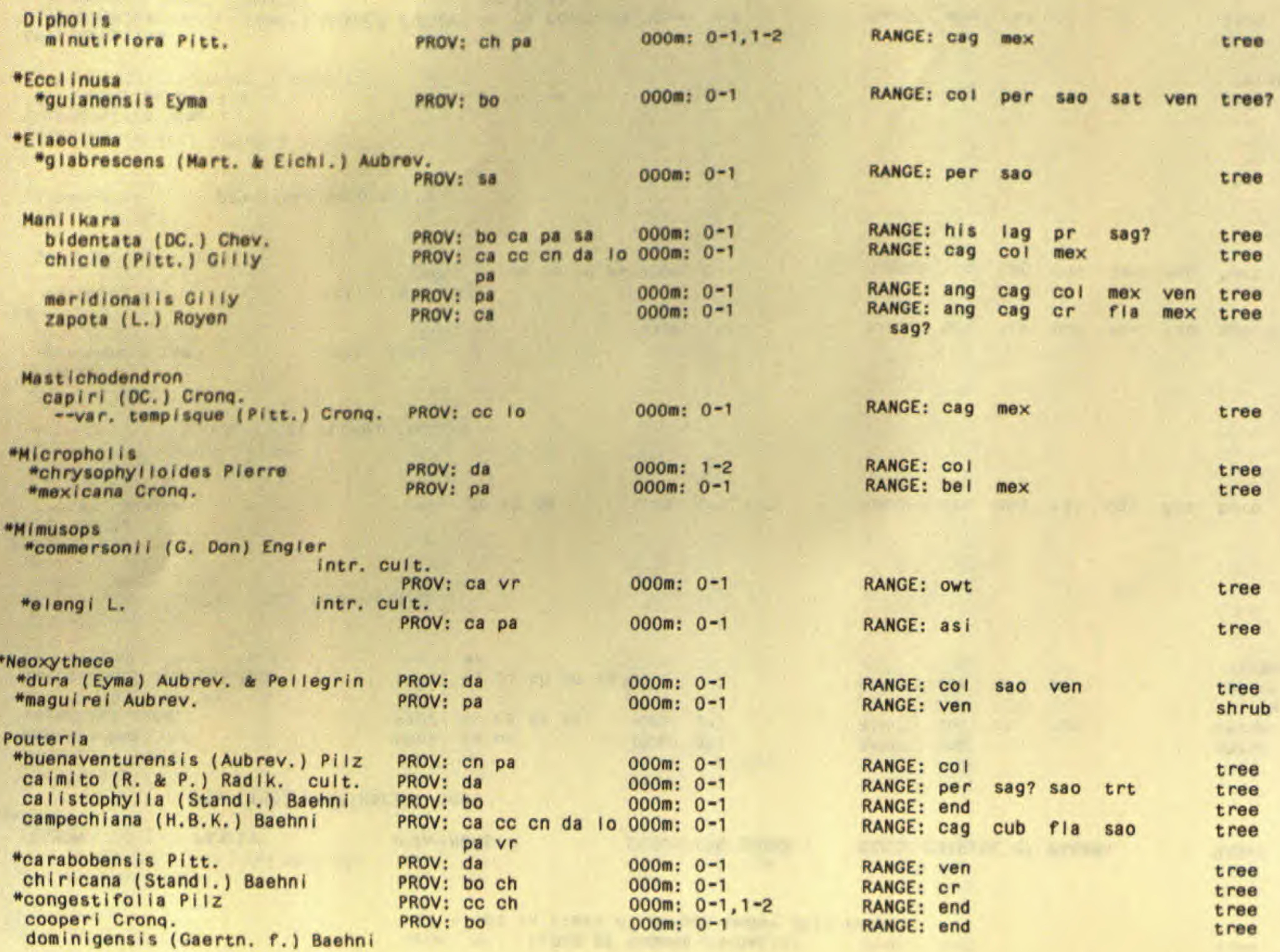


- -var. dominigensis

*durlandi i (Stand I.) Baehn i

*engleri Eyma

euryphylla (StandI.) Baehni

fossicola crong.

* leptopedicellata Pilz

Iucentifolia (StandI.) Baehni

*neglecta Cronq.

pentasperma (Stand I.) Baehni

sambuensis (Pitt.) Baehni

sapota (Jacq.) Moore \& Stearn cult.

sclerocarpa (Pitt.) Cronq.

stipitata Crong.

stylosa (Pierre) Dubard

subrotata Crong.

* ta rapotens is (Pierre) Baehni

*unilocularis (J.D. Sm, ) Baehni

PROV: ca

PROV: da

PROV: cn pa

PROV: bo

PROV: ca cc cn da

PROV: $\mathrm{cn}$

PROV: bo cc pa vr

PROV: ca da

PROV: bo da

PROV: da

000m: $0-1$

000m: $1-2$

000m: $0-1$

000m: $0-1$

000m: $0-1$

000m: $0-1$

000m: $0-1$

000m: $0-1$

000m: $0-1$

000m: $0-1$

PROV: ca cc ch da he 000m: 0-1 pa sa

PROV: sa

PROV: ca da pa

PROV: bo ca pa

PROV: cn da

PROV: pa

PROV: ca ch da pa 000m: $0-1$

000m: $0-1$

$000 \mathrm{~m}: 0-1$

$000 \mathrm{~m}: 0-1$

000m: $0-1$

$000 \mathrm{~m}: 0-1$

\section{Synsepa I um}

*dulcificum (Schum. \& Thonn.) Daniell

$$
\text { cult. PROV: ca }
$$

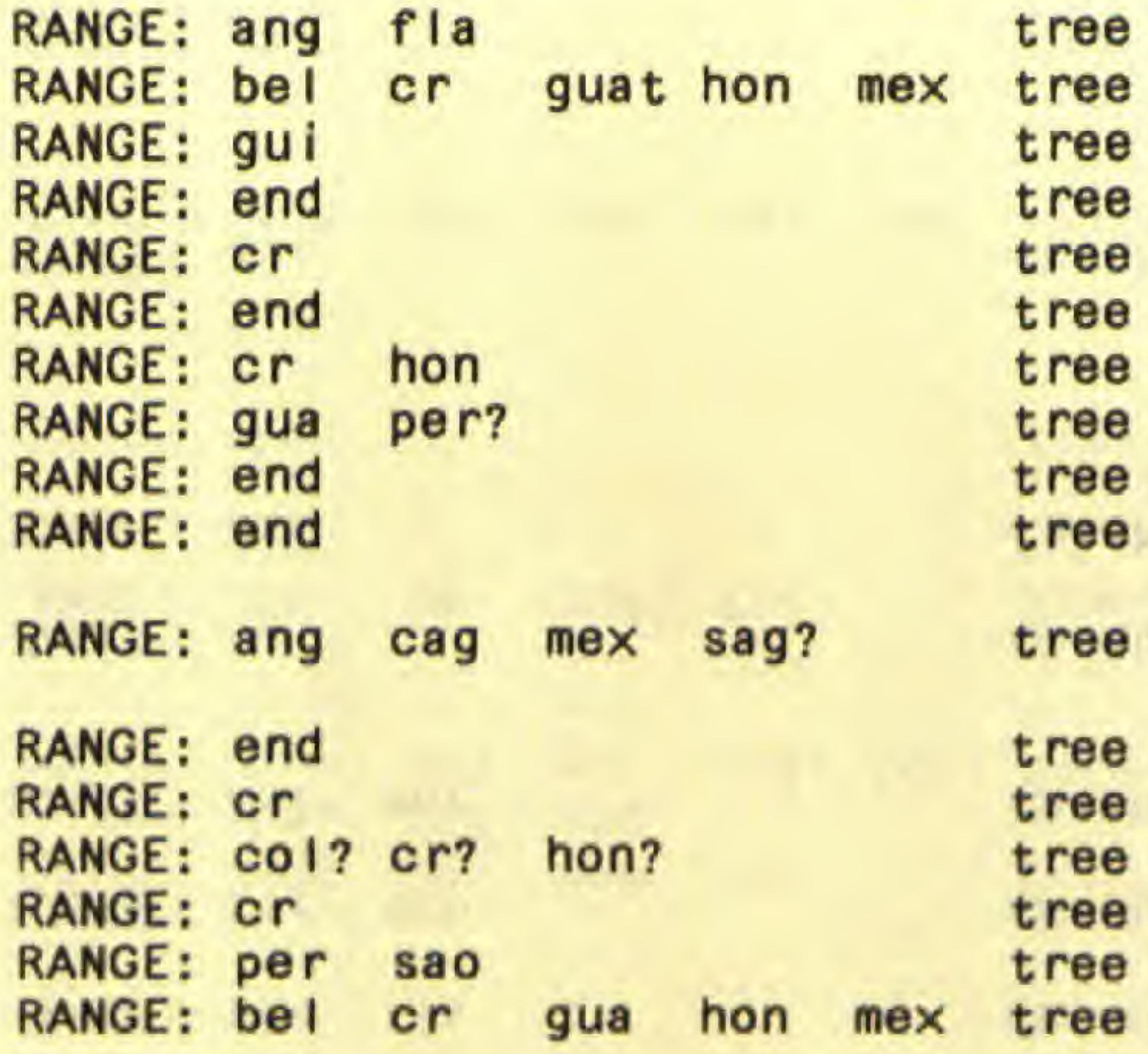

240 (155) EBENACEAE

Diospyros

artanthifolia Mart.

digyna Jacq.

* iscolor Ki.

inconstans Jacq.

salicifolia Willd.
PROV: ca cn da

PROV: $V r$

PROV: ca

PROV: da

PROV: ca da 000m: $0-1$

000m: $0-1$

000m: $0-1$

000m: $0-1$

000m: $0-1$
RANGE: per sag? sao

RANGE: cag col mex

RANGE: as $\mathbf{i}$

RANGE: ang

mex tree

tree

tree

tree

shrub

\section{1 (156) STYRACACEAE}

\section{Styrax}

argenteus Pres।

--var. a rgenteus

-var. micranthus D'Arcy

conterminus J.D. Sm.
PROV: cc ch da

PROV: ch cn

PROV: $\mathrm{ch}$ 000m: $0-1,1-2,2-3,3+$ RANGE: cag or mex

000m: 1 -2 tree tree 
TAXON

FAMILY

PROVINCES

ELEVATION (OOOM.)

RANGE OUTSIDE OF PANAMA

HAB I T

242 (157) SYMPLOCACEAE

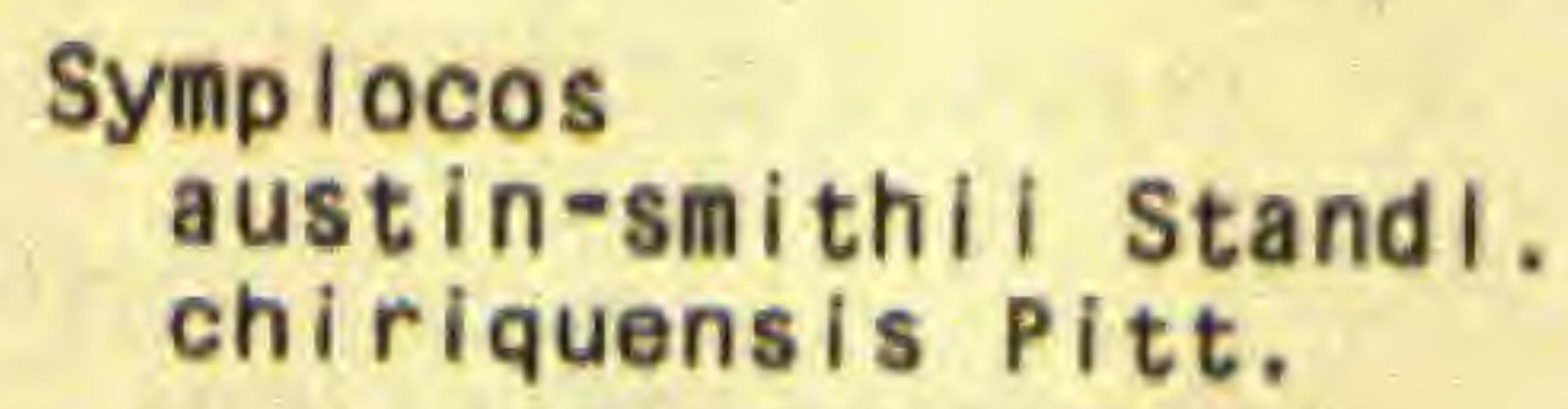

PROV: ch $000 \mathrm{~m}: 2-3$

PROV: ch PROV: ch pa

PROV: ch 000m: $0-1$

000m: $0-1$

000m: $3+$

000m: $0-1$

000m: $0-1$

000m: $0-1$

000m: $0-1$

000m: $0-1$
RANGE: $\mathrm{cr}$ RANGE: $\mathrm{Cr}$

RANGE: $\mathrm{cr}$ tree shrub tree tree

tree

tree

RANGE: $\mathrm{cr}$

shrub

RANGE: afr fla

shrub

RANGE: af $r$ sao

RANGE: asi eur

shrub

RANGE: as i

RANGE: as i

shrub

shrub

climber

RANGE: as $\mathrm{i}$

shrub

RANGE: af $r$ (n)

tree

245 (159) LOGANIACEAE

\section{Budd le ia}

americana $L$.

nitida Benth.
PROV: ca ch

PROV: ch 000m: $0-1,1-2$

000m: $1-2,2-3$
RANGE: bol cag mex per sat RANGE: cag mex tree tree 
245 (159) LOGANIACEAE cont.

\section{* skutchii Morton}

Mitreola petiolata (Gmel.) Torr. \& Gray

\section{* Pel tanthera}

*fioribunda Benth.

\section{Potalia}

ama ra Aubl.

\section{Spigel ia}

anthe Imia L.

humboldtiana Cham. \& Schlecht.

\section{Strychnos \\ brachistantha StandI. \\ * chlorantha Progel \\ * colombiensis Kruk. \& Barneby}

*croatii Kruk. \& Barneby

darienens is Seem.

*erichsoni i schomb.

nigricans Proge

panamensis seem.

panurensis Sprague \& Sandw.

*poeppigii Progel

toxifera Benth.
PROV: ch

PROV: ca he lo pa

$000 m: 2-3$

000m: $0-1$

PROV: da pa

000m: $0-1$

PROV: bo pa

000m: $0-1$

PROV: bo ca ch cn da 000m: $0-1$ lo pa sa vr

PROV: bo ca cc ch da 000m: $0-1$ pa sa

PROV: ca $\quad 000 \mathrm{~m}: 0-1$

PROV: pa sa $\quad 000 \mathrm{~m}: 0-1$

PROV: cn

000m: $0-1$

PROV: cn da pa sa 000m: $0-1$

PROV: ca cn da pa vr 000m: 0-1

PROV: da pa $\quad 000 \mathrm{~m}: 0-1$

PROV: ca 000m: $0-1$

PROV: ca cc ch cn da 000m: $0-1,1-2$

PROV: pa $v r$

000m: $0-1$

PROV: pa

PROV: ca da sa

000m: $0-1$ 000m: $0-1$
RANGE: mex

treelet

RANGE: ang cag nag per sag herb
Wdt

RANGE: per

tree

RANGE: cr per sag? sao shrub

RANGE: aff ang? asi cag? fla herb

mex per sag? sao

RANGE: cag mex sag sao herb

RANGE: bel gua mex nic shrub

RANGE: cag tree

RANGE: col per sat tree

RANGE: $c O$ I

shrub

tree

RANGE: bol cr gui nic per shrub

sag sao cr gui nic per climber

RANGE: per sao sat ven tree

RANGE: cag mex sao shrub

RANGE: cag col mex ven climber

RANGE: per shrub

climber

RANGE: bol col per sao sat climber

RANGE: $\mathrm{cr}$ ecu gui sao climber

246 (160) GENTIANACEAE

\begin{tabular}{|c|c|c|}
\hline $\begin{array}{l}\text { Apteria } \\
\text { *aphylla Small }\end{array}$ & PROV: da & 000m: $0-1$ \\
\hline $\begin{array}{l}\text { Centaurium } \\
\text { quitense (Kunth) Rob. }\end{array}$ & PROV: ca ch lo & $000 m: 0-1,1-2$ \\
\hline strictum (Schiede) Druce & PROV: ch & $000 \mathrm{~m}: 2-3,3+$ \\
\hline
\end{tabular}

RANGE: $f I a$

herb

RANGE: bah cag cr cub ecu herb gua hon mex nic per sat RANGE: cag mex

herb 


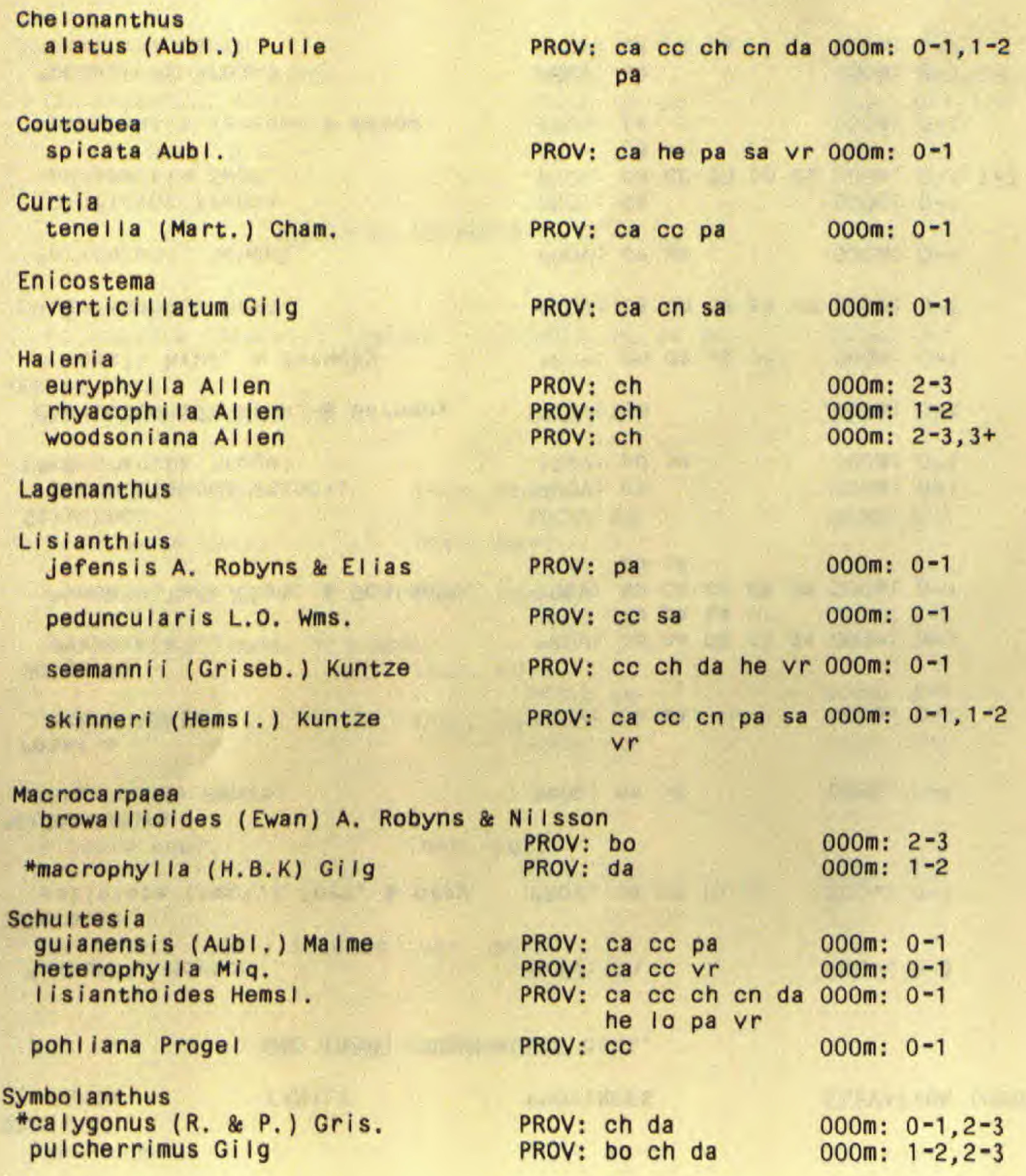

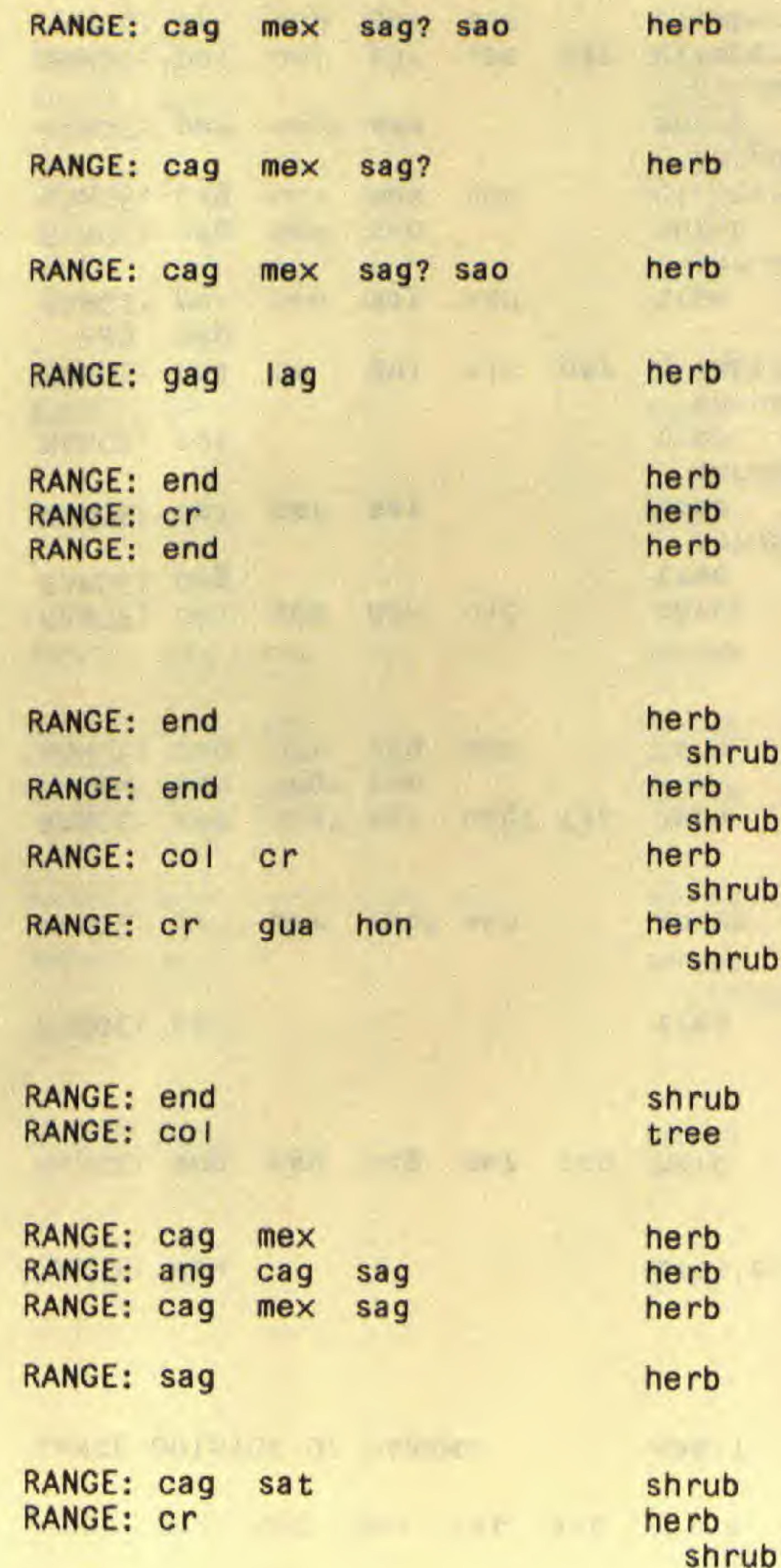




\section{Voyria}

aphylla (Jaca.) Pers.

aurantiaca Splitg.

* corymbosa Splitg.

*--subsp. a I ba (Stan

*f Iavescens Grisb.

pittieri (StandI.) L. O. Wms.

pulcherrima (StandI.) L.O. Wms.

*spruceana Benth.

tenella Hook.

PROV: bo CC da 000m: $0-1,1-2$

PROV: da pa sa 000m: $0-1$

PROV: ca cn pa sa $000 \mathrm{~m}: 0-1$

Maas

PROV: pa

PROV: bo

PROV: cc sa

PROV: sa

PROV: da sa

$0-1$

$00 \mathrm{~m}: 0-1$

$000 \mathrm{~m}: 0-1$

PROV: bo ca cn da pa 000m: 0-1

truncata (StandI.) StandI. \& Steyerm.

PROV: bo ca cc pa sa $000 \mathrm{~m}$ : $0-1,1-2$

*Voyriella

* parviflora (Miq.) Miq.

PROV: $\mathrm{cc}$

000m: $0-1$

246a (161) MENYANTHACEAE

Nymphoides

*indica (L.) Kuntze

PROV: ca cc cn pa

000m: $0-1$

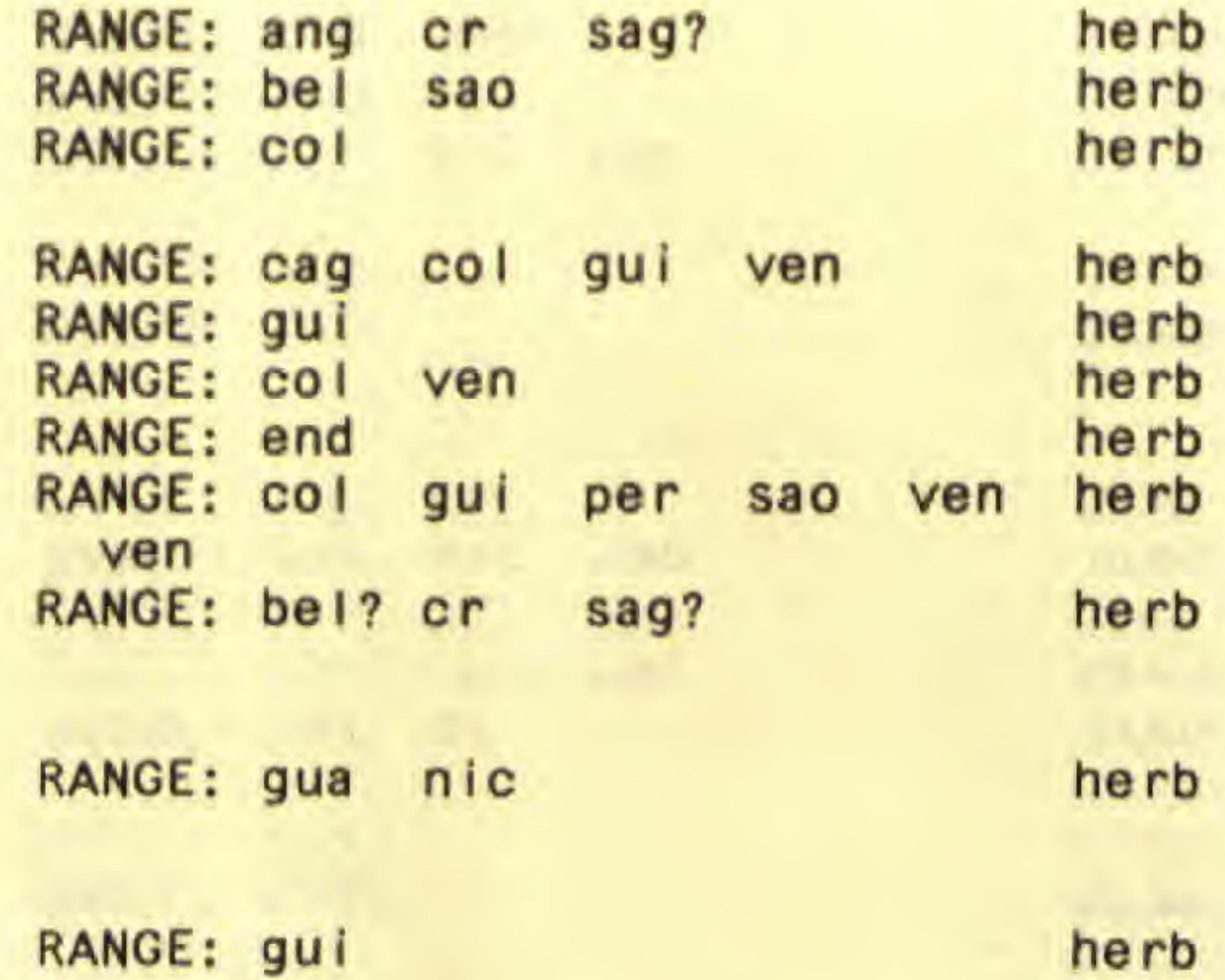

RANGE: cag gag mex sag trt herb

247 (162) APOCYNACEAE

Al l amanda

cathartica $\mathrm{L}$.

intr. cult.

PROV: bo ca ch cn da 000m: $0-1$ pa sa

Al lomarkgrafia

plumeri iflora Woods.

Anechites

nerium (Aubl.) Urb.

Aspidosperma

cruenta Woods.

* currani i Standl.

darienense Dwyer

* ma rcg ravianum Woods.

mega loca rpon Muel I. -Arg.
PROV: cn pa sa $\quad 000 \mathrm{~m}: 0-1$

PROV: bo da sa

000m: $0-1$

PROV: ca cn pa

PROV: da

PROV: da sa

PROV: ca ch da sa 000m: $0-1$

000m: $0-1$

000m: $0-1$

000m: $0-1$

000m: $0-1$
PROV: ca cn pa
RANGE: NWt

climber

RANGE: $\mathrm{col} \mathrm{cr}$

climber

RANGE: ang? col ecu

climber

RANGE: col gui sat ven

tree

RANGE: col

RANGE: end

RANGE: ven

RANGE: cag col cr mex tree

tree

tree 
247 (162) APOCYNACEAE cont.

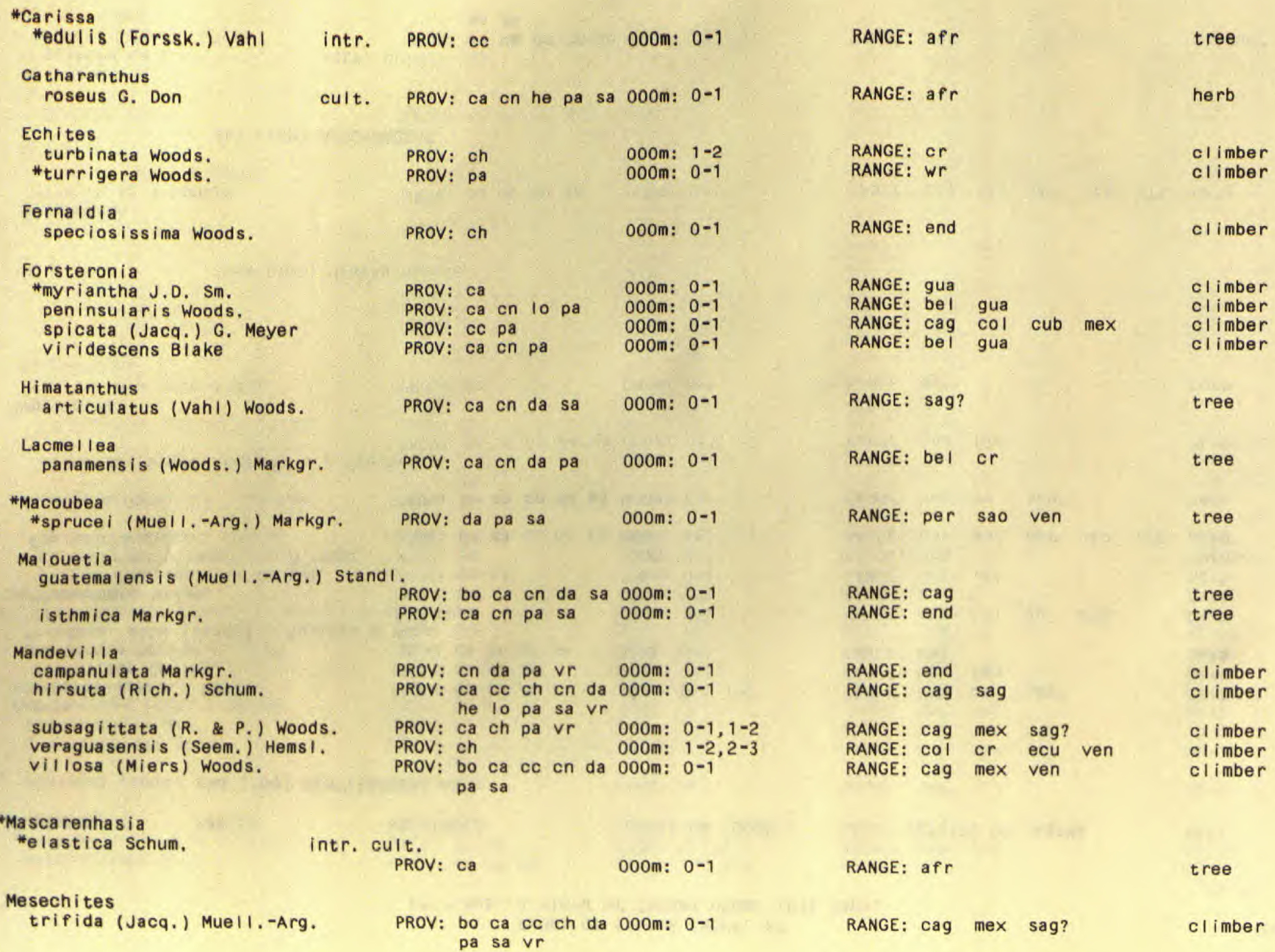


Odontadenia

* cognata (Stadelm.) Woods.

macrantha (R. \& S.) Markgr.

puncticulosa (Rich,) Pulle

Peltastes

colombianus Woods.

Plumeria

*acutifolia Poir.

* inodora Jacq.

pudica Jacq.

cult.

cult.

Prestonia

acutifolia (Benth.) Schum.

allenii Woods.

concolor (Blake) Woods.

exserta (A. DC.) Stand I.

ipomaeifolia A. DC.

lenticellata A. Gentry

*moll I is H.B.K.

obovata StandI.

portobellensis (Beurl,) Woods.

remediorum Woods.

tysonii A. Gentry

wedeli $i$ Woods.

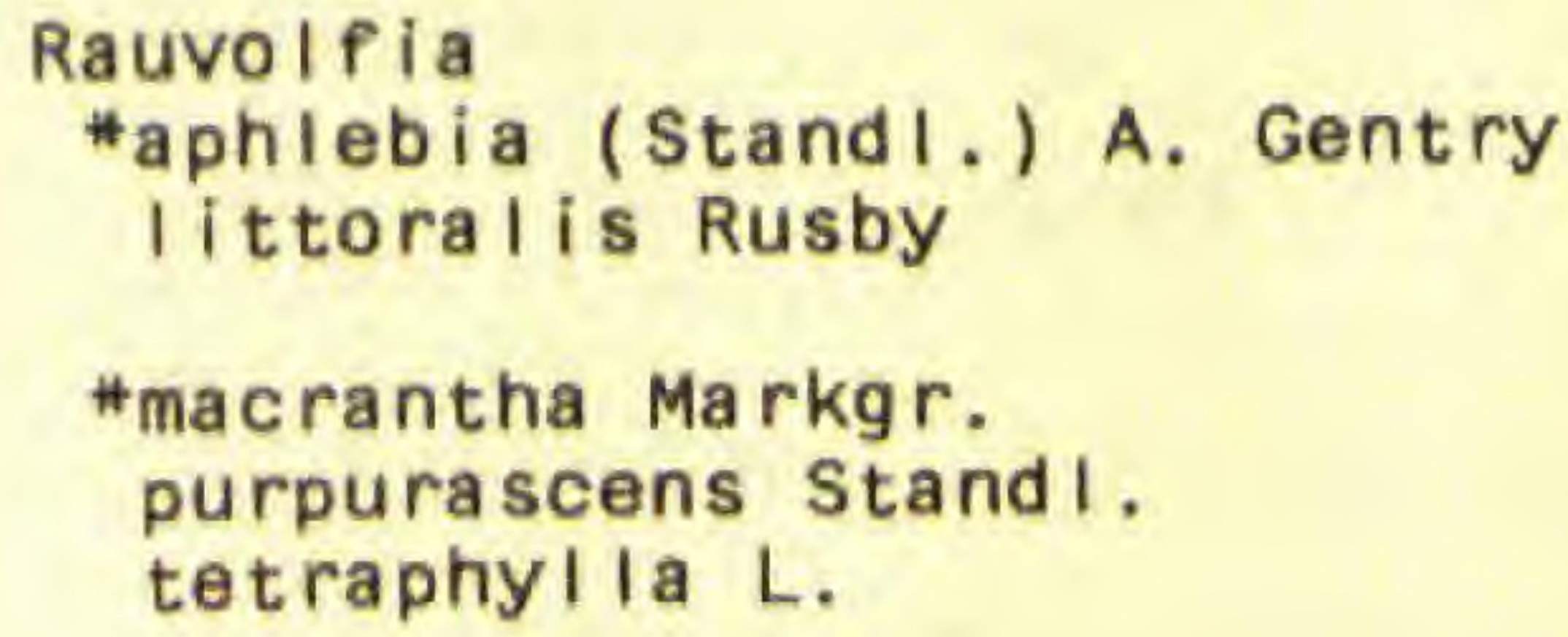

*macrantha Markgr. purpurascens Stand I. tetraphylla L.

Rhabdadenia

biflora (Jacq,) Muell. -Arg.

Stemmadenia

alfari (J,D. Sm.) Woods.

allenii Woods.

donnell-smithii (Rose) Woods.
PROV: bo ca da he $10000 \mathrm{~m}$ : $0-1$ pa sa

PROV: cn

000m: $0-1$

cn da pa sa vr 000m: $0-1$

PROV: $c$ a $\mathrm{CC}$ ch da he $000 \mathrm{~m}: 0-1$ pa sa

PROV: ca cn da pa vr 000m: 0-1

PROV: ca ch da pa 000m: $0-1$

000m: $0-1$

PROV: ca cc da pa Prov: ce cn pa sa 000m: $0-1$ PROV: bo $v r \quad 000 \mathrm{~m}: 0-1$

PROV: ca cc da pa $000 \mathrm{~m}: 0-1$

PROV: $c a$ cc da lo pa $000 \mathrm{~m}: 0-1$

PROV: cn pa $000 \mathrm{~m}: 0-1$

PROV: da 000m: we

PROV: ca cc ch cn da 000m: $0-1$ pa sa

PROV: bo ca cc ch cn 000m: 0-1 da he lo pa sa

PROV:

000m: $0-1$

PROV: $P$

000m: $0-1$

PROV: bo

000m: $0-1$

PROV: ch ch da sa vr 000m: $0-1,1-2$

PROV: ca cc ch cn da 000m: $0-1$

PROV: Ca

PROV: $\mathrm{CC}$

PROV: bo $v r \quad 000 \mathrm{~m}: 0-1$

PROV: ca cc da he lo $000 \mathrm{~m}: 0-1$

pa

PROV: bo ca ch cn da 000m: 0-1 pa sa

PROV: cc pa

000m: $0-1$

PROV: cc pa vr

$000 \mathrm{~m}: 0-1$

PROV: bo ch

000m: $0-1$
RANGE: $\mathrm{cr}$

RANGE: $c r$

sag?

limber

RANGE: $c r$ gui sao

climber

climber

RANGE: $\mathrm{cr}$

climber

$\begin{array}{lll}\text { RANGE: ang cag cr mex } & \text { tree } \\ \text { RANGE: col gui ven } & \text { tree } \\ \text { RANGE: col lag? ven } & \text { shrub } \\ & & \\ \text { RANGE: sag } & & \text { climber } \\ \text { RANGE: cr } & \text { climber } \\ \text { RANGE: bel } & \text { climber } \\ \text { RANGE: col trt ven } & \text { climber } \\ \text { RANGE: col gui } & \text { climber } \\ \text { RANGE: end } & \text { shrub } \\ \text { RANGE: ecu per sao } & \text { climber } \\ \text { RANGE: end } & & \text { climber } \\ \text { RANGE: cag mex } & \text { climber } \\ \text { RANGE: end } & & \text { climber } \\ \text { RANGE: end } & & \text { shrub } \\ \text { RANGE: end } & & \text { climber }\end{array}$

RANGE: $\mathrm{cOl} \mathrm{cr}$ herb

RANGE: $c O \mathrm{l}$ cr ecu shrub

RANGE: per sao tree

RANGE: end climber?

RANGE: ang asi cag mex oce shrub

sag?

RANGE: cag? fla gag sag? climber

RANGE: $\mathrm{cr} \quad$ tree

RANGE: end tree

RANGE: cag mex tree 
TAXON

grandiflora (Jacq.) Miers

lagunae Woods.

macrantha Stand I.

*minima A. Gentry

obovata (Benth, ) Woods.

\section{Stenosolen}

holothuria Markgr.

\section{Tabernaemontana \\ amygdal i fol ia Jacq.}

a rborea Rose

chrysocarpa Blake

*divaricata R. \& S.

* gentriana L. Al lorge

* longipes J.D. Sm.

*ma rckgarviana Macbr.

"panamens is (Markgr., Boit. \& A. Allorge) Leeuwenberg

cult. PROV: bo ca cc ch cn 000m: 0-1, 1-2
da pa sa vr

pendula Woods.

sananho R. \& $P$.

undulata VahI

Thevetia

ahoua i (L.) A. DC.

peruviana (Pers.) Schum. cult.

cult.

PROV: bo ca cc ch cn 000m: $0-1$

da he lo pa sa $v r$

PROV: bo $000 \mathrm{~m}: 0-1$

PROV: bo

000m: $0-1$

PROV: ca ce cn pa vr 000m: 0-1

PROV: 10 000m: $0-1$

PROV: da pa

000m: $0-1$

PROV: pa vr

000m: $0-1$

PROV: bo ca da pa sa $000 \mathrm{~m}: 0-1$

PROV: bo $\mathrm{cc}$ ch da $000 \mathrm{~m}$ : $0-1,1-2$

PROV: ca pa $\quad 000 \mathrm{~m}: 0-1$

PROV: ch pa 000m: $0-1,1-2$

PROV: $\mathrm{CC} \quad 000 \mathrm{~m}: 0-1$

000m: we

PROV: $\mathrm{cc}$ cn

PROV: sa

000m: $0-1$

000m: $0-1$

PROV: cc cn pa sa $000 \mathrm{~m}: 0-1$

PROV: bo ca ch cn da 000m: $0-1$ he lo pa sa vr

PROV: ca ce cn da pa 000m: 0-1 sa

PROV: ch

000m: 1 -2

longifolia (A. DC.) Markgr. 000m: $1-2$

Vinca

minor $L$. intr. cult.

PROV: WP 000m: $0-1$

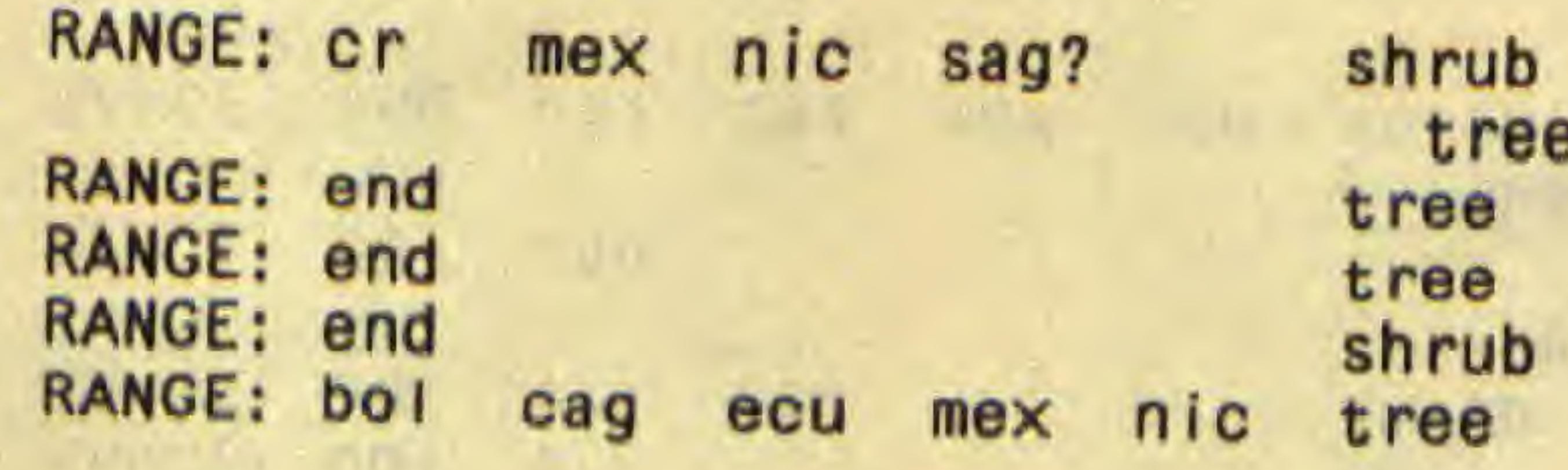

RANGE: end

shrub

RANGE: cag col mex ven

shrub

RANGE: bel gua

RANGE: cag

RANGE: as $\mathrm{i}$

RANGE: end

RANGE: $c r$

RANGE: per sao

RANGE: ang

RANGE: end

RANGE: sag

col ecu per sao

RANGE: $\mathrm{cr}$

gui per

trt

RANGE: cag mex sag?

RANGE: nWt

$$
\begin{gathered}
\text { shrub } \\
\text { tree } \\
\text { shrub } \\
\text { shrub } \\
\text { tree }
\end{gathered}
$$$$
\text { tree }
$$

RANGE: cag mex

RANGE: OWg

herb 
Asclepias

curassavica L.

*physocarpus Schltr.

Blepha rodon

mucronatum (Schlecht.) Decne.

PROV: bo ca cc ch cn 000m: $0-1,1-2$ da he lo pa sa vr

PROV: ca

000m: $0-1$

PROV: ca ce ch $\mathrm{cn}$ da 000m: $0-1,1-2$ pa $\mathrm{vr}$

\section{Calotropis}

procera (Ait.) Ait. f,

PROV: IO pa

000m: 0-1

\section{Cryptostegia}

"grandiflora R. Br. intr. cult.

rnanchum

cubense (A, Rich.) Woods.

glaberrimum (Woods.) L.0. Wms,

infimicola L.O. Wms.

magdalenicum Dugand

*montevidense Spr.

Fischeria

blepharopetala Blake

*brachyca Iyx L. O. Wms.

panamens is SpeIIman

* scandens DC.

\section{Gono lobus}

a lbomarginatus (Pitt.) Woods.

allenii Woods.

chiriquensis (Woods.) Woods.

edulis Hems!.

*eriocladon Benth.

fuscoviolaceus Woods.

\#heterophyllus (HemsI.) Stevens

inaequal is L.O. Wms.

lewisii L.O. Wms.

ophioglossa Woods.

* rothschuhii SchItr.

$\begin{array}{ll}\text { PROV: ca cc pa } & \text { 000m: } 0-1 \\ \text { PROV: ch } & 000 \mathrm{~m}: 1-2 \\ \text { PROV: ca cc pa } & 000 \mathrm{~m}: 0-1 \\ \text { PROV: ca pa } & 000 \mathrm{~m}: 0-1 \\ \text { PROV: pa } & 000 \mathrm{~m}: 0-1 \\ & \\ \text { PROV: ca cn da pa sa } 000 \mathrm{~m}: 0-1 \\ \text { PROV: vr } 000 \mathrm{~m}: 0-1,1-2 \\ \text { PROV: bo ca ce ch pa } 000 \mathrm{~m}: 0-1,1-2 \\ \text { PROV: da }\end{array}$

PROV: da

$000 \mathrm{~m}: 0-1,1-2$

$\begin{array}{ll}\text { PROV: bo ca cc vr } & 000 \mathrm{~m}: 0-1 \\ \text { PROV: ca cc pa } & 000 \mathrm{~m}: 0-1 \\ \text { PROV: ch } & 000 \mathrm{~m}: 1-2 \\ \text { PROV: ch } & 000 \mathrm{~m}: 1-2 \\ \text { PROV: cn } & 000 \mathrm{~m}: 0-1 \\ \text { PROV: ch } & 000 \mathrm{~m}: 1-2 \\ \text { PROV: da } & 000 \mathrm{~m}: 0-1 \\ \text { PROV: ca pa } & 000 \mathrm{~m}: 0-1 \\ \text { PROV: da lo } & 000 \mathrm{~m}: 0-1 \\ \text { PROV: bo cc cn pa vr 000m: } 0-1\end{array}$

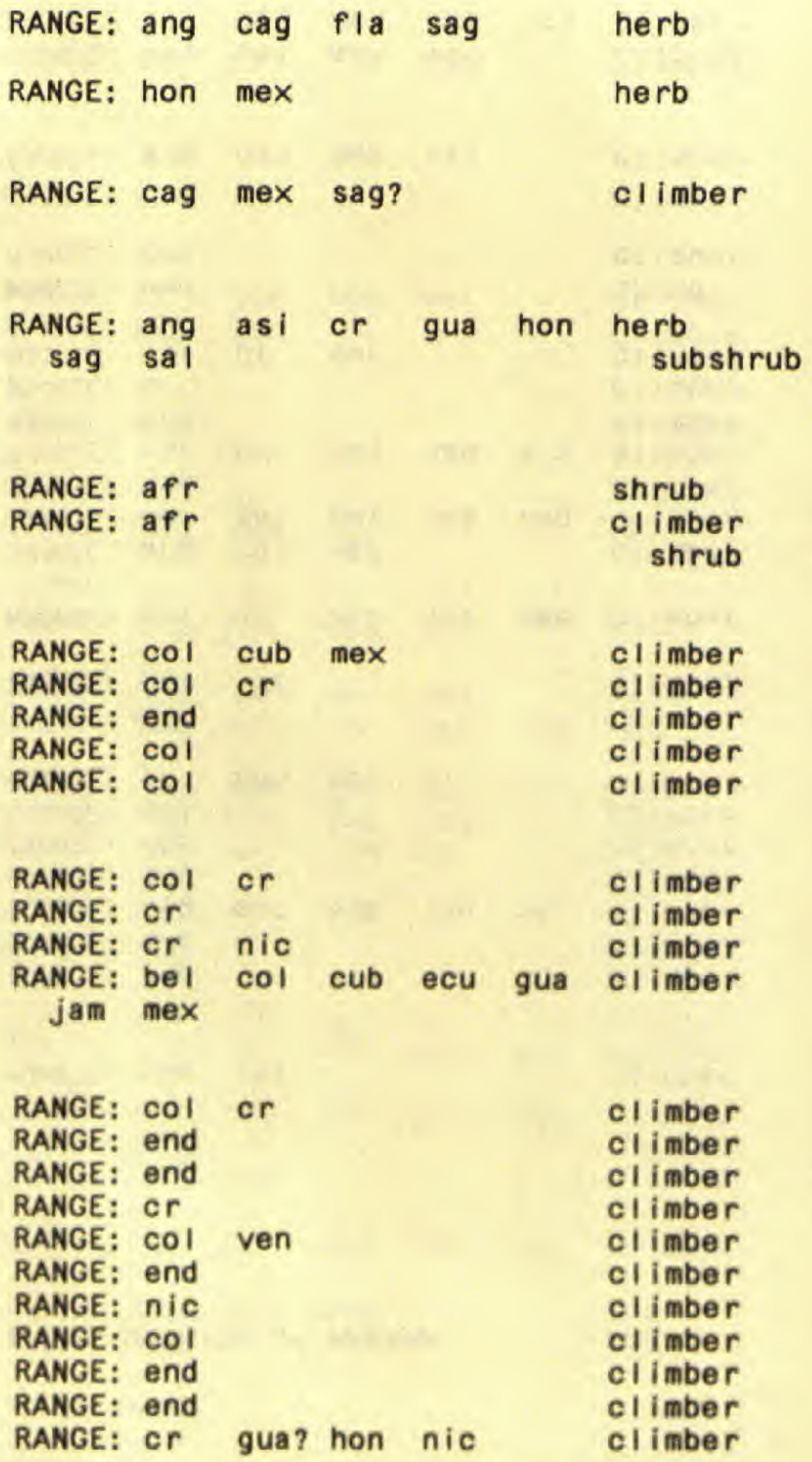


TAXON

Hoya

carnosa (L.) R. Br. intr, cult.

PROV: ca $\quad 000 \mathrm{~m}: 0-1$

Marsdenia

crassipes HemsI.

dressleri SpelIman

maculata Hook. f.

margaritaria Foster

panamensis SpelIman

rotheana Woods.

trivirgulata Bartlett

Matelea

brasiliensis (Schltr.) Spellman PROV: bo pa

PROV: ca pa sa 000m: $0-1$

PROV: pa 000m: $0-1$

PROV: bo ca da lo pa 000m: $0-1$

PROV: cc pa $000 \mathrm{~m}: 0-1$

PROV: $10 \quad 000 \mathrm{~m}: 0-1$

PROV: $\mathrm{CC} \quad$ O00m: $0-1$

PROV: ca he $000 \mathrm{~m}: 0-1$

*denticulata (VahI) Fontella \& Schwarz

PROV: bo ca ch da pa $000 \mathrm{~m}: 0-1$

maritima (Jacq.) Woods.

mediocris Woods.

panamensis SpelIman \& Dwyer

pinguifolia (Standl.) Woods.

pittieri (Standl.) Woods.

* pseudobarbata (Pitt.) Woods.

trianae (Trin.) SpelIman

tristis (Seem.) Spellman

*viridis (Mold.) Speliman

oxypeta I um

cordifolium (Vent.) Schltr.

PROV: $c c$ cn he lo pa 000m: $0-1$

PROV: cn pa $\quad 000 \mathrm{~m}: 0-1$

PROV: ce pa $\quad 000 \mathrm{~m}: 0-1$

PROV: ca ch pa $\quad 000 \mathrm{~m}: 0-1,1-2$

PROV: ch 000m: $1-2$

PROV: ch 000m: 1 -2

PROV: ca cc da lo pa 000m: $0-1$

PROV: $v r$

PROV: $V$

000m: $0-1$

$000 \mathrm{~m}: 0-1$

PROV: pa

000m: $0-1$

Sa rcos temma

bi lobum Hook. \& Arn.

clausum (Jacq.) R. \& S.

PROV: bo da pa 000m: $0-1$

PROV: bo ca cc ch cn 000m: $0-1$

glaucum H.B.K.

$$
\text { da he lo pa }
$$

PROV: cc da lo pa $000 \mathrm{~m}: 0-1$

Stephanotis

floribunda Brongn.

intr. cult.

PROV: ca 000m: $0-1$

$000 \mathrm{~m}: 0-1$

*obovata Decne.

PROV: ca pa
RANGE: cag sat

climber

RANGE: end

RANGE: end

RANGE: cag

RANGE: end

RANGE: end

RANGE: $\mathrm{COI}$

RANGE: $\mathrm{C}$

RANGE: per

sao

climber

col gua lag ven climber

climber

climber

climber

climber

climber

RANGE: COI

Ven

RANGE: ang

RANGE: $\mathrm{COI}$

RANGE: end

RANGE: COI

RANGE: end

RANGE: $\mathrm{cr}$

RANGE: COI

RANGE: end

RANGE: $\mathrm{CO}$ I

climber

cr ecu gui sao climber

col ven

gui per sag ven climber

climber

cr sag sao trt climber

climber

climber

climber

shrub

climber

RANGE: ang cag mex sag climber

RANGE: cag col mex ven climber

RANGE: ang cag mex nwt sag climber

RANGE: col or per ven climber

RANGE: afr ang

climber

RANGE: col cr sag sao climber 


\section{9 (164) CONVOLVULACEAE}

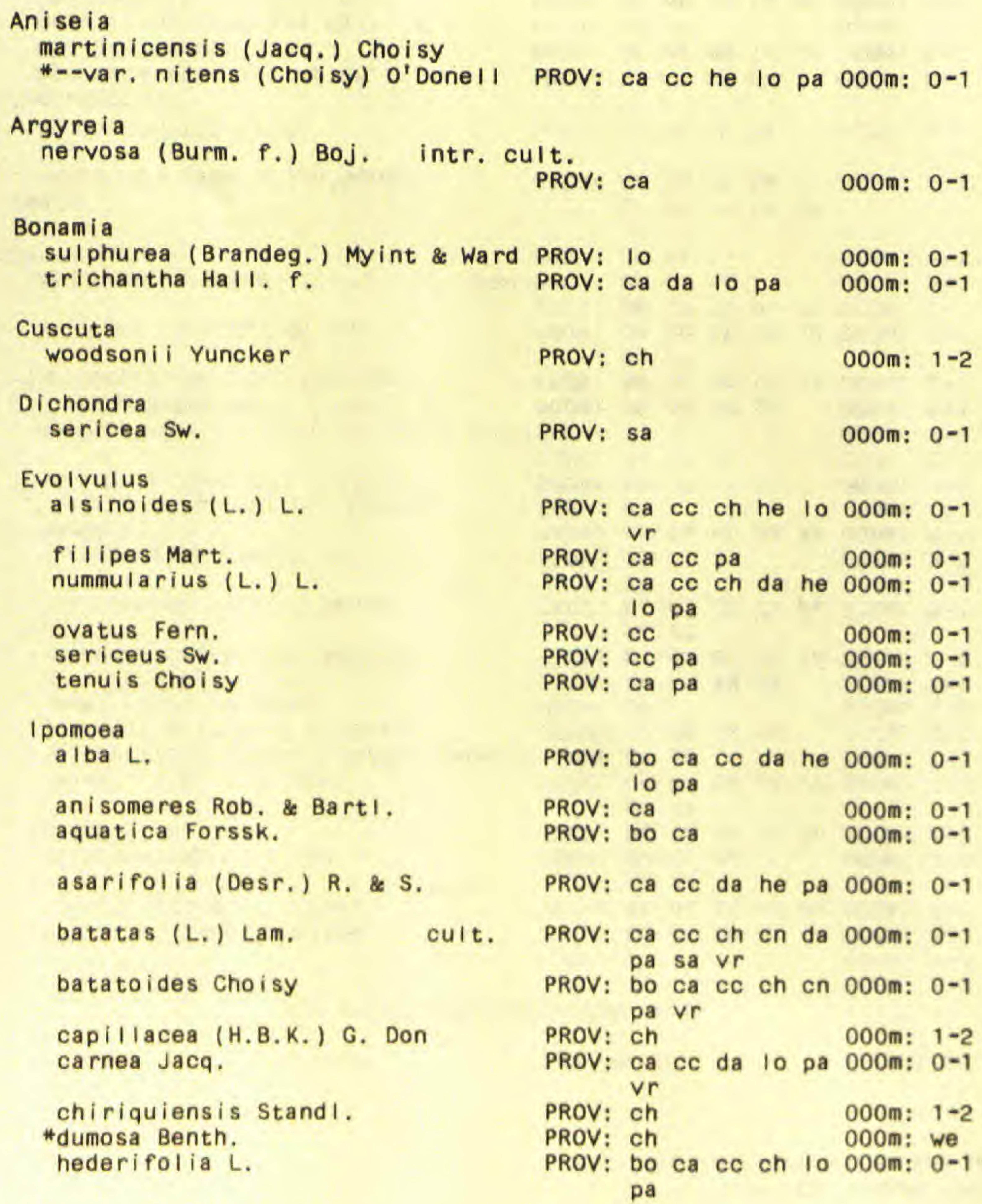

PROV: ca cc ch he lo $000 \mathrm{~m}: 0-1$ $\checkmark r$

PROV: ca cc pa 000m: 0-1 PROV: ca cc ch da he $000 \mathrm{~m}$ : $0-1$ lo pa

PROV: $C C$

000m: $0-1$ 000m: $0-1$

PROV: bo ca cc da he $000 \mathrm{~m}$ : $0-1$ lo pa

000m: $0-1$ PROV: ca 000m: $0-1$

PROV: ca cc da he pa 000m: $0-1$ PROV: ca cc ch cn da 000m: $0-1$ pa sa vr

PROV: bo ca cc ch cn 000m: 0-1 pa $v r$

PROV: ch 000m: 1 -2 PROV: ca cc da lo pa 000m: $0-1$ PROV: $\mathrm{ch}$

000m: $1-2$ 000m: we ch lo 000m: 0-1 pa

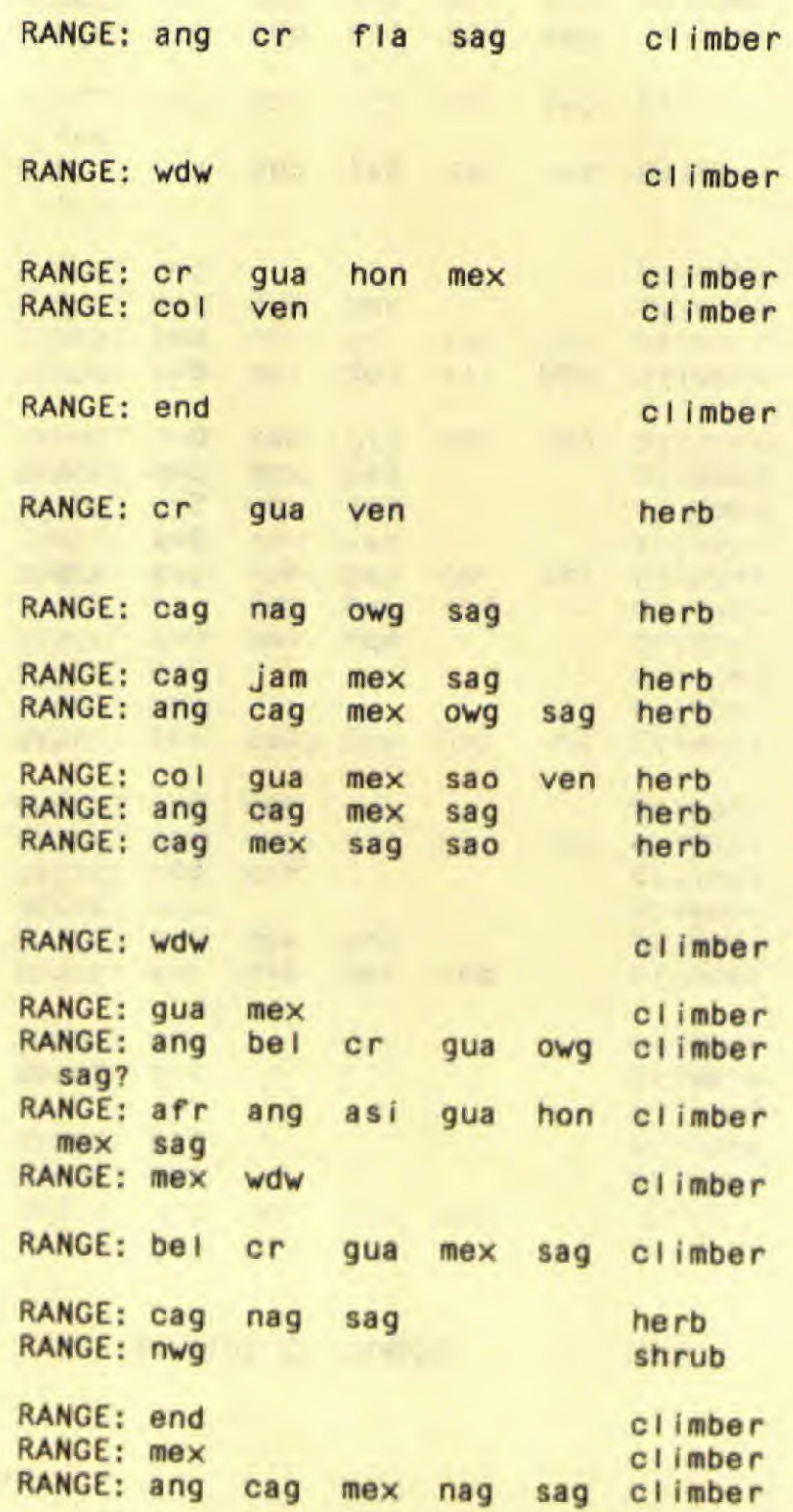


TAXON

249 (164) CONVOLVULACEAE cont,

* imperat I (VahI) Griseb. indica (Burm. f,) Merr.

Iindenii Mart, \& GaI mauritiana Jacq.

meyeri (Spr.) G. Don

minutiflora (Mart, \& Gal.) House mirandina (Pitt.) $O^{\prime}$ Donel I neei (Spr.) o'Donell

nil (L.) Roth

pes-caprae (L.) R. Br.

phillomega (VeII.) House

purpurea (L.) Roth

quamoclit $L$.

* ramossissima (Poir.) Choisy setifera Poir.

setosa Ker.

squamosa Choisy

tiliacea (Willd,) Choisy

trifida (H.B.K.) G. Don

tuxtlens is House

violacea $L$.

Iseia

Iuxurians (Moric.) O'DonelI

Jacquemont ia

agrestis (Choisy) Meisn.

cillata Sandw.

gracillima (Choisy) Hall. f. pentantha (Jacq.) G. Don

sphaerostigma (Cav.) Rusby

tamnifolia (L.) Griseb.

ROV: bo ca cn vr 000m: 0-1

PROV: bo ca ch cn da 000m: $0-1$

$$
\text { pa sa }
$$

PROV: ca lo pa 000m: $0-1,1-2$

PROV: bo ca ch cn da 000m: $0-1$ pa sa

PROV: ca ch he pa vr 000m: 0-1

PROV: ca pa $000 \mathrm{~m}: 0-1$

PROV: ca da pa vr $000 \mathrm{~m}: 0-1$

PROV: ch

000m: $1-2$

PROV: ca ch lo pa 000m: 0-1

PROV: bo ca ce cn lo $000 \mathrm{~m}$ : $0-1$ pa Vr

PROV: bo ca cn da pa 000m: $0-1$ PROV: $\mathrm{vr}$

$000 \mathrm{~m}: 0-1$

PROV: bo ca $\mathrm{ch}$ pa sa $000 \mathrm{~m}: 0-1$

PROV: da 000m: we

PROV: ch

000m: $0-1$

PROV: 10

000m: $0-1$

PROV: ca ch cn pa $000 \mathrm{~m}: 0-1$

PROV: bo $\mathrm{ca} c \mathrm{cc}$ he $000 \mathrm{~m}$ : $0-1$ sa $v r$

PROV: $\mathrm{ca} \mathrm{cc} \mathrm{ch}$ he lo 000m: $0-1$ pa

PROV: cn da pa $\quad 000 \mathrm{~m}: 0-1$ PROV: cn sa 000m: $0-1$

PROV: ca da he pa $000 \mathrm{~m}$ : $0-1$

PROV: $10 \quad 000 \mathrm{~m}: 0-1$ PROV: bo ca cn lo pa $000 \mathrm{~m}: 0-1$ PROV: $c c$ 000m: $0-1$ PROV: ca cc cn da he 000m: 0-1 lo pa vr

PROV: ca cc pa 000m: $0-1$

PROV: ca cc pa vr 000m: 0-1

\section{Maripa \\ * Iewisii Austin \\ nica raguens is Hems।.}

panamens is Hems I.
PROV: cn pa sa 000m: $0-1$

PROV: bo ca cc ch cn 000m: $0-1$ da pa $v r$

PROV: bo ca cn da pa 000m: $0-1$

RANGE: wdw
RANGE: sat


TAXON

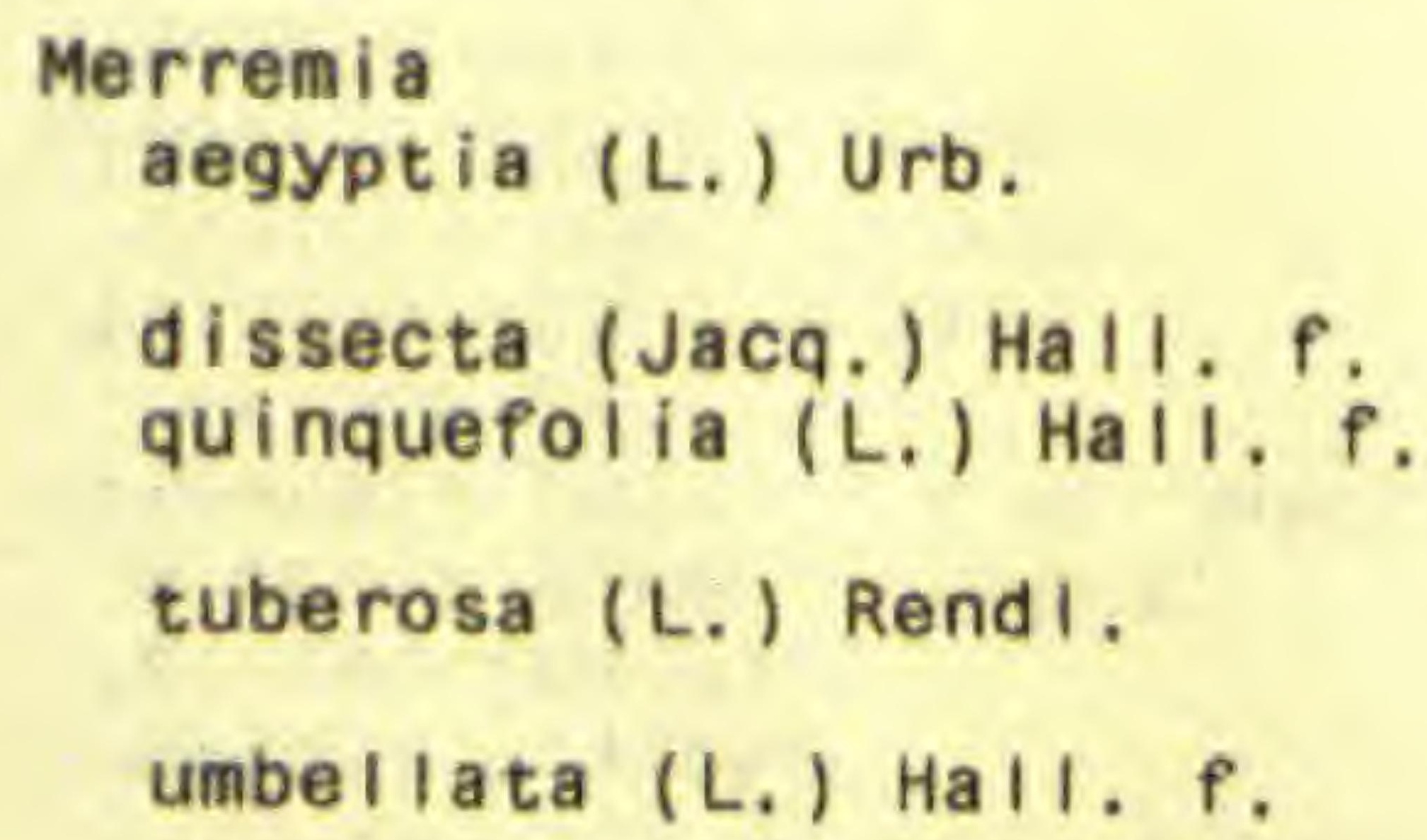

PROV: ca cc ch da he 000m: $0-1$ lo pa

PROV: bo ca ch 000m: $0-1$ PROV: ca cc ch he $10000 \mathrm{~m}: 0-1$ pa

PROV: bo ca cn pa 000m: $0-1$

PROV: bo ca ce ch cn 000m: $0-1$ da he lo pa sa

\section{* Odonellia}

*hirtiflora (Mart. \& Gal.) K. Robertson

PROV: ca ch da he pa 000m: $0-1$ $v r$

\section{Opercul ina}

codonantha (Benth.) Hall. f. PROV: ca

*hamiltoni $i$ (G. Don) Austin \& Staples

pteripes (G. Don) O'DonelI

PROV: ca pa $\mathrm{Vr}$

PROV: ca cc he pa

000m: $0-1$

000m: $0-1$

Stictocardia

tiliffolia (Desr.) Hall. f.

cult. PROV: pa 000m: $0-1$

Turbina

abutiloides (H.B.K.) O'Donell

corymbosa (L.) Raf.

PROV: $c c$ Io

PROV: bo ca da pa

000m: $0-1$ 000m: $0-1$

\section{0 (165) POLEMONIACEAE}

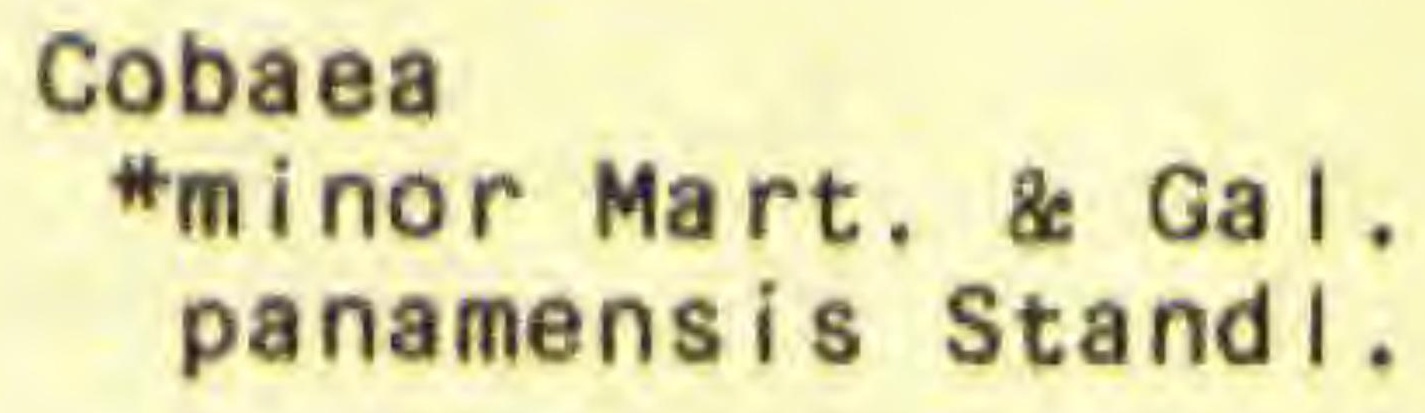

000m: $1-2$

000m: $1-2$

000m: $0-1$

PROV: $\mathrm{Ca} \mathrm{cC}$

PROV: ch

\begin{tabular}{|c|c|c|c|c|c|c|}
\hline RANGE: & ang & cag & $\operatorname{mex}$ & owg & sag & climber \\
\hline RANGE: & ang & cag & fla & $\operatorname{mex}$ & sag & cl imber \\
\hline RANGE: & ang & cag & fla & $\operatorname{mex}$ & sag & climber \\
\hline $\begin{array}{c}\text { RANGE: } \\
\text { sag }\end{array}$ & ang & cag & fla & $\operatorname{mex}$ & owg & climber \\
\hline $\begin{array}{c}\text { RANGE: } \\
\text { sag }\end{array}$ & ang & cag & fla & $\operatorname{mex}$ & owg & climber \\
\hline & & 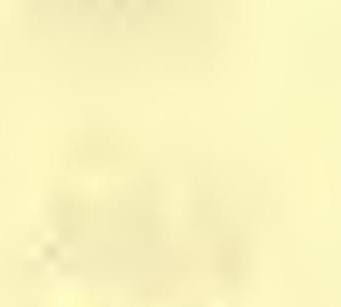 & & & & 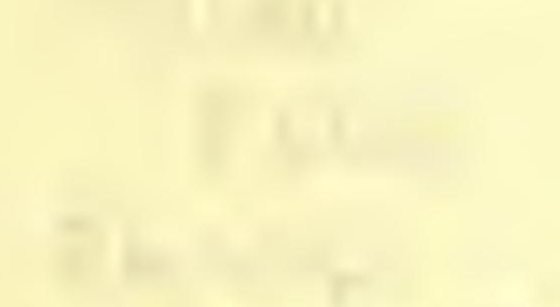 \\
\hline $\begin{array}{l}\text { RANGE: } \\
\text { mex }\end{array}$ & $\begin{array}{l}\text { bel } \\
\text { ven }\end{array}$ & $\mathrm{col}$ & $\mathrm{cr}$ & ecu & gua & climber \\
\hline & & $x^{3}+x^{4}$ & 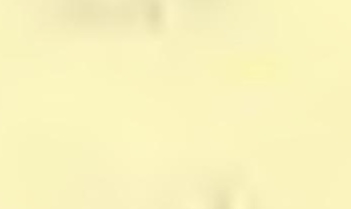 & 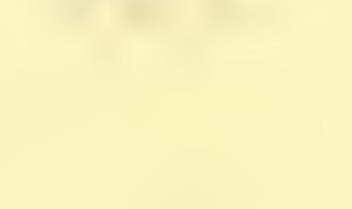 & & $x=2$ \\
\hline RANGE: & ecu & per & 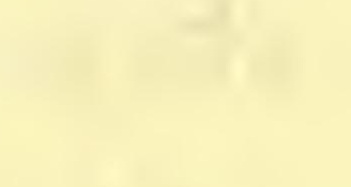 & & & climber \\
\hline RANGE: & ang & per & sao & & & climber \\
\hline RANGE: & cag & $\mathrm{col}$ & mex & per & & climber \\
\hline & at & vintin & & & & 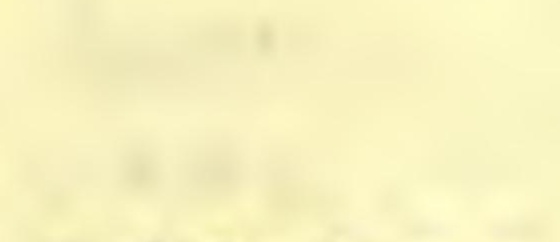 \\
\hline RANGE: & $w d w$ & & & & & climber \\
\hline RANGE: & col & ecu & ven & & & cl imber \\
\hline RANGE: & ang & cag & fla & mex & sag & climber \\
\hline th & 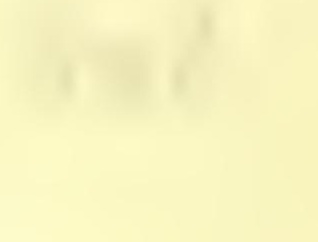 & Y & $=$ & $14-1$ & & int \\
\hline $\begin{array}{l}\text { RANGE: } \\
\text { RANGE: }\end{array}$ & $\begin{array}{l}\text { cr } \\
\text { nwt }\end{array}$ & 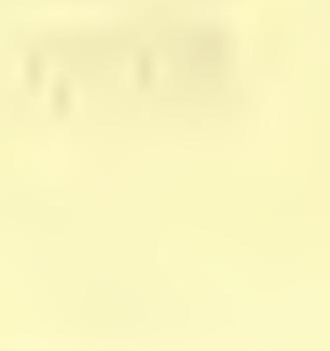 & 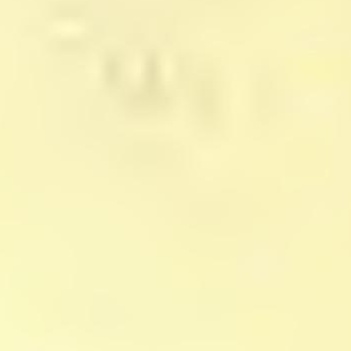 & & 10 & $\begin{array}{l}\text { climber } \\
\text { cl imber }\end{array}$ \\
\hline $\begin{array}{l}\text { RANGE: } \\
\text { RANGE: }\end{array}$ & cag & mex & nao & sag? & & shrub \\
\hline & cag & mex & sag? & & & shrub \\
\hline
\end{tabular}


TAXON

FAMILY

PROVINCES

ELEVATION (OOOM.)

RANGE OUTSIDE OF PANAMA

HABIT

251 (166) HYDROPHYLLACEAE

Hydrolea

spinosa L.

PROV: bo ca he pa

$000 \mathrm{~m}: 0-1,1-2$

Wigandia

*urens (R. \& P.) H.B.K.
PROV: ch da lo vr

$000 \mathrm{~m}: 0-1,1-2$

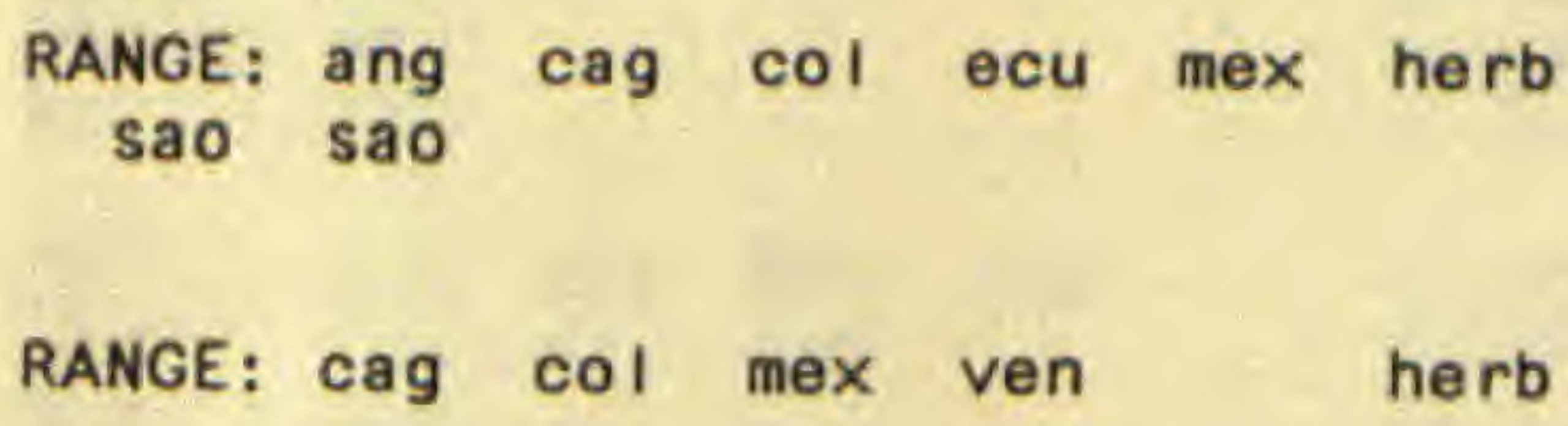

RANGE: cag col mex ven

herb

\section{2 (167) BORAGINACEAE}

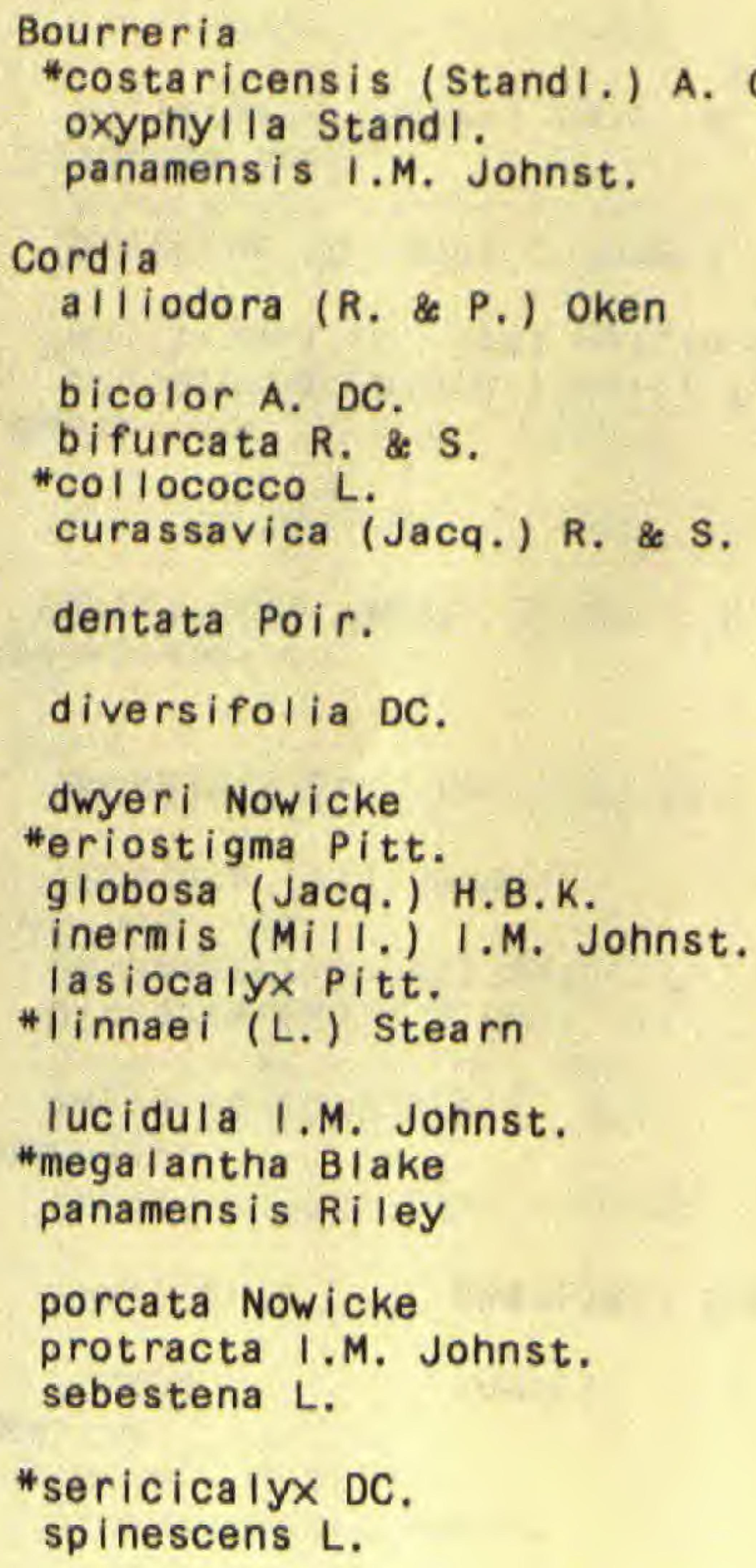

* sericica Iyx DC. spinescens L.

*toqueve Aubl. PROV: cn da sa $000 \mathrm{~m}: 0-1$ 000m: $0-1$

PROV: bo ca ch cn da 000m: $0-1$ he lo pa sa

PROV: ca da pa vr

PROV: bo da

000m: $0-1$

000m: $0-1$

PROV: $c c$

000m: $0-1$

PROV: bo ca cc cn he $000 \mathrm{~m}$ : $0-1$ lo pa sa vr

PROV: he lo vr

000m: $0-1$

PROV: bo ca

000m: $0-1$

PROV: cn cn pa 000m: $0-1$

PROV: cc pa 000m: $0-1$

PROV: pa $000 \mathrm{~m}: 0-1$

PROV: pa $000 \mathrm{~m}: 0-1$

PROV: ca cc da pa $000 \mathrm{~m}: 0-1$

PROV: ca cn da pa sa $000 \mathrm{~m}: 0-1$

vr

PROV: bo sa $\quad 000 \mathrm{~m}: 0=1$

PROV: $\mathrm{ch} \quad 000 \mathrm{~m}: 0-1$

PROV: ca cc ch cn da $000 \mathrm{~m}: 0-1$

lo pa sa vr

PROV: cc cn lo sa $000 \mathrm{~m}: 0-1$

PROV: sa $000 \mathrm{~m}: 0-1$

PROV: pa sa $000 \mathrm{~m}: 0-1$

PROV: cn pa 000m: 0-1

PROV: bo ca cc ch cn 000m: $0-1,1-2$

PROV: $c$

000m: $0-1$

\begin{tabular}{|c|c|c|c|c|c|c|}
\hline $\begin{array}{l}\text { RANGE: } \\
\text { RANGE: }\end{array}$ & $\begin{array}{l}\text { bel } \\
\text { end }\end{array}$ & hon & $x^{2}$ & & & $\begin{array}{l}\text { tree } \\
\text { tree }\end{array}$ \\
\hline & & & $=$ & 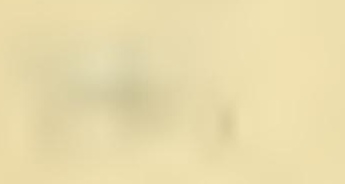 & & \\
\hline RANGE: & cag & $\operatorname{mex}$ & sat & & & tree \\
\hline RANGE: & ang? & cag & sag? & & & tree \\
\hline RANGE : & $\mathrm{cr}$ & sag? & & & & shrub \\
\hline RANGE: & ant & cag & mex & sat & & treelet \\
\hline RANGE: & ang & cag & $\operatorname{mex}$ & sag? & & $\begin{array}{l}\text { shrub } \\
\text { tree }\end{array}$ \\
\hline RANGE: & ang & cag & $\operatorname{mex}$ & sag? & & $\begin{array}{c}\text { shrub } \\
\text { tree }\end{array}$ \\
\hline RANGE: & cag & $\mathrm{col}$ & mex & & & $\begin{array}{c}\text { shrub } \\
\text { tree }\end{array}$ \\
\hline RANGE: & $\mathrm{col}$ & $\mathrm{cr}$ & 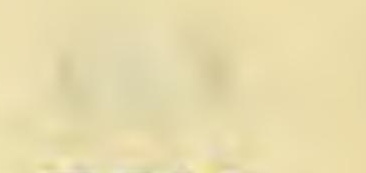 & ' & -2 & shrub \\
\hline RANGE: & cag & $\mathrm{col}$ & mex & & & shrub \\
\hline RANGE: & ang & cag & mex & sao & ven & shrub \\
\hline RANGE: & cag & $\mathrm{col}$ & $\operatorname{mex}$ & & & shrub \\
\hline $\begin{array}{l}\text { RANGE: } \\
\text { RANGF: }\end{array}$ & end & & & & & tree \\
\hline RANG & ang & cag & mex & sat & ats & shrub \\
\hline RAN & $\mathrm{cr}$ & & 't & 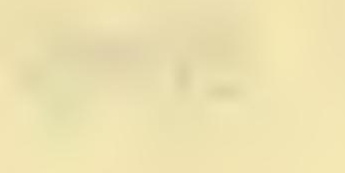 & & tree \\
\hline RANGE: & cag & $\operatorname{mex}$ & & & & tree \\
\hline RANGE: & cag & col? & ecu? & sal & & $\begin{array}{l}\text { shrub } \\
\text { tree }\end{array}$ \\
\hline $\begin{array}{l}\text { RANGE: } \\
\text { RANGE: }\end{array}$ & $\begin{array}{l}\text { end } \\
\text { end }\end{array}$ & & & & & $\begin{array}{l}\text { shrub } \\
\text { shrub }\end{array}$ \\
\hline RANGE: & ang & bah & cag & $\operatorname{mex}$ & ven & $\begin{array}{l}\text { shrub } \\
\text { tree }\end{array}$ \\
\hline $\begin{array}{l}\text { RANGE: } \\
\text { RANGE: }\end{array}$ & $\begin{array}{l}\text { sat } \\
\text { bol }\end{array}$ & a & caq & col & saa & tree \\
\hline RANGE: & & & & & & $\begin{array}{c}\text { tree } \\
\text { treelet }\end{array}$ \\
\hline
\end{tabular}


252 (167) BORAGINACEAE cont.

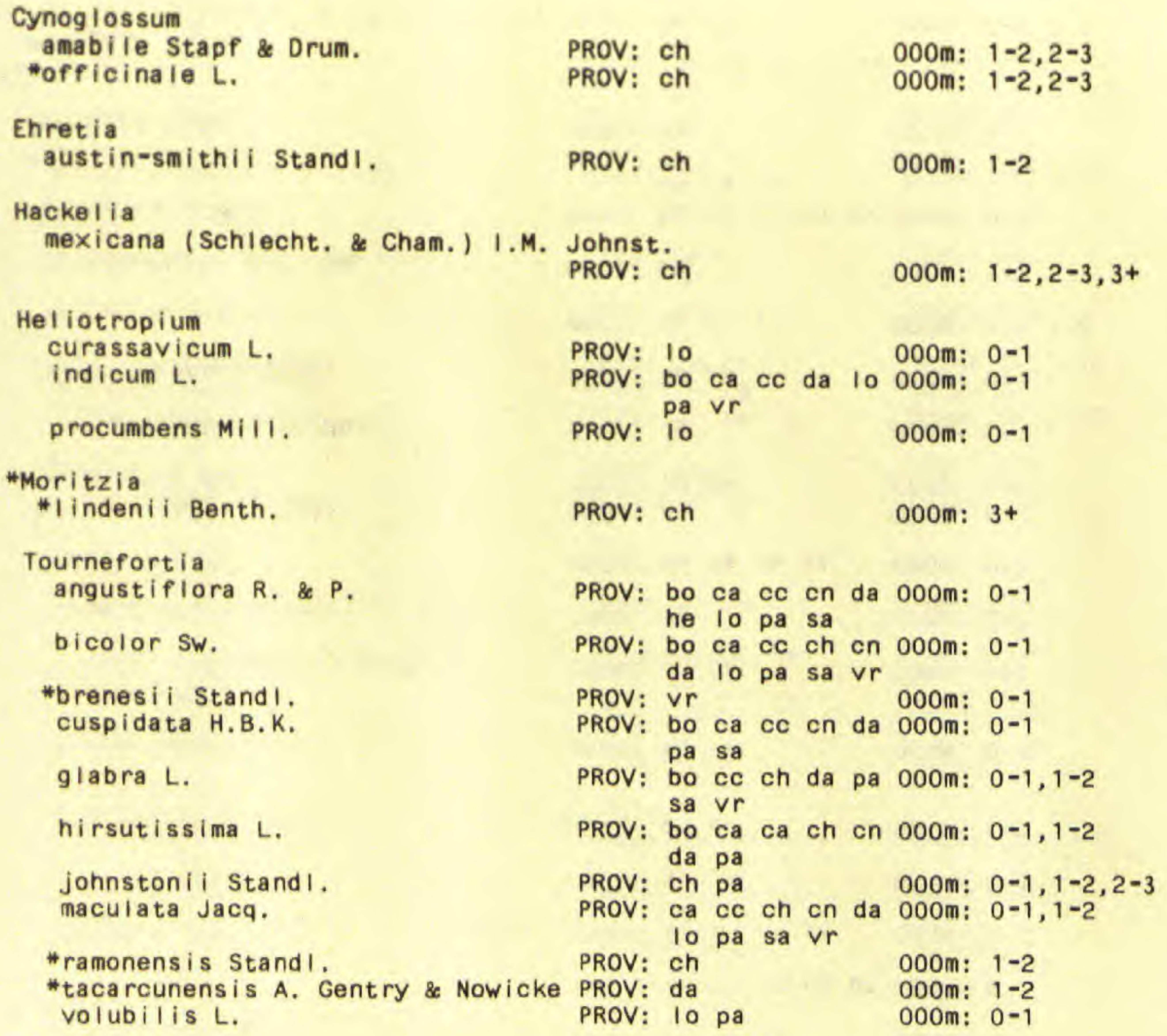

RANGE: as i cag

RANGE: eur

RANGE: $c r$

herb

herb

tree

RANGE: cag mex

herb

RANGE: NWg

RANGE: nwg

herb

herb

RANGE: nWg

herb

RANGE: $\mathrm{cr}$ ecu ven

herb

RANGE: cag? hon per sag shrub

RANGE: nWt

RANGE: $c r$

RANGE: ang cag sag

shrub

climber

shrub

shrub

RANGE: ang cag mex

shrub

tree

shrub

climber

RANGE: bol cag per ven

RANGE: ang cag mex per sag shrub

RANGE: $c r$ tree

shrub

RANGE: $c O$

herb

RANGE: ang

cag fla mex sag

$253(168,168 A)$ VERBENACEAE

Aegiphila

anomala Pitt.

cepha lophora StandI.

costaricensis Mold.
PROV: ca cc pa vr 000m: $0-1$

PROV: ca da pa

PROV: bo $\mathrm{cc}$ da 000m: $0-1,1-2$ 000m: $0-1,1-2$
RANGE: $c r$

RANGE: $\mathrm{CO} I \mathrm{cr}$

RANGE: $\mathrm{cr}$ mex ven 
TAXON

FAMILY

PROV I NCES

ELEVATION (OOOM.)

RANGE OUTSIDE OF PANAMA

HABIT

$253(168,168 A)$ VERBENACEAE cont.

deppeana Steud.

elata SW.

falcata J.D. Sm.

Pilipes Schau,

glandulifera Mold.

hirsutissima Mold.

hoehnei Mold.

--var. spectabilis Mold.

integrifolia Jacks.

laeta H,B.K.

* laevis (Aubl.) Gmel. magnifica Mold.

--var. pubescens Mold.

martinicens is Jacq.

mollis H,B, K.

odontophyIIa J,D, Sm.

panamensis Mold.

*pauciflora Standl. pendula Mold.
PROV: ch $10 \mathrm{vr}$

000m: $0-1$

PROV: ca ch en da pa 000m: 0-1

PROV: bo ca ce pa 000m: $0-1$

PROV: da pa

000m: $0-1$

PROV: bo ca

000m: $0-1$

PROV: da sa

000m: $0-1$

PROV: cn pa

000m: $0-1$

PROV: da

000m: $0-1$

PROV: ca da pa sa

000m: $0-1$

PROV: pa

PROV: bo pa

$000 \mathrm{~m}: 0-1$

000m: $0-1$

PROV: ca

000m: $0-1$

PROV: bo ca

000m: 0-1

PROV: ce ch vr

000m: $0-1,1-2$

PROV: bo

000m: $1-2$

PROV: bo ca cc ch cn 000m: $0-1$ da pa sa

PROV: da

000m: $1-2$

PROV: ca

000m: $0-1$

*Aloysia

*virgata juss.

*-var. platyphylla (Briq.) Mold. PROV: he pa

000m: $0-1$

Ca I I icarpa

acuminata $H, B, K$.

PROV: bo cn da pa sa $000 \mathrm{~m}: 0-1$

\begin{tabular}{|c|c|c|c|c|c|c|}
\hline $\begin{array}{l}\text { RANGE: } \\
\text { ven }\end{array}$ & cag & $\mathrm{col}$ & gui & mex & sag? & $\begin{array}{c}\text { shrub } \\
\text { tree }\end{array}$ \\
\hline RANGE: & ang & be I & cag & col & $\mathrm{cr}$ & shrub \\
\hline cub & gui & his & hon & $\operatorname{mex}$ & ven & climber \\
\hline RANGE: & cag & $\mathrm{col}$ & $\operatorname{mex}$ & 20 & $x_{x+1}$ & $\begin{array}{c}\text { shrub } \\
\text { tree }\end{array}$ \\
\hline RANGE: & bol & per & sag? & sao & & $\begin{array}{c}\text { shrub } \\
\text { tree }\end{array}$ \\
\hline RANGE: & per & sag? & sao & $=6$ & & $\begin{array}{l}\text { shrub } \\
\text { tree }\end{array}$ \\
\hline RANGE: & ven & +0 & $=-1$ & & & $\begin{array}{l}\text { climber } \\
\text { shrub }\end{array}$ \\
\hline 1978 & 30 & e & pratis & & & climber \\
\hline RANGE: & $\mathrm{col}$ & & & & & $\begin{array}{l}\text { shrub } \\
\text { cl imber }\end{array}$ \\
\hline RANGE: & bol & per & sao & trt & ven & $\begin{array}{c}\text { shrub } \\
\text { tree }\end{array}$ \\
\hline RANGE: & $\mathrm{col}$ & ven & & & & $\begin{array}{c}\text { shrub } \\
\text { tree }\end{array}$ \\
\hline RANGE: & gui & (6)" & ? & & & climber \\
\hline RANGE: & cag? & gua & & & & shrub \\
\hline RANGE: & $\mathrm{cr}$ & & & & & $\begin{array}{l}\text { shrub } \\
\text { cl imber }\end{array}$ \\
\hline RANGE: & ang & $\mathrm{col}$ & $\mathrm{cr}$ & trt & ven & $\begin{array}{l}\text { shrub } \\
\text { tree }\end{array}$ \\
\hline RANGE: & bol & sag? & & & & $\begin{array}{l}\text { shrub } \\
\text { tree }\end{array}$ \\
\hline RANGE: & $\mathrm{col}$ & $\mathrm{cr}$ & ven & & & $\begin{array}{l}\text { shrub } \\
\text { tree }\end{array}$ \\
\hline RANGE: & cag & $\mathrm{col}$ & cr & $\operatorname{mex}$ & & $\begin{array}{l}\text { shrub } \\
\text { tree }\end{array}$ \\
\hline $\begin{array}{l}\text { RANGE: } \\
\text { RANGE: }\end{array}$ & $\begin{array}{l}w r \\
\text { ecu }\end{array}$ & per & ven & ( & & $\begin{array}{l}\text { treelet } \\
\text { shrub }\end{array}$ \\
\hline 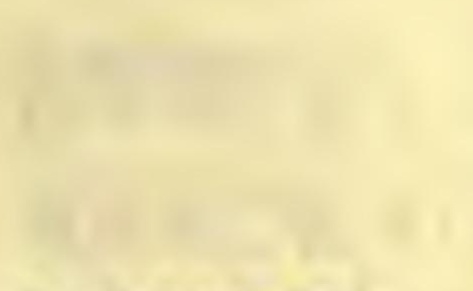 & 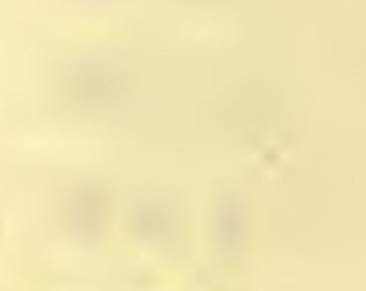 & r & (2) & & & $x^{12}+x$ \\
\hline RANGE: & sat & 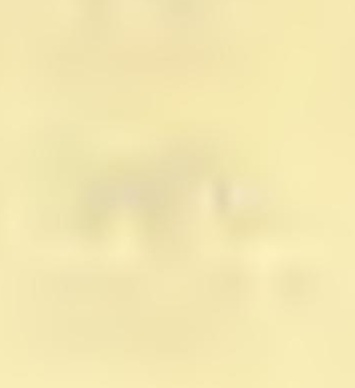 & & & & $\begin{array}{l}\text { herb } \\
\text { shrub }\end{array}$ \\
\hline RANGE: & 1 & cag? & $\operatorname{mex}$ & sag? & & $\begin{array}{l}\text { shrub } \\
\text { tree }\end{array}$ \\
\hline
\end{tabular}


$253(168,168$ A) VERBENACEAE cont.

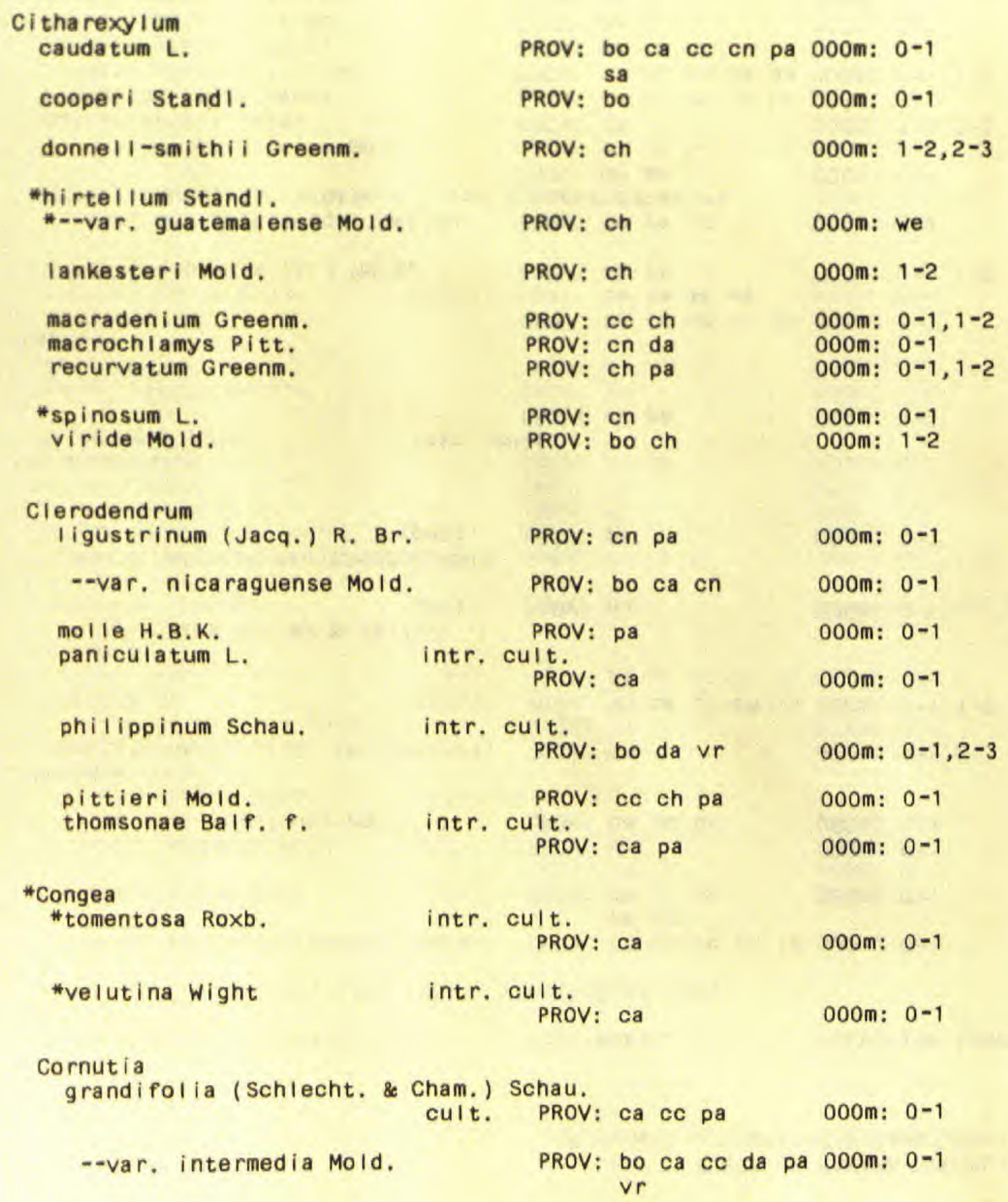

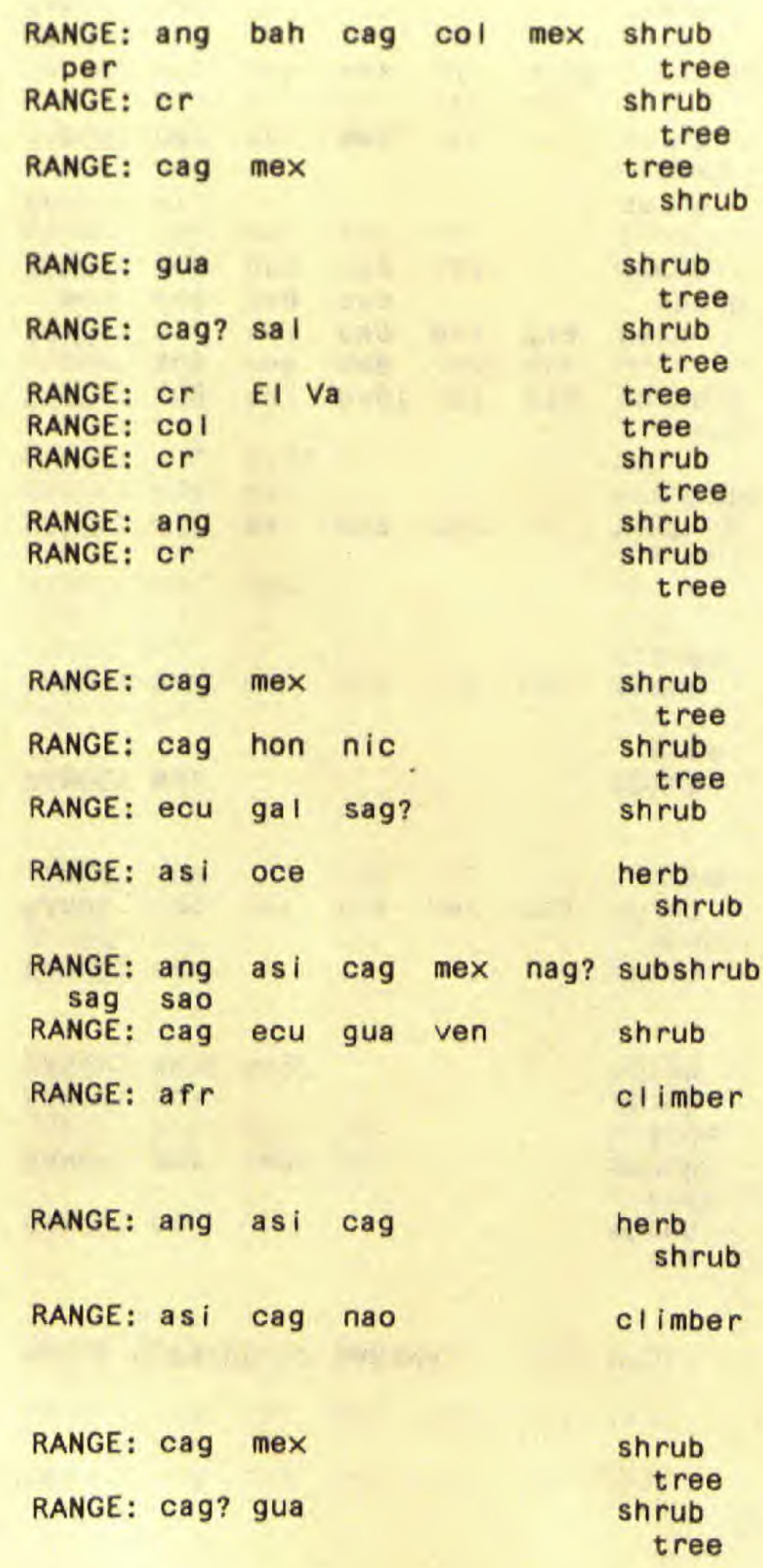


TAXON

FAMILY

PROVINCES

ELEVATION (OOOM.)

RANGE OUTSIDE OF PANAMA

HABIT

$253(168,168$ A) VERBENACEAE cont.

--var, normalis (Kuntze) Mold. PROV: bo ca ce ch lo 000m: 0-1

microcalycina Mold.

PRoV: da $\begin{array}{ll}\text { pa } & \\ \text { Pa } & 000 \mathrm{~m}: 1\end{array}$

- -var, anoma la Mold.

PROV: da 000m: $0-1$

--var. pulverulenta Mold.

PROV: ca cc da

000m: $0-1$

\section{Duranta}

costaricensis (J.D. Sm.) StandI. PROV: ch

$000 m: 1-2,2-3$

erecta $L$.

cult. PROV: bo ca cc ch lo $000 \mathrm{~m}$ : $0-1,1-2$

repens

pa

--var, a lba Bailey \& Bailey

cult. PROV: pa 000m: $0-1$

- -var. microphylla (Desf.) Mold.

cult. PROV: pa 000m: $0-1$

\section{*Holmskioldia}

"sanguinea Retz.

intr. cult.

PROV: cc pa

000m: $0-1$

\section{Lantana}

camara $L$.

--var. aculeata (L.) Mold.

PROV: ca cc en da he $000 \mathrm{~m}: 0-1$ lo pa sa vr

000m: $0-1,1-2$

--var. mista (L.) L.H. Bailey

PROV: ch pa

000m: $0-1$

*-var. moritziana (Otto \& Dietr.) Lopez-Palacios PROV: cn pa

000m: $0-1$

costaricensis Hayek

glandulosissima Hayek

--var. glandulosissima

*--var. grandis Mold.

*--var. parvifolia Mold. grosseserrata Mold.

hirta Graham

PROV: ch

000m: $1-2,2-3$

--var, pubescens Mold.

hispida H.B.K.

*--fo. parvifolia Mold. involucrata L.

- fo. rubella Mold. maxima Hayek

PROV: ca cc ch da pa 000m: $0-1,1-2$

PROV: ca cn 000m: $0-1$

PROV: pa

$000 \mathrm{~m}: 0-1$

000m: $0-1$

PROV: $\mathrm{cC}$

$000 \mathrm{~m}: 0-1,1-2$

PROV: ch

000m: we

PROV: bo ca ch cn da 000m: $0-1,1-2$

lo pa sa vr

PROV: cc pa vr

000m: we

PROV: sa

PROV: cc ch pa sa 000m: $0-1$

000m: $0-1,1-2$

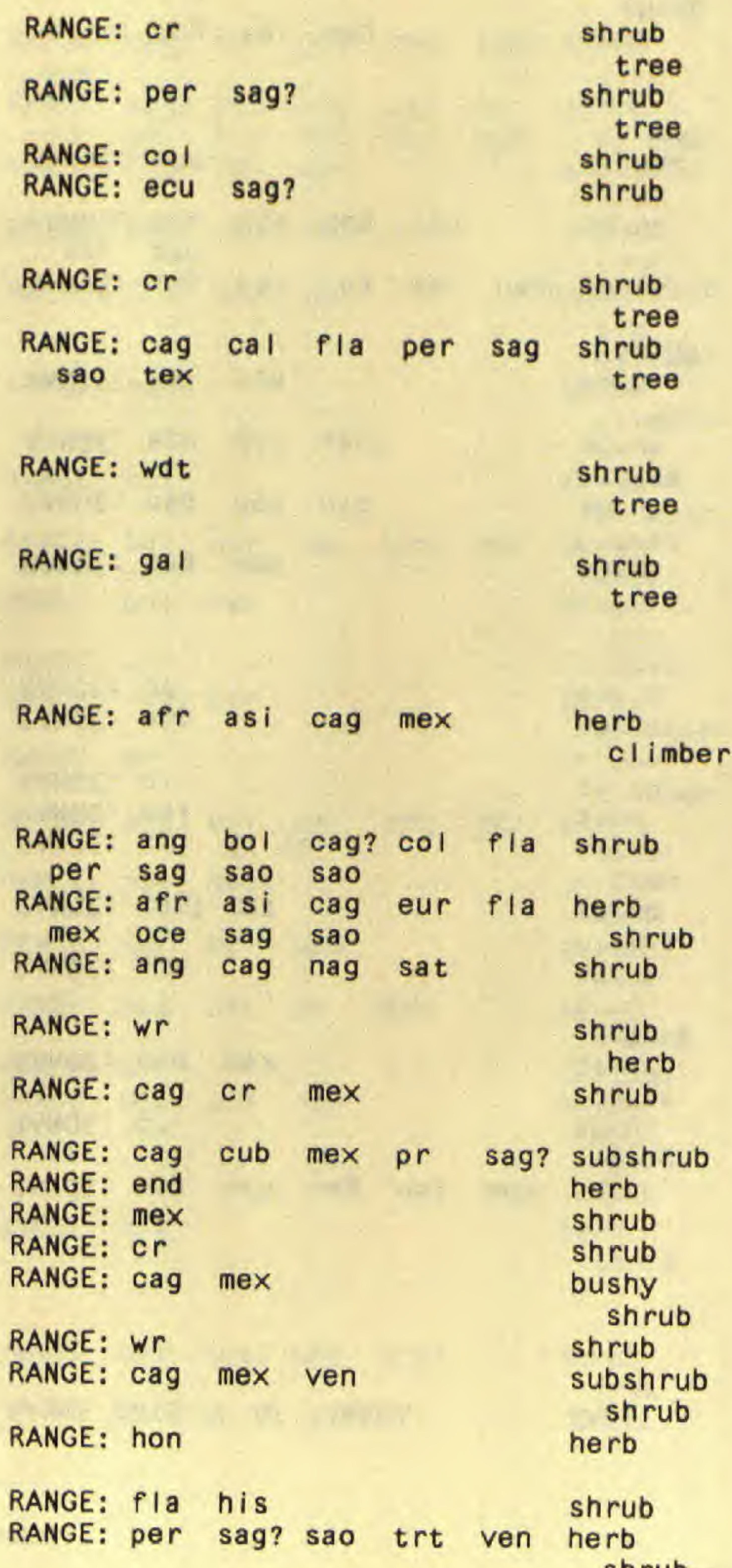


$253(168,168$ A) VERBENACEAE cont.

moritziana Otto \& Dietr.

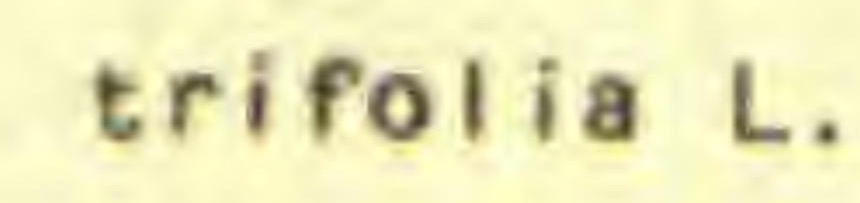

--fo. hirsuta Mold.

- -fo. oppositifolia Mold.

velutina Mart. \& Gal. -po, violacea Mold.

\section{Lippia}

alba Britt. \& Wils.

PROV: bo ca cc ch cn 000m: $0-1,1-2$ he pa

PROV: bo ca ch

000m: $0-1$

PROV: ch cn he pa sa $000 \mathrm{~m}: 0-1$

PROV: bo ca ch on he 000m: $0-1,1-2$ PROV: $\begin{aligned} & \text { pa } \\ & \text { ch } \\ & \text { pa }\end{aligned}$ PROV: pa

000m: $0-1,1-2$ 000m: $0-1$

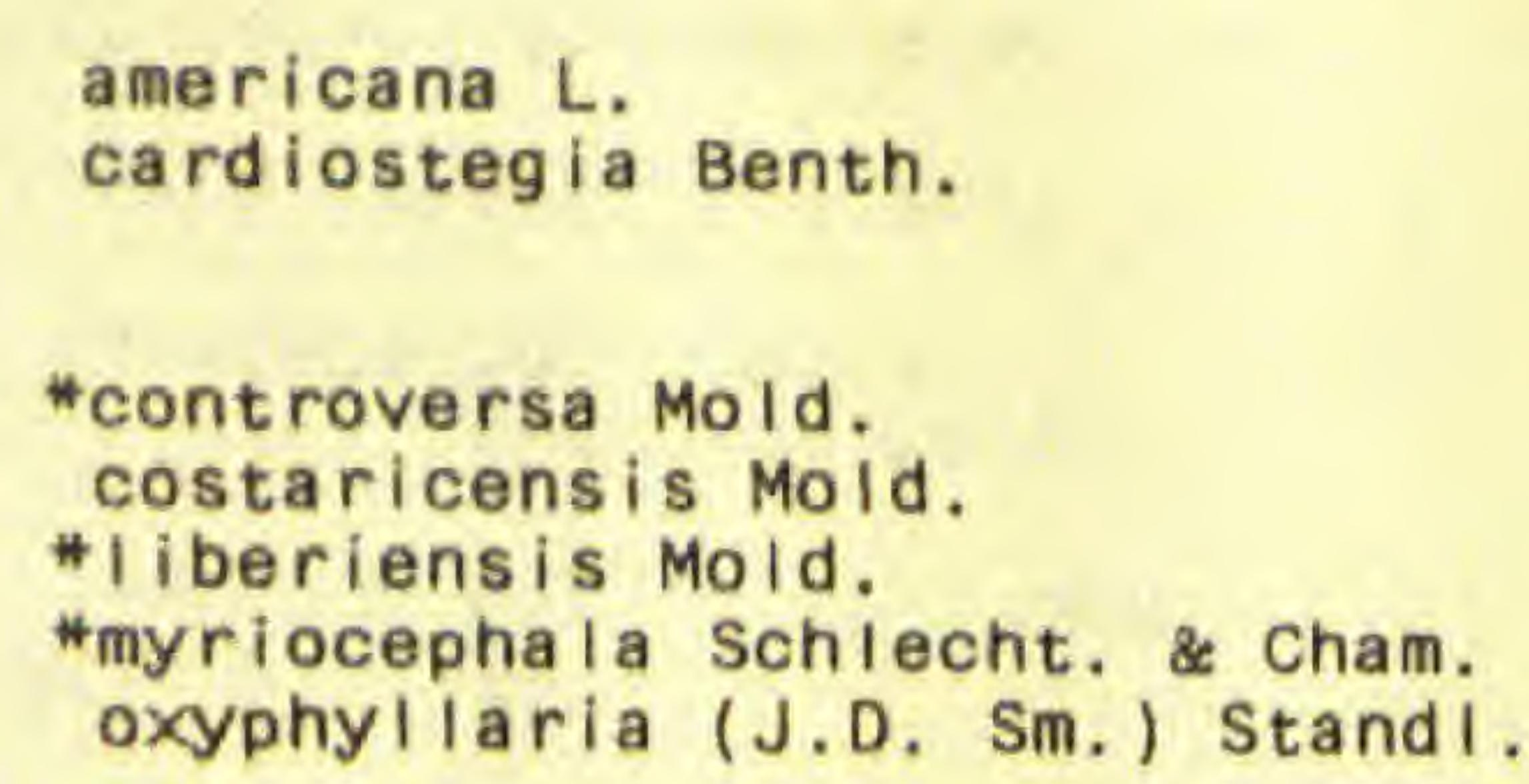

i

\section{PROV: ca da lo pa sa 000m: 0-1 $\mathrm{Vr}$} PROV: sa 000m: $0-1$ PROV: ca cn

000m: $0-1$

PROV: bo ca ch cn pa 000m: 0-1 vr
PROV: ca cn pa

\begin{tabular}{|c|c|c|c|c|c|c|}
\hline RANGE: & cag & $\operatorname{mex}$ & per & sag? & sao & $\begin{array}{l}\text { bushy } \\
\text { shrub }\end{array}$ \\
\hline $\begin{array}{c}\text { RANGE: } \\
\text { sag }\end{array}$ & $\begin{array}{l}\text { bol } \\
\text { saoo }\end{array}$ & cag & cub & $\operatorname{mex}$ & per & herb \\
\hline RANGE: & ang & as $i$ & bol & cag & cub & herb \\
\hline $\operatorname{mex}$ & nao & per & sag? & sao & & \\
\hline RANGE: & $w d t$ & & & & & herb \\
\hline $\begin{array}{l}\text { RANGE: } \\
\text { RANGE: }\end{array}$ & $\begin{array}{l}\text { cag } \\
\text { wdt }\end{array}$ & $\operatorname{mex}$ & tex & ven & & $\begin{array}{l}\text { shrub } \\
\text { herb }\end{array}$ \\
\hline RANGE: & ang & cag & mex & sag? & sao & $\begin{array}{l}\text { herb } \\
\text { shrub } \\
\text { subshrub }\end{array}$ \\
\hline RANGE: & per & sag? & & & & herb \\
\hline RANGE: & cag & $\operatorname{mex}$ & & & & $\begin{array}{c}\text { bushy } \\
\text { shrub } \\
\text { tree }\end{array}$ \\
\hline RANGE: & $\mathrm{cr}$ & $\operatorname{mex}$ & & & & shrub \\
\hline RANGE: & $\mathrm{cr}$ & & & & & tree \\
\hline $\begin{array}{l}\text { RANGE: } \\
\text { RANGE: }\end{array}$ & $\begin{array}{l}\text { Wr } \\
\text { cag? }\end{array}$ & & & & & shrub \\
\hline $\begin{array}{l}\text { RANGE: } \\
\text { RANGE: }\end{array}$ & $\begin{array}{l}\text { cag? } \\
\text { cr }\end{array}$ & $\mathrm{cr}$ & gua & non & $\operatorname{mex}$ & $\begin{array}{l}\text { tree } \\
\text { shrub }\end{array}$ \\
\hline RANGE: & $\mathrm{cr}$ & ' & & 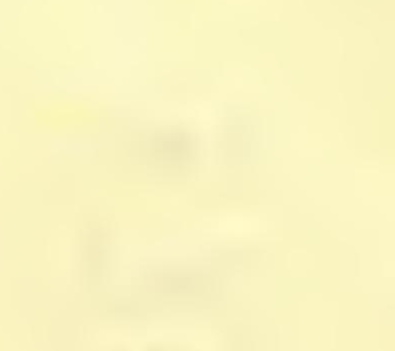 & $-x=1$ & $\begin{array}{l}\text { tree } \\
\text { tree }\end{array}$ \\
\hline RANGE: & ang & cag & sag? & sao & 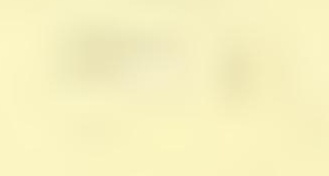 & climber \\
\hline $\begin{array}{l}\text { RANGE: } \\
\text { RANGE: }\end{array}$ & $\begin{array}{l}\text { nwt } \\
\text { end }\end{array}$ & 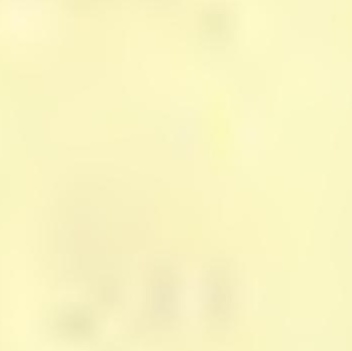 & & & & $\begin{array}{l}\text { shrub } \\
\text { cl imber }\end{array}$ \\
\hline RANGE: & sat? & & 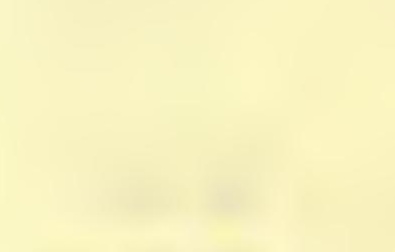 & (1) & $x_{2}$ & shrub \\
\hline $\begin{array}{l}\text { RANGE: } \\
\text { RANGE: }\end{array}$ & $\mathrm{col}$ & ecu & ven & & & shrub \\
\hline RANGE: & cag & cub & $\operatorname{mex}$ & & & $\begin{array}{l}\text { cl imber } \\
\text { subshrub } \\
\text { cl imber } \\
\text { subshrub }\end{array}$ \\
\hline
\end{tabular}

RANGE: bel cag? cub gua sag? herb SaO

RANGE: cag caI

RANGE: cag col mex nao per herb 
TAXON

FAMILY

PROVINCES

ELEVATION (OOOM.)

RANGE OUTSIDE OF PANAMA

HAB IT

$253(168,168$ A) VERBENACEAE cont.

- -var nodiflora

scaberrima (A, Juss,) Mold.

strigulosa (Mart. \& Gal.) Mold.

--var. sericea (Kuntze) Mold.

- -var. strigulosa

Priva

Iappulacea (L.) Pers.

-fo, a Ibifiora Moid.

- fo, I appulacea

Stachyta rpheta

cayennens is (Rich.) VahI

*--fo. purpurea Mold. guatema lens is Mold.

jama icensis (L.) Vah I

*--subsp, atrocoerulea Mold. *mutabilis (Jacq.) Vahl

\section{* Tamonea}

*spicata Aubl.

Tectona

grandis L. $f$.

intr, cult.

PROV: ca

Verbena

hybrida Rumpler

Iitoralis H.B.K.

inter, cult.

PROV: pa

PROV: ch pa

000m: $0-1,1-2,2-3$

* parvula Hayek

PROV: ch

PROV: ca ch da vr

PROV: da

PROV: ca da pa

PROV: da pa

intr. cult.

000m: $0-1$

000m: $0-1,1-2$

000m: 0-1

000m: $0-1$

000m: $0-1$

cooperi Stand I.

floridula Duchass, Walp.

masoniana Pitt.

parviflora A. Juss.
PROV: bo 000m: 0-1

000m: $0-1$

$000 m: 2-3$

000m: $0-1$

000m: $0-1$

000m: $0-1$

000m: $0-1$

000m: $0-1$

000m: $0-1$

000m: $0-1$

000m: $0-1$

RANGE: nwt owt wdt
RANGE: cag cub mex ven
RANGE: bah gag lag mex tex herb
trt ven
RANGE: ang bol cag cub gal herb
his mex sag? sao tex

RANGE: ang bol cag fla mex herb

nwt per sag sao sat tex

RANGE: ang bol cag fla mex herb

nwt per sag sao sat tex

RANGE: ang cag gag mex per herb

sag? sao shrub

shrub

RANGE: cag mex herb

RANGE: afr ang asi cag ecu herbub

mex nao oce sao cag ecu herb

RANGE: wr herb

RANGE: cag sat shrub

RANGE: gui herb

RANGE: asi or tree

RANGE: Wdw herb

RANGE: afr cag cal nag? nao herb

nao oce sag herb
ANGE: bol

RANGE: cag? gua tree

RANGE: boi sag? sao tree

RANGE: end tree

RANGE: $\mathrm{col}$ tree

RANGE: asi tree 


\section{3a (168a) AVICENNIACEAE}

Avicennia

bicolor Standi.

germinans (L.) L.

tonduzii Mold.

\section{4 (169) LABIATAE}

Cunila

polyantha Benth.

Hypt is

atrorubens Poit.

brachiata Briq.

brevipes Poit.

capitata Jacq.

conferta Benth.

dilatata Benth.

Iantanifolia Poit.

mutabilis (A. Rich.) Briq.

oblongifolia Benth.

obtusiflora Benth.

pectinata (L.) Poit.

pulegioides Benth.

recurvata Poit.

savanna rum Briq.

*sinuata Poh I

*spicigera Lam. suaveolens (L.) Poit.

*urticoides H.B.K. verticillata Jacq.
PROV: ca cc cn da lo 000m: $0-1$ pa

PROV: bo ca cc cn lo $000 \mathrm{~m}$ : $0-1$ pa sa

PROV: pa

$000 m: 0-1$

$\begin{array}{cllc}\text { RANGE: cag mex } & & \begin{array}{c}\text { shrub } \\ \text { tree }\end{array} \\ \text { RANGE: ang bah cag fla gal shrub } \\ \text { mex per sag? sao tex } & \text { tree } \\ \text { RANGE: col cr } & & & \text { tree }\end{array}$

RANGE: cag mex

shrub

PROV: bo 000m: $0-1$ PROV: ca $\mathrm{cc}$ ch he pa $000 \mathrm{~m}$ : $0-1$ PROV: ca

$000 \mathrm{~m}: 0-1$ PROV: bo ca cC ch da $000 \mathrm{~m}: 0-1$ PROV: bo ca ce ch da 000m: $0-1$ PROV: ch pa sa vr

000m: $0-1$

PROV: cc he vr 000m: $0-1$

PROV: cc ch pa

000m: $0-1$

PROV: ca cc ch da he 000m: $0-1$ lo pa vr

000m: $0-1$

PROV: ch pa

PROV: bo ce ch cn vr 000m: $0-1,1-2$

PROV: ca cc ch da he 000m: $0-1,1-2$ lo pa vr

PROV: ca ch pa $000 \mathrm{~m}: 0-1$ PROV: bo ca ce ch cn 000m: $0-1$ pa $\vee r$

000m: $0-1$

PROV: ch

000m: $1-2$

PROV: pa $\mathrm{Vr}$

000m: $0-1$

PROV: bo ca cc cc ch $000 \mathrm{~m}$ : $0-1$ da he pa $v r$

PROV: ch

000m: we

PROV: bo ca da pa sa $000 \mathrm{~m}: 0-1$

\begin{tabular}{|c|c|c|c|c|c|c|}
\hline $\begin{array}{l}\text { RANGE: } \\
\text { RANGE: }\end{array}$ & $\begin{array}{l}\text { af } r \\
\text { col }\end{array}$ & $\begin{array}{l}\text { ang } \\
\mathrm{cr}\end{array}$ & $\begin{array}{l}\text { gua } \\
\text { ven }\end{array}$ & $\operatorname{mex}$ & sag & $\begin{array}{l}\text { herb } \\
\text { herb } \\
\text { subshrub }\end{array}$ \\
\hline RANGE: & as i & mex & nwt & per & sao & herb \\
\hline $\begin{array}{l}\text { RANGE: } \\
\text { mex }\end{array}$ & $\begin{array}{l}\text { af } r \\
\text { per }\end{array}$ & $\begin{array}{l}\text { as i } \\
\text { ven }\end{array}$ & cag & $\mathrm{col}$ & ecu & herb \\
\hline RANGE: & $\begin{array}{l}\text { bol } \\
\text { ven }\end{array}$ & cag & $\mathrm{col}$ & mex & sao & herb \\
\hline $\begin{array}{c}\text { sat } \\
\text { RANGE: }\end{array}$ & $\begin{array}{l}\text { ven } \\
\text { col }\end{array}$ & sao & ven & & & $\begin{array}{l}\text { herb } \\
\text { subshrub }\end{array}$ \\
\hline $\begin{array}{c}\text { RANGE: } \\
\text { Sao }\end{array}$ & ang & bol & cag & $\operatorname{mex}$ & sag & herb \\
\hline RANGE: & af $r$ & nwt & per & & & herb \\
\hline ANGE: & $\mathrm{cr}$ & gua & hon & $\operatorname{mex}$ & nic & $\begin{array}{c}\text { shrub } \\
\text { tree }\end{array}$ \\
\hline $\begin{array}{c}\text { RANGE: } \\
\text { per }\end{array}$ & $\begin{array}{l}\text { bol } \\
\text { sag? }\end{array}$ & $\mathrm{col}$ & cr & ecu & nic & herb \\
\hline RANGE: & $n W t$ & owt & & & & herb \\
\hline $\begin{array}{l}\text { RANGE: } \\
\text { RANGE: }\end{array}$ & $\begin{array}{l}\text { cag } \\
\text { nwt }\end{array}$ & $\begin{array}{l}\text { col } \\
\text { per }\end{array}$ & sag & ven & & $\begin{array}{l}\text { herb } \\
\text { herb }\end{array}$ \\
\hline $\begin{array}{c}\text { RANGE: } \\
\text { Sal }\end{array}$ & $\mathrm{col}$ & $\mathrm{cr}$ & ecu & gua & per & herb \\
\hline RANGE: & bo ! & $\mathrm{col}$ & $\mathrm{cr}$ & gua & hon & herb \\
\hline mex & sa I & sao & sat & ven & & \\
\hline RANGE: & afr & as $i$ & $n w t$ & & & herb \\
\hline $\begin{array}{c}\text { RANGE: } \\
\text { per }\end{array}$ & $\begin{array}{l}\text { afr } \\
\text { sag }\end{array}$ & $\begin{array}{l}\text { ang } \\
\text { wdt }\end{array}$ & as i & cag & oce & herb \\
\hline RANGE: & cag & & & & & \\
\hline INGE: & nwt & & & & & herb \\
\hline
\end{tabular}


TAXON

254 (169) LABIATAE cont.

\section{"vilis Kunth \& Bouche}

Lepechinia

schiedeana (Schlecht.) Vatke

Marsypianthes

chamaedrys (VahI) Kuntze

Oc imum

*bas i I icum L.

*campechianum MilI.

canum sims

gratissimum L.

Salvia

a Ivajaca 0erst.

* lasiocephala Hook. \& Arn.

* eucantha Cav.

longimarginata Briq.

membranacea Benth.

micrantha Vahi

occidentalis SW.

polystachya ort.

pteroura Briq.

trichopes Epling

wagneriana Polak.

\section{Scutellaria}

* costaricana Wend I.

glabra Leonard

lewis iana Nowicke

*maxoni i Leonard

purpurascens SW.

\#uliginosa St. Hil.

*Solenostemon

*scutel larioides (L.) Coll.

cult. PROV: ca cc pa 000m: $0-1,1-2$

intr, cult. nat.

PROV: da sa 000m: $0-1$

PROV: bo ca da he lo $000 \mathrm{~m}$ : $0-1$ pa sa

PROV: da 000m: $0-1$

PROV: $v r \quad 000 \mathrm{~m}: 0-1$

PROV: $\mathrm{ch}$ da $000 \mathrm{~m}: 1-2$

PROV: ca ch da he $10000 \mathrm{~m}: 0-1,1-2$

cult. PROV: pa sa

000m: 2-3,3+

PROV: $c a \mathrm{cc}$ ch da $000 \mathrm{~m}$ : $0-1$

PROV: ch 000m: $0-1,1-2$

PROV: cn pa

$000 \mathrm{~m}: 0-1$

PROV: ca cc ch on da 000m: $0-1$ he lo pa vr

PROV: $\mathrm{cc}$ ch

000m: $0-1,1-2$

PROV: $\mathrm{CC} \mathrm{ch}$

000m: $0-1$

PROV: ch

000m: $1-2$

PROV: ch

000m: $1-2$

PROV: CC

000m: $0-1$

000m: $0-1$

000m: $0-1$

PROV: ch Vr

000m: $0-1,1-2$

PROV: bo ca cn da pa 000m: $0=1$

$$
\text { sa }
$$

000m: $0-1$

PROV: cn pa

$0-1,1-2$
PROV: ch

$000 \mathrm{~m}: 1-2$

PROV: ch

$000 m: 0-1,1-2$

PROV: ca ce ch cn lo 000m: $0-1$ pa $v r$

RANGE: or gua nic ven $\begin{gathered}\begin{array}{c}\text { subshrub } \\ \text { herb }\end{array} \\ \text { RANGE: cag }\end{gathered}$
herb

RANGE: cag

RANGE: ang cag mex per sag herb sao

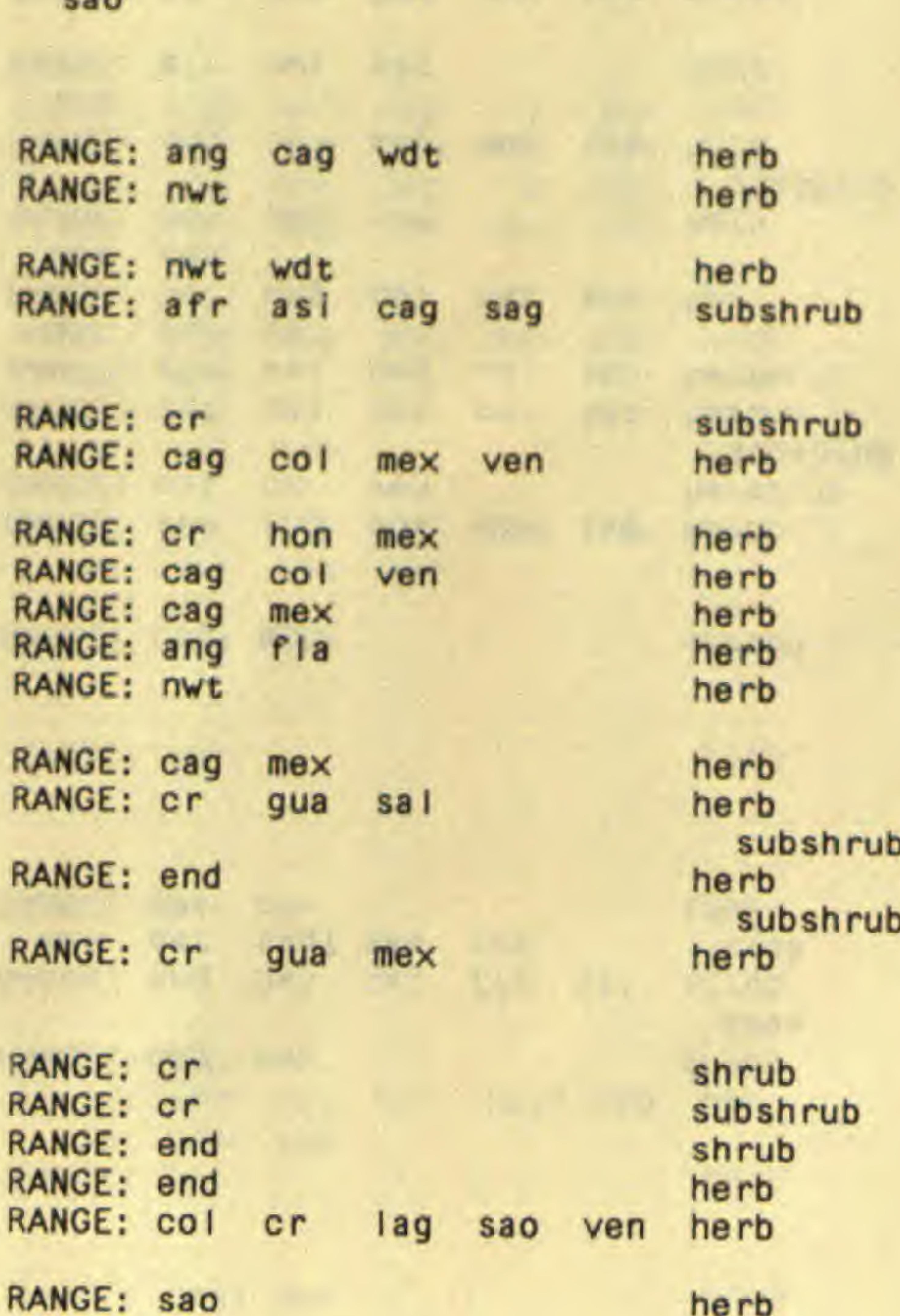

RANGE: as $i$

herb
PROV: ch $\quad 000 \mathrm{~m}: 0-1$

PROV: ch $\quad 000 m: 0-1$ costaricensis Briq. 
TAXON

$256(170)$ SOLANACEAE

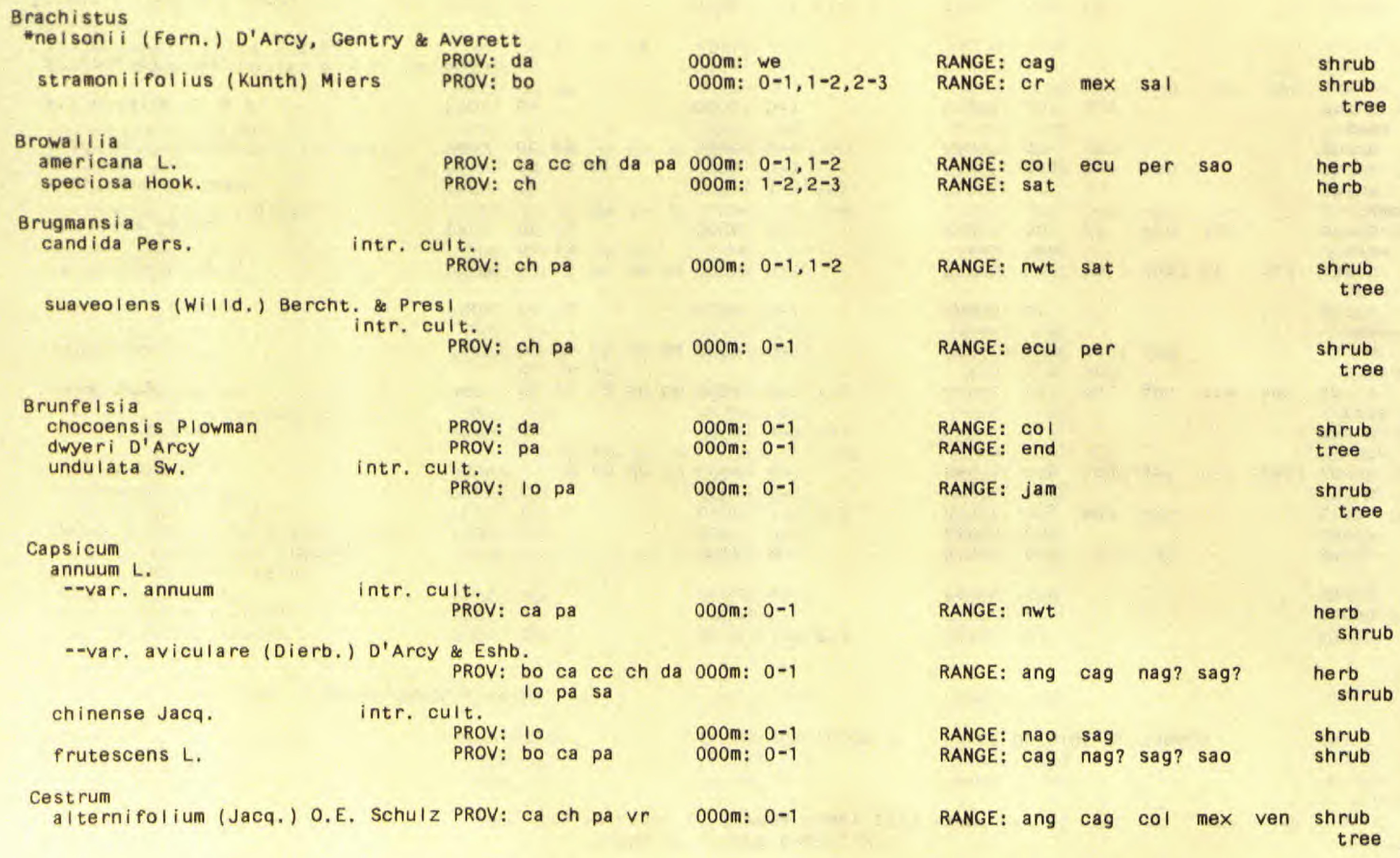


TAXON

FAMILY

PROV INCES

ELEVATION (000M.)

RANGE OUTSIDE OF PANAMA

HABIT

256 (170) SOLANACEAE cont

chiriquianum Francey

costaricense Francey

gracile Francey

* jaca I tenang i num Loesn. - -var, tomentosum Francey johnniegentrianum D'Arcy

lanatum Mart. \& Gal.

langeanum D'Arcy

latifolium Lam.

lewisil D'Arcy

mega lophyl Ium Dun.

nocturnum L.

pittieri Francey

racemosum R. \& P

reflexum sendt.

rugulosum Francey

scandens Vahl

standleyi francey

strigilatum R, \& P.

warszewiczii Ki.

* Cuatresia

* riparia (H.B.K.) Hunz.

\section{Cyphomand ra}

a I Iophyl Ia (Miers) Hems I.

* divaricata (Mart.) Sendt.

* dol ichocarpa Bitt.

hartwegii (Miers) Walp.

\section{Datura}

metel L.

stramonium L.

intr. cult.

PROV: ch

000m: $0-1$

PROV: ch $\quad 000 \mathrm{~m}: 1-2,2-3$

PROV: $V r$

$000 \mathrm{~m}: 1-2,2-3$

PROV: $v r$

$000 \mathrm{~m}$ : we

PROV: $v r$

PROV: CC

PROV: ch

000m: we

000m: $0=1$

PROV: $\mathrm{cn}$

$000 m: 1-2,2-3$

ca ch cn da lo $000 \mathrm{~m}: 0-1$

PROV: $\mathrm{ch}$

000m: $1-2,2-3$

PROV: bo ca ce ch cn 000m: $0-1,1-2$ da lo $\mathrm{vr}$

PROV: bo ca ch cn pa 000m: $0-1$

PROV: ch 10

000m: $0-1$

PROV: bo ca ce ch cn 000m: $0-1$

PROV: bo da

000m: $0-1$

PROV: ch

000m: $1-2,2-3$

ca ce

$000 \mathrm{~m}: 0-1$

PROV: $\mathrm{cc}$ pa

000m: $0-1$

PROV: pa

000m: $0-1$

PROV: ch

000m: $0=1$

PROV: ch

$000 m: 1-2,2-3$

PROV: ca da pa

$0-1$

PROV: bo pa sa

000m: $0-1$

PROV: ch

000m: $2-3$

PROV: bo ca ce ch cn 000m: $0-1$ da pa

\begin{tabular}{|c|c|c|c|c|c|c|}
\hline $\begin{array}{l}\text { RANGE: } \\
\text { RANGE: } \\
\text { RANGE: }\end{array}$ & $\begin{array}{l}\mathrm{cr} \\
\mathrm{cr} \\
\text { end }\end{array}$ & & & & & $\begin{array}{l}\text { shrub } \\
\text { shrub } \\
\text { shrub }\end{array}$ \\
\hline RANGE: & end & & & & & shrub \\
\hline RANGE: & end & & & & & shrub \\
\hline RANGE: & cag & $\operatorname{mex}$ & ven & & & tree \\
\hline RANGE: & end & & & & & shrub \\
\hline RANGE: & ang & cag? & gui & nic & sag? & shrub \\
\hline $\begin{array}{l}\text { sao } \\
\text { RANGE: }\end{array}$ & $\mathrm{cr}$ & & & & & $\begin{array}{c}\text { tree } \\
\text { shrub } \\
\text { tree }\end{array}$ \\
\hline RANGE: & bol & cag? & gua & lag & per & $\begin{array}{l}\text { tree } \\
\text { shrub }\end{array}$ \\
\hline sao & trt & ven & & & & tree \\
\hline RANGE: & ang & cag? & jam & & & $\begin{array}{l}\text { shrub } \\
\text { tree }\end{array}$ \\
\hline RANGE: & cr & & & & & $\begin{array}{c}\text { shrub } \\
\text { tree }\end{array}$ \\
\hline $\begin{array}{c}\text { RANGE: } \\
\text { sao }\end{array}$ & $\begin{array}{l}\text { bel } \\
\text { ven }\end{array}$ & bol & cag? & $\mathrm{cr}$ & per & $\begin{array}{l}\text { shrub } \\
\text { tree }\end{array}$ \\
\hline RANGE: & bol & $\mathrm{cr}$ & ecu & sao & & $\begin{array}{l}\text { shrub } \\
\text { climb }\end{array}$ \\
\hline RANGE: & $\mathrm{cr}$ & & & & & shrub \\
\hline RANGE: & cag? & gua & ven & 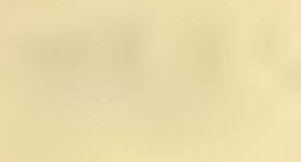 & & shrub \\
\hline RANGE: & $\mathrm{cr}$ & & & & & $\begin{array}{l}\text { shrub } \\
\text { tree }\end{array}$ \\
\hline RANGE: & per & sao & & & & \\
\hline RANGE: & cr & & & & & tree \\
\hline RANGE: & cag & $\mathrm{col}$ & & & & $\begin{array}{l}\text { shrub } \\
\text { tree }\end{array}$ \\
\hline RANGE: & $\mathrm{col}$ & $\mathrm{cr}$ & nic & & & herb \\
\hline $\begin{array}{l}\text { RANGE: } \\
\text { RANGE: }\end{array}$ & gua & ven & & & & tree \\
\hline $\begin{array}{l}\text { RANGE: } \\
\text { RANGE: }\end{array}$ & $\mathrm{cr}$ & & & & & tree \\
\hline $\begin{array}{c}\text { RANGE: } \\
\text { hon }\end{array}$ & $\begin{array}{l}\text { bol } \\
\text { sao }\end{array}$ & cag? & $\mathrm{col}$ & $\mathrm{cr}$ & ecu & shrub \\
\hline
\end{tabular}

RANGE: afr asi

RANGE: $w d W$

shrub

herb

Deprea

orinocensis (Kunth) Raf.

PROV: bo $\mathrm{ch}$

000m: $2-3$

RANGE: $c r$ ven 


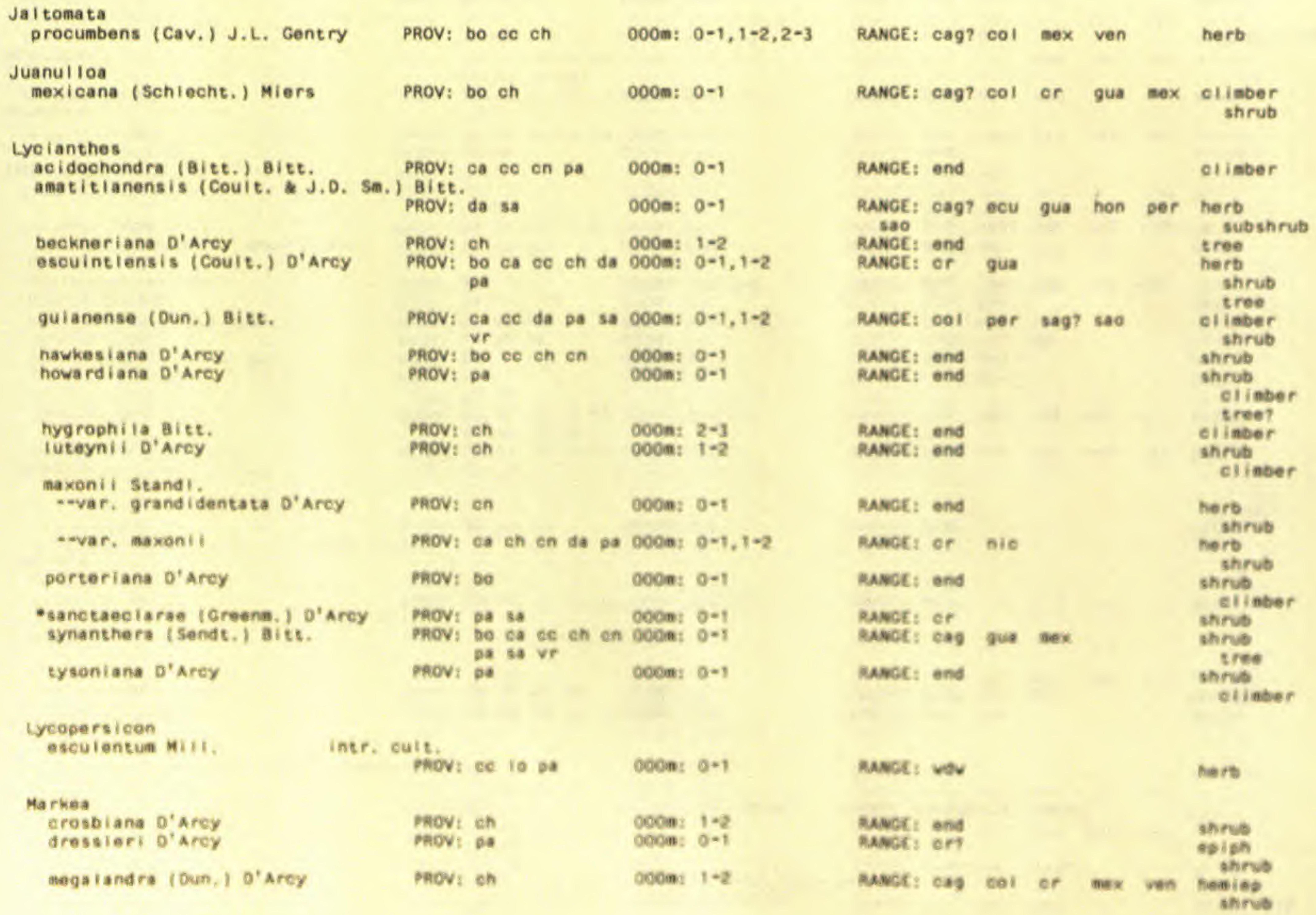


TAXON

\section{FAMILY}

PROVINCES

ELEVATION (OOOM.)

RANGE OUTSIDE OF PANAMA

$H A B \mid T$

256 (170) SOLANACEAE cont.

neurantha Hems I.

ulei (Damm,) Cuatr.

PROV: bo ch Vr

000m: $0-1$

\section{Nicotiana}

tabacum L.

intr. cult.

PROV: ch pa

000m: $0-1,2-3$

intr. cult.

PROV: pa

000m: $0-1$

PROV: bo ca cc ch cn 000m: $0-1$ da he lo pa

angulata $L$.

cordata Mill.

gracilis Miers

hirsuta Dun.

ignota Britt.

lagascae R, \& $S$.

minuta Griggs

philadelphica Lam.

pubescens $L$.

--var. hygrophila (Mart.) Dun.

- -var. pubescens

\section{Rahowardiana}

wardiana D'Arcy

\section{Schwenckia}

americana $L$.

Solandra

brachyca Iyx Kuntze

grandiflora SW.

intr. cult.

ch da 000m: $1-2,2-3$

PROV: ca cc pa

000m: $0-1$

maxima (Sesse Moc.) P.S. Green PROV: ch

000m: $1-2$

\section{Solanum}

accrescens Standl. \& Mort.

*acerifolium Dun, americanum Mill.
PROV: ca pa $000 \mathrm{~m}: 0=1$

PROV: ch 000m: $1-2$

PROV: bo ca cc ch cn 000m: $0-1,1-2$
RANGE: $c r$

RANGE: cag? col per

shrub

hemiep

shrub

RANGE: WdW

herb

RANGE: SaO

herb

shrub

RANGE: cag nag nao owg sag herb

RANGE: cag gag lag mex herb

RANGE : cag mex herb

RANGE: mex nao herb

RANGE: cag? cub gua herb

RANGE: bol cag nag herb

RANGE: cag mex herb

RANGE: ang cag? mex nag nao herb

RANGE: mex sao herb

RANGE: cag nag? nao owg sag? herb

sao

RANGE: end

shrub

RANGE: cag? cr

herb

RANGE: or gua

hemiep iphyte

RANGE: cag

RANGE: mex

hemiep

shrub

hemiep

shrub

RANGE: cr

shrub

RANGE: afr col or hon sao heree

RANGE: as $i$ bah eur jam mex herb 
256 (170) SOLANACEAE cont.

anceps R. \& P.

*aphyodendron Knapp

a rboreum Dun.

argenteum Poir.

asperum Rich.

\#brachystachys Dun.

"candidum Lindl.

canense Rydb.

caripense Dun.

*chrysotrichum schlecht.

cordavense Sesse Be Moc

*diodontum Bitt. erianthum D. Don

erythrotrichum Fern. evolvulifolium Greenm.

extensum Bitt.

fosbergianum D'Arcy

granelianum D'Arcy

hayesii Fern.

hazenii Britt,

hirtum VahI

imberbe $B i t t$.

incomptum Bitt.

ipomoea Sendt.

jama icense Miil.

lancaeifolium Jacq.

* I anceol a tum Cav.

leucoca rpum Dun.
PROV: cc da sa

PROV: ch

000m: $0-1,1-2$

000m: $0-1,1-2,2-3$

PROV: bo ca cc ch cn 000m: $0-1,1-2$ da lo pa sa

PROV: bo ca cc ch da 000m: $0-1,1-2$ pa

PROV: ca cc cn da pa 000m: 0-1

PROV: ch

PROV: ch

000m: 2-3

PROV: cc da pa

000m: $0-1$

PROV: ch

000m: $1-2,2-3$

PROV: $c h$

PROV: $\mathrm{ch}$

PROV: ch

PROV: WP

PROV: $c h$

PROV: bo $\mathrm{ch}$

000m: $0-1,1-2,2-3$

000m: $1-2$

000m: $1-2,2-3$

000m: $0-1$

000m: $1-2$

000m: $1-2,2-3$

PROV: ca pa sa $\quad 000 m: 0-1$

PROV: pa $000 m: 0-1$

PROV: da 000m: $1-2$

PROV: bo ca cc en pa 000m: $0-1$

PROV: ca cn da lo pa 000m: 0-1

PROV: ca da

000m: $0-1$

PROV: ca cn da pa sa $000 \mathrm{~m}$ : $0-1$

PROV: $\mathrm{ch}$ 000m: $1-2,2-3$

PROV: ca

000m: $0-1$

PROV: ca da he pa sa $000 \mathrm{~m}: 0=1$

$\checkmark r$

PROV: bo ca cc ch cn 000m: 0-1 da he lo pa vr

PROV: ch en

000m: $0-1,1-2$

PROV: da $\mathrm{vr}$

000m: $0-1$

\begin{tabular}{|c|c|c|c|c|c|c|}
\hline ANGE: & ecu & per & sao & & & herb \\
\hline RANGE: & cag & $\mathrm{col}$ & $\mathrm{cr}$. & $\operatorname{mex}$ & sat & $\begin{array}{c}\text { shrub } \\
\text { tree }\end{array}$ \\
\hline RANGE: & $\mathrm{col}$ & $\mathrm{cr}$ & $\operatorname{mex}$ & per & ven & $\begin{array}{c}\text { shrub } \\
\text { tree }\end{array}$ \\
\hline RANGE: & $\mathrm{col}$ & $\mathrm{cr}$ & sao & 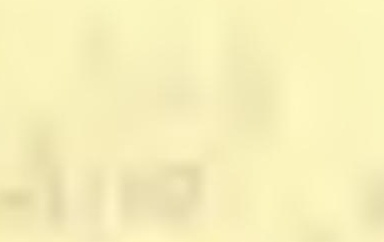 & & shrub \\
\hline RANGE: & bel & cag? & gui & sag? & sao & $\begin{array}{l}\text { shrub } \\
\text { tree }\end{array}$ \\
\hline NGE: & cag & $\operatorname{mex}$ & & $\tan$ & & shrub \\
\hline NGE: & bel & cag & $\mathrm{col}$ & $c r$ & ecu & herb \\
\hline $\begin{array}{l}\text { gua } \\
\text { trt }\end{array}$ & gua & $\operatorname{mex}$ & nic & per & sal & shrub \\
\hline RANGE: & cag? & ecu & gua & ven & & $\begin{array}{l}\text { herb } \\
\text { climber }\end{array}$ \\
\hline RANGE: & $\mathrm{cr}$ & per & ven & & & $\begin{array}{l}\text { herb } \\
\text { cl imber }\end{array}$ \\
\hline RANGE: & $\mathrm{col}$ & $\mathrm{cr}$ & & & & $\begin{array}{l}\text { shrub } \\
\text { tree }\end{array}$ \\
\hline RANGE: & bel & hon & in' & & & $\begin{array}{l}\text { shrub } \\
\text { tree }\end{array}$ \\
\hline $\begin{array}{l}\text { ANGE: } \\
\text { ANGE: }\end{array}$ & $\begin{array}{l}\text { ven } \\
\text { ang }\end{array}$ & cag? & fla & gui & $\operatorname{mex}$ & $\begin{array}{l}\text { herb } \\
\text { shrub }\end{array}$ \\
\hline $\begin{array}{r}\text { OWg? } \\
\text { RANGE: }\end{array}$ & $\begin{array}{l}\text { ven } \\
\text { gua }\end{array}$ & & & & & $\begin{array}{l}\text { tree } \\
\text { shrub }\end{array}$ \\
\hline RANGE: & col & cr & ecu? & the & & $\begin{array}{l}\text { ep iph } \\
\text { shrub } \\
\text { climber }\end{array}$ \\
\hline RANGE: & $\mathrm{col}$ & ( & $x^{2}=$ & the & & shrub \\
\hline RANGE & end & & & & & $\begin{array}{c}\text { shrub } \\
\text { tree }\end{array}$ \\
\hline RANGE: & end & & & & & climber \\
\hline RANC & cag? & nic & per & sag? & & $\begin{array}{l}\text { shrub } \\
\text { tree }\end{array}$ \\
\hline RANGE: & cag & col & $\operatorname{mex}$ & trt & & $\begin{array}{l}\text { shrub } \\
\text { tree }\end{array}$ \\
\hline $\begin{array}{c}\text { RANGE: } \\
\text { trt }\end{array}$ & & & gua & $\operatorname{mex}$ & nic & $\begin{array}{c}\text { shrub } \\
\text { tree }\end{array}$ \\
\hline RANGE: & $\mathrm{col}$ & ven & & & & shrub \\
\hline RANGE: & $\mathrm{cr}$ & & & & & $\begin{array}{c}\text { shrub } \\
\text { tree }\end{array}$ \\
\hline RANGE: & sao & & & & & climber \\
\hline RAN & ang & cag & fla & jam & $\operatorname{mex}$ & shrub \\
\hline & sag? & sao & & & & \\
\hline RANGE: & ang & cag & gua & $\operatorname{mex}$ & sag? & climber \\
\hline $\begin{array}{r}\text { Sao } \\
\text { RANGE: }\end{array}$ & $\mathrm{trt}$ & & & & & \\
\hline RANGE: & cag? & $\operatorname{mex}$ & sag? & & & $\begin{array}{c}\text { shrub } \\
\text { tree }\end{array}$ \\
\hline
\end{tabular}


TAXON FAMILY

PROVINCES

ELEVATION (OOOM.)

256 (170) SOLANACEAE cont.

mammosum $L$,

intr. cult.

PROV: ch da pa 000m: $0-1$

melongena $L$.

intr. cult.

microleprodes Bitt.

- -var. felicis D'Arcy

PROV: ca 000m: $0-1$

PROV: ch $\quad 000 \mathrm{~m}: 0-1$

--var. microleprodes

PROV: bo ch da $v r$ 000m: $0-1$

nigrescens Mart. \& Gal.

PROV: ca ch da

$000 \mathrm{~m}: 0-1,1-2,3+$

nudum Dun.

\section{oxyca rpum Schiede}

pectinatum Dun.

PROV: ca cc ch pa vr 000m: $0-1,1-2$

PROV: ch

000m: $1-2$

perattenuatum I.M. Johnst.

*pertenue Standl. \& Morton

phaseoloides Polak.

*physal ifolium Rusby

*--var. nitidibaccatum (Bitt.) Edmonds

RoV: ca cc ch lo pa $000 \mathrm{~m}: 0-1$

$\begin{array}{ll}\text { PROV: pa } & 000 \mathrm{~m}: 0-1 \\ \text { PROV: } \mathrm{ch} & 000 \mathrm{~m}: 1-2\end{array}$

PROV: ch $\quad 000 \mathrm{~m}: 1-2$

intr. nat.

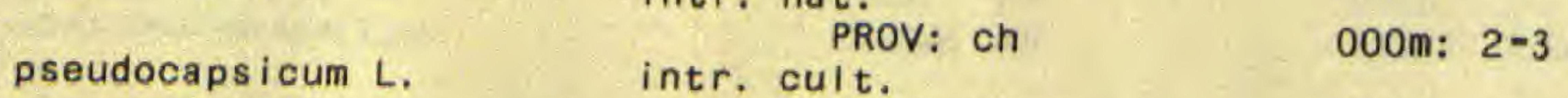

pulverulentum Pers. PROV: ch 000m: 1-2

PROV: $\mathrm{ch} \quad 000 \mathrm{~m}: 1-2$

quitoense Lam.

intr. cult. nat.

PROV: ce ch da pa 000m: $0-1,1-2$

* ramonense Morton \& Standley

PROV: bo ch $000 \mathrm{~m}: 0-1$

PROV: bo pa $000 \mathrm{~m}: 0-1$

*rudepannum Dun.

rugosum Dun.

schlechtenda I i anum Wa I p.

seaforthianum Andr.

*sessiliflorum Dun.

siparunoides Ewan

PROV: ca ce ch cn da 000m: 0-1

he lo pa sa vr

PROV: bo ca ce en da 000m: $0-1$ PRov: pa sa

PROV: bo ca cn da he 000m: $0-1,1-2$ lo pa

PROV: ca ch lo pa 000m: $0-1,1-2$

PROV: cc 000m: $0-1$

PROV: ca pa

$000 \mathrm{~m}: 0-1$

*stramoni ifolium Jacq.

PROV: bo

000m: $0-1$

subinerme Jacq.

PROV: ca cc pa sa $000 \mathrm{~m}$ : $0-1$

\begin{tabular}{|c|c|c|c|c|c|c|}
\hline RANGE: & nwt & & & 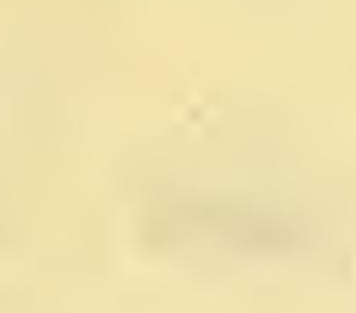 & & $\begin{array}{l}\text { herb } \\
\text { shrub }\end{array}$ \\
\hline RANGE: & his & owg & & 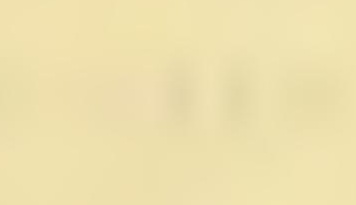 & & herb \\
\hline RANGE: & end & & 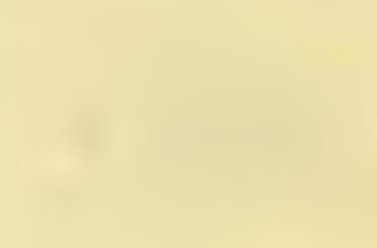 & & & shrub \\
\hline RANGE: & end & & & & & $\begin{array}{c}\text { tree } \\
\text { shrub } \\
\text { tree }\end{array}$ \\
\hline $\begin{array}{l}\text { RANGE: } \\
\text { mex }\end{array}$ & $\begin{array}{l}\text { bo I } \\
\text { nao }\end{array}$ & $\begin{array}{l}\text { cag? } \\
\text { sag? }\end{array}$ & $\begin{array}{l}\text { ca I } \\
\text { sao }\end{array}$ & fla & jam & herb \\
\hline RANGE : & cag & col & $\mathrm{cr}$ & cub & ecu & shrub \\
\hline & his & $\operatorname{mex}$ & $p r$ & & & tree \\
\hline RANGE: & cag & mex & & & & herb \\
\hline RANGE: & $\mathrm{col}$ & $\mathrm{cr}$ & per & & & $\begin{array}{l}\text { herb } \\
\text { shrub }\end{array}$ \\
\hline $\begin{array}{l}\text { RANGE: } \\
\text { RANGE: }\end{array}$ & $\begin{array}{l}\text { end } \\
\mathrm{cr}\end{array}$ & & & & & $\begin{array}{l}\text { shrub } \\
\text { shrub }\end{array}$ \\
\hline RANGE: & cag? & $\mathrm{cr}$ & gua & & & herb \\
\hline RANGE: & wdw & 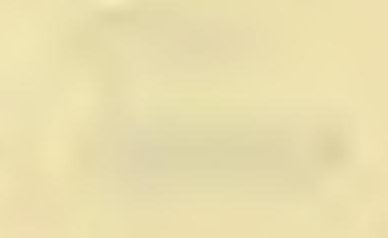 & $y=$ & & 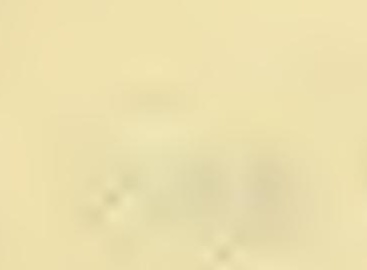 & herb \\
\hline RANGE: & end & & 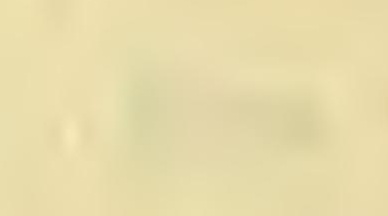 & 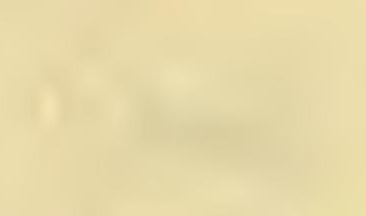 & & shrub \\
\hline RANGE: & cr & sat & in $4=$ & & $x^{2}$ & $\begin{array}{l}\text { shrub } \\
\text { tree }\end{array}$ \\
\hline RANGE: & $\mathrm{COI}$ & cr & ecu & per & & $\begin{array}{l}\text { herb } \\
\text { shrub }\end{array}$ \\
\hline RANGE: & $\mathrm{cr}$ & 3 & 21 & 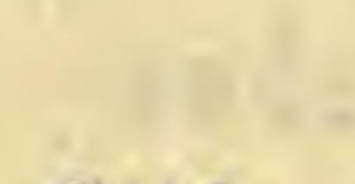 & 15: & shrub \\
\hline $\begin{array}{c}\text { RANGE: } \\
\text { per }\end{array}$ & cag & $\mathrm{col}$ & ecu & gua & mex & $\begin{array}{c}\text { shrub } \\
\text { tree }\end{array}$ \\
\hline RANGE: & cag & $\mathrm{col}$ & mex & 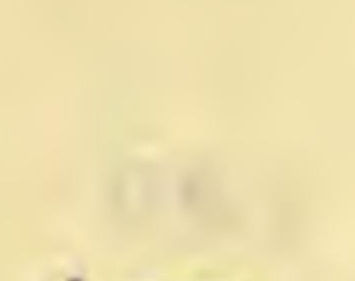 & 14 & $\begin{array}{l}\text { shrub } \\
\text { tree }\end{array}$ \\
\hline $\begin{array}{c}\text { RANGE: } \\
\text { SaO }\end{array}$ & bel & cag? & gag & lag & sag? & $\begin{array}{c}\text { shrub } \\
\text { tree }\end{array}$ \\
\hline $\begin{array}{l}\text { RANGE: } \\
\text { Sag? } \\
\text { RANGE: }\end{array}$ & $\begin{array}{l}\text { ang } \\
\text { ang }\end{array}$ & bel & cag? & cub & $\operatorname{mex}$ & $\begin{array}{l}\text { shrub } \\
\text { tree } \\
\text { cl imber }\end{array}$ \\
\hline $\begin{array}{c}\text { RANGE: } \\
\text { sat }\end{array}$ & $\mathrm{col}$ & $c r$ & ecu & per & sao & shrub \\
\hline RANGE: & $\mathrm{col}$ & ecu & per & $n$ & & $\begin{array}{r}\text { cl imber } \\
\text { shrub } \\
\text { tree? }\end{array}$ \\
\hline $\begin{array}{l}\text { RANGE: } \\
\text { Ven } \\
\text { RANGE: }\end{array}$ & $\mathrm{col}$ & ecu & gui & per & sat & shrub \\
\hline RANGE: & ang & cag & gui & mex & sag? & $\begin{array}{l}\text { shrub } \\
\text { tree }\end{array}$ \\
\hline
\end{tabular}


256 (170) SOLANACEAE cont.

torvum Sw.

trizygum Bitt.

PROV: bo ch he lo

000m: $0-1,1-2$

PROV: bo $\mathrm{ch}$

intr. cult.

tuberosum L.

umbe I la tum $\mathrm{Mi}$ II.

*vaccinifflorum Standl. \& Mort.

*valerianum Mort. \& Standl.

wend Iandi i Hook, f woodsonili Corr.

cult.

Witheringia

a sterotricha (Stand I.) Hunz.

bristaniana D'Arcy

coccoloboides (Damm,) Hunz.

correana D'Arcy

cuneata (Stand I.) Hunz.

*exiguiflora D'Arcy

hunzikeri D'Arcy

laxissima (Stand1.) D'Arcy maculata (Mort. \& StandI.) Hunz. *morí D'arcy

solanacea L'Her. $\begin{array}{ll}\text { PROV: ch } & 000 \mathrm{~m}: 1-2 \\ \text { PROV: bo ca ce ch cn } 000 \mathrm{~m}: 0-1 & \end{array}$

PROV: bo ca cc ch cn 000m: $0-1,1-2,2-3$ PROV: $\mathrm{ch}$ he lo pa vr

$000 m: 2-3,3+$

PROV: ch

PROV: $\mathrm{CC}$ ch

PROV: $\mathrm{ch}$

000m: $0-1$

000m: $0-1,1=2$

000m: $3^{+}$

PROV: bo cc ch pa

000m: $0-1,1-2$

PROV: da

PROV: ch pa

000m: $0-1,1-2$

000m: $0-1,1-2,2-3$

PROV: cn sa

PROV: bo ch

000m: $0-1$

000: $1-2$

PROV: da pa sa 000m: $0-1,1-2$

PROV: on

000m: $0-1$

PROV: ch

PROV: bo ch

PROV: ch

000an: $0-1$

000n: $1-2$

$000 \mathrm{~m}: 1-2$

ROV: bo ca ce ch ch 000m: $0-1,1-2$ da lo pa sa 000m: $2-3$

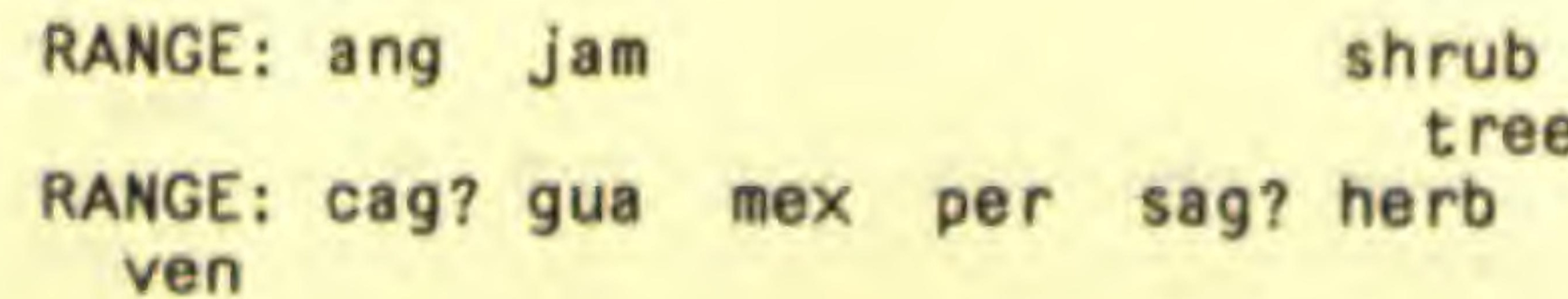

RANGE: $w d w$ RANGE: cag col cr gag mex shrub

RANGE: $c r$

tree

RANGE: $\mathrm{cr}$

RANGE: $\mathrm{cr}$

RANGE: ven$$
\text { tree }
$$

shrub

cl imber

herb

RANGE: er

herb

RANGE: end

RANGE: COI cr

RANGE: $c r$

RANGE: or

nic ven

shrub

shrub

herb

shrub

herb

herb

RANGE: $\mathrm{cr}$

RANGE: end

shrub

shrub

herb

subshrub

RANGE: end

shrub

herb

RANGE: Or

RANCE: ang

nex sag? sao

shrub

erb

shrub

tree

257 (171) SCROPHULARIACEAE

Aga I inis

hispidula (Mart.) D'Arcy

PROV: CC vr

000a: $0-1$

000n: $0-1$

lectra

aspera (Cham. schlecht.) L, O, Whs.

PROV: ca he pa

PROV: ch

000 : $1-2,2-3$

000m: 0-1 cult, PrOV: be ce pa 
TAXON

Bacopa

axillaris (Benth.) Stand I. bacopoides (Benth.) Pulle Iaxiflora (Benth.) EdwalI monnieri (L.) Penn.

monnierioides (Cham.) Rob. repens (SW.) Wettst.

salzmannif (Benth.) Edwal I sessiliflora (Benth.) Pulle

Benjaminia reflexa (Benth.) D'Arcy

Buchnera

pusilla H.B.K.

rosea H.B.K.

weberbaueri Diels

Ca Iceolaria

i razuensis J.D, Sm.

mexicana Benth.

perfoliata L. f.

tripartita R. \& P.

Capraria

biflora L.

peruviana Benth.

Castilleja arvens is Schlecht. \& Cham. quirosii Standi.

Digitalis

purpurea L.

Escobedia

grandiflora (L. f.) Kuntze
PROV: ca pa 000m: 0-1

PROV: ca cc cn lo pa $000 \mathrm{~m}: 0-1$

PROV: cc pa $\quad 000 \mathrm{~m}: 0-1$

PROV: bo ca ch cn pa 000m: 0-1

$$
\text { sa }
$$

PROV: ca cn pa sa vr 000m: $0-1$

PROV: cc da pa

$000 \mathrm{~m}: 0-1$

PROV: bo ca ce ch cn 000m: $0-1,1-2$

pa vr

PROV: ca ce cn lo pa 000m: 0-1

PROV: pa

000m: $0-1$

PROV: ca cc ch cn he 000m: $0-1,1-2$ pa $v r$

PROV: ca pa

PROV: $\mathrm{CC}$ ch

000m: $0-1$

000m: $0-1,1-2$

PROV: ch

PROV: bo $\mathrm{ch}$

000m: $2-3,3+$ 000m: $1-2,2-3$

PROV: ch

$000 \mathrm{~m}: 1-2,2-3,3+$

PROV: $\mathrm{ch}$

000m: $1-2,2-3,3+$

PROV: bo ca pa sa

000m: $0-1$

PROV: pa

000m: $0-1$

PROV: ch lo

PROV: ch

$000 \mathrm{~m}: 0-1,1-2,2-3$

000m: $1-2,2-3,3+$

PROV: ch

$000 m: 2-3$

000m: $1-2$

\begin{tabular}{|c|c|c|c|c|c|}
\hline $\begin{array}{l}\text { RANGE: } \\
\text { RANGE: } \\
\text { RANGE: } \\
\text { RANGE: }\end{array}$ & $\begin{array}{l}\text { col } \\
\text { bel } \\
\text { sao } \\
\text { jam }\end{array}$ & $\begin{array}{l}\text { gua } \\
\text { gua }\end{array}$ & gui & nic & sao \\
\hline RANGE: & cag? & gua & sag? & sao & \\
\hline $\begin{array}{r}\text { RANGE: } \\
\text { sag? }\end{array}$ & ang & cag? & col & jam & mex \\
\hline $\begin{array}{c}\text { RANGE: } \\
\text { Sao }\end{array}$ & ang & cag & $\mathrm{COl}$ & gui & mex \\
\hline RANGE: & cag & $\mathrm{col}$ & gua & gui & \\
\hline RANGE: & cag & cub & sat & & \\
\hline $\begin{array}{l}\text { RANGE: } \\
\text { sag? }\end{array}$ & $\begin{array}{l}\text { cag? } \\
\text { sao }\end{array}$ & $\mathrm{cr}$ & ecu & gua & mex \\
\hline $\begin{array}{l}\text { RANGE: } \\
\text { RANGE: }\end{array}$ & $\begin{array}{l}\mathrm{col} \\
\mathrm{bel}\end{array}$ & $\begin{array}{l}\text { per } \\
\text { cag? }\end{array}$ & $\begin{array}{l}\text { sag? } \\
\text { per }\end{array}$ & sag? & trt \\
\hline
\end{tabular}

RANGE: or herb

RANGE: bol cag col ecu gua herb mex per ven

RANGE: $\mathrm{COI} \mathrm{cr}$ ecu

RANGE: bol cag col ecu mex herb per sag sao

RANGE: ang cag fla owg sag shrub
sao

RANGE: col ecu per shrub

RANGE: cag gua his mex nao herb sag

RANGE: $\mathrm{cr}$

herb

shrub

RANGE: OWg

herb

RANGE : col cr mex per herb 
TAXON

257 (171) SCROPHULARIACEAE cont.

\begin{tabular}{|c|c|c|c|c|c|c|c|}
\hline ibsoniothamnus & & & & & & & \\
\hline $\begin{array}{l}\text { a latus A. Gentry } \\
\text { cornutus (J.D. Sm.) A. Gentry }\end{array}$ & PROV: & bo $\mathrm{c}$ & ch d & da & & 000m: & $0-1,1-2$ \\
\hline $\begin{array}{l}\text {--var, cornutus } \\
\text {--var, latidentatus (A. Gentry) }\end{array}$ & $\begin{array}{l}\text { PROV: } \\
\text { D'Arcy }\end{array}$ & ch & pa & & & 000m: & $0-1,1-2$ \\
\hline $\begin{array}{l}\text { mirificus A. Gentry } \\
\text { "truncatus A. Gentry }\end{array}$ & $\begin{array}{l}\text { PROV: } \\
\text { PROV: } \\
\text { PROV: }\end{array}$ & $\begin{array}{l}\text { pa } \\
\mathrm{cc} \\
\mathrm{cc}\end{array}$ & $\begin{array}{l}v r \\
\text { cn }\end{array}$ & & & $\begin{array}{l}\text { 000m: } \\
\text { 000m: } \\
\text { 000m: }\end{array}$ & $\begin{array}{l}0-1 \\
0-1 \\
0-1\end{array}$ \\
\hline Lamourouxia & & & & & & & \\
\hline gutierrezii oerst. & PROV: & $\mathrm{ch}$ & & & & 000m: & $1-2$ \\
\hline viscosa Kunth & PROV: & $\operatorname{ccc}$ & $\operatorname{ch} v$ & $v r$ & & $000 \mathrm{~m}:$ & $0-1,1-2$ \\
\hline Leucoca rpus & & & & & & & \\
\hline perfoliatus (H.B.K.) Benth. & PROV: & bo $c$ & $\mathrm{ch}$ & & & 000m: & $1-2,2-3$ \\
\hline Lindernia & & & & & & & \\
\hline crustacea (L.) F. MueII. & PROV: & $\begin{array}{l}\text { bo } c \\
\text { da }\end{array}$ & $\begin{array}{ll}\text { ca } & 0 \\
\text { lo } & p\end{array}$ & $\begin{array}{ll}\text { cc } & c \\
\text { pa } & s\end{array}$ & $\begin{array}{l}\text { ch cn } \\
\text { sa } \vee r\end{array}$ & 000m: & $0-1$ \\
\hline diffusa (L.) Wettst. & PROV: & bo & $\begin{array}{l}\mathrm{ca} \\
\mathrm{vr}\end{array}$ & cc c & en pa & 000m: & $0-1$ \\
\hline $\begin{array}{l}\text { dubia (L.) Penn. } \\
\text { H--subsp. anagali idea (Michx, ) Coo }\end{array}$ & $\begin{array}{l}\text { PROV: } \\
\text { Dperride } \\
\text { PROV: }\end{array}$ & ca & $\mathrm{ch}$ & pa & $=2 x$ & 000m: & $\begin{array}{l}0-1 \\
0-1\end{array}$ \\
\hline \#Mazus & & & & & & & \\
\hline *japonicus (Thunb.) Kuntze & PROV: & ch & & & $34 x+1$ & 000m: & $1-2$ \\
\hline $\begin{array}{l}\text { Mecardonia } \\
\text { procumbens }(M i), \text { ) Small }\end{array}$ & PROV: & bo & $\begin{array}{l}\text { ca } \\
\text { he }\end{array}$ & $\begin{array}{ll}\operatorname{cc} & 0 \\
10 & p\end{array}$ & $\begin{array}{l}\text { ch cn } \\
\text { pa sa }\end{array}$ & 000m: & $0-1$ \\
\hline $\begin{array}{l}\text { Micranthemum } \\
\text { pilosum Ernst }\end{array}$ & PROV: & ca & cc 1 & he $p$ & pa & 000m: & $0-1$ \\
\hline $\begin{array}{l}\text { Russelia } \\
\text { equisetiformis Schlecht. \& Cham. }\end{array}$ & & & & & & & \\
\hline sarmentosa Jaca. & $\begin{array}{l}\text { PROV: } \\
\text { PROV: }\end{array}$ & $\begin{array}{l}\text { ca } \\
\text { ca } \\
\text { he }\end{array}$ & $\begin{array}{l}\text { pa } \\
\text { cc } \\
\text { pa }\end{array}$ & $\begin{array}{l}\mathrm{ch} 0 \\
\mathrm{vr}\end{array}$ & cn da & $\begin{array}{l}\text { 000m: } \\
\text { 000m: }\end{array}$ & $\begin{array}{l}0-1 \\
0-1,1-2\end{array}$ \\
\hline $\begin{array}{l}\text { Scoparia } \\
\text { dulcis } L .\end{array}$ & PROV: & $\begin{array}{l}\text { bo } \\
\text { he }\end{array}$ & $\begin{array}{ll}\text { ca } \\
10\end{array}$ & $\begin{array}{ll}c c & c \\
p a & s\end{array}$ & $\begin{array}{l}\text { cn da } \\
\text { sa } v r\end{array}$ & 000m: & $0-1$ \\
\hline
\end{tabular}

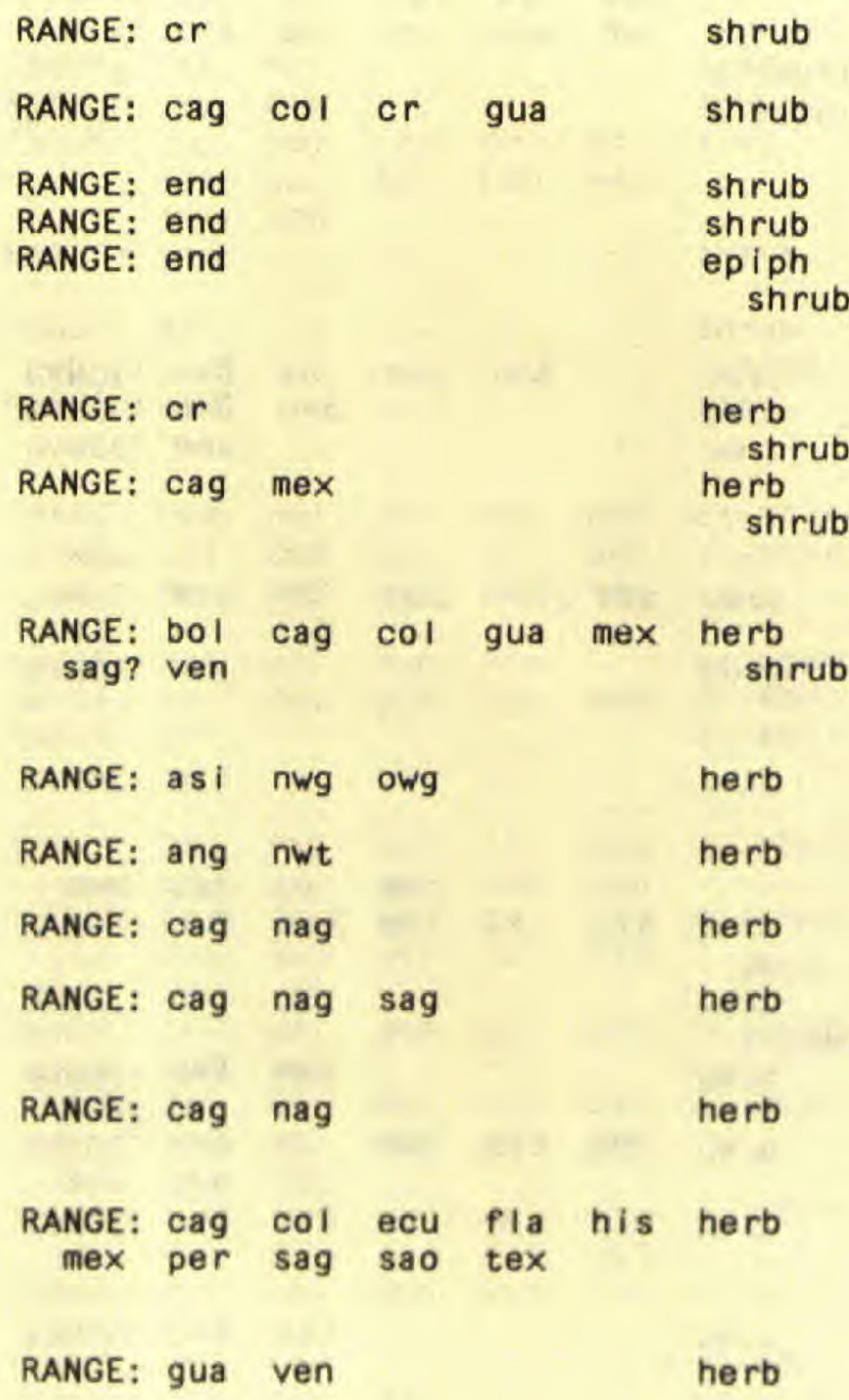

RANGE: jam nWt 
TAXON

257 (171) SCROPHULARIACEAE cont.

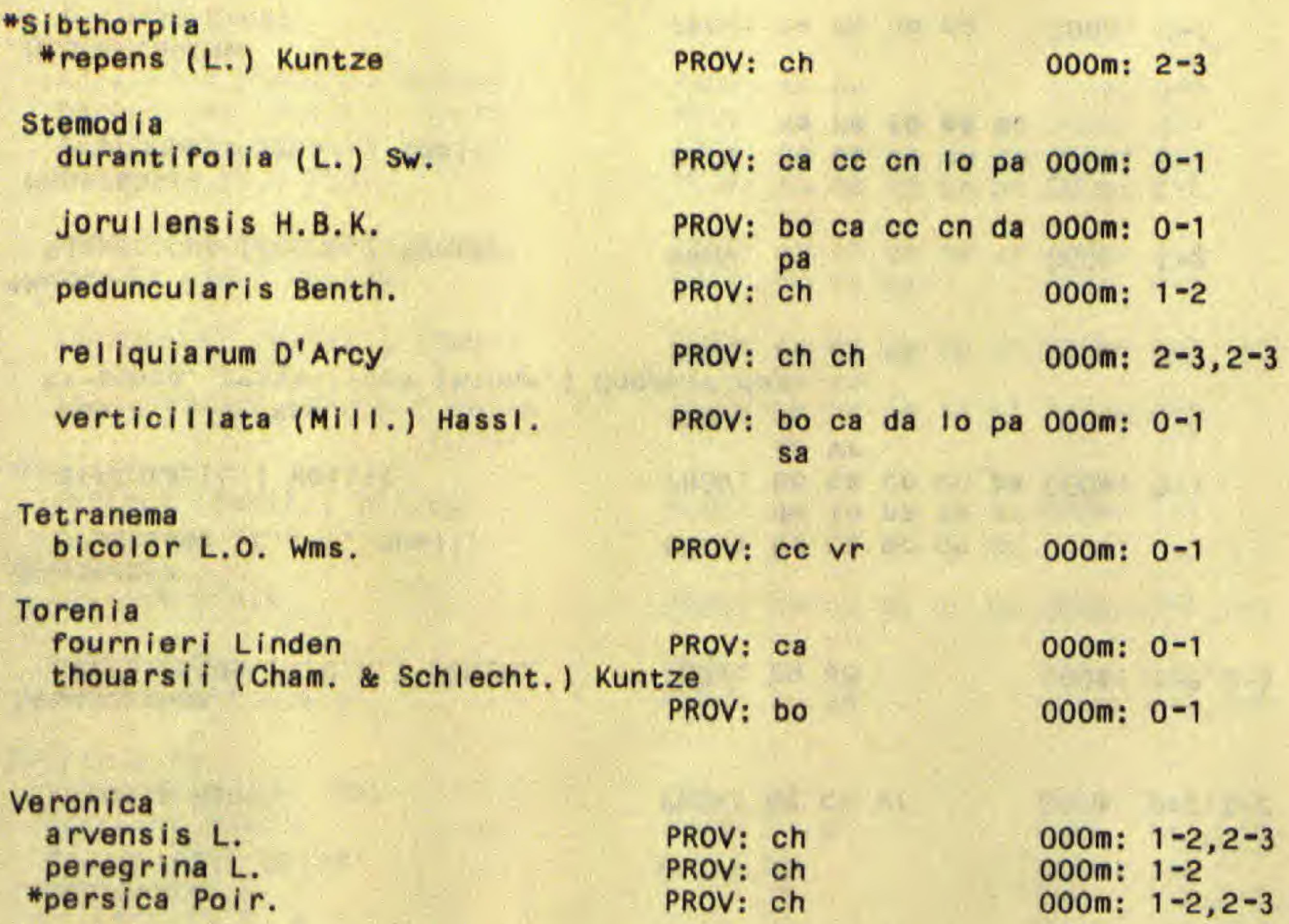

258 (172) BIGNONIACEAE
Adenoca I ymma

a rthropetiolatum A. Gentry *chocoens is A. Gentry

inundatum DC.

Amphil ophium

paniculatum (L.) H.B,K.

--var. molle (Schlecht. \& Cham.) StandI.

pannosum (DC.) Bur. \& Schum.
PROV: ca pa

PROV: da

000m: $0-1$

PROV: bo ca cc ch cn 000m: $0-1,1-2$ he lo pa sa $v r$
PROV: cn da

000m: $0-1$

000m: $0-1,1-2$

\begin{tabular}{|c|c|c|c|c|c|c|}
\hline RANGE: & cag & sat & & & & herb \\
\hline RANGE: & ang & caa & cal & his & & \\
\hline $\begin{array}{c}\text { KANGE: } \\
\text { sag }\end{array}$ & $\begin{array}{l}\text { ang } \\
\text { sao }\end{array}$ & cag & cal & his & nao & herb \\
\hline RANGE: & cag & $\mathrm{cr}$ & cub & gag & $\operatorname{mex}$ & herb \\
\hline $\begin{array}{l}\text { sag? } \\
\text { RANGE: }\end{array}$ & cag & $\operatorname{mex}$ & $=1$ & & $=-$ & herb \\
\hline 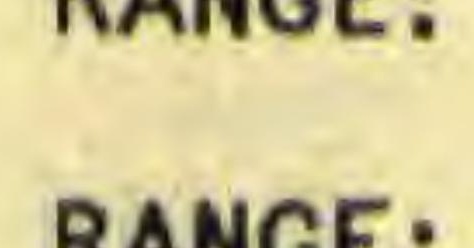 & & & & & & \\
\hline RANGE: & $\mathrm{cr}$ & $\mathrm{cr}$ & 213 & 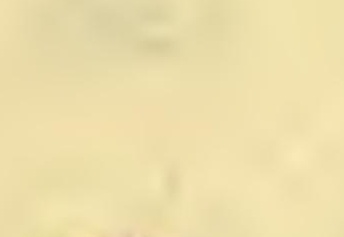 & 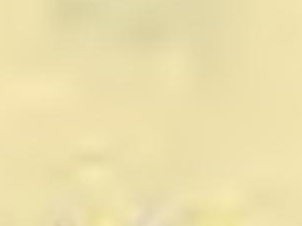 & $\begin{array}{l}\text { nerb } \\
\text { herb }\end{array}$ \\
\hline RANGE: & ang & $\mathrm{cag}$ & $\mathrm{col}$ & $\mathrm{cr}$ & his & herb \\
\hline $\operatorname{mex}$ & sag & sal & sao & & & \\
\hline RANGE: & nic & $4=9$ & 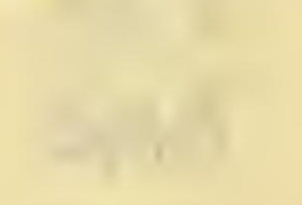 & & & herb \\
\hline & & he & $x^{2}-$ & $=$ & $2+2$ & \\
\hline RANGE: & as i & & & & & herb \\
\hline & asi & $\mathrm{cr}$ & nar & caa? & $t r t$ & harh \\
\hline $\begin{array}{c}\text { RANGE: } \\
\text { ven }\end{array}$ & & $\mathrm{Cr}$ & per & sag! & Trt & nero \\
\hline RANGE: & eur & 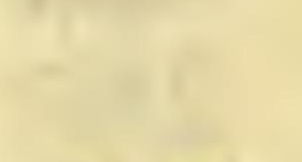 & 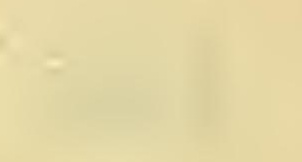 & (4) & y. & herb \\
\hline RANGE: & nwg & owg & & & & herb \\
\hline RANGE: & cag & eur & $\operatorname{mex}$ & nwg & & herb \\
\hline
\end{tabular}

RANGE: $\mathrm{COI}$

RANGE: $\mathrm{COI}$

RANGE: cag sat ven

climber

climber

climber

RANGE: ang bel bol cag col climber
cr cr ecu gua gui hon
mex nic per sag sal sao
trt ven




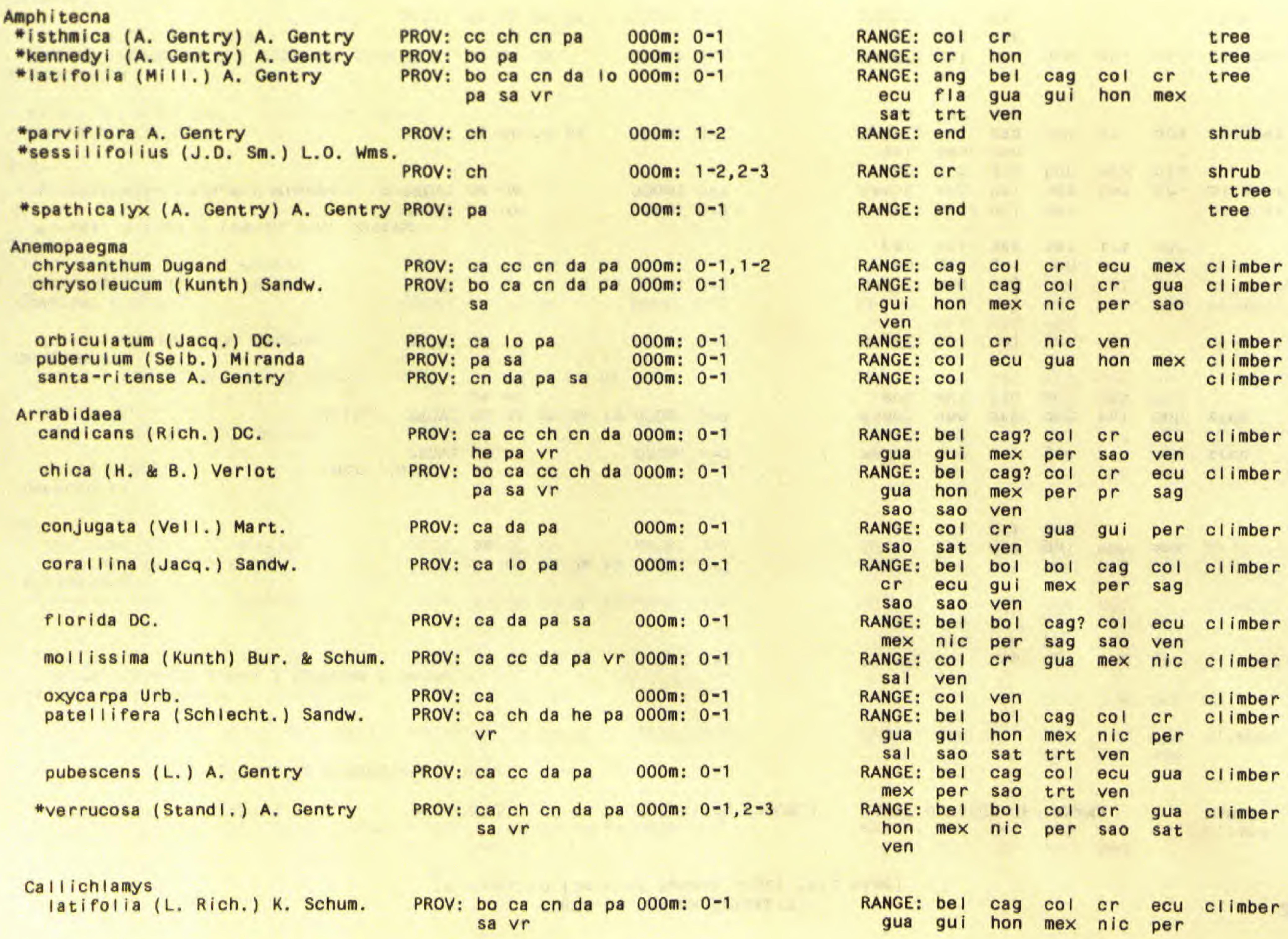


TAXON

\section{FAMILY}

PROVINCES

ELEVATION (OOOM.)

\section{RANGE OUTSIDE OF PANAMA}

258 (172) BIGNONIACEAE cont.

\section{Cera tophytum}

tetragonolobum (Jacq.) Sprague \& Sandw.

PROV: ca da pa sa $000 \mathrm{~m}: 0-1$

\section{Clytostoma \\ bina tum (Thunb.) Sandw.}

Crescentia

a lata H.B.K. intr. cult.

PROV: ch

$000 \mathrm{~m}: 0-1$

cujete L.

Cuspidaria

floribunda (DC, ) A. Gentry

cult. PROV: bo ca ch da lo 000m: 0-1 pa sa

PROV: da

000m: $0-1$

Cydista

aequinoctialis (L.) Miers

PROV: bo ca cc ch cn 000m: $0-1$ da he lo pa sa $\mathrm{vr}$

*--var. hirtella (Benth.) A. Gentry $000 \mathrm{~m}: 0-1$

heterophylla Seib.

PROV: ca da he pa $000 \mathrm{~m}: 0-1$

Distictella

magnoli ifolia (H.B.K.) Sandw.

PROV: ca cn da

000m: $0-1$

Godmania

aesculifolia (H.B.K.) Standl.

PROV: ca he pa $v r$

000m: $0-1$

\section{Haplophragma}

adenophyllum (G. Don) Dop intr. cult.

PROV: ca 000m: $0-1$

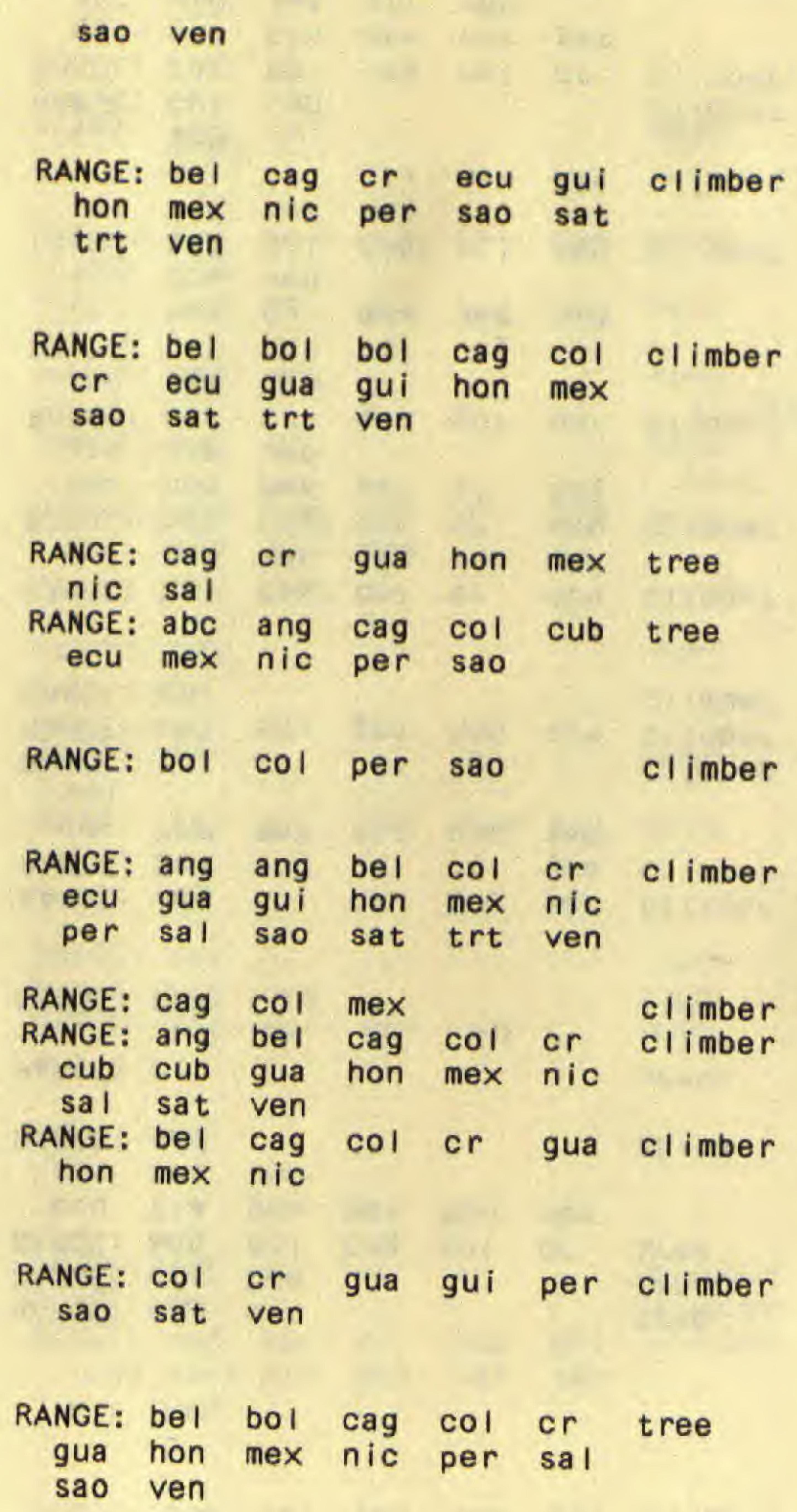


TAXON

--subsp. sandwithiana A. Gentry

copaia (AubI.) D. Don cult. PROV: ca ch da pa 000m: $0-1$ *-subsp. spectabilis (DC.) A. Gentry

PROV: bo ca cn da pa 000m: $0-1$

nimosifolia 0 . Don intr. cult.

PROV: ch

000m: $0-1$

Kigelia

-africana (Lam.) Benth. intr. cult.

PROV: ca $\quad 000 \mathrm{~m}: 0-1$

Lundia

corymbifera (Vahl) Sandw.

PROV: ch da pa $000 \mathrm{~m}: 0-1$

puberula Pitt.

PROV: ca cn da

000m: $0-1$

\section{Macfadyena}

uncata (Andr.) Sprague Sandw. PROV: bo ca ch cn da 000m: 0-1 pa sa

unguis-cati (L.) A. Gentry

PROV: ca pa

000m: $0-1$

\section{*Mansoa}

*hymenaea (DC.) A, Gentry

PROV: pa sa vr

000m: $0-1$

*kerere (AubI.) A. Gentry

PROV: bo ca cn da pa 000m: 0-1

-parvifolia (A. Gentry) A. Gentry PROV: bo da

000m: $0-1$

"standleyi (Steyerm.) A. Gentry

PROV: bo ch pa

000m: $0-1$

*verrucifera (Schlecht.) A. Gentry PROV: ca cn pa sa

000m: $0-1$

Martinella

obovata (H.B.K.) Bur. \& K. Schum. PROV: ca ch cn da pa 000m: 0-1

RANGE: col cr
RANGE: bel bol cag or ecu tree
gui nic per sao sat ven
RANGE: cr sao

RANGE: afr

tree

RANGE: bol col cr ecu gui climber
per sao trt ven
RANGE: bel cag col ecu mex climber
nic per sat ven

nic per sat ven RANGE: bel bol cag col cr climber
ecu gua gui gui mex per
Sao sat trt ven
RANGE: ang bel bol cag col climber
cr ecu gua gui his hon
lag mex nic per sao sat
ven

RANGE: bel col cr ecu gua climber
gui his mex nic per sal
Sao ven
RANGE: bel bol cag col cr climber
gua gui gui hon nic per
sao ven
RANGE: bol col ecu hon per climber
sao sat
RANGE: col cr ecu gua gui climber
per sat ven
RANGE: cag col cr ecu gua climber
gui gui hon mex per sao
trt ven
$\begin{aligned} & \text { RANGE: bel bol cag col cr climber } \\ & \text { gua gui per sao ven }\end{aligned}$


TAXON

FAMILY

PROVINCES

ELEVATION (OOOM, )

RANGE OUTSIDE OF PANAMA

HABIT

258 (172) BIGNONIACEAE cont.

Mussatia

hyacinthina (Stand I.) Sandw.

PROV: bo cn da pa vr 000m: $0-1$

\section{Paragonia \\ pyramidata (L, Rich.) Bur.}

\section{Pa rmentiera}

aculeata $(H, B, K$,$) Seem.$

cereifera Seem.

*dressleri A. Gentry

macrophyl la StandI.

*morii A. Gentry

Phryganocydia

corymbosa K. Schum.

phellosperma (Hemsi.) Sandw.

i thecocten ium

*crucigerum (L.) A. Gentry

Pleonotoma

variabilis (Jacq.) Miers

cult. PROV: ca

cult. PROV: ca

PROV: cn pa

PROV: bo $\mathrm{CC}$

PROV: pa

000m: $0-1$

000m: $0-1$

000m: $0-1$

000m: $0-1$

000m: $0-1$

PROV: ca ce cn da pa 000m: 0-1 sa

PROV: ca da pa

000m: $0-1$

PROV: bo ca ch cn da 000m: 0-1 pa sa $v r$

PROV: bo ca da lo pa 000m: 0-1 sa

Podranea

ricolasiana (Tanfani) Sprague

intr. cult.

PROV: $\mathrm{ch} \quad 000 \mathrm{~m}: 0-1$

\section{Pyrostegia}

venusta (Ker.) Miers intr. cult.

PROV: $\mathrm{ch} \quad 000 \mathrm{~m}: 0-1$

Saritaea

magnifica (van Steenis) Dugand

intr. cult.

PROV: ca 000m: $0-1$

PROV: ch 000m: $2-3$

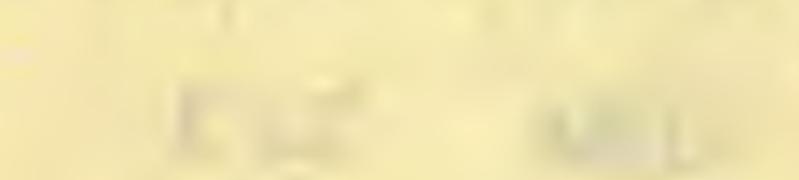

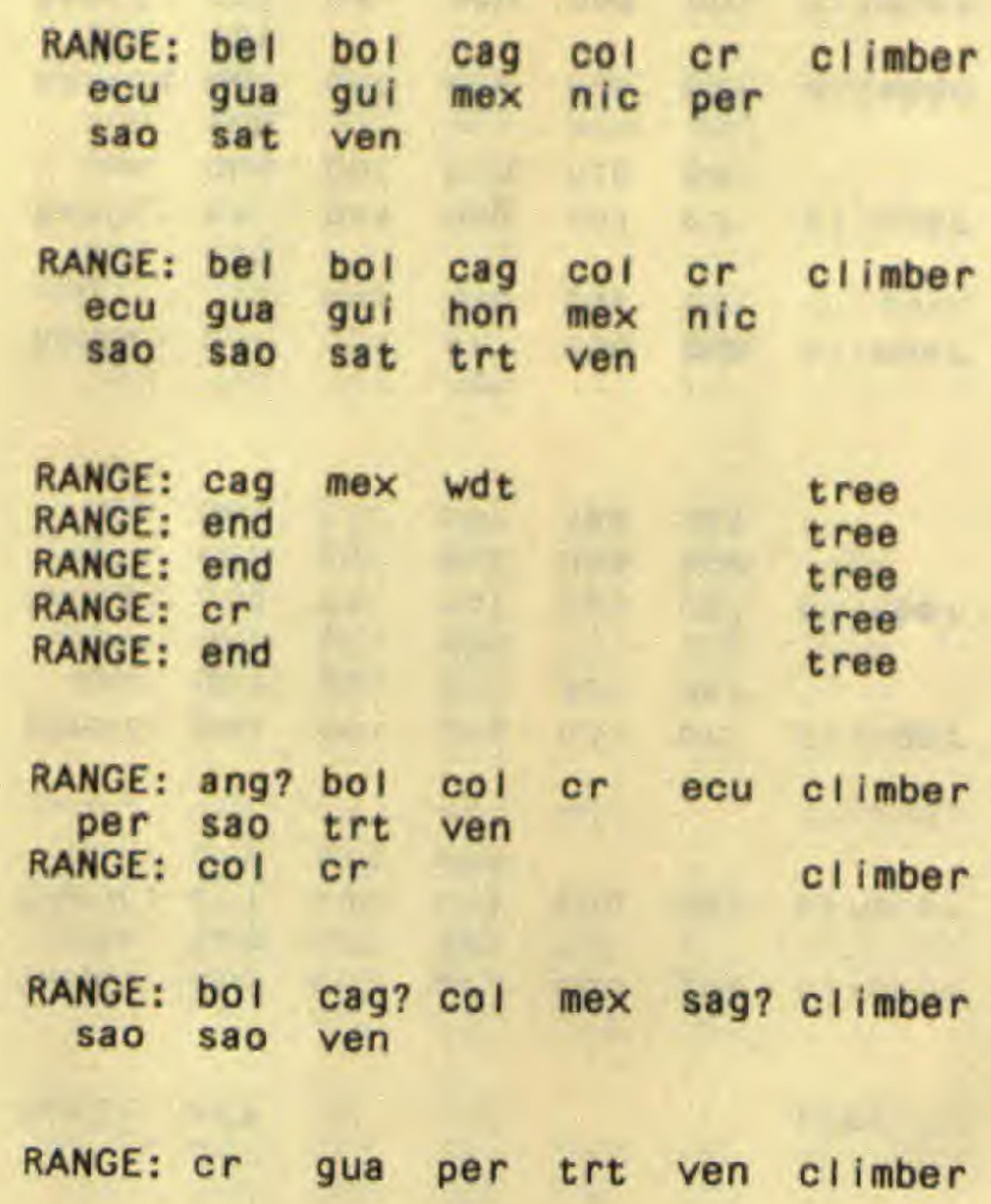

RANGE: af $r$

climber

RANGE: bol $\mathrm{cr}$ sag sao climber

RANGE: $\mathrm{COI}$ ecu

climber

RANGE: ang ven woody

hemiep

climber 
TAXON

FAMILY

PROVINCES

258 (172) BIGNONIACEAE cont. dressleri A. Gentry

fastigiata Schery

fuscata A. Gentry

nica raguensis Stand I.

parvifiora (Oerst.) Monachino
PROV: pa

000m: $0-1$

vr

PROV: cn da pa 000m: $1-2$

PROV: bo cn pa 000m: $0-1$

PROV: bo $\mathrm{cn}$ da pa sa $000 \mathrm{~m}$ : $0-1,1-2$

\section{Spathodea}

campanulata Beauvois

cult.

PROV: ca

000m: $0-1$

St izophy I I um

inaequilaterum Bur. \& K. Schum.

cult.

riparium (H.B.K.) Sandw.

PROV: ca cn da pa sa $000 \mathrm{~m}: 0-1,1-2$

PROV: bo ca ch cn da $000 \mathrm{~m}$ : $0-1$ pa sa vr

\section{Tabebuia}

chrysantha (Jacq.) Nichols.

PROV: ca ce ch cn da 000m: $0-1$ pa $\vee r$

guayacan (Seem.) Hems I.

PROV: ca ch cn da pa $000 \mathrm{~m}$ : $0-1$

\#heterophylla (DC.) Britt. intr. cult.

PROV: ca $\quad 000 \mathrm{~m}: 0-1$

impetiginosa (DC.) Standl.

PROV: da pa

000m: $0-1$

ochracea (Cham.) Stand I.

--subsp. neochrysantha (A. Gentry) A. Gentry

PROV: ca cc da he pa 000m: $0-1$

vr

ce da pa

palustris HemsI.

PROV: bo ca ce ch da 000m: 0-1

rosea (Bertol.) DC.

striata A. Gentry

he pa sa vr

000m: $0-1$

Tanaecium

nocturnum (Barb. Rodr.) Bur. \& K. Schum.

PROV: da $\quad 000 \mathrm{~m}: 0-1$

Tecoma

stans H.B.K. intr. cult.

PROV: ca cc ch cn da 000m: 0-1
RANGE OUTSIDE OF PANAMA

HAB IT

RANGE: COI

RANGE: cag? col ecu ecu gua climber

sag?

RANGE: $c r$

RANGE:

gua mex nic climber

RANGE: bel bel cag cr gua climber

hon nic per sao sat

RANGE: $a f r$

tree

RANGE: bol col cr ecu per climber

Ven
RANGE: bel bol cag? col cr climber

ecu gua gua hon mex nic

per sal sat trt ven

RANGE: bel col cr ecu gua tree hon jam mex nic per sal ven

RANGE: bel cag col cr gua tree

mex ven

RANGE: ang

shrub

ATCE: tree

mex nic per sag sal sao ven

RANGE: cag? col or hon nic tree sal trt ven

RANGE: col or ecu

shrub

RANGE: bel cag col cr ecu tree

gua hon mex nic sal ven

RANGE: $\mathrm{cOI}$

tree

RANGE: col gui per sao

cl imber

$\begin{array}{rllll}\text { RANGE: ang ang cag col cr } & \text { shrub } \\ \text { ecu fla gua hon mex nao } & \text { tree } \\ \text { nic per sag sal sao tex } & \end{array}$ 
TAXON

\section{FAMILY}

PROVINCES

ELEVATION (OOOM.)

RANGE OUTSIDE OF PANAMA

HAB I T

258 (172) BIGNONIACEAE cont.

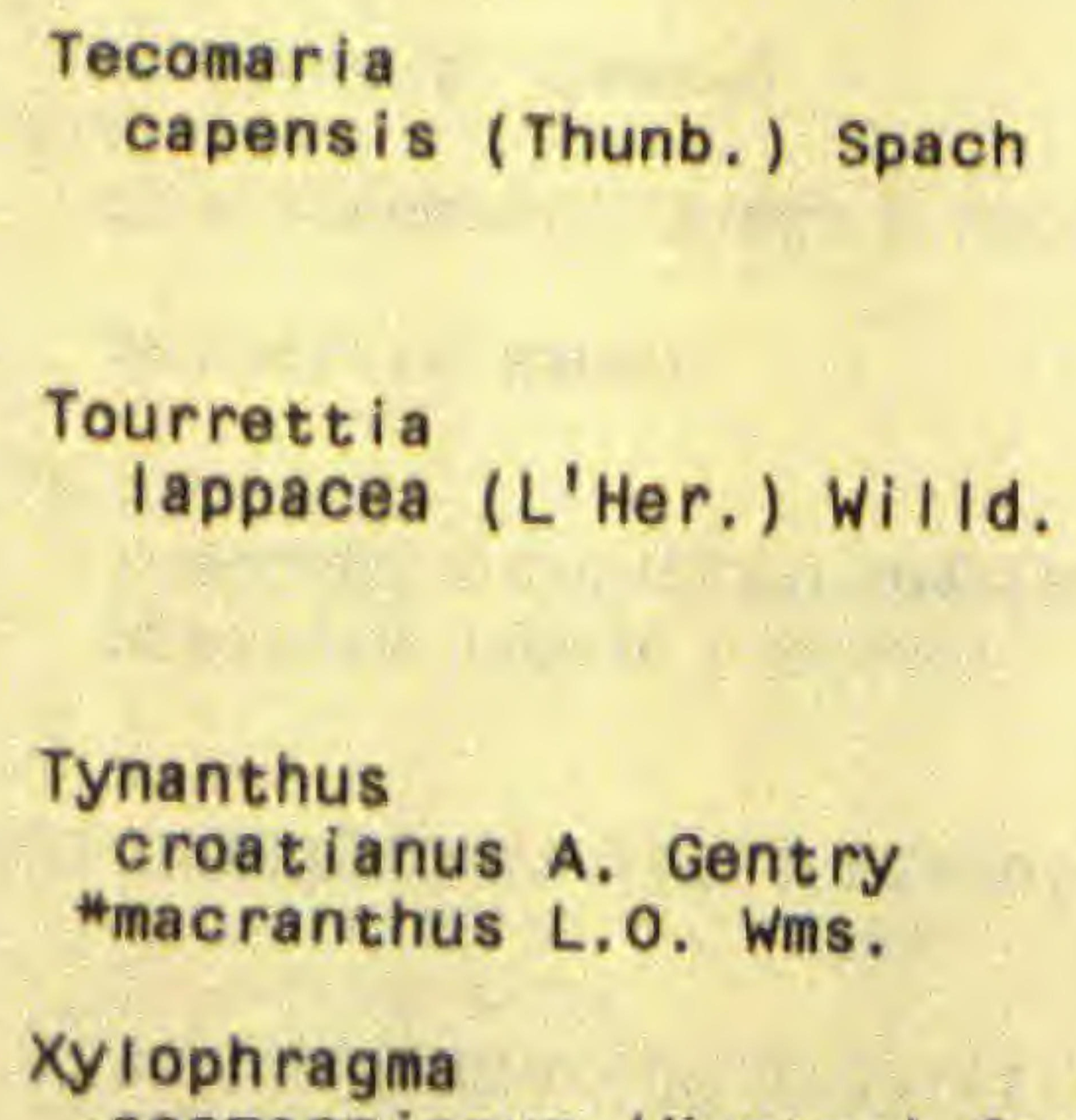

Tecoma ria

Tourrettia

Iappacea (L'Her.) Willd.

\section{Tynanthus}

croatianus A. Gentry

*macranthus L.O. Wms.

Xylophragma

capensis (Thunb.) Spach intr. cult.

PROV: pa $\quad 000 \mathrm{~m}: 0-1,1-2$

seemannianum (Kuntze) Sandw. PROV: ca cc ch da he 000m: 0-1
PROV: ch

000m: $0-1,1-2$

PROV: ca da pa

000m: $0-1$

000m: $0-1$ pa $v r$

\section{9 (173) PEDALIACEAE}

\section{Sesamum}

ind icum L.

intr. cult.

PROV: lo pa 000m: 0-1

\section{1 (174) OROBANCHACEAE}

\section{Conopholis \\ alpina Liebm. \\ --var. a lpina}

"americana (L.) WaII. f.

PROV: ch

PROV: ca ch

$000 \mathrm{~m}: 1-2,2-3$

$000 \mathrm{~m}: 0-1,2-3$

\section{2 (175) GESNERIACEAE}

\section{Ach imenes}

candida Lind I.

*erecta (Lam.) H.P. Fuchs

longiflora DC.

pedunculata Benth.

$\begin{array}{ll}\text { PROV: ch } & 000 \mathrm{~m}: 1-2 \\ \text { PROV: pa } & 000 \mathrm{~m}: 0-1,1-2 \\ \text { PROV: cc ch lo pa } & 000 \mathrm{~m} ; 0-1 \\ \text { PROV: ch } & 000 \mathrm{~m}: 1-2\end{array}$

RANGE: af $\mathrm{r} \mathrm{cr}$

shrub

subshrub

RANGE: bol cag col cr ecu climber
gua hon mex nic per sao

sat ven

RANGE: $\mathrm{COI}$

RANGE: $\mathrm{cr}$

climber

cl imber

RANGE: bel cag col cr gua climber
gui gui mex nic sal sat

$\begin{array}{lll}\text { RANGE: cr gua mex } & \text { herb } \\ \text { RANGE: cag } & & \\ \text { RANGE: cag cr mex } & \text { herb } \\ \text { RANGE: cag col gua mex } & \text { herb }\end{array}$




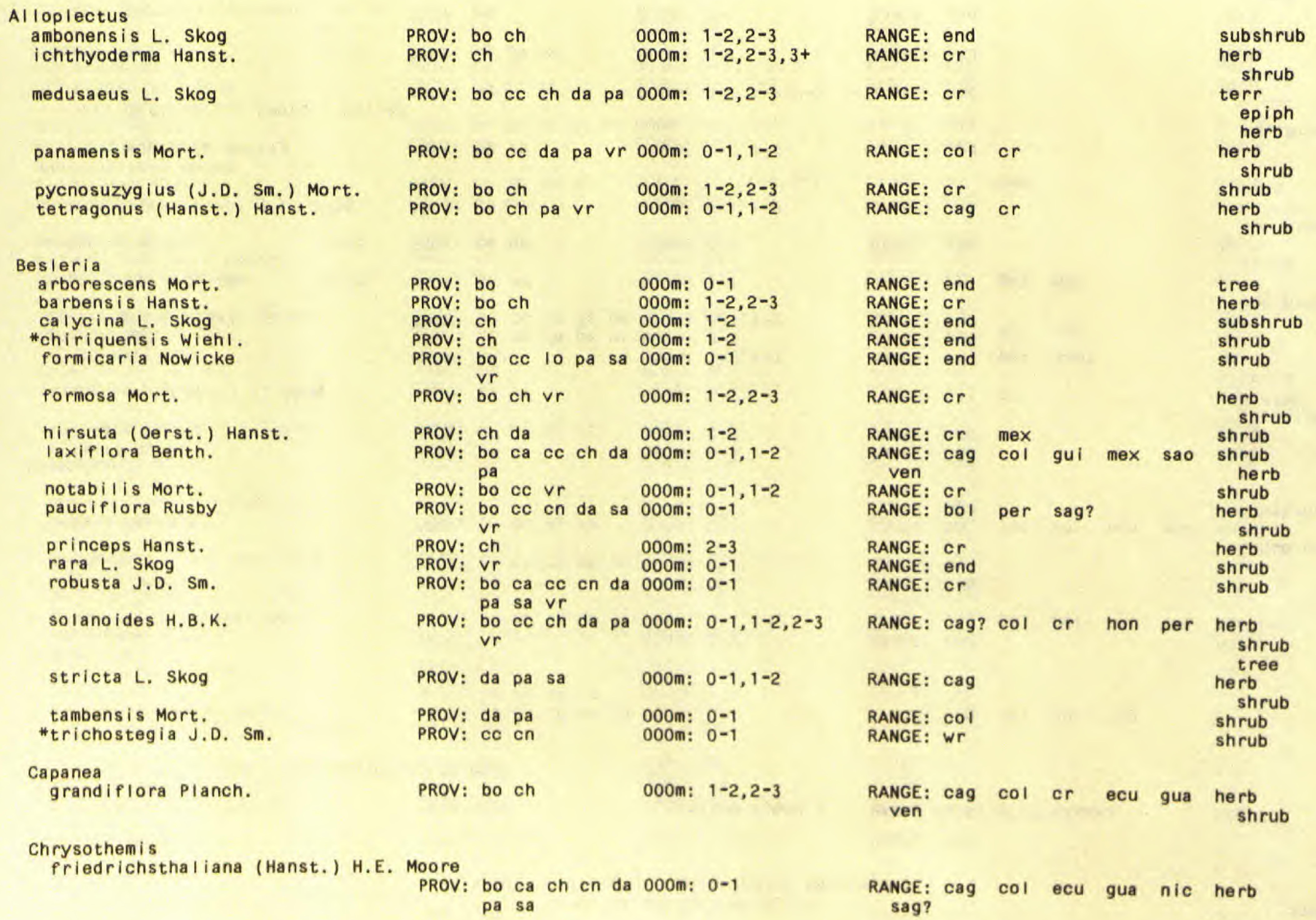


TAXON

\section{FAMILY PROVINCES}

ELEVATION (OOOM.)

262 (175) GESNERIACEAE cont.

pulchella (sims) Decne.

\section{Codonanthe}

chiricana Wiehl.

crassifolia (Focke) Mort.

Iuteola WiehI.

macradenia J.D. Sm.

uleana Fritsch

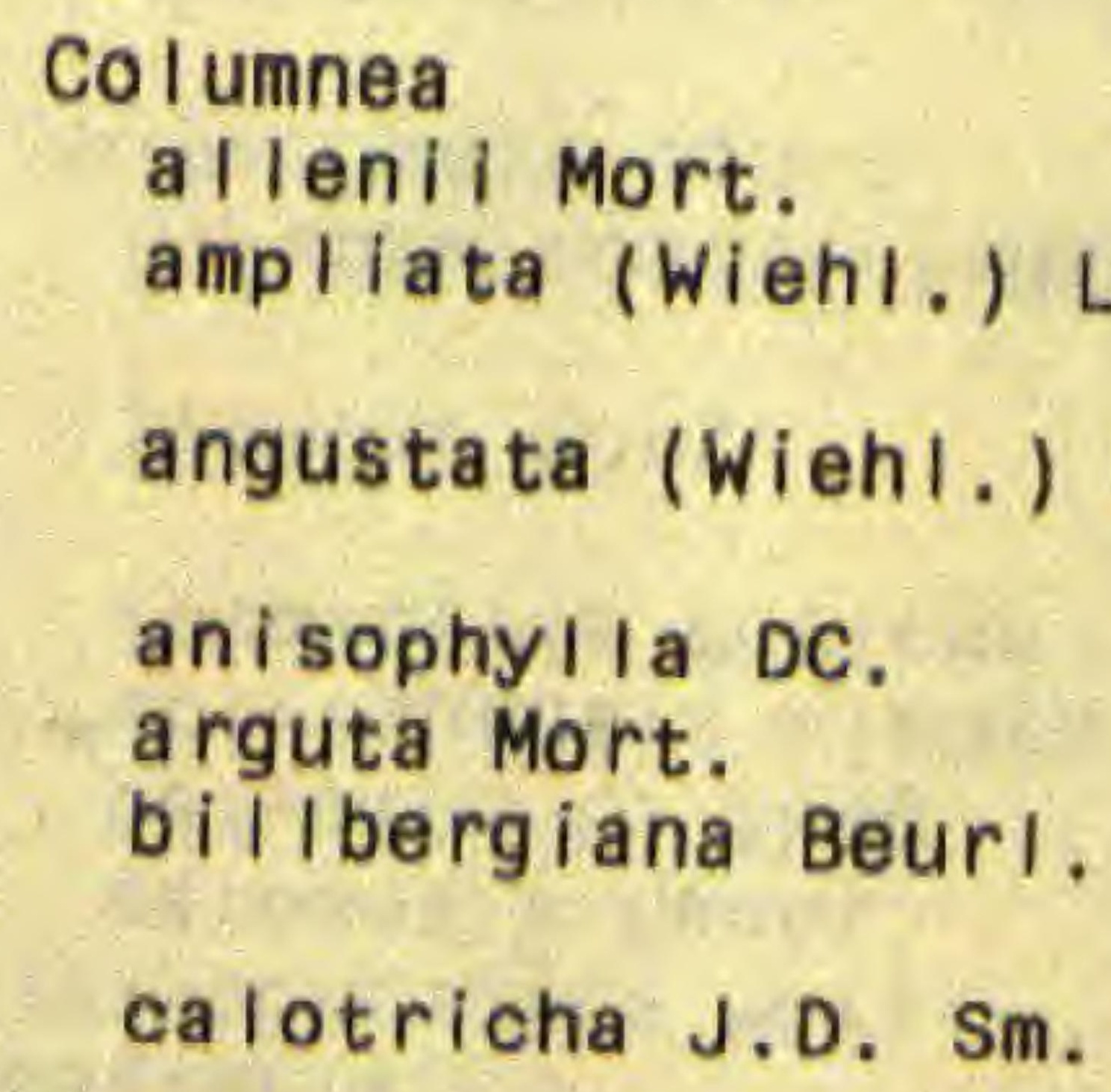

\section{cult}

cerropirrana (WiehI.) L. Skog

consanguinea Hanst.

--var, adpressa Morley

--var. darienensis (Mort.) Morley

PROV: da

crassa Mort.

cruenta Morley

dissimilis Mort.

dressleri Wiehl.

flaccida Seem.

florida Mort.

ga II icauda WiehI,

hiantiflora Wiehi.

hirsutissima Mort.

PROV: $\mathrm{CC}$

PROV: $V r$

PROV: ch

ult PROV:

PROV: da

PROV:
PROV: bo ca ch cn da 000m: $0=1$

$$
\text { lo pa sa vr }
$$

PROV: ch

000m: 1 -2

pa $\mathrm{Vr}$

000m: $0=1$

U: ca ch cn pa sa $000 \mathrm{~m}: 0-1$

000m: $0-1$

000m: $0-1$

PROV: da $v r$

000m: $0-1$

PROV: ca cc en da pa 000m: $0-1,1-2$

000m: $0-1$

000m: $0-1$

000m: $0-1,1-2$

PROV: bo $\mathrm{ch}$

$000 m: 1-2,2-3$

000m: $0-1,1-2,2-3,3+$ RANGE: end 000m: $0-1$

000m: $0-1$

000m: $0-1$

000m: $0-1,1-2$

000m: $2-3$

$000 \mathrm{~m}: 0-1,1-2$

000m: $0-1$

000m: $1-2$

000m: $0-1$
RANGE: end

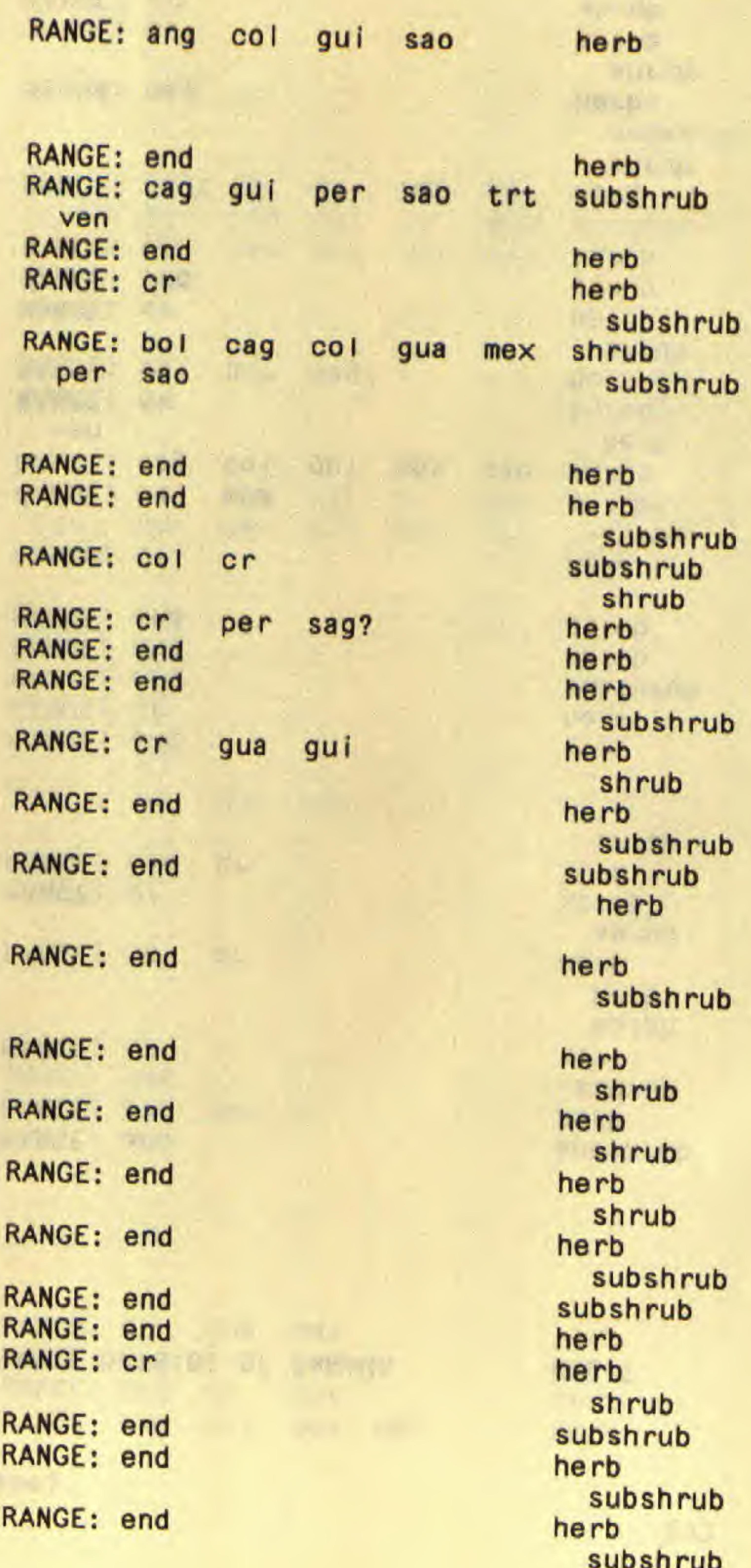


262 (175) GESNERIACEAE cont.

hirta KI. \& Hanst.

--var. hirta

--var. mortoni i (Raymond) Morley

PROV: $v r$

000m: we

RANGE: $c r$

herb

i I lepida Moore

cult. PROV: WP

incarnata Mort.

cult. PROV: pa

kah Ibreyeriana Mast.

: bo ch

RANGE: $w r$

RANGE: end

RANGE: $\mathrm{CO} I$

maculata Mort.

magnifica Oerst.

microcalyx Hanst.

mira Morley

PROV: bo cc ch pa $v r$ 000m: $0-1,1-2$

PROV: ch

$000 \mathrm{~m}: 2-3$

RANGE: end

RANGE: $c r$

PROV: $c c$ ch da $v r$ 000m: $0-1,1-2,2-3$

PROV: da pa

000m: $0-1,1-2$

RANGE: $c r$

RANGE: end

000m: $0-1$

nervosa (Oerst.) Hanst.

PROV: cn pa

000m: we

nicaraguens is Derst.

000m: $0-1$

ochroleuca (Oerst.) Hanst.

PROV: bo

$\begin{array}{ll}\text { PROV: } v r & 000 \mathrm{~m}: \text { we } \\ \text { PROV: } v r & 000 \mathrm{~m}: 0-1\end{array}$

oxyphyila J.D. Sm.

panamensis Mort.

$000 \mathrm{~m}: 0-1$

PROV: $\mathrm{CC}$

000m: $0-1$

PROV: ch

000m: $1-2,2-3$

parvifiora Mort.

PROV: cc da pa vr

000m: $0-1$

pectinata Mort.

000m: $0-1$

pendula (Oerst.) Hanst. perpulchra Mort.

PROV: $\mathrm{CC}$

000m: we

PROV: $v r$

$000 \mathrm{~m}: 0-1$

polyantha (WiehI.) L. Skog

PROV: ch

000m: $1-2,2-3,3+$

RANGE: end

RANGE: $\mathrm{cr}$

RANGE: $c r$

RANGE: $\mathrm{cr}$

RANGE: $c r$

nic

sag sao

RANGE: $w r$
RANGE: $\mathrm{cr}$

RANGE: $\mathrm{COI}$

RANGE: end

RANGE: $\mathrm{cr}$

RANGE: end

pulchra (WiehI.) L. Skog

PROV: pa

000m: $0-1$

RANGE: $\mathrm{cr}$

purpurata Hanst.

rubida (Mort.) Mort.

rubra Mort.

PROV: bo ca ch cn da 000m: $0-1,1-2$

PROV: $\mathrm{ch}$

PROV: cc pa vr $\quad$ 000m: $0-1,1-2$

sanguinolenta (Oerst.) Hanst.

PROV: bo

000m: $0-1$

PROV: ch

PROV: $v r$

PROV: da

000m: $0-1$

000m: we

$000 \mathrm{~m}: 0-1,1-2$

PROV: bo $\mathrm{ch}$

$000 m: 1-2,2-3$

RANGE: end

RANGE: $\mathrm{cr}$

RANGE: end

RANGE: end

RANGE: $\mathrm{cr}$

RANGE: $\mathrm{cr}$

RANGE: $c r$

RANGE: end

herb

herb

herb

herb

shrub

shrub

epiph

herb

herb

herb

herb

herb

herb

shrub

herb

shrub

herb

epiphyte

herb

shrub

herb

subshrub

herb

shrub

herb

herb

shrub

herb

shrub

herb

subshrub

herb

shrub

herb

herb

shrub

herb

shrub

herb

herb

shrub

herb

shrub

tulae Urb.

- -var. tomentulosa (Mort.) Morley 
TAXON

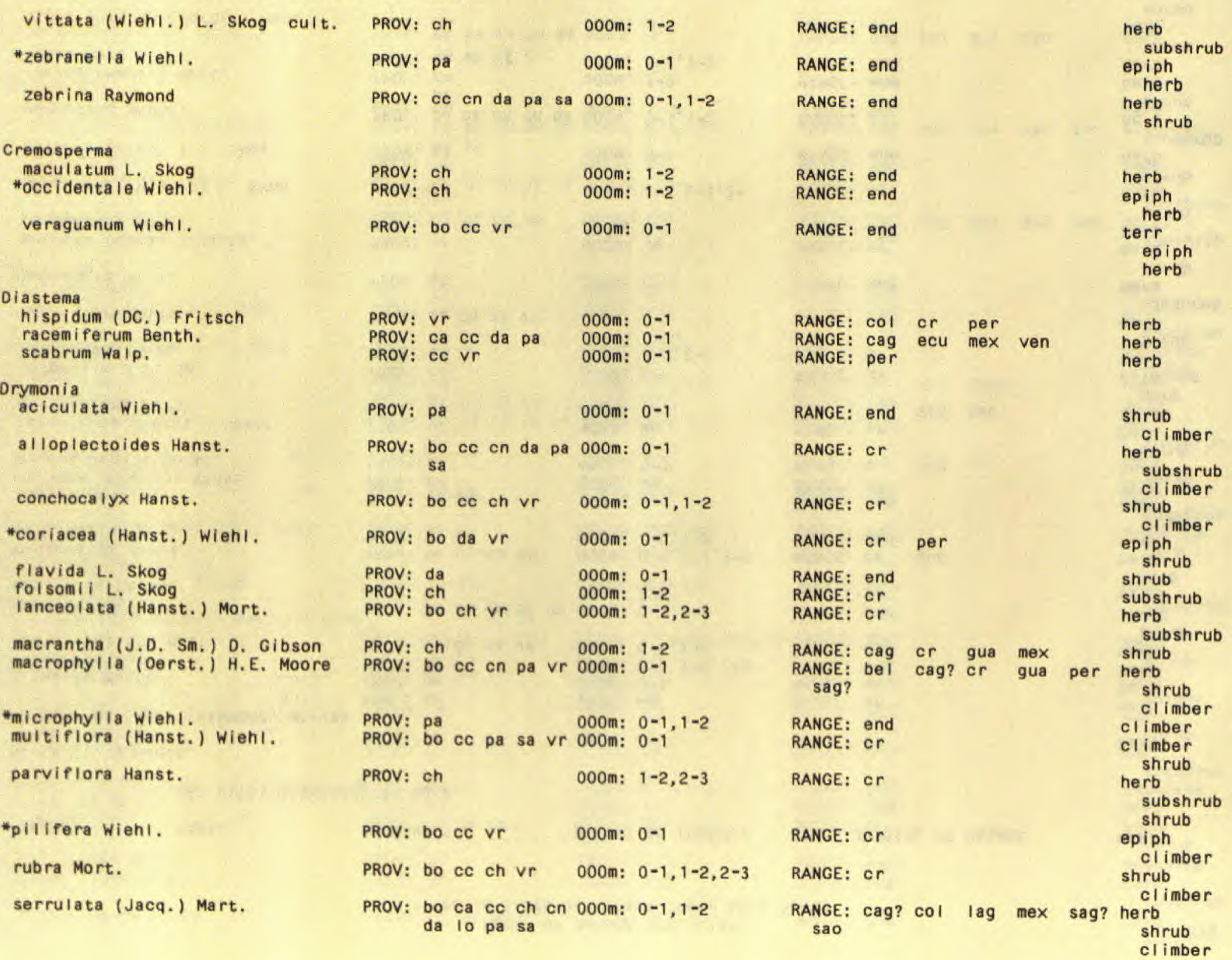


TAXON

stenophylla (J.D. Sm. ) H.E. Moore PROV. ch suiphurea Wiehi.

PROV: pa

000m: $0-1,1-2$ 000m: $0-1$

turrialvae Hanst.

\section{variegata Uribe}

warscewicziana Hanst.

PROV: bo $\mathrm{cc} c h$ da $\mathrm{vr} 000 \mathrm{~m}$ : $0-1,1-2$

PROV: da

000m: $1-2$

PROV: bo $\mathrm{cc}$ cn da $\mathrm{vr} 000 \mathrm{~m}$ : $0-1,1-2$

\section{Episcia}

cupreata (Hook.) Hanst. intr. cult.

I I lacina Hanst.

PROV: ca pa

PROV: $\mathrm{VO}$

\section{Gasteranthus}

acropodus (J,D. Sm.) WiehI.

delphinioides (Seem,) Wiehl.

dressleri wiehl.

imbricans (J,D. Sm.) Wiehl.

*maculatus Wiehl.

*tinctus (Mort.) Wiehl. wend I and ianus (Hanst.) WiehI.

Gloxinia

perennis (L.) Fritsch intr. cult. nat.

Koellikeria

erinoides (DC.) Mansf.

Kohleria

allenii Standl. \& L.0. Wms.

spicata (Kunth) Oerst.

tubiflora (Cav,) Hanst.

Monopyle

*grandiflora WiehI.

macrocarpa Benth.

maxonii Mort.
PROV: ca pa $\quad 000 m: 0-1$

PROV: bo cc cn pa sa $000 \mathrm{~m}: 0-1$

PROV: cn da pa sa $v r$ 000m: $0-1$

PROV: cn

PROV: bo

000m: $0-1$

000m: $0-1$

PROV: da

000m: $1-2$

PROV: da

PROV: bo ch da $\mathrm{vr}$

000m: $1-2$

000m: $0-1,1-2,2-3$

000m: $0-1,1-2$

PROV: $c c$ ch $v r$

$\begin{array}{ll}\text { PROV: ch } & 000 \mathrm{~m}: 0-1 \\ \text { PROV: bo ce ch } v r & 000 \mathrm{~m}: 0-1,1-2\end{array}$

PROV: ca cc cn da lo $000 \mathrm{~m}: 0-1$,

$$
\text { pa sa } v r
$$

PROV: da

000m: $1-2$

PROV: cc da pa sa

000m: 0-1

PROV: bo ch $v r$ 000m: $0-1,1-2$
RANGE: $c r$

RANGE: end

RANGE: $\mathrm{cr}$ ecu

RANGE: $\mathrm{COI}$

RANGE: $c r$

RANGE:

RANGE: col sao ven

RANGE: $\mathrm{COI} \mathrm{cr}$ nic

herb

RANGE: $c r$

RANGE: $\mathrm{COI} \mathrm{cr}$

RANGE: end

RANGE: $\mathrm{cr}$

RANGE: end

RANGE: $\mathrm{CO}$

RANGE: $\mathrm{cr}$

shrub
herb

subshrub

herb

shrub

herb

shrub

herb

shrub

climber

subshrub

shrub

herb

shrub

herb

subshrub

shrub

terr

herb

herb

RANGE: col per ven

herb

RANGE: bol col or ven herb

RANGE: $\mathrm{cr}$

RANGE: cag col or mex

RANGE: col cr ven mex herb

herb

RANGE: end

RANGE: col per

terr

herb

herb

subshrub

RANGE: $\mathrm{cr}$ shrub

herb 
TAXON

262 (175) GESNERIACEAE cont.

panamensis Mort.

\section{Moussonia}

ampla L. Skog

serrulata (Mort.) Wiehl.

\section{Napeanthus}

apodemus J.D. Sm.

Naut i loca Iyx

colombianus WiehI.

\section{*colonens is Wiehl. \\ dressleri Wiehl. \\ panamens is (Seem.) Seem. \\ *speciosus Wiehi.}

\section{Neomortonia \\ *nummularia (Hanst.) WiehI.}

rosea WiehI.

\section{Derstedina}

cerricola Wiehl.

suffrutescens L. Skog

\section{Pa radrymonia}

*alba Wiehl.

decurrens (Mort.) WiehI.

"flava WiehI.

hirta L. Skog

I ineata (Mort.) WiehI.

*macrophyI la WiehI.

metamorphophyIIa (J.D. Sm.) Wiehl.

ommata L. Skog

(J.D. Sm. ) Wiehl

pedunculata L. Skog

PROV: bo

PROV: da

PROV: ch 000m: $0-1$

$000 m: 1-2,2-3$

000m: $1-2,2-3$

PROV: ca ce cn da pa 000m: 0-1

$$
\text { sa vr }
$$

PROV: ca cn da sa

000m: $0-1$

PROV: cn

PROV: $c C \mathrm{vr}$

PROV: ca da pa

000m: $0-1$

$000 \mathrm{~m}: 0-1$

000m: $0-1$

000m: $0-1$

PROV: ch cn

000m: $0-1$

PROV: da pa

000m: $0-1$

PROV: bo $\mathrm{ch}$

$000 m: \quad 1-2,2-3$

PROV: $\mathrm{vr}$

000m: $0-1,1-2$

PROV: bo

$000 m: 0-1$

PROV: bo ce cn pa vr 000m: $0-1$

PROV: cc cn da pa vr 000m: $0-1$

PROV: da $000 \mathrm{~m}: 0-1$

PROV: cc $v r \quad 000 \mathrm{~m}: 0-1$

PROV: cc ch vr

$000 m: 0-1,1-2$

$000 m: \quad 1-2,2-3$

000m: $0-1$

000m: $0-1$

000m: $1-2$
RANGE: end

herb

RANGE: end

RANGE: end

shrub

subshrub shrub

RANGE: $\mathrm{col} \mathrm{cr}$

herb

RANGE: COI

RANGE: end

RANGE: end

RANGE: col mex

RANGE: end

$\operatorname{mex}$

RANGE: $\mathrm{cr}$ gua mex

RANGE: $\mathrm{COI} \mathrm{cr}$

RANGE: end

RANGE: end

RANGE: end

RANGE: $\mathrm{cr}$

RANGE: end

RANGE: $\mathrm{CO}$ I

RANGE: end

RANGE: end

RANGE: $\mathrm{cr}$

RANGE: end

RANGE: cag

herb

shrub

herb

herb

herb

herb

epiph

herb

herb

herb

subshrub subshrub

epiph

herb

herb

subshrub

epiphyte

herb

subshrub

herb

subshrub

epiph

herb

herb

subshrub

herb

subshrub

herb

herb

RANGE: COI 
TAXON

FAMILY

PROVINCES

262 (175) GESNERIACEAE cont.

lacerata Mort.

PROV: ch vr

000m: $0-1,1-2,2-3$

herb

Reldia

alternifolia Wiehl.

veraguens is Wiehl.

PROV: cn pa sa

000m: $0-1$

PROV: bo $\mathrm{cC} v \mathrm{r}$

000m: $0-1$

Rufodorsia

intermedia Wiehl.

major WiehI.

minor Wiehl.

Sinningia

inca rnata (Aub) I.) Denham

Solenophora

ca I ycosa J.D. Sm.
PROV: cc ch pa

$000 m: 0-1,1-2$

PROV: pa

000m: $0-1,1-2$

PROV: ec cn pa vr

000m: $0-1$

PROV: ca cc pa

000m: $0-1$

PROV: ch
$000 m: 1-2,2-3,3+$

RANGE: end

RANGE: end

herb

herb

RANGE: end

RANGE: end

herb

herb

RANGE: $\mathrm{cr}$

herb

subshrub

shrub

$\begin{gathered}\text { RANGE: cag col gui mex sao herb } \\ \text { ven }\end{gathered}$
RANGE: cr gua hon sal $\begin{gathered}\text { herb } \\ \text { shrub } \\ \text { tree }\end{gathered}$

264 (176) LENTIBULARIACEAE

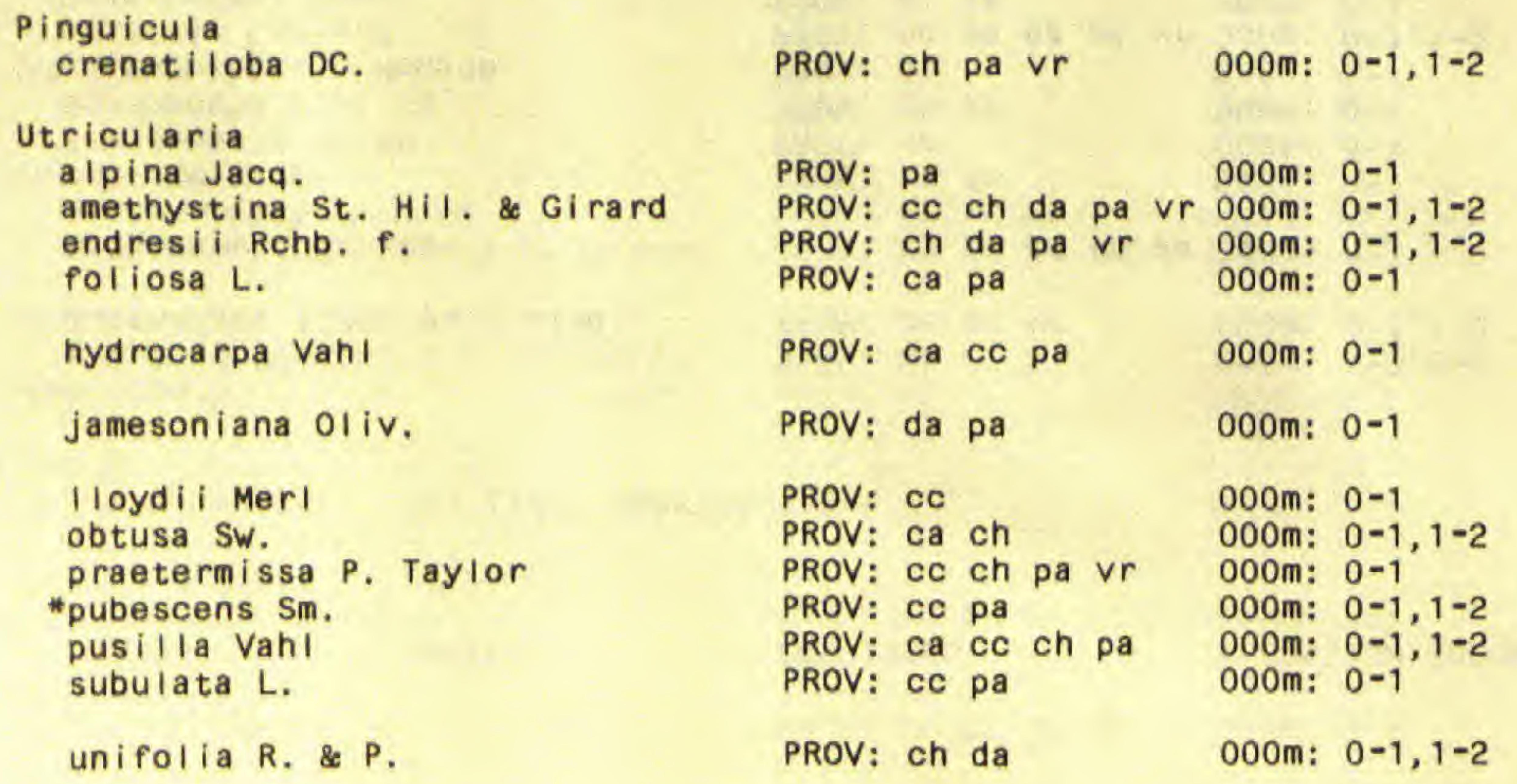




\section{6 (177) ACANTHACEAE}

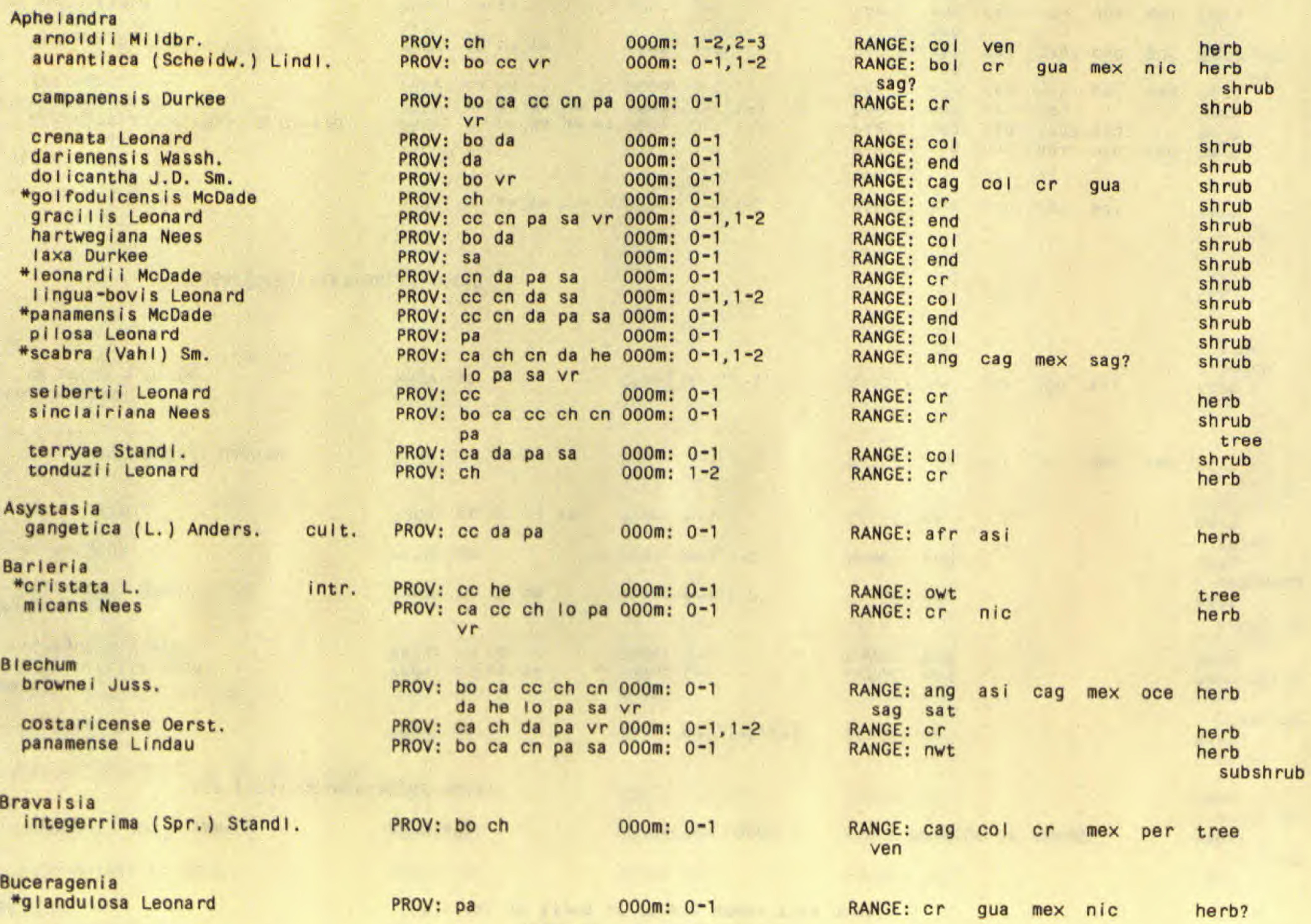


PROV: ca ch ch he lo 000m: $0-1$ pa $\vee r$

PROV: ca pa

000m: $0-1$

PROV: $\mathrm{ch}$

PROV: $c C$ Vr

PROV: bo ch

PROV: ch

ch da 10 000m: $0-1$ pa

PROV: pa

000m: $0-1$

PROV: ca cc ch on he 000m: $0-1,1-2$ lo pa

PROV: pa

000m: $0-1$

PROV: $v r$

000m: $0-1$

PROV: bo ch $v r$

000m: $1-2,2-3$

PROV: bo $\mathrm{ch}$

$000 \mathrm{~m}: 1-2,2-3$

000m: $0-1$

000m: 0-1

PROV: bo ca cn

000m: $0-1$

PROV: bo ca cc pa $v r$ 000m: $0-1$

PROV: pa

000m: $0-1$
RANGE: $w r$

herb

RANGE: COI

herb

RANGE: end

RANGE: $c r$

RANGE: $\mathrm{cr}$

RANGE: $\mathrm{cr}$

RANGE: ang col fla

herb

herb

shrub

herb

herb

RANGE: $\mathrm{cr}$

herb

shrub

RANGE: cag or nag? nic sag? herb sao

RANGE: af $r$

shrub

RANGE: cag mex

shrub

RANGE: end

RANGE: as $\mathrm{i}$ bel

herb

RANGE: cag mex

herb

RANGE: $\mathrm{cr}$

herb

RANGE: cag mex sag sao

herb

RANGE: oWt 
a IIenii (Leonard) Durkee aurea Schlecht.

carthaginens is Jacq

chamaephyton D. Gibson

comata (L.) Lam.

crenata (Leonard) Durkee graciliflora (StandI.) D. Gibson macrantha Benth.

metall ica Lindau

oerstedii Leonard

panamense Durkee

pectoralis Jacq.

--var, macrophyllus Durkee

- -var. pectoralis

refractifolia (Kuntze) Leonard

* refulgens Leona ro

secunda Vah!

tinctoria (Oerst.) D. Gibson cult.

trichotoma (Kuntze) Leonard urophylla (Lindau) D. Gibson

Ka Ibreyeriella

rostel lata Lindau

Louteridium

costaricense RadIk, \& J.D. Sm.

PROV: $\mathrm{CC} \quad$ 000m: $0-1,1-2$

PROV: $c c$ ch da sa $v r$ 000m: $0-1,1-2,2-3$

PROV: bo ca cc he pa $000 \mathrm{~m}: 0-1$

PROV: bo ch da sa $000 \mathrm{~m}: 0-1$

PROV: bo ca ch cn da 000m: $0-1,1-2$ he pa sa

PROV: bo

Pa $v$ 000m: $0-1,1-2$

PROV: $\mathrm{cc}$ ch $000 \mathrm{~m}: 0-1,1-2$

PROV: bo ca ch cn da $000 \mathrm{~m}: 0-1$

PROV: Cc ch cn pa 000m: $0-1,1-2$

PROV: pa 000m: $0-1$

PROV: da

000m: $0-1$

PROV: ca ch cn da pa 000m: $0-1$

PROV: bo en da pa sa $000 \mathrm{~m}$ : $0-1,1-2$

PROV: ch

$000 \mathrm{~m}: 1-2$

PROV: bo ca cc cn da $000 \mathrm{~m}$ : $0-1$ he pa

PROV: ch da pa 000m: $0-1,1-2$

PROV: bo da sa 000m: $0-1$

PROV: ch da vr $000 \mathrm{~m}: 0-1$

PROV: cn sa

000m: $0-1,1-2$

PROV: bo ca ce cn da 000m: 0-1

he lo pa sa

Megaskepa sma

erythrochlamys Lindau cult. PROV: ch

000m: 0-1

Mendoncia

brenesii Standl. \& Leonard

cordata Leonard

costaricana Oerst.

gracilis Turrill

hirsuta (P. \& E.) Nees

I indavii Rusby

I itoralis Leonard

retusa Turrill

tonduzii Turrill
PROV: $\mathrm{ch}$

PROV: bo $\mathrm{cn}$

PROV: $c h$

PROV: ca pa

PROV: da

PROV: ca cc da

PROV: ca

PROV: $\mathrm{cc}$ cn
PROV: bo ca da pa 000m: $1-2$

$000 \mathrm{~m}: 0-1$

$000 \mathrm{~m}: 1-2$

000m: $0-1$

000m: $0-1$

000m: $0-1,1-2$

000m: $0-1$

000m: $0-1$

000m: $0-1$
RANGE: end

RANGE: cag mex

RANGE: cag mex

RANGE: $\mathrm{cr}$

RANGE: $\mathrm{cr}$

jam nic

shrub

herb

herb

herb

RANGE: $c r$

RANGE: end

RANGE: cag

RANGE: or

cr mex

shrub

climber

shrub

herb

RANGE: $\mathrm{cr}$

RANGE: end

herb

herb

RANGE: end

RANGE: ang

sag

RANGE: $c r$

RANGE: end

RANGE: ang

ng

RANGE: cag

RANGE: $c r$

RANGE: $\mathrm{cr}$

RANGE: $C O I$

\section{herb}

cag or nic nwt herb

herb

shrub

herb

shrub

shrub

shrub

herb

subshrub

RANGE: $c r$

shrub

RANGE: $c r$ ven

shrub

RANGE: $\mathrm{cr}$

RANGE: $\mathrm{COI}$

RANGE: $\mathrm{cr}$

RANGE: $C O I$

RANGE: bOI

RANGE: bel

RANGE: $\mathrm{CO}$ I

gui per

cag? gua

RANGE: $c r$

RANGE: $c r$

climber
climber
climber
climber
bol gui per climber
clim? gua sag? climber
climber
climber
climber


NeI sonia

brunelloides (Lam.) Kuntze

\section{Neriacanthus}

grandiflorus Leonard

Odontonema

microphyl Ius Durkee

*tubiforme (Bertol.) Kuntze

\section{*Poikil acanthus \\ *macrantha Lindau}

Pseuderanthemum

atropurpureum (BuII) Bailey

$$
\text { cult. }
$$

cordatum (Nees) RadIk. cuspidatum (Nees) Radik. standleyi Leonard

\section{Razisea}

spicata Oerst.

\section{Ruellia}

anthracina Leonard

biolleyi Lindau

colonens is Wassh.

cooperi Leonard

fulgida Andr.

--var, angustissima (Hochr.) Leonard

--var. fulgida
geminifiora H.B.K.

geminiflora H.B.K.
inundata H.B.K.

leucobracteatus Durkee

macrophy I Ia VahI

ma I acosperma Greenm.

metal I ica Leonard
nudiflora (EngeIm. \& Gray) Urb.

--var. puberula Leonard

pittieri Lindau

praeclara Standl.
PROV: ca cc ch da lo 000m: 0-1 pa

PROV: $c h \mathrm{vr}$

$000 \mathrm{~m}: 0-1,1-2$

PROV: cn pa

000m: $0-1$

PROV: bo ca cc ch cn 000m: $0-1,1-2$ da pa pa sa vr

PROV: ch

000m: $1-2$

PROV: ca en pa

000m: $0-1$

PROV: ca cn da pa

PROV: ca da pa

000m: $0-1$

000m: $0-1$

PROV: $c h$

000m: $1-2$

PROV: bo ca cc ch da 000m: $0-1,1-2$

$$
\text { pa sa vr }
$$

PROV: cc pa $v r$

PROV: bo en da

PROV: cn

000m: $0-1$

000m: $0-1$

PROV: bo $000 \mathrm{~m}: 0-1$

PROV: $\mathrm{cc}$
PROV: ca cc da he $000 \mathrm{~m}: 0-1$
PROV:

$\begin{array}{lll}\text { PROV: ca cc da he } & 000 \mathrm{~m}: 0-1 \\ \text { PROV: ca cc pa } & 000 \mathrm{~m}: 0-1\end{array}$

PROV: $c a$ cc he lo pa 000m: $0-1$

$\begin{array}{ll}\text { PROV: sa } & 000 \mathrm{~m}: 0-1 \\ \text { PROV: } & 0-1\end{array}$

000m: $0-1$

PROV: pa 000m: $0-1$

PROV: bo ca ce cn da 000m: $0-1$

PROV: ca cc lo pa $000 \mathrm{~m}: 0-1$ PROV: bo $\mathrm{ch}$ cn da 000m: $0-1$ PROV: bo on sa $000 \mathrm{~m}: 0-1$

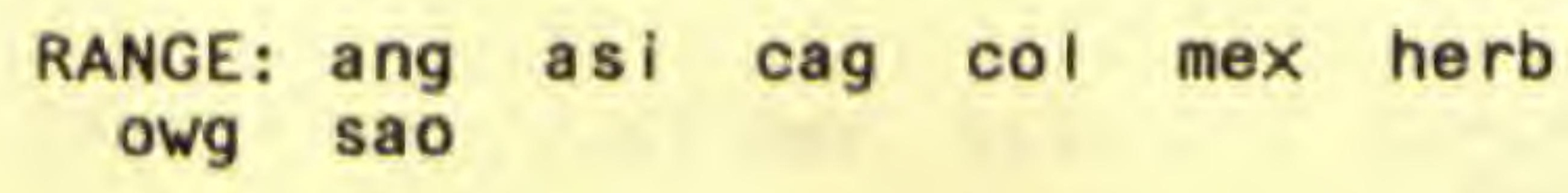

RANGE: col

shrub

RANGE: end

RANGE: cag or hon mex nic shrub

herb

RANGE: gua

shrub

RANGE: oce

RANGE: sao

RANGE:

RANGE: cag

mex sag

tree

herb

herb

herb

RANGE: col cr shrub

RANGE: end

RANGE: $\mathrm{cr}$

RANGE: end

RANGE: end

herb

herb

herb

herb

RANGE: col ven herb

RANGE: ang herb

RANGE: ang cag col herb

RANGE: cag col mex nic sao herb

RANGE: ang col per sao ven herb

RANGE: mex shrub

RANGE: or nic herb

RANGE: or gua herb

RANGE: $c r$ herb

RANGE: end shrub 


\section{TAXON}

\section{FAMILY}

PROVINCES

ELEVATION (OOOM.)

RANGE OUTSIDE OF PANAMA

HABIT

266 (177) ACANTHACEAE cont.

tonduzi i Lindau
*tuberosa L.

tubiflora H.B.K.

--var, tetrasticantha

- -var. tubiflora

Sanchezia

pennel i i Leonard

\section{Staurogyne}

agrest is Leonard

\section{* St reblacanthus}

"monospermus Kuntze

\section{Tel lostachya \\ a lopecuroidea (VahI) Nees}

\section{Tetramerium}

"nervosum Nees

Thunbergia

a lata Boj.

erecta (Benth.) T. Anders.

fragrans Roxb.

grandiflora Roxb.

cult.

cult.

$$
\text { intr. nat. }
$$

PROV: ch cn da

000m: 0-1

PROV: ca pa

000m: $0-1$

(Lindau) Leonard

PROV: bo ce cn da vr 000m: $0-1$

PROV: cn da pa 000m: $0-1,1-2$

PROV: da sa

000m: $0-1$

PROV: ca

000m: $0-1$

PROV: bo

000m: $0=1$

PROV: ca cc pa $v r$ 000m: $0-1$

PROV: ca cc he pa 000m: $0-1$

PROV: ca cc

PROV: ca pa

000m: $0-1$

Trichanthera

gigantea (H. \& B.) Nees
PROV: ca ce cn da lo 000m: 0-1 pa

\begin{tabular}{|c|c|c|c|c|c|c|}
\hline RANGE: & cr & 1.' & t & & & herb \\
\hline RANGE: & ang & cag? & & & & herb \\
\hline RANGE: & $\mathrm{col}$ & $\mathrm{cr}$ & & & & $\begin{array}{l}\text { herb } \\
\text { shrub }\end{array}$ \\
\hline RANGE: & $w r$ & & n & & & herb \\
\hline RANGE: & col & per & & & & herb \\
\hline RANGE: & $\mathrm{cr}$ & & & & & herb \\
\hline RANGE: & $\mathrm{cr}$ & y & $x^{2}+x^{\prime}$ & & & herb \\
\hline $\begin{array}{c}\text { RANGE: } \\
\text { sao }\end{array}$ & $\begin{array}{l}\text { ang } \\
\text { trt }\end{array}$ & $\begin{array}{l}\text { cag } \\
\text { ven }\end{array}$ & $\mathrm{col}$ & gui & per & herb \\
\hline RANGE: & $\mathrm{cag}$ & $\operatorname{mex}$ & sag? & sat & & herb \\
\hline RANGE: & $a f r$ & as $\mathrm{i}$ & cag & oce & sag & climber \\
\hline $\begin{array}{l}\text { RANGE: } \\
\text { RANGE: } \\
\text { RANGE: }\end{array}$ & $\begin{array}{l}\text { afr } \\
\text { as i } \\
\text { as i }\end{array}$ & $\mathrm{cr}$ & jam & D & 10 & $\begin{array}{l}\text { shrub } \\
\text { cl imber } \\
\text { climber }\end{array}$ \\
\hline RANGE: & $\mathrm{col}$ & $\mathrm{cr}$ & gui & ven & & $\begin{array}{l}\text { shrub } \\
\text { tree }\end{array}$ \\
\hline
\end{tabular}

269 (178) PLANTAGINACEAE
Plantago

australis Lam.

I anceolata L.

major $L$. $\begin{array}{ll} & \text { PROV: ch } \\ \text { intr. } & \text { PROV: ch } \\ \text { cult. } & \text { PROV: ca ch he }\end{array}$ 000m: $1-2$

000m: $1-2$

000m: $0-1,1-2,2-3$
RANGE: nWt

RANGE: eur wdW

RANGE: $w d w$ herb

herb herb 
270 (179) RUBIACEAE

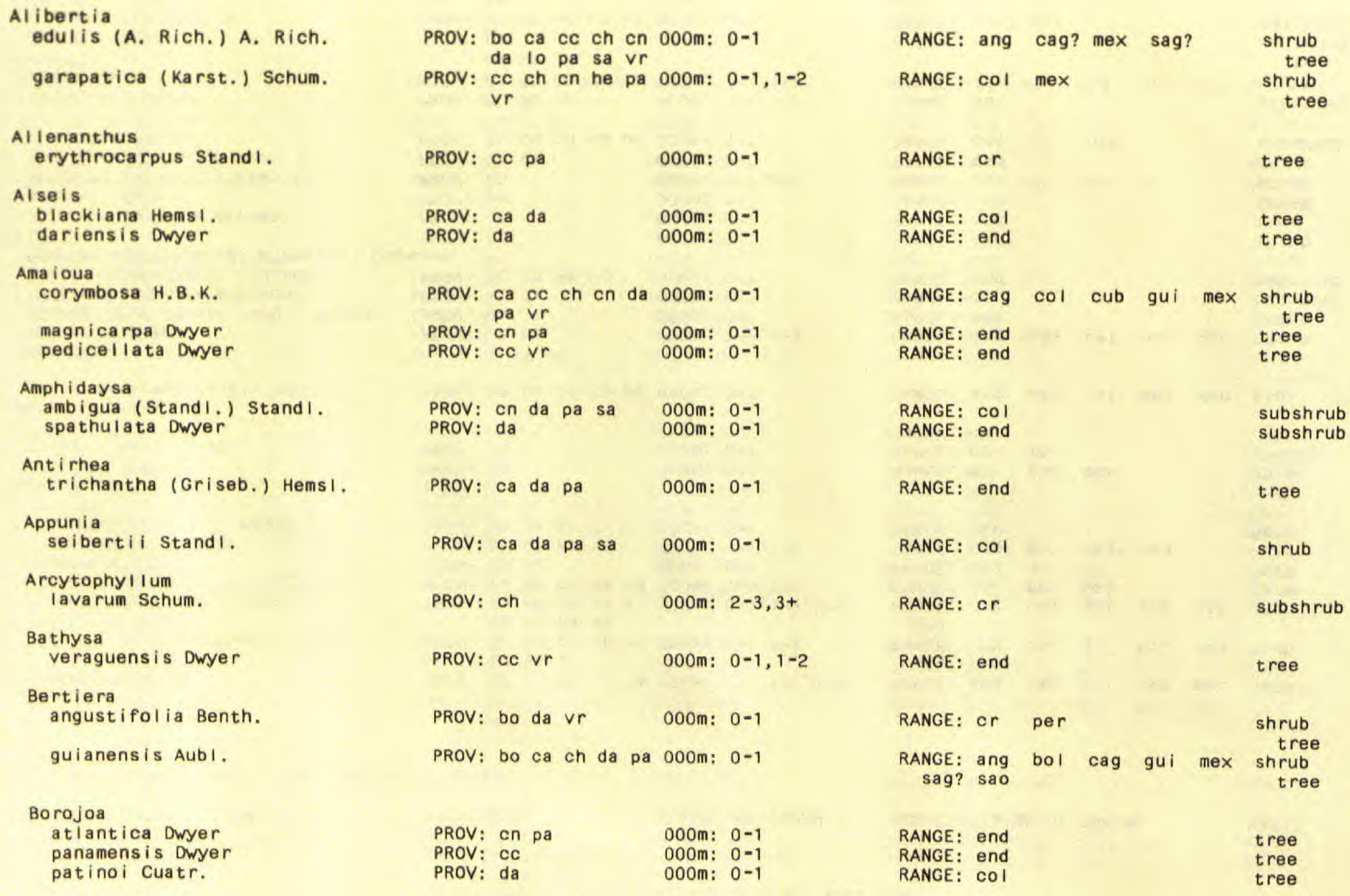


Borreria

densiflora DC.

laevis (Lam.) Griseb.

latifolia (Aubl.) Schum. ocimoides (Burm. P.) DC. pumilio Stand I.

suaveolens G. Meyer

*verticillata (L.) Meyer

\section{Bouva rdia}

glabra Polak.

* Iaevis Mart. \& Gal.

Ca Iycophy I I um candidissimum (VahI) DC.

\section{Cephael is}

axillaris SW.

*barcel lana (Muell. -Arg.) Stand I. bristanil Dwyer \& Hayden

camponutans Dwyer \& Hayden

*caput-anguis (Dwyer \& Hayden) Dwyer

correae Dwyer \& Hayden

croatii Dwyer

dichroa (Standl.) Standl.

dimorphandrioides Dwyer

discolor Polak.

dressleri Dwyer

elata SW.

gaugeri Dwyer

glomerulata J.D. Sm.

insueta Dwyer

ipecacuanha (Brot.) A. Rich.

kennedyi Dwyer

latistipulata Standl.

nana (Stand I.) Standi.

panamens is Dwyer

panchocoensis Dwyer

tomentosa (AubI.) VahI

vultusmimi Dwyer

twilliams ii StandI.
PROV: bo ca ch cn he $000 \mathrm{~m}: 0-1,1-2,2-3$ pa sa $v r$

PROV: bo ca cc ch cn 000m: $0-1,1-2$ da lo pa sa

PROV: ca ch cn pa vr 000m: $0-1,1-2,2-3$

PROV: ca cc ch da pa $000 \mathrm{~m}: 0-1,1-2$

PROV: $\mathrm{ca}$ cc $\quad 000 \mathrm{~m}: 0-1$

PROV: ca ch pa vr 000m: $0-1,1-2$

PROV: pa sa vr $000 \mathrm{~m}: 0-1$

PROV: ch $000 \mathrm{~m}: 0-1$

PROV: ch $000 \mathrm{~m}: 0-1$

PROV: ca cc da lo pa 000m: 0-1

PROV: $\mathrm{ch}$ da $\quad 000 \mathrm{~m}: 1-2,2-3$

PROV: da $000 \mathrm{~m}: 0-1$

PROV: bo da $000 \mathrm{~m}: 0-1$

PROV: bo cn pa vr 000m: $0-1$

PROV: pa 000m: $0-1$

PROV: $\mathrm{CC} \quad 000 \mathrm{~m}: 0-1$

PROV: da 000m: 1-2

PROV: ch 000m: $1-2,2-3$

PROV: ch Vr 000m: $0-1$

PROV: bo ca cn da pa $000 \mathrm{~m}$ : $0-1$$$
\mathrm{vr}
$$

PROV: cn da sa 000m: $0-1,1-2$

PROV: ca ce ch cn da 000m: $0-1,1-2$ pa sa vr

PROV: pa

PROV: bo ca $\mathrm{cc}$ cn pa $000 \mathrm{~m}: 0-1$ sa $v r$

PROV: $v r$

PROV: ca da sa

PROV: pa sa 000m: $0-1,1-2$

PROV: $\mathrm{ch}$

PROV: ca

PROV: cn

PROV: da

PROV: bo ca ce cn da 000m: $0-1,1-2$ lo pa sa vr

PROV: cn

PROV: cc pa 000m: $0=1$

000m: $0-1$

$000 \mathrm{~m}: 0-1$

000m: $0-1$ 000m: $0-1$

\section{RANGE: ang cag col jam mex herb \\ sag? ven \\ RANGE: ang cag gui his sag herb \\ Sao \\ RANGE: ang cag gui sag sao herb \\ RANGE: as $i$ mex wdt herb \\ RANGE: end herb \\ RANGE: cag? gui sag? sao herb \\ RANGE: nic herb \\ RANGE: $\mathrm{cr}$ gua mex shrub \\ RANGE: $\mathrm{cr}$ mex shrub \\ RANGE: ang cag col mex ven tree}

RANGE: ang cag col mex ven shrub

RANGE: sat shrub

RANGE: $\mathrm{cr}$ subshrub

RANGE: end subshrub

RANGE: end shrub

RANGE: end tree

RANGE: end shrub

RANGE: end shrub

RANGE: end

RANGE: $\mathrm{COI}$ cr nic subshrub

RANGE: col subshrub

RANGE: jam shrub

RANGE: end

RANGE: cag? gua subshrub

RANGE: end shrub

RANGE: nwt subshrub

RANGE: end subshrub

RANGE: $\mathrm{cr}$ subshrub

RANGE: nic subshrub

RANGE: end shrub?

RANGE: col subshrub

RANGE: cag? sag? shrub

RANGE: end subshrub

RANGE: per shrub 


\section{Chimarrhis \\ latifolia Standl.}

parviflora Standi.

\section{Chiococca}

a Iba (L.) Hitchc.

durifolia Dwyer

jefensis Dwyer

phaenostemon Schlecht.

\section{Chione}

buxifolia Dwyer \& Hayden

campanensis Dwyer

costaricensis Standi.

darienensis Dwyer

* mexicana Standi.

panamens is Steyerm.

\section{Chomel ia}

atlantica Dwyer

coclensis Dwyer

grandicarpa Dwyer

leucophylla Dwyer

panamensis (StandI, ) Dwyer

peninsularis Dwyer

*polyantha Blake

psilocarpa Dwyer \& Hayden

recordii standl.

--var. paucipubescens Dwyer

--var. recordi

spinosa Jacq.

\section{Cinchona}

pubescens VahI

Coccocypse I um

cordifolium Nees \& Mart.

herbaceum $\mathrm{P}$. Br,

hirsutum DC.

-var. glabrum (DC.) L.O. Wms.

$\begin{array}{ll}\text { PROV: ch } & 000 \mathrm{~m}: 0-1 \\ \text { PROV: bo pa vr } & 000 \mathrm{~m}: 0-1\end{array}$

PROV: bo ca ch cn da 000m: $0-1,1-2$ he lo pa sa

PROV: $c c$ cn pa 000m: $0-1$

PROV: pa

PROV: ch

000m: $0-1$

000m: $0-1,1-2$

$\begin{array}{ll}\text { PROV: pa } & 000 m: 0-1 \\ \text { PROV: pa } & 000 m: 0-1 \\ \text { PROV: cn } & 000 m: 0-1 \\ \text { PROV: da } & 000 m: 0-1 \\ \text { PROV: cc } & 000 m: 0-1 \\ \text { PROV: ch pa sa } & 000 m: 0-1,1-2\end{array}$

PROV: ch pa sa 000m: $0-1,1-2$

PROV: cn da 000m: $0-1$

PROV: cc 000m: $0-1$

PROV: cn 000m: $0-1$

PROV: Cn da

PROV: ch da lo pa sa 000m: $0-1$

PROV: ch $000 \mathrm{~m}: 0-1$

PROV:

000m: $0-1$

PROV: ca da

000m: $0-1$

PROV: ca

000m: $0-1$

PROV: da pa

000m: $0-1$

PROV: ca cc da he lo $000 \mathrm{~m}: 0=1$ pa $v r$

PROV: ch

000m: $1-2$

PROV: ch da 000m: $1-2$

PROV: bo $\mathrm{ca} \mathrm{cc}$ ch he 000m: $0-1,1-2$ pa sa $v r$

PROV: ch cn pa
RANGE: $c r$

RANGE: $\mathrm{cr}$

tree

tree

RANGE: ang bel cag gua sag climber
Sao tex
RANGE: col

RANGE: $\mathrm{cr}$ gua hon mex shrub

tree

RANGE: end

RANGE: end

RANGE: $\mathrm{cr}$

RANGE: end

RANGE: mex nic

RANGE: end

\section{shrub}

tree

tree

tree

tree

tree

shrub

RANGE: end

tree

RANGE: end

RANGE: end

RANGE: end

RANGE: $\mathrm{COI}$

RANGE: end

RANGE: per

RANGE: per

sat ven

RANGE: end

RANGE: $\mathrm{CO} I$

1

cr

gua nic

RANGE: cag col mex sao ven

shrub

shrub

tree

tree

tree

shrub

shrub

shrub

climber

shrub

tree

tree

shrub

RANGE: or sag?

tree

RANGE: cag gua mex sao herb

RANGE: ang cag sag herb

RANGE: bel gua mex nic herb 
TAXON

--var. hirsutum lanceoiatum (R. \& P.) Pers.

PROV: ca ch en pa sa $000 \mathrm{~m}$ : $0-1$

PROV: cc ch da he vr 000m: $0-1,1-2$

\section{Coffea}

a rabica $L$.

I iberica Hiern

Condaminea

corymbosa (R. \& P.) DC.

petiolata Dwyer

\section{Cosmibuena}

ovalis Stand $I$.

skinneri (Oerst.) Hems I.

intr. cult.

PROV: ce ch pa 000m: $0-1$

intr. cult.

PROV: cn $000 \mathrm{~m}: 0-1$

000m: $0-1 \quad$ RANGE: af $r$

000m: $0-1,1-2$

PROV: ch

000m: $0-1$

PROV: pa

000m: $0-1$

PROV: cc ch $000 \mathrm{~m}: 0-1$
PROV: ca cc cn da pa $000 \mathrm{~m}: 0-1$

sa

\section{Coussa rea}

cerroazulensis Dwyer \& Hayden

curvigemmia Dwyer

\section{durifolla Dwyer}

enneantha Stand I.

garciae Stand $I$.

\section{jefensis Dwyer}

latifol ia Standl.

Ioftonil (Dwyer \& Hayden) Dwyer

morii Dwyer

nebulosa Dwyer

neei Dwyer

paniculata (Vahl) Standl.

roseo-cremea Dwyer

veraguens is Dwyer

villosula Dwyer

\section{Coutarea}

hexandra (Jacq.) Schum.

PROV: pa

000m: $0-1$

cn da 000m: $0-1$

PROV: pa

PROV: bo cc da pa $000 \mathrm{~m}: 0-1,1-2$

PROV: $p$ a

PROV: bo cn pa $\quad 000 \mathrm{~m}: 0-1$

PROV: cn pa $000 \mathrm{~m}: 0-1$

PROV: da

PROV: $\mathrm{ch}$

PROV: da $v r$

000m: $0-1$

000m: $1-2$

000m: $0-1$

PROV: da

PROV: $p a$

PROV: $V r$

PROV: cc da

000m: $0-1$

000m: $0-1$

$000 \mathrm{~m}: 0-1$

000m: $0-1$

PROV: ca cc ch cn da 000m: 0-1 he pa
RANGE: bol cag col mex trt herb RANGE: bol cag per sag? sao herb

RANGE: afr

shrub

tree

shrub

tree

RANGE: bol or sag?

shrub

RANGE: end

tree

shrub

RANGE: $c r$

RANGE: $c a g$

col mex nic

tree

tree

RANGE: end

RANGE: $\mathrm{cr}$

RANGE: end

RANGE: end

RANGE: $\mathrm{COI}$

RANGE: end

RANGE: $c 01$

RANGE: end

RANGE: end

RANGE: end

RANGE: end

RANGE: gui

RANGE: end

RANGE: end

RANGE: end

sag? trt

tree

shrub

tree

tree

shrub

shrub

tree

tree

tree

shrub

tree

tree

shrub

tree

shrub

tree

shrub

tree

tree

RANGE: ang cag col mex per tree sag? sao

\section{Crusea}

coccinea DC.

--var. chiriquiensis W.R. Anderson 
TAXON

FAMILY

PROV I NCES

270 (179) RUBIACEAE cont.

longiflora (R. \& S.) W.R. Anderson

PROV: ch

000m: $1-2$

PROV: cc ch pa vr

PROV: $c h$

PROV: pa

grandiflora Schlecht.

panamens is Dwyer

Didymaea

alsinoides (Schlecht. \& Cham,) Standl.

PROV: ch

Didymoch I amys

connellii N.E. Br.

Diodia

denudata StandI.

sarmentosa SW.

teres WaIt.

Elaeagia

auriculata HemsI.

nitidifolia Dwyer

Exostema

mexicanum A. Gray

\section{Fa ramea}

altipetens Dwyer

bocataurens is Dwyer

bullata Standl.

caput-anguis Dwyer \& Hayden

eurycarpa J.D. Sm.

jefens is Dwyer \& Hayden

I iesneri Dwyer

I uteovirens Standl.

000m: $2-3$

000m: $0-1$

000m: $0-1$

PROV: cc sa

000m: $0-1$

000m: $0-1$

PROV: ca cn $v r$

PROV: $\mathrm{cc}$ ch he $\mathrm{vr}$

PROV: da

000m: $0-1$

PROV: da pa sa

PROV: ca pa

000m: $0-1$

PROV: da

PROV: bo

000m: $0-1$

PROV: pa

000m: $0-1$

PROV: cc cn da pa 000m: $0-1$

PROV: pa $000 \mathrm{~m}: 0-1$

PROV: pa

000m: $0-1$

PROV: ca ch cn da sa 000m: $0-1$
ELEVATION (OOOM.) RANGE OUTSIDE OF PANAMA

HABIT

RANGE: cag mex

herb

subshrub

000m: $0-1,1-2$

000m: $1-2,2-3$

000m: $0-1,1-2$

000m: $0-1,1-2$
RANGE: cag? mex sag? sao ven herb

RANGE: cag $\mathrm{cr}$ mex tree

RANGE: end

RANGE: $\mathrm{cr}$ herb

RANGE: gui ven herb

RANGE: col climber subshrub shrub

RANGE: afr ang asi cag jam herb

oce sag? shrub

RANGE: cag fla nag nao nao herb

sag tex

RANGE: $\mathrm{cr}$ hon

shrub

RANGE: end

ree

RANGE: bel or gua hon mex tree sal

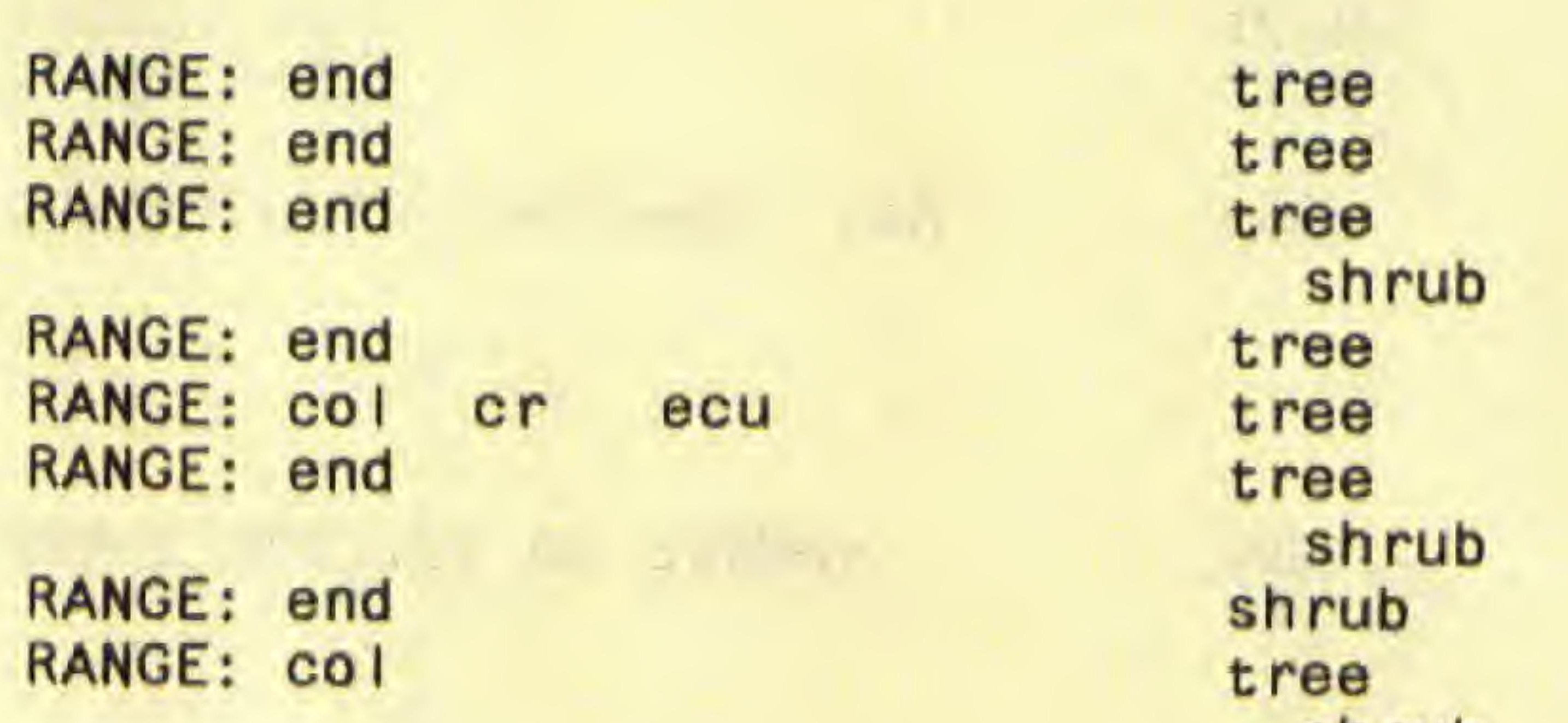


TAXON

FAMILY

PROVINCES

ELEVATION (OOOM.)

RANGE OUTSIDE OF PANAMA

HAB IT

270 (179) RUBIACEAE cont.

occidentalis (L,) A. Rich.

oval is Standl.

papulata Dwyer \& Hayden

pauciflora Dwyer

scalaris Standl.

suerrensis (J.D. Sm, ) J.D. Sm.

ta I amanca rum Stand I.

terreyeae Stand I.

woodsoni i Standi.

\section{Ferdinandusa}

panamens is StandI. \& Wms.

\section{Ga I i um}

aschenbornif Schauer

mexicanum H.B.K.

uncinulatum DC.

\section{Genipa}

americana $L$.

curviflora Dwyer

Williamsii StandI.

\section{Geophila}

croatii Steyerm.

macropoda (R. \& P.) DC

repens (L.) Johnst.

\section{Gonzalagunia}

bracteosa (J.D. Sm.) Rob.

ka Il unki i Dwyer

ovatifolia (J.D. Sm. ) Rob.

panamens is (Cav.) Schum.

rosea Standl.

rud is (Standi.) StandI.

veraguens is Dwyer

\section{Guet ta rda}

chiriquiensis Standl.

foliacea StandI.

macrosperma J.D. Sm.

odorata (Jacq.) Lam.
PROV: ca cc cn da lo $000 \mathrm{~m}: 0-1$ pa $v r$

PROV: ch 000m: $1-2$

PROV: pa $\quad 000 \mathrm{~m}: 0-1$

PROV: vr $\quad 000 \mathrm{~m}: 0-1$

PROV: ch 000m: 1-2

PROV: bo cc ch en vr 000m: $0-1,1-2$

PROV: bo cn da 000m: $0-1$

PROV: da 000m: $0-1$

PROV: cc pa sa $000 \mathrm{~m}: 0-1$

PROV: bo

000m: $0-1$

PROV: ch

PROV: ch

000m: $1-2,2-3,3+$

PROV: $\mathrm{ch}$

000m: $1-2,2-3$

PROV: bo ca ce ch da 000m: $0-1$ he lo pa vr

PROV: da pa sa $000 \mathrm{~m}: 0-1$

PROV: cc da pa $000 \mathrm{~m}: 0-1$

PROV: ca 000m: $0-1$

PROV: bo da pa sa $000 \mathrm{~m}: 0-1$

PROV: bo ca ch da lo $000 \mathrm{~m}: 0-1$ pa

PROV: cn da pa sa 000m: $0-1$ PROV: $v r \quad 000 \mathrm{~m}: 0-1$ PROV: bo cc sa $000 \mathrm{~m}: 0-1$

PROV: bo ca $\mathrm{cc}$ ch $\mathrm{cn} 000 \mathrm{~m}$ : $0-1$ he pa sa $v r$

PROV: bo ch da he vr 000m: $0-1,1-2$

PROV: ca ch cn da $10000 \mathrm{~m}: 0-1$ pa sa

PROV: vr

000m: $0-1$

PROV: cc ch pa $\quad 000 m: 0-1,1-2$

PROV: ca da pa vr 000m: $0-1$

PROV: ca cn da sa $000 \mathrm{~m}: 0-1$

PROV: $\mathrm{ca}$ cc ch da he $000 \mathrm{~m}$ : $0-1$ pa $\vee r$

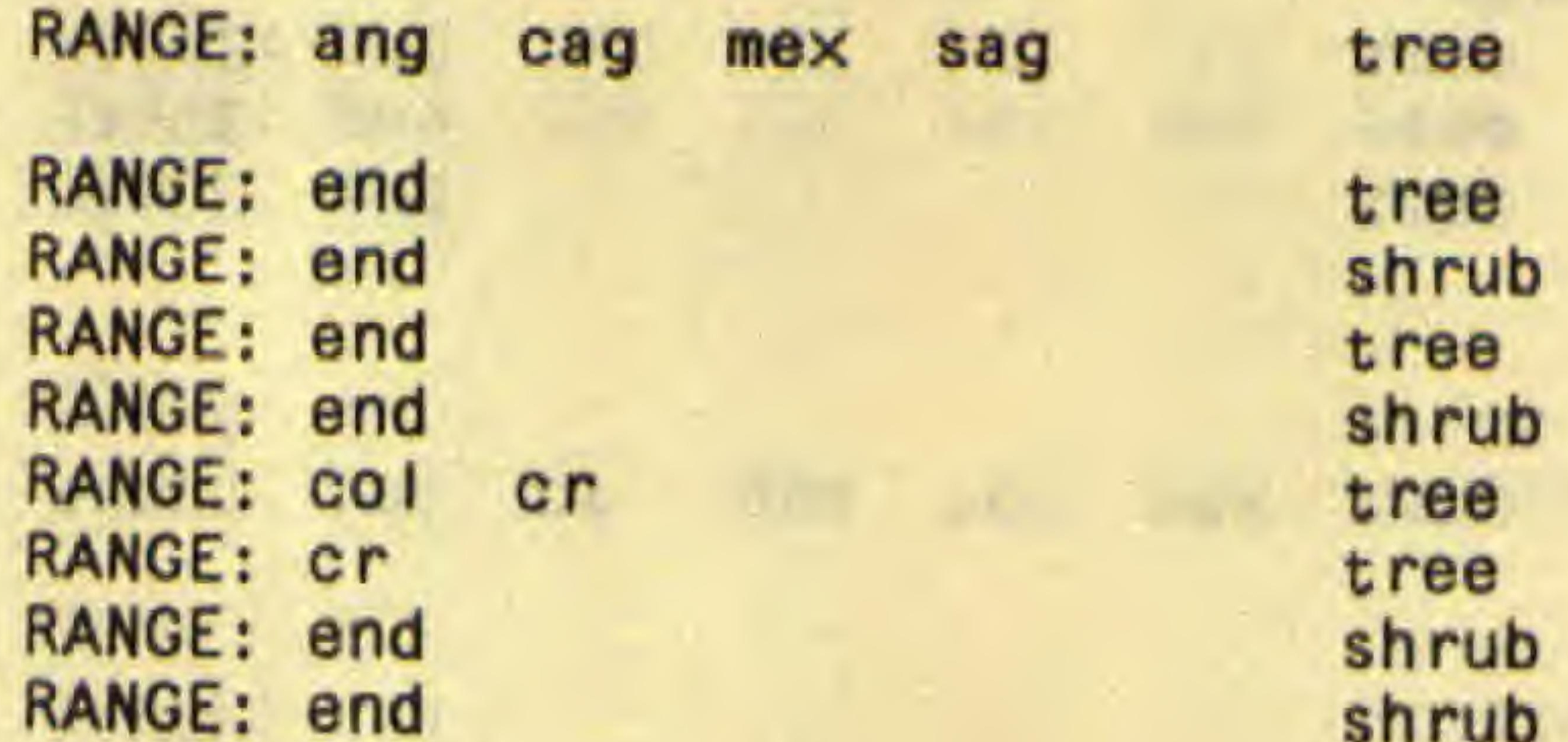

RANGE: end

tree

RANGE: cag mex climber

RANGE: cag mex climber

RANGE: cag mex nao tex herb

RANGE: ang cag col fla mex tree

sag sao ven

RANGE: end

RANGE: $\mathrm{COI}$

shrub

tree

tree

RANGE: nic

RANGE: col

RANGE: ang

mex per

$c r$

herb

herb

cag col gui jam herb

RANGE:

RANGE: end

RANGE: COI

RANGE: ang cag col cub mex

shrub

shrub

shrub

RANGE: $c r$

RANGE: $c O$ I

RANGE: end

tree

shrub

shrub

RANGE: COI

RANGE: COI

RANGE: cag gua

RANGE: ang? cub

sag?

tree

tree

tree

shrub 
270 (179) RUBIACEAE cont.

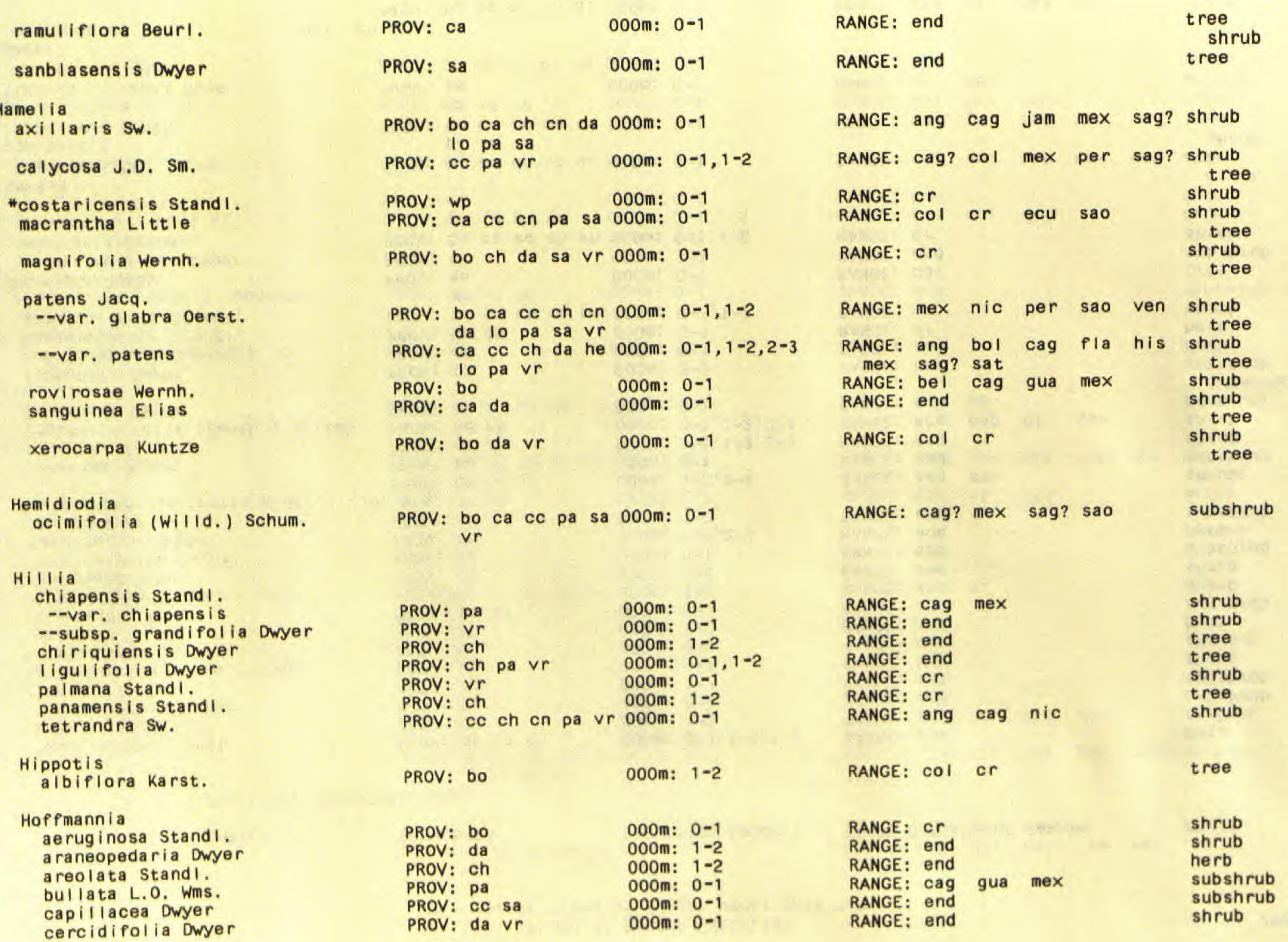


TAXON FAMILY PROVINCES

270 (179) RUBIACEAE cont.

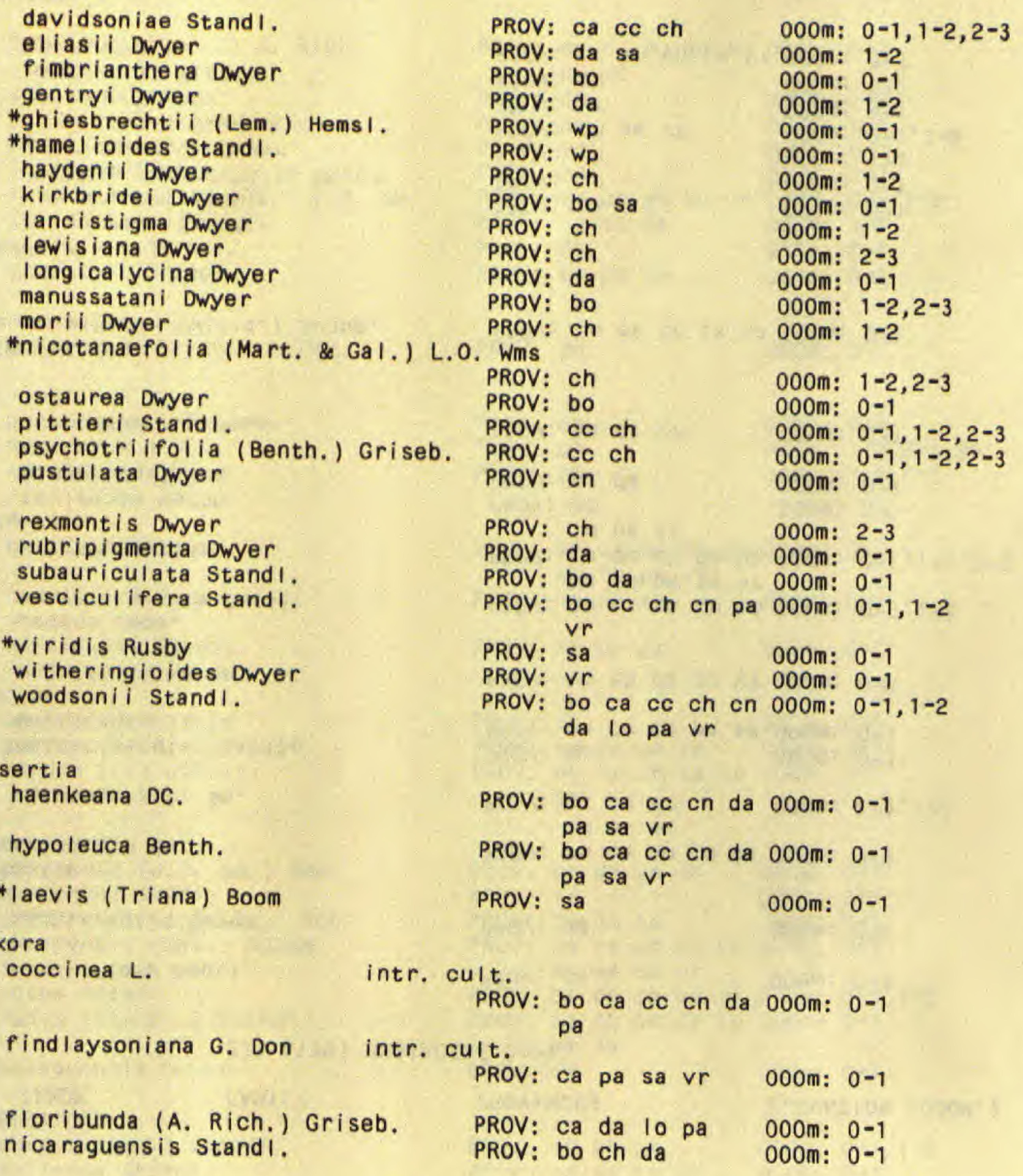

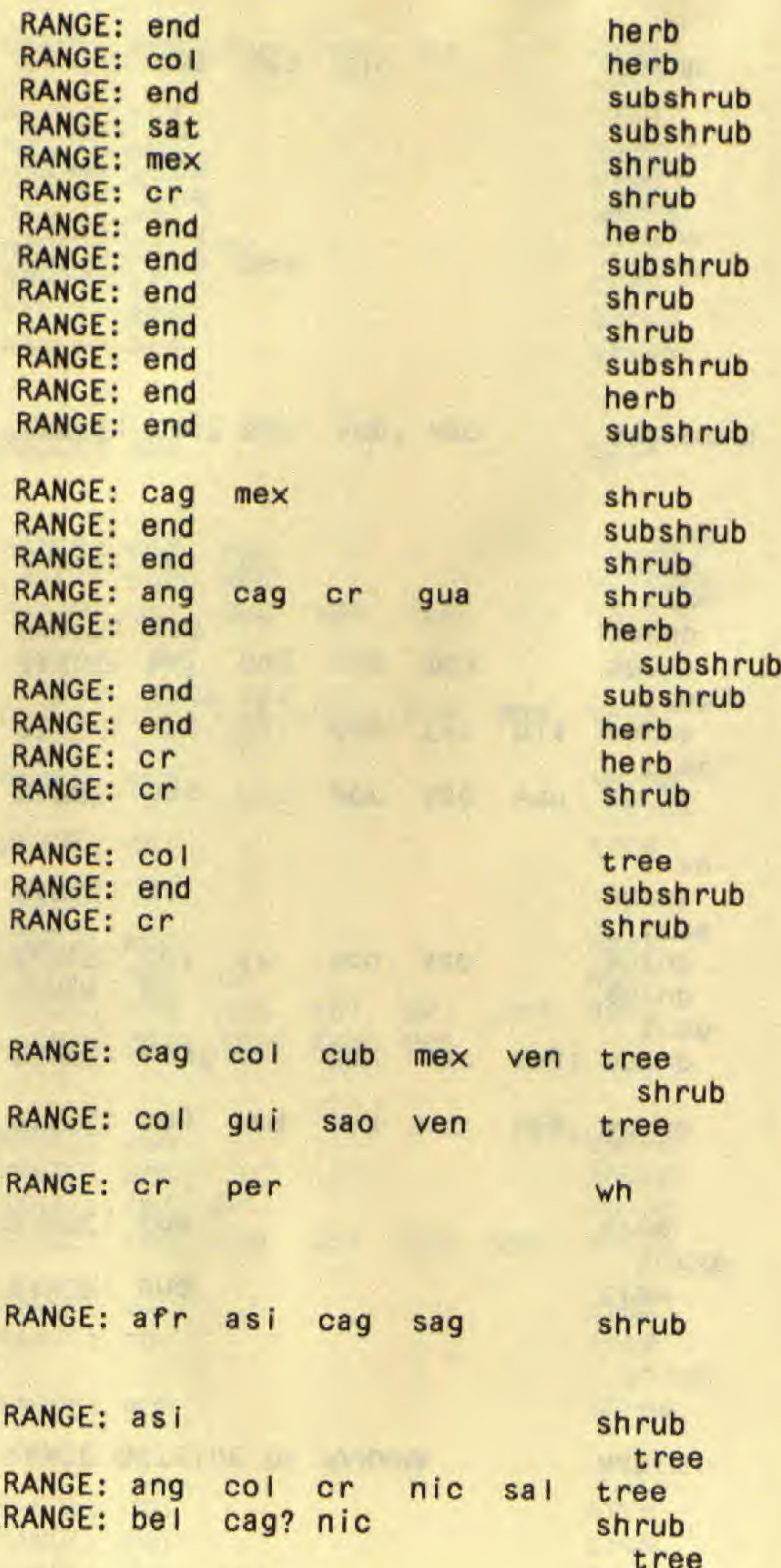


TAXON

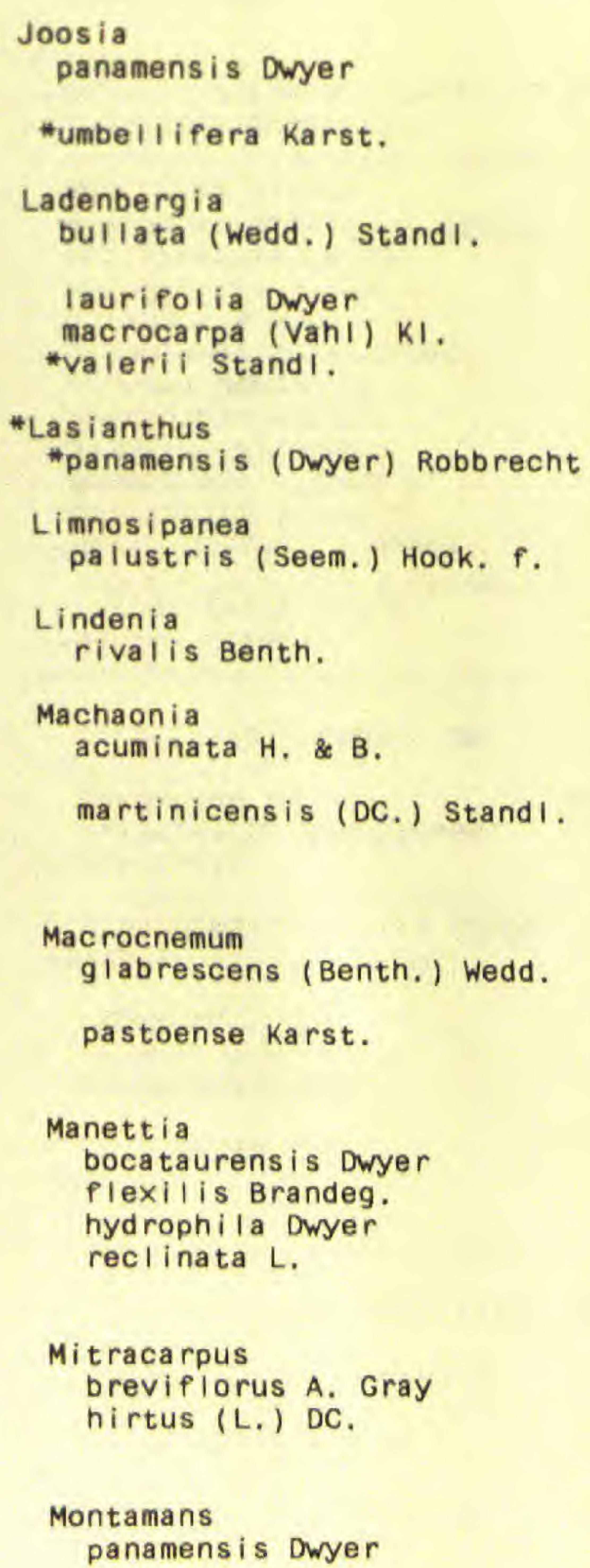

PROV: bo ce cn pa sa $000 \mathrm{~m}$ : $0-1$

Pr

000m: $1-2$

PROV: pa

000m: $0-1$

PROV: $v r$

PROV: da

PROV: pa

000m: $0-1$

000m: $0-1,1-2$

000m: $0-1,1-2$

PROV: cc cn pa

000m: $0-1$

PROV: WP

000m: we

PROV: ca da he pa 000m: $0-1$

PROV: ca cc cn da he 000m: $0-1$ pa sa vr

000m: $0-1$

PROV: bo ca $\mathrm{cc}$ ch $\mathrm{cn} 000 \mathrm{~m}$ : $0-1$ PRov. da pa vr

000m: $0-1$

$\begin{array}{ll}\text { PROV: bo } & 000 \mathrm{~m}: 0-1 \\ \text { PROV: ch } & 000 \mathrm{~m}: 1-2 \\ \text { PROV: } v r & 000 \mathrm{~m}: 0-1 \\ \text { PROV: bo ca ce ch da } 000 \mathrm{~m}: 0-1\end{array}$

PROV: bo ca cc ch da 000m: $0-1$ lo pa sa vr

PROV: ch

000m: $1-2$

PROV: bo ca ce ch da $000 \mathrm{~m}: 0-1$ pa $\vee r$

PROV: ch

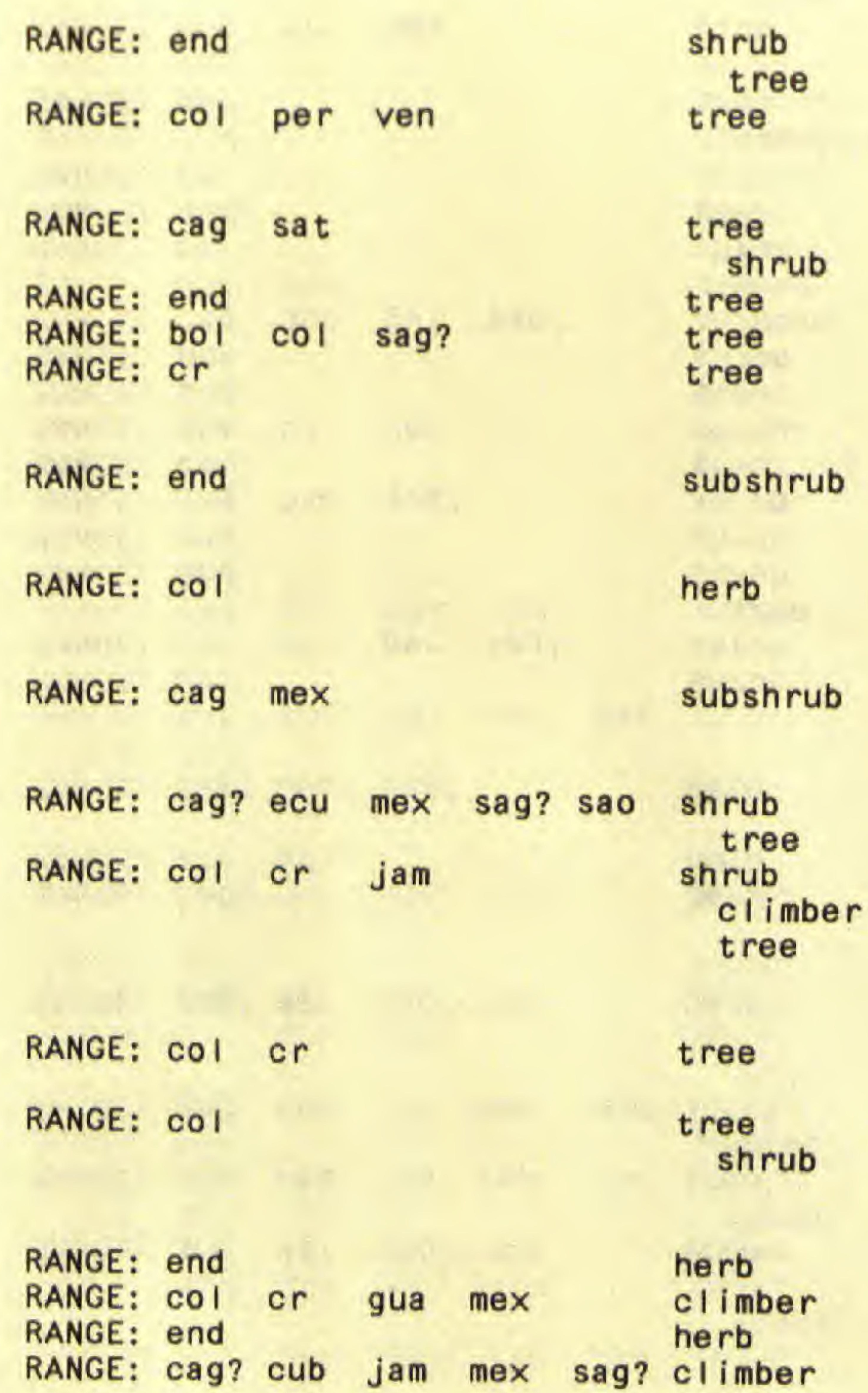

RANGE: $\mathrm{cr}$ mex nao herb

RANGE: ang asi cag jam mex herb 
TAXON

\section{Morinda}

citrifolia L.

panamensis Seem.

rojoc L.

\section{Nertera}

granadens is (L. P.) Druce

Oldenlandia

callitrichoides Griseb. corymbosa $\mathrm{L}$.

Iancifolia (Schum,) DC.

Palicourea

*adusta Standl.

angustifolia H, B. K.

bella (Stand I.) Dryer

chiriquina Stand I.

crocea (SW.) R. \& S.

*discolor Krause

fastigiata Benth.

gibbosa Dwyer

grandibracteata Dwyer

guianensis Aubl.

lasiorrachis 0erst.

montensis Dwyer

montivaga StandI.

ochnoides Dwyer

*padifolia (Roem.) Taylor \& Lorence

PROV: ch da $v r \quad 000 m: 0-1,1-2$

panamens is Stand I.

pauciflora Stand I.

rigidifolia (Dwyer \& Hayden) Dwyer

triphyl la (Mueil, -Arg.) DC.

tubuliflora Dwyer

tumidonodosa Dwyer

veraguens is Dwyer
PROV: bo ch da vr 000m: $0-1,1-2$ PROV: $\mathrm{ch} \quad 000 \mathrm{~m}: 2-3$

PROV: pa $v r$

$000 \mathrm{~m}: 0-1$

PROV: ch da 000m: 1-2

PROV: bo ca ce ch da 000m: $0-1$ he pa vr

PROV: bo ca cn sa 000m: $0-1$

PROV: ca cc en da pa 000m: 0-1

PROV: cn sa

000m: $0-1$

000m: $1-2,2-3$

000m: $0-1$

000m: $0-1$

000m: $0-1$

000m: 2-3

000m: $1-2,2-3$

Om: $0-1$

$000 \mathrm{~m}: 0-1$

000m: $1-2$

000m: $0-1$

PROV: bo ca cn da lo $000 \mathrm{~m}$ : $0-1$

PROV: pa sa vr

PROV: ch $\quad 000 \mathrm{~m}: 1-2$

000m: $0-1$

000m: $1-2$

$000 m: 0-1,1-2$

PROV: pa

PROV: da pa

000m: $0-1$

000m: $0-1,1-2$

000m: $1-2$ 000m: $1-2$

$\begin{array}{lllll}\text { RANGE: ang asi cag? oce } & \begin{array}{c}\text { tree } \\ \text { shrub } \\ \text { tree } \\ \text { shrub }\end{array} \\ \text { RANGE: ang cag fla mex } & \begin{array}{c}\text { shrub } \\ \text { RANGE: ang cag fla mex sag? shrub }\end{array}\end{array}$

RANGE: cag? mex sag? sao herb

RANGE: cub herb

RANGE: afr wdt herb

RANGE: cag? owg? sag? herb

RANGE: $\mathrm{cr}$ shrub

RANGE: col or per sag? shrub

RANGE; end tree

RANGE: end

RANGE: ang nic sag? shrub

RANGE: $\mathrm{cr}$ tree

RANGE: col or ven shrub

RANGE: end tree

RANGE: end shrub

RANGE: cag gua gui sag? shrub

RANGE: $c r$ shrub

RANGE: end tree

RANGE: $\mathrm{cr}$ shrub

tree

RANGE : cag $\mathrm{cr}$ mex tree

RANGE: end

RANGE: end $\quad$ shrub

RANGE: end shrub

RANGE: $\mathrm{cr}$

RANGE: cag? gui sag? tree

RANGE: end

RANGE: end

RANGE: end tree

tree 
270 (179) RUBIACEAE cont.

\section{vestita StandI.}

\section{Pentagonia \\ a I ba Dwyer \\ bocataurensis Dwyer \\ brachyot is (Stand $I$. ) Stand $I$.}

gymnopoda (StandI.) StandI.

macrophylla Benth.

nuciformis Dwyer

pinnatifida Seem.

tinajita Seem.

veraguens is Dwyer

wend $i$ and $i \mathrm{i}$ Hook.

Pogonopus

speciosus (Jacq.) Schum.

Posoqueria

latifolia (Rudge) R. \& S.

\section{Psychotria}

acuminata Benth.

- subsp. acuminata

--subsp. boraginoides Dwyer

aggregata Stand I.

a Iboviridula Krause

$$
\begin{aligned}
& \text { a I lenii Standl. } \\
& \text { bakeri Dwyer } \\
& \text { berteriana DC. }
\end{aligned}
$$

\section{boquetens is Dwyer}

brachiata SW.

brachybotrya Mue II, -Arg.

calophylla Standl.

capacifolia Dwyer

capitata R. \& $P$.

--subsp. capitata

--subsp. fissistipularis Dwyer
PROV: $\mathrm{CC}$ vr

000m: $0-1$

PROV: da

PROV: bo

PROV: cn da pa sa

000m: $0-1$

000m: $0-1$

000m: $0-1,1-2$

PROV: cn da pa

000m: $0-1$

PROV: bo ca cc cn da $000 \mathrm{~m}$ : $0-1$ pa sa

PROV: vr $000 \mathrm{~m}: 0-1$

PROV: da pa sa $\quad 000 \mathrm{~m}: 0-1$

PROV: ch $000 \mathrm{~m}: 0-1$

PROV: Vr

PROV: bo cn pa sa 000m: $0-1$

PROV: ca cn da pa $000 \mathrm{~m}$ : $0-1$

PROV: bo ca cc ch cn $000 \mathrm{~m}$ : $0-1,1-2$ da lo pa sa vr

PROV: ca $\mathrm{cc} \mathrm{cn}$ da he $000 \mathrm{~m}$ : $0-1,1-2$ lo pa sa vr

PROV: pa

PROV: bo cc ch pa 000m: $0-1,1-2,2-3$

PROV: da pa sa

000m: $0-1$

PROV: cc pa sa vr 000m: $0-1$

PROV: ch lo pa 000m: $0-1$

PROV: bo da vr $000 \mathrm{~m}: 0=1$

PROV: ch 000m: $1-2$

PROV: bo ca cc cn da 000m: $0-1$ lo pa sa vr

PROV: bo ca cc cn da 000m: $0-$ he pa

PROV: ca cn pa $000 \mathrm{~m}: 0-1$

PROV: bo da

000m: $0-1$

PROV: ca cc cn da pa 000m: 0-1$$
\text { sa } \mathrm{vr}
$$

PROV: ca cn

000m: 0-1
RANGE: $c r$

shrub

RANGE: end

RANGE: end

RANGE: end

RANGE: end

RANGE: $\mathrm{col}$ ecu

RANGE: end

RANGE: COI

RANGE: end

RANGE: end

RANGE: $\mathrm{cr}$

shrub

shrub?

shrub

tree

shrub

tree

tree

shrub

tree

shrub

shrub

subshrub

shrub

RANGE: cag col mex ven tree

RANGE: cag col gui mex per tree sao ven

RANGE: cag? mex per sag? shrub

RANGE: COI shrub

RANGE: end shrub

RANGE: col cr per shrub

RANGE: end tree

RANGE: end shrub

RANGE: ang cag or his hon shrub

mex $p r$ sag tree

RANGE: end shrub

RANGE: ang cag sag trt tree

RANGE: bol per sag? sao trt shrub

RANGE: $c r$

tree

RANGE: end

shrub

RANGE: cag sag trt

subshrub

RANGE: end shrub

tree

shrub 
TAXON

--subsp, rubra Dwyer

carnosocarpa Dwyer \& Hayden carthaginens is Jacq.

catillicalyx Dwyer chagrens is Stand I.

chiapens is Standl. chiriquina Stand $\mathrm{I}$.

chloritica Muell. -Arg.

cincta Standl.

cooperi Standl.

copens is Dwyer

copeyana StandI. \& L.O. Wms. croceovenosa Dwyer

davidsei Dwyer

deflexa DC.

dosbocensis Dwyer

dukei Dwyer

\section{durilancifolia Dwyer}

emetica L. $f$.

*epiphytica StandI.

\section{eugenifolia Dwyer}

"eurycarpa Standl.

fendieri Standi.

fruticetorum StandI.

furcata DC.

goldmani i Standi.

graciliflora Benth.

granadensis Benth.

grandis Sw.

\section{haematocarpa StandI.}

hammel ii Dwyer

horizontalis SW.

impatiens Dwyer
PROV: Wp

000m: $0-1$

PROV: bo

$000 \mathrm{~m}: 0-1$

PROV: bo ca cc ch da 000m: $0-1,1-2$ he $10 \mathrm{pa} v \mathrm{r}$

PROV: $v r$

000m: $0-1$

PROV: bo ca ce cn da 000m: $0-1$

pa sa

PROV: bo ch sa 000m: $0=1$

PROV: $\mathrm{ch}$ 000m: $0-1,1-2,2-3$

PROV: da

000m: $0-1$

PROV: bo cn pa sa vr 000m: $0-1$

PROV: cc vr

000m: $0-1$

PROV: cc pa

PROV: ch Vr

PROV: ch

000m: $0-1$

000m: $1-2$

000m: $1-2$

000m: $1-2$

PROV: bo ca cc da he $000 \mathrm{~m}: 0-1$ pa sa $v r$

PROV: $V r$

PROV: da pa vr $\quad 000 \mathrm{~m}: 0-1$

PROV: pa 000m: $0-1$

PROV: bo ca cn da pa 000m: $0-1$

PROV: da

$000 \mathrm{~m}: 0-1$

V

PROV: $\mathrm{ch}$ da

PROV: bo

PROV: ca cn

PROV: pa

000m: $1-2$

000m: $0-1$

000m: $0-1$

PROV: bo ca ce ch on 000m: $0-1$

da he lo pa sa

PROV: ch da pa

000m: $0-1,1-2,2-3$

PROV: ca ce ch da pa 000m: $0-1$

PROV: ca $\mathrm{vr}$

$000 \mathrm{~m}$ : $0-1$

PROV: bo ca ce ch cn 000m: $0-1$

$$
\text { da pa sa vr }
$$

PROV: cc cn da sa vr 000m: 0-1

PROV: bo $\mathrm{Vr}$ 000m: $1-2$

PROV: ca cc ch cn da 000m: $0-1,1-2$ he lo pa sa vr

PROV: da

\begin{tabular}{|c|c|c|c|c|c|c|}
\hline ANGE: & end & whr & tent & & & $\begin{array}{c}\text { shrub } \\
\text { tree }\end{array}$ \\
\hline RANGE: & end & t & & & & shrub \\
\hline $\begin{array}{c}\text { RANGE: } \\
\text { trt }\end{array}$ & cag & $\mathrm{col}$ & gui & per & sag & shrub \\
\hline RANGE: & end & & & & & tree \\
\hline RANGE: & cag & mex & & & & subshrub \\
\hline ANGE: & cag? & $\mathrm{cr}$ & mex & per & sag? & tree \\
\hline ANGE: & end & & & & & $\begin{array}{c}\text { shrub } \\
\text { tree }\end{array}$ \\
\hline RANGE: & $\mathrm{col}$ & per & sao & +1 & & $\begin{array}{c}\text { shrub } \\
\text { tree }\end{array}$ \\
\hline RANGE: & $\mathrm{col}$ & & & & & $\begin{array}{c}\text { shrub } \\
\text { tree }\end{array}$ \\
\hline RANGE: & $\mathrm{col}$ & 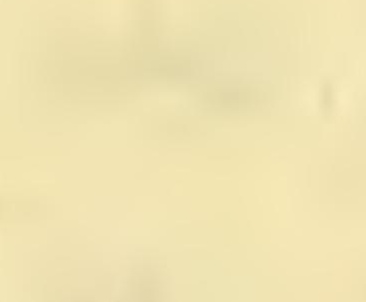 & 't & & & $\begin{array}{l}\text { tree } \\
\text { shrub }\end{array}$ \\
\hline ANGE: & end & 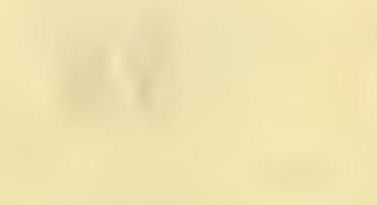 & 't & -34 & its & shrub \\
\hline 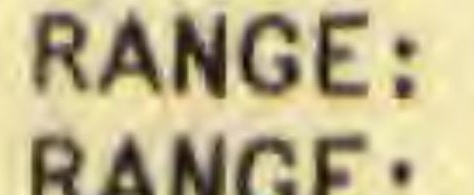 & $\begin{array}{l}\text { cr } \\
\text { enc }\end{array}$ & 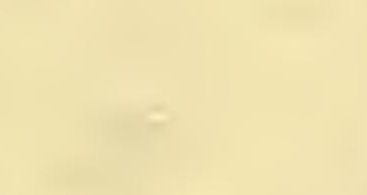 & & & & tree \\
\hline : & $\begin{array}{l}\text { end } \\
\text { end }\end{array}$ & in: & . & & & tree \\
\hline RANGE: & cag? & gui & sag? & & & $\begin{array}{l}\text { subshrub } \\
\text { shrub }\end{array}$ \\
\hline N & d & & & & & shrub \\
\hline & c & & & & & $\begin{array}{c}\text { subshrub } \\
\text { shrub }\end{array}$ \\
\hline NGE: & end & & & & & tree \\
\hline $\begin{array}{r}\text { ANC } \\
\text { sa }\end{array}$ & bol & cag? & col? & gua & per & subshrub \\
\hline IAN & ecu & per & & & & ep iphyte \\
\hline ANC & bel & $\mathrm{cr}$ & gui & sao & ven & shrub \\
\hline : & e & & & & & subshrub \\
\hline : & $\mathrm{cr}$ & & & & & tree \\
\hline : & end & & & & & subshrub \\
\hline NC & cag & hon & $\operatorname{mex}$ & & & shrub \\
\hline RANG & $\mathrm{col}$ & $\mathrm{cr}$ & & & & hrub \\
\hline ANGE & c & & & & & shrub \\
\hline : & g? & cr & hon & & & shrub \\
\hline RAN & cag & gua & nic & & & subshrub \\
\hline RANC & cag? & cr & jam & $\operatorname{mex}$ & sag? & $\begin{array}{l}\text { tree } \\
\text { shrub }\end{array}$ \\
\hline RANGE: & & $c r$ & & & & shrub \\
\hline ANGE: & er & & & & & subshrub \\
\hline ANG & ang & cag? & ecu & his & $\operatorname{mex}$ & shrub \\
\hline SANG & en & & & & & \\
\hline
\end{tabular}


270 (179) RUBIACEAE cont.

\section{indigotica Dwyer \\ insignis Standl. \\ liesneri Dwyer \\ * I imitanea Standi. \\ I imonens is Krause \\ longipedunculata Dwyer I uxurians Rusby}

macrophyIIa R. \& P.

magna Stand I.

marginata SW.

maxonil Standi.

micrantha H.B.K.

*microbotrys StandI.

microdon (DC.) Urb.

\section{mima Standi.}

moril Dwyer

mortoniana standi.

nervosa SW.

nubiphila Dwyer

oerestediana StandI.

officinalis Sandw.

\section{olgae Dwyer \& Hayden}

orchidea rum Stand 1 .

- -subsp. orchidea rum

- -subsp. persedens Dryer

\section{oresbia Dwyer}

ostaurea Dryer Hayden

panamens is Standl.

parvifolia 0erst.

perparva Dwyer

philacra Dwyer

pilosa R. \& P.

pithecobia stand I.

pittieri Standi.

- -subsp. oinochrophyila Dryer

--subsp. pittieri

$\begin{array}{ll}\text { PROV: pa } & 000 \mathrm{~m}: 0-1 \\ \text { PROV: sa } & 000 \mathrm{~m}: 0-1 \\ \text { PROV: pa } & 000 \mathrm{~m}: 0-1 \\ \text { PROV: da } & 000 \mathrm{~m}: 1-2\end{array}$

PROV: da $000 m: 1-2$

PROV: bo ca cn da sa $000 \mathrm{~m}$ : $0-1$

PROV: $v r \quad$ 000m: $0-1$

PROV: cc ch cn da pa 000m: $0-1,1-2$ sa $\mathrm{vr}$

PROV: bo $\mathrm{cc} c \mathrm{ch}$ cn da $000 \mathrm{~m}$ : $0-1,1-2$ pa $\vee r$

PROV: cn

000m: $0-1$

PROV: bo ca cc en da 000m: $0-1,1-2$ lo pa sa vr

PROV: bo Vr

000m: $0-1,1-2$

PROV: bo ca cc en da 000m: $0-1$

pa sa

PROV: $\mathrm{CC}$

000m: $0-1$

PROV: ca cc ch da lo 000m: 0-1

PROV: bo cn da pa vr 000m: $0-1,1-2$

PROV: Vr 000m: $0-1$

PROV: ch lo 000m: $0-1,1-2$

PROV: ca cc cn da pa 000m: $0-1$

PROV: ch $\mathrm{ch}$ 000m: 1 -2

PROV: bo ca cc sa 000m: $0-1$

PROV: bo ca $c c$ da pa $000 \mathrm{~m}$ : $0-1,1-2$

PROV $\mathrm{sa}$

000a: $0-1,1-2$

PROV: ca da pa sa 000m: $0-1,1-2$

PROV: $c$ c ch da pa sa 000m: $0-1,1-2$

vr

PROV: da

PROV: bo $\mathrm{CC}$ O00n: $1-2$

PROV: $\mathrm{Ca}$ cc ch cn da 000n: $0-1$

pa $V r$

PROV: ch

PROV: da pa

PROV: da

(2) $1-2$

000 : $: 0-1,1-2$

PROV: bo ce ch da pa 000m: $0-1$

sa $\mathrm{vr}$

000m: $1-2$

PROV: da

$000 \mathrm{~m}: 0-1,1-2$

PROV: bo ca ce ch cn 000m: $0-1$ da lo pa sa

\begin{tabular}{|c|c|c|c|c|c|c|}
\hline RANGE: & end & & & & & tree \\
\hline RANGE: & end & & & & & shrub \\
\hline RANGE: & end & & & & & shrub \\
\hline RANGE: & $\mathrm{col}$ & $y^{\prime}$ & & 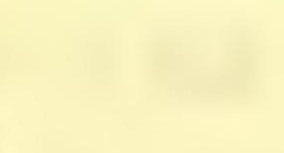 & & shrub \\
\hline RANGE: & cag & cr & sag? & & & $\begin{array}{c}\text { subshrub } \\
\text { shrub }\end{array}$ \\
\hline RANGE: & end & & & & & shrub \\
\hline RANGE: & bol & sag? & & & & $\begin{array}{l}\text { tree } \\
\text { shrub }\end{array}$ \\
\hline $\begin{array}{c}\text { RANGE: } \\
\text { per }\end{array}$ & $\begin{array}{l}\text { bol } \\
\text { sag? }\end{array}$ & cag? & col & gua & $\operatorname{mex}$ & shrub \\
\hline RANGE: & $\mathrm{cr}$ & & & & & shrub \\
\hline RANGE: & jam & & & & & shrub \\
\hline RANGE: & $\mathrm{cr}$ & & & & & subshrub \\
\hline RANGE: & col & ecu & nic & per & ven & $\begin{array}{c}\text { shrub } \\
\text { tree }\end{array}$ \\
\hline RANGE: & per & ven & & & & herb \\
\hline RANGE: & ang & cag? & cub & ecu & gui & shrub \\
\hline $\operatorname{mex}$ & sag? & & & & & tree \\
\hline RANGE: & col & sao & & & & tree \\
\hline RANGE: & end & & & & & shrub \\
\hline RANGE: & er & & & & & shrub \\
\hline RANGE: & ang & $\begin{array}{l}\text { bah } \\
\text { ven }\end{array}$ & cag & col & fla & snrub \\
\hline RANGE: & $\begin{array}{l}\text { pr } \\
\text { end }\end{array}$ & ven & & & & tree \\
\hline RANGE: & cag & or & $\operatorname{mex}$ & & & shrub \\
\hline RANGE: & cag & col & gui & & & shrub \\
\hline RANGE: & end & & & & & $\begin{array}{l}\text { tree } \\
\text { shrub }\end{array}$ \\
\hline RANGE: & cr & & & & & subshrub \\
\hline RANGE: & end & & & & & subshrub \\
\hline RANGE: & end & 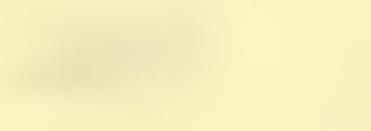 & & & & tree \\
\hline RANGE: & end & & & & & tree \\
\hline RANGE: & end & 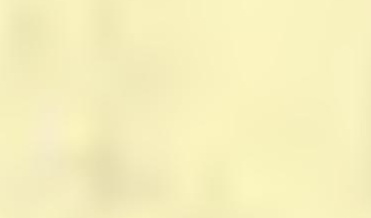 & 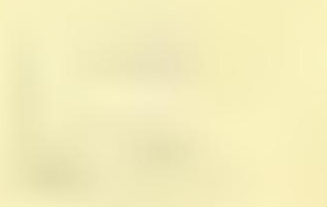 & 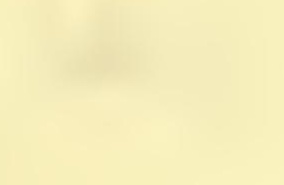 & & tree \\
\hline $\begin{array}{l}\text { RANGE: } \\
\text { RANGE: }\end{array}$ & cr & gus & & & & shrub \\
\hline $\begin{array}{l}\text { RANGE: } \\
\text { RANGE: }\end{array}$ & $\begin{array}{l}\text { end } \\
\text { end }\end{array}$ & & & & & herb \\
\hline RANGE: & cr & per & $\operatorname{sag} 7$ & & & $\begin{array}{l}\text { Shrub } \\
\text { shrub }\end{array}$ \\
\hline RANGE: & er & & & & & subshrub \\
\hline UANC & d & & & & & 51 \\
\hline & & cag? & & & & shrub \\
\hline
\end{tabular}


polyphlebia J.D. Sm.

psychotriaefolia (Seem.) Standl. pubescens SW.

\section{pumil i ocarpa Dwyer} quadrangulata Dwyer racemosa (AubI.) Raeusch.

\section{rosulatifolia Dwyer} sanfelicensis Dwyer santa-rosae Standl. santaritensis Dwyer siccorubra Dwyer

siggersiana Stand I. solitudinum Standl.

"suerrensis J.D. Sm. taca rcunens is Dwyer

taurina Dwyer

trichotoma Mart. \& Gal.

\section{tutensis Dwyer}

uliginosa Sw.

umbell iformis Dwyer \& Hayden

vallensis Dwyer

veraguensis Dwyer

viridis $R$, $P$.

wilburiana Dwyer

xerococcoides Dwyer

Randia

aculeata $L$.

armata (SW.) DC.

* diversi loba Stand I.

formosa (Jaca.) Schum.

gentryi Dwyer

*grandifolia StandI.

karstenii Polak.

las iantha (Standl.) StandI.

mega locarpa Dwyer

mi ra Dwyer

pepoformis Dwyer

"platysepala StandI.
PROV: bo da $v r$ 000m: $0=1$

PROV: bo ca ce ch cn 000m: $0-1$

$$
\text { da pa sa } v r
$$

PROV: bo ca cc ch cn 000m: 0-1, 1-2 da pa sa $v r$

PROV: $V r$

PROV: bo ca cc ch cn $000 \mathrm{~m}: 0-1$ da lo pa sa

PROV: da

PROV: ch

PROV: da

$000 \mathrm{~m}: 0-1,1-2$

000m: $1-2$

000m: $1-2$

PROV: cn pa

000m: $0-1$

PROV: ch $V r$

000m: $1-2$

PROV: ce da pa $v r$ 000m: $0-1$

PROV: bo cc da pa $v r$ 000m: $0-1,1-2$

PROV: bo ca cc cn pa 000m: $0-1$

PROV: da $000 \mathrm{~m}: 1-2$

000m: $1-2$

PROV: cc pa $v r \quad 000 m: 0-1,1-2$

PROV: $v r \quad$ 000m: $1-2$

PROV: bo ca cc ch cn 000m: $0-1,1-2$ da lo pa sa $v r$

PROV: bo ce cn pa $000 \mathrm{~m}$ : $0-1$

PROV: cc pa $v r \quad 000 \mathrm{~m}: 0-1$

PROV: $v r \quad 000 \mathrm{~m}: 0-1$

PROV: da pa sa $000 \mathrm{~m}: 0-1$

PROV: $v r \quad 000 \mathrm{~m}: 0-1$

PROV: da sa 000m: $1-2$

PROV: ca ce ch cn da 000m: $0-1$ he pa sa $v r$

PROV: bo ca cc cn da 000m: $0-1,1-2$ lo pa sa

PROV: sa $000 \mathrm{~m}: 0-1$

PROV: ca ch cn da pa $000 \mathrm{~m}: 0-1$

PROV: da 000m: 1 -2

PROV: cn $v r \quad$ 000m: $0-1,2-3$

PROV: ch $000 \mathrm{~m}: 0-1$

PROV: ca pa 000m: $0-1$

PROV: pa $000 \mathrm{~m}: 0=1$

PROV: pa $000 \mathrm{~m}: 0-1$

PROV: da pa sa $000 \mathrm{~m}: 0-1,1-2$

PROV: $\mathrm{ch}$ 000m: $1-2$

\begin{tabular}{|c|c|c|c|c|c|c|}
\hline RANGE: & $\mathrm{COI}$ & $\mathrm{cr}$ & gua & & & subshru \\
\hline RANGE: & col & $\mathrm{cr}$ & ecu & ven & & $\begin{array}{c}\text { shrub } \\
\text { tree }\end{array}$ \\
\hline RANGE: & cag & $\operatorname{mex}$ & & 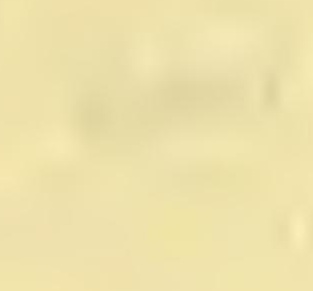 & & $\begin{array}{c}\text { shrub } \\
\text { tree }\end{array}$ \\
\hline RANGE: & end & & & & & shrub \\
\hline RANGE: & end & & & & & tree \\
\hline RANGE: & nwt & & & & & shrub \\
\hline RANGE: & end & & & & & herb \\
\hline RANGE: & end & & & & & shrub \\
\hline RANGE: & col & $14=$ & he & & & subshrub \\
\hline RANGE: & end & $t^{2}$ & $x^{2}$ & & & tree \\
\hline RANGE: & end & & & & & shrub \\
\hline RANGE: & cr & & & & & shrub \\
\hline ZANGE: & $\mathrm{col}$ & $\mathrm{cr}$ & $10=$ & $=2$ & 647 & $\begin{array}{l}\text { shrub } \\
\text { tree }\end{array}$ \\
\hline $\begin{array}{l}\text { RANGE: } \\
\text { RANGE: }\end{array}$ & $\begin{array}{l}\text { col } \\
\text { end }\end{array}$ & cr & nic & & & $\begin{array}{l}\text { shrub } \\
\text { shrub }\end{array}$ \\
\hline RANGE: & end & & & & & tree \\
\hline $\begin{array}{c}\text { RANGE: } \\
\text { ven }\end{array}$ & $\mathrm{col}$ & gua & hon & $\operatorname{mex}$ & nic & tree \\
\hline $\begin{array}{l}\text { RANGE: } \\
\text { RANGFF: }\end{array}$ & end & & & & & tree \\
\hline mex & ang & cag & $\mathrm{col}$ & ecu & Jam & suosnrub \\
\hline RANGE: & end & & & & & $\begin{array}{l}\text { shrub } \\
\text { tree }\end{array}$ \\
\hline $\begin{array}{l}\text { ANGE: } \\
\text { ANGE: }\end{array}$ & $\begin{array}{l}\text { end } \\
\text { end }\end{array}$ & & & & & $\begin{array}{l}\text { shrub } \\
\text { shrub }\end{array}$ \\
\hline RANGE: & bel & bol & cag? & col & $\mathrm{cr}$ & tree \\
\hline $\begin{array}{l}\text { cub } \\
\text { RANGE: } \\
\text { RANGE: }\end{array}$ & $\begin{array}{l}\text { sag? } \\
\text { end } \\
\text { end }\end{array}$ & & & & & $\begin{array}{l}\text { shrub } \\
\text { subshrub } \\
\text { subshrub }\end{array}$ \\
\hline NGE: & ang & cag & $\mathrm{col}$ & jam & $\operatorname{mex}$ & $\begin{array}{c}\text { shrub } \\
\text { tree }\end{array}$ \\
\hline $\begin{array}{l}\text { RANGE: } \\
\text { SaO }\end{array}$ & $\begin{array}{l}\text { cag } \\
\text { ven }\end{array}$ & $\mathrm{col}$ & gui & lag & $\operatorname{mex}$ & tree \\
\hline ANGE: & col & & & & & shrub \\
\hline $\begin{array}{l}\text { ANGE: } \\
\text { ANGE: }\end{array}$ & $\begin{array}{l}\mathrm{Col} \\
\mathrm{col}\end{array}$ & per & sag? & sao & & $\begin{array}{l}\text { shrub } \\
\text { tree }\end{array}$ \\
\hline ANGE & $\mathrm{cr}$ & $\operatorname{mex}$ & sa I & & & shrub \\
\hline ANGE & $\mathrm{cr}$ & 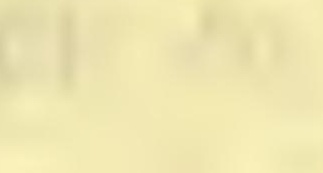 & r & trat & & tree \\
\hline ANG & $\mathrm{Cr}$ & & & & & tree \\
\hline ING & $\begin{array}{l}\text { end } \\
\text { end }\end{array}$ & & & & & shrub \\
\hline NC & $e$ & & & & & $\begin{array}{l}\text { tree } \\
\text { shrub }\end{array}$ \\
\hline ANG & & & & & & sh \\
\hline
\end{tabular}


TAXON

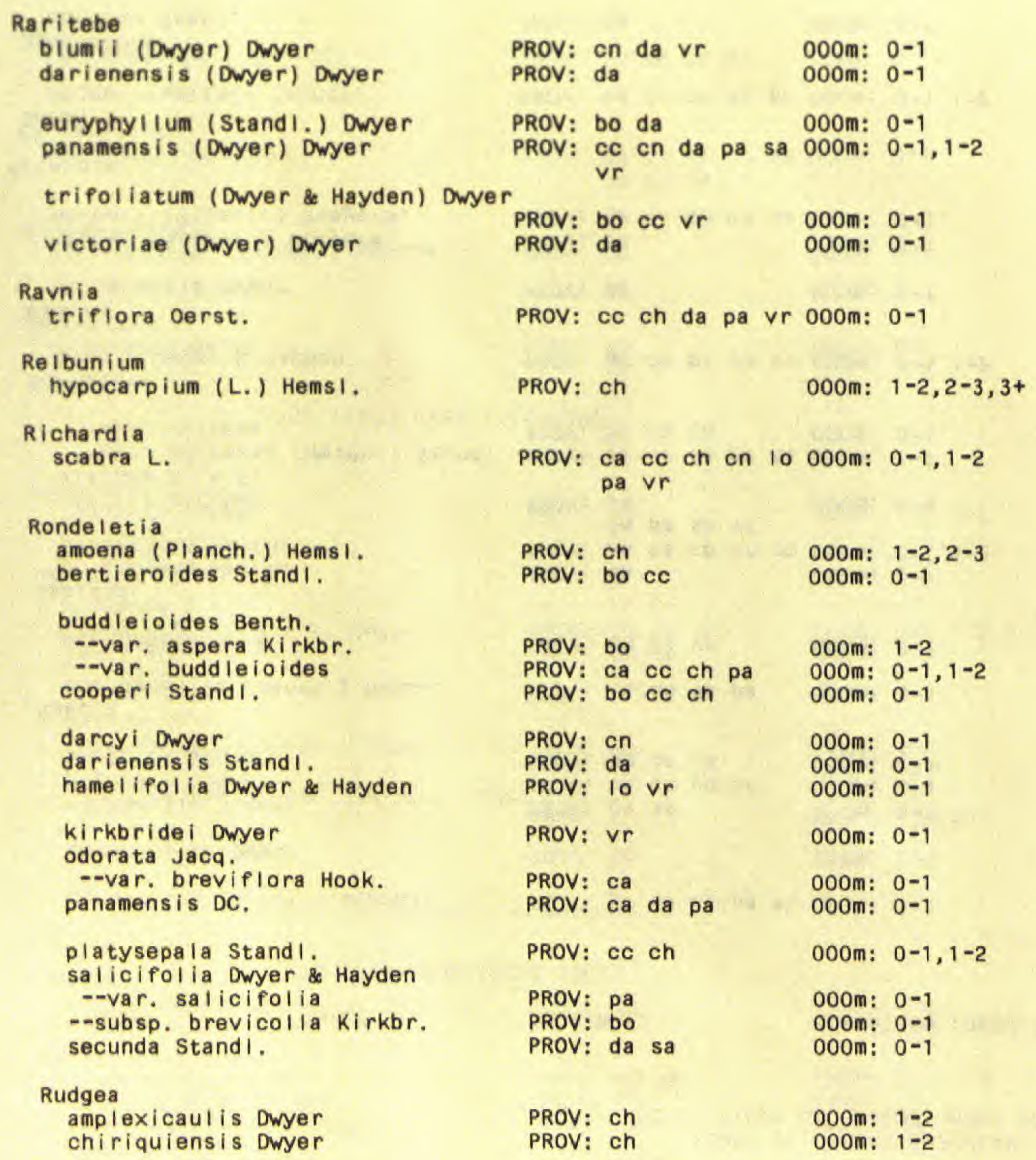

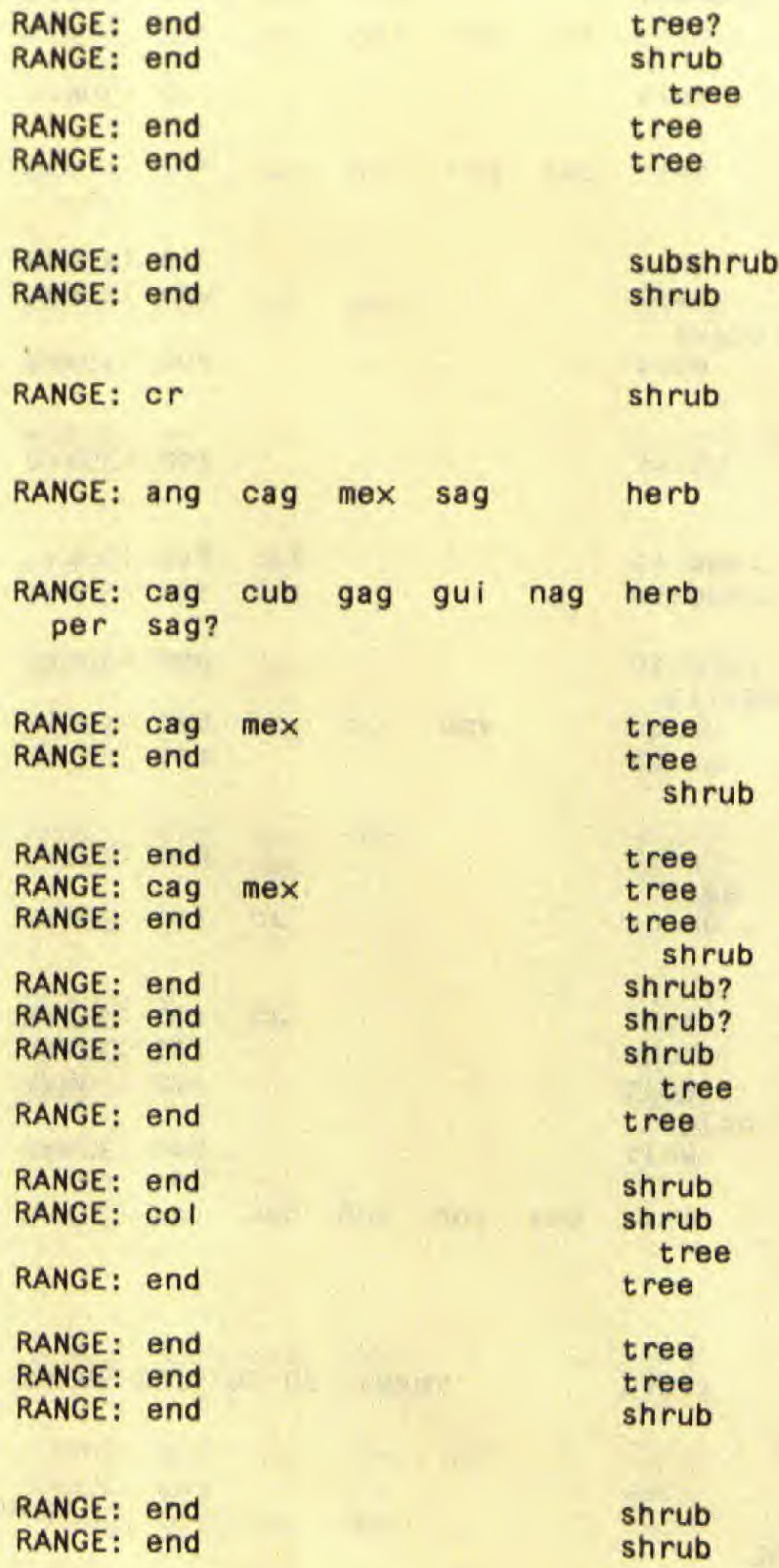


TAXON

FAMILY

PROVINCES

ELEVATION (OOOM.)

RANGE OUTSIDE OF PANAMA

HAB IT

270 (179) RUBIACEAE cont.

cornifolia (H.B.K.) StandI. coronicarpa Dwyer

isthmensis Standi.

pittieri Standl.

skutchi i Standi.

\section{Rustia}

occidentalis (Benth.) HemsI.

panamens is Dwyer

\section{Sabicea \\ * colombiana Wernh. \\ panamensis Wernh.}

stellaris Dwyer

villosa R. \& $S$.

- -var. adpressa (Wernh.) Standl.

--var, villosa

\section{Schradera \\ blumii Dwyer \& Hayden}

\section{Simira}

darienens is Dwyer

kluge i (Standl.) Steyerm.

maxonil (Standi.) Steyerm.

\section{*Sipanía}

\section{Sommera}

donnell-smithil standI.

\section{Spermacoce \\ confusa RendI.}

tenuior L.

Stachyarrhena

dichroa StandI.

Tobagoa

maleolens Urb.

Tocoyena

pittieri (Standl.) Stand..
PROV: bo ca da pa sa $000 \mathrm{~m}$ : $0-1$

PROV: $\mathrm{CC}$

000m: $0-1$

PROV: ca sa

PROV: bo ce pa sa $000 \mathrm{~m}: 0-1$

000m: $0-1$

PROV: ca en da pa $000 \mathrm{~m}: 0-1$

PROV: ch pa vr 000m: $0=1$

PROV: da 000m: $0-1$

PROV: bo ca cc ch cn 000m: $0-1,1-2$

da pa sa $v r$

000m: $0-1$

PROV: bo ca da pa sa $000 \mathrm{~m}: 0-1$

PROV: bo $\mathrm{cc}$ en $000 \mathrm{~m}$ : $0-1$

PROV: cc da pa sa $\mathrm{vr} 000 \mathrm{~m}: 0-1,1-2$

PROV: da

000m: $0-1$

PROV: pa

000m: $0-1$

PROV: bo ca ch en sa 000m: $0-1$

PROV: bo ce ch da pa 000m: $0-1,1-2$

PROV: ca

000m: $0-1$

PROV: ca pa sa

000m: $0-1$

PROV: cn da pa sa

000m: $0-1$

PROV: $\mathrm{CC}$

000m: $0-1$

PROV: ca da pa 000m: $0-1,1-2$

\begin{tabular}{|c|c|c|c|c|c|c|}
\hline $\begin{array}{c}\text { RANGE: } \\
\text { sao }\end{array}$ & $\begin{array}{l}\text { bol } \\
\text { ven }\end{array}$ & cag & gua & gui & sag & tree \\
\hline RANGE: & end & & $=$ & & & $\begin{array}{l}\text { tree } \\
\text { shrub }\end{array}$ \\
\hline $\begin{array}{l}\text { RANGE: } \\
\text { RANGE: }\end{array}$ & $\begin{array}{l}\text { end } \\
\text { col }\end{array}$ & & & & & $\begin{array}{l}\text { tree } \\
\text { shrub }\end{array}$ \\
\hline RANGE: & $\mathrm{col}$ & $\mathrm{cr}$ & & & & tree \\
\hline RANGE: & col & $\mathrm{cr}$ & & & & $\begin{array}{l}\text { shrub } \\
\text { tree }\end{array}$ \\
\hline RANGE: & end & & & & & tree \\
\hline RANGE: & col & & & & & shrub \\
\hline RANGE: & cag & $\mathrm{col}$ & $\mathrm{cr}$ & gua & & shrub \\
\hline RANGE: & end & & & & & climber \\
\hline RANGE: & $\mathrm{col}$ & per & 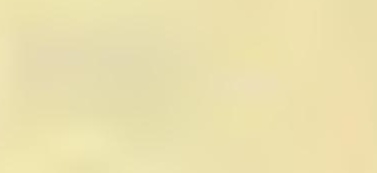 & i & 10 & climber \\
\hline RANGE: & cag & sag & & 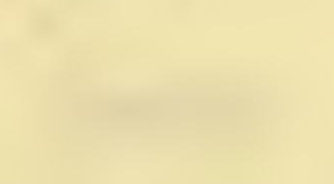 & 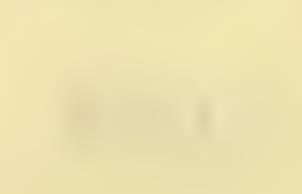 & climber \\
\hline RANGE: & $\mathrm{col}$ & $x^{2}$ & (1) & 1 & ax & shrub \\
\hline RANGE: & end & & & & & $\begin{array}{l}\text { tree } \\
\text { shrub }\end{array}$ \\
\hline RANGE: & ven & & & & & tree \\
\hline RANGE: & $\mathrm{cr}$ & 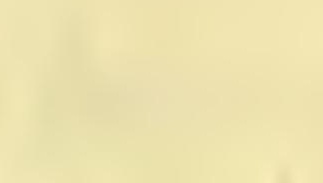 & $\frac{1}{2}$ & $x^{2}+2$ & 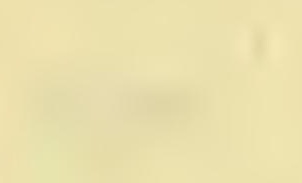 & tree \\
\hline RANGE: & er & & & & & tree \\
\hline RANGE: & cag? & fla & sag? & & & $\begin{array}{c}\text { subshrub } \\
\text { herb }\end{array}$ \\
\hline RANGE: & cag & nag & sag & sao & & subshrub \\
\hline RANGE: & end & & & & & tree \\
\hline RANGE: & ang & trt & ven & & & herb \\
\hline NGE: & 1 & $\mathrm{cr}$ & & & & tree \\
\hline
\end{tabular}


270 (179) RUBIACEAE cont.

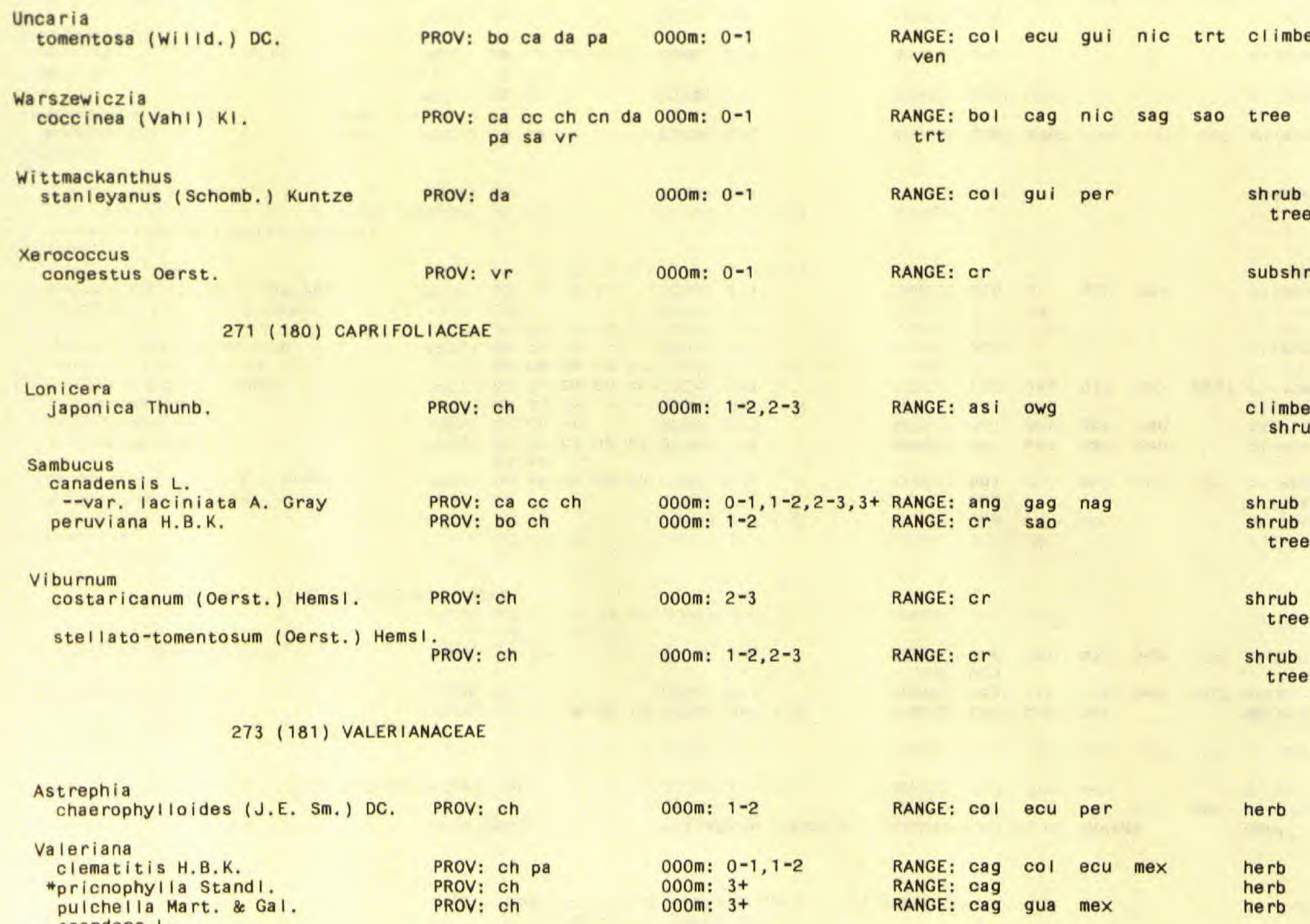


TAXON

273 (181) VALERIANACEAE cont.

-"var. candolleana (Gardn.) Muell.

--var. scandens

urticaefolia H.B.K.
PROV: ca $\mathrm{cc}$ ch da PROV: bo

PROV: ch 000m: $0-1,1-2$ 000m: $0-1$

000m: $1-2$

\section{5 (182) CUCURBITACEAE}

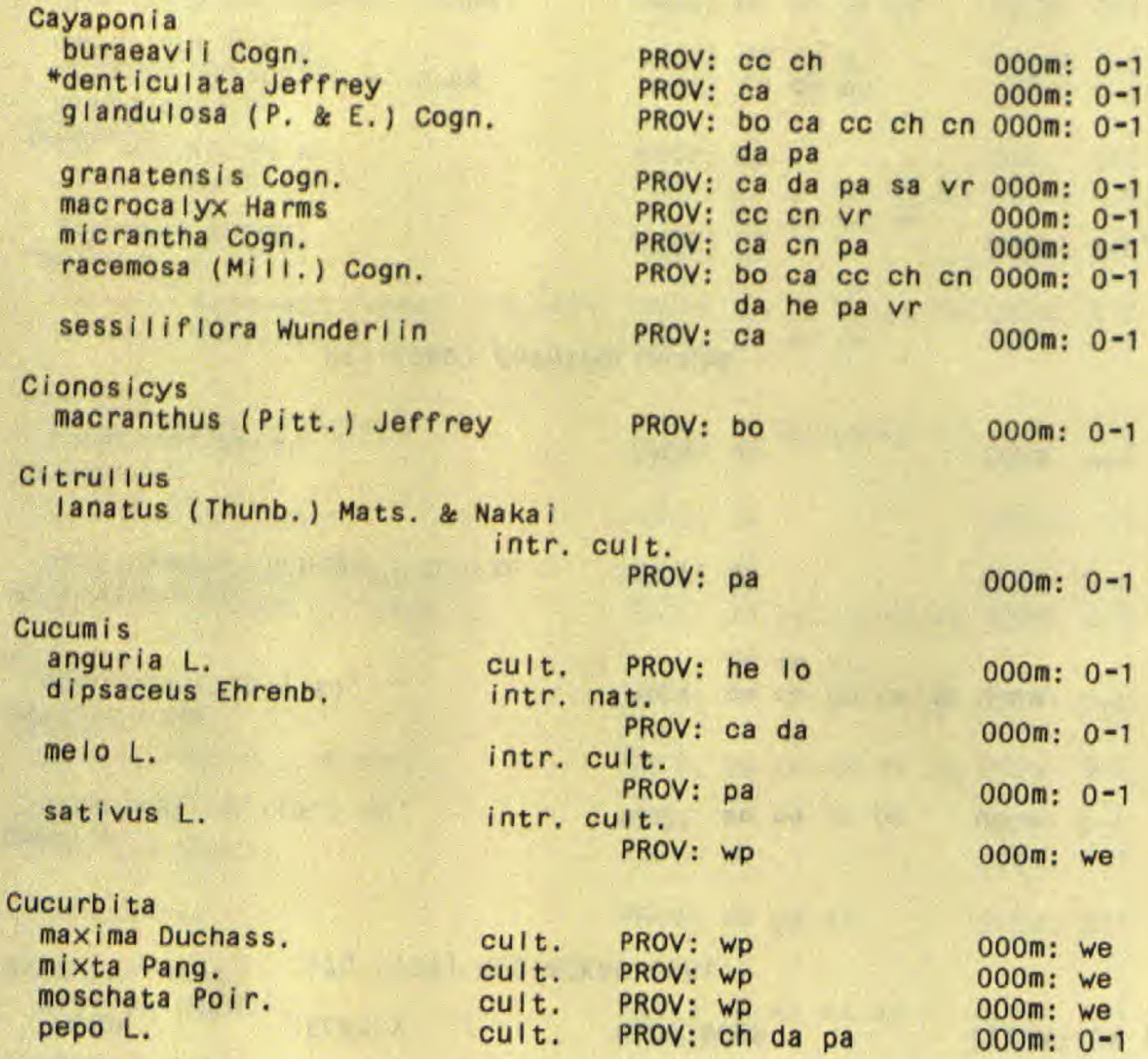

PROV: pa 000m: 0-1

$000 \mathrm{~m}: 0-1$

000m: $0-1$

000m: $0-1$

000m: $0-1$

000m: we

Cucurbita

maxima Duchass.

mixta Pang. moschata Poir. pepo L.

cult. PROV: Wp

cult. PROV: WP

$\begin{array}{ll}\text { cult. } & \text { PROV: WP } \\ \text { cult } & \text { PROV: ch da }\end{array}$

000m: we

000m: we

000m: we

000m: $0-1$

Cyclanthera dissecta (Torr. \& Gray) Arn. \& Hook.

dressleri Wunderlin
PROV: ch 000m: $0-1$ 000m: $1-2$

$\begin{array}{lllll}\text { RANGE: cr } & \text { gua mex } & \text { herb } \\ \text { RANGE: cag fla gag mex sag? herb } \\ \text { Sao ven } & & & & \\ \text { RANGE: cag col mex per } & \text { herb }\end{array}$

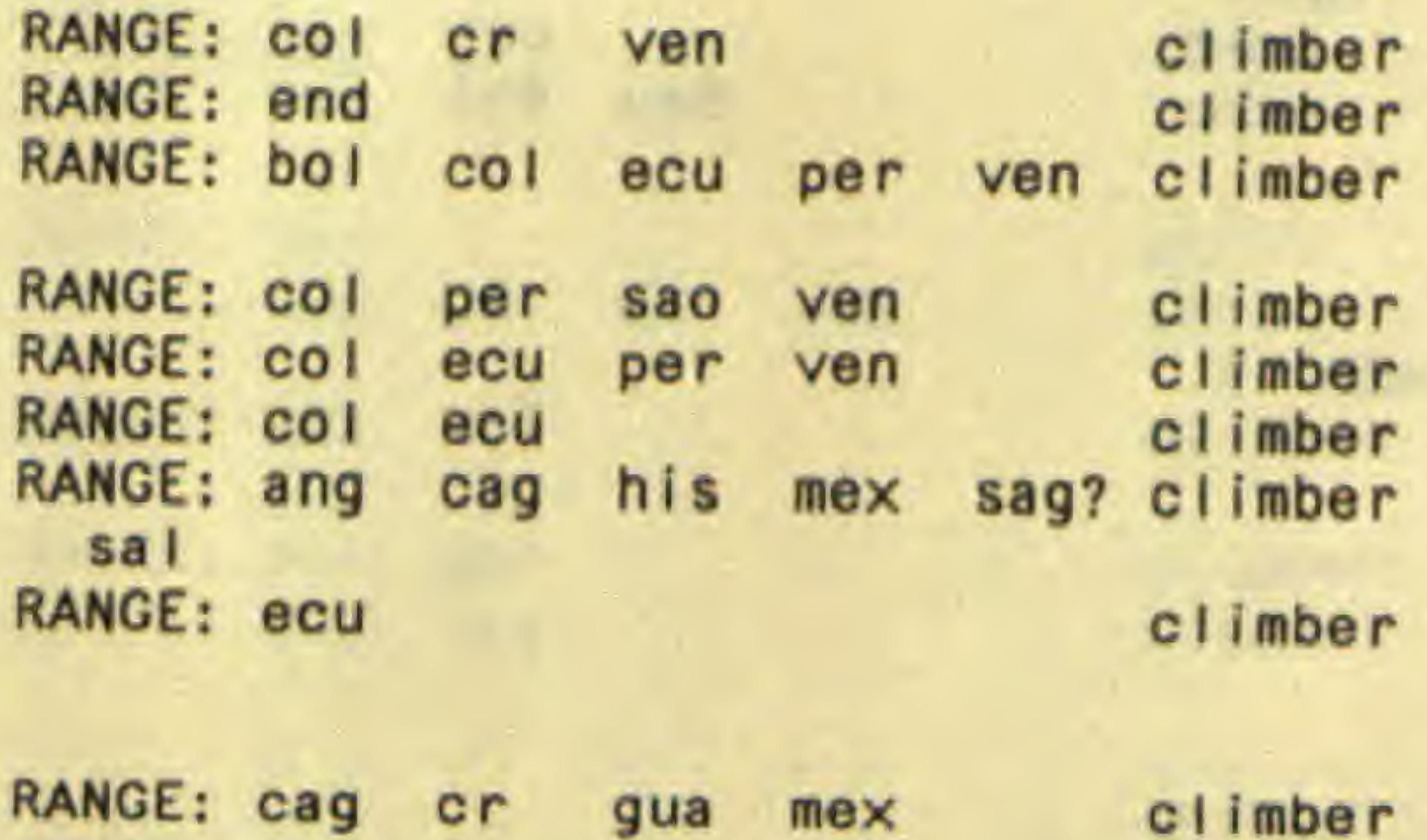

RANGE: af $r$

climber

RANGE: gua nwg?

climber

RANGE: owg? WdW?

climber

RANGE: owg

climber

RANGE: OWg

cl imber

RANGE: NWg OWg

RANGE: NWg OWg

RANGE: nWt OWg

RANGE: ? NWt

climber

climber

climber

climber

RANGE: cag mex nag? tex climber RANGE: end mex mag? tex 
TAXON

pedata (L.) Schrad.

cult. PROV: ca

000m: $0-1$

\section{Elateriopsis}

oerstedii (Cogn.) Pitt.

\section{Fevillea \\ cordifolia L.}

\section{Frantzia}

panamensis Wunderl in

pittieri (Cogn.) Pitt.

\section{Gurania}

coccinea Cogn.

eriantha (P. \& E.) Cogn.

makoyana (Lem.) Cogn.

meg istantha J.D. Sm.

seemaniana Cogn.

suberosa StandI.

*tubulosa Cogn.

\section{Lagenaria}

siceraria (Molina) Standl.

intr. cult.

\section{Luffa}

aegyptiaca Mill.

operculata (L.) Cogn.

Melothria

dulcis Wunderlin

pendula $\mathrm{L}$.

scabra Naud.

trilobata Cogn.

Momordica

cha rantia L.

$$
\text { PROV: cc en pa 000m: } 0-1
$$

PROV: bo cc cn da pa 000m: $0-1,1-2$ sa $\vee r$

PROV: ca ch da lo pa 000m: 0-1

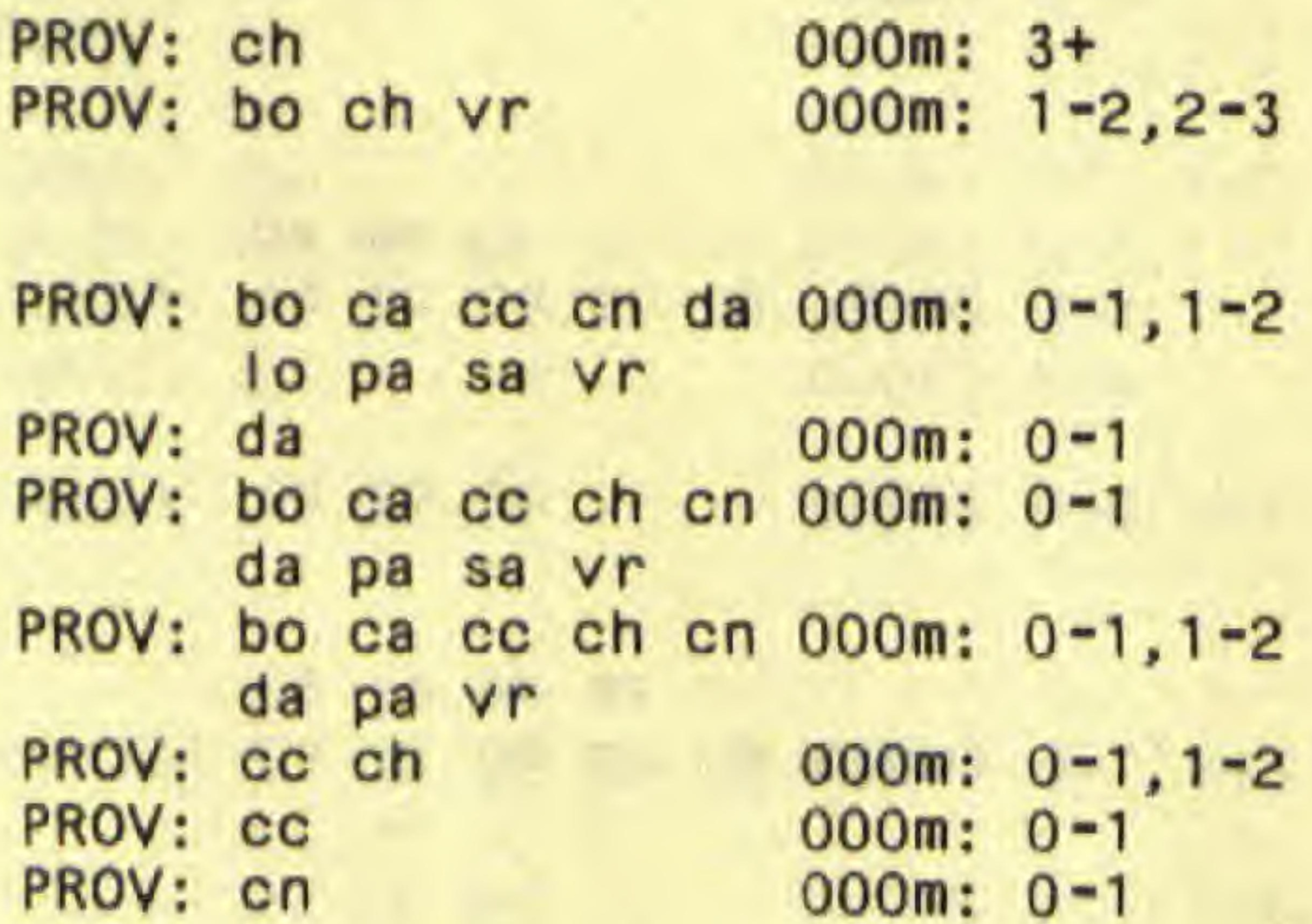

intr. cult. nat.

PROV: ca cc ch da he $000 \mathrm{~m}$ : $0-1$ lo pa sa vr

000m: $0-1$

PROV: bo ca ce en da 000m: 0-1

PROV: bo ca cc ch cn 000m: $0-1$

da he lo pa sa

PROV: bo ca cc ch pa 000m: $0-1,1-2$

PROV: bo ca cn da lo $000 \mathrm{~m}$ : $0-1$ pa sa

intr. nat.
RANGE: cag? ecu mex per sag? climber

RANGE: $\mathrm{cr}$

climber

RANGE: ang cag per sag? sao climber

RANGE: end

climber

RANGE: $c r$

climber

RANGE: $c r$

climber

RANGE: $\mathrm{cr}$ per

climber

RANGE: cag? gua

climber

RANGE: $\mathrm{cr}$

climber

RANGE: $\mathrm{cr}$

RANGE: $w r$

RANGE: $w r$

climber

climber

climber

RANGE: af $r$ sao

climber

RANGE: or owg?

climber

RANGE: cag ecu mex per sag climber

RANGE: end

climber

RANGE: ang cag nag sag sao climber

RANGE: cag gua mex climber

RANGE: cag col cr gui mex climber ven

RANGE: nWt wdw

climber 
TAXON

\section{Polyclathra}

cucumerina Bertol.

PROV: cc da pa

000m: $0-1$

\section{Posadaea}

sphaerocarpa Cogn.

PROV: ca pa

000m: $0-1$

Psiguria

bignoniacea (P. \& E.) Wunderlin PROV: bo ca cc cn da 000m: 0-1

longipedunculata (Cogn.) Wunderlin

warscewiczii (Hook, f, Wunderli lo pa $v r$

ROV: bo

$$
\text { da lo pa } v r
$$

\section{Ryt idostyl is}

carthaginens is (Jacq.) Kuntze

PROV: bo ca cc ch cn 000m: $0-1,1-2$ da lo pa sa

Sechium

edule (Jacq.) SW.

cult. PROV: ca cc ch

000m: $0-1$

Selysia

prunifera (P. \& E.) Cogn.

PROV: bo cc ch cn da 000m: $0-1$ pa sa

Sicana

odorifera (VeII.) Naud. intr. cult.

PROV: da pa $\quad 000 \mathrm{~m}: 0-1$

\section{Sicydium}

coriaceum cogn.

tamnifol ium (H.B.K.) Cogn.

PROV: ca

PROV: 000m: $0-1$ pa sa

\section{Sicyos}

*chiriquensis Hammel \& D'Arcy

PROV: ch

$000 \mathrm{~m}: 2-3$

276 (183) CAMPANULACEAE

\section{Burmeistera}

chiriquiensis Wilbur

cyclostigmata J.D. Sm.

darienensis Wilbur
PROV: ch

000m: $1-2$

PROV: bo $\mathrm{cc}$ ch da pa $000 \mathrm{~m}$ : $0-1,1-2,2-3$

PROV: $\mathrm{da}$

$000 m: 0-1,1-2$

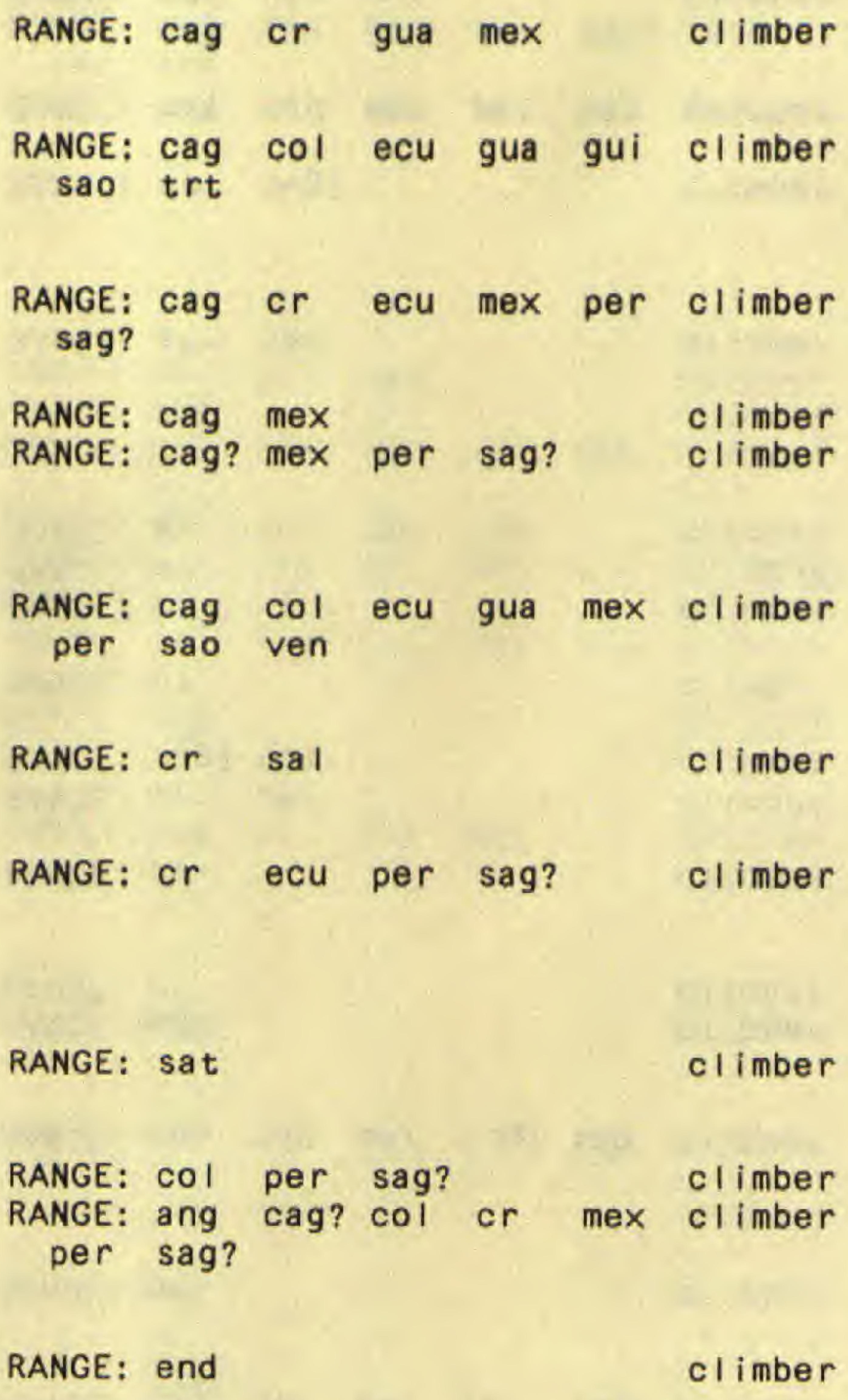

RANGE: $c r$

RANGE: col cr ecu

herb

shrublet herb

RANGE: end 
276 (183) CAMPANULACEAE cont.

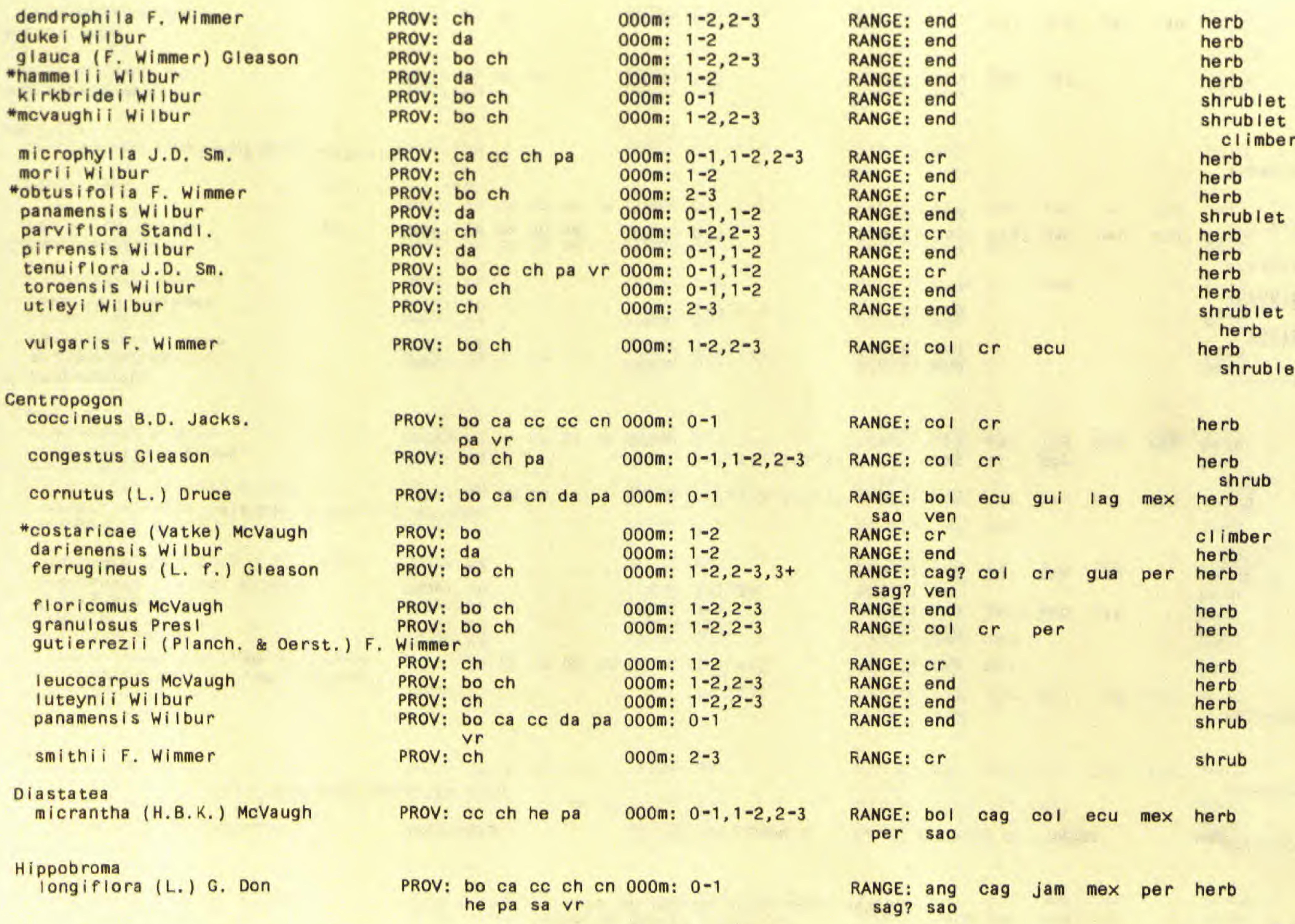


TAXON

FAMILY

PROVINCES

ELEVATION (OOOM.)

RANGE OUTSIDE OF PANAMA

HABIT

276 (183) CAMPANULACEAE cont.

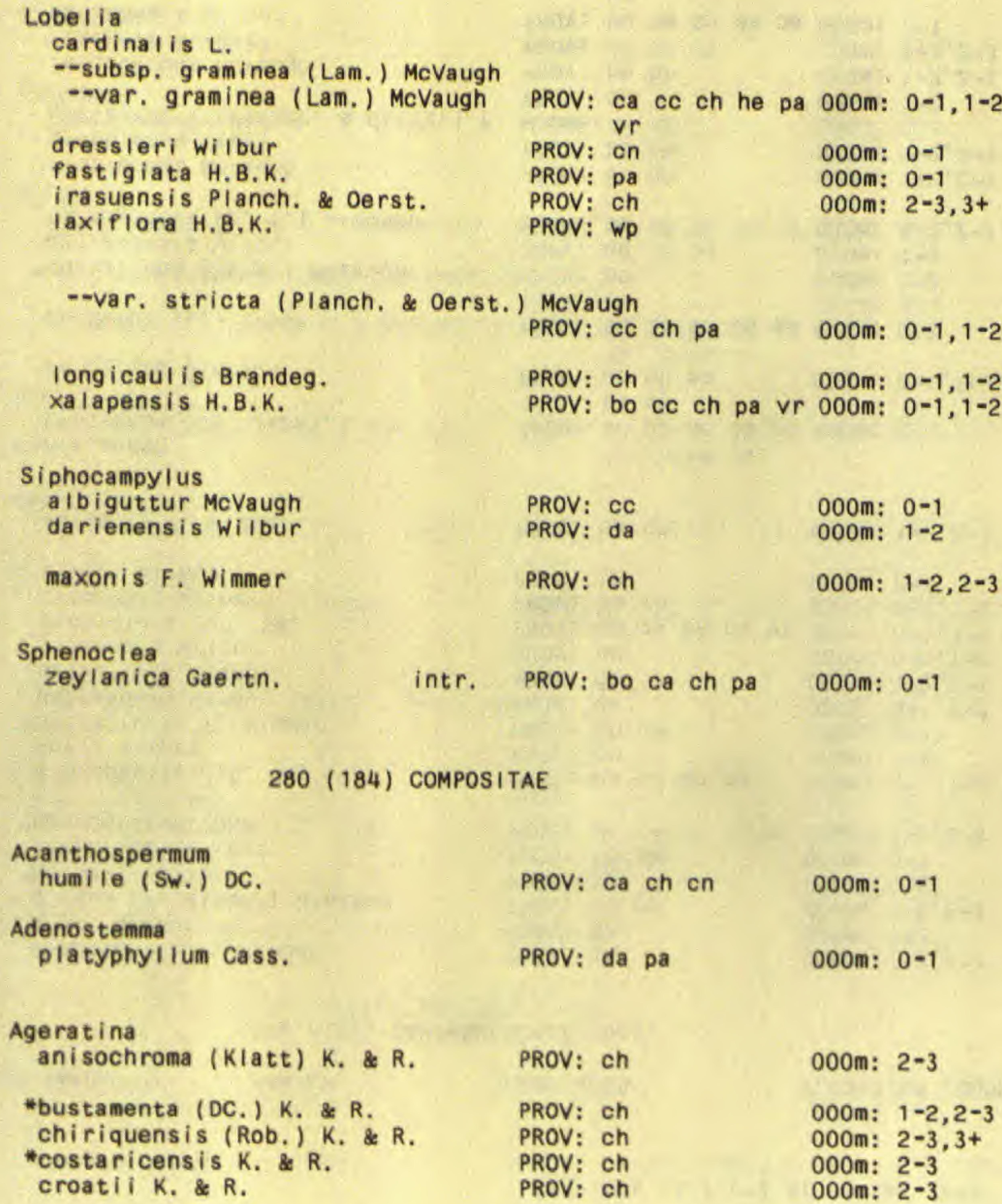

RANGE: cag mex

herb

RANGE: end

herb

RANGE: or herb

RANGE: cag col mex nao shrub

herb

PROV: ch

00m: $0-1,1-2,2-3,3+$ RANGE: cag col or gua mex shrub 000m: $0-1,1-2,2-3,3+$ RANGE: cag or mex herb

rb

RANGE: cag gal lag mex sag herb

Sao

RANGE: end

RANGE: end

herb

RANGE: end

herb

shrublet

herb shrublet

RANGE: cag? nag? nao owg sag? herb sao tex 
280 (184) COMPOSITAE cont.

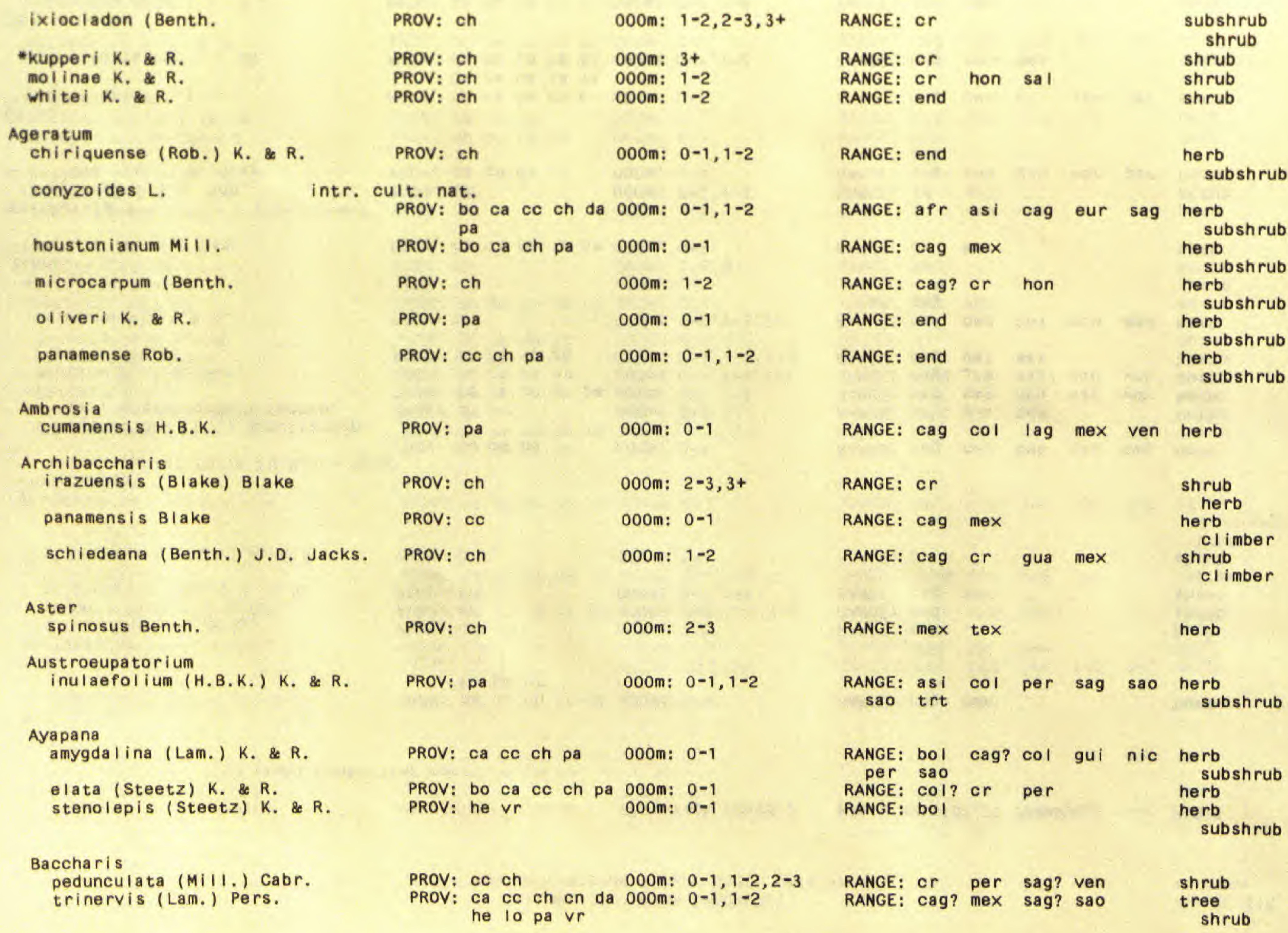


TAXON

FAMILY

PROVINCES

ELEVATION (OOOM.)

RANGE OUTSIDE OF PANAMA

HABIT

280 (184) COMPOSITAE cont.

Ba I timora
recta L.

\section{Bartlettina}

"chiriquensis K. \& R.

maxonil (Rob.) K. \& R.

platyphyila (Rob.) K. \& R.

prionophylla (Rob.) K. \& R.

Bidens

bipinnata

--var. cynapilfolia (H.B.K.) Maza

ostruthioides (DC.) Schultz-Bip.

- -var, costaricensis (Benth.

pilosa L.

reptans (L.) G. Don

riparia H.B.K.

triplinervia H.B.K.

BI umea

viscosa (Mill,) Bad.

Brickellia

*argyrolepis B, L. Rob.

diffusa (Vahl) A. Gray

Ca lea

jamaicensis (L.) L.

urticifolia (MIII,) DC.

Centratherum

punctatum Cass.

\section{Chaptalia}

nutans (L.) Polak.

runcinata $\mathrm{H}, \mathrm{B}, \mathrm{K}$.

\section{Chromolaena}

ivaefolia (L.) K. \& R.
PROV: ca ce ch cn da 000m: $0-1$ he pa $v r$

$\begin{array}{ll}\text { PROV: ch } & 000 \mathrm{~m}: 1-2 \\ \text { PROV: ch } & 000 \mathrm{~m}: 1-2 \\ \text { PROV: ch } & 000 \mathrm{~m}: 0-1,1-2 \\ \text { PROV: ch } & 000 \mathrm{~m}: 1-2\end{array}$

PROV: $\mathrm{ch}$

PROV: ch

PROV: bo ca cc ch pa 000m: $0-1,1-2$

PROV: cc ch pa $v r$ 000m: $0-1,1-2,2-3$

PROV: ca he lo pa $000 \mathrm{~m}: 0-1$

PROV: ch

$000 \mathrm{~m}: 1-2,2-3,3+$

PROV: ca cc he lo pa 000m: $0-1$

PROV: $\mathrm{ch}$

PROV: 000m: $2-3$

000m: $0-1$

PROV: bo ca cc ch cn 000m: 0-1

$$
\text { da he pa sa vr }
$$

PROV: ca cc ch he pa $000 \mathrm{~m}$ : $0-1,1-2$

PROV: ca cc ch he pa 000m: $0-1,1-2$

PROV: bo ca cc ch cn $000 \mathrm{~m}$ : $0-1,1-2$ da he lo pa sa

PROV: ch

000m: $1-2$

PROV: ch

000m: $1-2$
RANGE: cag mex

herb

RANGE: end

RANGE: end

RANGE: cag mex

RANGE: $\mathrm{cr}$

herb

shrub

shrub

tree

shrub

tree

RANGE: ang cag cub nao owg herb
RANGE: cr gua mex
RANGE: ang cag nag sat wdw herb
RANGE: cag? jam sag? sao ven herb
RANGE: cag gal sat
RANGE: bol cag col ecu mex herb
per sag ven

RANGE: or mex

herb

RANGE: $\mathrm{cr}$

RANGE: cag cub gag mex sag? sao

shrub

RANGE: cag col cr jam ven shrub

RANGE: cag or mex shrub

RANGE: sat wdt

herb

subshrub

RANGE: ang bol cag $\mathrm{cr}$ gua herb
mex nag sag sao tex
RANGE: bol col cr sao

RANGE: ang bol cag jam mex shrub
nag sag sao 


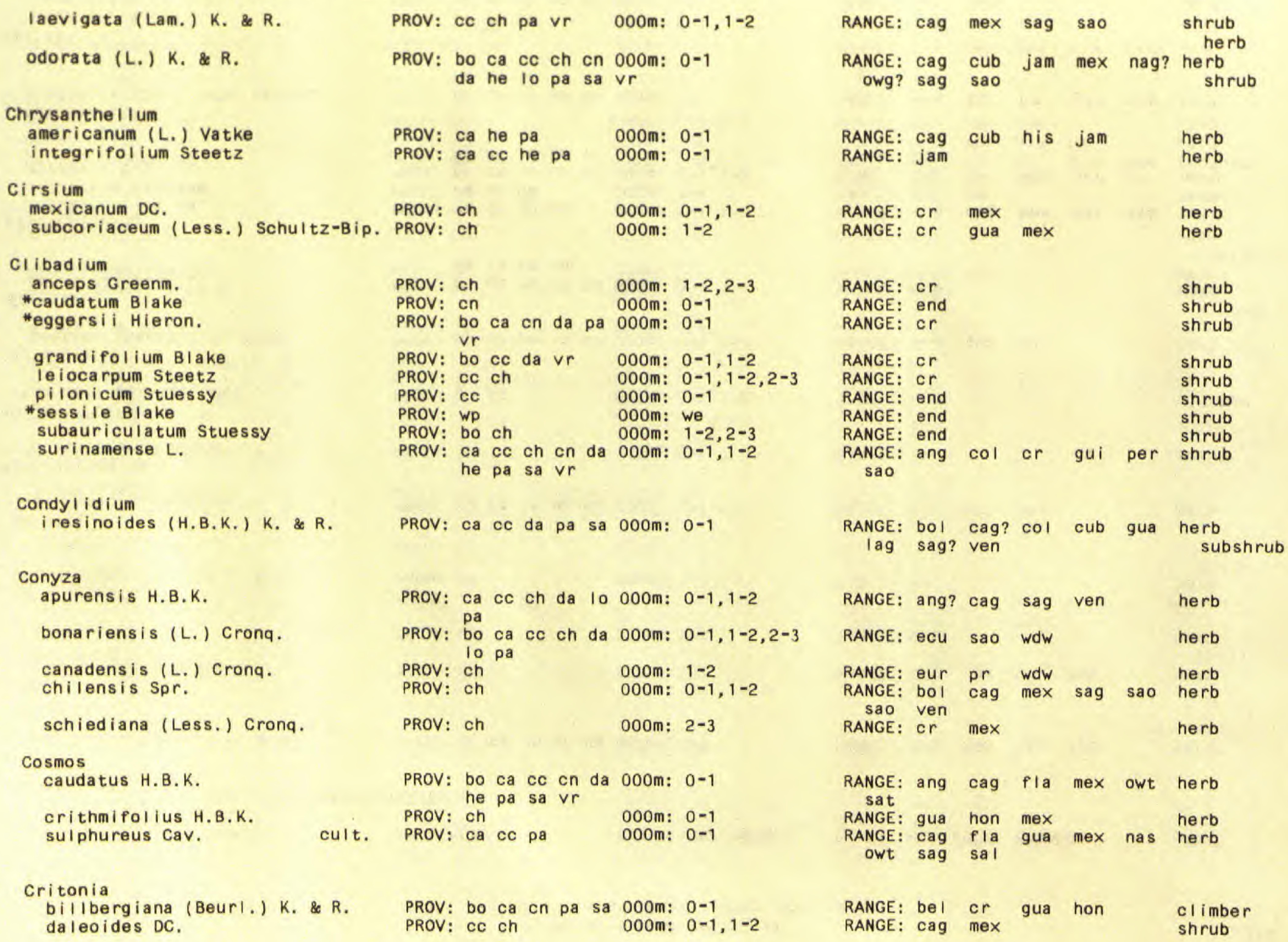


TAXON

280 (184) COMPOSITAE cont.

morifolia (Mill.) K. \& R.

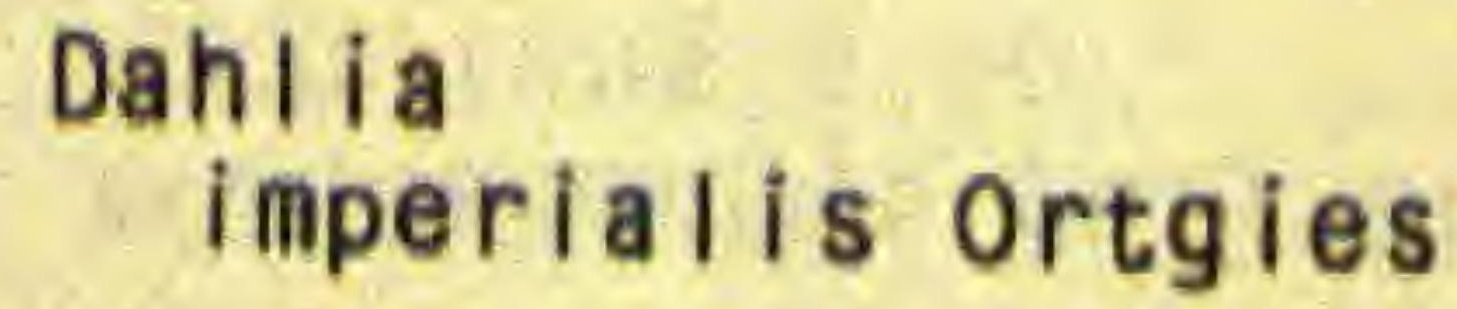

\section{Decachaeta}

thieleana (Klatt) $K$, \& $R$.

\section{Dellila}

biflora (L.) Kuntze

*Diplostephium

"costaricense Blake

\section{*Dresslerothamnus}

*angust iradiatus Rob.

Dyssodia

montana (Benth.) A. Gray

Eclipta

prostrata (L.) L.

Elephantopus

angustifolius SW.

dilatatus Gleason

moll is H.B.K.

\section{Eleutheranthera} ruderalis (SW.) Schultz-Bip.

\section{Emilia}

fosbergi i Nicols.

sonchifolia (L.) DC.

\section{*Erato}

"polymnioides DC.

Erechtites

hieracifolia DC.

--var. cacalioides (Spr.) Griseb.
PROV: bo ca cc ch da 000m: 0-1

PROV: $\mathrm{ch}$

$000 m: 1-2$

PROV: ch

000m: $1-2$

PROV: ch

000m: $1-2$

PROV: ca cc lo pa $\mathrm{vr}$ 000m: 0-1

PROV: ch

000m: $3+$

PROV: cc pa

$000 \mathrm{~m}: 0-1,1-2$

PROV: ca ce ch lo pa 000m: $0-1,1-2$

PROV: bo ca cc ch cn 000m: 0-1 da lo pa sa $\begin{array}{ll}\text { PROV: ca cc ch pa } & 000 \mathrm{~m}: 0-1,1-2 \\ \text { PROV: ca cn pa } & 000 \mathrm{~m}: 0-1\end{array}$

PROV: bo ca cc ch cn 000m: $0-1,1-2$ da he lo pa vr

PROV: bo ca cc ch da 000m: $0-1$ he pa sa

PROV: ca ch pa 000m: 0-1

PROV: bo ca ch cn da 000m: $0-1$

he pa sa

PROV: bo

000m: $1-2$
RANGE: end herb

shrub

tree

RANGE: cag col or mex

herb

shrub

RANGE: WdW

herb

RANGE: $\mathrm{cr}$

herb

shrub

RANGE: $\mathrm{COI} \mathrm{cr}$ mex

herb

RANGE: $\mathrm{cr}$

shrub

RANGE: end

climber

RANGE: cag gua mex

herb

RANGE: WdW

herb

RANGE: ang cag mex sag sao herb

RANGE: $c 01$ or herb

RANGE: col or mex per pr herb

ven Wdw?

RANGE: ang bol cr jam nas herb

per sao

RANGE: bah or owg herb

RANGE: $\mathrm{cr}$ owg herb

RANGE: ang or nag herb 
280 (184) COMPOSITAE cont.

\section{lo pa sa}

valerianaefolia (Wolf) DC.

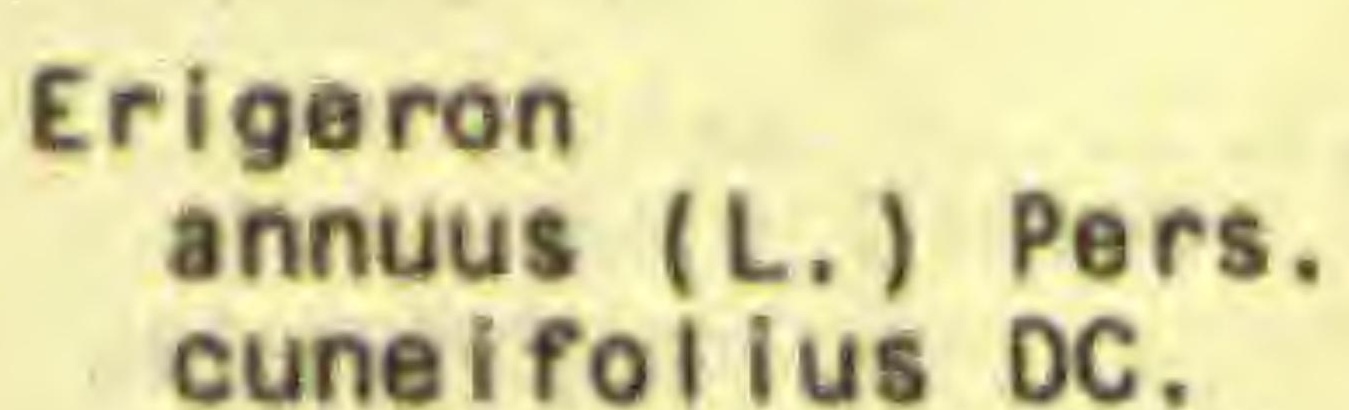

\section{Fleischmannia}

IIenii K. \& R.

\section{-capillipes (Benth. \\ chiriquensis $K$. \& $R$.}

croatii K. \& R.

hymenophyl Ia (KIatt) K. \& R microstemon (Cass.) K. \& $R$. misera (Rob.) K. \& R.

panamensis $K$. \& $R$.

pratensis (KIatt) K. \& R.

sideritidis (Benth.

sinclairii (Benth.

tysoni i K. \& R.

Ga I insoga

*quadriradiata R. \& P.

Garcilassa

rivularis P. BE.

Gerbera

jamesonii Bolus

Gnapha I i um

americanum $\mathrm{Mi}$ II.

attenua tum DC.

domingense Lam.

roseum H.B.K.

Gongrostylus

costaricensis (Kuntze) K. \& R.

PROV: ch
PROV: bo $\mathrm{ch}$

PROV: $c h$

PROV: ch

PROV: ch

PROV: ch

PROV: $v r$

PROV: $\mathrm{ch}$

PROV: ch

PROV: $\mathrm{cc} \mathrm{ch}$

$000 m: 0-1,1-2$

PROV: da

PROV: ce pa

000m: $1-2$

000m: $1-2,2-3$

000m: $1-2$

000m: $1-2,2-3,3+$

000m: $1-2$

000m: $0-1$

000m: $1-2,2-3$

000m: $2-3$

$000 \mathrm{~m}: 0-1$

000m: $0-1,1-2$

PROV: cc ch da he lo 000m: $0-1,1-2$ pa

PROV: ca cc en pa vr 000m: $0-1$

PROV: ca cc ch cn da 000m: $0-1,1-2$

he lo pa vr

000m: $2-3$

PROV: $\mathrm{cc}$ ch da

$000 \mathrm{~m}: 0-1,1-2,2-3$

PROV: bo ca ch da pa $000 \mathrm{~m}: 0-1,1-2$

intr. cult.

PROV: ch

PROV: ch

PROV: ch

PROV: $\mathrm{ch}$

PROV: sa $v r$ 000m: $1-2$

000m: $1-2,2-3,3+$

000m: $0-1,1-2,2-3$,

000m: $0-1,1-2,2-3$

000m: $2-3,3+$

000m: $0-1$
RANGE: asi cag? mex oce sag? herb sao

$\begin{array}{cll}\text { RANGE: eur nag nao } & \text { herb } \\ \text { RANGE: cub gag his pr } & \text { herb } \\ \text { RANGE: ang asi cr his mex subshrub } \\ \text { Owg per sao ven }\end{array}$

owg per sao ven

RANGE: end

herb

RANGE: mex nic

RANGE: end

RANGE: end

RANGE: $\mathrm{cr}$

RANGE: ang

RANGE: $c 0$ I

RANGE: end

RANGE: Cag

herb

herb

herb

RANGE: $c r$

RANGE: cag

RANGE: end

subshrub

herb

herb

climber

$\mathrm{rb}$

ubshrub

herb

herb

subshrub

herb

shrub

RANGE: cag col cr ecu eur herb mex nag per sag ven

RANGE: bol cr per sag? herb

RANGE: af $r$

herb

RANGE: Sao WdW?

herb

RANGE: mex

RANGE: his nwg? per pr

herb

herb

herb

RANGE: COI

RANGE: $\mathrm{cr}$ ecu

ep iphyte 
TAXON

FAMILY

PROVINCES

ELEVATION (OOOM.)

\section{Hebec I in ium}

costaricense $K$. \& R.

*knappi i K. \& R.

macrophy I Ium (L.) DC.

reedil K. \& $R$.

\section{Helichrysum}

bracteatum (Vent.) Andr. cult,

\section{Heliopsis}

buphtha Imoides (Jacq,) Dun.

\section{Heterocondylus}

vitalbae (DC.) K. \& R.

\section{Hida Igoa}

ternata Llave \& Lex.

\section{Hieracium \\ absissum Less. \\ gronovii L.}

irasuense Benth.

Hypochoeris
radicata $L$.

radicata $L$. intr. nat.

\section{Ichthyothere} scandens Blake

I soca rpha

oppositifolia (L.) Cass.

*--var. achyranthes (DC.) Keil \& Stuessy

PROV: cc he lo 000m: 0-1

\section{Jaegeria}

hirta (Lag.) Less.

Jung ia

ferruginea L. $\mathrm{f}$.
PROV: $c c$ ch pa

000m: $0-1,1-2$

$\begin{array}{lr}\text { PROV: co pa } & 000 \mathrm{~m}: 0-1,1-2 \\ \text { PROV: da } & 000 \mathrm{~m}: 0-1 \\ \text { PROV: bo ca ch da lo } 000 \mathrm{~m}: 0-1 \\ \text { pa } \\ \text { PROV: da } & 000 \mathrm{~m}: 0-1 \\ \text { PROV: ch } & 000 \mathrm{~m}: 1-2 \\ \text { PROV: ch } & 000 \mathrm{~m}: 1-2\end{array}$

PROV: ca cc ch da he 000m: $0-1,1-2$ lo pa vr

000m: $0-1,1-2$

000m: $1-2,2-3$

000m: $0-1$

$000 \mathrm{~m}: 1-2,2-3,3+$

000m: $1-2$

000m: $1-2$

PROV: ch pa

\begin{tabular}{|c|c|c|c|c|c|c|}
\hline $\begin{array}{l}\text { RANGE: } \\
\text { RANGE: }\end{array}$ & $\begin{array}{l}\mathrm{Cr} \\
\text { end }\end{array}$ & & & & & $\begin{array}{c}\text { shrub } \\
\text { tree } \\
\text { herb }\end{array}$ \\
\hline $\begin{array}{c}\text { RANGE: } \\
\text { SaO } \\
\text { RANGE: }\end{array}$ & $\begin{array}{l}\text { cag } \\
\text { sao } \\
\text { end }\end{array}$ & gui & jam & $\operatorname{mex}$ & sag & $\begin{array}{l}\text { herb } \\
\text { shrub } \\
\text { subshrub }\end{array}$ \\
\hline & & $y^{4}$ & & & st & . \\
\hline RANGE: & $\operatorname{mex}$ & owg & & & & herb \\
\hline $\begin{array}{c}\text { RANGE: } \\
\text { per }\end{array}$ & $\begin{array}{l}\text { bol } \\
\text { ven }\end{array}$ & cag & col & ecu & $\operatorname{mex}$ & herb \\
\hline $\begin{array}{r}\text { RANGE: } \\
\text { sag? }\end{array}$ & $\begin{array}{l}\text { cag? } \\
\text { sao }\end{array}$ & ecu & gui & hon & per & $\begin{array}{l}\text { herb } \\
\text { shrub }\end{array}$ \\
\hline $\begin{array}{c}\text { RANGE: } \\
\text { per }\end{array}$ & $\begin{array}{l}\text { col } \\
\text { ven }\end{array}$ & $\mathrm{cr}$ & ecu & gua & mex & climber \\
\hline $\begin{array}{l}\text { RANGE: } \\
\text { RANGE: } \\
\text { RANGE: }\end{array}$ & $\begin{array}{l}\text { cag } \\
\text { hon } \\
\text { end }\end{array}$ & $\begin{array}{l}\operatorname{mex} \\
\text { nag }\end{array}$ & & & & $\begin{array}{l}\text { herb } \\
\text { herb } \\
\text { herb }\end{array}$ \\
\hline RANGE: & eur & 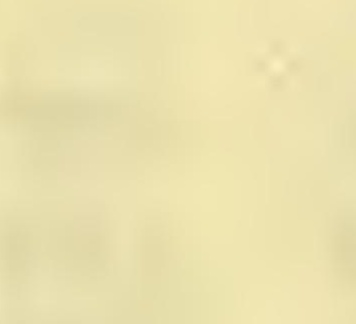 & & & 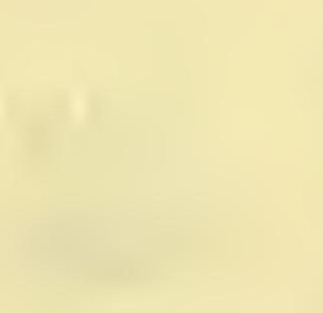 & herb \\
\hline RANGE: & col & int & (1) & & & shrub \\
\hline $\begin{array}{c}\text { RANGE: } \\
\text { jam }\end{array}$ & $\begin{array}{l}a b c \\
\text { mex }\end{array}$ & $\begin{array}{l}\text { ang } \\
\text { sag? }\end{array}$ & $\begin{array}{l}\text { cag } \\
\text { trt }\end{array}$ & $\begin{array}{l}\text { col } \\
\text { ven }\end{array}$ & gua & herb \\
\hline RANGE: & $\mathrm{cag}$ & mex & sag & sao & & herb \\
\hline
\end{tabular}

RANGE: per 
TAXON

\section{FAMILY}

PROV INCES

ELEVATION (OOOM.)

RANGE OUTSIDE OF PANAMA

HABIT

280 (184) COMPOSITAE cont.

KoanophyII on

dukei K. \& $R$.

panamense K. \& R.

PROV: ch 000m: $1-2$

PROV: bo ce pa

"pittieri (KIatt) K, \& R.

PROV: bo 000m: $0-1$

solldaginoides (H.B.K.) K. \& R.

PROV: ch pa

wetmorei (Rob.) K. \& R.

PROV: bo ca pa

000m: $0-1$

\section{*Laestadia}

*costaricensis Blake

PROV: ch

PROV: $\mathrm{ch}$

PROV: ch

*minuscula Cuatr.

panamensis Blake

\section{Lasianthaea}

fruticosa (L.) K.M. Becker

nowickeana $D^{\prime}$ Arcy

Leucanthemum

vulgare $L$.

intr. cult.

PROV: ce ch pa vr 000m: $0-1$

PROV: sa

000m: $0-1$

PROV: ch

000m: $0-1$

Li abum

bourgeaui Hieron.

*megacepha I um Schultz-Bip.

Lycoseris

latifolia (D. Don) Benth.

Me I ampodium

costaricense Stuessy

divaricatum (Rich.

paniculatum Gardn.

Melanthera

angustifolia A. Rich.

a spera (Jacq.) Sina II

--var, aspera
PROV: cc ch da 000m: $0-1,1-2$

PROV: ch

000m: $0-1$

PROV: ca ch da pa 000m: $0-1$

PROV: bo $\mathrm{ch} \quad$ 000m: 1 -2

PROV: bo ca cc da 10 000m: $0-1$

PROV: $\mathrm{Pa}$

000m: $2-3$

PROV: pa

000a: $0-1$

PROV: bo ca ce ch cn 000m: $0-1$ da lo pa sa vr

\begin{tabular}{|c|c|c|c|c|c|c|}
\hline $\begin{array}{l}\text { RANGE: } \\
\text { RANGE: } \\
\text { RANGE: }\end{array}$ & $\begin{array}{l}\mathrm{cr} \\
\mathrm{cr}\end{array}$ & & & & & $\begin{array}{l}\text { shrub } \\
\text { shrub } \\
\text { tree } \\
\text { shrub }\end{array}$ \\
\hline $\begin{array}{l}\text { RANGE: } \\
\text { mex }\end{array}$ & $\begin{array}{l}\text { cag } \\
\text { per }\end{array}$ & $\begin{array}{l}\text { cr } \\
\text { sag? }\end{array}$ & ecu & ga I & gua & $\begin{array}{l}\text { shrub } \\
\text { subshrub }\end{array}$ \\
\hline RANGE: & $\mathrm{cr}$ & & & & & $\begin{array}{l}\text { shrub } \\
\text { tree }\end{array}$ \\
\hline RANGE: & $\mathrm{cr}$ & Y & 't 't & 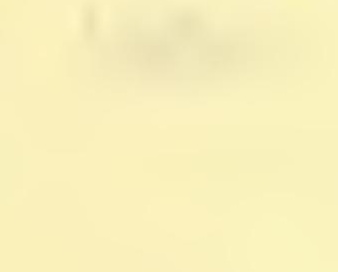 & $2=$ & herb \\
\hline $\begin{array}{l}\text { RANGE: } \\
\text { RANGE: }\end{array}$ & $\begin{array}{l}\text { end } \\
\text { end }\end{array}$ & 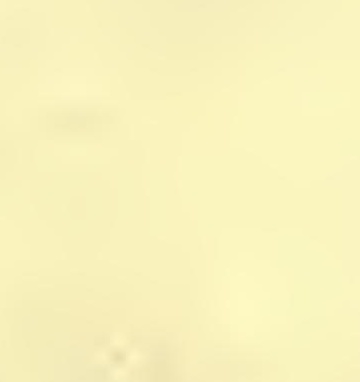 & & & & $\begin{array}{l}\text { herb } \\
\text { herb }\end{array}$ \\
\hline $\begin{array}{l}\text { RANGE: } \\
\text { RANGE: }\end{array}$ & $\begin{array}{l}\text { cag } \\
\text { end }\end{array}$ & er & $\operatorname{mex}$ & ven & & $\begin{array}{c}\text { shrub } \\
\text { tree } \\
\text { herb }\end{array}$ \\
\hline RANGE: & owg & 1 & & & & herb \\
\hline RANGE: & $\operatorname{mex}$ & & & & & herb \\
\hline RANCE: & col & mex & ter & & & herb \\
\hline RANGE: & cag? & col & gua & ven & & shrub \\
\hline RANCE: & er & & & & & herb \\
\hline $\begin{array}{c}\text { RANGE: } \\
\text { ven }\end{array}$ & cag & col & $\operatorname{mex}$ & OWt & sao & herb \\
\hline $\begin{array}{l}\text { RANGE: } \\
\text { mex }\end{array}$ & $\begin{array}{l}\text { cag } \\
\text { sai }\end{array}$ & $\begin{array}{l}\text { col } \\
\text { sao }\end{array}$ & $\mathrm{er}$ & gua & hon & herb \\
\hline $\begin{array}{c}\text { RANice: } \\
\text { his }\end{array}$ & $\begin{array}{l}\text { bah } \\
\text { hon }\end{array}$ & $\begin{array}{l}\text { cr } \\
\text { eex }\end{array}$ & cub & fia & gus & herb \\
\hline $\begin{array}{c}\text { RANGE: } \\
\text { sat: }\end{array}$ & $\begin{array}{l}\text { bah } \\
\text { ven }\end{array}$ & cag & cub & fia & $m=t$ & herb \\
\hline
\end{tabular}


TAXON

280 (184) COMPOSITAE cont.

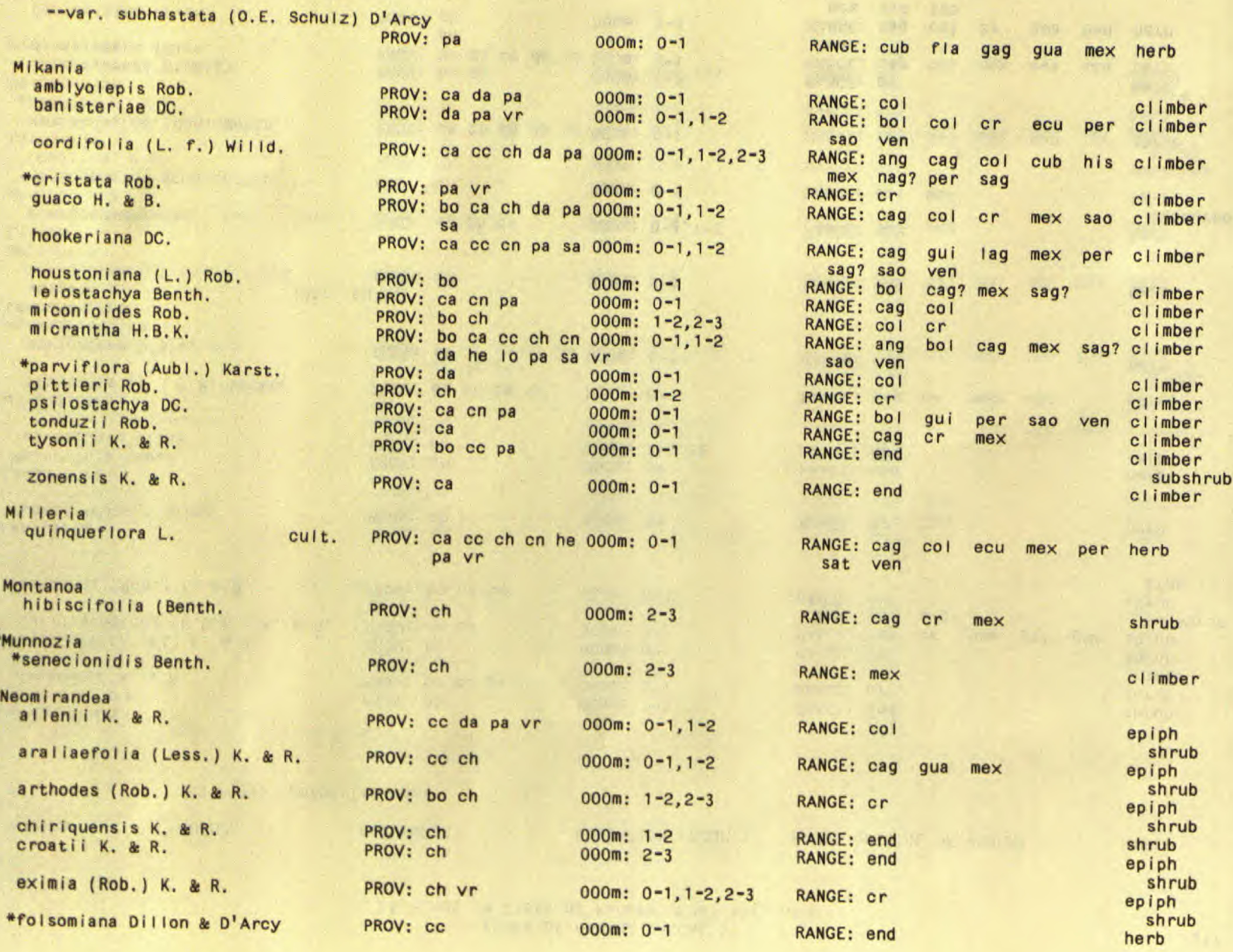


280 (184) COMPOSITAE cont.

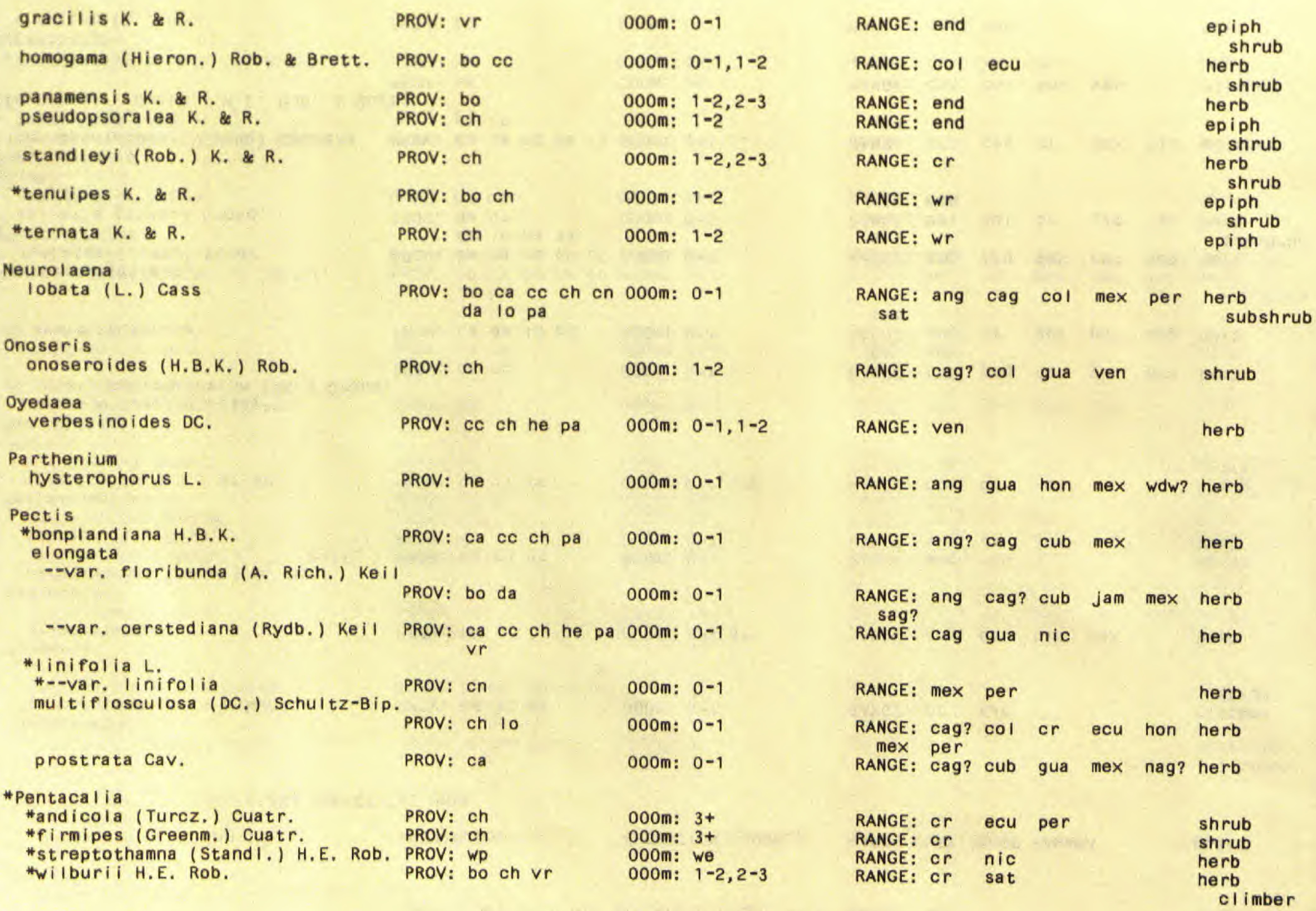


TAXON

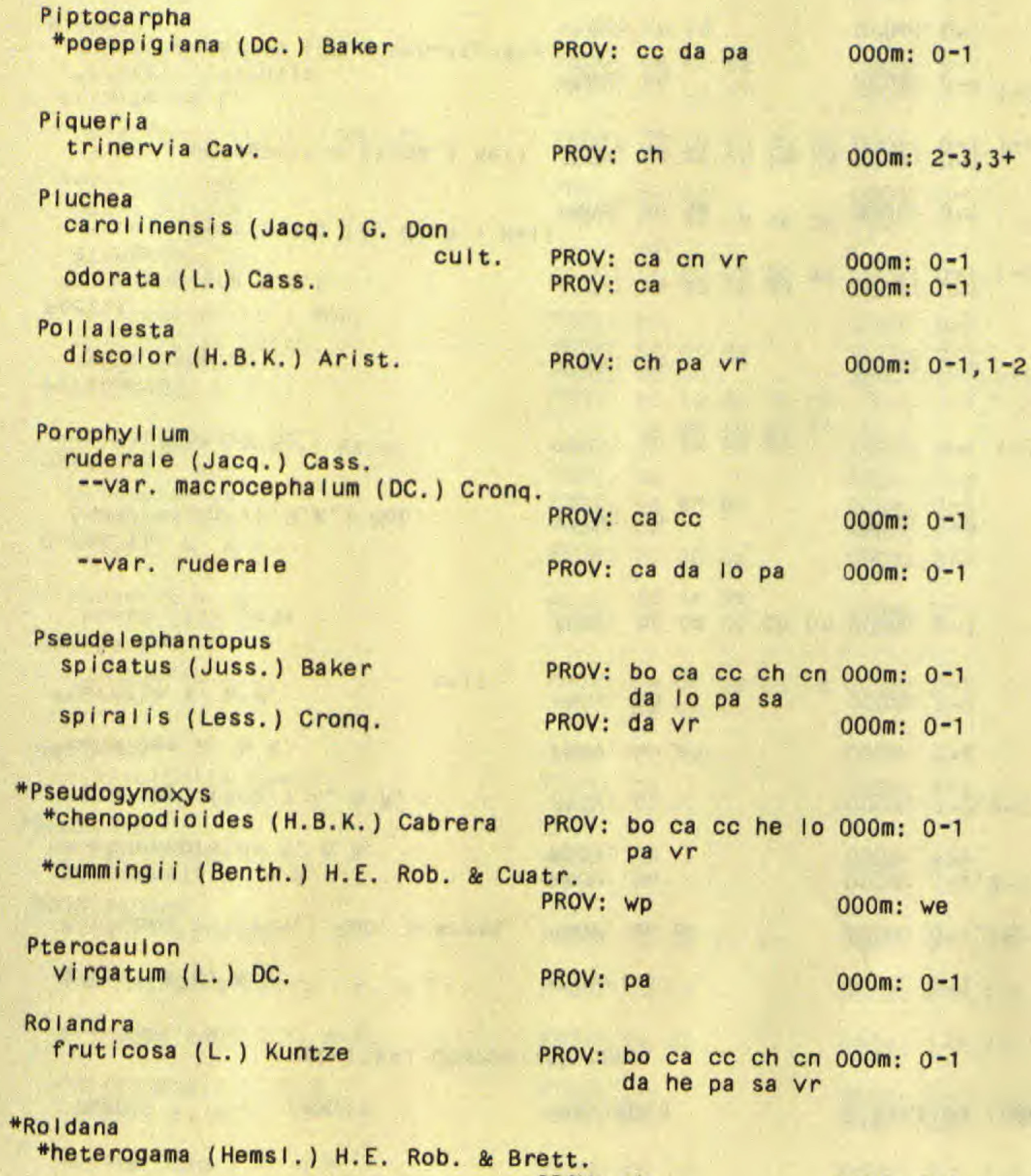

\section{*Pseudogynoxys}

*chenopodioides (H.B.K.) Cabrera PROV: bo ca cc he lo 000m: 0-1 * cummingi i (Benth.) H.E. Rob. \& Cuatr. pa Vr

PROV: wp 000m: we

Pterocaulon

virgatum (L.) DC.

PROV: pa

000m: $0-1$

Rol andra

fruticosa (L.) Kuntze

PROV: bo ca cc ch cn $000 \mathrm{~m}$ : $0-1$ da he pa sa $v r$

\section{*RoIdana}

*heterogama (Hems I.) H.E. Rob. \& Brett.

PROV: ch

000m: 2-3

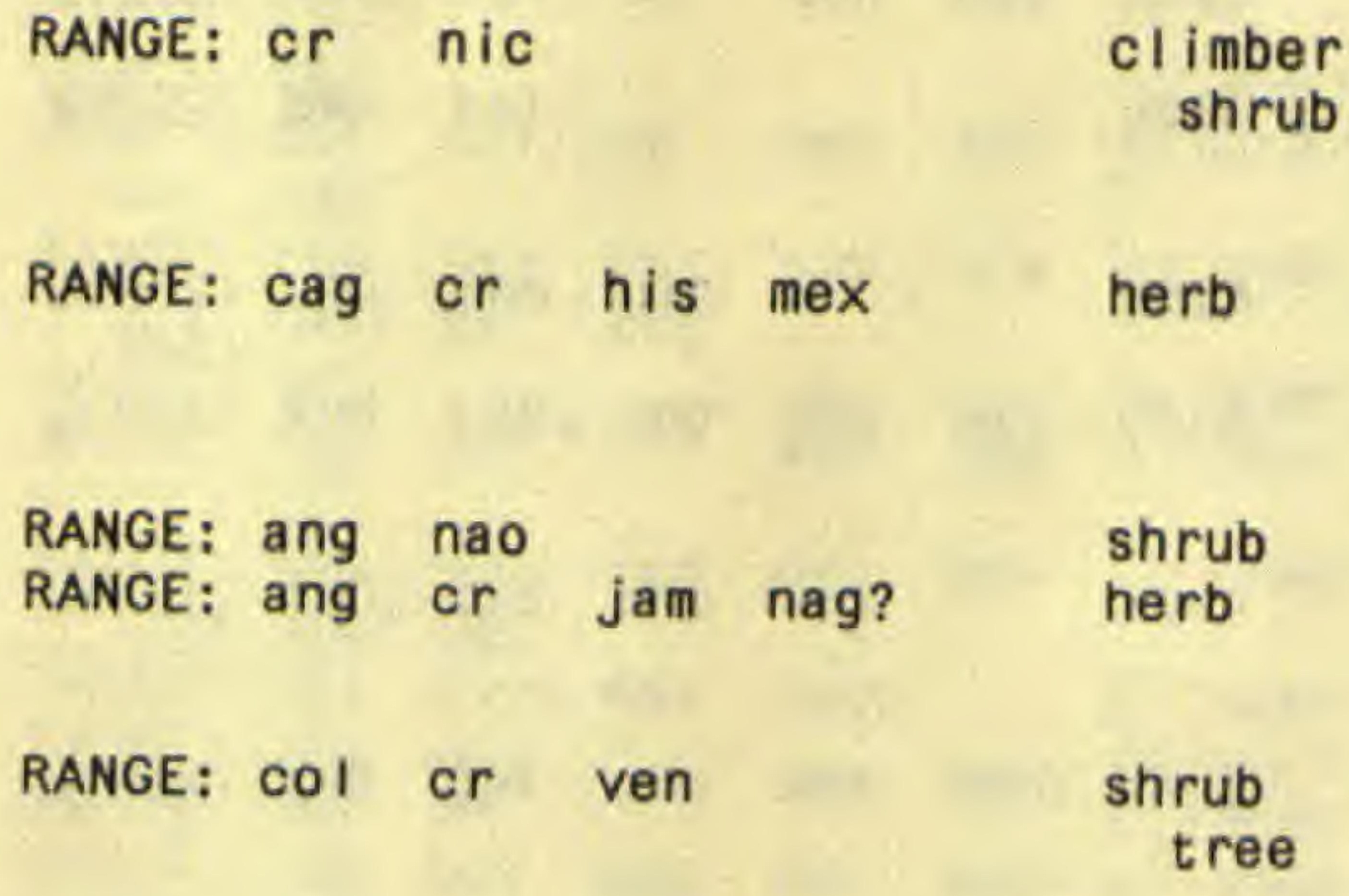

RANGE: $c r$

subshrub 
TAXON

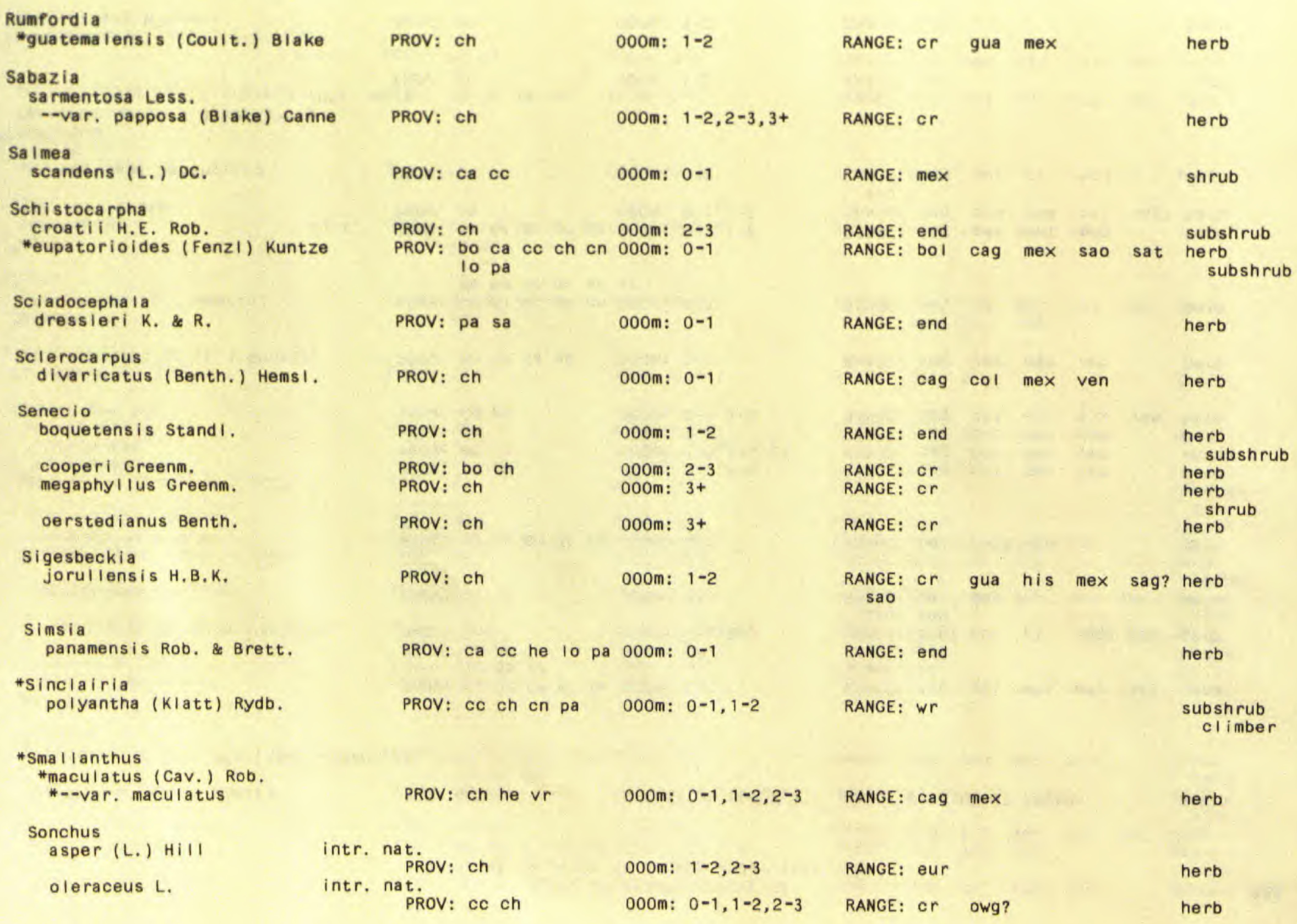


TAXON
FAMILY
PROVINCES

ELEVATION (000M.)

RANGE OUTSIDE OF PANAMA

HABIT

280 (184) COMPOSITAE cont.

Spl Ianthes

a lba L'Her.

diffusa P. \& E.

oppositifolia (Lam.) D'Arcy

\section{Spi racantha}

cornifolia H.B.K.

\section{Stevia \\ caracasana DC. \\ Iucida Lag. \\ ovata Willd. \\ trifiora DC.}

\section{Struchium}

sparganophorum (L.) Kuntze

\section{Synedrella}

nodiflora (L.) Gaertn.

\section{Tagetes}

erecta $L$.

filifolia Lag.

cult.

microglossa Benth. paniculata DC.

PROV: ca cc cn da he 000m: 0-1

lo pa vr

PROV: cc he

PROV: $c h$

000m: $0-1$

PROV: ca da

$000 \mathrm{~m}: 1-2,2-3$

000m: $0-1$

PROV: ca cc cn da he 000m: $0-1$ pa

PROV: $\mathrm{cc}$ ch

PROV: ch

PROV: ch

PROV: ch pa

000m: $0-1,1-2$

000m: $1-2,2-3,3+$

000m: $1-2$

$000 \mathrm{~m}: 0-1,1-2$

PROV: bo ca da pa $000 \mathrm{~m}$ : $0-1$

PROV: bo ca cc ch cn 000m: $0-1$

da he lo pa sa vr

PROV: bo ca ce ch pa 000m: $0-1,1-2$

PROV: $\mathrm{ch} \quad 000 \mathrm{~m}: 0-1,1-2$

PROV: ch vr

000m: $1-2$

\section{"Tanacetum}

"parthenium (L.) Schultz-Bip.

intr. cult.

PROV: ch 000m: $1-2$

PROV: ch

000m: $1-2$

$000 m: 1-2,2-3$

PROV: ch

PROV: ch da

\begin{tabular}{|c|c|c|c|c|c|c|}
\hline $\begin{array}{c}\text { RANGE: } \\
\text { ven }\end{array}$ & cag & col & $\operatorname{mex}$ & per & sag & herb \\
\hline RANGE: & per & & & & & herb \\
\hline RANGE: & cag? & $\mathrm{col}$ & er & nag? & nao & herb \\
\hline $\begin{array}{r}\text { sag? } \\
\text { RANGE: }\end{array}$ & $\begin{array}{l}\text { sao } \\
\text { bol }\end{array}$ & owg & & & & herb \\
\hline w & & & & nat & 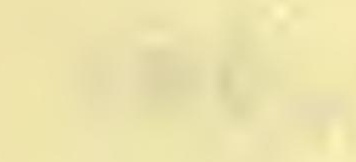 & \\
\hline RANGE: & bel & cag? & ven & & & herb \\
\hline RANGE: & cag & col & $\operatorname{mex}$ & ven & & herb \\
\hline RANGE: & cag & col & $\operatorname{mex}$ & ven & & \\
\hline RANGE: & cag & ecu & $\operatorname{mex}$ & ven & & erb \\
\hline RANGE: & cag & $\mathrm{col}$ & ecu & mex & ven & herb \\
\hline RANGE: & ang & cag & mex & sat & & herb \\
\hline RANGE: & $w w t$ & & & & & herb \\
\hline RANGE: & cag & $\operatorname{mex}$ & owt & sag & & herb \\
\hline $\begin{array}{c}\text { RANGE: } \\
\text { Sao }\end{array}$ & cag & ecu & mex & per & sag? & boun \\
\hline RANGE: & cag & col & $\mathrm{cr}$ & ecu & & herb \\
\hline
\end{tabular}

RANGE: eur

herb

RANGE: WdW

herb

RANGE: end

shrub

tree

RANGE: per tree shrub 


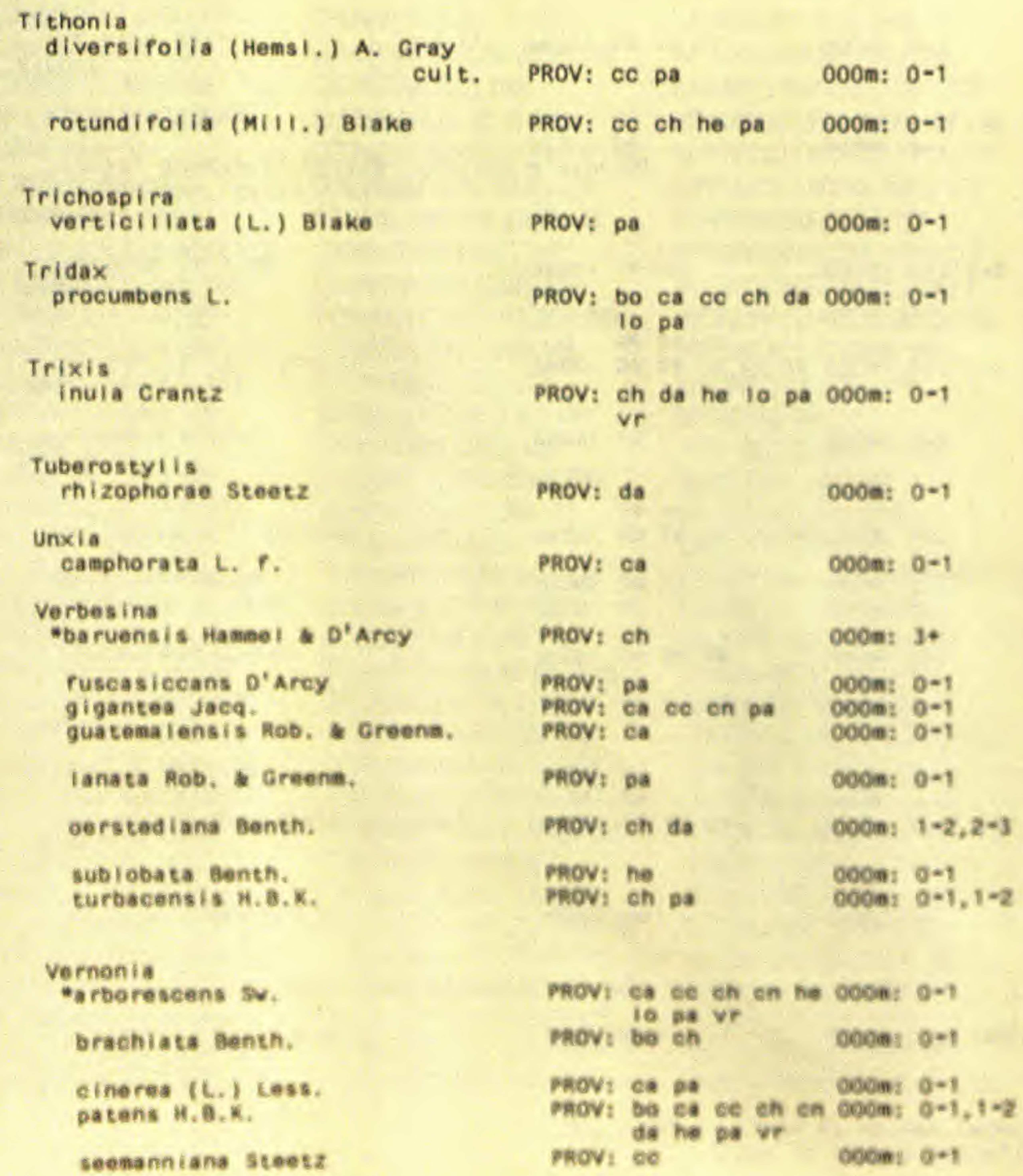

\begin{tabular}{|c|c|c|c|c|c|c|}
\hline $\begin{array}{c}\text { RANGE: } \\
\text { ven }\end{array}$ & ang & cag & gag & his & $\operatorname{mex}$ & herb \\
\hline $\begin{array}{c}\text { RANGE: } \\
\text { sat }\end{array}$ & ang & cag & col & $\operatorname{mex}$ & sao & herb \\
\hline RANGE: & $n w t$ & & & & ( & herb \\
\hline RANGE: & cag & col & $\operatorname{sex}$ & von? & & nerb \\
\hline $\begin{array}{c}\text { RANGE: } \\
\text { JaA }\end{array}$ & $\begin{array}{l}\text { ang } \\
\text { sex }\end{array}$ & $\begin{array}{l}\operatorname{cag} \\
\operatorname{tex}\end{array}$ & $\begin{array}{l}\text { col } \\
\text { ven }\end{array}$ & cub & gua & herb \\
\hline RAMCE: & col & ecu & & & & shrub \\
\hline RuNGe: & col & gui & $5 a 0$ & von & & herb \\
\hline RANCE: & end & & & & & $\begin{array}{c}\text { clinber } \\
\text { tree }\end{array}$ \\
\hline Ruce: & and & & & & & shrub \\
\hline RANCE: & $\cos$ & col & his & $\operatorname{mox}$ & & herb \\
\hline RANGE: & gua & han & nie & & & $\begin{array}{l}\text { herb } \\
\text { shrub }\end{array}$ \\
\hline $\begin{array}{c}\text { Mavot: } \\
\text { nio }\end{array}$ & bet & or & gus & hen & $\operatorname{sex} x$ & $\begin{array}{l}\text { shrub } \\
\text { tres }\end{array}$ \\
\hline RARGE: & er & 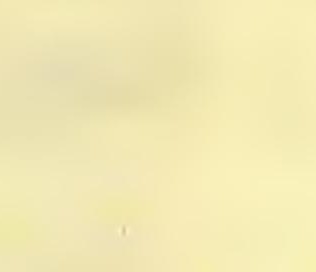 & & & 1 & $\begin{array}{l}\text { shrub } \\
\text { tree }\end{array}$ \\
\hline $2 \times w b t_{1}$ & gua & & & & & shros \\
\hline Rarse: & $\cos$ & col & $n \in x$ & $39 t$ & ven & $\begin{array}{l}\text { ahrvo } \\
\text { tres }\end{array}$ \\
\hline Roct: & $\operatorname{cag}$ & mex & ver & $\operatorname{seg}$ & & shrve \\
\hline DNGEI & er & & & & & $\begin{array}{l}\text { herb } \\
\text { shrub }\end{array}$ \\
\hline Wege: & $\cos$ & fis & nex & ove & 849 & nerb \\
\hline WAGts & $\cos$ & $\operatorname{nex} x$ & 309 & & & $\begin{array}{l}\text { thrus } \\
\text { tres }\end{array}$ \\
\hline
\end{tabular}


ACANTHACEAE (ACA) 284 AIZOACEAE (AIZ) 117 ALISMATACEAE (ALI) 2 AMARANTHACEAE (AMA) 116 AMARYLLIDACEAE (AML) 49 ANACARDIACEAE (ANA) 148 ANNONACEAE (ANN) 122 APOCYNACEAE (APO) 243 AQUIFOLIACEAE (AQF) 175 ARACEAE (ARA) 30 ARALIACEAE (ARL) 226 ARAUCARIACEAE (ARU) 1 ARISTOLOCHIACEAE (ARS) 112 ASCLEPIADACEAE (ASC) 247 AVICENNIACEAE (AVI) 259 BALANOPHORACEAE (BNP) 111 BALSAMINACEAE (BLS) 183 BASELLACEAE (BAS) 118 BATACEAE (BAT) 117 BEGONIACEAE (BEG) 206 BERBERIDACEAE (BER) 120 BETULACEAE (BET) 102 BIGNONIACEAE (BIG) 270 BIXACEAE (BIX) 200 BOMBACACEAE (BOM) 190 BORAGINACEAE (BOR) 252 BROMELIACEAE (BML) 41 BRUNELLIACEAE (BNL) 133 BURMANNIACEAE (BMN) 57 BURSERACEAE (BRS) 160 BUTOMACEAE (BUT) 3 BUXACEAE (BUX) 148 CACTACEAE (CAC) 206 CALLITRICHACEAE (CLL) 148 CAMPANULACEAE (CAM) 308 CANNACEAE (CNA) 55 CAPPARACEAE (CPP) 130 CAPRIFOLIACEAE (CPR) 305 CARICACEAE (CRC) 205
CARYOCARACEAE (CCR) 194 CARYOPHYLLACEAE (CRY) 118 CASUARINACEAE (CAS) 93 CELASTRACEAE (CEL) 176 CERATOPHYLLACEAE (CTP) 119 CHENOPODIACEAE (CHN) 114 CHLORANTHACEAE (CLR) 100 CISTACEAE (CIS) 199 CLETHRACEAE (CLE) 229 COMBRETACEAE (CMB) 212 COMMELINACEAE (CMM) 46 COMPOSITAE (CMP) 310 CONNARACEAE (CNN) 134 CONVOLVULACEAE (CNV) 249 CORIARIACEAE (CRR) 148 CORNACEAE (COR) 228 CRASSULACEAE (CRS) 132 CRUCIFERAE (CRU) 129 CUCURBITACEAE (CUC) 306 CUNONIACEAE (CUN) 133 CYCADACEAE (CCD) 1 CYCLANTHACEAE (CYC) 28 CYPERACEAE (CYP) 18 CYRILLACEAE (CYR) 175 DICHAPETALACEAE (DCH) 167 DILLENIACEAE (DLL) 192 DIOSCOREACEAE (DSC) 50 DROSERACEAE (DRS) 132 EBENACEAE (EBN) 239 ELAEOCARPACEAE (ELC) 184 ERICACEAE (ERI) 229 ERIOCAULACEAE (ERO) 40 ERYTHROXYLACEAE (ERX) 157 EUPHORBIACEAE (EUP) 168 FAGACEAE (FAG) 102 FLACOURTIACEAE (FLC) 201 FUMARIACEAE (FUM) 129 GENTIANACEAE (GEN) 241
GERANIACEAE (GER) 155 GESNERIACEAE (GSN) 276 GNETACEAE (GNE) 1 GRAMINEAE (GRM) 4 GUTTIFERAE (GUT) 196 HAEMODORACEAE (HAE) 49 HALORAGACEAE (HAL) 226 HAMAMELIDACEAE (HAM) 133 HERNANDIACEAE (HRN) 128 HIPPOCASTANACEAE (HCS) 179 HIPPOCRATEACEAE (HPC) 177 HUMIRIACEAE (HOU) 157 HYDROCARYACEAE (HCY) 215 HYDROCHARITACEAE (HDC) 3 HYDROPHYLLACEAE (HYD) 252 HYPERICACEAE (HYP) 199 ICACINACEAE (ICC) 178 IRIDACEAE (IRI) 51 JUGLANDACEAE (JUG) 101 JUNCACEAE (JUN) 48 JUNCAGINACEAE (JCG) 2 LABIATAE (LAB) 259 LACISTEMATACEAE (LCS) 101 LAURACEAE (LAU) 126 LECYTHIDACEAE (LCY) 210 LEGUMINOSAE (LEG) 136 LEMNACEAE (LMN) 39 LENTIBULARIACEAE (LNT) 253 LILIACEAE (LIL) 48 LINACEAE (LIN) 157 LOASACEAE (LOA) 206 LOGANIACEAE (LOG) 241 LORANTHACEAE (LOR) 108 LYTHRACEAE (LYT) 210 MAGNOLIACEAE (MAG) 122 MALPIGHIACEAE (MLP) 164 MALVACEAE (MLV) 186 MARANTACEAE (MRN) 55 MARCGRAVIACEAE (MRC) 194
MARTYNIACEAE (MAR) 276 MAYACACEAE (MAY) 40 MELASTOMATACEAE (MLS) 215 MELIACEAE (MEL) 161 MENISPERMACEAE (MNS) 120 MENYANTHACEAE (MNY) 243 MONIMIACEAE (MNM) 125 MORACEAE (MOR) 103 MORINGACEAE (MRG) 131 MUSACEAE (MUS) 51 MYRICACEAE (MYR) 101 MYRISTICACEAE (MYS) 124 MYRSINACEAE (MRS) 233 MYRTACEAE (MRT) 213 NAJADACEAE (NAJ) 2 NYCTAGINACEAE (NYC) 116 NYMPHAEACEAE (NYM) 119 OCHNACEAE (OCH) 194

OLACACEAE (OLC) 111

OLEACEAE (OLE) 240

ONAGRACEAE (ONA) 224

PASSIFLORACEAE (PAS) 204

PEDALIACEAE (PED) 276 PHYTOLACCACEAE (PHT) 117 PIPERACEAE (PIP) 93

PLANTAGINACEAE (PTG) 288

PLUMBAGINACEAE (PLB) 237 PODOCARPACEAE (PDC) 1 PODOSTEMACEAE (PDS) 132 POLEMONIACEAE (PLM) 251 POLYGALACEAE (PGL) 166 POLYGONACEAE (PLG) 112 PONTEDERIACEAE (PON) 47 PORTULACACEAE (POR) 118 POTAMOGETONACEAE (POT) 2 PRIMULACEAE (PRM) 237 PROTEACEAE (PRT) 108 QUIINACEAE (QII) 196 RAFFLESIACEAE (RAF) 112
RANUNCULACEAE (RAN) 120 RAPATEACEAE (RPT) 41 RESEDACEAE (RSD) 131 RHAMNACEAE (RHM) 183 RHIZOPHORACEAE (RHZ) 211 ROSACEAE (ROS) 134 RUBIACEAE (RUB) 288 RUTACEAE (RUT) 158 SABIACEAE (SAB) 182 SALICACEAE (SAL) 101 SAPINDACEAE (SAP) 179 SAPOTACEAE (SPT) 237 STYRACACEAE (STY) 239 SYMPLOCACEAE (SYM) 240

TACCACEAE (TAC) 50 THEACEAE (TEA) 196 THEOPHRASTACEAE (TEO) 233 THYMELAEACEAE (THY) 209

TILIACEAE (TIL) 184 TOVARIACEAE (TOV) 129 TRIGONIACEAE (TRG) 166 TRIURIDACEAE (TRI) 4 TROPAEOLACEAE (TRP) 157 TUhivERACEAE (TNR) 204 TYPHACEAE (TYP) 2 ULMACEAE (ULM) 102 UMBELLIFERAE (UMB) 227 URTICACEAE (URT) 106 VALERIANACEAE (VAL) 305 VELLOZIACEAE (VLL) 50 VERBENACEAE (VRB) 253 VIOLACEAE (VIO) 201 VITACEAE (VIT) 184 VOCHYSIACEAE (VOC) 166 WINTERACEAE (WIN) 122 XYRIDACEAE (XYR) 40 ZINGIBERACEAE (ZIN) 53 ZYGOPHYLLACEAE (ZYG) 158 


abc ... Aruba, Bonaire, Curaçao
afr... Africa
ang... Antilles (general)
asi... Asia
bah. .. Bahamas
bel .. Belize
bol ... Bolivia
cag .. Central America (general)
cal... Calitornia
col... Colombia
cr ... Costa Rica
cub .. Cuba

ecu .. Ecuador

end . endemic to Panama

eur ., Florida

exp ... to be expected in Panama

fla ... Florida

gag ... Greater Antilles (general)

gal .. Galapagos

gua .. Guatemala

gui ... Guianas

his... Hispaniola

hon .. Honduras

jam .. Jamaica

\author{
lag ... Lesser Antilles (general) \\ mex. . Mexico \\ nag .. North America \\ nao .. U.S.A. \\ nas .. Southern United States \\ nic... Nicaragua \\ not .. not in Panama \\ nwg .. New World (general) \\ nwt .. New World (tropical) \\ oce ... Oceania (incl. Australia) \\ owg .. Old World general \\ owt .. Old World tropical
}

\section{SYMBOLS FOR OCCURRENCES OUTSIDE OF PANAMA}

\section{PANAMA}

endemic

to be expected in Panama

not in Panama

\section{CENTRAL AMERICA}

Costa Rica .

Nicaragua . . . .....

El Salvador .............

Guatemala ............

Central America general

ral. cag

\section{ELEVATION SYMBOLS}

0 . below $1000 \mathrm{~m}$

1. $1,000-2,000 \mathrm{~m}$

3. $3,000 \mathrm{~m}$ and over

we. ,elevation not available wdt

$w d w$ Wr
2. $2,000-3,000 \mathrm{~m}$

\section{BY REGION.}

\section{ANTILLES}

Bahamas .......... bah

Cuba............. cub

Hispaniola ........... his

Puerto Rico......... pr pr

Trinidad, Tobago ...... trt

Aruba, Bonaire, Curaçao abc

Greater Antilles general. gag

Lesser Antilles general . lag

Antilles general....... ang

\section{OLD WORLD}

Africa ......... afr

Europe ........ eur

Asia .......... as

Oceania ......... oce

Old World general owg

Old World tropical owt

(North America other)

(North America general,

includes Canada)

\section{GENERAL}

Pantropical

(worldwide, tropical)

Cosmopolitan (worldwide)

Without range ......... Wr
NEW WORLD

New World tropical nWt New World general nwg 
LIST OF FAMILIES

Family Name

Acronym Dalla (Flora Family Name

(Weber Torre of

1982) \& Harms Panama)

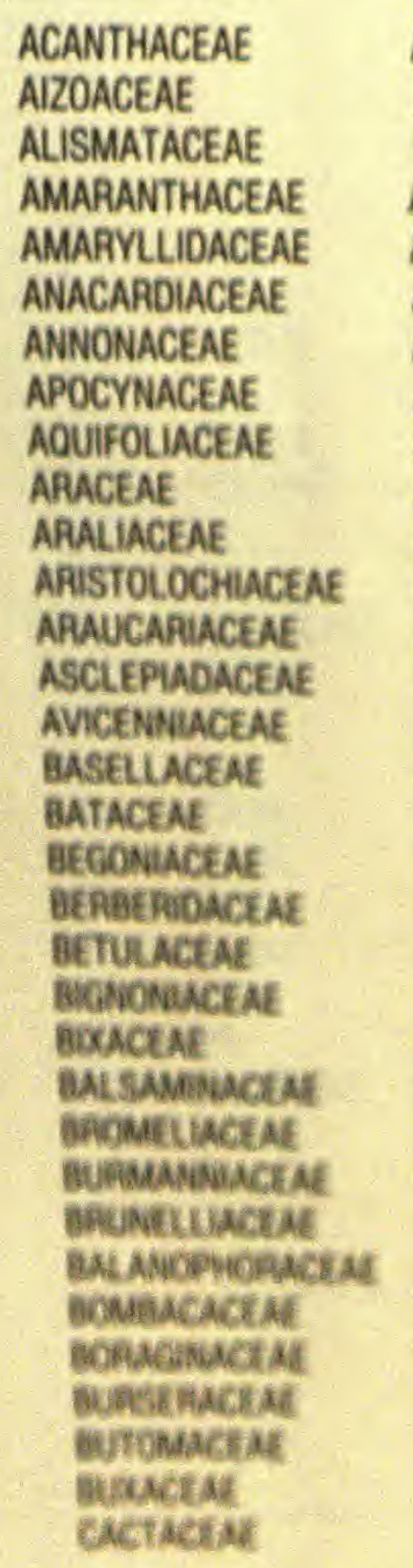

Acronym Dalla (Fora Family Name

Weber Torre of

1982) \& Harms Panama)
Acronym Dalla (Flora

(Weber Torre of

1982) \& Harms Panama)

\begin{tabular}{|c|c|c|c|c|c|}
\hline . & 266 & (177) & CAMPANULACEAE & CAM & 276 \\
\hline$A I Z$ & 84 & (57) & CASUARINACEAE & CAS & 51 \\
\hline ALI & 15 & (4) & CYCADACEAE & $\mathrm{CCO}$ & 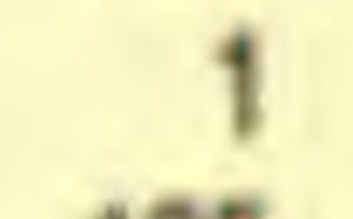 \\
\hline AMA & 79 & (53) & COCHLOSPERMACEAE & $\mathrm{CCH}$ & 195 \\
\hline ML & 40 & (24) & CARYOCARACEAE & CCA & 183 \\
\hline ANA & 153 & (101) & CELASTRACEAE & CEL & 158 \\
\hline ANN & 98 & (67) & CHENOPODLACEAE & CHN & 78 \\
\hline APO & 247 & (162) & CISTACEAE & CAS & 190 \\
\hline AQf & 157 & (102) & CLETHRACEAE & CLE & 230 \\
\hline ARA & 23 & (11) & CALUTRICHACEAE & $\mathrm{Cu}$ & 148 \\
\hline ARL & 227 & (144) & CHLOPANTHACEAE & QA & 54 \\
\hline ARS & 74 & (50) & COMBRETACEAE & CNE & 221 \\
\hline ARU & 6 & $(-)$ & COMMELANACEAE & CMM & 30 \\
\hline ASC & 248 & (163) & COMPOSITAE & CMP & 280 \\
\hline AVI & 2539 & (168a) & CANWACEAE & OU & 47 \\
\hline aAs & 6 & (589) & CONМИARACEAE & CaN & 127 \\
\hline BAT & 81 & (55) & CONVOUVULACEAE & CaN & 269 \\
\hline BEG & 206 & (i3) & CORALACEA & $\cos$ & $2 \pi$ \\
\hline aEA & $\boldsymbol{0}$ & (6) & CAPPARACEAE & $\cos$ & 107 \\
\hline Bet & 81 & (40) & CAPREOLUCEAE & $\mathrm{cm}$ & 21 \\
\hline gig & sa & (in) & vacucene & $c x$ & 205 \\
\hline$B x$ & 194 & (125) & COPLARACEA & cy & 151 \\
\hline 25 & 16 & (110) & CUSSUUACLE & $\cos$ & ins \\
\hline Bast. & $x$ & in) & chucirtat & Gey & 160 \\
\hline enis & 6 & an & chmoeminctae & CAV & \\
\hline Bes. & 16 & (a) & GReTomiacrae & ct? & \\
\hline 6 & $n$ & 16 & accuentacrac & $d x$ & $2 n$ \\
\hline Gous & (II) & (6) & CWNondaces & an & 100 \\
\hline 03 & aia & (xen) & crountracese & cre & \\
\hline 5 & 68 & (9) & CWRACIAL & cy & \\
\hline Wh & 4 & (9) & cyeisucrac & Con & 134 \\
\hline ast & 16 & कin & achortasia & Cos & 浀 \\
\hline ac: & 260 & cise & cuirvidial & ai & 策 \\
\hline
\end{tabular}

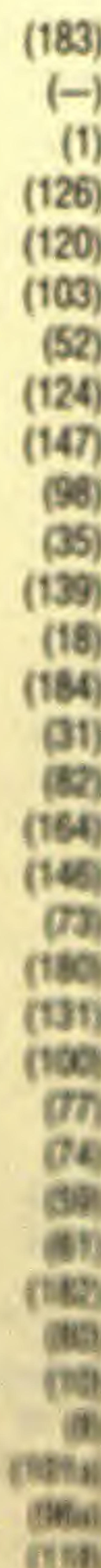

(183)

DROSERACEAE

DIOSCOREACEAE

EBENACEAE

ELAEOCARPACEAE

ERICACEAE

ERIOCAULACEAE

ERYTHROXYLACEAE

EUPHORBIACEAE

FAGACEAE

PLACOURTIACEAE

GEMTLUACEAE

GERADAACEAE

GNETACEAE

GRMAOVEAE

oESNERUCER

cumrrerue

HAEMCOONCEAE

Herongacere

HEPOCASTAUCEA

mbHoCUASTACE

Hansexcede

Herockurtactue

iemunouctut

mososmuctue

imiacecte

cachuctas

Acactse

resicicter

senceste

basure

iresaciat

Mebrtwatacest

ifertatcaceas
DRS 112

DSC 43

EBN 240

ELC 171

ERI 233

ERO 30

ERX 134

EUP 147

FAG 62

FC 199

GEN 246

GER 129

Gie

cas.

GS4

ext

WAE

He.

nos

$10 \mathrm{C}$

nov

noc

now

Mro

mis

cx:

at

ixs

an

Ua

Bas

ICs

IEr (76a)

(15)

$(160,161)$

(7)

(175)

(123)

(10)

(10)

(S)

972

(106)

oti

(ist)

(t)ase

(ด)

(9)

(3)

chin

(1)

(9)

(ist) 


\section{LIST OF FAMILIES (continued)}

\begin{tabular}{|c|c|c|c|c|c|c|c|c|c|c|c|}
\hline Family Name & $\begin{array}{c}\text { Acronym } \\
\text { (Weber } \\
1982)\end{array}$ & $\begin{array}{l}\text { Dalla } \\
\text { Torre } \\
\text { \& Harms }\end{array}$ & $\begin{array}{c}\text { (Flora } \\
\text { of } \\
\text { Panama) }\end{array}$ & Family Name & $\begin{array}{c}\text { Acronym } \\
\text { (Weber } \\
1982 \text { ) }\end{array}$ & $\begin{array}{l}\text { Dalla } \\
\text { Torre } \\
\text { \& Harms }\end{array}$ & $\begin{array}{c}\text { (Flora } \\
\text { of } \\
\text { Panama) }\end{array}$ & Family Name & $\begin{array}{c}\text { Acronym } \\
\text { (Weber } \\
1982)\end{array}$ & $\begin{array}{l}\text { Dalla } \\
\text { Torre } \\
\text { \& Harms }\end{array}$ & $\begin{array}{c}\text { (Flora } \\
\text { of } \\
\text { Panama) }\end{array}$ \\
\hline LEGUMINOSAE & LEG & 128 & (83) & OLEACEAE & OLE & 243 & (158) & RUTACEAE & RUT & 137 & (89) \\
\hline LILIACEAE & LIL & 38 & $(21,22)$ & ONAGRACEAE & ONA & 224 & (142) & CEAE & SAB & 166 & (109) \\
\hline LEMNACEAE & LMN & 24 & (12) & OPILIACEAE & OPI & 71 & (47) & SALICACEAE & SAL & 56 & (37) \\
\hline LENTIBULARIACEAE & LNT & 264 & (176) & ORCHIDACEAE & ORC & 50 & (33) & SAPINDACEAE & SAP & 165 & (108) \\
\hline LOASACEAE & LOA & 206 & (132) & OROBANCHACEAE & ORO & 261 & (174) & SAXIFRAGACEAE & SAX & 117 & (79) \\
\hline LOGANIACEAE & LOG & 245 & (159) & OXALIDACEAE & $0 X \mathrm{~L}$ & 130 & (84) & SCROPHULARIACEAE & SCR & 257 & (171) \\
\hline LORANTHACEAE & LOR & 67 & (46) & PALMAE & PAL & 21 & (9) & BACEAE & SMR & 138 & (90) \\
\hline LYTHR & LYT & 216 & (136) & PAPAVERACEAE & PAP & 104 & (72) & SO & SOL & 256 & (170) \\
\hline MAGNOLIACEAE & MAG & 95 & (65) & PASSIFLORACEAE & PAS & 203 & (130) & & SPT & 239 & (154) \\
\hline MARTYNIACEAE & MAR & 260 & $(-)$ & PODOCARPACEAE & PDC & 5 & 2) & ACEAE & STP & 161 & (105) \\
\hline MAYACACEAE & MAY & 28 & (13) & MACEAE & PDS & 113 & (78) & CEAE & STR & 178 & (117) \\
\hline MELIACEAE & MEL & 140 & (92) & CEAE & PED & 259 & (173) & CEAE & STY & 241 & (156) \\
\hline MALPIGHIACEAE & MLP & 141 & (93) & ACEAE & PGL & 145 & (96) & ACEAE & SYM & 242 & (157) \\
\hline MELASTOMATACEAE & MLS & 223 & (141) & ACEAE & PHT & 83 & (56) & & TEA & 186 & (122) \\
\hline MALVACEAE & MLV & 175 & (115) & & PIP & 53 & (34) & ASTACEAE & TEO & $135 a$ & (151) \\
\hline MONIMIACEAE & MNM & 101 & (69) & NACEAE & PLB & 238 & (153) & AEACEAE & THY & 214 & (135) \\
\hline ACEAE & MNS & 94 & (64) & CEAE & PLG & 77 & (51) & & TIL & 174 & (114) \\
\hline HACEAE & MNY & $546 a$ & (161) & EAAE & PLM & 250 & (165) & CEAE & TNR & 201 & (129) \\
\hline MORA & MOR & 64 & (43) & ACEAE & PON & 34 & (19) & & TOV & 106 & (73a) \\
\hline VIACEAE & MRC & 184 & (121) & ACEAE & POR & 85 & $(58$ & TF & TRG & 142 & (94) \\
\hline MORING & MRG & 109 & (76) & ETONACEAE & POT & 11 & $(3 a$ & & TRI & 18 & (6) \\
\hline MARAI & MRN & 48 & (32) & PRII & PRM & 237 & $(15$ & ACEAE & TRP & 131 & (86) \\
\hline MYRSINACEAE & MRS & 236 & (150) & EAE & PRT & 66 & (45) & & TYP & 8 & (3) \\
\hline MYRTACEAE & MRT & 222 & (140) & E & QII & 185 & (121a) & UL & ULM & 63 & (42) \\
\hline MUSACEAE & MUS & 45 & (29) & CEAE & RAF & 75 & (50a) & ERAE & UMB & 228 & (145) \\
\hline MYRICACEAE & MYR & 57 & (38) & ACEAE & RAN & 91 & (62) & KNOWN & UNK & 500 & $(-)$ \\
\hline IYRISTICACEAE & MYS & 99 & (68) & $\mathrm{AE}$ & RHM & 169 & (111) & FAMILY UNKNOWN & UNP & 300 & $(-)$ \\
\hline AJADACEAE & & 12 & (3B) & ORACEAE & $\mathrm{RHZ}$ & 220 & (138) & (PTERIDOPHYTE) & & & \\
\hline CTAGINACEAE & NYC & 80 & (54) & & ROS & 126 & (81) & NOT VASCULAR PLANT & UNV & 501 & $(-)$ \\
\hline EACEAE & NYM & 88 & (60) & EACEAE & RPT & 31 & (16) & URTICACEAE & URT & 65 & (44) \\
\hline HNAC & $\mathrm{OCH}$ & 182 & (119) & ICEAE & RSD & 108 & (75) & INACEAE & VAL & 273 & (181) \\
\hline OLACACEAE & OLC & 72 & (48) & RUBIACEAE & RUB & 270 & (179) & VIOLACEAE & VIO & 198 & (127) \\
\hline
\end{tabular}

